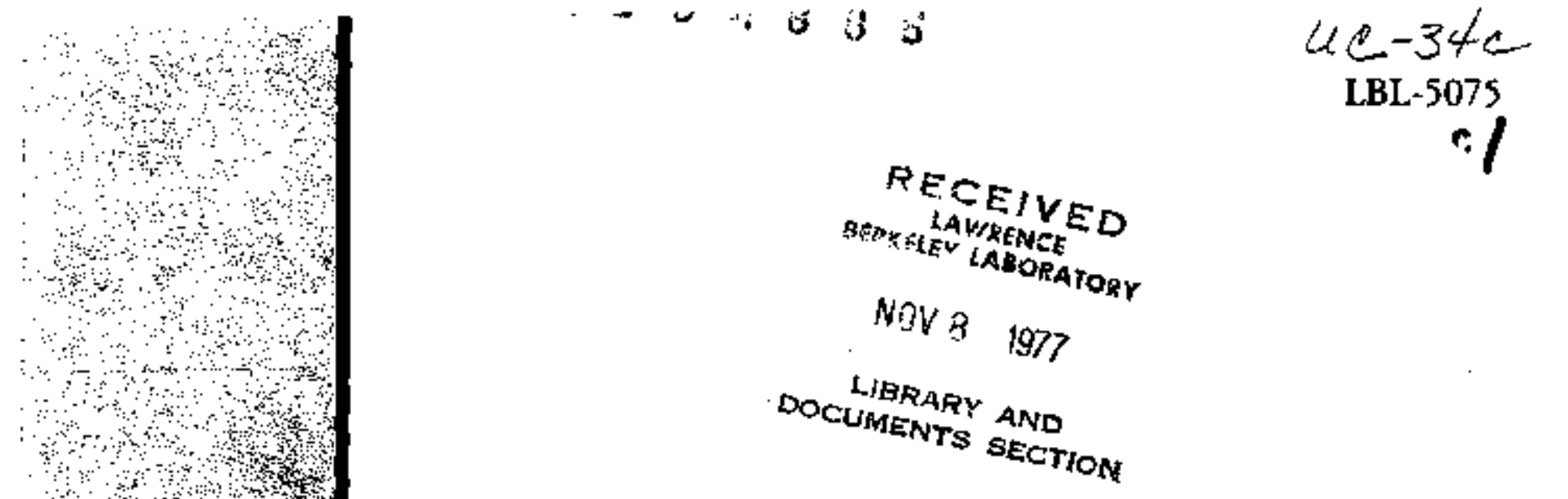

\title{
NUCLEAR SCIENCE
}

\section{Annual Report \\ 1975}

For Reference

Not to be taken from this room

Latorence Berkeley Laboratory

University of California/Berkeley

Prepared for the U.S. Energy Research and Development Administration under Contract $\$$-7405-ENG-48 


\section{LEGAL NOTICE}

This report was prepared as an actount of work spontored by the United \$tates Governiment. Naither the United \$tates now the Unitort States Energy Ressarch and Dovelopment Adininistration, nor any of their employes, nor any of their contractors, subcontractors, or ther employees, makes any warranty, express or implied, or essumes any Jegal liability or responsibility for the accuracy, oompteteness or usefuliness of any information, appartuts, product or process dilselosed, or represonts that its uso would not infing privately owned rights.

Printed in the United States of America Available from

National Technical Information Service U.S. Department of Commerce 5285 Port Royal Road

Springfield, Virginit 22161

Price: Printed Copy \$1 I.00 Microfiche \$3.00 
$00 \quad 14504387$

LBL-5075

UC. $34 \mathrm{c}$

Physics-Nuclear

TID 4500-R65

\title{
NUCLEAR SCIENCE
}

\section{Annual Report \\ 1975}

\author{
B.G. HARVEY \\ Director

\section{H.E. CONZETT \\ H.H. HECKMAN \\ W.D. MYERS \\ Editors}

來

Lawrence Berkeley Laboratory

University of California Berkeley 
ERRATUM for LBL Nuclear Science Report LBL-5075, Pg, 8 (1975). Garma and Alpha Decay from the 2.1 -msec Isomer ${ }^{213 \mathrm{~m}_{\text {Ra }}}$

D. G. Raich, H. R. Bowman, R. E. Eppley, and J. O. Rasmussen, Lawrence Berkeley Laboratory, University of California, Berkeley, California 94720

and

I. Rezanka

Heavy Ion Accelerator Laboratory, Yale University, New Haven, Connecticut 06520

An error was made in calculating the alpha-decay hindrance factors show in italics in Fig. 3 (p. 10) of the original axticle. Corrected values are given in the following table:

\begin{tabular}{|c|c|c|c|}
\hline \multirow{2}{*}{$\begin{array}{l}\text { Final state } \\
\text { in } 209 \mathrm{Rn} \\
(\mathrm{keV})\end{array}$} & \multicolumn{3}{|c|}{$\begin{array}{c}\text { Alpha Decay Hindrance Factors from } \\
\text { Initial State }\end{array}$} \\
\hline & $213 \mathrm{~m}_{\mathrm{Ra}}$ & $213 \mathrm{Ra}$ & \\
\hline 214.7 & $3.0 \times 10^{4}$ & 6.1 & $\vdots$ \\
\hline 110.1 & 2100 & 2.0 & \\
\hline 0. & 1700 & 5.7 & \\
\hline
\end{tabular}





\section{$00: 345$ 出}

GENERAL, DNTRODUCTIION

B. G. Harvey, Division Head

This is the first Annual Report of the Nuclear Science Division of Lawrence Borkeley Laboratory, The Nuclear Stience Division was formed on November 1, 1975 as part of an extensive reorganization of the Laboratory's scientific structure. Most of its components cane from the Nuclesr Chemistry Division which had ex1sted since 1946, initially under the leadership of Glenn T, Seaborg. Isadore Perlman became Dixector of the Division in 1958 and served in this capacity until Glenn Seaborg's retum in 1972 from his service as Chancellor of the thiversity of California and ther as Chairman of the Atomic Energy Comnission. Earl $k$. Hyde served as Deputy Director of the Division with Glenn Seaborg tuntil Hyde became Deputy Director of the Laboratory in 1973 .

During those 39 years, the Nuclear Genistry Division had grown into a multidisciplinary organization whose research ranged over an increasingly Iarge number of widely separated fields. The nuclear sclence conponents of the Nuclear Chemistry Division form the bulk of the Nuclear science Division; those were joined by the Heckman Group frow the Physics Division (now the Heckman/Greiner Group).

The new Division was charged with the responsibility of organizing all the Laboratory's researeh in nuclear science with the exception of heavy element chemistry and the medium energy nuclear science work in the Physics Division. It therefore has supervisory and financial responsibility for the high energy heavy ton muclear science (Bevalac) research of several groups who elected to renain in the Physics Division because the najor portion of their work is in high energy physics or mediun onergy nuclear sicence. In addition, the Nuclear Science Divisfon is responsfble for the operation of the 88-inch Dyclotron and its increasingly large Outside User program. (Operation of the SuperHILAC and Bevalac National Accelerators remains the responsibility of LBL's Accelerator Bivision).

The muciear science research is done by twelve groups in the Nuclear Science Bivision plus three Physies Division groups. Of this total of fifteen, $s 1 x$ groups work exclusively on high-energy heavy ion physics at the Bevalac, three work only at the 88 -inch Cyclotron, while four use beans fron both the Cyclotron and the SuperHILAC. The Muclear Theory Grow is involved in a wide range of activities with increasing enphasis upon high energy havy ion physics. The Table of Isotopes Group is nearing ccmplation of the Seventh Edition of the Table: the computer-prepared "manuscript" will go to the publisher early in 1977 .

The Mhiclear Science Division includes 44 professional permanent staff members, 47 post-doctoral fellows and U.S. visitors and 21 foreign visitors (these visitors come from Great Britain, Australin, Egypt, Mexico, France, Japan, Gerpany and India). The Divisdon also includes 27 graduate students, and in the period covered by this report, it awarded 3 Ph.D. degrees.

During 1976, the work of the Davision received two major reviews. The Division's Visiting Committe came to Berkeley in February and reported very favorably on our activities to the laboratory Director. As part of an ERDA review of puclear physics accelerators, the research at the 8B-inch Cyclotron received a penetrating study and again the conclusions were high favorable.

The research that is described in this first Annual Report of the Nuclear Science Division continues the high tradition of excellence that was established by the Nuclear Chenistry Division. Our closer association with our colleagues in the Physics Division is rapidly bringing about a broadening of perspectives and a grester appreciation of the essential unity of low and high energy research. Even though financial limits nay be placed upon the physical growth of the Nuciear Science Division, there are no restraints upon the intellectual developuent of its menhers. With the stimulus of our new colleagues and pushed by the new ideas that are continually exposed in our corridors and seminar rocms, the climate is indeed nost favorable for the new Division. 



\section{$00.3450: 383$}

\section{CONTENTS}

\section{i. EXPERIMENTAL RESEARCH}

\section{A. Nuclear Structure}

Long-Lived States at Hìgh Excitation Energies in ${ }^{8} \mathrm{Be}$ and ${ }^{10} \mathrm{~B}$

( $\mathrm{F}$, Ajzenherg-Selove, C, F. Mggulre, D. L. Hendrie, D. K. Scott and J. Mahoney) . . . . . 3

Energy levets of ${ }^{12} \mathrm{i}_{\mathrm{N}}$

(C. F. Maguire, D. L. Hendrie, D, K. Scott, J. Hahoney, and F. Ajzenberg-Selove) . . . . . 5

Gamma and Alpha Decay from the 2.1 rasec Iscminer $213 \pi \mathrm{Ra}$

(D. E. Raich, H. R. Bowman, R. E. Eppley, J. O. Resmussegr, and I. Rezanka) . . . . . . . . . 8

Extension of the $T_{-}=3 / 2$ Beta-Delayed Proton Precarsor Sertes to ${ }^{57} \mathrm{Zn}_{\pi}$

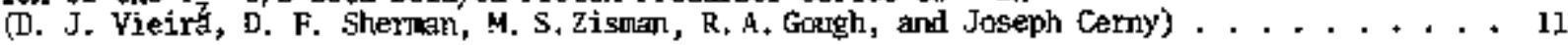

61 Ge: A Nen Beta-Delayed Protor Precursor

(D. J. Vielra, D. M. Moltz, ‥ S. Zisman, and Joseph Cerny) + . . . . . . . . . . . 13

Revised Single-Particle Energies in $\mathrm{N}=83$ Nuclei

(F. Kleinheinz, M. R. Maier, R. M. Dianord, F. S. Stephens, and R. K. Sheline) . . . . . . . 14

Triaxial Shapes in Light La Nuclei

(T. A. Butler, J. Moyer-ter-Yahri, D. Ward, H. Bartschat, P. Colombani,

R. M. Dianond, and F. S. Stephens) . . . . . . . . . . . . . . 16

High-\$pin States in 127,129 La; A Tesst of Backtending in the Even Ba and Ce Nuclei

(D. Ward, H. Bertschat, P. A. Butler, P. Colcmbani, R. M. Dianiond, and

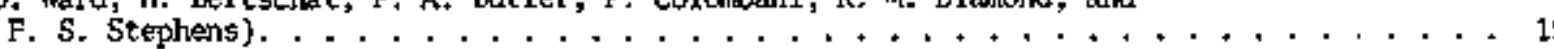

Rechxed Transition Probailities for High spin states in ${ }^{232} \mathrm{Th}^{*}$

(M. W. Guidry, P. A. Butler, P. Colombani, I. Y. Lee, D. Ward, R. M, Dianond,

F. S. Stephens, E. tichler, N. R. Johnson, and R. \$turn). . . . . . . . . 22

Coulomb Excitation of High-Spin States in $23 B_{U}$

(E. Grosse, J. de Roer, R. M. Diamund, F. S. Stephens, and P. Tjom) . . . . . . . . 24

High-Spin States of 174,176 Yb Studied in Coldamb Excitation with Kr and Xe Beamls

(D. Hard, P. Colmanani, I. Y. 1ee, P. A. Butler, R. S. Simon,

R. M. Diemingrd, and F. S. Stephens). . . . . . . . . . . . . . . .

Transitions Between High-Spin Muclear States

(M. V. Eanaschik, R. S. Simcin, F+ Colombant, F. S. Stephens, and R, M. Diamond) . . . . . . 29

Moments of Inertia in 162 rb at Very High Spins

(R. S. Simon, M. V. Eanaschik, P. Colombani, D. P. \$oroka, F. S. Stephens,

and R. H. Diantond). . . . . . . . . . . . . . . . . 31

Experimental Status of High-Spin States

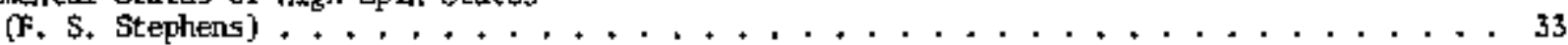

Continum Gamma kays Following (HIxnY) Reactions

(R. M. Diamond). . . . . . . . . . . . . . . . . . . . 34

40 Ar Bowbardments of Targets Aroutrol the $\mathrm{N}=126 \mathrm{Z}=\$ 2$ Closed \$hells

(A. Ghiorso, J. M, Nitschke, R, E. Leber). . . . . . . . . . . . . . . . . . 36

Search for Spontaneous Fission in Neutron Deficient Isotopes of Pu, Om, and Cf

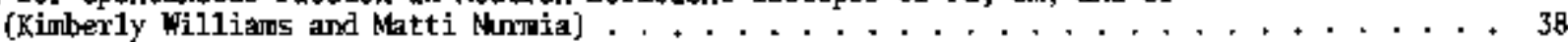

A 51-Second Spontaneous Fission Activity in the Reaction ${ }^{22} \mathrm{Ne}+{ }^{200} \mathrm{Bi}$

(L. P. Somerville, M. J. Muruia, and A. Ghiorso) . . . . . + . . + . . . . . . . . . 39

Hew Attemழt to Find the Dubna "0.1 260104 " Activity

(A. Ghiorso, P, A. Baisden. I, Binder, R. E. Leber, D. Lee, D. Morrissey,

J. M, Nitșchke, M. J. Nurmia, R. J. Otto, L. P. Sonterville, K. Thumas, K. E. Willians,

E. K. Hulet, R. W. Loughheed, J. F. Witd, J. H. Landrum, R. J. Silva, C. E, Benis,

and M. Fowler) . . . . . . . . . . . . . . . . . . . . . . . . . . 40 


\section{B. Nuclear Reactions and Scattering}

\section{Microscopic}

a. Light lons

Polarization Effects in the Final-State Intoraction Region of the p-d Brealap Reaction

(F. N. Rad, H. E. Conzett, R. Roy, and F. \$eiler) . . . . . . . . . . . . . . . . . . . . . 44

Vector Analyzing Power in the ${ }^{3} \mathrm{He}\left(\mathrm{d},{ }^{3} \mathrm{He}\right) \mathrm{np}$ Reaction

(R. Roy, F. N. Rad, H. E. Conzett, and F. Seiler) . . . . . . . . . . . . . . . 46

Polarization Phenonema in Fepw-Body Systegns

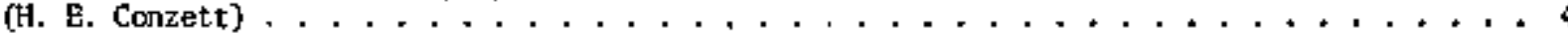

Vector Analyzing Power in Elastzc Deuteron-neuteron Scattering Between 20 and 40 MeV

(H. E. Conzett, W. Dahme, R. M. Larimer, Ch. Leentarn, and J. 5. C. MtKee) . . . . . . . . 50

The Vector Analyzing Power in the ${ }^{2} \mathrm{H}(\mathrm{d}, \mathrm{p})^{3} \mathrm{H}$ Reaction at $30 \mathrm{WeV}$

(H. E, Conzett, J. S. C. McKes, R. M, Larimer, and Ch, Leemann) . . . . . . . . . . . . . 51

Vector Analyzing Power in the ${ }^{2} \mathrm{H}(\mathrm{d}, \mathrm{p})^{3} \mathrm{H}$ Reaction between 15 and $25 \mathrm{MeV}$

(H. E. Conzett, R. M. Larimer, F. N. Rad, R. Roy, and F. Seller) + . + . . . . . . . . 52

Cross-Sections and Vector, Analyzing Powers in the ${ }^{3}$ He $(d, d)^{3}$ He and ${ }^{3}$ He $(d, p)^{4}$ He Reactions

Between 15 and 40 dey

(R. Roy, F. Seiler, H. E. Conzeptt, F, N. Rad, and R. M. Larimer). . . . . . + . + , + 53

Maximam Tensor Analyzing Power $A_{y y} \cdot 1$ in the ${ }^{3} \mathrm{He}(\mathrm{Q}, \mathrm{p})^{4} \mathrm{He}$ Reaction

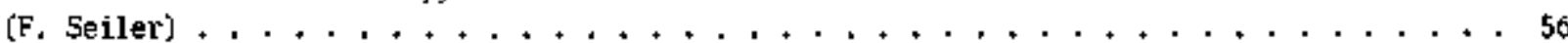

Points of Maximm Aralyzing Power in the "He(d,p) ${ }^{4} \mathrm{He}$ Reaction

(F. Seiler, R. Roy, H. E. Conzett, and F. N. Rad . . . . . + . . . . . . . . . 57

vector Aralyzing Power in d- ${ }^{4} \mathrm{He}$ Elastic 5cattering Between 15 and 45 us

(H. E. Conzett, H, Dahme, Ch. Leenarn, J. A. Macdonald, and J. P. Meuldexs) , . . + . . . 5B

Tensor Analyzing Power Ay in d - He Elastic scattering from 20 to $45 \mathrm{MkV}$

(E. J. Stephenson, H. E. Conzett, R. M. Larimer, B. T. leemann, and R. Roy) . . . . . + S9

Extreme Values $A_{y}= \pm 1$ of the Analyzing Power for Spin-1 Polarization

(F, Seiler, F. N. Rad, and H. F. Conzett) . . . . . . . . . +. . . . . . . . . . . . . . . . 61

Heasurement of the Vector Aralyzing Power Near the Value $A_{y}=1.0$ in d. ${ }^{4}$ He Elastic Stattering

(H. E. Conzett, F. Seiler, F. W. Rad, R. Roy, and R. M. Larimer'). . . . . . . . . 64

Extreme Values of the Analyzing Power in the ${ }^{6} \mathrm{Li}(\mathrm{d}, \alpha)^{4}$ the Reaction

(F. Seiler, F. M. Rad, H. E. Conzett, and R. Roy) . . . . . . . . . . . . . . . . . . 65

Points of Maximun Polarization Efficiency in ${ }^{4} \mathrm{He}(\mathrm{d}, \mathrm{d})^{4} \mathrm{He}$

(F. Seiler, H. E. Conzett, F, N, Rad, and R. Roy) , . . . . . . . . . . . . . 66

Deviations from Chargo-Symletry in tho Reaction ${ }^{4} \mathrm{He}(\mathrm{t}, \mathrm{t})^{3} \mathrm{He}$

(W. Dalyae, P. J. A. Buttle, H. E. Conzett, J. Arvieux, J. Birchall, and

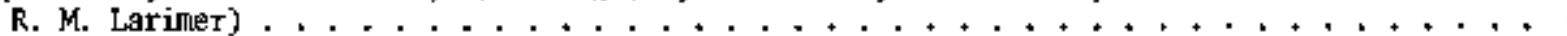

Polarization 5ymetries in Direct Reactions: ${ }^{3} \mathrm{He}(\vec{t}, \mathrm{~d})^{4} \mathrm{He}$ and ${ }^{2} \mathrm{H}(\mathrm{d}, \mathrm{p})^{3} \mathrm{H}$

(H. E. Conzett) . . . . . . . . . . . . . . . . . . . . . . . . . BB

Depolarization Parameter in $\vec{P}-10$ B EIastic Scattering and the Spin-Spin Interaction

(.T. Birchall, H. E. Conzett, F. N. Rad, S. Chintalapudi, and R. M. Larimer). . . . . . . 70

Cross Sections and Reaction Mechanisus of (p,pxn) Reactions on ${ }^{20 \mathrm{~B}_{\mathrm{Pb}}}$ in the $24-52$ MeV Range

(J. O. Raspussen, R. Donangelo, H, Kawakami, M. Koike, K. Kormra, M. Sakai,

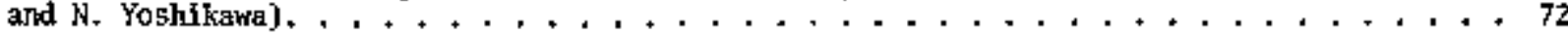

The $\left\{\alpha^{2} \mathrm{He}\right\}$ Reaction as a Spectroscopic Tool for Investigating High Spin States

(R. Jakn, G. J. Kozniak, I. P. Stahe1, and Joseph Cerny). . . . . . . . . . . . . 74 


\section{$03, j 4 j j .3,0$}

The $\left(a,{ }^{8}\right.$ e $)$ Reaction in the 1p She11

[G. 3. Woztiak, D, P, Stahe1, N, A. Je1ley, and Joseph Cemy). + . . . . . . . . . . . 7]

The $\left(a,{ }^{\mathrm{B}} \mathrm{Be}\right)$ Reaction in the 2s-1d Shell

(D. P. Stahel, 6. \$. tozniak, M. S. Zisalan, and Joseph Corny). . . . . . . . . . . . . . 79

Exotic Nass Megsurements Using the $\left({ }^{4} \mathrm{He},{ }^{8} \mathrm{He}\right)$ Reaction

(G. Kekolis, M. Zisman, D, Scott, R. Jalm, and Joseph Cerny) . . + . . + . . . . . . . 81

\section{b. Heavy lons}

Studles of ${ }^{4} \mathrm{H}$ and ${ }^{5} \mathrm{H}$ by the Two-Proton Pickng Reaction $\left({ }^{6} \mathrm{Li},{ }^{8} \mathrm{~B}\right)$

(R. B. Wolsomilier, K. H. Wilcox, D. Ashery, G. J. Wozndak, and Joseph Cerny) . . . . . . . 82

The $\left({ }^{9} \mathrm{He},{ }^{8} \mathrm{~B}\right)$ Reaction and the Unbound thuclide ${ }^{10_{\mathrm{Li}}}$

(K. H. Wilcox, R. B. Heisemiller, G. J. Wozriak, N. A. Jelley, It. Ashery,

and Joseph Cerny) . , . + . . . . . + . . + . . . . . + . + . . . . . . . 83

The ( $\left.{ }^{9} \mathrm{Be},{ }^{8} \mathrm{Be}\right)$ Reartion at $50 \mathrm{MeV}$

(D. P. Stahel, G. J. Wozmiak, B. D. Jeltena, M. S. Zisnan, R. Jahri,

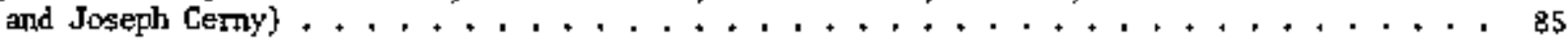

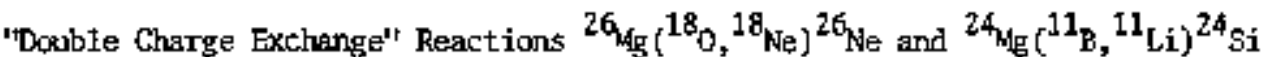

(G. Kekelis, M. Z1sman, Joseph Cerny, D. Scott, R. Jahr, F. Ajzenberg-Selove,

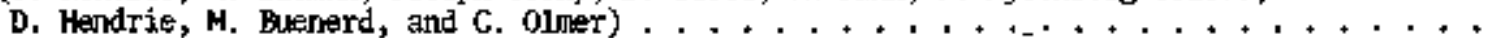

Prohection of ${ }^{11} \mathrm{Li}$ in the $\left({ }^{11_{B}},{ }^{11} \mathrm{Li}\right)$ Reaction on ${ }^{232}$ Th

(D. K. Scott, M. Buenerd, D. L. Hendrie, G. Kekelis, J. Mahoney, A. Menchaca-Rocha,

and $\mathrm{C}$. Olmer $\mathrm{K} . \ldots \ldots \ldots$

States at High Excitation in ${ }^{16} \mathrm{O}$ observed in the Reaction ${ }^{12} \mathrm{C}\left({ }^{20} \mathrm{Ne}^{16} \mathrm{O}\right){ }^{16} \mathrm{O}$

(A. Menchaça-Rocha, M. Esenerd, D. L. Hendrie, C. Olner, and D. K. Scott). , . , . . , . 91

The Contribution of Alpha Cluster Exchange to Elastic and Inelastic ${ }^{16} 0-{ }^{20}$ Ne Scattering

(R. Stock, W. F. W. Schneider, U. Jahnke, D. L. Hesdrie, J. Mahoney,

C. F. Mguire, D. K. Scott, and G. Wolschtn). . . . . . . . . . . . . . . . . . . . . . . 93

Excitation of Gient Resonances in ${ }^{197}$ Au and ${ }^{2 D B} \mathrm{pb}$ by Inelastic Scattering of Heavy Ions

(D. Ashery, M. S. Zisman, R. B. Weiseniller, A. Guterman, D. K. Scott,

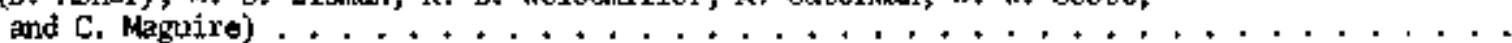

Argon Ion Reaction Studies on Tantaluk and Gold Near Coulomb Barrierr Energies

(R. C. Eggers, H. \$, Ribbe, and J, O. Rasmussen\}. . . . . . . . . . . . . . . . . 98

Seniclasșical and Queantun Mechanical Aspects of One- and Two-Mkcleon Transfer

(D. K. Scott, D. L. Hendrie, L. Krats, C. F. Maguire, J. Wahoney,

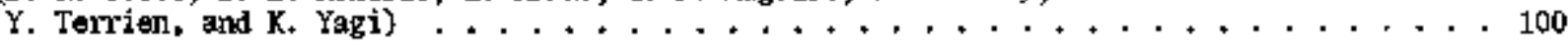

New Aspects of High Energy, Heavy-Ion Induced Transfer Reactions on Heavy Targets

(C. Olmer, M. Buenerd, C. K. Gelbke, D. L. Hendrie, J. Mahouney,

A. Menchaca-Roche, and D. K. Scott) . . . . . . . . . . . . . . . . . . . 102

Evidence for Shallow Strongly Absorbing Heavy-Ion Optical Potentials

(J. G. Cramer, R. h. DeVries, D. A. Goldberg, M. S. 21snan, and C. Maguire) +. + , , . , I07

${ }^{6} \mathrm{Li+}{ }^{28} \mathrm{Si}$ Flastic Scattering

(J. G. Cramer, R. M. DeVries, D, A. Goldberg, M. S. Zisman, and C. F. Mbgure) . + + . + . 109

${ }^{40} \mathrm{Ca}+{ }^{40} \mathrm{Ca}$ Elastic Scattering Resonamees

(C. F. Mguire, D. L. Hendrie, U. Jahnke, J. Mahonert, and D. K. Scott) . + . . . . . . 110

Elastic Scattering of ${ }^{14} \mathrm{~N}$ on ${ }^{12} \mathrm{C}$ at 81 and $100 \mathrm{HeV}$ and the Reaction ${ }^{12} \mathrm{C}{ }^{14} \mathrm{~N}^{13} \mathrm{Ng}{ }^{13} \mathrm{C}(3.09 \mathrm{MFV})$

C. Olmer, $\mathrm{A}$, Euenerd, G. Delic, D. L. Hendrio, A. Menchaca-Roche, and D. X. Scott) +..+. 111

Quasi-Elastic Collislons of $315-\mathrm{MeV} 16_{0}$ on Heavy Elements

(M. Buenerd, C. K. Gelbke, B. G. Harvey, D. L. Hendrie, J. Mahoney, A. Henchaca-Rocha,

C. Olmer, and D. K. Scott). . ..............+.+............. 115

Search for Shock Waves at Threshold Energy

(D. L. Hendrie, M. Buenerd, A. Alenchaca-Focha, C. Olmer, and D. K. Scott). . . . . . 117 


\section{Macroscopic}

Preliminary Measurement of the Light Charged Particles in Coincidence with Heavy-Ior Relaxation Thenomena

(G. J. Mathews, G. Wozniak, P. A. Russo, R, C. Jared, and L. G. Moretto). . . . . . . . . . 119

Fragment Correlation Approach to the Study of Partial Relaxation in Heavy-Ion Collisions

(4, Newman, E. Cauvin, P. Russo, R, Jared, and l. G. Moretto) . . . + . . . . . . . . . . 120

Reaction Products from the Interaction of 170 - and $252-\mathrm{Mey} 20$ Ne on a

(G. J. Pathews, B. Calvîn, R. Schnitt, R, C. Jared, and L. G, Moretto) . . . . . . . . . . . 123

Deep Inelastic Reaction of 17 S-HeV Ne with Au

(J. Moultun, G. Wozniak, B. Caunin, P. Russo, R. Jared, and L. G. Moretto). . . . . . . . + . 126

Systematics of the $40_{\mathrm{Ar}}+\mathrm{Cu}$ Reaction

(G. J. Mathews, B. Catwín, J. Moultat, R. Schmitt, R, C. Jared, and L. G. Moretto) . . . . . 127

Preliminary Heasument of Reaction Prohnets from the $40_{A r}+$ Ag Reaction at $170 \mathrm{MeV}$

(G. J. Mathews, R. Schmitt, P. Glässel, G. Wozniak, R, C. Jared, and L, G. Moretto) . . . . . 128

Measurement of Pre and Post Evaporation Masses of Deep Inelastic Collisions Fragments of $340 \mathrm{MeV} \mathrm{Ar}+\mathrm{Ag}$

(B. Cauvin, R. Jared, P. Russso, P. Gläşsel, R. Schnitt, and L. B. kkretto) . , . . . . . . . 129

Evidence of Irconplete Relaxation in the Reaction $107,109_{\mathrm{Ag}}+40 \mathrm{Ar}$ at 288 and $\$ 40 \mathrm{MeV}$

Bombarding Energies

[J. Galtn, L. G. Moretto, R. Babinet, R. Schmitt, R. Jared, and S. G. Thaupson] . . . . . . 132

Potential Anergy Effects and Diffusion in the Relaxed Components of the Reaction ${ }^{197}$ Aut $+{ }^{40} \mathrm{Ar}$

at 288 and $340 \mathrm{Mer}$ Bombarding Energies

(L. G. Moretto, J, Galin, R. Babinet, Z. Fraenkel, R. Schitt, R, Jared, and

S. G. Thoulsonj ...................................... 134

Nuclear Relaxation Pheromena and Diffusion in the Reaction $107,109 \mathrm{Ag}+{ }^{84} \mathrm{Kr}$ at $7.2 \mathrm{MeV} / \mathrm{Mhcleon}$

(R. P. Schmitt, F. Russo, R, Babinet, R. Jared, and L. G. Moretto). . . . . . . . . + . 136

Charged Fxagments Producet in the Interaction of ${ }^{159} \mathrm{~Tb}$ and ${ }^{86} \mathrm{Kr}$ at 620 MeV Bonbarding Energy

(G, J. Wozniak, P. Glässel, R. Schnitt, P. Russo, B, Carnin, R. Jared, and

l. G. Moretto) . . . . . . . . . . . . . . . . . . . . . . . . . . 138

Stuty of the Fragments Emitted in the Interaction Between ${ }^{181} \mathrm{I}_{\mathrm{T}}$ and ${ }^{86} \mathrm{Kr}$ at $620 \mathrm{MeV}$

Bcrubardirng Energy

(P. Glässel, B. Gaurin, G. Wozniak, P. Pusso, R. Schmitt, R. Jared, and

L. G. Moretto) . . . . . . . . . . . . . . . . .

Diffustve Phenamena in the Charge and Angular Distributions for the Reaction ${ }^{197} \mathrm{Au}+620 \mathrm{MeV}{ }^{86} \mathrm{Kr}$

(l. G. Moretto, B. Cauvin, P. G1asse1, R. Jared, P. Fusso, J. Svertek, and

G. Wozniak] . . . . . . . . . . . . . . . . . . . . . . . 142

Evidençe of Defomation and Incamplete Relaxation in a Nearly-Synmetric Heavy Systen:

979-MeV $\mathrm{Xe}+\mathrm{Tb}$

(P. Russo, 6. Wozniak, R. Schtuitt, R. Jared, P. Glässe1, and L. Moretto). . . . + . + . . 144

Binary Breakup (and Secondary Fission) of a Heavy Deformed System: 979-NeV Xe+Au

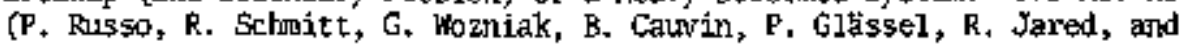

L. G, Moretto] . . . . . . . . . . . . . . . . . . . . . . . . . . . . . . . 147

Charge- and Hass-Distributions in the Reaction of $40 \mathrm{Ax}$ ions with ${ }^{23 \&}$ i

(J. V. Kratz, J. 0. Li1jenzin, A. E. Norris, and 6. T. Seaborg) . . . . . . . . . . . 1S0

Mass Yield Distributions in the Reaction of $136 x_{\mathrm{e}}$ Ions with $238 \mathrm{y}$

(R, J. Otto, M, M. Fowler, D. Lee, I. Binder and G. T. Seaborg) . . . . . . . . . . . IS3

Investigation of ${ }^{21 l_{A t}}$ Produced by $230 \mathrm{MeV} 40_{\mathrm{Ar}}$ an ${ }^{209} \mathrm{Bi}$

(D. J. Morrissey, R. J. Otto, S. Yashita, A. Ghiorso, and G. T. Seaborg). . + . . . . . . . 155

Heavy-Ion Reactions with Heavy, Non-Fissioning Targets

(I. Binder, M. M. Fowler, D. Lae, R, J. otto, and G, T. Seaborg]............. 158 


\section{$00,5450+351$}

Possible Mechanisms for $256_{\mathrm{Pn}}$ and $256_{\mathrm{Md}}$ Production in the Compound System. 264104

(L, P. Somervi1le, D. J. Morrissey, R, J. Otto, G. T, Seaborg, and A. Ghiorso) : . . . . . 160

A Radiochemical Study of the Mass Yield Distribution from the Reaction of $25.2-\mathrm{GeV}{ }^{12} \mathrm{C}$

Ions with Natural Uranium

(R. J, Otto, M. M. FowIer, I. Binder, D. Lee, and G. T, Seaborg). . . . . . . . . 165

Recoil Ranges of Heavy Products in the Quasi-Fission Reaction

(R. J. Otto, M. M. Fowler, I. Birder, D. Lee, and G. T. Seaborg). . . . . . . . . . . . 168

c. Relativistic Heavy lons

\section{Projectile Fragmentation}

Mmentum Distribations of Isotopes Produced by Fragmentation of Relativistic ${ }^{12} \mathrm{C}$ and ${ }^{16_{0}}$ Projectiles

(D. E, Greiner, P. J. Lindstron, H. H. Heckwan, Bruce Cork, and F, S. Bieser) . . +. . + 174

Isotope Production Cross Sections from the Fragneatation of ${ }^{16} 0$ and ${ }^{12} \mathrm{C}$ at Relativistic Erexgies

(P. J. Lindstrom, D. E. Greìner, H, H. Hexlman, Bruce Cork, and F, S. Biteser) . . . . . , 177

Pragunentation of ${ }^{40} \mathrm{Ar}$ at $100 \mathrm{GeV} / \mathrm{C}$

(P. J. LIndstram, D. E. Greiner, H, H. Heckman, Buce Cork, and F. S. Bieser) , . , . . 178

Fragnentation of ${ }^{4} \mathrm{He},{ }^{12} \mathrm{C},{ }^{14} \mathrm{~N}$, and ${ }^{16} \mathrm{o}$ Nuclei in Naclear Emulsion at $2,1 \mathrm{GeV} /$ hucleon

(H. H, Heckesn, D, E. Greiner, P/ J. Lindstram, and H, Stre] . . . . . . . . . . . . . 180

Contoub Dissociation of Relativistic ${ }^{12} \mathrm{C}$ and ${ }^{16} \mathrm{O}$ Khaclei

Harry H. Heckman and Peter J. Lindstrom . . . , . . . . . . . . . . . . . . . 185

Pratuction of Light Muclei in Mhacleus-Nucleus Collisions at 1.5 and $2,1 \mathrm{GeV} /$ Macleon

[J. Papp, J. Jaros, I. Schroeder, J. Staples, H. Steiner, A, hagner, and J. Wiss) . . . . . 187

Nucleus-Nucleus Total Cross Sections

(J. Jaros, O. Chamberlaîn, H. Stelner, A. Wagner, R. Fuzesy, 1. Schroeder, L. Anderson,

and G. Shapiro). . . . . . . . . . . . . . . . . . . . . . . . 189

Inclusive Plon Production in Collisions of Felativistic Protons, Deuterons, Alphas, and Carbon Ions with Nuclei

(J. Papp, J. Jaros, L. Sclroeder, J. Staples, H. Steiner, A, Wagner, and J. Wís) , . . . 190

Diffractive Scattering of Heavy Ions

(A. R, Clark, W. R, Holley, D. Keefe, L. T. Kerth, R. E. Morgado, M. Richardson,

G. Sclumpriacher, I. S, Schroder, and A. R. Zingher) . . . . . . . . . . . . . 195

Projectile Fragmentation and Asssociated Multiplicity Distributions in Relativistic Heavy

Ion Collișions

[J. Carro11, V. Perez-Mendez, E. Whipple, M. Gazzaly, J, Geaga, G, Igo,

J. McClelland, M. Nasser, A, Sagle, and F, Spinka I. . . . . . . . . . . . . . . . 194

\section{Target Fragmentation}

Nuclear Gamm Rays Following Interact fon with Relet1vistic Carbon Projectiles

(T. Shibata, H. Ejiri, R. Anholt, H. Bownan, J. G. Ioannou-Yanuou, J, O. Rasmussen,

E. Rauscher, S. Nagamiya, and K. Nakai]. . + . + . +. . . . . . . . . . . 196

Fragnents from C, Al, Ag, and U Irradiated by High Enestgy Protons

(R. G. Sextro, G. D. Westfal1, A. M. Poskanzer, and A. M.,Zebeluan) , . . . . . . . . 201

Counter Experiments in the Thin Target Area at LAMPF

(G. W. Butler, D. G. Perry, A. M. Poskanzer, J. B. Natowitz, F, Plasil, and

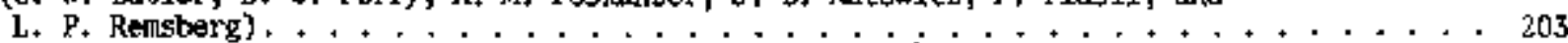

Evaporation-Like Fragnents of Uranium Irradiated by $2.1 \mathrm{GeV} / \mathrm{hucleon}{ }^{12} \mathrm{C}$ and 20 Ne Ions

(A. M. Poskanzer, A. Sandova1, R. G. Sextro, and H. H. Gutbrod) , . + + . . + + . . . . . 204

Search for Fragment Enission fran Nuclear Shock Weves

(A. M. Poskanzer, R. G. Sextro, A. M. Zebelman, H. H. Gutbrod, A. Sardoval,

and R. Stock). . . . . . . . . . . . . . . . . . . . . . . . . . . . . . . 206 
Central Collision of Relativistic Heary Ions

(H. H. Gutbrod, A, Sankorsl, J, Gosset, N. G. Myer, A. M. Poskanzer,

G. D. Westfali, and R. Stock]. . . . . . . . . . . . . . . . . . . . . . 208

Final State Interactions in Relativistic Havy-Ion Beactions

(P. J. Johansen, J. Gosset, H. H. Gutbrod, W. G. Heyer, A. M. Poskanzer,

A. Sandoval, and G, P. Kestfall) , . . . . . . . . . . . . . . . . . 209

D. Mesonic Atoms end Atomic Physics

Mesconic Atcons

(Clyde Wiegard, Gary Godfrey, and Gary lum) . . . . . . . . . . + . . + . . . . + 212

Nultiple Scattering and Energy Loss Straggiing of Heavy Ions

(B. G. Harvey and J, Mahoney) . . . . . . + . . . . . . . . . . . . . . 214

Measurement of Atamic Charge Exchange Cross Sections for High Energy Heavy Ions

(Fred Bieser, Bruce Cork, tank Crawford, Douglas Greiner, Harry Heckman,

Peter lindstron, Grart. Paisbeck, and Larce Wilson) . . . . . . . . . . . , . , 215

Atomic K-Vacancy Production with 3 GeV Carbon Ions

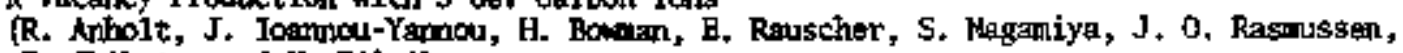

T. Shthata, and H. Ej(r). .............................

K Vacancy Production by 4.88 GeV Protons

(R. Anholt, S. Nagemiya, H, Boretan, J, Iognnou-Yandoch, E. Reuschër, and

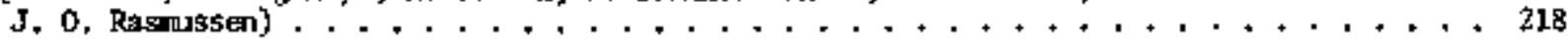

$X-R a y$ Comtinua Observed with $326-470$ NeVt Xe Ions

(R, Arholt and H, E, Meverhof). . . . . . . . . . . . . . . . . . . . . 219

A New Way of Predicting Isc Vacancy Production by Heavy Ions

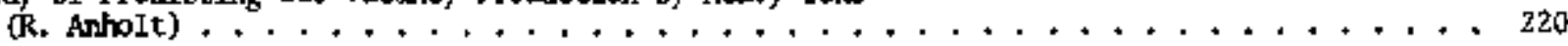

\section{THEORY}

\section{A. Nuclear Reactions}

\section{Microscopic}

Enhancesent of Forward-AngIe Cross Sections in Feavy-Ion Reactions Because of Projectile Excitation

(Norman K. Glendenting and Georg Wolschin), . . . . . . . . . . . . . . . . 225

Physical Basis for Erhaned Forward Cross Sections in $N i\left({ }^{18} \%,{ }^{16} 0\right)$ Reactions

(Nomian K. Glendenining and Georg kolschin). . . . . . . . . . . . . . . . . . .

Two-Particle Transfer Contributions to Elaștic and Inelastic Heavy Ion Scattering

(Norrman $k$. Glendenning and Georg Wolschin\}. . . . . . . . . . . . . . . . 228

Tables of Nuclear Structure Amplitudes for Two-Nucleon Transfer Imolving Light Projectiles $(A<4)$

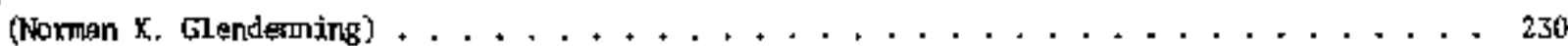

Polarization of Single-Particle States Involved in Heavy Ion Transfer Reactions

(K. Pruess, G. Delic, L. A. Charlton, and N. K. Glendeming), . . . . . . . . . . . . . 231

Polarization of Single Particle States in Hev' Ion Induced Nuclear Reactions Within

the Two Center Sheli Mode1; I. The Formalisn

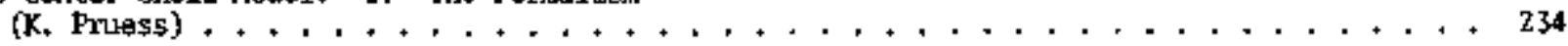

Polarization of Single Particle States in Heavy Ion Induced Bucloar Reactions Within

the Two Center Shell Model; II. Nmerical Results

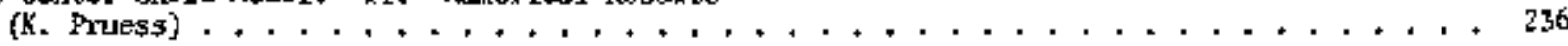

A New Approath to Artisymetrization and Rearrangenent in Nucleus-Nuxleus Collisions,

(K. Pruess and P. Lłehtner) . . . . . . . . . . . . . . . . . . 238 
Fintte Range Evaluation of (p-d, d-t) with Muntentm Space Techniques

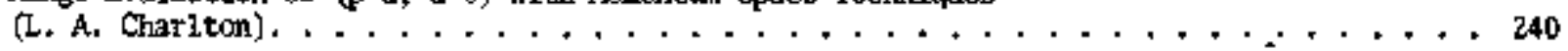

Finite-Range Effects in Haltistep $(p-d, d-n)$ Reactions

$(\mathrm{P}, \mathrm{D}$. Funz and L. A, Charlton $) \ldots \ldots \ldots \ldots \ldots \ldots$

Chebyshev Expansions and the Finite Range Distorted Mave Born Approximation for Muclear

Trtolsfer Reactions

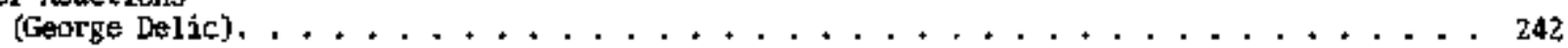

Optical Mode1 Analysis of $\mathrm{N}+\mathrm{C}$ and $\mathrm{C}+\mathrm{C}$ Elastic Scattering

(George Delic) . . . . . . . . . . . . . . . . . . . . . . . . 244

Computing the Finite Range D1storted Waves Born Approximation for ${ }^{11} \mathrm{~B}\left({ }^{16} \mathrm{O},{ }^{15} \mathrm{~N}\right){ }^{12} \mathrm{C}$

(George Delic). . . . . . . . . . . . . . . . . . . . 246

Finite Range Distorted Waves Born Approximation Calculations for ${ }^{13} \mathrm{C}\left({ }^{3} \mathrm{He},{ }^{6} \mathrm{He}\right){ }^{10} \mathrm{C}$

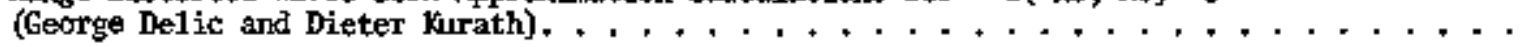

\section{Macroscopic}

A Theoretical Approach to the Problen of Partia1 Equilibration fi Heavy Ion Reactions

(L. G. Moretto and J. S. Sventek) . . . . . . . . . . . . . 251

Diffusive Phenontena Reflected in the Charge and Argular Distributions of $\mathrm{N}, \mathrm{Ne}, \mathrm{Ar}, \mathrm{Kr}$

Induced Reactions

(L. G. Moretto and J. S. Srentek) . . . . . . . . . . . . . . . 254

Diffusion Mode1 Predictions for the Reaction $620 \mathrm{MeV}{ }^{86} \mathrm{Kr}+{ }^{197} \mathrm{Au}$

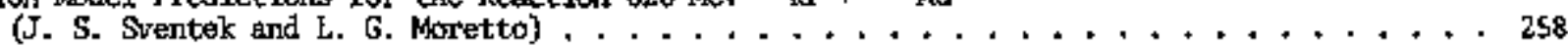

The Classical-Linit S-Matrix for Coulonb Excitation

(R. Donengelo, M. W. Qutdry, J. P. Boisson, and J. O. Rasmeussen) . . . . . . . . . 261

Theory of Coulombuthlear Interference for Heavy-Ior Excitation of Rotational States

(M. H. Guldry, H. Massnam, R. Dorangelo, and J. 0. Rasirussen). . . . . . . . . . 264

Barrier Penetration Theory in More Than One Dimension

(Peter Ring, John 0. Rassoussen, and Herbert Massmann) . . . . . . . . . . . 266

\section{Relativistio}

Nucleus-Nucieus Fragnentation Probabilities Assunigg Exact Isospin Symmettry

(Y. Karant and R. Malfliot) . . . . . . . . . . . . . . 269

Perłpheral High Energy Collision Between Complex tuclet

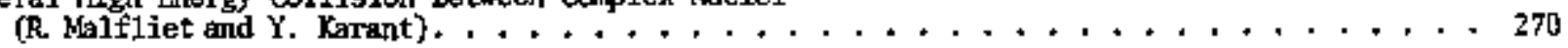

Relativistfc Heavy Ion Peripheral Reactions

(J. P. Boisson, T, Shibata, J. G. Ioarmou-Yannou and J. 0. Rasnussen) . . . . . . . 272

\section{B. Nuclear Structure}

\section{Microseople}

Evidence for Triaxlal Shapes in Pt Nuctei

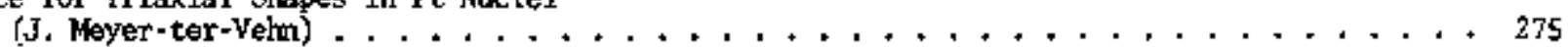

Sympetry Betwegr Particle and Hole level Systems in ${ }^{189} \mathrm{Aul}$

(J. L. Wood, R. W. Fink, and J. Meyer-ter-Vehn) . . . . . . . . . . . . 276

Crossing of Rotational Bands in the Cranking Model and High Spin States

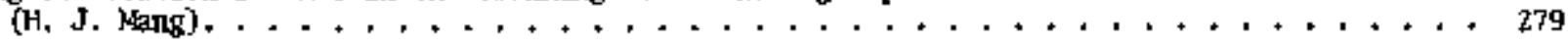

Simple Dynamical Models Including Pairing Residual Interaction

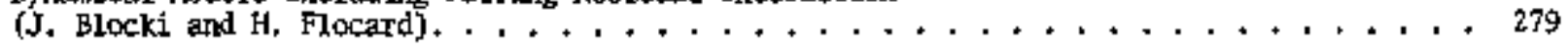

Time Dependent Hartree-Fock Calculation of Muclear Collective Motion

(J. Blocki and H. Flocard). . . . . . . . . . . . . . . . 280 
On the Solution of Constrained Hartres-Fock Bogolyubov Equations

(H. J. Mang, B. Samadi, and P, Ring $\ldots \ldots \ldots \ldots \ldots$. . . . . . . . . . .

Test of Many-Body Methods on an Exactly Soluble Model with Rackbending and Gapless

Superconhluctivity

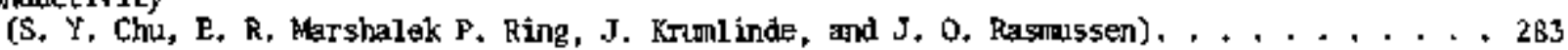

On the Shell Effect Contribution to the Mement of Inertia at Vexy High Angular Monentun

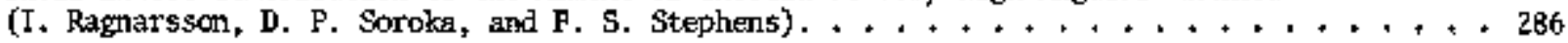

Sent-Microscopic Rotational Band Theory

(D. Sorokr, J. Rassolissen, and K. S. Tanabe). . . . . . . . . . . . 288

Generalized Particle Core Coupling

$\left(\mathrm{G}_{+} \mathrm{J}_{+}\right.$Mathews $) \ldots \ldots \ldots \ldots \ldots \ldots$

Theoretical Estimates of Magnetic Moments for Bxcites Muclei Shell and Pairing Effects

(L. G. Moretto). . . . . . . . . . . . . . . . . . . . . . 291

Nornalized Shell Mode1 Alpha Decay Theory AppIied to Unfavored Decay

[T. Fliessbach, H. J. Meng, and J, O. Rasumssen] . . . . . . . . . . . 294

Application of the Coupled Chamel Alpha Decay Theory to Odd-Wass Wuclei

(A. J. Soinski, E. A. Rauscher, J, O. Rasglussen, and D. G. Raich) . . . . . . . . . . 296

\section{Macroscopic}

Modified Thomes-Femmi Approximation for Mtountum-Dependent Interactions

(J. Randrup) . . . . . . . . . . . . . . . . . . . . . . 301

The Mxiear Surface Studied with a Mrmentum-Dependent Interaction in the Thrmas-Fermi

and Hartree Approximations

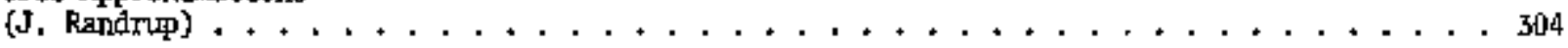

The Nuclear Interaction Potential in a Folded-Yukawa Model with Diffuse Densities

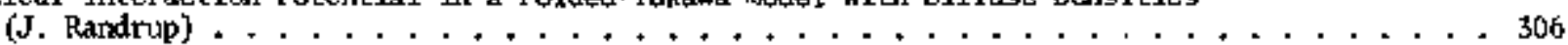

Macroscopic Aspects of Nucleus-Mucleus Interactions

(J. Blocki, \$, Randrap, w. L. Swiatecki, C. F. Tsang). . . . . . . . . . . . 310

Proximity Forces

(J. Blocki, J. Randrup, W. J. Swiatecki, and C. F. Tsang). . . . . . . . . 311

Heavy-Ion Potential Derived from a Velocity-Dependent Alucleonic Interact Ion

[. Rardrup] . . . . . . . . . . . . . . . . . . . . . . . 313

Convolution as a Geometrical Ansatz

(Willian D. Myers) . . . . . . . . . . . . . . . . . 314

The Nuclear Surface Diffuseness

(W. D. Myers and H. won Gropte $) \ldots \ldots \ldots \ldots$

The Droplet Model of the Giant Dipole Resonance

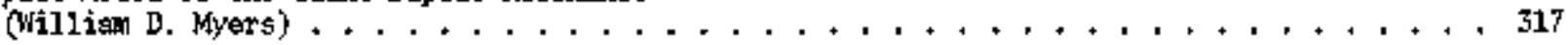

Proxinity Fission

(J. S. Sventek, L. 6. Moreptto, and W. J. Swiatecki). . . . . . . . . . . . . 320

C. Damping, Fission, General Relativity

Schrödinger Equation for Scattering with Energy Loss

(R. Lipperheide) . . . . . . . . . . . . . . . . . . . . $\ldots 22$

One Body Viscosity; Canparison Between Quantom Mechanical and Classical Descriptions

(J. Btocki, Y. Borteh, and J, Randrup).

One-Body Dissipation and a New Dynamies

(J. Blocki, J. Randrup, M. Robel, and W. J. Swiatecki) . . . . . . . . . . 322 


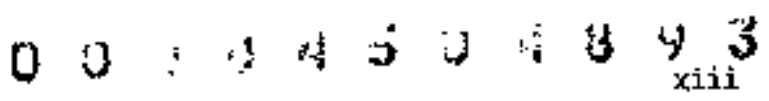

A Microscopic Method for Calculating the Intrinsic Excitation Occurring in Muclear Fission

(T. Boneh, J. P. Btocki, and W. D. Myers\}. . . . . . . . . . . . . . . . . . . . 32S

Microscopic and Macroscopic Calaulations of One-Body Dampling in Fission

(Y. Boneh, J, F. Blocki, and W. D. Btyers\}. . . . . . . . . . . . . . . . . . 327

Further Studies on \$pontaneous-Fission Half-lives for Even Huclei with $z ; 92$

(J. Randrup, S. E. Larsson, P. Moller, S. G. Nilsson, K. Potorski, and

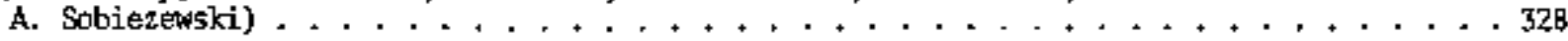

Interstellar Deuterium Abundance and the Closed Versus Open Universe

(E1izabeth A. Rauscher). .............................. 331

Ii). APPARATUS

\section{A. Acclerator Operations and Development}

88-Irch Cyclotron Operation, Development and Studies

(J. Bowen, D. J. Clark, P. E. Frazier, L. Glesgow, R. A. Golugh, D. L. Hendrie,

W. R. Hoiley, A. Jain, and D, Hortis) . . . . . . . . . . . . . . . . + . 337

150 Mev Proton Medical Cyclotrun Design Stukty

(R. J. Burleigh, D. J. Clark, and W. S. Floof) . . + . . . . + . + , , , . 339

Heavy-Ion Booster Cyclotron Design Studies at Berkeley

(R. J, Burleigh, D. J. Clark, and L. R. Glasgow) . . . . . . . . . . . . . . . . . . 341

A Fast-Closing Vacum Valve for the $88-$ Inch Cyclotron

(R. A. Gough, R. Lam, D. Morris, and C. bartine2). . . . . . . . . . + . . . . . 343

Heavy Ion Source Development

(Davfd J. Clark) . . . . . . . . . . . . . . . . . . . . . . . . . 345

SuperHLLAC - 1975 . . . . . . . . . . . . . . . . . . . . . . . . . 347

B. Nuclear Inștrumęntation

A Large Solid Angle "be Identifier

(G. J. Worniak, N. A. Jelley, D. P. Stahel, and Joseph Cemy). . . . . . . . . . . . . . . 349

Completion of a Recoil Atan Mass Analyzer (RAkA)

(D. M. Moltz, R. A. Gough, M. S. Zisman, D. J. Vieira, and J. Cerny) . . . . . . . . . , 351

A Proportional Camter Tine Zero System

(G. Keke1is, G. Gabor, D. K. Scott, and C. K. Gelbke) . . . . . . . . . . . . . . 352

Response of Pilot U Scintillator to Heavy Ions

(M. Buenerd, D. L. Hendrie, U. Jabnke, J. Mahoney, A. Menchaca-Rocha, C. Olner,

and D. K. Scott..................................... 353

Spectrometer Focal Plane Detector System

(B, G, Harvey, J. Mahoney, and R. F. Burton) . . . . . . . . . . . . . . . . 3\$4

Identification of Aton Nhmbers IJ to $Z=60$ in Fragments fron Heavy-Ion Reactions

(R. Jared, F. GlasseI, R. \$chmitt, P. Russo, and L. G. Moretto\}. . . . . . . . . . . . . . . 356

Energy Calibrations of 5olid State Counters for Heavy Ion Experiments

(P. Russo, R. Jared, R. Babinet, and L. G. Moretto). . . . . . . . . . . . . . . . 358

Wilczynski Plots: An Effective Way to Display Singles Date

(J. S. Sventek and L. G. Noretto) , . . . . . . . . . . . . . . . . . . 361

A High Intenșity Heary-Ion Recoil Target Şystem

(Joachim H. Nitschke). . . . . . . . . . . . . . . . . . . . . . . 364

A Heavy-Ion Spectrometer for the SuperHILAC

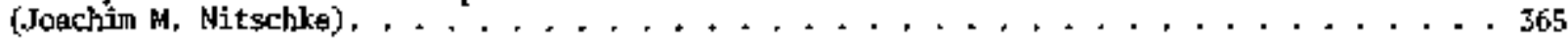


A. Versatile Sputtering System

(\$. W, Eckroad, R. E. Leber, A+ (Ghiorso). . + + + + + + + + + + . + . . + . . . 366

A Timlng Detector Utilizing Isochromous Transport of Secondary Blectrons

(A. M. Zebelman, W. G. Meyer, K. Halbach, A. M. Poskanzer, R. G. Sextro,

G. Gabor, and D. A. Landis] . . . . . . . . . . . . . . . . . . . , . . 367

An Easy-to-Build wire Chamber with Good Position and Pulse-Height Resolution

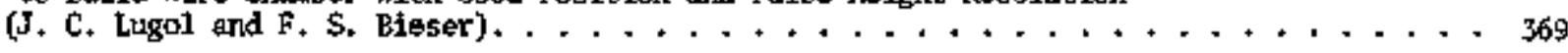

Drift Chambers for Heavy-Ion Cosnic Ray Experinent

(Fred Bieser and Douglas Greiner) +.............................. 370

An Entelligent MuIttehamel PDM Transmitter

[A. Ross Harrower, Cluick MeParland, and R. C. Z1nk] . . . . . . . . . . . . . . . . . 371

High Intersity Flux Measurerents Using Current to Frequency Converters

(A. Ross Hartower). . . . . . . . . . . . . . . . . . . + . . + . . . . 372

A Monte CarIo Caloulation of \&-Ray Effects on Drift Chamber Resolution

(Errostine Beleal, Douglas E, Grolner, and Fred Bieser] . . . . . . . . . . . . . . . 372

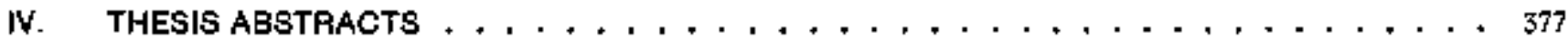

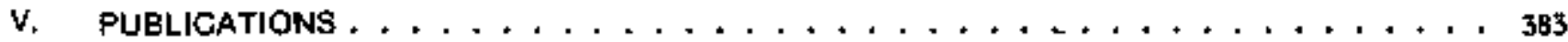

VI. AUTHOR INDEX ..........................................401 
$00 . \therefore 4$ JU,

\section{EXPERIMENTAL RESEARCH}





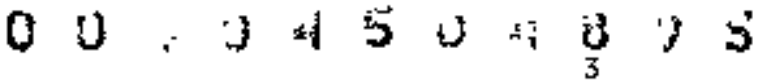

\section{A. NUCLEAR STRUCTURE}

\author{
LONG-LIVED STATES AT HIGH EXCITATION ENERGIES IN ${ }^{8} \mathrm{Be}$ AND ${ }^{10} \mathrm{~B}^{*}$
}

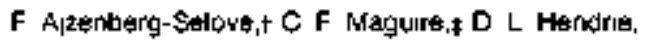

D K Scoll, and J Mahoney

This experiment was undertaken to study possibly narrow states at high excitation energies in ${ }_{\mathrm{Be}}$ and $10_{\mathrm{B}}$. Above $\mathrm{E}_{\mathrm{x}}-19.2 \mathrm{keV}$ in $\mathrm{B}_{\mathrm{Be}}$ all $\mathrm{krown}{ }^{\mathrm{I}}$ states have intrinsic widths $>0.7 \mathrm{M}$ V with the exception of the T - 2 state at $27.492 \mathrm{HeV}$, which has a $\mathbf{r} \approx 10 \mathrm{keV}$. One knows from the level structure of 8 Li that a share state [ $F<40 \mathrm{keV}]$ exists at $E_{x}=6.53 \pm 0.02 \mathrm{MeV}{ }^{3}$ Atterpts in a study of $6 \mathrm{Li}$ $(t, p){ }^{8}$ is to deteroine its $J^{\pi}$ pave been unsuccessfuI, 4 but Barker has predicted ${ }^{5}$ a $4^{+}$state whose paraneters [ including $y^{2} \sim 0$ ] are consistent with the experimental obseivations. Thus, ay isobaric analog $T=1$ state wrold bo expected in ${ }^{3} \mathrm{Be}$ at $\mathrm{E}_{\mathrm{X}}-23$ MeV. Such a $T=1$ state would be expected to be quite pure, since the nearest $\mathrm{T} \times 04^{+}$states, observed in the phase shift amalysist of ${ }^{4} \mathrm{He}(a, a)$, are Iocated at $E_{x}=19,9$ and $25,5 \mathrm{MeV}$. The only other reported sharp state in ${ }^{8} \mathrm{Li}$ is at an $\mathrm{E}_{\mathrm{X}}=7.1 \pm 0.1$ MeV [ I $250 \mathrm{keV}]$. Thus, we wished to study excited states with $E_{x}>22 \mathrm{MeV}$ in order to try to observe the expected two $T=1$ states.

The situation in the $A=10$ triad is sinflar: the level structure of $10 \mathrm{~B}$ is quite well known 1 below $E_{x}=9 \mathrm{MeV}$. At higher energies only the states 8 at $E_{x}=11,53 \pm 0,04$ and $12,57 \pm 0,03 \mathrm{MeV}$ are definitely known to be quite shaxp $(\Gamma=270 \pm 50$ and $90 \pm 30 \mathrm{keV}$. respectively), although there is indication of a rumber of relatively sharp resonances in ${ }^{9} \mathrm{~B}\left(\mathrm{p}, \mathrm{Y}_{0}\right)$ and $(p, Y 1)[\Gamma$ os 0.15 to $0.5 \mathrm{MeV}]$, corresponding to $10.8<\mathrm{E}_{x}<19.7 \mathrm{MeV}$ The results from $9 \mathrm{Be}\{\mathrm{p}, \mathrm{p}$,$\} ,$ $10 \mathrm{~B}_{\mathrm{B}}\left(e, e^{\dagger}\right), 11$ and $\mathrm{1}_{\mathrm{B}}(\mathrm{p}, \mathrm{d}) \mathrm{1} 2$ with respect to $\mathrm{ID}_{\mathrm{B}}$ states with $\mathrm{E}_{\mathrm{x}}>\mathrm{Il}$ keV are not conclusive. In the analog region in $10 \mathrm{Be}$, Anderson et a1, 13 have recently reported states fram a study of the

$9_{\text {Be }}(d, p)$ libe reaction at $B_{x}=9.27,9.4,10.57 \pm 0.03$, and $11.76 \pm 0.02 \mathrm{MeV}$. The levels lope* $(9.3,11.8)$ are also reported in a study 14 of $9 \mathrm{Be}\left(\mathrm{p}, \pi^{+}\right) 10 \mathrm{Be}$. The analog of these four states would be expectied to lie at $E_{x} \approx 11,0,11,1,12+3$, and $13.5 \mathrm{MeV}$ in $10 \mathrm{~B}$.

The ${ }^{9} \mathrm{Be}\left({ }^{3} \mathrm{He}, \mathrm{c}\right){ }^{8} \mathrm{Be}$ and ${ }^{1} 1_{\mathrm{B}}\left({ }^{3} \mathrm{He}, \mathrm{c}\right)^{10_{\mathrm{B}}}$ reactions have been stadied at $\mathrm{E}\left({ }^{3} \mathrm{He}\right)=49.3 \mathrm{keV}$ using the $88-$ inch cyclotron and the Berkeley OSD magnetic spectrometer ${ }_{+} 15$ The Identification depended on a measure ment of Bo using a resistive-wire position-sensitive proportional counter and a double $(\mathrm{dE} / \mathrm{dx}$ ) лoegsirement in the transmission proportional counters, combined with a measurement of the time of flight (a $\mathrm{W} / \mathrm{Q}$ ) betweon a scintillator foil at the entrance of the spectrometer and a plastic scintillator behind the focal plane detectors. The $E_{\alpha}$ vs chamel calibration was obtained by using a carbon target and moving the groups, from very well known bound states of ${ }^{11} \mathrm{C}$ (from ${ }^{12} \mathrm{C}\left({ }^{3} \mathrm{He}, \alpha\right.$ ) runs) were made at $\theta \mathrm{lab}=14,5^{\circ}, 30^{\circ}$, and $48^{\circ}$ and at magnetic fields with would match those of the $9_{\mathrm{Be}}$ and $1_{\mathrm{B}}$ runs, so that contaninant subtraction would be possible.
Al1 three targets were oriented at an angle of $30^{\circ}$ with the incident ${ }^{\mathrm{H}} \mathrm{e}$ beam. The $\mathrm{g}_{\mathrm{Be}}$ and ${ }^{1} \mathrm{H}_{\mathrm{B}}$ (98: enriched) targets hart a thicksess of 400 and $500 \mu g 0^{2}$, respectively, as measured by an $\alpha$ geuge (土1St). The thickness of the carbon target was not measured directly but from the shifting of chame 1 positions of identical groups when the carbon target and the Be target were used it appears that its thickness was aproximgtety $800 \mathrm{\mu g} / \mathrm{cm}^{2}$. The effective thickness of the $9 \mathrm{Be}$ and $\mathrm{I}_{\mathrm{B}}$ targets was 60 and $80 \mathrm{keV}$, respectively. The carbon contamination of the ${ }^{9} \mathrm{Be}$ target was $\sim 0.58$, while that of the ${ }^{11} \mathrm{~B}$ target was smail. Groups from $160(3 \mathrm{He}, \alpha) 150$ were observed in the $1 \overline{1}_{\mathbf{B}}\left(\hat{H}_{\mathrm{He}}, \mathrm{C}\right)$ spectra.

Spectra were obtained at $\theta 1 a b=14+5^{\circ}$ and $30^{\circ}$ covering $16<\mathrm{E}_{\mathrm{x}}<29 \mathrm{MeV}$ in $8 \mathrm{Be}$. Figure 1 shows a composite display of the two rums taken at $30^{\circ}$. The well-known states ${ }^{8} \mathrm{Be}(16.91,17,64,18.15$, and 19.22] are clearly seen: their [ J"; T ] are $\left[2^{+} ; 0+1\right]$, $\left[1^{+} ; 1\right],\left[1^{+} ; 0\right]$, and $\left[3^{+} ;\{1\}\right]$ respective $1 y$. The other $2^{+}$, mixed isospin state $\mathrm{Be}_{\mathrm{Be}}(16.63)$ is very

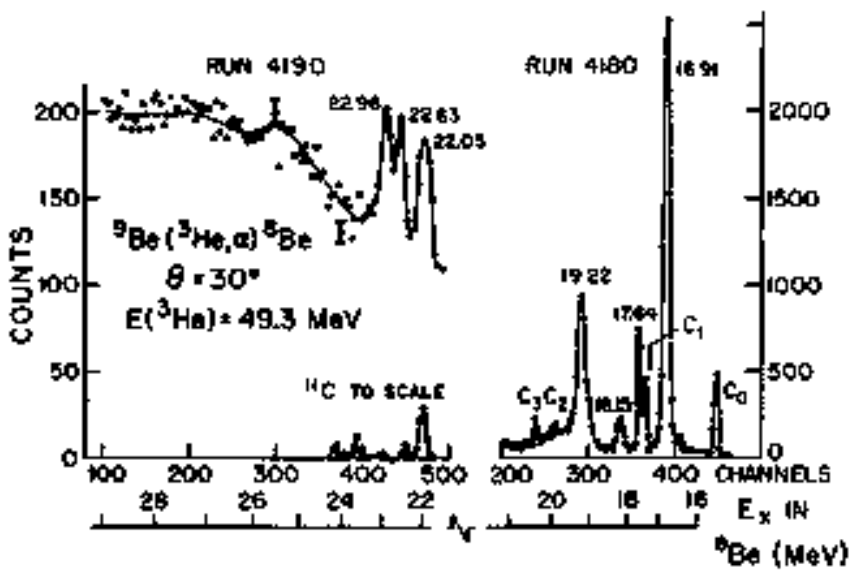

Fig. 1. Spectrum of the a particles from the $9_{\mathrm{Be}}$ $\left({ }^{3} \mathrm{He}, \alpha\right)^{8} \mathrm{Be}$ reaction at $\theta_{1 \mathrm{ab}}=30^{\circ}, \mathrm{E}\left[{ }^{3} \mathrm{He}\right)=49.3 \mathrm{MeV}$. Rum 4180 was made with a spectrograph frequency of $22.606 \mathrm{MHz}$ and a charge of $2.12 \mathrm{mC}$ : the right-hand ordinate shows the average number of coumts in a four-channel bin; the abscissa covers $I 6<E_{x}<21 \mathrm{keV}$ in ${ }^{8} \mathrm{Be}$. The groups labeled $\mathrm{C}_{\mathrm{O}}+\mathrm{C}_{3}$ corresponds to $11 c^{*}(0,2,00,4.32$, and 4.80$)$, Rum 4190 was carried out at a frequency of $21+000 \mathrm{Mez}$ and a charge of $1.71 \pi C$ : the left-hand ordinate atso represents the average nuber of counts in a four-channel bin; the abscissa covers $21.8<\mathrm{E}_{\alpha}<29 \mathrm{keV}$. The curve 1a. beled "llc" is plotted to scale frod a rum made thder analogous conditions with a carbon target, corrected for the $0.5 \%$ carbon contamingtion of the ${ }^{\mathrm{Be}}$ target: see text for details. The curve covers the region $6.2<\mathrm{E}_{\mathrm{X}}<8.8 \mathrm{kHV}$ in ${ }^{1} \mathbf{l}_{\mathrm{C}}$.

(XBL 766-8322) 
weakly populated both at this angle and at $14.5^{\circ}$, as is $8 \mathrm{Be}^{*}(18,91)$. The measured excitation energy of the $3^{+}$state is $19.22 \pm 0.03 \mathrm{MeV}$, suggesting little contribution from the known $3^{+}$state $8 \mathrm{Be}^{35}(19,06)$.

The spectra cleariy show the carbon contamination and the groups leading to $\operatorname{ll}^{*}(0,2,00,4.32$, and $4.80 \mathrm{j}$ : they provide both an energy reference and a calibration for the intensity of contaninant groups in the region of the higher excited states of $8 \mathrm{Be}$. The intrinsic width ${ }^{\prime}$ of $\delta_{\mathrm{Be}^{*}}(17,64)$ is known to be 10. $7 \pm 0.5 \mathrm{keV}$ : its measured full width at half maximu (FitA) was used first to determine the instrumental width [ $\approx 50 \mathrm{keV}]$; to check the widths of $\mathrm{Be}^{\star}(16.91,78.15$, and 19.22$)$; and then to determine the widths of the higher excited states of ${ }^{8}$ Be.

The three new states in ${ }^{8} \mathrm{Be}$ are shown in Fig.1 and thefr paraneters are displayed in Table $1_{+}$The errors in the $E_{X}$ and $\Gamma_{c, \mathbb{R}}$, are arbitrarily increased and reflect ucertainties in the energy calibration and in the subtraction of the many particle background. It should be roted that the carbon contatiinant peaks shown in this $\mathrm{E}_{\mathrm{x}}$ regiom on Fig. 1 of gourse shift kinematically more slowly than do the Be peaks: the $14.5^{\circ}$ results make ungmbiguous the assigrment of the three peaks to ${ }^{8} \mathrm{ge}$, There is some indication of a broad state at $E_{x} \sim 25 \mathrm{MeV}$ : such a state has been seen ${ }^{1}$ earlier. Hithin statistics we do not observe any influence on the two spectra of the $T=2$ state at $27.5 \mathrm{MeV}$. We feel that the present paper presents the first evidence for sharp states of ${ }^{8} \mathrm{Be}$ at these high $\mathrm{E}_{\mathrm{K}}$.

One and possibly tho of the states $\mathrm{Be}^{*}$ (22.05, 22.63 , and 22.98 ) should be the analogs of $8 \mathrm{Li}^{4}$ $(6.53,7.1)$. It is tenpting, on the basis of the smaller width, to assign $(22.63)$ to be the ant alog of $\mathrm{Li}^{\star}$ (6.53), and on the basis of the resuitant energy difference to assume that $8 \mathrm{Be}^{*}$ (23.0) and $8_{\mathrm{Li}^{*}}(7 . \mathrm{i})$ are analogs. The analog of $\mathrm{Be}_{\mathrm{Be}}(22.05)$ is not reported in $\mathrm{LLi}_{\mathrm{i}}$. This is clearly a guess. since the Jग of none of these states is established, and calculations of energy shifts are precluded. However, the ovidence for such long-lived states at very high excitation energies in 8 be suggests the neod for additional experínental and theoretical work.

Spectra inwolving states of $10 \mathrm{~B}$ were obtatjed at $\theta_{\mathrm{lab}}=14.5^{\circ}$ and $30^{\circ}$ for $6<\mathrm{Ex}_{\mathrm{X}}<30 \mathrm{kkV}$, at $20^{\circ}$, $25^{\circ}$, and $36^{\circ}$ for $6<E_{x}<15 \mathrm{NeV}$, and at $\theta=48^{\circ}$ and $55^{\circ}$ for $7<\mathrm{E}_{x}<14 \mathrm{HeV}$. Figure 2 shows a composite display of two of the runs taken at $30^{\circ}$. The thind run at $30^{\circ}$, which covered $24<E_{x}<30 \mathrm{HeV}$, and the run at 14.5 which covered 20<Ex MeV, did not show any sharp strusture ( $\Gamma<0.5 \mathrm{k} k \mathrm{~V})$ above the multiparticle breaku background.

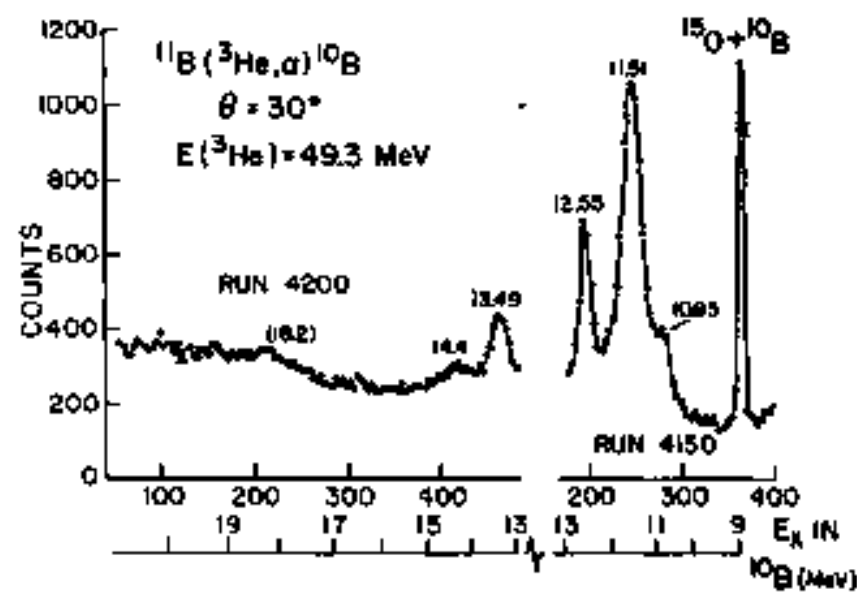

Fíf. 2. Spectrum of the a particles from the $11_{B}$ $(\text { He, } a)^{10} \mathrm{~B}$ reaction at $\mathrm{\theta}_{1 \mathrm{gb}}=30^{\circ}, \mathrm{E}\left({ }^{3} \mathrm{He}\right)=49,3 \mathrm{KeV}$. The ordinate shows the average minher of counts in a four-channel bin: statistical errors are shown for two representative points. Rum 4150 was made with a spectrogr,tph frequency of $22.606 \mathrm{MHz}$ and a charge of 1,42,mC: the abscissa covers $9<\mathrm{E}_{\mathrm{x}}<13 \mathrm{He}$ in $10_{\mathrm{B}}$. Eun 4200 was carried out at a frequency of 21. $000 \mathrm{MHz}$ and a charge of $1.44 \mathrm{mC}$ : the abscissa covers $13<\mathrm{E}_{\mathrm{x}}<20 \% \mathrm{deV}$. The peak labeled $150+10_{\mathrm{B}}$ $150+10_{\mathrm{B}}$ is a composite of $15 \mathrm{0}^{*}(6,18)$ (fru $\left.160\left({ }^{3} \mathrm{He}, \alpha\right)^{1} \mathrm{~S}_{0}\right)$ and $\mathrm{i}^{\mathrm{f}}$ one or more of the $10_{\mathrm{B}}$ states at $\mathrm{E}_{\mathrm{X}}=8.89 \mathrm{MeV}$. $1 \mathrm{O}^{*}$ (6.18) is resolved at other angles.

(XRL 766-8323)

The widths calculated for the $10_{\mathrm{B}}$ states were determined in the manner discussed previously using the experimental width of $10 \mathrm{~B}^{*}(6.56)$ whose intrinsic width is known to be $25.1 \mathrm{keV}$.

The stumary of urr results is shown in Table 1. (1) We confirm the existence of $10 \mathrm{~B}^{*}(10.85$, 11.51 , and 12.55$)$, although our width for $10 \mathrm{~B}^{\mathrm{h}}$ (11.51) is puzzlingly larger than previously reported. (2) he repart a new state $10 \mathrm{~B}^{\mathrm{*}}(13.49)$ and possibly two other wider states. Our work suggests that the $9 \mathrm{Ke}(p, y)$ work ${ }^{9}$ should be malyzed further. Comparison with the analog region in 10 Be shows four relatively sharp states in both nuclei: ${ }^{10} \mathrm{Be}^{*}[9.27$. $9.4,10.57$, and 11.76$)^{13}$ and $1 \mathrm{OB}^{ \pm}(10.85,11.51$, 12.55, and 13.49) (as sumtarized in Table 1). Since information on the $j^{\pi}$ of these states is not knowl with certainty at this time $\left[4^{*}\right)$ and $\left(2^{+}\right)$have bees suggested 1 for $10 \mathrm{Be}^{*}(9.27,9.4)$ and $\left[0^{*}, 1^{*}, 2^{+}\right)$would be assigned to $10_{\mathrm{B}}(12.55,13.49)$ if these are the states seen in the ${ }^{9} \mathrm{Be}(\mathrm{p}, \mathrm{\gamma})$ workl, definite analog assignmernts cannot be made.

Table 1, Smmary of the results.

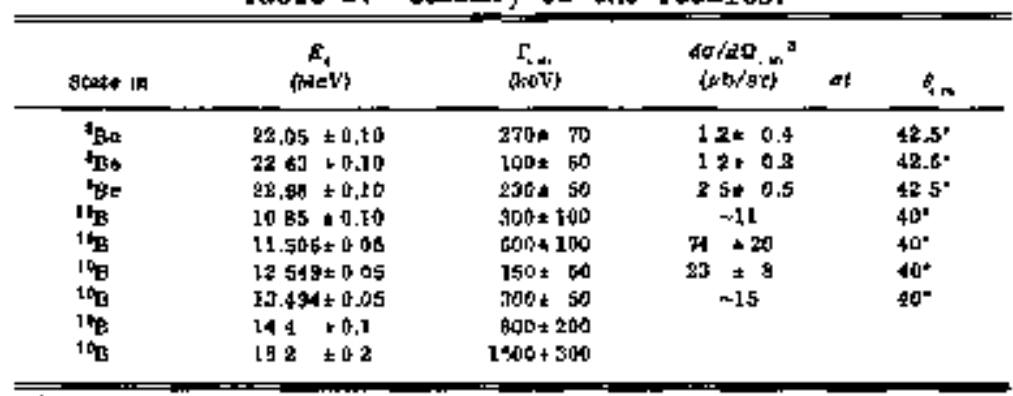

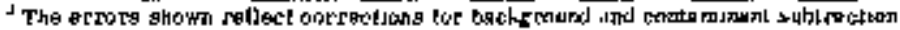




\section{$00: 2 ; \quad 5 \quad 6+\frac{1}{3} ; 5$}

\section{Footnotes and References}

*Condensed from Phys. Rev, C 13, 46 (1976).

tPormanent address: University of Pemsylvania, Philadelphia, Penri.

${ }^{\ddagger}$ Present address: Departnent of Physics, Vanderbilt University, Nashville, Tenuessee.

1. F, Ajzenberg-Selove and T, Lauritsent, Muç1. Phys. A227, 1 (1974).

2. R. G. H. Robertson, H, 5. Chien, and D. R. Goosman, Ptys. Rev. Lett. 34, 33 (1975).

3. J. H. Watson, F. Ajzenberg-Selove, and R. M1ddleton, Phys. Lett. 18, 302 (1965).

4. F. AJzerberg-Selove, E. R. F1ynn, J. D. Garrett, and 0. Hansen, Eull. Amer. Soc. 20, 596 (1975).

5. F, C. Barker, Nucl. Phys, 83, 418 (1966).

6. A. D. Bacher, F. G. Remini, H. E. Conzett, R. de Swinlarski, H. Meiner, and J. Ernst, Phys. Rev. Lett. 29, 1331 (1972).

7. D. G. Foster (pxivate communcation).
8. J, D, Purvis, $F$. Ajzenberg-Selove, and $L_{+} M$. Polsky, Phys. Rev, 162, 1005 (1967).

9. A. Fisher, Ph.D. thesis, Stanford University, 1970 (unpublished) (see Table I0.I1 of Ref. 1 for a sumbary of the reșults).

10. H. J. Votava, T. B. Clegg, E. J. Lutrig, and W. J. Thorepson, Nuc1. Phys. A204, 529 (1973); I. W. J. Wild ark G. B. Crinean, Aust. J. Phys. 27, $663(1974)$.

11. P, Kossany1-Demay and G, J, Van Praet, Murl. Phys, 81, 529 (1966).

12. D. Bachelier, M. Bermas, I. Brissadu, C. Detraz, and P. Radvanyi, Wucl. Phys. Al26, 60 (1969).

13. R. E. Anderson, J. J. Kraushaar, M. E. Rickey, and $\mathrm{W}, \mathrm{R}, \mathrm{2im}$ menman, Nucl, Phys. A236, 77 (1974).

14. 5. Dahlgren, P. Grafströ̀n, B. Hóistad, and A. Asberg, thucl. Phys. A204, 53 (1973).

15. B. G. Harvey, J. Mahoney, F. G. Puhlhofer, F. S. Goulding, D. A. Latidis, J. -C. Faivre, D. G. Kovar, M. S. Zisman, J, R, Meriwether, S. W. Cooper, and D. L. Hendrie, thucl. Instrum. Methods 104, $21(1972)$.

\section{ENERGY LEVELS OF ${ }^{1 \hat{2}} \mathbf{N}^{*}$ \\ C F Magullie.t D L Hendira, D K Scoll \\ $J$ Mahoney and $F$ Azentuerg-Selove\#}

\begin{abstract}
Comparison of the known 1,2 states in ${ }^{12} \mathrm{~B}$ with those in the nirror mucleus, $12 \mathrm{~N}$, jomediately reveals the incomplete nature of our wodorstanding of the structure of $12 \mathrm{~N}$ (see Table 1). The reasons for this are easy to understand. inile a wide vartety of reactions with convenient characteristics (i.e., high + (o-values, uutgoing charged particles) pop-

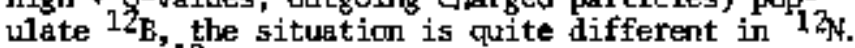
States in $12 \mathrm{~N}$ can, for all practical pirposes, be populated only by the reactions $10 \mathrm{~B}(3 \mathrm{He}, \mathrm{n})[1,569]$, ${ }^{2}{ }_{C}(p, n)(-18.126), 12 \mathrm{C}(3 \mathrm{He}, \mathrm{t})[-17.362)$, and $14 \mathrm{~N}(\mathrm{p}, \mathrm{t})$ [-22.141] [ 0 in trackets], 3 and by comparable reactions involving heavy ions. The difficalties of accurate neasurements of neutron energies and of obtaining the highly energetic, monoenergetic beaus of particles needed to overcome the negative Qvalues have only recently begun to be solved. With the exception of the $12 \mathrm{C}\left(3_{\mathrm{H}} \mathrm{e}, t\right)$ study of bali and $\operatorname{Cern}^{4}$ at $\mathrm{E}(\mathrm{H} \mathrm{He})=49.8 \mathrm{Mev}$ who reported on states with $\mathrm{E}_{\mathrm{K}}<5.3 \mathrm{MeV}$, jost of the evidence on the levels of $12 \mathrm{~N}$ derives from studies of $10_{\mathrm{B}}\left({ }^{3} \mathrm{He}, \mathrm{n}\right)$, particularly those by Fuchs et al. 5 at $E\left({ }^{3} \mathrm{He}\right)=12.5-13$ $\mathrm{MeV}$ and by Zafiracos et al.6 at $\mathrm{E}(\mathrm{He})=4.0$ and 5.8 MeV, (See Ref, 1 for a more complete listing of the experiments relating to I $2_{N}$.)
\end{abstract}

The ${ }^{12} \mathrm{C}\left(3_{\mathrm{He}}, \mathrm{t}\right)^{12_{\mathrm{N}}}$ has been studied at $\mathrm{E}\left({ }^{3} \mathrm{He}\right)=$
49.3 $\mathrm{MaV}$ using the 88-inch cyclotron and the Berkeley QSD magnetic spectrometer. 7 The detall of the procedures followed have been described earlier, 8 except that the $E_{t} v s$ channel calibration was made by using a carbon target and moving the groups from $12 \mathrm{~N} *(\mathrm{D} .964)$ across the length of the detector by varying the magnetic field.

The target used was an isotopic carbon target (98.98 12C) whose thicloess was $\sim 300 \mu \mathrm{g} / \mathrm{cm}^{2}$. If was oriented at $30^{\circ}$ and its thickness corresponded to all energ loss of $\sim 100 \mathrm{keV}$ for $49 \mathrm{kvV}$ the ions. Contaminat ion from $\mathrm{I}^{3} \mathrm{C}(3 \mathrm{He}, \mathrm{t})$ and $160\left({ }^{3} \mathrm{He}, \mathrm{t}\right)$ was not observed: on for these two reactions are $-2,239$ and $-15.44 \mathrm{BkeV}$ to the ground states of $13 \mathrm{~N}$ and 16. 3 This experiment was concerned with detemining the level strusture of $12 \mathrm{~N}$ with $0<\mathrm{E}<10 \mathrm{MeV}$ and; to some extent, the region with $E_{X} \times 15$ fov. Since Qf for the $12 \mathrm{C}(3 \mathrm{He}, \mathrm{t}) 12 \mathrm{~N}$ reaction is $-17.362 \mathrm{MeV}$, Would be concerned with contaminant graups in 13N with $14 \mathrm{E}_{\mathrm{X}} \mathrm{F}_{\mathrm{c}} 24 \mathrm{MeV}$ and with $16 \mathrm{~F}$ states with 2 \& $\mathrm{E}^{\text {th } 1 \text { ? }}$ Hev. The level structure of $16 \mathrm{~F}$ is very poorly knom but we have searched for all known energetically permissible states dive to 13C and 160 costamination, and we find evidence for none. 


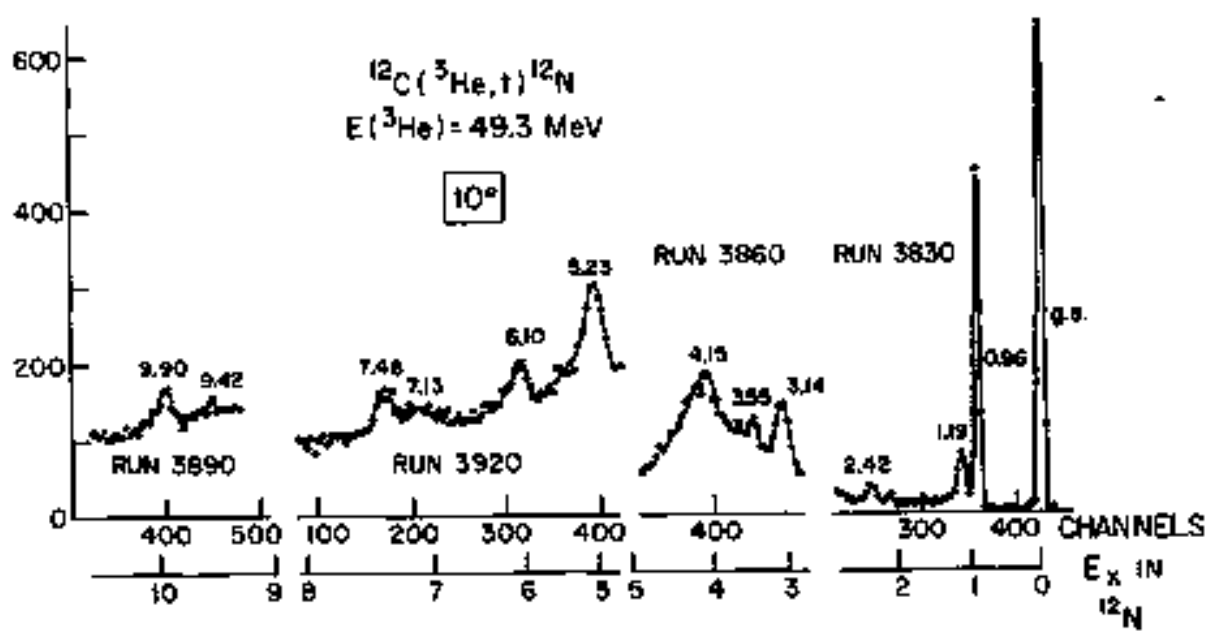

Pig, 1. Spectrum of the tritons from the $12_{\mathrm{C}}(3 \mathrm{He}, \mathrm{t})^{12} \mathrm{~N}$ reaction at $\theta_{1 \mathrm{ab}}=10^{\circ}$, $E\left(3^{3}\right)=49.3 \mathrm{MeV}$. This corposite figure shows the results from four runs: $3830[32.152 \mathrm{MHz}$, D. $235 \mathrm{mCl}]$, $\mathrm{rm} 3860[30.010 \mathrm{MHz}, 0.407 \mathrm{mC}]$, nu 3920 [29.302 $\mathrm{kHz}$, $1.14 \mathrm{mC}$ ] and ram 3890 [26.000 $\mathrm{MHz}, 1.30 \mathrm{mC}$ ] [frequency of spectrograph, incident

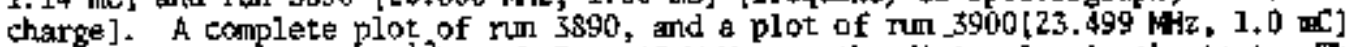
show no otleer groups in $12_{\mathrm{N}}$ with $\mathrm{E}_{\mathrm{x}}<15 \mathrm{MeV}$; see the discussion in the text. The ordinate shows the average number of counts in a four-channel bin; statistical errors are shom for four points. The abscisssa shows $E_{X}$ in $12_{\mathrm{N}}$ (in MeV).

(XBL $766-8320$ )

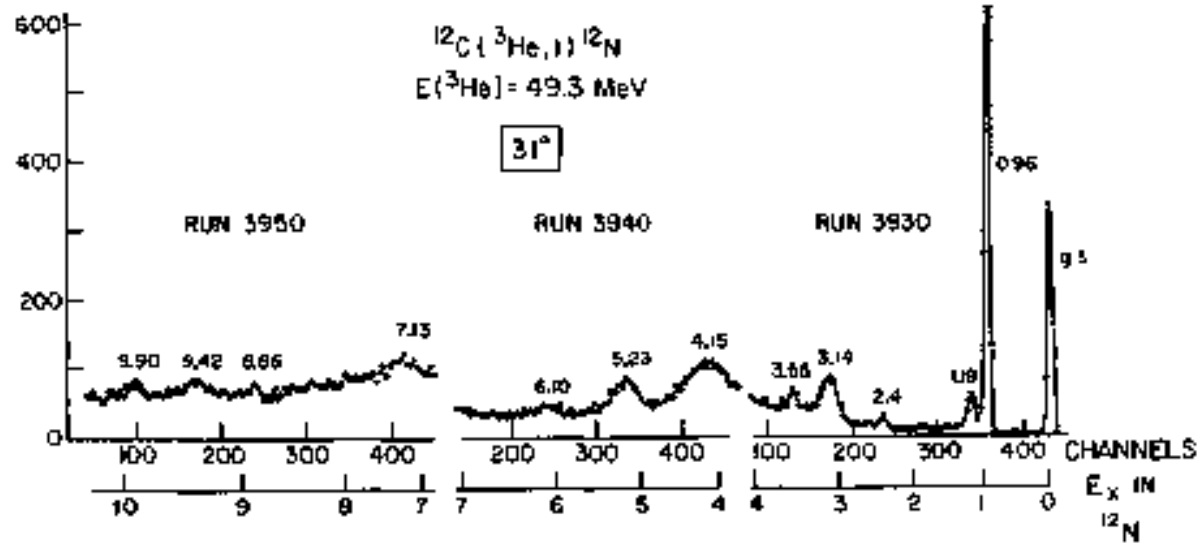

Fig. 2. Spectrun of the tritons at $\theta t a b=31^{\circ}$. This composite figure shows the results from ron 3930 [30.753 $\left.\mathrm{MHz}, 1 . \mathrm{tg}^{\mathrm{H}} \mathrm{\pi C}\right], 3940$ [28.296 $\left.\mathrm{MHz}, 1.37 \mathrm{nC}\right]$ and $3950[26,301 \mathrm{MHz}, 3.63 \mathrm{mC}]$. Plots of runs 3960 and 3970 which cover $10<\mathrm{E}_{\mathrm{x}}<13 \mathrm{kgV}$ in $12 N$ sliow no other groups. See also caption of Fig. 1.

(XBL $766-8321)$

The results we have obtained at $\theta_{1 a b}=10^{\circ}$ and $31^{\circ}$ are shown in Figs. 1 and 2 and displayed in Table 1 where they are cormared with previous results.

In conclusion, this investigation of the states of $12 \%$ has confirmed the existence of one doubtful state, 1 12/N* $(2,4)]$, casts doubt on another $\left[12 \mathrm{~N}^{*}(1.7)\right]$, reports the existence of four new states, raises questions concerning selective population by the ( $\left.{ }^{\mathrm{He}}, \mathrm{n}\right)$ and ( $\mathrm{He}, \mathrm{t}$ ) reactions, and bf congarison with the analog region in $12 \mathrm{~B}$, clearly shows that the level structure of $12 \mathrm{~N}$ is st:1l very poorly known. 


\section{$00 ?$ )}

Table 1. States in ${ }^{12} \mathrm{~s}$.

\begin{tabular}{|c|c|c|c|c|}
\hline \multicolumn{3}{|c|}{ Previcus result $\mathrm{s}^{\mathrm{a}}$} & \multicolumn{2}{|c|}{ Prosent results } \\
\hline $\begin{array}{c}\mathrm{E}_{\mathrm{x}} \\
\text { (nev }: \text { keV })\end{array}$ & $5 \pi$ & $\begin{array}{l}\Gamma_{\in \cdot m} . \\
(\mathrm{keV})\end{array}$ & $\begin{array}{c}E_{x} \\
(M e V \pm k e V)\end{array}$ & $\begin{array}{c}\Gamma \\
\text { c,m, } \\
(\mathrm{keV})\end{array}$ \\
\hline g.5. & $\mathrm{I}^{+}$ & Bound & g.s. & Bound \\
\hline $0.964 \pm 8$ & $z^{+}$ & $<35$ & $\equiv 0.964$ & $<20$ \\
\hline $1.192 \pm 9$ & $(2)^{-}$ & $140 \pm 35$ & $1,190 \pm 20$ & $80 \pm 30$ \\
\hline$(1.72 \pm 0.08)$ & & & $\mathbf{b}$ & \\
\hline $2.43 \pm 40$ & & & $2.415 \pm 20$ & $45 \pm 15$ \\
\hline $3.114 \pm 15$ & $\pi=+$ & $210 \pm 50$ & $3.136 \pm 30$ & $240 \pm 40$ \\
\hline $3.533 \pm 15$ & $(2)^{+}$ & $170 \pm 50$ & $3.550 \pm 50$ & $150 \pm 100$ \\
\hline $4.25 \div 30$ & & $290 \pm 70$ & $4,150 \pm 80^{C}$ & $650 \pm 100$ \\
\hline$\$ .320 \pm 12$ & & $180 \pm 20$ & $5.230 \pm 80^{C}$ & $400 \pm 80$ \\
\hline & & & $6.100 \pm 80$ & $300 \pm 100$ \\
\hline$(6.4)^{d}$ & & & & \\
\hline$(6.9)^{\mathrm{d}}$ & & & $7.130 \pm 100$ & $500 \pm 100$ \\
\hline $7.629 \pm 20$ & & $200 \pm 40$ & $7.480 \pm 100$ & $180 \pm 80$ \\
\hline $8.446 \pm 17$ & & $90 \pm 30$ & & \\
\hline & & & $(8.860) \pm 100)$ & $\sim 100$ \\
\hline $9.035 \pm 12$ & & $16 \pm 20$ & & \\
\hline$(9.2)^{\mathrm{d}}$ & & & $9.420 \pm 100$ & -200 \\
\hline & & & $9.900 \pm 100$ & $100 \pm 50$ \\
\hline
\end{tabular}

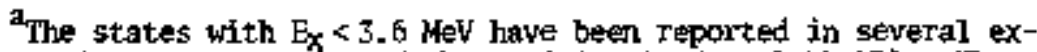
periments: see Re?. 1 (Tables 12,25,12,26, and 12,27). The

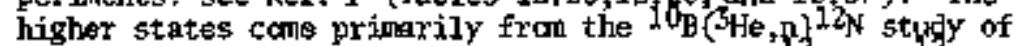
Ref. 5. Correspondence with analog states in $12 \mathrm{~B}$ and $12_{C}$ is established only for the first truee states and probably for I2)* (3.53). The other states are shown listed in line with that state in 12E which has most closely the same $E_{x}$.

$b_{\text {we see no evidence for this state. }}$

The ${ }^{A} \mathrm{~F}_{\mathrm{X}}$ of the 5.23 and $4.15 \mathrm{HeV}$ state is the same as the two states $5.32,4.25$ observed by Fuchs et al. 5 but the absolute values appear to be really $100 \mathrm{keV}$ lower (arr errors

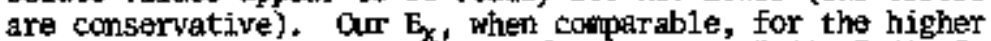
states always tend to be lower: for instance $7.48-7+63$. In the case of the $4.15-4.25$ and $5.23-5.32$ states the widths we observe are substantially larger: the (3ike,t) reaction may be exciting additional (unresolved) states.

$\mathrm{d}_{\text {Seo Ref. } 7 \text {. }}$

\section{Footrotes and References}

"Condensed fram Phys. Rev. C 13, 993 (1976).

†resent address: Department of Physics, Vanderbilt University, Nashrille, Ternessee.

${ }^{\ddagger}$ Permanent address: University of Pennsyluania, Philadolphia, Pennsylvania,
1. F, Ajzenberg-Selove, Mhisl. Physs. A 248, 1 (1975)

2. F. Ajzenberg-Selove, R. Middleton and J. D. Garrett, Phys. Rev. C 12, (197\$).

3. N. B. Gove and A. H. Wapstra, Muclear Data Tables $\underline{11}, 127$ (1972).

4. G. C. Eall and J. Cerny, Phys. Rev. 177, 1466 (1969). 
5. H. Fuchs, K, Grabisch, D, Hilscher, U. Jahoke, H. Kuge, T. Masterson and H. Morgenstern, Mucl. Plys. A 234, 61 (1974).

6. C. D. Zafiratos, F. Ajzenberg-Selove and F, S. Dietrich, Nucl. Phys, 77, 11 (1966); ibid. 89, 706 (1966).
7. B. G. Harvey, J. Mahoney, F. G. Ptihlhofer, F.S. Tourlding, D. A. Landis, J.-C. Faivre, D. G. Kovar, M. S. Zisuan, J. R. Meriwether, -5 . W. Copper, and D. L. Hendrie, Nucl. Instr, and Meth. I04, 21 (1972).

8. F. Ajzenberg-Selove, C. F. Mguire, D, L, Hendrie, D. K. Scott, and J. Mahoney, Phys. Rev. C $13,46(1976)$.

\title{
GAMMA AND ALPHA DECAY FROM THE 2.1 msec ISOMER ${ }^{219} \mathrm{Ra}$
}

\author{
D E Ralch.* H R Buwman. H E Eppley.t
}

J 0 Fasmussen, and I Rezankit

\begin{abstract}
The iscmer ${ }^{213} m_{\mathrm{Ra}}$ was produced by the reaction $209 \mathrm{Bi}(10 \mathrm{~B}, 6 \mathrm{n})$, as well as by bombertents of $12 \mathrm{C}$ on $\mathrm{Pb}$ isotopes, $14 \mathrm{~N}$ on $\mathrm{Bi}$, and 160 on natural $\mathrm{Hg}$. with projectile energies in the range $60-100 \mathrm{MeV}$. The isomer decays with a half-life of $2.1 \mathrm{~ms}$ both via ganma intermal transitions and via alpha branchirg to levels in 20 Rn. A level schente is proposed in which the iscmeric state is assigned as either $17 / 2^{-}$or $13 / 2^{+}$with shell-model configurations either of the $\mathrm{hg} / 2$ protons coupled to a $\mathrm{pl} / 2$ neutron hole, or of an uncorpled $i_{13 / 2}$ neutron hole. On the tasis of alpha dacay rate predictions from the new Fliessbach theory, the $17 / 2^{-}$isomeric assigniment is to be preferred.
\end{abstract}

In wost of the early experintental runs a heavy-metal target foil was placed at about a $45^{\circ}$ angle in the Yale HILAC bear. Comting was carried out simultaneously with irradiation, using a detector positioned $90^{3}$ from the beam line, obliquely facing the front of the target.

For the coincidence experiments a second Ge(Li) detector was placed $180^{\circ}$ frar the first, obliquely facing the back of the thin target. Three- and farrparaneter coincidence events (El,E2, nanosecond time between the two $\gamma$-rays, and sometimes millisecond tine since bean burst) were recorded serially on magnetic tape by the PDP-B/I for later computer sorting and analysis.

The alpha spectroscopy experiments wore perforined at Berkeley SuperHILAC using the helium-jet tochnique to catch recoiling reaction products and transfer the्ञा to a low-background counting chanber.

When we used the $206 \mathrm{ph}$-enriched target, we encountered strong interference from the twin doublets of the $214 \mathrm{Fr}$ and $21 \mathrm{~d}_{\mathrm{H}} \mathrm{Fr} \alpha$-groups, The francium erexgies are shown at the botton of Table 1; they are ideally positioned to mask our expected $213 \mathrm{R}_{\mathrm{R}}$ energies, especially since they also have nillisecond hatf-11ves, 5.5 and $3.6 \mathrm{~ms}$, respectively, for $214 \mathrm{Fr}$ and its isomer. The $214_{\mathrm{Fr}}$ is presumably produced both by reaction with the small $20 \% \mathrm{~Pb}$ fraction in the target- $v i a$ the direct p4n-aut regction which should go at nearly the same energy as $206 \mathrm{~Pb}$ $\left.{ }^{12} \mathrm{C}, 5 n\right)^{21}{ }_{\mathrm{Ra}}-$ and via the direct $\mathrm{p} 3 \mathrm{n}$-out reaction with $200 \mathrm{Fo}$.

We trere able to reduce the ${ }^{214} \mathrm{Fr}$ interference considerably by turning to ${ }^{204} \mathrm{~Pb}$-enriched target. The reaction $204 \mathrm{~Pb}\left({ }^{1} \mathrm{C}, 3 \mathrm{n}\right) \mathrm{IS}_{\mathrm{Ra}}$ was expected to peak at about 61 ikev, very near the coulomb tarrater. The 1or energy largely elivinate the competing $214 \mathrm{Fr}$ prodtuction since the pn-out reaction cross section is greatly reduced.

The results are summarized in Table 1.

Table 1. Results of alpha spectroscopy. (Probable error in last digit is given in parentheses.)

\begin{tabular}{|c|c|c|c|c|c|}
\hline \multirow[b]{2}{*}{$\begin{array}{l}\text { Isotope } \\
{\left[\mathrm{Q}_{\mathrm{a}}(\mathrm{MeV})^{\mathrm{a}}\right]}\end{array}$} & \multicolumn{2}{|c|}{ Present mork } & \multicolumn{3}{|c|}{$\begin{array}{l}\text { Reported in } \\
\text { literature }\end{array}$} \\
\hline & $\begin{array}{c}\mathrm{E}_{\alpha} \\
(\mathrm{MeV})\end{array}$ & $\begin{array}{c}\text { Decay } \\
\text { : }\end{array}$ & $\begin{array}{c}E_{a} \\
(\mathrm{MeV} V)\end{array}$ & $\begin{array}{c}\text { Decay } \\
8\end{array}$ & Ref. \\
\hline $213_{\mathrm{Ra}}$ & $6.731(5)$ & $46(3)$ & $6.730(5)$ & $45(2)$ & 2 \\
\hline \multirow[t]{3}{*}[6.893(5)]{} & $6.624(5)$ & $47(3)$ & $6.623(5)$ & $49(2)$ & \\
\hline & $6.522(5)$ & $6(1)$ & $0.520(5)$ & $6(1)$ & \\
\hline & $6.411(5)$ & $0.5(1)$ & 6.408 & 0.4 & \\
\hline $213 \mathrm{~m}_{\mathrm{Ra}}$ & $8.467(5)$ & $69(7)$ & & & \\
\hline \multirow[t]{2}{*}[8.663(5)]{} & $8.358(10)$ & $28(6)$ & & & \\
\hline & $8.266(10)$ & $3(2)$ & & & \\
\hline \multirow[t]{2}{*}{${ }^{214}{ }_{F r}$} & & & $8.426(5)$ & 93.0 & 3 \\
\hline & & & $8.358(5)$ & 4.7 & \\
\hline \multirow[t]{2}{*}{$214 \mathrm{~m}_{\mathrm{F}} \mathbf{r}$} & & & $8.549[8]$ & $51(2)$ & 4 \\
\hline & & & $8.477\{8\}$ & $49(2)$ & \\
\hline $\begin{array}{r}\text { Note: Rat } \\
\\
\\
\text { fno } \\
\text { fr }\end{array}$ & $\begin{array}{l}\text { io measure } \\
\text { t correcter } \\
\text { actioft. }\end{array}$ & $\begin{array}{l}\text { of } \frac{213}{213} \\
\text { for cel }\end{array}$ & $\frac{2}{a}$ a-deca) & $\begin{array}{l}=8.8 \\
\text { invery }\end{array}$ & 9) $\times 10^{-4}$ \\
\hline
\end{tabular}




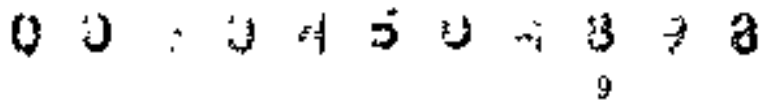

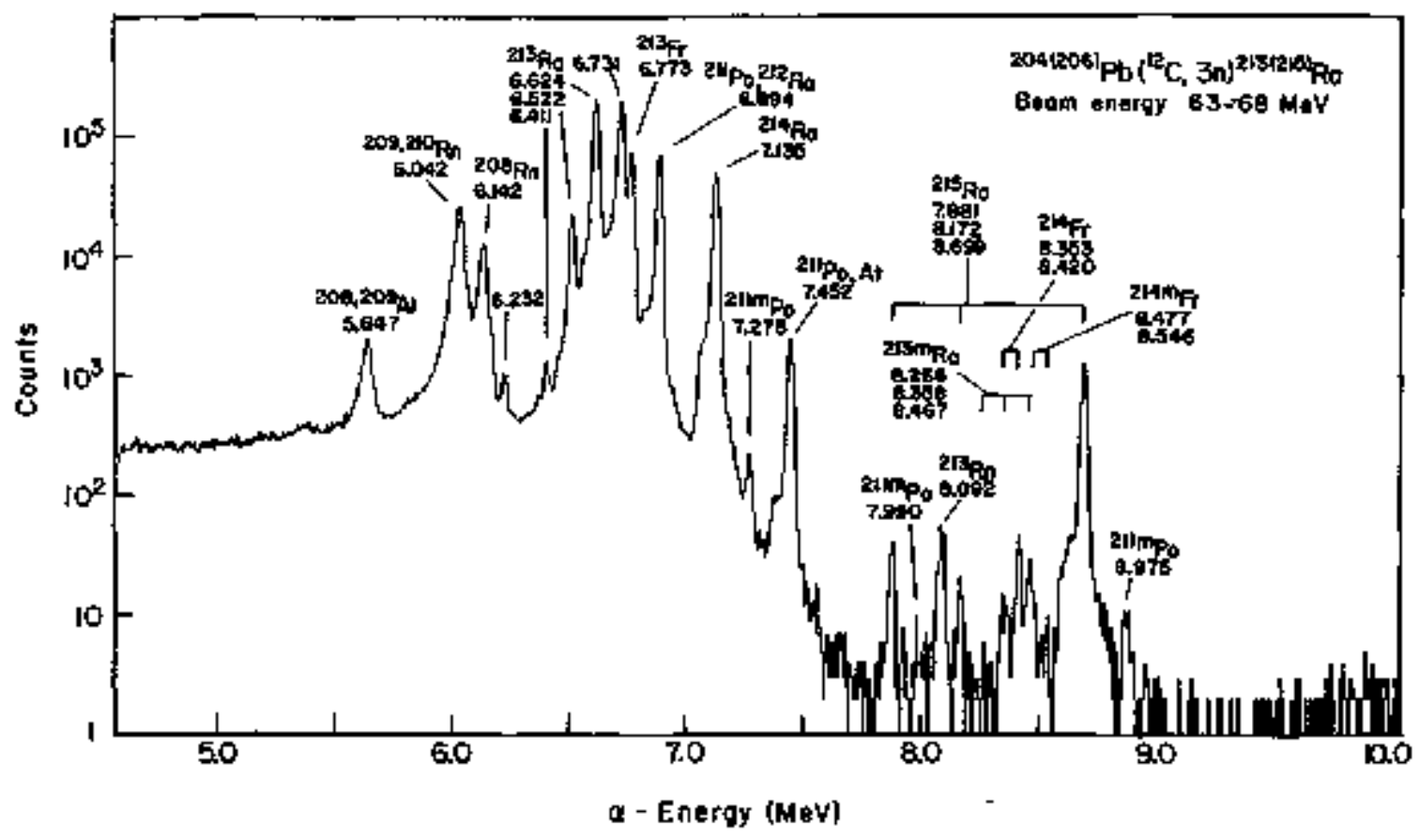

Fig. 1. Alpha-particIe spectrum showing ${ }^{2139 k a .}$ (XBL 761-41)

The umsorted alpha spectrum from our rums at 63- $6 \mathrm{~B}$ MeV is shown in Fig. 1, and a detail at tho enexgies of interest in two time intervals appears in Fig. 2,

We propose the level scheme ilfustrated in Fig. 3 of states populated in the 2.1-ns 2130 Ra decay. The 21 Re nutiens is sufficiently close to doublynagic $208 \mathrm{Fb}$ that the spherical single-particle model should predict lower energy levels fairly well. lie expect the single hole in the 126-neutron shell to dorinate the configurations of the first few states, resultting in a ground state with $\mathrm{J}=1 / 2^{*}$, and first two excited states $5 / 2$ and $3 / 2^{-}$(with the nettron hole strecessiveIy occupying $\mathrm{P}_{1 / 2}, \mathrm{f}_{5 / 2}, \mathrm{P}_{3 / 2}$ sheIl-model orbitaIs). Higher-seniority configurations inwolving the hig/2 protons are expected to become significant sorewhat above $1 \mathrm{MeV}$ and excitations of the $208 \mathrm{pb}$ core should not appear below $\sim 2.5 \mathrm{MaV}$.

Low-lying high-spin states are a comon cause of isomerisan and are readily populated in heavy-ion reactions. Two candidates for such a state suggest thenseIves. First, on the basis of level trends expect an $i_{13 / 2^{-1}}\left(13 / z^{+}\right)$nextrom-hole state near $1800 \mathrm{keV}$. Second in $214_{\mathrm{Rk}}$ the $8^{+} \pi\left(\mathrm{h}_{\mathrm{g}} / 2^{6}\right) 8^{+}$state at $1864 \mathrm{keV}$ is 1scateric, with an E2-transition halflife nuxh longer than that seen for other E' 2 's in similar cascajes, probably reflecting cancellation in the pairing factor $\left(U_{i} U_{f}-V_{i} V_{f}\right)^{2}$ near the halffilled $h_{g / 2}$ proton shell. This m(hg/2 $\left.{ }^{6}\right)_{8}$ state might couple in $213_{\mathrm{Ra}}$ with the $\mathrm{p}_{1} / 2{ }^{2}$ ngutron hole to produce a long-lived $17 / 2^{*}$ state of about the correct energy. In a separate report we test these two possabilities kith Filessbach's new nomaltzed $\alpha$-decay theory 1 by comparing prodictions of branching ratios to the daughter states from each of the mypothetlcal perent states. The pattern of intensities fits our a-spectroscopic data mach better

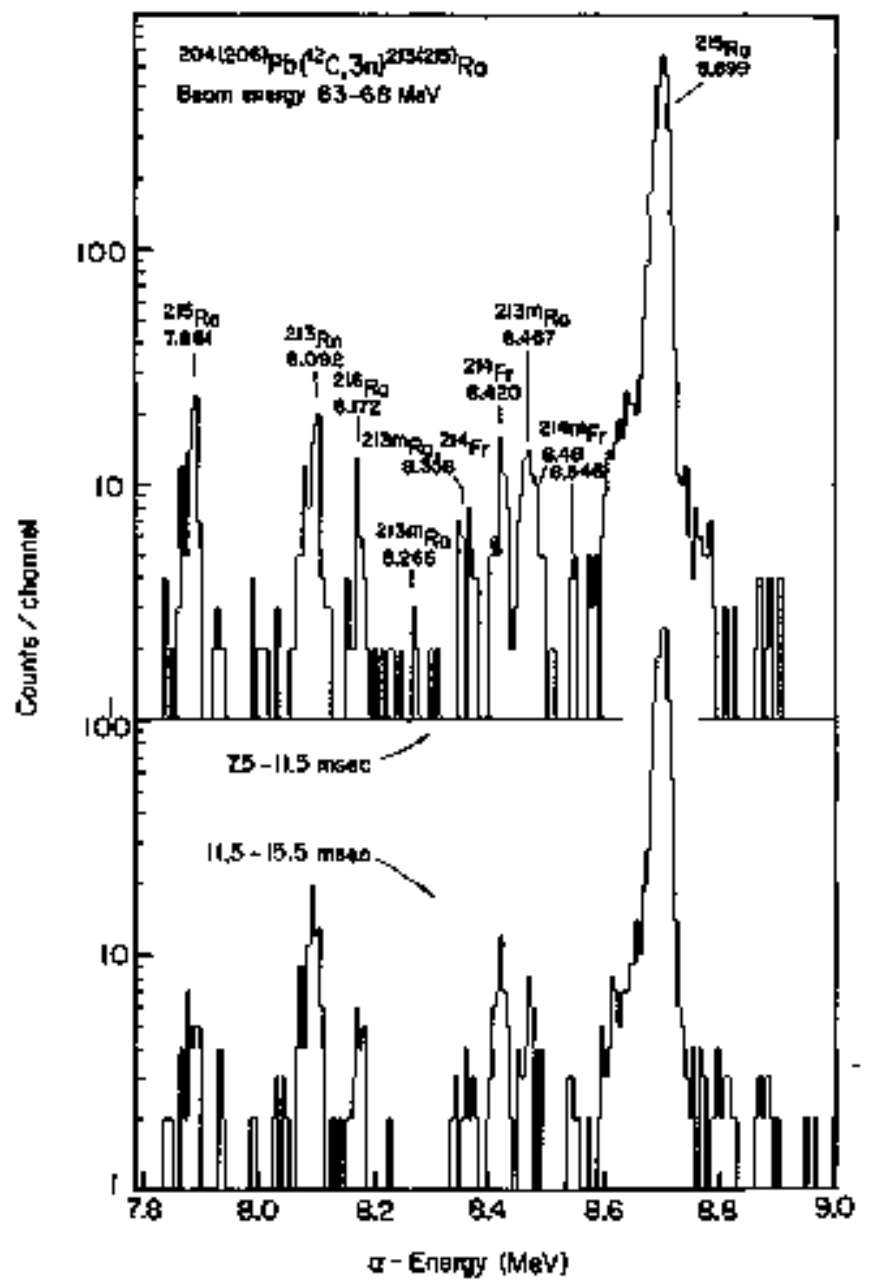

Fìg. 2. Detail of two the intervals contributing to the a-spectrut of Fig, 1. 


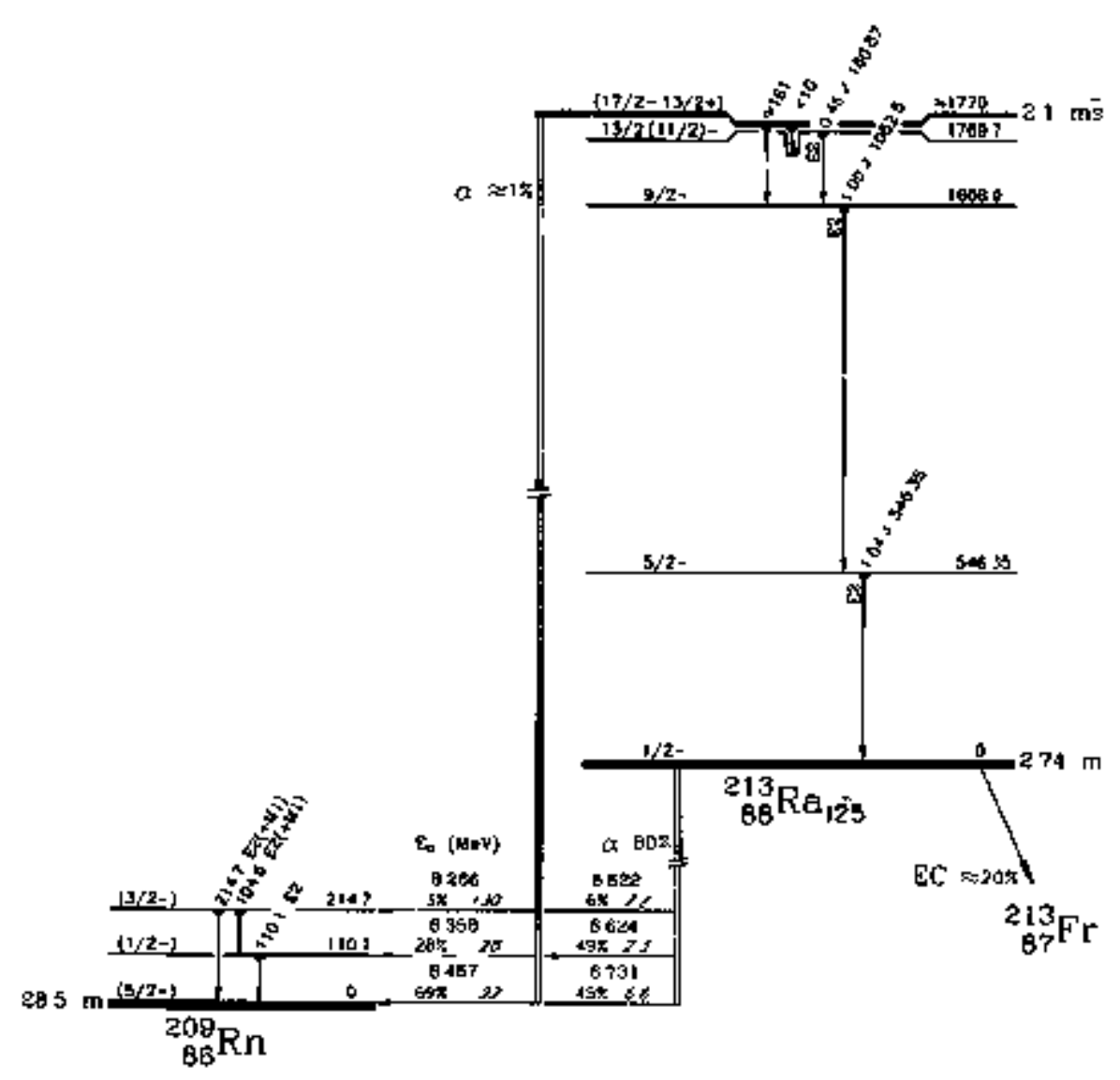

Fig. 3. Proposed ${ }^{213} m_{\mathrm{Ra}}$ decay scheme. Relative photon intensities (and uncertainties) are shown in front of transition energies. Aproximate hindramce factors follow a-feeding percelnages.

assuming a $17 / 2^{-}$iscmeric state, so we Ieport this as aur preferred interpretation.

\section{Foptnotes and References}

*Present address: Chenistry Division, Argctne National Laboratory, Argonne, TL 604\$9.

${ }^{\dagger}$ Present address: Monsanto Research Corp., Mound Laboratory, lefiamisburg, of 45342 .
Fresent adiress: Research Laboratory, Xerox Corp., Rochester, NY 14600

1. T, Fliessbach, Z. Phys. A272, 39 (1975).

2. K, Valli, w. Treyt1, E. K. Hyde, Phys. Rev. 161, $1284(1967)$.

3. D. F. Torgerson and R. D. Macfarlane, Phys. Rev. C2, 2309 (1970).

4. K. Valli and E. K. Hyde, Phys. Rev. 176, 1377 (1968). 


\title{
$01: 4$ if 50 is 899
}

\section{EXTENSION OF THE Tz $=-3 / 2$ BETA-DELAYED PROTON PRECURSOR SERIES TO ${ }^{57} \mathrm{Zn}^{*}$}

\author{
D J. Vieira, D F. Sherman, M. S. Zißman, \\ R. A, Gough, and Joseph Comy
}

The well-known series of $A=4 n+1, T_{z}=-3 / 2$ B-delayed proton prectursors has been completed from ${ }^{9} \mathrm{C}$ through $49 \mathrm{Fe}^{1}$ with the recent addition of $4 \mathrm{STr}_{\mathrm{Cr}}{ }^{2}$ Such studies both establish the decay properties of proton rich moklel far fron stability and, with the use of the isoberic multiplet mass equation, permft an excellent prediction of the precursor's mass. Although the $\beta$-delayed proton activity fron the heavior muclides in this serios, through ${ }^{41} \mathbf{T i}$, has been produced in reasconable yield $(\sim 30)-250 \mu b)$ by the (He, $2 n$ ) reaction on $T_{2}=0$ targets, extension of these studies fyrther into the fo she11 by enuploying the 32 s $(16,0,3 n)^{45} \mathrm{Cr}^{2}$ and $40_{\mathrm{Ca}}\left({ }^{12} \mathrm{C}, 3 \mathrm{n}\right)^{49} \mathrm{Fe},{ }^{3}$ teac tions has been hampered by much Jower cross sections ( $\sim 300-500 \mathrm{nb})$, He wish herein to report the obser vation of $53 \mathrm{Ni}$ via the $40 \mathrm{Ca}(160,3 \mathrm{n})$ reaction and of

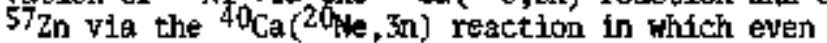
lower yields were observed $\{25$ and $-60 \mathrm{nb}$, respectively').

Beta-delayed protons emitted from precursors retained in the target were detected in a semiconductor counter telescops subtending an effective solid angle of $0.24 \mathrm{sx}$. These precyrsofs were produced by employing pulsed beams of 160 ( 60 and 65 $\mathrm{MeV}, \sim 100$ particle-rA) or $2 \mathrm{ONe}^{+4}(62$ and $70 \mathrm{NeV}$, $\sim 60$ particle-nA) fron the 88 -inch cyclotron in irmadiations of natural calcium targets rotated to a target angle of $70^{\circ}$. A slotted rotating wheel system, sinilar to that described in Ref. 4, cortrolled the pulsing of the beam and protected the detectors while the target was being irradiated. Several detector telescope combinations were used.

For completeness the $53 \mathrm{ki}$ results (show in Fig. 1a), discussed in the previous Anmul Report (LBl-4000), have been focluded. The large group at $1.59 \mathrm{MeV}$ (c.m.) is krown to arise from direct proton radioactivity of 530 4 produced via the competing $40 \mathrm{Ca}(160, \mathrm{p} 2 \mathrm{n})$ reaction, while the $1.94 \pm 0.05 \mathrm{MeV}$

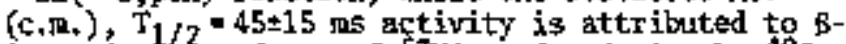
delayed protm decay of $53 \mathrm{Ni}$ produced via the $40 \mathrm{Ca}$ $(160,3 \pi)$ reaction (see decay scheme in Fig. 2b).

The proton spectrum resulting from the $70 \mathrm{MeV}$ 20 Ne babardient of a $1.8 \mathrm{mg} / \mathrm{cm}^{2}$ calciun target for $20,000 \mu \mathrm{C}$ (as $10^{+}$) is shown in Fig, 1b Again, 53 Co arising frm the competing ${ }^{4} \mathrm{Ca}(20)$, ap $\left.2 \mathrm{n}\right)$ reaction observad. Three proton groups at energies of $1.95 \pm .05,2.58 \pm .05$, and $4.65 \pm .05 \mathrm{MeV}$ (c.m.) were observed, corresponding to a total del ayedproton yield of $60 \mathrm{nb}$. The half-life of each group is consistent with the $40 \pm 10 \mathrm{~ms}$ value determined from the sur of all three groups. These activities are very conpatible with the B-delayed proton enission expected from $\mathrm{S}_{\mathrm{in}} \mathrm{inhose}$ predicted threshold via the $40 \mathrm{Ca}(20 \% \mathrm{k}, 3 \mathrm{n}$ ) reaction is $\sim 50.0 \mathrm{MeV}$ (as. suming a mass excess of $-32.77 \mathrm{MaV}$, calculated using the Kelson-Garvey charge syndetry relations 5 and two predicted $T_{2}=-1 / 2$ masses from Ref. 6 ). The pro-

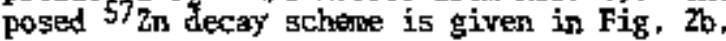

The groups observord at 4.65 $\mathrm{HW}$ and $1.95 \mathrm{MeV}$ can be assigned to the isospin-forbidden protom decay of the lowest $\mathrm{T}=3 / 2$ state of ${ }^{57} \mathrm{O}_{1}$ to the gromind state and first excited state of $\$ 6^{\mathrm{Ni}}$, respectively. This energy difference of $2.70 \mathrm{t} .07 \mathrm{keV}$ agrees yery well with the known separation between these $\$ 6$ Ni stetes of 2.702+.003 NeV.7 Based on these two groups, the excitation energy of the lowest $T=3 / 2$ state of $57 \mathrm{Ou}, 5.35 \pm .04 \mathrm{HeV}$ (assuming the ground state mass excess of ${ }^{57} \mathrm{Cu}$ is -47.31 Hev, ${ }^{6}$ canpares well with its lonown analog state in $57 \mathrm{Ni}$ at $5.23 \pm .01$ MeV. 8 The group at 2,58 MeV could be attributed to isospin-allowed proton decay of a $\mathrm{T}=1 / 2$ state at $3.28 \pm$.0S MeV in $57 \mathrm{cu}$, wich could be the sirror of one of the two kngwn $7 / 2^{-}$states in $5 / \mathrm{Ni}$ at 3.23 .02 and $3.36 \pm, 02 \mathrm{MeV} . \mathrm{g}$

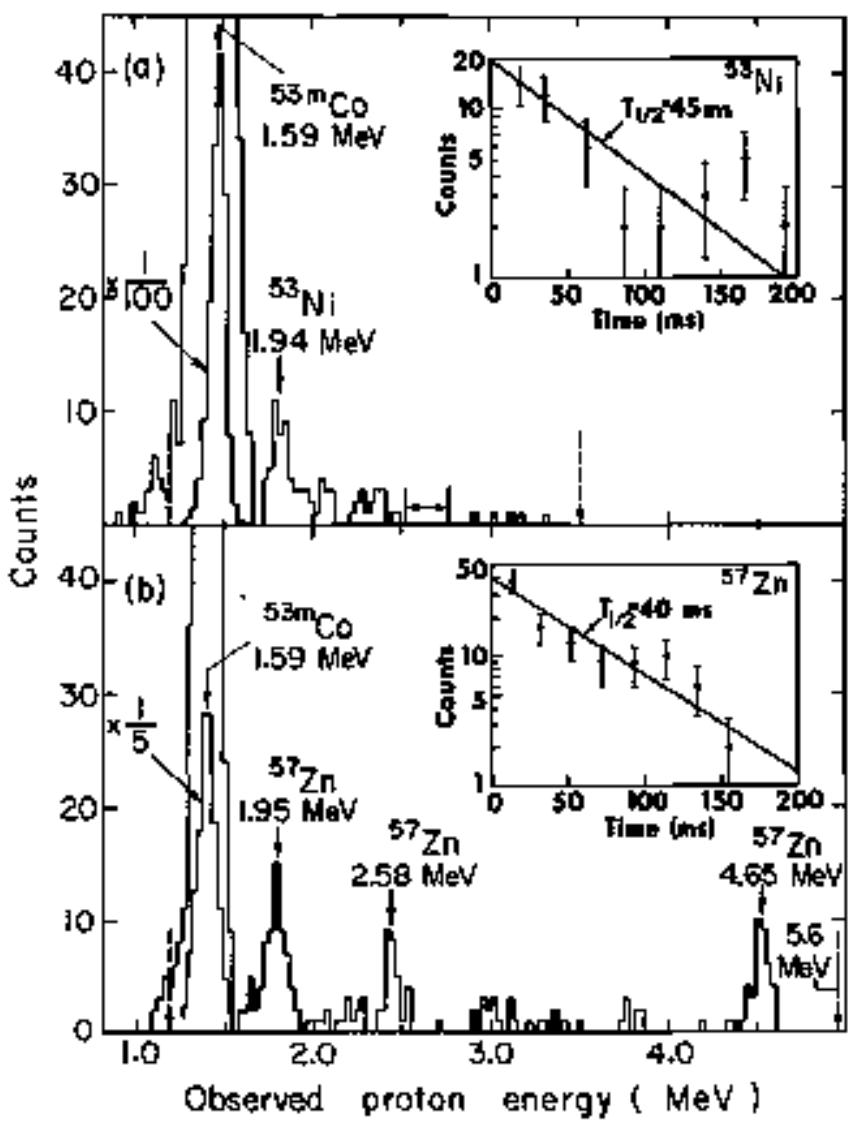

Fig. 1. Droton spectra observed following the bcinbaxtuest of calcium with (a) $65 \mathrm{MeV} I 60$ and (b) $70 \mathrm{MeV} 20 \mathrm{Ne}$. Dashed vertical arrows indicate the energy region in both spectra over which protons could be reliably observed. The precursor ard the center-of-mass energy are labeled above each proton. group. The horizontal arrows in (a) designate the energy region where proton decay to the ground state of $\$ 2$ Fe pould be expected (see text). Decay curves for the ${ }^{5}$ Nit group and the sum of the three $57_{\mathrm{Zn}}$ grcups are show in the insets. 


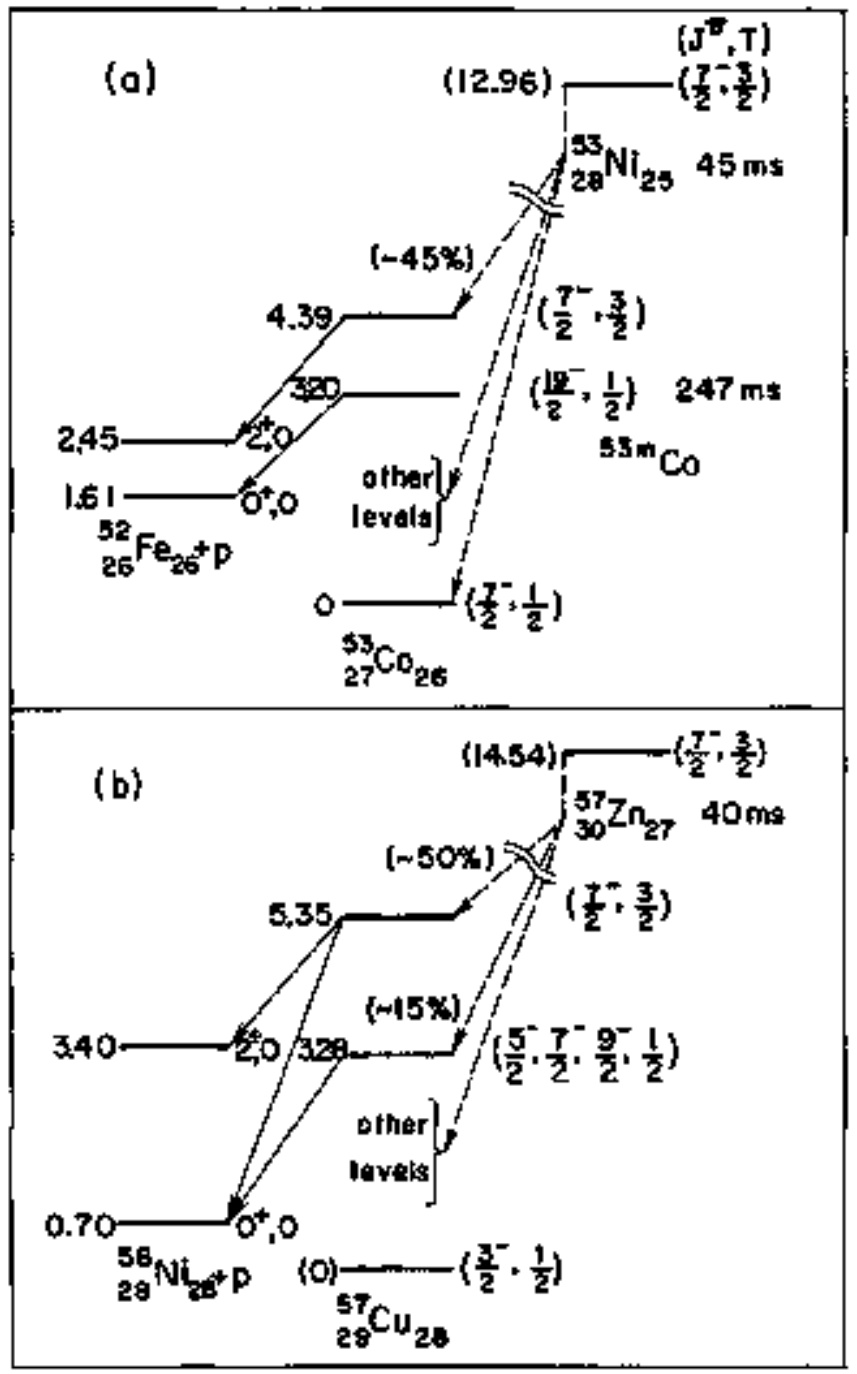

Fig. 2. The proposed decay schemes for (a) 53i and (b) $572 \mathrm{n}$. Inecays which have not been directly observed are indicated with dashed Iines. A11 energies are given in $\mathrm{MeV}$ retative to the ground state mass of the appropriate $\mathrm{T}_{z}=-1 / 2$ nuclide. Predicted energies, asstmed spin, parity and isospin values, and ostisạted branchîng ratios are shown in parentheses.

If the mass excesses of the lowest $T=3 / 2$ states in the $T_{z}=-1 / 2$ nuclei $5 \mathrm{Co}[-38.26 \pm .06 \mathrm{MeV})$ and $5 \mathrm{Cu}(-41.97 \pm .04 \mathrm{MeV})$, together with those of the correspording $\mathrm{T}-3 / 2$ states in the $\mathrm{T}_{z}=3 / 2$ and $+1 / 2$ members of each multiplet, are used in the isoberic nultiplet mass equation (TMN), the predicted mass excesses for $5{ }^{3} \mathrm{Nl}$ and $5 / \mathrm{Zn}$ are $-29 \pm .18 \mathrm{NeV}$ and $-32.63 \pm .13 \mathrm{HeV}$, respectively. For $53 \mathrm{Ni}$, good agreement betwem this prediction and the Kelson-Garvey type mass prediction of $=29.69 \mathrm{MeV}$ or the Coulonb energy caloulation of $-29.65 \mathrm{Mev}^{10}$ is found, Similar agreenent is obtaing between the IrME result for the mass excess of ${ }^{57} \mathrm{n}$ and the Kelsom-Garvey prediction (described earlier) of $-32.77 \mathrm{MeV}$.

Surveying the $\beta$-delayed proton decoy of the $J^{\pi}=7 / 2^{-}, T_{2}=-3 / 2$ ruclei from $4 \mathrm{Cr}$ to $5 / \mathrm{Zn}$, we see that only in the case of ${ }^{5} \mathrm{~T}_{\mathrm{Z}}$ has isospin-forbidder proton decay frcin the analog state (in the enitter) to the ground state of the $\mathrm{T}_{z}=0$ residual micleus been observed, a result which may largely beflue to the enhanced stability of the doubly magic 56 Ni. On the basis of penetrability calculations alome, an intensity ratio for proton decay fron the 5 thu $(T-3 / 2)$ state to the ground state $\left(0^{+}\right)$relative to the first excited state $\left(2^{+}\right)$in 5 Ni of $\sim 28 / 1$ would be expected. The observed ratio of $\sim 0.6 / 1$ indicates that the reduced width for (isospin-forbidien) proton decay to the ground state is $\pi 1 / 45$ of that for decay to the first excited state. Such a reduced width ratio is consistent with the limits observed 2,3 in the proton decays of the analog states in 45 , $4 \mathrm{~g}_{\mathrm{h}, \text { and }}{ }^{3} \mathrm{Co}$, which indicate $\gamma_{\mathrm{p}}{ }^{2}\left(0^{+}\right) / \gamma_{\mathrm{p}}{ }^{2}\left(2^{+}\right)<1 / 8$.

\section{Footnotes and References}

"Condensed from Physics letters 므, 261 (1976).

1. J. C. Hardy, in Nuclear Spectroscopy and Reactrons - Part C, ed, J. Carny, (Acaderuic Press, Hew York, 1974) p. 417.

2. K. P. Jackson, J. C. Hardy, H. Schmeing, R. L. Graham, J. S. Geiger, and K. W. Allen, Fhys, Lett. A9B, 341 (1974).

3. J. Cermy, C. U. Cardinal, H. C. Evans, K. P. Jackson, and N. A. Jelley, Phys. Rev. Lett. Z4, $1128(1970)$.

4. J. Cerny, R. A. Gough, R. G. Sextro, and J. E. Ester 1, Muc1. Phys. A188, 666 (1972).

5. I. Kelson and G. T, Garvey, Phys, Lett. 23, 689 (1966).

6. M. Harchol, A. A. Jaffe, J. Miron, 1. Lhma, and J. Zionj, Wucl. Phys, A90, 459 (1967).

7. H. Nann and w. Benenson, Phys. Rev, C 10, 1880 [1974].

B. F. M. Ectward's, J. J. Kraușharar, and B. W. Ridley, Shel. Phys. A199, 463 (1973).

9. D. Mueller, E. Kashy, H. Bemenson, and H. Narm, Phys. Rev. C 12, $\$ 1$ (1975).

10. F, D. Becchetti, D. Delmhard, and T. G. Dubay, Mucl, Phys, A168, 151 (1971). 


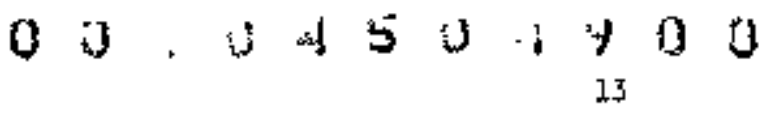

${ }^{87}$ Ge: A NEW BETA-DELAYED PROTON PRECURSOR

O J Vieira, D M Moliz.

u S Zisman, and Joseph Cermy

Further efforts to extend the series of $A=4 n$ $+1, T_{2}=-3 / 2$ i-delayed proton precursors have succeeded by observing $\beta$-delayed protons frow $61_{\mathrm{Ge}}$ produced via the $40 \mathrm{Ca}(24 \mathrm{Mg}, 3 \mathrm{n})$ reaction.

As in the $53_{\mathrm{Ni}}$ and $57_{\mathrm{Zn}}$ experiments 1 discussed in the previous report, B-delayed protons exicted from precursors retained in the target were detected by a connter telescope protected by a slotted rotating whee1 as illustated in Fig. 1, These precursors were produced by enploying a recently desceloped pulsed beam of $85 \mathrm{MeV} 24 \mathrm{Ng}^{44}(\sim 50$ particle-nA) fron the 88-inch cyclotron in irradiations of a $1,5 \mathrm{mg} \mathrm{cm}^{2}$ natural calctim target rotated to a target angle of $70^{\circ}$.

Figure 2 shows the identified proton spectrum observed in 25,000 $\mu \mathrm{C}$ of integrated beam using a 15-1m $\Delta E$ and 250-pm E detector telescope, A proton grout at an energy of $3.15 \pm .07 \mathrm{keV}$ (c.m.) was observed at a yield of $\sim 30$ nb with a half-life of $40 \pm 15 \mathrm{~ms}$. A much weaker group at $1.81 \pm .07 \mathrm{MeV}$ was also observed, but this activity exhibited a halfilfe much longer than the counting period of $120 \mathrm{~ms}$. This latter proton group is consistent with a large fluctuation accurring at 1.7 to $1,8 \mathrm{MgV}$ obsegred in the continum $\beta$-delayed proton spectrun of ${ }^{65} \mathrm{Ge}$ (Ep (c.m.) $=1.0=2.3 \mathrm{kev}, \mathrm{T}_{1} / 2=30.8$ sec ${ }^{2}$, which could be producet by $\left(24 \mathrm{~kg}, X_{n}\right)$ reactions with heavier isotopes present in the natural calcium target. Repoating the experiment at a bombarding energy of

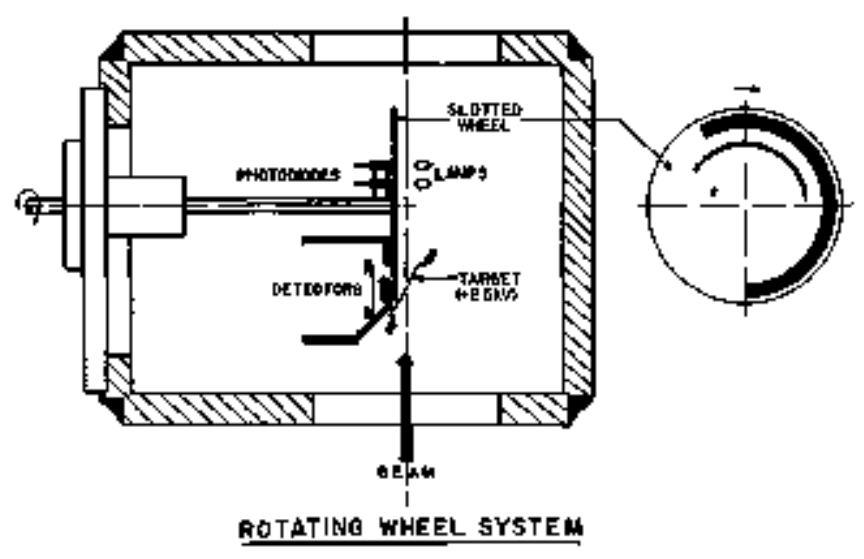

Fig. 1. Diagran of the slotted, rotating wheel. Photodiodes control pulsing of the cyclotron bear and provide tining signals for the counting period. A detector telescope backed by a reject detector is protected by the $1.5 \mathrm{mit}$ thick Ta whee 1 when the beard is on and allowed to view the target through a large slot in the wheol when the bean is off.

(XBL 743-605)

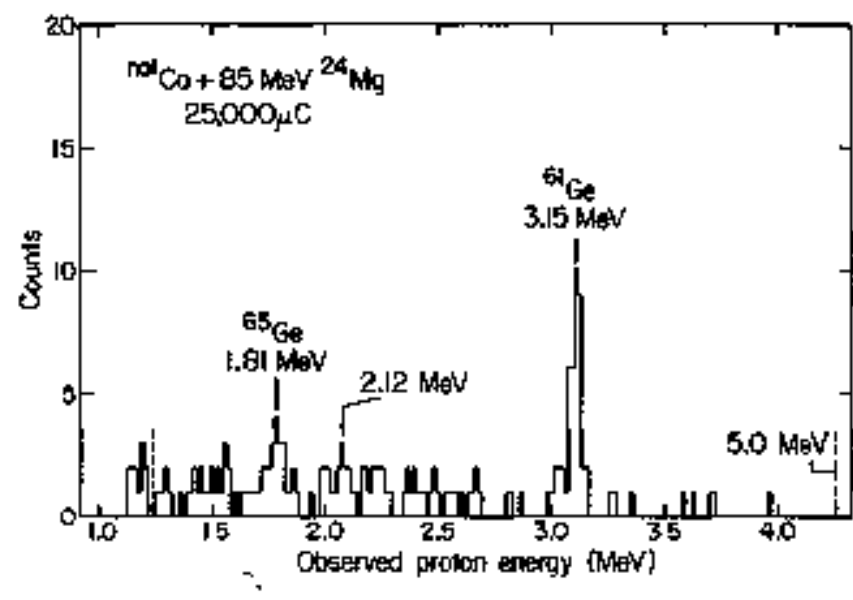

Fig. 2. The proton spectrum observed following the bombardment of calcium with $85 \mathrm{MkV} 24 \mathrm{hkg}$. Dashed vertical ajtows indicate the mergy region over which protons could be reliably identified. The precursor and the center-of-Jass energy are labeled above each proton group. For the possibIe source of the $2.12 \mathrm{MeV}$ group, see text.

(XBL 764-2739)

$78 \mathrm{MeV}$ produced sinilar results with the $1.81 \mathrm{MeV}$ activity increasing slightly in yield [supporting ${ }^{65} \mathrm{Ge}$ as its probabie source), while the $3.15 \mathrm{MeV}$ activity decreased in yield.

The $3.15 \mathrm{kel}$ activity is most reasonably explayned as arising from the B-delayed protor decay of ${ }^{2} \mathrm{Ge}$ produced via the $40 \mathrm{Ca}(24 \mathrm{Mg}, 3 \mathrm{n})$ reaction (threshold $\sim 61,3 \mathrm{MeV}$ assuming a mass excess of -34.7 MeV for ${ }^{6} \mathrm{l}_{\mathrm{Ge}}$ estimated from Coulomb energy systematics discussed below]. Since ${ }^{6}{ }_{[j}$ is sufficiently far frcil stability that the only previously known monber of this isospin quartet was the 6la ground state, it is necessary to estifrate the expected ex-

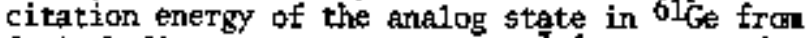
Coulanb displacenent energy data 3,4 adjusted for the mass 61 system. These calculations predict that the analog state should be near $3.53 \mathrm{Hev}$, which agrees well with an excitation energy of $3.47 \mathrm{MeV}$ obtained when the $3.15 \mathrm{MeV}$ activity is attributed to proton decay to the ground state of $60 \mathrm{Zn}$ [see $\mathrm{Fig}$. 3], Decay of this analog state to the first excited state of $60_{\mathrm{Zn}}$ would be expected at an energy of $2.15 \mathrm{MkV}$. Weak evidence for such a proton group is observed at $2.12 \mathrm{kgV}$, but dup to poor statistics and possible interference from ${ }^{6}{ }^{\circ} \mathrm{Ge}$, the sgurce of this grop is uncertain. If attributed to $6 \mathrm{I}_{\mathrm{Ge}}$, this branch would correspond to an upper linit of $\sim 20 \%$. Additional experiments on separated isotope ${ }^{40} \mathrm{Ca}$ targets will. be necessary to confirm the origin of this $2.12 \mathrm{HeV}$ group. 


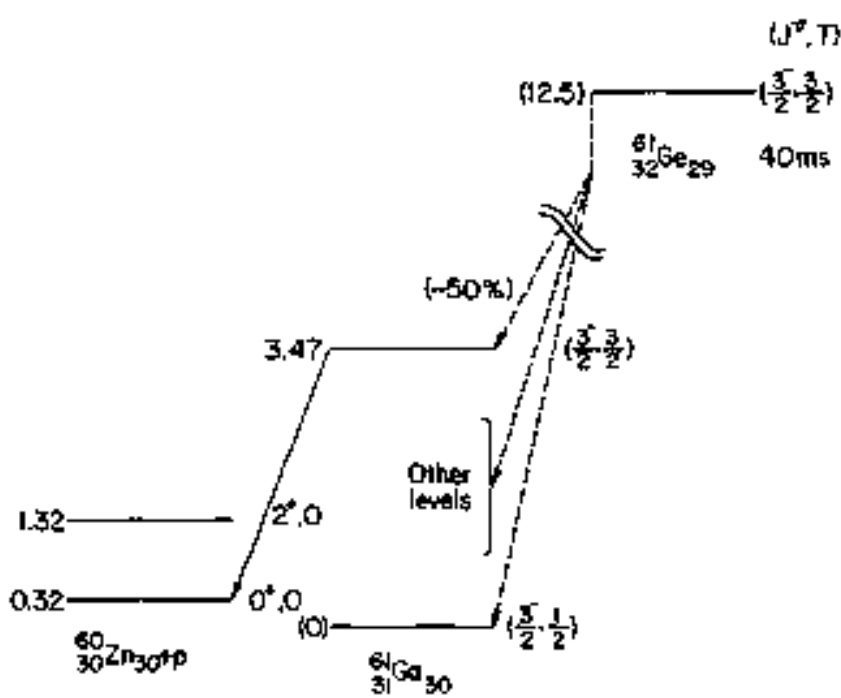

Fig. 3. The proliminary decay scheipe of ${ }^{61_{G e}}$. Decays which have not been directly observed are indicated with dashed lines. A11 energies are given in keV relative to the predicted mass excess of $-47.22 \mathrm{keV}$ for the ground state of $61 \mathrm{Ga} .4$ Predicted energies, assure spin, parity isospin values, and estinated branching ratios are shown in parenthoses.
( $\mathrm{XBL} 764-2740$ )

\section{References}

1. D. J, Vieira, D. F. Shemant, M. S. Ztsanan, R. A. Gough, and J. Cerny, Phys. Lett. 60B, 261 (1976).

2. J. A, Macdinald, H, Schmeing, J. C. Hardy, H. R. Andrews, J. \$. Geiger, R. L. Graham, ard R. P. Jackson, Cha1k River Nuclear Laboratories Report (1975) AECl. 5226 and J. C. Hardy, private communication.

3. W. J, Courtiney and J, D. Fox, Atoric Data and Nurclear Data Tables 15, 141 (1975).

4. M. Harcho1, A, A. Jaffe, J. Miron, I. Unna, and J. Zioni, Muc1. Phys. A90, 459 (1967).

\section{REVISED SINGLE-PARTICLE ENERGIES IN $\mathbf{N}=$ QS NUCLEI* \\ P. Kleinheanz+ M. R. Maier, R. M. Diamond, F. 5 Stephens, and R. K Shelire}

The single-neutron states in $\mathrm{N}=83$ muclei have been extensively investigated, ${ }^{1}$ mainly by detrterch stripping on targets of the stable isotones with $\mathrm{N}=82$. The deta suggest the 5 )stentic occurrence of three states in each rucleus populated by highangular momentum transfers; these levels vary tmoothty with proton nuber and 1ie below 2 Mor excitation. The $(d, p)$ angular distributiont for these states were assigned as 1- 5 transfers, and the leve1s therefore were interpreted as ho/2 fragnents. More recent $1 y$, radioactivity ${ }^{2}$ and $(a, M Y\}^{2}$ experiments 3 extended the systematics of these high-j states to 147 which is not accessible in single-neutron transfer. Except for ${ }^{139} \mathrm{Ba}$, where a recent $(\mathrm{d}, \mathrm{p})$, study conflicts with the oarlier investigations, 1 mone of these studies located the $i_{13 / 2}$ single par. ticle excitation which, ficm systematics, is oxpected to lie within the range of excitation covered by these experiments.

We have used the following compound muclear reactions followed by particle easssion to populate levels in the $\mathrm{N}-83$ isotgnes of $\mathrm{Ce}$, $1 \mathrm{~d}$, and $\mathrm{Gd}$

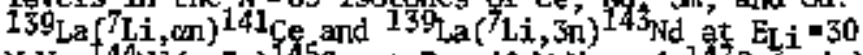
$\mathrm{MeV}$, $144_{N d}(a, 3 n)^{145} \sin E_{n}=40 \mathrm{MeV}$, and $147 \sin (a, 4 n)$ $147 \mathrm{Gd}$ at $\mathrm{E}_{\alpha}=57 \mathrm{HeV}$. The experiments were carried out at the berkeIey 8s-inch cyclotron, using standard in-beem $\psi$-ray spectroscopy techniques 5 with various combinations of $8 \mathrm{~cm}^{3}$ planar Ge, $30 \mathrm{~cm}^{3}$ and $50 \mathrm{~cm}^{3}$ coaxial Ge(Li) detectors. Measurements of $\gamma$ ray excitation functions and two-point angular dis- tributions as well as two-detector four-parameter coincidences were tised to establish the level schenes of Fig. 1. In each of these nuclei essentially the ontire decay intensity proceeds through the previously known lowest-1ying high-j level, but our measurements show these levels to be iscmeric with ranosecond half-lives. They decay via single $\gamma$-transitions to the well established ${ }^{6} f_{7 / 2}$ ground state of each mocieus. This fact is clearly inconsistent with a $9 / 2^{-}$assigmment for these states.

The half-lives were extracted fron the rotine spectra. They are listed in the table. Coroparison with the single-particle estimate ${ }^{7}$ classified the

Table 1. Half-lives of $i_{13 / 2}$ states in $N=83$ 1sotones.

\begin{tabular}{|c|c|c|c|}
\hline Nhecleos & energy (kel) & $\begin{array}{l}T_{1 / 2} \\
\text { (ns) }\end{array}$ & $\begin{array}{l}\text { Enhancerest } \\
\mathrm{T}_{1 / 2}^{S p} / \mathrm{T}_{1 / 2}\end{array}$ \\
\hline${ }^{141} \mathrm{Ce}$ & 1370.8 & $4 \pm 2$ & $9 \pm 4$ \\
\hline $143 \mathrm{hd}$ & 1230.0 & $6 \pm 2$ & $12 \pm 4$ \\
\hline 145 & 1105.8 & $12 \pm 2$ & $12 \div 2$ \\
\hline $147 \mathrm{Gd}$ & 997.4 & $20 \pm 3$ & $15 \pm 3$ \\
\hline
\end{tabular}



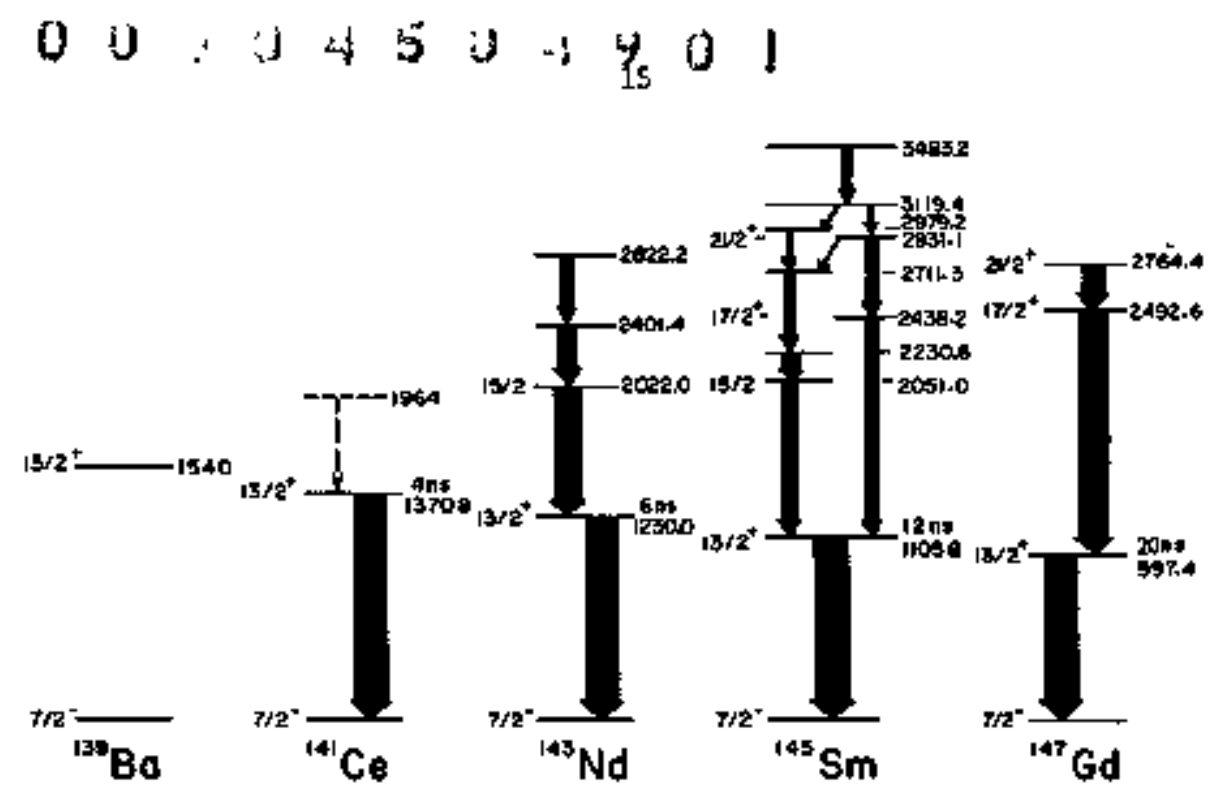

Fig. 1. Partial level schemes for $N=83$ isotones as deduced from the presernt work and from Ref. 4. The excitation energies are accurate to $1 \mathrm{keV}$.

(XBL 766-8231)

deexcitation transitions as E3, whereas MII tranșitions, as required by the previous assignments, wuld have to be 1 of times rotarded in alt cases. The life-tines, together with the observed strong transfer intensities, argue for the identification in all these maclei of the first high-j state as an $i_{13 / 2}$ single-neutron state, He have also included in $1 \mathrm{gg}$. I the $i_{13 / 2}$ state in ${ }^{139} \mathrm{Bg}$. The angularmomentum transfer to this level has been measured as $3=6$ in a $(d, p)$ experiment with 20 , feV desterons, 4 a regult which has been ignored in nore recent work, 2,8

The proposed $i_{13 / 2}$ assignients in the $N=83$ mulei renove a mober of discrepancies connected with the oarlier $9 / 2^{-}$assigmmontes. The stripping data into the $\mathrm{N}=83$ isotones of $\mathrm{Ba}, \mathrm{Ce}, \mathrm{Na}$, and $\mathrm{sin}$ quoted in Ref. 1 consistently gave spectroscopic strengths for $\tau=5$ transfer in excess of the sumrule limit for $\mathrm{hg} / 2$, in $\mathrm{Nd}$ and $\mathrm{sin}$ by as exich as 708 . With the $i_{13 / 2}$ assigminent fur the lowest state, the renaining observed $i=5$ strengths become conpatible with the sum-rule limit, and the reported cross sections to the high-j state of lowest energy are consistent with a predominant $1_{13 / 2}$ configuration. Wheover, in the $B^{+}$decay of the $h_{1} / 2$ isguer of 147T (Ref. 2), two high-lying states in $14 / \mathrm{Gd}$ were found to be populated with $\log \mathrm{ft}$ values $\times 4.4$ and assigned $9 / 2-$. The third high-j level (the one presently being considered), which from $(a, n)$ data was also assigned as $9 / 2^{*}$, is only populated with very small intensity in the $\beta^{+}$decay and this feature is explained by our $13 / 2^{+}$assigrment of this levet.

\section{Footnotes and References}

tondensed from Phys. Lett. 53B, 442 (1975).
Wow at Institut für Kernphysik, KFA JuIich, D-5170 Julich, W. Germany.

*Visitor fran Physik Department, Technische Iniversitat Munchen, D-8046 Garching, W. Germany.

The data also revezled a previousty unreported balf-life of $28 \pm 2$ ns for the $2101 \mathrm{kev} 4^{+}$level in 142 did which was populated by the $\left({ }^{\prime} \mathbf{L}, 4 n\right)$ reaction.

In contrast to the authors of Ref. 4 an $11 / 2^{+}$assigment has bean adopted for this level in a recent compilation, 8

1. E. Nemen, K. S. Toth, and 1, R. Williams, Phys. Rev. C7 290 (1973) and references given therein.

2. E. Newmen, K. S. Toth, D. C. Hensley, and W. D. Echuldt-0tt, Phys. Rev. C9 674 (1974).

3. J. Kownacki, H. Ryde, V. O. Sergejev, and $z$. sujkowski. Phystea Scripta 566 (1972).

4. \$. S. Jpson, W. Booth, and J. G. B. Haigh, Nucl. Phys. A2D6, 114 (1973).

5. J. Gizon ot al., Muxl. Phys. A222, 557 (1974).

6. C. Ekstron, S. Ingelman, $\mathbf{H}$. Olinats and B. Hamberg, Physica Scipta E, 1B1 (1972).

7. c. M. Lederer, J. M. Hollander, and I. Perlman, Table of Isotopes, Sixth Ed. (John Wiley \& Spons Inc., Hew York, London, Sidney 1968) p. 578.

8. L. R. Greemwood, Mret. Data Sheets 12, 139 (1974). 


\section{TRIAXIAL SHAPES IN LIGHT La NUCLEI*}

P A Gutber, J Neyer-ler-Wehin 0 Ward,g $H$ Bertschal,

$P$ Cokombanu, I R M Diamond, and F 5 silephens

The yrast levels of the light odit lanthanm isotopes have been identified ${ }^{1}$ as a decoupled band in which an $\mathrm{b}_{11 / 2}$ proton has its spin maximally aligned with the rutation axis of a prolate core. A rutber of such exprples have since beer observed, but in scme cases it has been shown ${ }^{2}$ that the data available on additiona1 states indicate deviations from axial symtetry. As a test of the triaxial description have magsure lifetimes for the $15 / 2$ and $19 / 2-$ levels in 129,131 La.

Wh have used the recoil-distance prothod to

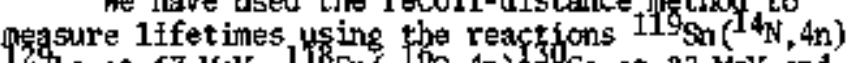

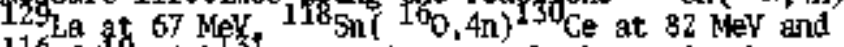
$116 \mathrm{CA}(19,4 n) \mathrm{Lt}_{\mathrm{La}}$ at $72 \mathrm{HeV}$. The heavy-ion beans were provided by the LRL 88-inch cyclotron. The Sn targets were stretched self-supporting foils of 0.75 me in thickness. The CA target was $0.6 \mathrm{mg} \mathrm{cm}^{-2}$ of enriched $\mathrm{Cd}$ evaporated on $0.2 \mathrm{mg} \mathrm{cm}^{-2}$ nickel. Ganna rays mre detected in a planar ó detector placed at [O with respect to the beam direction. For the lowest transitions in the yrast seculence, recoil-distance decay curves were derived by integrating the stopped $\left(I_{S}\right)$ and ropving $\left(I_{t h}\right) \gamma$-ray peaks and plottIng the ratio $I_{g} /\left(I_{S}+1_{11}\right)$ versus plimger setting (Fig. 1). The average recoil velocity was derived from the centroid shifts of stopped and moving $\gamma$ ray components, and after correction for the finito solid angle of the Ge detector was found to be $0.87 \%$

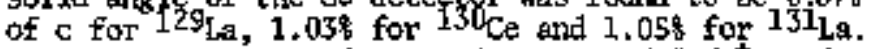
For each nucleus, lifetimes for the $19 / 2^{-}\left(4^{+}\right)$and $15 / 2^{*}\left(2^{+}\right)$levels were obtained by fitting simultaneously the two decay cunves using a least-squares fitting procedure. The behavior of the feeding ahead of the $19 / 2$ state, approximated by tar or more levels whose lifetines were tree parameters could be determited partly by the shape of the $23 / 2-$ $\rightarrow 19 / 2$ decay curve and partly by the constraint that al] the curves pass through a common origin. Where the experinantal relative intensities of the cascade transitions markedly decreased with increasing spin, feeding into the band from other levels was also taken into account. The regults are shown in Table 1. Where it can be seen that for $130 \mathrm{Ce}$, the only case where other measurements are available for coloparison, the present value for the $2 \rightarrow 0$ transition is in excellent agreenent with that measured by Dehnhardt et $91 . \$$

Calculated $B(E 2)$ values for transitions between the lonest yrast states of an $\mathrm{h}_{11 / 2}{ }^{-}$quasiparticle coupled to an asymetric core are show in Fig. 2. For the $11 / 2,15 / 2$, and $19 / 2$ states the total angurar formentum tends to localize about the intrânsfe 2 -axis foblate symetry axis at $\gamma=60^{\circ}$ ). If the total angolar monentur points along the 2 axis the Ez-transition probability can be approxinated by

$$
B(E 2)_{\text {class }} \equiv \frac{5}{16 \pi}\left(Q\left(\frac{\hat{2}}{22}\right)^{2}\right.
$$

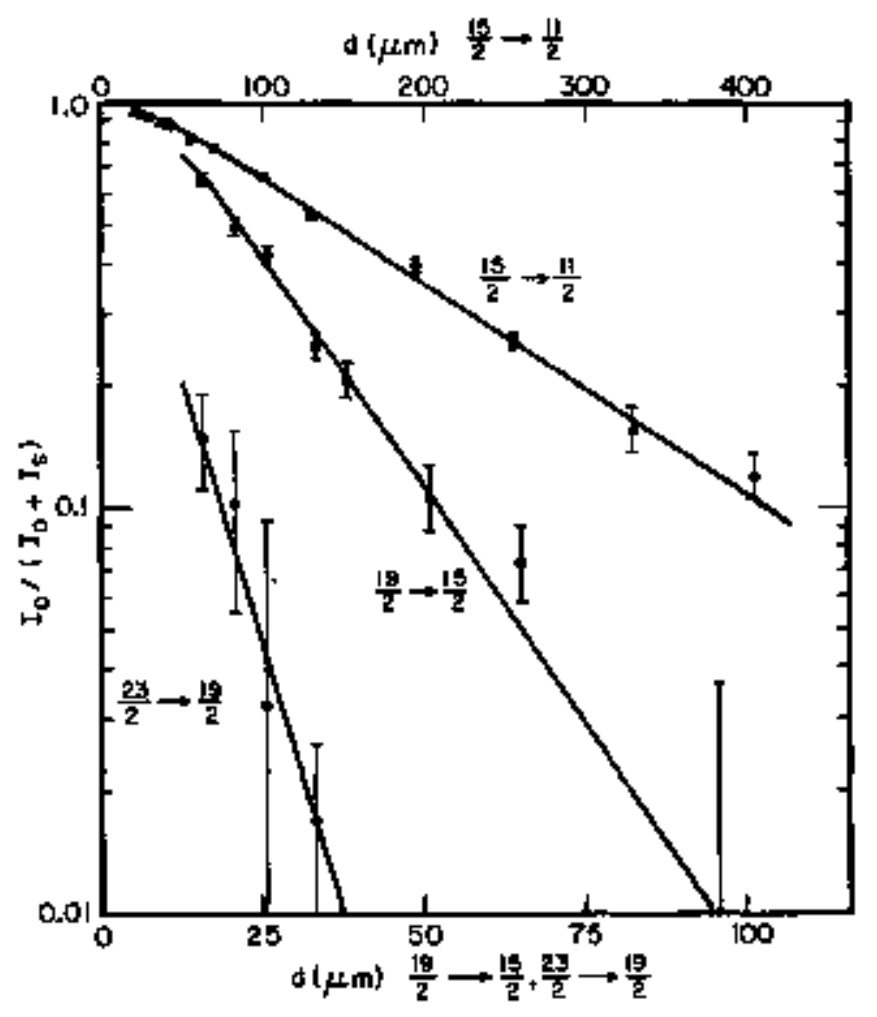

Fig. 1. Rocoil-äistance decay curve for ${ }^{131}$ La. (XBL $753-2430$ )

Where $Q \frac{(2)}{2} \cdot Q_{0} \cdot \sin \left(\gamma-60^{\circ}\right) / \sqrt{2}$ is the $\mu=2$ compoRent of the infrinsic quadropole tensor related to the 2-axts (1ower dashed IIne in Fig. 2). The exactly calculated $\mathrm{B}(\mathrm{E} 2)^{\prime} \mathrm{s}$ for the $15 / 2^{-}+11 / 2^{-}$and the $19 / 2+15 / 2^{2}$ transitions closely follow this classiçal linit over the range $10^{\circ}<\gamma<35^{\circ}$ (Fig. 2). . For states with $I>19 / 2$ the total angytar mosontur of the system tends to align with the 1-axis prolate symetry axis at $\gamma=0^{\circ}$, since this axis has the largest mowent of inertia in the triaxial reglon, and thereby minimizes the core rotational energy. This tendency now doninates over the preference of the particle for the 2 -axis. The $B(E 2)$ 's of higher transitions (e+8+, $27 / 2^{-}+23 / 2$ ) therefore peak near $Y=30^{\circ}$ where $Q\left\{j=0, \sin \left(\gamma+60^{\circ}\right) / \sqrt{2}\right.$ has its maximum (upper dashed line in Fig. 2).

The $B(\mathrm{E} 2)$ values talgulated from the asymotric rotor node1 with $\gamma=22 \quad(129 \mathrm{La})$ and $\gamma=23^{\circ}$ [131 La) are also shown in Table I together with predictions of the symetric rotor model and the weak coupling model. The experinental resuIts for the $15 / 2+11 / 2^{-}$and $19 / 2^{-}+15 / 2^{-}$transitions in $129 \mathrm{La}$

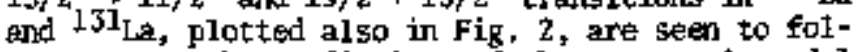
low closely the predictions of the asymetric model. The prediction of weak colpling for the $\mathrm{B}(\mathrm{EZ} ; 15 / 2$ 


\section{$0 \mathrm{~J}: \mathrm{S}$ :}

Table 1. Lifetines and B(E2) values in ${ }^{129}, 131_{\text {La }}$ and their respective core nuclei. The experimental results are compared with predictions from various theoretical models. References are given fin parestheses.

\begin{tabular}{|c|c|c|c|c|c|c|c|c|}
\hline \multirow[t]{2}{*}{ Mucleus } & \multirow[t]{2}{*}{$\begin{array}{c}\mathrm{E}_{\mathrm{y}} \\
\text { (keV) }\end{array}$} & \multirow[t]{2}{*}{$\underset{(p \operatorname{cec})}{\tau}$} & \multirow[t]{2}{*}{$\begin{array}{c}\mathrm{E}(\mathrm{E} 2) \downarrow \text { exp. } \\
\left(\mathrm{e}^{2} \mathrm{~b}^{2}\right)\end{array}$} & \multicolumn{2}{|c|}{$\begin{array}{l}\text { Strong } \\
\text { coupling }\end{array}$} & \multirow[t]{2}{*}{$\begin{array}{c}\text { Weak } \\
\text { coupling } \\
\end{array}$} & \multicolumn{2}{|c|}{$\begin{array}{c}\text { Rotation aligned } \\
\text { (triaxial core) }\end{array}$} \\
\hline & & & & $K=1 / 2$ & $K=11 / 2$ & & $Y=0$ & $Y_{\exp }{ }^{b}$ \\
\hline $\begin{array}{r}{ }_{2}^{128} \mathrm{Be} \\
+0\end{array}$ & 279 & $140 \pm 30(4)$ & $0.33 \pm 0.07$ & & & & & \\
\hline$\underset{2 \rightarrow 0}{130_{\mathrm{Ce}}}$ & 254 & $\begin{array}{l}225 \pm 19(3) \\
211 \pm 99\end{array}$ & $0.33 \pm 0.02$ & & $\because$ & & & \\
\hline $\begin{array}{l}129 \mathrm{La} \\
15 / 2+11 / 2 \\
19 / 2+15 / 2\end{array}$ & $\begin{array}{l}269 \\
475\end{array}$ & $\begin{array}{l}130 \pm 6^{\mathrm{a}} \\
8.7 \pm 1.3^{\mathrm{a}}\end{array}$ & $\begin{array}{l}0.42 \pm 0.02 \\
0.38 \pm 0.06\end{array}$ & $\begin{array}{l}0.53 \\
0.55\end{array}$ & $\begin{array}{l}0.07 \\
0.22\end{array}$ & 0.33 & $\begin{array}{l}0.50 \\
0.52\end{array}$ & $\begin{array}{l}y=22^{\circ} \\
0.37^{\circ} \\
0.42^{\mathrm{c}}\end{array}$ \\
\hline $\begin{array}{r}130_{\mathrm{Ba}} \\
2+0 \\
132_{\mathrm{Ce}}\end{array}$ & $\begin{array}{l}357 \\
325\end{array}$ & $67.9 \pm 9.5[3\}$ & $\begin{array}{l}0.24 \pm 0.08(5) \\
0.32 \pm 0.05\end{array}$ & & & & & \\
\hline $\begin{array}{l}24 \pm \mathrm{La} \\
15 / 2+11 / 2 \\
19 / 2+15 / 2\end{array}$ & $\begin{array}{l}336 \\
533\end{array}$ & $\begin{array}{c}55 \pm 2^{\mathrm{a}} \\
5.8 \pm 0.6^{\mathrm{a}}\end{array}$ & $\begin{array}{l}0.34 \pm 0.02 \\
0.33 \pm 0.03\end{array}$ & $\begin{array}{l}0.49 \\
0.50\end{array}$ & $\begin{array}{l}0.06 \\
0.20\end{array}$ & 0.30 & $\begin{array}{l}0.45 \\
0.47\end{array}$ & $\begin{array}{l}y=23^{0} \\
0.322^{d} \\
0.37^{d}\end{array}$ \\
\hline
\end{tabular}

Present results.

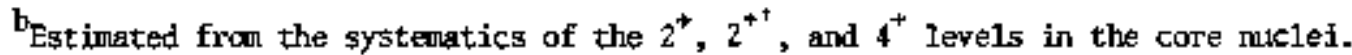

Gadel paraneters: $\beta=A^{2 / 3}=6.8$ derived from $B\left(E Z ; z^{+}+0^{+}\right)$of cor $\theta$ nuclei, Fervi energy $h^{0}\left(\varepsilon_{1}+\epsilon_{2}\right) / 2$ estimated frofl Nilsson level scheme ( $\epsilon_{y}$ s.p. energies of $h_{11 / 2}$ shell), $\Delta=1.0$ Met from bdd-eden mass differences.

Wodel paraneters: $B \cdot A^{2 / 3}-6.4, \lambda_{F}=\left(\varepsilon_{1}+\varepsilon_{2}\right) / 2, A=1.0 \mathrm{NeV}$ derived in the same way as for 129 La. 


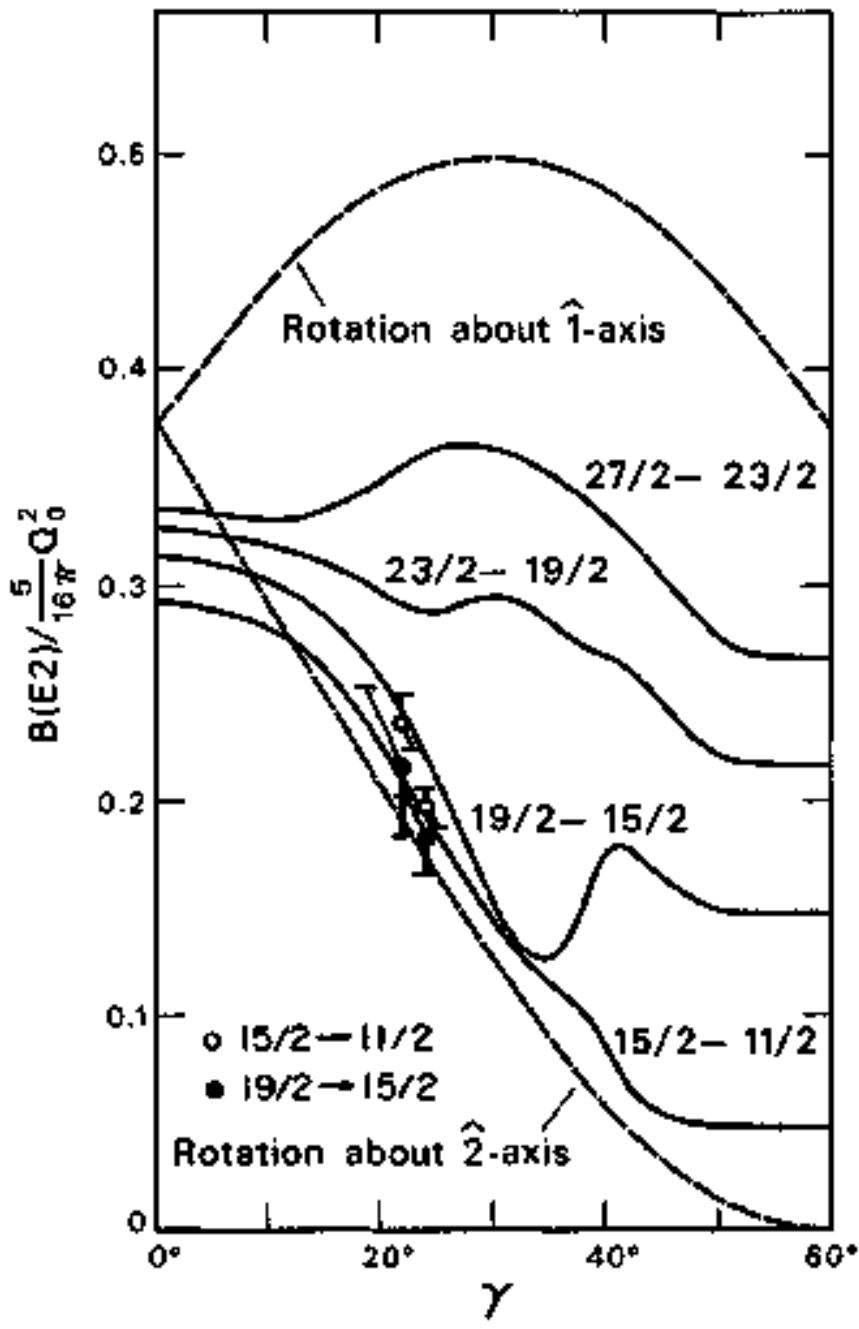

Fig. 2. Caloulated transition probabilities as functions of the core asymetry $\gamma$. The calculations are accurate to wthin about 5 s for a range of $B \cdot A^{2 / 3}$ given by $4<B \cdot A^{2 / 3}<7$ and a range of $\lambda_{F}$ given by $\varepsilon_{1}<\lambda_{F}<\varepsilon_{2}$. The broken lines give classical linits for $B(E 2)$. Show also are the experimental date for $129 \mathrm{La}\left(\gamma=23^{\circ}\right)$. The transition probabilities are givepn in terms of $\frac{5}{16-\pi} 0_{0}^{2}$ is given

$$
Q_{0}=\frac{3}{\sqrt{5 \pi}}(1.2)^{2} 2 B A^{2 / 3}(f n)^{2} .
$$

(XPL 751-4646)
- 11/2 ${ }^{+}$) values are next best, but they differ sig" nificantly from the experinental results, being sys teratically smallex in magnitude, The lack of precise experimental data on the core $4+2$ transition makes further quantitative coluparison with the weak coupling model difficult, but with the additional assingtion of a simple rotatlonal or vibretional core one would obtain a value of respectively 1.4 and 2.0 for the ratio $\mathrm{B}\left(\mathrm{E} 2{ }^{\circ} ; 19 / 2^{-} \rightarrow 15 / 2\right)$ : B(E2; $15 / 2^{-}+11 / 2^{-}$) in contradiction to the experimental evidence.

\section{Fontmotes and References}

"Condensed from Phys. Lett. 56B, 453 (1975).

†t.K. S.R.C./N.A.T.O. Ferlor.

*Persanent address: TU Minchen, 8046 Garching, West Gemeny.

${ }^{5}$ Pejmanent address: Chalk River Nuctear Laboratories, Ontario, Canada.

D.A.A.D. Fellow; perminent address: Hahn-NfeituerInstitut, BerIin, West Germary.

"Permanent address: Inștitut de Physique Nucléaire, 91406 Orsay, France.

1. J. R. Leigh, K. Naka1, K. H. Maler, F. Ptihlhofer, F. S. Stephens, and R. H. Djambond, Nact. Phys. A213, 1 (1973); F. S. Stephens, R. M. Diamond, J. R. Leigh, T. Kammuri, and R. Makai, Phys. Rev. Lett. 29, 438 (1972).

2. J. Meyer-ter-Vehm, F. S. Stephens, and R. M. Dizatond, Phys. Revr. Lett. 32, 1383 (1974).

3. If, Dehwharit, S. J. Mills, M. Mtiler-Veggian,

U. Netntarin, D. Pelte, G. Poggi, B. Povh, and

P. Taras, Nuc1. Phys. 2225, 1 (1974). 


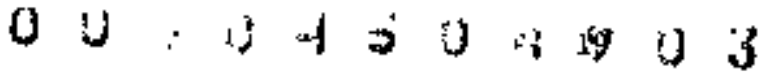 \\ HIGH-SPIN STATES IN ${ }^{127}$, 129 La; A TEST OF BACKBENDING IN THE EVEN Ba AND CE NUCLEI*}

D Ward. $+\mathrm{H}$ Bernschat, $\mathrm{P}$ A Buller, P Colombanı,

$R$ M Diamond, and F S Stephens

The rotation-aligned coupling schemel has had considerable success in describing the collective bands in a muber of weakly deformed odd-mass nuclei. It has also been pointed out that the presence of backbending in an odd-mass rare-earth nucleus is an immortant clue as to the cansse of backbending in the adjacent doubly-even nuclei. If a particular rotation-aligned particle delays or prevents the occurrence of backbending in the ad-mass case, it is likeIy that this particle is urvolved in the structure of the band whose intersection with the ground band couses the backbending in the doubly-even: nucleus. 2 Conversely, if the odd-particle band does backbend, this particle must not be such as inportant component. $\$$ Application of this idea has showh the importance of $i_{13 / 2}$ neutrons in the backbending behavior observed in the 1ighter deformed muclei $\mathrm{Dy},{ }^{4} \mathrm{Er},{ }^{2}$ and $\mathrm{Yb}^{5}$. Very recently the inportance of rotation-alisned $\mathrm{h}_{\mathrm{g} / 2}$ protons in the backbending of the heavier deformed $\mathrm{Hf}, \mathrm{W}$ and $0 \mathrm{~s}$ netci hos been demonstrated by studying $167_{\mathrm{L} 4{ }^{6}}$ and $18 \mathrm{I}_{\mathrm{Re}}, 181,1830 \mathrm{Os}$ ?

In this paper we repart a study of the highspin decoupled states of $127,129 \mathrm{La}$ from which we can draw conclustons concerning the causes of the backbending observet in the neighboring $\mathrm{Ba}$ and $\mathrm{Ce}$ muclei, Previous studies of high-spin states in I26, $12 \mathrm{Ba}^{3}$ and $13 \mathrm{O}_{\mathrm{Ce}} 9$ have shown that the ground bands backbend around spin $10^{+}$. Thiscorresponds to a spin of $31 / 2^{-}$in the $11 / 2^{-}$band in La, but the earlier mork on $127,129 a^{1}$ only assigned levels as high as $27 / 2^{-}$. In the present work we have identified Ievels to spin $35 / 2^{\circ}$, just beyond the critical region. To obtain as conplete a camparison aspossible, we have also studied the ground band in $12{ }^{\circ} \mathrm{Ce}$, since it was previously momli only to spin $8^{+}$.

The experiments were performed at the LBI 83 inch cyclotron, using the reactions $117 \mathrm{G}\left(14 \mathrm{~N}, 4 \mathrm{n}_{\mathrm{r}}\right)$

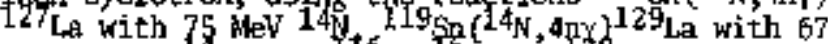
end $75 \mathrm{MeV} 14_{\mathrm{N}}$, and $116_{\mathrm{Sn}}\left(16_{0}, 4 \pi y\right)^{12} \mathrm{Ce}$ with $88 \mathrm{MeV}$ 160. The targets were made by evaporating the $t i n$ isotope $t o \sim 50 \mathrm{mg} / \mathrm{om}^{2}$ of lead. Gamula rays were detected both in-singles and in coincidence mode using two 45 cc coaxial Ge(Li) detectors. The $0^{\circ}$ to $90^{\circ}$ anisotropy of the $\gamma$-rays was measured to test the main cascade sequences were indeed stretched $\mathbf{E 2}$ trarsitions.

Typical coincidence spectra for ${ }^{129} \mathrm{La}$ are shown in Fig. 1, and the cascade secuences deduced from individual gates set on the known ground-band

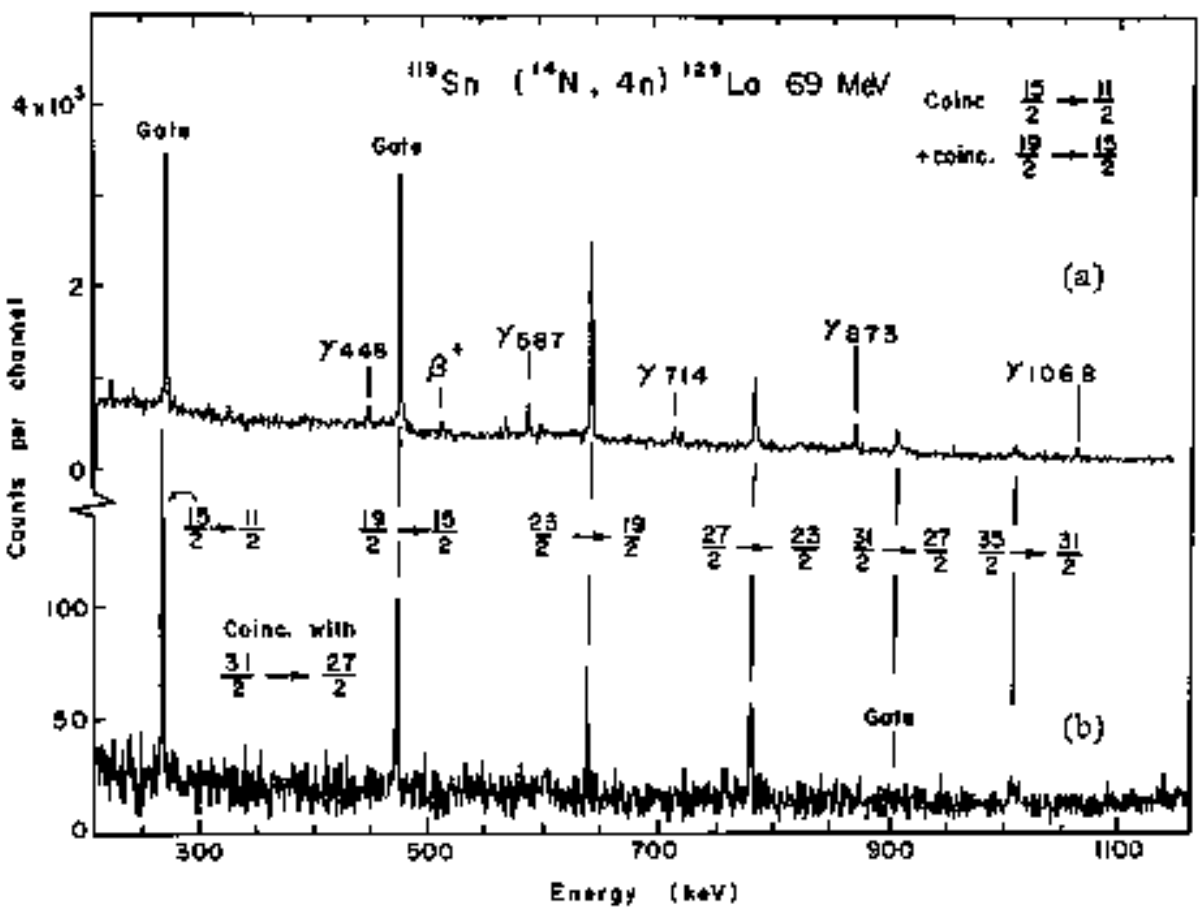

Fig. 1. Coincidence spectra for 129 La, spectrup (a) is for the sun of gates set on the lowest two rotational transitions, (b) is for the $31 / 2+27 / 2$ transition. Spectra corresponding to background events have been subtracted in an appropriate way.

(XAl 7412-8448) 
transitions are sumbrized in Table 1. The Az values derived frcm the anisotropy measurents assuming $A_{4}=-0.05 \pm 0.05$ are consistent with stretchet E2 assignitents (Table 1). The levol scherues are in Fig. 2 .

To conmare the systematics of the core states in $127,129 \mathrm{La}$ with those of the corresponding $\mathrm{Ba}$ and Ca nuclei we have calculated the moments of inertia, Fand the rotational frequencies, w, according to the formulae given in [3]. PIots of $6 \mathrm{vs}$. $w^{2}$ are show in Fig. 3. It can be seen that the $11 / 2$ decoupled band in $\mathrm{La}$ does not backbend, even for rotational frequencies appreciably higher than those for which the Ea and Co nuclei do backberd. This fact suggests that a rotgeion aligrnent of two sich $h_{11 / 2}$ protons is involved in the backbending in the even barîum and cerìn תuklei. In fact it seems likely that the backteending is caused by an intersection of the groumd band with a band conmosed of two bilo grotons axinally aligned with the rotation axis. It is of interest not only that the backbenking is caused by protons rather than netstrons in this region, but also that it is nost likely twe to a rotation-aligpment effect like that observed in the rare-earth region, rather then to a shape change as is the case for the netutron-deficient Hoclei. It appears that the technique of stuntying an even-even case by observing its behavior with various particle orbits blocked, is applicable to this Ba-Ce region, as well as to the deformed rarenarth region, and can probably be applied rather generally.

Table 1. Silmary of the rotational cascades in 127,129 La and 528 Ce observed in this experiment. Assigments in la nuclei to spin $27 / 2^{-}\left(8^{+}\right)$have been made previously 10 Values for $126,12 \mathrm{~s}_{\mathrm{Ba}}$ and $130 \mathrm{Ce}$ are from previous work; $128_{\mathrm{Ce}}$ assignments ${ }^{8}, 9$ to spin $8^{+}$have been made previously. 11 Uncertainties in the relative intensities and $A_{2}$ coeffictents are shown in parentheses.

\begin{tabular}{|c|c|c|c|c|c|c|c|}
\hline \multirow{2}{*}{$\frac{\text { Spin }}{I \text { (or } I-11 / 2}$} & \multirow{2}{*}{$\frac{{ }^{128} \mathrm{ka}^{\mathrm{a}}}{\mathrm{E}_{\mathrm{y}}(\mathrm{keV})}$} & \multicolumn{3}{|c|}{${ }^{129}{ }_{12 \mathrm{~A}}(75 \mathrm{MeV})$} & \multirow{2}{*}{\multicolumn{2}{|c|}{$\frac{130 \mathrm{Ce}^{\mathrm{b}}}{\mathrm{E}_{\mathrm{Y}}}$}} & \\
\hline & & $\mathbf{E}_{\gamma}$ & Int & $A_{2}$ & & & \\
\hline 2 & 284 & 269.3 & 100 & $0.35(3)$ & \multicolumn{3}{|c|}{254.1} \\
\hline 4 & 479 & 474.8 & $81(8)$ & $0.32(3)$ & \multicolumn{3}{|c|}{456.7} \\
\hline 6 & 644 & 641.9 & $55^{\circ}(16)$ & $0.35(3)$ & \multicolumn{3}{|c|}{614.2} \\
\hline 8 & 782 & 785.6 & $29(3)$ & $0.31(4)$ & \multicolumn{3}{|c|}{729.6} \\
\hline 10 & 894 & 910.5 & $11^{c}(2)$ & $0.26(15)$ & \multicolumn{3}{|c|}{757.0} \\
\hline 12 & 906 & 1013.5 & $8^{C}(2)$ & $0.02(30)$ & \multicolumn{3}{|c|}{503.3} \\
\hline 14 & & & & & \multicolumn{3}{|c|}{549.0} \\
\hline 16 & & & & & \multicolumn{3}{|c|}{$693 . S$} \\
\hline Spin & $126_{\mathrm{Ba}} 8$ & \multicolumn{3}{|c|}{$127 \mathrm{La}(76 \mathrm{MeV})$} & \multicolumn{3}{|c|}{${ }^{128} \mathrm{Ce}(88 \mathrm{MeV})$} \\
\hline I $\cos I-11 / 2$ & $\mathrm{E}_{Y}(\mathrm{kel})$ & $\mathbf{E}_{\gamma}$ & Int. & $A_{2}$ & $\mathbf{E}_{\gamma}$ & Int & $A_{2}$ \\
\hline 2 & 256 & 252.4 & 100 & $0.19[3]$ & 207.3 & 100 & $0.26(3)$ \\
\hline 4 & 455 & 458.6 & $73(7)$ & $0.28(3)$ & 399.6 & $88(9)$ & $0.23(3)$ \\
\hline 6 & 621 & 630.8 & $48(5)$ & $0.23(3)$ & 550.6 & $8 B(9)$ & $0.32(3)$ \\
\hline 8 & 757 & 780.3 & $31(4)$ & $0.13(4)$ & 662,6 & $50(5)$ & $0.26(4)$ \\
\hline 10 & 852 & 908 & $8(2)$ & $0.10(15)$ & 711,1 & $33(3)$ & $0.31(6)$ \\
\hline 12 & 805 & 1003 & $\$(1)$ & $0,16(30)$ & 576.5 & $28(3)$ & $0.25(6)$ \\
\hline 14 & 672 & & & & 560.6 & $22^{\mathrm{C}}(2)$ & $0.24(6)$ \\
\hline 16 & 825 & & & & 689.9 & $14^{c}(z)$ & $0.38(10)$ \\
\hline
\end{tabular}

a. Ref. 8 .

b. Ref. 9 .

c. Not single lines in the direct spectra. Relative intensities taken from spectra in colncidence with the $2^{+}+0^{+}\left(15 / 2^{-}+11 / 2^{-}\right)$transitions. 


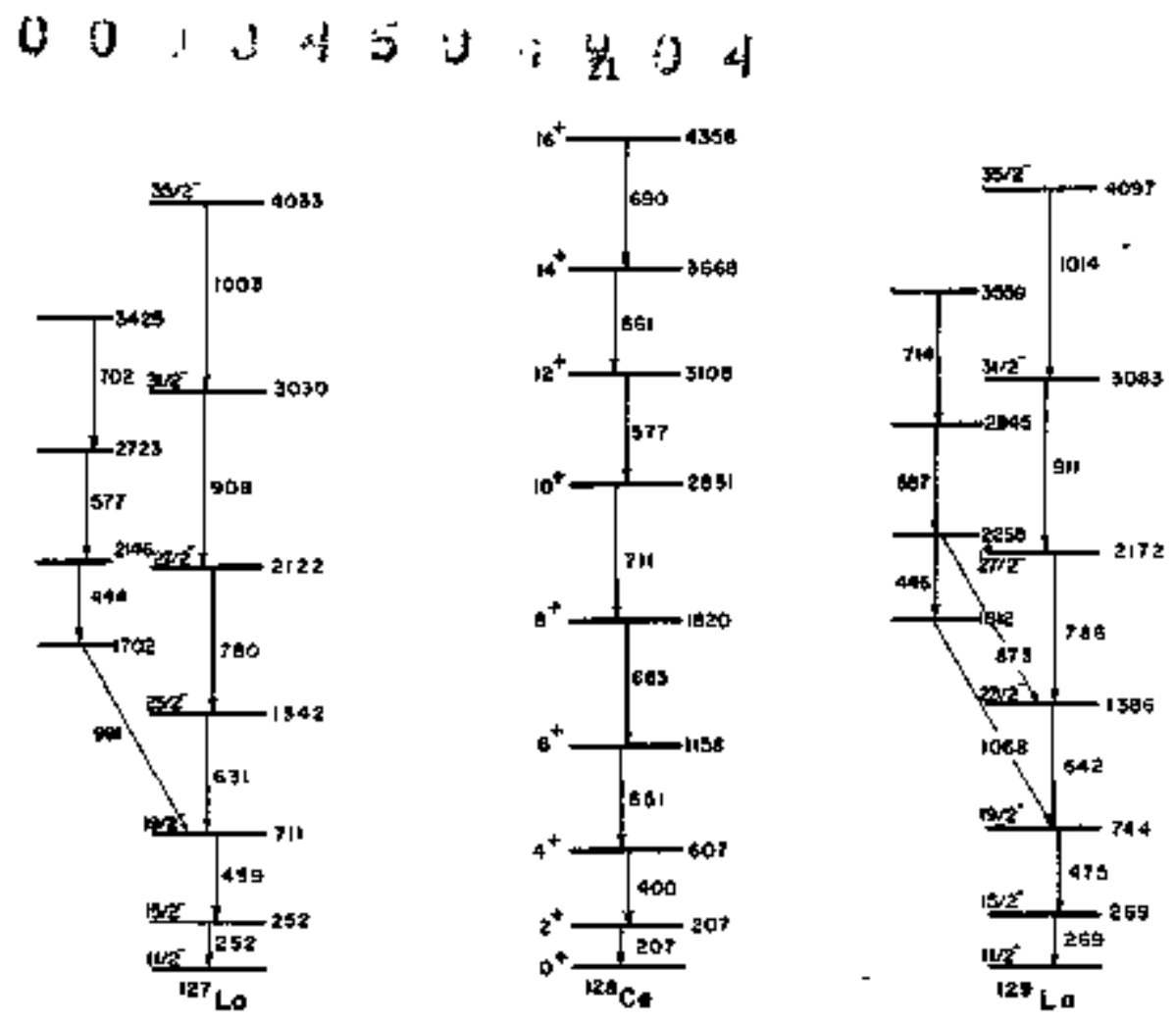

Fig. 2. Level schene for ${ }^{127,129} \mathrm{La}$ and ${ }^{128_{\mathrm{Ce}}}$. (XBL, 7412-8447)

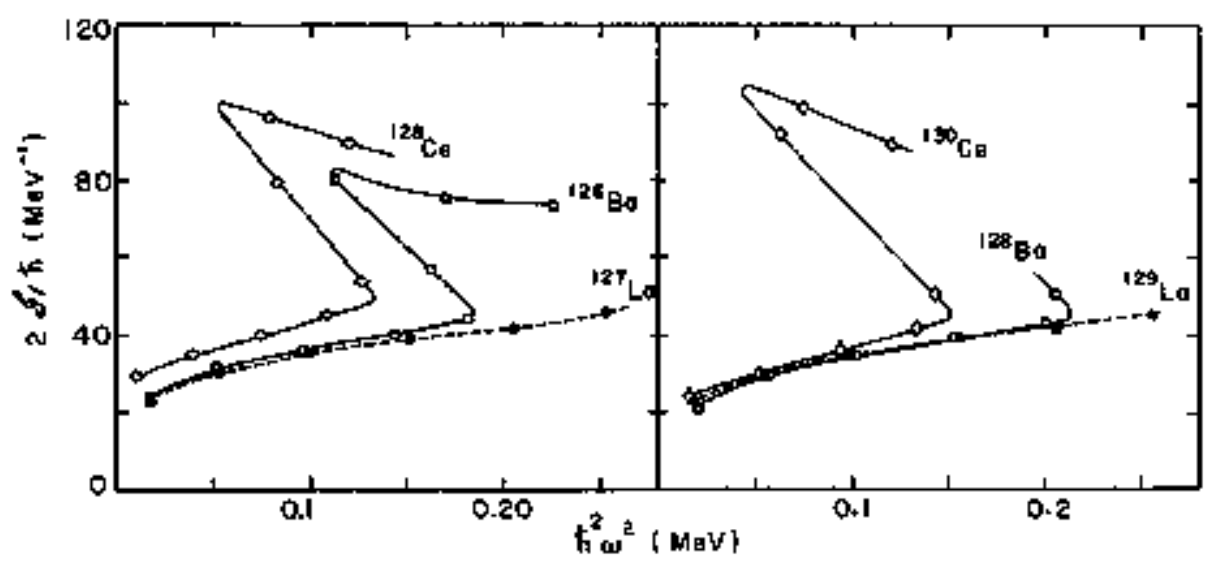

Fig. 3. Comparison of backbending plots for dore atatas in 127,129 La with adjacent oven muctei.

(XBL 7412-8446)

\section{Footnotes and References}

*Condensed from Phys. letters 565, 139 (1975).

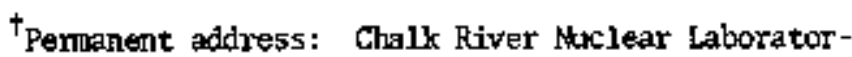
ies, Ontario, Canada.

FDAAD-Fellow; pemanent address: Hahn-Institut, Berlin, Geriany.

${ }^{5}$ Penmanent address: Institut de Physique Nucléaire, 91406 Orsay, France.

1. F. S. Stephens, R. M. Dianond, and S. G. Nilsson, Phys. Lett. 44B, 429 (1973).
2. E. Grosse, F. S. Stephens, and R. M. Dianomd, Phys. Rev. Lett. 31, B 40 (1973).

3. E. Grosse, F. 5. Stephens, and R. M. Diamond, Phys. Rer. Lett. 32, 74 (1974).

4. W. F. Iavidson, R. M. Lieder, H. Betscher, A, Neskakis, and C. Mayer-Boricke, Proceedings of the Intermational Conference on Reactions between Con-. plex Nuclei, eds. R. L. Robirison. F. K. KeCowan, J, B, Ball, and H, J. Hanil ton (North-Holland, Austerian, 1974) Vol. 1, p. 190 .

5. L. L. Riedinger, G. J. Sigith, P. H. Stelson, E. Eichler, G. B. Hagentam, D. C. Hensley, N. R. Johnsan, R. L. Robinson, and R. 0. Sayer, Phys. Rev. Lett. 33,1346 (1974). 
6. C. Foin and D. Barneoud, Phys. Rev. Lett. 33, 1049 (1974).

7. W. F, Davidsn, R. M, lieder, H, Bequschor, A. Neskakis, and C. Mayer-Bórtcke, preprint. International Conference on Nuclear Structure and Spectroscopy, Ansterdam, 1974.

8. C. F1aum, D. Cline, A. H. Suryar, and D. C. Kistner, Phys, Rev, lett. 33, 975 (1974).
9. D. Hard, H. R. Andrews, G. J. Costa, J. S. Geiger, R, L, Grahem, and P. Taras, Anerican Phys. Soc. Bull. 19, $502(1974)$.

10. J. R. Leigh, K. Nakai, K. H. Maier, F.

Pihuthofer, F. \$. Stepherts, and R. M. Diamond, Nucl. Phrs. A213, 1 (1973).

11. D. What, R. M. Dianond, and F. \$. Stephens, Muct. Phys. Al17, 309 (1968).

\section{REOUCED TRANSITION PROBABILITIES FOA HIGH SPIN STATES IN ${ }^{232}{ }^{2}{ }^{*}$}

M W Gudry, $P \wedge$ Buller.t $P$ Colombent, I Y Lee, D Wiard,

R M Guamond, F S Stephens, E Eichler, S N R Johnson.s and R Sturm

The measurement of lifetines, or equivalently, reduced transition probabilities, for high-spin states is of considerable interest because these measurenents provide a stringent test of muclear models. These lifetimes normally lie outside the range of comventional electronic nethods, bat are of ten amenable to determinations by three complementary techniques: 1) the Doppler-shift recoil-distance method (RD), 2) the Doppler-broadened lineshape method (DBLS), and 3) multiple Coulconb excitation methods (COILEX). We have applied a variety of these methods to measurement of the 1ifetipes and reduced transition probabilities for spins $6^{+}-18^{+}$of the ground state rotational band in $232 \mathrm{Th}$.
In Fig, 1 the rehuced transition probability

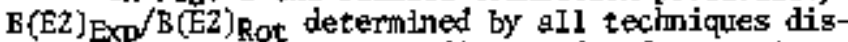
cussed in this report are displayed, Por cantleteness we have also plotted ratios from previously plablished recoil-distance measurements with ${ }^{40}$ Ar profectiles. 1 The matual consistency of the results and their agrement with the rotational model is striking. In Fig. 2 we plot the weighted average of all B(G2) ratios determined by lifetine messurements in the present work. For all states that have been studied, the average $B(E 2)$ values are those of a good rotor, within the linits of experinental uncertainty.

Insofar as was practical, the various aralyses

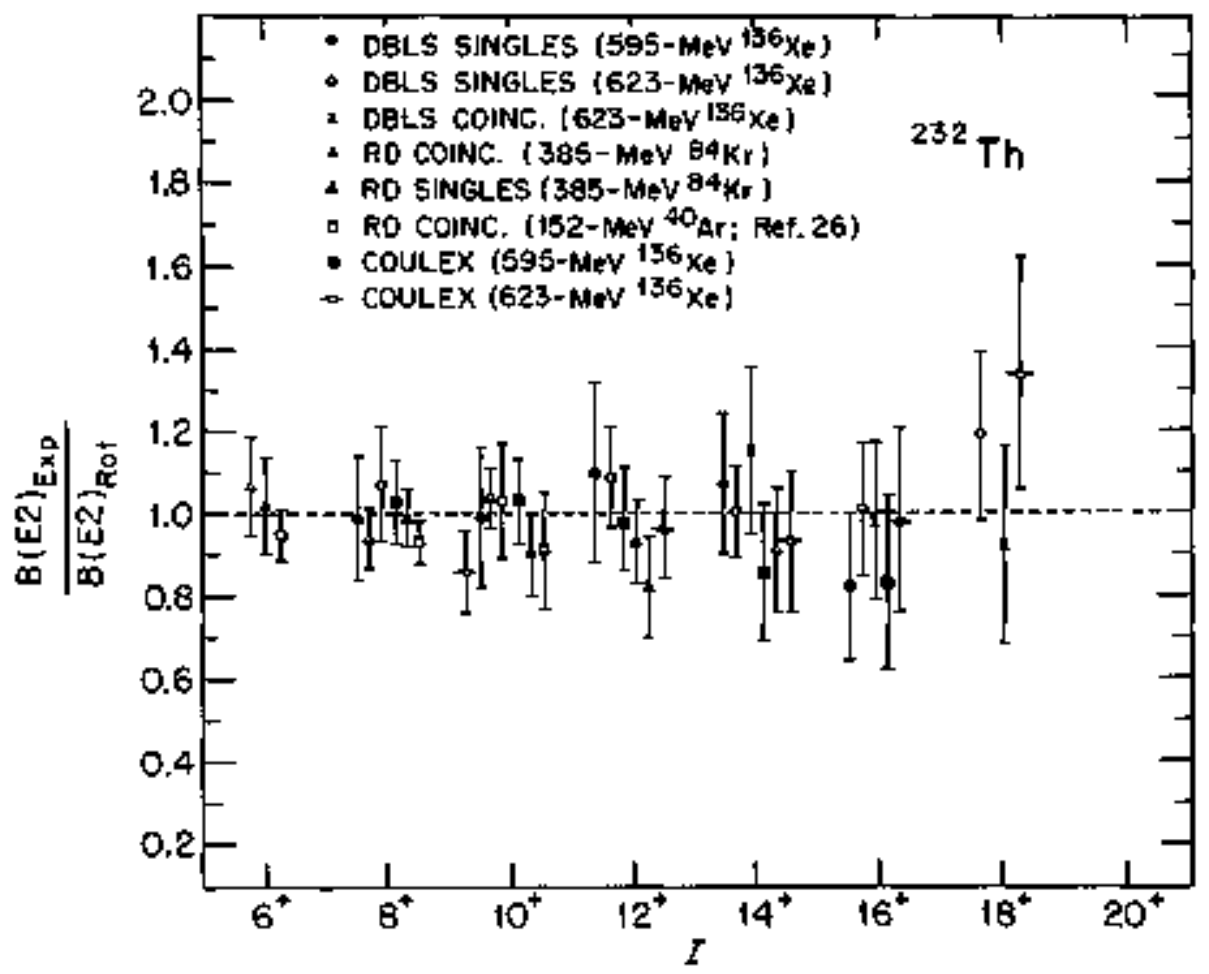

Fig. 1. Values of the ratio $6(E 2)_{\text {exd }} / B(E 2)_{\text {rot }}$ for states in ${ }^{232}$ Th determined in this experiment and in previous (XOL) 765-1804) 


\section{$00 ! 430 \div 403$}

23

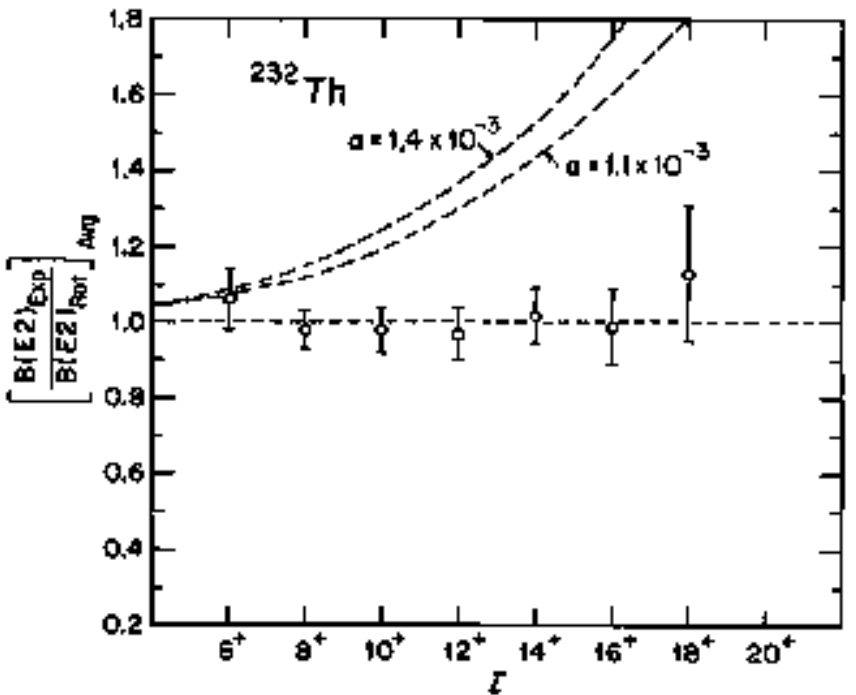

Fig. 2, Weighted average of the ratio $B(E 2)_{\exp }$ $B(\mathrm{E} 2)_{\mathrm{Tpt}}$ determined fram $\mathrm{RD}$ methads ${ }^{84}{ }_{\mathrm{Kr}}$ projectiles in backscatter coincidence and in singles), and from the DBLS experinent ( $136 \%$ projectiles in singles and backscatter coincidence rodes). The dashed 1 ines represent the $B(E 2)$ ratios expected from simple centrifugal stretching and B-bound wixíng models, as discussed in the text.

(KBL 765-1803)

described hero were carried out independently. There fore the results displayed in Figs. 1 and 2 provide criteria for judging the ourtual consistency of the methods employed, wich is seen to be puite good.

Phenomenologically, the mowent-of-inertia for deformed nuclei is known to be spin dependent. 2 The variation of the manent-of-inertis with angular momentum manifests itsolf in the rotational energy23ye1 spacings. Thus the energy of the $16^{+}$state in 232 Th is $\sim 158$ below that of the rigid-rotor prediction using the energy of the $2^{+}$state. The simboth deviations of the energy levels Eram those of a pure rotor have generally been described with a formalism tinploying some conblnation of 4th-order cranking, Corfolis anti-pairing, centrifugal stretching, or band-mixing calculations. These suggest that the monent-of-inertia depends both on the pairing and the deforation, while we expect the transition probabilities, to first order, to be sensitive to the deformation only. Therefore non-rotetioneI energy spacings need not be attended by non-rotational transition probabilities.

If one considers a 1st-order band-mixing modeI, the $\mathrm{B}(\mathrm{ES})$ values for the grotind bard can be written as $^{3}$

$$
\begin{aligned}
& B(E 2, I+I-Z)=E(E 2, I+I-2) \text { fot } \\
& \left\{\frac{1+1 / 2 a I+1)+(I-2)(I-1)}{1+3 \alpha}\right\}^{2}
\end{aligned}
$$

The paraneter a may be spectfied if explicit așumptions are made about the națure of the bands being mixed into the ground band, 3 Thus, if we assume the rixing of a pure $\beta$-vibration into the ground band, the parameter $\alpha$ is fixed by two E2 matrix elements connecting the ground and $\mathrm{B}$-bands. Using $\mathrm{B}(\mathrm{E} 2$, $\left.a_{\mathrm{g}} \rightarrow 2_{\theta}\right)$ and $\mathrm{B}\left(\mathrm{E2}, \mathrm{2}^{2} \rightarrow \mathrm{0}_{6}\right)$ of Mc(Bom, et al. ${ }^{4}$ we

Sirdilarly, if one attributes deviations of the ground band moment-of-inertia to centrifugal stretching, $\alpha$ may be approsimated from the energy spacings in the groumd band, 3 and has a value in this case of $c$ of $1,4 \times 10^{-3}$. In Fig. 2 plot the B(E2) ratios expected fron lst-order wixing of the ground and $B-$ bands using values of a deternined from the groundto-B matrix elements and from the energy spacings. Clearly neither curve is consistent with the experjomental data.

The failure of the curve deteranined from the centrifugal-stretching madel to fit the lifetime data is not difficult to understand, since the monent-of -inertia is sensitive to other than centrif ugal-stretching effects. For instance, alterations in the pairing interaction brought about by coriolis anti-pairing effects will have sipnificant effect on the mament-of-inertia, but should have Iittie influence on the nuclear deformention and on the transition probabilities, which are deternatned primarily by the deformation. In addition one sust consider the possible admixture of bands other than the $B$. band, such as higher-lying $K=0$ bands and the $K=2$ $\gamma$-band, into the ground band.

The a parameter derived from the matrix elements is less model-dependent than that derived fros the energy spacings since it is related directly to transition probabilities between the ground and higher-lying band, In Ref. 4 it is conctuded that the lowest excited $0^{+}$level is of $\beta$-vibrational char. acter. The matrix elements used to derive only comect the lowest two members of the ground and $6-$ bands. Therefore, our results are consistent with adnixture of a pure B-bard at low spins since the gall deviations of the $B(E 2)$ from rotationd values at low sping lie within experimental uncertainty. At higher spins olir results are incospatible with contimued adinixture of a single band with the same characteristics as that detemined by the unatrix elements of Ref. 4.

We may use Eq. (1) and the experinemital $\mathrm{B}(\mathrm{P} 2)$ values phenomenologically to set upper limit of $|a|$ s $2 \times 10^{-4}$ for the higher-spin region. Asstering the collective B(E2) values to depend only on the nuclear shape we have ${ }^{3}$

$$
\frac{A B_{2}(J)}{B_{2}(0)}=[I(1+1)
$$

and on the basis of the present data we conclude $\Delta \beta_{2}\left(16^{+}\right) / \beta_{2}(0)<0.06$. This lack of softmess against further quadrupole deformation for welldeformed nuclei is consistent with the behevior of many rare-earth muclei in recent deformed shellmodel calculations.

\section{Footnotes and References}

* Cordensed from LBL 4038, Nucl. Phys. A to be published). 
†U.K. S.R.C./N.A.T.O. Fellow.

${ }^{\text {}}$ Permanent address: Institut de Fhysique Nuclequire, 91406 Orsay, France.

*Preșent addresక̧: Chalk River Mkc1ear Laboratories, Dntario, Conada.

${ }^{5}$ Cak Ridge National Laboratory, Dak Ridge, Temmesses.

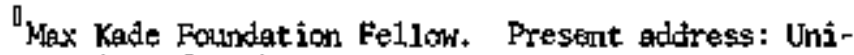
versity of Marburg, Marburg, Гenzany.

1. N, R. Johnson, R. J. Sturth, E, Eichler, M, H, Guidry, G. D. O; Kelley, R. O. Sayer, D. C. Hensley, N. C. Singhal, and J. H. Hamilton, Phys. Rev. C 12,
1927 (1975),

2. M. A. Mariscotti, G. Scharf-Goldhaber, and B. Buck, Phys. Rey. 178, 1864 (1969).

3. R. M. Dianond, G. D, Synons, J. L, Quebert, K. H. Maier, J. R. Leigh, and F. S. Stephens, Mucl. Phys. A184, 481 (1972).

4. F, K. McGowar, C. E. Benis, Jr., W. T. Milnọr, J. L. C. Ford, ఫr., R. L. Robinson, and P. H. Stelson, Phys. Rev. C 19, 1146 (1974).

S. R. Bengtsson, S. E. Larsson, G. Leander, $P$. Miller, S, D, Nilsson, S. Aberg, Z, Szytanșiki, Preprint, Jue 1975.

\section{COULOMB EXCITATION OF HIGH-SPIN STATES IN ${ }^{23{ }^{\circ}} \mathrm{U}^{*}$}

E Grosse, $\uparrow$ de Bder,t R M Diamond.

$F$ S stephents, and $P$ TJoms

The strong electronagnetic interaction produced in the encounter of a deformed target nuxleus by a heavy projectile excites high-spin states in the ground-state rotational hand and in rotational bands buift on collective states colpled to the ground-state band. Such miltiple Caulonb excitation is a powerful tool for Investigating collective nuclear properties at high rotatima] frequencies, of special interest are the rotational bands of the strongly defomed actinide nuclot, which cannot be studied by (HI, XrY) reactions due to the strong fission cappetition. We have studied levels in the groud-state and octupole-vibrational bands of $238 \mathrm{u}$ excited by $\mathrm{kr}$ and Xe ions up to $\mathrm{I} \sim 20 \mathrm{~h}$,

Thitck metallic targets, enriched in $238 \mathrm{~L}$, were bombarded at the LBL sumerHIAC with bears of ' $84 \mathrm{Kr}$ ( $385 \pm 5 \mathrm{MeV}),{ }^{6} 6_{\mathrm{Kr}}(394 \pm 6 \mathrm{MeV}), 132 \mathrm{Ke}(605 \pm 20 \mathrm{MeV})$, and $136 \mathrm{Ke}(640 \pm 40 \mathrm{MeV})$, The decay $\mathrm{r}$-rays were observed by two ( 40 cc) coaxial Ge(Li) detectors, at $\theta_{Y}=0^{\circ}$ and $\theta_{Y}=90^{\circ}$ with respect to the beam direction and 4-5 con from the target. Singles, $Y$-backscattered projectile and $\gamma^{-\gamma} \gamma$ coincidences were simultaneously stored. The low-energy portion of the singles $\gamma$-ray spectnm and the $\gamma-\gamma$ coincidence spectrul is show in Fig. 1 for the $136_{\text {Xe borbard- }}$ ment. Almost alt the tines stown here betong to the

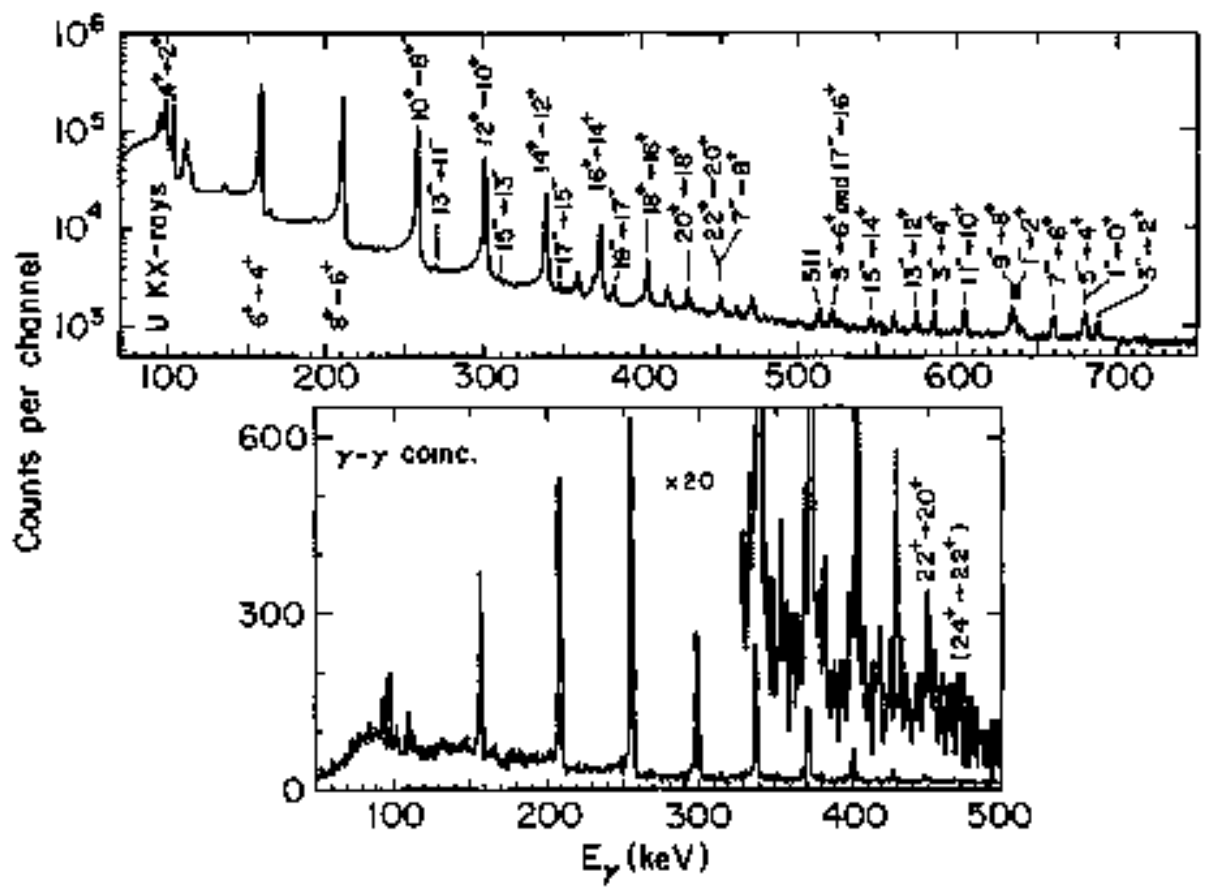

Fig. 1. Portions of the $\gamma-r a y$ singles spectrum (top) and $\gamma-y$ coincidence spectrnm (botton) from a thick $236 \mathrm{~J}$ target Coutan excited with $640 \mathrm{Mov} 136 \mathrm{x}$ ions. The $\gamma-Y$ coincidence spectrum is the sun of spectra gated by the $6-4,8-6,10-8,12-10$, and 14-12 transitions. 


\section{$00 ; 3$ a $50 ; 006$

gEgund-ștate and lowest-energy actumpole bands of

230u. The resulting decay schere is shown in Fig. 2 .

The moments-of-inertia of the ground and octupole bards are shown as a function of the sulare of the rotational frequency in Fig. 3a. This type of plot corresponds to that ienerally made to display beckbending behavior of nupigi in the rare-earth region. The ground band of 238 does not backbend up to spin 22 (tentatively 24) even though the nomentof-inertia increases by $\sim 508$. It is obvious that the octupole band has a 1arger apparent moment-ofinertia than the ground band, and behaves in a scolowhat unusial manuer with increasing rotational frequency. The nature of this band has been of interest for some time. The very general occurrence of collective octurpole vibrational states, having a frequency to $* 15 / \mathrm{A}^{1 / 3} \mathrm{MeV}$, is $11 \mathrm{known}$ and the sharp drop in energy of components of this state at the begining of the rare-earth and expecially the actinide deformed regions is understood and has been qualitatively reproduced in recent calculations 1,2 Four low-1ying ostupoile bands are expected in $23 \mathrm{~B}_{\mathrm{U}}$ having $k$ values $0,1,2$, and 3 , and three collective negative-perity bands are know ${ }^{3}$ below $1200 \mathrm{keV}$ which day correspond to the $K=0,1$, and 2 bands. The lowest observed band is the one shown in Fig. 2 , which has (initially) a predominant $K$ value of zero. There are at lenst three plansible types of behavior for these octupole bands at higher rotational fre-

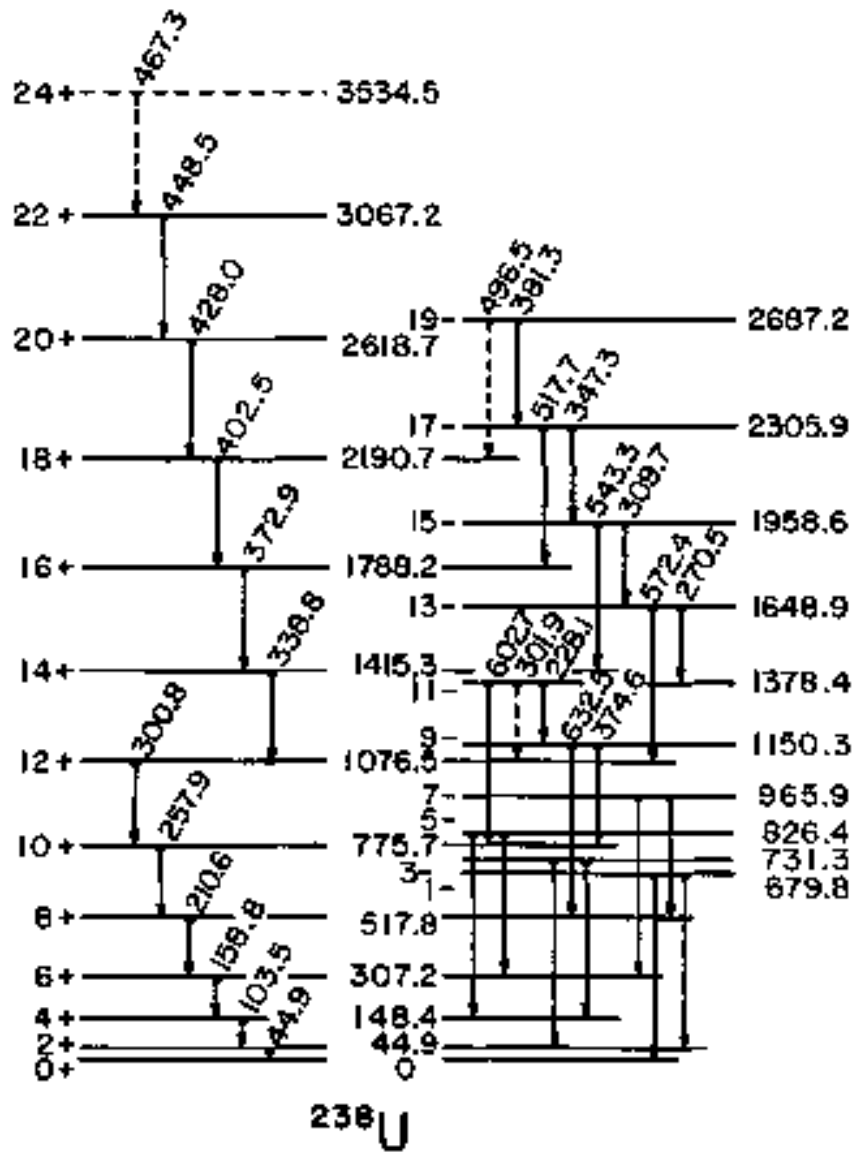

Fig. 2, Leve1 scheme for the ground and 1owestenergy octumole bands of $238 \mathrm{U}$.

(XBL $755 \cdot 30383$

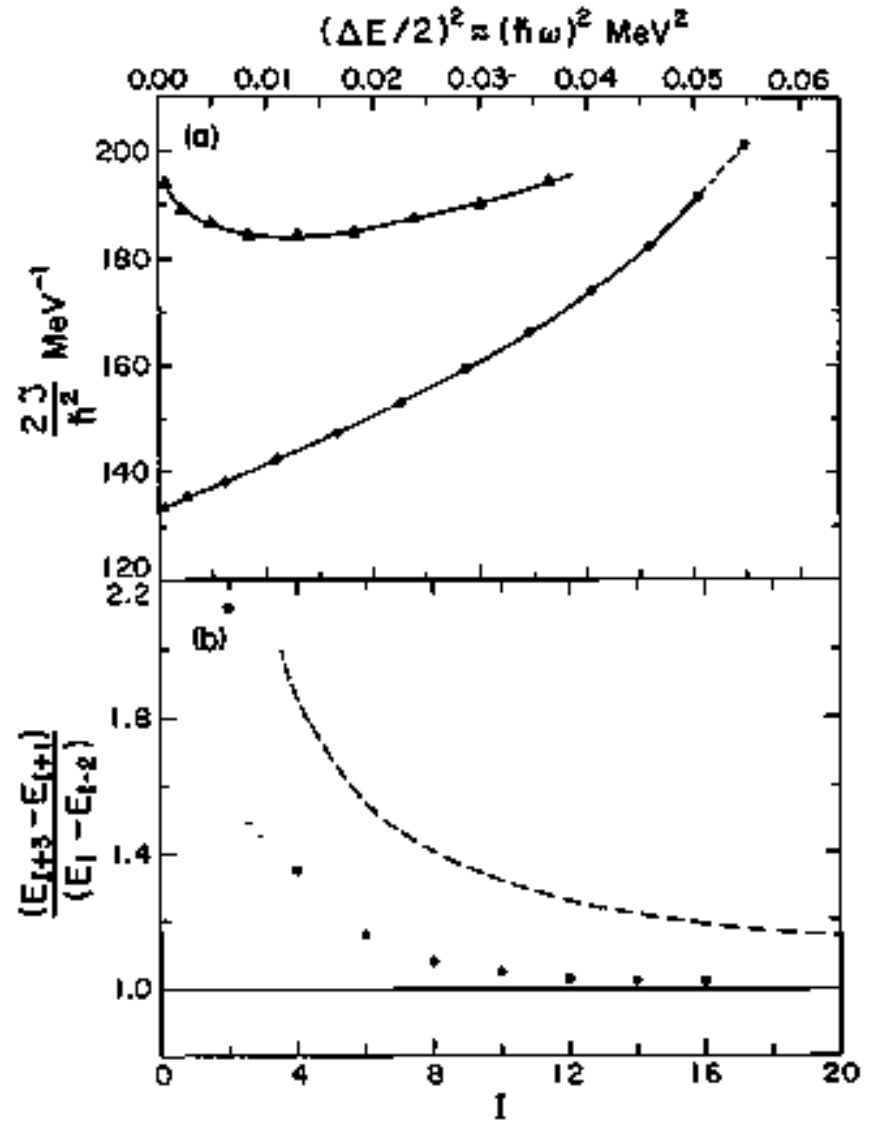

Fig. 3. (a) Backbending plots for the grome and octupole bands of 238t. The ordinate is $2 \mathrm{~d} / \mathrm{A}^{2}=$ (41-2)/(E $\left.E_{T}-E_{T-2}\right)$ and the abscissa is $(n+1)^{2}$. $\left(\mathrm{E}_{\mathrm{I}}-\mathrm{E}_{\mathrm{I}-\mathrm{j}}\right)^{2 / 4}$. (b) Comperison of the data for the acturpole hand (dots) with the aligned-vibrator prediction [solid line] and the single-band prediction (dashed line).

CKBL 755-3039)

quencles. If the other rotation-vibration interactions are weak, then the Coriotis forte inil align the vibrational angular monenttr along the rotation axis, producing a lowest band with energy spacings identical to those of the ground band, but having spins 3th higher, Or, the octupole deformation might be stabilized by the rotation, resulting in the even and odd spin states mergling into one band having the usual I( $T+1)$ energy spacings. A third possibitity is that the collective actupole character of the band might be destroyed, for exarple by independent aligr ment of its particle (and/or hole) corponents, leading to irregular level spacings.

The experimental level energies of the lowest. odd-parity band in 23iv are coupared with these possibilities in Fig. 3b. The plot is constrixcted such that the rotation-aligned vibrator prediction is unity; whereas, the dashed line is the stable octupole-shaped rotor (one-band) prediction. The data are very regular end converge rather convincingly to the rotation-aligned limit. This result is in excellent accord with explanations that have been given for the coupression (large noments-of-inertia) of the lowest few states of this octupote band, explantions involving Coridis mixing of the $K=0,1$, 
2, and 3 octupole component. 1 It is also interesting to considor the effect on the E3 transitions. At sufficiently high spin values each rotationaligned state has a near ly unique value of core angular monentum, R. The ES transitions cannot change $R$, so that the $I_{\text {gnd }} \rightarrow(I+3)$ oct transitions to the aligned (lonest) band pick up all the E3 strength, and the other transitions between these two bands becone sunal1. The previous Coriolis calculations, 2 have show this tendercy in the $0^{+} \rightarrow 3^{-}$transitions, in agrement with the experimental data,

The present Coulonb excitation experiments provide much the highest spin values know in actinide-region ruxlei. The data show that 23 \& does not backbesh up to spin 22\% (probably not to 24h); though it is not yet clear that it will not backbend at still higher spin values. The evidence suggeșts that at the higher spin values observed, the octupole band aligns its angular jocmentum (35) with that of the rotating core. This concept of the Coriolis force aligning an angular momentur vector along the rotation axis has previously been applied to one-particle states in weakly deformed nuclei, 4 and to two-particli states in strongly, deformed even-even nuclet. 5 one sees from the $23 \mathrm{U}_{\mathrm{U}}$ case that this idea may apply to amy angular monentum vector in a rotating system, including, in some circumstances, collective angular monenta.

\section{Footintes and References}

“Condensed fron Phys. Rov. lett. 35, 565 (1975).

tOn leave frora Max-PLanck-Instìtut fir Kernphysik, 69 Heidelberg, Gemany; supported by the Dentscher Akadenischer fustauschd ienst.

${ }^{*}$ On leave from Lutwig-Waximilians Unfversität, ufimchen, Gentwan; supported by the Eurdesninisteriun ftir Forschumg and Tochnoiogie.

${ }^{8}$ Dn leave from the University of 0slo, Norway.

1. K. Neergard and P. Vogel, Nucl, Phys. At45, 33 (1970).

2. K. Neergaird and P. Togel, Nuc1, Phys. Al49, 217 $(1970)$.

3. F. K. McGowern, C. E. Bends, Jr., W. T. Milner, J. L. C. Ford, R. L. Robinson, and P. H. Stelson, Phys. Rer, C 10, $1446(1 \$ 74)$.

4. F. S. Stephens, R. M. Diarond, J. R. Lelgh, I. Kammuri, and K. Nakai, Phys. Rev. Lett. 29, 438 (1972).

5. F. 5. Stephens and R. Simon, thacl. Phys. A183, $257(1972)$.

\section{HIGH-SPIN STATES OF ${ }^{174}, 176$ Y STUOIED IN COULOMB EXCITATION WITH Kr AND Xe BEAMS*}

D Ward, $\mathrm{P}$ Colambani,q I $Y$ Lee, P A Butler, s

R 5 5imon," $A$ is Damord, and $F$ S Stephens

The availability of beans of $\mathrm{Kr}$ and $\mathrm{Xe}$ at energies up to $8.5 \mathrm{MeV} /$ nucleon has opsined up the possibility of Coulomb exciting high-spin states in stable and near-stable defomed mulei. In the actinide region, states up to spin $18^{*}$ for 232 Th arot $22^{+}$for $238 \mathrm{U}$ have been observed by Coulamb excitation with $\mathrm{Kr}$ and $\mathrm{Xe}$ beams.1-3 In recent studies of the rare-earth region, Kearns et a1 4 have identified some $12^{+}$and $14^{+}$states using $84 \mathrm{Kr}$ ions at about 350 ker. Coulomb excitation of rare earth nuclej with projectiles sufficiently heavy so that states of angular mantentum larger than 16 are populated is quite interesting since: (i) the method of populating states is different than from a (HI, xin) reaction, so that additional states may be seen (of particular interest in this region is the study of backbending muclei) and (ii) sorne muclei that cannot be reachod by (HI, xm) reactions can be coulomb excited. Examples of this latter type wuld be the actinides and the neutron rith rare earth moclei, including $174 \mathrm{Yb}$ and $176 \mathrm{Yb}$. Also the high recoil momentum of the target nuclei following coulomb excitation with heavy iorts makes them ideal for lifetime studies using analyses of the doppler-broatened line shapes.

In addition to studying the $\gamma$-spectrom in singles with a Ge(Li) detector, we have used a jew type of annular particle detector and have detected $y$-rays in colncidence with specified ranges of the particle scattering ande. A description of this apparatus has been given in the more complete report of this work. 5 The sensitivity of the method was improwed further by demanding an additional $y$-ray event to be detected in either of tho $7.5 \times 7,5 \mathrm{~cm}$ NaI (T1) detectors placed at $\pm 90^{\circ}$. AIl eronts in the range 100 \& $E_{\gamma}<900 \mathrm{keV}$ were accepted frct the NaI (Tl) detectors. This adoitional $\gamma$-ray condition had the effect of suppressing low-multiplicity events, whilst y-rays in paths of high miltiplicity (e. $50^{+}+18$ ) were not affected. The spectra for $176 \mathrm{yb}$ and $174 \mathrm{yb}$ are shown in Figs. 1 and 2 .

Details for the extraction of lifetimes car be obtained from the full report of this work. 5 A summary of the experimental lifetimes is shown in Fig. 3 where they are crapared to the rotational values. For the 174 Yb $8^{+}+6^{+}, 10^{+}+8^{4}$ and $12^{+}+$ $10^{*}$ transitions, the present measurenents agree with the second set of Menchester values 6 within their combined errors; however, the present adopted valwes tend to be systematically about 8 i higher. The Manchester data were obtained using a $84 \mathrm{kr}$ beam with $\gamma$-rays detected in singles, and if we me the conparison with the present $86 \mathrm{Kr}$ singles data only, the systemat ic discrepancy is reduced to $+5 \%$, of which about $+3 t$ can be attributed to the different choice 
of stopping powers. To within an accuracy of $\sim 13 \%$ the average of the measured transitions in both $174 \mathrm{Yb}$ and $176_{\mathrm{Yb}}$ are consistent with the rotational model. However, there might be a systenatic trend for the measured ratios to decrease with increasing spin.

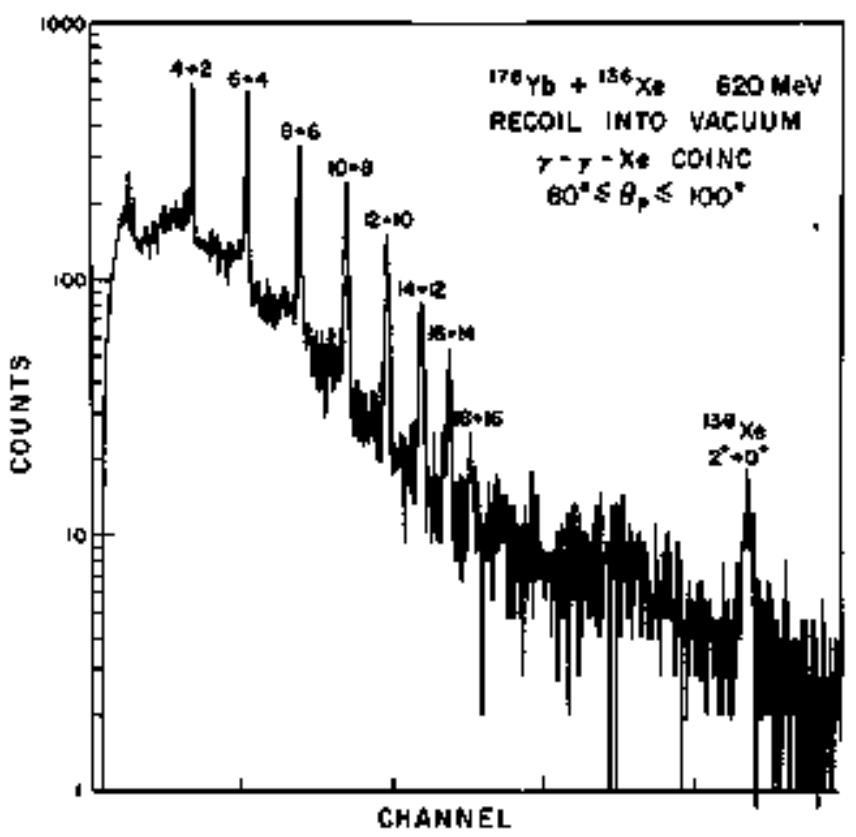

Fig. 1. Gaund rays in coincidence with ${ }^{13} 6_{\text {Xe }}$ iong scatiered in the atmulus $80^{\circ}<\theta<100^{\circ}$. The target of $176 \mathrm{rb}$ was about $1 \mathrm{mg} \mathrm{cm}^{-2}$ and inclined at $45^{\circ}$ to the beam direction. There is an additional $\psi$-ray coincidence (NaI) requirement on this spectrus,

[XBL $765-1800$ ]

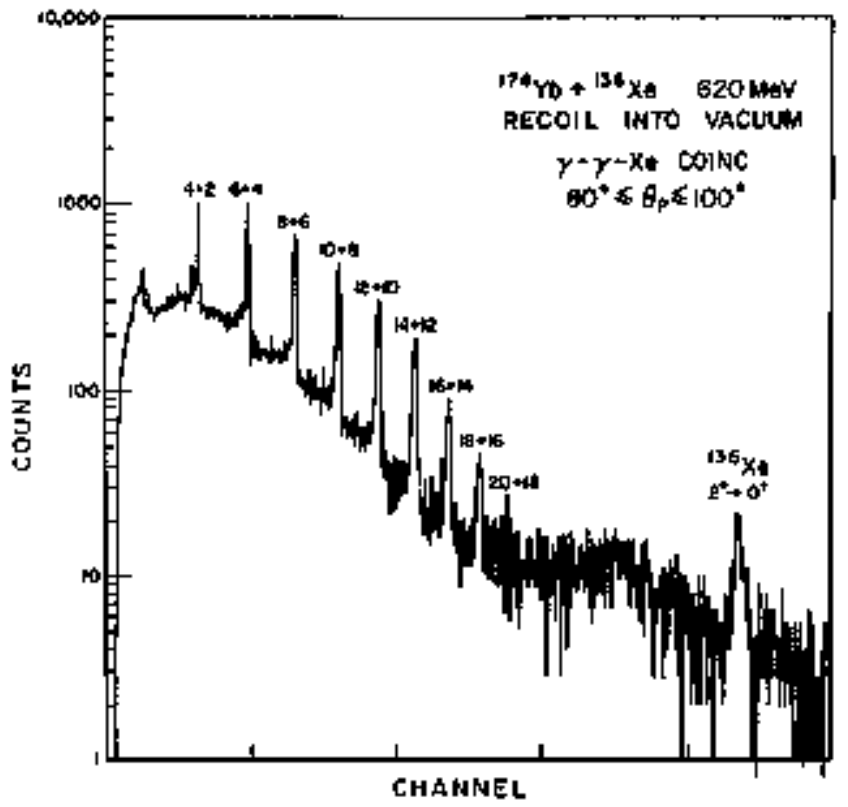

Fig- 2 Ganm-rays in coincidence with 136 Xe ions for ${ }^{174} \mathrm{Yb}$. The arrangenent was the sann as that deseribed for Fig. 1.

(XBL 766-8328)

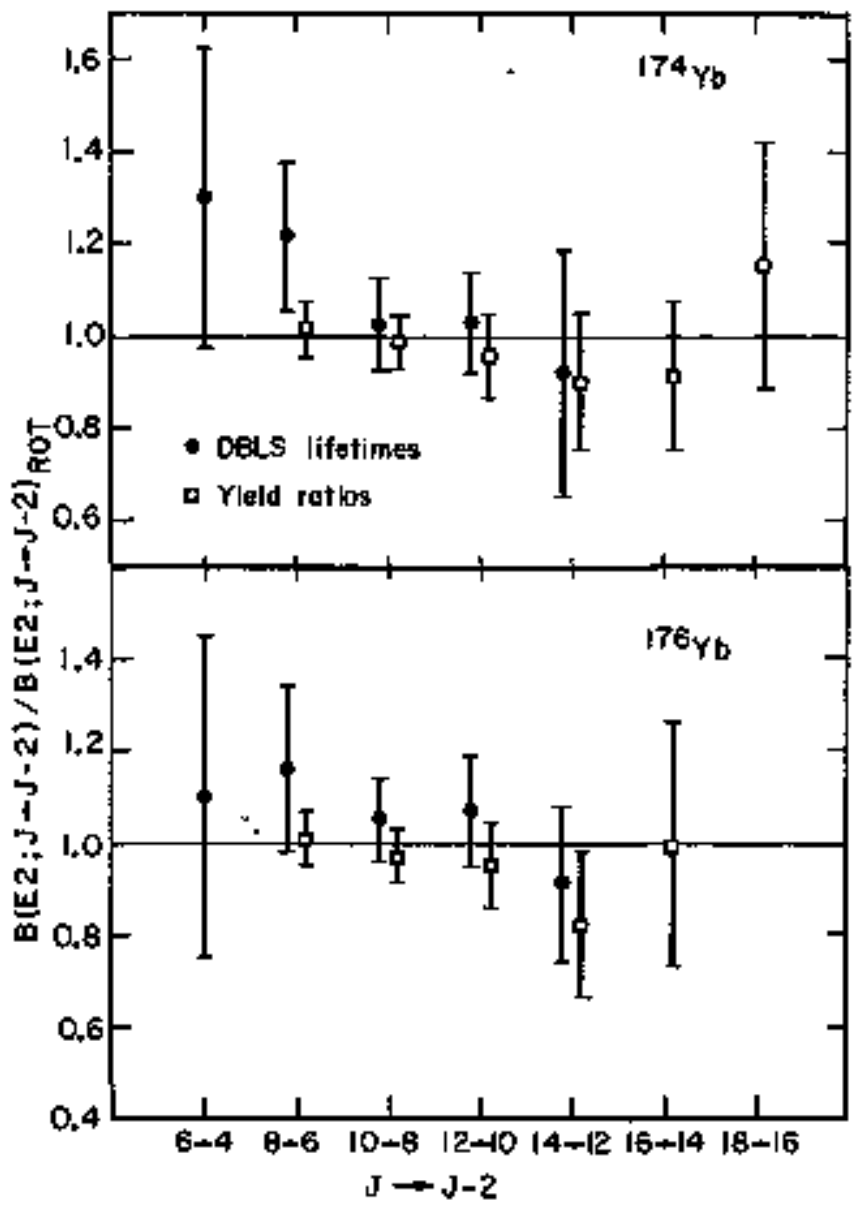

Fig. 3. Comparison of the lifetines obtained by Doppler-broadened line analysis with the rotationa1 model. The yield ratio $R$ (see the full report) is also approxinately equal to $\mathrm{B}(E 2)_{\exp } / \mathrm{B}(E 2)$ Totationa1.

(XBL $763-5228$ )

There is now considerable interest in highspin states, due to the prowounced anortalies (backberdings) that have been observed around $I=16$ in the ground state rotational bands of somb rare erth nuclei. At least three explanatlums have been considered for these effects: 1) a collapse of the poitring correlations; ${ }^{7}$ 2) a change in shape of the nuclei; 8 and 3 ) an aIigrument of the angular monenta of two particlalar high-j nucleons $\left\langle i_{13 / 2}\right.$ neutrons in the 1ight rare earth region) with the rotational angular momentum of the core. 9 Most of the information on these high-spin states has conte from studies of the gannua-ray de-excitation of (HI, xn) produets. The present work has, however, prowided the first date on neutron-rich rare earth wuclel. The information on the high-spin states of the yb nuclei from mass 164 to 176 is show on the usial backbending type of plot in Fig. 4. Tho interesting features are apparent in these plots. The first is that $168 \mathrm{Yb}$ shows less backbending than its neighbors, . $166 \mathrm{Yb}$ and $17 \mathrm{dp}$. This has been previousiy noted by Faessler ${ }^{10}$ and ascribed by him as a pairing effect due to the 10 Ievel density at just this neutron number (98). This kind of pairing effect was previous1y suggested 11 to be responsible for the soasewhat larger moments of inertia (compared with the 


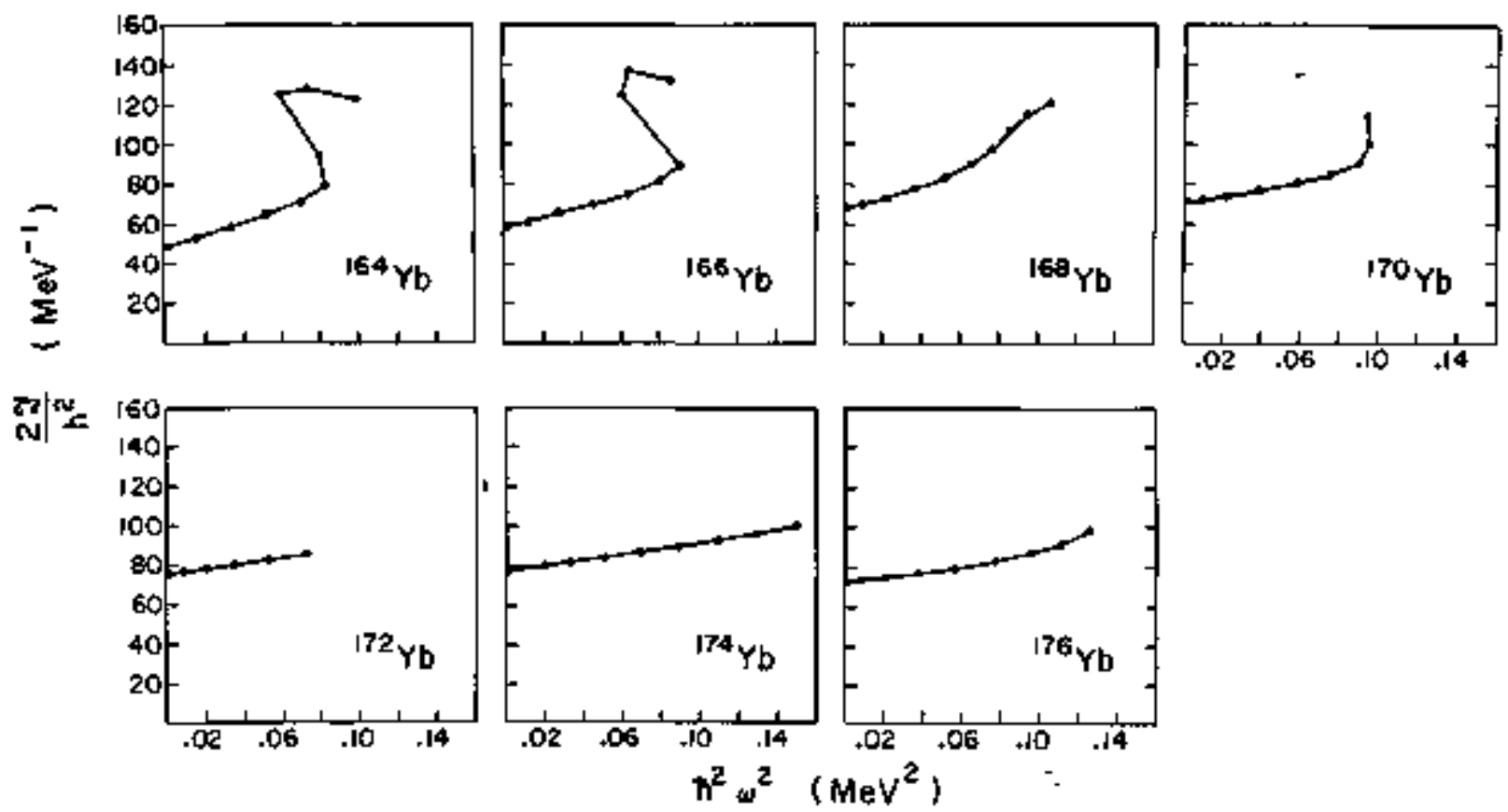

Fig. 4. Plots of the nonemt-of-inertia versus rotational frequency (squared) for the Ylb isotopes.

XRL 765-1802)

neighboring malei) of the $98-n e u t r o n$ HF and $W$ isotopes This explanation of the redixted backbending in $168 \mathrm{Yb}$ is still consistent with the gerrerally accepted idea that the backbending in thís mass region is caused mainly by the alinguent of two $i_{13 / 2}$ nettrons; since, in the aligment mechamisan, the moment of inertia plays a strong role, and a larger gromend band enment of inertia would certainly go in the direction of weakening the backbending. Thus a pairing explanation for the weak backbending of 168y seems Iikely to bo correct, altbough it is not clear whether the weaker pairing in $108 \mathrm{yb}$ prevents the alignient of the $i_{13 / 2}$ neutrons in the yrast states or just motifies it sombewhat.

The other interesting feature in Fig. 4 is the Yery regular behavior of the high-spin states in 174,17 Yo compared with that of the lighter Yb isotopes. This behavior is easy to understand in the aligrnent rode1, and was predicted to occur,9 It happens becanise the aligrment requires the low-st comonents fran a $j$-sholl, and (for a prolats shape) these essential couponents lic higher in energy as the shell fills. For example, the Fermi level is near the $A=5 / 2$ corponent of the $i_{13 / 2}$ shell in $167 \mathrm{yb}$; wereas, it is up to $0-9 / 2$ in $17 \mathrm{Yb}$. Thus one car easily imderstand why the aligupent of 50 $i_{13 / 2}$ neutrons would be atfficult in $1 / 4 \mathrm{Yb}$ or $176 \mathrm{qb}$. However, backberding does recar in the wand 0 s rus clei barjig neutson pumbers similar to 174,176\%, In these cases, it has been shom that the alignment of tho hg/2 protons play a rajor role in the backberding, and this process is less likely to occur in 174, $176 \mathrm{Yb}$ because: 1) Yb has fewer protons; 2) the monent-of-inertia is larger in the rb region; and 3) $\varepsilon_{4}$ is less positive (e 4 less negative) for the $\mathrm{y}^{2}$ isotopes 12 Thus the lack of backbending in $1 / 4,176 \mathrm{rb}$ sems quite consistent with our present understanding of this process.

\section{Footootes and Referencess}

Condensed from LEL-4375, Nuxel. Phys. B (to be poblished).

$\dagger_{\text {Perik? }}$ tories, Ontario, Carada.

*Permanent address: Institut de Physique Nucifaire, 91406 Orsay, Frapce.

E.K. S.R.C./N.A.T.O. Fellow.

"On leave from Seltion Physik der Ludwig-NaximitiansUniversität lifinchen, 8046 Garching, Germany; sponsored by the Bendespinisterium fur Forschung und Tecthologie.

1. E. Grosse, J, Dekoer, R, M. Dìmond, F. S. Stephens, and P, Tjom, Phys. Rerv, Lett. 35, 565 (1975).

2. P. Colqubani, J, C. Jacmart, N. Poffe, M. Ricu, P. P. Singh, C. Stephen, and A. Weidinger, Phys. Lett. B $48 \mathrm{~B}, 315[1974]$.

3. M. W. Guidry, P, A. Bestler, P. Colombani, I. Y. Lee, R. N. Diamond, D. Wart, F+ S. Stephers, E. Eichler, N. R. Johnson, and $k$. Stum, to be pubilshed (1976).

4. F. Kerns, 6. Dracoulis, T. Inanura, J. C. Lisle, and J. C. MHlinot, J, Phys. A7, 111 (1976).

5. A Winther and J. DeBoer, Coplagl Excitation (Acadenic Press, New York, 1966), p. 303. 


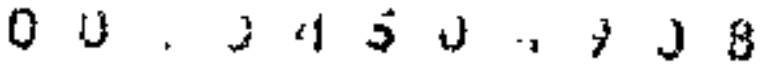

6. T. Inentra, F, Kearns, ఫ. C. Lisle, G. Varley, and J. C. Millsott, Argalal Report I974, Schuster lab., University of Hanchester, p. 75.

7. B. R. Mottelson and J.-G. Valatin, Phys. Lett. 5, \$11 (1960).

8. P, Thiebxrger, Phys. Lett. $45 \mathrm{~B}, 417$ (1973),

9. F. 5. Stephens and R. S. Sinon, Nucl. Phys.
A138, 257 (1972)

10. A. Faessler, F, Gritwem, "L. Lin, and J. Urbano, Phys. Lett. A138, 87 (1974).

11. F. S. Stephens, N. Lark, and R. M. Dianond, Phys. Rev. Lett. 12, 225 (1964).

12. P. S. Stephens, P. Kleinheinz, R. K. Sheline, and R. S. Sirion, Nucl. Phys. A222, 235 (1974).

\section{TRANSITIONS BETWEEN HIGH-SPIN NUCLEAR STATES*}

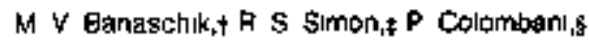

$F$ S Stephens, and $A M$ Dlamond

Studies of transitions between high-spin nthclear gtates can give inforgation about manents of inertia, shapes, and other structural features of such nucles. High sptn values (up to $80 \mathrm{n}$ ) can be brought into ecmound nuclei following heavy-ton re actions; howerer, $Y$-ray studies following such reactions have thus for produced information mainly or states having spins below $-20 \mathrm{c}$. The reason is that all the transitions between higher-spin states are too weak individually to be resolved, and thus coruprise an apparent continum The There some eariy attempts to study this continulu, 1,2 but these studies have only recentty been resumed. 3-6 In the present work, the energies, angular correlations, and number of continum $y$ rays have been measured, and the information obtained from these quantities has been related diroctly to nuclear monents of inertia at angular moenta up to $60 \mathrm{~h}$.

We have stidied mainly the reactions $\$ 2 \mathrm{Se}(40 \mathrm{Ar}$,

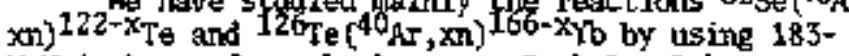
Hel Ar beams fron the Iawrence Berkeley Laboratory SperHILAC. In addition, a limited smvey over target $\left(A 1, C, A s, A g, S n\right.$, and $\left.S_{0}\right\}$ and projectile $\left\{{ }^{60}\right.$ and ${ }^{4} O_{A T}$ ) at several bonbarding mergies was made. The targets were $-1 / \mathrm{qg}_{\mathrm{g}} / \mathrm{or}^{2}$ thick, and were evaporated onto 25-1s $\mathrm{Pb}$ backings, which stopped the beam and recolling nuclei with very little background. To measure the continun $y$ rays, we used three $7.5-0 m$ $\times 7.5$ - CII NaI(T1) detextors at angles of 0,45 , and $9 t^{\circ}$ to the beam direction. Each detector bad an absorber of $0.32-\mathrm{ca} \mathrm{Pb}$ and $0.32-\mathrm{cm} \mathrm{Cl}$, snd was placed 60 cn from the target. This Iong flight path to the MaI detector permitted an almost ccamplete separation between neutrons and $\psi$ rays in the tine spectrum. The three NaI spectra were recorded event by arent in coincidence with pulses from a Ge detector which was $5 \mathrm{~cm}$ fram the target at an angle of $225^{\circ}$ to the bean direction. The Ge singles spectrum wis stored simaltaneously. To obtain the trtue NaI $\gamma$-ray spectrow, $N\left(E_{\gamma}\right)$, the pulse-height distribution wust be corrected for the detector response(unfolded). The raw and unfolded of NaI-detector spectra in coincidence with the full Ge-detector spectrum are shom for three targets in Fig. 1.

For the heavier product nutclei $(z \geq 50$ or A $z 110)$, tho features of the contimum spectra are clear. Above -3 wev the intensity falls of appraxinately exponentially with increasing energy, where

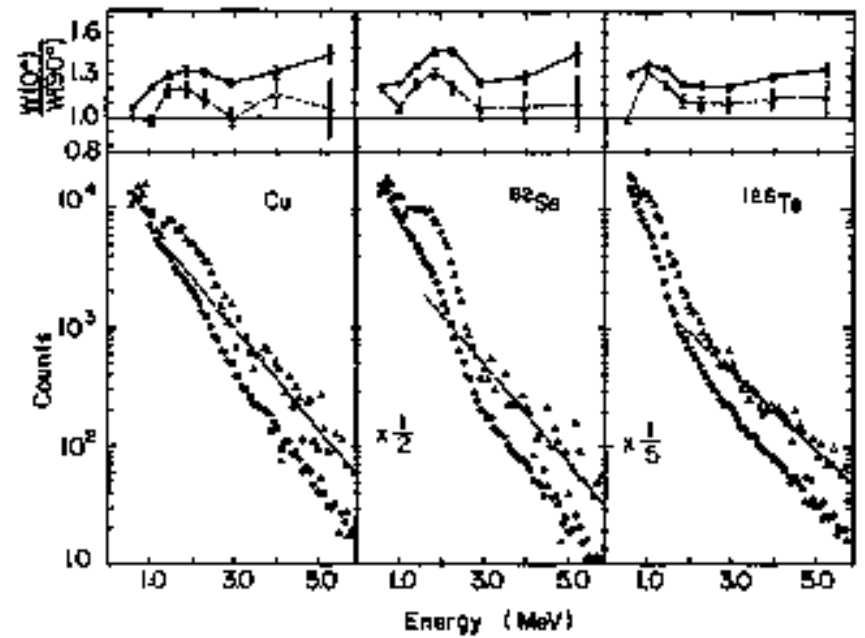

Fig. 1. The raw (dots) and unfolded (triangles) continum spectra in coincidence with the full Gedetector spectrom for 183 - $\mathrm{keV}$ ${ }^{\mathrm{Ar}} \mathrm{projectiles}$ on natural $\mathrm{Cu}, 82 \mathrm{Se}$, and ${ }^{126} \mathrm{Te}$ targets. The stralght lines are fitted to the unfolded spectron between 3.2 and $6.2 \mathrm{kbV}$ (solid portion) and extrapolated to lower energies (dashed protion). The upper plots show the $0^{\circ}-90^{\circ}$ ratios for the row data (dots) and for the unfolded data corrected for recoil rotion (triangles), The error bars indicated statistical errors only.

(XBL 751-2146)

as scmewhere below this mergy a prominent bulu ocars. These features seen very likely to result fron the statistical and yrast cascades as has been predicted, 7 and recently observed in to nuclei with 100 projectiles, ${ }^{6}$ The slope of the exponential part of the spectrum is rather similar for all the targets we have studied and corresponds to "temperatures" ranging frol 1 to $1.5 \mathrm{MeV}[\mathrm{T}=-\mathrm{dE} / \mathrm{d}$ inN] in good agresment with the expectations for a purely statistical cascade. The buplis show by the angur lar correlations to be canposed almost coupletely of stretched E2 transitions whose energies are rather low and decrease with increasing prodict nass in a very systejuatic way, suggestive of a monent-ofinertia effect. Also, the eneroby and the intensity of the stretched-E2 compontint of the but generally 
increase with angular-momentum 144ut. These properties strong1y suggest identifleation of this bump with the yrast cascade, though there is not yet any direct experimental evidence that this eascade occurs close to the yrast line. The yrast bump is generaily less prominent for products below $Z \sim 50$ as is inlustrated by the cunve for a $\mathrm{Cu}$ target in Fig. 1 .

Hore detailed information about yrast energles comes fron studies of the individual reaction channels. This is itlustrated in Fig. 2, whith shows raw and unfolded spectra from the reaction $85_{S e}{ }^{40}{ }_{A r}$,

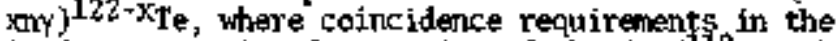
Ge detector allowed selection of the $4 \mathrm{n}\left(11 \mathrm{~B}_{\mathrm{Te}} ; 605\right.$, $601,615$, and $753 \mathrm{keV})$ and fin (116 Te; 679,681, 643, and $771 \mathrm{kev}$ ) reaction channels. 8 These spectra each have a rather sharp drop, but at quite different onergies: $2.4 \mathrm{MeV}$ for the $4 \mathrm{n}$ reaction and $1.7 \mathrm{MeV}$ for the on reaction. An estimate of the angular momentwin in each chamel can be made from the $\overline{\mathrm{F}}_{Y}$ values, which are 29,20 , and 13 , for the $4 n, 5 n$, and on reactions. Assuming that the few nissed transitions below $0.6 \mathrm{deV}$ compensate for the dipoles present, we can estimate the average angular momentum in these channe1s to be $2 \pi \mathbb{N}_{\text {, }}$ or 58,40 , end 2 ft. This procedure has been sholm in other cases 5 to be consis. tent with estinnates of the channel angtlar monentur based on cross-section measurements. Since the highest yalue of the angular momentum having appreciable intensity, rather than with the average value, we must estimate this "bighest" value. Por the on reaction, a point midway between the on and in average values (3) ] seens unlikely to be in error by more than -15 s. The 4n value should be rather near the measured average, and 64h (the kass-nodel ${ }^{9}$ dyravic limit for this reaction) sems likely to be correct within $\rightarrow 158$. These estimates give $24 / \mathrm{h} 2$ values of $75 \mathrm{MeV}^{-1}$ for $I \sim 33$ and $110 \mathrm{mev}^{-1}$ for $I \sim 64$. For

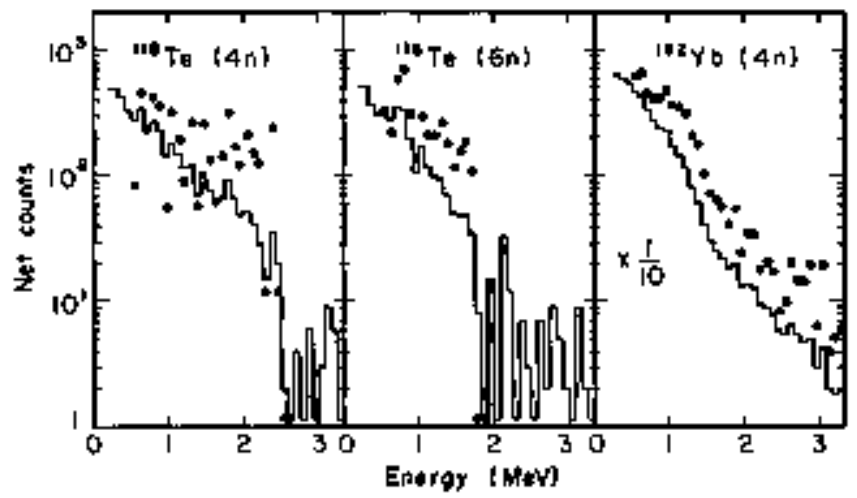

Fig. 2. The histograns show the raw continum spectra in coincidence with the (backgroum-corrected) $Y$-ray lines frora specific reaction products (labeled). Negative or zero counts are plotted at the bottom of the figure. The dots show the unfolded spectra in regions where the statisticel variations are not too large.

(XBL. $752 \cdot 2239$ camparisch, the value of $2 \mathrm{~A}^{2}$ is $39 \mathrm{MeV}^{-1}$ for the

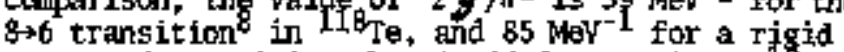
sphere of wass 118. The liquid-drop estinates 10 for $I-33$ and 64 in $118 \mathrm{Te}$ are 91 and $104 \mathrm{Mov}-\mathrm{I}$. The spectrum for ${ }^{162} \mathrm{Yb}$ from the ${ }^{40} \mathrm{Arr}+126 \mathrm{Te}$ reaction is also shown in Fig. 2, and an estfuste sinilar to those above gives $2 \mathrm{~A}^{2} \sim 140 \mathrm{HeV}^{-1}$ at $\mathrm{I} \sim 48$, The rigid-sphere value for this case is $141 \mathrm{MeV}^{-1}$ and the 1iquid-drom estimate is $154 \mathrm{kel}-1$. There is, no doubt, muxh yet to be learned about extracting moments of inertia fros these spectra; however, it seems likely that these transition energies and associated spins are roughly correct.

\section{Footnotes and References}

*Condensed from Phys. Fev. Lett. 34, 892 (197\$).

tરATO Fellow, Institut fïr Strahlen- und Kernphysik, Bonn, West Germany.

\$Sektion Physik Universität Minchent, Garching, Nest Gervany.

${ }^{5}$ Permanent address: Institut de Physique Nhcleaire, 9I406 Orsay, France.

1. J. F. Mollenauer, Phys, Rev, 127, 867 (1962).

2. Yu. Ts. Oganesyan, Yu, V. Lobanov, B. N. Markov, and G. N. Flerow, th. Eksp. Teor. Fiz. 44, 1171

(I963) [ Sov. Phys. JETP 17, 791 (I963).

3. E. der Katoosian, $O$. C. Kistner, and A. W. Sunyar, Fhys, Rev, Lett. 33, 596 (1974).

4. G. B. Hagemann, R. Brods, B. Herskind, M. Ishthara, and $\mathrm{H}$. Fyde, in Proccedings of the Internotional Conference on Reactions between Complex Nuclei, Nashrille, Ternessee, 1974, edited by

R, L. Robinson, F. K. BkCowein, J. B. Vall, and J. H, Haniliton (North-Holland, drosterdam, 1974), Vol. 1 , p. 110 .

5. P. 0. Tjकm, F. \$. Stepheris, R. M. Diamond, f. De Boer and H, E. Meyerhof, Phys. Rev, Lett. $33,593(1974)$.

6. J. 0. Newton, J. C. Lisle, G. D. Dracoulis, J. R. Lejgh, and D. C. Neisser, Phys. Rev, Lett, 34, $99(1975)$.

7. J. R. Grover and J. Gilat, Phys. Rev. 157, 802, 814 (1967).

8. A. Lutukko, A. Kerek, I. Rezanka, and C. J, Herrlander, Nucl. Phys, A135, 49 (1969).

9. R. Bass, Phys, Lett. 47B, 139 (1973), and Nucl. Phys. A231, 45 (1974).

10. D. P. Soroka and W. J. Swiatecki, private comminication. 


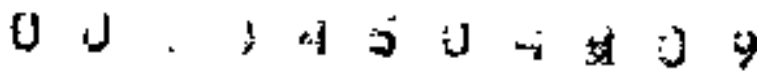

\section{MOMENTS OF INTERTIA IN ${ }^{162}$ Yb AT VERY HIGH SPINS'}

\author{
A \& Smon,t it V Banaschk, $\$$ P Colombenı,s \\ - O P Soroka, F S Stephens, and F M Diamond
}

The purpose of this rork was to obtain nuclear moments of inertia at vory high spins by studying the $\gamma$-ray spectrum following ( $\mathrm{HI}, \mathrm{kTY})$ reactions leading to the final pucleus $162 \mathrm{y}$. Two reactions were in-

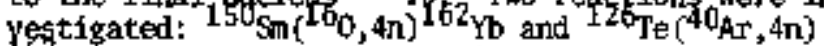
$162 \mathrm{Yb}$. He neasured the continuk spectron in three $7,5 \times 7,5-\mathrm{cm} \mathrm{NaI}(\mathrm{Tl})$ detectors at $0^{\circ}, 45^{\circ}$ and $90^{\circ}$ with respect to the beam direction and 60 an from the target. These detectors were gated by coincident pulses Erom a Ge detector at $225^{\circ}$ to the beam and 5 con froth the target. The energy distribution of the continum gamma rays was obtained from the observed NoI pulse-height spectra by an unfolding procedure 1 using a carefully adjusted rosponse function and the reasured total efficiency curve of the NaI detecotors, with a small correction for the motion of the recoiling proxuct mucleus, By normalizing to the nuber of singles events in the gating lines of the Ge detector, the isotropic unfolded spectrum can be given in absolute events per decay and way be integrated to yield the average gajpm-ray multiplicity, $\vec{F}$, of the reaction. For the 40 ar reaction at $181 \mathrm{MeV}$, raw and umfolded spectra are shown in Fig. 1 , as well as the ratio of events at $\phi^{\circ}$ to those at $9 \phi^{\circ}$.

The anisotropy (Fig. 1) suggests that most of the buap transitions are of stretched $E 2$ cheracter, so that an estimate of the average angular mo mentum, $\bar{c}$, in the charmel can be obtained. Așsaming no angular thomentum is carrled off by neutrons or the statistical cascade, we subtract the statistical transitions (a11 those in the exponential tail plus an estinated backgroumd underneath the buprp-a total of $\sim 4$ transitions in a11 cases] and multiply the rest by $\mathrm{th}$. Both this estinated $\bar{q}$ and the total

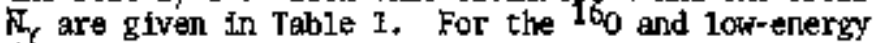
$40_{A x}$ cases, these $\bar{c}$ estirdates are in excellent agreement with average values obtained from measured reaction chapnel cross sections, $\bar{l}$ (d), using the sharpcut-off mode1. Therefore, in these cases the upper boumdary angular momentam, $t_{\mathbf{u}}$. given by the cross= section measureinents seeals very likely to be the

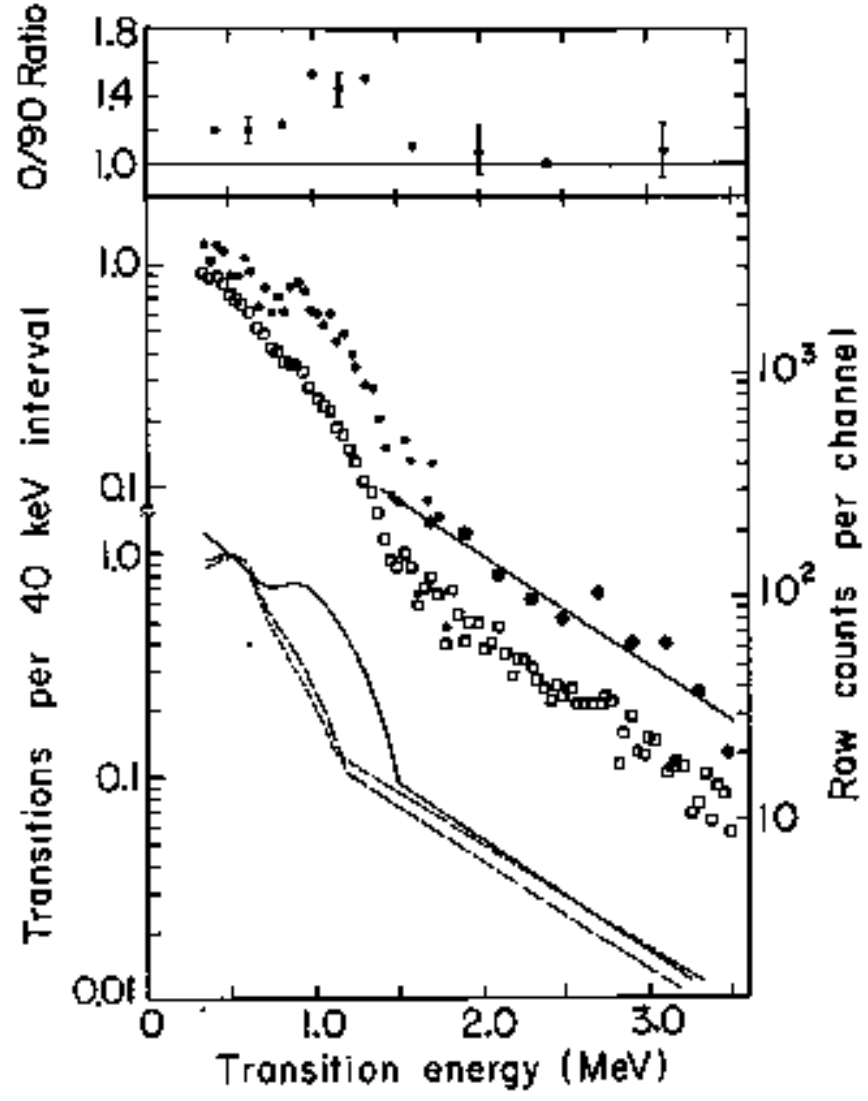

Fig. 1. RaW (口) and unfolded ( 1 ) continum gamuna spectra from the $12 G_{\mathrm{Te}}\left({ }^{40} \mathrm{Ar}, 4 \mathrm{n}\right) 162 \mathrm{Vb}$ reaction at $181 \mathrm{kef}$. The larger solid dots represent 5 chamel averages. Also shom is the 0/90 ratio for the unfolded spectrtm. At the bottom are schematic spectre for this case (solid ine), the 12 Te $(40 A r, 4 n)$ $162 \mathrm{Y}$ - Teaction at $157 \mathrm{MeV}$ (10mer-dashed line) and the $150 \mathrm{~m}(100,4 \mathrm{n}){ }^{162} \mathrm{Yb}$ reaction at $87 \mathrm{MeV}$ (shorterdashed line).

(XEL $7511-9561$ )

Table 1, Data used to obtain maments of inertia,

\begin{tabular}{|c|c|c|c|c|c|c|c|c|}
\hline Reaction & $\underbrace{}_{\text {proj }}$ & $N_{Y}$ & $\begin{array}{l}\bar{t} \\
\bar{b}\end{array}$ & $\begin{array}{l}E_{\text {edge }} \\
\text { Mel }\end{array}$ & $\begin{array}{l}\sigma(4 \pi) \\
\text { mbam }\end{array}$ & $\sum_{x=4}^{6} 0(x+)^{a}$ & $\ln _{n}(4 n)$ & $\begin{array}{c}\bar{q}(\sigma)^{b} \\
h\end{array}$ \\
\hline $150_{\text {S月 }}+1$ & 87 & 17 & 26 & 1.2 & 540 & 760 & 36 & 27 \\
\hline $126_{\mathrm{Te}}+4$ & 157 & 17 & 27 & 1.16 & 220 & 280 & 39 & 28 \\
\hline $126_{\mathrm{Te}}+4$ & 181 & 23 & 39 & 1.40 & 220 & 620 & 63 & 56 \\
\hline
\end{tabular}

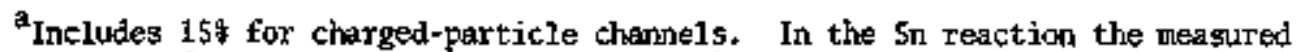
$i_{13 / 2}$ band population was increased by $35 \%$ to allow for other bands.

$b_{\bar{\lambda}(0)}=0.67\left[e_{u}^{3}(4 n)-e_{u}^{3}[5 n)\right] /\left[q_{u}^{2}(4 n)-x_{u}^{2}(5 n)\right]$. 
Jaxirm angular momentum in the yrast (collective) cascade. For the $181 \mathrm{M}$ V 40 \& case the two $\bar{x}$ vaiues do not apree, possibly indicating a net angular mbintu carried by the neutrons and/or the statistical cascade. For our purposes the $\bar{I}$ based on the cufolded spectrom is more relevent, and the maxisum angular monentum in the yrast cascide is estimated (based on the other toro cases) to be about 1ith largex than this $\bar{d}$, giving a value of $\sim 50 \mathrm{~h}$. This number, hrover, is less certain then that for the other two cases.

There are two methods for obtaining effective monents of inertia, $\$$, from these data. One depends on relating a transition energy, $E_{t}$, to the corresponding spin, I, according to the approximate rela

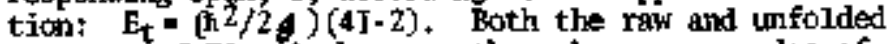
spectrue of Fig. 1 show a rather sharp upper edge of the bum. This edge is found to be lower for the 160 and low-energy $40_{\mathrm{Ar}}$ cases (show scheriatically in the bottom part of Fig. 1) where less angular ncouentum is brought into the system. This guggests that the energies of the edge can be associated with ganla transttions between the highest spin states in the yrast cascade (estinated above). Three values for $2 / \mathrm{h}^{2}$ can be obtained in this way and are plotted on Fig. 2 against $(t+4)^{2}$ in the usual backbending type of plot, where ho is taken to be $E_{t} / 2$. A1so show are the moments of inertia of the known low-spin states in $162 \mathrm{Yb}$ and, for comparison, the low-spin data for the isotone $160 \mathrm{Er}$.

The same method can be applied for transitions In a reglur of the spectrum corresponding to $l$ valus below which there is no appreciable direct population into the chamel of interest. This region is likely to bolow 30-35 $\mathrm{m}$ for the an channel in the $181 \mathrm{MeV} 4 \mathrm{dAr}_{\mathrm{Ar}}$ case (Fig. 1) since most of the population with lower spins goes into the 5 or $6 n$ cham nels, bat if pould be less than $20 \mathrm{~h}$ for the ${ }^{10} \mathrm{O}$ and low-energy $40_{A r}$ cases. Provided there is a monotonic increase of transition energy with spin (no backbending), a spin value for each transition energy can be obtained by saning all the transitions (less the estimated statistical cascade background) up to that transition energy and multiplying by two. This method is spplicable between $\sim 0.7$ and $1.0 \mathrm{MeV}$ in Fig. 1, leading to moments of inertia given by the dots connected by a splid line in Fig, 2 ,

The preceding method is an "integral" one, and thus is not very sensitive to tocal variations in the mothent of inertia. The secood method is a "itifferential" one, and can show such local varlations. Each point on the unfolded spectrun of Fig. 1 gives the nobuer of transitions per $40 \mathrm{keV}$ energy interval. The reciprocal of this is the difference, $\Delta_{t}$, between transition energies and is related to the mowlont-of-Înertia by

$$
\Delta E_{t}+\frac{\theta^{2}}{2 t}-2 E_{t} \frac{d \ln \theta}{d I}
$$

where $E_{t}$ is the transition onergy for which $A E_{t}$ is evaluated. This method also repuires the full popwhation in the channel, and thus can only be applied below $30-351$. for the $181 \mathrm{Mev} 40 \mathrm{Ar}$ case. For the region $0.7-1.0 \mathrm{keV}$ in Fig. 1 , is nearly constant, so that the last tem of Eq. (i) can bo neglected. giving $2 \& / \mathrm{H}^{2} \approx 8 / \mathrm{A} \mathrm{E}_{\mathrm{t}}$. This procedure leads to the

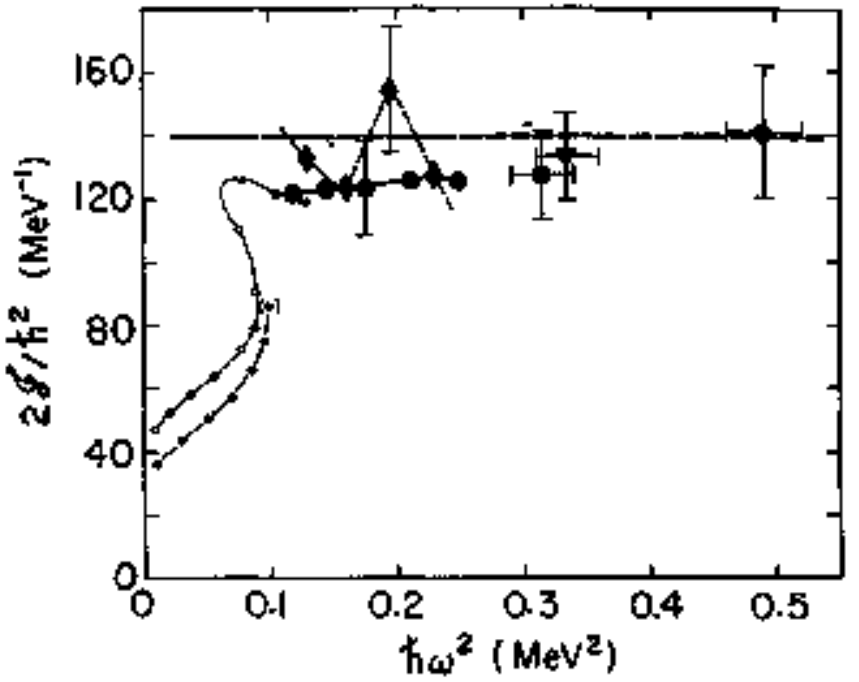

Fig. 2. Backbending plot for ${ }^{162} \mathrm{Yb}$. The salnal soljid dots correspond to the kntum low-spin states of $1{ }^{2} \mathrm{Yb}$, whereas the open circles are for the isotorie $160 \mathrm{Fr}$. The large dotg correspond to values derrived by the integral method fram the $181 \mathrm{MeV}$ $40 \mathrm{Ar}$ dats The triangle and square come from the $157 \mathrm{HeV}{ }^{40} \mathrm{Ar}$ and $87 \mathrm{MeV} 100$ spectra using the same method. The dianonds are values from the differential method applied to the $181 \mathrm{MeV} 4 \mathrm{OAT}$ case. The horizontal dashed line is the momint-of-inertia of a rigid sphere with $A=162$.

(XBL 7511-9562)

diamonds and dashed Iine in Fig. 2. The results are in good agreestent with those of the integral method and suggest that there might be a local increase in the momint of inertia around $(\mathrm{n} \omega)^{2}-0.2 \mathrm{Me \textrm {V } ^ { 2 }}$ or $E_{t}=0.9$ Motf. This possible rise can be seen directly th both the infolded and raw spectra of Fig. 1, but it is not clearly outside the prosent uncertain ty lomits.

The offective moment-of-inertia values measured by the techniques described above are ccmpared in Fig. 2 with that of a rigid diffuse sphere of mass 162, having an equivalont his radfus of 6.71 fm. The deformed rigid-body vatue for the momentof-inertia would be roughly 10 targer than this rigid-sphere value. It will obviously be of interest to Iraprove these methods in order to see more details of these manents of inertia and to extend the measure nents to other nuclei.

\section{Footnotes and References}

Condensed from Phys. Rev. Letters 36, 359 (1976).

ton leave from Sektion Physik der Iudwig-Naximilians Universität Winchen, 8046 Garching, Germany, sponsored by the bundesministerium futs Forschung und Tochnologie.

*Present address: Techulsche fochschule, Dasmstadt, Germiny.

${ }^{5}$ Ferminient address: Institut de Physique Nucleaire, 94106 Orsay, France. 


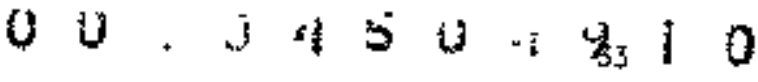

1. J. F. Mollenauer, Lawrence Radiation Laboratory Report LKC:L-9748 (1961).
2. W. D. Meyers, Nuc1. Phys. A204, 465 (1973).

\section{EXPERIMENTAL STATUS OF HIGH-SPIN STATES*}

\author{
F s Slephens
}

High-Spin nuclear states are interesting at the present time, both because they are experinenta1ly accessisble, and because there have recently been several rather dranatic changes observed at high spins, and others even pore dramatic are predicted. I want to describe very briefly just what these are, what we now know about them, and what we are trying to find out about them.

One of the changes occurring in high-spin states is the reduction and eventual quenching of the pairing correlations, There is now stron evidence ${ }^{2}$ that the pairing is, indeed, decreasing with fncreasing spin even at the lowest spin values. However, it is also reasonably clear that it has not disappeared by spin $20 \mathrm{~h}$ in toeny rare-earth nuclei. It is certainly an interesting experimental probleal to determine just where and how the pairing is quenched, and we have recently obtained scme evidence that suggests there nay still be sone pairing left at $-35 \mathrm{~h}$ in at least one case,

Changeg in the nuclear shape are a second type of effect expected to occur with increasing spin. Small increases in deformation are krowom 3 to occur at 10w ( $₹ 124$ ) spin values in a number of nuxlei with moderate or small deformation, and a rather sudden change frow slightly oblate to rather strongly prolate shapes has been observed $4,5,6$ in severd 1 ilght Hg nuclei. The liquid trop model (with rigid-body norkents of inertia) predicts ablate shapes to be nost stable us to high spin values (50 to $70 \mathrm{n}$ for $A * 160$ ) and them triaxial shapes with increasing deformation leading to fissiom (at 70-80 z). Accorting to recent calculations, 9 the shell corrections do not change the basic liquid-drop tendencles. The prolate-deformed rare earth nuclel, for example, are predicted to becane (or tond to became) oblate via triakial shapes at moderate spin values $(30-50 h)$ and then follow the liqu⿰d drop estimates. Two interesting conclusions from both the liquid-drop and the rotating-deformed-oscillator calculations are that there should be very high-spin isaneric states in the oblate region and also rapidly increasing monemts of inertia in the triaxial region preceding fission. These effects are being searched for at the presernt time.

A third phenomeron that is expected to ocaur at high-spin values is the alignment of individual particle angular joomenta. This is the dominant strutture of the low-energy high-spin states in spherical and near-spherical nuclei, as is well known, and this process should camete with collective motion at high er spin yalues in the more deformed nuclei. Rocently a madel 10 involving the aligrment of the angular momentum of an odd nucleon with that due to the core rotation has had considerable success for mamy weakly deformed naclei. However, it is not yet entirely clear to what extent the core motion aust be purely rotational in auch a mode1. Also, it now seems rather likely that the "backbending" which has been observell in scase of the light rare earth maclei is due to the aligment 12 of two particular maxleons $(13 / 2$ neutrons in this mass region, with the core rotation. If the oblate shapes predicted by the liquid drop model are reached, all the angular mcanentibe would be carried by aligned particles. This would be sigmaled rather unaiblquously by the occurrence of the high-spin isomeric states mentioned above. Thus, at least three effects--10ss of pairing, shape changes, and atignment--are known to be oocurring in muclei as the spins increase, and the interplay and coupetition of these (and perhaps still other) processes inakes the study of high-spin states rather exciting at the present time.

\section{Footnotes and References}

"Sumary of invited paper, Proceedings of the Conference on Highly Excited States in Nuclei, Vol. Z, Julich, Germany, Segt. 1975, to be published.

1. B. R. Mbttelson and J. G. Valatin, Phys, Rev, Lett. 5, 51l (1960).

2. R. A. Sorenson, Rers. Mod. Phys. 45, 353 (1973), and references thereitn.

3. R. M. Dianond, F, S. Stephens, K. Nakai, and R. Nordhdgen, Phy's. Rev, C 3, 344 (1971).

4. D. Proetel, R. M. Diamond, P. Kienle, J. R. leigh, M. R. Maier, and F. \$. Stephens, Phys, Rev, Lett. 31,890 (1973).

5. N. Rud, D. Ward, H. R. Andrews, R. L. Graham, and J. S. Geiger, Phys. Rev, Lett. 31, 1421 (1973).

6. D. Proetel, R, M. Diamond, and F. S. Stephens, Phys. Letters 4 BB, 102 (1974).

7. S, Cohen, P. Plasil, and W. J. Seriatecki, Annals of Phystes 8z, 557 (1974).

8. R. Bengtsson, 5. E. Larsson, G. Leander, P. Hïller, S. G. Nilssson, S. Agerg, and Z. Szymanski, preprint, ऑune 1975.

9. A. Foessler, prīvate comomication, July 1975 .

10. F. S. Stephens, Rev. Mod. Phys. 47, 43 (1975), and references therein.

11. A. Johnson, H. Ryde, and J. Sztarkier, Phys. lett. 34B, 605 (1971).

12. F. S. Stephens and k. S. Simon, Shacl. Phys. A183, 257 (1972). 
F M Diemond

Fron simple considerations one can estinate the angular momentum brought to a reaction by a herpy ion,

$$
\ell_{\text {flax }} h=[2 \mu(E-\sqrt{ })]^{1 / 2}\left(R_{p}+R_{t}\right)
$$

where $i$ is the reduced mass, $E$ and $V$ are the energy and Coulomb barrier in the center-of-mass systen, and $R_{p}$ and $R_{t}$ are the projectile and target radii, respectively. To estimate the angular momentum actually retained in the campound systen and to what extent it is formed requires a madel-dependent approach. But whether linited by entrance-channel restrietions ${ }^{1}$ or by fission, 2 maxlei with upto 70 are expected in the rass region 100-180 with Ar or $\mathrm{kr}$ projectiles of the appropriate energy. Such a value is far more than can be accounted for by the discrete $\gamma$-ray transitions. A very large anount of angular monentun must be carried off by the contimum $\gamma$-rays that procede the discrete transitions.

By the use of a coincidence sycter 3-8 usually MaI-Ge, the reaction product can be specified by gates in the Ge detector spectrum, and the average number of gantua rays in the cascades passing through the gates, the average $\psi$-ray multiplicity for that reaction charuel, can be determined. Reșolts of our early measurement on the 166yb conpoud-mulear systen are given in Table 1. Listed are the values of the average $\gamma^{-}$ray multiplicities of transitions above $0.6 \mathrm{MoV}$; for the total average $\gamma$ - $x$ ay nultiplicity, one onust add the minber of coincident r-rays below 0.6 $\mathrm{MeV}$, mesing essentially the average number of discrete ground-band transitions observed.

The striking feature of Table 1 is the great variation in the value of $\mathrm{A}_{30}$, for different reactions. We bolieve this is a hatural result of angular moneritu considerations. Muclei with a large amount of angular momentum tend to enit fewer neutrons and have a Ionger yrast cascade, while those with littie angular moosintur enit more neutropis but fewer cascade $\gamma$ rays. The average mutiplicity, $\hat{N}_{>0}, 6$, should then be proportional to the average angular momentum of the nuclei leading to that product. Figure 1 shows that there is such a rough proportionality with the value of id determined from the cross section for that reaction, and that the angular momentum is far more decisive in deternining the value of 3.0 .6 than is the nature of the projectile or the number of neutrons encitted. We belier'e that this is the most inportant conclusion to come out of our first study; a secondary conclusion is that thare

Table 1. Average number of contzinm $\gamma$-rays above 0.6 MeV.

\begin{tabular}{|c|c|c|c|c|c|c|}
\hline \multirow[b]{2}{*}{ E(keV) } & \multirow[b]{2}{*}{$I_{i} \rightarrow I_{f}$} & \multirow{2}{*}{$\frac{347 \mathrm{MeV}{ }^{84} \mathrm{Kr}+{ }^{82} \mathrm{Se}}{\mathbb{8}_{>0.6}}$} & \multirow{2}{*}{$\frac{88 \mathrm{MeV}{ }^{16} \mathrm{O}^{150} \mathrm{Sn}}{\mathrm{H}>0.6}$} & \multirow[b]{2}{*}{ H(kev) } & \multirow[b]{2}{*}{$I_{I}+I_{E}$} & \multirow{2}{*}{$\frac{26 \mathrm{MeV}^{1} \mathrm{H+}^{165} \mathrm{Ho}}{N>0,6}$} \\
\hline & & & & & & \\
\hline & & ${ }^{162} \mathrm{Yb}(4 \mathrm{n})$ & & & ${ }^{163} \mathrm{Er}(3 n)$ & \\
\hline 166 & $2+0$ & $\$ 1$ & 8 & 127 & $13 / 2^{+}+9 / 2^{+}$ & 1,2 \\
\hline 320 & $4+2$ & 14 & 10 & 165 & $15 / 2^{+}+13 / 2^{+}$ & 2.2 \\
\hline 437 & $6+4$ & 12 & 9 & 171 & $13 / 2^{-} \rightarrow 11 / 2^{-}$ & 2.3 \\
\hline 521 & $8-6$ & 12 & 9 & 190 & $9 / 2^{-}+7 / 2^{-}$ & 1,3 \\
\hline \multirow[t]{3}{*}{579} & $10+8$ & & 9 & 213 & $15 / 2^{+}-11 / 2^{+}$ & 1.3 \\
\hline & & & & 218 & $17 / 2^{+}+13 / 2^{+}$ & 1.7 \\
\hline & & $163 \mathrm{db}(\mathrm{m})$ & & 236 & $11 / 2^{*}+7 / 2^{-}$ & 1.6 \\
\hline 202 & $17 / 2^{+}+13 / 2^{+}$ & 20 & 14 & & & \\
\hline \multirow[t]{3}{*}{345} & $2 \mathrm{I} / 2^{+}+17 / 2^{+}$ & 19 & 9 & & ${ }^{164} \operatorname{er}(2 n)$ & \\
\hline & & & & 208 & $4 \rightarrow 2$ & 3.7 \\
\hline & & ${ }^{16} 1_{\mathrm{Yb}(5 \mathrm{n})}$ & & $3: 4$ & $6+4$ & 3.3 \\
\hline 232 & $17 / 2^{+} \rightarrow 13 / 2^{+}$ & 10 & 5 & 410 & $8+6$ & 3.2 \\
\hline
\end{tabular}




\section{○U:}

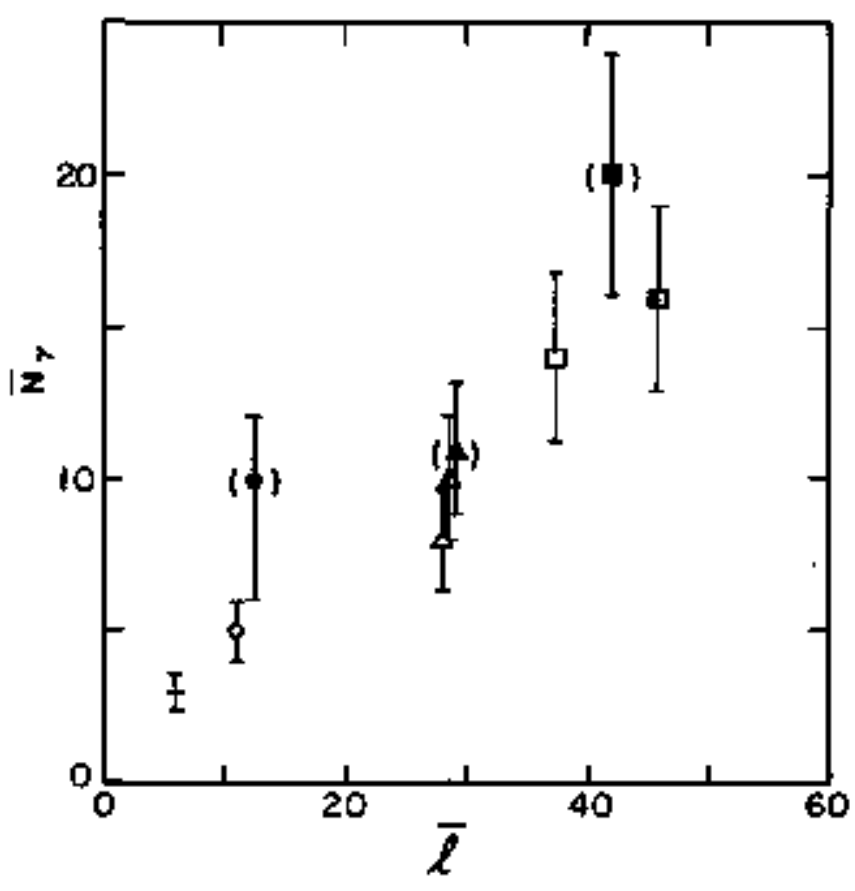

Fig. 1. Average number of continum $\gamma$-rays with $E_{y}>0.6 \mathrm{MeV}$ coincident with the lowest observed discrete transition $\left(2^{+}+0^{+}\right.$or $\left.17 / 2^{+}+13 / 2^{+}\right)$vs. the initial average angular modtentum of the compound rucleus leading to that reaction The cross is for the reactions $165_{\mathrm{Ho}}\left({ }_{\mathrm{H}}, \mathrm{xur}\right) 163,164 \mathrm{Er}$, and the circles, triangles and squares are for the $5 \mathrm{n}, 4 n$, and 3n reactions fromk $160+150$ in (open], ${ }^{84} \mathrm{Kr}^{+}{ }^{8}{ }_{\mathrm{Se}}$ (closed), $40_{\mathrm{AT}^{+}} 126 \mathrm{Te}$ (half-closed). The parentheses on the ${ }^{84} \mathrm{Kr}$ data indicate that considerable w1certainty in the values of $\bar{l}$ is introduced by the target thicluess in this case.

(XBL 746-3366)

does appear to be some fractionation by spin into the different $x$ reactions.

The points lin Fig. I detenine a curve with a slope of $\sim 1 / 2$, Such a value with the known cascade feeding times of $<10$ picosecond is consistent only if the majority of the continum $\gamma$-rays are stretched E2 transitions. To check this, we have measured the angular correlation of cho $\psi$-rays absorbed in the Nal counters in coincidence with the entire spectrom. in the Ge counter. The results for three nuclei made by irradiating the Iisted targets with $183 \mathrm{MkV} 40_{\mathrm{Ar}}$ are shom in the top of Fig. 2 . The ratio $w\left(0^{\circ}\right) /$ $w\left(90^{\circ}\right)$ for the raw polse leight spectra is given as solid lines, but after unfolding and correcting for Doppler shift at $0^{\circ}$, these points are replotted as dashed lines. At the lower $\gamma$-ray energies, where most of the transitions are concentrated (note logarithic scale for bottin part of figure glving the spectra) the anisotropy does indicate nostly stretch ed E2 transitions.

The spectra in Fig. 2 also illustrate that there is a gross structure in the continulu spectra, namely a division into a high-energy, rearly exponential tail, and a low-energy bump containing nost of the intensity and consisting predoratnantly of stratched E2 transitions. It secons most natural to

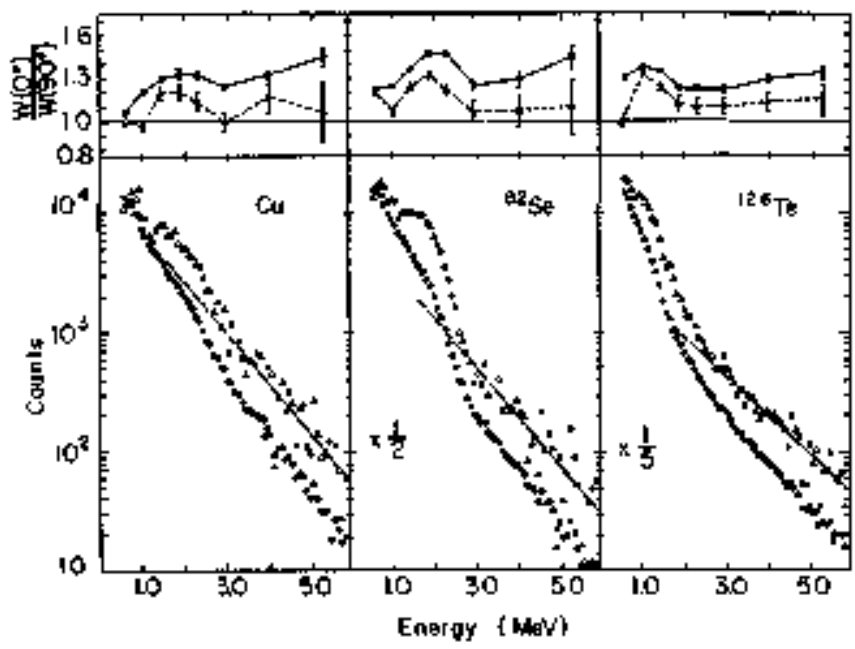

Fig. 2. The raw (dots) and unfolded (triangles) contintnm spectra in coincidence with the full Ge detector spect fu for $183 \mathrm{MeV} 40 \mathrm{Ar}$ projectjles on $\mathrm{O},{ }^{6} \mathrm{Se}$, and ${ }^{126} \mathrm{Te}$ targets. The straight lines are fitted to the wafolded spectrum between 3.2 and $6.2 \mathrm{MeV}$ (solid portion) and extrapolated to lower energies (dashed portion). The upper plots show the of $/ 90^{\circ}$ intensity ratios for the raw data (dots) and for the unfolded data corrected for recoil notion (triangles). The encor bars indicate statistical errors only.

\{XBL 751-2146)

associate the exponential tail with the first 3-4 transitions that follow neutron enission and constitute a statistical gammanay cascade. But after a few such $Y$-rays, of a few Mev each, the yrast region is reached and now the nucleus is hard-pressed to lase argular somentum with a minimum loss in excitation energy. So the rature of the transitions changes to stretched E2 $\gamma$-rays of moderate ( $\sim 1 \mathrm{MeV}$ ) energy; this is the yrast cascade. If only a single sequence of $\gamma$-rays were involved, they wowld have enough intensity to be seen as discrete lines so there must be a number of such sequences. And because of the short de-excitation times for the whole cascade, the E2 transitions must be enhanced and form members of (several collective bands. Te have unate preI infinary studfes of the dependence of the yrast bump on the projectile size, projectile energy, and compound-nuclear nाass munber.

Although much is yet to be learned about the dependencies just mentioned, information of another type can be extracted from the data to give some clues as to the nature of the hath-spin states that are at the top of the rrast cascade. In particular, values of effective moments of inertia can be determined from the expression, $E_{t}=\frac{b 2}{2}$ (4I-2), if corresponding values of $I$ and the transition energy, $\mathrm{E}_{t}$ can be determined. With the assumption that the transition energies fncrease monotonlcally with spin (no backbending) the break in the curve indicating the end of the yrast cascade for a particular reaction thannel is the highest onergy transition, and corresponds to the highest spin state. An estimate of a spin can be obtained from the muItiplicity, and 
so yields a value for $\frac{24}{t}$. Three values so obtairad in $162 y b$ made by an ${ }^{\hbar 2^{+}} 160$ and two $\$ 0_{A r}$ bombardment are shown in Fig. 3 plotterd against (hus ${ }^{2}$ as in the usual backbending figure, along with the values detentined $\mathrm{fr}^{\prime} \mathrm{m}$ the ground band and from the ground band in the 1 sotone $160 \mathrm{Er}$. Such measirements seem capable of giving very useful information on the nature and properties of these highspin states, that canmot be reached by any other method at present.

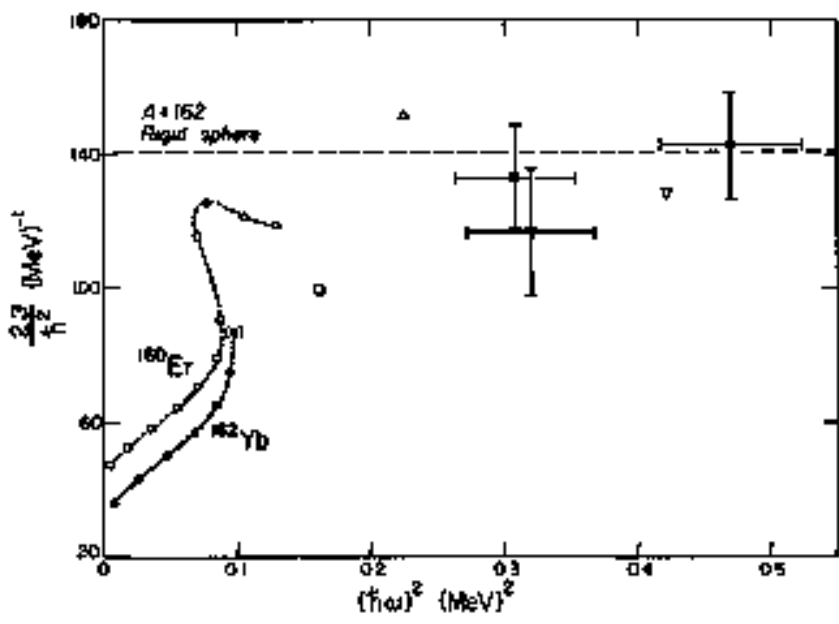

Fig. 3. Plot of $\frac{2}{5}$ vs. $(1+\omega)^{2}$ for ${ }^{162} \mathrm{rb}$. The filled square and triangles are determined as described in the text; the eircles are the discrete transitions in the ground bankl. The open circles are the discrete ground-band transitions in the isotoge ${ }^{160} \mathrm{Er}$, which backbends; it seems likely that $162 \mathrm{Yb}$ will also backbend at higher spith. The dashed line is the rigid-sphere value.

(XBL 756-\$235)

\section{Footmotes and References}

* Shortened version of LBL-4081, 1ectures given at the Eighth Masurian School in Nuclear Physics, MikolajkI, Foland, Nukleoniks 2I, 29 (1976).

1. J. Kilczynski, Nhe1. Phys. A216, 386 (1973).

2. S. Cohen, F. P1asil, w. S. Swiatecki, in Proceedings of the Third Conference on Reactions between Corplex Mucloi, ed. by A, Ghiorso, R. M. Diamord, and H. E. Conzett, (Univ. of Calif. Press, Berkeley, Calif, , 1963) p, 325 .

3. E, dẹr Mateosian, O. C. Kistner, and A. W. Stunyar, Phys. Rev. Lett. 33, S96 (1974).

4. G. B. Hggenann, R. Broda, B. Herskind, M. Ishthare, \$, Ogaza, and H. Ryde, Nuc1. Phys. A245, $166(1975)$.

5. W. Fenzl and O. W. B. Schult, Z. Physik 272, 207 (1975).

6. P. O. Tj\&m, F. 5. Stephens, R. M. Diamond, J. te Boer, and w. E. Meyerhof, Phys. Rev. Lett. 33. 593 (1974).

7. M. V. Banaschik, R. S. Simon, P. Colombani, D. P. Soroka, F. S. Stephens, and R. M. Diamorki, Plys. Rev. Lett. 34, 892 (1975).

B. J, 0. Newton, J, C. Lisle, G. D. Bracoulis, J. R. Leigh, and D. C. Weisser, Phys. Rer. Lett. 34, $99(1975)$.

\section{${ }^{10}$ Ar BOMBARDMENTS OF TARGETS AROUND THE $N=126 Z=82$ CLOSED SHELLS}

A Ghırso J M Notschike, and R E Leber

To assess the inpact of shell closure and shape excursions on codpound-nucleus excitation and, more specifically $y_{h 0}$ (H.I., , 罚) reactions, a series of

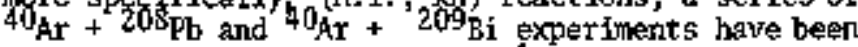
performed. Dganessian et al.,1 reported recently that the nagicity of lear isotopes couplat with the dissipative shepe transitions to deformed componmd nuclei provide reaction $Q$ values of sufficient1y greater magnitude to substantially lower the excitation energy of the compound interpediate.

Through detection of spontaneous-fission events, the Dubna roctup concIuded that two-or threeneutron eraporative dexay is the most likely (H.I., xi) process in this regline, with charged-particle enission being neglixibly small. Their work includes the production of 246 Fith a 7 nanobart cross sec tion and $24{ }^{2}$ with 1.5 nanobarns via $20 f_{\mathrm{Pb}}\left({ }^{4} 0_{\mathrm{Ar}}, \mathrm{xn}\right)$ reactions, where $\mathrm{xn}=\mathrm{z}$ and $4 \mathrm{n}$, respectively. (The protuct of the $x=3 n$ reaction, $245_{F}$, was not observed since it lacks a fission branch.) Also reported is the fomation of $256104,2 n$-evaporation residue from the bombardement of $20 \mathrm{~B} p$ with magic 50 i.

Clearly, bbtaining nuclei comparably "cool" to these systems has important implications for the heavy/superbeary elentent search; horever, the Dubna observations differ appreciably from calculations. The model presented by Alonso, 2 for example, predicts (for $R_{n}=1,208 \mathrm{fm}$ ) that the cross section for the $x_{n}=$ In reaction will be roughly two orders of magnitude larger than that $f \sigma r \quad x \sigma_{1}=2 \pi$. To attempt resoluting of this discrepanch it was decidect to buthard $203_{\mathrm{Pb}}$ with $4 \mathrm{O}_{\mathrm{Ar}}$.

The procedure for this investigation was, as 
detailed elsenhere, 3 to do alpha spectroscopy on recoil products transferred to a low-backsround conting area through a helfun-jet capillary. The activity is deposited on a vertical whes which is stepped at a determined rate to position the activity spots in front of gold surface-barrier silicon detectors, Data are passed through a ckuk interface to a PDP-9 CCoputer for on-line sorting and magnetic tape storage for stibsequent off-tine sorting on LBLs $\operatorname{coc} 7600$ computor.

In theșe experiments ${ }^{246} \mathrm{P}$ R $\left(\mathrm{xg}_{\mathrm{n}}-2 \mathrm{n}\right)$ could not be seen above a detection lioit of about $1 \mathrm{nb}$. However, ${ }^{245} \mathrm{Fm}$ was observed and characterized by half iffe and excitation fuction; moreover, the agreement between erpirical and predicted excitation functions is relatively good (Fig. 1). (Scobe difficulties were encounteref in specifying projectile onergies due to straggling and possible local helium density variations from beant heating.)

Therefore, tentatively conclude that, insofar as the two-neutron evaporation cross section is not at approximate parity with the 3n-out process, the extent of "cooling" inparted to the compound ru-

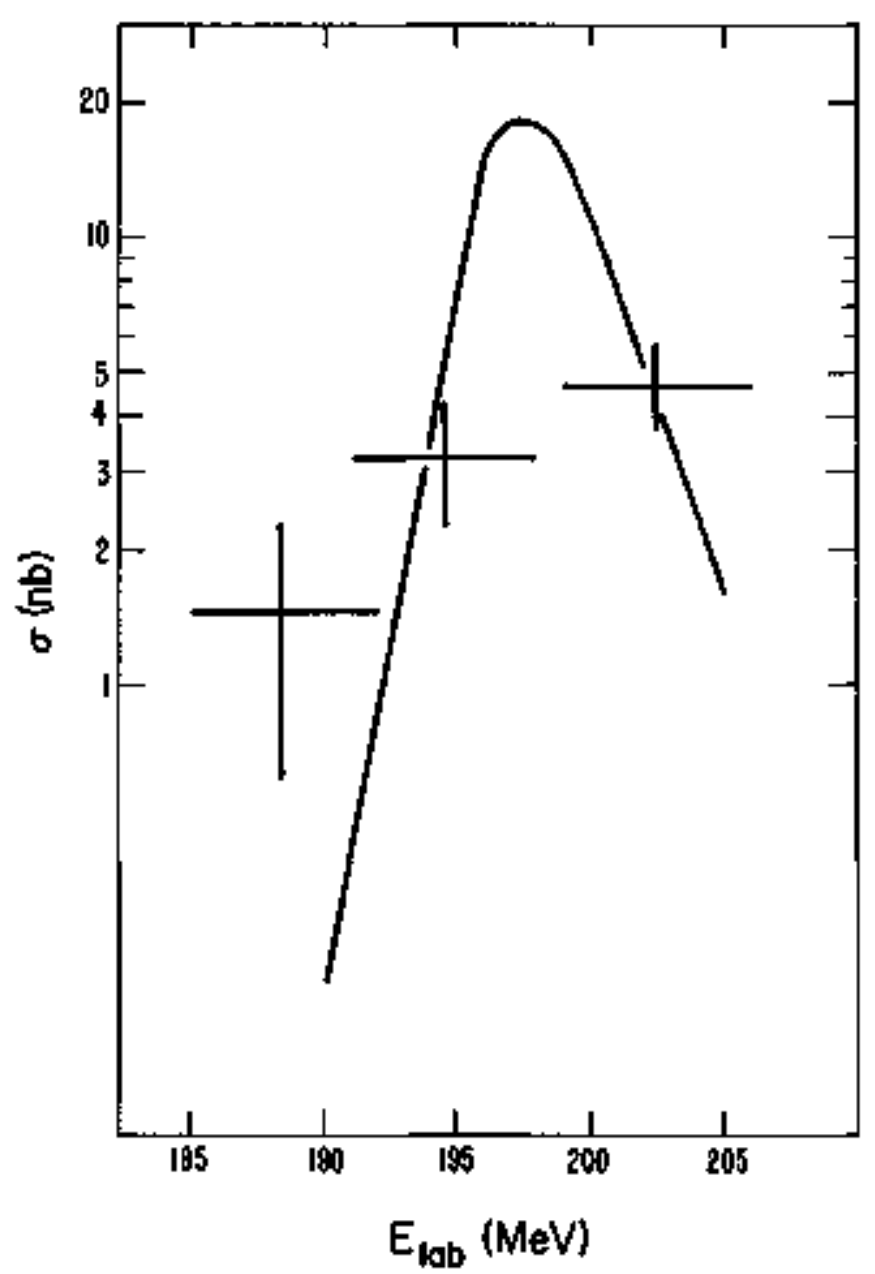

Fig. 1. Excitation function for the ${ }^{208} \mathrm{pb}\left({ }^{40} \mathrm{Ar}, 3 \mathrm{~s}\right)$ ${ }^{24}$ Fm reaction I solid line is theoretical $\left(\mathrm{R}_{0}-1.208\right.$ fin) function].

(XEL. $766-8247$ ) cleus through shape changes may not be of the degree claimed elsewhore.

of particular interest in the $209_{\mathrm{Bi}}+40_{\mathrm{Ar}}$ experiments is an alpha line at $7.90 \pm 0.05 \mathrm{HeV}$, with a half life of $21 * 4$ secs, good evidence for $243 \mathrm{Es}$ $\left(\mathrm{E}_{\mathrm{g}}=7.90 \mathrm{Her}, \mathrm{T}_{1 / 2}=20\right.$ secs\}. Produced in a $20 F_{B i}\left(40_{k r}, \alpha 2 n\right)$ reaction, this activity--while not a direct contradiction of the above mentioned statement in Ref. 1 , since the rass defect of 208 P exceeds that of $209 \mathrm{Bi}$ by some $3.5 \mathrm{MeV}-$-indicates that charged-particle emission camnot be easily assumed negligible or disregarded in this transuranium region.

Transfer products near the lead and bismeth targets were produced in abundance, in these experingents. Muctides observed were: $2 \mathrm{II}_{\mathrm{Bi}}, 2 \mathrm{I} 1,21 \mathrm{Im}_{\mathrm{P}}$, $212 \mathrm{Po}, 211_{\mathrm{At}}$, and $212,212 \mathrm{mAt}$. In smaller yields

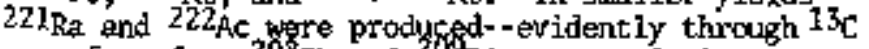
transfer-from $20 \mathrm{pb}$ and $20 \mathrm{Hi}_{\mathrm{Hi}}$, respect Ively.

Restricting the helium flow through the target chamber so as to limit recoil collectton to an acceptance angle of about $10^{\circ}$ with respect to the bodn axis gave an interesting result: for 211 po, popriation of the ground state tended to be more forward peaked than the distribution of the isomer, 2101 po. This misy be evidence for different entrance-chansel mechanisms (quasi-e1astfe vs. a deep-inelastic process for 211 ro and 21 impo, respectivety).

Noteworthy, too, is the propensity for ${ }^{13} \mathrm{C}$ transfer, responsible for $22 \mathrm{I}_{\mathrm{R} a}$ with the $208 \mathrm{P}$ target and $222,22 \mathrm{mAc}$ with ${ }^{209} \mathrm{Bi}$. These activities, identified by time correlation with their respective daughters and granddaughters, were reproducible and ontually exclusive. Further, there was no apparent evidence for $14 \mathrm{C}$ transfer.

Becrause the melting point of lead netal (3280 is too low to use the elementally pure material as a target, a lead coapound from which to make a șiitable, chemically and mechanically stable target had to be fokmd. Initial trials were with natural and separatod Pbs. Prepared by precipitation with hydron gen from the nitrate solution, Phs could be obtained in good yield as a finely divided black poiter. As a target, however, the sulfide proved inadequate when beam heeting caused the target temperature to exceed $490^{\circ} \mathrm{C}$. At temperatures in excess of about $450^{\circ} \mathrm{C}$, the sulfur atoms tend to leave their lattice sites and diffuse out of the crrstal. 4 Such a variable stoichionetry is untenable for our application since the target composition contimously changes and the lead meta1 residue is relatively volatile.

Lead monoxide proved a more suitable alternative. Precipltation of the hydroxide from a ritric acid solution followed by delnydration of the $\mathrm{Pb}(\mathrm{CH})_{2}$ in a vacunin furnace gave either one or both of the allotropic forms of $\mathrm{PgO}$. The thermodynalics of this material, hrower, were such that it was subject to reduction by the molybdenum backing foil at elevated tenperatures. Effective solutions to this problen. incIude comvective cooling of the target by helinm flow through a double window and vapor depositing onto the ifo a thin ( $250 \mathrm{\mu g} / \mathrm{cm}^{2}$ ) Pd film onto which the $\mathrm{PbO}$ is esaporated. 


\section{References}

1. Yu. Ts. Oganessian, Yu. P, Jretyakov, A. S. I1j inov, A. G. Dertin, A. A. Pleve, S. P. Tretyakova. V. M. Plotko, H. P. Ivanov, N. A. Danilov, Ylo. S. Korotkin, and G, N. Flerow, Jח奋, D7-8099 (1974).

2. J. R. Alonso, Gmelin, Handbuch der Anorganischen thesrie, Band $7 b$, Teil Al, p. 28.

3. A Ghiorso, J. M, Nitschke, J. R, Alonso, C, T. Alonso, M. Nurmia, and G. T. Seaborg, Phys. Rev. Lett. $33,1490(1974)$.

4. W. W. Scanlon, Phys. Rev. 92, 1573 (1953).

\section{SEARCH FOA SPONTANEOUS FISSION IN NEUTRON DEFICIENT ISOTOPES OF PU, Cm, AND Cf}

Kumberty Willams and Mattı Nurma

The systematics of the spontaneaus fission half-1ives for even-even isotopes of elements from plutonium to nobelium are shown in Fig. 1. Not only does the 152-nelttron subshel1 protuce a well-knom effect on the spontanedus fission half-1ives, but it also shows up as a characteristic kink in the alpha energy systematics with an alpha energy minimin around 252 reutrons (Fig, 2). There is a similar kink at 145 neutrons (which suggests there may be another nelitron subsheli at 144 neutrons, \& neutrons below the 152-netutron subshe11.

Since a subshe11 ranifests itself as a rap in the Nilsson diagram at a perticular value of nuclear defomation, it is "valid" over only a limited area in the mutide chart. Thus, the assumed 144-neutron subshell should no longer necessarily affect the Pnt isotopes, just as no effect is seen of the 152 -net1tron subshell in the spontaneous $f$ ission systematics of the heary Pu isotopes. The spontaneous fission half-1ives of the light Pu, om, and Cf isotopes, therefore, my not decrease as drastically as those of Fin.

Figure 1 shows purely systematic predictions fitting a parabola of the form $\mathrm{Log} \mathrm{T}_{1} / 2 \mathrm{SF}=\mathrm{A}+\mathrm{B}\left(\mathrm{N}-\mathrm{N}_{0}\right)^{2}$

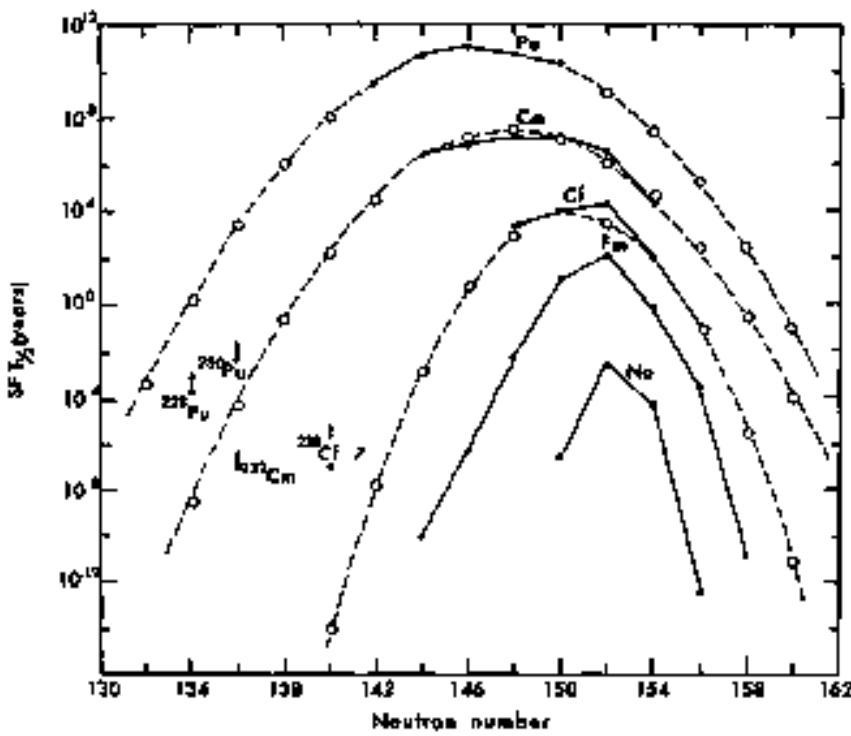

Fig. 1. Spontaneolds fission half-1fves vs, neutron momber for the indicated elements.

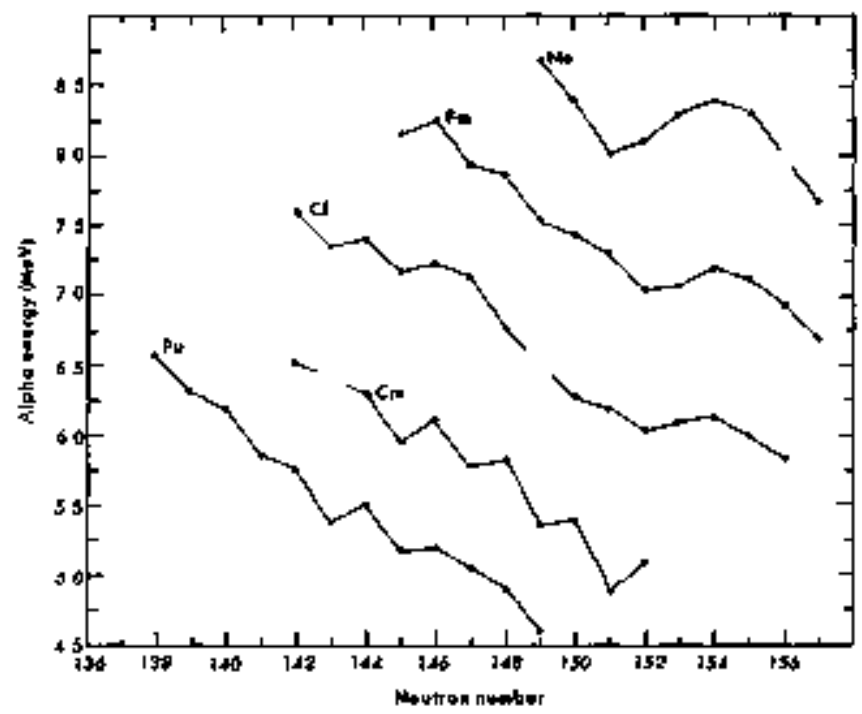

Fig. 2. Alpha-particle onergies vs, neutron number for the indicated elements.

(XBL 766-8234)

to known points for Pu, On, and Cf. These predictions do not take into account the fact that the light isotopes, Pu in particular, are expected to approach spherical shape and, therefore, probably do not have spontareous fission half-lives as short as these. In fart, it is not ureasonable to $\mathrm{ex}-$ pect to observe a jininum spontaneous fission half. Iife somewhere between 130 and 135 neutrons, the spontaneous fission haIf-lives begining to increase again as the 126-neutron subșhell is approachled.

In an effort to observe the spontareous $\mathrm{fis}$ sion decay of some of these light isotopes, the experiments listed in Table 1 were performed at the 88-inch cyelotron using a rotating drum system equipped with uica detectors.1 Experinental upper linits for the percentage of spontaneous fission branching for the listed isotopes are obtained by assuming a proctuction cross-section. Lower livits For the spontaneous fission half-Iife are based on the experimental spontaneous fission branching ratio and an estimated total half-life obtained by extrapolation of the known alpha and electron capture tatf-lives for the ever-ever isptopes. With the possible exception of the $40 \mathrm{Ar}+\mathrm{Hg}$ banbardment, no fissions other than a normal backpround were detected. Future work will include repeating the 


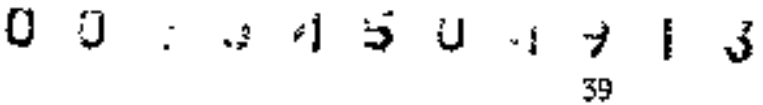

Table 1. Spontanecus fission (SF) of light isotopes of $\mathrm{Fu}, \mathrm{Om}$, and $\mathrm{Cf}$.

\begin{tabular}{|c|c|c|c|c|c|}
\hline Reaction & Isotope & ${ }^{0} \mathrm{Calc}^{\mathrm{a}}$ & ${ }^{5 F}$ Expt $^{b}$ & $\mathrm{~T}_{1 / 2 \mathrm{SF}-\mathrm{Expt}^{\mathrm{b}}}$ & $\mathrm{T}_{1 / 2 \mathrm{SF}} \cdot \mathrm{Pred}^{\mathrm{C}}$ \\
\hline $3_{\mathrm{He}}+233_{\mathrm{U}}$ & ${ }^{228} \mathrm{Pu}$ & $1.24 \mathrm{nb}$ & $<498$ & $>1.93 \mathrm{~m}$ & $1.5 \mathrm{y}$ \\
\hline $24 \mathrm{Mg}+\mathrm{Pb}$ & $228_{\mathrm{Pu}}$ & 11 ט & 9.884 & $>1.8 \mathrm{~h}$ & \\
\hline${ }^{3} \mathrm{He}+{ }^{233} \mathrm{u}$ & ${ }^{230} \mathrm{Pu}$ & $140 \mathrm{nb}$ & $0.34 \%$ & $>1.8 \mathrm{a}$ & $2.1 \times 10^{3} \mathrm{\gamma}$ \\
\hline $28_{\mathrm{Si}}+\mathrm{Pb}$ & ${ }^{232} \mathrm{Om}$ & $220 \mathrm{nb}$ & $<15 \%$ & $>3.3 \mathrm{~s}$ & $28 \mathrm{~m}$ \\
\hline $40_{\mathrm{Ar}}+\mathrm{Hg}$ & ${ }^{238} \mathrm{Cf}$ & $20 \mathrm{nb}$ & $<808$ & $>\sim s$ & $260 \mathrm{~ns}$ \\
\hline
\end{tabular}

These calculations are bosed on the model used by J. R. Alonso, Grelin, Handbuch der Anorganischen Chenie, Band Fb, Teil Al. p. 28 .

bThese limits are based on the assumed cross section and assured total half-life.

These values are obtained from the parabolic fits shown in Fig. 1.

$40_{\mathrm{Ar}} \mathrm{Hg}$ experiment and using other target/projectile conbinations to produce the light om and $\mathrm{CF}$ isotopes.

\section{Reference}

1. For a description of the equipment, see $E, K$. Hulet et al. , Phys, Rev. Lett, 26, 253 (1971),

\section{A 51-SECOND SPONTANEOUS FISSION ACTIVITY IN THE REACTION ${ }^{22} \mathrm{Ne}+{ }^{209} \mathrm{~B}$ 1}

L P Somervile, M J Nummia, and A Ghowso

The one-minute spontangexs ftssion activity produced from the reaction $22 \mathrm{Ne}+209_{\mathrm{Bi}}$ by Dubna experimenters Kuznetsov et al. 1 has been verified git the Berkeley 88-inch cyclotron, where 115 MeV 22 fle ions struck a rotating Bi target mounted over the ontire, circumference of a brass wheel (see Fig. 1). The 2 ide lons struck the wheel at $72^{\circ}$ from normal incidence, instead of at nomal incidence, to deposit the recoils in a thin layer of the target so that a calculated spontaneopus fission detection efficiency of $90 \%$ could be obtained. Spontaneous fission events were recorded by fixed 3.1-onl-wide mica detectors about a millinater from the spinning $B$ i target wheel, roteting at $1.1 \mathrm{~A} \mathrm{~cm} / \mathrm{sec}$. A total of 138 spontanoous fission tracks were recorded by the mich detectors for an exposrre of 8.3 $\mu^{\mathrm{A}}$-hrs $22 \mathrm{Ne}^{+} 5$ with an inherent detection efficiency of 100\%. The tracks were enlerged to a few microns in size by chemical etching in $\mathbf{4 8 4}$ bydrofluoric acidd for about one hour at $60^{\circ} \mathrm{C}$. The mica sheets were scamed for fission tracks using a $\times 100$ power microscope with -70 s measured efficiency, The beam intensity was measured by using the $209_{\mathrm{Bi}}$ target whel as a Faraday cup. Using the width of the excitation function (corrected for energy loss in the target) obtained by the Dubna group of shoust $3.5 \mathrm{mg} / \mathrm{cm}^{2}$ of $209_{\mathrm{Bi}}$, a cross section of $0.66 \pm 0.33 \mathrm{nb}$ at the peak of the excitation function was obtained. This value

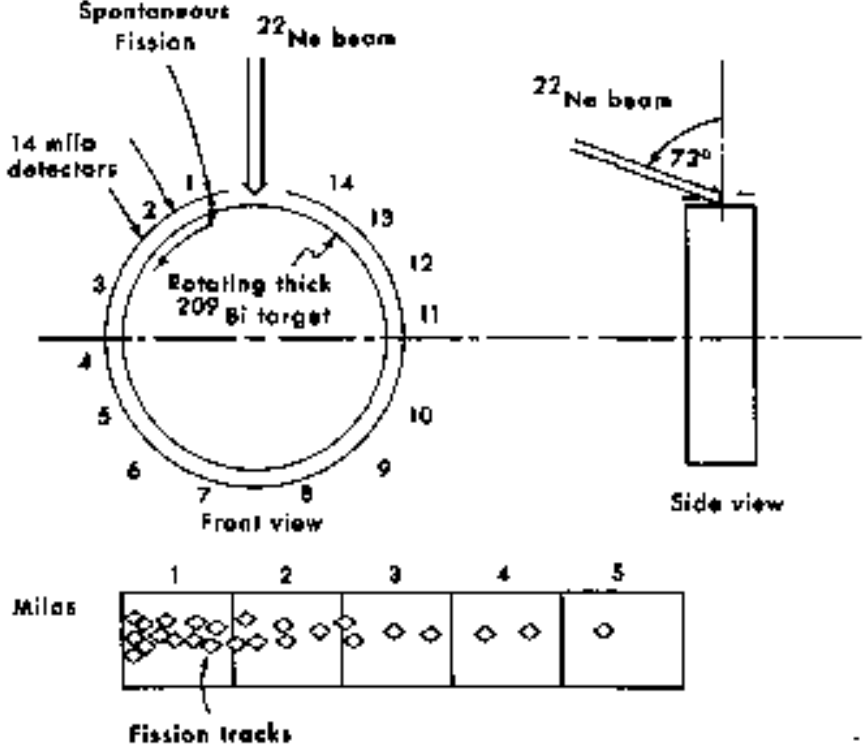

Fig. 1. Fotating drum assently for detecting spontaneous fission. The beam intensity was neasured by using the target as a Faraday cup.

(XBL $7611-4380$ ) 
compares with the Dubra measurentent of $0.45 \pm 0.08 \mathrm{nb}$. The half-1ife of this spontaneous fission activity was obtained by least squares fit of the decay points

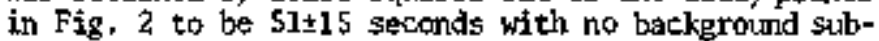
tracted. The Dubna value of $60 \pm 5$ seconds is in agreement within experimental error with this result.

The Dubna group corrcluded on the basis of the oretical argunents that the observed fission activ-

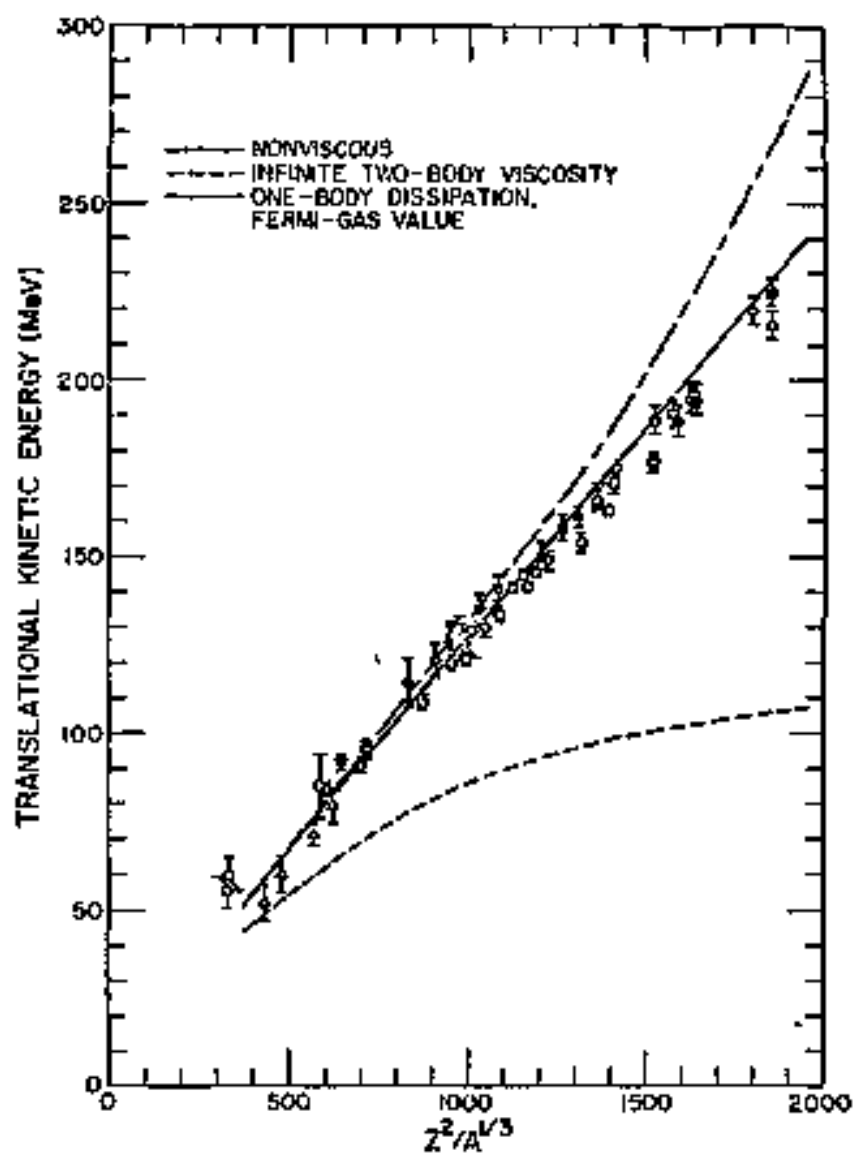

Figure 2. Decay curve for the spontaneous f1ssion activity.

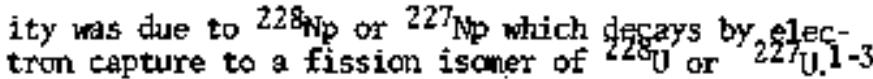
This hypothetical decay mode was called "delayed fis sion." They excluded the possibility of a primary production reaction in which a proton is enitted by showing that the spontaneous fission activity is not produced tn the reaction $22 \mathrm{Ne}+$ Pb. A reaction in which an alpha particle is emitted was also excluhded basted on the absence of spontaneous fission activity from the reaction $180+209 \mathrm{Bi}$.

Future experiments may be attempted in Berkeley to look for rare uransum $x$-rays from the electron capture decay of $22 \mathrm{~s}_{\mathrm{Np}}$ or $227 \mathrm{~Np}$ followed $b y$ coincidence courting of spontaneous fission events. Such a direct verification of "deleyed fission" has not yet been accouplished. Upper linits on the spontaneaus fission branching ratio have been set by the non-obseryation of gpontapeous fission frem

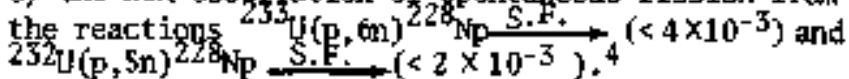

Population of a fission isconer state of a nuclide elsewhere in the regich of atomic nutiers near the target prohuced by a noncalpound nucleus reaction is also a possibility. However, no such flssign iscmers have been found in reactifins $20 \mathrm{Ne}+209 \mathrm{Bt}$ ( $<.1, \mathrm{nb}), 160+n \mathrm{nt} \cdot \mathrm{Pb}, 2 \mathrm{ONe}_{\mathrm{Ne}}+207 \mathrm{pb}, 2 \mathrm{~N}_{\mathrm{Ne}}+\mathrm{nat} \cdot \mathrm{Pb}$, and $24 \mathrm{Hg}+$ nat. $\mathrm{Pb}$. The possibility of a 51 -second fission isomer of $22{ }_{\text {Np }}$ has not been ruled out, but the lorgest-1Ived known fission iscmer, $242 \mathrm{Ama}_{\mathrm{Am}}$, has a half-life of only 13,7 milliseconds.

\section{References}

1. V. I. Kuznetsov, N. K. Skobeler, and G. N, Flexov, J. Mucl. Phys. (USSR) 4, 279 (1966).

2. N. K. Skobelev, Sov. J. Mhel. Phyrs, 15, 249 $(1972)$

3. E. E. Berlovich and Yu. P. Novikov, Sov. Physics-Dok. 14, 349 (1969).

4. R. L. Hahn, Oak Ridge National Laboratory, private commanication.

\section{NEW ATTENPT TO FINO THE DUBNA "0.1-\$ ${ }^{260} 104^{N}$ ACTIVITY}

A. Ghwrso. P. A. Baisden, I. Binder, A. E. Leter, D. Lee, D. Morristey,

J. M. Nilschke. M, J Nurmia, F. J Dtto, L P. Somervilte, $\mathbf{X}$ Thomas, K. E. Wiliams, E. K. Hulal," R. W, Loughheed," J. F. Whid," J H. Landrum,* R. J. Silva,t C. E. Bomi\$,t and $\mathbf{m}$ Fowm|erł

The followng paper details recent experinents designed to settle with finality whether or not the isotope $260_{104}$ is a spontaneous-fission emitter with half-life of about $0.1 \mathrm{sec}$ as claimed by the Dubna heavy elenents group. 1 These experivunts were conducted at the 88 -inch cyclotron by a tean of scientists from lawrence Livernore, Oak Ridge kational Laboratory, Los Alanos Scientific Laboratory, and Lawrence Berkeley Laboratory. Nore details are available in LBL reports.
The principal reaction used in our recent attelpts to produce the Dubra $\sim 0,1-\$ e c$ SF activity was $249_{\mathrm{gk}}[15 \mathrm{~N}, 4 \mathrm{n}]{ }^{260} 104$. As in the earlier experiments? which were also tusiccessful, the reaction recoits mexe caught in racun on the periphery of a rotating drum. These products wore quidkly carried next to thin mica sheets so that spontaneous fission events could be spatially detected. A completely new apparatus was built for these experiments with an important difforence being the use of a longer 


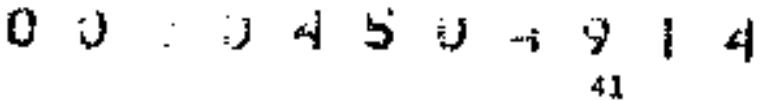

drum to sprezd wot the long-lived $5 F$ background activity fros $256 \mathrm{Fm}$ groduced in the bombardments frum Its e.c.-mother, $250 \mathrm{Md}$. The $249 \mathrm{Bk}$ target used con-

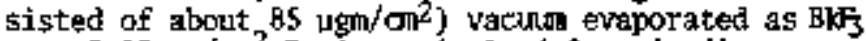
unto $2.08 \mathrm{mg} / \mathrm{cm}^{2}$ Be in a circle $4.8 \mathrm{~mm}$ in diameter. The target was plaçed $21 \mathrm{~mm}$ from the surface of a 254-Jig dianeter water-cooled drum, which was rotated typieally at 60 revolutions per minute. The nuclearreaction recoils passed in vaculn through an opening 20-лn square to strike the drum surface. The 360 nin long drum was scanued axially, usualiy at a speed of $7.7 \mathrm{Jm} / \mathrm{sec}$. The mica fission-track detectors, 31 Ina wide and 50 ma long, were held in a curved holder about 0.8 won athay from the drum surface and complete ly surrounded the dint in a 31 -nitio slice.

The $15 \mathrm{~N}$ ipn energies used were obtajned by three settings of the 88-inch cyclotron and the use of Be-foil degraders. The nominal values of the energies out of the cyclotion, calculated from the cyclotron frequencies and fields, were $91,6,93,6$ and $105 \mathrm{MeV}$, thought to be accurate to better than 0.5 Mev. In the 93.6-Mov case, a speciat analysis was made by $\mathbf{D}$. Hendrie using the large beam analyzer magnet and the energy was found to be $93.74 \pm 0.01 \mathrm{MeV}$. The Be degraders used to obtain lower energtes yero weighed and the final eneries at the $0.5 \mathrm{mg} / \mathrm{cm}^{2}$ $249 \mathrm{Kk}$ target were obtained by calculation. The drop in energy of the $1 S_{\mathrm{N}}$ ions through the $\mathrm{Bk}$ itself was about 0.5 MeY. The early experiments showed that the beam profile attained a needlis-sharp focus when tumed for reaximum bean current, and jndeed we melted seall holes through two old targets with currents in the vicinity of 3-5 charge microamperes. A threen phase 60-Hz wobler was instelled alout $5 \mathrm{~m}$ upstream follow ing the last beam quadrupole magnet. The wobbler was singly the stator of an induction notor and succeeded in moving the beam in a small circle at a $60-\mathrm{Hz}$ rate with the loss of abcut 158 of the bean intensity. Wath this arrargenent, wo were able to use bean currents as high as 4 k , brit nomally we linited the beam to abcut 3 uA to have a rargin of safety. An infrared detection system was installed to guard agatnst any unusual excursions of the beard and was similar to the one used in the Berkeley elenent 106 experinents. 3

We found it advantageous to put a very thin (26 $\mathrm{\mu g} / \mathrm{cm}^{2}$ ) aluminum foil between the target and the drut to prevent any of the target material frombeing knoked out by the beam onto the drum surface and thus increasing the spontaneous fission background. This also served as a rough monitor of the bean current since as much as $5+$ of the 259 did that was produced stopped in these foils.

In addition to the regular drun experinents we made neasurements of the long-lived recoil products directly. This was accomplished with a special holder murnted next to the target. In thesse experiments, an exterral Faraday cup was used to give achorate beam reatings. An excitation function for 256 w. which is made with a large cross section, is shown in Fig. 1 and was obtained by stopping its recoils in $1.0 \mathrm{mg} / \mathrm{an}^{2}$ Al and counting the foils directly in a fission counter. The growth and decay in one of these runs, a $1.65 \mu \mathrm{A}-\mathrm{hr}$ bormbardment at $88 \mathrm{MeV}$ for 2,0 hrs show in Fig. 2, shows quite clearly that very little ${ }^{256 \mathrm{Fn}}$ is produced directly since the data can be fitted by a curve, asstaing that only 256ud is produced. The berkelium target was prepared at the Lawrence Livermore Laboratory after being chemically separated from its califorriun daughter just a few days before the first bombardient. This timing of the separation is iljportant because the jalf life of ${ }^{249} \mathrm{BK}$ is only 314 days and it very soon

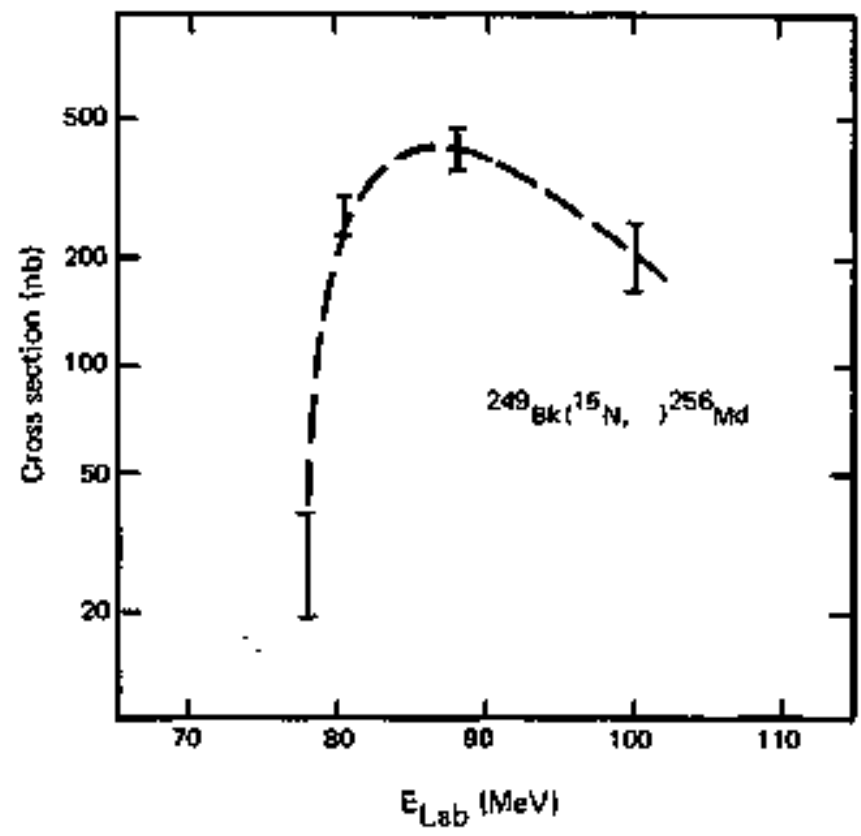

Fig. 1. Excitation futction for 256 a. Cross section in nandbarns.

OCBL $766-8241$ )

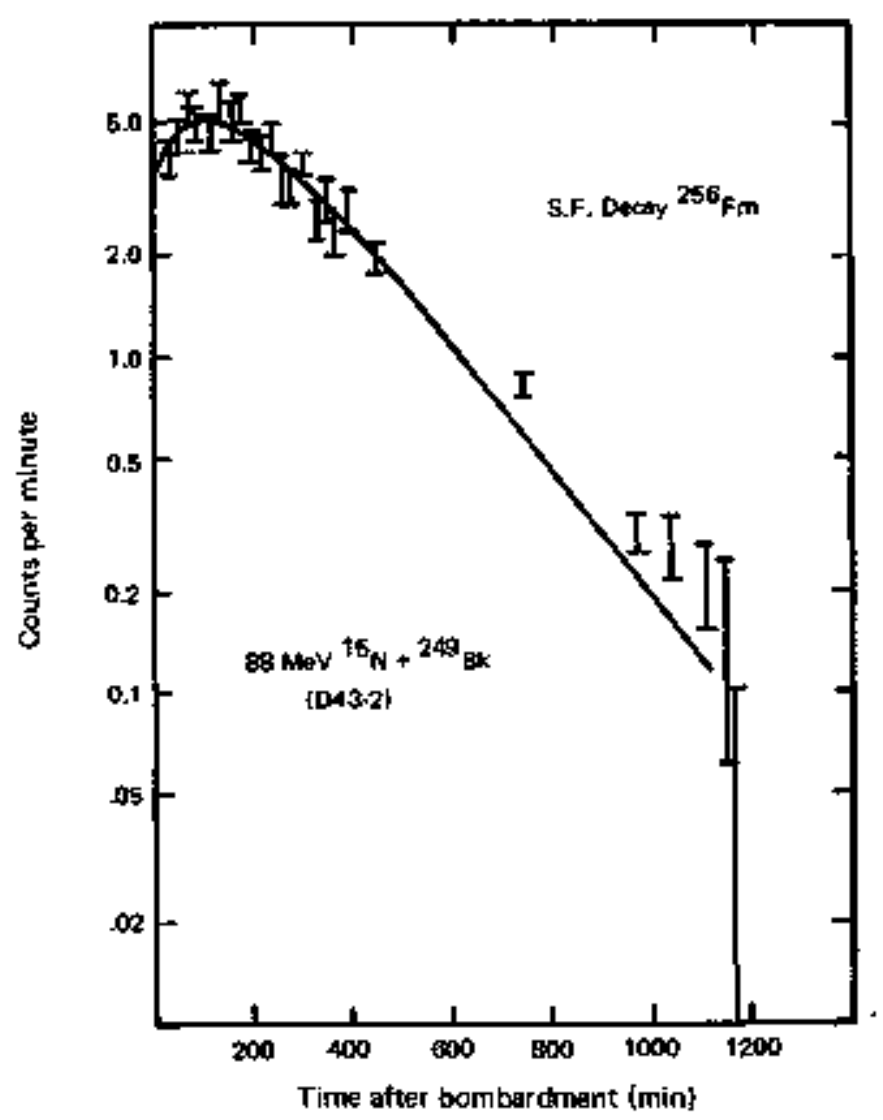

Fig. 2. Growth and decay curve of $256 \mathrm{Fm}$ (From 25 (X) ). (XEl 766-8243) 
bectmes contaninated by its 360-yr daughter. The series of bombardments were then carried aut with the chief objective being the attenpt to see whether an $\sim 0.1$-sec SF activity was produced. It was not and we decided to make as many cross checks as we could so that there would be no question of the validity of our attempts.

Bombardments were made with $78,82,86,88$ and $100 \mathrm{MeV} 1 \mathrm{~N}_{\mathrm{N}}$ ions entering the target. There is gerleral agrement that the cross section for the $24 \mathrm{Bk}$ $(15 \mathrm{~N}, 4 \mathrm{H}) 2 \mathrm{C}_{104}$ Teaction showld peak at about $82 \mathrm{MeV}$ so this series adequately covers this range. Approximately $40 \mu a-h r s$ of beam were devoted at each of these energies in the search for the 80-masec activity, The results of the bonbardment at $82 \mathrm{MeV}$ are shown in the decay curve in Fig. 3. A calculated computer fit is also plotted, As can be seen, the maximum anount of a hypothetica] 80 -msec ${ }^{4}$ activity that can be extracted from these data is very șnal1. It corresponds to a cross section of less than 0.5 nanobarns if an excursion $2 \mathrm{a}$ higher than observed is allowed.

These decay curves were extracted from the raw scanning data by the following method. An early test w with a thicks $241_{\text {m }}$ target to produce the $2.3-\mathrm{sec}$ 25 No via the $^{15} \mathrm{~N}, 4 \mathrm{n}$ reaction at $82 \mathrm{MeV}$ gave us an actual situation of recoiling ruclei made by complete fusion. The angular distribution of these recoils is very narrow, being broadened mostly by neutron boil-off and multiple scattering withill the target. When the tracks were sumed in slices along the direction of rotation of the drum, the distribution shown in Fig. 4 was obtained; such a distribortion indicates that most of the fusion-produced nuclei can be measured by selecting a relatively narrow "window' in the transverse direction. Because in the case of the berkelitm bonterdments the

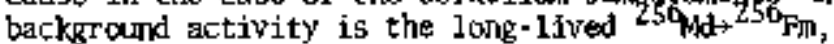
which is spread odt on the entire drum surface, we gained about a factor of $Z$ in signal-to-backgrand ratio.

To make sure that our track counting efficiency was normal, a few test exposures of our mica were made from a ${ }^{25}$ Cf source with and without an aluminum foil $250 \mathrm{\mu g} / \mathrm{cm}^{2}$ in thickness, which is equal to the calculated $\mathrm{CN}$-recoil range. Our average efficiency was 90 t.

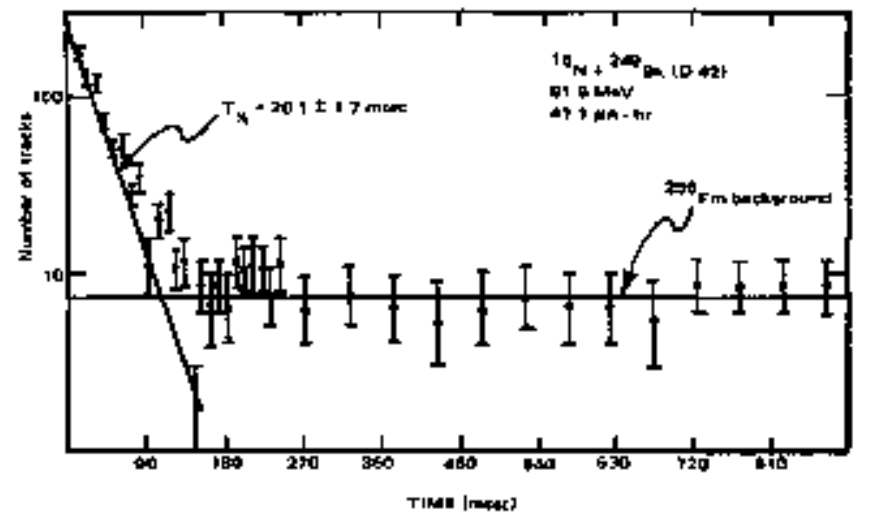

Fig. 3. Decay curve for spontaneous-fission events detected at $81.6 \mathrm{MeV}$. Solid lines represent comouter fit to the data.

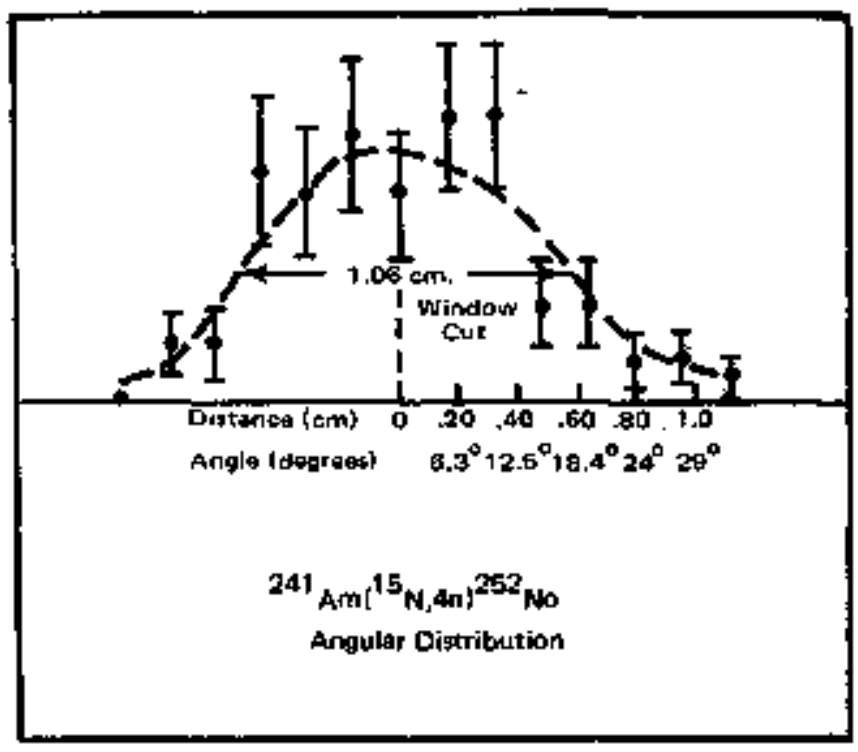

Fig, 4, Trapsyersedistribution of 252 No fission

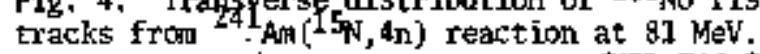

(XBL 760-8244)

Though our decay curves show no hint of the so-called 80-msec 5 activity, they very clearly show one with a half life of $220 \mathrm{msec}$. This same activity was first reported by Ghiorso at the Welch Conference in 1969 (Ref. 2, p. 148).

The maximu cross section for producing the +t $\sim 20$ msec" activity is about $10 \mathrm{nb}$ when made by $1 \mathrm{~N}_{\mathrm{N}}$ ion bxombariment of $249_{\mathrm{Bk}}$ and has the excitation function shown in Fig. 5, However, on the basis of this data alone we cannot exclude the possibility that the yields include some fission from the 13.7-mser $5 F$ iscmer, $242 m_{\mathrm{Am}}$, froduced by some sort of transfer

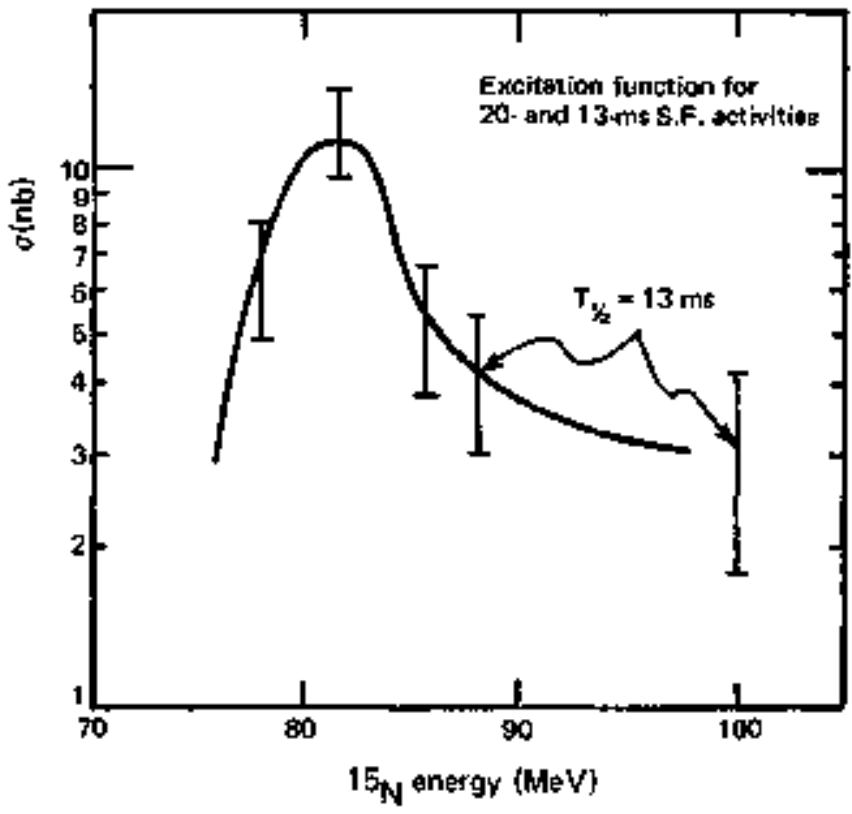

Fig. 5. Excitation function of the " 20 -msec" spontaneous-fission activity, (KBL 766-8245) 


\section{0 ป . .}

or pther reaction. The transverse track distribution for the " $\sim 20$-msec" activity is remarkably narrow at 83 HeW (ses Fig. 6). These data would seem to point to a complete fusion reaction except that its production $\sigma$ is sonewhat lower then expected (see below), Just prior to undertaking the berkelium. boubarduents, we boribarded $248 \mathrm{Om}$ with 160 ions and $246 \mathrm{G}$ with 100 ions at about $93 \mathrm{MoV}$ and once again pbserved the " 20 -msec" SF activity without observIn the hypothetical 80-nosec Dubna activity. Ho excitation function was measured becarse of lack of tifate. It is possible that the "w 20 -msect" activity includes $260_{104}$ since it passes some of the tests required. It may take some tine before we can state with certainty whether or not wo have observed this muclide since there is the distinct possibility that this activity is due to $261 \mathrm{lr}$ or similar maclide. This sibuation points up again the great difficulties that are encountered when working with spontaneous fisșion activities when the only paraneter that is measured is half-life.

\section{Although we set a naxtmu cross section for} the 15 , $4 n$ reaction on $249 \mathrm{BK}$ to produce the $80-n s e c$ activity which was more than an onder of magnitude less then the $5 \mathrm{ab}$ claisaed by Dutes, it should be remembered that the actual cross section to produce 260104 by this mechanism is expected to be as mach as $25 \mathrm{nb}$. The reasoning is as follows: the cross section for the identical reaction to nake 260105 in bombarthonts of 249 ch has been found experimentally to be $-12 \mathrm{nb}$. This rew value was obtained by a reevaluation of tho old Berkeley data by Pirkko Eskola

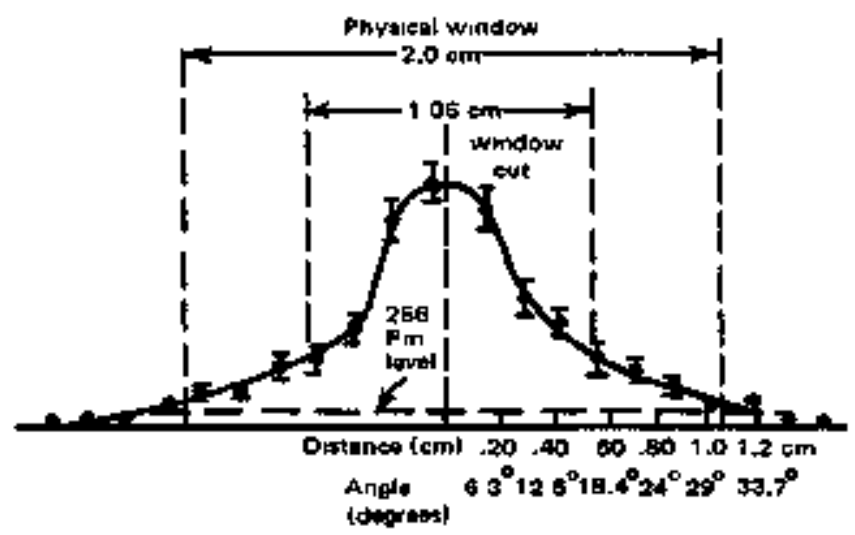

Fig, 6. Transwerse track distribation of the to $\rightarrow 20$-msece" spontanequs-fission activity at 81.6 MeV.

(XBL 766-8245) and appears in her recent thosis at the University of Helskinki. The cxoss section to make 260104 by the same reaction should be larger since the values of $\Gamma_{n} /\left(\Gamma_{n}+\Gamma_{f}\right)$ and $R_{t}$ are nore favorable, Inteed a calculation adjusted to the 260105 production cross section shows that the production of 26004 will be about twice as large. Our limit on the anount of the 80-msec activity that is made in the $249 \mathrm{gk}$ plus $15 \mathrm{~N}$ reaction is this almost two orders of ragmitude below that expected if it is due to 260104.

There is no escape from the conclusion that can be drawn on the basis of opur data. The nass 260 isotope of element 104, rutherfordium, does not decay by spontaneous fissich with a half-ilife of " 0.3 $\sec , "$ or "0.1 sec, " or "\$0 msec."

We extery arr thanks to the staff and operations rew of the 88 -inch cyclotron. Their help was invaruable to the soccess of these experiments.

He would like to acknowledge with gratitude the concentrated effort by mom colleagues that wont into the task of making these latest experiments so definitive. In particular, we would litke to thark Glem T. Seaborg for following developments very closely and providing inspiration and seientiflc guidance.

\section{Footnotes and References}

tarrence Livermore Laboratory.

†oak Ridge National Laboratory.

FLos Alamos Scientific Laboratory,

1. G. N. Flerow et al., Atonnaya Energiya 17,310 (1964); Sov. J. Atamic Energy 17, 1046 (1964); Phys. Lett, 13, 73 (1964).

2. A. Shiorsp, Proc. of the Folt. A. Welch Foumdation Conf. on Transuranium Elements, Houston, Nov. 1969 (p. 107).

3. A. Ghiorso et al., Phys, Rev, lett. 33, 1490 (1974),

4. The "k-0.1-sec" SF activity has recently been designated by tubna as having an $80-m s e c T_{1 / 2}$.

5. I. Zvara, private comumication. 


\title{
B. NUCLEAR REAC'TIONS AND SCATTERING
}

\section{Microscopic}

\author{
a. Light Ions
}

\section{POLARIZARION EFFECTS IN THE FINAL-STATE INTERACTION REGION OF THE p-d BREAKUP REACTION ${ }^{*}$}

F N Radf.t H E Conzelt, $A$ Roy.ł and F Sellers

\begin{abstract}
Anong the experimental obserwables in the three-ruscleon system, the elastic cross section, inelastic cross section, and elastic analyzing power have been intensively fnvestigated. 1 Inelastic analyzing powers comprise one class of observables which has received vory little attention. That is, on1y a few exporiments have been done which even show the presence of polarization effects, and theoretical interpretation and predictions via exsct three-body calculations have not, as yet, been made. The three-nucleon calaulations based on the Faddeev equations with separable potentials for the $1_{50}$. ${ }^{3} \mathrm{~S}_{1}-\mathrm{B}_{1}$, and $\mathrm{P}_{2}$-weve compongrts of the nucleon-nucleon interaction, 2,3 have prowided predictions of the vector and tensor polarizations which aro in good agreement with the experinental data in the elastic channel, 4-6 whereas the $S$-wave forces alone had been sufficient to give agreenent with the differential cross-section data, Sinilarly, calculations which have bes successful in fitting inelastic crosssection data, have been restricted to two-macleon: S-wave interactions 7 and, thws, carmot predict ana1yzing powers for incident polarized protons or deutorons. It seens that experimental evidence of substantial polarization effects, such as reported here, are required in order to encourage or even cappel the addition of the tensor force and P-wave contributions to three-body caloulations.
\end{abstract}

The polarized proton or deuteron bean from the Berkeley 88-inch cyclotron was passed through a gas target in a 36-in. diameter scattering chamber. A description of the experinental arrangenent and of the data acquisition procedure has been given elsewhere, 5

our experimental results for the proton analyzing power $A y(\theta)$ in the ${ }^{2} H(\vec{p}, p) d^{*}$ reaction at $E_{p}=22.7$ MoV are shomin in Fig, 1, where the errors inditated are purely statistical. Here, d* denotes final-state pairs with rolative energy $\mathbf{E}_{\mathrm{TP}}<1 \mathrm{MeV}$, in both singlet and triplet states. For coraparison the smooth curve represents the elastic scattering analyzing powers measured in the sane experiment. As can be seer, $A_{y}(\theta)$ in the breaky reaction regches substantial values at angles greater than $\theta_{\mathrm{C} . \mathrm{m}}=70^{\circ}$, and its angular distribution is quite stotiar to that of the elastic analyzing power. Figme $Z$ shows our experimental results for the deuteron vector analyzing power in the $\mathrm{H}(\mathrm{d}, \mathrm{p}) \mathrm{d}$ * reaction at $\mathrm{E}_{\mathrm{d}}=45.4 \mathrm{MeV}$, corresponding to the same center-of-mass ener $y$ as in the $4(\vec{p}, \mathbf{p}) d^{*}$ reaction. Agatn the extors indicated are prrely statistical and the solid line is a snooth arve drawn through the elastic scattering

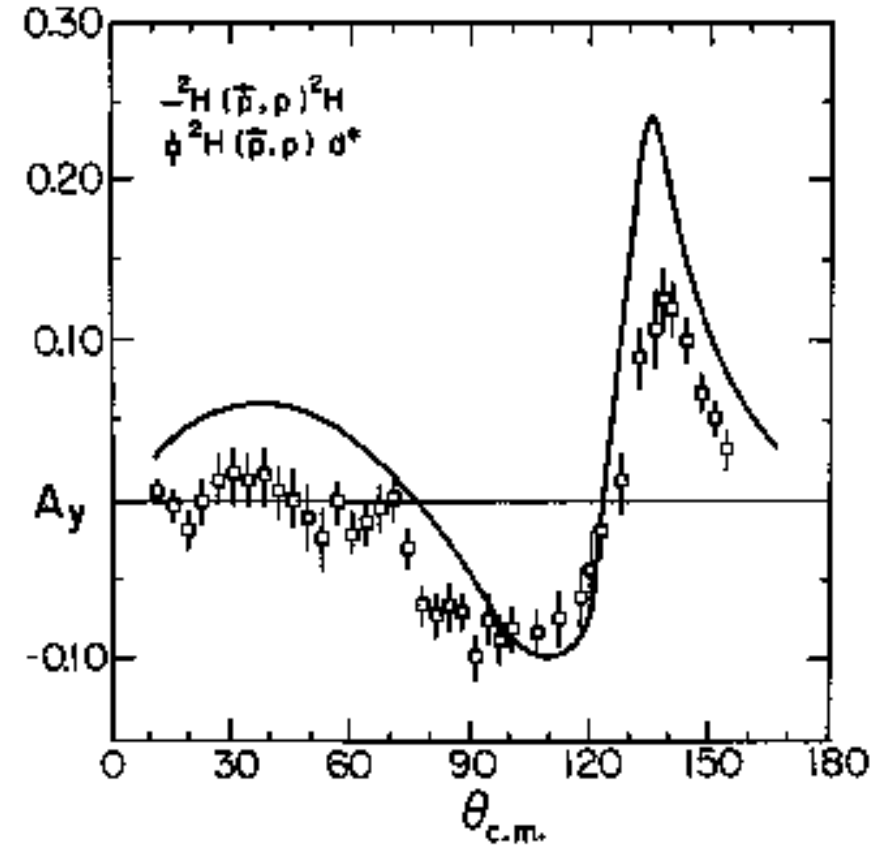

Fig, 1. The proton analyzing power Ay( $(\theta)$ in the

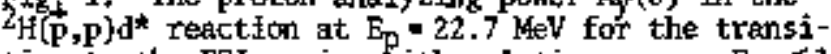
tion to the FSI region with relative enexgy $E_{n p}<1$ HeV. The smooth curve represents the elastic scattering analyzing powers measured in the same experIment.

(XBL 753-2612)

results. 5 Here also, the similarly between the inelastic and elastic anaIyzing powers is quite def inite.

This similarity between the angular distribu" tion of the analyzing powers for the elastic and inelastic scattering is rather umexpectod in view of the results reported by Brichman et al. 8 in the ana-

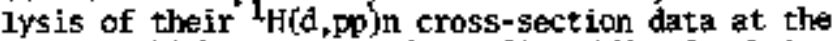
slifhtly higher energy of Ea-52,3 Met . In their analysis they used the Matson FSI factorization assumption ${ }^{9}$ to deternd the the separate contributions to the $\mathrm{l}_{\mathrm{H}}\left(\mathrm{d}, \mathrm{d}^{*}\right)_{\mathrm{P}}$ cross section fron the production of singlet and triplet np pairs.

$$
\left(\mathrm{d}^{\prime} / \mathrm{d} \Omega\right)_{\mathrm{d}^{*}}=(\mathrm{d} / \mathrm{d} /)_{\mathrm{d}^{*}}^{\mathrm{s}}+(\mathrm{d} \sigma / d \Omega)_{\mathrm{d}^{*}}^{\mathrm{t}}
$$




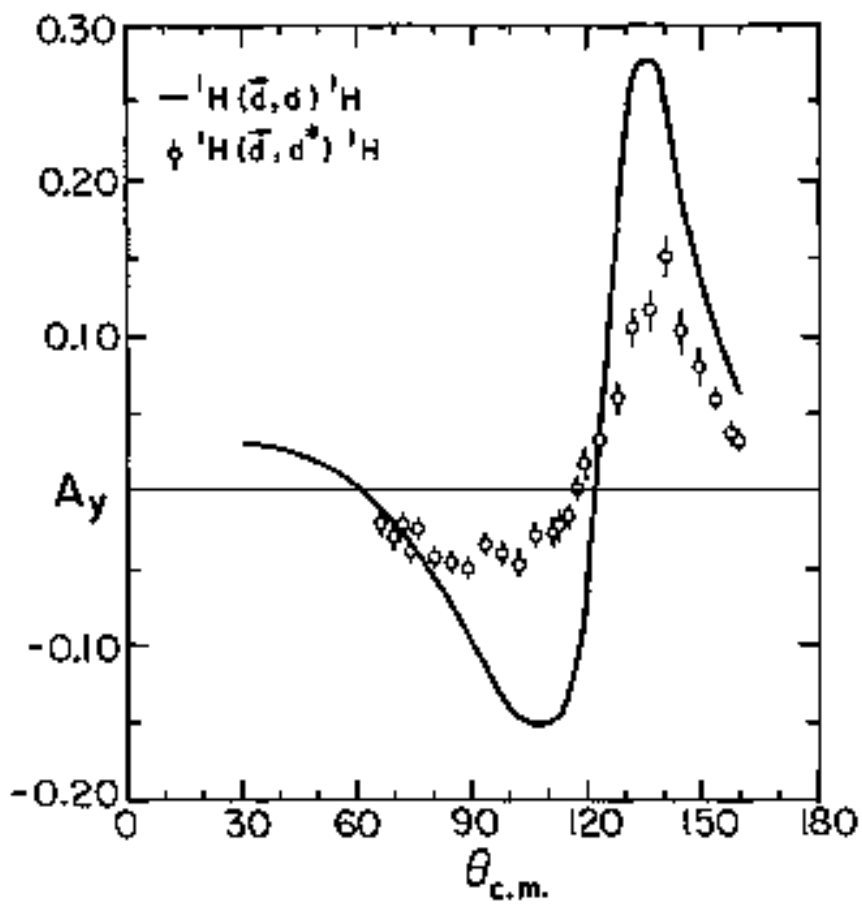

Fig, 2. The deuteron analyz ing power $A$, $[\theta]$ in the $I_{H}\left(d, d^{*}\right)^{I_{H}}$ requction at $E_{f}=45.4 \mathrm{MgV}$ for the transi$t$ ion to the FSI region with relative energy $E_{m}<1$ WeV. The smooth curve represents the deuteron elas $t i c$ scattering analyzing powers of Ref. 5.

(XRL 753-2613)

They found that between $\theta_{c}=85^{\circ}$ and $150^{\circ}$ the production of singlet pairs extededed that of triplet pairs for $E_{n y}<1$ kew, with the ratio $\mathrm{R}=$ (do/da) $\mathrm{d} * /$ (do/da)t reaching a value of about 10 near $\theta_{\mathrm{C} . m .}$ $120^{\circ}$. The angular dependence of $(\mathrm{d} \sigma / \mathrm{d}(\mathrm{l}) \mathrm{d}$. was $\mathrm{sin}-$ ilar in shape to that of the flastic cróss section, and their Bom approximation calaulation, in which the final-state np wave function used was effectively that of a deuteron with binding energy $\mathrm{B}_{\mathrm{B}}=\mathrm{D}$, was in excellent agreenent with (do/da) t Howerer, (do/dh) s. was quite different, with the regult that the tombined singlet plus triptet crosssection showed little resemblance to the elastic angular distribution. The more recent exact threebody calculations of rluge et al.,10 using spindependent separable potentials for the relative $S$ state of the two nucleons, substantiate the earlier findings of Britckman et ai. in the determination of the separate singlet and triplet $\mathrm{d}^{*}$ production cross sections. Furthemore their caloulated ratio $\left|T^{5}\right| 2 /\left|T^{t}\right|^{2}=(d \sigma / d \sigma) d^{*} /(d \sigma / d \Omega) t$ for $E_{T p}=0$ is in good agreerent with the ratio $\mathrm{R}$ deduced of Brichman et al., from the analysis of their data. This ratio was shown to be as high as 20 in the backward angular region.

We can, in the same way, express oux analyzing power results as the incoherent sum of the singlet and triplet d* prohuction contributions,

$$
\mathrm{A}^{\mathrm{d}^{*}}=\frac{\mathrm{A}_{\mathrm{y}}^{\mathrm{s}}(\mathrm{d \sigma /d} / \mathrm{d}) \mathrm{d}_{\mathrm{d}^{*}}^{\mathrm{s}}+\mathrm{A}^{t}(\mathrm{d \sigma} / \mathrm{d} \theta)_{\mathrm{d}^{*}}^{\mathrm{t}}}{(\mathrm{d \sigma} / \mathrm{d} \Omega)_{\mathrm{d}^{*}}^{5}+(\mathrm{d \sigma} / \mathrm{d} \Omega)_{\mathrm{d}^{*}}^{t}}
$$

Where As and At are the analyzing powers for production of np $p$ ings in the $1 S_{0}$ and $S_{1}$ states, respectively. If triplet $\mathrm{d}^{*}$ production were the major contribution to the cross section, one could expect the similarity between the elastic ant inelastic vector analyzing powwers seen in Figs, 1 and 2. However, it is the singlet d" production wich is the major contribution to the cross section in just the backward angular region of naximan analysing powex. In view of the fact that the ratio, $R$, is found to be quite large over a considerable re glum of backward angles, $\mathrm{Eq}_{\text {. }}$ (2) there reduces to $A_{C}{ }^{*}=A$, Implying that the angular distribution of the vector analyzing power in the elastic chamel is sinilar to that in the breakn channel with the np pair in the singlet state. This condition is quite inexpected, especjally in view of the marked dissimilarity between (do/di) fo and (do/dn)t for $\mathrm{d}^{*}$ prowiction. AIthough the exact three-boty calculations very nicely reproduce the singlet and triplet $d^{n}$ production cross sections, there is an obvious need to include the incre realistie mucleon-macleon tensor and $P$-wave interactions in an effect to explain the measurements of the analyzing power results presented here.

\section{Footnotes and References}

*Condensed framl Phys. Rev. Lett. 35, 1134 (1975).

thuclear science Lab., M.Y.T., Cambridge, Mass.

Ftyysics Dept., Laval Univ, Quebec, Canada.

sPhysics Dept., University of BaseI, Switzerland

1. H. E, Conzett, in Proc. Int'I Conf. on Few Body Problens in Nuclear and Particle Physics (Les Presses De L'Jniverstte Laval, (huébec, 1975).

2. P. Dolescha11, Muc1. Phys. A220, 491 (1974); Nucl. Phys, A201, 264 (1973).

3. S. C. Pieper, hucl. Fhys. A193, 529 (1973),

4. J. S. C. McKee, H, E. Conzett, R. M. Larimer, and Ch. Leemann, Phys. Rev. Lett. 29, 1613 (1972); A. Fiore, J. Arvieux, $N$. Van Ser, G. Perrin, $F$. Merchez, J. C. Gondrand, C. Perrín, J, L, Duratd, and $k$, Darves Elanc, Bhys. Rev. C 트, 2019 (1973)

5. F. N. Rad, J. Birchall, H. E. Contett, S. Chintalapudi, and R. Roy. Phys, Rev, Lett, 33, 1227 (1974].

6. F. N. Rad, J. Birchall, H. E. Concett, and R. Roy. Phys, Rev, Lett. 33, 1579 (1974).

7. R. T. Cahil1 and J. H, S10an, Mrc1, Phy's. A165, 161 (1971); W. Ebentöh, Nucl. Phy's. A191, 97 (1972)

8. H. Britclewenn, K. Kluge, H. Mathäy, L. Schänzler, and K. Wick, Hucl. Phys. Ais7, 209 (1970).

9. M. C. Goldberger and K. M, Matson, Collision Theory (filey, New York, 1964) p. S40.

to. W. Kluge, R. Schlüifter, and w, Ebenhöh, Nucl, Phys. A228, 29 (1974). 
During the past decade, substantial investigations of polarization effects have been made in the three-micieon system, 1 The polarization in muleondeuteron elastic scattering has received the principal attention of both the experinental and the theoretical effort, while the study of such effects in the breaknp reaction has received very hittle attertion. The exact three-body calctalations using the Faddeev equations have shom a renarkably good agreenemt with the proton and deuteron analyzing powers in $\mathrm{p}^{-d}$ elastic scattering. 1 Howerer, in the breakup channel the theoretical calculations have been restricted to the use of sucleon-macleon swave forces and, theriefore, cannot predict any polar izations. Until recently, experingintal medosurements of polarization effects in the treakup reaction had yietded values which were very salall or consistent with zero. ${ }^{3}$ The first evidence of significant polarizations was seen by Rad et al,4,5 in their megsurements of the vector analyzing power $A_{y}$ for the transition to the no final state interaction (FSI) region. The $1_{H}(\vec{d}, \mathrm{p}) \mathrm{mp}$ and ${ }_{\mathrm{H}}(\hat{\mathrm{p}}, \mathrm{p}) \mathrm{mp}$ re. actions, studied at the same center-of-mass ensrgy, showed not only significant values of the vector analyzing powers, but also a definite sinilarity of its angular distribution with that of the elastic chame1.

Since the spip structure of the deuteron breakup on ${ }^{3} \mathrm{He}$ and $\mathrm{l}_{H}$ is the sane, the nondynamical properties of the two reactions are identical. Thus, it is of intereşt to look for simâlar polarization. effects in the ${ }^{3} \mathrm{He}\left(\mathrm{d},{ }^{3} \mathrm{He}\right) \mathrm{d}^{*}$ reaction, where $\mathrm{d}^{\boldsymbol{*}} \mathrm{de}-$ notes flngl-state np pairs with low relative energy $E_{n p}$, in both singlet and triplet states. he report here measurements of the vector analyzing power $A_{y}$ in this reaction at $E_{\mathrm{d}}=30,35$, and $40 \mathrm{MeV}$ for $E_{\mathrm{T} p}<2.0 \mathrm{HeV}$. Our results are shom in Fig. 1. The statistical errors are snaller than the symbols. For comparison, the analyzing powers in d-He elastic scattering at the sane energies are shown as the smooth eurves. It is seen that $A_{y}$ in the $3 \mathrm{He}\left(\mathrm{d},{ }^{3}+\mathrm{l}\right.$ ) $) \mathrm{d}$ reaction reaches substantial values and follows that of the elastic thamel at $d^{*}$ production angles beyond $90^{\circ} \mathrm{c}, \mathrm{m}$. The peak values near ${ }^{\theta} \mathrm{c}, \mathrm{m}=135^{\circ}$ and $155^{\circ}$ are quite constant in magnitude and position over the 10-MeV energy interwal studied. In a comperison of our regilts with the previous measureraents, 4 near $\theta_{c, m}=135^{\circ}$ the ratio $A y$ (elastic)/Ay $\left(d^{*}\right)$ is 3 fn both the He $\left(d, d^{*}\right)^{3}$ he and ${ }_{H}\left(d_{1}, d^{*}\right)$ $t_{H}$ reactions, and the ratio is $\approx 1.4$ and a 1.9 , respectively, near $\theta_{\text {c.m. }}=155^{\circ}$. We are presently involved in a comparison of these data with DWRA calculations of $A_{y}(\theta)$ in transitions which produce both the $1_{S_{0}}$ and ${ }^{3} S_{1}$ final state $d^{*}$.

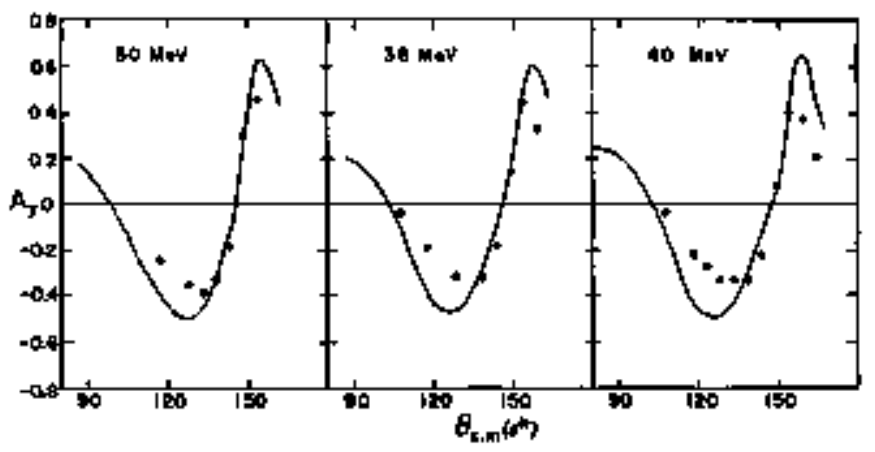

Fig. 1. The yector analyzing power, $A_{y}(\theta)$, in the reaction ${ }^{3} \mathrm{He}\left(\mathrm{d}, \mathrm{He}^{3} \mathrm{~d}^{*}\right.$, The \$nosth curves represent the malyzing powers in d-3e elastic scattering at the same energies.

(XEL 756-3182)

\section{Footnotes and References}

*Proc, Fourth Int'1 Symposium on Polarizetion Phenomena in Nuclear Reactions (Bİrkhäuser Verlag. Base1, 1976).

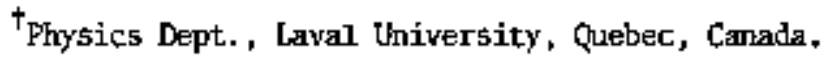

${ }^{*}$ Nixclear Science Lab., MT, Canbridge, Mass.

${ }^{5}$ Physics Dept., Univerșity of Rasel, Switzerlend.

1. H. E. Conzett, in Proc. Int'1 Conf. an Few Body Problens in Nuclear and Particle Physics (Les Presses de 1'Thiversite Laval, Qúbec, 1975).

2. R. T. Cahill and I. H. S1om, Nuc1. Fhys. A165 (1971) 161; w. Ebentö̈h, Nuc1. Phys. A191 〔1972) 97.

3. F. N. Rad, L. C. Northeliffe, J. G. Rogers, and D. P. Saylort Phys. Rev. Lett. 31 (1973) 57; Phys. Rev. C6 [1973) 1248; J. Arvieur, et al, Nucl. Phys. A150 (1970) 75 ; C. 0. Blyth, et al., Proc. Int ${ }^{\dagger} 1$ Conf. on Few Body Problems in Muclear and Particle Physics (Les Presses de 1'thiversitł Lava1, québec, 1975).

4. F. N. Rad, R. Roy, H. E. Conzett, J. Birchall, F. Seiler, and R. M. Lariner, Bull. Am. Phys. Soc. 20 (ig75) 578 .

5. F. N. Rad, H. E. Conzett, R. Roy, and F. Seiler, Fourth Polarization Symposium. 


\title{
O) . 3 \&
}

\section{POLARIZATION PHENOMENA IN FEW-BODY SYSTEMS"}

\author{
H E Conzett
}

My intention is to survey the recent developments in the study of polarization effects in the two, three-, and several-rucleon systems.1,2 Because these meastrements or calculations essentially have to do with cross sections for particular selected spin states, it is clear that these studies provide more detailed information and test more detailed calculations than is possible with the usual spin-averaged cross sections.

One of the micleon-nucleon discussfon sessions at the Quebec conference considered experinents for the rext decade, with a stress on polarization experiments, ${ }^{3}$ Included among the several suggestions for future experiments were: (1) a need for more acarrate $p-p$ cross-section and polarization data below $20 \mathrm{Met}$ because of significant discrepancies ancug the existing data; (2) measurements of the neutron to proton polarization transfer $\left(D_{t}\right)$ or the spincorrelation coefficłent, $C_{n n}$, in $n-p$ scattering at $50 \mathrm{MeV}$, in order to make a substantial improvennent in the determination of $\varepsilon_{1}$, the ${ }^{3} S_{1}-3_{1}$ mixing parameter, since the avallable data left $\varepsilon_{1}$ undetermined between $-10^{\circ}$ to $+5^{\circ}$ at $50 \mathrm{MeV} ;{ }^{4}$ and (3) a general request for measurements above the inelastic threshold of total cross sections for various spin orientations of both bean and target, and for measurements of the analyzing powers and spin correlation coefficients, at the higher energies above the inelastic threshold. I an happy to report tho significant progress that has already been made in these three areas at just the beginning of that decade,

Hutton et al, 5 have recently reported on measuredents of the analyzing power in p-p scattering at $10 \mathrm{MeV}$. Their results are shown in Fig. 1. Note that the maximm (negative) value is $2 \times 10^{-3}$ and the typlcal error is 10 of that, or $2 \times 10^{-4}$, so these are accirlate data, with more thon an order of magתitude better precision than had been attained previously. Traditionally, the low energy p-wawe phase

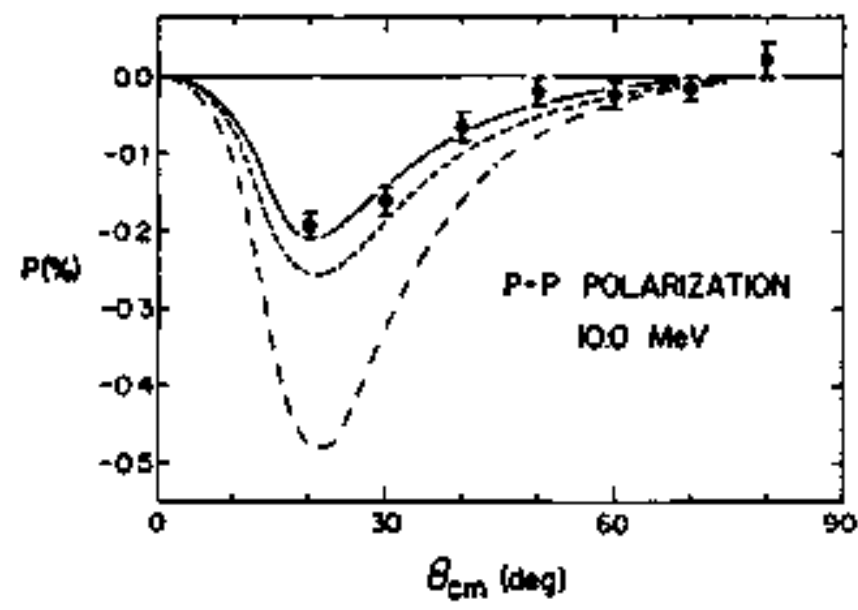

Fig. 1. Analyzing power in $\overrightarrow{\mathrm{p}}-\mathrm{p}$ scattering at 10 MeV (Ref. 5). shifts have been abtained with phornomenological $\mathrm{x}$ trapolations from higher energies. The dot-dash curve in Fig. 1 is the analyzing power calculated from the phase shifts resulting frod the $1-27.6$ MeV analysis of Arndt et al., ${ }^{6}$ in wich the overall nornalizations of the cross-section data were kept fixed. That is clearly inconsistent with theșe data, whereas an analysis with the nonmalizations taken as adjustable paraneters resulted in calou" lated analyzing powers that are in reasonable agreement with the data. The solld curve is the calculated analyzing power from a phase-shift analysis of these and nearby $9.9 \mathrm{MkV}$ cross-section results. The $s^{-}$and $p$-wave phase shifts determined in this analysis ${ }^{5}$ are the first model independert determinations made below $25 \mathrm{MeV}$.

With respect to the n-p raixing parameter $\varepsilon_{1}$, Johnson et aI. 7 reported at zurich on their measurements at si hev of the $n-p$ spin correlation parameter $k_{y y}$ (equal to $C_{m}$ for parity conserving or time-revertsal invariant interactions). Their regults are shown in Fig. 2, along with curves calculated from phase-shift solutions with the indicated values of $E_{1}$. The $-8^{\circ}$ value sems to be ruled out, but an additional overail nomalization uncertainty of $=2 \$ \$$ leaves the error on $\varepsilon_{1}$ considerably larger than the $\pm 1^{\circ}$ enyisioned by Binstock and Bryan. Their calculations, 4 in fact, whith $\varepsilon_{1}=2.5^{b}$ gives $A_{y}$ (1.e. $C_{\mathrm{Tn}}$ ) $=0.38$ at $\theta_{\mathrm{Cm}}=120^{\circ}$, which is a value 35 larger than that shown as the calculated regult in Fig. 2 , This discrepancy between the two caloulations must certainly be resolved, tot these measiatenents, requiring both a polarized feutrun beam and polarized proton target, clearly demonstrate an inpressive experinental achievenent.

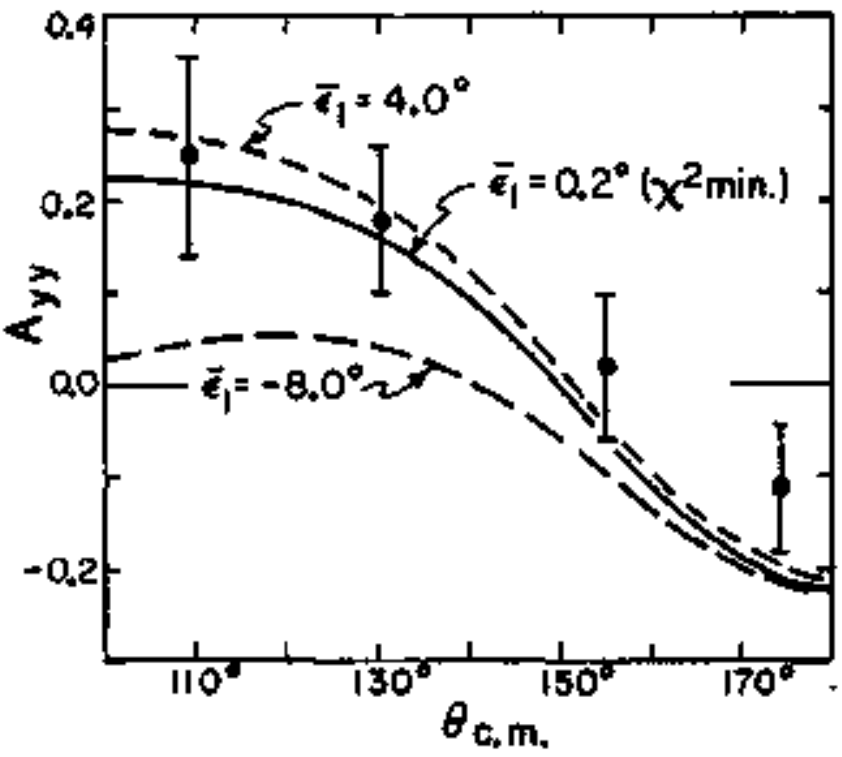

Fig. 2. The $n-p$ spin correlation parameter Arr at $50 \mathrm{MeV}(\operatorname{Ref}, 7)$. 
Hopeful1y, this experinent will be funther pursoled in order to reduce the experimental errors and thats rectuce the uncertainty in $E_{1}$.

Concerming polarization effects in the $\mathrm{N}$-d breaku reaction, there have been recent notertorthy reports of both theoretical and experimental resilts, Rad et al., 8 have reported on measurements, at $\mathbf{E}_{\mathbf{p}}=$ $22,7 \mathrm{MeV}$, of both the proton and deuteron (vector) analyzing powers in the p-d breakup transition to the np funal-state interaction (FSI) region of the three-body spectrum. These data are shown in Fig. 3, where the smooth colloves respresent the elastic p-d proton and deuteron analyzing powers. The substantial values of these inelastic analyzing powers demr onstrated a clear need for the exact $\mathrm{N}-\mathrm{d}$ breaku calculations to include moro than the simple s-wave $N-N$ interactions that had boen sufficient to fit the cross-section data. Such calculations are now underway. The fixst of these at $\mathrm{E}_{\mathrm{n}}=22.7 \mathrm{KeV}$, is reported in a contribution to this confererce by Bruinsma and van Wageningen, 9 Their work shows that the tensor force affects the cross section in the region of the FSI peak but has little influence in the quasifree scatterinc region. Their calonlated deuteron tensor analyzing powers show vory substantial values, an example is show in Fig. 4. In particular, $T_{20}$ exceeds 0.35 in the FSI region for the particwlar $\theta_{1}, \theta_{2}$ ingte conbination chosen here. These predictions of substantial tensor analyzing powers will certainly encourage experimentalists to measure them. The calculated deluteron vector analyzing porers are less than 0.05 for this angle combination, so the situation seems to be comparable to that of the elastic $\mathrm{N}-\mathrm{d}$ case, where the ${ }^{3} \mathrm{~S}_{1}-\mathrm{B}_{1}$ tensor force was chiefly responsible for the observed tensor polarizations while the $N-N$ p-wave interactions were the source of the vector polarizations. It is

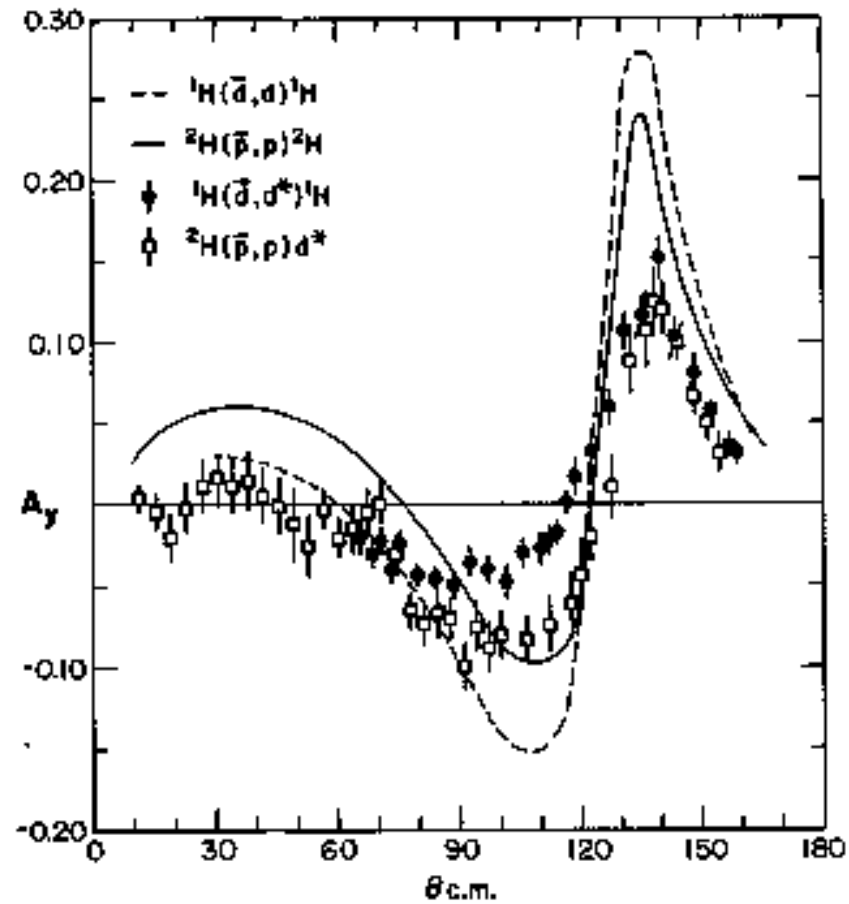

Fig. 3, The proton and deuteron analyzing powers $A y$ in the p-d brealap reaction at $22.7 \mathrm{MeV}$ (Ref. 8). CXEL 753-2664)

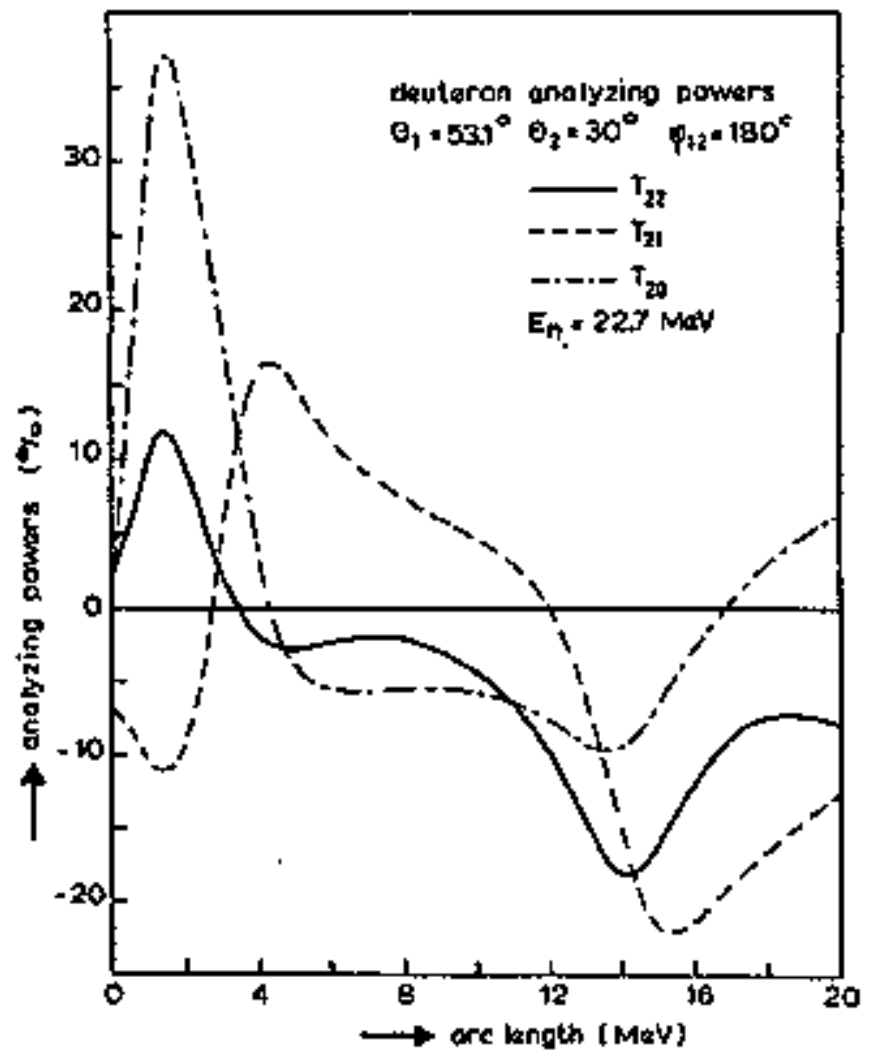

Fig. 4. Calculated deuteron tensor analyzing powers $\mathrm{T}_{2 \mathrm{q}}$ in the $\mathrm{n}-\mathrm{d}$ breakup reaction at 22.7 MeV (Ref. 9).

(XBL 7512-10060)

planned to next add the p-wave $\mathrm{N}-\mathrm{N}$ interactions in these breakip calculations. There is now a real probise that the very fruitful interchange which ocrurred between theory and experiment with respect to polarization observables in elastic $\mathrm{N}$-d scattering can now be repeated for the breakup reaction.

There have been two rather recent developients that show the consequent effects on polarizations that follow frow particular symetries of the nuclear interaction. The first of these involves charge-syumetry or isospin congervation. Experiments on the $3 \mathrm{H}(\mathrm{p}, \mathrm{n}){ }^{3} \mathrm{He}$ and $/ \mathrm{L}$ i $(\mathrm{p}, \mathrm{n})^{7} \mathrm{Be}$ reactions 10 had shown that the neutron polarizations pr and the protor analyzing powers $A_{y}$ wore essentially gqual, with lifoited exceptions. This equatity was seen to result frow charge-symetry, 11 and it is an approximate equality becausse of the presence of the Coulotb interaction. However, it is in just those regions of șignificant difference between $p y$ and $A_{y}$ that useful information can be terived on the structure of the nuclei studied,12 Recent studies of $\mathrm{Py}$ * Ay in $(p, n)$ reactions on other juclei were reported at Zixich. Figure $S$ shows the data of Lisowsk et al., 13 on ${ }^{9} \mathrm{Be}$. At $8.1 \mathrm{MeV}$ the older $\mathbf{P}_{\mathrm{y}}$ values (crosges) are quite different frop those reported here (dots), and the latter are close to the measured $A_{y}$ values (trianglos). Final corrections to the Ay values from background subtractions are expected to irprove the agreesient. As is suggested by the comparlson at $9.1 \mathrm{kWV}$, new mearurements w1ll be made there of $p$, as a check on the old values (crosses). 


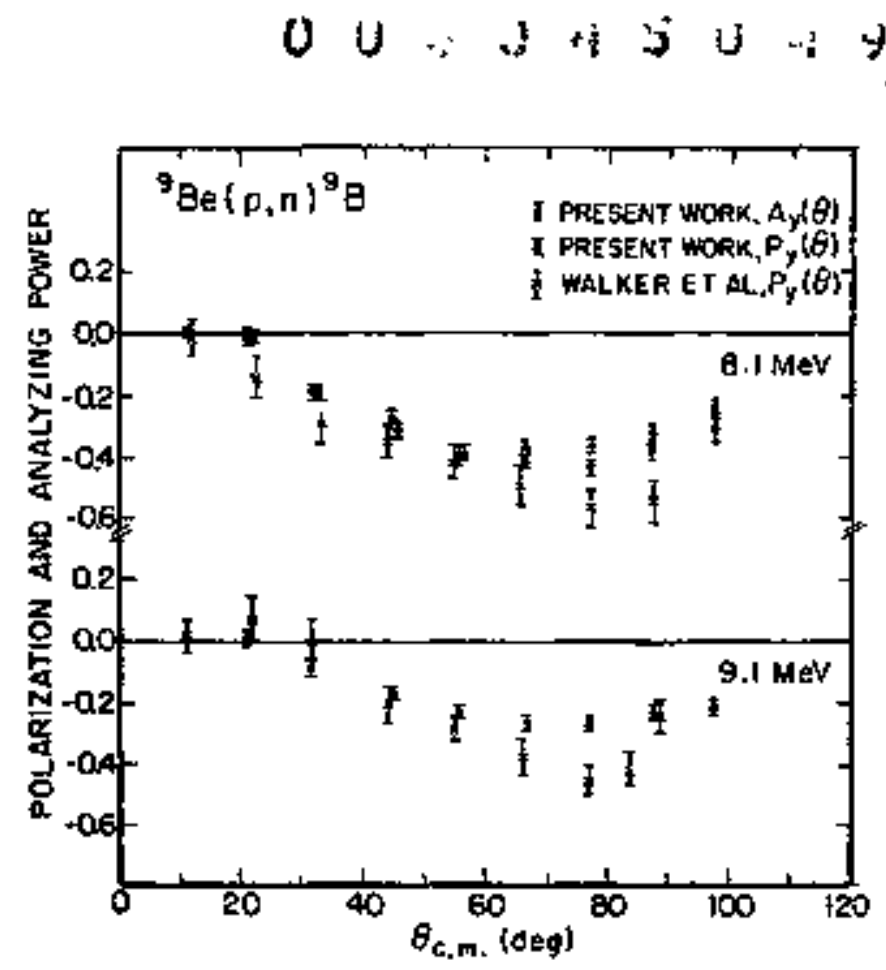

Fig. 5. Polarizations $p$ and analyzing powers $A_{y}$ in the $9 \mathrm{Be}(p, n\} 9_{\mathrm{B}}$ reaction at $\mathrm{B}, 1$ and $9.1 \mathrm{MeV}$ (Ref. 13).

(XBL. 7512-10061)

The second development shows that for reactions with tdentical or charge-symotric particles in the initial state, the angular-distribution synmetry of the analyzing powers can provilde a clear siguature of the reaction mechanism. 14

In sxmenary, recent polarization studies in $\mathrm{N}-\mathrm{N}$ scattering at and belor 50 otev have provided specific and significant inprovements in the phaseshift paraneters. High energy investigations with both polarized proton beans and targets have shown unexpectedly large spin effects, and this provides a challenge for theoretical effort to explain these results. Experinental and theoretica1 work on the three-nucleon problem continues to yield new and interesting results, with the omphasis now shifting to polarization studies in the breaku reaction. Ongoing work on several-nucleon systems contipues to provide polerization data for general analyses, nuclear structure infonation, or specific rescnance effects. Finally, the basic interaction symetries cont lnwe to have tuique and important consequences for polarization observabies.
Footnotes and References

*Excerpted from Proc. Seventl Int'1. Conference on Few-Body Problems in Nuclear and Particle Physics, Delizi. India, Dec. 1975, to be published.

1. Few Body Problens in Nuclear and Particle Physics, ds. R. J. Slobotrian, B. Cujec; and $\mathrm{K}$. Ramavataram (Les Presses de l'Universite Learal, (quebec, 1975).

2. Proc. Fourth Int'l Sympositin on Polarization Phenomeia in thiclear Reactions, zurich, kugust 1975 (Birlchauser Verlag, Basel), to be published.

3. J, Juvanovitch and J. Edgington, Ref. 1, p. 170.

4. J. Binstock and R, Bryan, Phys, Rev, I으 (1974) 1528 .

5. J. D. Hutton, W. Habber1i, L. D. Knutson, and P. Signell, Phys. Rev. Lett. 35 (1975) 429, and Ref. 2.

6. R, A. Arndt, R. H, Hackman, and l. B. Roper, Phys. Rev. C9 (1974) 555.

7. S. W. Johnson, F. P. Brady, N. S. P. King, M. W. MeNaughton, and P. Signell, Ref. 2.

8. F. N. Rad, H. E. Conzett, R. Roy, and F. Seiler, Phys, Rev, Lett. 35 (1975) 1134.

9. J, Bruinsma and R. van Wagentingen, 1975, Proc, Seventh Int'1. Conference on Few-Body Problents in hiclear and Particle Physics, Delhi, India to be published).

10. R. C. flaight, J, J. Jarmer, J. E. Simucns, J, C. Martin, and T. R. Donoghus, Phys, Rev, Lett. 28 (1972) 1587; Phys. Rev. C 9 (1974) 1292; it. Rohrer and L, Brown, Kucl. Phys. Az21 (1974) 325.

11. H. E. Conzett, Phys, Lett. S1B (1974) 445;

L. G. Amold, Ref. 2 .

12. L. G. Atroold, R, G. Seyler, T, R. Dronoghue, L. Brown, and U. Rokter, Phys. Rev, Lett. 32 (1974) 310.

13. P. W. Lisowski, G. Mack, R. C. Byrd, W. Tornow, 5. 5. Sloubic, R. L. Walter, and T. E. Clegg, Ref. 2.

14. H. E. Conzett, Ref. 2 . 


\title{
VECTOR ANALYZING POWER IN ELASTIC DEUTERON-DEUTERON SCATTERING BETWEEN 20 AND $40 \mathrm{MOV}$
}

\author{
HE Corzenl, W Oehme, $R$ ho Larimer, \\ Ch Legmann, ark, J $S C$ McKeef
}

The subject of d-d elastic scattering has not received very mulh attention in the past. The cross sections are quite silooth functions of energy in the region thp to 20 Mev where there are the most data available. However, the complexity of the spin structure and the low theshold for inelastic procosses has made any meaningful phase-shift analysis impossible because of the large nuber of paraneters involved even with the restrictive assumption of chanuel-spin conservation, 1 A reschlating-group calculation ${ }^{2}$ has obtained good agreement with the crosssection data between 5 and $20 \mathrm{keV}$, but the use of a purely central nucleon-mucleon potential precludes the prediction of any spin-polarizat for observables.

From an experimental point of view, the polarization experiments in elastic d-d scattering have raised a qualítative question. Previous measurements of the vector aralyzing power iT 11 in d-d scattering have been made at soveral energles below $12 \mathrm{keV}, \lambda, 3$ and at $21,4 \mathrm{MeV}, 4$ Nonzero but very small values of $\mathrm{tT}_{11}$ Nere obtained, reaching a maximm value of about 0.04 at $21.4 \mathrm{MeV}$. These values are almost an order of magnitude smaller than the nucleon and deuteron vector analyzing powers foumd in other elastic procesges involving few nucleon systens, e.g., p $+2 \mathrm{~F},{ }^{3} \mathrm{He},{ }^{4} \mathrm{He}$ and $\mathrm{d}+{ }^{3} \mathrm{He},{ }^{4} \mathrm{He}$. Since sizable contributions of $\$, p$, and D-wayes were required to fit the d-d data, 12 the rather insignificant polarization effects could not be explained as a consequence of a predcalinance of $S$-wave scattering. Thus, its reason remajnet mexplained.

We have extended the meașurements of vector analyzing powers in dे-d scattering to $40 \mathrm{MeV}$ to examine whether or not 1ts ankmilously sall talue persists at these higher energies. Also, another determination ntar $20 \mathrm{HeV}$ was desired, since the older measurement at $2 \mathrm{I}, 4 \mathrm{HeV}$ was rather uncertain because of lack of ksorledge of the beam polarizaticn. We used the oxialiy injected vector-polarized deuteron bean from the Berkeley 88-inch cyciotron. left-right asymetry data were taken sinultanecusly at two angles separated by $20^{\circ}$, using pairs of $A \mathrm{E}-\mathrm{E}$ silicon detectar telescopes. A polarimeter, consisting of a gas target and a pair of $\triangle E-E$ counter telescopes, was placed downsttrean of the matn scattering chamber and provided continuwus monitoring of the bean polarization. The analyzer used was 4Ho, whose vector analyzing power in $\mathrm{d}-4$ He elastic scettering has been measured in detail. 5 The differential cross section for vector-polarized dedrterons is given by

$$
\sigma(\theta)=\sigma_{0}(\theta)\left[1+2\left(\text { it }_{11}\right)\left(\mathrm{IT}_{1 I}\right)\right] .
$$

where $\sigma_{o}(\theta)$ is the differential cross section for unpolarized dexterons and it 11 is the bear polarization. A left-right asymetry measurement gives

$$
\varepsilon(\theta)=2\left(\mathrm{it}_{11}\right)\left(\mathrm{iT} \mathrm{T}_{11}\right)
$$

and the simaltanecus determination of the beam polarization yields the vector analyzing puters ir 11 . Figure 1 shows our data at $\mathrm{E}_{\mathrm{d}}=20,30$, and $40 \mathrm{MeV}$; the particle symetry requires that $i T_{11}^{(\theta)}=-\mathbf{i} T_{11}$ $(\pi-\theta)$. Orr $20-\mathrm{MeV}$ values are a factor of 2 larger than the previous results at $21.4 \mathrm{MeV}$, and clearly the vector analyzing powers increase rapidly with increasing energy. These values, when conpared with the analyzing powers in d-p elastic-scattering measured at comparable center-of-mass energies, 6 can no longer be considered ancmalously small.

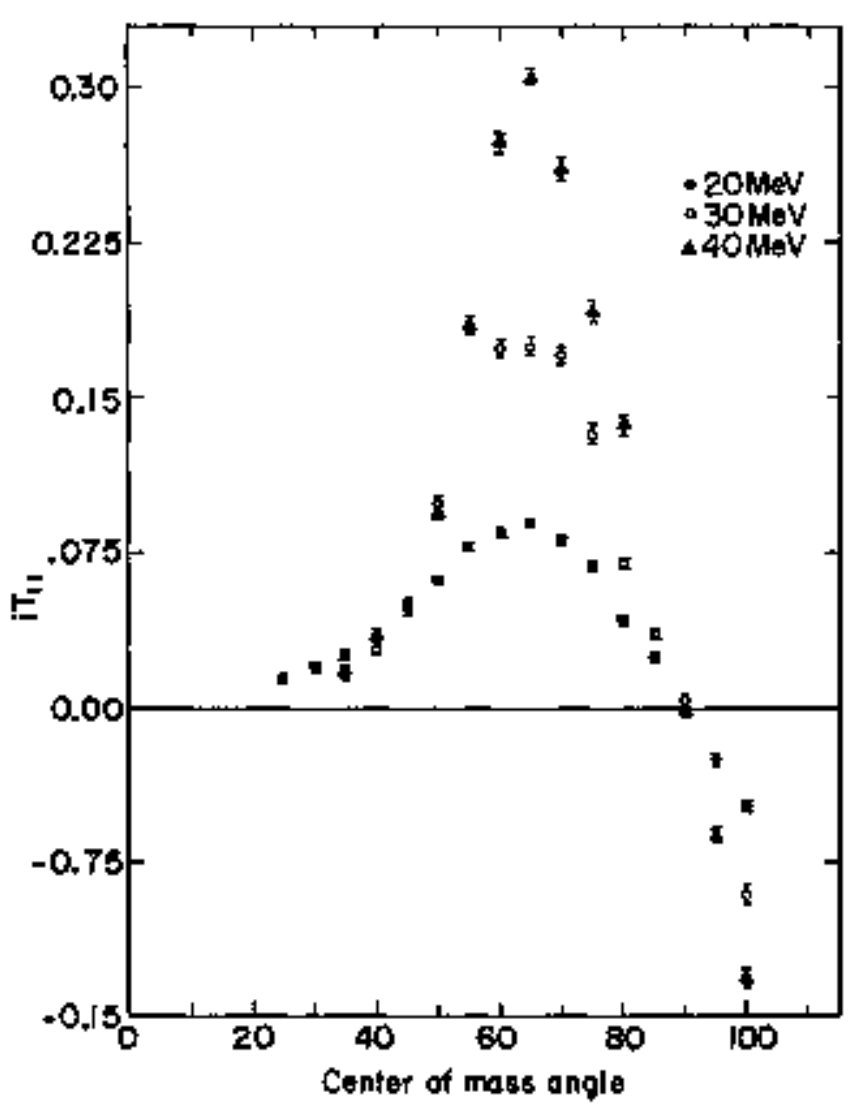

Fig. 1. Vector analyzing power, $\mathrm{iT}_{11}$, in $\mathbf{d}-\mathrm{d}$ scattering between 20 and $40 \mathrm{Mej}$.

(XRE $728-3846)$

\section{Footnotes and References}

"Proc. Fourth Int'1. Symposiun or Polarization Phenomena in kuclear Reactions (Brrkhüuser Verlag, Resel, 1976).

†Sektion Physik der Universität Hinchen, 8046 Gerching, Germany.

"University of Manitoba, Winnipeg, Manitoba, Canada. 


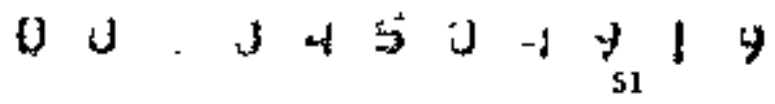

1. G. R, Plattmer and L. G. Keller, Phys, Letters 30B, (1969) 327; P. D. Lien, Nucl. Phys. A178 (1972) 375; H. 0. Meyer ard P. Schiemenz, Muc1. Phys. A197 (1972) 259 .

2. F. S. Chwieroth, Y, C. Tang, and D. R. Thompson, Nucl. Phys. A189 (1972) 1.

3. W. Griebler, Y. Könik, R. Risler, P. A. Schoelzbach, R, E, White, and P. Marmier, thecl. Phys. A193
(1972) 149,

4. J. Arviewx, J, Goudergues, B. Mayer, and A. Papineau, Phys. Letters 22 (1966) 610 .

5. H. E, Conzett, W. Dahure, Ch. Eeengan, J. A. Macdonald, and J. P. Meulders, Fourth Polarization Syalp.

6. J. S, C. HcKee, H. E. Conzett, R. M. Larimer, ant Ch, Leemann, Phys. Rev. Lett. 29 (1972) 1613.

\section{THE VECTOR ANALYZING POWER IN THE ${ }^{2} H(\vec{d}, p)^{3} \mathrm{H}$ REACTION AT $30 \mathrm{MeV}$}

H E Conzélt J S C Mckę †

$R M$ Larımer, and Ch Leemann

The ${ }^{2} H(d, p)^{3} H$ reaction has been studied at energies up to $12 \mathrm{MeV}$ by a muber of groups. 1 Grthebler etal., in particular have measured the differential cross sections and the deuteron analyzing powers at 9 energies between 3.0 and $11.5 \mathrm{MeV}$, and have analyzed their data in terms of possible resanances in $4 \mathrm{He}$. Their analysis of the coefficients in a legendre expansion fit to the data shoned that no simple isolated state of this system exists betweegl 24 and 30 Mev excitation of ${ }^{4} \mathrm{He}$ since relatively strong interference effects were observed.

The present experinent was utadertaken at $\mathrm{E}_{3}=30$ $\mathrm{MeV}$ in order to study the ${ }^{2} \mathbf{H}(\mathbf{d}, \mathrm{p})^{3} \mathrm{H}$ reaction in a region of considerably higher excitation of $4 \mathrm{He}$. Also, it is to be oxpected that the dfrect nucleon transfer mode should be enhanced with respect to the conpound-nucleus reaction mechanisin at this higher energy. Maasurements were rade of the differential cross-section and the vector analyzing power iTil. The iT $_{11}$ results are shom in Fig. 1, ard the surprising feature is the approximate antisymmetry of the data with respect to $\theta_{\text {glt }}=90^{\circ}$. The degres of symintry observed is quite renarkable because the iTl1 data at $\mathrm{E}_{\mathrm{d}}=11.5 \mathrm{MeV}$ show 11ttle symmetry of any kind and are almost uniformily positivo in sign.

The entrance chamel particle identity rewuires that $\sigma(\theta)=\sigma(\pi-\theta)$, and in the inverse ${ }_{\mathrm{H}} \mathrm{H}(\overrightarrow{\mathrm{p}}, \mathrm{d})^{2} \mathrm{H}$ reaction the exit-channel particle 1dentity requires that $A_{y}[\theta]=-A_{y}(\pi-\theta)$ for the proton analyzing power. 2 There is, a priom, no such symmetry condittion inoposed on the analyzing powers in the ${ }^{2} \mathrm{H}(\mathrm{d}, p)+\mathrm{H}$ reaction. However, it can be shown that if the reaction mechaniss is entirely that of direct nucleon transfer, the indistinguishability of the neutron transfer's between the two deuterons results in exact symetries in the deuteron analyzing powers. ${ }^{3}$ In particular for our purposes

$$
\text { iT }_{11}(\theta)=-\mathrm{iT}_{11}(\pi+\theta) \text {. }
$$

so the near antisyranetry of our date is clear evidence of a predominantly direct nucleon-transfer reaction mode at this higher evergy. of course, the ositatin conclusion is that deviations from Eq. (i) show that othex than the direct nucleon"transfer process is contributing to the reaction. It is possible, in principle, for the corqound-nucleus reac- tion mechanion to give the result (1) if the reaction should proceed entirely through a single state or through states of the same parity so that only the even-L terms would contribute in the Legendre expansion

$$
\sigma(\theta) \mathbf{i t}_{11}(\theta)=\sum \mathrm{a}_{\mathrm{L}} \mathrm{P}_{\mathrm{L}}^{\mathrm{l}}(\cos \theta)
$$

In view of the data and analysis of Grïbbler at al., this circumstance is most unlikely in this reaction.

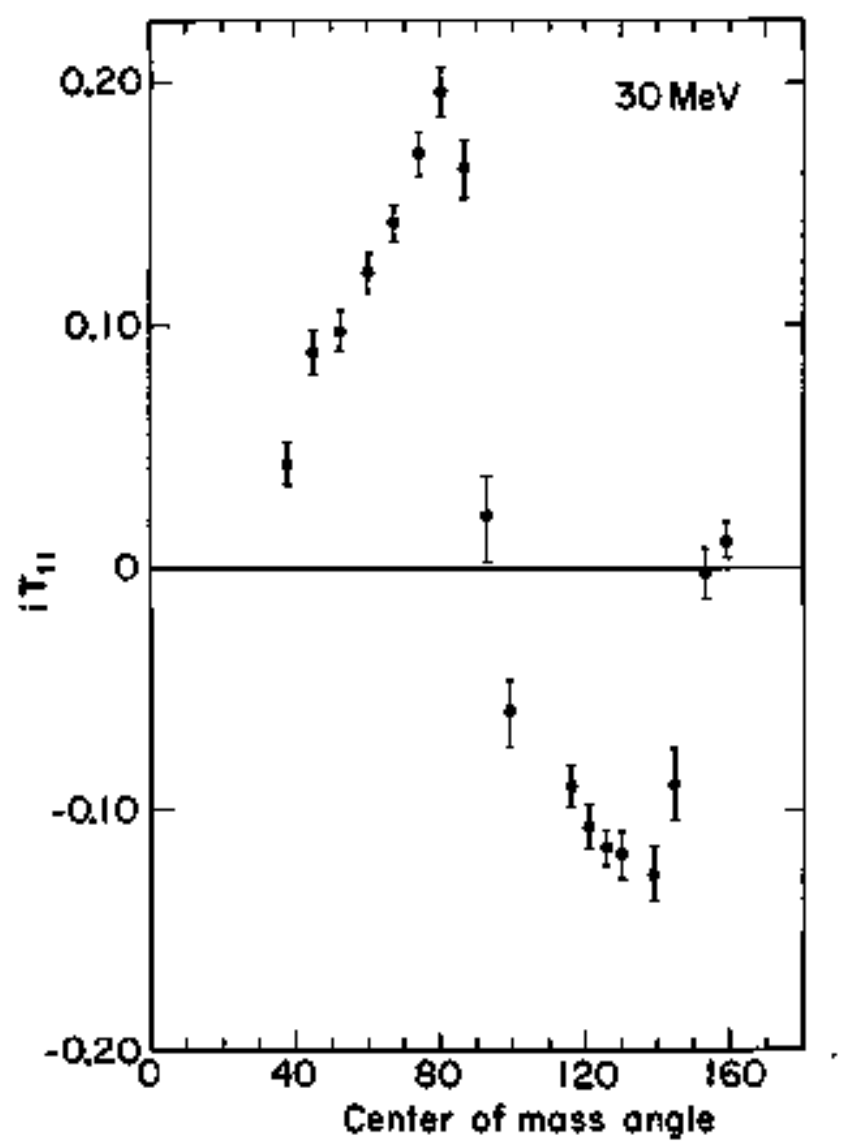

Fig. 1. Vector analyzing power, $1 \mathrm{~T} 11$, in the ${ }^{2} \mathrm{H}(\mathrm{d}, \mathrm{p})^{3} \mathrm{H}$ reaction at $30 \mathrm{Mer}$ (XBL $\left.72 \mathrm{~s}-3848\right)$ 
In summary, we heve found in the ${ }^{2} \mathrm{H}[\mathrm{d}, p]^{3} \mathrm{H}$ reaction that the entrance-channel particle identity imposes definite syonetries on the polarization observables that are clear signatures of the direct-reaction process. He know of no other example of a condition by which this process can be identified so elearly.

\section{Footnotes and References}

Proc, Fourth Int'l. Symposium on Polerization Phencunena in éfuclear Reactions (Bìrkhäușer Verlag, Base1, 1976).
${ }^{\dagger}$ University of Maritoba, Winnipeg, Manitoba, Canada.

1. W. Grüebler et al., Nucl, Phys. A193 (1972) 129, and references therein.

2. He follow the ladison convention, Polarization Phenomera in Muclear Reactions, eds. H. H, Earschal 1 and W. Habberli (tritiv, of Wisc. Press, Madison. 1971) p. $x \times x$.

3. H, E, Conzett, J. \$. C. McKee, R, M. Lartner, and Ch. Leeman, to be published.

\section{VECTOR ANALYZING POWER IN THE ${ }^{2} H(d, p)^{3} H$ REACTION BETWEEN 15 AND $25 \mathrm{MeV}^{*}$}

H E Conzett, f $M$ Larumer, F N Fad, $t$

$R$ Roy.t and $F$ Selless

The charge-syumetric reactions ${ }^{2} \mathrm{H}\{\mathrm{a}, \mathrm{p}\}^{3} \mathrm{H}$ and $2 \mathrm{H}(\mathrm{d}, \mathrm{n})^{3} \mathrm{He}$ have been studied in considerable detail at energies up to 15 Mev, 1,2 Difforential cross sections, nucleon polarizations, and deuteron vector and tensor analyzing powers have ben measured, sond differences in these observables for the two reactions have been exanined for evidence of a possible deviation from the charge sympetry of the nuclear interactions. The more recent conparisons and calculations ${ }^{3}$ have been able to explain the observed differences between the two reactions in terms of the Coulcab effect, including the Q-value difference.

The reactions, in addition to being charge symmetric, each possess the additional symetry of entrance-channel particle identity. This requires that $\sigma(\theta)-\sigma(\pi-\theta)$ and $p(\theta)=-p(n-\theta)$ for the dif ferential cross sections and the nucleon polarizations, respectively. In general, no conparable symbetry is imposed on the angular distributions of the deuteron analyzing-power couponents, and, indeed, no stgggestion of symmetry is seen in the data at delateron energies up to $11.5 \mathrm{MeV} .2$ However, recent jeasurements of the vector analyzing power in the ${ }^{2} \mathrm{H}(\mathrm{d}, \mathrm{p})^{3} \mathrm{H}$ readtion at $30 \mathrm{NoW}$ have disclosed the surprisng restlt that, there, the symetry

$$
A_{y}(\theta)=-A_{y}(\pi-\theta)
$$

is approximately fulfilled. ${ }^{4} \mathrm{Al}=0$, it has been shown that the condition (1) holds exactly if the reaction should proceed entirely by way of the direct nuclem-transfer process. 5 Thus, in this particular case deviations from the symenetry (1) colstitute clear qualitative evidence that the reaction proceeds (also) via the intennediate (compound) 4he. Thrs, at the lower energies analyses of the data in terms of states in "He are certainly appropriate.

He report here on measurinents of $A_{y}(\theta)$ in the ${ }_{2} H(d, p){ }^{5} \mathrm{H}$ reaction at 15,20 , and $25 \mathrm{MeV}$ which were made in order to exanine the transition from the complete lack of symlonetry at $11.5 \mathrm{MeV}$ toward that of
(1) at $30 \mathrm{NeV}$. Our results, which are displayed in Fig. 1, show that the triansition is a gradual one. Thus, the change from the compound-nuxilus reaction mechanisal to the predoninantly direct nucleon-transfer reaction mode is correspondingly gradual, and at $30 \mathrm{MeV}$ and above andiysis in terms of the directreaction process is clearly appropriate.

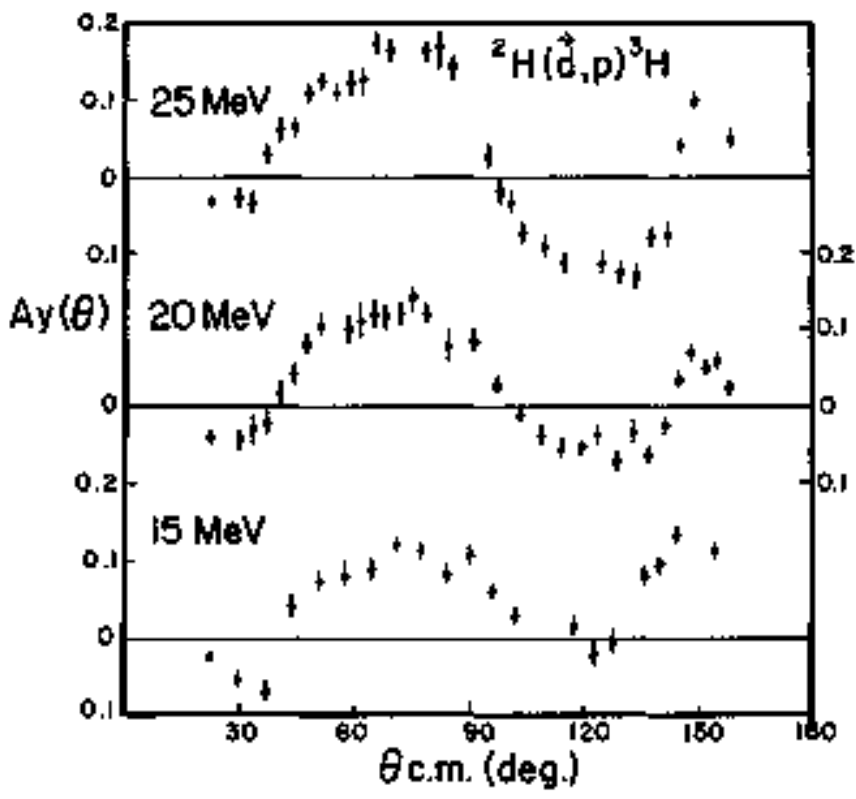

Fig. 1. Vector aralyzing power, $A_{y}\left(=2 / \sqrt{3} \mathrm{IT}_{11}\right)$, in the ${ }^{2} \mathrm{H}(a, p)^{3} \mathrm{H}$ reaction between 15 and $25 \mathrm{MeS}$.

(XBL 756-1551)

\section{Footnotes and Refexences}

"Proc. Fourth Int'1. Symposium on Polarization Phercomena in Nuclear Reactions (Birkháuser Verlag, Basel, 1976]. 


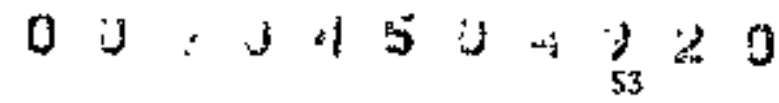

${ }^{\dagger}$ Present address: Nuclear Science Lab, , M.I.T., Cambridge, Mass.

*Fresक्तt address: Physics Dept., Laval Iniversity, Quebec, Canada.

${ }^{\mathbf{6}}$ Present address: Physics Dept., University of Resel, Shitzerland,

1. R. A, Hardepopf, R. L. Waltor, and T. B, Clegg, Phys. Rev. Lett, 28 (1972) 760 and references therein.

2. W. Grüebler et al., Nucl. Phys. 193 (1972) 129.

3. Reference 41 (unpublished) of Ref. 2 .

4. H. E. Conzett, J. S. C. McKee, R. M. Lariner, and Ch. Lemann, Fourth Polarization Symp.

5. H, E. Conzett, J. S, C. McKee, R, M, Larimer, and Ch. Leemanit, to be pubtished.

\section{CAOSS-SECTIONS AND VECTOA ANALYZING POWERS IN THE

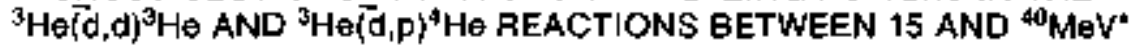

\author{
R Roy.t $F$ Seller, H E Conzeal. \\ F N Aad,s and $R$ M Larimer
}

Differential cross-sections and angular distributions of tho vector analyzing power iT $_{11}$ were obtained for the $3 \mathrm{He}(\mathrm{d}, \mathrm{d}){ }^{3} \mathrm{He}$ and $\mathrm{s} \mathrm{He}(\mathrm{d}, \mathrm{p})^{4} \mathrm{He}$ reactions at intervals of $5 \mathrm{MeV}$ between 15 and $40 \mathrm{MeV}$. At each energy data taken at 25-35 angles. Figure 1 shows the ${ }^{3} \mathrm{He}(\mathrm{d}, \mathrm{p})^{4} \mathrm{He}$ results. The statistical errors are shown wherever they are larger than the symbols. The scale of the cross-sections is sub. ject to a systematic error, estimated to be less than 69 . The data at $15 \mathrm{MeV}$ join smoothly to measurewents of lawer energies. 1 Fits were made to these data in terms of Legendre polynominals, 2

$$
\begin{aligned}
& \frac{d \sigma}{d L^{2}}=\frac{\sigma_{\mathrm{TOT}}}{4 \pi} x^{2} \sum d_{00}(\mathrm{~L}) p_{\mathrm{L}}(\cos \theta) \\
& \left(\mathrm{iT} \mathrm{T}_{11}\right) \times\left(\frac{\mathrm{d} \sigma}{\mathrm{d} \sigma}\right)=\frac{0 \mathrm{TOT}}{4 \pi} \hbar^{2} \sum_{\mathrm{L}} \mathrm{d}_{11}(1) P_{L, 1}(\cos \theta) .
\end{aligned}
$$

Plots of the energy dependerte of the coefficients $d_{00}(L)$ and $d_{11}(L)$ aro show in Fig, 2 .
The coefficlents of the cross-section for thpolarized particles doo(L) for even degree $L$ show some evidence of a broad structure near 20 and 40 $\mathrm{MeV}$, while the odd-dogree coefflcients repeat only the $20 \mathrm{MeV}$ structure. This coincidos with a shift away from a predominance of the coefficients $d_{11}$ (2) to d1 (1). The Iatter indicates large interference terms between reaction matrix elements of opposite parity. This is also visible in the angular distribxtions of iT 11 , which shift from antisymetry with respect to $90^{\circ}$, to a more symetric distribution. This observation adds support to the result of two recent analyses 2,3 which postulate mostly interfererce betwegn d-wave levels below $11.5 \mathrm{MeV}$ and a strong $\mathrm{d}_{7 / 2^{+}}=\mathrm{f}_{7 / 2^{-}}$interference at higher energies,

The Legendre coefficients from an analysis of the ${ }^{3} \mathrm{He}(\mathrm{d}, \mathrm{d}){ }^{3}$ He elastic scattering data lend support to these tentative conclusions because similar variations are found near 20 and $40 \mathrm{MeV}$.

In view of the quite smooth energy dependence of these date, we are making optical model analyses of the elastic scattering results and DWBA analyses of the $(a, p)$ data. 

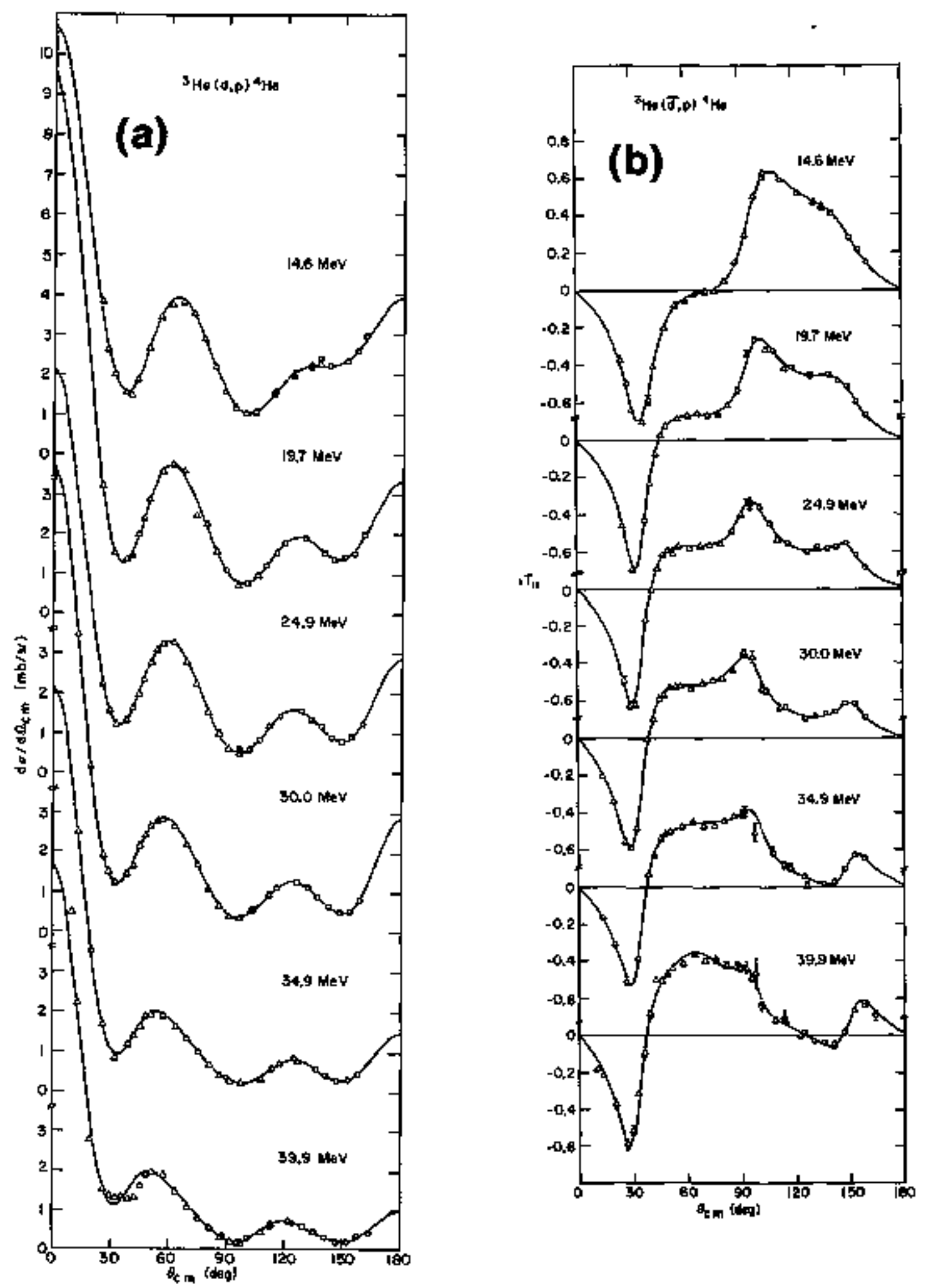

Fig. 1. Angular distributions of (a) the cross sections and (b) the vector analyzing

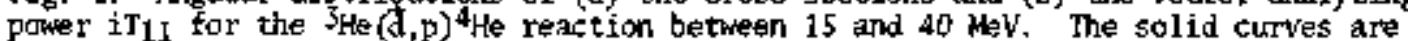
Legendre polynomial fits ta the data.

(1a: XEL 7512-9207) (1b: XBL 7512-9209) 


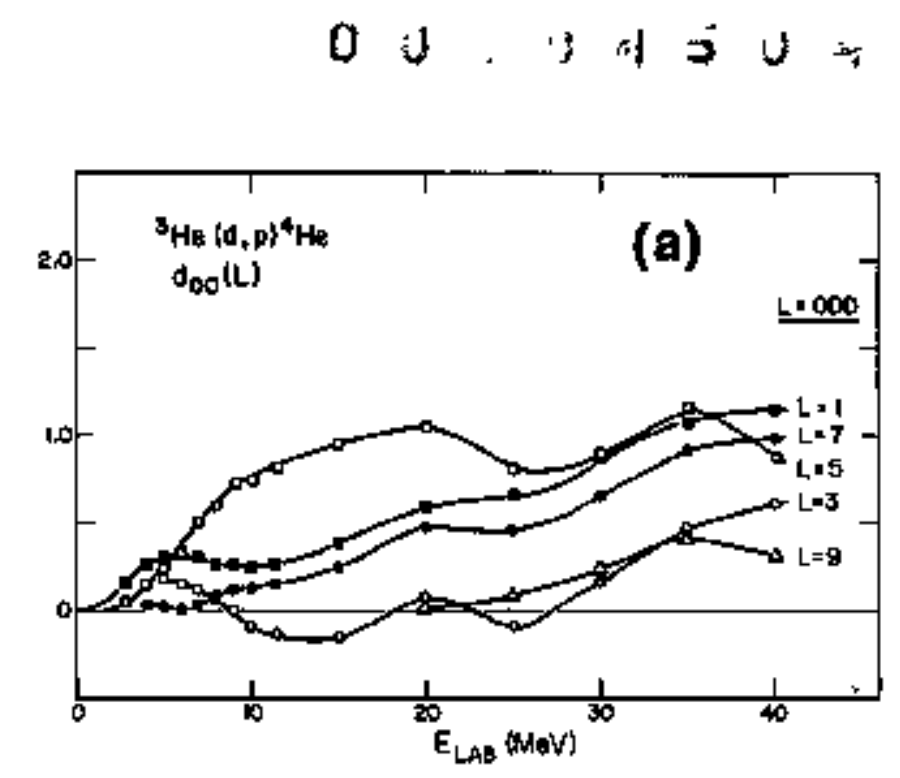

\section{1}
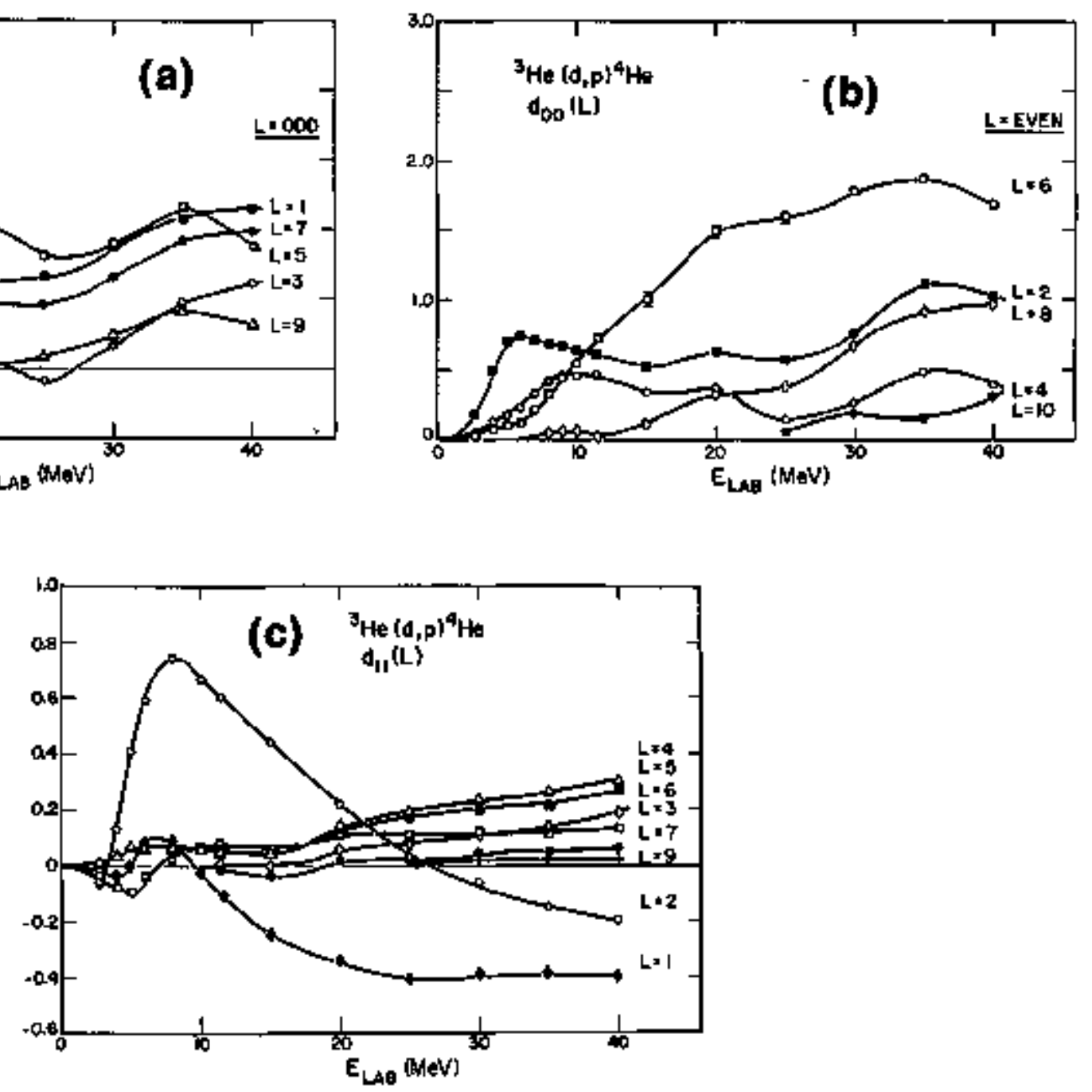

Fig. 2. The coefficients $d_{00}(L)$ and $d_{11}(L)$ of the Legendre polynonial expansion fits of Fig. 1. (2a: XBL 756-3193) (2b: XBL 756-3189) (2c: XBL 756-3194)

\section{Footnotes and References}

*Proc. Fourth Int'l. Symposiurs on Polarization Phenomena in Wictear Reactions (Birkhäuser Verlag, Base1, 1976).

†Present address: Physics Dept., Laval Uhiversity, Quebec, Canada,

"Present address: Physics Dept., University of Baset, Switzerland.
${ }^{\$}$ Present address: Nuclear Science Lab., M.I.T., Cambridge, Mass.

1. W. Grütebler, V. Konig, k, Rush, P, A. Schelzbach, R. E. White, and P. Marmier, Nuc1. Phys. A176 (1971) 631 .

2. F. Seílex, Mucl. Phys, A244 (1975) 236.

3. D. D. Dodder and G. M. Hale, Fourth Polarization Sylip. 


\section{MAXIMUAM TENSOA ANALYZING POWER AYY $=1$ IN THE ${ }^{3} \mathrm{He}(\vec{d}, \mathrm{p})^{4} \mathrm{He}$ REACTION"}

F Selkert

Maximum possible values of the analyzing power have been found in the elastic scattering of both spin-1/2 and spin-1 particles fram spin 0 nuclei, and have rapidly becane the basis for the calibration of particle polarizations. Plat tner and Bacher showed analytically that values $A_{y}= \pm 1$ must ocour at sevoral energies in nucleon-4 $\mathrm{He}$ and ${ }^{3} \mathrm{He}-$ ${ }^{4} \mathrm{He}$ elastic scatterjing. In the elastic scatterfing of deuterons from $\mathrm{H}_{\mathrm{He}}$, a similar procedure was used by Grüebler at al., to identify three points $\left(\mathbf{E}_{0} ; \theta\right)$ below $12 \mathrm{MeV}$ whexe the terssor analyzing power $\mathrm{Ayy}$ is unity. ${ }^{2}$ It has thus been demonstrated that extremse values of polarization efficiencies do occur in etas. tic scattering with the spin configurations $1 / 2+0+$ $1 / 2+0$ and $1+0+1+0$. It is the purpose of this letter to fumish considerable evidence for the first $A_{y y}=1$ point observed in a nuclear reaction (as opposed to elastic scattering) and to point out that such points are quite generally of 1 mportance in the analysis of the process.

An inspection of the ${ }^{3} \mathrm{He}(\mathrm{A}, \mathrm{p})^{4} \mathrm{He}$ data obtained by Griebler et al. ${ }^{3}$ between $2, B$ and $11.5 \mathrm{MeV}$ shows sizeable values for the spherical tersor moments $\mathrm{T}_{\mathrm{kg}}(\theta)$ but none approach the physical linits closely. The Cartesian efficiency

$$
A_{Y \gamma}(\theta)=-\frac{1}{2} \sqrt{2}\left\{T_{2 \theta}(\theta)+\sqrt{6} \mathrm{~T}_{22}(\theta)\right)
$$

as coniputed from the data of Griebler et al., shows large positive values neas $10 \mathrm{MeV}$ for both forward and backward angles (Fig, 1). Thas the data available slow only that the largest values of $A_{y y}(E, 0)$ should be found near $E_{d}=9 \mathrm{Mg} /$ and $\theta 0 \mathrm{ON}=27^{\circ} \mathrm{y}$ and should lie very cloge to unity.

For the spin space $1 / 2+1 \rightarrow 1 / 2+0$ of the ${ }_{\mathrm{H}}(\mathrm{d}, \mathrm{p})^{\mathrm{d}} \mathrm{He}$ reaction, the conditions for $\mathrm{A}_{\mathrm{y}}=1$ are

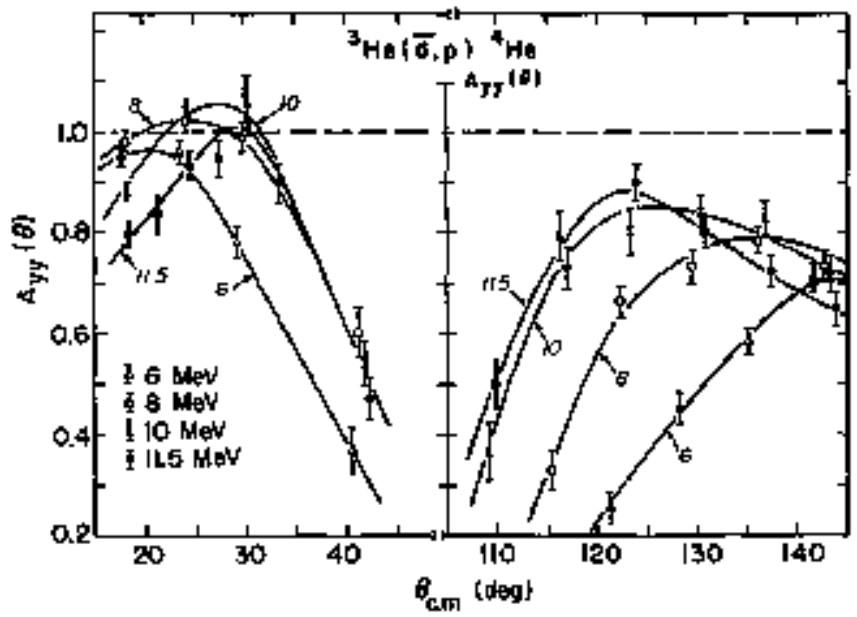

Fig. 1. Tensor analyzing power Ayy for the ${ }^{3}$ te (d,p) 4 the reaction betreen 6 and $11.5 \mathrm{kfe}$, calculated froil the data of Reference 3 .

$$
\begin{aligned}
& M_{1,1 / 2 ; 1 / 2}=-M_{-1,1 / 2 ; 1 / 2,} \\
& M_{1,-1 / 2 ; 1 / 2}=-M_{-1,-1 / 2 ; 1 / 2,}
\end{aligned}
$$

where the indices denote the spin projections of the deuteron, ${ }^{3} \mathrm{He}$ and proton respectively. It should be noted that the proof, usually given for the existence of an extreme value of the analyzing porer, does not hold here. While only one cotplex anplitude must be zero for the elastic scattering processes discussed up to now, 1,2 Eas. (2) require that two amplitudes must vanish sidultanequsily. Thus all that can possibly be shown is that Ap is equal to unity within certain Iimits. For practical purposes, however, it should be quite sufficient if a close approximation to the extreme value can be demonstrated.

Finally, it should be exphasized that the conditions imposed on the transition matrix at an extroste point of the analyzing power are linear in the amplitudes. This holds independent of the spin space of the process and the rank of the rolarization observable. These linear constraints are similar to those obtatned by the requirement of parity consenvation or tine refersal invariance, except that they only hold for a particular energy and angle. of course, any value of the analyzing power inposes cunstraints on the reaction anplitudes, but these are usually highly nonlinear. At an extrene point $\left(\mathrm{E}_{0}, \mathrm{\theta}_{0}\right)$, however, simple linear condin tion result. These can be very useful in an analysis of the process, stnce they are additionn 1 to the usual set of the bilinear equations. By analyoing dats taken at and near such a point, these constraints cen be imposed directly on the analysis, considerably restricting the solution space. Even in the simple cases, where the existence of the extrene can be proven directly, 1,2 this may be of scme importance since the quality of the analysis needed for that kind of proof is not very high. In view of the difficulties encomtered quite genera11y in the analysis of miclear processes between particles with spin, this use of extreme points of the analyzing power may well be more inportant than the more obvious spplication as absolute calibration points for particle polarizations.

\section{Footnotes and References}

"Condensed from Phys. Lett, 61ㅌ, 144 (1976).

Fresent address: Physics Dept., Univorsity of Rasel, 5witzerland.

1. 6. R. Plattner and A. D. Racher, Phys. Lett. 36B (1971) 211.

2. W. Grutebler et a1., Nuc1. Phys. A242 (1975) 285.

3. W. Grifebler, V. Künig, and P. A. Schmelzbach, Results of neasurements and aralyses of muclear re actions induced by polarized and uopolarized deaterons, Eidg. Techm. Hochschule Zịnich, internal report, May 1973. 


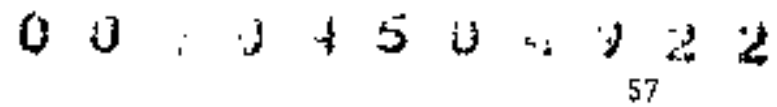

\section{POINTS OF MAXIMUM ANALYZING POWER IN THE $\left.{ }^{3} \mathrm{He} \overrightarrow{i d}, p\right)^{4} \mathrm{H}$ 舀 REACTION*}

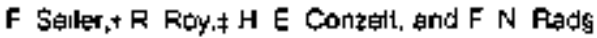

The ${ }^{3}$ He (d,p) ${ }^{4}$ he reaction has been the first process, other than elastic schettering, in which a point $\left[\mathrm{E}_{0}, \hat{\theta}_{0}\right]$ of maximm possibfe tensor analyzing porrer $A_{y \gamma}=1$ has been proposed. 1 An inspection of the comblete deuteron polarization data of Griebler et al., 2 at $11,5 \mathrm{keV}$ and of the $1 \mathrm{~T}_{11}$ measurements of Roy et $a_{+}, 3$ at 15 Hev indicates the possibility of large positive values of both $A_{y}$ and $A_{y y}$ near

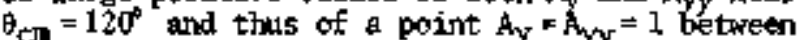
these energies. The necessary but hot sufficient conditions $A_{X X}=A_{z z}=1 / 2$ and $A_{x z}=0$ are nearly fulfilled at 11.5 Mler. 1,4 Unfortmately these tensor observables are not available at higher energies. For the investigation and possible identificetion of such a point, the relevant condittors on the M-matrix hrve to be verified. Using the representation $5,6,7$

$$
M=\frac{1}{\sqrt{2}}\left(\begin{array}{rrrrrr}
-\mathrm{iA}-\mathrm{D} & \sqrt{2} \mathrm{~F} & -\mathrm{i} A+\mathrm{D} & -\mathrm{B}-\mathrm{C} & \sqrt{2} \mathrm{E}-\mathrm{B}+\mathrm{C} \\
\mathrm{B}-\mathrm{C} & \sqrt{2} \mathrm{E} & \mathrm{B}+\mathrm{C} & -\mathrm{i} A+\mathrm{D} & \sqrt{2} \mathrm{~F} & -\mathrm{iA}-\mathrm{D}
\end{array}\right)
$$

they are $A=B=0$ for an extreme value $A y=1$; for $A_{y}= \pm 1$ they are $A=B=0, C=7$ if. Imposing these conditions on the formulae for the observables 6,7 gives the following

$$
\begin{aligned}
& \text { A } \quad= \pm 1 \\
& A_{y y}=x_{0, y}^{y^{\prime}}=1 \text {, } \\
& A_{\mathrm{xx}}-A_{z 2}=-1 / 2 \\
& A_{x z}=k_{x z}^{y^{\prime}}=C_{x z, y}=0 \\
& \mathrm{~K}_{\mathrm{x}}^{\mathrm{x}^{\prime}}=\mathrm{k}_{z}^{x^{\prime}}=\mathrm{K}_{\mathrm{xy}}^{\mathrm{x}^{+}}=\mathrm{K}_{\mathrm{y}^{\prime}}^{\mathrm{x}^{\prime}}=0 \\
& \mathrm{~K}_{\mathrm{x}}^{z^{\prime}}=\mathrm{K}_{z}^{z^{\prime}}=\mathrm{K}_{\mathrm{xy}}^{\mathrm{z}^{\prime}}-\mathrm{K}_{\mathrm{yz}^{\prime}}^{z^{\prime}}=0 \\
& \mathrm{C}_{\mathrm{X}, \mathrm{x}} \cdot \mathrm{C}_{\mathrm{z}, \mathrm{x}}=\mathrm{C}_{x y, \mathrm{x}}-\mathrm{C}_{\mathrm{y}, z \mathrm{x}}=0 \text {, } \\
& C_{x^{\prime} z}=C_{z, z}=C_{x y, z}=c_{y z, z}=0 \text {, } \\
& \mathrm{p}^{y^{\prime}}-\mathrm{A}_{\mathrm{D}, y}=\mathrm{K}_{y^{\gamma^{\dagger}}}=-\mathrm{C}_{y y, y}=\mathrm{t} \text {, } \\
& \mathrm{K}_{\mathrm{Y}}^{\mathrm{Y}^{1}} \quad \mathrm{x}-\mathrm{C}, \mathrm{y}=\mathrm{t} \text {, } \\
& C_{x x, y}=-K_{x x}^{y^{\prime}}=C_{z z, y}=-K_{z z}^{y^{\dagger}}=1 / 2 t \text {, } \\
& K_{0, x}^{x^{\dagger}}=-x_{0, z}^{z^{\prime}}=u \text {, } \\
& \mathrm{K}_{\mathrm{o}, \mathrm{z}}^{\mathrm{x}^{\mathrm{i}}}=\mathrm{K}_{\mathrm{o}, \mathrm{x}}^{\mathrm{\prime}^{\prime}}=v \text {. }
\end{aligned}
$$

Here $\sigma_{0}, A, P, K$, and $C$ denote the unpolarized cross-\$ection, emalyzing power, particle polariza- tions, polarization transfer coefficients and fificiency correlation coefficients, respectively. The first subscripted index stands for the berm, the second for the target polarization. Thus 24 polarization obsenvables involving two or less particle polaritations are numerically determined, while the other 14 are given by the three parameters $t, u$, and $v$. With the cross-section for unpolarized particles there are thas four parmeters that can be detenmined experimentally. By a careful selection of the experisents, through an inspection of the general formala, 5,6 a verification of an extreme point of the conponents $A_{y}$ and $A_{y y}$ should be feasible. The establishment of such a point would be very important in an analysis of the process, due to the restrictions imposed on scrie elements of the M-matrix.

\section{Footnotes and References}

'Proc. Fourth Int'1. Syleposium on Polarization Phencmena in Nuclear Reactions (Birkhauser Verlag, Besel, 1976].

${ }^{\dagger}$ Present address: Physics Dept., University of Basel, Switzerland.

"Present address: Physics Dept., Laval University. Queber, Canada.

${ }^{5}$ Present address: Nhelear Science Lab+, M. I.T, , Cambridge, Kass.

1, F, Sefler, Phys. Lett, 61E, $144(1976)$.

2. W. Grübler, V. König, A. Ruh. P. A. Sclvnelzbach, R. E. White, and P. Manmier, Nucl. Phys. Al76 (1971) 631 .

3. R. Roy, F, Seiler, H. E. Conzett, F. N. Rad, and R. M. Lariner, Fourth Polarization Symp.

4. F. Seiler, F. N. Rad, and H. E. Conzett, LBL-report, LALL-\$496.

5. P. L. Csonka, M. J. Moravcsik, and M.D. Scadron, Phys. Rev. 143 (1966) 775.

6. G. G. Ohlisen, Rep. Prog. Phys. 35 (1972) 717 .

7. J. L. Gammel, P. W, Keaton, and G. G. Ohlsen, Los Alamos Scitritific Laboratory, Report No. LA-4492-14S, July I97D. 


\section{VECTOR ANALYZING POWER IN $\mathrm{d}$ - ${ }^{4} \mathrm{He}$ ELASTIC SCATTERING BETWEEN 15 AND $45 \mathrm{MeV}^{*}$}

HE Conzell, wr Dahme.t Ch Leemann.

$J$ A Macdonate a and J P Meuderss

Phase shift andyses of d - ${ }^{4} \mathrm{He}$ elastic scattering data below $17 \mathrm{MeV}$ have led progressively to inproved agreement with the data and to the determination of level parameters for those states of ${ }_{\mathrm{Li}}$ that appear as resonances in the $\mathrm{d}+4 \mathrm{He}$ channel. 1,2 These data have shown substantial values of the vector and tensor analyzing powers, so we selected $\mathrm{He}$ as a potentially good andyzer for the higher energy polarized deuteron beams. Also, two independent determinations $2,3,4$ of the absolute vertor analyzing power $A_{y}\left(E_{\mathrm{d}}, \theta_{\text {con }}\right)$ have been made which are in very good agreement. In the scattering of a purely vector-polarized deuteron beam the measured left-right asymetry is given by

$$
E(\theta)=[3 / 2) \mathrm{P}_{y} A_{y}(\theta),
$$

where $p$ is the bean polarization. Asymmetry data were taken at $5 \mathrm{NaV}$ intervals from 15 to 45 latev at center-of-jass angles from $30^{\circ}$ to $16^{\circ}$. At all angles the elastically sccattered deuterons were detecterd with $\Delta E-E$ counter telescopes placed at equal angles to the left and right of the bears axis. Particle identification served to separate deuterons from protons wich came from the deuteron breakup reaction. A gas target of $\mathrm{He}$ at $=1$ ath pressire was used with beams typically of $50 \mathrm{nA}$. A polarimeter, consisting sinilarly of a gas target and a left-right peir of counter telescopes, was placed downstrear of the main scattering chanber, and it provided continuous manitoring of the bean polarization during the course of the experinent.

Absolute norsalization of our data to the calibration point $A_{h}\left(11.5 \mathrm{keV}, 118^{\circ}\right)=-0.410 \pm 0.010$ (Refs. 2,3) was achieved in the following mamer. The bean of erergy $\mathrm{E}_{1}$ (e.g., $15 \mathrm{or} 20 \mathrm{MsV}$ ) incident at the first taryet was degraded in aluminum to an energy $\mathrm{E}_{2}=11.5$ at the polarineter where the asymmetry $\varepsilon_{2}\left(11.5,11 g^{\circ}\right)$ was measured. At the sane tifne, $\varepsilon_{1}\left(\mathrm{I}_{1}, \theta\right)$ was measured in the main scattering chamber, so frovt (2)

$$
A_{y}\left(E_{1}, \theta\right)=A_{y}\left(11,5,118^{\circ}\right) \varepsilon_{1} / \varepsilon_{2}
$$

which gives $A_{y}\left(E_{l}, \theta\right)$ In terms of the measured asymmetries and the $A_{y}$ calibration value, At higher energies for $\mathrm{B}_{1}$ a previously deterinined value for $A_{y}\left(E_{2}, \theta_{2}\right)$ served as a reference polarimeter analyzing power. We also used the corplete angular distribution of Ref. 5 at $15 \mathrm{MeV}$ for an índependent absolute norualization of our $15 \mathrm{kkV}$ data. Wh found this to correspond to a value of $\mathrm{Ay}_{\mathrm{y}}(11.5 \mathrm{MeV}$, $118^{\circ} \mathrm{y}=-0,415 \pm 010$, in very good agreement with the other calibration value.

Our 20-45 $\mathrm{keV}$ results are show in Fig. 1, and our 15-MoV data are in excellent agreoment with those of Ref. 5. It is seen that d-4 $\mathrm{He}$ elastic scattering serves as a very good vector-polarization

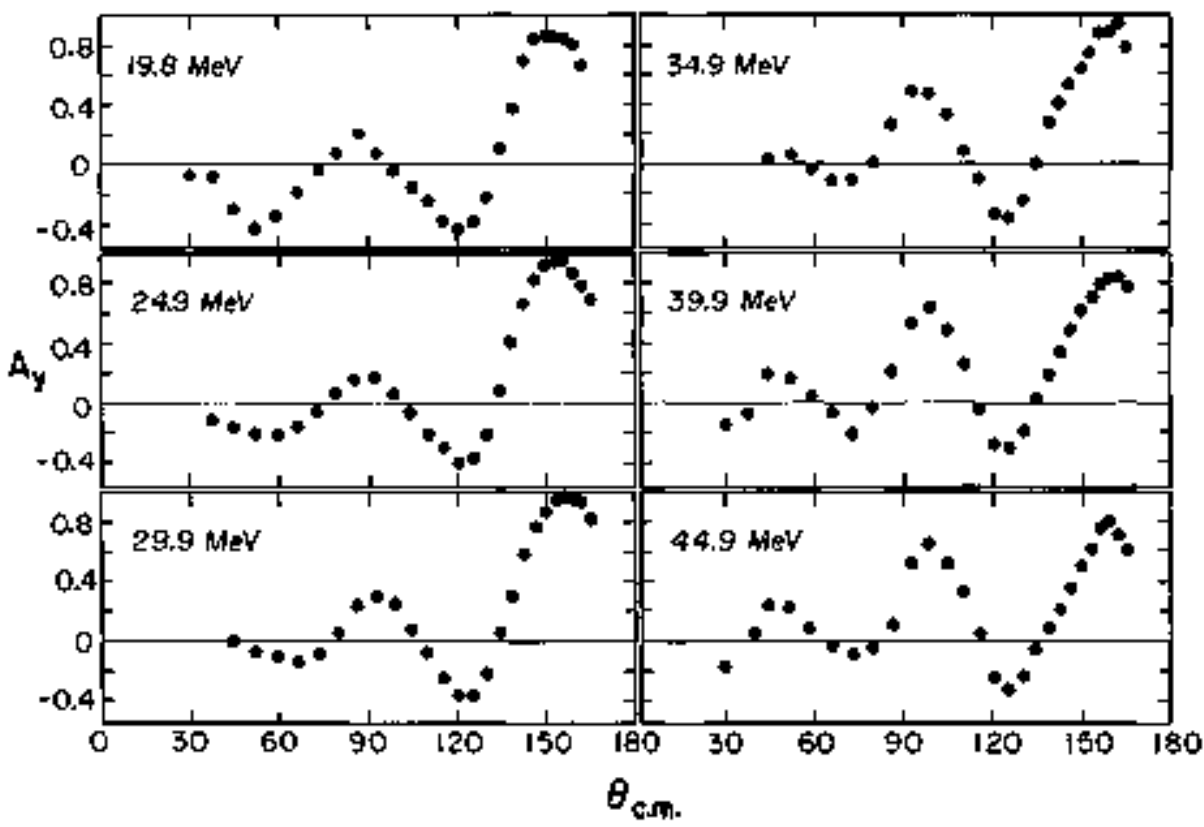

Fig. 1. Vector analyzing powers, $A_{y}(\theta)$, in t . Afe elastic scattering between 20 and $45 \mathrm{MeV}$.

(XBL 756-3155) 


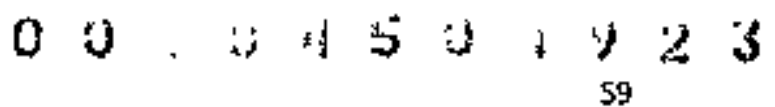

analyzer over a wide range of deuteron energies.

Of particular interest is the fact that $\boldsymbol{A}_{\mathrm{y}}$ $\left(155^{\circ}\right)$ reaches values near 1,0 at 24.9 and 29.9 meV. It has been shown recently that at an energy and an gle for which $A_{y}(\mathbf{E}, \theta)= \pm 1.0$ in o - ${ }^{4} \mathrm{He}$ scattering, all the analyzing-power components are fixed and detemined. 6 This result has been extended to apply to all tw-body reactions with lncident polarized deuterons, and it is independent of the spins of the target and reaction products. 7 In view of our results, a point near $\theta_{\mathrm{cm}}=155^{\circ}$ between 25 and $30 \mathrm{keV}$ in $\mathrm{d}-{ }^{4} \mathrm{He}$ scattering is the best candidate for reaching the extrewae value Av1 (Ref. 8). The possibility of an $\mathrm{Ay}=-1$ value at $\theta_{\mathrm{con}}=55^{\circ}$ at 11.88 HeV $^{6}$ is not supported by the $A_{y}(E, 0)$ data in that resion. 4

\section{Foptnotes and References}

*Proc. Fourth Int'1. Sympositum on Polarization Phencmena in huclear Reactions (Birkhäuser Verlag, Basel, 1976 ].

${ }^{\dagger}$ Sektion Physik der Universität Mitmchen, B046 Garching, Gerrany.
*Chalk River Aluclear Laboratories, Chalk, River, Ontario, Canada

${ }^{5}$ Centre de Physique Nucleaire, Universite de Lowrain, Belgiur,

1. P. A. Schoelzbach et al, thucl. Fhys. A784

(1972) 193, and Refs, therein.

2. W. Grtiebler et a1., Muc1. Myys. A242 265 .

3. W. GriebIer et al., thucl. Phys. A134 (1969) 686; V. Konig et al. , Nurcl, Phys. Albु \{1971) 393.

4. G. G, Ohlsen et al., Phys, Rev. CB [1973) 1262 ,

5. R. A. Hardekopf et ai., Los Alamos Report LA-5051 (1972).

6. W. Grïibler et af., Muc1. Phys, A242 (1975) 285.

7. F. Sefler, F. N. Red and H. E. Conzett, Fourth Polarization Symp.

8. H. E. Conzett, F. Seiler, F, N. Rad, R. Roy, and R. M. Lariner, Fourth Polarization Symp.

\section{TENSOR ANALYZING POWER AYY IN $\bar{d}-{ }^{4} \mathrm{He}$ ELASTIC SCATTERING FROM 20 TO $45 \mathrm{MeV}$}

\author{
E J Stephenson, H E Conzelt, R M Larumer, \\ B T Lèmogn, entad R Roy
}

Evern though tensor-polarized deltercon beans at energies above $20 \mathrm{MoV}$ have been arailable for scome time, there are no measurements of tensor andyzing powers in this energy region suitable for use as a secondary polarization standard. This peper reports measurenents of angular distributions of the tensor analyz hng power $A_{y y}$ in $d-{ }^{4} \mathrm{H}$ elastic scattering from 20 to 45 aleV decteron botbarding energy. The target, ${ }^{4} \mathrm{He}$, was chosen because it is a simple system with analyzing poners that vary slowly with energy and angle, and because $\mathrm{He}$ has proved in the past to have large analyzing powers for a variety of polarized projectiles.

The analyzing powers discussed in this paper are defined in accordance with the Madion Comvention. 1 The tensor-polarized deuteron beam was produced by an atomic bean polarized-1on scurce that injected the bean axially into the 88 -inch cyclotron. The tensor analyzing power was detemined by çकाएaring the count rates heasured with the polarizing interunediate-field $\because$.f. transition alternately on and off. Because of a vector-polarized camponent in the beat, it was necessary to observe the count rate sinouttaneously on either side of the bean to tancel the effect of the vector analyzing power. From these four count rates, $Y$, the tensor anglyzing power is given by

$$
A_{y y}=\frac{1}{P_{y y}}\left\{\frac{Y_{L P}}{Y_{L U}}+\frac{\mathrm{Y}_{R P}}{Y_{R U}}-2\right\}
$$

hhere L and R derrote the two sides of the bean, P and $U$ denote polarized and unpolarized, and $\mathrm{PrV}$ is the tensor beam polarization.

In the experiment, the betan passed flist through the main scattering chamber where the angular distributions were measured and then through a saller chamber used as a polarineter, Each chanber contained a 4 He gas target. During the experIment the beam energy was raised from 20 to $45 \mathrm{keV}$ In 5 kev steps. At each new energy, the polarimeter detectors were set at the angle and energy of an analyzing power point measured previously at a lower energy, The beain energy was restuced after the main scattering chouber by placing an alvminan absorber in the beam. Folarimeter calibration points were chosen at 17,25 , and $35 \mathrm{keV}$ and at laboratory angles between $80^{\circ}$ and $90^{\circ}$.

The inttial value of the tensor aralyzing power Ay at 17 MeV was taken from Ohlsen's neasure Joents, 2 thich are accurate to within 0.02 . (The 
acctmulation of statistical errors in our method increases this uncertainty to 0.03 at $35 \mathrm{keV}$.) Figure I shows angular distributions of $A_{y}, A_{y}$, and $A_{x x}$ measured at $17 \mathrm{beV}$ from this experiment and the earlifer measurenents of Ohlsen. Using the angular ¡istribution of $A_{y y}$ as a calibration standard, good agreenent has obtained with the magritude of all the analyz ing powers. The origin of the discrepancy in $A_{x C x}$ at forward angles is not tinderstood. A comparisor with the tensor calibration point ${ }^{3}$ at $E_{;}=11.9$ Mer where Ay $=1$ was ascurate to within $5 \%$.

The measured angular distributions from 20 to $45 \mathrm{lew}$ (see Fig. 2) show large values of Ayy at all energies. The last negative and positive excursions

$$
{ }^{4} \mathrm{He}(\bar{d}, \mathrm{~d}){ }^{4} \mathrm{He} \quad \mathrm{E}_{\mathrm{d}}=17 \mathrm{MeV}
$$

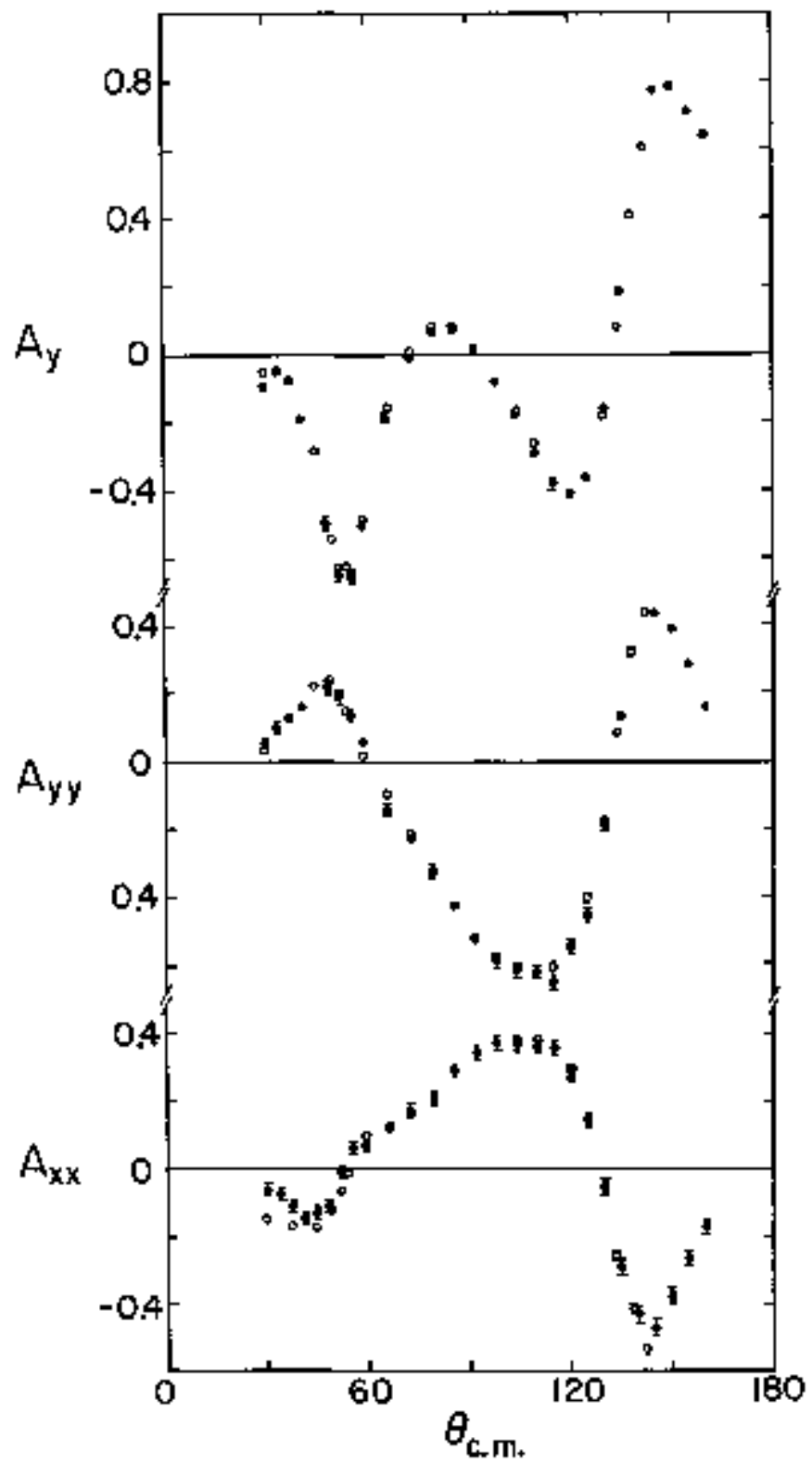

Fìg. 1. Measurements of three antyzing powers in id $n$ He olastic scattering at $\mathrm{E}_{\mathrm{d}}=17 \mathrm{MV}$. The solid eircles are measurentents from this experjoent and the open circles are from Ref. $z$.

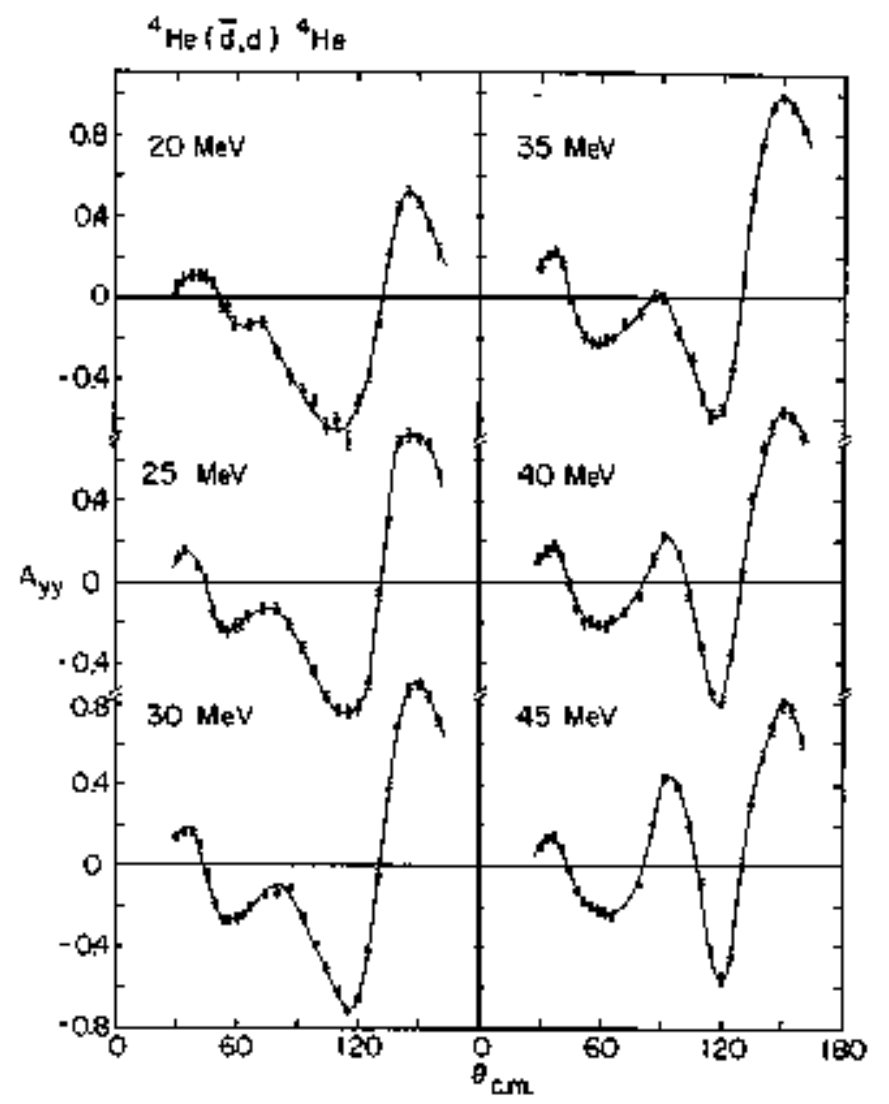

F䗆. 2. Measurements of Ary in $\mathrm{d}-{ }^{4} \mathrm{He}$ elastic scattering. The desuteron bcrmbarding energy is notes. The curves are a guide to the eys,

(XEL 764-2664)

in each angular distribution are good candidates for use in a polarimeter. The analyzing power varies slowly in energy and angle, making the geconetric corrections sulall.

The efficierey of a polarimeter, given approxinately by $\mathrm{Ayy}_{\sigma}{ }_{\sigma}$, is shown in Fig, 3 for the last tho excursions as a function of energy. A polarimeter observing particles at the angle of the positive peak is more efficient over rost of the mergy range, even though the cross section there is staller by a factor of about 3. If instead one observes the recoil toparticles from this positive peak, the of ficiency increases for the salne detection reconetry by a factor between 5 and 10, However, in this case the energy resolution must be good in order to cleanly separate $\alpha$-particles which origingte from elastic scattering and the deuteron breakip reaction.

From this experiment it is clear that the observation of deuterphs elastically scattered fran $4_{\mathrm{He}}$ is suitable as a polarization standard in the trergy region from $20^{\circ}$ to $45 \mathrm{MeV}$. Mepasmements of angular distribtions of the tensor analyzing porer Axx are planted. With these measurements and the measurements of the vector anslyzing power $A y$, enough data should be avajlable to perform a phaseshift analysis of $4 \mathrm{He}(d, d){ }^{4}$ elastic scattering in this mergy region. Such an analysis would indicate 


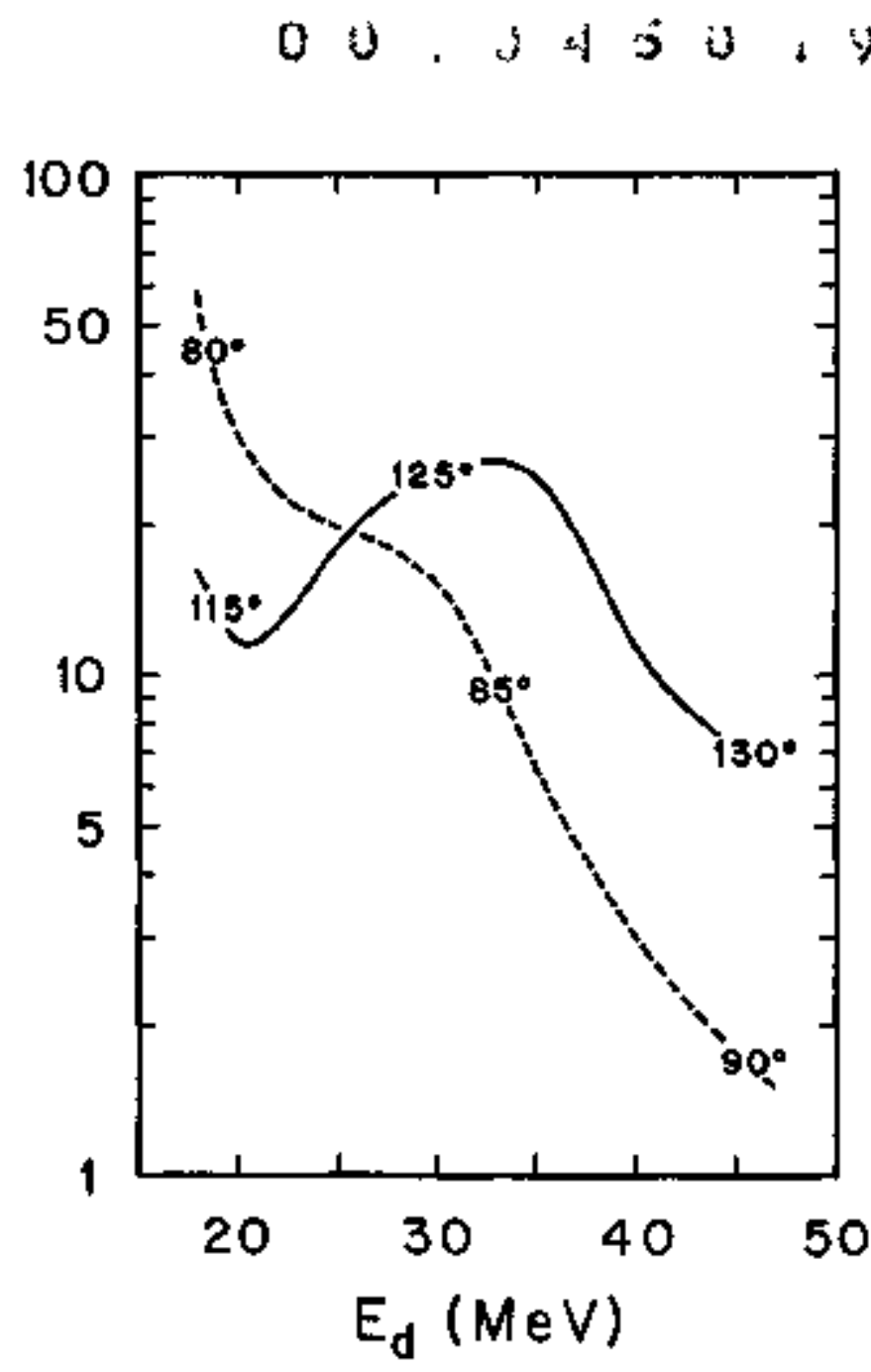

Fig. 3. Polarimeter efficiency, Ayy ${ }^{2} \sigma$, for the center-of-nass scattering angles noted on each curve. These angles correspond to extreme values of Ayr. Measurenents at different energies are cometed by a spooth curve. The $y$-axis scale is arbitrary.

(XBL 764-1385)

whether the tensor analyzing power $A_{x y}$ reaches its noximal value of $I$ in the vicinity of $\mathrm{E}_{\mathrm{d}}-35 \mathrm{MeV}$ and $\theta_{\text {c. }}=150^{\circ}$. If it does, this point would bectme a primary standard for the meastriement of the tensor bean polarization.

\section{Footnote and References}

"Present address: Physics Dept., Laval University, Quebec, Canada.

1. Polarization Phenomena in Muclear Reactions, ed.

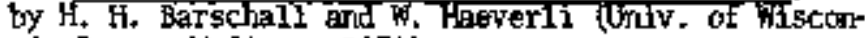
sin Press, Madison, 1971) p, xxy.

2. G. G. Ohisen, P. A. Lovoi, G. C. Satzman, U. Meyer-Berkhout, C. K. Mitche11, and W. Gruebler, Phys. Rev. C8, 1262 (1973).

3. W. Grüebler, P. A. Schmelzbach, V. Kởitg, R. Risler, B, Jenney, and D. Bowme, Nucl. Fhys, A242, $2 B 5(1975)$.

\section{EXTRENE VAL.UES AY $= \pm 1$ OF THE ANALYZING POWER FOR SPIN-1 POLARIZATION*}

F Soliler, $F$ N Rad.f and H E Conzett

Maxinde possible values of the analyzing porrer have been identified at several energies and angles in the elastic scattering of spin- $1 / 2$ and spin-1 particles from spin-0 muclei, 1,2 and evidence for at least one extreme value of the tensor component $A_{y}$
$=1$ has been foumd in the $3 \mathrm{He}(\mathrm{d}, \mathrm{p})^{4} \mathrm{He}$ reaction. The recent analysis by Grüebler et al. 2 of d- 4 He elastic scattering data between 3 and $17 \mathrm{Hov}$ has proved the existence of points at which the conmonent $A_{x y}$ of the spin-1 aralyzing power reaches its maximun possible value of unity. Also, for points of maximum vector amalyzing power, $A_{y}= \pm 1$, they exanined the resulting conditions son elenents of the transition matrix $M$ which connocts initial and final spin states, $X_{f}=M_{X i}$. At such a point three equations oust be satisfied anong the N-batrix amplitudes, with the consequence, then, that all the remaining analyzing powers are determined:

$$
A_{y y}=+1, \quad A_{x x}=A_{z z}=-\frac{1}{2}, A_{x z}=0 .
$$

In addition to its usefulness in an absolute calibration of deuteron vector polarization, the ocarrence of stuch a mique and restrictive point culd clearly be of great interest and value, since there the relative values of all the M-natrix elements are determind.

We show in this paper that the necessary, but not sufficient, conditions (1) for the existence of a point $A_{y}= \pm I$ are valid for reactions with the general spin structure $1+a \rightarrow b+c$, where $a, b$, and $c$ are arbitrary spins. This follows from a property of the spin-1 derisity matrix, and exantnation of the $3(2 a+1)$ by $(2 b+1)(2 c+1) M-\pi a t r i x$ is not required.

Mathods of specifying a 'degree of polarization" for an ensemble of polarized spin-1 particles produced in a muclear roaction have been given by Lakin, $\$$ Fano, and Minnaert. 7 They are based on the fact that the density matrix of the ensemble is positive semidefinite. Its expansion in terms of an 
irreacible set of tensor operators ${ }^{\tau} \mathbf{k q}$ immposes conditions on the tensor moments $\left.t_{\mathrm{kg}}=\mathrm{vq}_{\mathrm{kg}}\right\rangle_{*}, 5,7$

Identical limitations apply for the polarization efficiencies $T_{k q}$ due to tine reversal invariance. They are particularly simple in a transverse coordjuate system $S^{\dagger}$ with the $2^{\prime}=a x i s$ perpendicular to the reraction plane.

$$
\left(T_{20}^{\prime}+r^{2}\right)^{2}=3\left(T_{10}^{\prime}\right)^{2}+6\left|T_{22}^{\prime}\right|^{2}
$$

In the space (THo, $\sqrt{2}\left|T_{22}\right|$, T 10 ), Eq. (2) defines the interior of a cone tifig. 1). Points on the surface or the base $\left[\mathrm{T}_{20}=\frac{1}{2} \sqrt{2}\right.$ ) of the Lakin cone corrospond to restricted cubinations of the maximm possible values of the $\mathrm{T}_{\mathrm{kg}}$. In teijas of the observables $\mathrm{T}_{\mathrm{kg}}$ or $\mathrm{A}_{\mathrm{if}}$ defined $\mathrm{by}$ the Madison comention, the $\mathrm{r}_{\mathrm{kq}}$ ere:

$$
\begin{aligned}
\mathrm{T}_{10}^{\prime} & =\sqrt{2} \mathrm{i} \mathrm{T}_{11}=\frac{1}{2} \sqrt{6} A_{y^{\prime}} \\
\mathrm{T}_{20}^{+} & =-\frac{1}{2}\left(\mathrm{~T}_{20}+\sqrt{6} \mathrm{~T}_{22}\right)=\frac{1}{2} \sqrt{2} \mathrm{~A}_{Y y^{\prime}} \\
\mathrm{T}_{22}^{\prime} & =\frac{1}{4}\left[\sqrt{6} \mathrm{~T}_{20}-2 \mathrm{~T}_{22}\right)-i \mathrm{~T}_{21} \\
& =\frac{1}{6} \sqrt{3}\left[\left(A_{\mathrm{z}_{2}}-A_{\mathrm{x} x}\right)+i 2 A_{\mathrm{x} 2}\right]
\end{aligned}
$$

Thus points with $A_{y}=1$ lie on the base of the Lakin cone. Only there, ralues of $A_{y}= \pm$ can be attained (Fig, 1), points $A$ and $B$ ), and then only if $\left|T_{2}\right|=0$. This results in the conditions of Eqs. (1). As noted, this was shown for d-4 $\mathrm{Fe}$ elastic scattering by direct calculation with the appropriate 3 by 3 N-matrix, 2 Fron the derivation given here, it is clear that Eqs. (1) are valid in general and are independent of the spin space of the process. For all reactions involving polarized spin-l particles they therefore provide a clear signature of points where $A_{y}= \pm 1$ is possible.

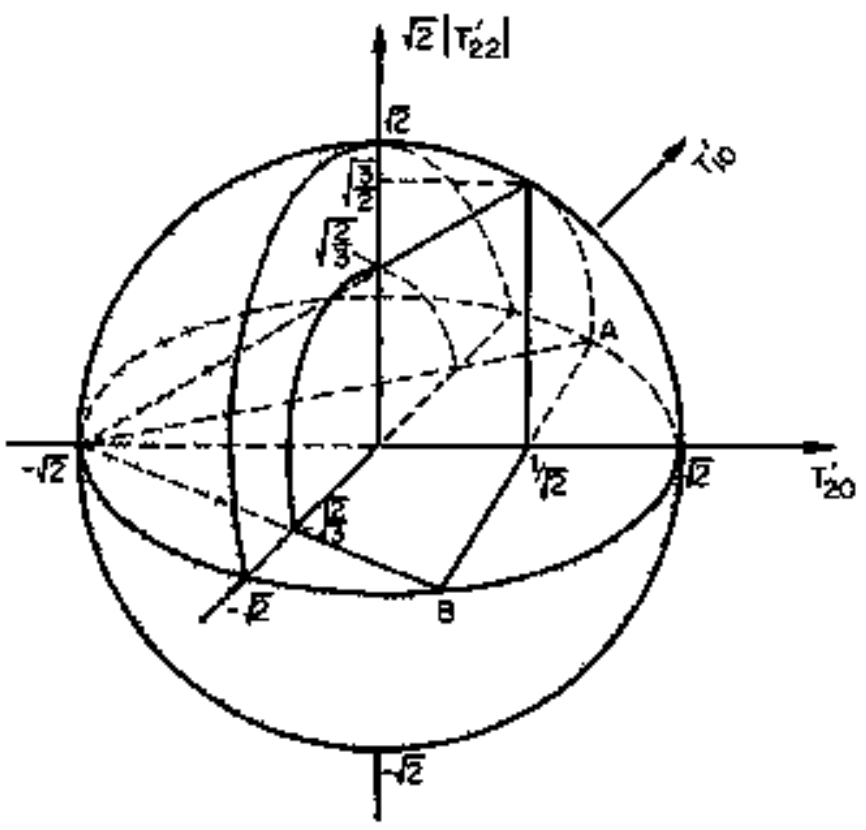

Fig. 1. Cutaway drawing of the cone defined by Ea. (2). The points $A$ and $B$ correspond to the extrene values $A_{y}= \pm 1$.

(XBL 754-2652)
It should be noted that the agrespont of the experimental date with Eqs. (1) are necossary but not sufficient conditions for the existence of an extrene value of the efficiency $A_{y}$. Ary point along the line $A B$ in Fig. 1 satisfies Eqs. (1). Using a beam with calibrated tensor polarization, 2 it is possible to determine that a point on the line $A B$ is reached. Howerer, the calibration of spin-1 rector polarizations is stil1 so uncertain that it cannot be show expertuental1y that point $A$ or $B$ is indeed sttaines. Although such a unique and restrictive point is a priori quite imlikely, an experinertal value of $A_{Y}>0.98$ lias been measured in d-4 He elastic scattering at $E_{d}=26.8$ weV near $\theta_{\mathrm{l}}=$ $135^{\circ}$ (Ref. 4). Hence, these criteria are of prac. tical interest.

${ }^{6} \mathrm{Li}(\mathrm{d}, \mathrm{a})^{4} \mathrm{He}$, Measurements by Griebler et al 10

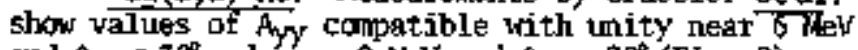
and $\hat{\theta}_{\text {ort }}=30^{\circ}$ and near $8 \mathrm{MeV}$ and $\theta_{\text {cin }}-90^{\circ}$ (Fig. 2). Hear a weV only Ayy can reach unity, since the particlo symmetry in the exit chanmel requires that $A_{y}\left(90^{\circ}\right)=0$. Near $6 \mathrm{NeV}$ thore are drastic changes in the angular distributions of alt efficiencies, which nakes a comparison wíth Eqs. (1) difficult. The comportent $A_{y}$ near $30^{\circ}$ varies rapidly from negatlve values at $4 \mathrm{MeV}$ to large positive values at 6 MeV, decreasing aga in above $7 \mathrm{MeV}$. 10 it is this possible that the value $A_{y}=1$ is attained. The component Axz changes sign at forwart angles between 6 and $7 \mathrm{Mer}$, while the ralues of $A_{x x}$ and $A_{z z}$ are close to $-1 / 2$. In this region the contitions necessary for $A_{y}=1$ sag therefore be fulfilled.

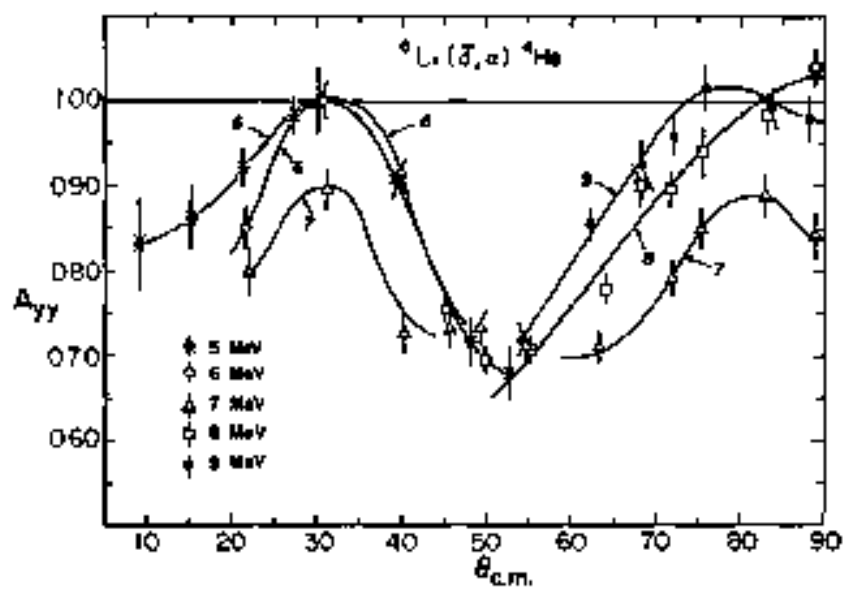

Pig, 2. Values of the efficiency tyy in the Di $[d, \alpha)^{4} \mathrm{He}$ reaction at $5,6,7,8$, and $9 \mathrm{MeV}$, showing two points with $A_{y y} \simeq 1$.

(XBL 753-2614)

$3_{\mathrm{He}}\left(\mathrm{d}_{1},\right)^{4} \mathrm{He}, \quad$ one point with $\mathrm{Ay}_{\mathrm{y}}=1$ has been tentat ively identified near $9 \mathrm{MeV}$ and $\theta$, $=27^{\circ}, 3$ Since $A_{2 z}=-1, A_{X x} \approx 0$ and $A_{x z}=-1.1,10^{\circ}$ it is clear that Ay cannot reach the value -1 . The situation is different for the possible $A_{3 y}$ maximum above 11.5 HeV, where no masurements are available. At 11,5 $\mathrm{MeV}$ a value of $\mathrm{A}_{\mathrm{r}}=0.9$ is futud near $\theta_{\mathrm{cm}}=120^{\circ}$ (see Fig. 1 of Rat. 3). The vector analyzing power Ay 15 tatge and positive both at $11.5 \mathrm{NeV}$ and 15 Mev, 10,11 The values for $A_{x x}$ and $A_{x z}$ below 11.5 


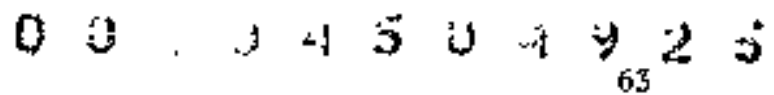

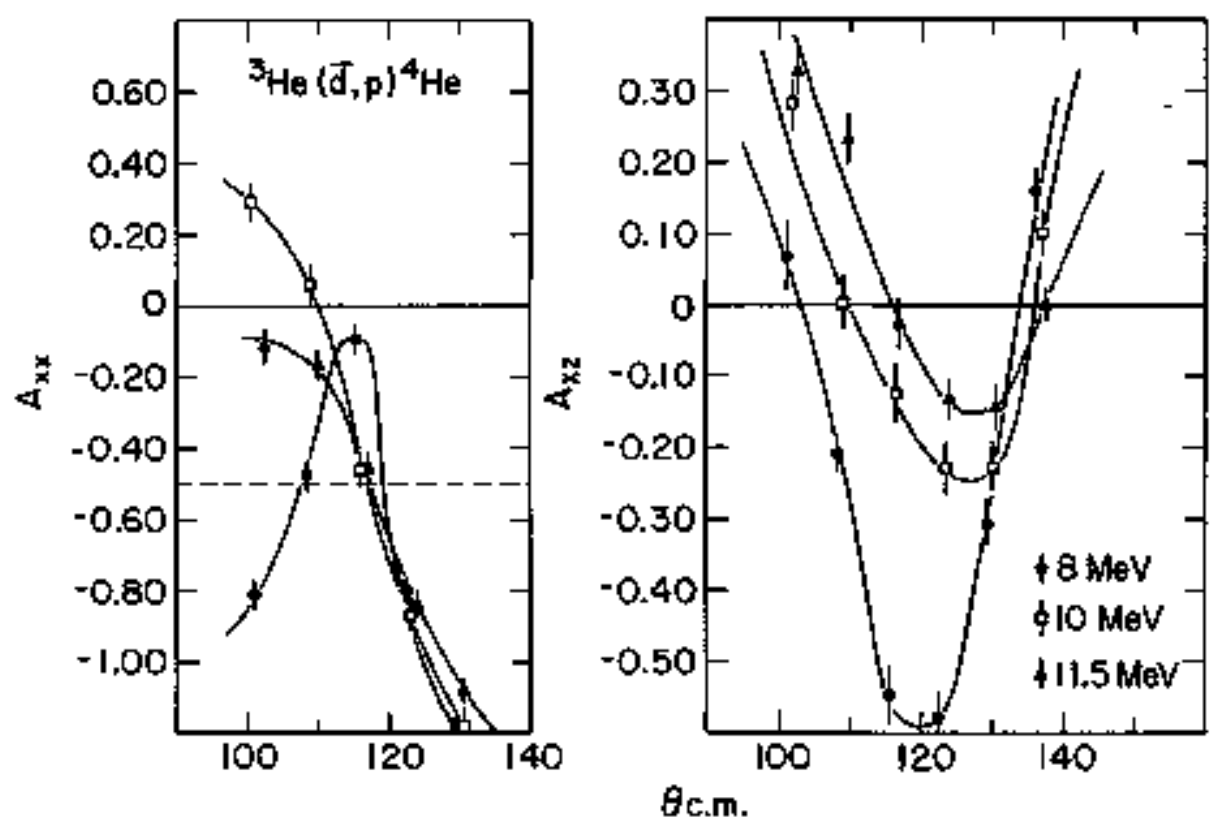

Fig. 3. Components $A_{x x}$ and $A_{X z}$ of the analyzing power in the $3_{1 e(d, p)}{ }^{4} H e$ reaction below 11.5 met.

(XBL 754-2663)

MeV are plotted in Fig, 3, Clearly Axx and, dus to the condition $A_{x y}+A_{y y}+A_{z z}=0$, also $A_{z z}$ lie near $-1 / 2$ at $120^{\circ}$, while $A_{K z}=0$ obtains at angles moving toward $120^{\circ}$ as the enery increases. This the necessary conditions for Ay $=1$ may be met near this angle at some energy between 12 and $15 \mathrm{MeV}$.

\section{Footnotes and References}

'Condensed from t.BL-3496, subsitted to Phys. Rev. for publication.

†Prosent address: Physics Dept., University of Base1, \$w1tzer1and.

Fresent address: Nuclear Science Lab. , M.I.T., Cambridge, Mass.

1. G, R. Plattmer and A. D. Bacher, Phys, Lett, $36 \mathrm{~B}, 2 \mathrm{I} 1(1971)$.

2. W, Grïlebler, P. A. Schmelzbach, V. Kớnìn, R. Risler, B. Jenny, and D. Boenma, Nucl. Phys, A242, 285 (1975).

3. F, Seiler, Phys, Letters 61B, I 44 (I976),
4. H. E. Conzett, F. Seller, F. N. Rard, R. Roy, and R. M. Larìner, Proc. Fourth Int ${ }^{\dagger} 1$. Symp. on Polarization Phencmena in Nuclear Reactions (Birkthuser Ver1ag, Basel, 1976).

5. W. Lakin, Phys, Rev. 98, 139 (1955).

6. [. Fano, Rev. Mod. Phys. 29, 74 (1957).

7. P. Minnaert, Prys. Rev. Lett. 16, 672 (1966).

8. Polarization phenomema in nuclear reactions, H. H. Rarschall and W. Haeber11 eds. (University of Wisconsin press, 1971\}, p. xov.

9. F. Seiler, R. Roy, H. E. Conzett, and F. N. Rad, to be published.

10. W, Griiebler, V. König, and P. A. Schnelzbach, Results of measurements and andyses of muclear reactions inhuced by polarized and unpolarized deuterons, Eidgenössische Teclmische Hochschule Ztirich, internal report, May 1973 .

11. R. Roy, F. Seiler, H. E. Conzett, F. N. Rad, and R. M. Larimer, Proc. Fourth Int'1. Syup. on Polarization Phencmena in Nuclear Reactions (Birktäuser Yerlag, Basel, 1976). 


\title{
MEASUREMENT OF THE VECTOR ANALYZING POWER NEAR THE VALUE AY $=1.0 \overrightarrow{\mathrm{d}}-{ }^{4} \mathrm{H} \oplus$ ELASTIC SCATTEAING*
}

\author{
H E Conzẹll. F Setler, $F$ F Rid
}

$A$ foy, ${ }^{\frac{f}{3}}$ and $R$ M Lofimer

Points in energy and angle (E,, ) at wich a camponent of the andyzing power for spin-polarization reaches its theoretical maxifrm are of particular interest and importance. Experifentally, they provide valuable absolute calibrations. Theoretically, there exist at such polnts 1mportant canditions on, or relations ancong, the N-antrix taplitudes. As a consequence, other polarization observables are determined, and in reactions with particwlarly simple spin structure it is possible that all such observables are determined.

The simplest case is that with the spin structures $1 / 2+0+1 / 2+0$, for which it has been shown that points for which $A_{y}(E, 6)= \pm 1$ do exist in $\mathrm{N}-\mathrm{A}_{\mathrm{He}}$ and $3 \mathrm{fl}^{4} \mathrm{He}$ elastic scattering, 1 The resulting condition on the two $M$-matrix gniplitudes $f$ and $g$ is that $g= \pm i f$, and the other polarization observable, the spin rotation argle, is determined. Sinzlar investigations in $\mathrm{d}-4 \mathrm{He}$ elastic scattering $(1+0 \rightarrow 1+0)$ have led to identification of points for which, the tensor analyzing-pormar corponent Ayy $=1$ (Ref, 2 ). The corresponding linear relations anong the $M$-mnatrix elements at points $A y=1$ and $A_{y}= \pm 1$ have been given, 2,3 and it was shown 2 ) that $A_{v y}=1$ is a necessary condition for the existence|of a polnt of $A_{y}= \pm 1$. This latter result has now been proved to bi valid more generalty, that is, it is valid for all reactions with the spin structure $1+a+b+c$, where $a, b$, and $c$ are arbitrary spins. 4 Relations anong, and deternjuations of, the other polarization observgbles have also been given for several reac. tions.

Since a point of $\mathrm{A}_{y}= \pm 1$ for spin 1 is so wique and restrictive, and a pricri perhaps so ulike ly, the location and identification of such a point would be of considerable value. The best candidate for such a point was suggested in the megsurements of $A_{y}(\theta)$ in d-4 ${ }^{4}$ elastic scattering betneen 15 and $45 \mathrm{M}=V^{6} \mathrm{~b}$ There, values of $\mathrm{A}_{\mathrm{Y}}\left[\theta_{\mathrm{L}}=135^{\circ}\right)>0.97$ were found near 25 and 30 Mell. Therefore, we have examined this energy region to determine the ruaximum value of $A_{y}(E, \theta)$. The experimental details and the calibration procedures are described elsewhere. ${ }^{6}$ We find that $A_{y}$ reaches its maximum value near 28.6 MeV, and we show in Fig. 1 our preliminary results for $A_{y}\left(28,6 \mathrm{keV}, \theta_{L}\right)$. Although finite geofetry corrections and polynconial fits to all our data in this energy and angular region nust be made before the maximon value of $A_{y}$ is detemulned, it is elear fron Fig. I that $A y$ does indeed reach a value very close to unity near $\theta_{\mathrm{L}}=135^{\circ}$ at $28.6 \mathrm{MeV}$.

We have elso mogsured $A y,(E, \theta)$ over the same regions, using the ${ }^{\mathrm{He}}(\mathrm{d}, \mathrm{d}) \mathrm{H}^{4} \mathrm{Ay}$ data of Ref, 3 at $17 \mathrm{Mov}$ for the determination of the tensor polarization of our beam. Again, our preliminary reaslt is that Ayy reaches a maximn value approaching untty near $28.6 \mathrm{HeV}$ and $\theta_{1}=135^{\circ}$, as would be required to

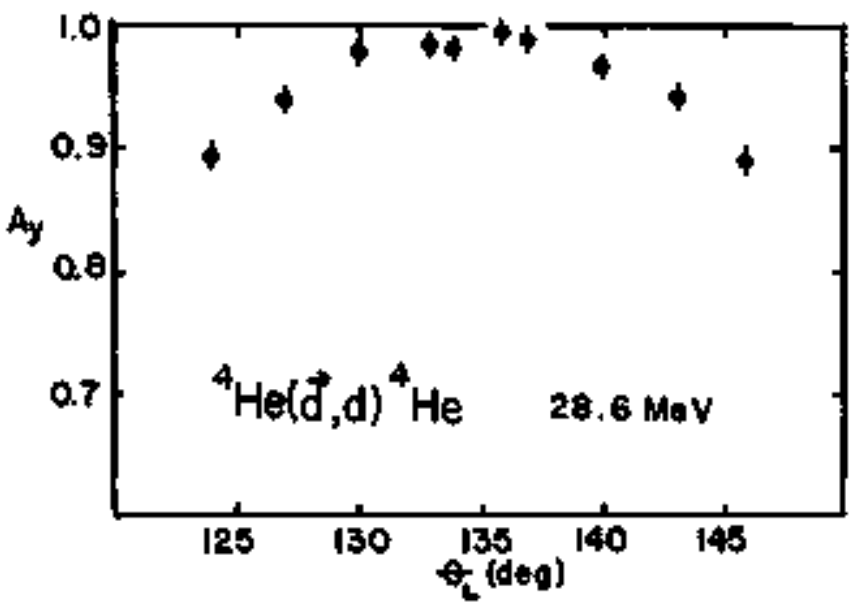

Fig. 1. The vector anklyzing power, $A y(\theta)$, in d-4he elastic scattering at $28,6 \mathrm{MgV}$.

(XBL. $756-1531$ )

fulfill the necessary condition that $A_{y y}=1$ at a point for which $A_{y}=1$.

\section{Footootes and References}

*Proc. Fourth Int”1. Syliposium on Polarization Phendema in Nuclear feactions (Birkhaser Verlag, Basel, 1976).

†Present address: Physics Dept., University of Base1, Switzerland.

FPresent adiress: Mucloar Science Lab., M.I.T., Canbridge, Mass.

${ }^{6}$ Presegnt address: Physics Dept., Laval University, Quebec, Calada.

1. G. R. Plattner and A+ D. Bacher, Phys, Lett. $36 \mathrm{~B}$ (1971) 211 .

2. H. Grübbler et al., Muc1. Phys. A242 (1975) 285.

3. G, G. Oh1sen et al., Phys, Rev, C 8 (1973) 1262.

4. F. Seiler, F. N. Rad, and H. E. Conzett, Fourth Polarization Sylnp.

5. F, Seiler, Pourth Polarization Symp., F. Seiler, H. E. Conzett, F. N. Rad and R. Roy, ibid, ; F. Sefler, H. E, Conzett, and F. N, Rad, ibid.; F. Sejler, F. N, Rad, H, E, Conzett and R. Roy, ibjd.

6. H. E. Conzett et al, Fanth Polarization Syug. 
DU 1 औ di

EXTREME VALUES OF the ANALYZING POWER IN THE ${ }^{6} \mathrm{Li}(\mathrm{d}, \alpha)^{4} \mathrm{He}$ REACTION*

F Seller, F N Rad, t H E Conzenl, and R Roys

Extrene values of the analyzing power have been identifled in $\vec{p}^{-4} \mathrm{He}$ and $\mathbf{d}-{ }^{4} \mathrm{He}$ elastic scettering. $T, 2$ Recently it has been proposed that similar points xist also in reactions. ${ }^{3}$ A calculation of the cartesian tensor Ay from the $6 \mathrm{Li}(\mathrm{d}, 0)$ He data of Grüiebler ot al, 4 shows experimental values with Ayy $\simeq 1$ at two points (Fig. 1). Although more data are clearty needed, extrene values $A_{y y}=1$ are possible. A definite identification requires the elenents of the M-gatrix to fulfill certatn conditions. For Ayy $=I$ the $M-m a t r i x^{5}$

$$
\begin{gathered}
M=-\frac{1}{2}(a-e, \sqrt{2} k, \text { a+e; } \sqrt{2} h,-2 k,-\sqrt{2} h ; \\
a+e, \sqrt{2} f, a-e)
\end{gathered}
$$

mest satisfy $a=0$. If an analysis is not available, the influmice of this condition on certain observables con be tested. Bestde the efficiencles $A_{1 j}$ for deuteron polarization and $A_{k 1}^{2}$ for ${ }_{L} \overline{1}$ polar ization, only the officiency correlation coefficients $C_{i j} \mathrm{kl}$ are available. The 25 linearly independent ợservables ${ }^{5}$ yield

$$
\begin{aligned}
& A_{y y}^{1}=A_{y}^{2}=1 \\
& C_{x, z}=C_{z, x}=C_{x, x}=C_{z, z}=0 \\
& C_{x y, x}=C_{x y, z}=C_{y z, x}=C_{y z, z}=0 .
\end{aligned}
$$

Near $6 \mathrm{HeV}$ all efficiencies undergo rapid changes and near 30 $\mathrm{A}^{\circ}$ is large and positive. The possibility $A_{y}=1$ (kef, 6) should therefore be investigated with closely spaced efficiency measurements. If $A_{y}=1$ is found, the conditions

$$
a=0, \quad h= \pm \text { ie, } \quad k= \pm \text { if }
$$

shotild be tested. Experimentally this yields

$$
\begin{aligned}
& A_{y= \pm 1}^{1}=A_{y}^{z}=1 \\
& A_{z x}^{1}=A_{z z}^{1}=-1 / 2 \\
& A_{x z}^{1}=C_{x z, y}=C_{x z, x z}=C_{x z, z z}=0 \\
& 2 C_{z z, y}= \pm C_{y, y} \\
& 2 C_{z z, x z}= \pm C_{y, x z} \\
& 2 C_{2 z, z z}= \pm C_{y, z z},
\end{aligned}
$$

in addition to Bqs. (3) and (4). Since efficiency correlation experinents are difficult, it is likely that a veriflcation of the conditions will be done usjing the results of an amalysis. The conditions above nay help in the selection of the data base necessary, since measurements near a suspected

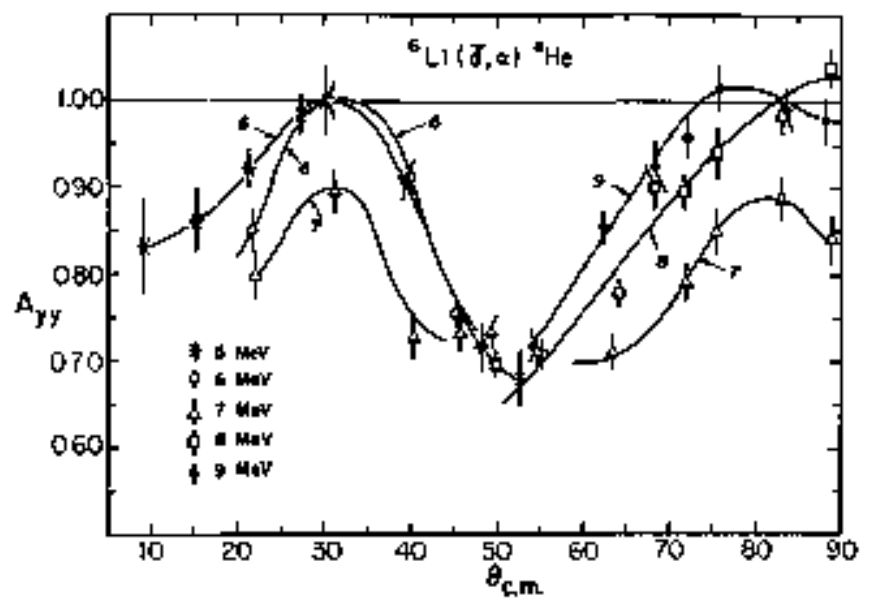

Fig. 1. Regions of $A_{y y} \simeq 1$ in the $\sigma_{L i}(d, a)^{4} \mathrm{He}$ reaction (Ref, 4).

(XBL 753-2614)

extrese point are a powerful tool in an analysis, ${ }^{3}$ It is aIso very likely that similarly large values of $A_{y}$ and $A_{y}$ will be found for polarized tL.t-besins, since these maxina seen correlated with certain excited states in the intermediate systen.

\section{Footnotes and References}

"Proc. Folrth Int'1. Symposium on Polarization Phenomema in Nuclear Reactions (BIrkbäuser Verlag, Basel, 1976 j.

tPresent address: Physics Dept., University of Basel, Switzerland,

"Present address: Noclear Science Lab., M.I.T., Caubridge, Mass.

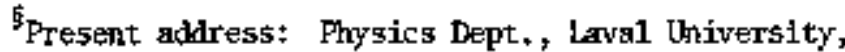
Quebec, Canada.

1. G. R. Plattorer and A, D. Bacher, Fhys. Lett. $36 \mathrm{~B}(1971) 211$.

2. W. Grtiebler ot al., Mucl. Phys, A242 [1975) 285 .

3. F. Seilor, Phys. Lett. 61B (1976) 144.

4. W, Grijebler et al., Results of Measurements and Andyses, Eidgenossische Technische Hochschulle, Ztrich, May 1973.

5. W. E. Kohler and D. Fick, 2, fïr Phys. 215 (1968) 408.

6. F. Seiler, F. N. Rad and H. F. Conzett, LBLreport, LBL-3496, and to be published. 
Several points $\left(\mathbb{E}_{i}, \hat{\theta}_{j}\right)$ of maximhn possible analyzing power have recently been identified in d-4he elastic scattering. From an malys is of data between 3 and $17 \mathrm{MeV}$, three points with $\mathrm{A}_{y y}=1$ were found below $12 \mathrm{MoV}$ and another whth $\mathrm{A}_{\mathrm{y}}=-\mathrm{I}$ may exist above that energy. 1 An experifnental indica. tion that extrome values of the analyzing power $A_{y}$ may occur cowes from measurements near $\mathrm{E}_{\mathrm{i}}-28 \mathrm{MeV}$ and $\theta_{C_{1} m_{+}}=154^{\circ}$, where $A_{y} \simeq A_{Y y} \simeq 1.2$

Reaching an extreme value of scme compcnent of the analyzing power inposes certain conditions on the elements of the trinsition matrix $\mathrm{M}$. In the parametrization of ohlisen et al., 3

$$
M=\frac{1}{2}\left(\begin{array}{crcc}
A-B & \sqrt{2} & D & -A-B \\
-\sqrt{2} D & 2 & C & \sqrt{2} D \\
-A-B & -\sqrt{2} & D & A-B
\end{array}\right) \text {, }
$$

an extrene value of $A y y=1$ requires $B=0$. For an extreme value $A y= \pm l$, however, $A=C \times \pm i D$ and $B=0$ is necessary. Thus $A_{y}=1$ is a prerequisite for $A_{y}= \pm 1,1,4$ and both components tust peak at the same critical point $\left(\mathrm{e}_{j}, \vartheta_{j}\right)$. A definfte identification of points with extreme values of the analyzing power can be carried out by using a phase-shift analysis to campute the arditudes A through D and to verify the validity of the relevant conditions. In an alternate approach ${ }^{5}$ these conditions are introtuced into the formulas for the observables ${ }^{3}$ and can then be checked experinenta11y. The requirement $B=0$ for $A_{y y}=1$ yields

$$
\begin{aligned}
& \mathrm{A}_{y y}=\mathrm{p}^{y^{\prime} y^{\prime}}=\mathbf{k}_{y y^{*}}^{y^{*} y^{\prime}}=1 \\
& A_{x x}=p^{x^{\prime} x^{+}}=K_{y y^{x^{+} x^{+}}}=\mathrm{x}_{\mathrm{xx}}^{\mathrm{y}^{+} \mathrm{y}^{\prime}} \\
& A_{z z}=p^{z^{\prime} z^{\prime}}=k_{z 2}^{y^{\prime} y^{\prime}}=k_{y y}^{z^{\dagger} z^{\prime}} \\
& \mathrm{k}_{\mathrm{x}}^{\mathrm{x}^{\mathrm{r}}}=\mathrm{K}_{\mathrm{z}}^{\mathrm{x}^{\prime}}-\mathrm{k}_{\mathrm{xy}}^{\mathrm{x}^{+}}=\mathrm{K}_{\mathrm{yz}}^{\mathrm{x}^{\mathbf{1}}}=0 \\
& \mathrm{~K}_{x}^{z^{+}}=\mathrm{k}_{z}^{z^{\dagger}}=\mathrm{k}_{x y}^{2^{+}}=\mathrm{k}_{y z}^{z^{\dagger}}=0
\end{aligned}
$$

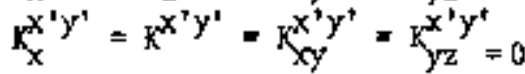

$$
\begin{aligned}
& \mathrm{H}_{\mathrm{X}}^{y^{\prime} z^{\prime}}=\mathrm{K}_{z}^{\mathrm{y}^{1} z^{\prime}}=\mathrm{K}_{\mathrm{xy}}^{\mathrm{y}^{7} \mathrm{z}^{\prime}}=\mathrm{K}_{\mathrm{y}^{\prime}}^{\mathrm{y}^{\prime} z^{\prime}}=0 \text {. }
\end{aligned}
$$

Equations (4) to (7) are particularly inportant, since a value of zero at $\left(E_{i}, \theta_{i}\right)$ does not depend on the calibration of any polarizations. For an extreme value $A= \pm 1$, tho relative walues of all $\mathrm{M}$ Jatrix elements are deternined and the combon comstant is given by the cross section $\sigma_{\text {a }}$ at $\left(E_{i}, \theta_{i}\right)$. Thus all $\mathbf{5 1}$ polarization observables are mumerically determined, and in addition to Eqs. (4) to (7) the following conditions hold

$$
\begin{aligned}
& \mathrm{A}_{\mathrm{y}}=\mathrm{p}^{\mathrm{y}^{\top}}=\mathrm{K}_{\mathrm{y}}^{\mathrm{y}^{\top} \mathrm{y}^{\top}}=\mathrm{K}_{y \gamma}^{y^{\prime}}= \pm 1 \\
& A_{y y}=p^{y^{\top} y^{+}}=\mathrm{K}_{y}^{y^{\prime}}=\mathrm{K}_{y y^{\gamma^{\prime}} y^{\prime}=1} \\
& A_{x z}=p^{x^{\prime} z^{\prime}}=k_{x z}^{x^{\prime} z^{\prime}}=0 \\
& \mathrm{~K}_{\mathrm{Xz}}^{y^{+}}-\mathrm{k}_{\mathrm{xz}}^{\mathrm{x}^{+} \mathrm{x}^{1}}-\mathrm{k}_{\mathrm{xz}}^{\mathrm{y}^{+} \mathrm{y}^{+}}-\mathrm{k}_{\mathrm{xz}}^{\mathrm{z}^{1} \mathrm{z}^{1}}=0 \\
& K_{y}^{X^{\prime} z^{\prime}}=K_{x x}^{x^{\prime} z^{1}}=K_{y y^{\prime}}^{x^{\prime} z^{\prime}}=K_{z z}^{x^{+} z^{\prime}}=0 \\
& K_{y}^{z^{1} z^{\prime}}=k_{z z}^{y^{\prime}}=K_{y}^{x^{\prime} x^{\dagger}}=K_{x \alpha}^{y^{\prime}}=\mp 1 / 2 \\
& K_{z z}^{z^{\prime} z^{*}}=K_{x x}^{z^{\prime} z^{\prime}}=K_{z z}^{x^{\prime} X^{\prime}}=K_{x x}^{x^{\prime} X^{\prime}}=1 / 4 \\
& A_{x x}=A_{z z}=k_{x x}^{y^{\prime} y^{\prime}}=k_{z z}^{y^{\prime} y^{\prime}}=-1 / 2
\end{aligned}
$$

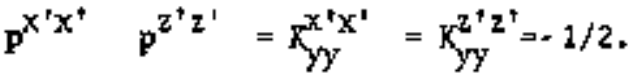

The quantities $p, A$ and $K$ denote the polarizetion of the scattered denterons, the anglyzing power and the polarization transfer coefflcients respectively. With these conditions a straightforward experimental identification of a point $a_{y}= \pm 1$ or $A_{y} y^{=1}$ is possibie. Care should be taken to rerify each condition in several ways, choosing the observables by an inspection of the general formulae and taking into account the relations between observables recquired by other symmetries. ${ }^{3}$

\section{Footiotes and References}

*Proc. Fourth Int'1. Symposium on Polerization Phemonera in Nuclear Regctions (Birkhäuser Verlag, Basel, 1976).

TPresent address; Physics Dept., University of Base1, Sritzerland.

tPresent address: Nuclear Science Lab., M. F.T., Cambridge, Mass.

${ }^{5}$ Present address; Plysics Dept., Laval Iniversity, Quebec, Camada.

1. W. Grïtebler, P. A. Schmelzbach, V. Rönig, R. Risler, B. Jenry, and D. Boerma, Muc1. Phys. A242 (1975) 285 .

2. H. E. Conzett, F, Seiler, F, N. Rad, R. Roy, and R. N. Larimer, Fourth Polarization Symp.

3. G. G. Ohlșen, J. L. Gammel, and P. W. Keaton, Phys, Rev. C5 (1972) 120 .

4, F. Seiler, F. N. Fad, and H. E, Conzett, Fourth Poplarization Symp.

5. F. Seiler, Phys. Lett. 61B (1976) 144 . 


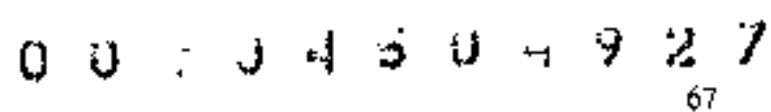

DEVIATIONS FROM CHARGE-SYMMETRY IN THE REACTION ${ }^{4} \mathrm{He}(\boldsymbol{d}, \mathrm{t})^{3} \mathrm{He}^{*}$

W Dahrne.t P I A Buttele. H E Conzentt, J Aurvegux.

I Euchall and R M Laruner

For nuclear reactions in which the final ruclei belong to the same isospin multiplet, Simoniugl showed that the concept of charge symetry of the nurlear forces requires the angular distributions of the cross section to be symetric and the vector an alyzing power to be antisymetric with respect to $90^{\circ}$ in the c.w. systent. Experimental evidence of deviations from this theorem was reported by Gross et al. 2 for the cross section of the reaction ${ }^{2} \mathrm{H}(\alpha$, t) ${ }^{3} \mathrm{He}$. In order to provide additiongl information for a more detailed study of the symetry-violating mechanist we masured the vector enalyzing powers ${ }_{1} \mathrm{~T}_{11}$ of the mirror reactions $4_{\mathrm{He}}(\mathrm{d}, \mathrm{t})^{3} \mathrm{Fe}$ and ${ }^{4} \mathrm{He}$ (d, ${ }^{3} \mathrm{He}^{3} \mathrm{H}$ at $\mathrm{E}_{\mathrm{d}} 1 \mathrm{ah}=32.0$ and $41.0 \mathrm{MeV} .3$ The ampular distributions of ${ }^{1 \mathrm{~T}} 11$ for the ${ }^{3} \mathrm{He}$ and triton channe1 differ by as wuch as 0.08 (Fig. 1).

The simplest method of taking into account coulomb effects is a coherent superposition of slightly different proton and neutron transfer amplitudes in a DWBA calculatiç, as also considered by Gross et al.: $T\left(\vec{k}_{f}\right)=T_{p}\left(k_{f}\right)+T_{n}\left(-k_{f}\right)$. Choosing the coordinate system according to the Madison Convention we get, using satchter" $s^{4}$ notation:

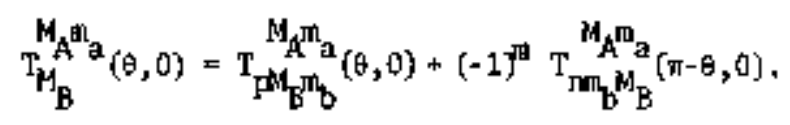

The reaction in question also is of a spin type not usually treated in DWBA formalisus:

$1+0+1 / 2+1 / 2$. Becatuse of the similarity of ${ }^{3}$ He and $3 \mathrm{H}$ we asstme their LS interactions to be Identical and use thanrel spin $\frac{t}{S}$ in the final chan. nel. The zero-range DWRA code DaECK was modified to sum over $S=0,1$ and to add the toro transfer amplitudes coherently.

The optical mode1 parameters used in these INBs calculations were obtained by fitting to the
15 to 45 MeV elastic d+4 He vector analyzing power data of Leentern et al. 5 along with the cross sections of Willmes ${ }^{6}$ and the 6 to $32 \mathrm{MeV}$ elastic ${ }^{3}+{ }^{3} \mathrm{H}$ cross section data of Bacher et al. 7 and Batten et al. ${ }^{8}$ No ${ }^{3}+{ }^{3} \mathrm{H}$ analyzing power data aro available at present. The derived paraneters are listed in Table 1 for the toro energies of interest here. Good fits are achfeved over the entire energy region of the d+4e data. Fits of less quality were obtained for the Hot+. $\mathrm{F}$ data. The bound state form factors were calculated as by Gross et al.

Figure 1 shows a comparison of our proliminary calculations with the experimental data. The structure is reprotuced but the quantitative agreement is not satisfactory. The fit to the cross secttom is of sinilar quality. At present, the discrepancies. seem to be tue to the uncertainties of the 3 to+3 optical potential.

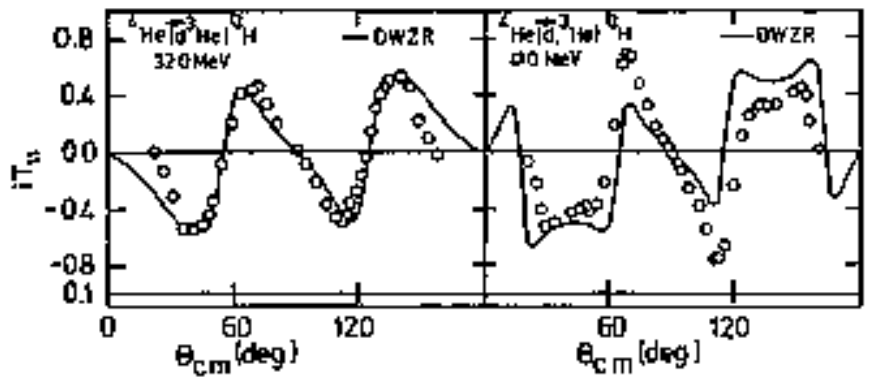

Fig. 1, Vector analyzing powers, iT 1 , in the ${ }^{4} \mathrm{He}\left(\mathrm{d}, 3_{\mathrm{He}}\right){ }_{\mathrm{H}}$ reaction at 32 and $41 \mathrm{MeV}$. The curves are from DHBA calculations.

(XBL 766-8273)

Table 1. Optical model parameters $\left(r_{c}=1.3 \mathrm{fm}\right)$.

\begin{tabular}{|c|c|c|c|c|c|c|c|c|c|c|c|}
\hline & $\mathrm{E}_{\mathrm{d}}$ & $v_{0}$ & $\mathbf{r}_{0}$ & $a_{0}$ & $W$ & $W_{\mathrm{S}}$ & $\mathbf{r}_{\mathrm{I}}$ & ${ }^{a} I$ & $\mathrm{Y}_{\mathrm{LS}}$ & $\mathbf{r}_{1 S}$ & is \\
\hline \multirow[t]{2}{*}{$d+{ }^{4} \mathrm{He}$} & 32.0 & 68.7 & 1.18 & 0.56 & 0.0 & 2,55 & 2.56 & 0.5 & 4.6 & 1.18 & 0.5 \\
\hline & 41.0 & 62.2 & 1.18 & 0.56 & 0.0 & 3.27 & 2,56 & 0.5 & 4.6 & 1.18 & 0.5 \\
\hline \multirow[t]{2}{*}{${ }^{3} \mathrm{H}^{3} \mathrm{He}$} & 32.0 & 192.5 & $1+30$ & 0.60 & 0.74 & 0.0 & 1.60 & 0.5 & 8.0 & 1.30 & 0.5 \\
\hline & 41.0 & 205.0 & 1.30 & 0.60 & 17.5 & 0.0 & 1.60 & D. 5 & 8.0 & 1.30 & 0.5 \\
\hline
\end{tabular}




\section{Footnotes and References}

"Proc. Fourth Int'l, Symposium on Polarization Phenchena in Nhclear Reactions (Birkhäuser Verlag, Easel, 1976).

'Present address: Sektion Physik der Universitat Menchen, Garching, Germany.

FPermanent address: University of Manchester, England,

פPresent adidress: I.S.N., University of Grencble, France,

"Present address: University of BaseI, Switzerland.
1. M. Shtomius, Phys, Lettors 37B (1971) 446.

2. E. E. Gross et a1., Phys. Rev. C5 (1972) 602.

3. W. Dahme at al., Proc. Int. Conf. on Mucl. Phys., Mmich 1973 (North Folland, Amsterdam 1973) p. 444.

A. G. R. Satchler, Nucl. Phys, 55 (1964) 1.

S. Ch. Laemann et a1., Bull. An. Phys. Soc. 17 (1972) 562.

6. H. Willmes, private comumication.

7. A. D. Bacher et a1., Nucl. Phys. Al19 (1968) 481.

8. R. J. Batten et al., Nucl. Phys. A151 (1970) 56.

\section{POLARIZATION SYMHETRIES IN DIRECT REACTIONS: \\ ${ }^{3} \mathrm{He}(\overrightarrow{\mathrm{t}}, \mathrm{d})^{4} \mathrm{He}$ AND ${ }^{2} \mathrm{H}\left(\overrightarrow{\mathrm{d}}, \mathrm{p},{ }^{3} \mathrm{H}^{*}\right.$}

H E Conzest

The observable consequences of particle symmetry or change syllotecy in reactions of the type $b(a, c) c^{\prime}$ are well known. Here, the final-state particles $c$ and $c^{*}$ are the charge-symmetric members of an isospin doublet or are identical particles, $c c^{\prime}$. The symuetries imposed on the angular distributions of the cross section and the zmalyzing powers have been given by Barshay and Temert and Simoniuls: 2

$$
\begin{array}{ll}
\sigma(\theta)=\sigma(\pi-\theta) & \text { for a state of } \\
\mathrm{T}_{\mathbf{k q}}(\theta)=(-1)^{q} \mathrm{~T}_{\mathbf{k q}}(\pi-\theta) & \text { deftnite isospin. }
\end{array}
$$

These symotrios are exact for $\mathrm{C}=\mathrm{c}^{*}$, but significant devietions from (I) have been observed in the ${ }^{4} \mathrm{He}(\mathrm{d}, \mathrm{t})^{3} \mathrm{He}$ reaction (or its inverse) at derteron energies ranging from threshold (2I.5 MeV) to 41 HeV. Nocken et al, ${ }^{3}$ have concluded that the lower energy cross-section data fros the inverse reaction are explained in terms of the corupoumd-nucleus reaction mechanish, and they suggest that the deviations from symetry about $\theta=\pi / 2$ result from isospin mixing in the " $\mathrm{Li}$ intemediate nucleus. At the higher mergies $E_{d} * 32$ and $41 \mathrm{MeV}$, deviations fron the symmetxies (1) in both the cross-section ${ }^{5}$ and in the deaterron vector analyzing powers have, in large measure, been explained via DWBA calculations as resulting from the slightly different proton and neutron transfer amplitudes, 5,7,8 This explanation in terms of expected Coulotib effects thus retatis the concept of basic charge-symbetry at these higher energies.

There is no symetry condition such as (IB) imposed on the polarizations, $t_{\mathrm{kg}}(\theta)$, of the outgoing particle in the $b(a, c) c$ reaction; so, it follows that in a reaction $a^{\dagger}(\vec{a}, c) d$, where the identical particles ( $\left.\mathrm{a}^{\prime}=\mathrm{a}\right)$ or isospin partners are in the initial state, the condition (1B) on the anal$y z$ ing powers does, in general, not apply. However, in this paper it is shown that if the reaction mechanissm is a purely direct transfer process, conditicas (1B) is imposed on the analyzing powers in the $a^{\prime}(\mathrm{A}, \mathrm{d})$ d reaction. This symunetry then becomes, in this class of reactions, a clear signature of the direct transfer machanisat as contrasted with the compotnd-nucleus, or intertuediate state, process.

Figure 1 shows the tow direct transfer amplitudes which are added coherentiy; $\mathrm{N}_{1}$ and $\mathrm{N}_{2}$ are the transferred nycleons or mucleon clusters. As oxamples, in the ${ }^{2} \mathrm{H}\left(\mathrm{d}_{\mathrm{p}}\right)^{3}{ }_{\mathrm{H}}$ reaction $\mathrm{N}_{1}=N_{2}=n$, and in the $3_{\mathrm{He}}(\bar{t}, \mathrm{~d})^{4} \mathrm{He}$ reaction $\mathrm{N}_{1}=n$ and $\mathrm{N}_{2}=\mathrm{p}$. As is noted in Fig. I, the M-matrix amplitude(z) for the transfer of $N_{2}$, producing particle a at the angle $\pi-\theta$, is described in the coordinate system with the $y$-axis along $\overrightarrow{\mathbf{k}}_{\mathbf{i}} \times\left(-\vec{k}_{\mathbf{f}}\right)$. Rotation of the coordinate system throragh an angle $\pi$ aroand the z-axis, 59, that the $y$-axis is along $\vec{k}_{1} \times \vec{k}_{f}$, gives $(-1)^{m}$

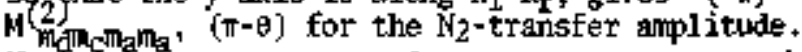

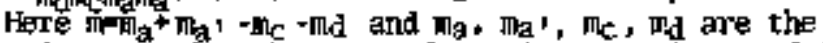
spin magnetic substates of particles $a, a^{p}, c$, and $d$. The camplete M-matrix anplitude is then

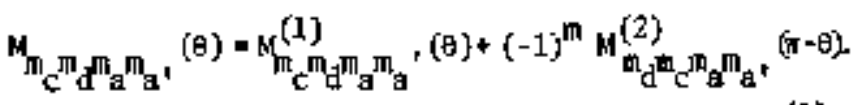

The particle identity or charge syatietry provides that

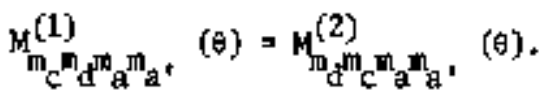

In terns of the transition natrix $\mathrm{M}$, the analyzing powers are given by the expression 


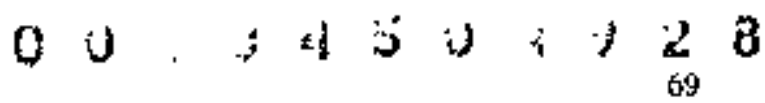

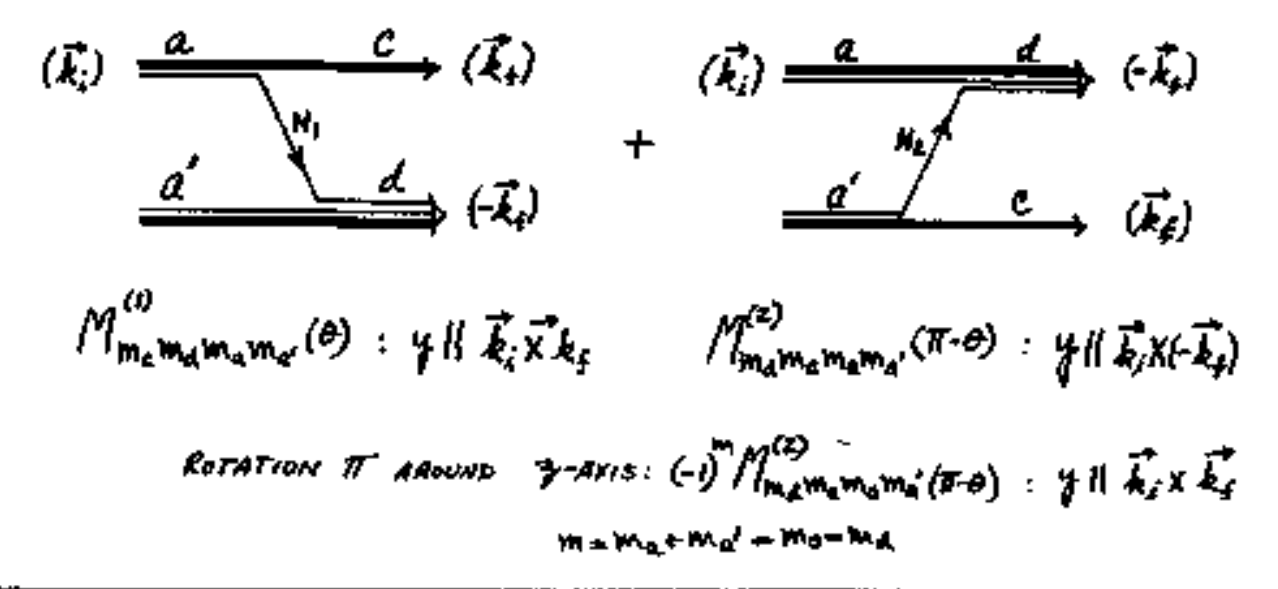

Fig. 1. The two direct-transfer amp1itudes which add coherent1y in the a' $[a, c]$ d reaction.

(XBL, 758-1867)

$$
\sigma(\theta) \mathrm{T}_{\mathrm{kq}}(\theta)=\operatorname{Tr}+(\theta) \tau_{\mathrm{kq}} \mathrm{M}^{\dagger}(\theta),
$$

where the $\mathrm{T}_{\mathrm{kg}}$ are spherical tensor operators. 9,10 Using (1) ard (2) in Eq. (3) th has been shownll that

$$
\mathrm{T}_{\mathrm{kq}}(\theta) \times(-1)^{\mathrm{q}} \mathrm{T}_{\mathrm{kq}}(\pi \quad \theta) \text { for } \mathrm{a}^{\mathrm{r}=\mathrm{a}} \text {. }
$$

The symmetry (10) thus provides clear indication of a purely direct trensfer process. of course, if the reaction should proceed through a single $J^{\text {TI }}$ state or states of the sane parity in the compouml macleus, this symetry condition could also ressult. Hextever, an exanination of the energy dependence of $T_{k g}(\theta)$ should resolve any ambigutty. Also, the excitation energies of the intermediate nuclex which are accessible in this class of reactions with polarized beans would, almost certainly, be in regions of overlapping levels.

As a clear example of these considerations, Fig. 2 shows angular distributions of the vector analyzing power $A_{y}(\theta)=(2 / / J) 1 T_{11}(\theta)$ in the $2 \mathrm{H}(\mathrm{d}, \mathrm{p})$ $3^{3} \mathrm{H}$ reaction at energies from 11.5 to $30 \mathrm{MeV}$. The $11.5 \mathrm{MeV}$ data are from zürieh, 12 and the other data are fram Berkeley, 13 The corresponding range of excitation energies in $4 \mathrm{He}$ is 29.6 to $38.8 \mathrm{MeV}$, and ons sees the obvious transition from a camplete lack of syntetry in the angular distribertion to one of near antisymmetry with respect to $\theta=\pi / 2$, as given by Eq. (10). The Zürich data range dom to He excitation energles of $24.2 \mathrm{MkV}$, and all the antalyzinis power ccomonents $\mathrm{T}_{\mathrm{kq}}(\theta)$ are consistent in that no such symmetry exists. Hence, analysis of these data in terms of $4 \mathrm{He}$ internediate states is required at the lower deuteron energies, whereas analysis in terns of the direct mucleon-transfer process is appropriate above $30 \mathrm{MeV}$. It shoutd be noted from the data of Fig. 2, that evidence persists for some contribution from the corpoundnucleus process at energies above that of the highest suggested excited state of $4 \mathrm{He}$, $\mathrm{fte}^{*}(32 \mathrm{MeV})$ corresponding to $E_{d}=16.5 \mathrm{MeV} .14$

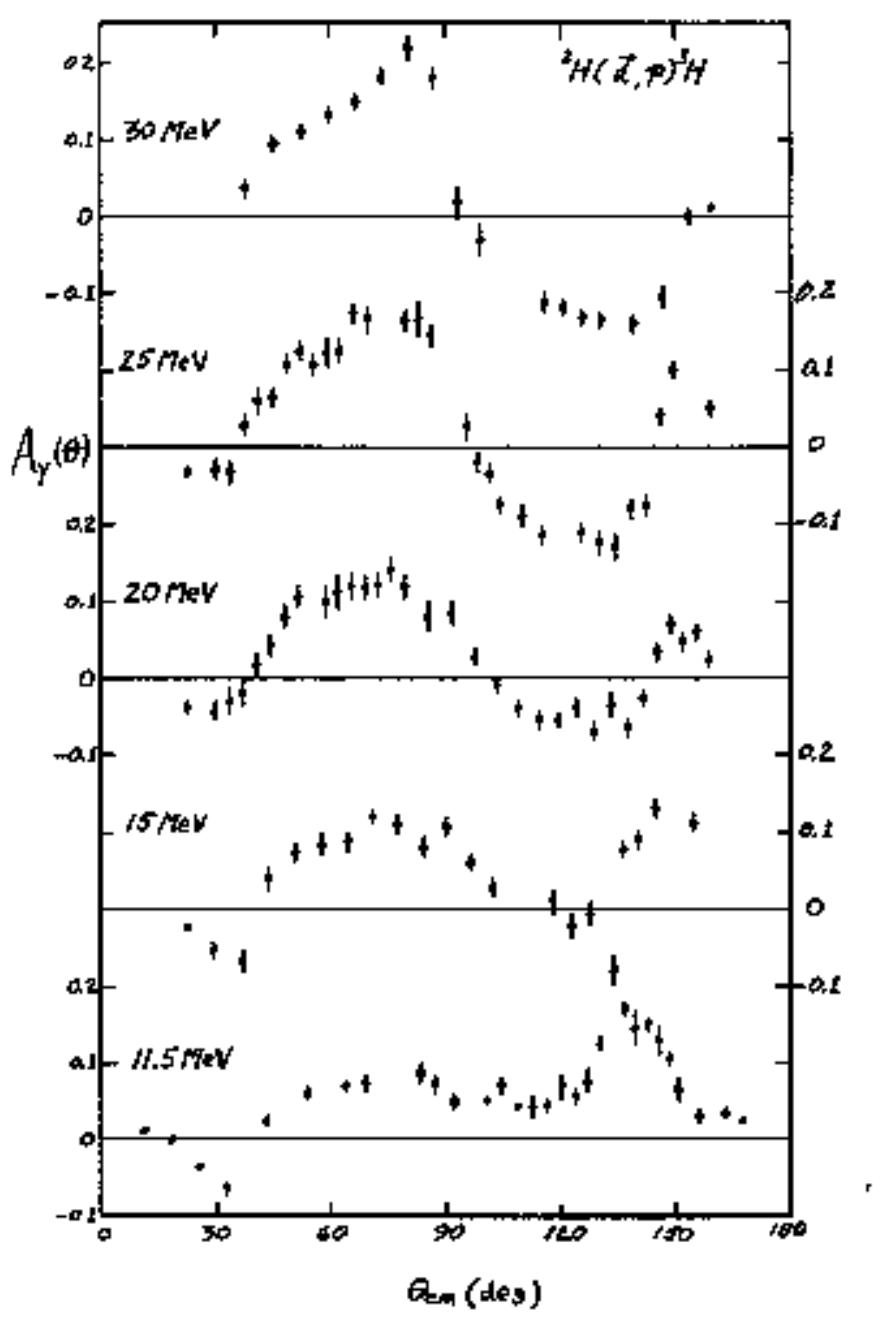

Fig, 2, The vector aralyzing power $A_{y}(\theta)$ in the ${ }^{2} \mathrm{H}(\mathrm{d}, \mathrm{p})^{3} \mathrm{H}$ reaction betheen 11,5 and $30 \mathrm{keV}$. The 11. 5-MeV data are from Ref. 12 .

(XBL 758-1865) 
Angther case of interest is the ${ }^{3} \mathrm{He}(t, \mathrm{~d})^{4} \mathrm{He}$ (or ${ }^{3} \mathrm{H}\left({ }^{3} \mathrm{He}, \mathrm{d}\right)^{4} \mathrm{He}$ ) reaction. As was noted before, it has been reported that the lower energy cross section data saggest isospin mixing in the compound mucleus "Li. Support for this conclusion colld be provided by measurements at those energies of the analyzing power $\mathrm{iT}_{11}(\theta)$ in the ${ }_{\mathrm{He}}(\overrightarrow{\mathrm{t}}, \mathrm{d})^{\mathbf{4}} \mathrm{He}$ reaction. The lack of syumetry of the form (4) would be confiming evidence for the compound-rucleus reaction mechanism. With respect to the higher energies, a DHBA calculation has beet made of the analyzing power in the $3_{\mathrm{H}}(t, \mathrm{~d}) \mathrm{H}$ e reaction at an energy gquivalent to $\mathrm{E}_{\mathrm{d}}=32 \mathrm{MeV}$ in the inverșe reaction. The parameter yalues were those used by Dabute, Ruttle et $a I$, 8 in their fits to $g(\theta)$ and $i_{11}^{(\theta)}$ in that inverse reaction 4 He $(d, t)^{3} \mathrm{He}$. The near antisyronet $T y$ of $A_{y}(\theta)$ about $B=\pi / 2$ is distinct.

Considering the presentiy available polarized beams, we list in Table 1 examples of reactions in which measurements of $\mathrm{T}_{\mathrm{kg}}(\theta)$ may provide unambigunis information on the reacton mecharism.

Table 1.

$$
\begin{aligned}
& { }^{2} \mathrm{H}(\mathrm{d}, \mathrm{p}){ }^{3} \mathrm{H} \\
& { }^{3} \mathrm{He}(\overline{\mathrm{t}}, \mathrm{d})^{4} \mathrm{He} \\
& { }^{3} \mathrm{He}(\overrightarrow{\mathrm{t}}, \mathrm{p}){ }^{5} \mathrm{He} \\
& { }^{3} \mathrm{He}\left({ }^{3} \overrightarrow{\mathrm{He}}, \mathrm{p}\right)^{5} \mathrm{Li}
\end{aligned}
$$

$$
\begin{aligned}
& { }^{6} \mathrm{Li}\left({ }^{6 \mathrm{~L} i, \mathrm{p}}\right)^{11_{B}} \\
& \left.{ }^{6}{ }_{\mathrm{Li}}{ }^{6}{ }_{\mathrm{Li}} \mathrm{d}\right){ }^{10}{ }_{\mathrm{B}} \\
& { }^{6} \mathrm{Li}\left({ }^{6} \mathrm{Li},{ }^{3} \mathrm{He}\right){ }^{9} \mathrm{Be} \\
& { }^{6} \mathrm{Li}\left({ }^{6} \mathrm{Li}, \alpha\right){ }^{8} \mathrm{Be} \\
& { }^{6} \mathrm{Li}\left({ }^{6} \mathrm{t}_{i},{ }^{2}{ }_{\mathrm{LI}}\right){ }^{5} \mathrm{Li} \\
& 6_{\mathrm{L}, \mathrm{i}}\left({ }^{6 \mathrm{GI}},{ }^{7}{ }_{\mathrm{Be}}\right)^{5} \mathrm{He}
\end{aligned}
$$

The ${ }^{6} \mathrm{Li}$ + ${ }^{6} \mathrm{Li}$ reactions, for example, could be particularly interesting, since deviations fran the synlatery (a) would provifia qualitative information about states in $12_{C}$ which have stome owerlap with the appropriate cluster comfigurations.

In sumary, we find that the anaiyzing powers, in reactions with identical or charge-symmetric particles in the initial state, cam provide definite identification of the react ion mechanism, whereas no such information is available from cross-section data alone.

\section{Footnote and References}

*Condensed from invited paper, Pros. Fourth Int'1. Symposium or Polarization Phenomena in Nuclear Reactions (Birkhäuser Verlag, Basel, 1976).

1. S. Barshay and G. M+ Tediner, Fhys, Rev, Letters 12 (1964) 728 .

2. M. Simonius, Phys. Lesters $37 \mathrm{~B}$ (1971) 446.

3. U. Hocken et a1., Nuc1. Phys. A213 (1973) 97,

4. G. J. Wagner et al., Few Particle Problems in the Nuclear Intoration, eds. I. \$1aus tot al. (North Holland, Abssterdam, 1972) p. 747 .

5. E. E. Gross et al, Phys, Rov. C5 (1972) 602.

6. W. Dakme et al., Proc. Int. Conf. on Mucl. Phys. (Mmich 1973) Vol 1, 444.

7. S. Edwards et a1., Phys. Rev, CB (1973) 456.

8. W. Dabme et a1, Fourth Polarization Sprep.

9. See, e.g., M. Simonius, Lect. Notes in Physics 30 (Springer-Verlag, Heidelberg 1974) p. 38.

10. W. Lakin, Fhys. Rev, 98 (1955) 139.

11. H. E. Conzett, Fourth,Polarization Symp.

12, W. Grílebler et a1., Nhel. Phys. A193 (1972) 129

13. H. E, Conzett, J. S. C. Mckee et a1., Fourth Folarization Symp, H. E. Conzett, K. M. Larimer et al, , Fourth Polarization Sysup.

14. E. X. Lin et a1., Mucl. Phys. A179 (1972) 65; E. L, Haase et al., Nucl. Phys. AI88 (1972) 89.

\section{DEPOLARIZATION PARAMETER IN $\vec{p}-{ }^{10} \mathrm{~B}$ ELASTIO SCATTEAING AND THE SPIN-SPIN INTERACTION*}

$\checkmark$ Birchall, $\mathrm{H}$ E Conzett, F N Rad, ;

S Chintalapudı, S and $R$ M Larımer

Sherif and Hussein $\mathbf{1}^{1}$ have pointed put that the depolarization parameter D is a sensitive probe of the spin-spin interaction in mucleon-nucleus elastic scattering. Other parameters, such as the crosssection and asymetry, polarization and spin rotation parameters, are relatively insensitive.

Two types of spin-spin force have been comsidered. A spherical term:

$$
\mathrm{U}_{\mathrm{ss}}(r)=\mathrm{V}_{\mathrm{ss}} \mathrm{F}_{0}(r) \underline{\sigma} \cdot \underline{\mathrm{I}}
$$

and a tensor terrot:

$$
U_{s t}(r)=-1 / 2 V_{s t} F_{t}(r)[3(\underline{\underline{a}} \cdot \underline{\hat{H}})(\underline{I} \cdot \underline{\hat{P}}]-\underline{\sigma} \cdot \underline{I}\} \text {, }
$$

where $v$ and 1 are the spins of the incident protion and the target nucleus, respectively, and it a unit vector in the direction of a lire connecting the centers of the projectile and target. The depth $V_{55}$ and the form factor $F_{0}(r)$ of the spherical term can be estinated from the nucleon-mucleon 


\section{QU.}

spin-spin interaction and the single nuxiecn wave function in the target nucleus, 2 The form and strength of the tensor interaction hare not yet been estimated, so a phencmenological hoods-saxcon form was taken for $F_{t}(r)$ and the strength $v_{s t}$ of the interaction was treated as a free parameter in the calcoilated fits to the available sparse depolarization data. 3 it is clear that more measurements of D $(\theta)$ to good acarracy over wider anghlar ronges are needed in a continuing study of the effects of the target spin in nurlean-nucleus elastic scattering.

We have measured $D(\theta)$ at several angles in the elastic scattering of 26-MeV polarized protons from ${ }^{10} \mathrm{~B}$. The polarization of the scattered protons is given by

$$
p(\theta)=\left\{A(\theta)+D(\theta) p_{o}\right] /\left[1+p_{o} A(\theta)\right\},
$$

where $P_{0}$ is the beam polarization and $A(\theta)$ is the target Rnalyzing power. The polarization of the beam was continuously monitored by scattering frcm a ${ }^{4} \mathrm{He}$ gas target downtream from the $10 \mathrm{~B}$. The $\mathrm{po}-$ larization of the elastically scattered protons was measured by a potarineter with high figure of merit and good energy resolution. 4 The polarimeter used a I-nim thick silicon solid state detector as polarization aralyzer and two side detectors at \pm 27 to the polarimeter axis. Protons which passed unscattered through the analyzer detector were stopped in a "zero degrea" detector. The zero degree collination had the same angular width as the analyzer, with respect to the target center, but nuch resuced angular height. The analyzing power of the target was deduced from the spin up--spin down count ratio in the zero-degree detector.

Geopetrical errors in the determination of $D$ were minimized by careful monitoring and adjustment of beam aligmment during the runs, by dexucing D from spin-up/spin-down ratios in each side detextor and by obtaining results with the silicon polarineter placed on each side of the beam. As a check on these procedures tho D-parameter of $12 \mathrm{C}$ was measured at a number of angles (D for elastic scattering from a spin zero nucleus should be identically 1.0). Values of $D$ consistent with 1.0 were found in each case.

Results of our D-parameter measurements are shown in Fig. 1. The curves are not fits to our deta. They are calculations fron Ref. 1, where the values of $\mathrm{V}_{\text {st }}$ were chosen to reproduce a data point from Saclay at $65^{\circ} \mathrm{c}$.m. and $19.8 \mathrm{MeV}$. It was pointed out by Sherif and Hussein that the tensor strengths $V_{\text {st }}$ extracted in their fits to the data were rather large. As a rosult, very recent theoretical effort has disclosed another contribution to deviations from unity of $D(\theta)$, which has been termed the quadrupole spin-flip effect. ${ }^{2}$ This effect can be present for nuclei that have ground-state quadrupole deformations, and, as such, I $>1$. Hence, further investigations are required to determine the separate effects from the explicit spin-spin interaction and from the quadrupole deformations.

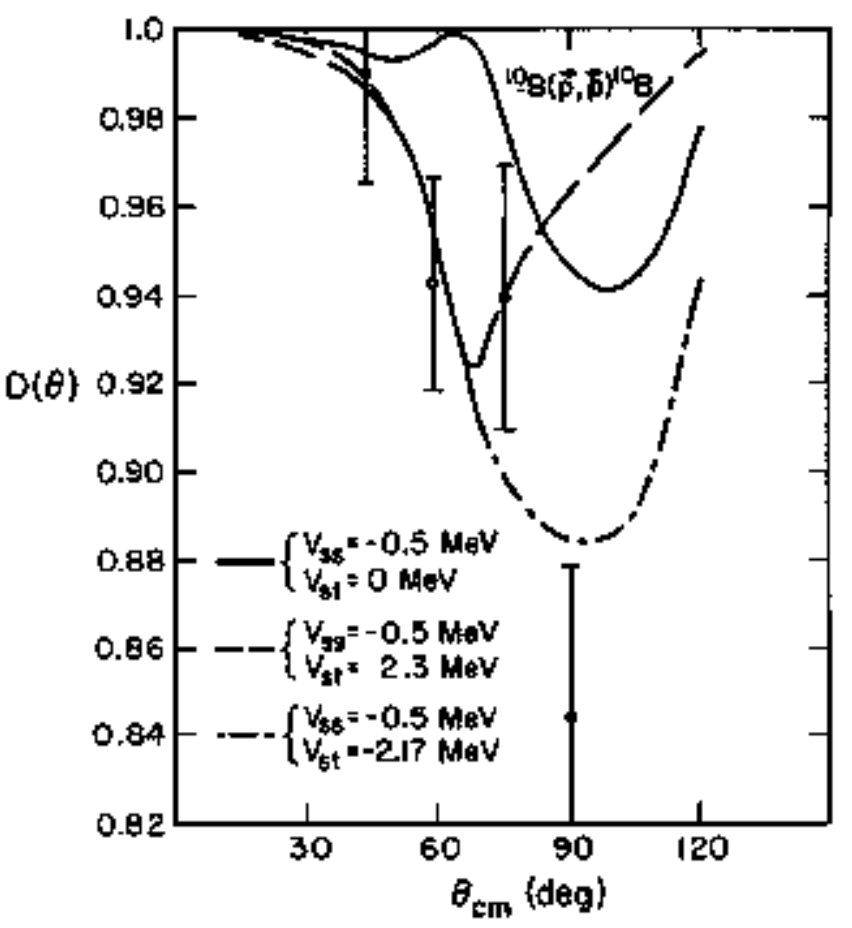

Fig. 1. Depolarization pararseter, $\mathrm{D}(\mathrm{\theta})$, in $\mathrm{p}^{\mathbf{1 0}} \mathrm{B}$ elastic scattering at $26 \mathrm{keV}$. The calculated curves are from Reference 1.

(XBL 743-2523)

\section{Foptnotes and References}

*Proc. Fourth Int'I. Symposium on Polarization Phencmena in Nuclear Reactions (Blrthăliser Vertag, BaseI, 1976 ).

†University of Basel, Switzerland.

thuclear Science Lab, M.I.T., Cambridge, Mass.

${ }^{5}$ Whabha Atconic Research Centre, Trombay, India.

1. H. 5. Sherif and A. H+ Husșein, Phys. lett. 41B (1972) 465; Phys, Res, C8 [1973] 518.

2. G. R, Satchler, Particles and Nurleli I (1971) 397.

3. J. Bircha11 et al., Phys. Lett. 53B (1974) 165, and references therein.

4. J. Birchall et al., Nucl. Instr. and Meth. 123 (1975) 105.

5. R. Beurtey, P. Cattillon and P. Schnabel, J. do Phys. 31. Supp. C2 (197(t) 96; P, Schuabel, thesis, Uriversity of Paris, 1971 (unpublished).

6. J, 5. Blairr and H. S. Sher if, Butl. Amer. Phys. Soc. 19 (1974) 1010; J. S. Blair, M. P. Baker, and H. S. Sherif, Phys. Lett, 60D (1975) 25. 


\title{
CROSS SECTIONS AND REACTION MECHANISMS OF (p.pKn) REACTIONS ON ${ }^{208} \mathrm{~PB}$ IN THE 24-52 MEV RANGE*
}

\author{
J O Rasmussen, A Donangelo, H Kawakamr,t it Konke,t
}

$K$ Konura.† M Sakal,t and N Yoshokawat

\begin{abstract}
The cross section for the reactions ${ }^{208} \mathrm{~Pb}$

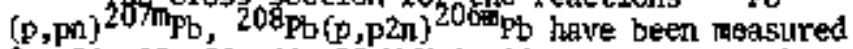
for 24, 28, 36, 44, $52 \mathrm{MeV}$ incident protons. The experimental results are shown to be consistent with the clean knockout mechanisan for the (p,pn) reaction and with knockout of one nelitron followed by evaporation of another for the $(p, p 2 n)$ reaction. We deduce a theoretical formila for the cross section: for these reactions.
\end{abstract}

The target used was metallic foil of an enriched isotope $(99.98)$ of $208 \mathrm{~Pb}$. A target foil of $8.3 \mathrm{mg} / \mathrm{cm}^{2}$ thickness prepared by electrodeposin tion. The Fi cyclotron pulse width of 0,078 ms was used, with delayed gamma rays counted in the 3.06 ins interval betmeen beam pulses.

The dolayod $802.9 \mathrm{keV}\left(2_{1}^{+}+0_{\mathrm{p}}^{+}\right) \gamma$-rays were counted from 0.053 to $0.352 \mathrm{~ms}(0.299 \mathrm{~ms})$ in the de. cay curve after the beam palse. The prompt $Y$-rays completely decayed out earlier than $0.053 \mathrm{~ms}$. The cross section for fojmation of the $0.12 \mathrm{~ms} 7-$ isomer at $2.2 \mathrm{MeV}$ in $200 \mathrm{~Pb}$ was calculated from the intensity of the 802.9-keV gamma ray.

The excitation function for the reaction ${ }^{208} \mathrm{~Pb}$ $(p, p n)^{207 m_{p}\left(13 / 2^{*}\right.}$ isomer of 0.80$)$ sec balf- 1 ife at $1.623 \mathrm{MeV}$ ) was measured with the 1063.7 and $569.8-$ keV $Y$-rays.

5ingles $Y$-ray spectra were also observed with the debunched proton beam. The cross section associated with the $2^{+}$state in $206 \mathrm{~Pb}$ was estimated from the $802.9 \mathrm{keV}\left(2 \mathrm{j}_{1}^{+} \rightarrow 0^{+}\right)$\% ray, and similarly the $569.8 \mathrm{keV}\left(5 / 2^{-}=1 / 2\right)^{\mathrm{g}}$ transition in $207 \mathrm{pb}$ was used to calculate the $5 / 2$ state cross section. The nunerical values for these cross sections are given in Table 1.

We w11l classify the possible reaction processes fin two broad eategories: (a) those in which the incidert proton interacts strongly with only one or two nutelear netutrons which leave the nucleus with

Table 1. Excitation fuptions for the reactions

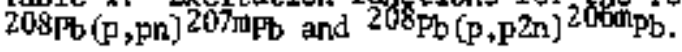

\begin{tabular}{|c|c|c|c|c|}
\hline & \multicolumn{2}{|c|}{$207 \mathrm{~m} \mathrm{pb}$} & \multicolumn{2}{|c|}{$206 m_{\mathrm{Pb}}$} \\
\hline$\underset{\text { (neV) }}{\mathbf{E}}$ & $\begin{array}{l}13 / 2^{+} \\
0(\mathrm{mb})\end{array}$ & $\begin{array}{l}5 / 2^{-} \\
\sigma(\mathrm{mb})\end{array}$ & $\begin{array}{l}7^{-} \\
\sigma^{-}(\mathrm{mb})\end{array}$ & $\begin{array}{c}2^{+} \\
\sigma(m b)\end{array}$ \\
\hline 24 & 2114 & & $2 \div 1$ & \\
\hline 28 & $45 \pm 9$ & & $8 \pm 3$ & \\
\hline 36 & $58 \pm 12$ & & $34 \div 11$ & \\
\hline 44 & $58 \pm 12$ & $120 \pm 25$ & $57 \pm 18$ & $95 \pm 20$ \\
\hline 52 & $65 \pm 13$ & & $57 \pm 18$ & \\
\hline
\end{tabular}

little or no excitation, and (b) those where the incident proton leaves the nucleus in an excited state above the neutron binding energy (after naybe lnocking out a neutron in the process). The excited nucleus usually decays subsequent1y by the evaporation of que neutron.

Using the notation of Grover and Carettol we will designate as Clean Knockout (CKO) the process described in (a) and as Inelastic Scattering follod by Evaporation of a neutron (ISE) the one described in (b).

We conclude then that the iscmer ratios for isotopes one and tho netitions less than the taxget are consistent with a reaction mechanisn consisting of one direct neutron ejection by the incosing proton with (CKO) knockout from orbitals in the first she11 below the Fermi energy leading to the (p,pn) reaction and (KOP) knockotut followed by evaporation from the second shell below the Fermi energy leating to the $(p, p 2 n)$ reaction.

The fact that the isomer yteld ratlos suggest certain processes does not ajply that the athers do not ocarr at all, but only that their contribution is small. For this same reason we have not considored other processes, such as comporind nucleus for mation, which give a snall contribution to the total cross section.

The conclusions we reached here are in agroement with the analysis made by Rao and Yaffe 2 on the proton induced reaction in 181 Ta.

From anr discussion on the isomber ratio evidence in part 2 we reached the conclusion that these reactions are periphera1. We now attempt to formulate a quartitative expressicm for their cross sections.

According to $\mathrm{Wong}^{3}$ the total charged particle nuclear reaction cross section can be expressed approximately as

$$
\nabla_{W}(E)=\left(R_{0}^{2} \hbar_{\omega_{0}} / 2 E\right) R_{n}\left\{1+\exp \left[2 \pi\left(E-E_{0}\right) / h_{w_{0}}\right]\right\}
$$

where $E$ is the charged particle energy

$\mathrm{E}_{\mathrm{o}} \quad$ is the height of the Coulonb barrier

$\boldsymbol{h}_{\omega_{0}}=\hbar\left[d^{2} \mathrm{~V}(\mathbf{r}) /\left.\mathrm{dr}^{2}\right|_{\left.\mathrm{R}_{0} / \mu\right]^{1 / 2}}\right.$

$\mu \quad$ is the reduced mass

$V(r)$ the effective [nuclear + Coolcmib] potential for the reaction

$R_{0} \quad$ is the nuclear radius, calculated from $\mathrm{dV}(\mathbf{r}) /\left.\mathrm{dr}\right|_{\mathrm{R}_{0}}=0$. 
Using the facts that the reactions are peripherat and that the cross section is domfnated by the exit proton penetrability derive the expression for the cross section:

$$
\begin{aligned}
\sigma\left(E_{i n}\right) & =\left(\Delta R / E_{1 n}\right)\left\{R_{0} h_{\omega} \ln \left(1+\exp \left(2 \pi\left(E_{-1} E_{0}\right) / \Delta \omega_{0}\right)\right]\right. \\
& \left.+\pi R_{0} E_{0}\left[1+\exp \left(2 \pi\left(E_{-}-E_{0}\right\} / \omega_{0}\right)\right]^{-1}\right\} .
\end{aligned}
$$

The energies $E_{i n}$ and $E_{\text {out }}$ proton energies before and after reaction, are related through the efected neutrons separation energies

$$
\begin{aligned}
& (p, p)+E_{\text {in }}=E_{\text {out }}+a S_{n_{1}} \\
& (p, p 2 n)+E_{\text {in }}=E_{\text {out }}+a^{\prime} S_{n_{1}}+S_{n_{2}}
\end{aligned}
$$

$a$ and $a^{+}\left(1<a, a^{\top}<2\right)$ are parameters included to take into acculunt that more onergy than the thresbotd binding energies is required on the everage to remove a neutrur. $R_{0}$ and $h_{\text {ino }}$ were detersuned by taking a potential

$$
V(r)=\left(2 e^{2} / r\right)-v_{0}\{1+\exp [(r-R) / b]\}-1 .
$$

The values taken for the parmeters in this potential are

$$
\begin{aligned}
& \mathbf{R}=1.25 \mathrm{~A}^{1 / 3} \mathrm{Fm} \\
& \mathbf{b}=0.65 \mathrm{Fin} \\
& v_{0}=30 \mathrm{MeVt}
\end{aligned}
$$

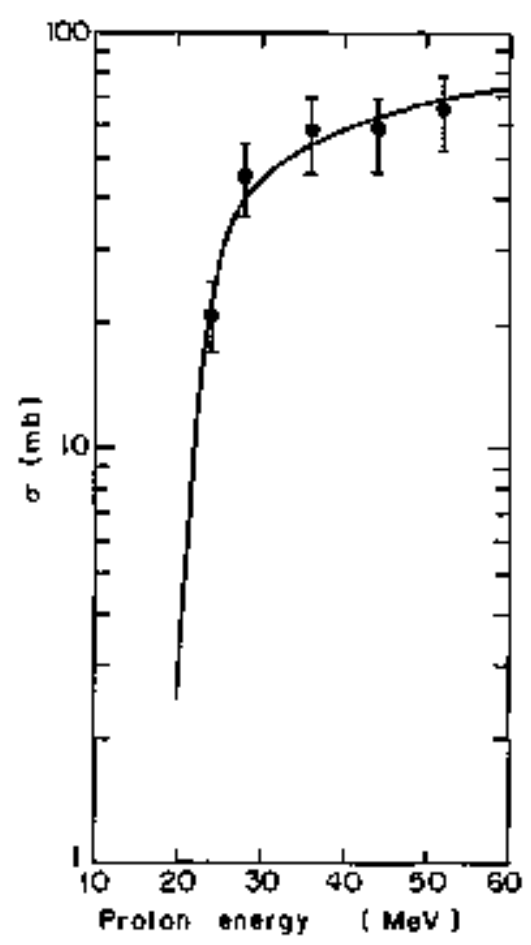

Fig. 1. Theoretical excitation curve for the $200 \mathrm{pb}(p, \mathrm{p})^{203 \mathrm{mp}}$ reaction $\left(13 / 2^{+}\right.$iscaler $)$. The date are from this work.

(XBL 7\$9-4075)
The parameters $a, a^{\prime}$ and $t_{R}$ were fitted to the experimental results. Besides our 208 gp data we fitted the Rao and Yaffe 2 -data for the $181_{\mathrm{Ta}}[\mathrm{p}$. p) $180 \mathrm{~m}$ Ta reaction. The cross sections are plotted in Figs. 1-3.

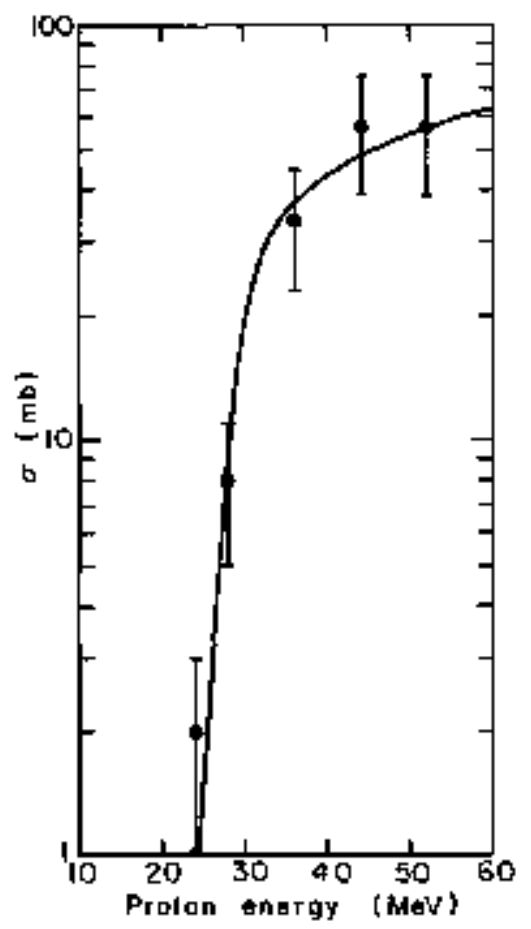

Fis. 2. Theoretical excitation curve for the

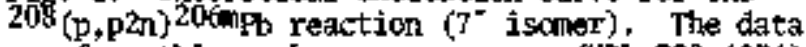
are from this nork.

(XBL 759-4076)

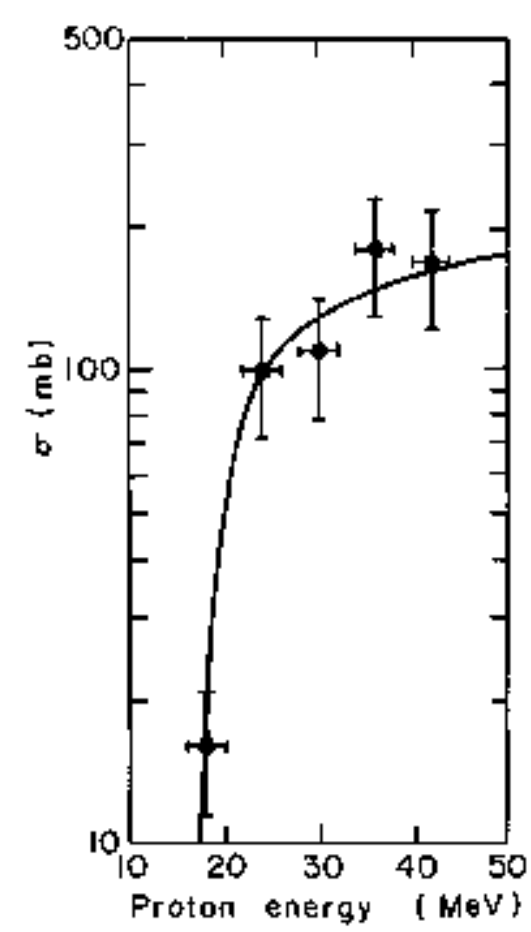

Fig. 3. Theoretical excitation ourve for the $18 T_{a}(p, p n) 180$ ta reaction. Data from Rao and Yaffe. 2 
That this simple expression fits the energy dependence of the cross sections is mainly a consequence of the dominant Intportance of barrier transfission by the outgoing proton, which must be at least lower in energy than the incoming proton by the neutron binding energy, regardless of the exact mechanism. The excess of a or a' over unity represonts the finite kinetic energy carried off by the neutrons (s) on the average. The fact that $\alpha$ is as large as 1.5 for the (p,pi) reaction points to several MeV average kinetic energy of the neutrons and favors the CKD mechanism as dominant over ISE.
Footnotes and References

"Condensed from Nucl. Phys. A262, 52 (1976).

'Institute for Nuclear Studies, Univ. of Tokyo, Tanashi, Tokyo, Japan.

1. J. R. Grover and A. A. Caretto, Jr., Ann. Rev. Nucl. Sci. 14 (1964), 51.

2. C, L, Rac and L, Yaffe, Car. Journ. Chem. 41 $[1963), 2516$.

3. C. Y. Wong, Phys, Rev, Lett. 31 (1973), 766.

4. M. A. Preston, Nulear Physics (Addison-Nesley, 1962), p. 547 .

\section{THE $\left(\alpha,{ }_{r}^{2} \mathrm{HE}\right)$ REACTION AS A SPECTROSCOPIC TOOL FOR INVESTIGATING HIGH SPIN STATES*}

A Jahn, $G \mathrm{~J}$ Woznuak,

Q P Slahel, and Joseph Cerny

Experimental systems capable of detecting nu" cIear reaction products in resonant final states with good efficiency and energy resolution can open ur a wide range of unexplored nuclear reactions. Although at present such stgudies are largely chonfined to the detection of ${ }^{\mathrm{B} e}$ nuclei, Robson has pointed out many other interesting resonant 5 ystems which can be detected as reaction products. Add1tionally, the well known final state irteraction in the two-mucleon $T=1$ system can be utilized; in particular, this interaction in the ${ }^{2} \mathrm{He}$ system locar izes the tho breakt protons into a narrow come. Thus ${ }^{2} \mathrm{He}$ can readily be detected with two proton detectors arranged in an appropriate geomotry.

A very interesting reaction which can be studied at reasonably high bombarding energies with such a detection system is that of $\left(\alpha_{3} 2 \mathrm{He}\right)$, potentially a direct $2 n$-transfer reaction very sinilar to the direct np-transfer reaction $(\alpha, d)$. The damonstrated selectivity of the $(\alpha, d]$ reaction 2,3 makes it a valwable spectroscopic tool with which to investigate high spin states in muclei with $T_{z}=T_{z}$ (terget), and therefore one cas anticipate that the $\left(\alpha,{ }^{2} \mathrm{Hk}\right)$ reaction might selectivity populate high spin states in suclei with $\mathbf{T}_{\mathbf{z}}=\mathbf{T}_{\mathbf{z}}$ (target) +1 . This reaction is particularly appealing due to the unavailability of high energy triton beams, so that the analogous $(t, p)$ raxction has not been investigated under conditions of large angular monientum transfer. We report here the results of this inftisl observation of the $\left(\alpha,{ }^{2} \mathrm{He}\right)$ ragction on ${ }^{12} \mathrm{C},{ }^{13} \mathrm{C}$ and $16_{0}$ (as $\mathrm{SiO}_{2}$ ) targets induced by $65 \mathrm{HeV}$ o-particles from the 88 -inch cyclotron. These data establish the expected high selectivity of this reaction and its usefulness as an inportant spectroscopic tool.

Figure 1 (a) shows the ${ }^{2} \mathrm{He}$ detection systern, consisting of two $A \mathrm{E}-\mathrm{P}$ counter telescopes. The $\Delta \mathrm{E}$ detectors were phosphorus-diffused Si. $380 \mu \mathrm{m}$ thick and the E detectors ware Si (Li), 5 ma thick, all having the same area of $1 \times 1.4$ an 2 Two collimator slits separated by a post were employed, so that the syster subtended a $15^{\circ}$ vertical and a $4^{\circ}$ horizonta1 acceptance angle. 2 He events were identified by using standard particle identification techniques as well as subngnosecond fast timing between the two $\Delta E$ coututers, which drastically rethiced random events. In addition, fast pileup rejection was utilized so that high singles counting rates $(30 \mathrm{kHz})$ could be tolerated in the $A E$ counters.

Figrre 1 (b) shows the relative time distribution of observed proton coincidences from the reaction $13 \mathrm{C}(a, 2 \mathrm{He}) 15^{\circ}$ at $13^{\circ}$ lab angle. The observad maximm difference in the time- of -flight of the two protons of $1.1 \mathrm{~ns}$ (FWIM) agrees with prediction based on the assumption of $400 \mathrm{keV}$ breakup energy. The coincidence comting rate was measured at different geometries obtained by varying the distance between the target and the collinator. Figure I (c) depicts the relative experimental efficiency versus the calculated efficiency for three different ${ }^{2} \mathrm{He}$ energies at three distances. The experimental data, norinalized at $10 \mathrm{~cm}$, are well reproduced by the calculations. The agreement between the calculated and experimertal relative efflciencies and the narrow $\triangle T O F$ peak (random coincidences frook a single beam burst would have spanned up to 12 ns FinM) require that the detected po coincidences are from the breakup of the unbound $2 \mathrm{He}$ system.

Fiqure 2 presents representative spectra from the $(a, 2 \mathrm{He})$ reaction on $12 \mathrm{c}, 1^{3} \mathrm{C}$, and 150 at forward angles. The experimental energy resolution of 350 kel was principally determined by kinematic broadening due to the $4^{\circ}$ acceptance angle, As can be seen in the spectra, the $(\omega, 2 H e)$ reaction is $e x$ " trewely selective, only very few states in the residial muclei are populated. In 14C strong transitions we observed to \& $3^{-}$level at $6.73 \mathrm{MeV}$ and 


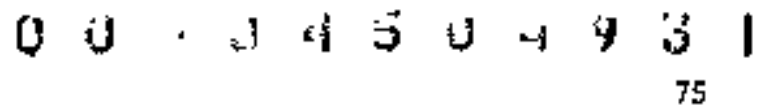

to $4^{+}$leve1 at $10.55 \mathrm{MeV}$ with weaker transitions to the ground state and to a level at $14.67 \mathrm{NeV}$. The 15C spectrum shows only strong transitions to the $5 / 2^{+}$level at $0.74 \mathrm{heV}$ and to two states at 6.85 HeV and $7.35 \mathrm{MeV}$ excitation energy, while in 180 only selective population of the $4^{+}$level at 3.55 MeV was observod (weak transitions whuld be obscured due to the reactions on the Si in the target).

Preferential population of high spin states has been observed in the $[x, d)$ reaction induced by 40-53 $\mathrm{MgV}$ a-particles on thany light nuclei; 2,3 the selectively porulated levels $\left[\mathrm{e} . \mathrm{g}+\right.$, of $\left(\mathrm{d}_{\mathrm{S}} / 2\right)_{5_{+}}^{2}$ or (f7/2) 2 character] correspond to particular kinematicalfy-fatroxed transitions in which the np pair can be simply captured in a relative triplot state about an undisturbed target core. Since sítilar. kinematic behavior and Q-values occur in the ( $\alpha, 4 \mathrm{He})$ reaction, one also expects to observe prediuninantly high spin states, but now those in which the nin pair is ceptured in a relative singlet state. At $65 \mathrm{Mov}$ bombsrding energy, tho transferred angular momentum in a surface interaction on these targets is about 4- $5 \mathrm{~h}$ and thus transitions to levels formed by capturing the two stripped neutrons into d-orbitals with configurations of $\left(\mathrm{d}_{5 / 2}\right)_{4}^{2}+$ should be entanced.

(a) ${ }^{2}$ He detection system
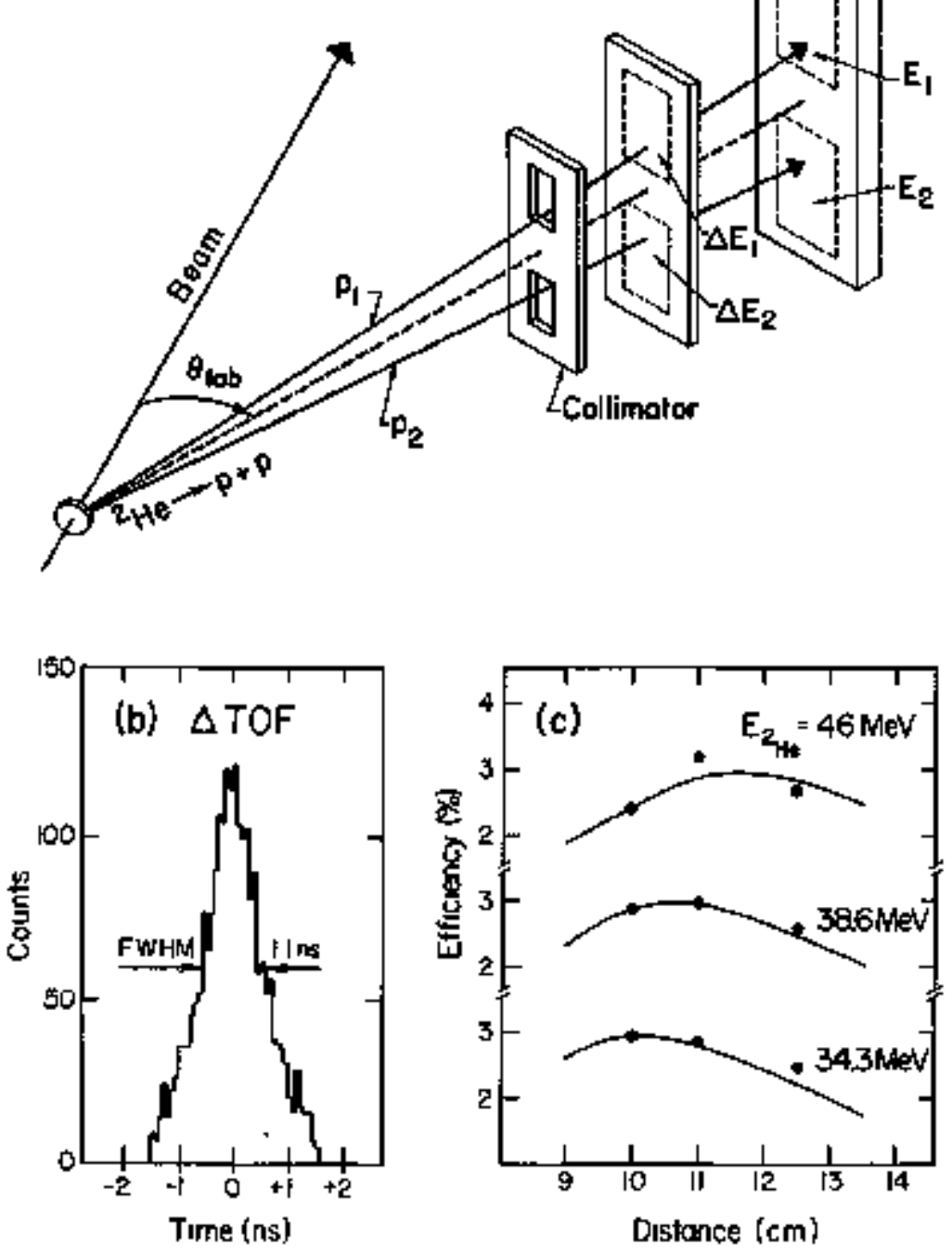

Fig. I. (a) Schenatic diagram of the ${ }^{2} \mathrm{Ha}$ detection system. (b) Spectrum of the time of flight differ. ence ATOF between the two brealap protons. (c) Comparison of experimental (dots) and theoretical (solid lines) $2 \mathrm{He}$ detection efficiencies as a function of the distance between target and collimator. The exporimental efficioncies have boon nonmalized to the calculations at a distance of $10 \mathrm{~cm}$.

(XBL 766-2941) 


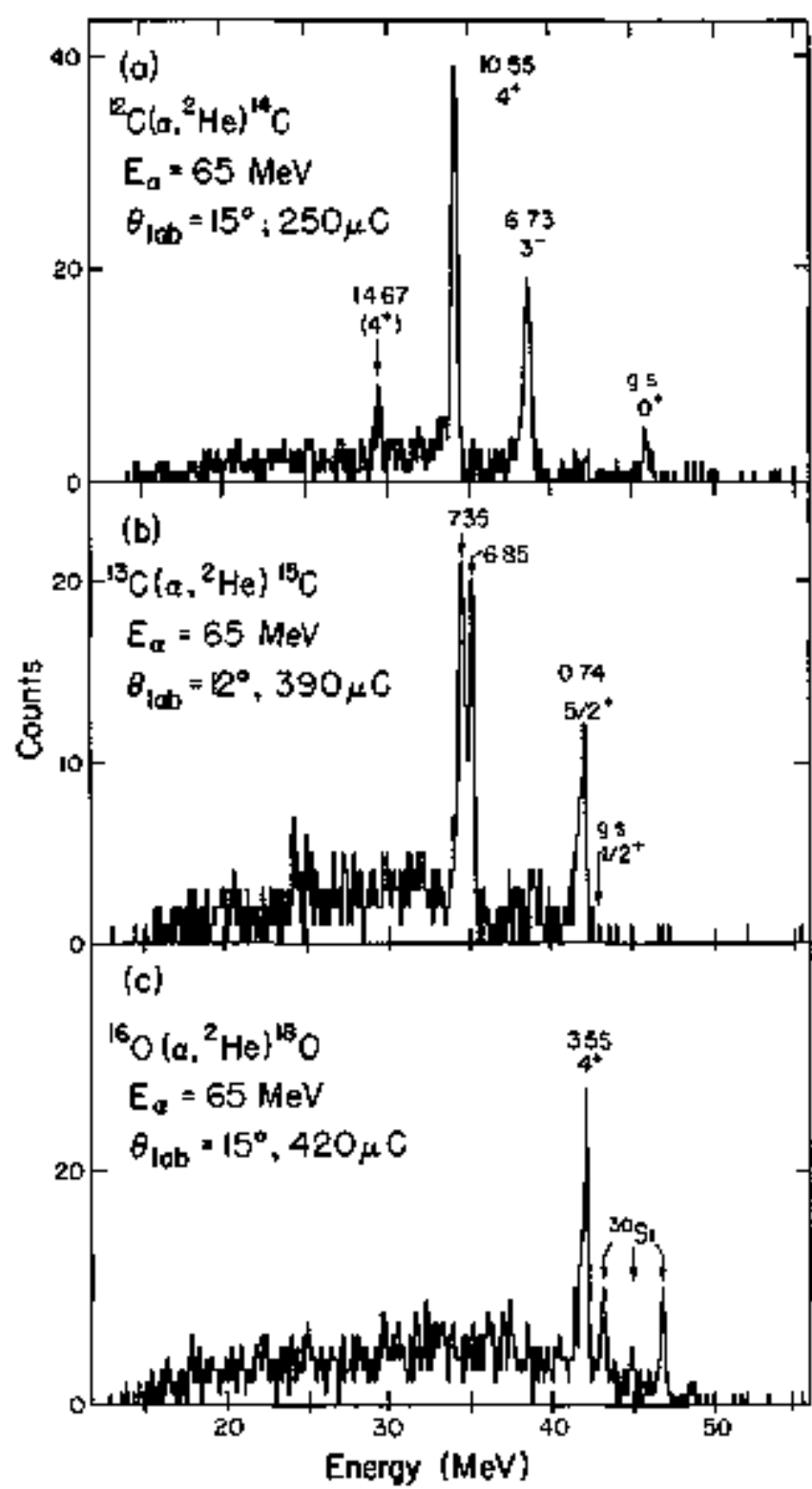

Fis. 2. 2he energy spectra obtained from the reac-

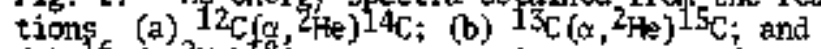
(c) $160\left(\alpha,{ }^{2} \mathrm{He}\right)^{180}$ at an $\alpha$-particle energy of $65 \mathrm{heV}$.

(XIL 766-2940)

The observed strong poptulation of the $4^{+}$states in ${ }^{4} \mathrm{C}$ and $18_{0}$, which have substantial $\left(d_{5 / 2}\right)_{4}^{2}$ character, $4,5,6$ is in agreenent with this sibiple picture.

Notfng Fig. 2 (a), except for the weak population of the ${ }^{14} \mathrm{C}$ ground state, transitions to the other observed states in $14 \mathrm{C}$ can be expleined as kinematically favored transitions to $5=0$ contonents in the known $6.73 \mathrm{MeV}\left(d_{5} / 2 \mathrm{p}_{1} / 2\right)_{3}=$ state $^{4}$ and in the 14.67 Mev state l possibly $4+7$ of $\left(\mathrm{d}_{5 / 2} \mathrm{~d}_{3 / 2}\right)_{4+}$ character ${ }^{4}$ though without additional calculations (ds/2 f7/2) 5 - camot be exciuded.1
Since the ${ }^{12} \mathrm{C}$ and ${ }^{13_{C}}$ targets only differ by a $\mathrm{P}_{1 / 2}$ neutrom, one expects the $\left(\alpha,{ }^{2} \mathrm{He}\right)$ reaction on ${ }_{13} 1 / 2$ poptiate preferentially the same two-neatron configurations originally observed in reactions on ${ }^{12} \mathrm{C}$, but now coupled to the $1 / 2^{-}$core. Thus the states observed in the $14 \mathrm{C}$ spectrum should be split into two corponents in the 15C spectrum, as has observed in the analogous $[\alpha, d)$ reactions on ${ }^{1} \mathrm{C}$ and 13C. Noting Fig. 2(b), then the doublet observed at $6.85 \mathrm{MeV}-7.35 \mathrm{HeV}$ in $15_{\mathrm{C}}$ can be interpreted ascomfiguration $\left\{\left[{ }^{12} \mathrm{C}\left(0^{+}\right) \mathrm{P}_{1 / 2}\right\}_{1 / 2} \otimes\left(\mathrm{d}_{5 / 2}\right)_{4}^{2}\right]_{7 / 2^{-}}, \mathrm{g}^{-} \mathrm{2}^{-} \mathrm{Al-}$ though this model does not predict the as'sigrnment of spins to the two components of the split state, relative enhancement via the $(2 \mathrm{~J}+1)$ statistical factor inqlies that the $7.35 \mathrm{keV}$ level might have the hight er spin ( $/ 2^{-}$) becalse of its larger cross section. Since the $5 / 2^{+}$state at $0.74 \mathrm{Mel}$ has a configuration $\left.\left[{ }^{1}{ }^{2} \mathrm{C}\left(0^{+}\right) \mathrm{p}_{1 / 2}\right\}_{1 / 2^{-}} \mathrm{p}_{1 / 2} \mathrm{~d}_{5 / 2}\right]_{5 / 2^{+}}$, the $\mathrm{p} 1 / 2$ neutron of $13 \mathrm{C}$ and the trafsferred $\mathrm{p} / 2$ neutron must couple to spin 0 and no splitting can arise. These throe ${ }^{15} \mathrm{C}\left(\mathrm{c},{ }^{2} \mathrm{He}\right)^{15} \mathrm{C}$ transitions and the $160\left(a,{ }^{2} \mathrm{He}\right) 180$ transition to the $3.55 \mathrm{MeV}\left(\mathrm{d}_{\mathrm{S} / 2}\right)^{2}$ state all show the expected forward peaked, structureless angular distributions, In addition, the observed cross sections of the transitions to the known $4^{+}$state in $14 \mathrm{C}(10.55 \mathrm{NeV})$, the sor of the split states in $15 \mathrm{C}$, and the $4^{+}$state in $10_{0}$ are all equal within errors, which is further evidence for the assuption of a comion $\left(d_{s} / 2\right)_{4}^{2}$ configuration for these states.

These results clearly demonstrate the utility of the $(\alpha, 2 \mathrm{He})$ reaction as a new spectroscopic tool capable of locating nany unobserved two-noutron states of high spin in the $2 \mathrm{~s}-1 \mathrm{~d}$ and higher shells. Furthermore, extension of this approach towards studying resomant final systems as reaction prochets to other cases seans particularly practical and fruitful.

\section{Footnotes and References}

condensed fram LBL-5038, Fhys, Rer, lott. 37, 812 (1976).

${ }^{t}$ DAAD Fellow, on leave from Institut firr Strahlenund Kemphysik der Universität Eom, Germany.

1. D. Robson, Nuc1. Phys., A204, 523 (1973).

2. E. Rivet, R. H. Pẹhl, J. Cermy, and B. G. Harvey, Phys. Rev, 14l, 1021 (1966).

3. C. C. Lu, M. 5. Zisman, and B. G. Harvey, Phys. Rev. 186, 1086 (1969),

4. W. W. True, Plys. Rer. 130, $1530(1963)$.

5. 5, Lie, Mucl. Phy's, Al81, 517 (1972).

6. T. T, S. Kow and G. E. Brown, thucl. Plys. 85, $40(1966)$.

7. N. Anyras-Theiss, et.a1., Phys. Lott. 12C, 201 (1974). 


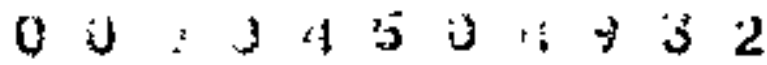 \\ 77
}

THE $\left(\alpha,{ }^{8} \mathrm{Be}\right)$ REACTION IN THE 1p SHELL*

\author{
G JWoznak, D P Stahel, \\ $N$ A Jelley, and Joseph carmy
}

The existence and the Importance of multimucleon correlations in maclei, and in particular of o-like four nucleon correlations, hes intrigued physicists for decades. Recently, detailed theoret lcal calculations have been mage of the extent of $\alpha=c l u s t e r i n g$ in 1 ight nuclei 1,2 and the $\left(d, 6_{L I}\right)$, ( $\left.{ }^{3} \mathrm{He},{ }^{7} \mathrm{Be}\right)$ and $(\alpha, 2 \alpha$ ) reactions have been, ewployed to verify experimentally these predictions. 3,4

To complens?t the infornation acqutred with the above three reactions, a very detailed study on 1p shell targets has been inade with the $\left(\alpha^{8} \mathrm{Be}\right)$ reaction. This reaction has an a priori simplicity

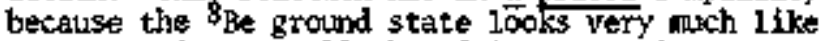
two a perticles weakly boind in a relative s-state; firther, since the projectile, the transfferred a particle and the $\mathrm{B}_{\mathrm{Be}}$ ground state all have zero spin, simple selection rules result Although ${ }^{\mathrm{Be}}$ is particle-unstable $\left[t_{1 / 2} \sim 10^{-16_{3}}\right\}$, its ground state is long-1ived compared with nuclear transit times, and one should be able to treat it as a single nucleus in a direct reaction.

The present investigation was carried out at moderately high botmbarding energies (65-72.5 $\mathrm{MkV}$ ) where it was hoped that direct processes would doninte and thus make possible the extraction of experimental spectroscopic factors. All stable lp shell targets wore investigated and the data were analyzed in the framework of the exact finite-range distorted-wave Borm-approxinatlon (EPR-ENRA) .

In the distorted-wave Born approxination, the differential cross section for the reaction $\mathrm{B}\left(\mathrm{a},{ }^{8} \mathrm{Be}\right) \mathrm{A}$ is given by:

$$
\frac{d \sigma}{\alpha}(\theta)=\sum_{L} s^{L}(B+A+\alpha) s(B+\alpha+\alpha) \sigma_{D W B A}^{L}(\theta)
$$

Where $L$ nus ower all the allowed anglar mulentun transfers. The kinematic part of the cross section, ol was calculated using DeVries' EFR-Died code IfHA, 5 The optical model potentials needed to generate the distorted waves in the entrance and exit channels were determined by fitting tabulated gparticle elastic scattoring data on $12 \mathrm{C}, 13 \mathrm{C}, 14 \mathrm{~N}$, and $15 \mathrm{~N}$ at $40.5 \mathrm{Ke}^{6}$ and on 160 at $65 \mathrm{keV}^{7}$ with the search code GENOA. ${ }^{B}$ The scattering data for each tar. get were fitted individually with woods-Saxon potentials haring both reat and inaginary volune tems. For the ${ }^{\mathrm{Be}}, \mathrm{10}_{\mathrm{B}}$ and $\mathrm{l}_{\mathrm{B}}$ tangets no tabulated scattering data were available in the appropriate region. Thus a potential from the 1iterature 9 sinilar to 13C (see Tple 1), which reproduced 46 MeV $\alpha$-scattering on ${ }^{3}$, was usot.

In order to approximate a potential for the particle-unstable $\mathrm{Be}$, GENah was used to determine an optical potential which reptoduced $50 \mathrm{MeV}^{-\mathrm{Be}}$ elastic scattering date on ${ }^{12} \mathrm{C}$ (potential $A A$ in Table 1). Since the $16 \mathrm{O}\left(\alpha,{ }_{\mathrm{Be}}{ }^{12} \mathrm{C}\right.$ reaction cala1lations were found to be relatively insensitive to

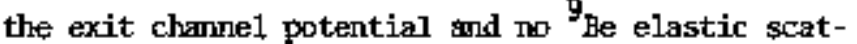
tering data existed for the other exit channels, potential AA was used to generate the distorted waves in the ${ }^{B}$ Be channel for all of the $1 p$ shell targets.

The bound state wavo functions, which describe the notion of an a eluster in the target nucleus $B$ and in ${ }^{8} \mathrm{Be}$, wre caloulated in the usual way using a real hoods-Saxon potential whose we11-depth rozs adjusted to give the observed a binding energy. The radius of the Noods-Saxpon well describing the torget nuclei was chosen to be $R=r_{\mathrm{C}} \mathrm{h}^{1 / 3}$. An $r_{0}$ of 2.0 was tsed for all targets; this give a radius which was larger than the physical size of the core $A$. This larger radius could correspond to the transferred a-particle existing at the surface of the core.

Table 1. Optical model potentials used in the DFBA calculations.

\begin{tabular}{|c|c|c|c|c|c|c|c|c|c|c|}
\hline Tanget & Projectile & Eroj. $_{\text {MeV }}$ & $\begin{array}{c}\mathrm{V} \\
\mathrm{MeV}\end{array}$ & $\begin{array}{l}r_{\mathrm{R}}^{a} \\
\mathrm{Em} \\
\end{array}$ & $\begin{array}{l}{ }_{R} \\
\text { fll } \\
\end{array}$ & $\begin{array}{c}\mathrm{N} \\
\mathrm{NeV}\end{array}$ & $\begin{array}{l}\mathbf{r}_{1}^{\mathrm{a}} \\
\mathbf{f} \\
\end{array}$ & $\begin{array}{l}{ }_{T} \\
f_{m}\end{array}$ & $\begin{array}{l}\mathbf{r}_{\mathrm{C}}^{a} \\
\underline{\mathrm{f}}\end{array}$ & Potential \\
\hline 160 & $a$ & 65 & 89.3 & 1.56 & .57 & 27.7 & 1,39 & .72 & 1.2 & $A^{b}$ \\
\hline $\mathrm{IS}_{\mathrm{kN}}$ & 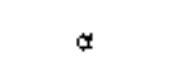 & 40.5 & 279 & 1.22 & .65 & 17.6 & 1.55 & .65 & 1.2 & $\mathrm{~B}^{\mathrm{C}}$ \\
\hline $14 \mathrm{~N}$ & $\alpha$ & 40.5 & 279 & 1.22 & .65 & 17.6 & 1.55 & .65 & I. 2 & $\mathrm{~B}^{\mathrm{C}}$ \\
\hline${ }^{13} \mathrm{C}$ & † & 40.5 & 170 & 1.47 & +55 & 20.8 & 1.56 & .35 & 1.2 & $c^{C}$ \\
\hline${ }^{12} c$ & $\approx$ & 40.5 & 36.7 & 1.80 & .41 & 7.6 & 1.96 & .66 & 1.2 & $D^{C}$ \\
\hline${ }^{11}{ }_{B},{ }^{10}{ }_{B},{ }^{9} \mathrm{Be}$ & 6 & 46 & 194 & 1,38 & .60 & 24 & 1.60 & .60 & 1.2 & $\mathbf{E}^{d}$ \\
\hline $12_{\mathrm{C}}$ & ${ }^{9} \mathrm{Be}$ & 50 & 35.2 & 1.72 & .92 & 12 & 2.65 & .50 & 1.2 & $\mathrm{AA}^{\circ}$ \\
\hline
\end{tabular}


Decreasing $r_{0}$ from 2.0 to 1.2 had only a snall effect or the shape of the fits but caused a strong decrease in the magnitude of the cross section.

Although ${ }^{8}{ }_{B}$ is unboumd by $92 \mathrm{kev}$, it is effectively bound by its Coulanb barrier during the reaction time. To generate a bound state wave function for the calculations, it was assumed that the Be intemal wave function varied smoothly and slowly when its binding energy was changed from $-92 \mathrm{kev}$ to $+10 \mathrm{ket}$. The ife interqal wave function was calculated for an a particle bound to a second one by $10 \mathrm{keV}$ in a Woods-Sakon we11 with a radius of 3.2 fro. (Changing the binding emergy fran $100 \mathrm{keV}$ to $10 \mathrm{keV}$ proxuced no change in the shape of the calculated cross sections and only a $7 t$ decrease in their magnitudes.)

In deriving experimental spectroscopic factors, we have tried to maintain consistent criteria for choosing the center-of-mass angle at which to relate experiment and theory, since the shapes of the calculated and experimental cross sections are not identical. If an experimental saximum existod in the angular distribution, the theoretical and experimental yields were compared at this angle. For the flatter angular distributions, the spectroscopic factor was calculated at a data point between $\theta_{\text {c.s. }}$. $=25$ and $35^{\circ}$. The values of the theoretical $\alpha-$ particle spectroscopic factors $\mathrm{S}\left(\mathrm{S}+\alpha^{+}+\alpha\right)$ in Eq. $]$ were taken fram Rotter ${ }^{1}$ and from Kurath. ${ }^{2}$ For $\mathrm{S}\left[{ }^{8}[\mathrm{~s}+\mathrm{a})\right.$ the theoretical value of 1.5 was taken from firrath. 2 The calculated cross sections and the experimentat data for al1 targets are given in Ref. 11 wile only the 160 target data and fits are discussed belor.

A compariscon of the $\left[\alpha,{ }^{B} \mathrm{Be}\right.$ ) experimental (symbols) and absolute calculated (solid curyes) cross sections for transitions populating the ${ }^{2} \mathrm{C}$ ground state and several excited states is shown in Fig. 1. The similar magnitudes of the experimental and calculated cross sections demonstrate good agreement between the theoretical a-particle spectroscopic factors and experiment. The shapes of the theoretical cross sections reproduce some of the features of the experinental data - most notably the relative spacing and magnitute of the two forward maxims in the ground state angular distribution. Furthemore, the damping of the ascillatory character observad experimentally in the $\mathrm{L}=2$ and $\mathrm{L}=4$ angular distributions, compared to that of the $L=0$ grownd state, is also reprobiced by the calctations.

In Fig. 2 are shown ratips $\mathbf{R}^{\text {abs }}$ of experimental to theorotical $\mathrm{s}_{\mathrm{x}}$ where $\mathrm{k}^{\text {abs }}$ is defined by:

$$
\mathrm{R}^{\text {abs }}=\frac{S(\exp )}{S(\text { theory })}=\frac{d \sigma / d \Omega(\theta) \exp }{d \sigma / d \delta(\theta))_{\text {th }}}+
$$

For consistency, Kurath's theoretical $\alpha$-particle spectroscopic factors ${ }^{2}$ are used for all targets. In general these ratios lie below the dashed line at Kabs -1.0 , but deviate from it by less than 50 . (of course this corperison is very sensitive to systenatic errors either in the experimental data or in the reaction calplations; an example of the lattor is that the magnitude of the calculated cross section is affected by the value of $r_{0}$ used in calculating the baind state wave functions). The 6ti.

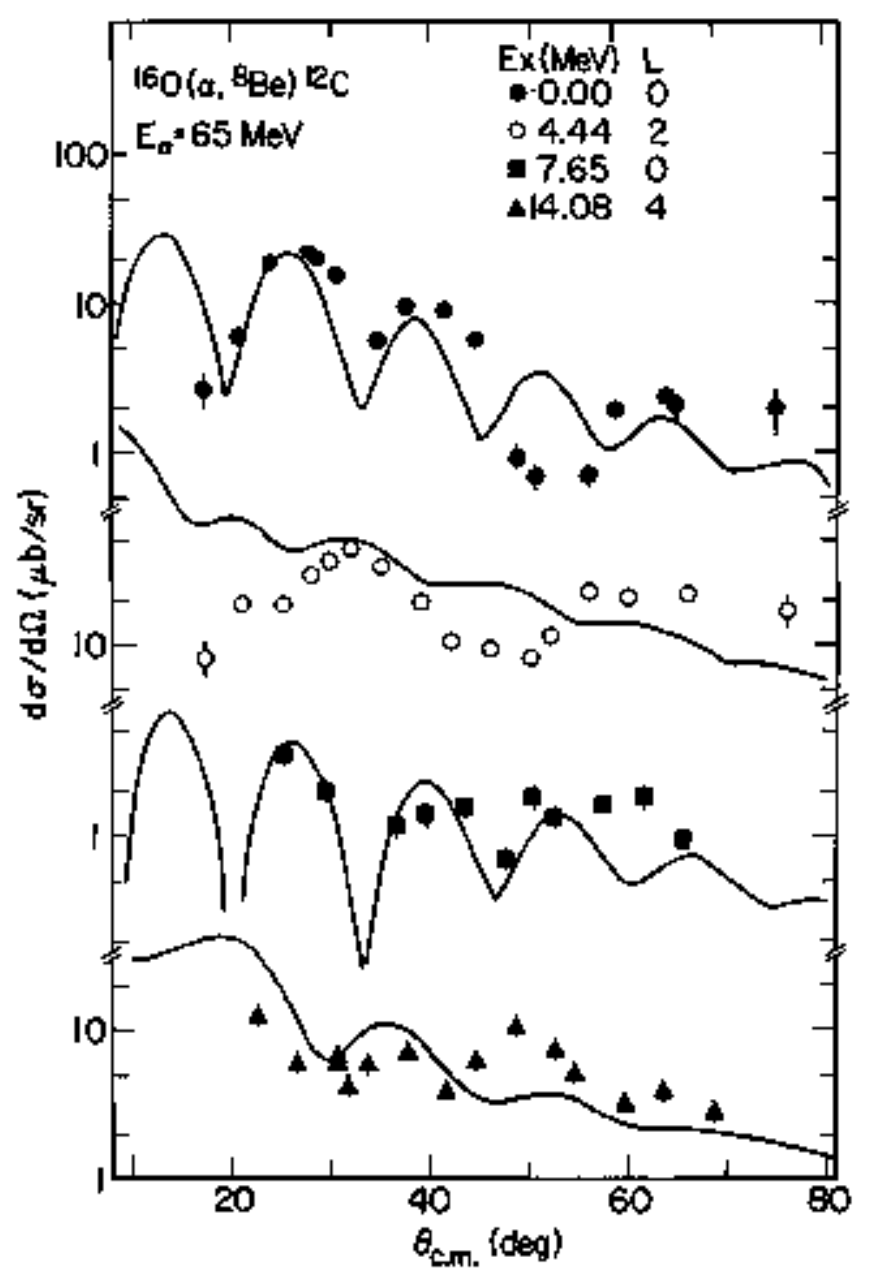

Fig. 1. Absolute experimental [symbols) and calailated (solid curves) (a, ${ }^{8} \mathrm{Be}$ ) cross sections at $\mathrm{E}_{\mathrm{\alpha}}=65 \mathrm{MeV}$ for transitions populating the ${ }^{12 \mathrm{C}(\mathrm{g} . \mathrm{s} .)}$ and several exchted states. A statistical error is given if it exceeds the height of the data point. In addition, the width of each data point corresponds to the angular acceptance of the ${ }^{8} \mathrm{Be}$ identifíer used in measuring that point.

(XBR. 7512-9930)

(g.s.) point is off-scale because of its very șlalI theoretical $S_{0}$.

In order to minimize systematic errors, the above ratio of spertroscopic factors, $\mathrm{R}^{a b s}$, was divided by the ratio for the gramid state transition. The ratio $\mathrm{R}^{\mathrm{rel}}$ is defined by:

$$
\mathbf{R}^{\mathrm{Te1}}=\frac{\mathrm{R}^{\mathrm{abs}}(\mathrm{B}+\mathrm{A}+\alpha)}{\mathbf{R}^{\mathrm{abs}}\left(\mathrm{B}+\mathrm{A}\left(\mathrm{g}, \mathrm{s}_{-}\right)+a\right)} .
$$

Part $b$ of Fig. 2 presents this relative ratio of $S_{a}$; $\mathrm{k}^{\mathrm{rel}}$ is again plotted against the final state populated, Better agrement between oxperiment and theory is sen in Fig, $2 \mathrm{~b}$ in that the values of $\mathrm{R}^{\mathrm{rel}}$ cIuster closer to 1 than those for $\mathrm{R}^{\mathrm{abs}}$ in Fig. 2a with onIy four values of $\mathrm{R}^{\mathrm{reI}}$ farther than $\pm 50 \%$ from wity; the 6il (g.s) point is again off scale. 


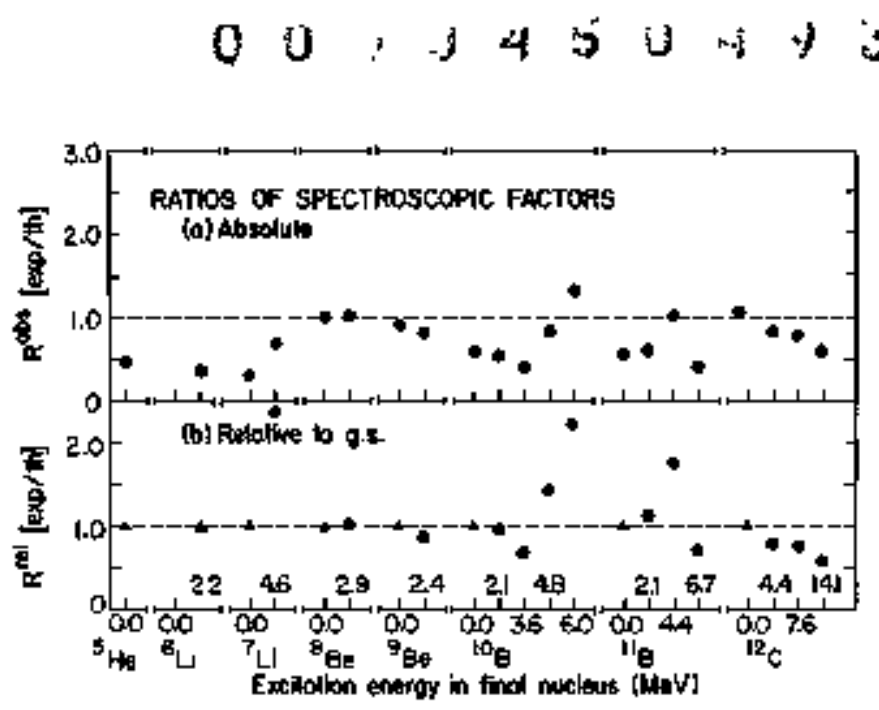

Fig. 2. (a) A comariscr of the ratios of experimental to wurath's ${ }^{2}$ thooretical spectroscopic factors [ $\mathrm{R}^{\mathrm{abs}} \equiv \mathrm{S}$ (exp)/S(theory)]. (b) A colpparison of these ratios relative to the ground state ratio for each target (see discussion in tert). Note that the ratio (Rrel) of $S_{a}$ refative to the $2.2 \mathrm{MeV}$ state in $6_{\mathrm{Li}}$ is given $\mathrm{for}_{\mathrm{r}} 10_{\mathrm{B}} \rightarrow 6_{\mathrm{LI}}+\alpha$. In both parts (a) and (b), the bLi(g.s.) point is off-scale because of its very smati theoretical S.

(XBL 7512-9926)

The selectivity and good quantitative agreement with theoretical predictions illustrate that the $\left(a, \mathrm{~B}_{\mathrm{Be}}\right)$ reaction is a useful spectroscopic probe with which to medsre the extent of $\alpha$-clustering in nuclei.

\section{Footnotes and Reforences}

"Condensed frọm LBL-4368, Phys. Rev.C 14, 815 (1976).

11. G. J. Wozniak, D. P. Stahel, Joseph Cerny, and N. A. Jelley, Phys. Rev. C14, 815 (1976).

'Present address: Muclear Physics Laboratory, University of Oxford, EngIand.

1. I. Rotter, Fortschr. Phys. 16, 195 (1968).

2. D. Kurath, Phys, Rev. C7, 1390 (1973).

3. K. Bethge, Agn. Rev. of hucl. Sci. 20, $255(1970)$.

4. J. D. Garrett in the Procesings of the Symposiuso on Tho-Nucleon Transfer and Pairing Excitations, Argorve Physics Division Informal Report PtYY-1972H $232(1972)$.

5. R. M. DeVries, Phys, Rev. CA, 951 (1973),

6. B. G. Harvey, J. R. Meriwether, J. Mahoney, A. Bhissiere De Nercy and D. J, Horen, Phys. Rev, 146, 712 (1966). See University of California Radiation Laboratory Report-16573 for tabulated data.

7. B. G. Hervey, E, J-M, Rivet, A, Springer, J. R. Werimether, W. B. Johes, J. H. Elliott, and P. Darriulat, Nuct. Phys. A52, 465 (1964). D. L. Hendrie, private combunlcation.

8. F. G. Perey, optical-model search code (unpiblished].

9. B. Zeidman, H, T, Fortune, and A. Richter, Phys. Rev, C2, 1612 (1970).

10. D. P. Stahe1, G. J. Wozniak, B. D. Jelteng, M. S. Zisuan, R, Jahn, and J. Cerry, The ( ${ }^{\mathrm{B} e},{ }^{8} \mathrm{Be}$ ) Reaction at $50 \mathrm{MgV}$, in this Armual Report.

\section{THE $\left(\alpha,{ }^{8}\right.$ Be) REACTION IN THE 2s-1d SHELL}

O P Stanel, G J Woznalk,

M S Zisman, and hoseph Cerny

In recent years, there have been several calculations 1.5 of $\alpha$-spectroscopic factors $S_{\text {te }}$ of $2 s$ Id shell nuclei with sol(3) shell model wave functions. 6 A comparison of these theoretical values with experimental ones should be a sensitive test of these predictions and thus the validity of the gU(3) shell model. Since the $\left(a,{ }^{8}\right.$ Be $)$ reaction has been successfully utiIized to doteymine experimental $S_{1}$ values for ip shell nuclei, our study of this reaction has been extended to $2 s-1 d$ sheil targets.

Targets of ${ }^{24} \mathrm{~kg},{ }^{28} \mathrm{Si}$, and ${ }^{40} \mathrm{Ca}$.e bombarded with $60 \mathrm{MeV} \alpha$-particles from the 88-inch cyclotron. The Be detection systeng employed has been described elsewhere. Typical Be energy spectra are show in Fig. 1. spectra frum the 24 dg and ${ }^{28}$ Si targets exhibit preferential population of the ground state rotational band and some menbers of higher-lying bands in the residual nuclei. This is consistent with the results of St (3) she11 mode1 calculations ${ }^{2}$ which show that in a good \$O (3) nucleus the a strength can be highly cuicentrated in a few rotational bands.

In the 20 Ne spectrum, there is evidence for population of merchers of the ground state rotational band up to spin $8^{+}$. No pogulation of the $z^{-}$ state of the $K=2$ band is observed at this angle. At other angles, however, there is some evidence for weak population of this umatural parity state, indicating thit multi-step reacticr processes could be present. ${ }^{6}$ In the 36 Ar spectrom only the ground state and the first $2^{+}$and $4^{+}$ states are excited. There is no significant population of higher menbers of this "gramd state rotational band" that were predleted in the SJ(3) scherve, 3 although the kinematics would favor transitions to these high spin states. Hipwever, in the upper half of the 2s-1d she11, SU(3) way not be a 

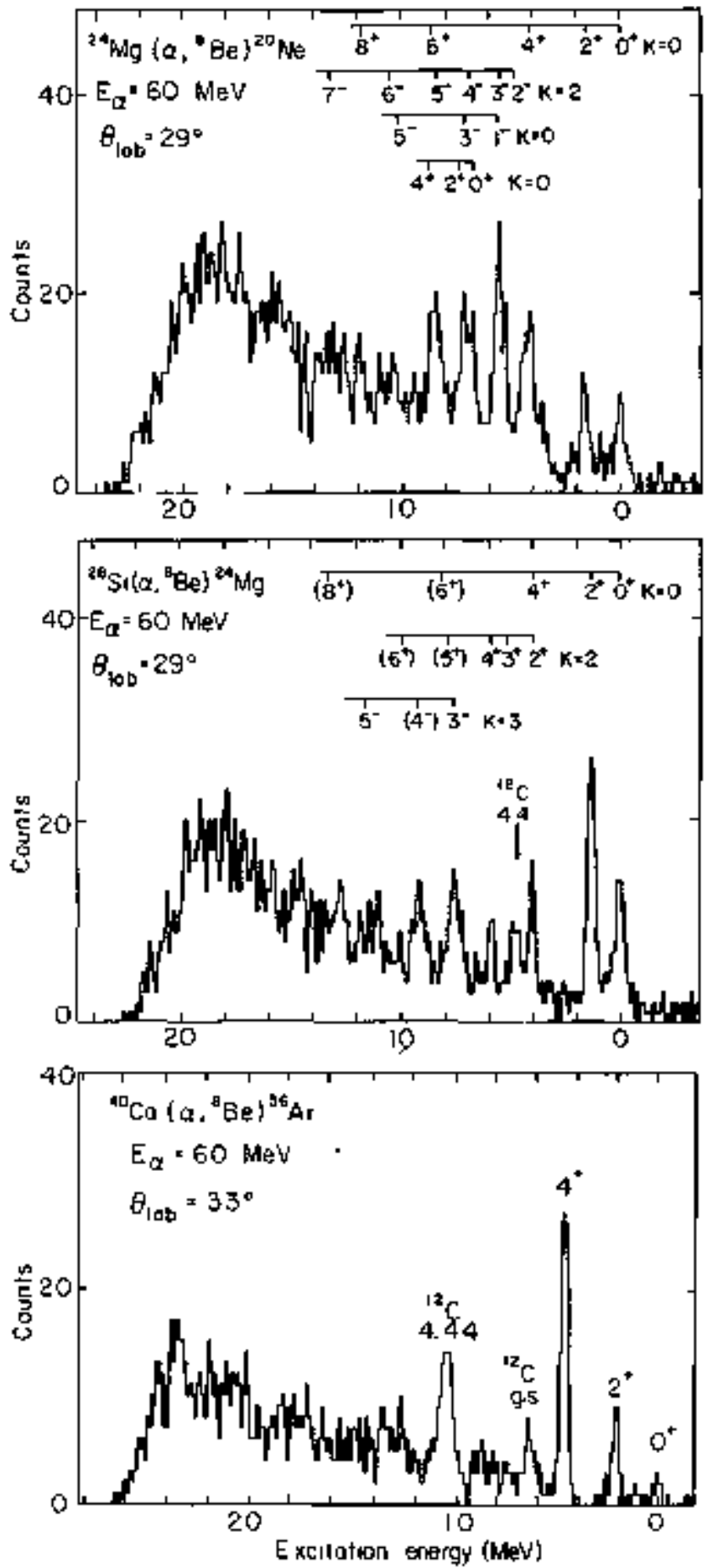

Fig. 1. Engrgy soptra of ${ }^{8}$ Be from the $\left(a,{ }^{8} \mathrm{Be}\right)$ reactions on $24 \mathrm{HB},{ }^{2} \mathrm{Si}$ and $40_{\mathrm{Ca}}$, (CRL 767-8316)

good symulty.

Angular distributions were mensured for the transitions to the gramd and several excited states and are shown in Pig. 2. They show fortard peaking and diffraction structure that tends to be washed out for the larger 2-transfers. Typical peak cross sections of $1-5 \mathrm{~kb} / \mathrm{sr}$ are observed. For the ground state $i=0$ transitions, there is a rather Iarge angular mngentum sisnatch of about fi for the $24 \mathrm{Ag}$ and abort th for the ${ }^{28} \mathrm{Si}$ and ${ }^{40} \mathrm{Ca}$ targets, which causes the cross sections to be ninch smaller than for the $\left(\alpha, \beta_{B}\right)$ reaction on even-even ip shell targets.? The distributions were analyzed using the exact finite-range DWRA code $10 L A,{ }^{9}$ assuming a simple ground state $\alpha$-eluster transfer mechanism. The cotical model parameters for the entrance charmels were taken from published elastic a-scattering data. For the exit channels, the parameters were determined from elastic ${ }^{9} \mathrm{Be}$ scattering data. ${ }^{\circ}$ The results of some preliminary calculations are shown in Fig. 2. Although the shapes of the distributions are reproducel fairly well, they are out of phase with respect to the data, and the extracted relative

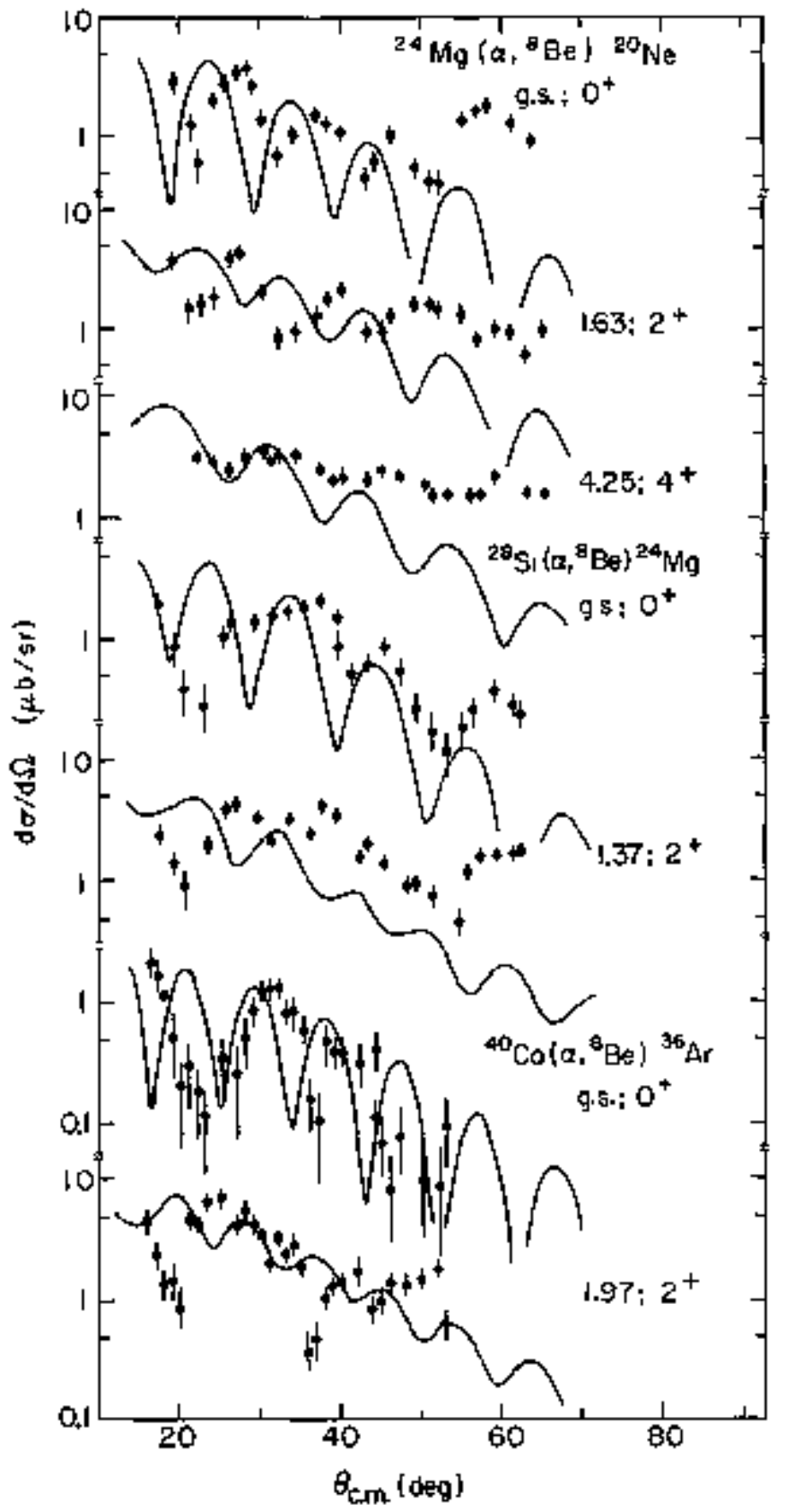

Fig. 2. Angular distributions of ${ }^{8} \mathrm{Be}$ from the (a. ${ }_{\mathrm{Be}}$ ) reaction on $24 \mathrm{Mg}, 2 \mathrm{~S}_{\mathrm{S}} \mathrm{i}$ and $4 \mathrm{OCa}$. The solid curves are exact finito-range DWBA calculations. 


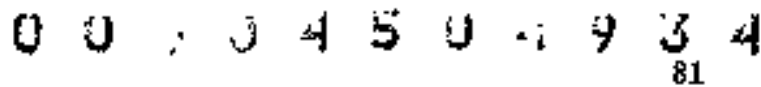

$S_{\text {t }}$ values are only in voderate agrement with the Si (3) predictions, as shown in Table 1. This could be due to the monentun misnatch which makes the calculations rather sensitivo to variations of paraneters. Furthermore, in reactions imolving deformed, collective muclei, rulti-step processes, which cannot be handled by first-order InBA, may play an important role. Thus, a more elaborate (and expensive) coupled-channels analysis may be required before quantitative structure information can be extracted from date such as these.

Table 1. क-spectroscopic factors.

\begin{tabular}{|c|c|c|c|c|c|}
\hline \multirow{2}{*}{$\begin{array}{l}\text { Target } \\
\text { macleus }\end{array}$} & \multirow{2}{*}{$\begin{array}{c}\text { Final } \\
\text { mucleus }\end{array}$} & \multirow{2}{*}{$\begin{array}{l}\text { Level } \\
\text { (Mol) }\end{array}$} & \multirow{2}{*}{$J^{\pi}$} & \multicolumn{2}{|c|}{ Relative $S_{a}$} \\
\hline & & & & $\left(\alpha_{,}^{B} \mathrm{Be}\right)$ & $\mathrm{Su}(3)^{\mathrm{a}}$ \\
\hline \multirow[t]{3}{*}{${ }^{24} \mathrm{~kg}$} & $20 \mathrm{Ne}$ & g.5. & $0^{+}$ & $\$ .00$ & 1.00 \\
\hline & & 1.63 & $2^{+}$ & 1.29 & .13 \\
\hline & & 4.25 & $4^{+}$ & 1.62 & .80 \\
\hline \multirow[t]{2}{*}{${ }^{28} \mathrm{Si}$} & $24 \mathrm{Mg}$ & g.5. & $0^{+}$ & 1.00 & 1.00 \\
\hline & & 1.37 & $2^{+}$ & 1.27 & 1.20 \\
\hline \multirow[t]{2}{*}{$40 \mathrm{Ca}$} & ${ }^{36} 6_{\mathrm{AT}}$ & g.s. & $0^{+}$ & 1,00 & 1.00 \\
\hline & & 1.97 & $2^{+}$ & 3.95 & 1.17 \\
\hline$a_{\text {. }}$ & $r^{24} \mathrm{Mg}$ & ${ }^{2 B} \mathrm{Si}$ & & $o r{ }^{40} \mathrm{C}$ & \\
\hline
\end{tabular}

\section{References}

1. A. Arima et al., in Actv, in Nucl. Phys, Vol. 5, M. Baranger and $E$, Vogt, eds, (Plenum Press, New York, 1972).

2. M. Tehtmura et al., Nucl. Phys. A204, 225 $(1973)$.

3. J. B. MeGrory, Phys. Lett. 476, 481 (1973).

4. J. P. Draayer, Nucl. Phys, A237, 157 [1975].

5. K. T. Hecht and D. Bramschoreig, Pharl. Phys. A.244, $365(1975)$.

6. M. Harvey, in Adv. in Mucl. Phys, Vol, 1, W. Baranger and E. Vogt, eds. (Plemm Pregs, York, 1968).

7. G. J. Wozniak, D. P, Stahel, N. A. Je11ey, and $J_{+}$Corny, preceding report.

8. J. R. Confort et al., Phys, lett. 40E, 456 (1972).

9. R. M. Dęlvies, Phys. Revv. C ㄹ, 951 (1973).

10. D. P. Stahel, G. J. Wozntak, B. D. Jeltefia, M. S. Zinan, R. Jahn, and J. Cerry, The ( $\left.{ }^{\mathrm{Be}},{ }^{8} \mathrm{Be}\right)$ Reaction at $50 \mathrm{KeV}$, in this Annual Report.

\section{EXOTIC MASS MEASUREMENTS USING THE $\left({ }^{4} \mathrm{He},{ }^{8} \mathrm{He}\right)$ REACTION}

G. KeKelis. M, Zișman, D. Scolt.

R. Jahn, and Joseph Cerry

The focm neutron pick-up reaction ( ${ }^{4} \mathrm{He},{ }^{8} \mathrm{He}$ ) is a valuable tool for reaching nuclei far frtil stability. 1 The 88 -inch cyclotron is ideally suited to the measurement of these reactions for two reasons. First, the cyclotron produces sufficientenergy al pha iefms to perforin reactions with $Q$-values of -50 to -60 HeV. Second, the magnetic spectrometer provides the large solid angle and sophisticated detection system required to measure cross sections in the $1-\mathrm{nb} / \mathrm{sr} \mathrm{region}$.

Most of the enthasis of the present experitur tal program has been directed to rehuchig extraneous backgrcumd in the magnetic spectroneter to the subnanobarn level. This background is produced by three processes. First, $\gamma$-rays and nesutrons proarced at the Faraday cup cause risidentification in tipe-of-flight measirements by producing betwembeam-burst counts in the plastic scintiliator "start" sigral of the Focal plane and the ME11l- photomultiplier "stop" signal. The problen has been greatly alleviated by using a gas proportional coumter" as the "stop" signal since tt is relatively insensitive to $Y$ rays and neutrons. The second contribution to the barkground is the intense elastic scattering. At small angles the elastics produce count rate problems as well as scattering from the walls of the spectrotieter, thereby entering the focal plane detector with peculiar trajectories. The last identif feation problem is die to high energy protons, deuterons, and tritons which are 11ghtIy ionizing and easily misidentified. These last two problens have been elfuinated by introducing a new focal plare detector designed by $B$. Harvey 3 which allows double $\mathrm{AE}$ and double posftion (trajectory) measuremontsts.

The $58_{\mathrm{Hi} i}\left(\mathrm{He}^{4}{ }^{8} \mathrm{He}\right)^{54}{ }_{\mathrm{Hi}}$ reaction was investigated with the abovs system before the new focal plane detactor and proportional counter time-zero 
system were introduced. The resulting ${ }^{8} \mathrm{He}$ spectrun is show in Fig. I. Background in this spectrum is on the ortier of the cross section of interest, that is $\$-10 \mathrm{nb} / \mathrm{sr}$. However, it does appear that a peak

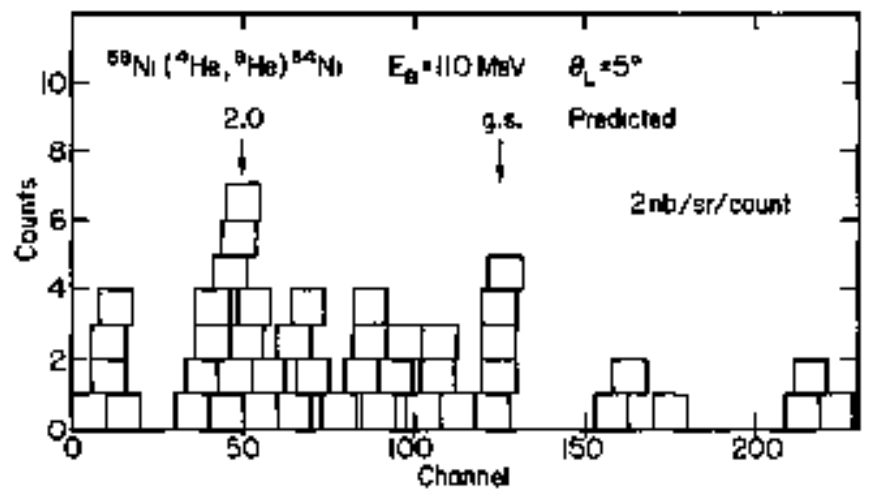

Fig. 1. Energy spectrm of the ${ }^{58} \mathrm{Ni}\left({ }^{4} \mathrm{He},{ }^{8} \mathrm{He}\right)^{54} \mathrm{Ni}$ reaction at $\mathrm{E}=1 \pm 0$ Mev. The predictet ground state location was taken from Ref. 4.

(XBL 764-2744) with a cross section of about $\$ \mathrm{nb} / \mathrm{sr}$ is located at the expected position, as well as a possible excited state at $2 \mathrm{kkV}$ excitation. The peak location is based on the mass of $54 \mathrm{Ni}$ as predicted by Harchol et $a 1 ., 4$ This experiment is being repeated using the new focal plane detector and time zero systems in order to produce a clean spectrum.

\section{Roferences}

1. J. Cerny, in Proceeding of the International Conference on Reactions Betwreen Conplex Nuclei, edited by R. L. Robinson et al., Nrorth-bolland, Ansterdam, 1974), Vol. 2, p. 483 .

2. G. Kekelis, G. Gabor, D. Scott, and C. K. Gelbke, A Proportional Counter Tine Zero System, in this Antulal Report.

3. B. G. Harvey, J. Mahoney, and R. F. Burton, Spectrometer Focal Plans Detector System, in this Arrmal Report.

\section{b. Heavy Ions}

\section{STUDIES OF ${ }^{4} \mathrm{H}$ AND ${ }^{5} \mathrm{H}$ BY THE TWO-PRONTON PICKUP REACTION $\left({ }^{\circ} \mathrm{Li}^{\circ}{ }^{\circ} \mathrm{B}\right)$}

A B Weisemiller, $\mathrm{K} \mathrm{H}$ Widcox, D Ashery.t $G 」$ Wozrak, and Joseph Cerny

Although the heavy hydrogen isotope ${ }^{4} \mathrm{H}$ has been the focus of numerous theoretical and experinental investigations, considerably less inforpa $\bar{z}$ tion is available on the level structure of $\mathrm{S}_{\mathrm{H}}, \mathbf{1}, 2$ In an attempt to obtain further inforgation on ${ }^{5} \mathrm{H}$, we have studied and congared results from the Gi (8i, $\left.{ }^{8}\right)^{4} \mathrm{H}$ and ${ }^{7} \mathrm{Li}\left({ }^{6} \mathrm{LI},{ }_{\mathrm{B}}\right)_{\mathrm{H}}$ reactions. This thoproton transfer reaction, which has been extensively investigated in the lp-shell region, 3 offers a par. ticularly simple way of probing these isotopes.

Lithium ions (produced in an internal PIG im. source) were accelerated in the 8s-inch cyclotron. A 93.3-MeV bean energy was chosen for this experimont because of the extrembe kinesatic conditions and the highly negative Q-values (which for the simplest particle breakup thresholds correspond to -17.77 MeV for the ${ }^{4} \mathrm{H}$ syster and $-25.02 \mathrm{MeV}$ for the $S_{H}$ systent), ${ }_{B}$ reaction products were detected in a standard three-counter-telescope; adequate ldenti fication of the ${ }^{8} \mathrm{~B}$ isotopes was accomplisted off. line by cross-gating the ratio of the two independent particle identification slgnals generated by each of the two thin transaission detectors. At each set of angles data were collected sequentially from a $7_{L i},{ }^{6} i,{ }^{2} \mathrm{C}$, and 100 target. Since carbon and oxygen are fojor target conteminants, this procedore assured an accurate calibration of the spectra from reactions induced on lithiu.

An energy spectrum of the ${ }^{6} L i\left({ }^{6} L 1,{ }_{B}\right)^{4} \mathrm{H}$ reac- tion is show in Fig. Ia. The smooth curve corresponds to the phase-space distribution for the three body breakup $6 \mathrm{Li}+6 \mathrm{Li}+8_{\mathrm{B}}+\mathrm{t}+\mathrm{n}$; the thresholds for other roulti-body breakuy modes are Indicated. The observed enhancement above this phase-space distribution can be attributed to the know $1+n$ final state interaction corresponding to transitions to the 2 ground state of ${ }^{\mathrm{H}}$ with possible contributions, from transitions to probable 1 and $0^{\circ}$ levels in ${ }_{H}$ (these states are a11 broad). This enhancement was seen with appropriate kinospatics at all of the forr angles studied, and assuming that all of the conts above phese space correspond to this transitior, then the observed yield is $-4 \mathrm{\mu b} / \mathrm{sr}$ (c.m.).

In contrast to these results, the spectrun of the $7_{\mathrm{Li}}\left({ }^{6} \mathrm{~L},{ }^{8} \mathrm{~B}\right) \mathrm{S}_{\mathrm{H}}$ reaction, displayed in $\mathrm{Pig}$. $1 \mathrm{~b}$, shows no obvious evidence for a strong final-state interaction in the $S_{H}$ system; this spactrum was accurulated for a total of $6200 \mu \mathrm{C}\left(\mathrm{Li}^{3+}\right)$, a factor of 6.5 more than for the $4 \mathrm{H}$ spectrum. The counts seen akove the solooth curve drawn in Fig. Ib, which corresponds to the phase space distribution for the four-body breakm $6 \mathrm{Li}+7_{\mathrm{Li}}+{ }^{\mathrm{B}}+\mathbf{t}+\mathbf{n}+\mathbf{n}$, can arise from contributions from other multi-body breakup chamels, such as the three-body breakup $8_{B}+t+$ (2n) or $8_{B}+4 \mathrm{H}+\mathrm{n}$, or fron the $\left({ }_{\mathrm{Li}}, \mathrm{B}_{\mathrm{B}}\right.$ ) reaction on carbon and oxygen contaninants in the ${ }^{7} \mathrm{Li}$ target. This yield above the phase-space curve corresponds to $100 \mathrm{nb} / \mathrm{sT}-\mathrm{MeV}\left(\mathrm{c}, \mathrm{a}_{4}\right)$ in contrast to the ${ }^{4} \mathrm{H}$

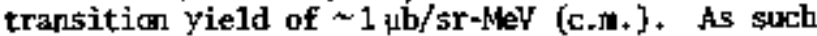
there is no indication fn these data, or in the 


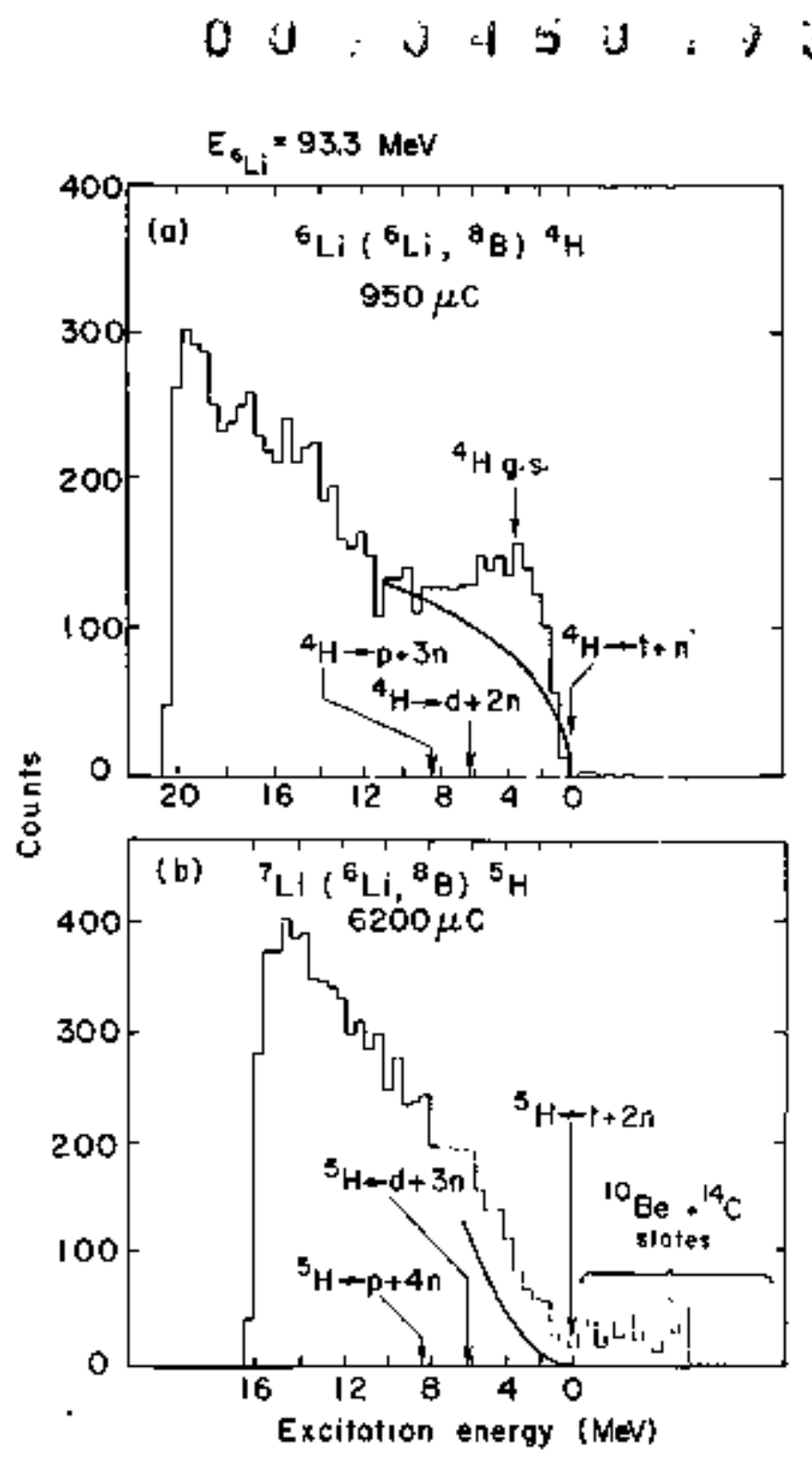

investigation at three other angles, for any narrow $\mathrm{SH}$ state, which agrees with the results frcm studies of the ${ }^{9}\left(a,{ }_{\mathrm{B}}\right)^{5} \mathrm{~F}_{\mathrm{H}}$ reaction ${ }^{4}$ and the ${ }_{\mathrm{H}}(t, p){ }^{5} \mathrm{H}$ reaction. ${ }^{5}$

\section{Footnotes and References}

PTesent address: Energy and Resources Groum, University of California, Berkeley, CA 94705 .

${ }^{\dagger}$ Present address: Џniversity of Tel-Aviv, IsrasI.

1. For mass 4 see S. Fiarman and W. E, Meyerhof, Nucl. Phys. A206, 1 (1973).

2. For mass 5 see F. Ajzenberg-Selove and $T$. Isuritsen, Nuc1. Phys. A227, 1 (1974) and references therein.

3. R. B. Weisenmillex, N, A, JeIIey, K. H. Wilcox, G. J. Wozniak, and J. Cerny, Phys. Rev. C13, 1330 (1976).

4. R. L. McGrath, J. Cerny, and S. W. Cosper, Phys. Rer. 165,1126 (1968).

5. P, G, Young, R. H. Stokes, and G. G. Ohlsen, Phys. Rer. 173, 949 (1968).

Fig. 1. (a) An energy spectrum of the ${ }^{6} \mathrm{Li}\left({ }^{6} \mathrm{Li},{ }_{\mathrm{B}}^{8}\right)$ ${ }^{4} \mathrm{H}$ reaction $\left(\mathrm{E}\left({ }^{6} \mathbf{L i}\right)=93.3 \mathrm{MeV}\right)$ collected at $\theta_{1}=14.7^{\circ}$ for $950 \mu \mathrm{C}$. These data are four channe1 stiph and the curve corresponds ta three-body phasespace (see text). (b) an energy spectron of the $\gamma_{\mathrm{Li}}\left({ }_{\mathrm{Li}},{ }_{\mathrm{B}\}}{ }_{\mathrm{H}}\right.$ reaction $\left(\mathrm{E}\left({ }^{6} \mathrm{Li}\right)=93.3 \mathrm{MeV}\right)$ collected at $\theta_{1 \mathrm{~h}}=14.7^{\circ}$ for $6200 \mathrm{\mu C}$. These dats are four chennel sums and the curve corresponds to four-body phase-space. The $10_{\mathrm{Be}}$ and $14_{\mathrm{C}}$ levels arise from ${ }^{2} \mathrm{C}$ and 160 contaminants in the target (see text).

(XBL $768-3907)$

\section{THE $\left({ }^{9} \mathrm{~B},{ }^{8} \mathrm{~B}\right)$ REACTION AND THE UNBOUND NUCLIDE ${ }^{10} \mathrm{Li}$}

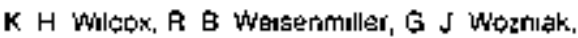

$N A$ Jelly, $D$ Ashory, $\neq$ and Joseph Cerny

Mass measurements of new highly neutron-excess nuclel provide important tests of the theoretical relations used to predict the limits of nuclear stability and the onset of new nuclear decay modes. 1 Experimental masses in the very light nuclei have proved particularly interesting since large discreparkies have been found between predicted and observed mass-excesses. If one excluxtes manyneutrom systems and hydrogen isotopes, all $\mathrm{T}_{z}=2$ nuclei in the Iight elements are nuxleon stable and have knowat masses with the exception of $10 \mathrm{Lj}$, which has been established to be particle umbound 2,3 hut for which a moss-excess has not been determined. By emloying the $\left|\Delta T_{7}\right|=3 / 2$ reartangement ryaction, $\left.{ }^{9} \mathrm{Be}, 8 \mathrm{~B}\right)$, we have pröduced lo Li via the $\mathrm{Ge}\left({ }_{\mathrm{Be}},{ }^{8} \mathrm{~B}\right)$ $\mathrm{tD} \mathrm{E}$ reaction.
A 121 Hey ${ }^{9} \mathrm{Be}^{+3}$ bean frod the lawrence Berkeley laboratory \&g-inch cyclotron was used to bombard a 0,68 m/s/on ${ }^{2}{ }_{\mathrm{Be}}$ foil. Beryllium vapor was supplied to the arc by support-gas-incuced sputtering of a piece of Be netal located just outside of the arc. ${ }^{3} \mathrm{~B}$ exit particles were identified by a detector telescope, subtending a solid angle of $0.3 \mathrm{msr}$, consisting of tho transmission (AE) detectors, 53 and 45 jm thick, a $210-\mu m \mathrm{E}$ detector, and a S00-um reject detector. The method of data handling has been described previous $1 y^{A}$ and inwolves in part a comparisen of two particle identification signals to reduce background; this comparison eliminated $\sim 25 \%$ of the ${ }^{9} \mathrm{Bo}-$ induced events.

Due to the low y leld of the $\left({ }^{9} \mathrm{Be},{ }^{8} \mathrm{Be}\right) \mathrm{re}-$ 
action, it was necessary to roduce the backsround from target contaminants and randon pulse pileup by additionolly requiring the $8 \mathrm{~g}$ particles to be in coincidence with an event in a recoil telescope. The recoil telescope subtender a solid angle of $15 \mathrm{msr}$ and consisted of $\mathrm{SE}$ and $\mathrm{E}$ detectors of thickness 11 and 48 Lin, respectively, backed by a 500-un reject detector. Figure 1 shows schematically the location of the detectors in the scattering chamber. A large recoil solid angle is required becasuse the recoiling ${ }^{2} \mathrm{Li}$, nucleous decays in flight

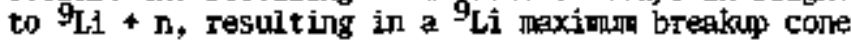
angle of about 10 degrees in the laboratory. The thickness of the $\mathrm{Ae}$ detector was chosen to eliminate background fromb the ${ }^{12} \mathrm{C}\left({ }^{9} \mathrm{Be},{ }^{8} \mathrm{~B}\right){ }^{13} \mathrm{~B}$ and $16_{0}\left({ }_{\mathrm{Be}}, \mathrm{B}_{\mathrm{B}}\right) 1 \mathrm{~N}_{\mathrm{N}}$ reactions, induced on target contaminants, by stopping the $13_{\mathrm{B}}$ and $17 \mathrm{~N}$ recoil nuclei while permitting the ${ }^{9}$ Li recoil danghters to pass through into the E detector. Fast timing techniques were used in establishing the coincidence between the $\mathrm{B}_{\mathrm{B}}$ and its associated recoil and to eliminate inter-bean-burst pilenp. (A more detailed discasston of the experimental technique is given in Ref. 5.).

An energy calibration for the $\left({ }^{9} \mathrm{Be},{ }^{8} \mathrm{~B}\right)$ reaction was abtainad by observing at smati angles the ${ }^{4} \mathrm{~B} \mathrm{Ca}\left({ }^{6} \mathrm{Li},{ }^{B} \mathrm{~B}\right){ }^{38_{A}}$ reaction to the ground and first excited (2.17 $\mathrm{kov}$ ) states of $38 \mathrm{Ar}$, initiated by an $80.6 \mathrm{keV} 6 \mathrm{~L}^{+2}$ beam. In addition the ${ }^{2} 8_{\mathrm{S}}\left({ }^{6} \mathrm{Li},{ }^{8} \mathrm{~B}\right){ }^{26} \mathrm{~kg}$ and ${ }^{16} \mathrm{OO}\left({ }^{6} \mathrm{Li},{ }_{\mathrm{B}}\right)^{14} \mathrm{C}$ ground state reactions were used. These reactions have reasonable cross section $(5-10 \mu \mathrm{b} / 5 \mathrm{r})$ and populate discrete states yielding ${ }^{8} \mathbf{B}$ with energies between 62 and $71 \mathrm{MVV}$, elose to the entergy of $-75 \mathrm{MeV}$ expected from the ${ }^{\mathrm{Be}}\left({ }^{9} \mathrm{Be},{ }_{\mathrm{B}}{ }^{10} \mathrm{li}\right.$ ground state reaction at $14^{\mathrm{a}}$.

The ${ }^{12} \mathrm{C}\left({ }^{9} \mathrm{Be},{ }^{8} \mathrm{~B}\right)^{13} \mathrm{~B}$ reaction was also studied separately, both to obtain a calibration point at higher energies and to study the yield of the ( ${ }^{9} \mathrm{Be}$,

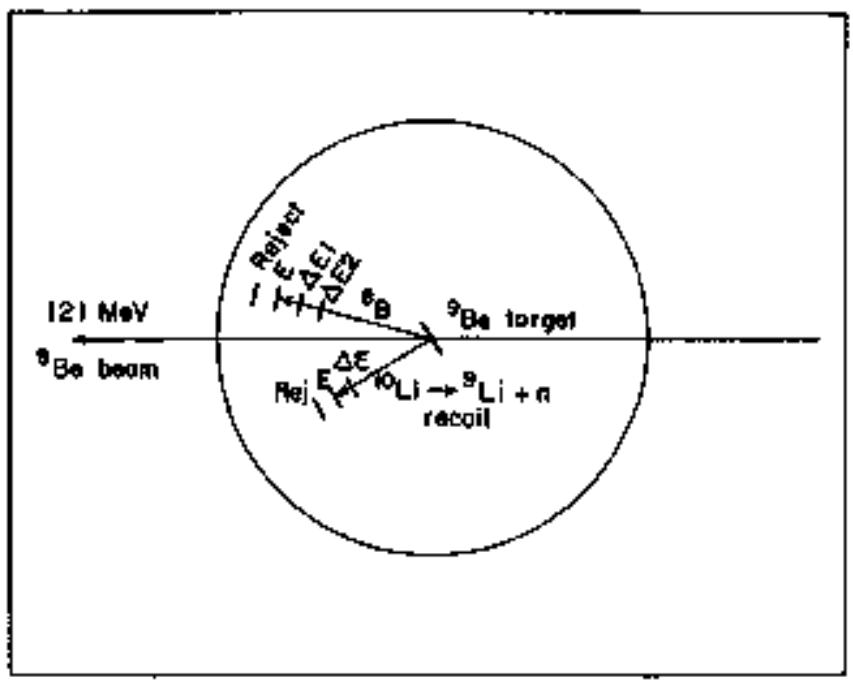

Fig. 1. Schematic draning of the scattering chamber setup showing orientation of the detectors and target; the solid angles of the ${ }^{8} \mathrm{~B}$ and recoil telescopes were 0.3 and I5 msr, respectively.

(XBL 758-3663) [Part (a) only]
$B_{\mathrm{B})}$ reaction on a major target contaminant. A $8_{\mathrm{B}}$ energy spectrum from this reaction at $14^{\circ}$ is shown in Fig. 2 (a). The ground state popslation is too low ( $200 \mathrm{rb} / 5 \mathrm{r}$ ) to be usefur for calibration purposes. However, the sharp peak at $E_{1}=81 \mathrm{MeV}$ provided a useful point. This, together with the ( ${ }_{\mathrm{Li}},{ }_{\mathrm{B}}$ ) data, established an accurate energy calibration.

A spectrom of all the $8_{\mathrm{B}}$ data frow resctions on the $9 \mathrm{Be}$ target, representing 6950 uC of $\mathrm{Be}$ bear (fully stripped), is shown in Fig. Z(b). The same data, with the additional requirerent that each coldnt be in coincidence with an event in the recosl detector (located at $33^{\circ}$ in the laboratory), are seon it Fig. 2 (c). With this requirement, elimination of ${ }_{\mathrm{B}}$ muclei arising from reactions or major target contaminants was essentially complete. Further, aithough isotope resolution in the recoil particle identification spectra was poor, it was adequate to show that all the associated recoils from the events in Fig. 2 (c) appear in the region expected for ${ }^{\mathrm{Li}}$ nuclei.

Wh have assumed of necessity that the observed veak arises predominantly from the population of 10́Li in its ground state. (Based on sinfule particle-particle, hole-hole theorests, the two lowest states of ${ }^{10} \mathrm{Ll}$ could be vory similar to those of ${ }^{12} \mathrm{~B}$, in which they are separated by $0.95 \mathrm{HeV}+$ ) The width of this peak, approximately $1,2 \pm 0.3 \mathrm{MeV}$ FAMM, (c.m.), is of the correct order of magnitude for a single uboumd state on the

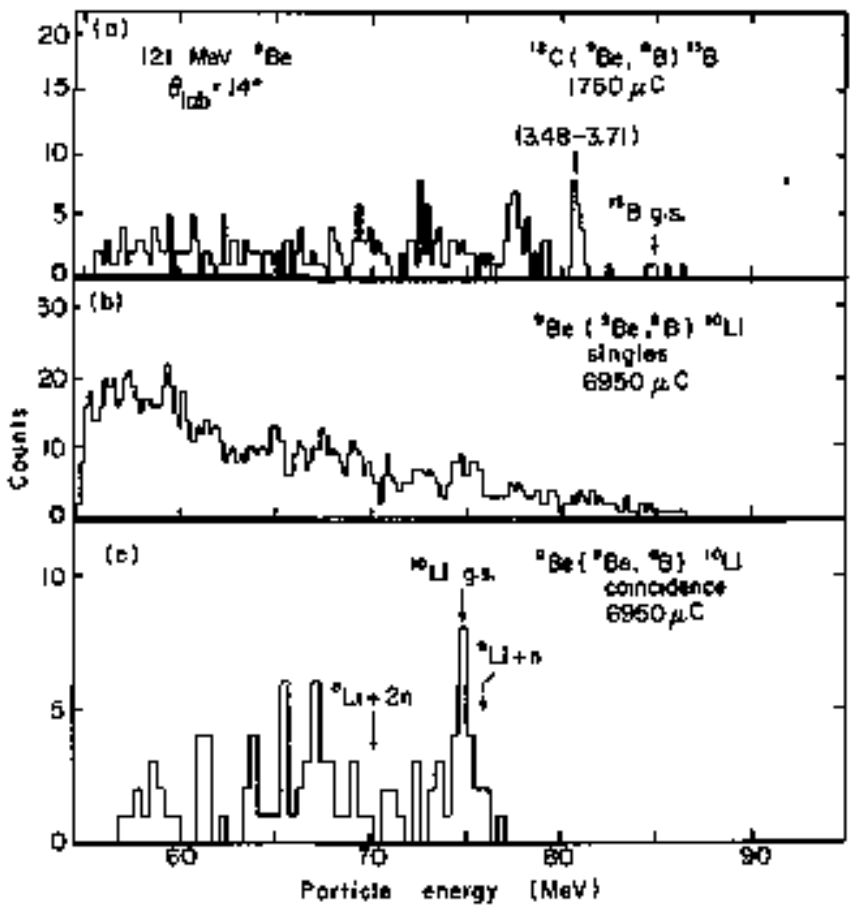

F1g. 2. Several energy spectra resulting frot the bonbardiaent of ${ }^{12} \mathrm{C}$ and $9 \mathrm{Be}$ targets by a $121 \mathrm{kHY}$ $9_{\mathrm{Be}}$ beam and observed at $74^{\circ}$ in the 1aboratory; (a) The $12 \mathrm{C}\left({ }^{9} \mathrm{Ae},{ }^{8} \mathrm{~B}\right) 13 \mathrm{~B}$ reaction; (b) the $9_{\mathrm{Be}}\left({ }^{9} \mathrm{Be},{ }^{8}\right){ }^{10} \mathrm{~L}$ reactich sipgles events, and (c) a two-channel sim of the $\mathrm{ge}\left(9_{\mathrm{Be}}, 8 \mathrm{~B}\right) 10_{\mathrm{Li}}$ reaction coincidence events.

XEL 758-3664) 
basis of neutron-tumeling calculations. At this angle of $14^{\circ}$ the cross section for population of the ground state of $10 \mathrm{Li}$ was $30 \mathrm{nb} / \mathrm{sr}$ (c.m. The observed Q value for the ${ }_{\mathrm{Be}}\left({ }_{\mathrm{Be}},{ }_{\mathrm{B}} \mathrm{C}_{\mathrm{L}} \mathrm{i}\right.$ ground state reaction has $-34.06 \pm 0.25 \mathrm{MeV}$,

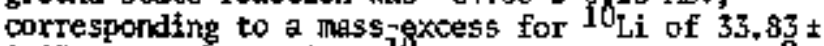
$0.25 \mathrm{MeV}$. The nucleus ${ }^{10} \mathrm{Li}$ is thts tonbound to $9_{\mathrm{Li}}$ plus a neutrog by $0.80 \pm 0+25 \mathrm{MeV}$ (using the recent revieasurement of the ${ }^{9}$ Li mass), somewhat mort unbound than the current prediction of $0.21 \mathrm{MeV}$ based on the Garvey-Kelson method.

for attempt was also made with the $\left({ }^{9} \mathrm{Be},{ }_{\mathrm{B}}\right)$ reaction to deternine the nass-exiess of the $\mathrm{T}_{Z}=$ $5 / 2$ nuclide $15_{B}$ by reactions on a $14_{C}$ target. A $121 \mathrm{NeV}{ }^{9} \mathrm{Be}$ bean was again used, bombarding a $100 \mathrm{\mu g} / \mathrm{cm}^{2}$ carbon target enriched to $\sim 80 \%$ in $14_{C}$. The experimental technjque was similar to that described for the ${ }^{9}\left({ }^{9} \mathrm{Be},{ }_{\mathrm{B}}{ }^{10}{ }_{\mathrm{Li}}\right.$ reaction, except that a single recoil counter of about 20 lin thickness, backed by a reject detector, ws used. ${ }^{8} \mathrm{~B}$ particles from the relatively high-yield $12 \mathrm{C} /{ }_{\mathrm{Be}}$. $\left.{ }_{\mathrm{B}}\right)^{13} \mathrm{~B}$ reaction were not sufficiently eliminated by the recoil angle requirement to perrit positive identification of any peaks due to the formation of $15 \mathrm{~B}$. An upper 1init of $50 \mathrm{nb} / \mathrm{sr}$ (c.m.) can be set for the ${ }^{1} \mathrm{C} C\left({ }^{9} \mathrm{Be},{ }_{\mathrm{B}}\right){ }^{1} \mathrm{~S}_{\mathrm{B}}$ ground state reaction at $14^{\circ}$ in the laboratory. (Also see Ref. 5.)

These results on ${ }^{10} \mathrm{Li}$ add another $\left|\Delta \mathrm{T}_{2}\right|=3 / 2$ nuclear rearrangement reaction as demonstrated tools to elploy (albeit not always successfully) in studying the spectroscopy of new highly neutronexcess light muclei.

\section{Footnotes and References}

*Condensed from Phys. Letters 59B, 142 (1975).

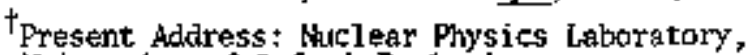
University of Oxford, England.

Femanent Address: Tel-Aviv University, Israel.

1. J. Cemy, Proc. Int. Conf. Reactions between conplex Nuxlei, dashvillê, 1974, ea. $R$. L. Pobioson, F. R. NcGowan, J. B. Ball, and J. H. Hamilton, (North-Holland, Ams terdara/American Elsevier, N. Y., 1974) Vo1. 2, p. 483.

2. A. M. Poskanzer, S, W, Cosper, E. K. Hyde, and J. Cerny, Phys, Rev, Letters 17, 1271 (1966).

3. J. D. Bowmen, A+ M. Poskanzer, R. G. Korteling, and G. W. Butler, Phys. Rev. C9, 836 (1974).

4. K.H. Wilcox, N. A.Jelley, G. J. Wozniak, R. B. Wefsermillor, H. L. Harney, and J. Cerryy, Phys. Rev. Letters 30, 866 (1973).

5. K. H. Wilcox, Lawrence Berkeley Laboratory Report No. LEL-4029 (Ph. D. Thesis), 1975 (uptulished.

6. R. B. Weisenut1ler, K. H. Wilcox, N. A. Jelley, G. J. Mozniak, and J. Cerry, to be published.

7. E. Kashy, W. Benenson, D. Mue1ler, R. G. H. Robertscin, and D. R. Goosman, Phys. Rev. CIl, $1959(1975)$.

8. N. A. Jelley, J. Cerny, D. P. Stahe1, and $K_{+} H_{+}$ Wilcox, Phys. Rev. C11, 2049 (1975).

\section{THE ( $\left.{ }^{9} \mathrm{Be},{ }^{8} \mathrm{Be}\right)$ REACTION AT $50 \mathrm{MeV}$}

D P Slahil. G J Wozniak, B D Jellema,

MI S Zsman. A Jahn, and Joseph Cerry

In recent years, single nuxleon transfer reactions induced by light heavy ions, such as ${ }^{7} \mathrm{Li}$, ${ }^{12} \mathrm{C},{ }^{13} \mathrm{C}, 14 \mathrm{~N}$, and 160 , have gained increased attention for muclear reaction and structure investigations. The ${ }^{9} \mathrm{He},{ }^{\mathrm{Be}}$ ) reaction has not been studied, mainly because of a lack of a suitable 8Be detection system. This reaction, however, has several experimental advantages over other hesvy ion one-neutron stripping reactions. Because the

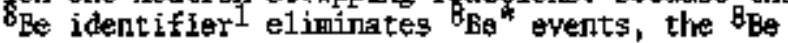
energy spectra lack the shadow peaks observed in other reactions that produce exit particles with wound excited states. Furthermore, the $8 \mathrm{Be}$ events can be clearly distinguished from the opore copiouselasticaly and inelastically scattered 9 Be particles. With these arivantages in mind, we have surveyed the ( ${ }_{\mathrm{Be}},{ }_{\mathrm{B}} \mathrm{B}$ ) reaction on several ip and 2s-1d shell targets.

The ${ }^{9} \mathrm{Be}\left(2^{+}\right)$ions, obtained from a PIG source, were accelerated by the 88 -inch ovilotion to 50 HeV. Beam currents of 100-200 nd (as 4+) were readily delivered on target. The ${ }^{3}$ Be particles $\left(\tau-10-16_{s}\right.$ ) were detected by mears of their breaktp a particles. A position sensitive detector (PSD) was used to measure the direction and energy of the original $B_{B e}$ and split af detectors were enployed to retuce random a-a background. An energy resolution of $\sim 4 \mathrm{D}$ (h kev (FWH) was achieved with an angular acceptance of $\leadsto 1^{\circ}$.

Sincultaneously with the ${ }^{8} \mathrm{Be}$ particles, ${ }^{9} \mathrm{Be}$ elastic scattering was measured utilizing a singlo $5 \times 1 \mathrm{~cm}^{2}$ PSD. The collifhator consisted of a tantalual plate with $B$ vertical slits, each with a horfzontal acceptance angle of $\sim 0.5^{b}$. The energy sigral was routed by the position slgnal thus allowing us to simultaneously measure elastic scattering at 8 angles each separated by $1^{\circ}$.

${ }^{8}$ Be energy spectra of the $\left({ }^{\mathrm{Be}}, 8_{\mathrm{Be}}\right.$ ) reaction on $12_{\mathrm{C}}, 160,28 \mathrm{Si}$ and $40 \mathrm{Ca}$ are shown in $\mathrm{Fig} .1$. They exhibit clearly the high selectivity of the reaction. only states with an appreciable singleparticle strength as known frow $(d, p)$ reactions are populated. In order to test whether current direct reaction madels, such as the EMRA, can account for 

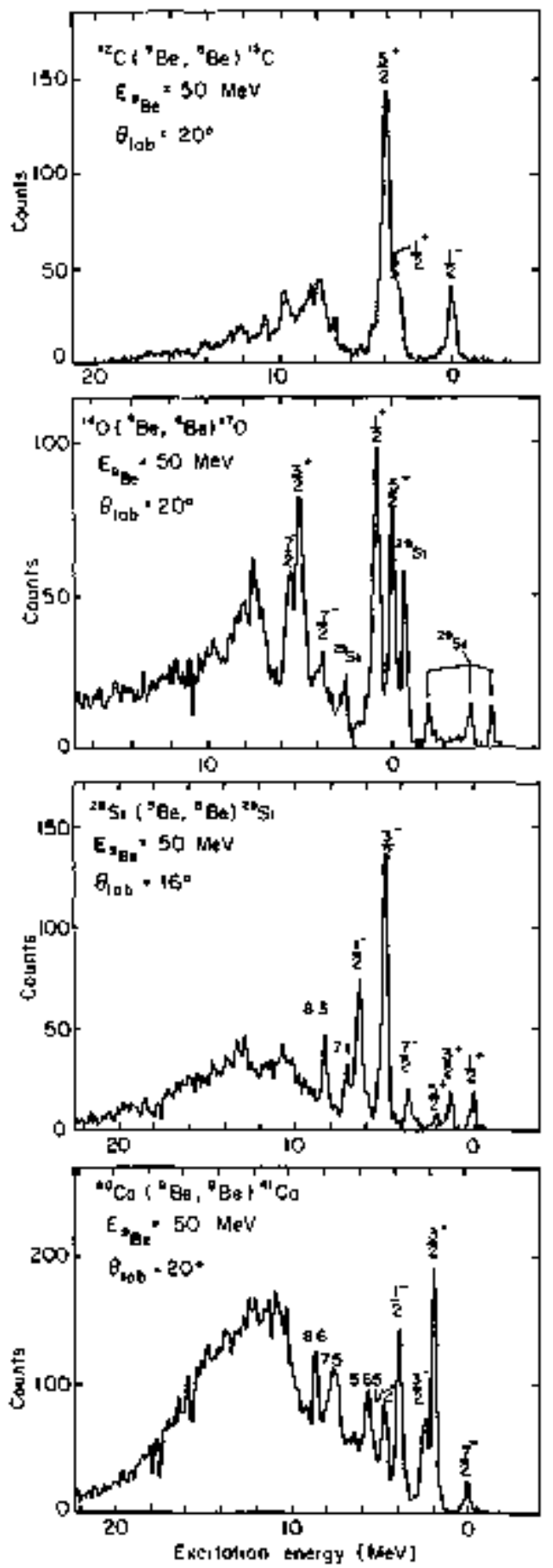

Fig. 1. Energy spectra of the ( $\left.{ }^{9} \mathrm{Be},{ }^{8} \mathrm{Be}\right)$ reaction on $12 \mathrm{C}, 160,2 B \mathrm{si}$ and $40 \mathrm{Ca}$. (XBL 767-8313)

the kinematics of the reaction, angular distributions for the $28 \mathrm{si}$ (9 ${ }_{\mathrm{Be}}, 8_{\mathrm{Be}}$ ) $29 \mathrm{Si}$ and the $40 \mathrm{Ca}\left({ }^{9} \mathrm{Be}, 8_{\mathrm{Be}}\right) 41_{\mathrm{Ca}}$ reactions were measured and are show in Figs. 2 and 3. Whereas for the $2 \mathrm{~g}_{\mathrm{Si}}$ target the distributions display some diffraction structure, those for ${ }^{40 \mathrm{Ga}}$ are almost featureless, as is typical of low-eniergy heavy-ion reactions. The distributions were analyzed in terms of the exact finite-range distored-wave Born-approxination using the computer code LoLA. 2 The optical model

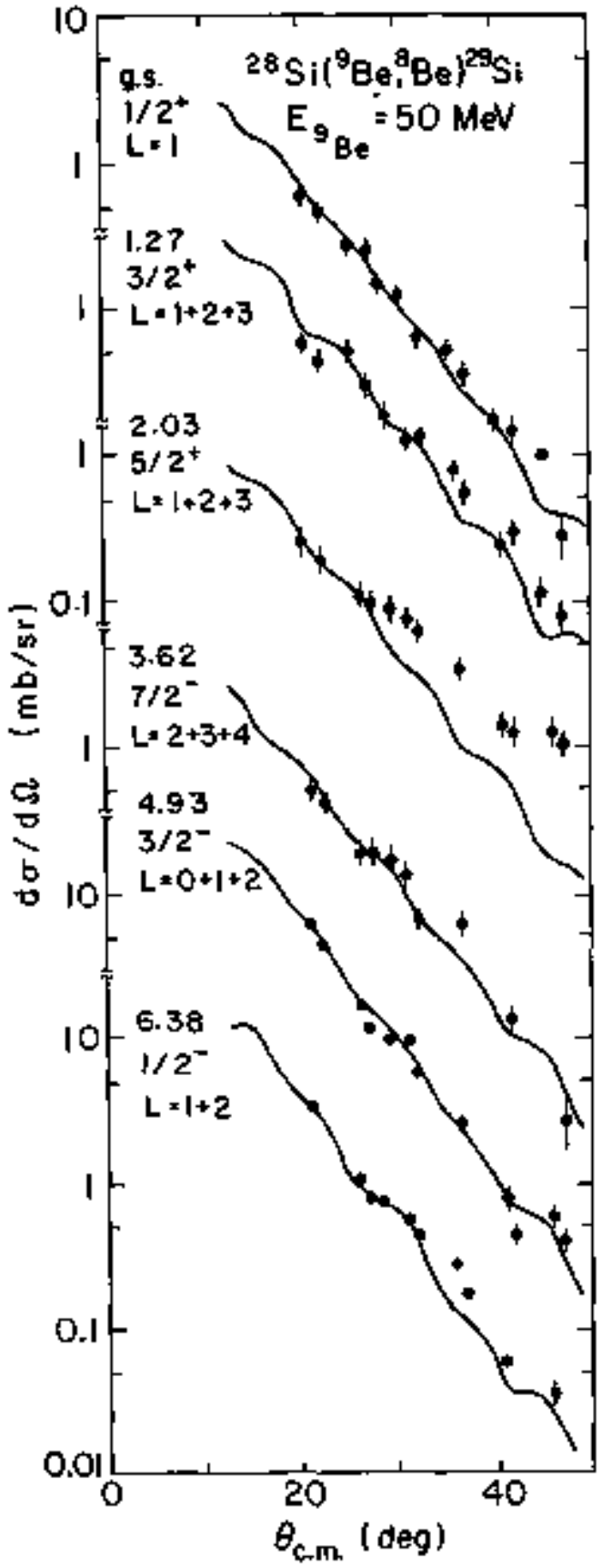

Fig. 2. Angular distributions of ${ }^{8} \mathrm{Be}$ from the ${ }^{2} \mathrm{Si}\left({ }_{\mathrm{Be}},{ }^{\mathrm{Be}}\right)^{29} \mathrm{Si}$ reaction. The solid lines represent exact finite-range DWRA calculations ustng optical model potential șet $v$.

XEL 755 -8314)

parameters were deteroined by fitting orr measured elastic scattering data with the searth code GENOA. ${ }^{3} \mathrm{~A}$ list of the extracted potentials is given in Table 1. In the DWRA colculations, the same parameter set was used for the initial and final channels. The results are shrown in Figs. 2 and 3, and the extracted spectroscopic factors are fisted in Table 2. For the spectroscopic fector of Be, the theoretical value 4 of 0.58 was used. For the 28 Si target, the shapes of the distributions are we1l reproduced, and the absolute and relative 


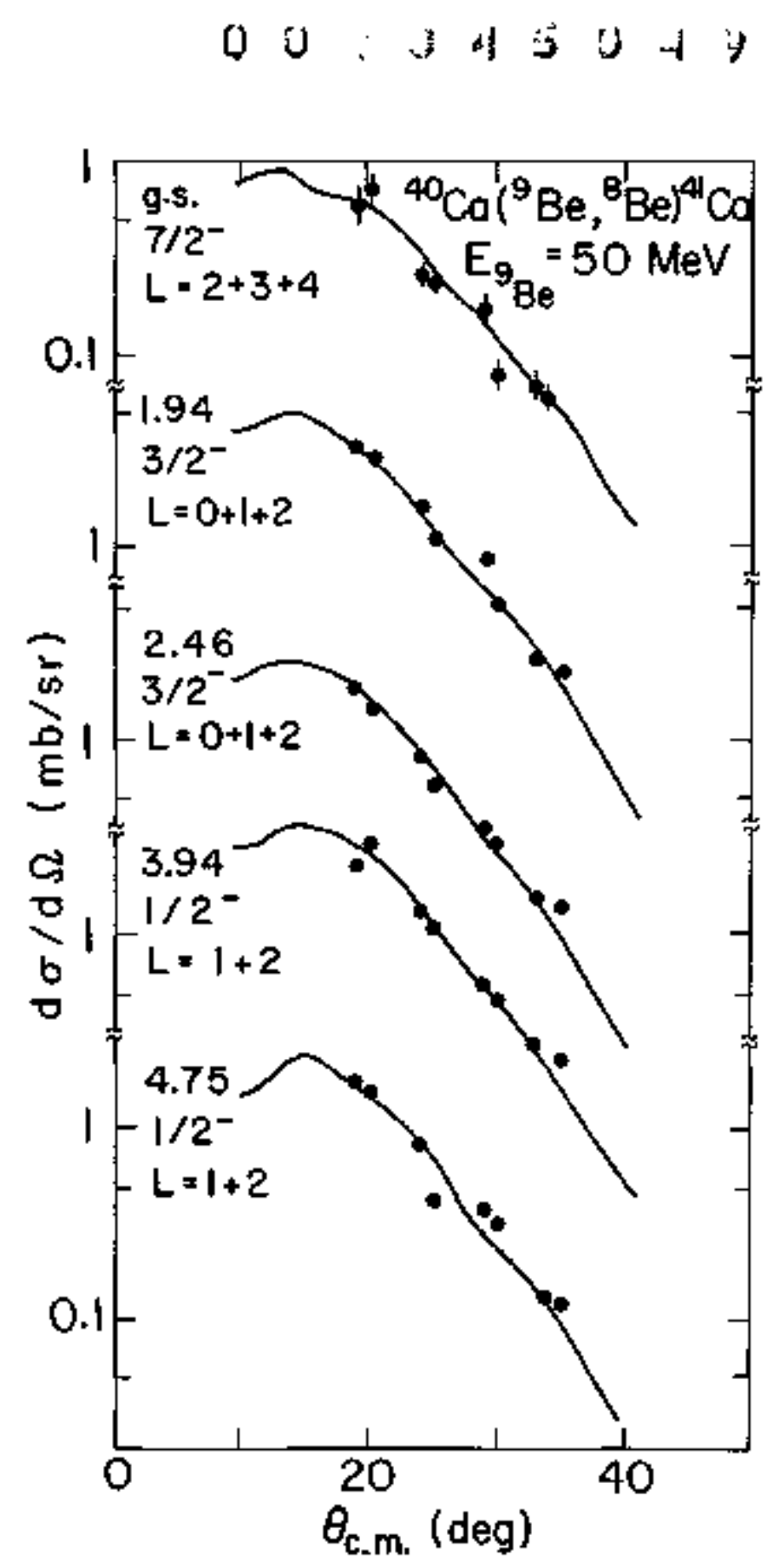

Fig. 3. Anpular distributions of ${ }^{8} \mathrm{Be}$ fron the $40 \mathrm{Ca}\left({ }_{\mathrm{Be}},{ }_{\mathrm{Be}}\right){ }^{4} \mathrm{Ca}$ reaction. The solid lines represent exact finite-range INBh calculations using optical nodel potential set VII.

(XRL $766-8325$ )

spectroscopic factors are in good agrement with the values determined from $(d, p)$ data. 5 Although for the $40 \mathrm{Ca}$ target the calculated shepes of the distributions fit the data well and the relative spectroscopic factors agree with the $(d, p)$ values, the magnitude of the predicted cross sections is too large, which is reflected in absolute spectroscopic factors about 3.5 times smaller than the corresponding $(\mathrm{d}, \mathrm{p})$ values, $\mathrm{A}$ possible explanation for this discrepancy is the fart that the momalization could not be done at the grazing angle of $\sim 12^{\circ}$ (c.m.), where the cross soction peaks, because of mechanical restrictions. It is kown from other heavy ion reactions that INBA calculations
Table 1. Optical model parangters for 50-kev elastic "Be scactering.

\begin{tabular}{|c|c|c|c|c|c|c|c|c|}
\hline Target & $\begin{array}{c}\mathbf{v} \\
0 \leqslant \mathrm{~V}]\end{array}$ & $\begin{array}{l}T_{\mathrm{DR}}{ }^{\mathrm{s}} \\
\text { (fin) }\end{array}$ & $\begin{array}{c}a_{R} \\
\left(\mathrm{fin}^{\prime}\right)\end{array}$ & $\underset{(N \leq V)}{n}$ & ${ }^{-} \tau_{0 I}$ & $\begin{array}{c}\mathrm{a}_{1} \\
\text { (fii) }\end{array}$ & $\begin{array}{l}r_{0 c}{ }^{7} \\
\text { (f) }\end{array}$ & Set \\
\hline \multirow[t]{3}{*}{${ }^{12} \mathrm{C}$} & 30,9 & 1.84 & 0.98 & s.50 & 2.78 & 0.45 & $1 . z$ & $t$ \\
\hline & 85.5 & 1.93 & 0.64 & 23.7 & 1.81 & 0.86 & 1.2 & J \\
\hline & 114.1 & 1. 记 & $\mathrm{D}, \mathrm{aE}$ & $\mathrm{H} 2,0$ & 2.64 & 0.52 & 1.2 & III \\
\hline \multirow[t]{3}{*}{$2 \mathrm{~B}_{\mathrm{Si}}$} & 35.2 & 1.83 & 0,70 & 6.5 & 2.66 & 0.70 & 1.2 & IV \\
\hline & 35.8 & 1.68 & 0.64 & 15.8 & 2.07 & $6 . \$ 5$ & 1.2 & $\mathrm{~V}$ \\
\hline & 209.8 & 1.28 & 0.71 & 38.2 & 1.74 & 0.93 & 1.2 & vI \\
\hline \multirow[t]{2}{*}{${ }^{40} \mathrm{cos}_{\mathrm{c}}$} & 70.8 & 1.42 & 0.73 & 9.7 & 2.06 & 0.82 & 1.2 & VII \\
\hline & 322.7 & 1.03 & 0.77 & 22,0 & 1.93 & 0.89 & 1.2 & YIIII \\
\hline
\end{tabular}

Table 2. Spectroscopic factors.

\begin{tabular}{|c|c|c|c|c|c|c|}
\hline Muclesus & $\begin{array}{l}\text { Level } \\
\text { (RteV) }\end{array}$ & $J^{\pi}$ & $\mathrm{S}_{\mathrm{abs}}^{9 \mathrm{Ba}}$ & $\begin{array}{l}{ }^{8} \text { te) } \\
s_{\text {rel }}\end{array}$ & $S_{a b s}$ & $\mathrm{p}_{\mathrm{r} \rightarrow 1}^{9}$ \\
\hline \multirow[t]{6}{*}{$29 \$ i$} & g.s. & $1 / 2^{+}$ & 0.42 & $1+00$ & 0.53 & 1.00 \\
\hline & 1.27 & $3 / 2^{+}$ & 0.70 & 1.67 & 0.74 & 1.40 \\
\hline & 2.03 & $5 / 2^{+}$ & 0.11 & 0.26 & 0.12 & 0.23 \\
\hline & 3.62 & $7 / 2^{\circ}$ & 0.30 & 0.71 & 0.38 & 0.72 \\
\hline & 4.93 & $3 / 2^{-}$ & 0.48 & 1.14 & 0.56 & 1.06 \\
\hline & 6.38 & $1 / 2^{-}$ & 0.51 & 1.21 & 0.53 & 1.00 \\
\hline \multirow[t]{5}{*}{${ }^{41} \mathrm{Ca}$} & g.5 & $7 / 2^{-}$ & 0.27 & 1.00 & 0.95 & 1.00 \\
\hline & 1.95 & $3 / 2^{-}$ & 0.23 & 0.85 & 0.70 & 0.74 \\
\hline & 2.46 & $3 / 2^{-}$ & 0.10 & 0.37 & 0.25 & 0.26 \\
\hline & 3.94 & $1 / 2^{*}$ & 0.20 & 0.74 & 0,67 & 0.71 \\
\hline & 4.75 & $1 / 2^{-}$ & 0.10 & 0.37 & 0.19 & 0.20 \\
\hline
\end{tabular}

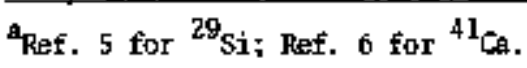

frequently overestimate the cross section at angles greater than the grazing angle. In spite of this probIem, the DhRA reproduces correctly the observed Q-value and 2 -transfer dependence of the cross sectimn. There is a clear enhancement for tranșitions with Q-values arolmd Qopt $=0$. Since the typical ground state $\mathrm{Q}$ values for the $\left({ }_{\mathrm{Be}}, 8_{\mathrm{Be}}\right)$ reaction are positive, a preferential population of excited states can be observed. In $29 \mathrm{Si}$ and $41 \mathrm{Ca}$ states up to $\sim 8 \mathrm{MeV}$ were strongly populated. The angular monentum matching is such that transitions involving sma11 $l$-transfers are favored, and the mismatch for large $z$-transfers results in a significant reduction of the yield, as seen in the reaction to the groumd state of $41 \mathrm{Ca}$ for which $t=2,3$, and 4 .

Thus, the $\left({ }^{9} \mathrm{Be},{ }^{8} \mathrm{Be}\right)$ reaction pronises to be a useful spectroscopic tool for studying low-spin single-particle states at high excitation, even at tandem energies where the majority of heavy ion neutron transfer reactlons to not give large yields. 
Rmferences

1. C. J. Hozriak, N. A. Jelley, D. P. Stahel, and J. Cermy, A Large Solld Angle Be Identifier, this Anrual Report.

2. R. M. DeVries, Phys. Rev. C 8, 951 (1973).

3. F. G. Perey, Optical-kbdel Search Code Gemoa (urmublished).

4. S. Cohen and D, Kurath, Nucl, Phys. Alol, 1 (1967).

5. M. C. Mermaz et a1., Phys. Rev. C 4, 1778 (197I).

6. D. C. Kocher and W. Haeberli, Muc1. Phys, A196, 225 (1972)

\section{"DOUBLE CHARGE EXCHANGE" REACTIONS ${ }^{26} \mathrm{Mg}\left({ }^{18} \mathrm{O},{ }^{16} \mathrm{Ne}\right)$ ${ }^{26} \mathrm{Ne}$ AND ${ }^{24} \mathrm{Mg}\left({ }^{11} \mathrm{~B},{ }^{11} \mathrm{Li}\right)^{24} \mathrm{Si}$}

G KeKelss, HI Zismen, Joseph Cermy. D Scont. R Jahn,

$f$ Alzenberg-Seilove, $D$ Hendre, M Buenerd, and $C$ Olmer

When measuring nuclear masses fat from stability, a potentially important techinique for reaching unlonown masses is the "double charge exchange" reaction. Two such reactions are $\left({ }^{1} 1_{B}, I_{L i}\right)$ and $\left(1 B_{0}, l_{k}\right)$. Q-values for these reactions are -40 to -60 HeV for the former and -20 to $-30 \mathrm{Mev}$ for the latter.

These reactions have been attempted uging the ongnetic spectrometer at the 88-inch cyclotron: the first to measure the mass of $26 \mathrm{kl}$ and the second to measure the mass of $245 \overline{\mathbf{i}}$. Prior to attempting the $264 \mathrm{gr}(18 \mathrm{0}, 18 \mathrm{Ne}) 26 \mathrm{Ne}$ reaction, the 24 $4 \mathrm{k}$ (180, $18 \mathrm{kl}) 24 \mathrm{ke}$ reaction was observed in order to evaluate the reaction cross section for double charge exchange, As shown in Fig, 1, the cross section for the $24 \mathrm{bg}\left(18_{0}, 18 \mathrm{ke}\right) 24 \mathrm{ke}$ reaction to the ground state of $24 \mathrm{Ne}$ was found to be about $800 \mathrm{nb} / \mathrm{sr}$. In Fig. 2 the spectrum obtained from the $26 \mathrm{pg}(180,18 \mathrm{ke}) 26 \mathrm{ge}$ reaction is shown. The ground state eross soction has dropped to about $100 \mathrm{nb} / \mathrm{sr}$. The predicted ground state location is between two possible peaks, makjng a positive identification of the ground state difflcult, especially since fither poak would correspond to a sulbstartial error in the mass predictions of Jelley et al. 1 or those of Garvey and Kelson. 2 lue largely to the high background, the experiment will be repeated,

The ${ }^{24} \mathrm{~Hz}\left({ }^{I} 1_{\mathrm{B}},{ }^{\left.11_{\mathrm{Li}}\right)^{24}}{ }^{24}\right.$ was a150 performed at $114 \mathrm{kl}$ to atteint to measure the mass of $24 \mathrm{Si}$. Although $15,000 \mu \mathrm{C}$ of charge were collected at $0_{\mathrm{I}}=5^{\circ}$ and $6^{\circ}$, no discernible peak could be identified. An upper linit for the cross section of the $\left(1_{B}, 11_{L}\right)$ double charge exchange reaction to the ground state of $24 \mathrm{Si}$ can therefore be set at $3 \mathrm{nb} / \mathrm{sr}$.

Reactions of this type still appear to us to be very pronjising for measuring masses such as 120 , 30 $\mathrm{kg}$ and $345 i$. The major difficulty thus $f$ ar has been in maintaining sufficient beam on target for tho long periods of time required to measure nanobarn cross sections. Exforts are currently being devoted to increasing the amount of "steady-state" bean and to inproving our ability to take data at more formard lab angles, 3 where the cross sections are expected to he larger.

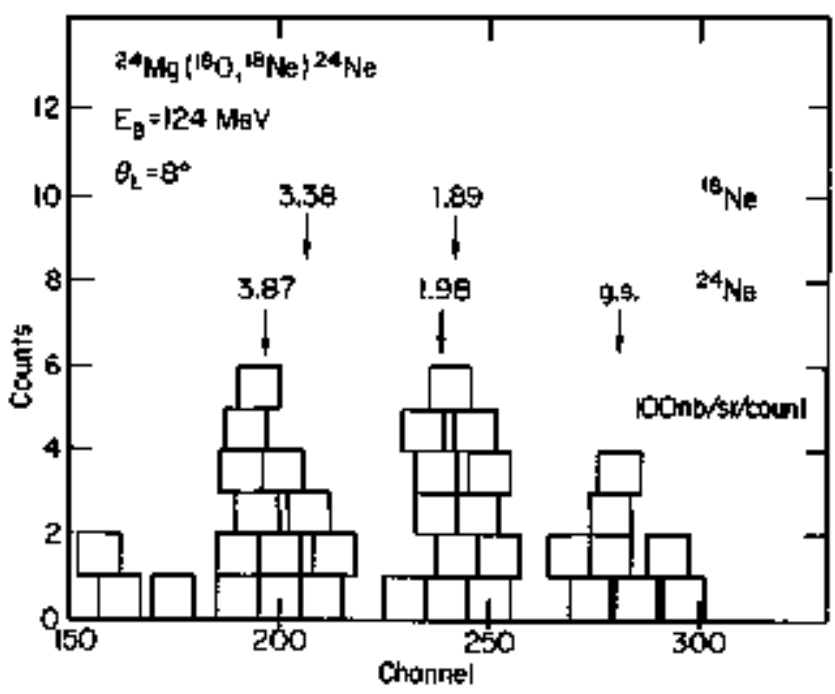

Fig. 1. Energy spectrun of the ${ }^{24} \mathrm{Mg}\left(18 \mathrm{o},{ }^{18} \mathrm{~N}_{\mathrm{Ne}}\right)^{24} \mathrm{Ne}$ reaction at $\mathrm{E}_{\mathbf{B}}=124 \mathrm{keW}$. (XBL'764-2746)

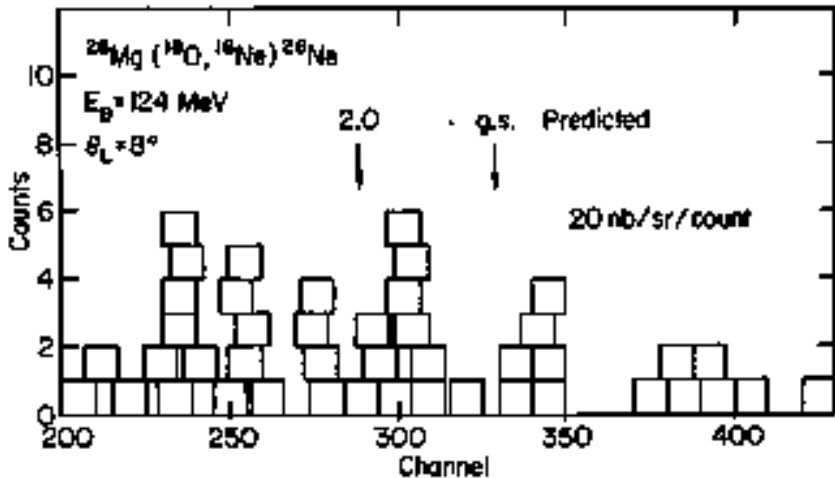

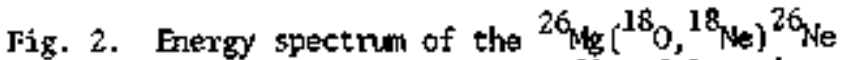
reaction at $\mathrm{E}_{\mathrm{B}}=12 \mathrm{deV}$. The predicted location was calculated using the $26 \mathrm{Ne}$ mass prediction of Jelley et al. 1

(XBL 764-2745) 


\section{Referencers}

1. N. Jelley, J. Cermy, D. Stahe1, and K. Wilcox, Physs. Rev. C4, 2049 (1975).

2. G. Garvey, W. Gerace, R. Jaffe, I. Talmi, and
I. Kelson, Rev. Mod. Phys, 41, s1 (1969); I. Kelsọn and G. Garvey, Phys. Letts. 23,689 (1966).

3. G. Kekelis, G. Gabor, D, Scott, and C. K. Gelbke, A Proportional Counter Tino Zero System, in this Annual Report.

\section{PRODUCTION OF "Li IN THE ("B, ${ }^{1 \dagger}$ Li) REACTION ON ${ }^{232} \mathrm{Th}$}

D K Scoti. M, Buenerd.' O L Henarle, G KeKelis, J. Mahoney, A menchaca-Focha,t and C Olmer

Since the theoretical predictions of the masses of exotic light ntelei are based on extrapolations from the valley of stability, it is clear that the most stringent test of these theories will cone from the study of nuclei close to the lintt of stability. Although spectacular aduances in detemining this $11 \mathrm{mit}$ for neutron-excess light maclei have been achieved by means of reactions on hegvy nuclei induced by bigh energy protons 1 and medilun energy heavy ions, 2 the measurement of nuclear masses even three or four neartrons removed from stability hes progressed very slowly. 3 In a recent experiment to measure the quss-excess of $29 \mathrm{dg}$ by the In transfer reaction $\left(11_{\mathrm{B}}, 8_{\mathrm{B}}\right)$ on $26 \mathrm{jg}$ the ground state cross section was only $15 \mathrm{nb} / \mathrm{sr}$. Because of the systematic decresse of cross sections with the transfer of a dreater number of incorrelated nucleons onto light targets, an extenșion to heavier isotapes is not promising.

The larger cross sections observed with heavy targets have recently been exploited to measure the nass of $210-$ also throe neutrons resoved from stability - by observing the grownd state

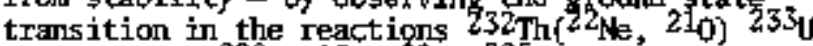
(Ref. 5) and $208 \mathrm{~Pb}(180,210$ ) $205 \mathrm{pt}$ (Ref. 6), in wich the cross sections were of order 1 kb/sr. An interesting question now is whether these methods can be extended to make mass neasurements at the 1init of stability, with currently available experimental techniques. In order to explore this possibility we have studied the proctuction of $11_{L i}$, the last stable isotope of 1 ithium, by the $\left(-2 p^{+2 n)}\right.$ transfer reaction $232 \mathrm{Th}\left({ }^{11} \mathbf{B},{ }^{1}{ }_{\mathrm{Li}}\right) 232 \mathrm{U}$ for which the ground-state Q value is accurately lonown from the measured mass-excess of ${ }^{11} \mathrm{Li}$. The g.s. cross section is coupared to that for producing 220 by essentially the same $(-2 p+2 n)$ transfer process $232 \mathrm{Th}\left({ }^{2} 2 \mathrm{Ne}, 220\right) 232 \mathrm{~J}$ which was used recentiys to measure the mass-extess of 220 .

The QSD spectroneter with a solid angle of 1.3 msr, was used to detect reactiong.prodicts arising from the bombardment of a 232 Th target, $4.7 \mathrm{mg} / \mathrm{cm}^{2}$ thick, with a $11_{\mathrm{B}}$ been of $114 \mathrm{MkV}$ produced at the 88-inch cyclotron. In order to identify ${ }^{1} 1_{L i}$ particles, protuced with cross sections of order nb/sr, it was necessary to make several independent particle identifications, The ragnetic rigidity (Bo) was measured with a position-sensitive Borkowski-Kopp counter, and the specific energy loss ( $d E / d x$ ) in a second propor. tional counter, In addition, the time-of-flight
(TOF) was detersined by a "tida-zero" detoctor consisting of an $870 \mathrm{\mu g} / \mathrm{cm}^{2}$ foil of $\mathrm{NE} 111$ at the entrance of the spextrometer and a large plastic scintillator mouted behind the focal plare proportional counters. An identification spectrum is shown in Fig. 1(a). Al though the $11_{\mathrm{Li}}$ events appear to be clearly separated, they are contaminated by a FOF tail from the adjacent, more ebudant $\mathrm{Li}^{+}$group. Theșe tails are clearly seen

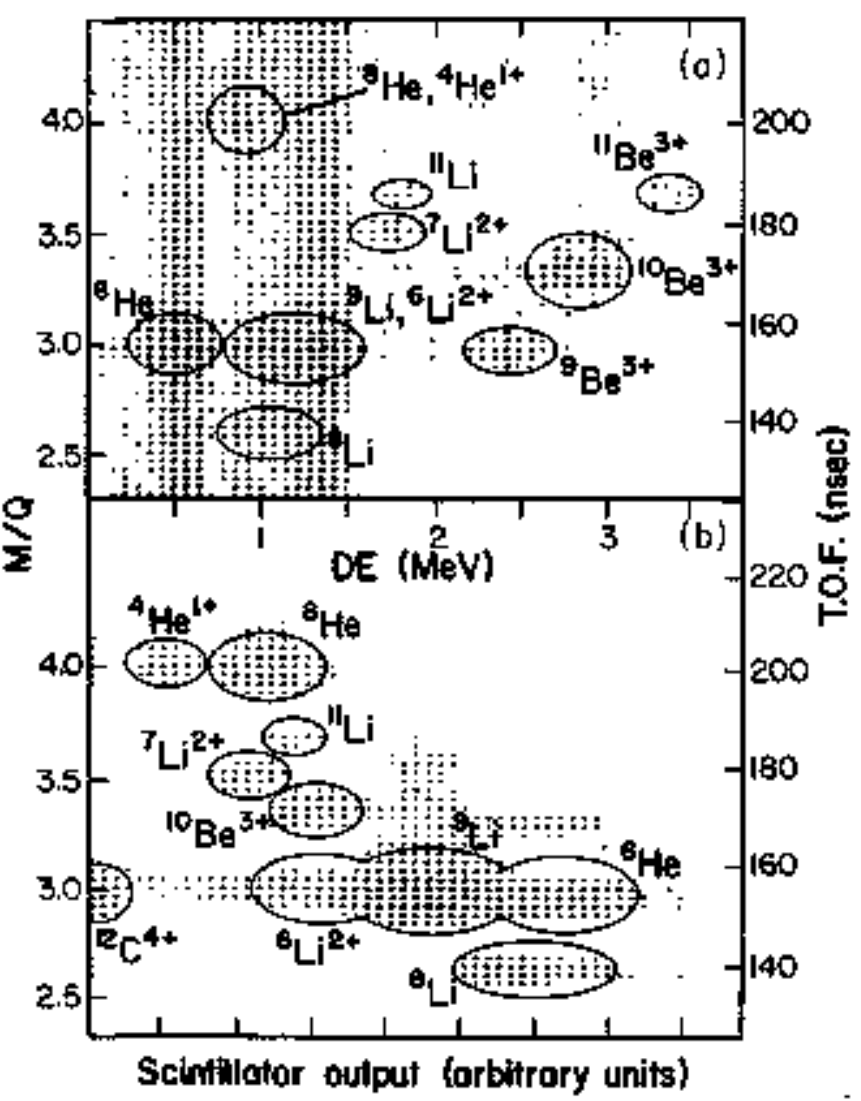

Fig. 1. Identification of particles in the reaction $1 \mathbf{1}_{\mathbf{B}}+{ }^{232} \mathrm{Th}$ at $11 \mathrm{~d} \mathrm{keV}$ and $20^{\circ}:$ (a) on the vertical axis is plotted TOF \& $\mathrm{M} / \mathrm{Q}$ and on the horizontal is $\mathrm{DE} \times \mathrm{M}^{2} \mathrm{Z}^{2} / \mathrm{Q}^{2}$; (b) plot of TOF versus $\mathrm{E}$ as measured by a scintillator in the focal plane. The tail due to pile-up in the TDF, evident in (a), is renoved by fast pilenap rejecticn.

(XEL 762-2322) 
for the high-yield reaction products ${ }^{9} \mathrm{Li}$, ${ }^{6} \mathrm{He}$ and

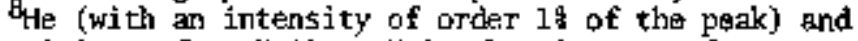
originate from "pile-up" in the time-zero detector. The contamination was eliginated by fast pile-up rejection as illustrated in Fig. 2(b), which also shows how the reliability of identification was further improved by measuring the residual energy in the focal plane scintillator. The energy spectra for different particle groups were obtained by multiparameter gating of these 2D-spectra.

Energy spectra for the $\left({ }^{11_{B}},{ }^{9}{ }_{11}\right)$ and $\left({ }^{11} \mathrm{~B}, 1 \mathrm{ll}_{\mathrm{Li}}\right)$ reactions on $23 \mathrm{~T}_{\mathrm{Th}}$ are show in Fig. 2 at $20^{\circ}$. In spite of the poor resolution of the experiment $(\approx 0.5 \mathrm{~W} \mathrm{eV})$, it is clear that the two proton transfer selectively populates the g. 5. of $234 \mathrm{U}$ (in which there art 10 levels in the first MeV of excitation). The differential cross section for this group was $5.8 \mu \mathrm{b} / 5 \mathrm{r}$. The spectrum for $\left[11_{B}, 1_{L}(i)\right.$ is shown in Fig. 2 (b) with the predicted ground state for a Q-value of $\approx-31$ hev corresponding to the measured mass-4xcess 5 of -40.94 for ${ }^{1} I_{\mathrm{L} i}$. The spectrum extends to the ground state region, wil the a cross section of $2.7 \mathrm{nb} / \mathrm{sr}$. In view of the practuction cross section in the g.s of $1.2 \mathrm{ub} / \mathrm{sr}$ for the $\left(22_{\mathrm{Ne}}, 22_{0}\right)$ reaction it $1 \mathrm{~s}$ interesting to see if this variation of three orders of magnitude in the "double-exchange" cross

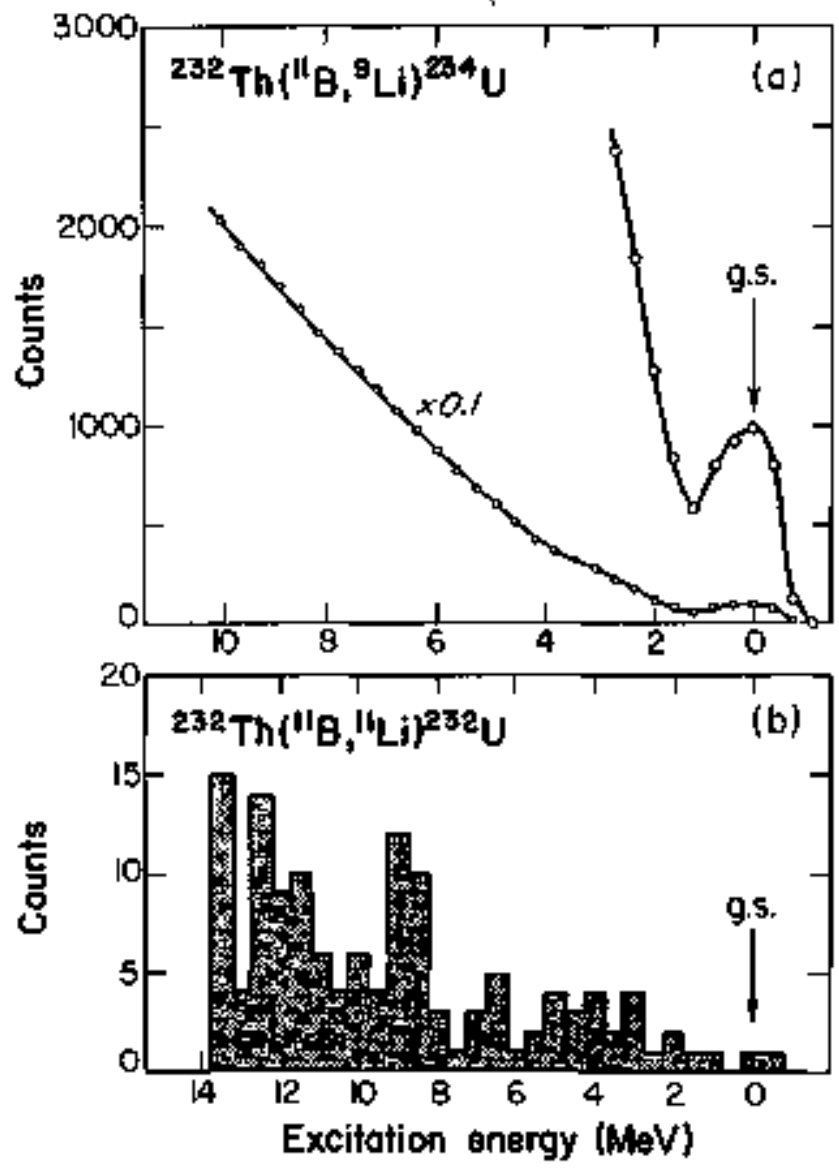

Fig. 2 . Energy spectra for the reactions

(a) $232 \mathrm{Th}(11 \mathrm{~B}, 9 \mathrm{Lj}) 234 \mathrm{U}$ and

(b) $232 \mathrm{Th}\left(1 \mathrm{i}_{\mathrm{B}}, 1 \mathrm{i}\right.$ i) $232 \mathrm{~J}$ at $114 \mathrm{MeV}$ and $20^{\circ}$, The arrows locate the production of the final nuclei in their ground states. section can be accounted for theoretically, in prater to test the feasibility of extending the technique to the measurement of other moclei at the limit of stability.

The reaction mechanism for multinucleon transfer reactions of the type discussed here contains both direct and statistical features. Although the differential cross sections peak at the grazing angle for direct transfer, the observation that the total production cross section at this angle for a series of isotopes depends exponentially an the ground state Q-value has a natural explanation in the statistical model; 8 then for the reaction $x+A+y+B$,

$$
\left.\sigma=\sigma_{0} \exp \left[\left(\frac{a_{x}+a_{A}}{E_{i}-V_{x k}}\right)^{1 / 2}{ }_{Q_{y B}}+\Delta E_{c}\right)\right]
$$

where " $a$ " is 0.05 tinnes the mass number of $x$ or $A$, $E_{i}$ is the incident energy, $V_{x g}$ the Coulonb barrier, Qup the ground state Q-value for the chamel $y+B$, and $\Delta \mathrm{E}_{c}$ is the change in Coulanb energy between the initial and final thannels. In the production of a series of isotopes, only Q changes, accolunting for the exponential dependence. wo use $\mathrm{Eq}$. (1) as a rough guide for the systertatics of $\mathrm{g}+\mathrm{g}$. cross sections; obviously in transitions to individua? states, variations due to shell and pairing energy effects will be encountered.

For reactions induced by ${ }^{22} \mathrm{Ne}$ at $174 \mathrm{MeV}$, and ${ }^{11} \mathrm{E}$ at $114 \mathrm{MeV}$ or $232 \mathrm{Th}$, the coeffictent $\left[\left(a_{x}+a_{A} / E_{j}-\gamma_{X_{A}}\right)\right]^{l_{2}}$ has approximately the same value of 0.42 . This value is also in reasonable agreesent with the enpirical value of 0.58 determined by Eq. (1) from the ratio of approximately 50 for the total and ground state cross sections observed for the production of 200 and 220 . We therefort also use the value of 0.58 to predict a ratio for the reactions $\left(11_{B}, 9_{L} i\right)$ and $\left(11_{B}, 11_{L} i\right)$, with Q-values of $=-18$ and $-31 \mathrm{MeV}$ respectively, of 1800 in good agreement with our experimental result of $5.8 \mathrm{~b} / 2.7 \mathrm{nb} .22150$.

Accepting $\mathrm{do} / \mathrm{d} \Omega \approx \mathrm{nb} \mid \mathrm{sr}$ as an experinental limit, it seems that multinucleon transfer reactions induced by nelutron excess heavy-ion bearss on heavy targets present a feasible method of measuring the mass excess of exotic 11ght nutiei at the ifnit of stability.

\section{Footnotes and References}

"Pemanent Address: Instituto de Fisica, U.N.A.M., Pexico.

PPemaruent Address: Institute des Sciences Nucleaires, Grenoble, France.

1. J. D. Bowman, A. M. Poskanzer, R. G. Korleting and G. W. Butler, Phys. Rev. C9, 836 (1974) and refs. therein.

2. A, 6. Artukh, V. V. Andeichikov, G. F. Gridnev, V. L. Wikhoev, V. V. Volkov, and J, Wilcymski, elac1. Phys, Al76, 284 (1971) and refs, therein. 


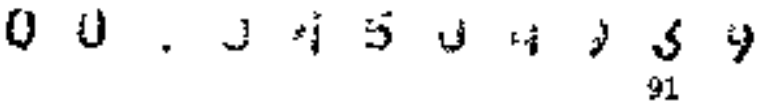

3. For a rexent review, see J. Cerrny, Reactions. Between Complex Nuclei, ed. R. C. Robinson, F. K. HeGowan and J. B. Ball (korth Hotland, Ansterdan, (1974\} Vol. 2, p. 483 .

4. D. K. Scott, B. G, Harvey, D. I. Hẹndrie, L. Xraus, C. F, Maguire, J, Mahoney, Y. Terrien and $K$, Yagi, Phys. Rev. Lett. 33, 1343 (1974).

5. A. G. Artukh, G. F. Gridnev, V. L. Mikheev, V. V. Volkov and J. Wilzynski, Nocl. Phys, Alg2
(1970) 170.

6. G, C. Bal1, W, G. Davies, Jf S. Forster and

H. R. Andrews, Phys, Lett, 60B, 265 (1970).

7. C. ThibauIt, R. Klapisch, C. Rigaud, A. M. Poskanzer, R. Preils, l. Hessand and W. Reisdorf, Phys. Rev. C 12, $644(1975)$.

8. A. Y. Abul-kagd, K. El-fbed and M. E1-Nadi, Phys. Latt. 39B (I972) 166.

\section{STATES AT HIGH EXCITATION IN ${ }^{16} \mathrm{O}$ OBSERVED IN THE REACTION ${ }^{12} \mathrm{C}\left({ }^{20} \mathrm{Ne},{ }^{18} \mathrm{O}\right){ }^{18} \mathrm{O}$}

\author{
A Menchace-Rocha, M Euenerd,t D L Hendrie, \\ C Olmes, and D K Scoll
}

\begin{abstract}
Heavy-ion induced multimucleon-transfer reactions have been widely used to locate high spin states. A1though the angular distributions of such reactions are, in general, structureless and lead mainly to a detemrination of the reaction mechanism, the strong selectivity of the reactions for populating sipple states at high excitation has led to interesting comparisons with the predictions of she11 and fiuster structure models. In studying the ${ }^{12} \mathrm{C}\left(20 \mathrm{ve}, 16_{0)} 10_{0}\right.$ reaction at incident energies between 150 and $294 \mathrm{MeV}$, we find a strong variation with incident energy of the cross section of a given state that is characteristic of the spin of the state. The incident energy dependence of states with known $\mathrm{J}$-values is found to be in agreenent with predfctions of a seniclassical model. This technique is used to investigate the J-values of three new states which we observe in 160 in the 21 to $24 \mathrm{KeV}$ excitation region.
\end{abstract}

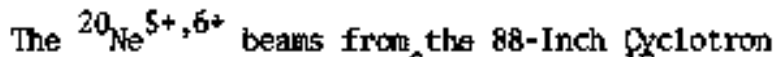
were used to bombard $100 \mathrm{\mu g} / \mathrm{cm}^{2}$ targets of $\mathrm{IZ}_{\mathrm{C}}$. The reaction products were detected and identified using the QSD magnotic spectroneter.

The energy spectra (Fig. 1) show groups at positions correspondini to we11-known 160 states at $6.1\left(J^{\pi}=0^{+}, 3^{-}\right), 6.9\left(2^{+}, 1^{-}\right), 10.4\left(4^{+}\right), 14.6\left(5^{-}\right)$, $16.2\left(6^{+}\right), 20.8^{\prime}\left(7^{*}\right) \mathrm{HeV}$. States are also observed at $21.7,22,2$ and $23.5 \mathrm{HeV}$. The engular distributions do not have any proninent diffraction structure and fall off exponentially with angle. Figure 2 shows the differential cross sections for the various groups, integrated over the observed angular range and plotted as a function of the incldent energy. In the following section we briefly describe a semiclassical mode1 whose predictions correspond closely to the spooth energy dependence of the experimental cross-sections for states of tonown $J^{\mathrm{x}}$.

In a seniclassical description of heary ion reactions a cluster transferred frcyn a state with orbital and magnetic quantun numers $t_{1}, \lambda_{1}$ in the projectile to a state Iabelled by $\ell_{2}+h_{2}$ in the residual mecleus has a transition probability of the ford : 1

$$
\begin{aligned}
P\left(e_{1} \lambda_{1}, e_{2} \lambda_{2}\right) & =P_{0}(R) S_{1}^{2} S_{2}^{2}\left|Y_{\ell_{1}}^{\lambda_{1}}\left(\frac{\pi}{2}, 0\right)\right|\left|Y_{\ell_{2}}^{\lambda_{2}}\left(\frac{\pi}{2}, 0\right)\right|^{2} \\
& \cdot \exp \left[-\left(\frac{\mathrm{RA}}{\pi}\right)^{2}-\left(\frac{(\Delta \mathrm{L})}{\sqrt{\mathrm{K}}}\right)^{2}\right]
\end{aligned}
$$

Where $\mathrm{R}=\mathrm{R}_{1}+\mathrm{R}_{2} ; Y$ is related to an average of the binding enefgies $E$ of the initigl and final states, according to $\gamma^{2}=2 m_{E} / h^{2}$, where in is the transferred mass. The quantities oL and $O K$ are defined by:

$$
\begin{aligned}
& \Delta L=\lambda_{2}-\lambda_{1}+\frac{1}{2} k_{0}\left(R_{1}-R_{2}\right)+Q_{e f f} / A V \\
& \Delta k=k_{0}-\lambda_{1} / R_{1}-\lambda_{2} / R_{2}
\end{aligned}
$$

Here $v$ is the relative velocity of the cores in the region of transfer, $\mathrm{k}_{0}=\mathrm{mW} / \mathrm{h}$ and $\mathrm{Qeff}_{\mathrm{e}}=\mathrm{Q}$ $\left(z_{1} \mathbf{f}_{2} \mathbf{f}-z_{1}^{i_{2}} z_{2}^{\mathbf{i}}\right) e^{2} / R, s_{1}$ and $s_{2}$ are spectroscopic factors of the cluster in the initial and final nuclei and $\mathrm{P}_{0}(\mathrm{R})$ depends on the radial wavefunctions of the inital and final changels at the point $R$; for the purpose of obtaining relative cross sections we use the value $\left|\psi_{2}(R)\right|^{2}$ where $\psi_{2}(R)$ is the wavefunction of the transferred cluster at the surface of the residual nucleus.

The curves in Fig, 2 show the predicted values of $P$ as a function of energy for various states; the absolute values were nomalized to fit the corresponding experimental data. As seen in the figure, the states at 21,7 and 22,2 are consistent with the trend for $\mathrm{d}$ a 6 . From angular correlatiom stadies of the a-decay of this state excited in the (6Li,d) reaction, a spin of 6 has been assigned. 2 However our observed eneray dependence agrees better with $\rfloor=B, 9$, The $23.5 \mathrm{MaV}$ groups follows a similat trend, but due to the large error bars, a spin as low as $J=5$ is not inconsistent with the data. 


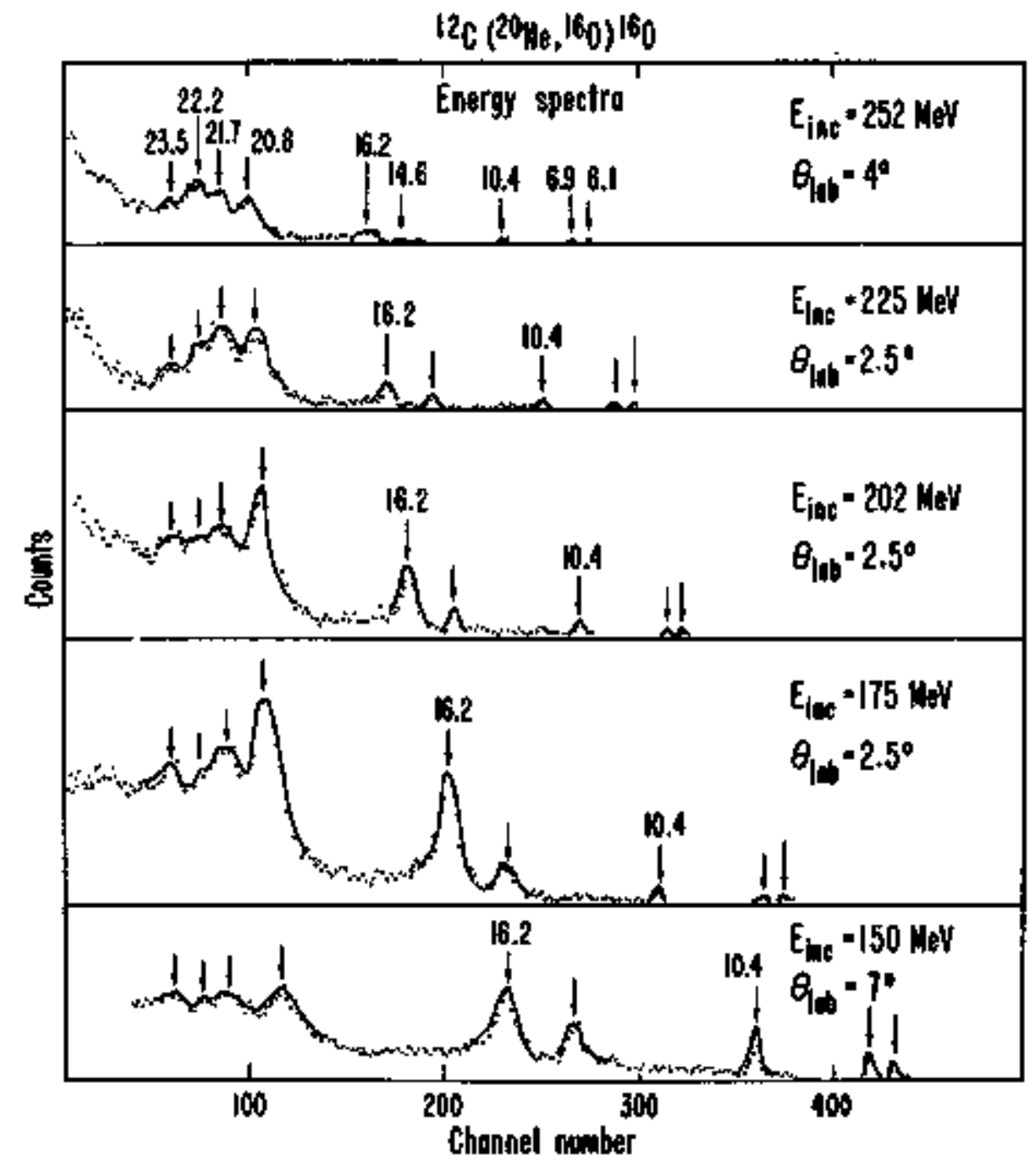

Fig. 1. Energy spoctra for the 12 ( $20 \%$ Ne, 160$)$ 160 reaction at incident energies between 150 and $252 \mathrm{keV}$.

(XBL 763-7537)

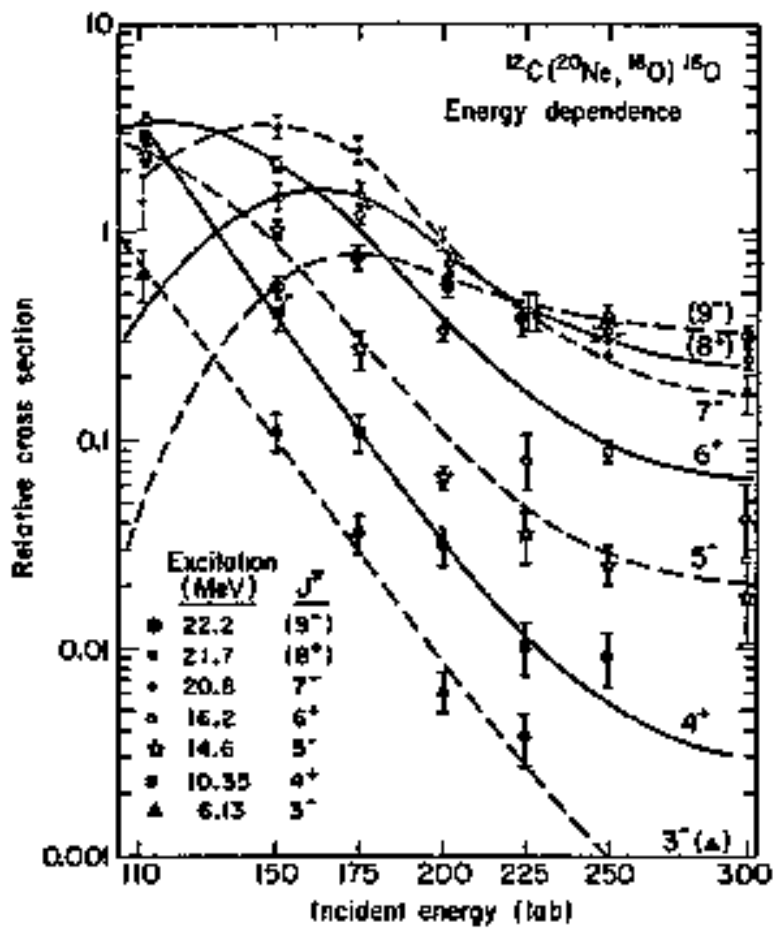

An interesting fegture of the present data is that the (20ke, 160$)$ reaction does not populate the $4_{2}^{+}$[1.1.1 $\left.\mathrm{kgV}\right]$ state - which is populated in other a-transfer reactions - whose structure is predicted to be of a complicated nature involving a ${ }^{12} \mathrm{C}_{4}^{x}{ }^{\left(2^{+}\right)}$core. The interpret this result as evidestef for a pure a-transfor nechanism, Both the calibration and the energy dependence for the state observed at 14.6 keV is conșistent with the $5^{*}$ assigment ${ }^{3}$ whtle the states at $14.39\left(4^{+}\right)$and $14.82\left(6^{4}\right)$, of dominant $2 \mathrm{p}$ - 2h configurations, are not favored, as expected in a direct $\alpha$-transfer model. Since we observe groups corresponding to all known members of the $K=0^{+}$(80) band, the state at $21.7 \mathrm{k} \mathrm{k}$, which appears to follow the trend of a $J=8$ state, is a good candidate to be the $8^{+}$ menber of this band. Theoretical predictions place it at an excitatian energy between 20 and $30 \mathrm{keV}$. Various atterpts to locate this state at an excicar tion energy ip to $21 \mathrm{MeV}$ have been unsuscessful. The systeratics indicate that the state at $22.2 \mathrm{MeV}$

Fig. 2. Observed energy dependence for the excitation of states in the ${ }^{12} \mathrm{C}$ ( $20 \mathrm{ke}$, 160$) 160$ reaction as a function of incident energy. (XEL 763-2531) 
is of higher sptn. The passibility of g- necessitates a configuration involving an odd nuber of muxleoms in the fp shell, i.e. (sd) ${ }^{3}$ (fp) $^{1}$ or [sd) ${ }^{1}$ (fp) ${ }^{3}$, although such configurations have not been needed so far to explain the experinental data at lower energies where there is a good matching with

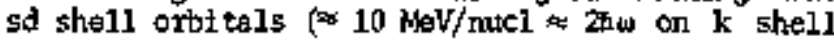
targets); at the present energies of up to $300 \mathrm{HeV}$ ( $15 \mathrm{HeV} / \mathrm{A}$ os 3 w on $p$ shell targets), fp shell orbits are better watched and states having configurations that include nucleons tn the fp she1l might be exhanced,

We have shown that the energy dependence of Inultinucleon transfer reactions might be a useful method for locating cluster states. Uling this method and the sermiclassical formalisn have slade tentative spin assignments to three new states in 160 , one of which $(22,2 \mathrm{MeV}, J * B)$ is a good candifate for the $K=0^{+}\left(80^{\circ}\right)$ rotational bard of 160 .

\section{Footnotes and References}

*Permanent Aởress: Institurtô de Fisica, I.N.A.M., Mexico.

'Permanent Address: Inștitut des Sciences Nucléaires, Granoble, France.

1. N. Anyas-Heiss, J. C. Come11, P. S. Fisher, P. N. Hudson, A, Menchaca-Rocha, D. J, Millener, A. D. Panagiotou, D. K. Scott, D. Strottnan, D. M. Brink, B. Buck, P. J. Ellis, and T. Engeland, Phys. Reports 12C, 201 [1974].

2. M. Avril, M. Lepareux, N. Sanier, A. Foti, G. Pappalard and A. Strazzeri, J. de Phys., (letters) 36, L-229 (1975).

3. K. P. Artenow, V. Z, Goldberg, I, P. Petrov, V. P. Rindakov, I. N. Serikov and V, A. Timofeev, Phys, Lett. 37B, 61 (1971).

\section{THE CONTAIBUTION OF ALPHA CLUSTEA EXCHANGE TO ELASTIC AND INELASTIC ${ }^{16} \mathrm{O}^{20} \mathrm{Ne}$ SCATTERING}

R Stock, W F w schnepder, $U$ U Jahnke, S O L Hendrie,

$J$ Mahoney, C F Magulte, D K Scotl, and G Welschur

Several recent stutios of the $20 \mathrm{Ne}$ ground state rotational band in uicroscipic a-cluster plus core models heve led to the conclusion that it mery be characterized as an alpha-particle-like configuration of s-d-shell mucleons rotating around a spherical 160 core. 1 Furthersore, the core may be, to a good aporoximation, identifled with the ground stite of 160 . In the scattering of 20 Ne from 160 , the exphange of the $\alpha$-cluster botheen two identical $16_{0}$ cores should then contribute to the observed cross sections. Viened in a simple one-step direct-reaction mechanist, the cluster can remain with the 160 core of the incident 20 Je projectile leading to elastic or inelastic scattering; in the latter case, the angular monentum transfor, $i$, of the reaction arises fron the excitation of cluster rotation with angular momentum $L=\&$ leading to a final 20 the state with $J \cdot L$. This part of the reaction may be described by optical model and DirBA arplitudes, respectively. In a coupled channel treatment, the corresponding anplitudes include the effect of virtual transitions between the rotational states of 20 ke in the reaction. On the other hand, the clister fory be exchanged to the 160 target mucleus forming one of the $20 \mathrm{wo}$ rotational states. Then the cross section at backward angles where the interference with the nonexchange contribution may be neglected is proportionel to $S^{2}$, the square of the spectroscopic amplitude. Furtherjuore, the interference structure at intermediate angles is very sensitive to $s_{a}$. This quantity can thus be deternined frov masured absolute cross sections more sensitively than in $a$ transfer reaction like $160\left({ }^{6} \mathrm{Li}, \mathrm{d}\right) 20 \mathrm{Ne}$.

Int order to test the prediction of microscopic models that the mesibers of the 20 ke groumd state rotational band are alpha cluster configurations with more or less constant elpha particle spectroscopic factors, we have measured angular distributions of the reactions

$20 \mathrm{Ne}+160+160$ (g.s.) $+20 \mathrm{Ne}\left(0^{+}(\mathrm{g} .5.) 2^{+} 4^{+}\right)$at an incident 20 he energy of 50 Nev which is slightly above the coulonb barrier. Self suppriting targets of aluminus - and rickel - oxide, with thicknesses of 250 and $100 \mathrm{\mu g} / \mathrm{cm}^{2}$ respectively, were mounted in a scattering chamber equipped with a liquid nitrogen cooling finger close to the target as a trap for pump oil vapors, in orilar to reduce the carton buildup on the target during the experiment. Two $A E-E-c o u n t e r$ telescopes with $5 \mu$ ORTEC BE transmission detectors and a solid angle of $2 \cdot 10^{-4} \mathrm{sr}$ mere used along with a monitor detector. The telescope events were sent to a conluter, stored in $h \mathrm{E}-(\mathrm{E}+\not \mathrm{E})$ arrays and analyzed off-îtne with a program for energy calibration, peak integration, peak unfolding and background subtraction. Energy calibration of the spectra was obteined frow the peak positions of the elestic 20 he scattering from $\mathrm{Ni}, 27 \mathrm{Al}$ and 160 . For each scattering angle and telescope, both the spectra of outgoing 200 and 160 (g.5.) products were obtained. Figure 1 shows these spectra for $\theta=29^{\circ}$. Since the 160 (g.s) angular distrfbuttions at forwart angles correspond to the anguifer distributions of the elastic and inelastic $20 \mathrm{Ne}$ backward scattering it was sufficlent to cover the forward angles.

The rosulting angular distributions for the ground state, $2^{+}$and $4^{7}$ states of 20 ke are shown in Fig. 2. The error bars include errors due to statistics, backgrumd correction, and uncertainties in the unfolding of impurity peaks. For 


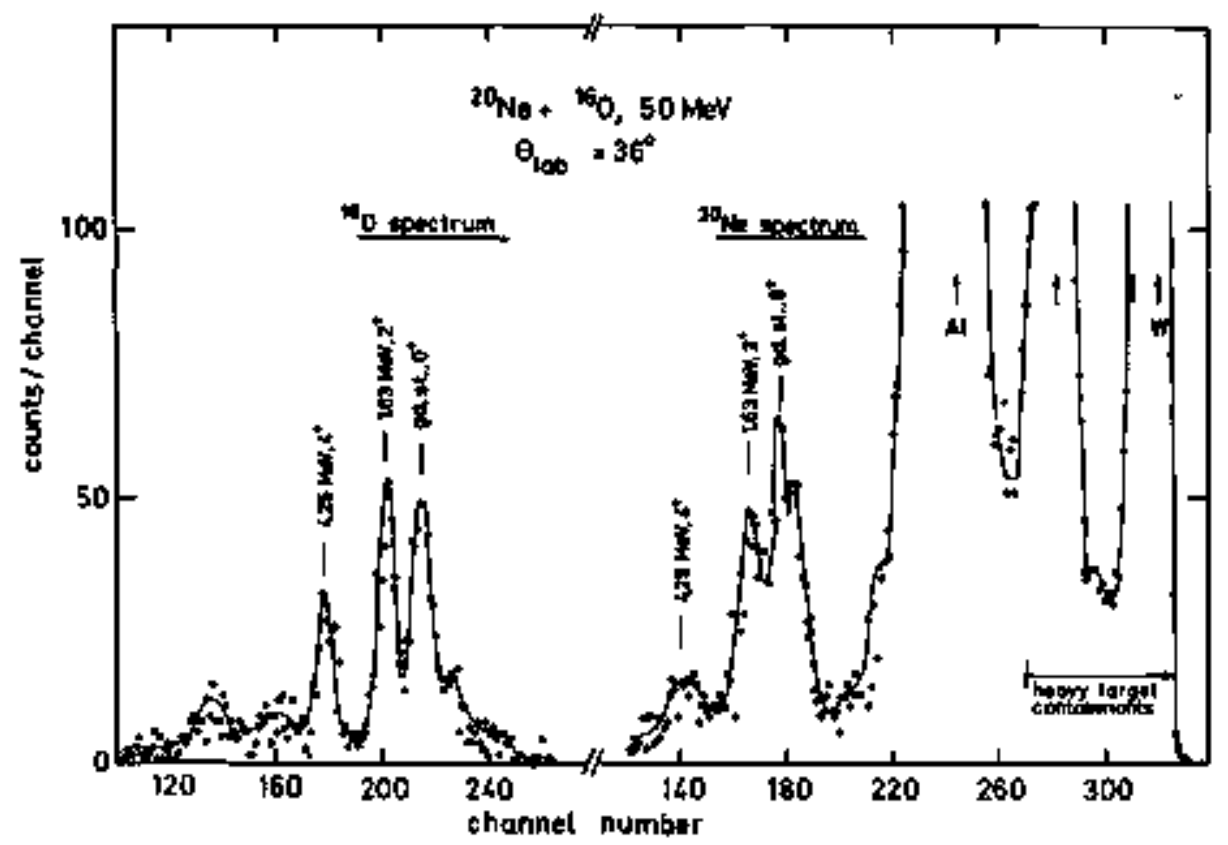

Fig. 1. Spectra of outgoing $20_{\mathrm{Ne}}$ and $16_{\mathrm{O} \text { at }} \mathrm{\theta}_{\mathrm{Iab}}=36^{\circ}$.

(XBL $766-8503)$

(a)

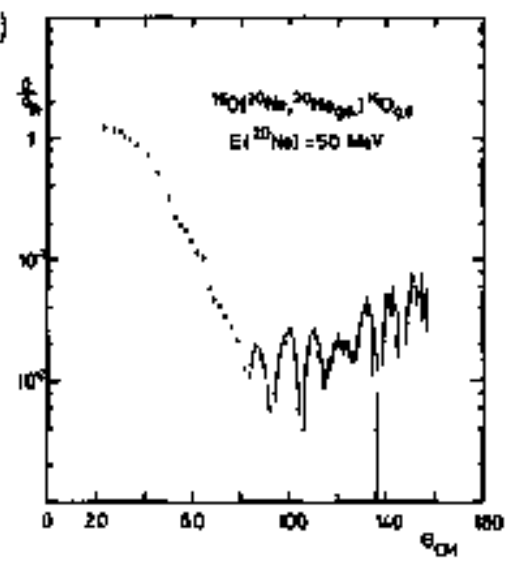

(b)

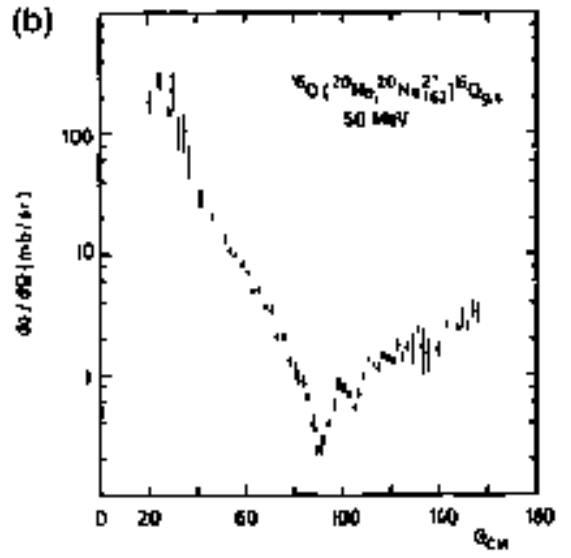

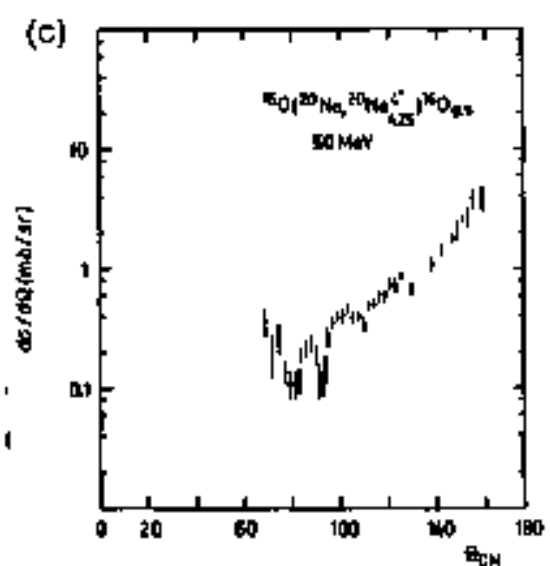

Fig. 2. Angular distributions for elastic and inelastic 20 Ne +160 scattering to the ground state, $z^{+}(1.63 \mathrm{MeV})$ and $4^{+}(4.25 \mathrm{MeV})$ states of 20 the. The sjooth curve in the ground state transition is drawn to guide the eye.

(XBL 766-8527)

the $4^{+}$transition, the peaks could only be identifind in the 160 spectra with sufficient reliability, thus ligiting the angular distribution to $\theta_{\text {c.m. }} \geq 70^{\circ}$.

A backward rise is observed in all three angular distributions, supgesting that a mecharism difforent from dirsect elastic or inelastic scattering takes over at angles beyond about $90^{\circ}$. The pronounced oscillations at intermediate angles, observed for the ground state transition, appear denmed in the inelastic reactions.

The ${ }^{16} \mathrm{O}_{\mathrm{r}}{ }^{20}$ Ne ground state transition was calculated as a coherent stperposition of elastic scattering and ciuster transfer using the code $B R I N I L D$ of Braun-hameinger et at. 2 In the reaction amplitude

$$
\mathrm{T}(\theta)=\mathrm{T}(\theta)_{\mathrm{\alpha}}+\mathrm{s}_{\mathbf{\alpha}} \mathrm{T}_{\mathrm{NHA}}(\pi-\theta)
$$

the relative phase is fixed by the symuetry of the system. 3 The set of optical model paraneters used In both anplitudes, was $v_{0}=100 \mathrm{kNV}, W=35 \mathrm{keV}$, $r_{V}=r_{W}=1.2 \mathrm{fm}, a_{V}=0.49 \mathrm{fm}, a_{w}=0.32$ fil, терre sonting a strongly absorbing potential. No R-dependence of the absorption was taken into account because enough direct exit charnels are available to carry the grazing angular momentum $l_{0}$ : 10 ; among these the low-Iying collective states are 


\section{Dป: j,}

particularly strongly excited in inelastic scattering.

Cluster formfactors are shom in Fig. 3 , according to Tomoda et a1. 4 and Hiura et al. 5 together with Woods-Saxon formfactors obtained with $r_{0}=1.4$ fil that are nomalized so as to fit the asyiptotic parts ( $R \geqslant \$$ fm). In the nucleat interiox the nicroscopic formfactors are drastically reduced due to the antisynotrization, and the major part of the alpha particle probability anplitude is pusted into the surface region. The fornfactor of Hitra et al. with 5c.m. = 0.45 exhibits the same surface amplitude as the woods-5axol formfactor with $r_{0}=1.4$ and a normalization $\$^{n+5+*}=0.8$. The latter will be used in the DwRA analysts.
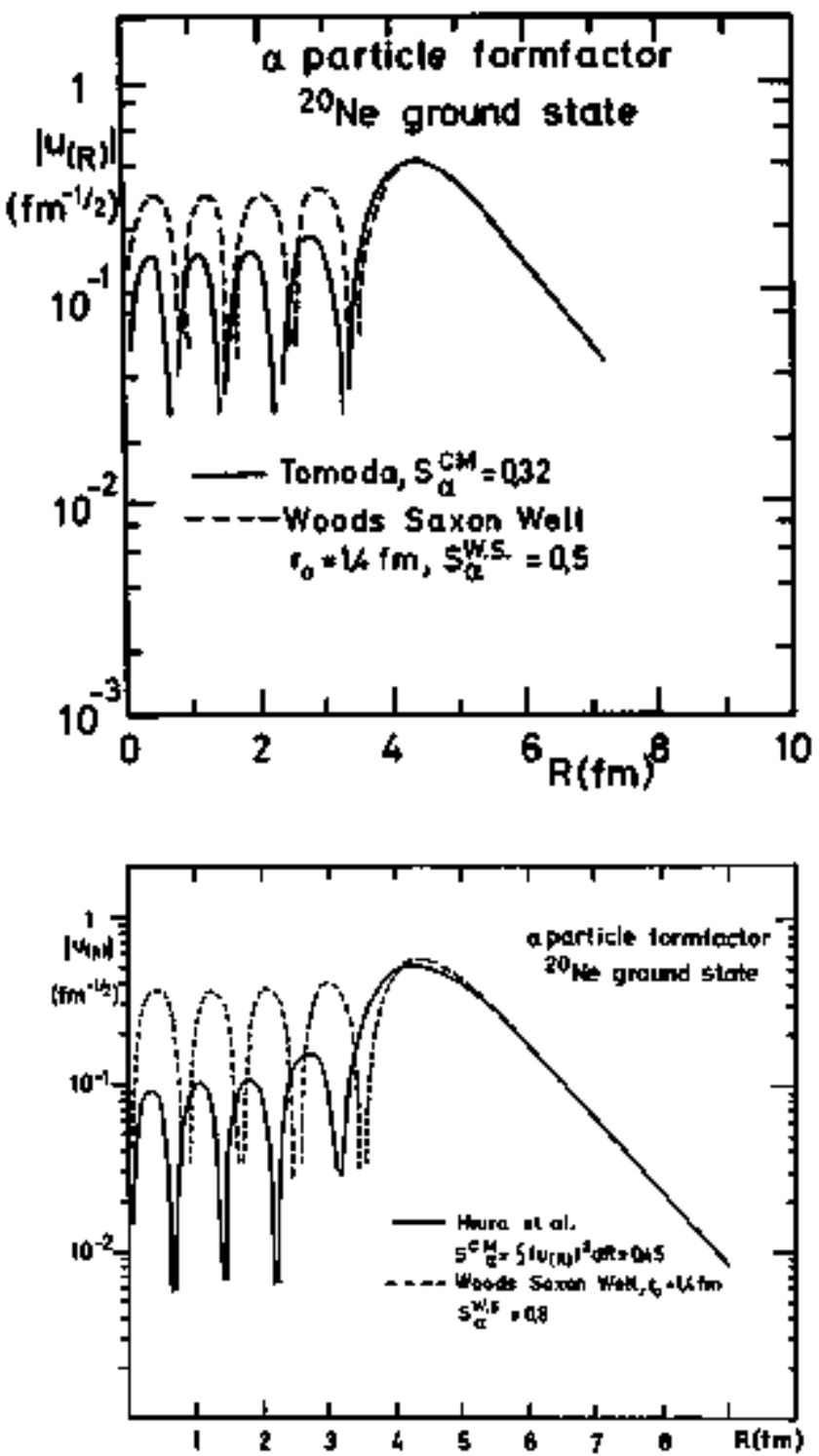

Fig. 3. Alpha particle relative motion fomfactors for the 20 Ne ground state. Microscopic calculations of Tomoda and Arima (Ref. 5; upper part) and Hiura et al. (Ref. 4; lower part) are matched by Woods-\$axon solutions in the external region (R> 5 fin).

(XB) 766-8526)
The results of the optical model and elastic transfer calculations are conpared with the ground state transition data in Fig. 4. Both fits are identical for $\theta_{c . m .} \leq 80^{\circ}$; "in this domain, the data are well reprodiced. The oN cross section continues to fall off towards larger angles, in a manner typica1 for stromgly absorbing potentials, whereas interference oscillations and a backward rise develop in the elastic transfer angular distribution for $\theta_{c, m}>80^{\circ}$. However, the oxperimental cross sections are largely underestinated at intemindiate angles, where also the phase of the oscillations is not well reproduced.

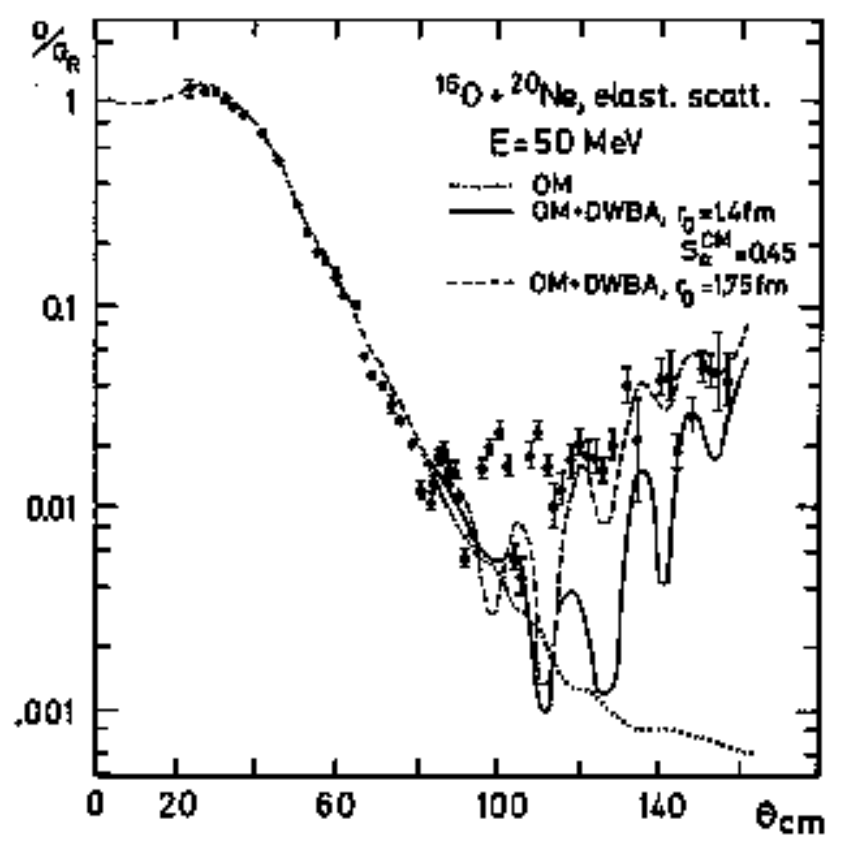

Fig. 4. Results of pptical model (a) and elastic transfer (ON+ DWBA) calculations for the $20 \mathrm{Ne}+160$ ground state transition.

(XBL 766-8504)

A variation of the optical nodel parameters did not yield significant improvements insofar as a better agreenent with the backward oscillatory pattern could only be achieved at the expense of fit quality at forward angles. Apparentiy, the DNBA anplitude falls off tro rapidaly from backward angles towards $90^{\circ}$, or the of amplitude is inadequate for $\theta>80^{\circ}$. Attempts to inprove the fit by scaling the IDBA asplitude with a larger bound state for factor nomblization were unsuccessful. Fpr expmple, an arbitrary normalization to $S^{4} . S_{*}=2$ shifted the anpular distribution unward by a unfform factor of about four for $63^{3} 100^{\circ}$, improving the agreement at intermediate angles but far overestimating the cross sections at the very backward angles. $A$ scmewhat better overall fit was obtained by increasing the hoods-Saxon well radits from $r_{0}=1.4 \mathrm{fm}$, as prescribed by the nicroscopic models, to $\mathrm{IO}_{0}=1.75$ fra, at the same time reductug the overall normalization to $\mathrm{SW}^{\mathrm{S}} \mathrm{S}_{+}=0.5$. The corresponding fit is a1so shom in Fig. 4. However, this procedure inplies an alpha particle surface reduced width twice as high as given $b y$ the microscopic we functions appropriate to the 20 Ne ground state. This corresponds to 
an alpha cluster localization far beyond the 11rits indicated for 1ow-1ying 20) ste stes by nicroscopic models, 1

It was therefore concluded thet the firstorder elastic scattering and exchange mochanism, underlying the 0N plus D Whe aproximation to elastic transfer, is not appropriate in the $160+20$ he case. A second-order calculation would have to include virtual transitions to the excited, collective states of 20 ire both in the entrance and exit channels, as well as the transfer of the alpha cluster amoong excited states. No cchmputer code was ayailable to the attthors that would take all these trans. ittons into account, along with an appropriate treatment of recoil effects.

In order to demonstrate the second order effects on the optical podel amplitude alone, a coupled channel calculation was done for elestic and inelastic $160+20$ scattering using the code of Ascuitto and Glenderming. No exchange contributions were taken into account. The relevant deformation constants, associated with a radius $R$ (projectile)

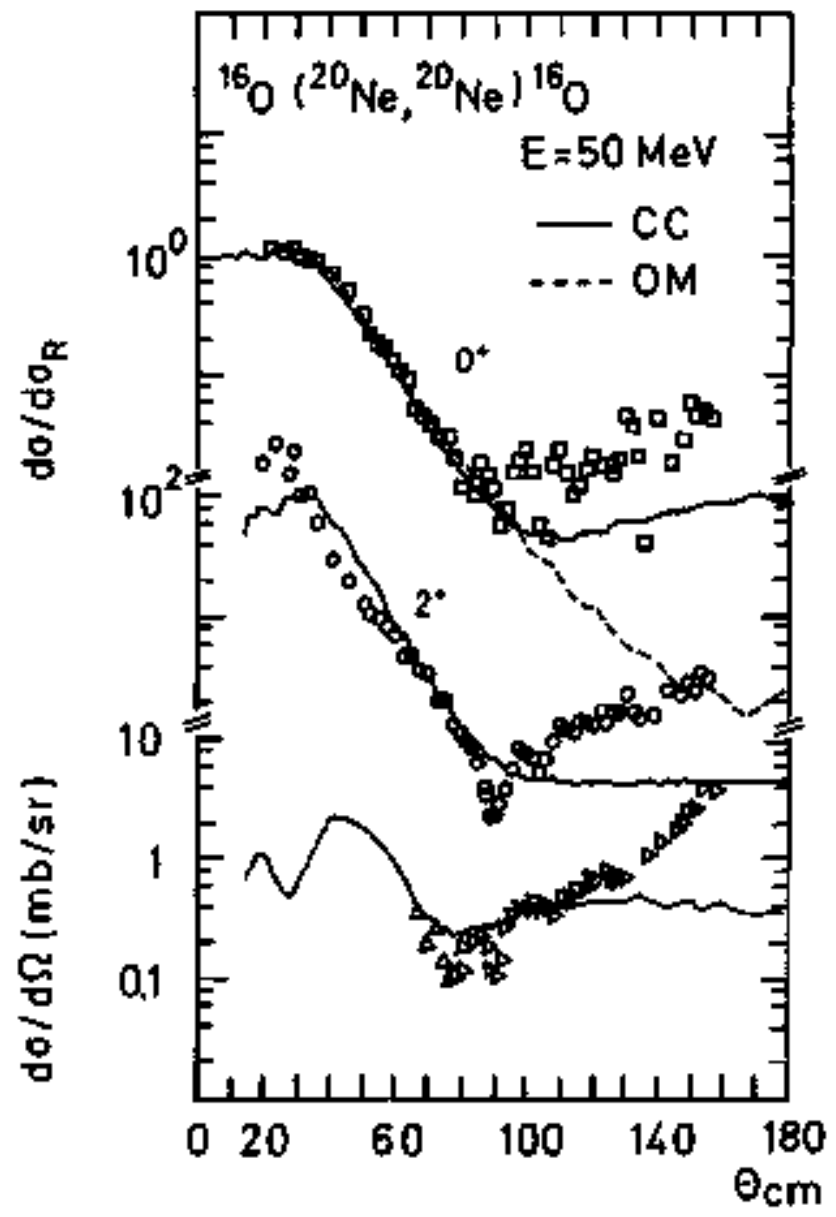

Fig. 5. Results of opticat model (OM) and colpled oume1 (CC, no exchange) calculations for $20 \mathrm{No}_{0}+160$ scattering to the first three states in 20ple. Potential parameters are the same as in the elastic transfer calculation except $W=15 \mathrm{NEV}$. Deformation paraneters are $\mathrm{BN}_{2}=0.43, B \mathrm{~B}=0.26$, $B \frac{X}{2}=0.8$ and $B=0.4$.

(XEl 756-8505) a $1.2(20)^{1 / 3}$ were deduced from the deformation length in Ref. 7. The Coulonb deformation $\beta$. was reduced slightly from the value. 0.87 reported by Stelscr and Grodzins, 7 becanse second order cotpling was included. The results of this calculation are shown in Fig. 5, which also gives the optical nodel fit (without chemnel coupling) for elastic scattering. The effect of charme1 corpling for the ground state transition is clearly exhibited. At intermediate scattering angles where the elastic transfer calculation (Fig. 4) was systematically underestinating the observed cross section, the $\alpha C$ cross sections are mich larger than the oul cross sections, the $2^{+}$transition cross section is underastinated at the very forkard angIes. It should be noted, howevier, that we did not attenpt any parameter optimization, and that the experimental error bars are large in this region (c.f. Fig. 2) dut to normalization and backsround carrection uncertainties. The coupled channel calculation for the pround state transition indicates that the ov arplituxle is too small at backward angles. Thiss nay in part explain the low cross sections and wrong phases obtained in the intermediate angle elastic transfer calculations. Oae alght, therefore, try to improve the model replacing the ou arolitute in by a coupled channeled amplitude. This was not done for the following reasons:

(a) the second order effects showld be sinalarly ingortant for the DiBA amplitude at intermediate angles, and (b) the model would be still unsatisfactory as long as 20 Ne is treated macroscopleally in the $C$ arplftude and rocroscoplcally in the DNRA allipitude.

\section{Footnotes and References}

*Condensed from LBL-\$0\$1

${ }^{4}$ Permanent Adtress: Fachbereich Physłk, Loiversität Narturg, W. Germany.

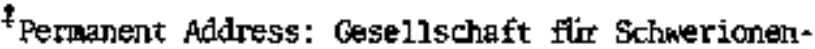
forsching, Darmistadt, W. Germany.

${ }^{5}$ Permenent Address: Hahn-keitner Institut fïr Kernforschung, Berlin, W. Gemany.

1. See for example, A. Arima, Proc. Int. Conf. Nucl. Phys, Murich 1973, ed. by $J$. deBoer and H, J, hang, North Holland (1973), Vol. $2, \mathrm{P}, 183$.

2. P. Brald-ktnzinger, H. L. Harney and S. Mennels, Nucl. Phys. A255, $190(1974)$.

3. P. Braun-bmzinger ot al , Z. Phys, A276, 107 $(1976)$.

4. T. Tomoda and A. Arima, Proc. IAS-IPCR Syuposiun on Cluster Structure, at. H, Kamitsubo et al., Tokyo, 1975, p. 90 and private condurication.

5. J. Hiura, F. Newoto and $\mathrm{F}_{+}$Bando, Pxog. Th. Phys. Supp1. 52, $173(1973)$ and private cos.mrication by $F$. Ternoto.

6. R. J. Ascuitto and H. K. Glendenning, Phys. Rev. 181, 1396 (1969).

7. R. H. Stelson and L. Grodzins, Mhacl. Data Sec. Al, 21 (1965). 


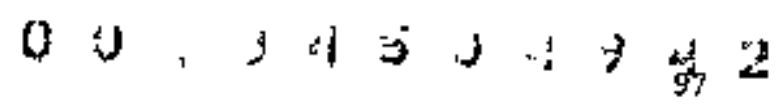

\section{EXCITATION OF GIANT RESONANCES IN ${ }^{197}$ AU AND ${ }^{200} \mathrm{~Pb}$ BY INELASTIC SCATTERING OF HEAVY IONS}

D Ashery, M S Zsman, it 6 Wielsenminler.

A Gulerman D K Scott and C Magulfe

The region of excitation energy in nuxclei where the giant monopolo and giant quadrupole resonances are expected to be, has bes extensively investlgated in recent years. Enhrncenents in the cross sections were observed in experiments in which photons, electrons, protons and 4 he ions mere used. hile the existence of the resonances was established and various propertios were studied, $a$ definite conciusion about the multipolarity of the resconances observed has not been reached; the tendency is to believe that they are of E2 character. The population of these resonamces by inelastic scattering of heavy ions through tho coulonb excitation process should help in identification of their nultipolarity since only the $2^{\dagger}$ component will be exritect.

\section{Self-supporting ${ }^{197} \mathrm{Au}$ and ${ }^{208} \mathrm{~Pb}$ targets wert} bombarded with 104-MeV 160 and $130-\mathrm{MeV} 20$ he beans at the $88-i n c h$ cyclotron. The inelastically scattered ions were detected at the foxal plane of the magnetic spectroneter, placed ot $55^{\circ}$ and $60^{\circ}$. These angIes are 11 below the grazing angle and the coulonb excitation requirement should still be reasonably satisfled. The expected cross sections for population of the giant quadruole resonance, asștning a $B(E 2)$ value equal to 100 of of the energy weighted sum-rule, is of the order of $1-2 \mathrm{hb} / \mathrm{sr}$. Due to the large background observed in the expertment, only upper linits could be set and are listed in Table 1, since calculations of the coulomb excitation process indicate that it tends to shift the position of the peak to a sonewhat lower excitation enorgy and also to make it a little narrower, the upper linits are quoted per MeV of the width in the expected position. The upper lintits are taken as tor stardard deviations of the beckBround.

Measurements wore also taken with a $140-\mathrm{HeV}$ 160 bean bombarding 197 Au and the inelastically scattered ions detected at $43^{\circ}$ and $54^{\circ}$. Under these conditions, we are well above the coulomb barrier. A broad peak clearly observed at both angles at the expected excitation energy for the $2^{++}$(or $0^{+}$) giant resonance. The spectrim obtained at $43^{\circ}$ is shown in Fig. 1, and is the first observation of this resonance by inelastic scattering of heavy ions. The observed width is abourt $3.5 \mathrm{MeV}$ but the enhancement could include some cortribution from the adjacent giant E1 rescrance. The cross sections, integrated over the observed peak, aro listed in Table 1.

Table 1.

\begin{tabular}{|c|c|c|c|c|}
\hline Target & Projectile & $\begin{array}{l}\text { Energy } \\
\text { (NeV) }\end{array}$ & $\theta_{1 \mathrm{ab}}$ & $\begin{array}{l}\text { Cross section } \\
\text { or upper limit } \\
\text { (ab/sr) }\end{array}$ \\
\hline $197 \mathrm{Aud}$ & 160 & 103.15 & $55^{\circ}$ & $<3.3 / \mathrm{MAV}$ \\
\hline $19 ?_{\text {Ard }}$ & $16_{0}$ & 103.15 & $60^{\circ}$ & $<7.5 /$ MeV \\
\hline${ }^{201} \delta_{\mathrm{Pb}}$ & 160 & 105.15 & $55^{\circ}$ & $<2.2 \mathrm{MeV}$ \\
\hline 197 Alu & $20 \mathrm{He}$ & 128.96 & $55^{\circ}$ & $<3,0 / \mathrm{MbV}$ \\
\hline${ }^{208} \mathrm{~Pb}$ & 20 kie & 128.96 & $55^{\circ}$ & $<7.0 / \mathrm{MeV}$ \\
\hline $197_{\mathrm{Aur}}$ & ${ }^{16} 0$ & 139.21 & $43^{\circ}$ & 670 \\
\hline $197_{\mathrm{Aur}}$ & $16_{0}$ & 139.21 & $54^{\circ}$ & 85 \\
\hline
\end{tabular}

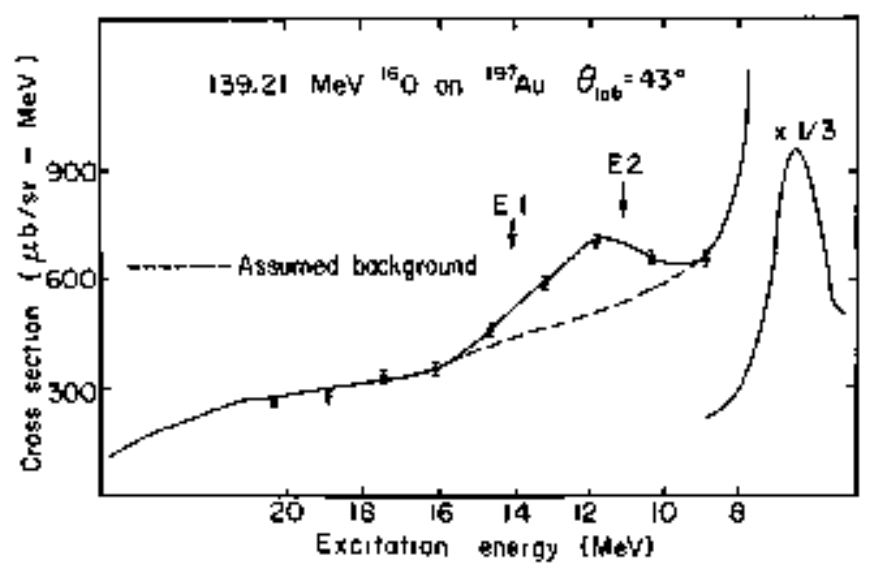

Fig. 1. $16_{0+197}$ Am inelastic scattering at ${ }_{1 \mathrm{AB}}=43^{\circ}$.

(XBl 766-8271) 


\section{ARGON ION REACTION STUDIES ON TANTALUM AND GOLD NEAF COULOMB BARRIER ENERGIES}

$R$ C Eggers, W 5 Rubbe, and J O Ragmussen

Gold and tantalup targets we brubarded with a $40 \mathrm{Ar}$ beam at 183 and $209 \mathrm{MeV}$, close to the Coulomb barrier height. Angular distributions of re. coiling reaction products were neasured by collexting an Al catcher foils. The radidactivity was counted after the irradiation by Ge(Li) detectors. One-neutron and one- and two-proton transfer prophrts were proninent. Simple semiclassical transfer theory adequately fits the backward peaking angular distributians at or below the Coulath barrier but falls at bombarding energies above the barrier. Yields of some products nore than two nass numbers renoved from reactants were observed in thicker targets at 235 MeV, and their significance is discussed,

To see what differences thete might be between spherical and sphemidal targets we chose to $\mathrm{rm}$ catcher foil experiments at the Berkeley Super Heavy Ion Accelerator. As targets we chose as spherical melei, gold, and as defonmed, tantalum. These muclei are as close in mass and charge as feasible, since the boundary between strongly deformed and spherical nuclei is not sharp in this region. The I⿰n our experinant at energies close to the Coulonb barrier to make it possible to compare theories involving nucleon tuneling with the projectile not doviating wuch from a Putherford trajectory. We made our measurements at 183,209, and $235 \mathrm{MeV} 2 \mathrm{ab}$ energy. For the two lower energtes we used a $400 \mathrm{\mu g} / \mathrm{cm}^{2}$ gold target and a $99 \mu \mathrm{g} / \mathrm{cm}^{2}$ tantolum target on $100 \mathrm{\mu g} / \mathrm{cm}^{2}$ carbon backing. At the $235 \mathrm{kgV}$ energy we used a $5 \mathrm{mg} / \mathrm{cm}^{2}$ gold target and a $10 \mathrm{mg} / \mathrm{cm}^{2}$ tantalum target. Table 1 gives a list of the differential reaction cross-sections by product

\section{One-Neutron Transfer Results and Discussion}

Let us look at the evidence for differences (angle and energy integrated) between spherical and deformed nuclei. Te have compared our $41_{\mathrm{Ar}}$ angular distrabutions to theoretical calculations using the Semi-Classical Trantsfer Theory (SCTT), expressions of Alder, Horf, Pauli, and Trautmoun.

Their forwitation goes beyond that of Breit and Ebe $1^{2}$ notably in the inclusion of dependence on the angular monenta of the particular shellIoodel orbitals involyed. For energies above the barrier Alder et al. 1 suggest that one may still bo able to apply the theory by replacing the Putherford cross section factor by an experimental or optical. model elastic cross section.

Figure 1 shrws excellent agreenent at the lowest energy point, $183 \mathrm{MeV}$, for gold. There is only a slight dip in cross section at the most backward angle, indicating a slight absorption into other chanels for the nearly head-on collisions.

Table 1. Transfer reaction cross sectlons for argon-40 bean (mb/sr c.m.)

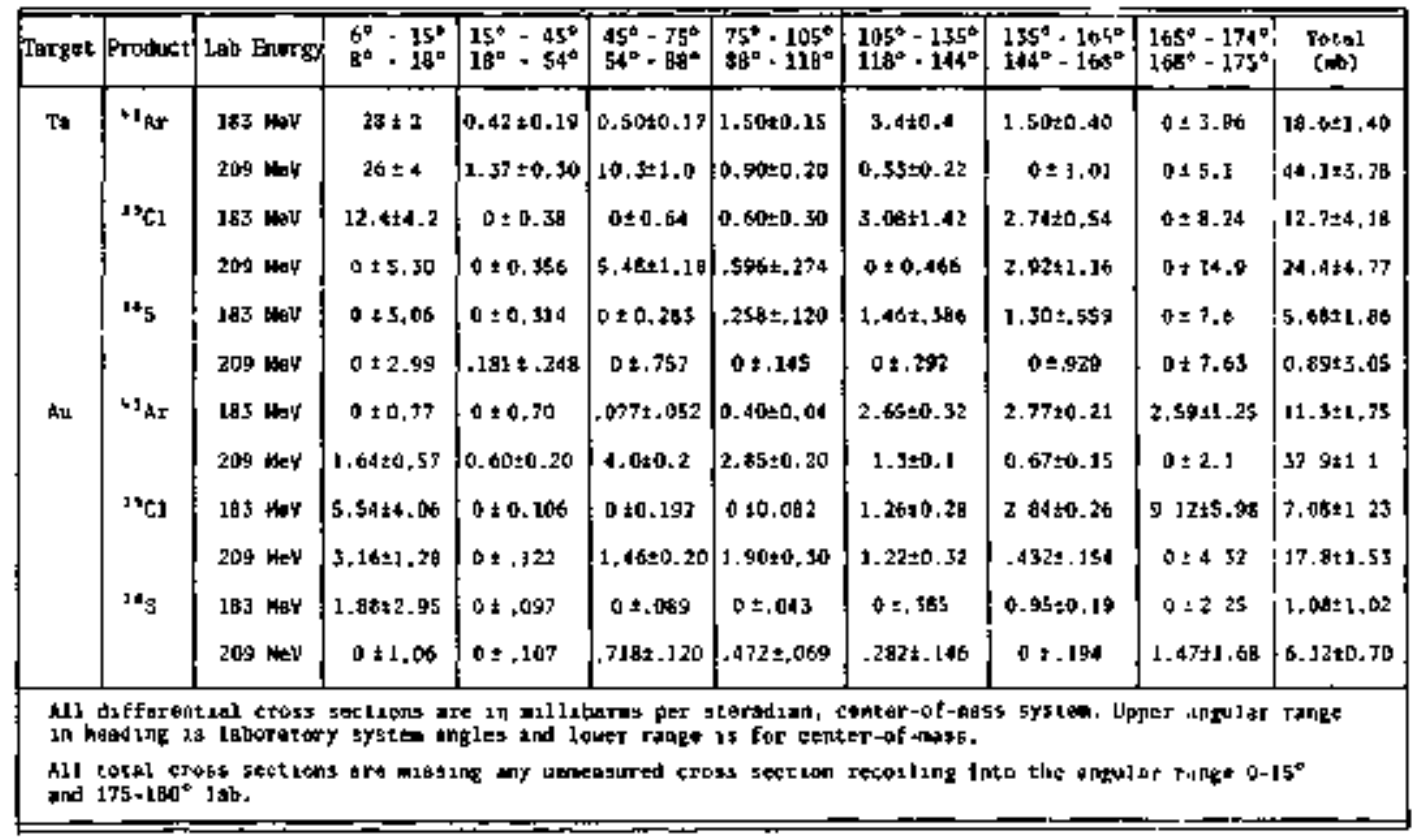




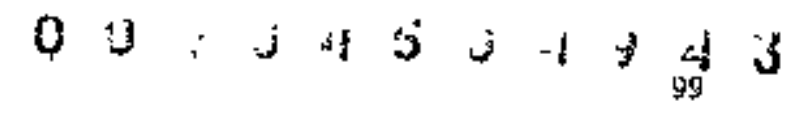

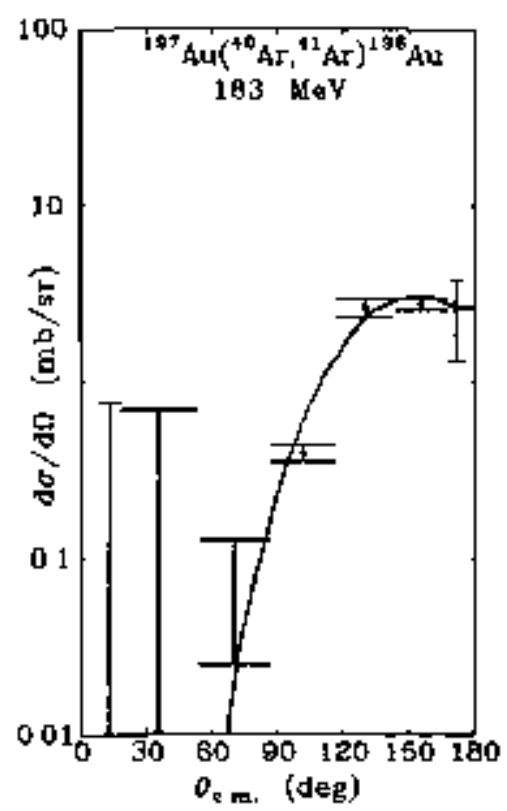

Fig, 1. Angular distribution of ${ }^{41_{A r}}$ produced from the reaction of $40_{A r}$ on $197_{A u}$ at $183 \mathrm{MeV}$ (1ab). The solid itne is the SCTT theory ${ }^{d}$ with an elastic cross section taken from an optical inodel progrand with real and imaginary Hoods-Saxon potential of radius constant 1.28 fm and diffuseness 0.4 tm. The po. tential depth is taken as $70 \mathrm{keV}$ for the real part and $15 \mathrm{kev}$ for the imaginary. Note that the theory fits the angular distribution rather w11. The theoretical curve here is for the $f_{7 / 2}$ neutron orbita1 in argon and $\mathrm{p3} / 2$ in gold, but the angular distribution is essentlally the same for gold orbitals of other $j$ values. The absolute theoretical cross section has been normalized uwward by a factor of $8+0$, but absolute cross sections depend sensitively on the radius constant used in the SCTT theory, here taken as 1.25 fn.

(XRL 743-2714)

In Fig. 2 for $\mathrm{Ta}$ at $183 \mathrm{keV}$, the agreement of SCTT theory and our data is not as good, in that the experinental angular distribution away from the peak does not drop as far as the theoretical. The same flattening trend is even more pronounced for the higher energy points, Figs. 3 and 4.

Clearly the semi-classical transfer theory patched by the above-barrier elastic scattering cross section is not adequate for the angular distriburtions we measured. It is likely that much of the excess cross section away from the classical grazing angle arises from inelastic transfer, where the ${ }^{4 A_{A}}$ and $i$ ts complement $196_{A 12}$, are left in higher excited states.

There are no systematic differences between the Au and Ta results that we can attribute silply to deformaticn. Evidently only a locallzed zone on the spherotdal surface is active for transfer, and the angular distribution is no less sharp than for sphericel nuclet.

The angular distributions for ${ }^{39} \mathrm{Cl}$ and ${ }^{38_{5}}$ can be seen from Table 1 to be rather sinilar to those for ${ }^{4} 1_{\mathrm{Ar}}$.

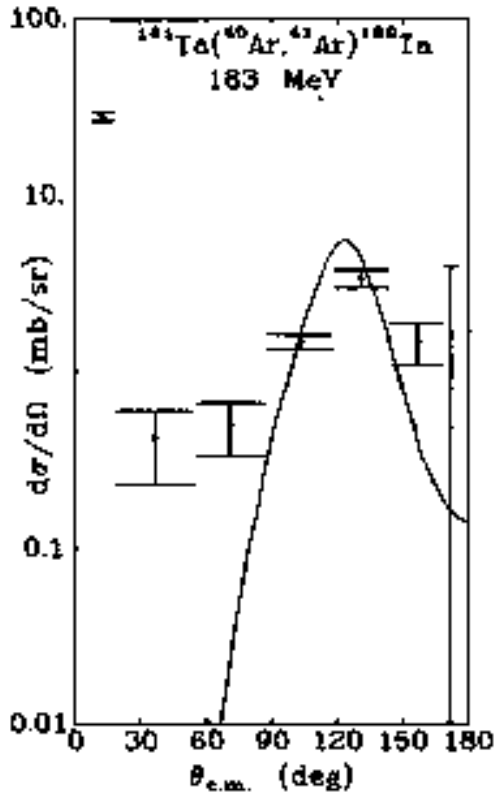

Fig. 2. Angular distribution of $41_{\mathrm{Ar}}$ produced from the reaction of $40_{\mathrm{Ar}}$ on ${ }^{181} \mathrm{Ta}$ at $183 \mathrm{keV}$. The solid line is the SCTT theorf 4 with an elastic cross section calculated in the same mennet as for Fig. 1, but with renormalizaticn factor of 2.0 and $i 11 / 2$ neutron orbital. The most forward angle point is unreliable, since $40_{A T}$ scattered into the catcher foll might transfer there.

(XBL 743-2711)

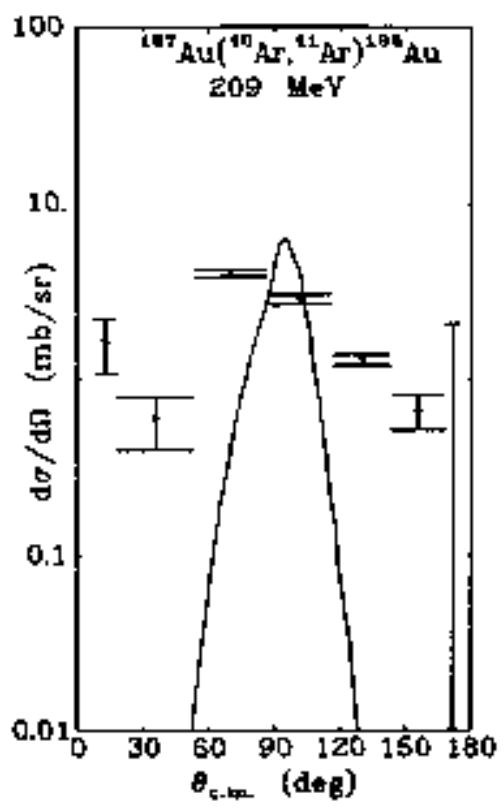

Fig. 3. Angular distribution similar to Fig. 1, except for $209 \mathrm{kev}$. Note that there is a marked disagreement between the theory and the experinent as to the width of the peak at the classical grazing angle. The theoretical curve has been renormalized upward by a factor of 8,4 .
(XAL, 743-2715) 


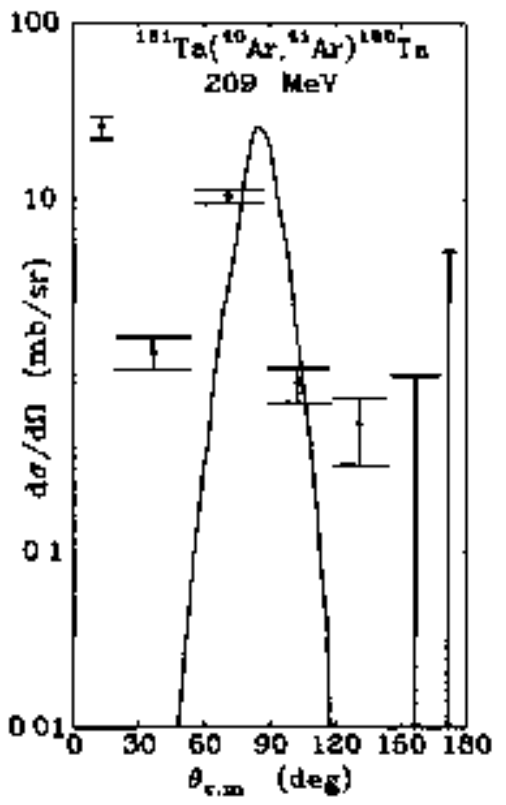

Fig. 4. This is a sirilar angular distribution to Fig. 2, except that the energy here is $209 \mathrm{BkV}$. The theoretical curve is normalized upward by a factor of 10. Sote that there is again a marked disagreement between the theory and the experiment as to the width of the peaking at the classical grazing angle.

(XBL 743-2712)

\section{Footnotes and References}

*Condensed from Nucl. Phys A263, (1976) 500.

${ }^{\dagger}$ Present Address: Cyclotron Institute, Texas AFM University, College Station, Texas.

${ }^{\ddagger}$ Present Address: Stadt Rudolf-Virchow-Krankenthaus, Strablungsabteilung, West Gemany.

1. K, AIder, R. Morf, M. Pauli ant D. Trautmagr, Hucl. Phys. A191 (1972) 399.

2. G. Ereit and M. E. Ebel, Phys, Rev. 103 (1956) 679 .

\section{SEMICLASSICAL AND QUANTUM MECHANICAL ASPECTS OF ONE- AND TWO-NUCLEON TRANSFER*}

C K Scon, D L Hendren, L Kraust, of F Maguire.

$J$ Mahonsy, $Y$ Terrien, $\neq$ and $K$ Yagis

The criterion for the validity of the seriaclassical approach to heavy-ion reactions is of ton expressed by the fact that the wavelength of relative motion of the cooliding ions is short tompared to the interaction radius, and consequently the particles are Iocalized on classical trajectories. Although this condition is usually well fulfilled for heavy-ion reactions at incident energies above the Cotlonst barrier, the observed onset of quantuin mechanical effects in going from ono- to two-mucleon trensfer in this energy ragion shuws that other criteria are necessary. Here give an andiytical description of the transition between quanturn mechanical and classical behavior in terms of the nunber of partial waves contributing significantly to the reactions, and illustrate the rescults by comparing one- and tho-nucleon transfer reactions induced by $78-\mathrm{HeV}_{\mathrm{eV}} 12 \mathrm{C}$ ions on 144.

We begin by making the partial-wave expansion of the scattering amplitude:

$$
f(\theta)=(2 i k)^{-1} \Sigma_{\ell}(21+1) n_{\ell} \exp \left(2 i \delta_{\ell}\right) P_{\ell}(\cos \theta) \text {. }
$$

On accont of the peripheral nature of heavy ion collisions at high energy, it has been shown ${ }^{1}$ that the magnitude of $n_{\ell}$ can be well described by

$$
n_{l}=n_{\ell_{0}} \exp -\left(R-R_{0}\right)^{2} /(\Delta l)^{2}
$$

which has a maximm for partial wave of $-\mathrm{kR}$, where $R$ is the interaction radius. The spread of contributing $\ell$ values, $h \mathrm{l}$, is determined by strong absorption $\left(\ell<t_{0}\right)$ and by the decay of the form factor $\left(l>\mathbb{R}_{0}\right)$.

For $t_{2}$, wake a Taylor expansion:

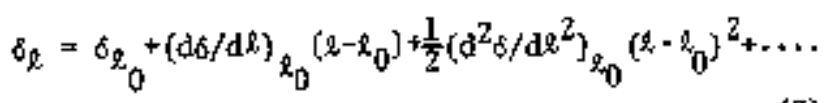

Using the WKB formalisn, the phase shift of and the stattering angle $\theta_{2}$, associated with partial wave $\mathrm{k}$, are related by ${ }^{\prime} \theta_{\mathrm{l}}=2\left[d \delta_{\mathrm{R}} / \mathrm{d} \Omega\right]$. Substituting into Eq. (3), obtain

$$
\delta_{l}=\delta_{Q_{0}}+\frac{1}{2} \theta_{0}\left(l-l_{0}\right)+\frac{1}{4}\left(\mathrm{~d} \theta_{l} / d\right)_{Q_{0}}\left(l-l_{0}\right)^{2} \ldots \text {, }
$$

where 8 is the angle of deflection of the trajectory corresponding to the grazing partial wave $\ell_{0}$. The scattering anplitude $f(\theta)$ in $B q_{+}$(1) can be evaluated with the above expressions for $\hat{\sigma}_{\ell}$ and $\eta_{l}$, together with the asysuptotic form of $P_{l}^{l}$ valid for large $\ell$, and by replacing the sum over $\ell$ by an integral. These additional simplifications depend on the conditions of $>$ at $>1$, valid for $a$ periphexal reaction at high incident 'energy. ${ }_{3}$ For tho differential cross section obtained 3

$$
\frac{d \sigma}{d \theta}=|f(\theta)|^{2}=\exp \frac{-\left(\theta-\theta_{0}\right)^{2}}{(\Delta \theta)^{2}}+\exp \frac{-\left(\theta+\theta_{0}\right)^{2}}{(\Delta \theta)^{2}}
$$$$
+ \text { (interference terms) . }
$$ 
This equation can be interpreted as the superposition of two "classical" distributions centered arvond the grazing trajectories at $\pm \theta_{0}$, with an interference term in the region of overlap. For the purposes of this work, we ignore the effects of the interforence which are discussed ir Ref. 2 and consider only the first tern in Eq. (S), This tern corresponds to a distribution centered at the argle $\theta_{0}$, of width $\Delta \theta$ :

$$
(\Delta \theta)^{2}-2 /(\Delta l)^{2}+\frac{1}{2}\left(d \theta_{Q} / d l\right)_{l_{0}}^{2}(\Delta t)^{2} .
$$

Wh now use the strietly slasateal result of a Rutherford orbit, that $l=\eta \cot (\theta / 2)$, where $\eta$ is the Somberfeld parameter, and therefore

$$
\left(d \theta_{2} / d z\right)_{g_{0}}=-2 T^{-1} \sin ^{2}\left(\theta_{0} / 2\right)
$$

The curve of $4 \theta$ versus al derived from Eas. (6) and (7) is shown in Fig. 1 for a typical reaction of $78-k_{\text {av }}{ }^{12} \mathrm{C}$ ions on $1444 d$, in which $(\mathrm{d \theta} / \mathrm{dt})_{\mathrm{t}_{0}}=$ 0.013 . This curve has a mininam value at

$$
\Delta s=\left[2\left(d \lambda / d \theta_{0}\right)_{\ell_{0}}\right]^{1 / 2}=\sqrt{\pi} \csc \left(\theta_{0} / 2\right)
$$

defining the transition at $\Delta l \approx 12$ between a region of quintal dispersion, $\Delta x \ll \sqrt{\pi} \csc \left(\theta_{0} / 2\right)$, where $\Delta \theta$ decreases with increasing $\Delta l$, and a classically dispersive region, $\Delta 2 \gg \sqrt{\eta} \csc \left(\theta_{0} / 2\right)$, whe re $\Delta \theta$ increases with increasing ah. Also, shown in Fig1 are separate curves corresponding to the two dispersive terms in Eq. (6).

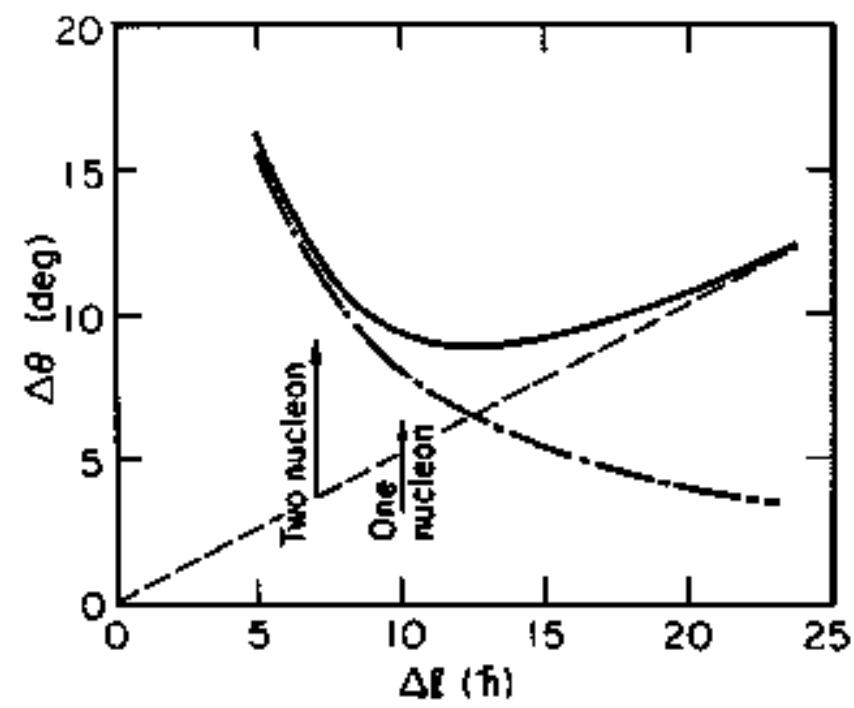

Fig. 1. The variation of to with $\Delta \mathrm{f}$ predicted from Eq. (6). The dashed and dot-doshed curves represent the linits of $\mathrm{E}$. ( $(6)$, obtained by setting the first and second dispersive terms to zero, respectively. The Al values derived fron fitting Eq. (2) to the IWRA reaction anplitudes for the one- and two-noutron transfer data of Fig. 2 are marked in the figure.

(XBL 752-2266)
The values of de pertaining to the one- and two-deutron transfer reactions $144 \mathrm{Nd}(12 \mathrm{C}, 13 \mathrm{C}) 143 \mathrm{i}$.

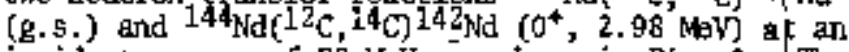
incident enersy of $78 \mathrm{KoV}$ are shown in $\mathrm{Fig}_{\mathrm{g}} \mathrm{2}$. The Aq values were cbtained from I:HR calculations using a typical set of optical-model parameters (potential $A$ of Table 1). For a single-nucleon transfer a $A=10$, and the corresponding value of $\Delta \theta=9.2^{\circ}$ is almost exactly equal to the observed half-width of the differential cross section at $1 / \mathrm{e}$ of the maximen. Dur theoretical curve for $\Delta t$ versus $\Delta \theta$ is therefore in good agrement with the data for one-nucieon transfer. Furthermore, since at lies close to the mirimun of the carve, our modal predicts that the width of the differentipl cross section for one-nucleon transfer should be relatively stable against variations of $\Delta R$ brought about, for example, by changes in the optical-mpde1 potential. This prediction is illustrated in Fig. 2 (a), by comparing [NRA calculations with potential $A$ and potential $B$, which is another typical potential from the literature. Both potentials lead to differential cross sections which are alowst

indistinguishable in the region of the bell-shaped raximum. Our nodel therefore gives a physical interpretation of the perșistençe in single-nucleon

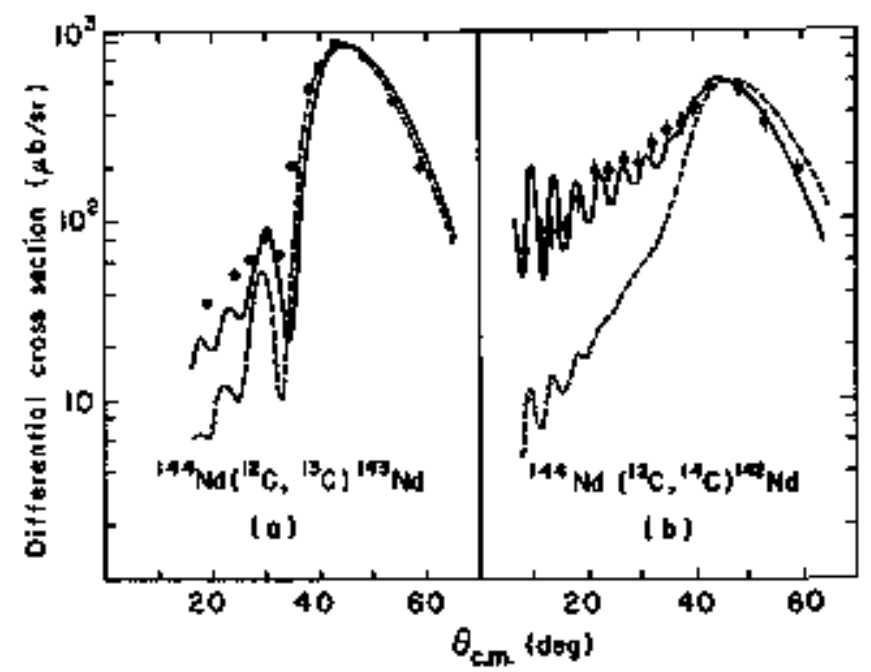

F1g. 2. Differential cross sections for (a) the reaction 144 d (12 1 C, $13 \mathrm{C}$ ) 143 kt and (b) the reaction $144 \mathrm{Nd}\left({ }_{\mathrm{C}}, 14_{\mathrm{C}}\right) 142 \mathrm{kd}$ at an incident energy of 78 MeV. The theoretical aurves in (a) are DWBA predictions rormalized to the data using the optical potentials of Table Iः potential A (solid line) and potential B (dashed Iine). In (b) both predictions use a potential of type $A$, with $r_{W}=1.26 \mathrm{fn}$ ( $\mathrm{solid}$ line) and 1.35 fur (dashed line).

(XBL 752-2268)

Table 1 Optical parameters used in analysis of $12^{2}+144 \mathrm{kd}$ at $78 \mathrm{HeV}$.

\begin{tabular}{ccccccc}
\hline \hline Potential & $V$ & $W$ & $r_{v}$ & $T_{W}$ & $a_{v}$ & $a_{W}$ \\
\hline$A$ & -40 & -15 & 1.31 & 1.31 & 0.45 & 0.45 \\
$B$ & -100 & -40 & 1.22 & 1.22 & 0.49 & 0.60 \\
\hline
\end{tabular}


trahsfer of the ambiguity in optical-model paramotors which is woll honon for elastic scattering 4,5

The ambiguity is less for the two-neutron tronsfer data as illustrated in Fig. 2(b) which corlpares two calculations with potentials of type $A$, and values of $r_{w}=1.36$ and 1.26 fr [the result obtained with potential B gave en almost identical distribution to potential $A$ with $r_{W}=1.36 \mathrm{fm}$, so the coruparison is essentially the same as in Fig, 2(a)j. We wish to mmphasize the sensitivity to the parameter $r_{w}$ in which a reduction of a few percent increases the forward-angle cross section by a factor of 10 . From an intuitive viempoint, the greater sensitivity of two-nucleon transfer to details of the optical potential has been ascribed to the sharper fall-off of the form factor which renders the forward cross section more sensitive to close trajectories deflected forwent by the at hactive nuclear potential.

In our model the greater sensitivity of the two-nucleon transfer cross section is due to the fact that the associated $\Delta l$ walues of approximately th lije on the steeply rișing portion of the aurve in Fig. 1. For these reactions then, whereas the one-rucleon transfer lies in the transition region between semiclassical and quantud mecharics, the two-nucleon trinsfer lies we11 in the quantum-Jechanical region. To define a classical trajectory, a certain spread of contributing $t$ values is necessary, and the model gives an analytical determination of this 1 init. It falls, however, to reproduce exactly the observed width of the distribution for two-nucleon transfer, since $\Delta R=$ oh corresponds to $\Delta \theta=12^{\prime}$, compared to the experimental value of $16^{\circ}$. For this case the form of $\eta_{\ell}$ and $t_{l}$ deduced fron the DWha phase shifts showed that, although the Gasssian parametrization of $\eta_{\mathfrak{f}}$ in Eq. (2) was still adequate, the expansion of ${ }^{6}{ }_{\mathrm{p}}$ in $\mathrm{Eq}+$ (3) hat to be carried to the next order. The inclusion of a third-order term has indeed been shown theoretically to lead to a broader angular distribution and to the asymetric rise at forward angles observed in our data for twomention transfer.

The analytical model we have describet for peripheral heavy-ion reactions at incident energies well above the Coulonb barrier establishes the regions of sentclassical and quantum mochanical behavior. It was used to give physical insight into the form of differential cross sections for one- and two-muclem transfer.

\section{Footnoties and Referencess}

"Condensed from Phys. Rev, Lett. 36, 226 (1976).

'on leave from Centre de Recherches Nucléaires et Université Pasteur, Strasboung, France.

${ }^{\dagger}$ On leave from Centre d'Etudes Nucleaires de Saclay, Saclay, Frence.

On leave fron Osaka Iniversity, Osaka, Japan. Present address: Tsukubs Iniversity, Ibaraki, Japan. 1. Y. M. Strutinskii, Phys. Lett. 44B, 245 (1973). 2. W. A, Friednan, W. K. KeVoy, and G. W. T. Shuy, Phys. Rew. Lett. 33, 308 (1974).

3. S. Kabana, in Reactong setween Complex Molef, edited by R. L. Robinson, F. K. MaGowan, J. B. Ba11, and $J . H_{*}$ Hanilton (Akorth-Holland, Ansterdan, 1974), Vol, 2, p.189.

4. N. K. Gendenning, In Reactions Between Coxplex Wtioled, edited by R. L, Robinson, F. K. Mklowan, 3. B. Ball, and J. H. Hamilton (North-Holland, Amsterdan, 1974], vol. 2, p.137.

5. G. R. Satchier, in heactions Betueen Corwtert ithotei, edited by R. L. Robinson et al. (WorthHalland, Ansterdam, 1974), Vol, 2, p.17l.

6. R. J. Ascuitto and N. K. Glendenning, Phys, Lett. 48B, 6 (1974).

\section{NEW ASPECTS OF HIGH ENERGY, HEAVY-ION INDUCED TRANSFER FEACTIONS ON HEAVY TARGETS}

C Olmer w Buenerd, C K Gẹloke. D L Hẹndrile,

J Manoney, A Mencheca-Rocha,t and D K Scol1

Single and multi-nucleon transfer reactions induced by high-energy heavy ions on heavy targets are currently being investigated using $20 \mathrm{k}$-V/amu beams developed at the 88-Inch Cyclotron. Experiments have been perforted using 312-bv 160 and $250-M e V ~ 14 N$ projectiles incident on targets of $208 \mathrm{pb}, 197 \mathrm{Au}$ and $232 \mathrm{Th}$. The reaction products were detected with the QSD nagnetic spectrometer and focal plane detection systen, pernitting the separation and identification of incividul isotopes.

Our motivation for compencing this work was provided by earlier studies 1 at the $88-$ Inch Cyclotron of heavy-ion induced single-proton stripping reactichs at loker incident energies. Typical spectra obtained near the grazing angles for the $20 \mathrm{SPb}(160,15 \mathrm{~N}) 209_{\mathrm{Bi}}$ reaction at 104,140 and 217 WeV are show in Fig. 1(a-c). Prominent in these spectra are transitions to well-knom proton sjngle particle states in $209 \mathrm{Bi}$, and these are labelled by their shell model orbitals. Thess spectra, and others measured for sinitar transfer reactions. exhibit a j-dependence of the transfer cross section, such that the proton rempved from a $j=2-1 / 2=j$, arbital (as in 160 ) would then be preferentially transferred to a $\mathrm{j}=\mathrm{\imath}+\mathrm{l} / 2=\mathrm{j}$, orbital in the residual nucleus, kn explanation of this observation, and its apparent disapperance 

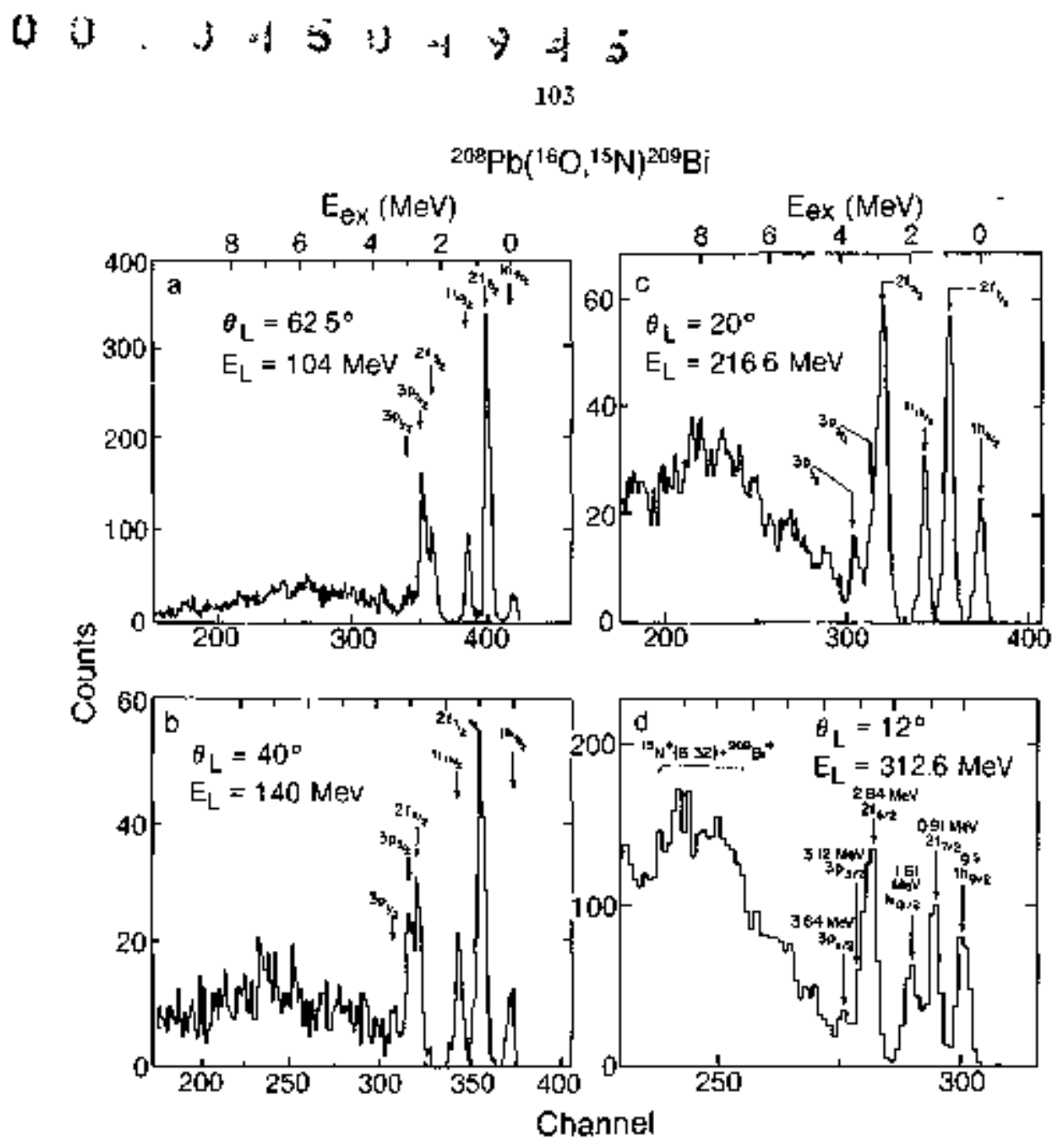

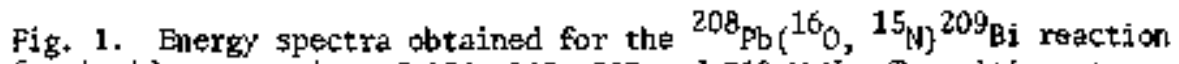
for incident energies of $104,140,217$ and $312 \mathrm{kV}$. Transitions to known proton single particle states are labelled according to thier she1l model orbitals.

(XBL 7612-4S18)

at higher incident energies (as evidenced by the equal population of the $2 f_{7 / 2}$ and $2 f_{5 / 2}$ levels), was found by invoking recold effects in the [WWA analysis, thereby providing experimental eqidence for the major inportance of a previcusly neglected theoretical consideration.

Interest in the cunrent work was sparked by the observation, within theșe measured data, of a broad structure centered at $\sim 8 \mathrm{keV}$ in excitation, the strength of whith appears to increase relative to the single particle strength as the incident energy was increased. The fact that this peak appeared to be constant in excitation energy over a wide range of bonbarding energy indicated that this phemcoenom was not the result of kinentatic factors favoring the population of different final states, but may reflect the increpsingly stronger population of the same individual state(5) in the residual micleus. To investigate the nature of this structure, we bave examined this same reaction, and other complomentary transfer reactions, at higher jincideant onergies where a more favorable population of the structure appears to be indicated.

A contracted spectrum of the ${ }^{20 \mathrm{~B}} \mathrm{~Pb}(16 \mathrm{O}, 15 \mathrm{~N})$
$209 \mathrm{Bi}$ reaction obtained near the grazing angle for an incident energy of $312 \mathrm{MeV}$ is shown in Fig. 1d for comparison with the data at lower incident energies. Several features in the current spectrum are of interest. First, the population of known high-spin proton single particle states is again inficated, and the experimental cross sections for these transitions are similar in magnitude to those obtained at the lower energies. Thus the population of the wide structure, observed again at $\sim 8 \mathrm{KaV}$ in excitation, appears to be strongly energy dependent (unlike the discrete states) and to increase rapidly with increasing incident energy.

An additional feature in these spectra is. the apparent reversal of the $j$-dependence seen at the lower bombarding mergies. Wheregs the pop-

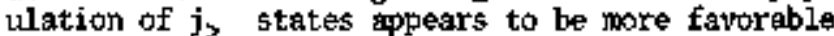
at 104 and 140 $\mathrm{MeV}$, and to equal the population of $j_{5}$ states at $217 \mathrm{MkV}$, the present high-energy work indicates a favoring of $j<$ over $j>$ final states. Semiclassical arguments, in the framework of the Brink model, 2 can explain this effect by fnvoking monertum conservation, as viewed 
schentatically in Fig. 2. A maximun transfer probability will occur if the component of monentur of the transferred macleon (of mass in) along the beara direction is almost conserved, l.e.

$$
h \Delta k-p_{i}-P_{f}=m v-h\left(\frac{\lambda_{i}}{R_{i}}+\frac{\lambda_{f}}{k_{f}}\right) \Leftrightarrow 0 .
$$

Thus for sall incident energies, $\lambda_{j}$ and $\lambda_{\text {f }}$ are of opposite sign (for the equality to hold) so $_{0}$ that the previously observed $f$-dependence will be seen, namely that $j_{3}+j_{\zeta}$ and $j_{\zeta}+j_{3}$. For higher ixcident energies, however, $\lambda_{i}$ and $\lambda_{f}$ are of the same sign so that a reversal of the $j$-dependence will be observed, namely that $j_{3}+j_{\rangle}$and $j_{\zeta}+j_{<+}$ Finite range, full recoil [WBA calculations are alreacy in progress to investigate whether a more complete analysis can sinilarly accotent for this enercy dependence.

A larger portion of the spectrul displayed in Fig. 1d is shown in Fig. 3, together with other (I60, $15 \mathrm{~N}$ ) spectra obtained with $197_{\mathrm{Au}}$ and $232 \mathrm{Th}$ targets. The excitation of several wide structures in the residual nuclei is indicated, and relativeIy intense yields are observed, In particular, several such peaks appes in the $197 \mathrm{Au} \rightarrow 198 \mathrm{Hg}$ spectrum, in addition to the expected ground state peak. The $208 \mathrm{~Pb}$ and $197 \mathrm{Al}$ targets were 0.7 and $2.7 \mathrm{mg} / \mathrm{cm}^{2}$ thick, respectively, and the 232 Th target was 5 moto ${ }^{2}$ thick so that comparable structure in the $23^{2} \mathrm{Th}+233 \mathrm{~Pa}$ spoctrum may be toresolved.

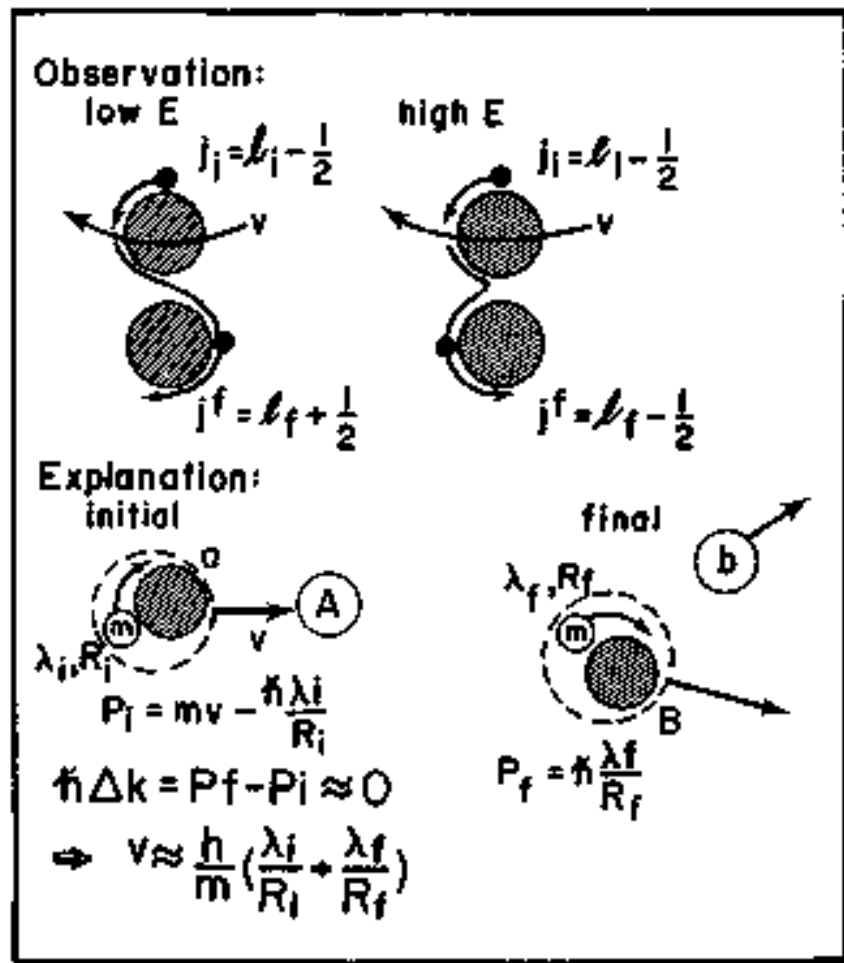

Fig. 2. Schentatic diagram of the $A(a, b) B$ transfer reaction, explaining the changing $j$-dependence of the cross section in ternts of different orbit matching at low and high incident energies.

(XBL 766-8324)

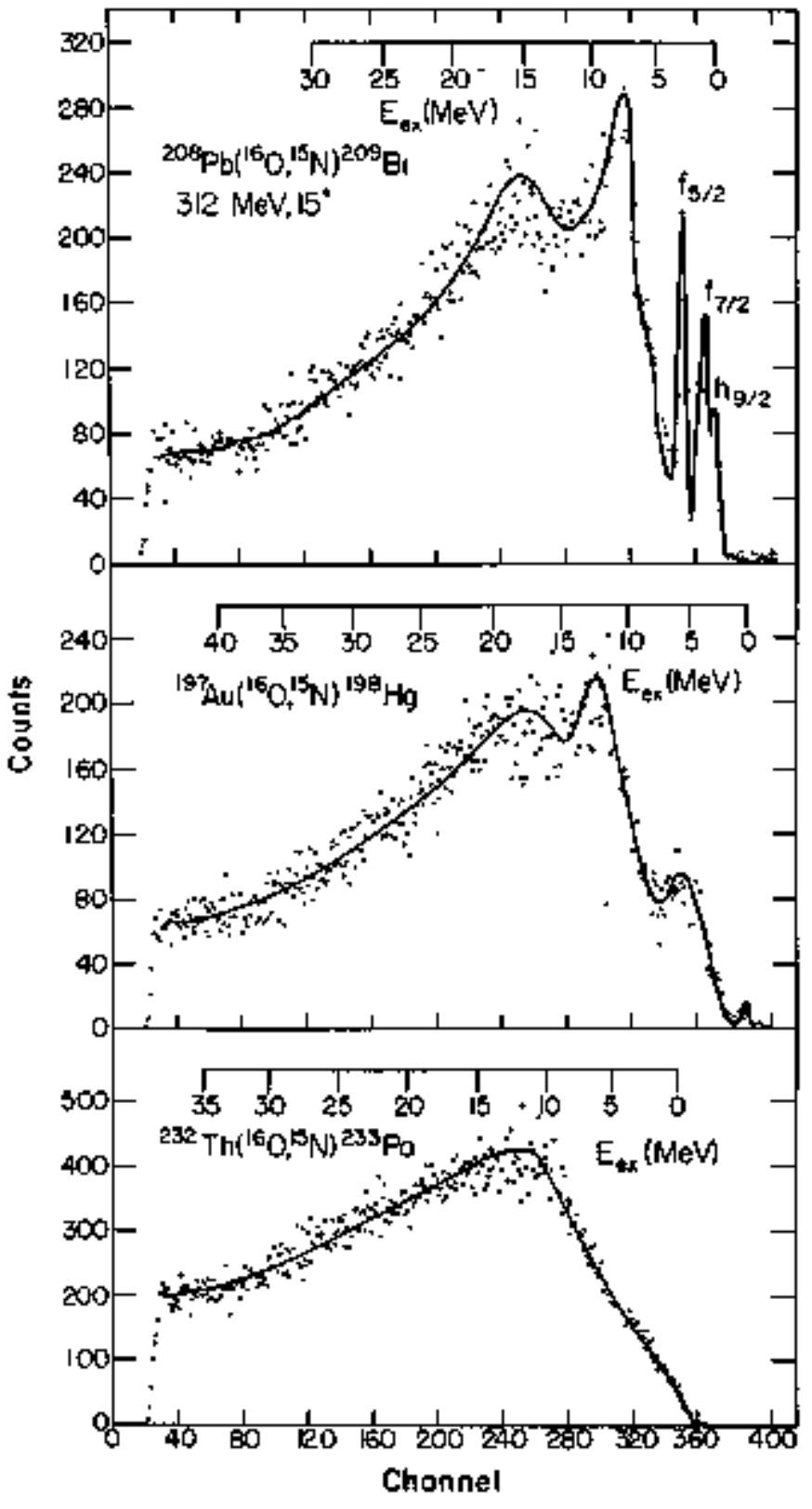

Fig. 3. Energy spectra at $15^{\circ}$ for the $\left({ }^{16} 0,15 \mathrm{~N}\right)$ reaction jnduced by $312 \mathrm{keV} 160$ on targets of $208 \mathrm{pb}, 197 \mathrm{Au}$ and $232 \mathrm{Th}$.

[XBL 763-2562)

Spectra for the corresponding $\left(16_{0}, 15_{0}\right)$ neutron tranşfer reaction are shown in Fig. 4. Again, the strong population of several broad structures is evident, some of which occur at nearly the same excitation energy as obseryed in the proton transfer spectre. As in the $(160,15 \mathrm{~N})$ results, the intensitios of these broad structures has increased strongly both absolutely and relative to the single particle transitions for inctdent energfes between 104 and $312 \mathrm{keV}$. The internse population of high spin single particle states is seen here also, especially for the ${ }^{208} \mathrm{~Pb}+209 \mathrm{pb}$ spectrum. Also evident in the spectrum is a candidate for the j15/2 fragment state, which was observed at the same excitation energy, but only weakly populated in the neutron transfer sturies 
$00.3450-1 \% \frac{1}{405}$

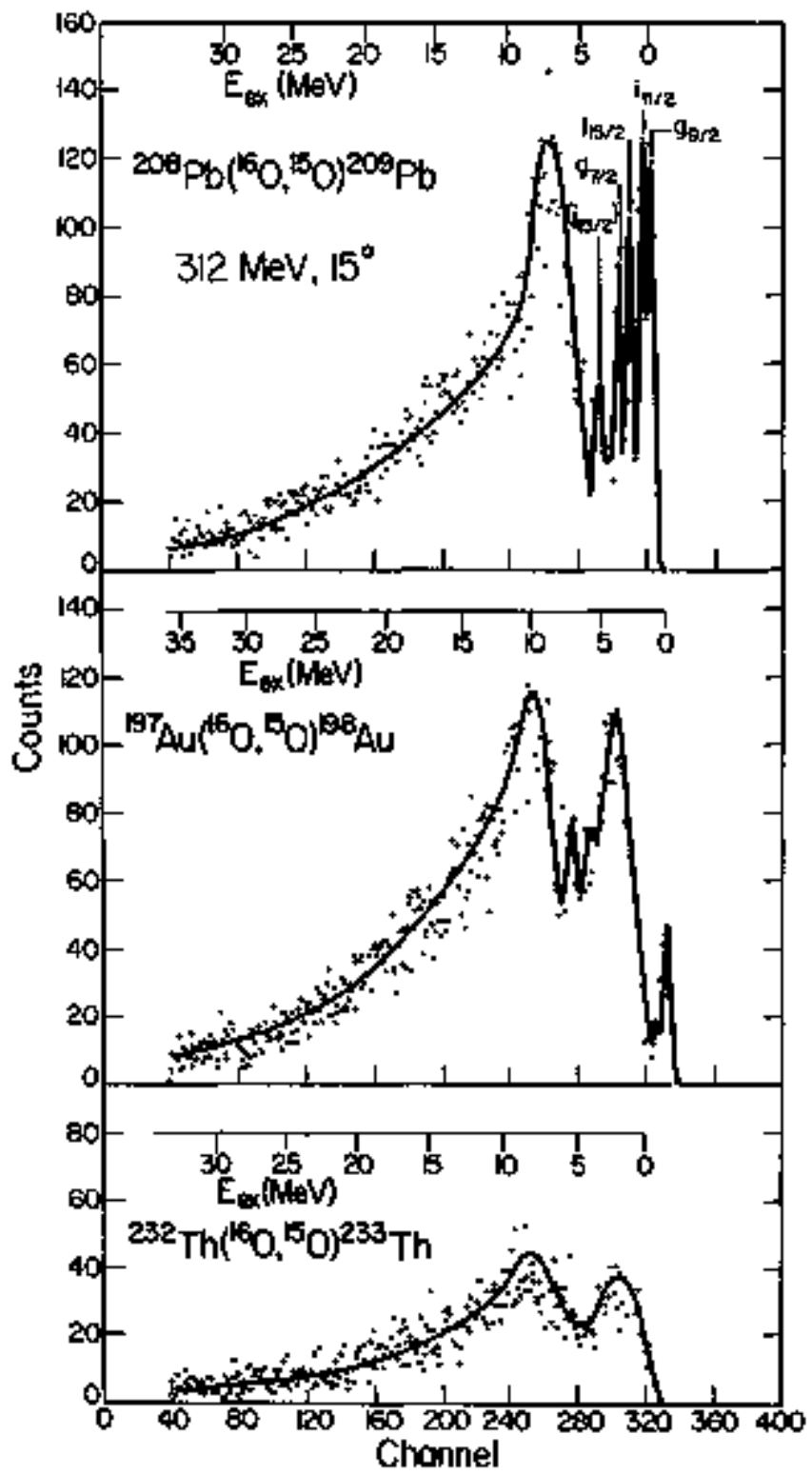

ous exeitation of the residual nuxleus and the $15 \mathrm{~N}$ or 150 reaction products, most probably the P3/2 hole state at 6.32 and 6.1B HeV, respectively. The subsequent in-flight gapuna ray decay of the reaction product would then give rise to a Iopplerbroadened peak in the spectrm. In order to investigate this possibility, proton and neutron transfer reactions were perfomed on $208 \mathrm{pb}, 197_{\mathrm{Au}}$ and $232 \mathrm{Th}$ targets using a $250 \mathrm{NeV}$ incident $14 \mathrm{~N}$ beam. Since $13 \mathrm{~N}$ has no particle-stable excited states, significant differences between the $(160,15 \mathrm{~N})$ and $(14 \mathrm{~N}, 13 \mathrm{~N})$ spectra may indicate that the $160 \mathrm{0}-$ induced transfer data contain peaks originating from the excitation of $15 \mathrm{~N}$ and 150 , together with a lower excitation of the residual ruclei.

Spectra for the neutron and proton transfer reactions induced by $14 \mathrm{~N}$ are shown in Figs. 5 and

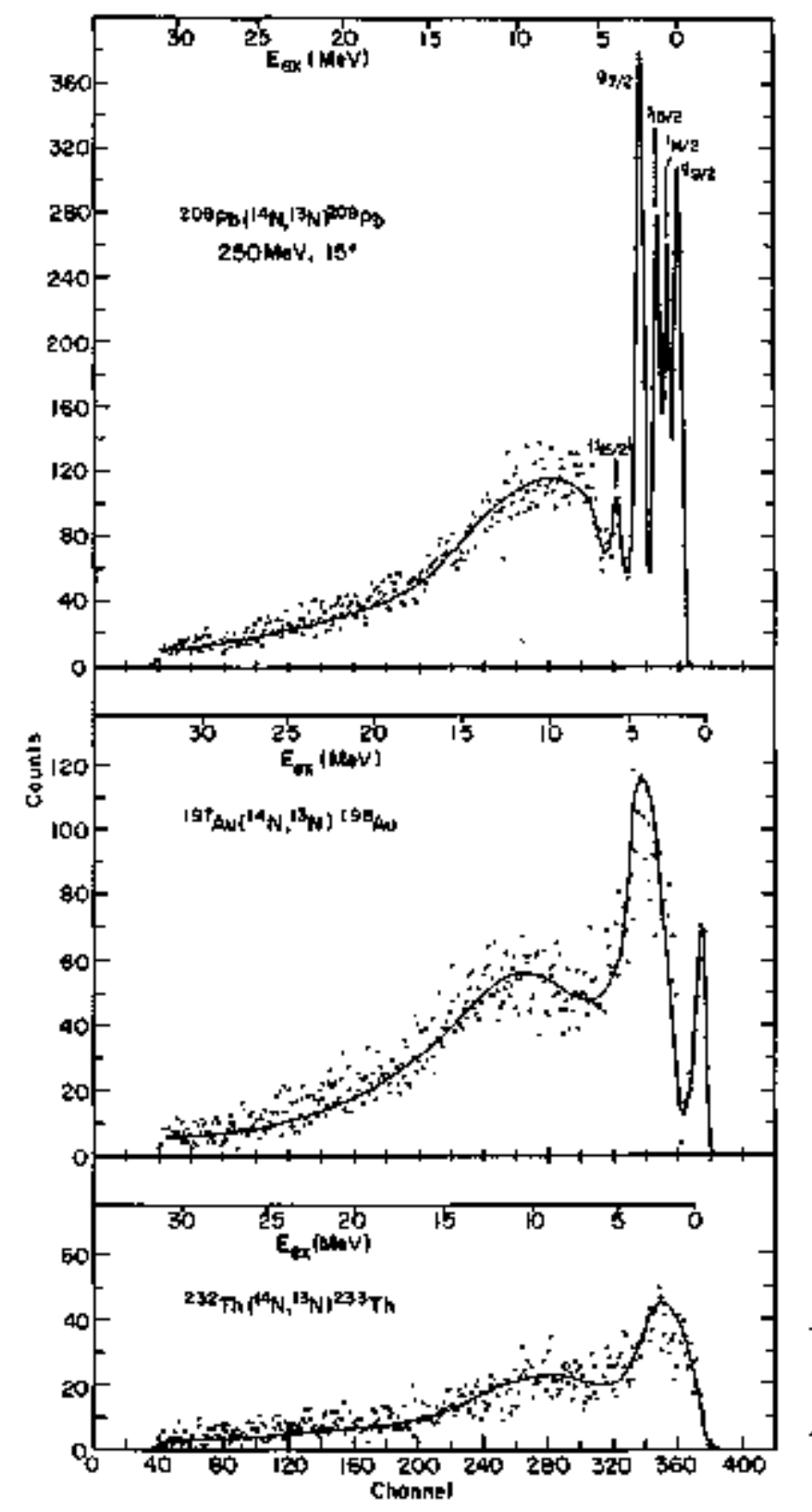

Fig. 5. Energy spectra at $15^{\circ}$ for the $(14 \mathrm{~N}, 13 \mathrm{~N})$ Teaction induced by $250 \mathrm{MeV} 14 \mathrm{~N}$ on targets of $208 \mathrm{~Pb}, 197_{\mathrm{Au}}$ and $232 \mathrm{Th}$.

(X) 763-5322 
6. A conparison of the $(160,150)$ and $(14 \mathrm{~N}, 13 \mathrm{~N})$ spectra shows remarkable símilarities, and suggests that ejectile excitation is not a major problem the present work, although manbigurs evidence for this conclusion is not yet ayaileble. A further similarity between 160 -and $14 \mathrm{~N}$-indured transfer reactions is observed in the population of discrete states from single nucleon transfer on $208 \mathrm{pb}$. As in the $(160,15)$ reaction data, the enhanced population of the $f_{5 / 2}$ state relative to the $f_{7 / 2}$ state is observed. In addition, for the (14 $\mathrm{N}, 13 \mathrm{~N}$ ) reaction, a conparable population of the $\mathrm{gg} / 2$ and $\mathrm{g}_{7 / 2}$ levels is seen, and the suspected $j_{15 / 2}$ fragment state also appears.

Differences among thașe spectra also exist, and several mysteries in these data still remain.

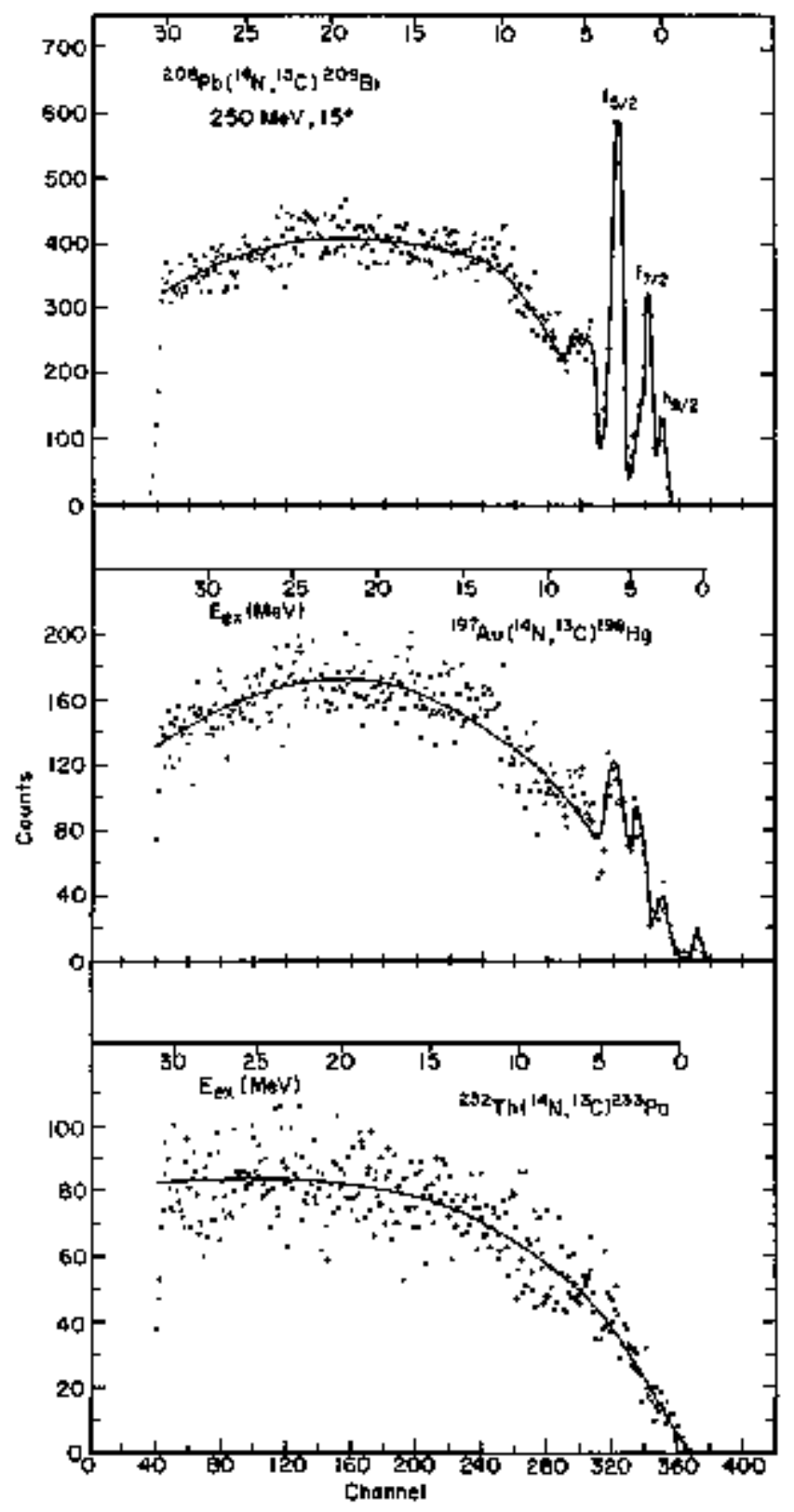

Fig. 6. Energy spectra at $15^{\circ}$ for the (14v, ${ }^{13} \mathrm{C}$ ) reaction incuced by $250 \mathrm{MeV} 14 \mathrm{~N}$ on targets of $20 \mathrm{~Pb}, 19 \mathrm{~F}_{\mathrm{Au}}$ and $232 \mathrm{Th}$.

(XBL 763-5321)
First, a marked difference is observed between neutron and proton transfer spectra. For the (14j, 13C) spectra especially and, to a lesser degree, for the $\left(160,1 \mathcal{F}_{\mathrm{N}}\right)$ spectra, a significant, under1ying continum-type background exists which does not appear for neutron transfer spectra. Although the shape of this strutture is reminiscent of that expected from projectile fragoentation. it is not at all clear why only proton and not neutron decay of the projectile whuld be obserryed in the present work.

An additional feature in these data, which is not understood at present, is the microscopic natare of the broad structures which appear in all the spectra. It is tantalizing to note that the observed centroids of many of these structures correspond alnost exactly to known giant resonance states, seen, for example, in inelastic eloctron, proton. अ recent $\left(p, p^{+}\right)$work of Bertrand and Kocher ${ }^{4}$ indicates a resonance at $\sim 4 \mathrm{MeV}$ excitation (FWHS $\sim 2 \mathrm{keV}$ ) and a wider resonence at $-12 \mathrm{kt} \mathrm{V}$

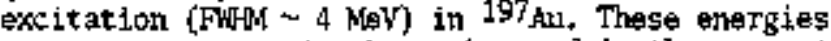
match almost exactly those obseryed in the present single nucleon transfer deta on $197 \mathrm{Aul}$, and similar correspondences of the obseryed ${ }^{209} \mathrm{Fb}$ and $2 \mathrm{~d}_{\mathrm{Bi}}$ peaks to known giant resonances in ${ }^{20} \mathrm{~Pb}$ can also be rade. It is premature at this stage to attribute the observed broad transitions to giapt resonances states al though, if recently obtained $16_{0}$ inelastic scattering data should exhibit these same excitations, then such a conclusion would not sem unreasonable,

The single nutleon transfer data reported here represent only a portion of the total data accumblated during the experiments. In particular, data for severaI angles over a wide excitation energy range for many different isotopes (from $6_{\mathrm{Li}}$ to ${ }^{1}$ ) are presently being processed. It is hoped that all these data, together with the inelastic scattering data, will be able to regrlve the question as to whether glant resonances are being populated in the present nork. This information will be especially useful in the recent investigations by many experimental and theoretical grous concerning the microscopic nature of deep inelastic processes. In particular, the theory of Broglia et aI, 5 irvokes just these giant resonances to explain the large energy, mess and charge losses which are experimentaly observed and, in a macroscopic framelork, attributed to frictional forces.

\section{Footnotes and References}

* Permanent addiress: Institur des Sciences Nucléaires, Grenoble, France.

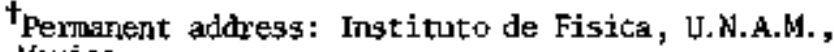
Mexico.

1. D. G. Kovar, F, D. Becchetti, B. 6. Harvey, F. Pthlhofer, J. Mahoney, D. W. Miller and

M. S. Ziman, Phys. Rev. Lett. 29, 1023 (1972);

D. G. Kovar, B. G. Harvey, F. D. Becche tti, J. Wahoney and M. S. Nagarajan, Nuclear Ghemistry Arnual Report (1972) bBL-1666, P, 90.

2. D. M, Brink, Phys. Lett. 40B, 37 (1972). 


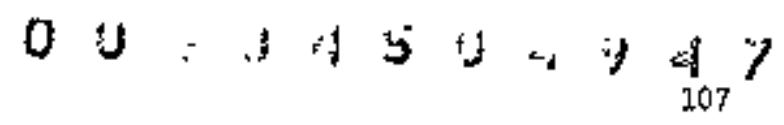

3. F. D. Becchetti, B. G. Harvey, D. Kovar, J. Whaneyer, C. Maguire and D. K. Scott, Ptyss. Rev. C 12, B94 (1975).

4. F. E. Bertrand and D, C. Kocher, subitted to
Phys. Rev. C.

5. R. A. Broglia, C. H. Dasso- and A. Winther, Pliys. Latt. G1B, 113 (1976).

\section{EVIDENCE FOR SHALLOW STRONGLY ABSORBING HEAVY-ION OPTICAL POTENTIALS}

J G Cramer, ${ }^{\prime}$ R M Devries, O A Goldberg.:

M S Zisman, and C Magure

Over the past several years we bave conhucted a detailed imrestigation of the elastic scattering of 160 fron $28 \mathrm{Si}$ and sinilar targets over a range of energies. We have concluded from this study that there are three principal regions of the nuclear potential which are probed by this type of elastic scattering analysis: [a] the extreme tall region of the potential, which is probed by elastic scattering performed very near the Coulomb barriex; (b) the strong absorption radius (or more quantitatively, the seni-classical turaing radius of the grazing partial wave), which is probed by scattering at essentially all bonbarting energies between the coulonb barrier and about $100 \mathrm{HeV}$; and (c) the surface region and "outer interior," which are probed by scattering at bombarding energies gbove about $100 \mathrm{keV}$, where enough energy is avllable to observe nuclear "rainbow" scattering.

Thus if the muclear potential is assumed to be independent of energy, it can bo mapped by simaltaneously fitting data of types (a), (b), and (c), as described above. In en effort to enlarge the infordation frotin the interior region, we have in a collaborative experiment studisa the scattering of 160 from $28 \mathrm{Si}$ at a laboratory bonbayding energy of $215.2 \mathrm{HeV}$, using the 160 beam fron the LBL 88-inch cyclotron. These data, together with data collected at lower energies ${ }_{\text {were fitted with a }}$ global analysis, requiring the same energy-independent six-parameter Woods-Saxon potential to fit both high and low energy data. The results are shom in F1g. 1. The potentials used in generating the curves in Fig. 1 are given in Table I.

There is a clear preference for the shallow potentials in this analysis. While it is possible to find deeper potentials that can fit either the low energy data or the high energy data, we have found no deep potential capable of fitting both low and high energy data at the sathe time. This fit is "unique" only in the context of energyindependent loods-saxon potentials.

It should perhaps be mentioned that Satchler has recently analyzed the drta set shown in Fig. 1 fron the point of view of the folding mode1. ${ }^{2} \mathrm{~B}$ e has started with a realistic nucleon-nucleon potential and obtained an effective real potential with a double-folding procedure while using an empirical Woods-Saxon imaginary potential. It was found to be necessary to renommalize the real potential and to we an energy-dependernt geometry for the imaginary potential, but with these modifications, it was possible to obtain quite a good fit to these data. Thus it is elear that deeper nomWoxds-Saxon energy-dependent potentials are quite capable of fitting the data set.

The results presented hexe pose an interesting question: Does the preference which has bexir found for a $10 \mathrm{MeV}$ real wet depth really mean that overlapping 160 and ${ }^{28}$ Si ruclei bave such a weak interactim? Even at our highest incident energies, "hotch-perturbation" tests of potential

Table 1. Dorived optical roodel potentials.

\begin{tabular}{|c|c|c|c|c|c|c|c|c|}
\hline Labol & $v_{0}$ & ${ }^{r_{0}}$ & ${ }^{a_{0}}$ & $\stackrel{\mathrm{w}_{0}}{\text { (rolins) }}$ & ${ }^{r} I$ & $a_{I}$ & $\begin{array}{c}x^{2} / N \\
(2 I 5.2 \mathrm{MEV})\end{array}$ & $\begin{array}{c}x^{2} / N \\
(3 \$, 0 \mathrm{HeV})\end{array}$ \\
\hline E18 & 10 & 1.35 & 0.618 & 23.4 & 1.23 & 0.552 & 4.9 & 1.2 \\
\hline A23 & 100 & 0.932 & 0.797 & 165 & 0.890 & 0.764 & 8.6 & 15 \\
\hline S75 & 100 & 1.06 & 0.640 & 42,0 & 1.06 & 0.640 & $1.3 \times 10^{5}$ & 2.0 \\
\hline
\end{tabular}



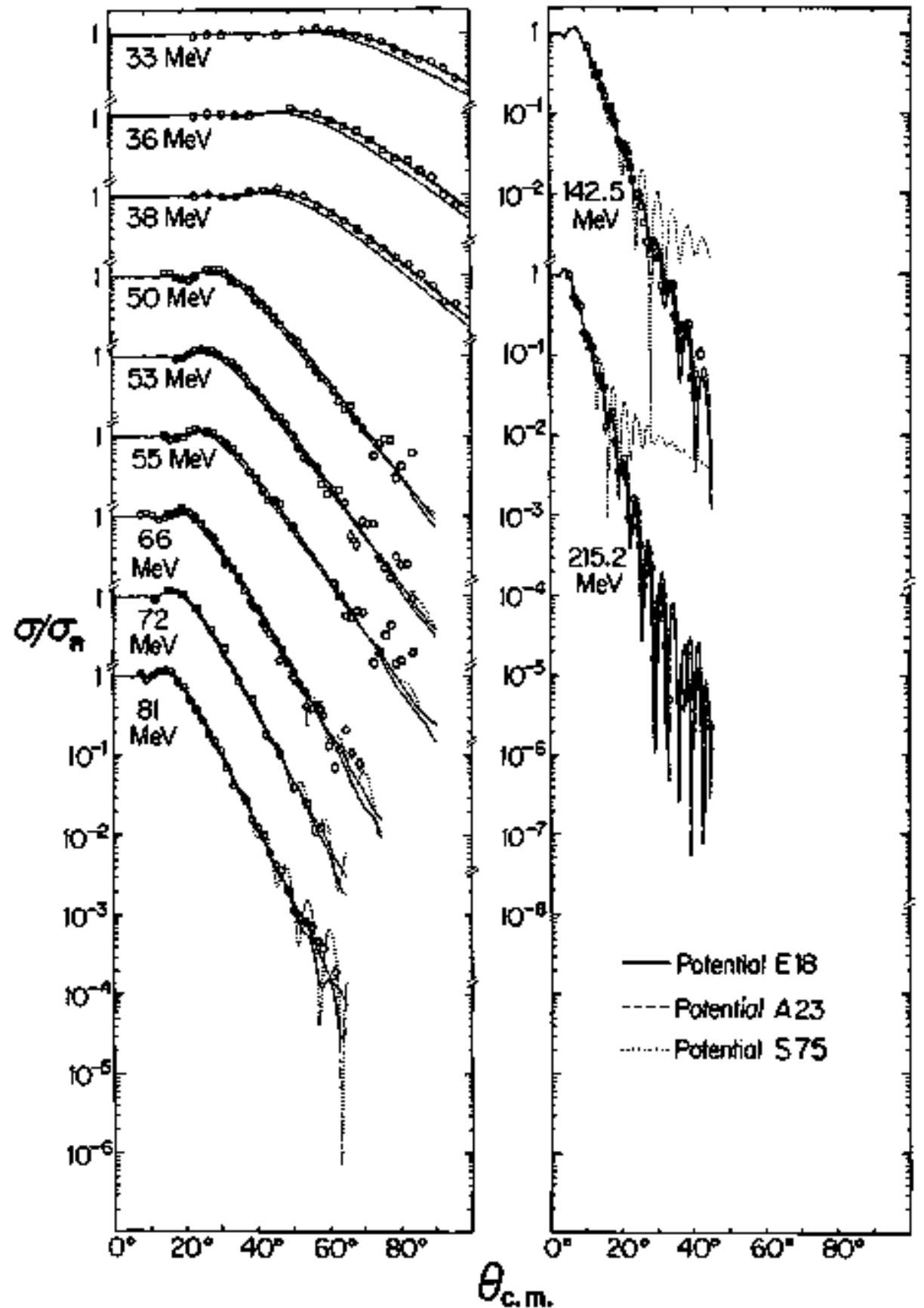

Fig. 1. Elastic ${ }^{16} \mathrm{O}+{ }^{28} \mathrm{Si}$ scattering at the labeled incident energies. The lines are optical model calculations using the parameters sets indicated and listed in Table $I$.

(XBL 766-8272)

sensjtivity indicete no contributions to the scattering from separation distances of less than 5.5 fm. 3 This the derived $v=10 \mathrm{MeV}$ value only indicates the potential's behevior in the nuclear surface region in terms of a Woods-Saxon shape and does not preclude the existence of a soft reputsive core, for example. Horegver, the eneroy required to separate $44 \mathrm{Ti}$ into $16 \mathrm{O}+28 \mathrm{Si}$ is $11.48 \mathrm{MeV}$. Therefore, in the zero incident energy limit, the bound-state pottential for this system would be considerably in excess of $10 \mathrm{MkV}$. Even with these qualifications, the derived $V=10 \mathrm{MEV}$ value for
160 ions represents a striking departure from the monotonic increase in reai wil depths foumd for light-ions, i.t., $50 \mathrm{keV}$ for nuxleons, $80-100$ $\mathrm{HoV}$ for deuterons, 110-130 $\mathrm{HeV}$ for alphas,

It is clear that more theoretical work on the potentials appropriate to heavy-ion interactions is indicated. More experinental data are needed on the elastic scattering at high and low energies of projectiles in the mas5 region $4<A<16$ so that optical potentials in this critical tromsition region can be determined. 


\section{Footnotes and References}

"Department of Physics, Univerșity of Washizgtom, Seattle, WA 98195 .

†Department of Physics, Lniversity of Rochester, Rochester, NY 14627.

'Iepartment of Phystes, University of Maryland,
College Park, Mil 20742.

1. I. G. Gramer et al. Nuxléar Physics Laboratory Afunal Report, University of Washlngton (1975),p.128.

2. G. R. Satchler, private comminication.

3. J. 6. Craner, private commication.

\section{${ }^{6} \mathrm{~L},+{ }^{28}$ Si ELASTIC SCATTERING \\ J $G$ Cramer, $R$ M Deunes, $D$ A Goldoerg.t \\ M S Zismen, and C F Magulre}

The ressuits of our study of the ${ }^{16} 0+{ }^{28} 5 i$ optical potential (see preceding paper) indicate that the 160 potential is radicelly different fron light in potentials. Clearly a study of Intennediate projectiles is of interest, concentrating on obtaining a large data set including both high and low energy data. For this reason we measured at the LBL 88 -Inch Cyclotron $135.1 \mathrm{keV}$ Li (the highest Elengy obtainable anywhere at this tine) scattering from $28 \$ 1$ as shown in Fig. 1. Data from Iowa 1 existed at $13 \mathrm{MeV}$ and are also show in $\mathrm{Fig}$. 1. Subsequently we have measured $18 \mathrm{keV}$ and, prelibjuarily, $44 \mathrm{keV}$ (11 MeV on temina1) data at Rochester - see Fig. 1. The Rochester measurements were made with the Rochester Havy Ion Detector us. ing a miti-slit aperture at the entrance to the Enge split-pole.

The anatysis follows the same line as was used for the 160 data even though the real potential is not expected to be as emergy independent. 2 The value of $V_{0}$ was griddet $p$, while the other five noodsSaxon paraneters were free in a simltaneous search on the $135.1 \mathrm{keV}$ and $13 \mathrm{HeV}$ data sets. Good fits could not be obtained for $\mathrm{V}_{0} \leq 25 \mathrm{MeV}$ while the fits for $V_{0} z 40 \mathrm{MeV}$ wero all about equally good for both data sets - see Fig. 1 for $V_{0}=40,100,150$ keV fits to the $135,1 \mathrm{MeV}$ data. Therefore, at this time we have not succested in eliminating the discrete ambiguities for Gi optical potentials. are:

The paramoters for the $V_{0}=100 \mathrm{NeV}$ potential

$v_{0}=100, \quad r_{0}-1,30, s_{0}=0.784, w_{\text {vol }}-36.7$

$r_{I}=1.57, a_{I}=0.883$, where $R=I+2 g^{1 / 3}$.

Most of the other potentials shared the following characteristics of this $V_{0}=100 \mathrm{HeV}$ potential :

a) $W_{0} / N_{0}$ much less than for $16_{0}$ and similar to that observed for $4 \mathrm{He}$;

b) $r_{I}>r_{0}, a_{I}>a_{0}$; this is opposite to what was found for 160. This behavior is a well-known feature of $6_{1}$ i optical paraneters.

c) The fits to the intermediate energies $[18 \mathrm{keV}$ and $44 \mathrm{kV}$ ) are not acceptable, perhaps indicating that an energy,dependence exists again in contrast to the 160 results. the tentatively conclude, therefore, that ${ }^{6}$ i behaves more like a "light" than a "heavy" ion.

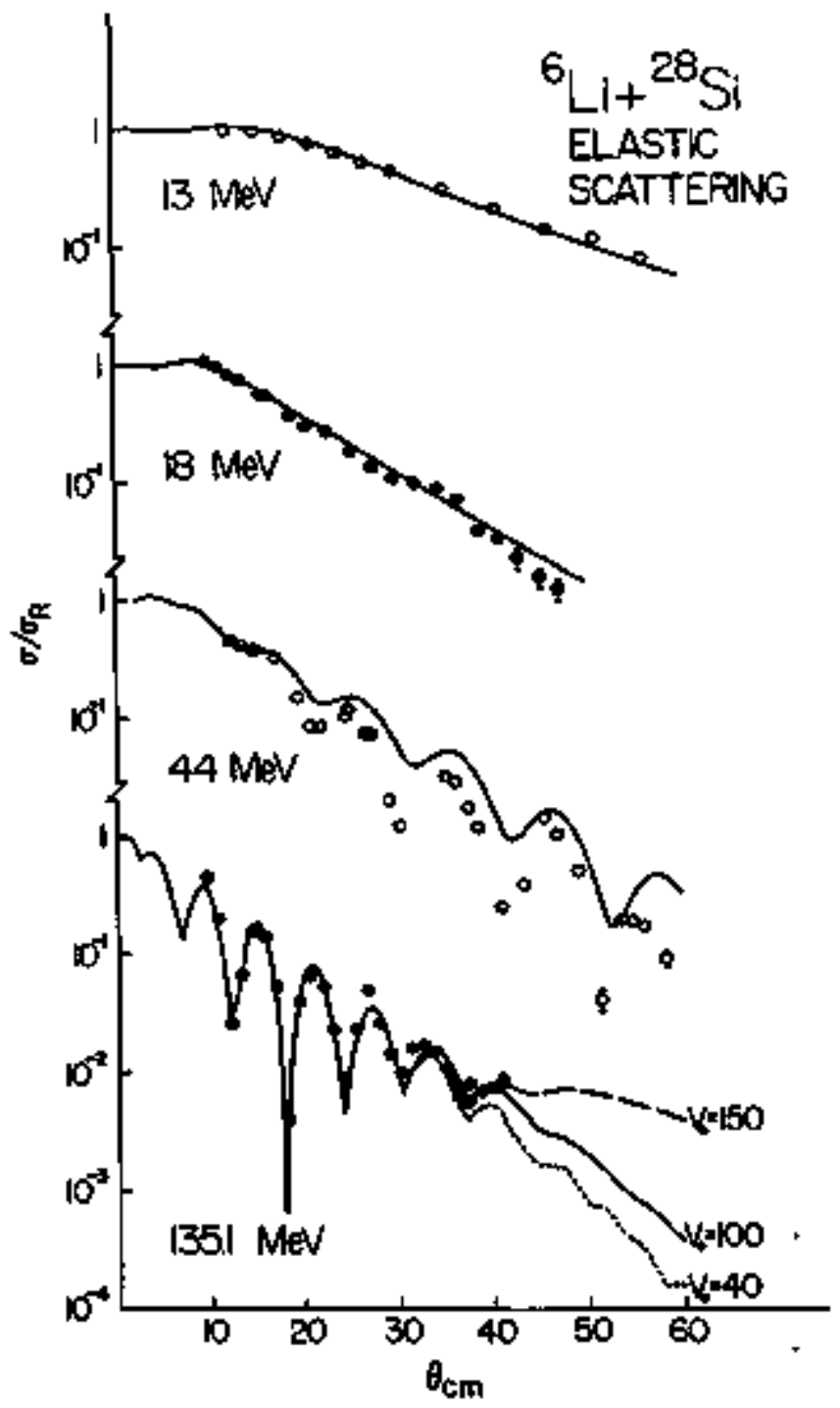

Fig, 1. Flastic scattering of ${ }^{6} \mathrm{Ii}+{ }^{28} \mathrm{Si}$ at the indicated laboratory enerbies. The curves are optical model fits (see text).
(XBL 366-8295) 
Finally, on the basis of the large angle differences in the predictions with the $v_{0}=40$, 100 and $150 \mathrm{kt}$ potentials, it appears possible to reșolve the discrete anbiguities by taking more data at $135.1 \mathrm{~kW}$. We plan to do this along with other intermediate energy measurements.

\section{Footnotes and References}

"Physics Department, liniversity of Washington, Seattle, iA 98195 .
†Physics Departnent, University of Rochester, Rochester, NY 14627,

†pysics Department, University of Maryland, College Park, ind 20742 .

1. J. E. Poling, E. Norbeck, and R. R. Corlson, Phys. Rev. C 13, 648 (1976).

2. D. F, Jackson and R, C. Johnson, Phys. Lett. $49 \mathrm{~B}, 249(1974)$.

\title{
${ }^{10} \mathrm{Ca}+{ }^{40} \mathrm{Ca}$ ELASTIC SCATTERING RESONANCES
}

\author{
C.. F. Magulre; D. L. Hendrie, U. Jahnke.t
}

J. Mahoney, and D. K. Scott

The gross structure observed in ${ }^{16} 0+16_{0}$ elastic scattering excitation functions, 1 but not observed in other systems, 2,3 can be explained by an $\ell$-dependent term in the inaginary part of an optical potential. 4 This model also predicts that resonance effects, although much restuced, should appear in the elastic scattering of $40 \mathrm{Ca}+40 \mathrm{Ca}$. Such effects were looked for by noubre et al, s at the Orsay Tandern from 110 to $150 \mathrm{MeV}$, and at 170 to $300 \mathrm{MeV}$ with the Orsay cyclotron, ALICE. Al though they saw no effect, sone aspects of their work may have reduced their chances of observing the effect. In particular, the $t$-dependent optical model predicts the largest effect near $160 \mathrm{MeV}$, just between the capabilities of their two machines.

The used the 88" Cyclotron to produce beams of 150 - to $173+4=140 \mathrm{Ca}$, beams; up to 50 na on target were measured. The QSD spectroneter was used to detect and identify the outgoing $40 \mathrm{Ca}$. Since the detector length spamed coly two charge states of the 40Ca, onIy $40-45 *$ of the scattered $\mathrm{Ca}$ could be used for the measurements. Because the distribution of charge states of the Ca leaving the target was energy dependent (and sliphtly target-dependent) tedious nomalization nos wore made to deteruine the distributions, in order to normalize the date and span the most prolific charge states (amound $\mathrm{z}=16 \mathrm{j}$. Energy dependent corrections to Faraday ctp and ronitor cgunter readings were also required.

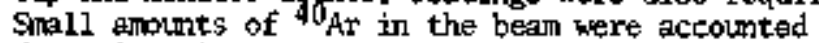
for. Sope inelastic events, especially to the region of the $2^{+}$at 3.90 and $3^{-}$at $3.73 \mathrm{kkV}$ were seen, but with too low a cross section in general to get intaningful data.

An elastic angular distribution was measured at $163 \mathrm{kgV}$, in order to extract an ( $l$-independent) optical potential (Fig. 1). No large sensitivity to cptical potential parameters that fit the angular distributions was noted in the excitation work. Additional data points were taken between 26 and $56^{\circ}$ (CM) with a solid-state detector, in close agredment with the spectrometer data and confinulng the nomalization corrections.

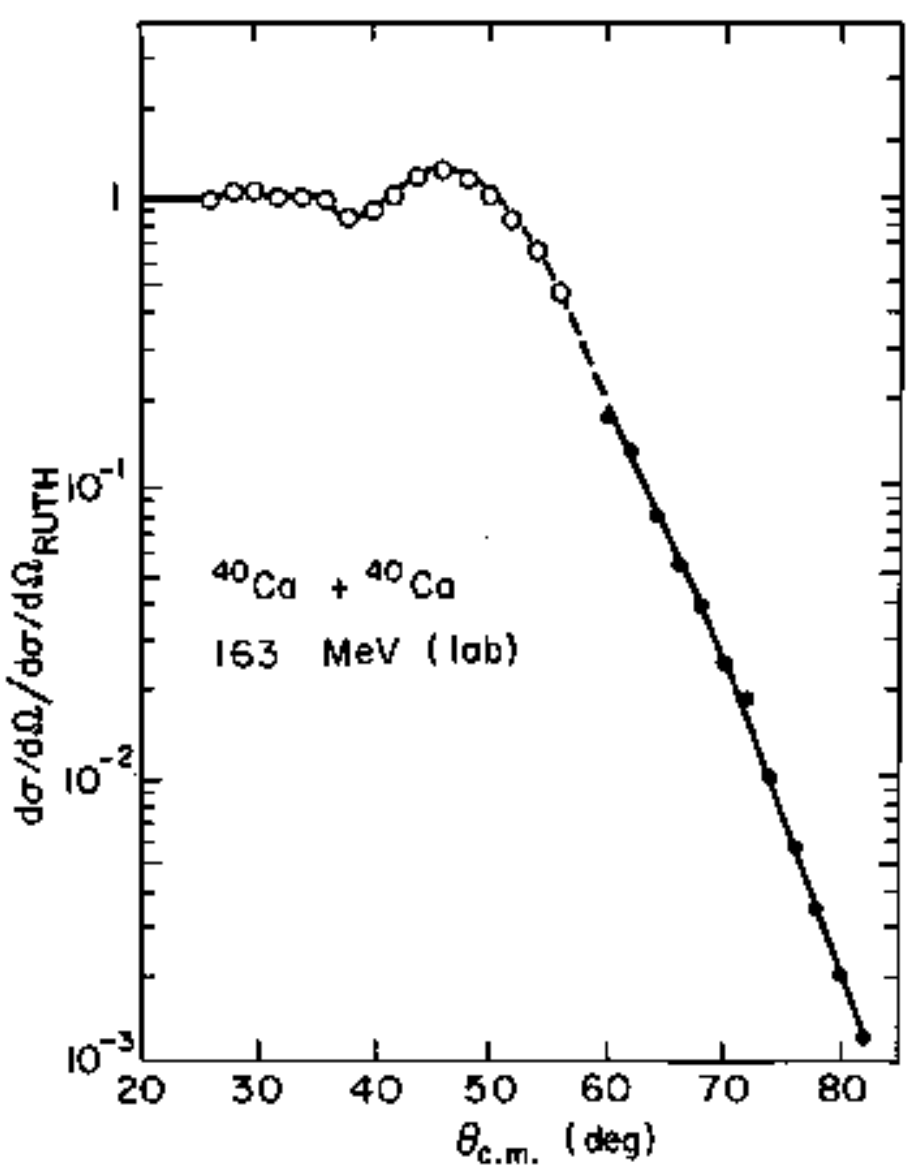

Fig. 1. Elastic scattering angular distribution at $163 \mathrm{HeV}$.

The excitation function data are seen in Fig. 2 , along with the optical model predictions. The $\mathrm{l}$-independent prediction clearly misses the observed resonant effects by as much as a factor of two. 


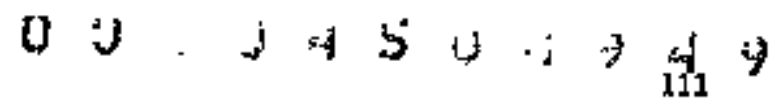

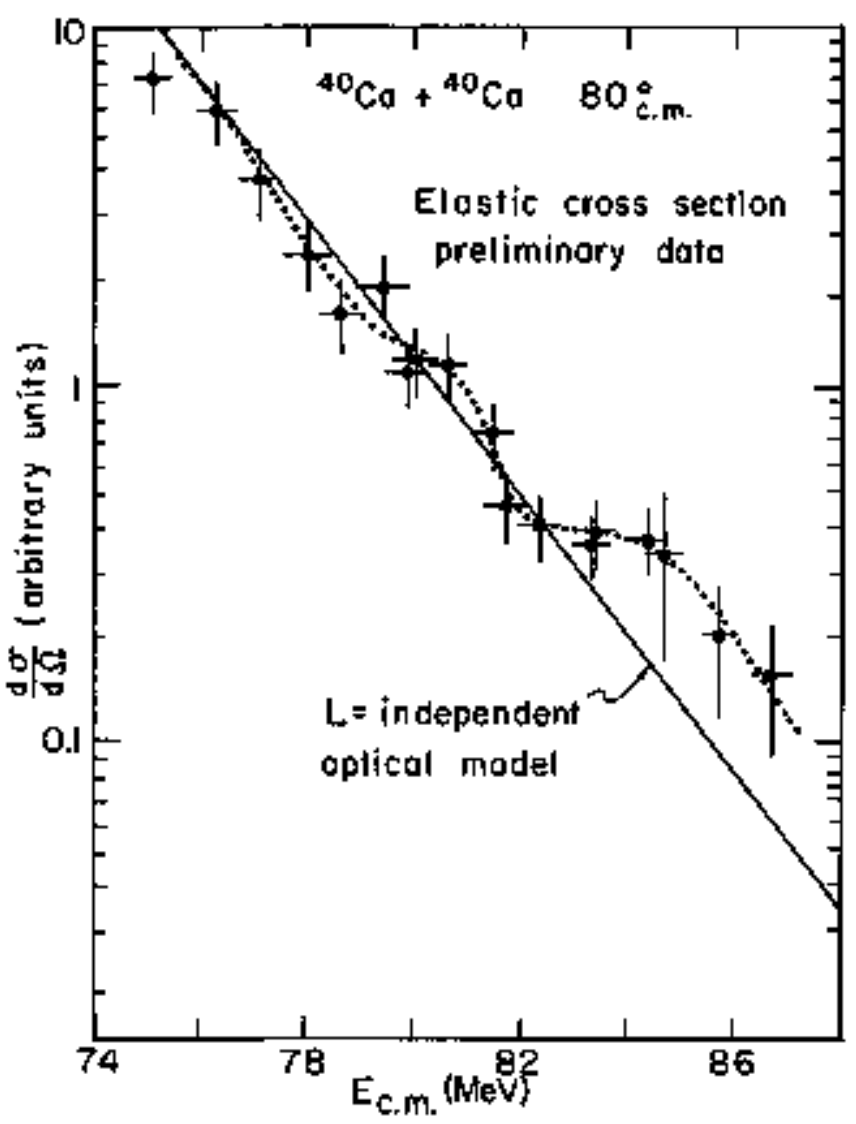

Fig. 2, Excitation function of ${ }^{40} \mathrm{Ca}+{ }^{40} \mathrm{Ca}$, with 2-independent model predictions (solia line).

Because we have not made final adjustments to the date to corvert the cross section into absolute values or made comprehensive checks of the $\ell$-dependent model, our conclusion thet weak resongnces appear to be present in the data is tentative. Since the excitation functions were not measured at $90^{\circ}$, it is possible that the observed variation is due to sweeping over oscilla tory structure in the differential cross sections as a function of energy.

The meașured differential croșs sections at $163 \mathrm{MeV}$ however gave no evidence for oscillatory structure. Our preliminary \&-dependent optical model calculations predict effects too sall to be measured by us at a $O M$ angle of $70^{\circ}$, the largest angle measured using AlICE.

\section{Footnotes and References}

Present address: Vanderbilt University, Nashville, Tenn.

†Present address: Halun-Meitner Institut, Berlłn.

1. R. H. Sleussen, J. V. Maher, A. Weldinger, and D. A. Bronley, Phys. Rev. Lott. 19, 369 (1967), and $20,175(1968)$; J. V. Wather, M. W. Sachs, R. H. Sienssen, A. Weidinger, and D. A. Bronley, Phys. Rev, 188, 1665 (1969).

2. R. H. Sienssen, in Proceedings of the Symosium on Heavy-Ion Scattering, Argome National Iaboratory Report No. ANi 7837, 1971 (urpublished), P. I45.

3. R. W. Shaw, R, Vanderbosch, and M. K. Mehta, Phys. Rev. Lett. 25, 457 (1970).

4. R. A, Chatwin, J, S. Eck, D. Robson, and A. Richter, Phys. Rev. C 1, 795 (1970).

S. H. Doubre, J. C. Roynette, J. C. Jacmart, N. Poffe, M. Riou, E. plaghol and $P$. de Sajntignon, Phys. Rer. Lett. 35, 508 (1975).

\section{ELASTIC SCATTERING OF ${ }^{14} \mathrm{~N}$ ON ${ }^{12} \mathrm{C}$ AT 81 AND $100 \mathrm{MEV}$ AND THE REACTION $\left.{ }^{12} \mathrm{Cl}^{14} \mathrm{~N},{ }^{13} \mathrm{~N}\right){ }^{13} \mathrm{C}^{*}(3.09 \mathrm{MeV})$}

$C$ Oliner M Buenerd, 3 Delrc, D L Hendre. A. Mencheca-Rochis and D K scotl

The observation ${ }^{1}$ of an anomalous angular distribution in the single ntreleon transfer reaction ${ }^{12} \mathrm{C}\left(\mathrm{P}_{\mathrm{N}}, 1 \mathrm{~N}_{\mathrm{N}} 1 \mathrm{1}_{\mathrm{C}}\right.$ to the $2 \mathrm{~s}$ (2 state in $13_{\mathrm{C}}$ at $3.09 \mathrm{hov}$ has provided the stianulls for similar work from several laboratories.2.5, Essentially, the anculaly consists of distorted-wave Born approxination calculations predicting an angular distribution that is out of phase with the observed features of the differential cross section when enploying optiçal model (OM) paraneters that reproduce the appropriate elastle scattering angular distributions.

The aim of tha pressent study was to irvestigate the $12 \mathrm{C}\left(14_{\mathrm{N}}, 1 \mathrm{~K}_{\mathrm{N}}\right) \mathrm{1}_{\mathrm{C}} \mathrm{C}$ reaction after perforning detailed an analyses of the $14_{\mathrm{N}}+{ }^{2} \mathrm{C}$ elastic seat. tering. The motivation for this was that in the work of DeVrles et at. 1 the elastic scattering data measured were too sparse. In the present work the elastic scattering measurements were performed using $100.3-\% \mathrm{EV} 1 \mathrm{~N}^{4+}$ and $80.7-\mathrm{MeV} 14 \mathrm{~N}^{3+}$ beams produced at the 88-Inch Cyclotron. The reaction products scattered frow the 1.07-me/ $/ \mathrm{Cu}^{2}{ }^{2}{ }^{2} \mathrm{C}$ target were detected in four $1.5 \mathrm{~mm}$ lithilotedrifted silicon detectors placed 2 apart, each with an angular accoptance of $0.25^{\circ}$. Data were accumulated over the angular range $3^{\circ}$ to $35^{\circ}$ (representing approximateIy, six orders of magnitude in the differential cross section), with an angular spacing of $0.5^{\circ}$.

The present experiment, because of the high density of data points as a function of angle 
combined with the wider angular range, reveals features of the elastic ratio-to-Rtitherford cross section which are not apparent in the earlier mea-

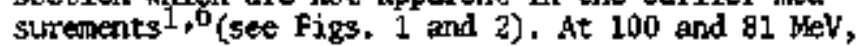
the cross section has three distinct regions:

(a) at forward angles it falls rapidly away from the Rutherford value by about an order of magnitude to the second miniman, (b) it them oscillates rapidly with a large anplitude (approximately through an order of magnitude), and (c) with increasing angle these oscillations are demped almost completely.

OM parameter searches were performed of the tho sets of data with the search code SOPHIE ${ }^{f}$ and Table 1 stmmarizes the results of the paraneter searches so far. Paraneter sets labelled 81A to D and $100 \mathrm{~A}$ to $D$ were obtained from searches on the data measured in the present experinent wille sets $81 E$ and $100 E$ were obtained froil searches on earlier data, 1,6 which were either too sparse or confined to a narrower range in angle. The ou predictions corresponding to the parameters of Tabte 1 aro shown in Figs. 1 and 2 for the 100- and 81-MeV data, respectively. While data in the backward heipisphere could be expected to have an important effect on the of parameters resulting from a search, it is clear from the present work that the $\mathrm{M}$ does predict the quali tative features of the elastic scattering cross section throughout the measured angular range. Furthenore, fits of equivalent quality are obtained with either a
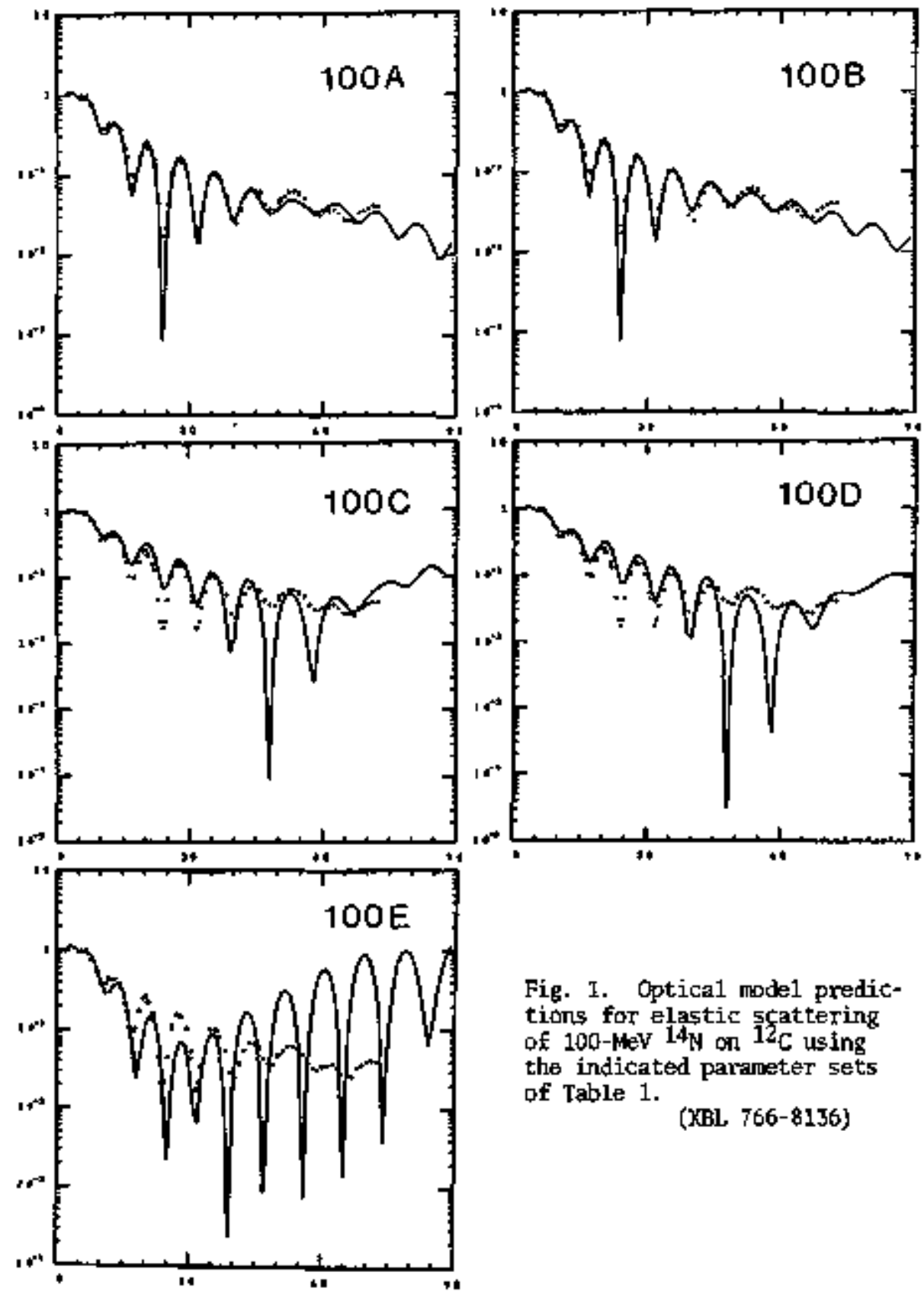

Fig. I. Optical mode1 predictions for elastic schattering of $100-\mathrm{HeV} 14_{\mathrm{N}}$ on $12 \mathrm{C}$ using the indicated paraneter sets of Table 1.

(XBl 766-8136) 

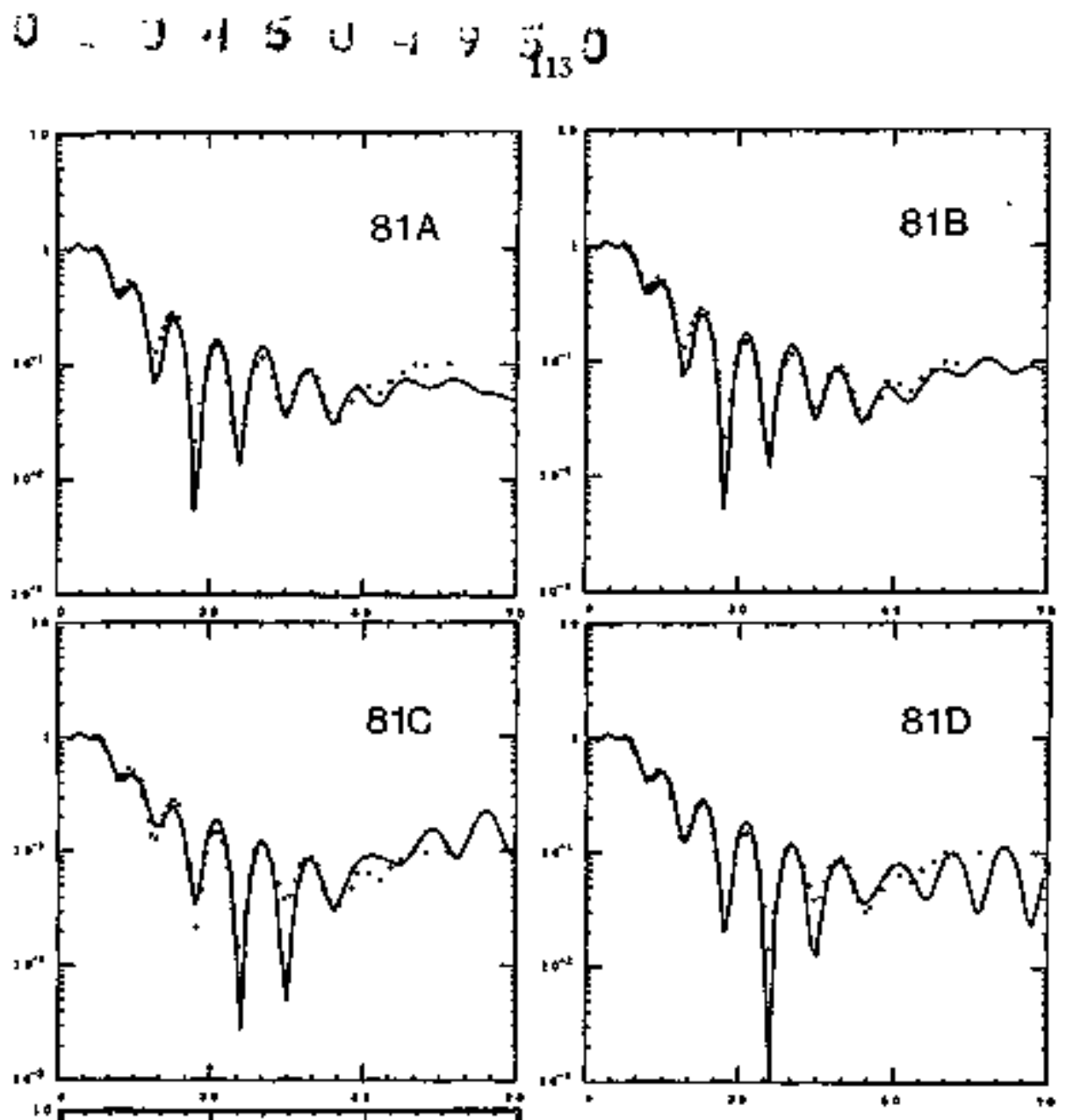

volume or a surface type absorbing potentilal for example compare potentials $100 \mathrm{~A}$ and 100B which have nearly identical real potentials.

The potentials listed in Table 1 were employed in INPA calculations for the reaction

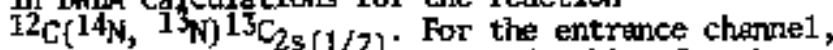
the $100-\mathrm{Mel}$ potenttals were used, while for the exit charmet the $81-\mathrm{NeV} 14 \mathrm{~N}+12_{\mathrm{C}}$ parameters wero enmloyed. The basis of the latter asstmption is that the $0 M$ has a smooth mass nuber dependence and provides sinilar elastic scattering predictions for systens with simflar values of $\eta$ (Sommerfeld parameter) and $k$ (Asyototic waye numer). Fqr the two systems ${ }^{14} \mathbf{N}+{ }^{12} \mathrm{C}$ (at $81 \mathrm{NeV}$ ) and $13_{\mathrm{N}}+{ }^{13} \mathrm{C}$ in

the exit channel of the reactions the respective values are 2.75 and 2.76 for $n$ and 3.39 and $3.41 \mathrm{fm}^{-1}$ for $k$. Some INBA results are shown in Fig. 3. Ox potentials of the type $\mathrm{E}$ produce cross sextions in phase with the reaction data, but so far, potentials that fit the elastic data and predict the correct phase of the reaction data have not been found. However, a closer inspaction of F1g. 3 shows that the IirRA cross section is vory sersitive to the type of potentials exployed. If (o) potentials of type $\mathrm{C}$, with imaginary diffuseness $\sim 0,1$ fr, are introduced then the DHEA cross section shows large changes in the angular region spenmed by the availabie reaction data - even though all the potentials A to $\mathbf{D}$ crne close to reproductng the elastic data well. 
Table 1. Optical nodel parameters for ${ }^{14} \mathrm{~N}+{ }^{12} \mathrm{C}$ elastic scattering.

\begin{tabular}{|c|c|c|c|c|c|c|c|c|c|c|}
\hline$\frac{\mathbf{E}^{1 \mathrm{ab}}}{(\mathrm{M} \mathbf{k V})}$ & Jabel & $\begin{array}{c}\mathbf{r}_{v} \\
(\mathrm{fin})\end{array}$ & $\begin{array}{c}a_{v} \\
\text { (fm) }\end{array}$ & $\begin{array}{c}V \\
(\mathrm{NeV})\end{array}$ & $\begin{array}{c}\mathrm{I}_{\mathrm{W}} \\
\text { (fi) }\end{array}$ & $\begin{array}{l}a_{H} \\
\text { (fin) }\end{array}$ & $\mathrm{w}^{\mathrm{a}}$ & $\mathbf{r}_{(\mathrm{f}}$ & $\mathrm{wW}_{\text {(tan) }}$ & $\mathrm{w}_{\mathrm{V}}$ \\
\hline \multirow[t]{5}{*}{81} & $81 \mathrm{~A}$ & 1.020 & 0.5564 & 166.4 & 0.9216 & 0.3757 & 47.14 & & & \\
\hline & $81 B$ & 1.041 & 0.5578 & 132.8 & & & & \multirow[t]{4}{*}{0.8571} & \multirow[t]{4}{*}{0.6303} & \multirow[t]{4}{*}{22,30} \\
\hline & $81 C$ & 1.296 & 0.3144 & 93.52 & 0.9778 & 0.1089 & 126.4 & & & \\
\hline & $81 \mathrm{D}$ & 1.177 & 0.4562 & 79.80 & 1.002 & 0.1895 & 318.4 & & & \\
\hline & 8IE & 1.069 & $0 . \$ 397$ & 86.78 & 0.8967 & 0.1137 & 123.6 & & & \\
\hline \multirow[t]{5}{*}{100} & $100 \mathrm{~A}$ & 1.115 & 0.5304 & 100.5 & 1.088 & 0.3162 & 54,44 & \multirow{5}{*}{1.159} & & \\
\hline & 1008 & 1.096 & 0.5563 & 100.1 & & & & & \multirow[t]{4}{*}{0.3306} & \multirow[t]{4}{*}{62.75} \\
\hline & $100 \mathrm{C}$ & 1.270 & 0.3100 & 118.0 & 1.140 & 0.1050 & 200.0 & & & \\
\hline & 1000 & 1.280 & 0.2920 & 116.0 & 1,140 & 0.1070 & 210.0 & & & \\
\hline & $100 \mathrm{E}$ & 1.065 & 0.5221 & 89.36 & 0. 8901 & 0.1133 & 265.8 & & & \\
\hline
\end{tabular}

a) Surface type imaginary potential $w$ is as used in Ref. 7; Volune type inaginary potential is as usisally defined.
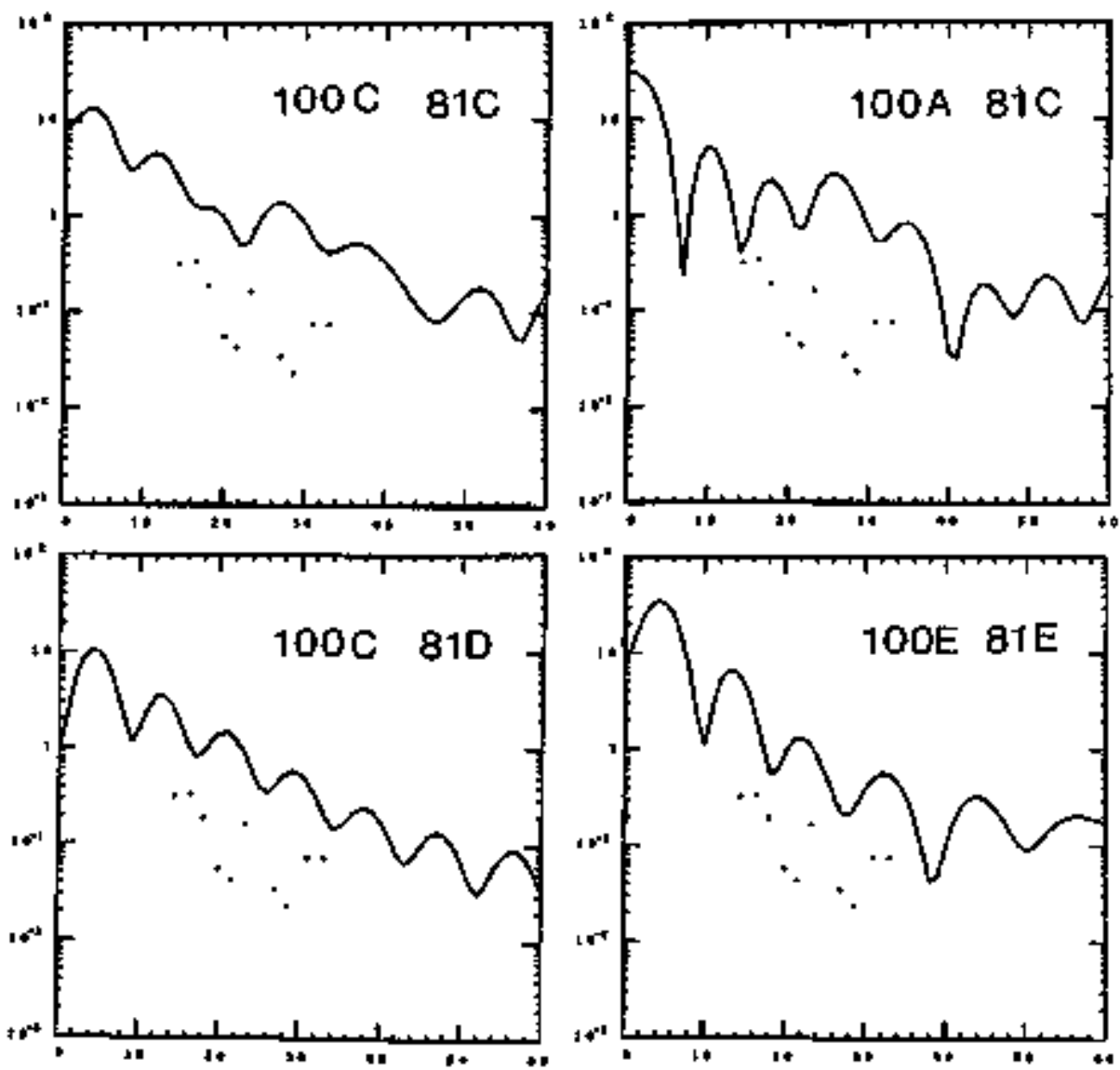

Fig. 3. Distorted-wave Bork approximation calculations with kINDRY for the reaction $12 \mathrm{C}\left({ }^{14} \mathrm{~N}, 13 \mathrm{~N}\right){ }^{13} \mathrm{C}_{25}$ (1/2) using the paraneters of Table 1. The cross sections are in mibr for unit spectroscoptc factors; the data are those of Ref. 1.

(XBL 766-813B) 


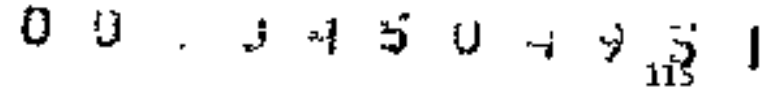

\section{Footrote and References}

"Work in progress.

1. R. M. DeVries, M. S. Zisman, J. G. Cramer,

K. L. Liu, F. D. Becthetti, B. G. Harvey,

H. Homeyer, D. G. Kovar, J, Mahioney and W. von Dertzen, Phys. Rev, Lett. 32, 680 (1974).

2. K. G. Nair, H. Woit, M. Hamm, C. Towsley and

K. Nagatani, Phys. Rev. 33, 1588 (1974).

3, K. G. Nair, H. Voit, C. W. Towsley, H. Hamm, J. D. Bromsor and K. Nagatani, Phys. Rev. C12, $1575(1975)$.

4. P. D. Bond, C. Chasman, J. D. Garrett, C. K.
Gelbke, 0. Itansen, M. J. LeVine, A. 2. Schwarzschild and C. E. Thorn, Phys. Rev, Lett. 36, 300 (1976),

5. T. Motobayashi, I. Kohno, K. Katori, M. Yoshie, T. Ohi and H. Kagitsubo, Phys, Rev. Lett. 36. 390 (1976).

6. H. von Oertzen, M. Liu, C. Caverzasio, J. C. Jacmart, F. Poughech, M. Riou, J. C. Roynette and C. Stephan, Nuc1. Phys, A143, 34 (1970).

7. G. Delic, Phys. Rev, lett. 34, 1468 (1975).

8. K. I. Kubo, K. G. Nair, and K. Nagatani, Phys. Rev. Lett. 37, 222 (1976).

\section{QUASI-ELASTIC COLLISIONS OF 315-MEV ${ }^{16} \mathrm{O}$ ON HEAVY ELEMENTS}

\author{
M Buenerd," C K Gelbke, a $\mathrm{G}$ Harvay. D L Hendrle,
}

$J$ datwoney, A Menchace-Pocha, $\mathrm{C}$ OMrner, and $\mathrm{O} K$ scott

In the course of the last few years, particle transfer reactions between heavy ions at incident energies $>100$ bev have provtded a large anount of data revealing the existence of two new reaction phemomena-the deep inelastic comporemt (DIC) and the quasi-elastic $(\mathrm{QBC})$ collisions. The kinetic onergy distribution from these reactions is dominated by two components: 1) a high-energy peak (quasi-elastic), located well above the coulonb barrier, which is observed only when a fow particles are transforred and at forward angles (the stury of this process reveals both statistical and direct reaction features]: and 2) the deep inelastic peak at an energy close to the Coulomb barrier and tnterpreted as a fully relaxed process. These two features were interpreted as two aspects of the sane phenorenon in the Wilczynski picture. 1 In the exper. iments reported here, beans of $315 \mathrm{kel} 160$ were used to bonbard targets of $197 \mathrm{Au}$ ( $\left.2 \mathrm{\pi g} / \mathrm{cm}^{2}\right), 208 \mathrm{pb}(1.2 \mathrm{~m} / \mathrm{s}$ $\left.\mathrm{cm}^{2}\right)$ and $232 \mathrm{Th}\left(10 \mathrm{mg} / \mathrm{on}^{2}\right)$ in order to study the quasi-elastic processes around an incident energy of $10 \mathrm{MeV} / \mathrm{A}$.

The experiments were carried out at the $88-$ inch cyclotron using the QSD spectrometer for high energy resolution and good particle identification but with limited energy range, and silicon $\Delta \mathrm{E}-\mathrm{E}$ connter telescopes providing broad energy range spectra. The thickness of the $A \mathrm{E}$ counter was chosen so as to permit both good $z$ identification and as broad an energy range as possible. The $\Delta E$ thickness ranged from $35 \mu$ to $100 \mathrm{p}$ and permitted the identiflcation of fragments between $z=2$ and $Z=9$, but with some wicertainties in mass resplution for higher $z$.

The kinetic energy distributions obtained with the silicon counter telescopes exhibit a strong quasi-elastic component (the fully relaxed rogion of the spectra could not be observed in the experiment on account of the sE counter thickness). Figure lows a sanple of energy spectra from the 100 + $208 \mathrm{pb}$ reaction. For each spectrum, the optirus final kinetic energy corresponding to the centrotd of the OEC poak has been extracted and the corresponding differential cross section. In wost of the cases the shape of the peak can be

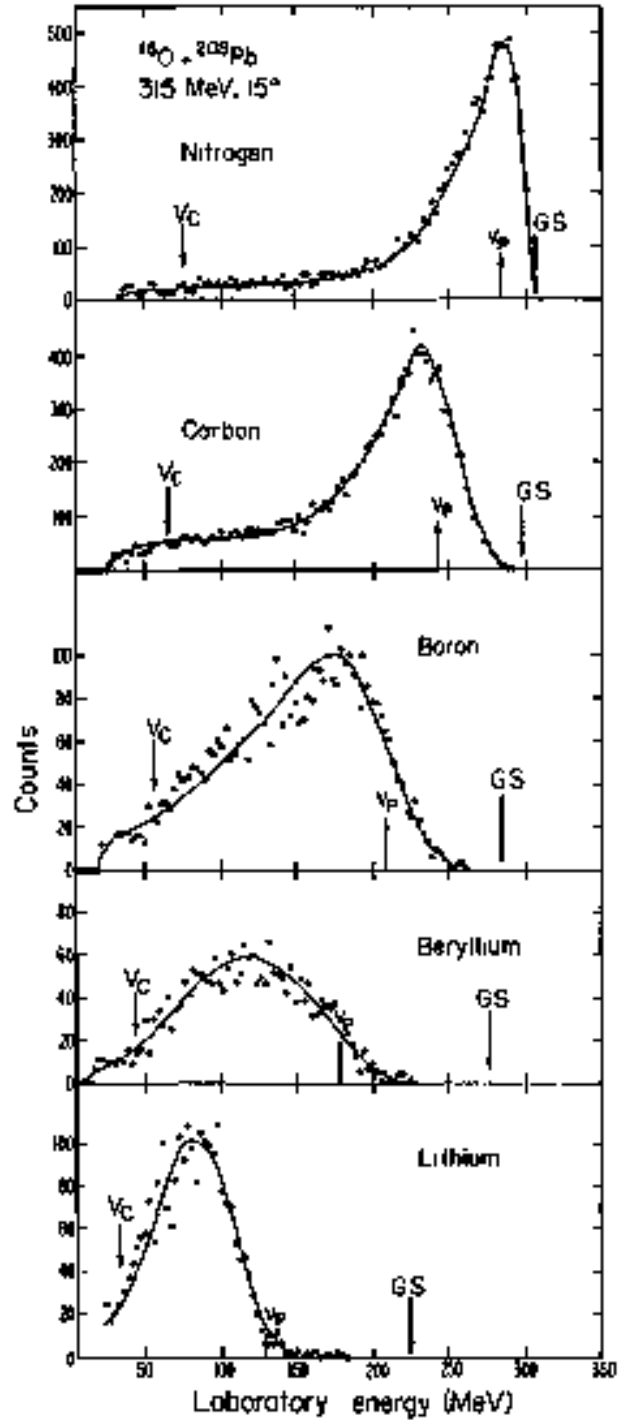

Fin. 1. Typical quasj-elastic energy spectra for the reaction $160+208 \mathrm{~Pb}$ at $315 \mathrm{keV}$. The arrows label points corresponding to: $V_{C}$, the exit Colllamb carrier; $V_{p}$, the beal velocity/G, 5 , the ground state transition.

(XBL. 768-3359) 


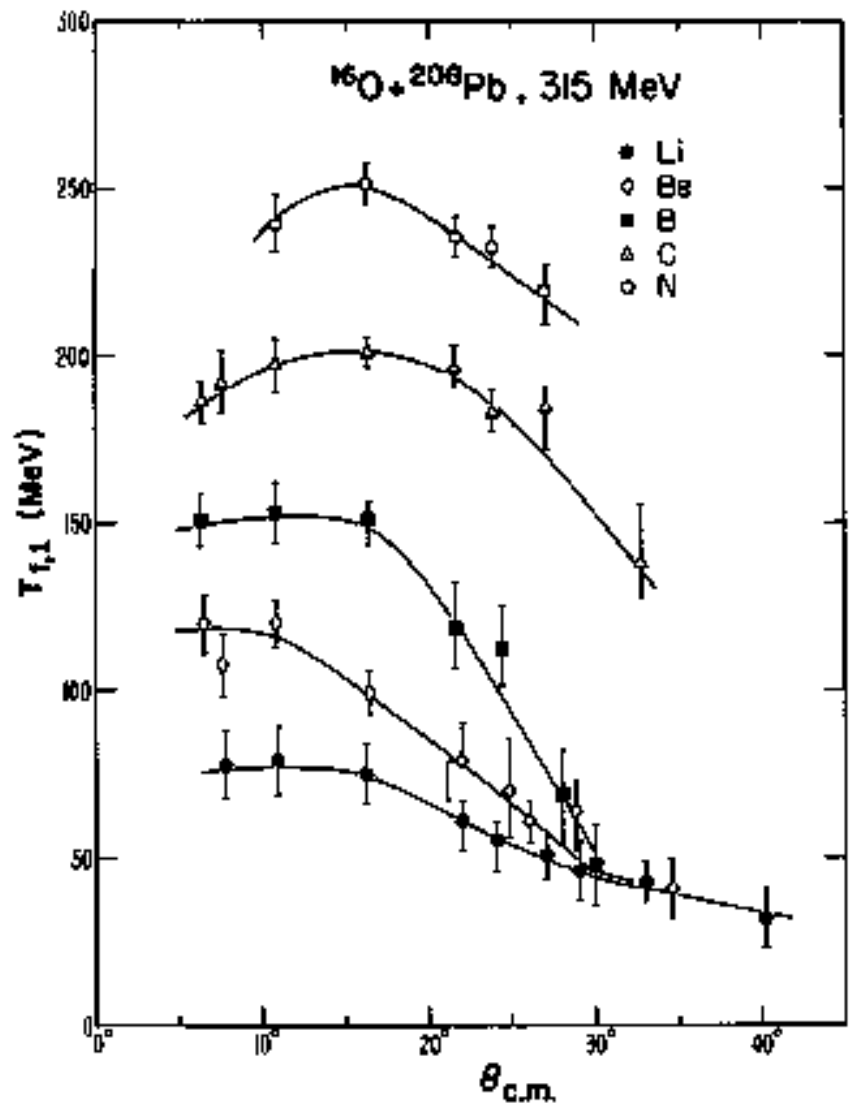

Fig. 2. Angular variation of the maximm in energy of the quasi-elastic peak for outgoing produsts in reactions induced by 160 on $208 \mathrm{pb}$, at $315 \mathrm{Mav}$.

(XBL 769-4055)

reproduced with a Gaussian plus a low energr exponential tail. As shown in Fig. 2, the optimm kinetic energy of the OEC peak exhibits a strong angular dependence. This effect is not observed at lower incident energy, al though the results of Ref. 2 shows a slight tendency to vary the same way. The neasured Q-values are roughly the same for the three targets used.

Preliminary angular distributions for the $160+20 \mathrm{~Pb}$, reactions are plotted in Fig. 3; they fall off approximately exponentially with the angle as expected from a direct reaction process. The average slope decreases with the number of charge units transferred.

The data are also sinilar to the high energy frapmegtation cross sections measured by Lindstrom et al. for $160+203 \mathrm{pb}$ at an energy of $33.6 \mathrm{GeV}$ (2,1 GeV/ruxleon). Figure 4 shows that the relative cross sections for the production of different isotopes are generally of the same magnitude in the two experiments, with a general trend towards larger cross sections for the production of more neutron deficient isotopes at the higher energy. Wote, however, that the relative elefment yields are identical within the experimental errors. This is a most umexpected result, since similar experiments at energies only a few atev/nucleon above the barrier give entirely different distributions of reaction products. 4 These results may indicate

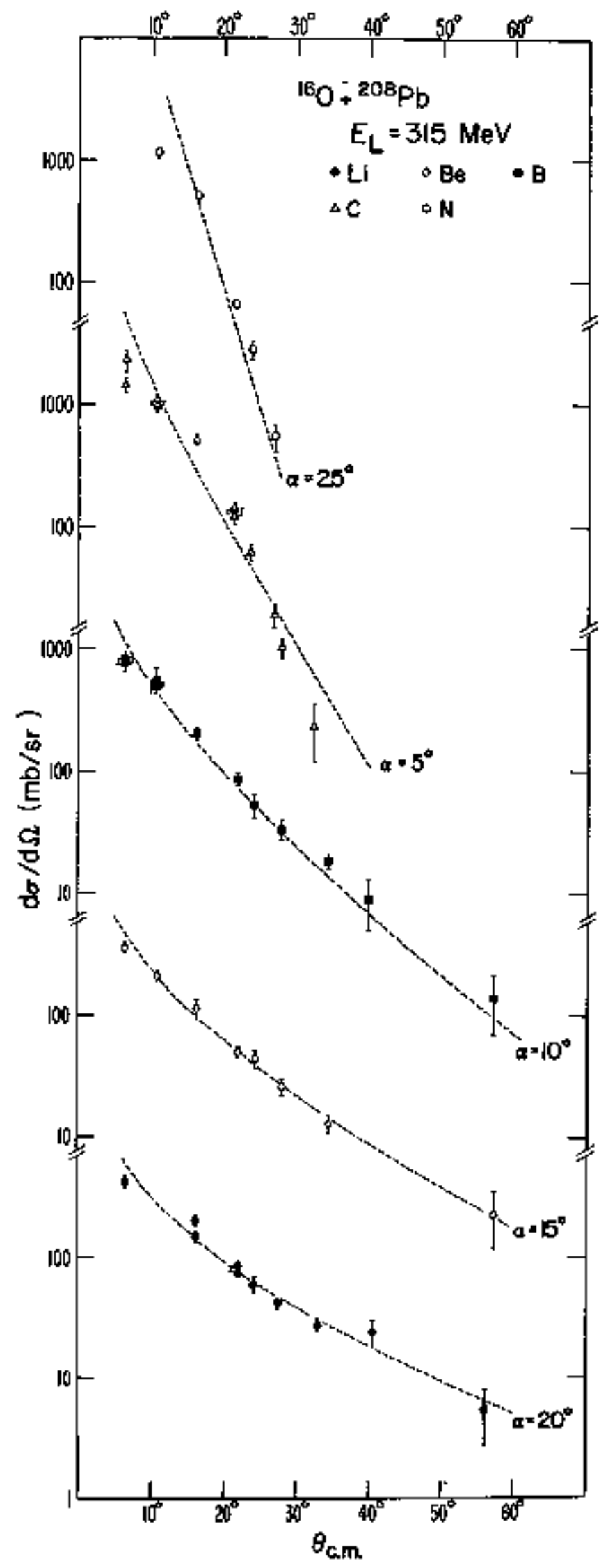

Fig. 3. Different cross sectfons of the guasielastic component in reactions with $160,268 \mathrm{~Pb}$ at $315 \mathrm{ufeV}$. The dotted arves are exponential decays with the indicated decay angles. 


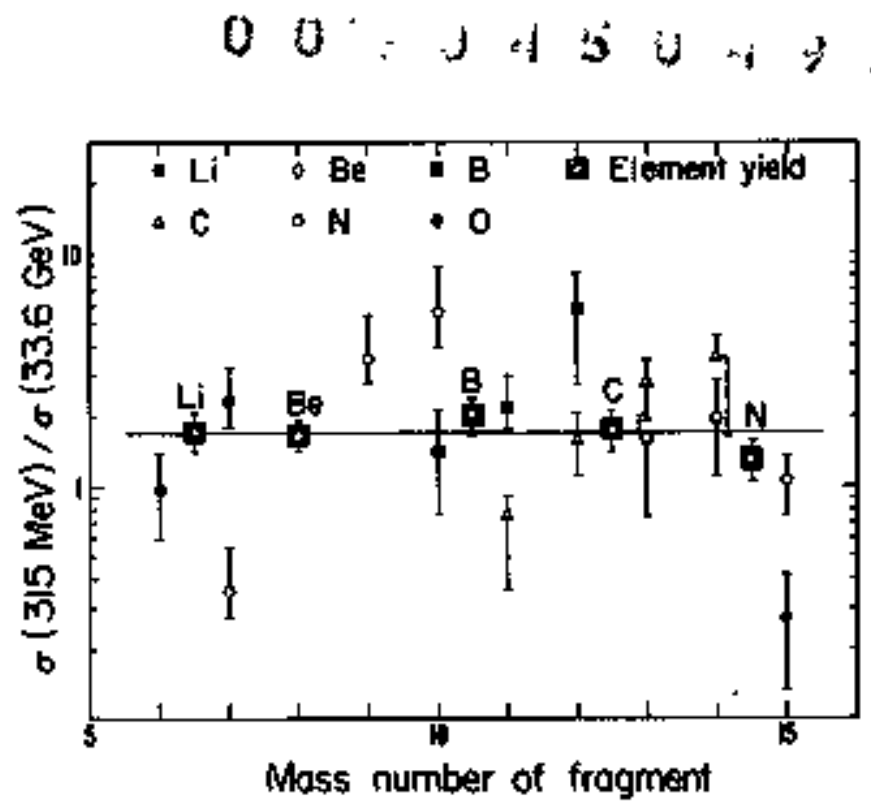

Fig. 4. Ratios of cross sections measured for the reactions $160+208 \mathrm{~Pb}$ at $315 \mathrm{MeV}$ and $33.6 \mathrm{GeV}$. laboratory energies. that the link between high and low energy phenomena can be wade in tho energy range of a few tens of MeV/nucleon where the ion velocities are comparable to the Fenmi velocity.

\section{Footnotes and References}

*Pemanent address: Institut dos scionces Nucléaires, Grenoble, France.

'Perranent address: Instituto de Fisica, U.N.A.M., Mexico.

1. J. Wilczynski, Phys. Lett. 47B, 484 (1973).

2. H, Kamitsuto, Proc. of Symposium on Atacroscopic Features of Heavy-ion Collislons, Arganne, I1linais (1976) Vol, I, p. 177.

3. P. J. Lindstron et al, LBL-3650 (1975).

4. A, G, Artukh et al., Nucl. Phys. A160, 511 (1971).

\section{SEARCH FOR SHOCK WAVES AT THRESHOLD ENERGY}

D L Hendre, M Buenerd." A Menchaca-Rochạ,s

C Olmer, and $0 \mathrm{~K}$ Sctot

At suffteiently high relative velocities, the central collision of two massive nuclei has been predicted to give rise to a new phenomenon. The high nuclear density at the point of contact was predicted to propagate through a massive nuxleus in a fashion conceptualized as a nuxlear compressional shock wave. Various models at relativistic energies have suggested differing posșible manifestations of a shock process. Several investigations at relativistic energies have yielded conflicting, but probably negative results. ${ }^{1}$ A calculation by Tsang ${ }^{2}$ suggested that the conditions under which shock phenomena would occur still exist at onergies accessibie to the 88-Inch Cyclotron, The much higher bean intensities available there makes possible much more sensitive experimental tests for the effect.

Beapls of $312-\mathrm{HeY} 160,[19.5 \mathrm{mbV} / \mathrm{a})$ were directed onto an $8-\mathrm{mg} / \mathrm{cm}^{2} 232$ Th target; intensities of $50 \mathrm{nA}\left(5 \times 10^{10} \mathrm{particles} / \mathrm{sec}\right)$ we routinely available. Two independently moveable solid-state conoter telascopes wre used to detect and identify Emitted fragyents, both singly and in coincidence. Farly results indicated that heavy fragment enission tas quite small and gave rise to very few coincidences. Since our counters were inapproprigto for singly charged ejectiles, and ${ }^{3} \mathrm{He}$ particles were always more than an order of magnitude reduced from $4 \mathrm{He}$, the bulk of our data and all of this report will be on entission of alpha particles.

Figure 1 shows a sample of single alpha particle spectra at several angles, In wich (at least) two components can be discussed. At large angles, the spoctra show a peak at the coulonb barrier, and art exponentially decreasing tail. Under the simplest poșsible assumptions, this

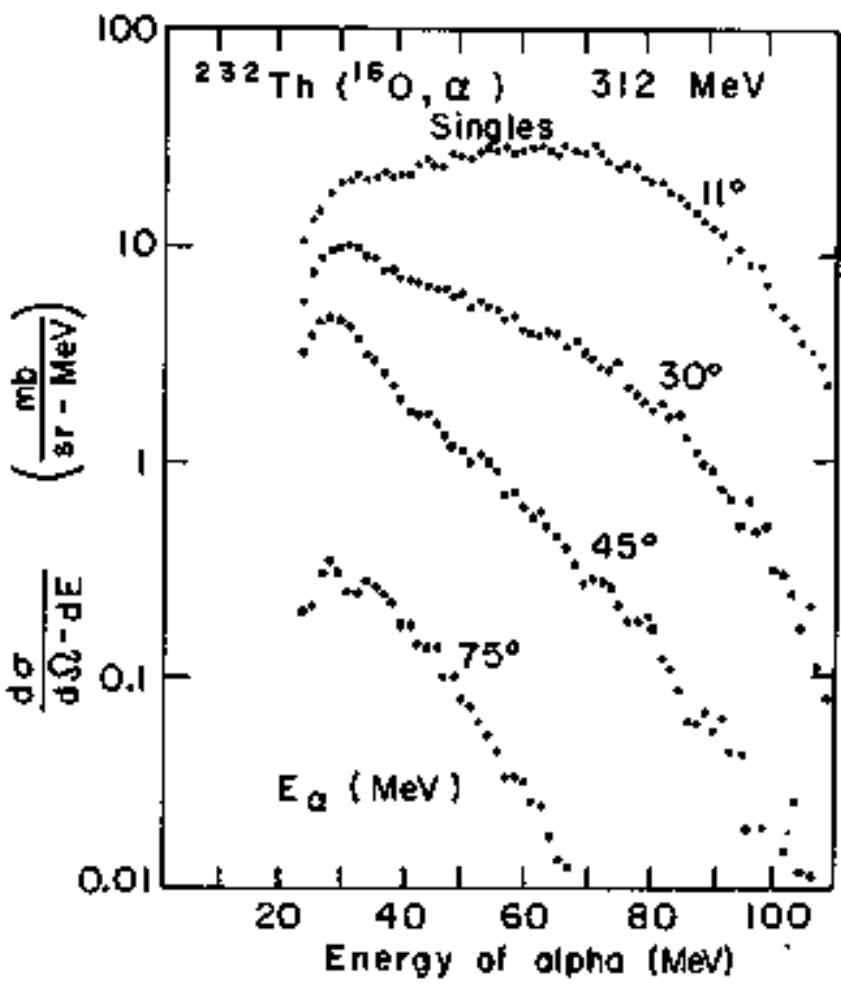

Fig. I. Energy spectra of alpha particles detected in a counter telescope placed at angles from $11^{\circ}$ to $75^{\circ}$.

XBL 766-8325) 
could be attributed to compound nuclear decay, with a charactoristic temperature of about $9 \mathrm{MeV}$. This is a much higher temperature than that expected by extrapolating from lower energy work. As yet, no additional effects such as muti-particle emission or effects of critical angular momenta in the absorption have beerl calculated. At forward angles, a direct component is seen, with a broad peak at $75 \mathrm{MeV}$. Very cride calculations indicate that this is consistent in cross section and energy and angular distributions with the projectile fragnentation seen by Heckman and coworkers at relativistic onergies. 3

Fimmes 2 and 3 show results of coincidences of two alphas on opposite sides of the inconding beam. In Fig. 2, both counters are moved ejumetrically about the beam axis; in Fig, 3, one

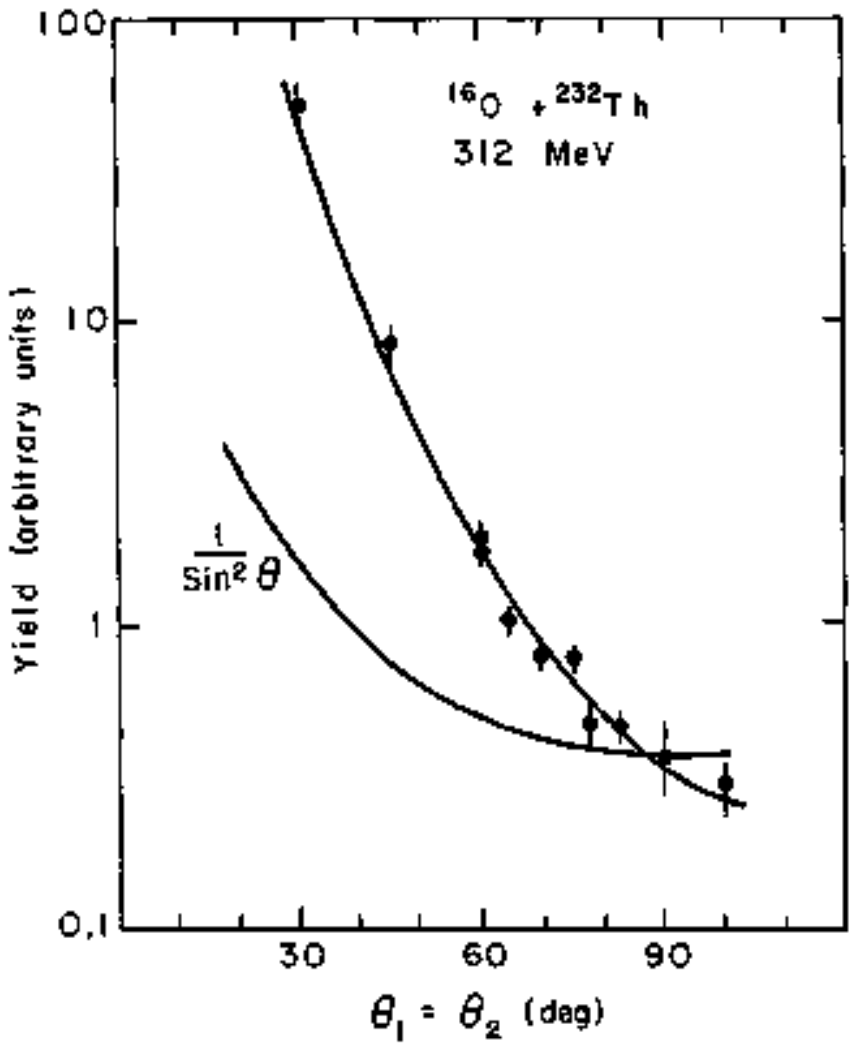

Fig. 2. Angular distribution of alpha particles de. tected in coincidence in two counter telescopes placed at symetrical angles about the bean axis. An arbitrarily normalized $\left(1 / \sin _{\theta}{ }_{\theta}\right)$ curve is shom for comparison to the data.

(XBL 766.8326)

\section{Footmotes and References}

*Present address: Institut des Sciences Nucl fatres, Grenoble, France.

tPresent address: Inst1tuto de Fisica, U.N.A.M. , Hexico. counter is held fixed, and the other moved. In these preliminary plots, the alpha spectra are sumbed over all energles, and the results were not corrected to obtain absolute cross sections.

Chance coincidences were a minor contribution and are subtracted.

Interpretation of the colncidence data are consistent with the Ideas from the singles data. 掟 evidence for shock effects are seen. The cross sections with at least one of these counters at forward angles nust be corrected if the fragnentation bypothesis is correct, for the fact that 160 fragrents into four alphas, nily tho of which are detected. Decorposing the coincident alpha spectra in several energy regions will significantly ald the interpretation. The high level of coincidences at large angles points out the need, not surprisingly, to take into account multi-alpha emission.

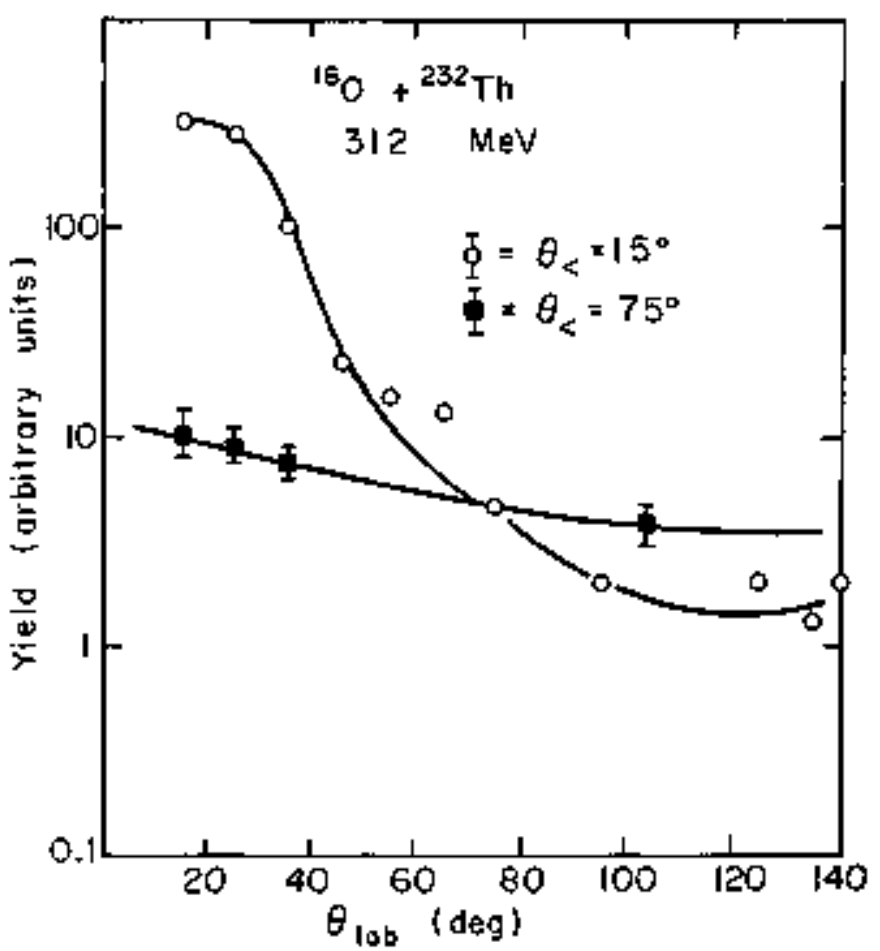

Fig. 3. Angular distrfbution of alpha particles detected in coincidence in two counter telescopes placed at asymmotrical angles about the bear axis.

(XRL 766-8327)

1. A. M. Poskanzer et al., Phys. Rev. Lett, 35 1701 (1975); H. G. Bumgardt et al., Z. Phys, A 273, 359 (1975).

2. C. F. Tsang, private commonication.

3. D. E. Greiner et al., Phys. Rev, Lett. 35, $152(1975)$. 


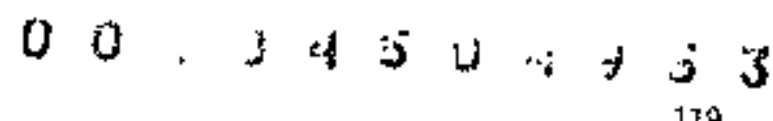

\section{Macroscopic}

\section{PRELIMINARY MEASUREMENT OF THE LIGHT CHARGED PARTICLES IN COINCIDENCE WITH HEAVY-ION RELAXATION PHENOMENA}

O J Malhews. G Woznalat, $P$ \& Russo.

R C Jared, and L G Moretto

Heavy-ion relaxation reactions are generally characterized by a complete dissipation of the incident kinetic energy, substantial mass transfer, and a nearly binary separaticn of the final fragnents. Recent measurements of the angular correlation of the fragments, 1 and measurements of coincident ganma roy's, ${ }^{2}$ however, indicate that substantial quartities of enitted protons, neutrons, and alpha particles also characterize these interactions. We report here sore results of a preliminary measurems of the feasibility of detecting, and the qualitative physies involved in, the light charged particles (LCP) in coincidence with such heavy-ion interactions.

The measurement has been attempted in the following way. A position-sensitive detector (PSD) is used to view the angular distribution of the LCP in coincidence with a $\Delta E-E$ telescope to identify a fragment $z$. With the PSD fixed and the telescope varied in angle, one should expect different qualitative features to appear in the PSD depending upon the nature of the parture of the particle emission environment. In particular, one can envision throe possible situations which wight be observed:

(1) If the particles are enittad isotropically in the center of yass system of scrins quasisphertcal intermediate complex, one would expect no correlation between the final fragnent angles and the LCP angular distribution.

(2) If the particles are enitted during the separation of tho fragments, then (as in ternary fission) one would expect coulorb focusing to resolt in an anticorrelation between the final fragment angles and the particle angular distribution.

(3) Particles which arise from the excited fraglents after separation should be expected to oxhibit some correlation between fragnent angles and LCP distribution.

One can suppose that the energy spectra of the LCP from these possibilities may also exhibit qualitative differences.

So what one wishes to do then is to exanine the PSD angular distribution in coincidence with a given fraguent as the telescope angle changes. As the correlation angle for the partmer $z$ passes through the PSD a change in the LCP angutar distribution should indlcate something of the nature of the enviromment from which the LCP were amitted.

The experiment, which has been perfomed to examane these phenconena, consisted of a thin ou target bonbarded by $252-\mathrm{kt} V 20 \mathrm{ke}$. An aluminum absorber was piaced before the PSD with sufficient thichess to stop the elastically scattered $20 \mathrm{Ne}$, but thin enough to allow the LCP to enter the detector. A crude separation of the proton and heavier particle contribution was possible in the resulting PSD energy spectru. Roughly equivalent

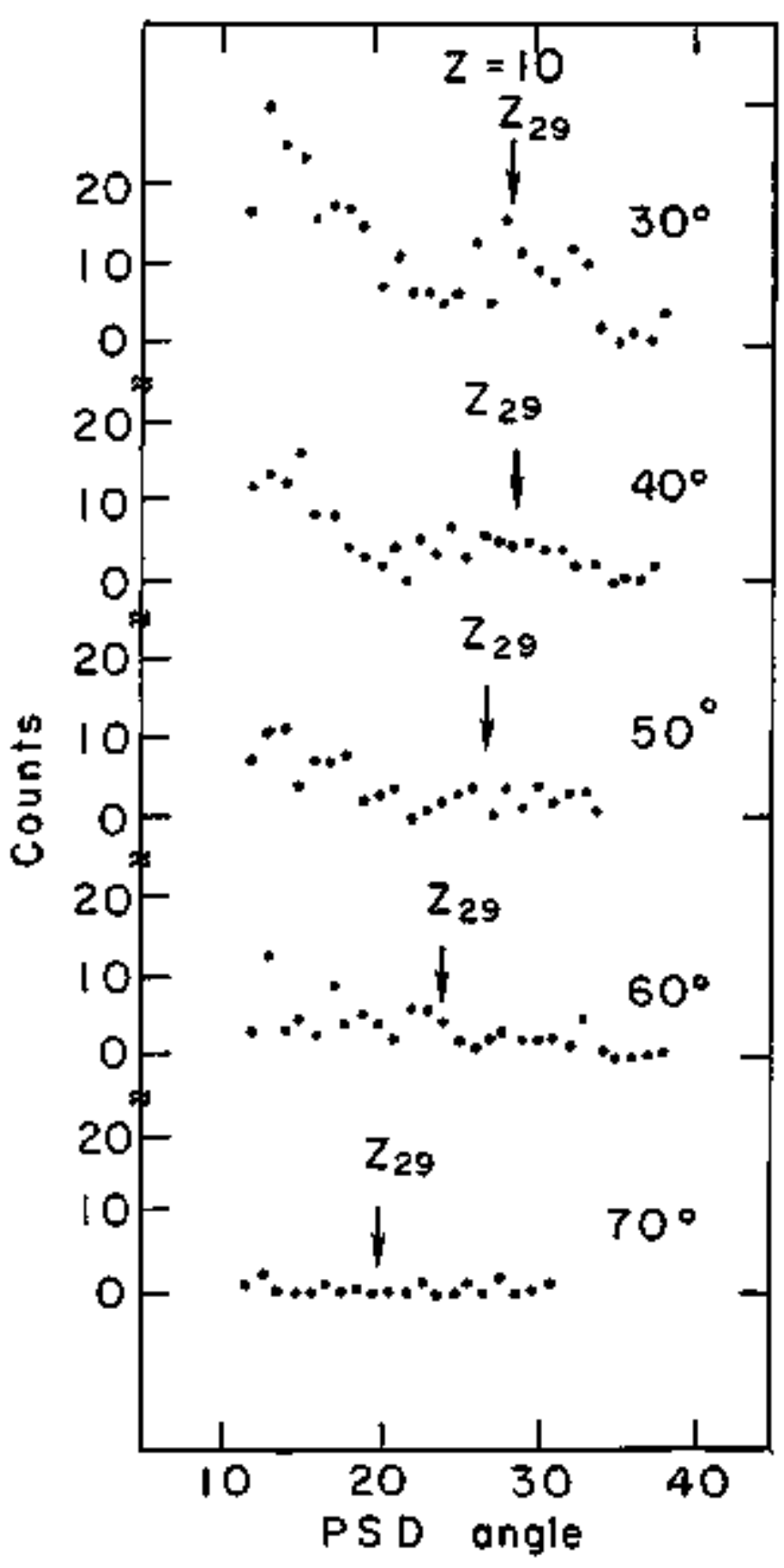

F1g. 1. PSO angular distributions for the LCP in coincidence with $Z \sim 10$ in the AE-E telescope. The predicted correlation angle of the heavy partuer is indicated by the arrows.

(XBL 766-8301) 
numbers of protons and heavier particles were observed. To enhance statistics, bends of several $Z$ 's in the telescope were sumed to obtain coincident PSD angular distributions.

Events in the PSD as a function of telescope angle for $2^{\prime} s$ near 10 and 23 are shom in Figs. 1 and 2. The expected correlation angle of the heavy partser fragthent is also shown on tis figure. The qualitative features are soine forward peaking at forward telescope angles which flattens at more backward telescope angles. Though it sents that there is, indeed, some change in the LCF angular distributions, it is not yet clear what these distributions indicate. To elarify the situation one needs to Iook at heviler 2's in the telescope, better statistics axe needed at backward angles, and perhaps, the $L C P$ kinetic energy spectre as we2l. Nevertheioss, it seens that something other than the uninteresting evaporation from the separted fragments say appear in these angular distributions.

Future experiments are planned which shall erploy a large solita angle position sensitive $\Delta \mathrm{E}-\mathrm{E}$ detector, currently being developed by our group. In this arrangenent the LCP can be more clearly separated and identified. Also, the increase in solid angle will facilitate the potainment of the much needed additional statistics at backward angles, and for higher $Z$ 's.

In conclusion, we have leamed from this preliminary study of the feasibility of the detec. tion of the light charged particles in coincidence with heavy-ion interactions. Most important1y, there is sone indication that the ensuing angular distributions may contain interesting structure which could yield new insight into the details of the interaction mechanism.

\section{References}

1. P. A. Russo, B. Cawin, G. Kozniak, M, Meuman, R. Schnitt, R. C. Jared, and L. G. Moretto, to be published.

2. K. Van Bibber, R. LeDoux, S. G. Steadman, F. Videback, G. Young and G. Flaum, in Synm. on Macroscopic Features of Heavy Ion Collisions, Argonne National Laboratory (1976).

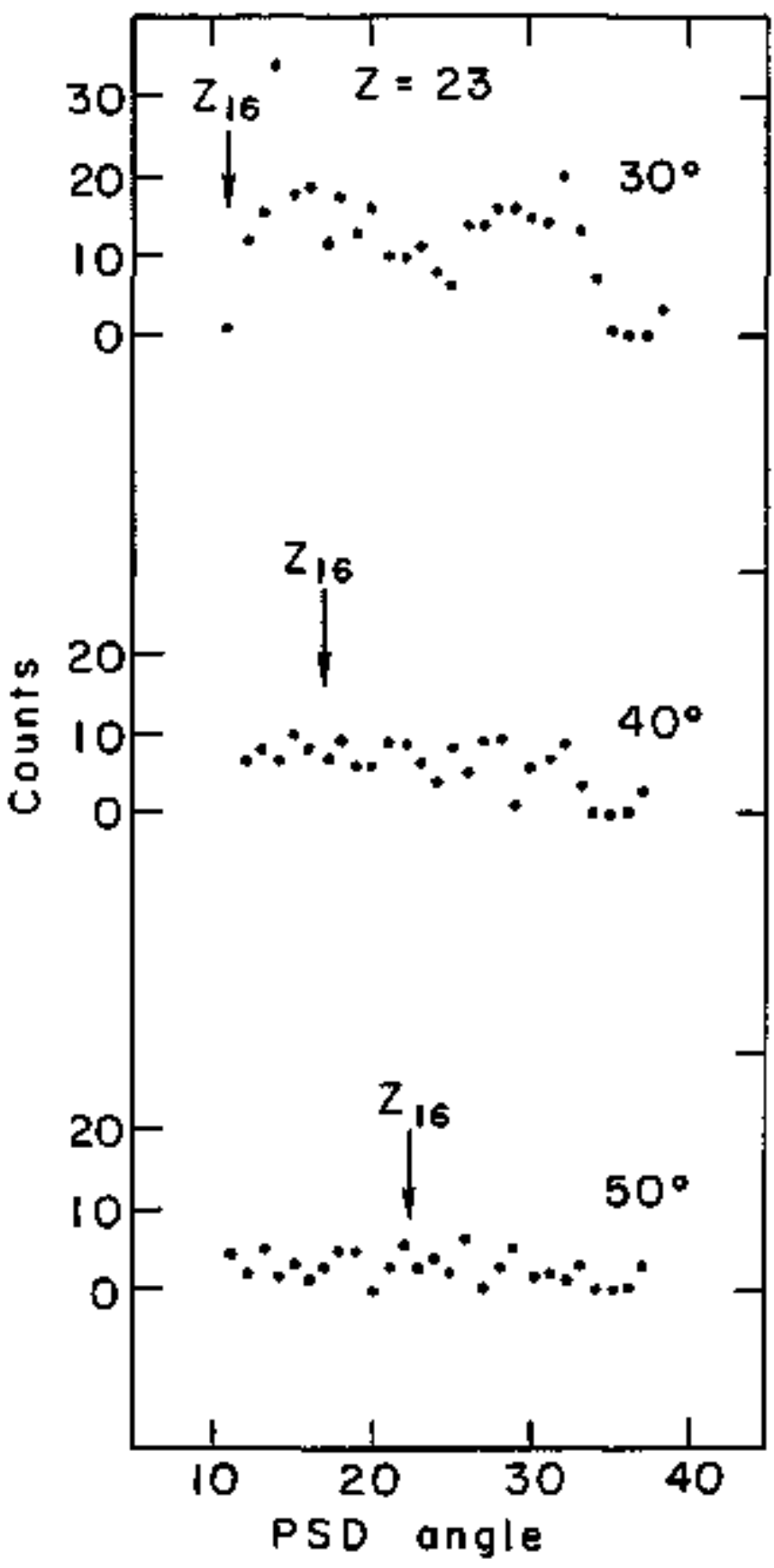

Fig. 2. PSD angular distributions for the LCP in coincidence with $Z, 23$ in the $\Delta E-E$ telescope. The prejicted correlation angle of the partner fragnent is indicatesd by the arrows. (XBL 766-8302)

\section{FRAGMENT CORRELATION APPROACH TO THE STUDY OF PARTIAL RELAXATION IN HEAVY-ION COLLISIONS}

h Newiman. B Cauvi,* P Rusșo.

R Jared, and L G Moretlo

In heavy-ion reactions a significant portion of the reaction cross-section appears in a conponent which has not fully equilibrated. That is, a compound nucleus does not form al thrugh the en- ergy of the relative wotion is essentially dissipated into the intermal modes during the inter. action. This is the "relaxed" component which has been ubiquitous in heavy-ion experiments. 
In our 1st generation of heavy ion experiments he looked at uncorrelated events by neans of an $E-\Delta E$ telescope. The singles data provided evidence for the formation of a short-lived intermedilate complex with wel1-defined mass-asysmetry, 1 on this basis we began a second generation of heavyion studies in which two fraghents produced in the decay of the intermediate colplex are detected. A positicin-sensitive detector (PSD) is used in conjunction with an E- $\Lambda E$ telestope. 2 In those experiments a number of special difficulties are enconntered. In partictular, correctton for gignalnonIinearity and pulse-height defect has been made. 3 Because angular correlations are measured, one can make confinuation of a binary decay. More partmeters corcerning each event are measured in the coincidence experiment so that one can provide a more detailed description of the systers. Bnergy dissipation and $\mathrm{N} / \mathrm{Z}$ equilibration (perhass at various stages of the interaction) can be studied more fully.

We here report on the coincidence study of the systers $107,109 \mathrm{Ag}+252 \mathrm{MkV} 2 \mathrm{O}^{2}$ at the 88 -inch crclotron. one telescope and 16 fie PSD were tused. For practical reasons identified the 1ight fragment in the telescope and detected the heavy partuer in the PSD. The telescope angle was fixed and the PSD was ineved to various angular settings. The PSD was positioned in the reaction plane at $40^{\circ}, 55^{\circ}$ and $70^{\circ}$ to the bean axis and the telescope always sat at $40^{\circ}$ in the opposite henisphere.

In a typical angular correlation (Fig. 1) we see that the heavy partner is found in a distribu$t$ icn of correlation angles, peaked about an average correlation angle. Another interesting feature is the broadness of the correlation.

Figure 2 shows the trends in the angular correlation parameters. We notica a nearly linear in-

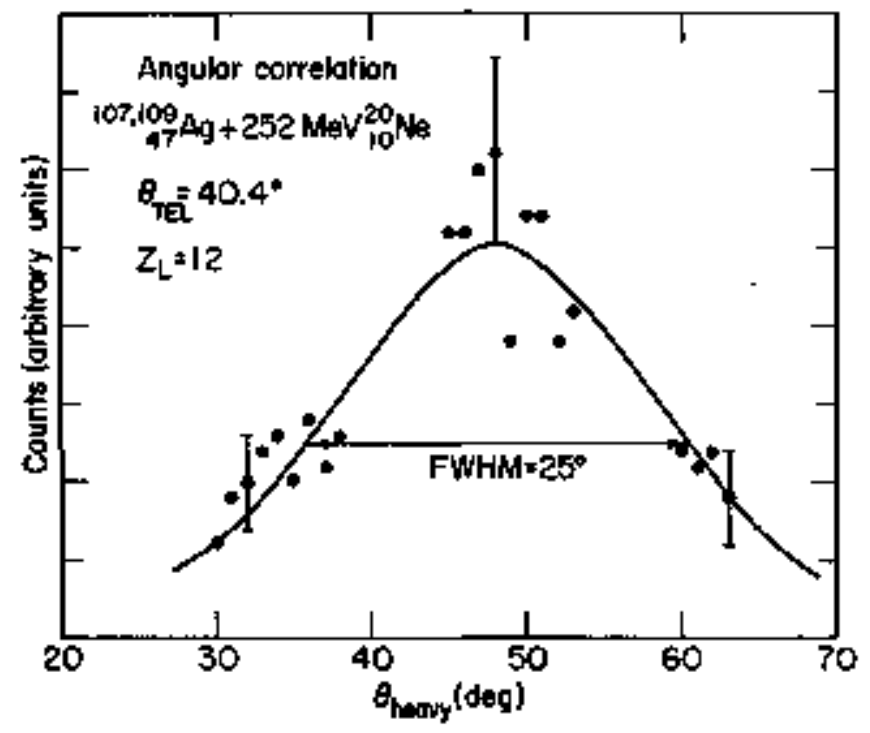

Fig. 1. Angular correlation for fragnents in the PSD in coincldence with $\mathrm{Z}=12$ fragients pbserved in the telescope at $40^{\circ}$. Data points and typical errors are shown, The solid curve is a gaussian fit to the data, (XBL 764-2650)

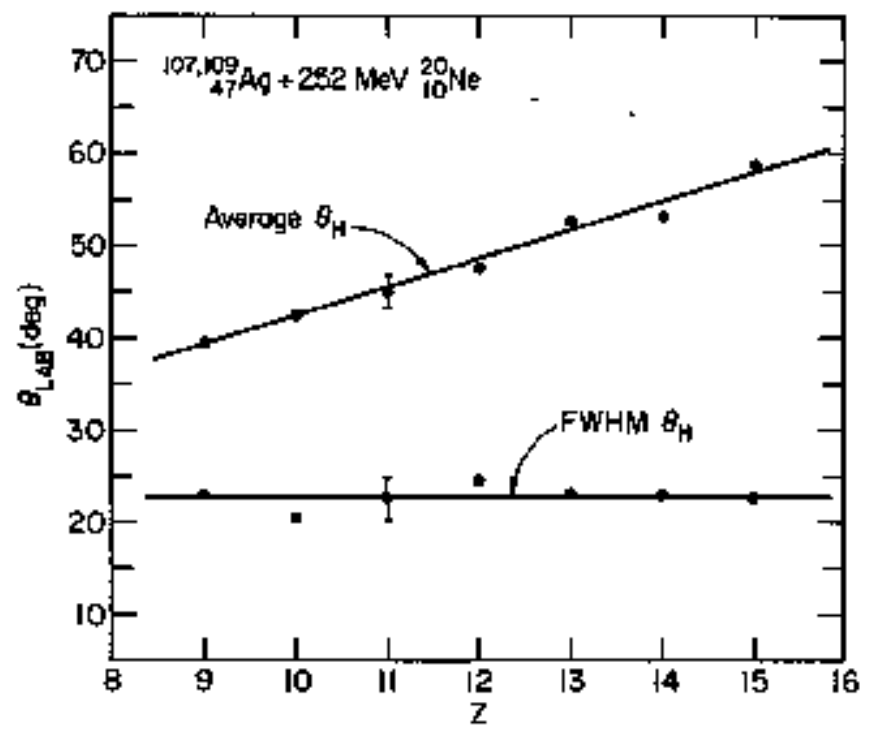

Fig. 2. Angular correlation parameters as a function of the $z$ in the telescopo. Data points are hown along with tines to guide the oye.

(XBL 764-2652)

crease in average correlation angle with $z$. This same trend is predicted if one asstmes an inelastic collision followed by the binary docay of two towching spherical fraguents. The angular correlation widths are essemtially constant with $z$.

For two-body kinersatics, conservation of linear monentum requires this relation:

$E_{1} M_{L}=\frac{E_{\alpha^{M}} \sin ^{2}\left(\theta_{H}\right)}{\sin ^{2}\left(\theta_{L}+\theta_{H}\right)}$. In the present experinent $E_{0}, M_{0}$, and $\theta_{L}$ are fixed so that the correlation angle is a function of only the mass and energy of the light fraginent. From other experiments 4,5 we know the variance in the energy and the mass of the light fragstent and one can calculate the expected correlation width. However, only about $10 \mathrm{~s}$ of the observed width can be accointed for in this manner. so it aypears that one must ascribe the bullk of the observed width to particle evaporation frote the two hot fraguents. Since the present systen is neutron rich and charged particles messt overcone a coulonb barrier, we expect neutron enission to to dominate this assumption has been corroborated by an evaporation code).

In the center of mass (c.m.) energy distribution (Fig. 3) we observe that the c.a. kinetic energies for both singles and coincidence events (approxinately coulonb energies of two touxhing spheres) are the same. The sane types of reactions are seen in both singles and coincidence.

In comparing the singIes and coincidence yields (Fig. 4) we see that they are equal within error. This means that for every fragrient entering the telescope, a second fragment can be detected. in coincionen in the PSD in a weIl-defined distribution of correlation angles.

The sharing of the exc1tation energy of the intermediate complex between the two fragments may 


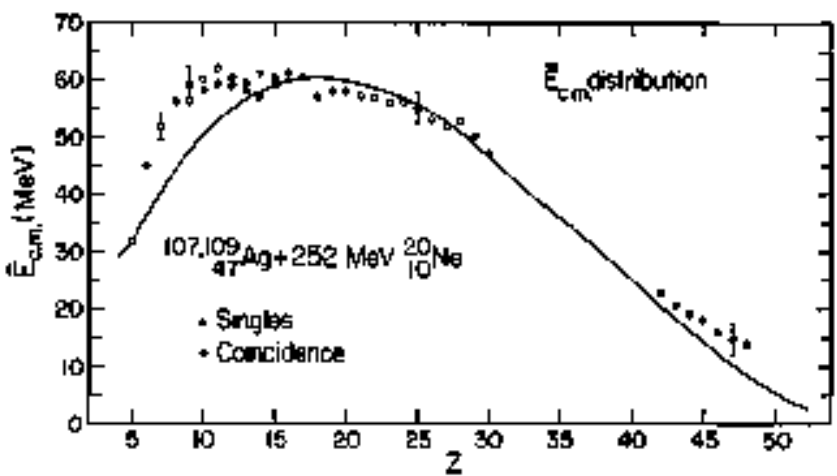

Fig. 3. Center of mass kinetic energies as a function of the fripwent atomic nuber. Open circles show the singles data and the closed circles show the coincidence data. The solid line is the kinetic energy as a function of $Z$ predicted for two touching spheres which are repulsed by their Coulonb field (not a fit).

(XBL 764-2654)

be inferred from the number of secondary particles onitted by each fragnent as it deexcites. The correlation between the fragment mass and atomic nuber is also of particular interest. In the coincidence experiment, since the energies and angles for both fragments axe measured, we soe essentially the complete kinematics of the reaction, In orter to determine the fragment masses before secondary enission and the number of nucleons enitted by each fragithent, we must solve a system of four equations ensuring energy, mass and linear momentur conservation in the reaction plane. These equations are nonlinear so that an iterative calculation is reguired in order to pbtain a unique result for the

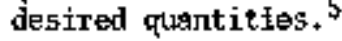

In this mamer we have determined the average number of neutrons exitted by a fragment of a given atomic nuber (Fip. 5). The number of neutrons shows a fairly smooth increase with the fraguent 2 . The linearity of the neutron yield is comsistent With the assumption of equal tomperatures. From this it appears that the intermediate complex is in thersma 1 equilibrium at the time of decay,

The experimerntal fragment masses before secondary emission have also been determined (Fig. 6). Compare the experimental masses of the light fragment with two simple assumptions: 1) the neutronto-proton ratio $\mathrm{N} / \mathrm{Z}$ of the projectile 20 le since the 2 of the light fragment is about the same as the $Z$ of the projectile; and 2 ) the $N / 2$ ratio of the hypothetical conpound mucleus. We see that the experimertal masses lie systematically along the $\mathrm{N} / 2$ line of the compound nucleus. This does not necessarily mean that a compound mucleus has been formed. The equitibrium $\mathrm{N} / \mathrm{Z}$ ratio must be determined by ninimization of the potential energy of the conplex at fixed mass asymmetry. In this case the equilibrium $1 / 2$ is only nomanaly different than that for a compound mucleus. One can say that the $\mathrm{N} / \mathrm{Z}$ ratio appears to be equilibrated throughout the intermediate system during the tine of the reaction. Analogous results for the heavy fragnent are shown in Fig. 6b and the same conclusion is drawn. We

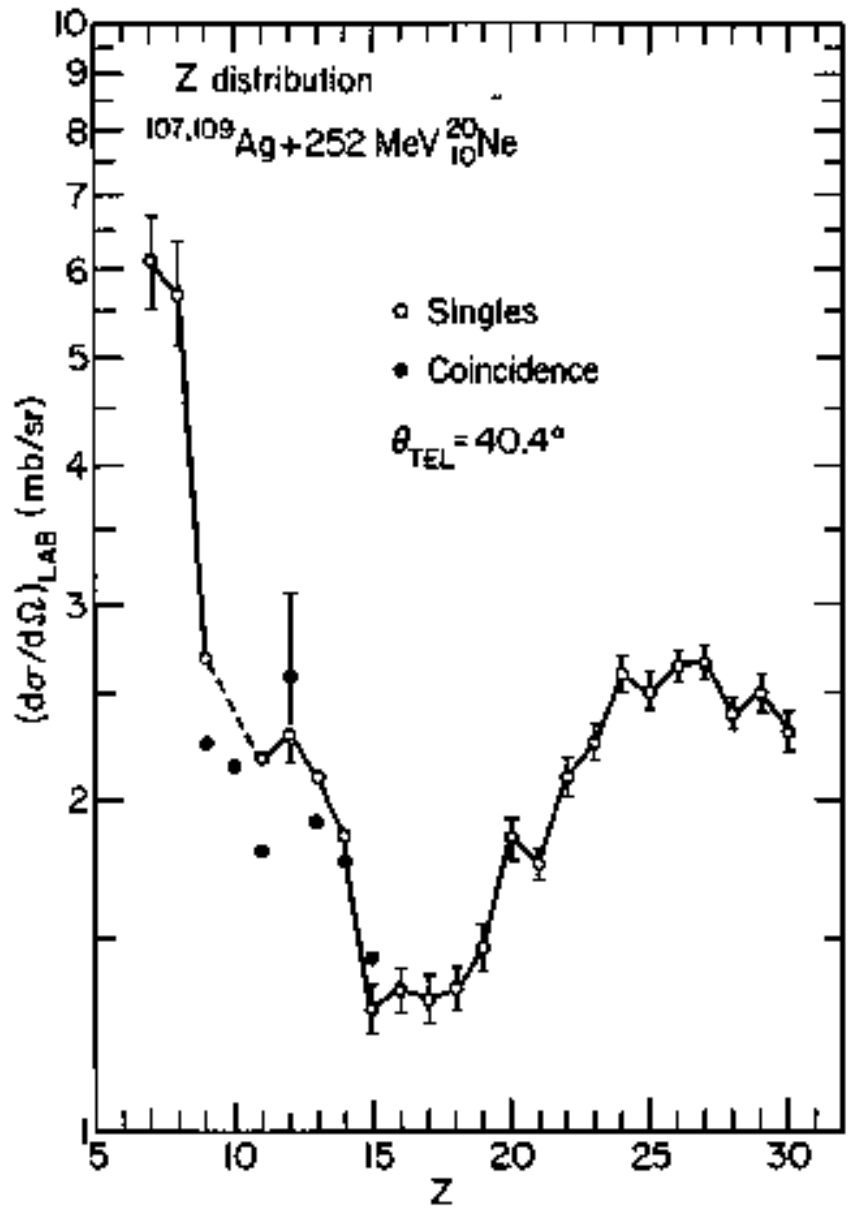

Fig, 4. Singles and coincidence cross-sections as a function of $z$. Dati points and typlcal error bars are shown. For $z=10$, the singles yleld of the relaxed component could not be adequately measured.

(XBL 764-2653)

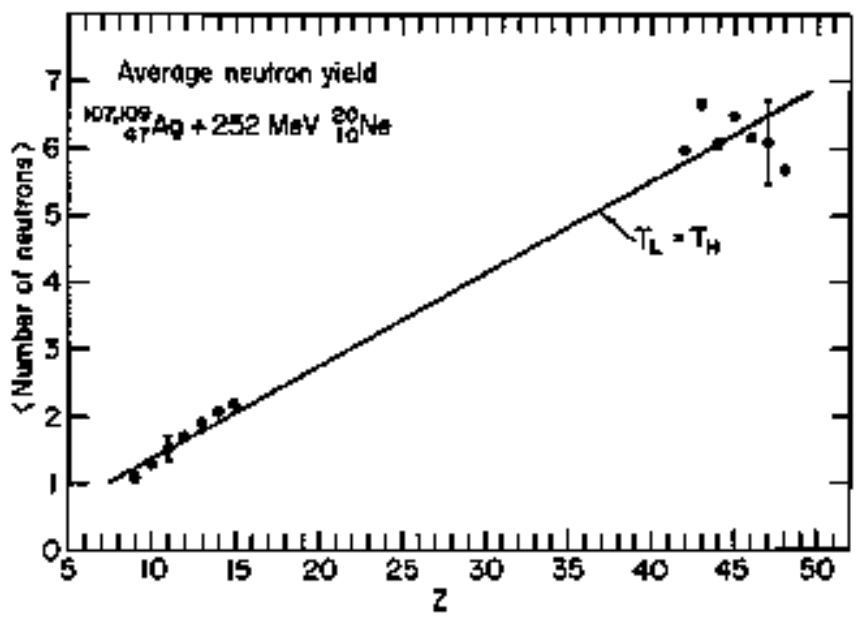

Fig. 5. Awerago number of neutrons enitted by a fragment of a given atcouic rumber. Dața points and typical errors are shown. The solid line shows the number of neutrons expected if the temperature of the light fragment and the temperature of the heavy fragnent are equal.
(XRI 764-2651) 


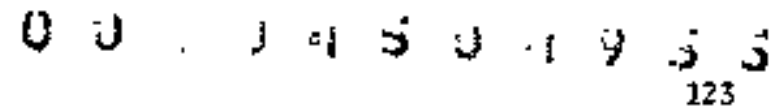

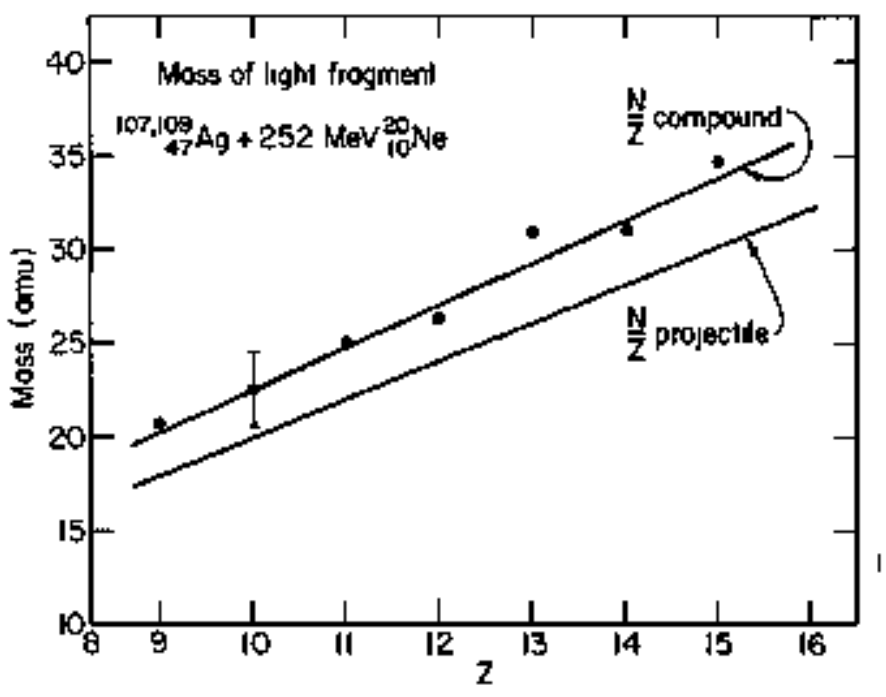

Fig. 6. (a) Mass of the light frament before seccondary emission as a function of the $z$ of the light fragnent. Data points and a typical error are shom. The bottom line depicts the neutron-to-proton ratio, $N / Z$, of the projectile. The top line represents the $N / Z$ ratio of the hypothettcal com-

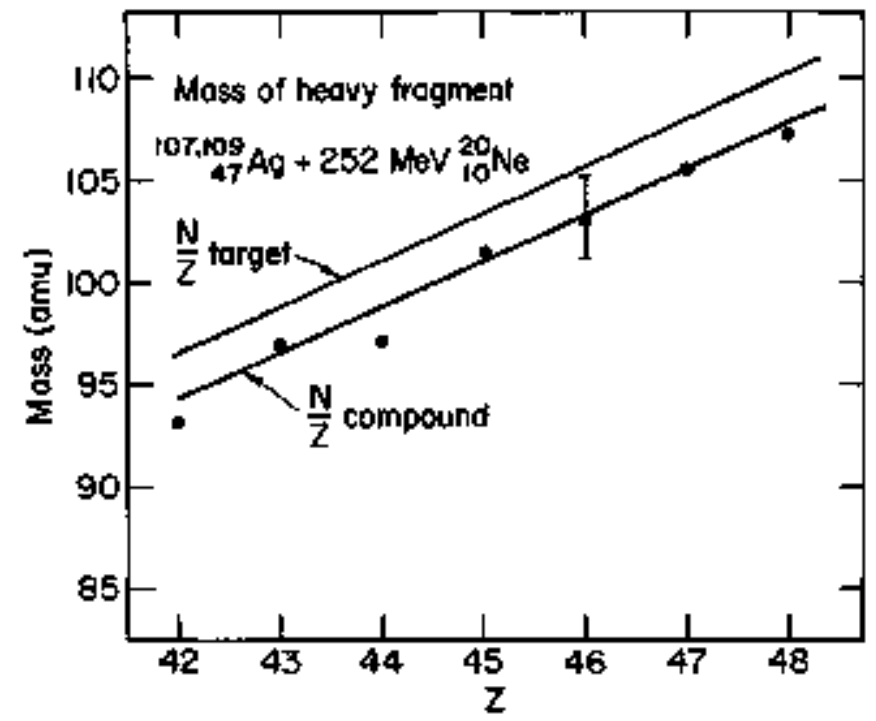

pound nuclews. (b) Hass of the heavy fragnent as a function of the $z$ of the heavy fragment. The top line shows the $N / Z$ ratio of the bypothetical corourand nucleus and the bottor tine is the $\mathrm{N} / 2$ ratio for the target. (a) (XBL 764-2648)

(b) (XRL, 764-2649) know that the system is not fully equilibrated along the mass-asymetry degree of freedom, but it appears to have reached equilibrium in the $N / 2$ coordinate.

In the near future we plan to use this same technique of correlated fraploents to stubly partial relaxation with varlous projectile, target and beam mergy combinations (especially lirypton and xench induced reactions), Azimathal widths should not be sensitive to the intrinsic oscillations of the conplex and might give a more sensitive mogsure of secondary emission from the fragments. light particle emission fror the interndiate complex would distort the kinematics in a complicated way and needs to be studied, as do secondary fission processes in heavier systems.

\section{Footnote and References}

* on leave from DPh-N/NF CEN, Saclar, France.

1. L. G. Moretto, R. P. Babinet, J, Galin and

s. G. Thowpson, Phys. Lett., 5BB 31 (1975).
2. R. P. Babinet, $Z$. Frankel, P. Pusso, R. Jared, L. G. Horetto and 5. G. Thompson, Mucl, Chen. Aumul Report 1974 , LBL-4000, p. 108.

3. P. Russo, R. Jared, R. Rabinet, and L. G. Moretto, 'Energy Calibration of Solid State Counters for Heavy Ion Experiments", this report.

4. R. Babinet, L. G. Moretto, J. Galin, R. Jared, J. Moulton and S. G. Thoupson, Hec1. Phys. A258 $172(1976)$.

5. B. Gatty, D. Guerreau, M. 1efort, J, Pouthas, X. Tarrago, J. Galin, B. Cunvin, J. Girard, and H. Nifenecker, Z. Physik A273 65 (1975).

6. B. Caurin, R. Jared, P. Pussoso, P. Glasse1, R. Schottt, and L. G. Horetto, 'Measurement of Pre and Post Eveporation basses of Deep Inelastic Collisions Fragments of 340-MteV Ar + Ag," this report.

\section{REACTION PRODUCTS FROM THE INTERACTION OF 170- AND 252-MeV ${ }^{20}$ Ne ON Cu}

G J Mallews, B Cauvin,' R Schmul,

$R$ L Jared and $L$ G Morstio

This study is a continution of efforts to systomatize the yields in heavy-jon reactions, 1 The particular interest in the 20 秋 $+C u$ systen is that with the availablo charge resolution, products on both sides of a symuetric mass split can be clearly identified. A slight disadvantage of this system, however, is that kinenatic compression provents the detection of large $Z^{\prime} s$ at large $\mathrm{f} . \mathrm{m}$. angles. 
Thin targets $\left(-400 \mu \mathrm{g} / \mathrm{cm}^{2}\right)$ of $\mathrm{Cu}$ have been bondarded with $170-1 \mathrm{eV} 20 \mathrm{Ne}$. The results of the borbardinent with $252-\mathrm{NeV} 20$ ke have been reported previously. ${ }^{2}$ As in earlier experiments ${ }^{3}$ the product 2 's were identifled with a $A \mathrm{E}-\mathrm{E}$ gas imization counter telescope. The data were stored serially on magnetic tape for later sorting and analysis.

The c.n. kinetic energy spectra for 170-NeV incident 20 he is show in $\mathrm{Fl}$. 1 for several product 7 's. The features of the relaxed conponent at all angles, for all charges, and the quast-

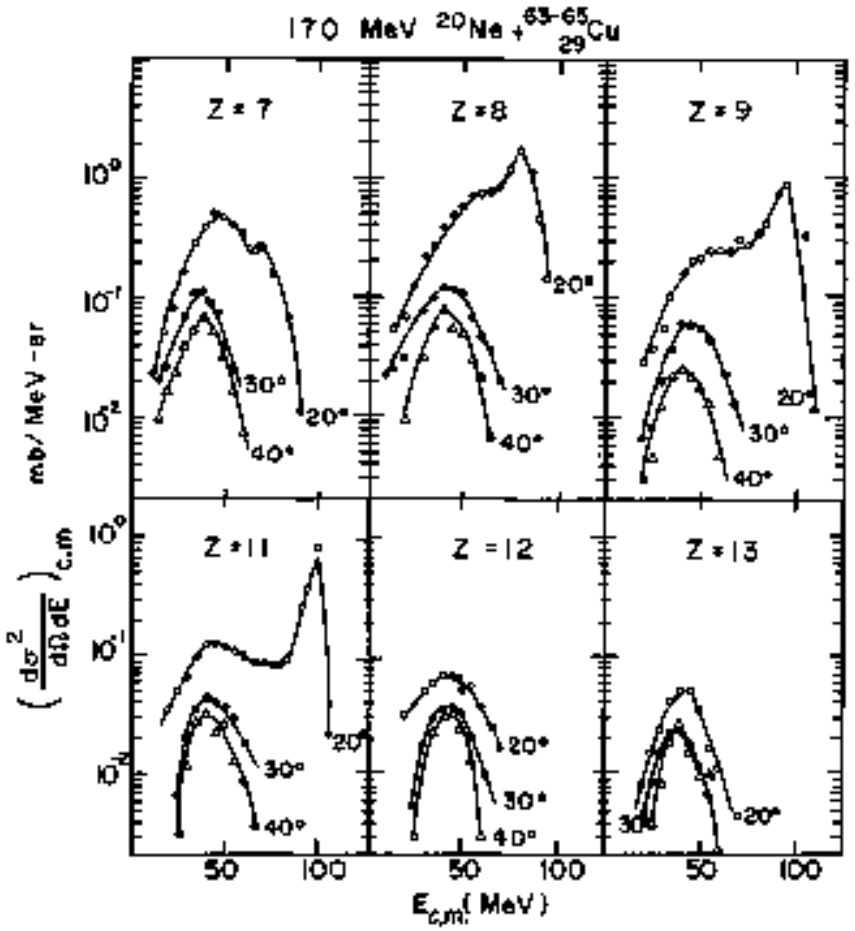

Fig. 1. Some c.n. kinetic energy spectra for products from 170-HeV incident $20 \mathrm{ke}$ on Cu. Quasielastic and relaxpd components are visible.

(XBL 766-8303) elastic codponent near the grazing angle, 2 's close to the projectile, are clearly visible. The cross sections for the relaxed component in these spectra were tntegrated and separated frot the quasi-elastic peak by a Gussian fitting procedure.

Laboratony cross sections for the relaxed com ponent of the detected product 2's at different mgles are shown in Figs. 2 and 3 . As expected, 1 the general features of these figures are;

(1) a decrease in cross section with decreasing energy, as fewer 1-waves penetrate to contribute to the relaxed couponent; (2) a relatively flat charge distribution showing no substantial preference for a symetric split, with considexably increased cross sections for proctuts lighter thar the projectile these effects cin be understood in terns of the mass-asymetry potential-energy surface at the point of injection), and (3) the odd-even effect.

Center of mass angular distributions are shown in Figs. 4 and 5 . The restults are again as

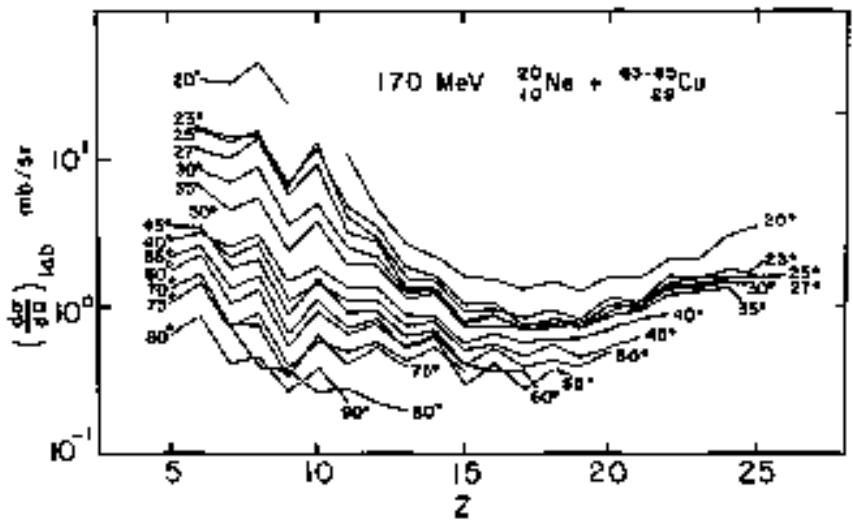

Fig. 2. Labcratory cross sections as a function of $z$ at various angles for the relaxed component: in the reaction of $270-$ Mov 20 te $+C u$.

(XBL 766-8304)

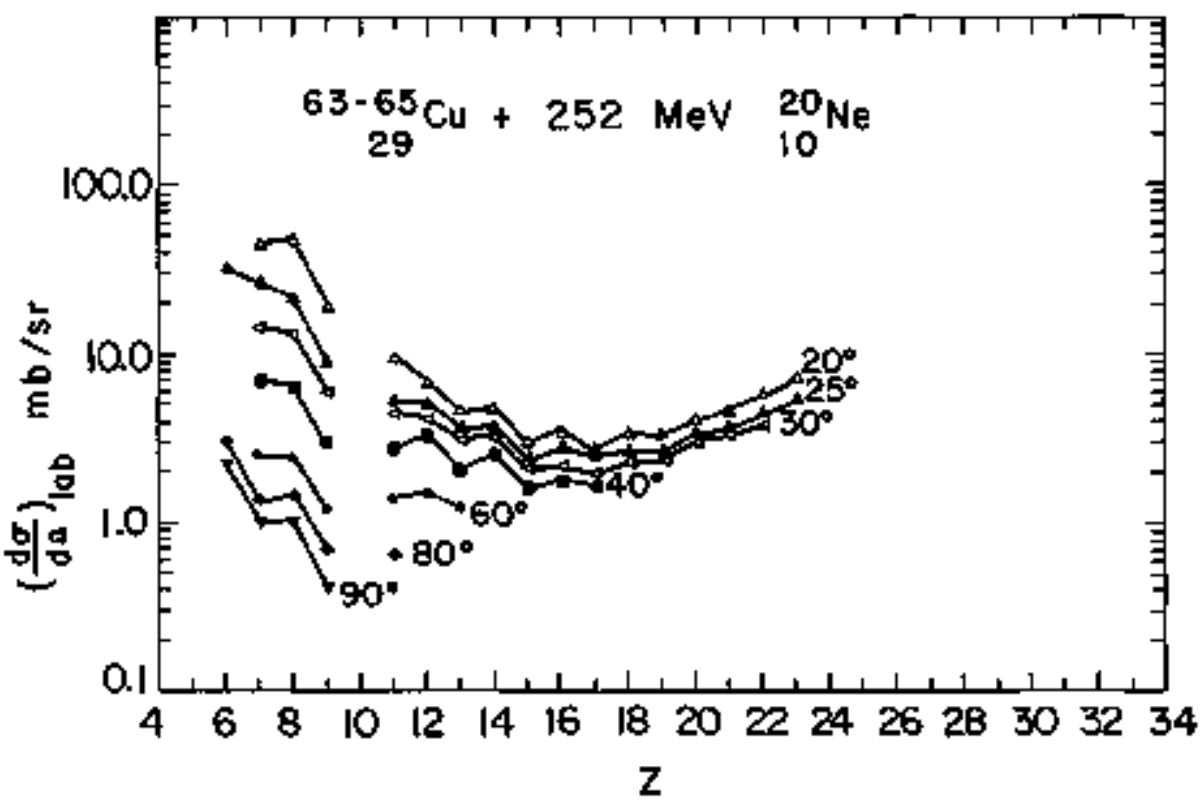

Fig. 3. Laboratory cross sections as a function of $Z$ at various angles for the relaxed component in the reaction of 252-14av 2015e. (XBL 752-2299) 


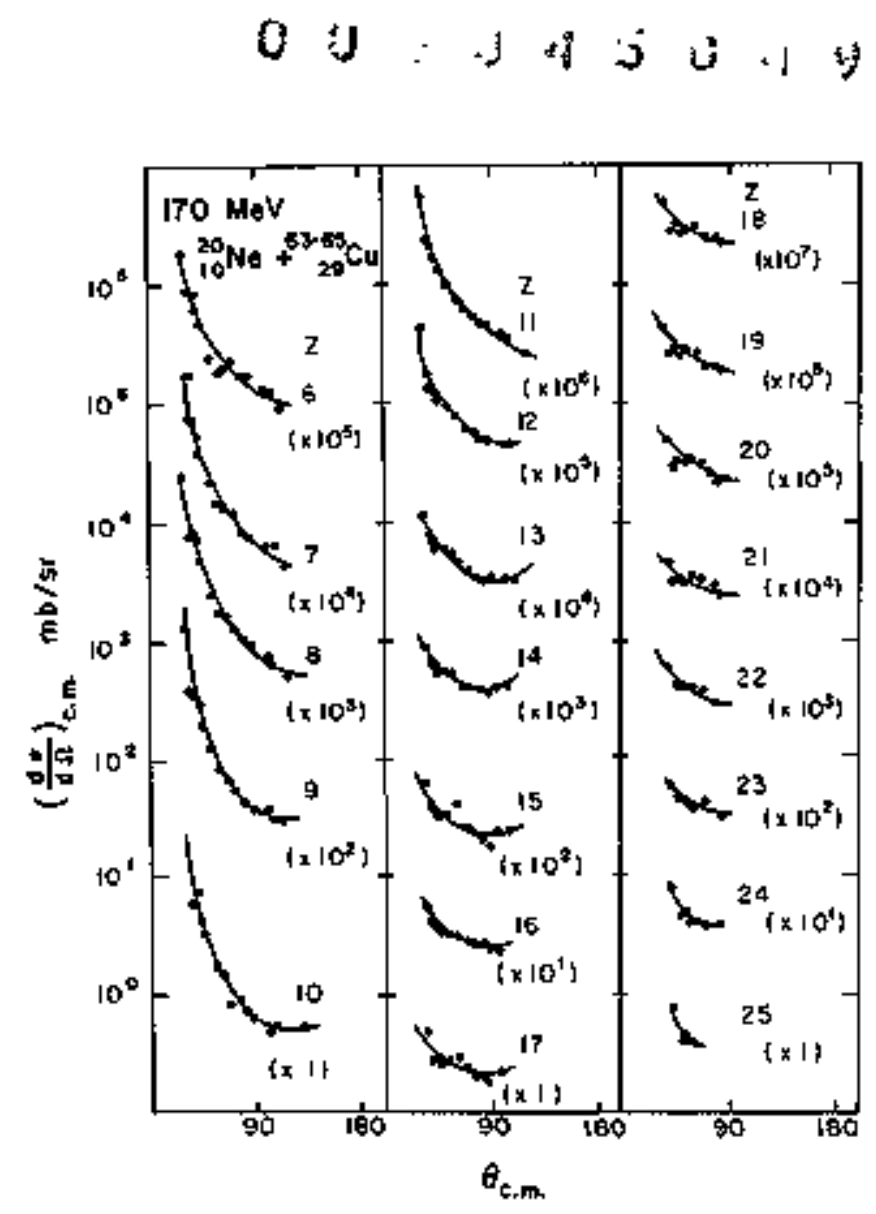

$\frac{j}{125}$

expected. The angular distributions exhibit forward peaking for products near the projectile which gradually converts to a $1 / 5 i n$ distribution due to the Ionger tines associated with greater mass transfer.

In conclusion, it seems that the qualitative features of the $20 \mathrm{He}+\mathrm{Cu}$ reaction are velI understood. What is nesded now are quantitative calculations to test the validity of corrent theoriogs.

\section{Footnote and References}

${ }^{*}$ On leave frin DPh $N / \mathrm{MF}$ GNN, Saclay, France.

1. L. G. Moretto, and J. S. Svantek, Preprint, LBL-S006 (1976).

2. R. Jared, L. G. Mbratto, R. Aabinet, J. Galin, J. Herter, R. Schritt, J. Houtlon, and S. G. Thompson, Nucleat Cheritstry Amuzil Report [1974], LBl-4000, p. 103.

3. R. Babinet, L. G. Moretto, J. Galin, J. Henter, $R$. Schmitt, J, Moulton, and S. G. Thospson, Mucl. Phys. A258, 172 (1976).

Fig, f. Center of mass angular distributions for the $20 \mathrm{Ne}+\alpha_{1}$ reaction at $170 \mathrm{MeV}$ laboratory energy.

(XBL 766-B305)

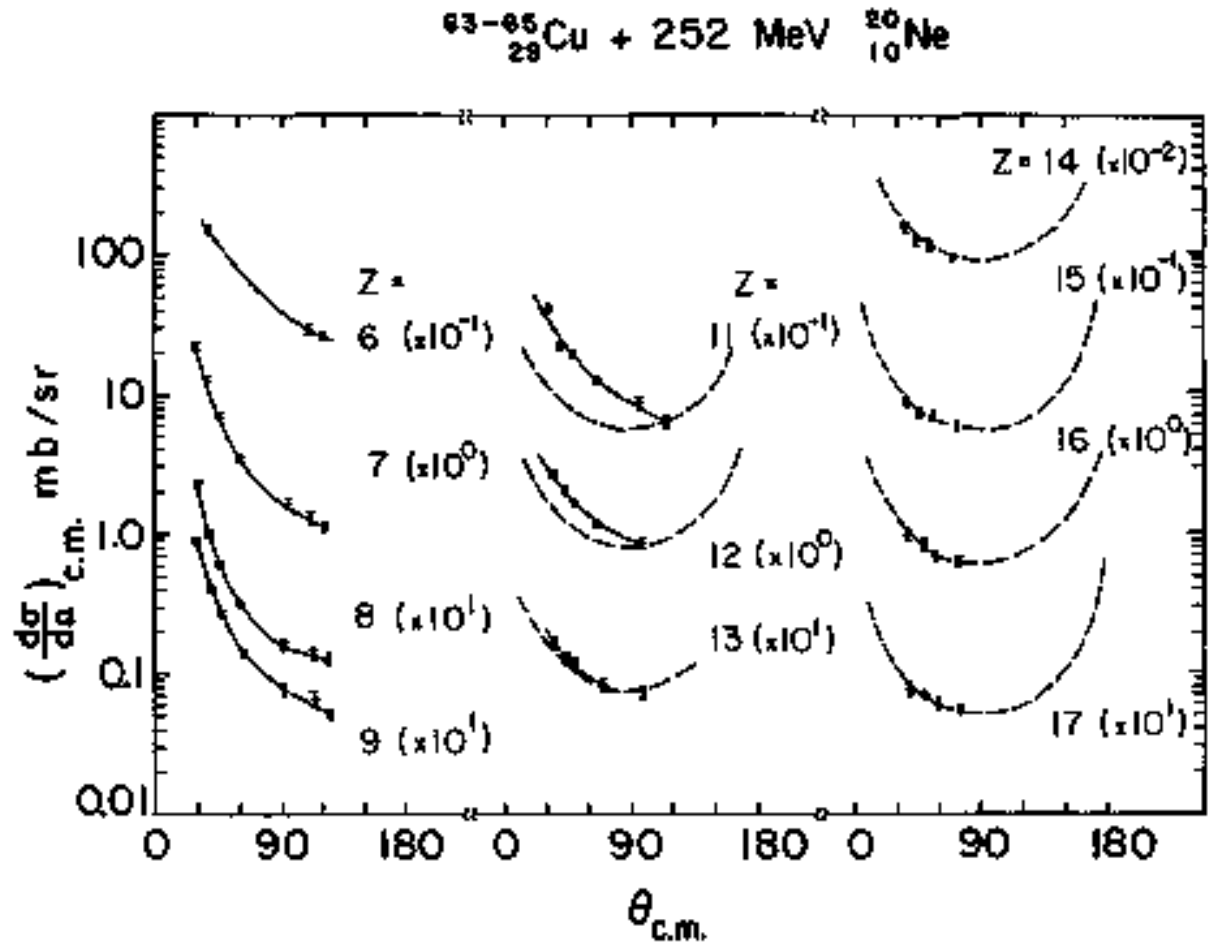

Fig. 5. Laboratory cross sections as a function of $Z$ at various angles for the relaxed component in the reaction of $252-\mathrm{Nov} 20 \mathrm{He}$.

(XBL 752-2300) 
DEEP INELASTIC REACTION OF 175-MeV Ne WITH AU

ل Moulton, G Wozniak, B Cauvn,' P Hus50,

P Jared, and L G Morello

The reaction of $\mathrm{Ne}$ with An has been perfomed at the 88 -inch cyclotron. The bean is focused on a thin retal target $\left(300\right.$ to $\left.800 \mathrm{lg} / \mathrm{cm}^{2}\right)$, and the $Z$ and kinetic energy of the reaction products are measured in E-dE detectors. The $\Delta \mathrm{E}$ counter is a gridded methane-gas ionization chamber developed by our group. I The $\mathbf{E}$ counter is a surface-barrler Si detector, With the low energy projectiles in this series of experiments, products up to about $Z$. 32 can be identified. (Mich heavier products can be identifled with more energetic projectiles.) The data pulses are anplified by a modular electronic system, converted to digital fom in a 4096-channel $A D C$, and stored on magnetic tape by a PDP-5 conputer. An elaborate sequence of data analysis programs have been developed, which sort events according to 2 , and calculate 1 ab and center-ofmass energies and cross sections. This data analysis is performed on our own PDP-9 combuter.

The products of the the plus Au reaction have center-of-mass kinetic energies which are essentially equal to the Coulanb repulsion between the two separating fragments (see Fig. 1). The kinetic energy brought in by the profectile has relaxed into internal degrees of freedom. Actually, products observed around the grazing angle and lighter than $z=11$ have broadened or two-lobed kinetic energy spoctra, arising fron reactions in which the energr has not completely relaxed. Products produced far from the grazing angle or differing appreciably frim the projectile mass have fully rolaxed, singlepeaked $\mathrm{kE}$ spectra at all angles. Prudtucts observed far from the grazing angle or, which are quite different from the projectile had to rotate and diffuse longer to reach the mass asymetry at which they eventubly split. Thus their kinetic energy spectra are relaxed and angle-independent.

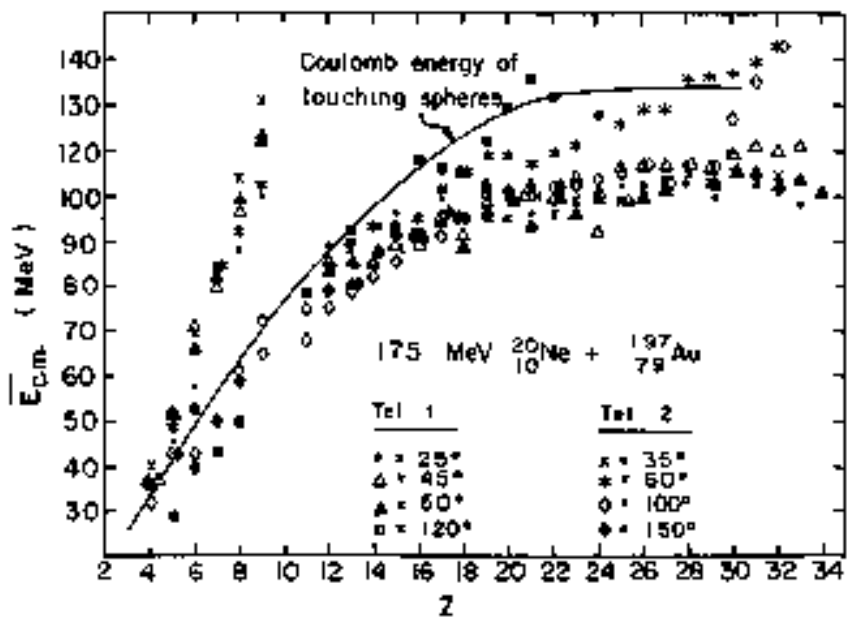

Fig. 1. Mean center-of-mass kinetic energy versus $Z$ for various lab angles. The solid line is che coulanb repulsion energ for too spheres calculated as $\mathrm{E}=1.44 \mathrm{z}_{1} \mathrm{z}_{2} / 1.4\left(\mathrm{~A}_{\mathrm{I}} / 3^{2}+\mathrm{Az}^{1 / 3}\right)$.

(XBL $766-8200)$
The lab cross sections in Fig. 2 present a striking picture. Considering those products and angles for which the kinettc energies are fully relaxed, see a great rise in cross sectton from $Z=10$ to $Z=32$, cowering three orders of magnitude. The cross section for energetically relaxed prowicts rises by one factor of 10 from $Z=9$ to $Z=4$. The cross section for products lighter than $\mathrm{Ke}$ whose kinetic energies are significantly ahove the Coulomb energy is quite large, indicating a fast, non-equilibrium transfer process.

For a better understanding of the significance of these results, it is useful to conpare then with those of two other regctions: 175-keV Ne on $\mathrm{Ag}^{2}$ and $286-\mathrm{MeV}$ Ar on Aus. ${ }^{3}$ For both of these reactions the center-of-mass kinetic energies of the prodicts generglly agree with the Coulanb energy of the separating fraglwents, as was seen for Ne plus dus. This indicates that in all of the reactions the inftial translational kinetic energy has relaxed into intemal degrees of freedom. However, important differences are observed in the lab cross sections for the range of elements detected.

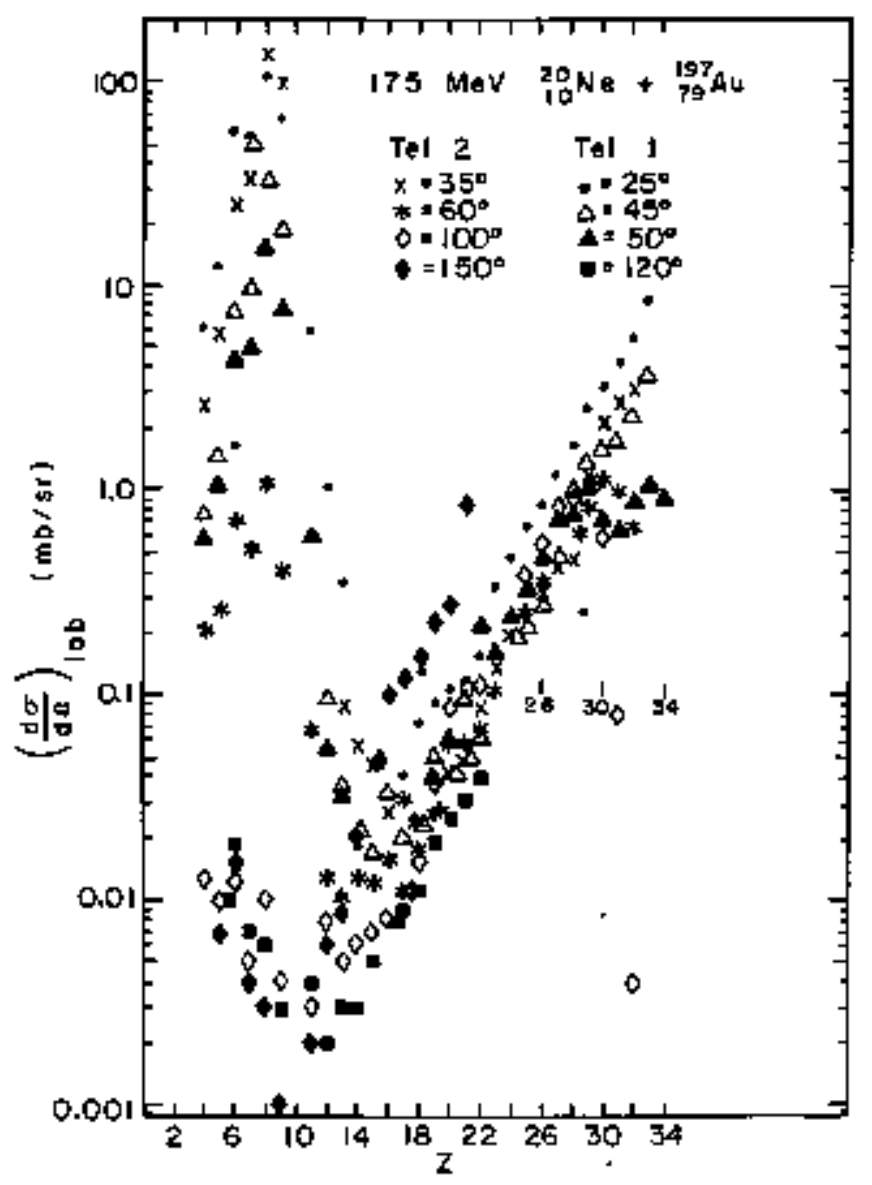

Fig. 2. Lat differential cross section versus $Z$ for various lab angles. 
In the He-Ag system, the cross section rises abruptly for elenents lighter than the projectile, and uuch more gently from $z-11$ to $z=29$. This is consistent with a picture of the reaction complex as two spheres stukk together and exchanging nucleons. The resulting 2 distribution approaches what would be expected for a diffusion process controlled by a liquid drop mass asymetry potential. The low mass of the projectile makes the starting asymetry quite large, so the system finds itself trapped on a downslope leading to greater asydmetry. Thus the high yleld for products lighter then Ne. With the large angular powenta of these reactions, (about 80 ) a dip in the potential exists at symetry, causing increased cross sec. tion around syuretric splitting for an equilibrated $z$ distribution. The rise in yield towards symotry indicates that the system has mored significantly towards equilibration.

The reaction of Ar on An is qualitatively similar. The $\mathrm{Z}$ distribution rices steadily with $\mathrm{Z}$, which may arise from the very pronounced depression at symetry in the mass asyunetry potential. There is Iittle rise in cross section for low $Z$ 's. The initial projectile-target mass asynnetry probably lies on the inside of the fump which separates extreme asyouretry from symetric splitting. This pulis the system "downhifl'" towards symetry nore strongly than in the reaction of $\mathrm{Ne}$ on $\mathrm{Ag}$. Therefore one understands why one sees little rise in the cross section corresponding to the great drop in the potential for produxts lighter than about 2 - 12 . Also the center-of-mass angular distributions for all products 4 to $Z=24$ are strongly forward peaked, with a minimln as far beck as $120^{\circ}$. This angular asyometry remains but diminishes through $Z=28^{\circ}$. Only for $Z$ w 29 does the angular distribution follow $1 / \sin \theta$. This forward peaking indicates rapid decay of the complex, in a time somewhat less than a rotatoinal period. The system quickly populates the $Z$ 's "downhill" from the initial projectile-target mass ashniletry, namely 2 's from 18 up to symuetry. Thore is only a low probability that the systen will get over the potential energy hurp to populate $Z^{+} \mathrm{s}$ below 12 before breakup of the complex. However, it is possible that the cross section for 2's abowe Ar may arise in part from fission of a colpound nucleus or of the target itself. This would also lead to an yield increasing with $z$, and could supply the $1 / \sin \theta$ component of the angular distribution of all $z^{\dagger} s$ observed.

The difference between the $z$ distribution of the Ne-Ag and the Ne-Au systens is quite remarkable. In the fomer, the initial projectile-tanget Hass asymunetry charneled rach of the cross section to $Z^{\dagger} s$ below $\mathrm{Ne}$, with lower, slowty rising cross section towards symmetry. In the latter case, nost of the cross section is for elemetats above Ne, with much less rise in yield for light products.

One needs to examine the angular distributions fron the Ne-Au reaction to determine the extent to which the strong population of heavy products is the result of a diffusive mechanism. Production of elenemts above the projectile by ftssion of a compound nucleus, of the target enriched by absorption of several mocleon frot the projectile, or even of the target itself, would seen to be quite possible. Hewever, the Ar-Au reaction denonstrated a significant ron-fission component for products up to $z=28$. A major complicating factor in these reactions is that different angular momentum wirdows may be involved in these reactions. Work is continuing in this series of expertrents, including analysis of a higher energy

\section{Footnote and References}

ton leave from DFh/MF CEN, Saclay, France

1. Balcoln Fowler et al., kucl. Inst. Moth. 24:341 (1975).

2. Regis Babinet et al., Macl. Phys A25B:172 (1976).

3. Lutiano Moretto et a1., Muc1. Phys, A259;173 (1976).

\title{
SYSTEMATICS OF THE ${ }^{40}$ Ar + CU REACTION
}

\author{
G J Mathews, B Cauvin, J Moutton, B Schmill,
} $R C$ Jared, and $L$ o Moretto

Preliminary reșults of the stuxty of this reaction with 288 - and 340 NeV incident 40 Ar were reported previously. 1 the report hers an extonsion of the systemetics of this system to $170 \mathrm{MeV}$. The present study includes wore complete charge and angular distributions.

Reaction products from the interaction of incident $40 \mathrm{kr}$ on this $\left(400 \mu \mathrm{g} / \mathrm{om}^{2}\right)$ Cu targets we identified in a $A \mathrm{E}-\mathrm{E}$ gas ionlzation counter telescope.

Kinetic energy spectra for sume of the reaction products at $170 \mathrm{hteV}$ are shown in Fig. 1. These exhibit the familiar strengly-danged component. The quasi-elastic contribution is also visible for products near the grazing angle and the projectile $Z$, although at these energies the quast-elastic and relaxed components are difficult to distinguish.

Laboratory charge distributions at $170 \mathrm{MeV}$ are shown in Fig. 2 . These cross sections exhìbit the expected features of an odd-even effect, a decrease in ragitude fron the higher energy data, and a relatively flat tharge distribution. The peaking of the cross sections near the projectile is the to the quasi-elastic component which was not separated out. 


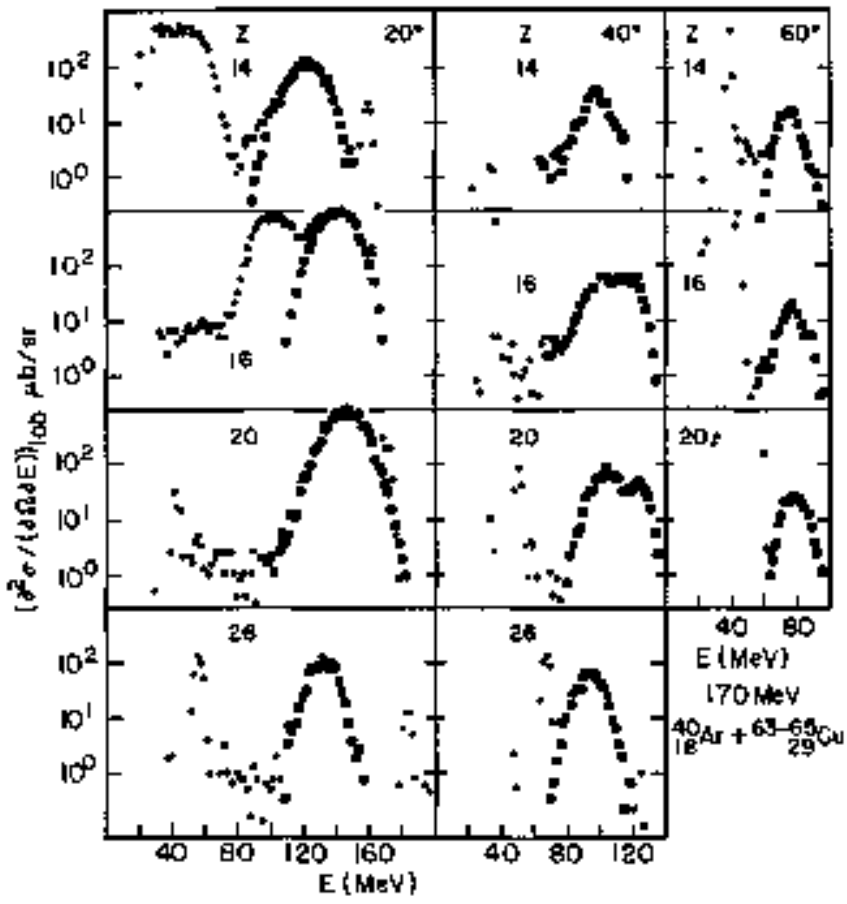

Fig- 1. Center-of-mass kinetic energy spectra for the $40_{\mathrm{Ar}}+$ ou reaction at $170 \mathrm{HeV}$. (XEL 7610-9377)

The center-of-ntass angular distributions, not shown, exhibit the forward-peaking characteristic of the short interaction times insolved in the formation of products near the projectife. The distributions approach $1 / \sin \theta$ for products requiring nore mass transfer.

In conclusion, it sects that the systenatics of the $40_{\mathrm{Ar}}+\mathrm{OM}$ reaction are consistent with current understanding of heavy-ion interactions.?

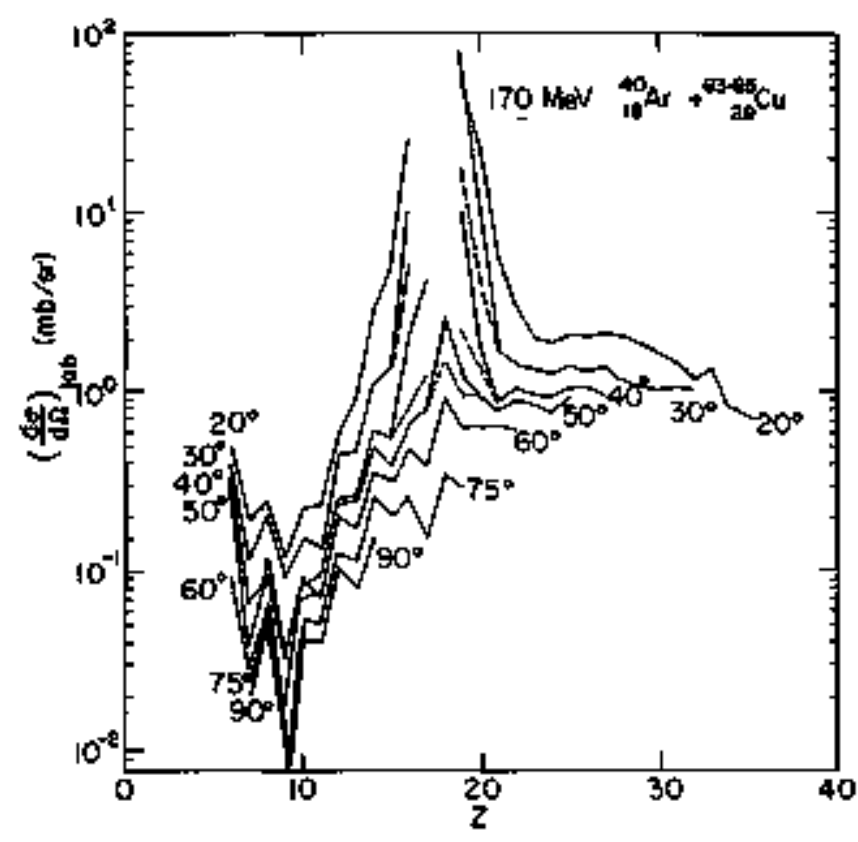

Fig. 2. Laboratery cross sections for products fron the $40 \mathrm{Ar}+$ ar reaction at $170 \mathrm{HeV}$.

(XBL 769-4064)

\section{Footnote and References}

"On leave fron IPh-N/MF CEN, Saclay, France.

1. l. G. Moretto, R. C. Jared, R. Babtnet, J. Galin, J. Hunter, and S. G. Thüuson, Nuclear Ohemistry Amual Report (1974), LBL-4000, p. 104.

2. L. G. Noretto, and $\rfloor$. S. Sventeck, Preprint, LBL -5006 (1976).

\section{PRELIMINARY MEASUREMENT OF REACTION PRODUCTS FROM THE ${ }^{40} \mathrm{Ar}+$ Ag REACTION AT $170 \mathrm{MeV}$}

G J Maltiews, A Schmity. P Glassek, $G$ Woznlak.

F C Jared, and $\mathrm{L} G$ Moretto

This reaction is of interest because at such low energr sbove the Coulowb barrier a separation of the strongly damed product distribution into two conponents has been rocently observed for the similar system of $40 \mathrm{Ar}+\mathrm{Al}$ at $220 \mathrm{MeV}$. According to the results of Reference 1 , the cross section for the relaxed prodxcts were observed to peak both near the projectile and near mass symmetry at angles close to the grazing angle. This was taken as an indication that two separate compontents contribute to the relaxed process. However, at these energies, the relaxed component is kinematically nearly indistinguishable frcm the quasielastlc products, and hence may be responsible for the observed rise in cross section near the projectile.
Laboratory cross sections are shown in Fig. 1 The experinental technique to derive these data is described in detail elsewhere. 2 In Fig. 1 the eross section does appear to rise near the projectile and near symetry, although the separation of the relaxed and quasi-elastic conponents is, indeed, not possible close to the projectile. To examine possible influence frotn the quasi-elastic contribution to the kinetic energy spectra, we plot in Fig. 2 the centroid of the c.il. energy spectrun for the rolaxed plus quasi-elastic. In Fit. 3, the $C_{*} m$. Fin of the peaks are also plotted. The c.n. energy and Fin tend to fail higher for prodicts close to the projectile, consistent with an interp. rotation that the qussi-elastic products are largely responsible for the rise in cross section. 


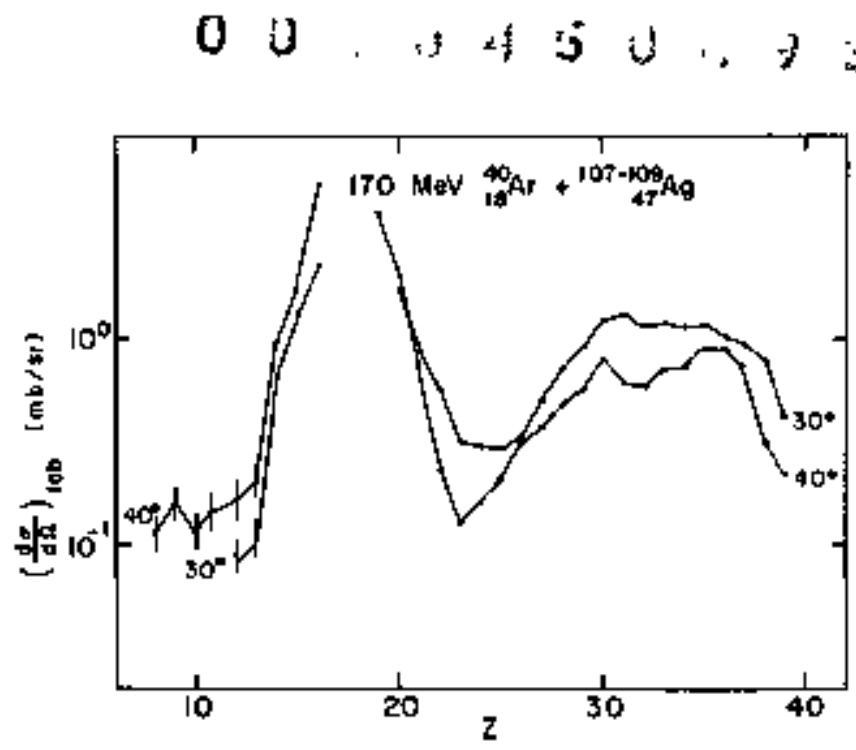

Fig. 1 Laboratory cross sections for ${ }^{40} \mathrm{Ar}+\mathrm{Ag}$ at $170 \mathrm{MeV}$.

(XBL 766-8306)

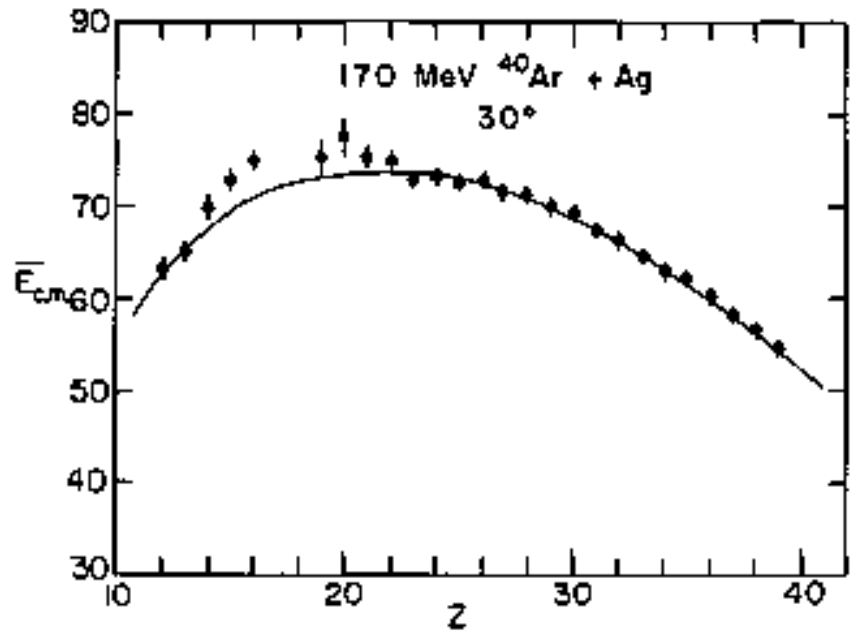

Fig. 2. The centroids of the c.m. relaxed quasiolastic energy spectra at $30^{\circ}$ 1ab, (XBL 766-8307)

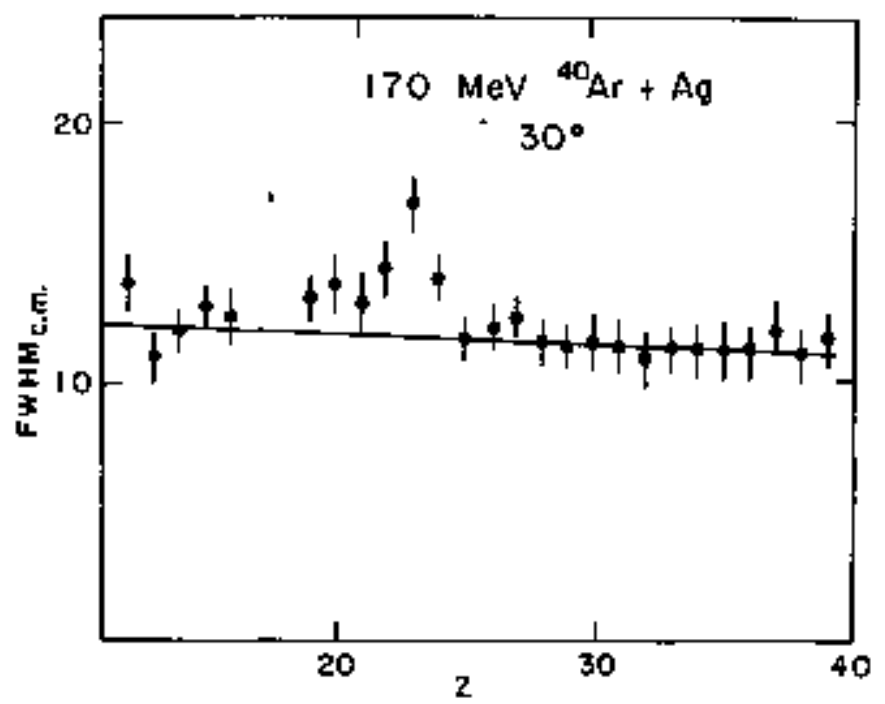

Fig. 3. Fint's of the c.m. kinetic energy spectra at $30^{\circ} \mathrm{lab}$.

(XEL 766-8308)

Future experiments are plamed to obtain a IBre corqplete angular distribution which should shed additiunal light on this problen.

\section{Footnote and References}

NATO Fellow.

1. C. Ngo, Oui Chaoui, F. Plas11, J. Peter, B, Tamain, Berlanger, Hanappe, private conmonication.

2. J. Galin, L. G. Bbretto, R. Babinet, R. Schmitt, R. Jared, and S. G. Thompson, hucl. Phys, AZ55, 472 (1975).

\section{MEASUREMENT OF PRE AND POST EVAPORATION MASSES OF DEEP INELASTIO COLLISIONS FRAGMENTS OF 340 MeV Ar + Ag}

B Cauvin, F Jared, P Fusso, P Glastsel,

A Schmilt, and $\mathbf{L} \mathbf{G}$ Moretio

Several groups have attenpted to determine the nass of the fraguents in deep inelastic collisions [D.I.C) of heavy ions by neans of various techriques, Some measurements of the fragnent masses following particle evaporation have beep performed in time of flight 1 and mass spectrueter experiments. Also coincidence experiments whith gtve the erergies and angles of the correlated fragnents permit the determination of the messes 3,4 before evaporation through the kinematics, provided the energies are corrected for neutron enission. This correction is generally performed by estimating the total nutrber of neutrons from the energy balance and sharing them according to the masses of the fragments. This nethod is linited by the lack of knowledge of the fragment atomic nubers, which makes it difficult to estimate the total binding energies of the fraguents.

The measurement of the $z$ of one of the fragwerts along with the energies and angle of both fragments provides the needid paraneters to deterwine accurately these binding emergies.

A coincidence experiment for the reaction $340 \mathrm{HeV}$ Ar + Ag was performed at the SupertILAC. In this experingent a $A E-E$ teloscope fixed at $40^{\circ}$. measures the $Z$ of one of the fragments, A position-sensit Ifve detector (PSD) was placed successively at $30^{\circ}$ and $50^{\circ}$ (Fig. 1). The measured energies of both light and heavy fragments were corrected for pulse height defects. ${ }^{5}$ A prelininary 


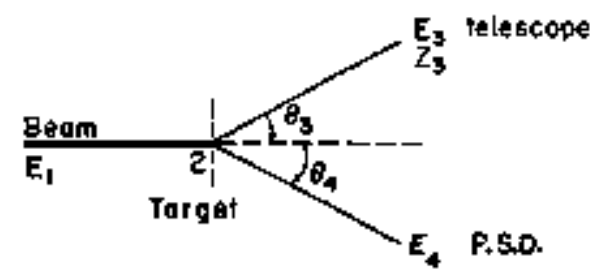

Fig. 1. Schematic diagram of the experinantal set-lp with the definitions of fragment numbers, energies and angles in the laboratory systent. (XBL 766-8208)

analysis based upon the average values of the mergy and angular distributions has beef made. $A$ wore detailed event-by-event treetment of the data is in progress.

These data were first analyzed in the vicinity of $z=32$ for the light fragnent. This corresponds to an almost symuetric splitting of the cumposite systeml. Near mass symetry it is ressonable to assume that the ratio of the number of evaporated particles $\left(v^{7}\right)$ to the mass before evaporation $\left(A^{*}\right)$ is the sane for both fragments. With this assumption and the following equations we calculated conservation of finear momentum in the 1ahoratory system [ Fas. (1) and (2)] conservation of mass [Eq. (3)] and charge [Eq. (4)], the masses of both fragments $\left(A_{3}^{*}, 4\right)$ priar to particle evaparation, and the total number of particles eveporated by each fragrnent $\left(v f_{3}\right)$. Superscript * denotes preevaporatim. (See Fig. 1 for the definition of the parameters and subscripts)

$$
\begin{aligned}
& \left.\frac{E_{3}}{1-\frac{v_{3}^{T}}{A_{3}^{k}}} A_{3}^{*}=E_{1} A_{1} \frac{\sin ^{2} \theta_{4}}{\sin ^{2}\left(\theta_{3}+\theta_{4}\right)}\right] \\
& \left.\frac{E_{4}}{T} A_{4}^{*}=E_{1} A_{1} \frac{\sin ^{2} \theta_{3}}{v^{2} \theta_{3} \theta_{4}}\right\} \text { linear momentum } \\
& 1-\frac{y_{4}}{A_{4}^{*}} \\
& A_{3}^{*}+A_{4}^{\star}=A_{1}+A_{2} \quad \text { conșgrvation } \\
& z_{3}^{*}+z_{4}^{*}=z_{1}+z_{2} \quad \text { conservation }
\end{aligned}
$$

The results obtained with the assumption that no charged particle is eveporated by the fragnents are shom in $\mathrm{Fig}$. 2a. Since good agreenent was obtained for the elastic scattering masses, 5 it appears that the systematic shift observed between the masses of the light and the heavy fragment is not due to experimental difficulties. 7 it is more $11 \mathrm{kely}$ the indication of charged particle evapora. tion from the light fragment. Since the fragments should be identical at symotry the number of evaporated charges necessary to make the two lines agree at syumetry is given by the shift projected onto the 2 axis. This number is 1.3 charge unit per fragment. In Fig. $2 b$ the masses have been

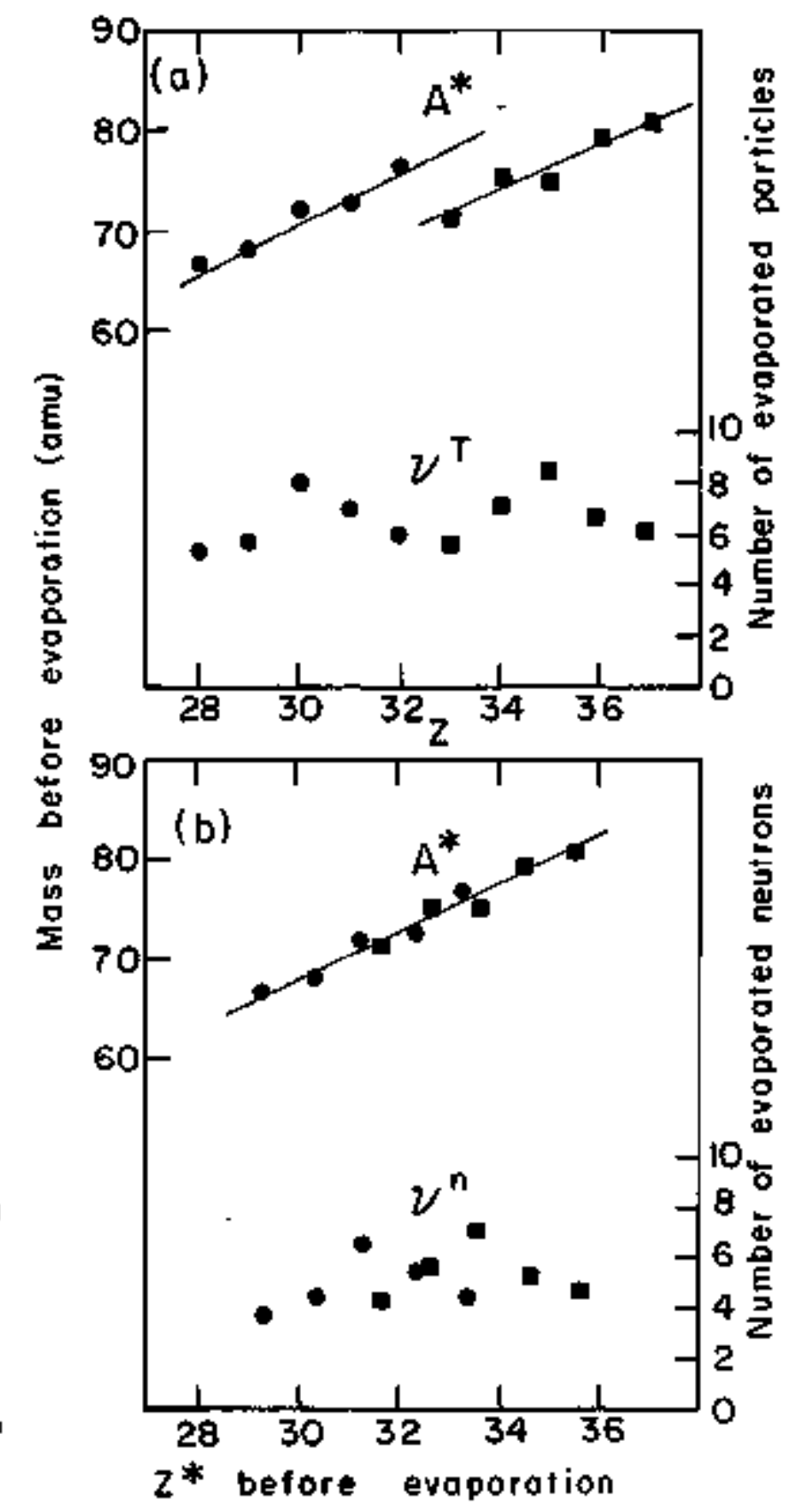

Fig. 2 (a). Masses before evaporation and total number of evaporated particles for fach fraguent from the reaction of $340 \mathrm{MkX} \mathrm{Ar}+\mathrm{Ag}$, with the asstelption that $\left(v_{7} / A_{3}^{*}=v_{4}^{*} / A_{4}^{*}\right)$. The solid circles $r e f e r$ to the light tragrent and the solid squares to the heavy fragment; no charged particIe evaporation is taken into account. fragments.

(b). I.3 protons were evaporated from both

(XRL 766-8209)

calculated with an average of 1.3 charge mit evaporated by the light fragnent. The neutrors nubbers $v^{n}$ mere obtained by subtracting from ${ }^{T} T$ the experimental value of 1.3 chargo unit. This inplies that the evaporated charged particles are mainly protons.

In order to analyze the data away frof symmetry, we can no longer assine that ${ }^{4} \mathrm{~T}^{\mathrm{A}} \mathrm{A}^{\mathrm{k}}$ is the 


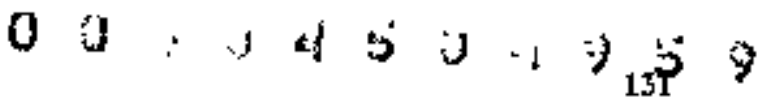

same for both fragments. Instead we con use another equation [ Eq. (5]] which expresses the conservation of energy in the laboratory systeat

$$
\begin{aligned}
& E_{1}+B\left(A_{I}, Z_{1}\right)+B\left(A_{2}, Z_{2}\right)=E_{3}^{*}+E_{4}^{*}+B\left(A_{3}^{*}, z_{3}^{*}\right) \\
& +B\left(A_{4}^{*}, Z_{4}^{*}\right)+E_{\gamma}^{T}+E_{n}^{T}+E_{p}^{T} .
\end{aligned}
$$

$\mathbf{E}^{\mathbf{T}}$, $\mathbf{E}^{\mathbf{T}}$ and $\mathbf{E}^{\mathbf{T}}$ are respectively the total energies astociated with gamm, reutron, and proton emission. The total binding mergies $B\left(A^{*}, Z^{*}\right)$ are taken the droplet mass table of Myers. 6

Using this equation, along with Eqs. (1) to (4) where v $3=v_{4}+v_{3}$ we developed a self-consisteit prockdre to obtain the masses and the number of evaporated neutrons for each fragnent. This procedure assumes a binary process and that no particles are enitted by the composite system before the separation of the fraemerts ( Eqs. (3) and [4]). $E^{T}$ is the sum of the individual rotational emergies of the fragments calculated from a simple model of two rigidly rotating spheres. Scme competition with neutron emission is included by adding to these energies a fraction of the birxing energy of the last neutron enitted in the averagg cascade. Since the excitation energies of the fragments are relatively high, it seens unlikely that strong shell effects would be present. The level desntiy parameter was simply taken to be $A^{k} / 8$ in the determination of the average kinetic energies of the evaporated particles.

The above set of equations is highly ronlinear preventing a direct solution, An iterative procediure was developed to obtain the solution. starting with a reasonable guess for $A_{4}^{*}, A_{3}^{\pi} v_{4}^{n}, w^{n}$;

$$
\text { (Step 1) The ratio } \frac{v_{3}}{v_{4}^{n}} \text { is extracted from }
$$

Bqs. (1] and [2];
(Step 2) $v_{3}^{n}$ and $v_{4}^{n}$ are obtained from Eq. (5);

(Step 3) With these values Eqs. (1) and (2) give the ratio $\frac{A_{3}^{*}}{A_{4}^{*}}$.

(Step 4) $A_{3}^{*}$ and $A_{4}^{*}$ are then calculated frow the ratio $\frac{A_{3}^{*}}{A_{4}^{*}}$ and Eq. (3).

(Step S) If the convergence is not obtained we start again at Step 1. Between Steps 1 and 2 the excitation energies, nuclear temperatnres, and kinetic energies of neutrons and protons are calculated.

The nunber of evaporated protons is a free parameter in the program and is not part of the iteration. In the analysis of the reaction

$A r+A g,\left|\nu^{p}\right|$ was estimaterd from the evraporation code of Dostrovsky et al. and renomalized with the procedure explained later. In these evaporation calculations we used $A / I 0$ for the Ievel density parameter and 1.5 fermi for the radius parameter. It was assuesd that the total excitation energy is shared by the two frapguents according to their mass. The initial masses $A^{\text {* }}$ were calculated by mitimizing the potential energy of two touching spheres 1 of given doass asynwetry with respect to the charge asyumetry degree of freedom, This evaporation code was developed by lostrovshy et al, 6 for the analysis of compound nucleus reactions leading to rasses and excitation energies strilar to those of the D. $1 . C$. fragnents. No angular momentum is taken into account in this code. We to not expect this to affect the results substantially since in the D.I.C. ons expects most of the initial angular monentum to go into relative motion. Table 1 summarizes the results of the calculations for the light fragments of the reaction. At symetry these calculations predict an average number of evaporated changes $\left(\mathrm{w}+2 w^{\mathrm{a}}\right]$ of

\begin{tabular}{|c|c|c|c|c|c|c|c|c|c|c|c|}
\hline$z^{*}$ & $A^{*}$ & $v^{n}$ & $v^{\tilde{p}}$ & $v^{\alpha}$ & $\begin{array}{c}v^{p}+2 v^{a} \\
\text { (nomal- } \\
\text { ized) }\end{array}$ & $z^{*}$ & $A^{*}$ & $v^{n}$ & ${ }_{\Downarrow} \mathbf{P}$ & $v^{\alpha}$ & $\begin{array}{c}P+2 v^{\alpha} \\
\text { (nolmal. } \\
\text { ized) }\end{array}$ \\
\hline 10 & 22 & 0.98 & $\sim 0$ & 0.02 & 0.05 & 22 & 49 & 2.52 & 0.39 & 0.03 & 0.58 \\
\hline 11 & 24 & 0.95 & 0.05 & 0.16 & 0.47 & 23 & 52 & 2.89 & 0.41 & 0.05 & 0.66 \\
\hline 12 & 27 & 1.53 & 0.02 & 0.01 & 0.05 & 24 & 54 & 3.03 & 0.38 & 0.08 & 0.69 \\
\hline 13 & 29 & 1.85 & 0.02 & 0.01 & 0.05 & 25 & 56 & 3.28 & 0.44 & 0.10 & 0.82 \\
\hline 14 & $3 I$ & 1.84 & 0.09 & 0.02 & 0.17 & 26 & 59 & 3.92 & 0.23 & 0,09 & 0.53 \\
\hline 15 & 33 & 1.82 & 0.10 & 0.03 & 0.20 & 27 & 61 & 3.90 & 0.47 & 0.07 & 0.78 \\
\hline 16 & 36 & 1,88 & 0.04 & 0.06 & 0.20 & 28 & 63 & 4.03 & 0.37 & 0.13 & 0,81 \\
\hline 17 & 38 & 1.97 & 0.10 & 0.18 & 0.59 & 29 & 66 & 4.16 & 0.44 & 0.09 & 0.80 \\
\hline 18 & 40 & 2.04 & 0.13 & 0.14 & 0.53 & 30 & $60^{\circ}$ & 3.99 & 0.48 & 0.20 & 1.13 \\
\hline 19 & 43 & 2.57 & 0.13 & 0.09 & 0.40 & 31 & 71 & 4.14 & 0.53 & 0.20 & 1.20 \\
\hline 20 & 45 & 2.36 & 0.22 & 0.09 & 0.51 & 32 & 73 & 4.25 & 0.61 & 0.20 & 1.29 \\
\hline 21 & 47 & 2.51 & 0.19 & 0.06 & 0.40 & 33 & 75 & 4.10 & 0.74 & 0.19 & 1,45 \\
\hline
\end{tabular}
1.05. This numer is close to the experimenta1 value

Table 1 , 
of 1.3. These calculated values were normalized to agree with the experimental rumber of 1.3 at symunetry.

Using the normalized values of Table 1 for $\mathbf{W}_{4}$ in the full iterative calculation, we bbtained the masses and neutron nubers of Fig. 3. Around

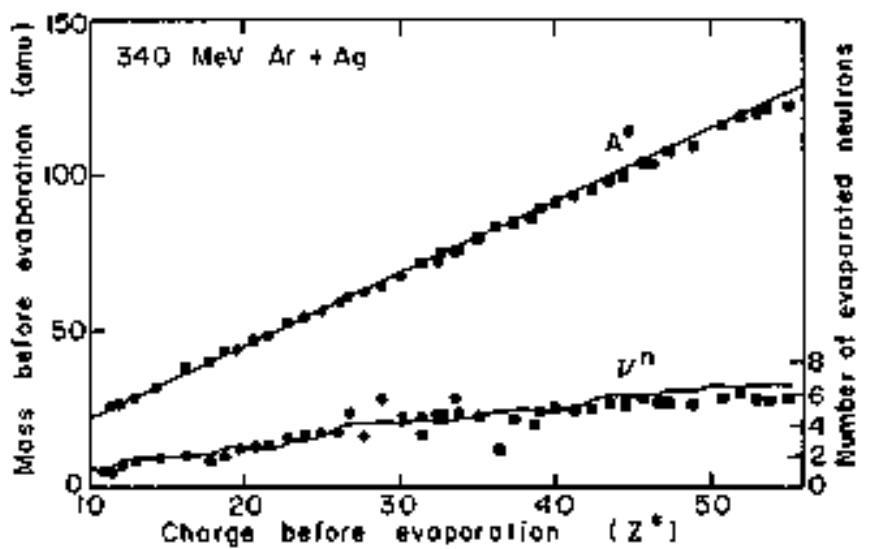

Fig. 3. Masses before evaporation and number of evaporated neutrons for each fragment calculated with the full iterative procedure. Symbols are the same as in Fig.2. The upper solid line is the prediction of the charge equilibriun model described in the text. The lower solid line represents the number of reoutrons $y^{M}$ obtained with the evaporation code of Ref. 6 assuming that the total excitation energy is shared by the fragments accorting to their mass.

(XBL 766-8210)

synnetry the agreement with the simple andysis done before (Fig - 2b) is good. The tpper solid line represents the masses calculated by assuning charge equilibration between the two fragnents. The good agreenent obtained with this nodel confirms the conclusions of Gatty et al. I for the comparable systen $A r+\mathrm{Ni}$, nanely that charge equilibrium is indied achieved in D.I.C. for the part of the angular distribution beyond the grazing angle. The Iower solid line represents the calculated values of |v in frou Table 1 extended towards higher atonic numbers. The good agreesnent with the experimental values suggest that thermal equilibritm is achievot between the fragoents as wo postulated when establishing the input parameters of the evaporation calculations. Further analysis will allow us to "explore" the behavior of the systen (wasses and excitation energles) as it relaxes along the energy degree of freedor.

\section{Footnotes and References}

"On leave from DPh/MF CWN, Saclay, France.

† PATO Fellow.

1. B. Gatty, D. Guerredu, M. Lefort, X. Tarrago, J. Galin and B. Caurin, J. Cjirent, H. Nifenecker, Nuc1. Phys. A253, 511 (1975).

2. J. C. Jacmart, P. Colombani, H. Dotubre, N. Frascaria, N. Poffé, M. Riou, J. C. Roymette and C. Stephan, Ntucl. Phys. A242, 175 (1975).

3. F. Hannappe, M. Lofort, C. Ngo, J. Peter, and B. Tamain, Phys, Rev, Lett. $\underline{32}, 738$ (1974).

4. K, L. Holf, J. P. Fink, J. R. Hulzenga, J. Birkelumd, H. Freiesleben and V. E. Viola, Phys. Rev. Lett. 33, 1105 (1974).

5. P. Russo, R. Jared, R. Babinet, and L. G. Moretto, Energy Calibrations of Solid State Counters for Heavy Ion Experiments, in this Annual Report.

6. W. D. Nyers, LBL Report LEL-3428,

7. I. Dostrovsky, z. Fraenkel, and G. Friedlander, Phys, Rev. 116, 683 (1959).

\section{EVIDENCE OF INCOMPLETE RELAXATION IN THE REACTION $107,109 \mathrm{Ag}={ }^{40} \mathrm{AT}$ AT 288 AND $340 \mathrm{MQV}$ BOMBARDING ENEAGIES"}

J Gátro.t L G Moretto,t R Babinets

F Jared, and $\$ 9$ Thompan

$107,109_{\mathrm{Ag}}+4 \mathrm{Ar}_{\mathrm{Ar}}$ at 288 and $340 \mathrm{kkV}$ hase been studied. Using a $\Delta E-E$ telescope, 1 it was possible to identify particles with atofuic mumers as high as $Z=30$. Kinetic energy spectra for these fragments have been studied over a braad range of laboratory angles. Two distinct components are visible in these distributions: one is at nearly elastic energies, the "quasi-elastic" component; the other is near the expected Coulomb energy, the "relaxod" component. The quasi-elastic component is confined to a narrow angular region around the critical angle and is restricted to $2^{\prime} s$ close to that of the projectile. The relaxed component is present for all atonic mobers and at all angles.

In the center-of-mass systen, the kinetic enexgy distributions are Gaussian in appearance. For fixed $\mathrm{Z}$, the first and second moments are fairly constant over a wide angular range. The mean energies are close to that of two touching spherical fragments. The FWM are approxinately $25 \mathrm{kbV}$ at the lower bombarding energy and about $30 \mathrm{MeV}$ at the higher energy.

The laboratory yields of the relaxed component are given tn Fig. 1. The cross section terds to in- 


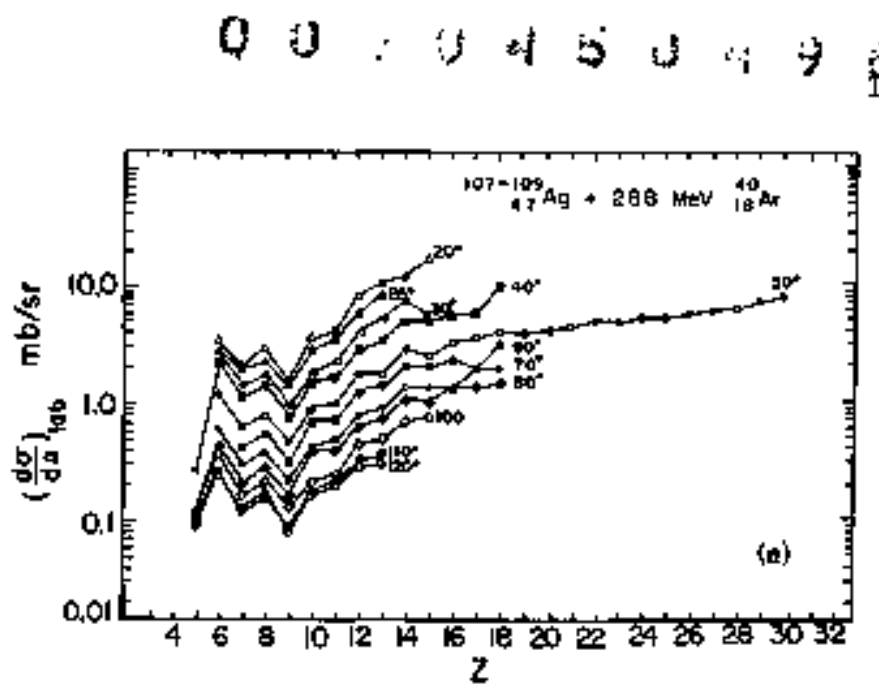

Fig. 1. Laboratory differential cross sections for various 2 's and for various angles at both bonbarding entrgies.

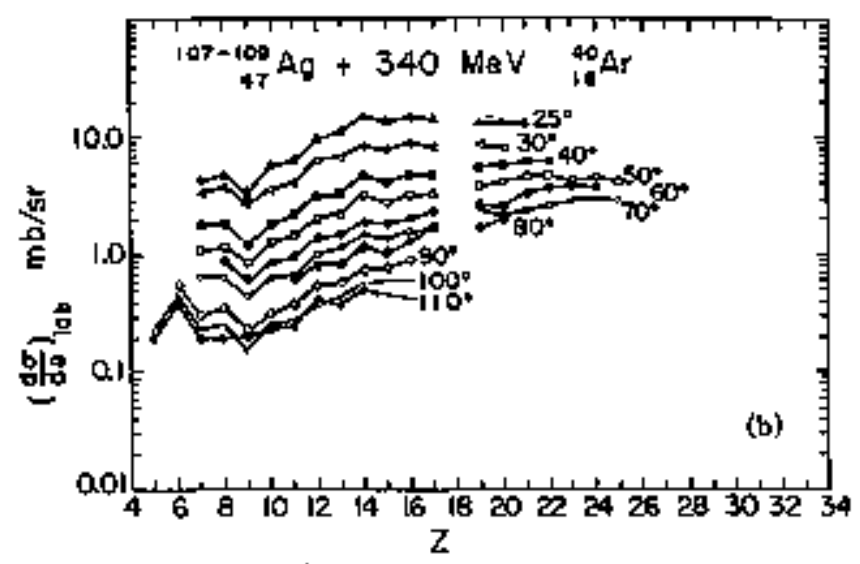

(a) XBL 7411-8216-A, (b) XBL 752-2301

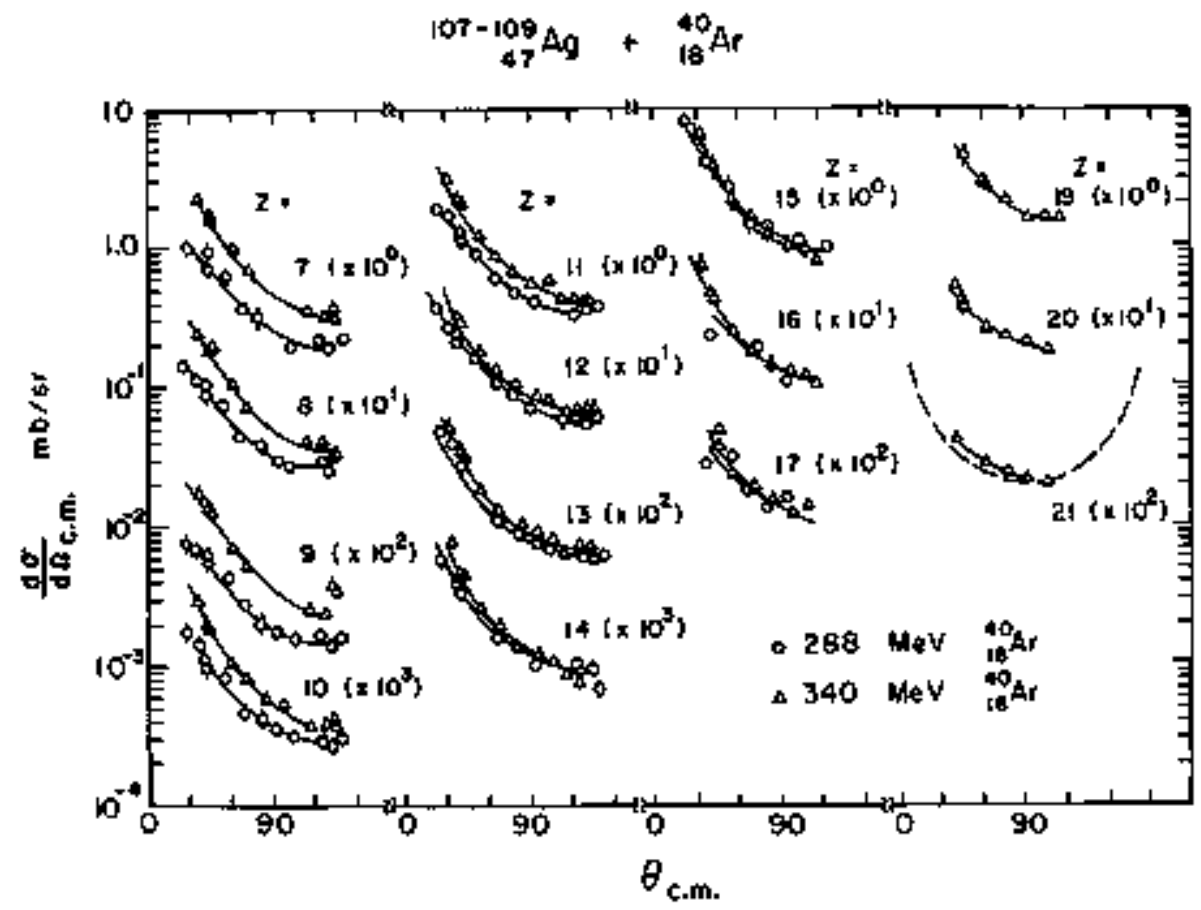

Fid. 2. Center-of-mass angular distributions for various charges at both bonbarding energies. The dashed curve at $Z=21$ corresponds to a $1 / s i n g$ distribution.

(XAL 752-2302)

crease with $z$ for $z>9$. The increase is somewhat more prontunced at the lower energy.

The center-of-mass angular distritutions (Fig. 2) are all forward peaked in excess of $1 / \sin \theta$, the distribution expected fron the decay of a rapidly rotating compound nuclelts. The forward peaking has been interpreted in terms of a short-lived intenmediate cooplex, consisting of to touching fragnents. 2,3 The exchange of charge has been described as a diffusion process along the charge asymetry coordinate of this complex. The predicted charge and angular distributions are in very good agresment with the experimental results.

\section{Footnotess and References}

"Comdensed from LBL-4064 and hucl. Phys. A25S 472 (1975).

${ }^{\dagger}$ Present address: Institut de Physique NuclEaire, Orsay, France.

"Sloan Fellow 1974-1976.

Freșent addres5: DPh/MF-CEN, Saclay, France.

1. H, M. Fowler, R. C. Jared, Mucl, Inst, Meth. $124,341(1975)$.

2. L. G. Woretto, R, P, Babinet, J. Galin and

S. G. Thompso1, Phys. Lett. 58B, 31 (1975).

3. L. G, Moretto and J. S. Sventek, Phys. Lett. $58 \mathrm{~B}, 26(1975)$. 


\section{POTENTIAL ENERGY EFFECTS AND DIFFUSION IN THE RELAXED COMPONENTS OF THE REACTION ${ }^{197}$ AU $+{ }^{40}$ AT AT 288 AND 340 MAV BOMBARDING ENERGIES"}

L G Morelto,t J Galun,t R Bebanets Z Fraenikel,

$A$ Schmilt, $A$ lared, and $S G$ Thompson

The fractints produced in the reaction $197 \mathrm{Au}+40_{\mathrm{Ar}}$ have been studied at 288 and $340 \mathrm{MeV}$ bombarding energies. It was possible to resolve individual atoalic numbers up to $2=32$ wtth a $\triangle E-E$ telescope, $\mathbf{1}$ Kinetic energy spectra and laboratory cross sections obtained over a bread range of angles. The energy spectra of reaction products are similar to thoso obtained with $N$ and Ne projectiles, 2,3 for Z's close to that of the projectile and for angles close to grazing,

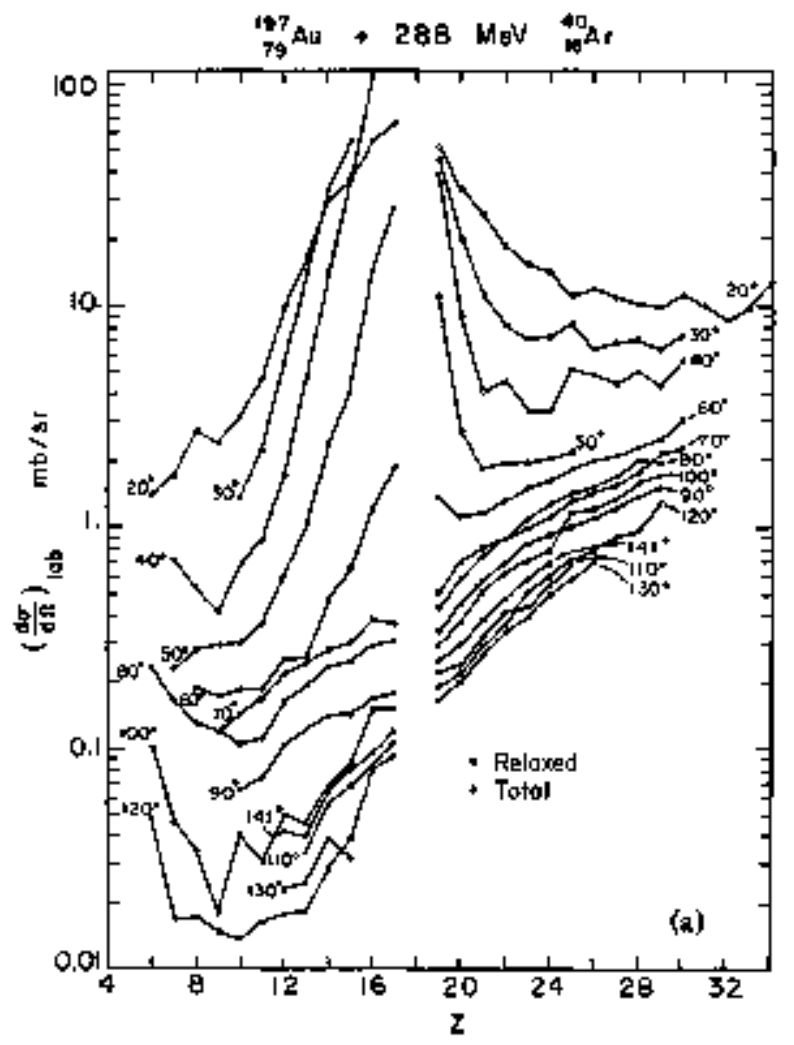

a high energy or "quasi-elastic" corponent is present. In addition, a "relaxed" couponent is visible at energies cIose to the expected Coulconb energy for two touching spherical framents. The relaxod component is present for all 2'5 and presists through the angular range of the measurements. For angles near the grazing and $2<18$, the two conponents are not readly distinguishable, and the laboratory charge distributions (Fig. l) peak near the projectile. For more backward angles the yields increase with $z$ above $z=9$.

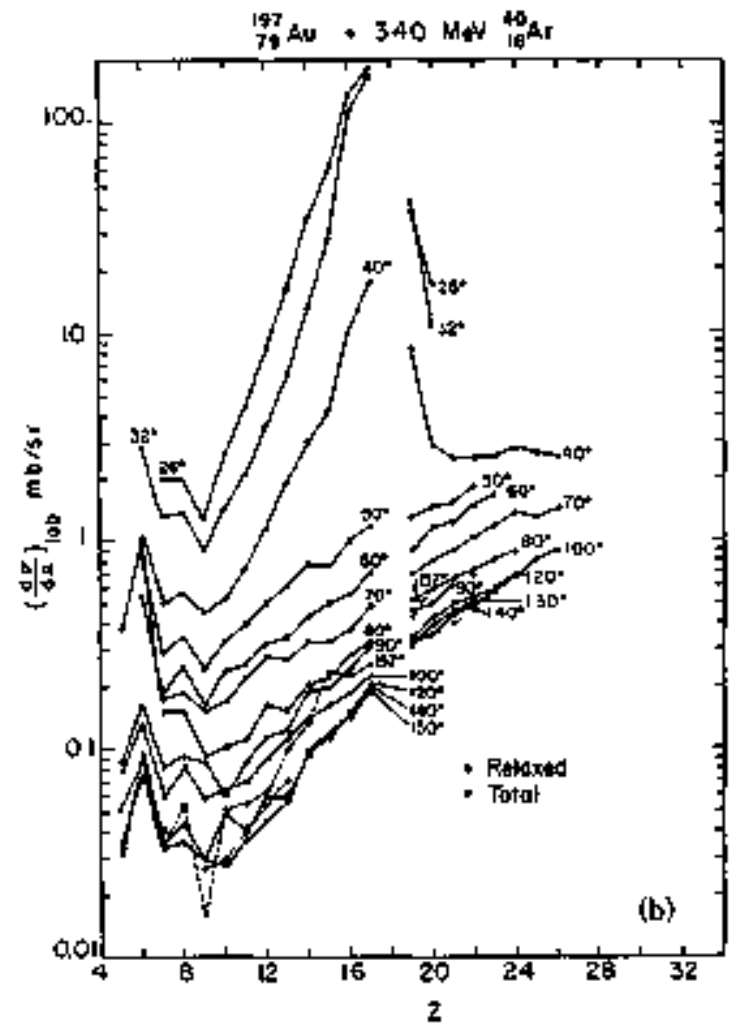

Fis. 1. Laboratory differential cross sections [do/da / lap) as a function of atomic number for various tab angles. Filled points indicete that the cross sections were obtained by integrating a relaxed kinetic energy distribution. Open points indicate either the presence of quasi-elastic conponent or that the kinetic energy distribution was umusually borad. Nottce how the peak around $Z=18$ disappears at backward angles, a (Xit 752-2305),

b (XBL 752-2306)

The center-of-mass angular distributions are depleted in Fig. 2. Afl the distributions are forward peeked in excess of $1 / \sin \theta$ supporting the notion that the Intermediate conplex formed in the reaction is short-lived.4,S 5uch forward peaking has been observed in other heavy ion sys- tems like $\mathrm{Ag}+\mathrm{N}$, Ne and $\mathrm{Ar}, 2,3,6$ Intike distributions obtained for these lighter systens, where the forward peaking yielded to 1/sing fout or five atomic number units above the projectile, the forward peaking persists for atcric rumbers as large as $Z=29$, 11 atcintic numbers above tho pro- 

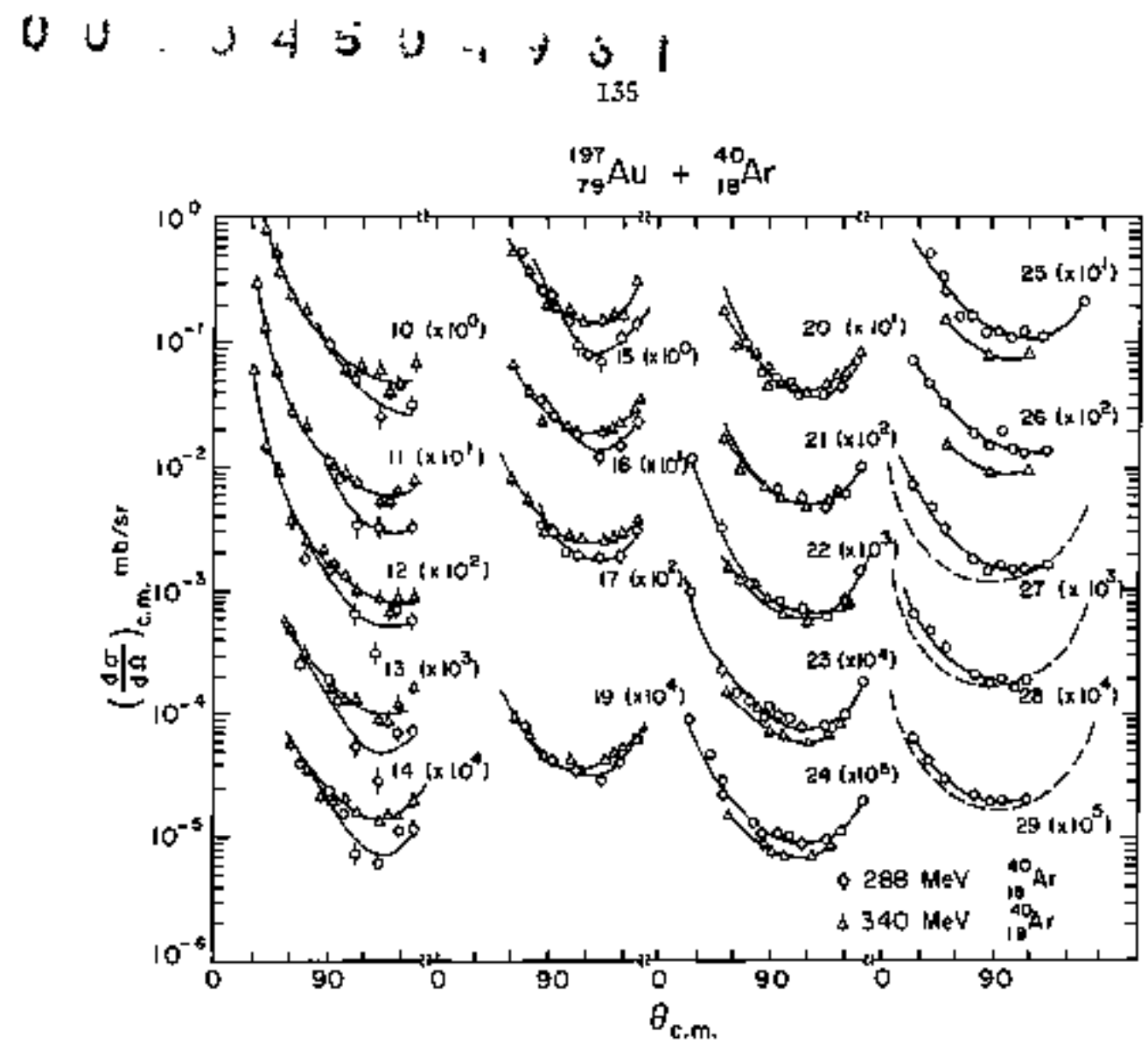

Fig. 2. Center-of-mass angular distributions for various atonic mutors. The quast-elastic contributions have beenl subtracted when present as a distinct peak. The dashed curves plotted for $z=27$, 28 and 29 correspond to $1 / \sin \theta$ distributions.

(XBL 752-2307A)

jectile. This "anomalous" behavior has been interpreted in terns of an entanced diffusion towards syumetry, pronoted by the potential entrgy which drives the intemediate comblex consisting of tor touching fraguents towart more symbetric configurations.

\section{Footnotes and References}

"Condensed from LBL-4984 and Mxel. Phys, A259, 173 (1976).

†Sloan Fellow 1974-1976.

${ }^{\dagger}$ Present address: Inștitut de Physique Mucleaire, Grsay, France.

"Present address; DPh/MF-CEN, Sarlay, Françe. Hresent address: weigan Institute, Israel.
1. M. M, Fowler and R. C. Jared, Nuc1. Inst. Meth. 124,341 (1975).

2. L. G. Moretto, S. K. Kataria, R. C. Jered, R. Schonitt and 5. G. Thompsan, Nacl. Phys. A255, 491 (1975).

3. R. Babinet, L. G. Wretto, J. Galin, R. Jared, 1. Moutton and S. G. Thoupsar, Kuel, Phys. A258, $172(1976)$.

4. L+ G. Moretto, R. P. Babinet, J, Galin, and \$. G. Throupson, Phys. Lett. 58B, 31 (1975).

5. L. G. Moretto and J. S. Sweqntek, Mrys. Lett. 58B, $26(1975)$.

6. J. Galin, L. G. Woretto, R. Babinet, R, Schnitt, R. Jared and S. G. Thoripson, Hell. Phys. A255, 472 (1975). 


\section{NUCLEAF RELAXATION PHENOMENA AND DIFFUSION IN THE REACTION ${ }^{107},{ }^{109} \mathrm{Ag}+{ }^{64} \mathrm{Kr}$ AT $7.2 \mathrm{MEV} / \mathrm{NUCLEON}$}

R P Schmtu, P Fuseo, F, Babmet '

$R$ Jared, and $L$ G Mọrelltot

Early regction studies with heavy projectiles and large $A$ targets like $B i ~+~ K r$ have rewealed the existence of what was considered a new mechanisth, 'quasi-fission." 1,2 The products of the quasi-fission process exhibit the following characteristics: fission-1ike kinetic energies, narrow mass distrifutions peaked near the projectile nass and sidepeaked angular distributions. Since only very massive systems have been studied, it is worthwile investigating the behavior of a systen obtained with the same large projectiles but with a lighter target.

Fragments proctuced in the reaction Ag and a $7.2 \mathrm{keV} /$ mucleon $\mathrm{Kr}$ beam were detected with $\mathrm{E}-\Delta \mathrm{E}$ telescopes. 3 it was possible to resolve individuat changes lo to $Z$ a 50 . The ktnetic energy distributions of the proditets are very similer to those obtained with $\mathrm{N}, \mathrm{Ne}$ and Ar.4-7 Near the grazing angle quisi-elastic and relaxed conponents are present as two distinct peaks for $Z^{\prime}$ 's close to 36 . Far forwart of the critical angle, the two corponents overlap. At more backward angles only a relaxed fission-1ike component is visible. The centrods of the relaxel component are somowhat

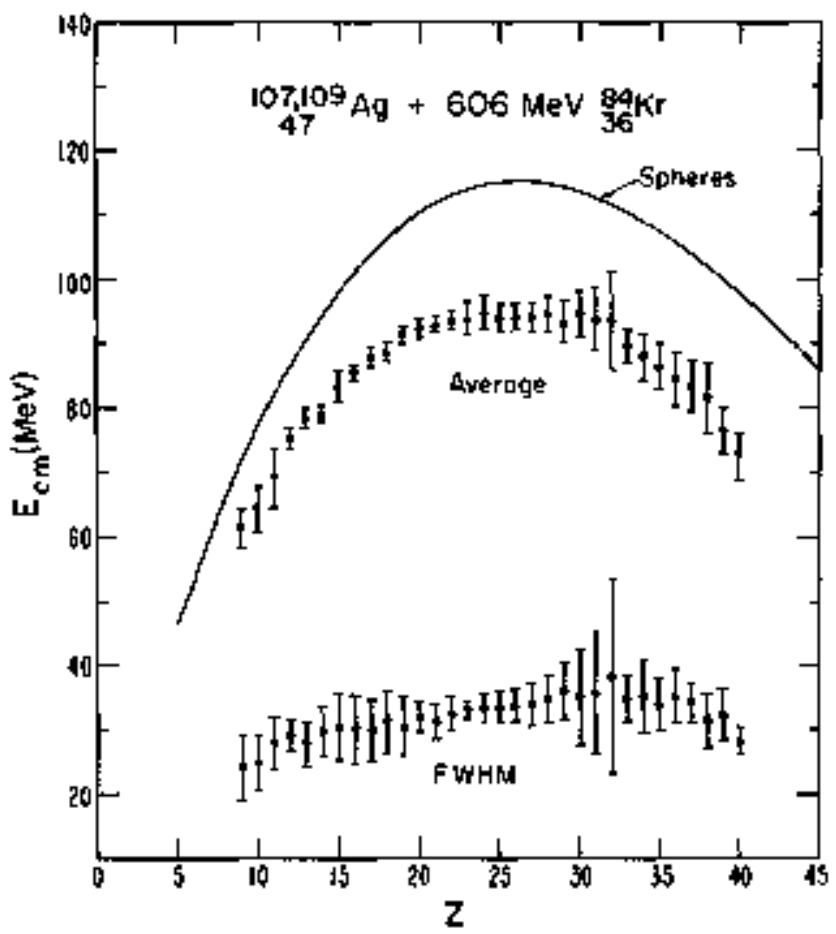

Fig. 1. kverage mean center-of-hass kinetic energies and FuM for the relaxed components. The averages have been made over the range 24-7t6 in the $1 \mathrm{ab}$. Error bars are the standard deviations from the mean.

(XBL 763-2479) lower than the coulonb energy of touching spheres (Fir. 1).

For backward angles the laboratory cherge distributions (Fig. 2) appear to rise steadily towards symetry, unlike those obtained with massive targets but similar to those obtained in Ar induced reactions. For angles inside the grazing angle, it is difficult to separate the relaxed and quasi-elastic components. As a result sowe peaking around the projectile is visible in the charge distribution for $24^{\circ}$.

The center-of-mass angular distributions are given in Fig. 3. The side pesking typical of heavier systems is conspicuously absent. Instead, the distributions are forward peaked in excess of $1 / \sin$ near the projectile, tending toward $1 / \sin \theta$ as one moves away from $z=\$ 6$.

The patterns of the charge and angular distri butions are not substantially different from those observed in Ar induced reactions and can be interpreted in terns of the diffusion model originally introduced to explain results observed with lighter projectiles. \&, 9

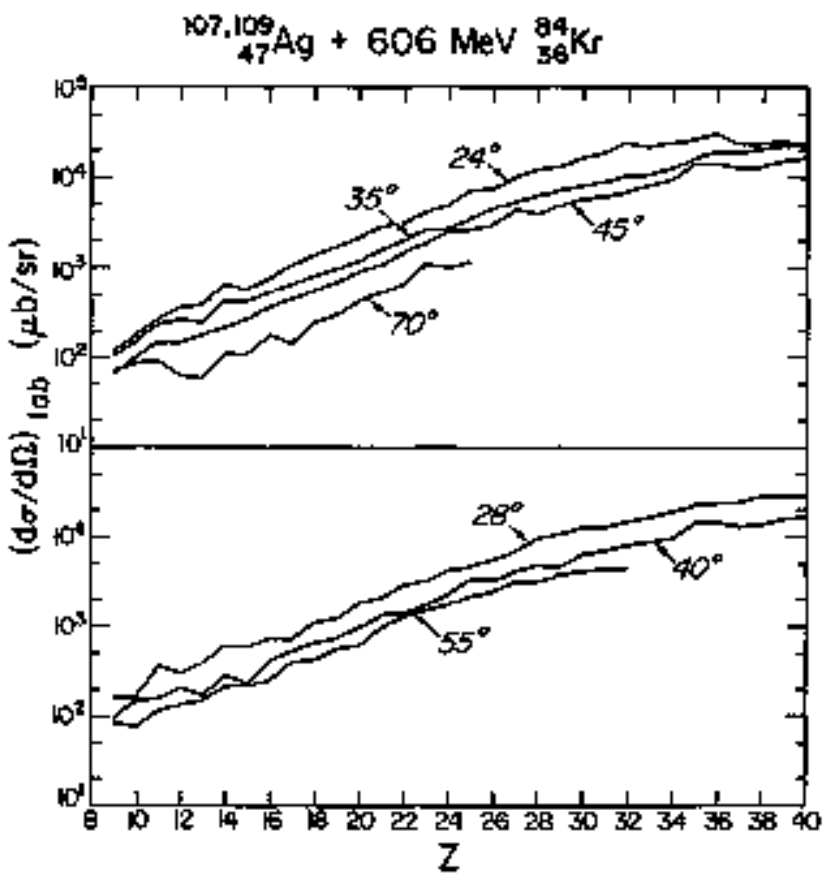

Fir. 2. Laboratory charge distributions at various $1 \mathrm{ab}$ angtes.
(XBL 763-2501) 


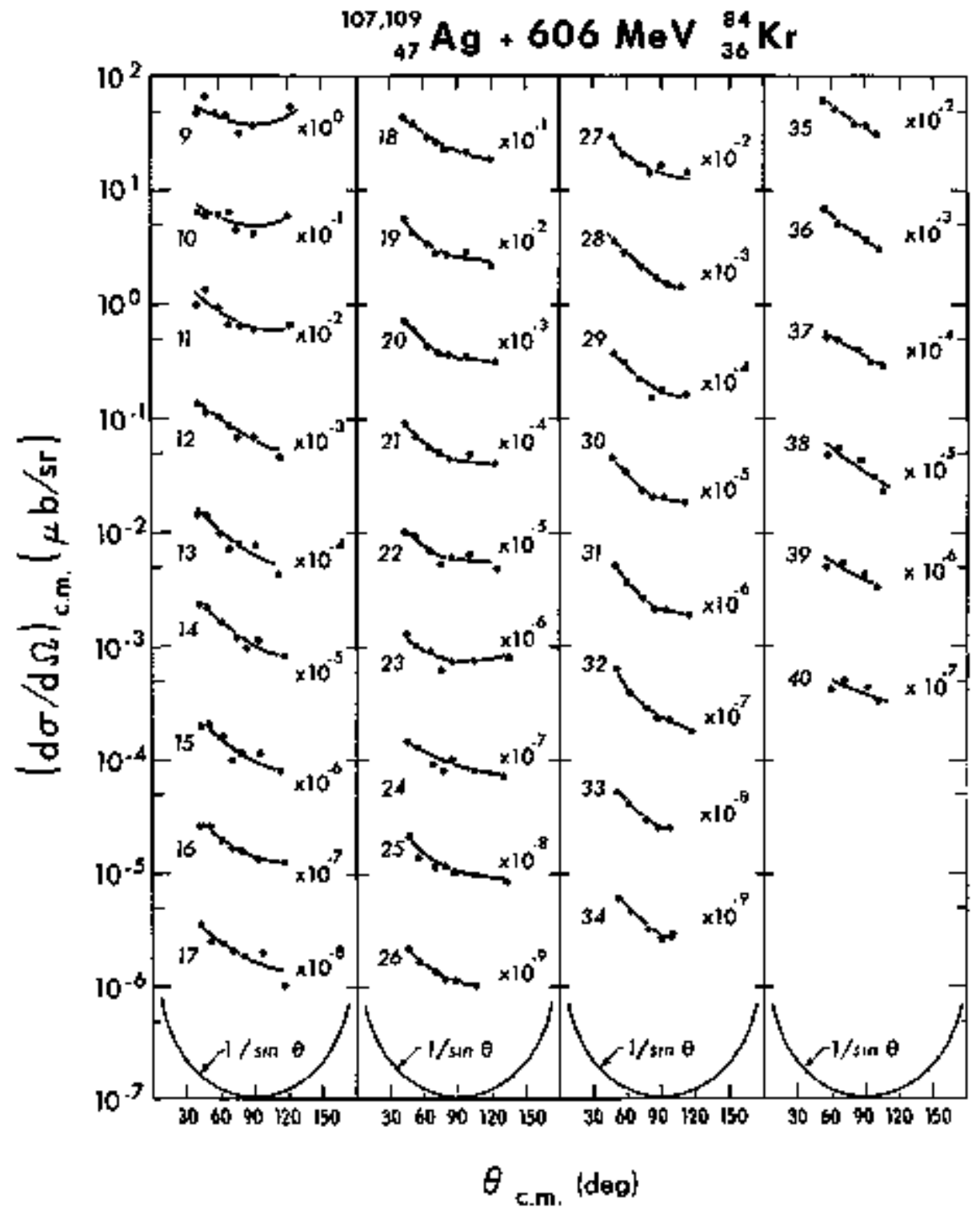

Fig. 3. Center-of-mass angular distributions for various atcitic rumbers. [XBL 763-2478]

\section{Foptontes and Refermices}

*Present address: DPb/MF-CEN, Saclay, France

† Sloan Fellow 1974-1976.

1. F. Hanappe, $\boldsymbol{H}$. Lefort, C. Ngo, J. Peter and

B. Tamain, Phys. Rev. Latt. 32,738 (1974).

2. K. L. Wo1f, J. P. Unzk, J. P. Haizenga, J. Birkelund, H. Freiesleben and W. E. Viola, Phys. Rev. Lett, 33, 1105 (1974).

3. M. M. Fowler and R. C. Jared Mucl. Inst, Method. 124,341 (1975).

4. L. G. Horetto, S. K. Kataria, R. C. Jared, R. Schritt and S. G. Thompson, Nure1. Phys, A255, 491 (1975).
5. R. Babinet, L. G. Moretto, J. Jalin, R. Jared, J. Koulton and S. G. Thongson, Nuc1. Phys. A258, 172 (1976).

6. J. Galin, L. G. Noretto, R, Rabinet, R. Sctmitt, R. Jared and S, G. Thompson, Mucl. Phys. A255, 472 (1975).

7. L. G. Mretto, J. Galin, R. Babinet, Z, Fraenkel, R. Setwitt, R. Jared and S, E. Thompson, Nuc1. Inys. A259, 173 (1976).

8. L. G. Moretto, R. P. Babinet, J. Galin and S. G. Thompson, Phys. Lett. 58B, 31 (1975).

9. L. G. Moretto and J. S. Sventek, Phys. Lett. 58B, 26 (1975).

10. L. G. Moretto, B. Canvin, P. Glassel, R. Jared, P. Russo, J. Sventek, and G. Wozniak, Phys. Rev. Lett. 36, 1069 (1976). 


\title{
CHARGED FRAGMENTS PRODUCED IN THE INTERACTION OF ${ }^{156} \mathrm{~T}$ D AND ${ }^{86} \mathrm{Kr}$ AT 620 MEV BOMBAFDING ENERGY
}

\author{
G J Wotnıak. P Glasssęl, R Schmit, P Russo,
} $B$ Caumn, $P$ Jared, and $L$ G Moretlo

Early studies 1,2 of $\mathrm{Kr}$ induced resetions yielded results apparently very different from those observed in heavy systems involving lighter projectiles and prompted the clain that a new mechanisn, "quasi-fissiom", was coming into play, In the system $\mathrm{Kr}+\mathrm{Bi}$ the mass distributions were sharply peaked about the projectile and the angular distributions for the fission-lłke products were side peaked. Detailed systematics of several $\mathrm{Kr}$ induced reactions tend to discount the claim for a new mechaniss (see this report). While peaked charge and angular distributions were observed with heavy targets like $\mathrm{Au}$ and $\mathrm{Ta}$, the system $\mathrm{Kr}+\mathrm{Ag}$ revealed characteristics reminiscent of $14 \mathrm{~N}, 20_{\mathrm{Ne}}$ and $40_{\mathrm{AT}}$ induced reactions. In an attempt to locate the tronsition region betwen the $197 \mathrm{Au}$ and $10 \%, 109_{\mathrm{Ag}}$ targets we imestigated the behavior of the $\mathrm{Kr}+15 \mathrm{~Tb}$ syston.

Fragments produred in the reaction $159 \mathrm{~Tb}$ $+620-\mathrm{MeW}_{\mathrm{K}} \mathrm{r}$ were identified with an E-bE telescope, 3 Utilizing this technique, it was possible to identify fragnents with $Z^{\dagger} s$ up to a maximus of 50. The fragment kinetic energy distributions contained the two components camon to surf heavy ion systems: quasi-elastic and relaxed.
The distributions of atomic mabers (z) for the relaxed component for several lab angles are showen in Fig. 1. Comparing these $z$ distributions to those obtained in studies with $197 \mathrm{AN}, 181_{\mathrm{T}}$ and $107,109 \mathrm{Ag}$ targets, one observes that the widths are intermediate to those of the $\mathrm{Kr}+181 \mathrm{Ta}$ and $\mathrm{Kr}+\mathrm{Ag}$ systems. Prelininary theoretical calculations indicate that this behavior pattern is consistent with the assumption of a diffusive nechanisa with respect to the charge asymmetry degree of freedon. Fotential energy surfaces for the $\mathrm{Kr}$ systens are given in Fig. ?.

Angular distributions for this systern (Fig. 3) are also intermediate to those obtained with light and heavy targets. The stromig focusing effects observed with $\mathrm{T}$ a and Au have becone quite weak for Tb and are confined to 2 's very near the projectile, For $2^{\dagger} s$ further from the projectile the angular distributions are more strongly forwarded peaked than in the case of $\mathrm{Ag}+\mathbb{K r}$. This implies that the ratio of the lifetime of the system to the rotational period is sma1ler than that of the $\mathrm{Ag}+\mathrm{Kr}$ system. As a result, on the average the intermediate complex undergoes less rotation and yields more forwand peaked distribution for

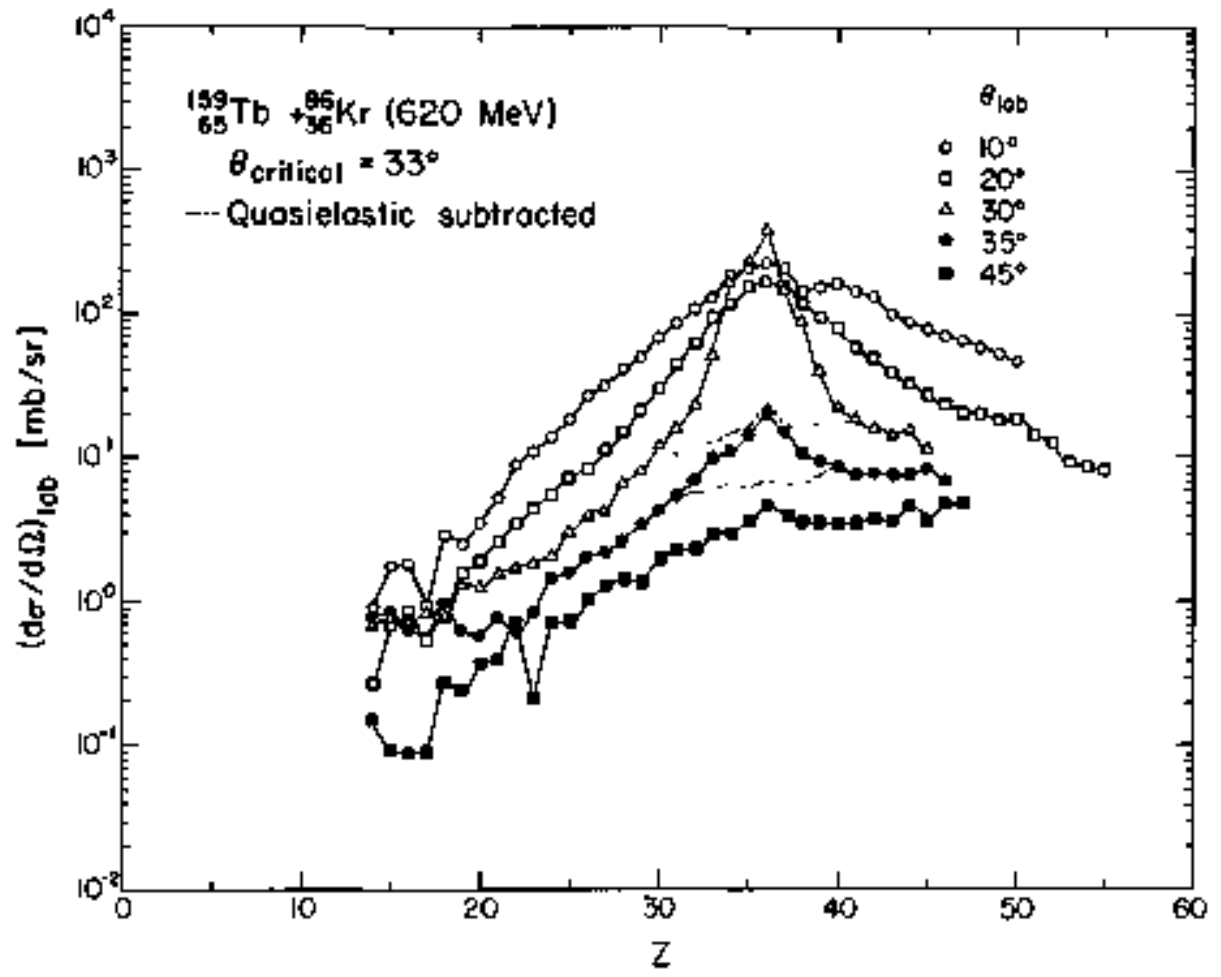

Fig. 1. Laboratory yields of the various fragnents for several angles. Near the grazing the quasielastic and relaxed components are we11 separated in energy. The dashed curves for $30^{\circ}$ and $35^{\circ}$ represent only the relaxed contribution. Notice that in these cases the peaking around $z=36$ almost completely disappears. 


\section{$00 \div 345+933$}

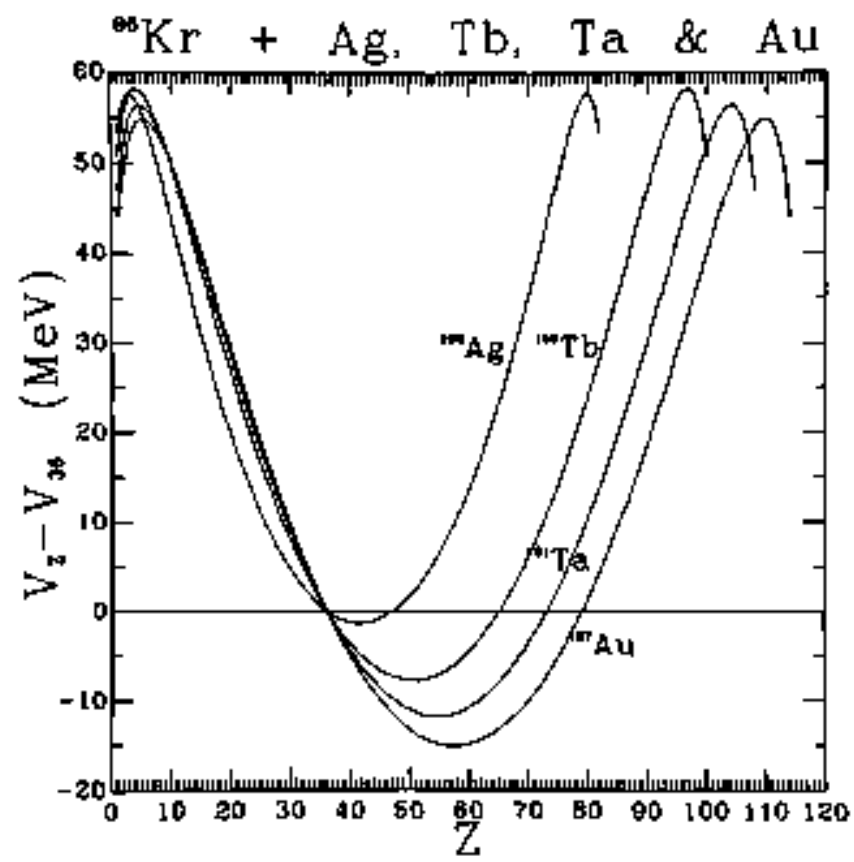

F1g. 2. Potential energy surfaces for an intermediate complex consisting of two touching ppheres relative to the entrance charmel for various $\mathbf{K r}$ systems.

(XBL 766-8174)

$k_{T}+$ Th. Sirce the mall charge transfer intolve even short times, the forward peaking yields to a slight side peaking for ' 2 's near the projectile.

If one assunes that the intermediate complex consists for two touching spherical fragnents fotating rigidly, the rotational period for the r.m.s. angular mpinentum tends to increase in going from $\mathrm{Ag}$ to Au targets. If the lifetine of the complex were constant and the complex is as described above, the gross features of the angular distributions would be reproduced. Long rotational periods would result in side-peaked distributions whils shorter periods would give rise to forward-peaked

distributions. Hower, there is no real evidence to support the notion that the lifetine of the complex is constant. In fact, the narrowed charge distributions obtained for the most massive systejus seen to indicate that the lifetine of the complex is de. creasing. Incomplete energy relakation for the heavier systents supports this hypothesis. A detailed theoretical analysis of such a wide variety of systems should provide us with reliable estimates of the decay properties of the intermodiate complex.

\section{Footnotes and References}

"MaTo Fellow.

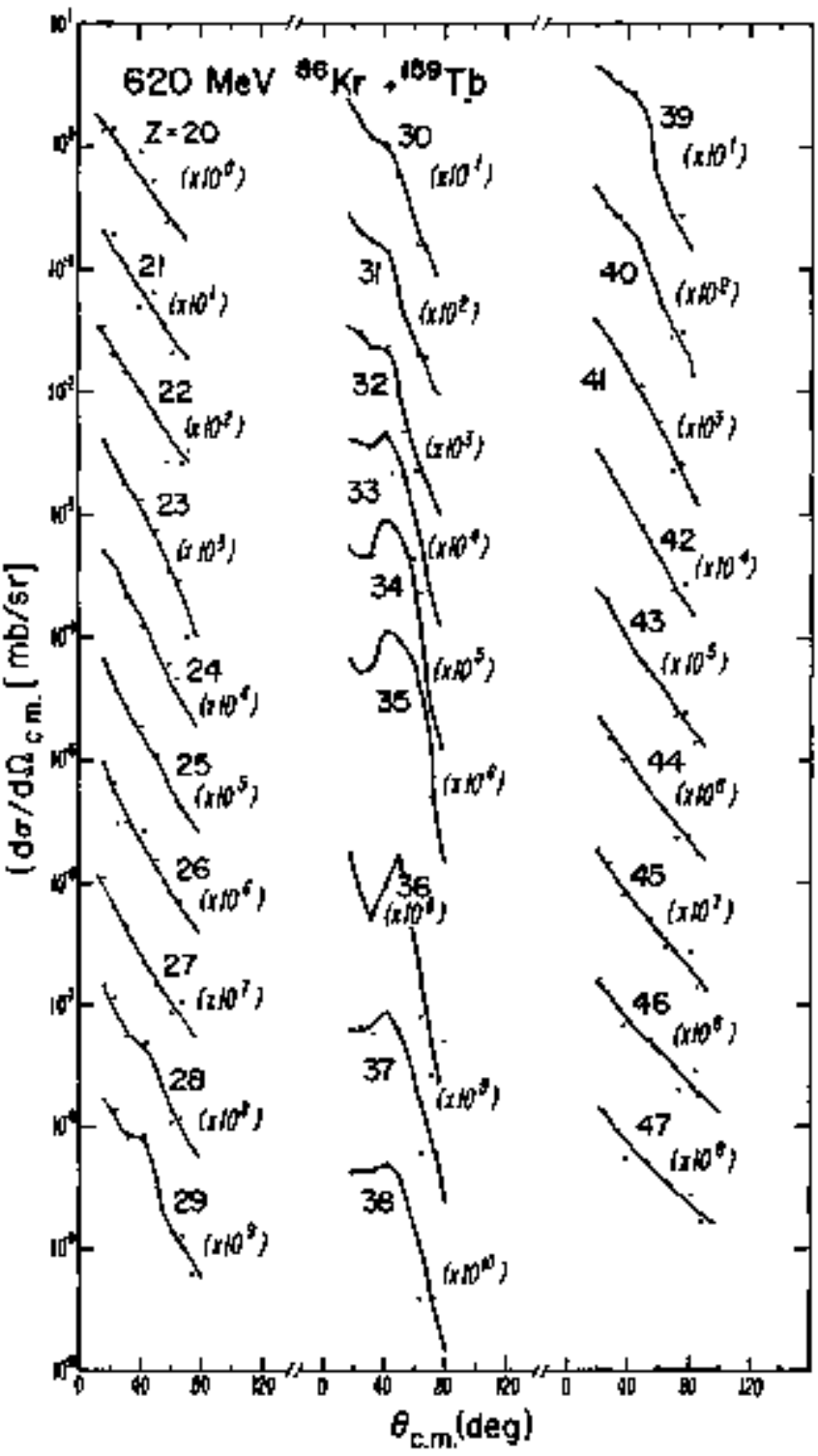

Fíg. 3. Angular distributions for $T b+K r$ for a variety of atomic nubers.

(XERL 766-3008)

$\mathrm{F}_{\mathrm{On}}$ leave from IPMN/MF-CEN, SacIay, France * Slagn Fellow 1974-1976.

1. F. Hanappe, M. Lefort, G. Ng6, J. Peter and

B. Tarain, Phys. Rev. Lett. 32738 (1974).

2. K. L. Wolf, J. P. Unik, J. R. Huizenga, J. Birkelund, H. Freiesleben and V. E. Viala, Phys. Rev. Lett. 33, 1105 (1974).

3. M. M. Fowier and R. C. Jared, Muec1. Instr. Weth. 124, 341 (1975). 
P Glasegl.; B Cauvin,t G Wornak, P Rugso.

R Schmort, $P$ Jarred, and $L$ G Morello

The diffusion model proposid by Moretto and sventekl has proved to be highiy successful when applied to heavy ion reaction involving 14N, 20Ne and $4 \mathrm{DAr}$ projectiles. Recent studies with $\mathrm{Kr}$ projectiles ${ }^{2}$ (i.e. Al $+K r$ ) seem to indicate that the model has a broader range of applicability. To further check the diffusion iodel the systematics of KI induced reactions have been investigated over a broad range of targets.

The experinental technique enployed is that of fragment z-identification. Individual atomic nembers with $Z$ as large as 50 have been identified by reans of $\mathrm{B}-\Delta \mathrm{H}$ telescopes. ${ }^{3}$ The resulting kinetlc energy distributions showed the usual quasi-elastic transfer component at near-elastic onergles and the relaxed conponent at near-Coulomb energies.

In Fig. I the mean c,w, kinetic energies of the relaxed components are plotted vs. 2. For comparison, the Coulank repulsion of two touching spheres is given. In gereral, the trends and magnituxies agree, except near the projectile at the grazing angle were the relaxed and quasi-elastic contribution could not be separated. The widths of the kinetic energy distributions, whth are also shown in

Fig. 1, are rather constant, with the same exception as above.

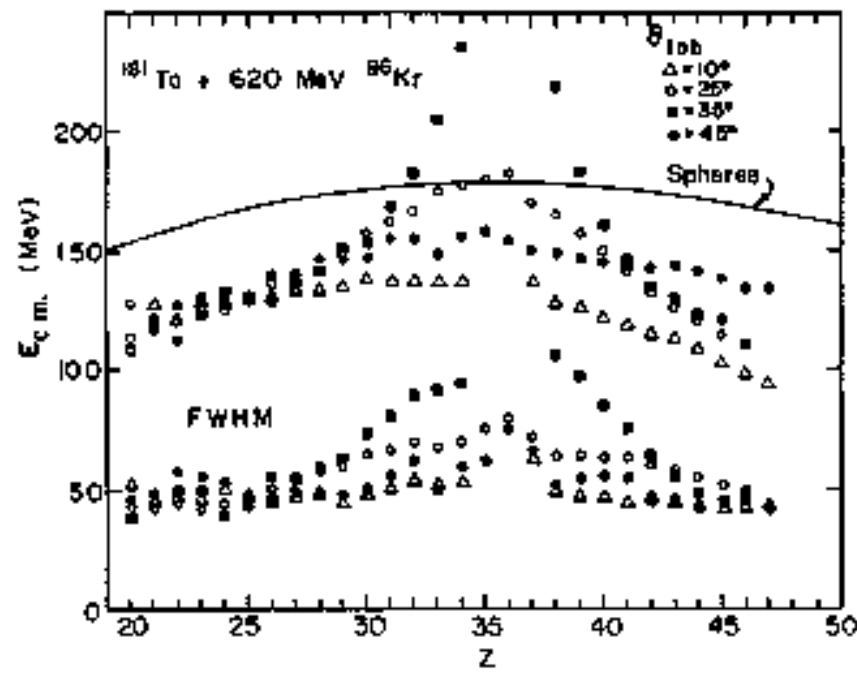

Fig. 1. Mean center-of-mass kinetic energłes and FWM for the relaved component as a fumction of 2 for various lab angles.

(XBL 766-8215)

The 2-distributions in Fig. 2 are very sfidilar to those obtained for Alu $+\mathrm{KI}$. The yield tends to peak near the projectile $Z$ and exhibits the same general behavior with angle as observed with Au ssee Ref. 2 and this report?.

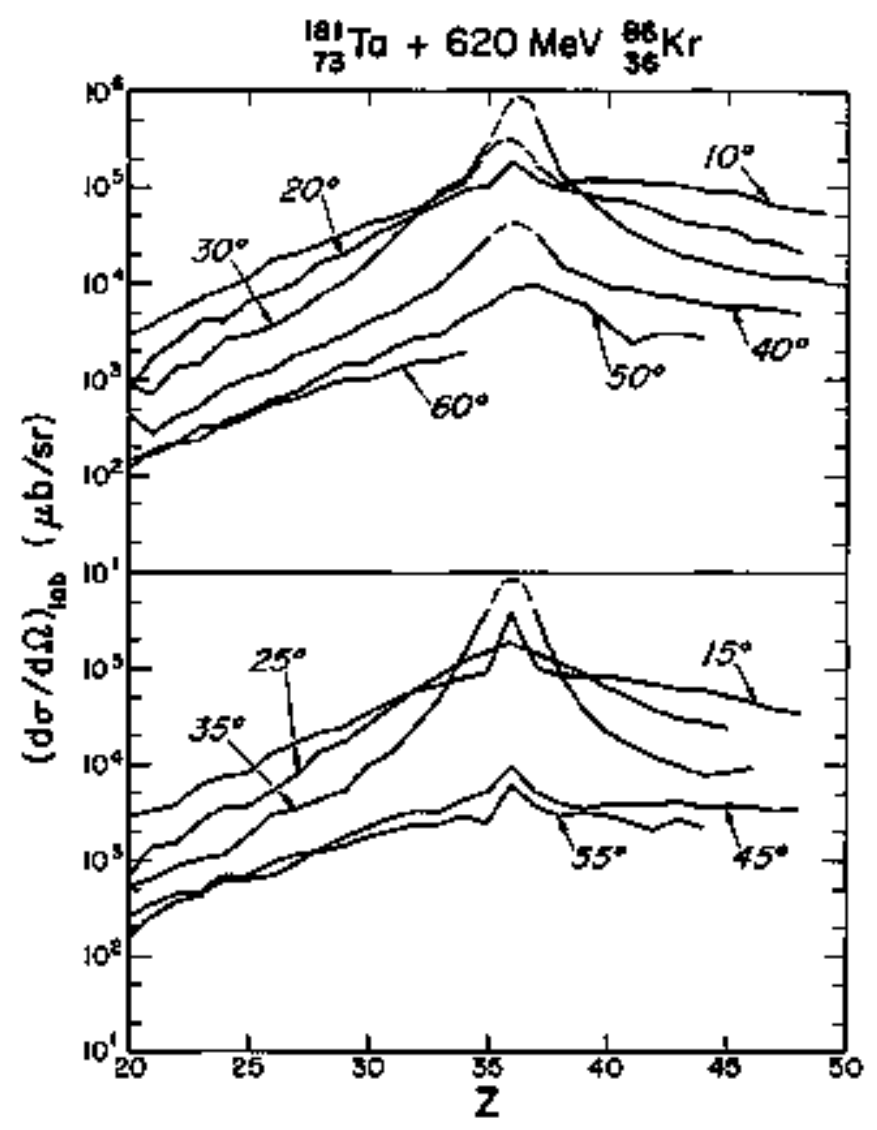

Fig. 2. Relaxed laboratory cross sections as a function of $Z$ for several lab angles. For argles close to the grazing and 2's near the projectile, the deconposition of the relaxed and quasi-elastic copponents is sconenthat umertain as the dashed curves indicate.

(XBL 763-2502)

Center of mass angular distributions for the relaxed components are given in Fig. 3. like $\mathrm{Au}+\mathrm{Kr}$, the distributions show strong side peaking for atomic numbers in the vicinity of the projectlle indicating that the intermediate conplex formed in the reaction decays before it rotates nuch past the grazing angle. This side-peaking gradually decreases for atonic numbers farther removed from the projectile, since it takes roore time for the diffusion process to reach these masses thus allowing the craples to notate farther and eventualty even cross $0^{\circ}$. The strong forward peaking persists for atomic numbers far away from the projectile, indicating that the lifetine of the intemediate conplex is not sufficient for it to rotate more than about half a revolution.

While the system $\mathrm{Ta}+\mathrm{Kr}$ is quite similar to that of Ald + Kr there are sipnificant differences: the charge distributions are not quite as 


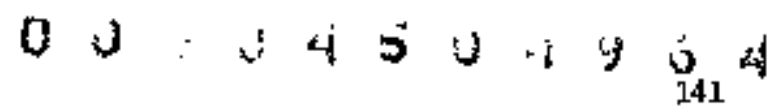

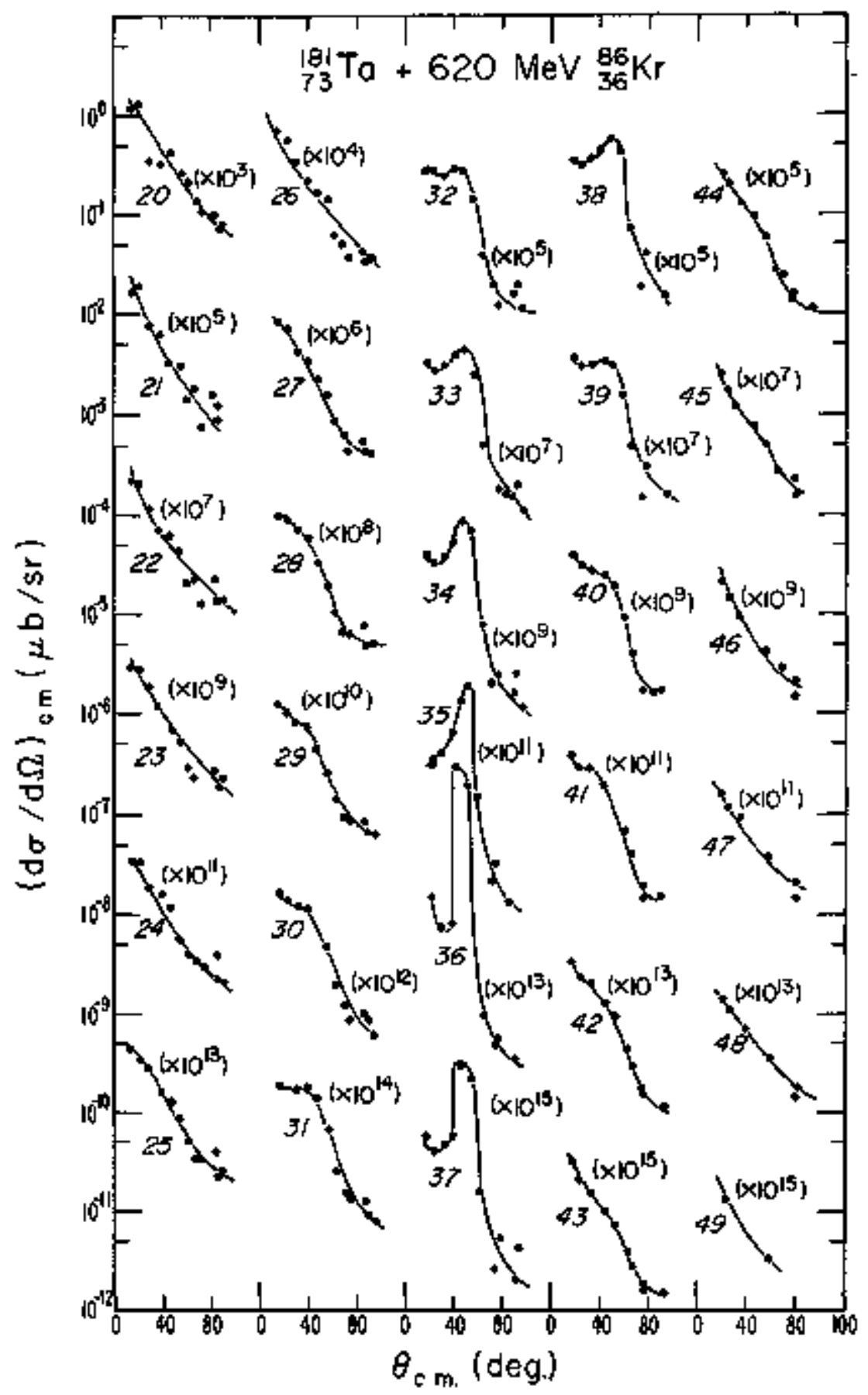

Fig. 3. Angular distributions for the relaxed component. Fhe quasi-elastic and relaxed components are not well separated for $z=36$. (XBL $763-2499$ )

peaked in the case of $\mathrm{Ta}$ and the side-peaking in the angular distributions tends to die out more quickly with $z$ than it does with Au. Prestmably, the differences are due to the difference in the coulomb field (an $8 \%$ effect). Since the sidepeaking seens so semsitive to the entmace chanme1, it is hoped that one will be able to theoretically extract the details of the nuclear transport phemomena inwluded in heavy ion systens.

\section{Footnotes and References}

$\dagger_{\text {On }}$ leave fron DPh/MF-CD, Saclay, France.

tsloan Fellow 1974-1976.

1. L. G. Woretto and J. S. Sventek, Phys. Lett. 58B, $26(197 \$)$.

2. 1. G. Moretto, B. Canvin, P. Glassel, R. Jared, P. Russo, J. Sventek, and G. Hozniak, Phys, Rev. lett. 36,1069 (1976),

3. M. M. Fowler and R. C. Jared, Mucl. Inst, Meth. 124 (1975) 341 . 


\section{DIFFUSIVE PHENOMENA IN THE CHARGE AND ANGULAF DISTRIBUTIONS \\ FOR THE REACTION ${ }^{197} \mathrm{Au}+620 \mathrm{MeV}^{86} \mathrm{Kr}^{*}$}

L G Morelto,t B Cauwn, P Gassel, $\$$ P Jared.

$P$ Fussa, I Swentek, and $G$ Woznsk

Tharged fragnents produced in the reaction $197 \mathrm{Au}+620 \mathrm{MeV} 86_{\mathrm{Kr}}$ have been studied by neans of $\mathrm{AE}-\mathrm{E}$ telescopes, 1 Individual atomic numbers haw been resolved us to $Z=50$. The kinetic energy distributions of the frapments are similar to those obtained with lighter projectiles like $\mathrm{N}$, Ne and Ar. $2,3,4,5$ at near-elastic energies and atomic numbers near that of the projectile, there is a quasi-elastic component. This component is confined to a narrow angular range close to the critical angle. At lower energies there is a relaxed component which persists throughout the angular range of the ressurements. The centroids of the relaxed peaks for the varlous fragments are close to the Coulonb repulsion energy expected for binary division (Fig, 1).

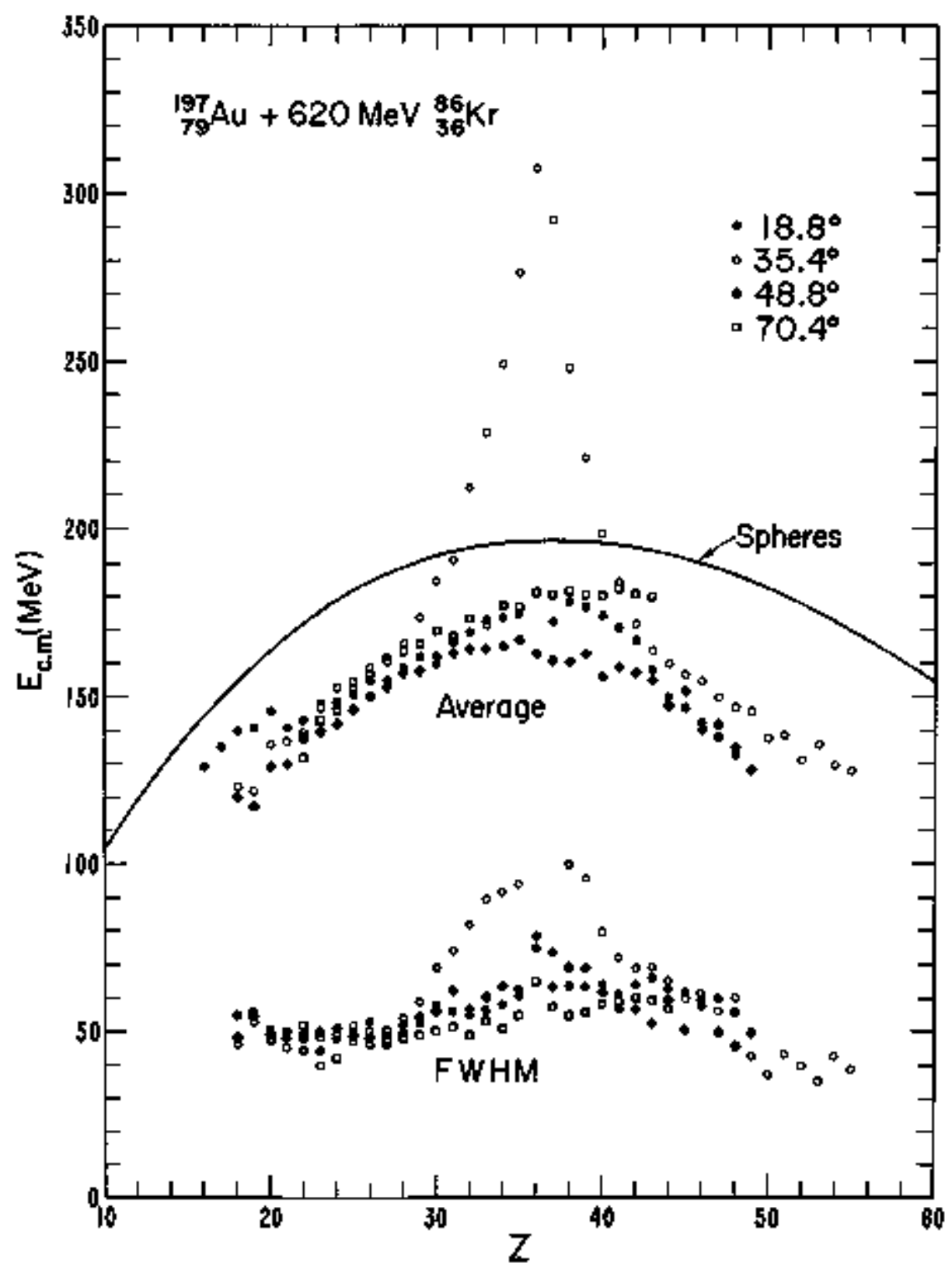

Fig. 1. Kean center-of-mass kinetic energies for the relaxed component. The solid curve is the expected Coulonb energy for touthing spheres. (XBL 7512-9903) 


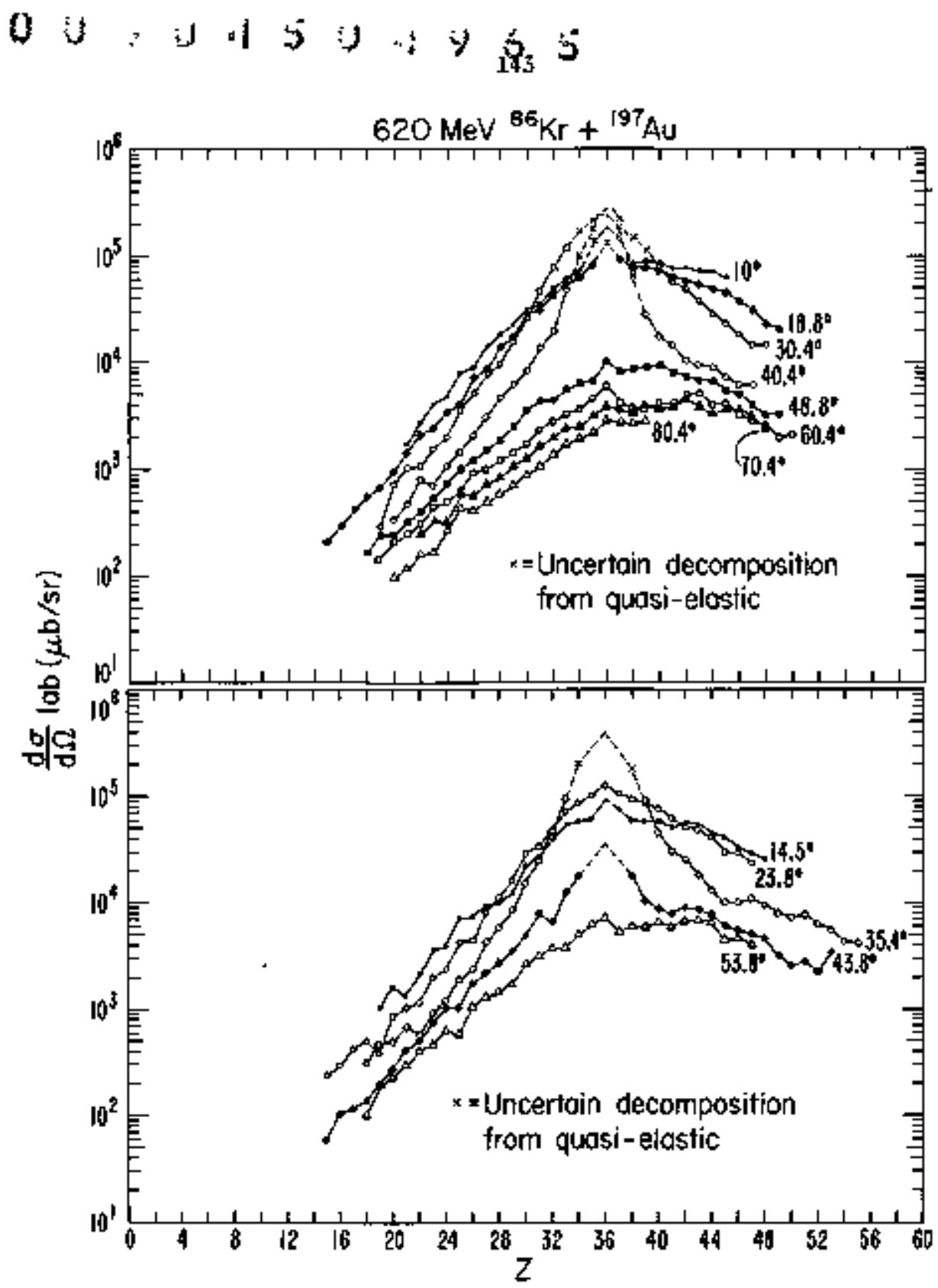

Fig. 2. Laboratory 2 distributions for various $19 \mathrm{~b}$ angles.

(XBi $761-2088$ )

In apparent contrast to reactions tnduxed by lighter projectiles, the charge distributions for the relaxed peak are strongly concentrated about the incident profectlle (Fig. 2). Horeover, the shape of the charge distribution is strongly dependent on the angle of observation: for backward angles the distributions are broad; for very forward angles the distributions are somewhat narrower; finally, at intermediate angles the distributions are sharply peaked. The width of the charge distributions has been interpreted in terms of the age of the intersediate cosplex at the tine of decay. Hazrow or "yourg" distributions result from short decay times, before substantial charge transfer has occurred. Broad or "old" distributions are produced when long decay tines pormit latge antounts of mass to be transferred.

The angular distributions are glso quite different from those obtained with lighter projec- tiles [Fig. 3]. For $z$ close to that of the projectile, the angular distributions aro side peakad rather than forward peaked, This seems to indicate that the lifetime of the composite system is substantially shorter relative to its rotational period, 6,7 As one woves away from the projectile $Z$, the side peaking rolaxes into forward peaking, thus corroborating the notion that longer times are involved in populating these $z^{\dagger} s$.

These experimental data have been interpreted in tarens of the diffision model developed from the study of reactions induced by tighter ions, 1.e. $\mathrm{N}$, Ne and $\mathrm{Ar}$. The successful application of this model ilulies that so-called quasi-fission ohernonena observed in other systems 1 ike $209_{\mathrm{B}}+84_{\mathrm{KT}}$ do not represent a new mechanisn.8,9 It seents likely that the diffusion model is capable of a unified explanation of charge and angular distributions observed in heavy-ion bombardments. 


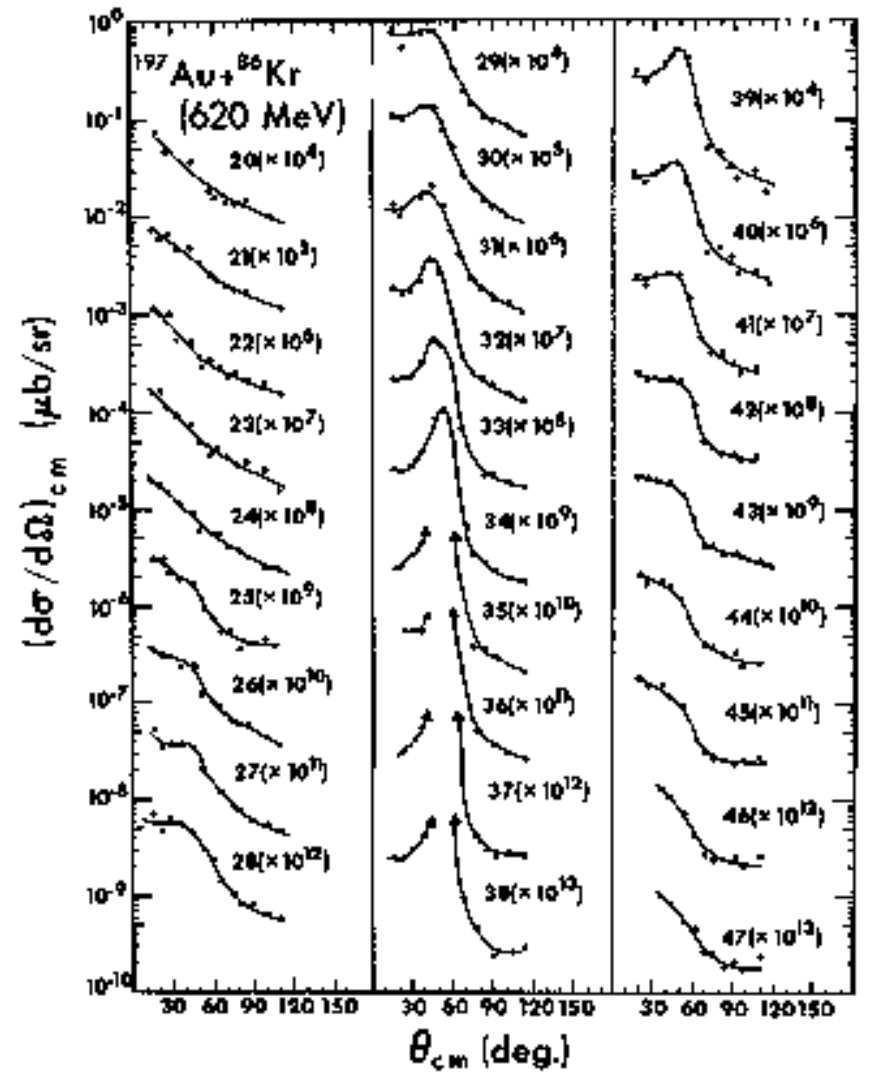

Fig. 3. Center-of-mass angular distributions for various atcmic numbers,

\section{Footnotes and References}

“Condensed fron LBL-4362 and Physs. Rev, Lett. 36, 1069 (1976).

tsloan Fe11ow 1974-1976.

ton leave froth DPhin/MP-CEN, Saclay, France. INATO Fellow.

1. M. M. Fowler and R.C. Jared, klcl, Inst, Neth. 124,341 (1975).

2. L. G. Moretto, 5. K. Kataria, R. C. Jared, R. Schraitt, and \$. G. Thompson, Hacl. Phys, A255, 491 (1975),

3. R. Babinet, L, G. Mretto, J. Galin, R. Jared, J. Wulton and S. G. Thanpson, Nucl. Phys. A258, $172(1976)$.

4. J. Galin, L. G. Moretto, R. Babinet, R. Schnitt, R. Jared and S. G. Thompson, Mux1. Phys. A255, $472(1975)$.

5. L. G. Moretto, J. Galin, R. Babinet, Z. Fraenkel, R. Schnitt, R. Jared, and S. G. Thompson, Mxc1. Phys. A259, 173 (1976).

6. L. G. Noretto, R. F. Babinet, J. Galin, and S. G. Tholpson, Phys, Lett. 58B, 31 (1975).

7. L. G. Moretto and J. Svantek, Phys. Lett. 58B, $26(1975)$.

8. F, Hanappe, M. Lefort, C. Ngo, J. Peter and B. Tan:in, Phys. Rev. Lett. 32, 738 (1974).

9. K, L. Wolf, J, P, Uhik, J, R, Kuizenga, J. Birkelund, H. Frelesleber and V. H. Viola, Phys. Rev. Lett. 33, 1105 (1974).

\title{
EVIDENGE OF DEFORMATION AND INCOMPLETE RELAXATION IN A NEARLY-SYMMETRIC HEAVY SYSTEM: $979-M e V X e+T b$
}

\author{
P Rusşa, o Woznıak, A Schmit, R Jarred. \\ $P$ Giässel:* and L $B$ Horetto
}

Relaxation processes in maclei have been studied through experimental imestigations of in elastic collisfons between heavy targets and projectiles. The use of a gas ionization $A E$ counter 1 with a solid state $\mathrm{E}$ detector emables the identification of elements up to and beyond the projectile $z$ and provides extensive information on the systematic features of these processes fron singles neasurements of the heavy ion products, ${ }^{2}$ Such meastreetents have been performed with projectiles as hoav' as $\mathrm{Kr}$ and have sore recently been extended to inclinde $\mathrm{xe}$ projectiles.

The ability of the telescope to retain $z$-resolution above atomic muber $\mathbf{5 0}$ had not been tested prior to the Xe experiments, A very short exploratory run was performad with the $X e$ beam at lower energies to estimate the $Z$ resolution in the region of atcitic number 54. A further inprovement
In the process of 2 -identification was obtained by neans of a couputerized method for the autorntic statistical identification and subsequent fitting of the Z-1 ines in the E-AE map. These techniques were used exclusively in the analysis of the $X e$ data extending the 2 -resolution to above 60 . The detector performance and $Z$-identification process are described elsewhere in this report. 3

A $979-(49 V$ 136) bean from the super-HILAC bonbaxded self-sppporting natural terbiun foils 0.6 to $0.7 \mathrm{mg} / \mathrm{con}^{2}$ thick. Pileup considerations inited the beam current to 5 to $10 \mathrm{nk}$ at the most forward angles but approxinately $50 \mathrm{~mA}$ were utilized at backward angles, Each of the two scattering chamber anns supported a mount which held two counter telescopes separated by $15^{\circ}$ on one arm and $20^{\circ}$ on the other. The arrangenent was swch that the telescope at the most becknard angle was positioned 


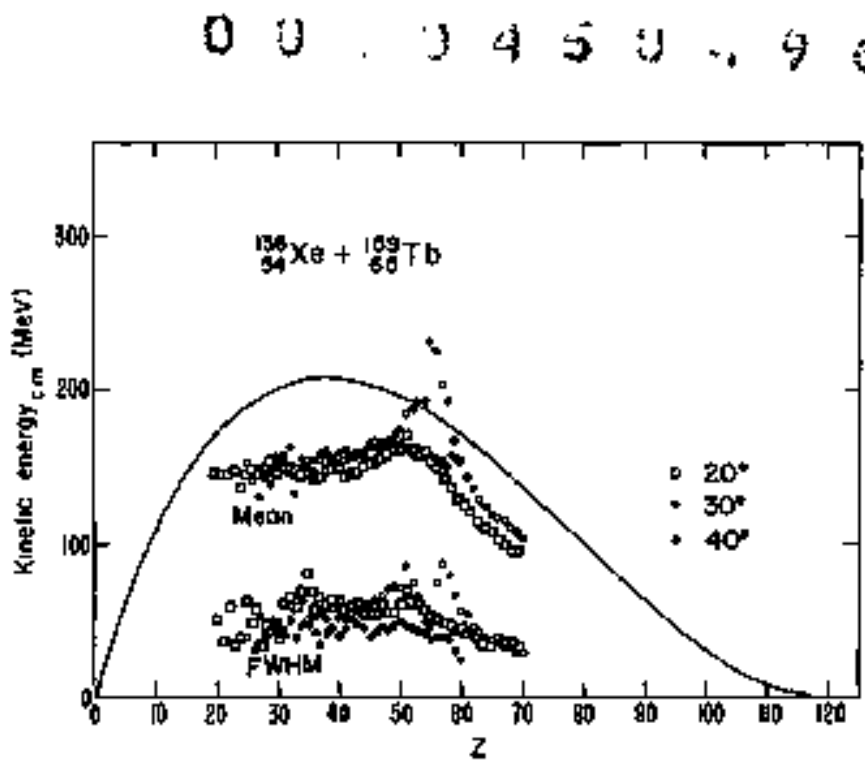

Fig. 1. Average c.m. Kinetic energies and widths of the kinetic energy distributions for elements produced in the $979+\mathrm{eV}$ Xo+Tt expertmont.

(XBC 766-2979)

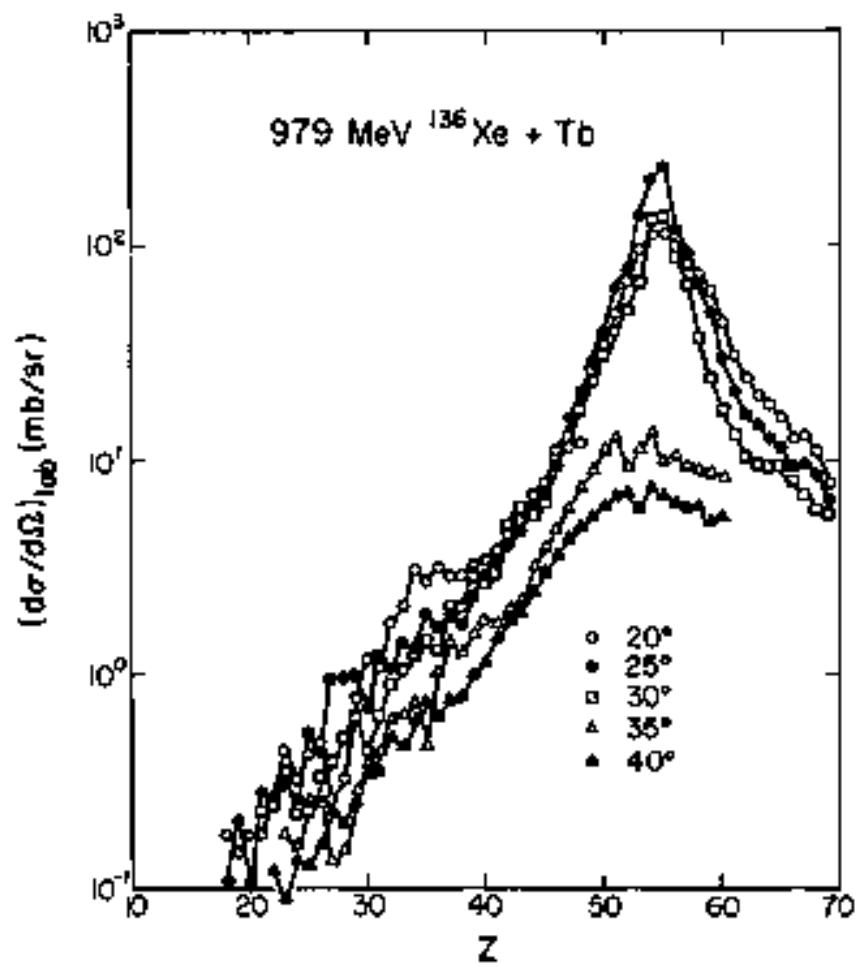

F1g. 2, Laboratory differential cross sections as a function of $z$ for five laboratory angles in the experiment 979-HeV $\mathrm{Xe}+\mathrm{Tb}$. The data includes quasi-elastic events which are inseparable fron deep irelastic events near the grazing angle.

(x) $766-2966$ )

closest to the target to minimize differences in count xates ancing the four colnters. The gas counter entrance windows were $0.28 \mathrm{mg} / \mathrm{cm}^{2}$ polycarbonete (Kimfol) foils at forward angles and $0.040 \mathrm{mg} / \mathrm{cm}^{2}$ Formvar foils at nore backward angles. Gas pressures in the forwart and backward pair of counters were independently regulated.

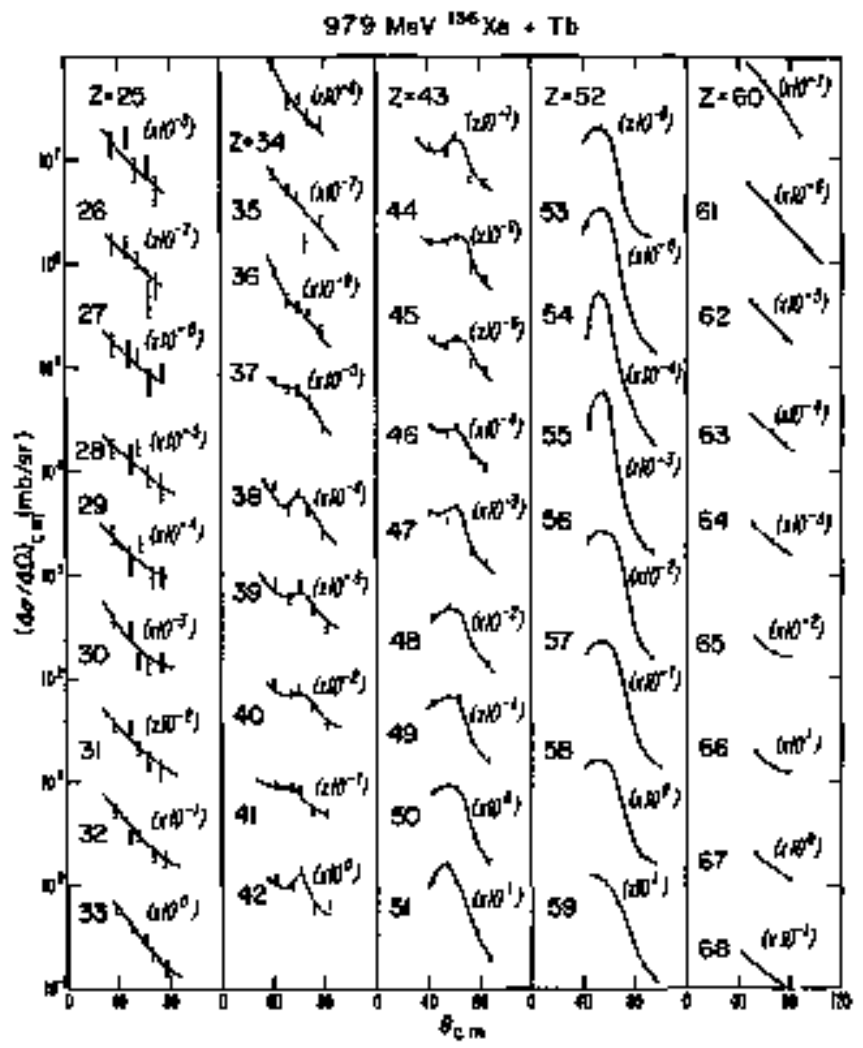

Fig. 3. Angular distributions in the center-of-mass for individuat elenents producer in the $979+\mathrm{HeV}$ Xe+Tb experdment.

(XBL 766-2975)

Periodically during the torbardment of terbium targets with $X_{e}$, a silver target with a thick nickel degrader foil was ingerted to provide callbration points at $Z=28,47$ and 54 . This calibration was most useful backward of the critical angle where the projectile $z$ Iive is weak. The data analysis techniques are described in detail elsewhere. 3

The 1aboratory differential cross sections were deterwined for each element for the deep inelastic processes. The cross sections were transformed to the center of mass assuming charge-equilibrated fragment masses for the intermediate conplex at fixed mass asymetry. A relatively stall correction (1ess then 10 s of the taboratory energy) due to evaporation of neutrons from the fragunents has been neglected in this transformation.

At the grazing angle and for $Z$ 's near the projectile, the widths of the kinetic energy distritutions are broadened as the process of energy relaxation becoutes less conplete and the inelastic and transfer products becono almost indistinguishable. These featurss are illustrated in Fig. 1 which is a graph of the average center of -mass (c.,m.) kinetit erergies for each element and the widths of the kinetic energy distributions. Relative to Coulonb energies for touching sphere, the observed c+m. kinetic energies away frat the grazing. angle and the projectile are lower by approximately 25\%. Some irplications of this will be discussed below. Near the projectile the energies increase sharply and the width broadert as energy equilibration diminishes. 
Figure 2 shows the charge distributions obtained for measurements at five Iaboratory angles. The cross sections peak strongly at the projectile 2 and are dominated by the $30^{\circ}$ data whteh is closest to the grazing angle $\left(31^{\circ}\right)$ for this reaction. Far below the projectila $Z$, the cross section decreases with increasing laboratory angle.

Relative to Coulomb energies for spheres, the c.m. kinetic onexgies are significantly lower (25\% compared to $15 \%$ ) for the $\chi_{\mathrm{A}}+\mathrm{Tb}$ data than for the Kr+AU data 2 with nearly the same composite $A$ and 2 . The higher temperature in the former case (the total c.m. kinette energy is approxinately zoo theV for $979 \mathrm{MeV} \mathrm{Xe+Tb} \mathrm{and} 150 \mathrm{kgV}$ for $620 \mathrm{MeV} \mathrm{Kr}+\mathrm{Au}$ ) will cause more particle evaporation in the $x_{e}+1$ to experiment. The apparent c. J. kiretic energy is less than the true energy if particle evaporaticn is neglected in the transformation from laboratory to c.n., but this effect is suall coopared with the magnftude of the difference between the $\mathrm{Xe}+\mathrm{Tb}$ and the $\mathrm{Kr}+\mathrm{Au}$ results. It is likely that deformation is responsible for much of the reduction in kinetic energy for the $\mathrm{Xe}_{e}+\mathrm{Tb}$ system, although reasons for larger fragnent deformations for $\mathrm{Xe}+\mathrm{Tb}$ are unclear. Thus for the two systeis of corparable mass, a large differeace in the noments of inertia might be expected. Therefore, while the coulomb energy for the nearly syumetric $\mathrm{X}_{\mathrm{f}}+\mathrm{Tb}$ pair is larger than the Coulomb energy for the $\mathrm{K}_{\mathrm{T}}+\mathrm{Aul}$ pair for spherical fragnents, deformation may equalize the repulsive forces in the two systens and increase the rotational period of the higher average l-wave systen (Ke-Tb). The result is, perhaps, conparable broakip tines and rotational periods for the toro systens.

If fragnent deformation is not larger in the $\chi_{e}+\mathrm{Tb}$ systen than in $\mathrm{Kr}+\mathrm{Mu}$, the experimental results might appear quite different due to large differences in the coulionb energies (and, therefort, the complex lifetimes) and the avsrage l-value (and, therefore, the rotational period). However, the side peakting near the grazing angle in the angular distributions is about equally persistent into $Z$ 's above and below the profectile for both $\mathrm{Xe}_{\mathrm{T}} \mathrm{Tb}$ (Fig. 3) and $\mathrm{Kr}+\mathrm{Au}(\mathrm{kef}$. 4 or 5]. Even if mass transfer is more rapid for $\mathrm{Kr}+\mathrm{Aul}$ which might be expected this far from mass symmetry, the increased deformation for Ke-Tb may reduce the Coulonb energies and increase the rotaticnal period enough that the same side peaking over a couparable range of masses is observed. The similarity in the wdths of the charge distribtitions (Fig. 2 and Ref. 4) for both systems gives further support to this idea.

A rore dranatic comparism between the two near $1 \mathrm{y}-$ symetric systens, $\mathrm{Kr}+\mathrm{Ag}$ and $\mathrm{Xe}+\mathrm{Tb}$, ilustrates lasge differences in the relative relaxgtion along the mass asymetry coordinate. The angular distributions for $620-\mathrm{k} k \mathrm{VV} \mathrm{Kr}+\mathrm{kg}$ (Ref. $\mathrm{Z}$ or 6) show tho evidence of side peaking and are in fact forwart pesked suggesting that the lifetimes are comparable to the rotationai period. The nore relaxed charge distributions for $\mathbf{K r} \rightarrow \mathrm{Ag}$ (Ref. 2 or 6) are extremely broad compared with $\mathrm{Xe}+\mathrm{Tb}$ (Fig. 2) and are peaked not at the projectile but, perhaps, several 2's above the projectile which is closer to syumetry. In both systems, the potential energy governing the exchange of rogss is fairly flat since the systemas are cloge to symmetric and so the differences in the charge distributions rust reflect the shorter lifetime for the higher $\mathrm{Z} X \mathrm{Xe}+\mathrm{Tb}$ corplex. The difference in the average 1ifetims of these two systens Inst be comparable to the time constant for mass equilibration to effect such a large difference in the character of the charge distributions.

An extension of the $X_{e}+T b$ data to more forward angles is prosently being undertaken. This will better define the structure of the angular distribution and give inproved estimates of the kinetic energies of the more completely relaxed process.

\section{Foothote and References}

*NATO Fellow.

I. M. Fowler and R. Jared, Natel. Instr, and wothod $124,341(1975)$.

2. L. G. Horetto and J. S. Swentek, LAL,-5006, Lawrence Đerkeley Laboratory (1976).

3. R. Jared, P. Glissel, R. Schmitt, P. Russo, and L. G. Horetto, Identification of Atom Nuntbers ux to $z=60$ in Fragments from Heavy Ion Reactions, this Annul Report.

4. L. G. Horetto, B. Cauvin, F. Glussel, R. Jared, P. Russo, J. Sventek, and G. Wozrilak, Phys. Rew. Lett., 36, 1069 (1976).

5. L. G. Woretto, G. Cannin, P. GIHsse1, R. Jared, P. Russo, J. Sventek, and G. Hotniak, Diffusive Phenomena in the Charge and Augular Distributions for Reaction $197 \mathrm{Ap}+620-\mathrm{MeV} 86 \mathrm{Kr}$, this Annmat Report.

6. R. P, Sclmitt, P. kusșo, R. Babinet, R. Jargd, and 1. G. Noretto, Nuclear Relaxation Phenonena and Diffusion in the Resction 10 , $100 \mathrm{Ag}+84 \mathrm{Kr}$ at $7.2 \mathrm{MeV} / \mathrm{Nkx}$ leon, this Annual Report. 


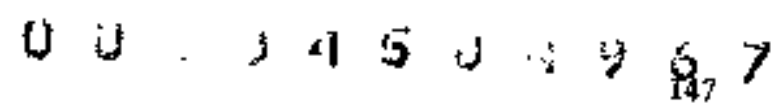

\section{BINARY BAEAKUP (AND SECONDARY FISSION) OF A HEAVY \\ DEFORMED SYSTEM: 979-MaV Xe + Au}

P Russo, R Schmitt, G Wozn|ak, B Cauyin,

$P$ Glassel, $P$, Jared, and L G Moretio

Investigation of the binary deep inelastic process in heavy 1on experiments performed by our group have recently been expanded to include xenon projectiles incident on nedium to heavy targets. While coulomb energtes are larger for the higher 2 systembs, the experimenta 1 results appear to be mokluated by sigmificant fragment defortations.

The present experiments were performed with 979-MeV 136 Xe bears incident on 0.6 to 0.7 me $/ \mathrm{m}^{2}$ gold foll targets. The experinental details have been discussed elsewhere. 1

The laboratory kinetic energy distributions for individual elentents produced in the $\mathrm{Xe}+\mathrm{Au}$ experijents are unique in that twit well-resolved peaks in the kinetic energy spectra are gpparent at forward angles up to $35^{\circ}$, particularly near $2=40$. Figure 1 shows the resolution and relative contributions of the two components observed in the $30^{\circ}$ measurements. Above $Z=48$, the lower energy peak has practically vanished. The energy of the lower energy component decreases at more forward laboratory angles so that at $2 \theta^{\circ}$ nearly half of the kinetic energy distribution is below the experinental threshold. Further backward than $35^{\circ}$ in the laboratory the energy difference between the two coupchents decreases, ard between 50 and $60^{\circ}$ the twr peaks merge and cross over.

The laboratory differential cross sections are plotted as a function of $Z$ in Figs. 2 and 4. Figure 2 shows the charge distributions for the high-emergy component of the kinetic energy spectrum showl in Fig. 1 for laboratory angles of $35^{\circ}$ and forward (Fig. 2A) and for $35^{\circ}$ and backward (Fig. 2B). The strong peak around the projectile and the dominance of the cross section near the grazing angle suggests that this component is associated with the binary inealstic process similat to that observed in the $X_{\mathrm{e}}+\mathrm{Tb}$ datal as well as the $\mathrm{Kr} \rightarrow \mathrm{Arl}$ and $\mathrm{Kr}+\mathrm{Ta}$ data. 2 This (binary, inelastic) coreponent in the Xe+Au data is characterized by side peaked angular distributions and incorplete relaxgtion of the total kinetic energies near the profectile. The center-of-mass kinetic energies obtained for these events by a transfommation which assumes a binary brealap of the complex resenbles strongly the Ye+Tb center-of-mass kinetic energy distributions (Ref. 1, Fig. 3). The kinetic energfes lie approxinately 304 below the Coulonb energies for spherical fragments with energy relaxation diminishing steeply around the projectile and near the grazing angle.

Isolation of the deep inelastic component from the $60^{\circ}$ date and all data below $z=48$ at $50^{\circ}$ and $b \in 10 w 2=42$ at $40^{\circ}$ was not possible, and so so this data does not appear in the charge distributions. At 70 and $75^{\circ}$ the binary inelastic energies extend below the experinentel threshold.
The markedly low center-of-mass kinetic energies of the binary inelastic fraguents suggest large froginent deformations. The charge distributions are equally broad if not broader than those observed

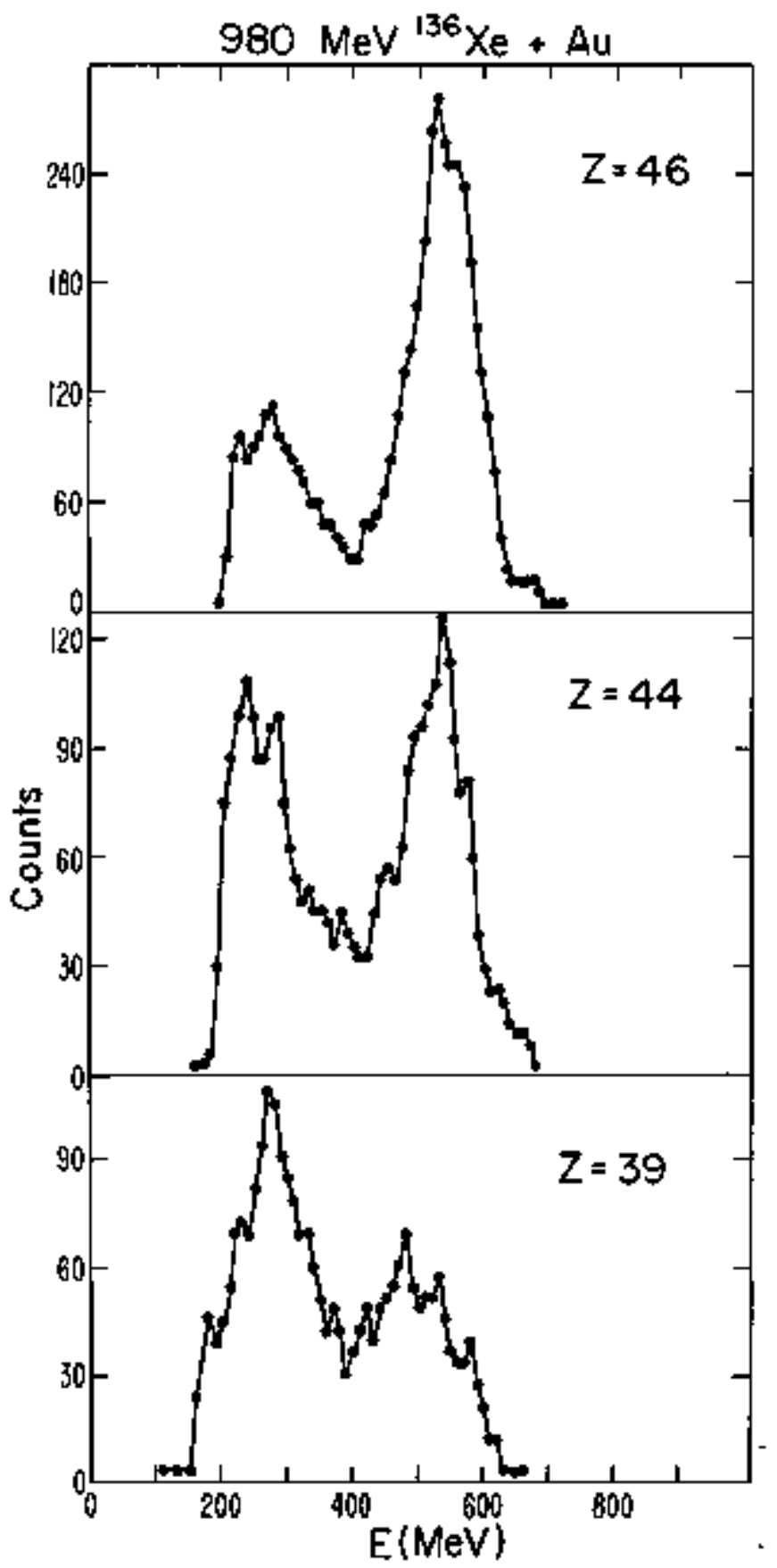

Fig. 1. Laboratory kinetic energy spectra obtained at $30^{\circ}$ in the reaction 979-k⿰V Xe+Ait. The resolution of two components ls shown for three different elements.
(XRL 766-2987) 

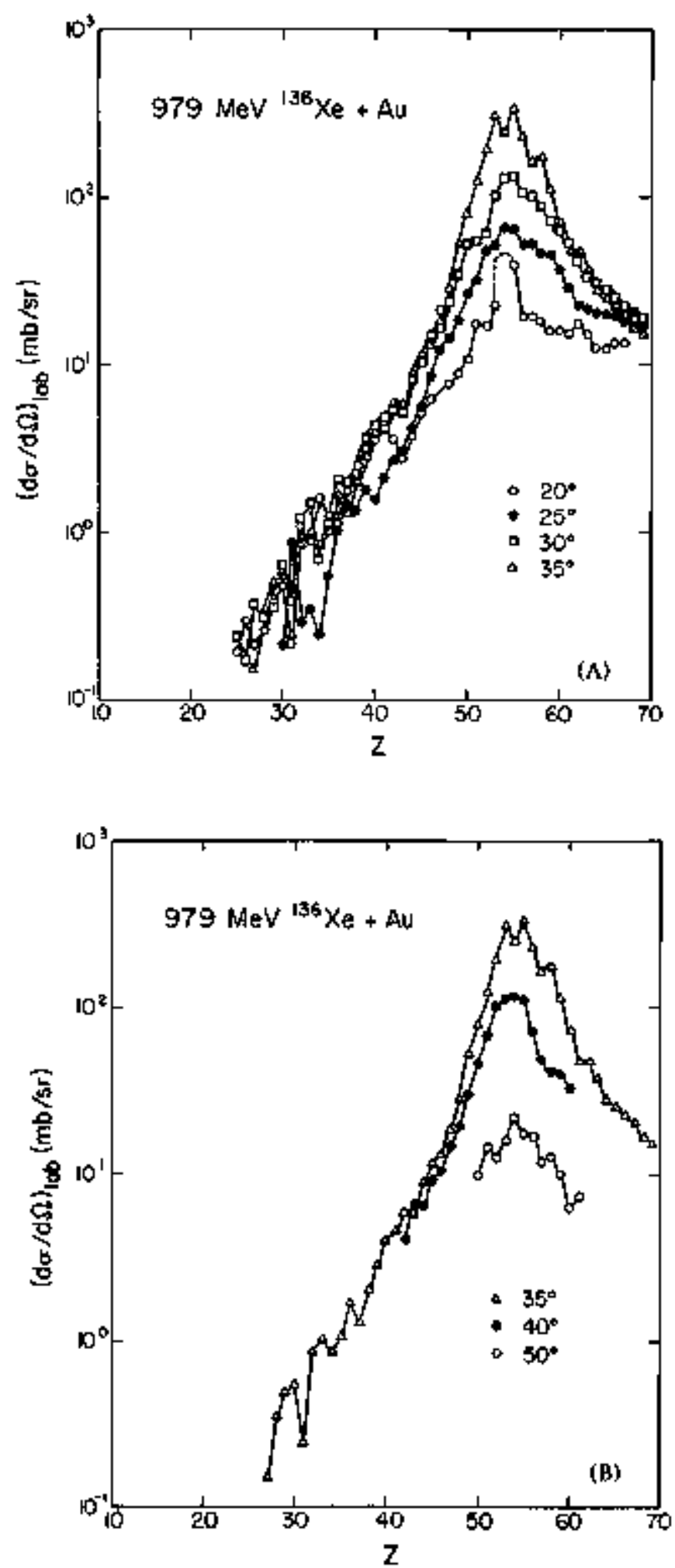

Fig. 2. Laboratory differential cross sections as a function of $z$ for the deep inelastic component from the experiment 979-MeV Ke+Au.

(A) Deata forward of (including) $35^{\circ}$.

(XBL 766-2982)

(B) Data backward of (including) $35^{\circ}$.

(XBL, 766-2980) for the krypton experirents with gold targets. This observation was also made frop the results of the xenon experiment with terbium targets. ${ }^{1}$ Although Coulonb energies are largest for the Xe+Au systen, increased deformtion reduces the rejulsive force so that break-up times nay not be sigrificantly roduced leaving the charge distribution widths unchanged. Furthenmore the extended shapes increase the rotatiol time so that even the slower processes (involving large mass trinsfer) may be side-peaked. The center-of-jtass angular distributions shoin in Fig. 3 reflest this possibllity. The persistence of the side peak for the entire range of elements is unique to this data and lends support to the previous argument that the average rotational period is increased, above that for the kTypton experiments for exauple. A second feature of the angutar distributions is tho forwand shift in the angle of the side peak for elements far-removed from the projectile. The persistence of the side peak tue to an increased rotational period implies that the side peak is composed of events produced by a broader range of corplex lifetimes. The angular distribution of events produced by a lonperlived conplex will be shifted formand since the rotation has proceeded further, and it is the long-lived conplex which gives rise to greater mpss transfer. Thus the xenon-gold system nay provide a megrs to stunty the time-evolution of the mass transfer process since the time constants for both mass transfer and rotation appear to be conparable in this case. Further measurements to include more forward and backward angles as well as interuediate angles are in progress.

Figure 4 shows the charge distributions for the resolved low energy component of the kinetic energy spectra shown in Fig. 1. These are peaked at approxinately one-half the target $Z$ suggesting that this component may originate fror secondary fisston of the target or perhaps ternary break-up of the complex. It is reasonable that the charge distributions for the products of fission of the gold-like fragurght show peaks above $Z=40$ since it may not be gold but in fact the more fissicable products of mass transfer away fron the projectile which contribute to this phenonenon. The taboratory kinetic enpergies of this component, averaged for three $z ' 5$ (36, 37 and 38) near the peak of the charge distribution are plotted in Fig. 5 as a function of laboratory angle. A parabolic curve peaked near $\$ 0^{\circ}$ glves a reasonable approximation to the data points. This strong kinenatic dependence of the kinetic enersy tin angle provides evidence that secondary fission of target-like products is the origin of the second component. The cross-section for the deep inelastic gold-like produets of binary break-up peaks near $50^{\circ}$ in the laboratory, consistent with a maximpn in the kinetic energy distribution nerr $50^{\circ}$ if secondary brealap is assumed. The cross section for these events, henceforth referred to as the fission component, increases slowly toward forwart angles as is shown in Fig. 4. Forward of $30^{\circ}$ in the laboratory, the cross section for the fission component cannot be determaned due to the experinental low energy cutoff. However, a peak in the fission cross section more forward than the most probable 


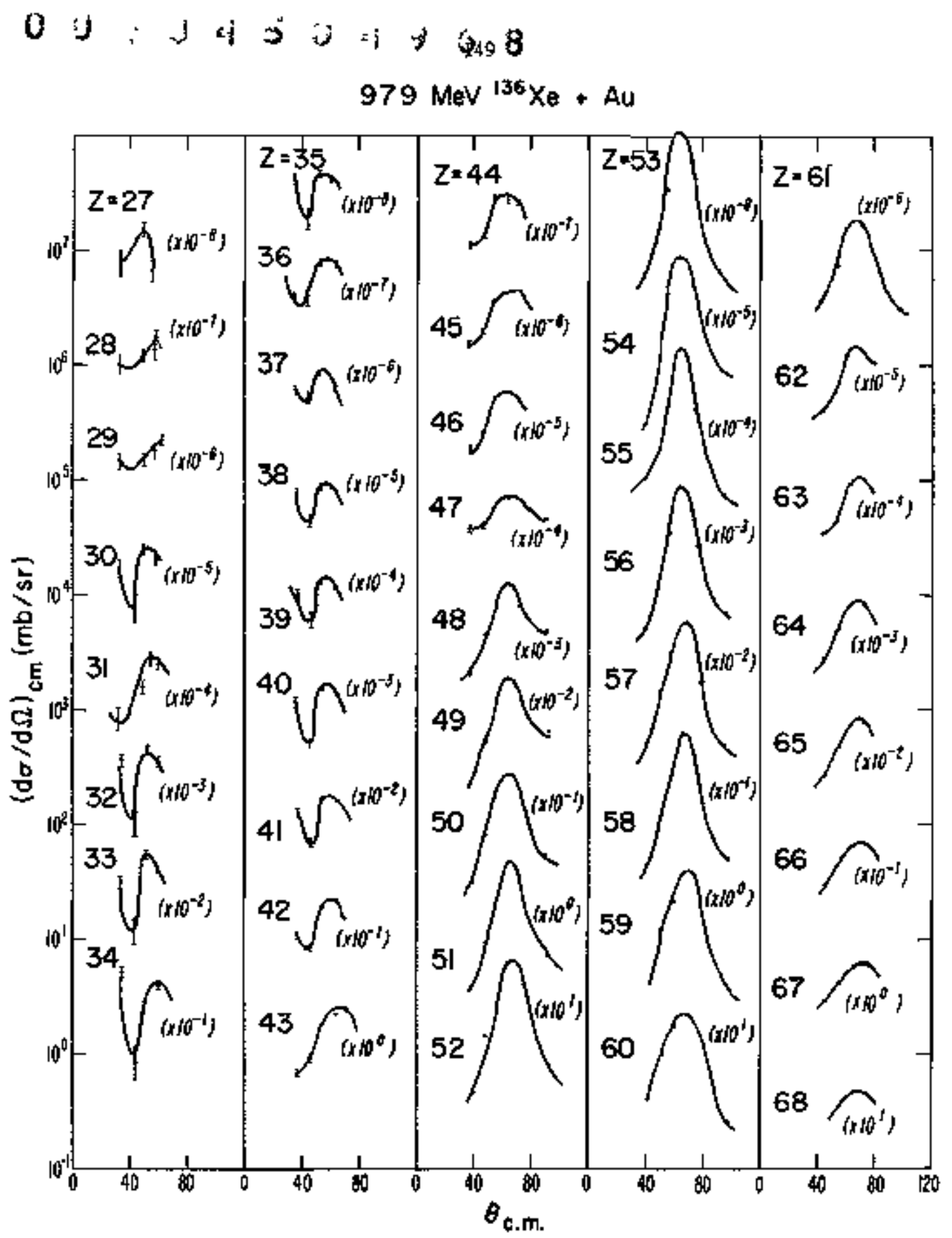

F1g. 3. Angular distributions of individual elements for the deep inelastic component in the experiment 979-(MeV Xe+Alu. (KBL 766-3007)

angle for the exission of target-like fragments is not sumprising if the center-of-plass moratanta of the fission fragments is a major fraction of that for the binary inelastic gold-13ke product as would be the case with the secondary break-up procoss.

The energy spectra for the fission component further forward than $30^{\circ}$ will provide more definitive infonation concerning the origin of these events.

\section{Footnotes and References}

*or leave from DPh-N/MF CEN, Saclay, France. † HATO Felloto,
1. P. Russo, G. Hozriak, R, Schmitt, R. Jared, P. Gitssel, and L. G. Moretto, Evidence of Deformation and Inconplete Relaxation in a Nearly Symetric Hegvy Systera; 979-NeV Xe+Tb, this report.

2. L. G. Woretto, B. Cauvin, P. GLsssel, R, Jared, P. Rusșo, J. Svontek and G. Wozniak, Diffusive Phenomena in the Charge and Angular Distributions for the Reaction $197 \mathrm{Au}+620-\mathrm{HeV} 9 b_{\mathrm{Kr}}$, this Annual Report. P, Glassel, B. Cauvin, G. Woeniak, P, Russo, R. Schmitt, R. Jared, and L. G. Moretto, Study of Fragments Enitted in the Interaction betweer 18l Ta and $86 \mathrm{Kr}$ at $620 \mathrm{KeV}$ Boubsarding Energy, this Anintal Report. 


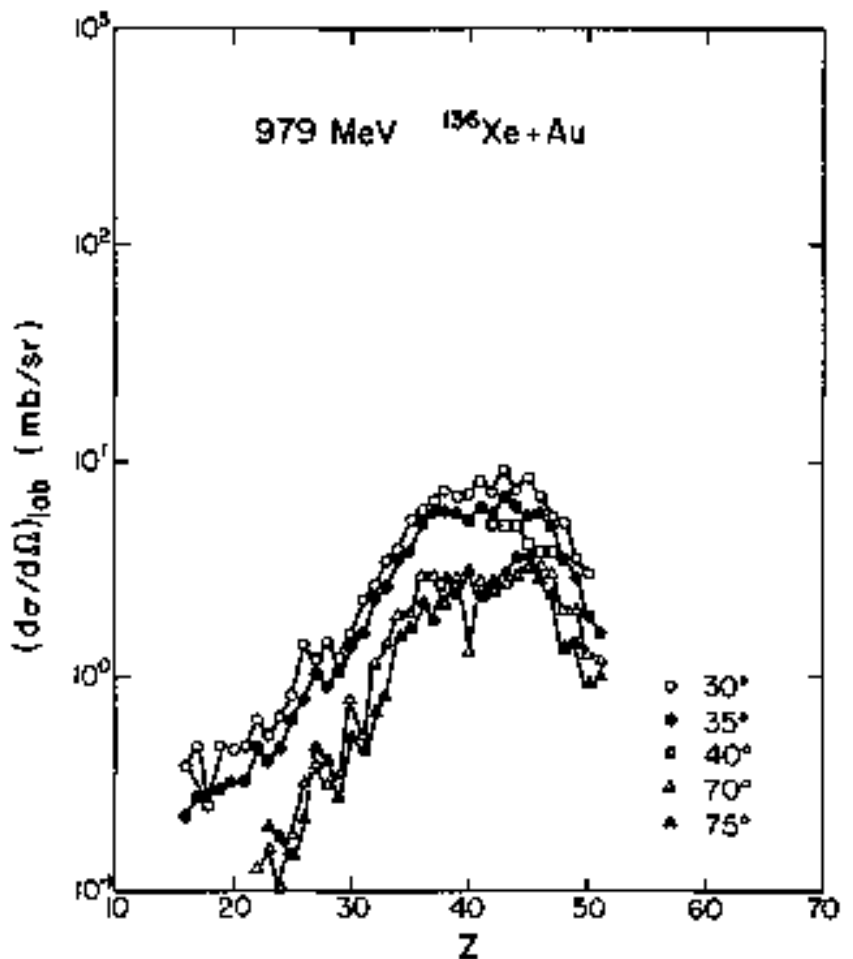

F1g. 4. Laboratory differentia1 cross sectims as a function of 2 for the second (other than deep inelastic) cosponent from the 979- MeV Xe+Au data.

[XBL 766-2981]

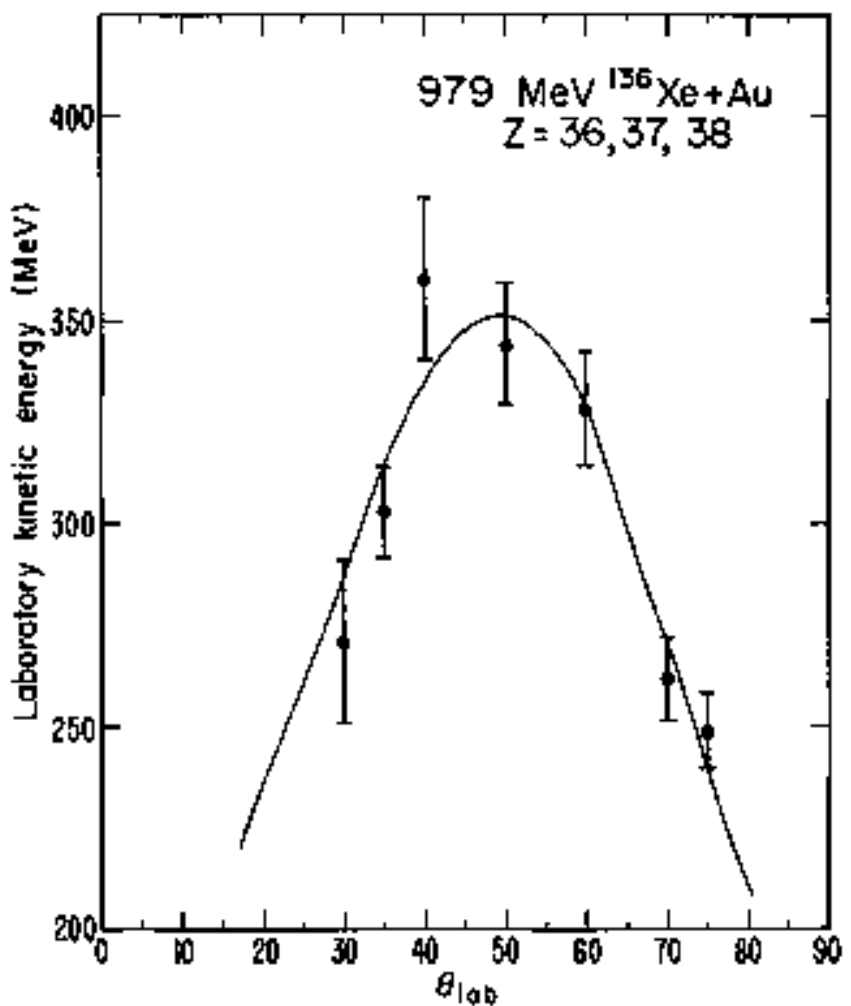

Fig. 5. Laboratory kinetic energies of the fission component as a function of laboratory angle fror the 979-HeV Xe+Au experiment.

(XEL 766-2977)

\author{
CHARGE- AND MASS-DISTRIEUTIONS IN THE REACTION \\ OF ${ }^{40}$ Ar IONS WITH ${ }^{238} \mathrm{U}$ * \\ $J$ Kratż J 0 Lipenzin \\ A E Norits sf and $G T$ Teaborg
}

The reaction of $288-140{ }^{40} \mathrm{Ar}$ iuns with thick 238U targets has been studied experimentally with radiochenical tectriques. The formation cross sections of 130 radioactive nuclides were neașued using thetr characteristic gama-ray enission. The data have been used to delineate charge and mass distribution.

The independent and partial cumelative yields are plotted vs. mass number (after particle evapporation] in Fig. 1(a). The apparent scatter in the data in Fig. 1(a) occurs because independent yields, and even many of the cundutative yields, represent only a fraction of the total mass yields. Figure 1 (b) is a contour map of the independent yields in a $Z$-A plane, indicating yield locations relative to the stability line, the structure reveated by the isopleths in Fig. 1(b) indicates that several yield distributions with different chargeand nass-dispersions, and hence different origins, are superimposed on each other. The overall distribution is doninated (1) by the htgh yields for transfer protucts near $A=40$ and $A=238$ ("rabbit ears," components $E$ and $F$ in Fig. $1[b])$, and (2) by a broad fission product distribution centered around $A \approx 133$. This distritution is not a single colpment, but consists of produxts from fusionfission (commonent A), from fission of heavy products formed by transfer of a few nucleons and șluali amounts of excitation energy (Component B), and from high energy fission of heary products from deep inelastic processes (shaded area D). Furthermore, there are neutron-rich products ranging from low $Z$ vaIues up to $Z \neq 26$ or ever higher, that are attributed to deep inelastic transfer reactlons (component C). 


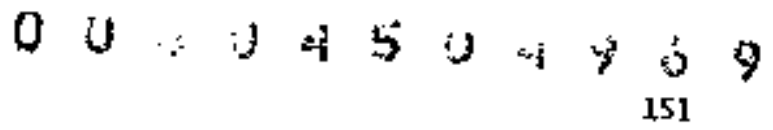
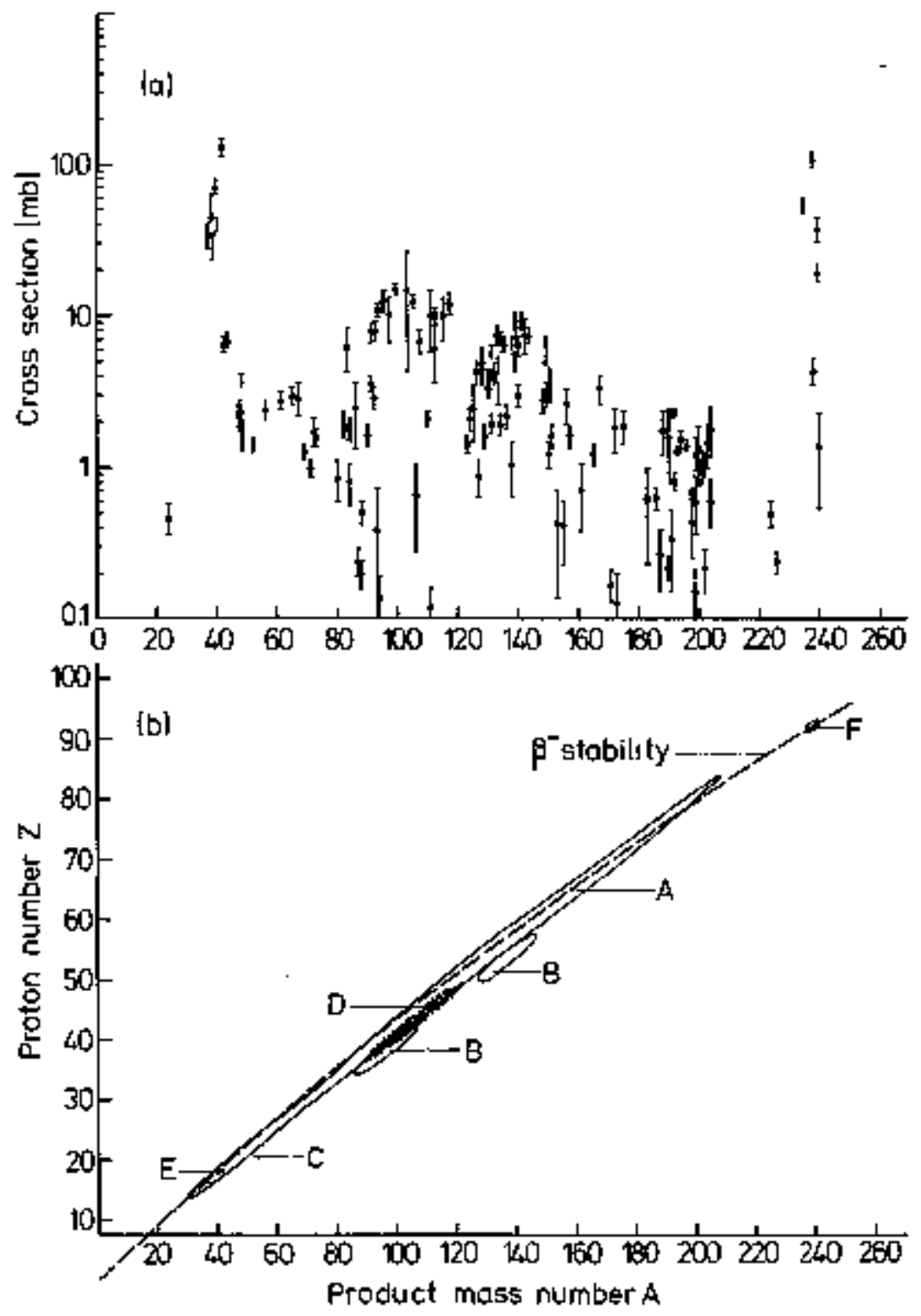

Fig. 1(a) Independent and cumplative formation cross sections of individuel muclides produced in the boglardment of a thick $238 \mathrm{y}$ target wtth $28 B \mathrm{MeV} 40 \mathrm{Ar}$ iols.

(b) Contour lines for equal independent formation cross sections in a $Z, A$ plane. Isoploths of $1 \mathrm{mb}$ are shown for components $A, B$ and $C$. The isopleths for components $\mathrm{E}$ and $\mathrm{F}$ refer to 10 sib cross sections.

(XBL 7511-8972)

For the dexuction of the total mass yields from the yields of single isotopes, we used experimentaly determined charge-disperșion curves for the mass chains. ( In Fig. 1(b) a charge-dispersion curve would correspond to a cut through the indicated "yield mountains" at a fixed mass number.) Then the experimentally determined independent or cuadative yields were related to the total soss yield by correcting for the independent yields of unobserved menbers of the chain. To arrive at the total nass yiold for a given nass, it was necessary to integrate the charge dispersion curve;
I.e., sum of the independent yields of all isotopes with mass $R$.

Figure 2 shows the total integrated chain mass yields (upper and lower limits are indicated at those mass numbers for which experimental data were obtained) and their deccomposition into individual components: (A) corplete fusion-fission $(620+150 \mathrm{mb})$, (B) transfer-induced fission $(150 \pm 30 \mathrm{~b})$, (C) deep inelastic transfer $(100 \pm 50 \mathrm{mb})$, (E) and (F) quasi-elastic transfer ("rabbit sars," $400 \pm 120$ no and approximately $200 \mathrm{mb}$, respectively). 


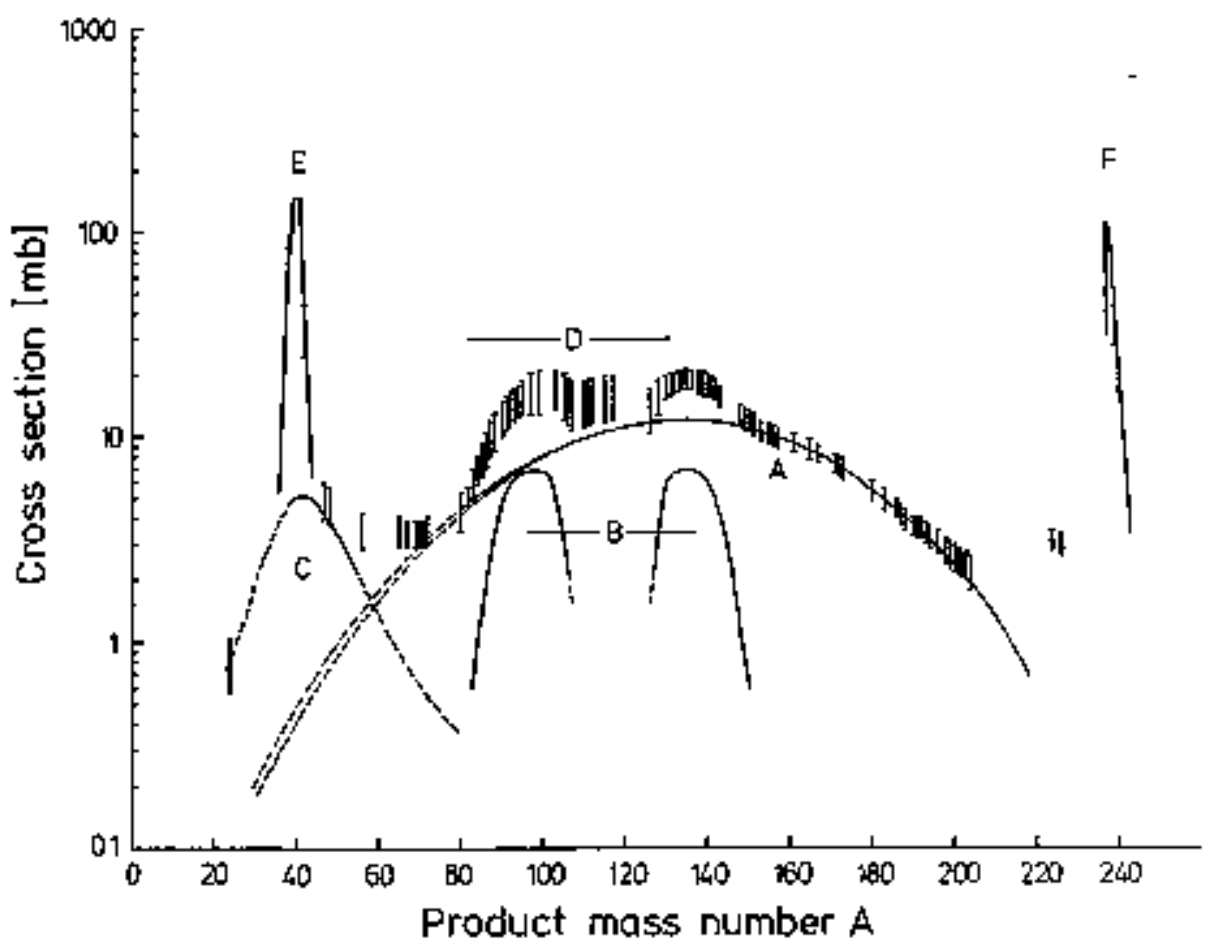

Fig. 2. Total integrated mass yields produced in the brmbardment of a thick 238 $40_{A r}$ ions. Conponent $A$ corresponds to $620 \mathrm{rab}$, $B$ to $150 \mathrm{mb}, C$ to $100 \mathrm{mb}, D$ not determined, $E$ to $\$ 00$ mb and $F$ to $220 \mathrm{mb}$.

(XEL 75i1-8975)

The existence of products from the sequential fission of heary fracments formed in deep inelastic collisions (D) is also indicated; however, are unable to dectuce a coaplete mass distribution for this conponent.

The results obtained from this survey experiment can be summarized as follows:

1. In the energy interval investigated, fusion-fission accounts for about 55 s of the total reaction cross section, while $35 \%$ quasi-elastic and $10 \%$ deep inelastic transfer are observed. The nass distributions of these corponents are show in $\mathrm{Fig}$. 2. The total reaction eross section anounts to about 1100 ib.

2. Evidence is presented for fission of heavy miclei after transfer reactions. Quasi-ulastic transfer leads to a doxble thamed fission product distribution signifying the transfer of relatively small amounts of excitation energy in grazing collisions. Fission after deep inelastic collisions seems to lead to a fission fragment mass distribution (incompletely determined) which is typical for higher excitation energles of the fissioning nucleus.
3. Proton pick-up reactions are observed leading to a butid-up of prohucts with $Z=25$; $i$,e., the mass distribution for multi-nucteon transfer and fusion-fission overlap considerably.

4. It is shown that the mass to charge ratios for both multi-muleon transfer- and fusion-fission frapments are equilibrated. Ho equilibration of this degree of freedom is attained for products formed by quasi-elastic transfer.

\section{Footrotes}

*Condensed from Phys. Rev. C 13, 2347 (1976).

${ }^{\dagger}$ on leave fron Institut für Kernchemie, Universität Mainz, Germany. Present address: Gesellschaft für Schwerionenforschung, GSI, ilarmstadt, Gertuany.

\# Department of Nuclear Chemistry, Chalmers University of Technology, Goteborg, Sweden.

${ }^{5}$ Los Alamos Scientific Leboratory, Uniyersity of California, Los Alamos, New Mexico 87545. 


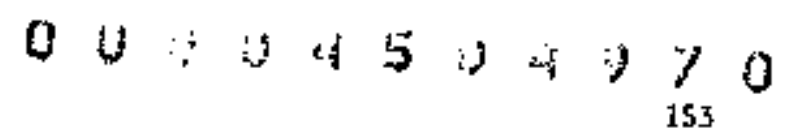

\title{
MASS YIELD DISTRIBUTIONS IN THE REACTION DF ${ }^{136} \times$ B IONS WITH ${ }^{238} \mathrm{U}^{*}$
}

\author{
A. J. Otto, M. M. Fowher, D. Lê, \\ I. Binder, and G. T. Seabor's
}

rields of 131 nuclides were measured radiochemically to defineate theress distribution in the reaction of 1150-kev $136 \mathrm{X}_{\text {e with a thick }} 238 \mathrm{y}$ target. Figure $1(a)$ shows the independent and partial candative yield cross sections for the muclides identified by gamen ray analysis of $\bar{s} \bar{x}$ chemical fractions. Figure l(b) depicts contours in the Z-A plane of constant independent yield and the most probable Isobaric charge lines dertved from the firal charge distributions. Beginning with ar approximate charye dispersicn description an iterative fitting procedure was used to arriwe at a final charge and mass distribution. Figure 1 (c) shows the final integrated chain mass yield distributions. Six coapchents were identified and associated with either the quasi-fission or the quasi-elastic tronsfer mechonlin.

Corponent $A$ is shown as an upper limit for the complete fusion-fission process. The quasielastic transfer mechanism can be assigned to conponents $E$ and $F$. The asymmetrfe fission distribution, component $B$, is the result of low energy fisston of the heavy elastic transfer proxhuct with mass numbers near that of 230 . The reass distribution of the quasi-Xe (deep inelastic cooponent) formed in the quasi-fissicn process is identified as conponent $C$. About 758 of the complementary quasi-l fiagments undergo high energy binary fission resulting in the broad symentric fission distribution shom as conponent D. Component G, collopuially labeled as the "gold finger" and not explained in the initial tescription of the $\mathrm{Kr}+\mathrm{J}$ experiments, 1 can be attributed to the quasi-fission (deep inelastic) reaction rechanisu. This interpretation of the conponents is supported by a mass balance of the composite Jass distribution. Components $H$ and $I$ are the result of reactions with contaminants in the target. 2

Further evidence that the "gold finter" represents quasi-U products that have strvived de-excitation without undergoing fission can be found by comparing the $\mathrm{Kr}+\mathrm{Bi}$ nass distribution ${ }^{3}$ with the $\mathrm{X}_{\mathrm{e}}+\mathrm{U}$ results. The quasi-Bi yiolds show the same trend as component $G$ except that a much smaller fraction of the quasi-Bi fraginents undergo highenergy binaty fission.

Table 1 gives the cross sections for the various components identified in the reaction of $40 \mathrm{Ar}, 4$ ${ }^{8} 4_{K}, 1$ and $136 \times$ e with $230 \mathrm{~J}$. Comparison of the fusion-fission croșs section and the quasi-fission cross section (deep inelastic process) shouts that for heavy ion reactions with uraniun the transition from fusion-fission to quasi-fission occurs between argon and krypton upon increasing the mass of the projectiles from argon to krypton to xencm.

A second experiment was done using $1150 \mathrm{HeV}$ 136 Xe with a thick $238 \mathrm{y}$ target. Diret gamma-ray analysis of the bombarded target foil without

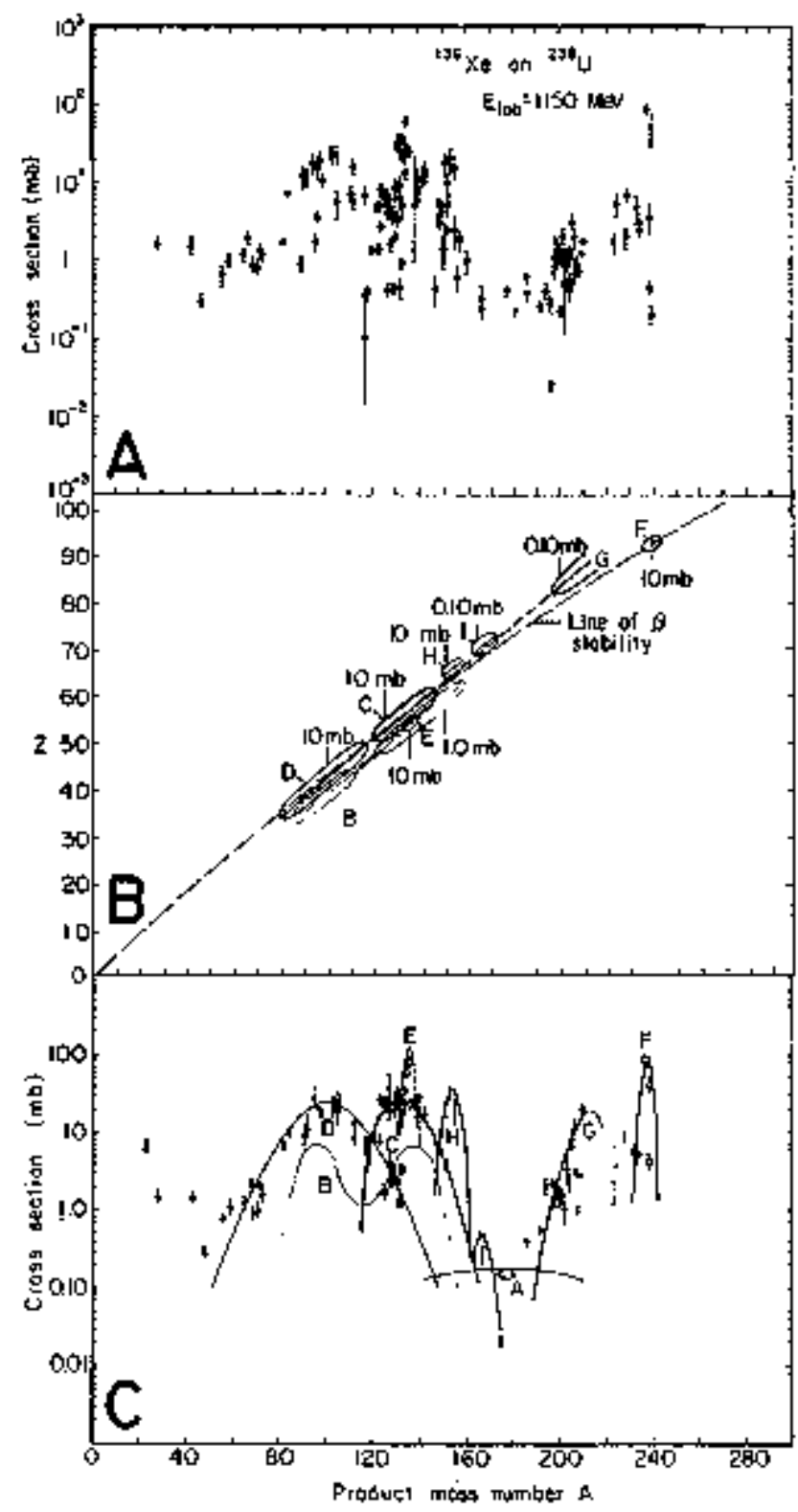

Fig. 1. Mas yield distribution for $1150 \mathrm{MVV}$

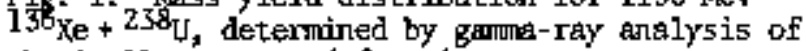
cherically separated fractions.

(a) Independent and cumblative yield formation cross sections.

(b) Contour lines for equal independent yields and lines of most probable charge for each component.

(c) Total integrated mass yields.

(XBL $758-3746 \mathrm{~B})$ 
Table 1. Cross Section Conparison

\begin{tabular}{|c|c|c|c|c|}
\hline \multirow[b]{2}{*}{ 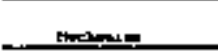 } & \multirow{2}{*}{ 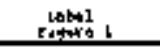 } & \multicolumn{3}{|c|}{ cron Intion } \\
\hline & & 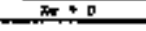 & Kx & $A r+C$ \\
\hline 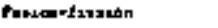 & $\omega$ & $<20=$ & 33+1s & 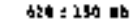 \\
\hline 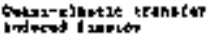 & 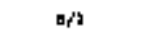 & $=163 n$ & $200+40=$ & 134,340 \\
\hline 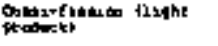 & c & $M 0 \pm 12 L n$ & ATh $170=$ & $104,30=$ \\
\hline Daniz-terant trason & $0 / 2$ & bם בa + 1900 & $\sin +40=0$ & 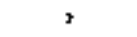 \\
\hline 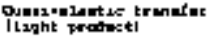 & $\mathbf{r}$ & No no & $79+10+\mathrm{NH}$ & AB I $230 \mathrm{~Pb}$ \\
\hline 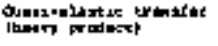 & 8 & $=$ ats nis & a dit 4 & - b \\
\hline 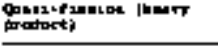 & c & +1400 & 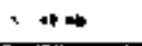 & m+11 \\
\hline 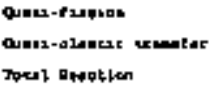 & 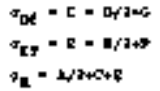 & 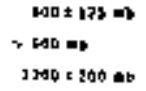 & $\begin{array}{c}17+170 \mathrm{mb} \\
96+11 \neq+\mathrm{mb} \\
1963+203 \mathrm{mb}\end{array}$ & 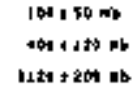 \\
\hline
\end{tabular}

chemical separations gave the partial cumblative and independent yields shown in Fìg. 2. Although the total integrated mass yleld distribution must be determined from the cross sections shown in Fig. 2 in order to afford a conplete analysis, these cross sections corresponding to independent and partial cumblative yields are consistert with the final rass distribution shown in Fig. $1(c)$.

Several conclusictus can be drawn from these experiments. First, the probability is slight that part of the quasi-U fragpents that were formed (and lost by undergoing fisstion) were in the superheavy element region. Second, it can be noted that component $D$ has a full width at half maximam of 20 to 25 mess units. Component $D$ is a conposite of many Gaussian mass distributions of fission fragments from those products above astatine in the quasi-uranium mass distribution. However, the full width at half maximum of the quași-Xe distribution (component C) appears to be only half as wide as component D. In such a situation the full width half raximum of component $D$ is only slightly larger then the full width half maximul of any one of the individual curves making op the compostte. Thus the width of component $D$ is a direct moasure of the excitation energy deposited in the quasi-uraniuno fragments. Third, the larger cross sections for producing the "gold finger" in the xenor reactions as compared with the krypton reaction is the result of the relative neutron richness of the 136 Xe projectile. This last conclusion follows if, as wighth be expected, the heavy quasi-fission fragnents are nore neutron excessive for the xenon reaction than for the kryptom reaction and thus have greater stability against fission.

\section{Footnotes and References}

*Condensed froun Phys. Rev. Lett. 36, 135 (1976).

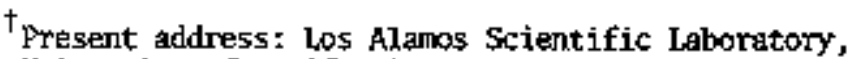
thiversity of California, Los Alamos, New Kexico 87545 .

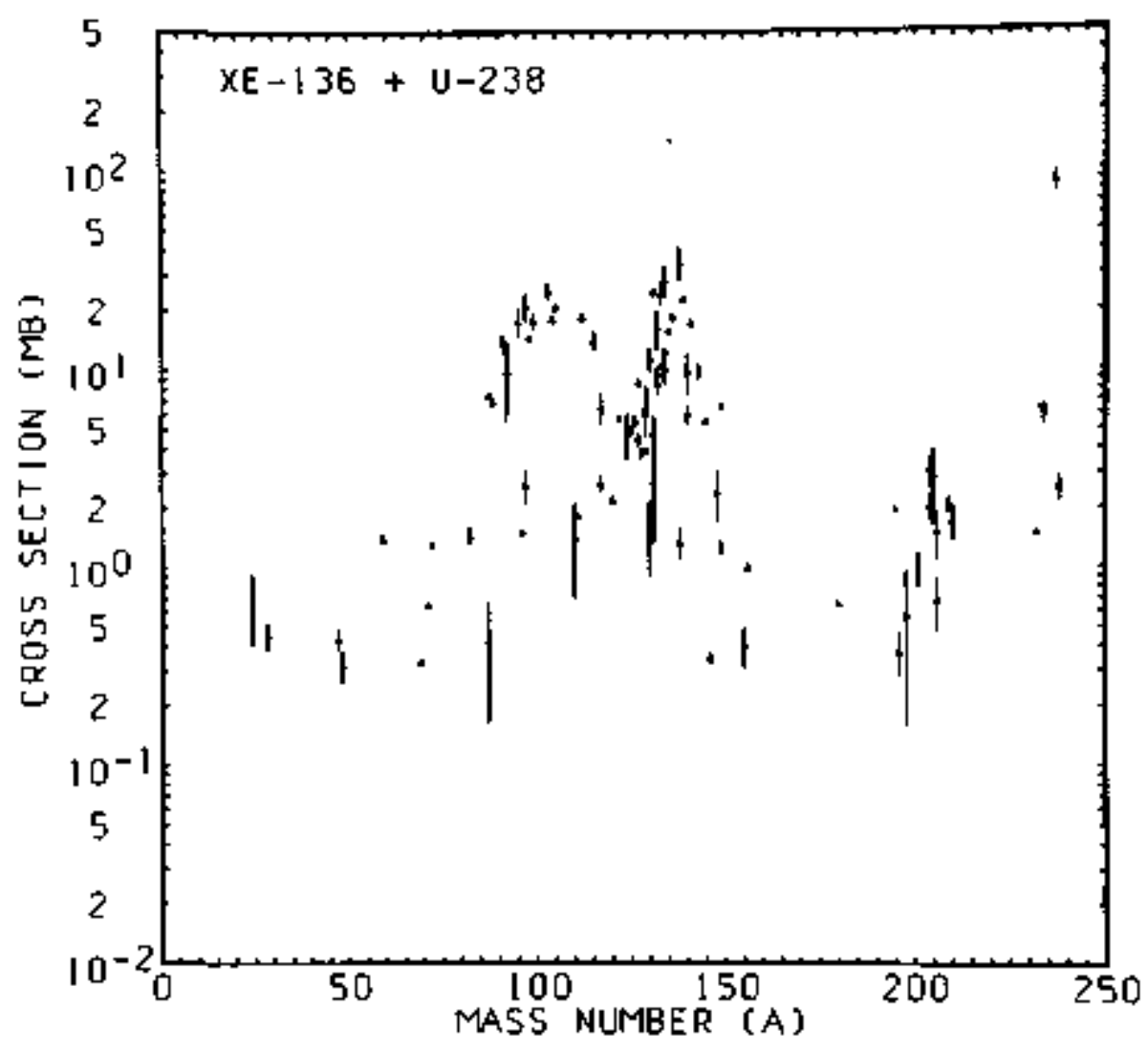

Fig. 2. Parial cumblative and independent yigld cross sections for the reaction of $1150 \mathrm{keV} 130 \mathrm{Xe}+$ 23\% the target without chemical separations.

(XEL 766-B093) 


\title{
0 j.
}

1. J. V. Kratz, A. E. Norris and G. T. Seaborg, Phys. Rev. Lett. 33, 502 (1974).

2. Al, Mg and AT jopurities in the target and target backing account for components $H$ and $I$. The target was made by sputtering U onto an Al backing in an aroon atmosphere. A thin Al deposit containing some ho was used to cover the U to prevent oxidation.
3. I, Binder, M. M. Fowder, D. Lee, R. J. Otto and G. T. Seaborg, "Heavy-Ion Reactions with Heavy, Non-Fissioning Targets," in this Anmual Report.

4. J. V. Kratz, J. O. Liljenzin, A, E. Norris and G. T. Seaborg, LBL 4339 and Phys. Rev. C/3, 2347 (1976), and "Charge- and Mass-Distributions in the Reaction of $4{ }_{A T}$ Ions with $238 \mathrm{r}$ in this Aumual Report.

INVESTIGATION OF ${ }^{21}$ At PRODUCED BY $230 \mathrm{MeV}{ }^{40} \mathrm{Ar}$ on ${ }^{209} \mathrm{~B}$ |

\author{
D J Morriegey, A J OHd, 5 Yashita, \\ A Ghiorse, and G $T$ \$peborg
}

It has been known for son years that the cross gection for the production of $211_{\text {At }}$ by the reaction $40 \mathrm{Ar}$ on $20 \mathrm{~S}_{\mathrm{Bi}}$ is on the order of millibarns. $111_{\text {At }}$ is a readily measured reaction product because it decays by alpha particle emission

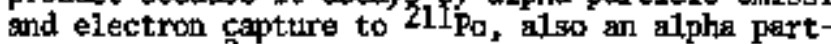
icle enitter, ${ }^{2}$ thus giving ons alpha panticle per atom formed. The Orsay group has used this property along with similar properties of $149 \mathrm{gTb}, 150 \mathrm{Dy}$ and $151 \mathrm{Dy}$ to investigate the "quasifission" reaction mechanism. 1,3 Bímbot ot aj, perforned a series of expertments, using a thin $209 \mathrm{Bi}$ target, in which they measured the excitation finction, and recoll range of the $211_{\text {At }}$ product. They found that the median range decreased as the bombarding energy increased, opposite to the trend expected for a coopound nucleus evaporation reaction, and thus their conclusion was that the $211_{\text {At }}$ was produced in a characterigtically non-c口apound nucleus reaction.l There are two reaction mechanisos that could oxplain or fit these experimental observations: they are the quasffission or multinucleon transfer dwechaxism, or a quasi-elastic transfer process. In our experiment we attenpted to locecure the recoil range and angular distribution of $211_{\text {At }}$ simultaneously and thereby could delineate these two mechanisus.

In a sijple model of quasifission, ole assunes conplete daming of the energy in the entrance channel followed by separation of tw fragments with the coulonb energy of two towching spheroids. Using this wode1, the angular and energy distribution of the products can be calculated by a two-step vector model shown in Fig. 1(b). These two vectors are the velocity of the center of mass and the velocity of the flssion fragment in the center of the mass system. Also shown in Fig. 1(a) is the calculated distribution in an aluminum stopper of $211_{A t}$ produced by $230 \mathrm{MeV} 40 \mathrm{Ar}$ on $209_{\mathrm{Bi}}$. In this reactlon the velocity of the $\mathrm{OS}$ is larger than the coulowb separation onsrg; therefore, all the $211_{A t}$ fuxlei have a forward velocity in the laboratory,

One can imagine that superimposed on the energy-angular distribution curve is a probability distribution. This is, of course, not shown. This distribution wi11 be different for the two reaction wechanisins. Moretto ot al. have noasured
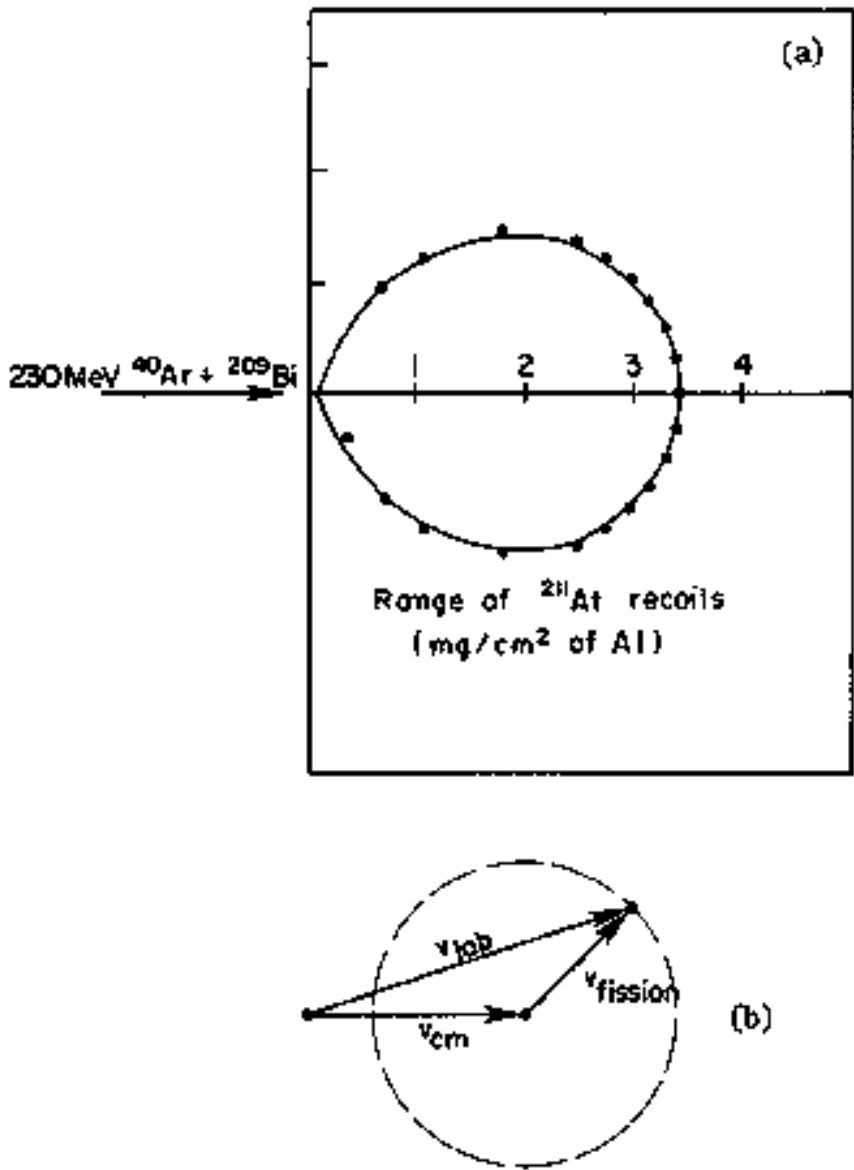

(b)

Fig. 1 [a), The computer calculated range curve for recoiling $211_{\text {kt }}$ produced by the simple vector model. (b). Representation of simple vector rodel used to calculate curve in Fig. 1(a).

(ẌBl 766-2910)

the distribution for the complimentary light fragThey found that the $\mathrm{dr} / \mathrm{d}$ c curve for $\mathrm{z}=16 \mathrm{had}$ a strong $1 / \mathrm{sin}$ component with sone forward peaking 


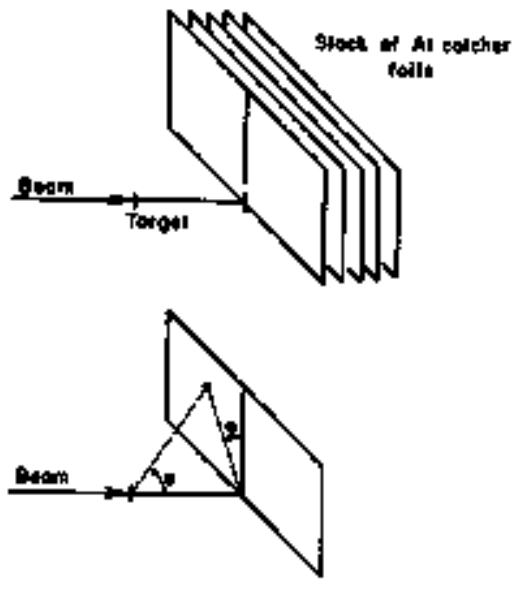

Fi. . 2. Schenatic representation of target and catcher foils with the orientation of laberatory angles $\theta$ and $\phi$.

(XBL 766-2911]

in the center of mass systen. Assuning the same type of angular distribution for $210_{\mathrm{Ar}}+209_{\mathrm{Bi}}$, this would force our At $\{z=85\}$ distribution to be 1/sine but be backward peaked in the center of nass systen. When converted to a distribution in the laboratory, this would be seen as an almost constant probability along the curve (since do/dr - constant), with sape rise in the probability of lor laboratory energy $211_{\text {At }}$ recoils.

On the other hand, if the reaction mechanism is quasi-elastic transfer, the $211_{\text {At }}$ should be found at the classical grazing angle with little or none found elsewhere.

A che quarter inch dianeter $0.116 \mathrm{ng} / \mathrm{cm}^{2}$ $\mathrm{Bi}_{2} \mathrm{~S}_{3}$ target, vacuson evaporsted onto $\mathrm{Be}$, was irvadiated with $228 \mathrm{MeV} 40_{A x}$ ions from the SupertILAC. This was the calculated energy in the center of the $B i_{2} S_{3}$ target after passing through the $B e$ target backing.

The recosling $211_{\text {At }}$ nucles were caught by a stack of $\sim 1 \mathrm{mg} / \mathrm{ch}^{2}$ Al foils. These foils located domstream and off axis from the target to allow a spatial separation of the recolis from the bean. This is shown schematically in Fig. 2. After the borbardment, each foil was divided in half to facilitate two counting methods. One half was placed in an ionization connter and the alpha half tife followed. The other half was used to rake a radioautograph.

Copies of the radioautographs of the sacond and third foils are show in Figs, 3(a) and (b) respectively. These show econentrit rings of alpha activity, vith the ring on the third foil inside that on the seccond. These radioautographs were transformed into angular and energy distributions by projecting slide coptes onto a ruled rask and cotnting the spots. In the case of foils 2 and 3 , radioautographs of shorter expossure time use used because the interse bands were not resolvable.

The decay curve for foll 3 is shom in Fig. 4 . Thas shows the half life of the alpha activity to
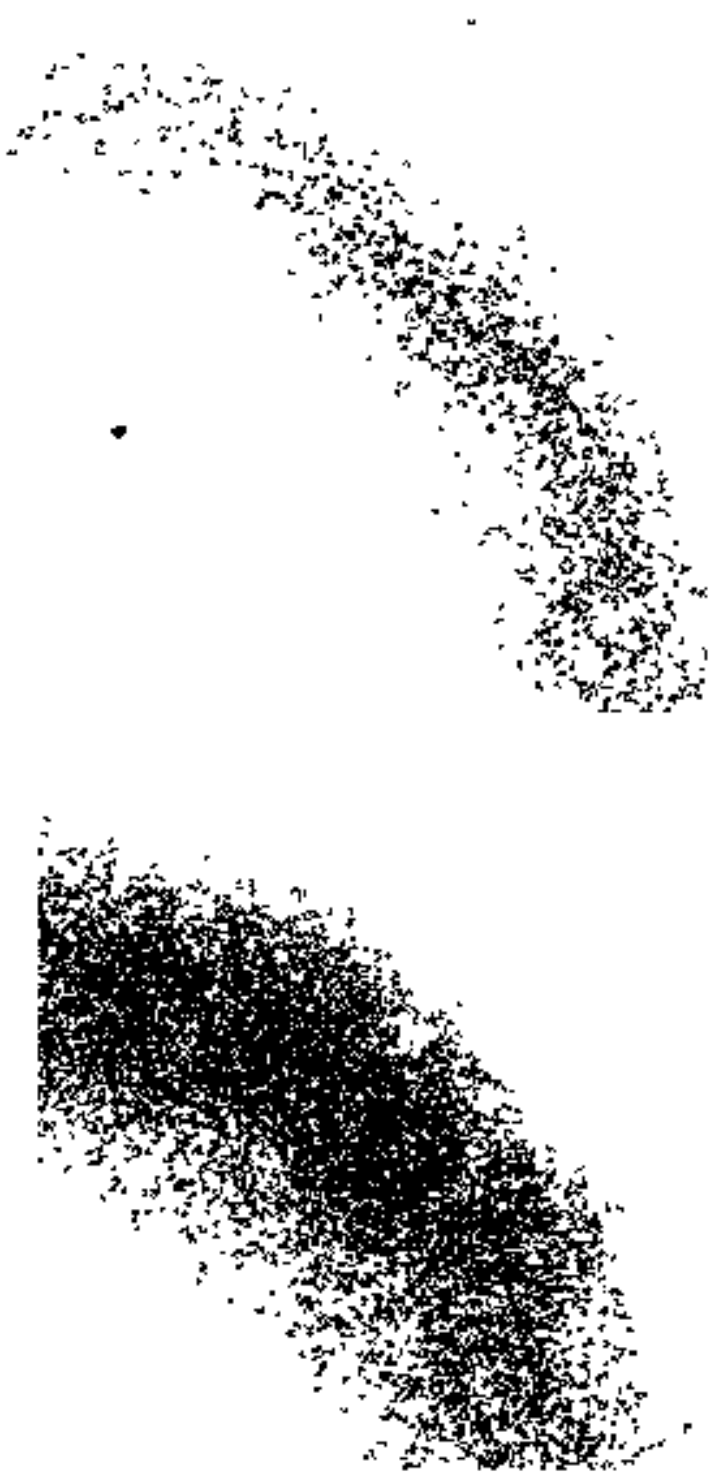

Fig. 3(a). Copy of a radioautograph of eatcher foll 2 there dark spots result from alphe particle emission. (XBB 765-4462)

(b). Copy of a radioantograph of catcher foil 3 where dark spots result from alpha particle emission.

(XBB 765-4461)

be 7.7 hours and allows us to attribute it exclusively to $211_{\text {At. }}$

The resutts of the angular and energy distribution analysis can be seen in Fig. 5 . Here the energy distribution is not explicit but can be discerned by remenbering that these were stacked Al foils. Thus activity un the later foils had to pass through larger and larger amounts of Al and thus had to be higher energy recoils. The highest activity was found in the second foil in the angular range of $40-45^{\circ}$ in the laboratory. This corresponds to a velocity vector that would be assoclated with an elastically recoiling $209_{\mathrm{B} 1}$ nuclei. Thes lends 


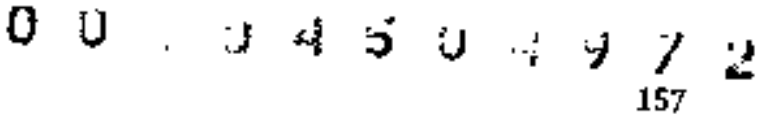

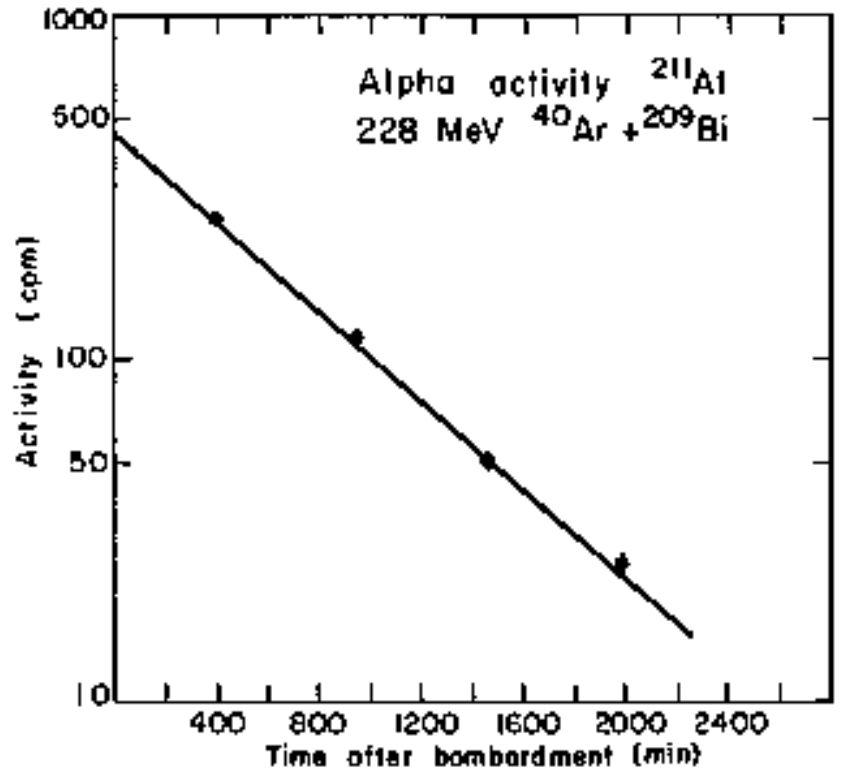

Fig. 4. Decay curve of catcher foil 3.

(XBL 766-2912)

support to the idea that the majority of the $211_{\text {At }}$ is produced in an elastic type event. Folls 1 and 4 are shown here in an expanded scrle. It is in these two foils that any $1 / \sin \theta$ commonent would be prominent. Foil 4 semls to show an increase in cont rate as one goes to maller angles in the laboratory. This is what Binbot et al. have seer for similar nog-contuound nucleus systems.
Foil 1, on the other hand, shows a decrease in count rate with decrease in lab angle. The activity in Foil 4 has a distribution corresponding on the curve show in Flg. l(a); thus we weth attribute it to a quasifission mechanism. If true, we would further expect a simalar contribution all along the curve of Fig. 1. Foil 1 does not fulfill this expectation, in fact it seents to show the opposite to be true. However, this 1ack of 10w energy fragnents at low laboratory argles may be due to the thiclmess of the target.

We feel that Fig. 5 slogys the production of $211_{\text {At }}$ fron $228 \mathrm{MeV} 40 \mathrm{Ar}$ on $209_{\mathrm{Bi}}$ to follow a quasielastic transfor type necharism with some lesser contribution fron a quasifissian mechanism. Thas sing11 contribution is seen at low angles in Foil 4. The note that the activity is down 3 orders of magnitude from that in the quasi-elastic transfer region.

\section{References}

1. R. Bipbot, D. Gardes and M. F. Rivet, Phys, Rev. C4, 2180 (1971).

2. M. 1ederer, J. Hollander and I. Perlenm, Table of Isotopes, 6th ed. J. Willey s sons (1967).

3. R. Binbot, D. Gardes, R. L. Ham, Y. De Horas, M. F. Rivet, Nucl. Phys, A228, 85 (1974).

4. l. G. Wretto, J. Galin, R, Babinet, $Z$, Fraenke1, R. Schmitt, R. Jared and S. G. Thorpson, LBL-4084 (1975).

5. L. C. Northrop and R. F. Schliling, Nacl, Data A7, 233 (1970),

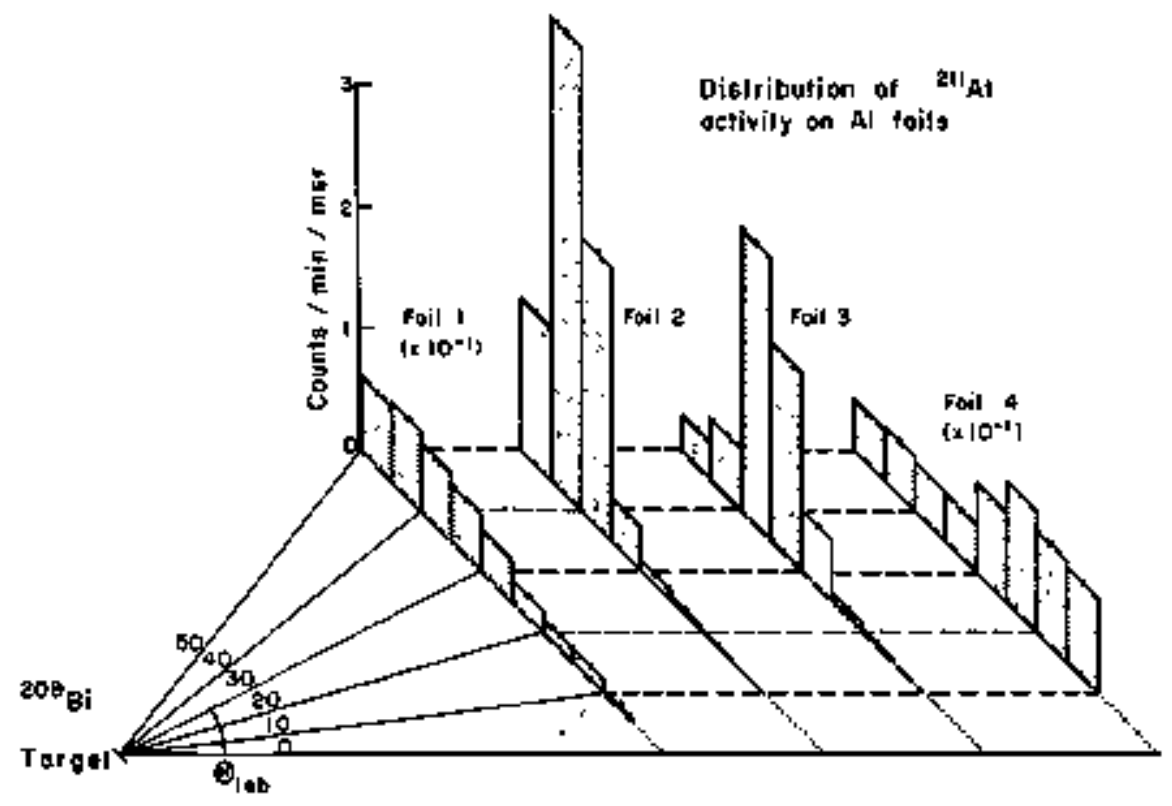

Pig. 5. Atpha activity distribution orl catcher foils 1 through 4 (covers per minute per millisteradian vs $\theta_{1 \mathrm{ab}}$ ). Mote that foils 1 and 4 are shown with an expanded scale. 


\section{HEAVY-ION REACTIONS WITH HEAVY, NON-FISSIONING TARGETS}

I Binder, M M Fowler, D Lee,

$F+$ oro, and $G T$ Seaborg

As part of the progran to stukty the reactions of heavy-ion projectiles (by radiochetrical methods), targets of bismuth and gold have been bombarderd with $\mathrm{Kr}$ and $\mathrm{Ar}$ beans available at the Berkeley SupertIlAC. The goal has been to investigate the yields of prontucts in the nefghbortood of the target jucleus withcut severe depletion of such produxts dute to fission. Both low-energy asymbatric and high-energy symotric fisston are seen to occur when urgulu is the targot material; also seen ambog the uranium reaction products is the so-called "gold-finger."1,2

Thick Au and Bi foils thave been hombarded with Kr and Ar ions. Scine target foils were counted directly for ganda rays using large-voludue Ge(Li) detectors and for $X$-rays using an intrinsic $G e$ detector. In this way uncertainties due to chemical yield losses are elirinated for all products so that absolute cross sections can be computed, and shorter-lived activities are accessible. Some of the forls were dissolved and chentcally processed using methots sinilar to those developed for uranium targets. 3 This producet samples consisting of simple groups of el ements. These samples were then examined for ganma-rays and alphe particles. In addition, sone of these experiments included spectfic chemical procedures to isolate one particular element or one special group of elements (e.g., a lanthanide sample.)

The nuxlides protuced were identified using the krowledge of gamm-ray or alpha-particle energy, half life, relative intensities of ganula rays originating from one nuclide, and chenical characteristics of the element.

More than 130 nuclides nere identified from the reaction $705 \mathrm{HeV} 64 \mathbf{K r}+209 \mathrm{Bi}$. Garge dispersion corves were obtajned for varfous regions of prohucts, and this enabled integration of isobaric yields giving the total yield for each nass number. The integrated result can be seen in Fig. 1.

The total yield curre has been factored into coruponents corresponding to yields from the vartous reaction rechanisms occurring. \$een are components ${ }^{4}$ of quasi-elastic trensfer (components $E$ and $F$ ), deep-inelastic transfer or quasifission (components $C$ and $G$ ) and high-energy symmetric fission, "quasiternary fission" (component D). The high energy symmetric flssion takes place for nuclides in the reighborhood of and above the ${ }^{209} \mathrm{Bi}$ target. It is not possible to distinguish a fusion-fission corponent, but this outgoing reaction charmel camnot be very large and an upper limit estinate is shown as component A. Table 1 contains the various cross sections for each of the identifled components. The relative error for the cross sections in Table 1 is about $15 \%$.

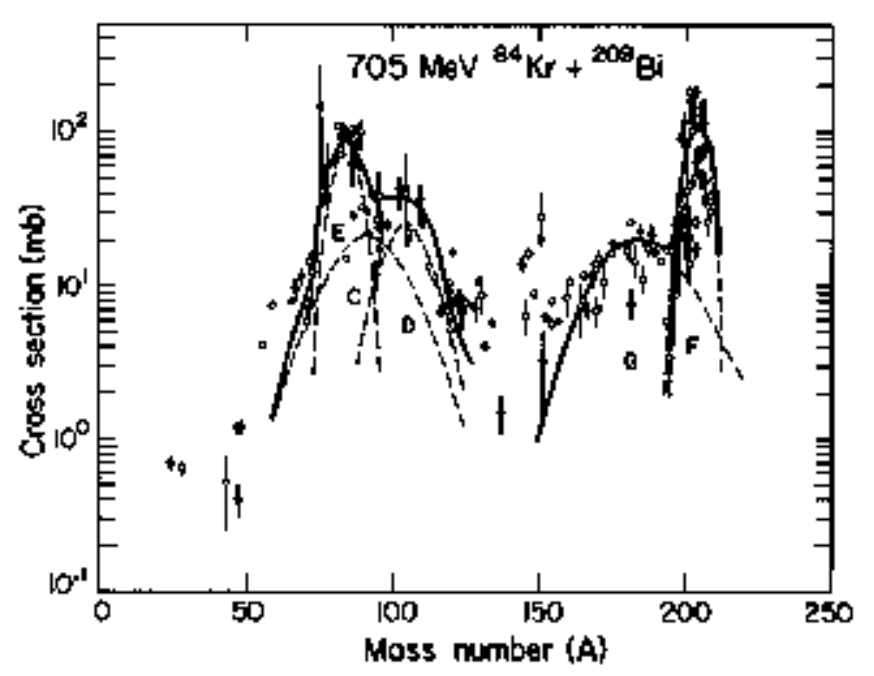

Fig. 1. Wass vield distribution ${ }^{4}$ for the reaction $705 \mathrm{KeV} \mathrm{s}_{\mathrm{Kr}}+209_{\mathrm{Bi}}$.

(XBL 766-8254)

Table 1. ${ }^{84} \mathrm{Kr}+{ }^{209}$ Bi yields.

\begin{tabular}{|c|c|c|}
\hline Nechanistu & $\frac{\text { label }}{[\text { Figure } 1)^{4}}$ & $\begin{array}{l}\text { Cross } \\
\text { Section }\end{array}$ \\
\hline Fusion-Fisston & $A / 2$ & $\leqslant 120 \mathrm{rb}$ \\
\hline $\begin{array}{l}\text { Quasi-Fission (deep } \\
\text { IneIastic light product] }\end{array}$ & $\mathrm{C}$ & $680 \mathrm{mb}$ \\
\hline $\begin{array}{l}\text { Quasi-Fission (deep } \\
\text { inelastic heavy product) }\end{array}$ & $G$ & 460) תit \\
\hline $\begin{array}{l}\text { Quasi-Elastic Transfer } \\
\text { (light product) }\end{array}$ & $\mathbf{E}$ & $880 \mathrm{mb}$ \\
\hline $\begin{array}{l}\text { Quasi-Elastic Transfer } \\
\text { (heavy protuct) }\end{array}$ & $\mathbf{F}$ & $850 \mathrm{mb}$ \\
\hline Quasi-Temary Fission & $\mathrm{D} / 2$ & $265 \mathrm{mb}$ \\
\hline Quasi-Fisșion & $o_{\mathrm{QP}}=\mathrm{C}=\mathrm{D} / \mathrm{Z}+\mathrm{G}$ & $\sim 700$ лा \\
\hline Quasi-Elastic Transfer & $\mathrm{v}_{\mathrm{QET}}=\mathrm{E}=\mathbf{F}$ & $-865 \mathrm{nb}$ \\
\hline Total Reaction & $\sigma_{\mathrm{R}}=\sigma_{\mathrm{QF}}=\sigma_{\mathrm{QET}}$ & $\sim 1600$ ș \\
\hline
\end{tabular}

As first noterd in the reaction $84 \mathrm{Kr}+197 \mathrm{Au}$ and later confirmed in the case of ${ }^{4} \mathrm{Kr}_{+}{ }^{209} \mathrm{Bi}$, 


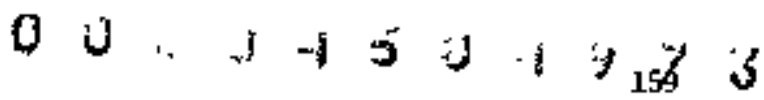

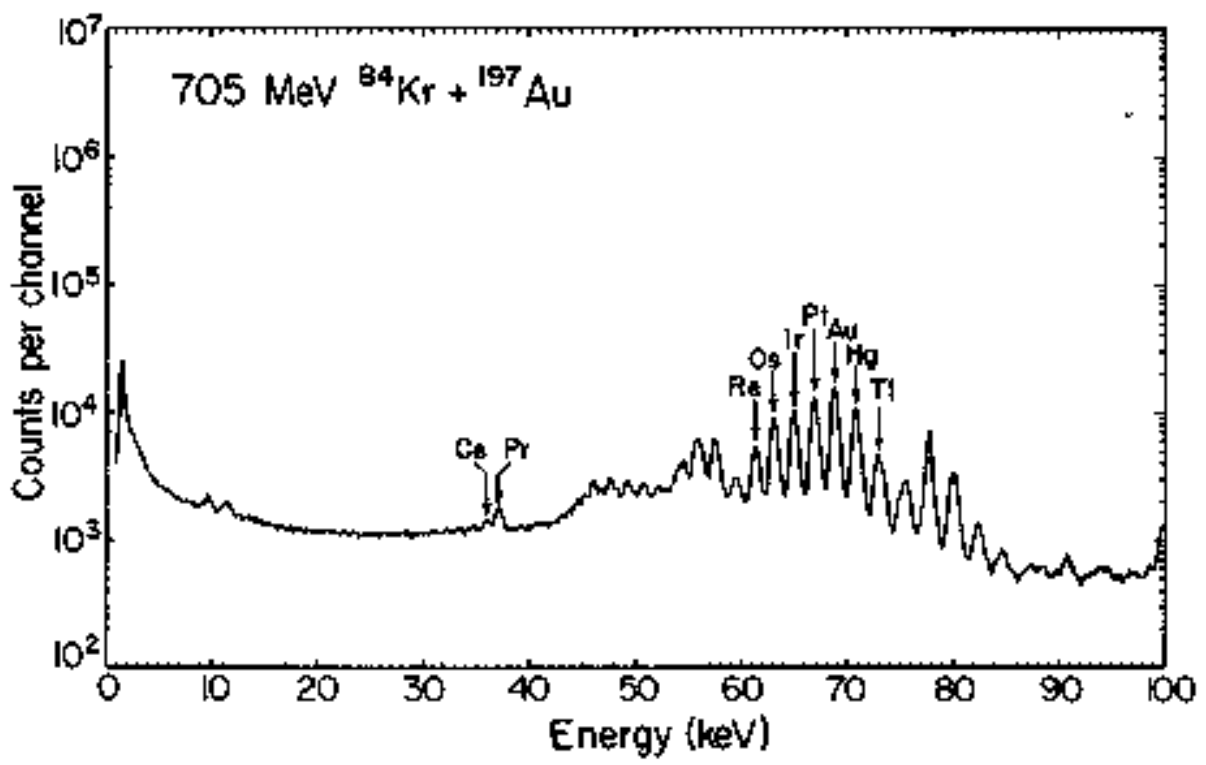

Fìg. 2. $X$-ray spectyum of 197 Au target foll irradiated with 714 ifeV ${ }_{\mathrm{KT}}$.

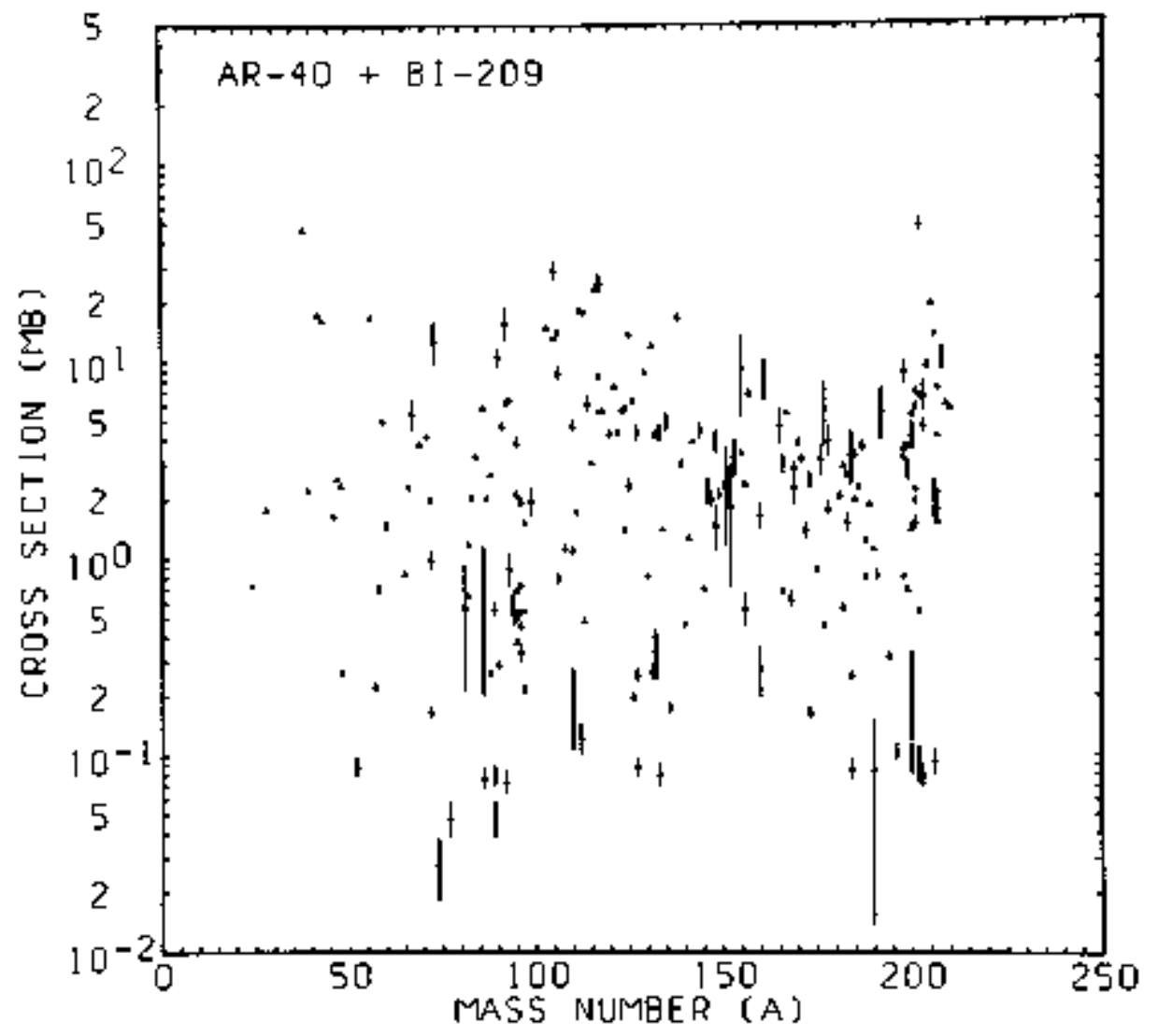

Fig. 3. Partial cumulative and independent yields fros the reaction of $340 \mathrm{MeV}{ }^{40} \mathrm{AT}+269_{\mathrm{Bi}}$.

(XBL 766-8092) 
products from the deep inelastic reactions extend forty to fifty mass units below the originating target nuclepus. In addition, products in the region of lead are depleted he to high-energy, symmetric fission of the initial pre-evaporation products above bisminth. The fission harriers are signifi. cantly lower for these heavfer products above the closed shells. These two factors * yields up to fifty mass units below the target nuclous and fission above the lead shell clostre -- are now believed to be responsibIe for the "goldfinger" products found in the reactions of heavy tons with uranium targets.

Figur 2 shows an $x$-ray spectrum taken of a gold foif that was bombarded with $714-k+V k r$ ions. Yields are raptdly declining above the target nucleus, but extend down to lanthanum isotopes. This is consistent with the nore quantitative mass iistribution deterwined by gama-ray anslysis.

The balance of protons and neutrons in the inconing and outgoing chanrels in the kryptrm reactions shows that mass is preferentially transferred fron the target nucleus to the $\mathrm{Kr}$ projectile in a deep-inelastic transfor reaction.

Results for the $340 \mathrm{MeV} 40_{\mathrm{Ar}}+209_{\mathrm{Bi}}$ reaction are given in Fig. 3, which shows the partial cowllative and independent yields of isotopes idmtified by galla-ray analysis. These results represent the first part of a conplete ress distribution anaIysis in which swooth chain yield cross sections will finally be obtained. The scatter in the cross sections shown resuIts from the fact that each cross section represents a fraction of the chein yield. A broad symmtric fission mass distribution would account for the generally high yields around $A \approx 110$. A complete fusion-fission reaction mechanista must thus make un a large part of the total reaction cross section. There is also evidence for a deep inelastic quasi-bisntuth comporent in the increasing yields betweer $A \approx 180$ and $A \approx 200$. These results are consistent with kinematic studles of the same target, projectille conbinations, both in the overall mass distribution and in showing that the transition fron complete fusion-fission to quasifission occurs when the moss of the projectile is increased from that of Ar to that of $\mathrm{Kr}$.

\section{Fontrotes and References}

Present address: Los Alamos Scientific Laboratory, University of Californta, Los Alamos, New Hexico 87545.

1. J. V. Krat 2, A. E. Norris and G. T. Seaborg, Phys, Rev, Lett. 33, 502 (1974).

2. R. J. Otto, M. M. Fowler, D. Lee and G. T. Seaborg, Fhys, Rev. Lett. 36, 135 (1976).

3. J. V. Kratz, J. O. LiIjenzin and G. T. Seaborg, Inorg. Nucl. Chem. lett. 10, 951 (1974).

4. The letters used to identify the various conponents in the mass distribution are consistent with their usage in references 1 and 2 . Letter $B$ has been assoclated with quasi-elastic induced fission of the uranium target and is this not required for Bi and All targets.

\title{
POSSIBLE MECHANISMS FOR ${ }^{266} \mathrm{Fm}$ AND ${ }^{265_{\text {Md }}}$ PROOUCTION IN THE COMPOUND SYSTEM $2{ }^{24_{104}}$
}

\author{
L. P. Somervile, D. J. Morriesey, A. J. Otlo, \\ 3. T. Seaborg, and A. Ghicrso
}

In heavy ion reactions designed to syntheșize the heaviest elenepps ( $2>100)$, spontaneous fission from the 177 dain $25{ }^{6} \mathrm{Fm}$ is often observed. It may be protuced either directly or as the daughter of 77-rin 256ht which dectrys by electron capture to ${ }^{256} \mathrm{Fm}$ which then udergoes spontaneous fission. The observed spontaneous fission activity in these reactions is often dominated by that of $25{ }^{\circ} \mathrm{Fm}$ which has been fortad to hove cross sections between $20 \mathrm{nb}$ and $0.2 \mathrm{mb}$. It wuld be desirable to know the mechanism of how this nuclide is protuced so that its yield could be estinated in fitture hervy olement sypthesis reactions. Also, a knowledge of how $25{ }^{\circ}$ in is produced in these reactions would give a better understanding of how nuclei with atonic numbers between those of the target and compound nucleus are mede.

In this report, experimental data on the cross sections for production of $25 \%$ prom several heavy 10in reactions lișted in Table 1 are presented. These data are conpared with kirematic studies of the reaction of $22_{\text {he }}+232 \mathrm{Th}$. 1
Table 1. Nuclear reactions studied.

\begin{tabular}{|c|c|c|c|}
\hline Reaction & $\begin{array}{l}\text { Coripound } \\
\text { system }\end{array}$ & $\begin{array}{l}\text { Rans } \\
\text { ener } \\
\text { studie }\end{array}$ & $\begin{array}{l}\text { ge of } \\
\text { rgies } \\
\text { ed (Ney) }\end{array}$ \\
\hline${ }^{13} \mathrm{c}+{ }^{249} \mathrm{Cf}$ & 262104 & $7 \$ .7$ & \\
\hline $15_{\mathrm{N}}+249_{\mathrm{Bk}}$ & ${ }^{264} 104$ & 77.9 & $=100$ \\
\hline${ }^{10} 0_{\mathrm{B}}+{ }^{253} \mathrm{Es}$ & 263104 & 67 & \\
\hline $11_{B}+253_{E s}$ & ${ }^{264} \mathrm{I04}$ & 55 & - I15 \\
\hline $16_{0}+{ }^{248} \mathrm{Cm}_{\mathrm{n}}$ & $264_{104}$ & 95.4 & \\
\hline${ }^{12} \mathrm{C}+{ }^{252} \mathrm{Cf}$ & ${ }^{264} 104$ & $5 S$ & $-\$ 15$ \\
\hline${ }^{13} \mathrm{C}+{ }^{252} \mathrm{Cf}$ & $265_{104}$ & 66 & $=89$ \\
\hline $18_{0}+{ }^{248} 0 \mathrm{~m}$ & $256_{104}$ & 98,7 & \\
\hline
\end{tabular}




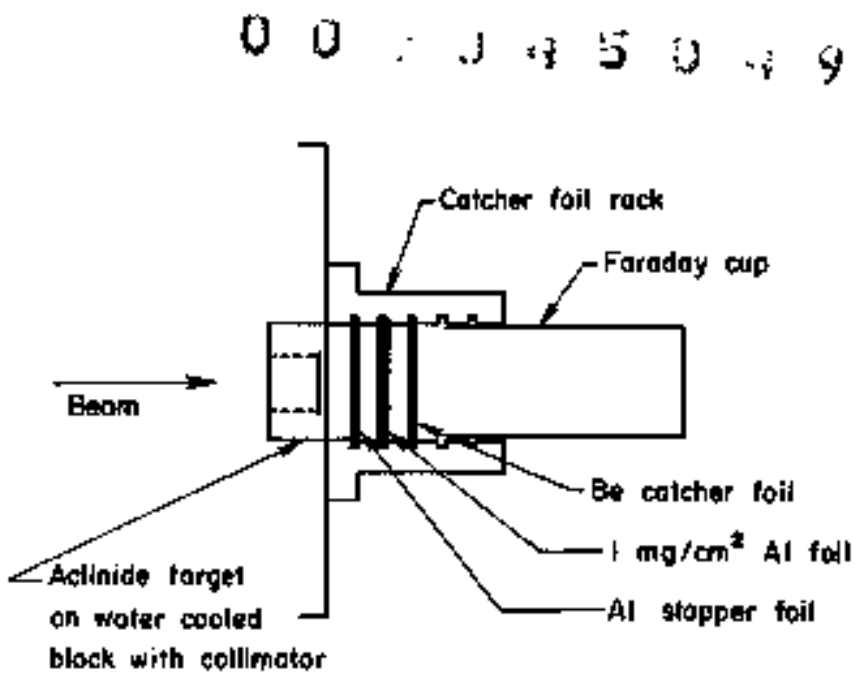

Fig. 1. Typical sot-p for catcher foil experiments. The relative positions of the Al stopper foil (20-40 $\mu_{g} / \mathrm{cm}^{2}$ ) and Al catcher foil (1 $\mathrm{mg} / \mathrm{om}^{2}$ ) are shown. A 1 mil fie foil was used in the $15 \mathrm{~N}+249 \mathrm{Bk}$ experiments. Greater than $95 \%$ of the 256Fu activity was found in the Al catcher foll.

(XEL 766-8579)

In a series of bcmbardments devoted to a segrch for 260104 the cross sections to produce $256{ }_{\mathrm{F}}$ were ineasured by Ghiorso and co-workers during the periat 1963 to 1976. The exporiments were conducted at the SupertIIAC and recently at the 88-inch cyctotron. The recoil nuclides from these bombardments were caught by a thin catcher foil in the path of the bean (Fig. 1). A I mg/ $/ \mathrm{cm}^{2}$ Al foil was used as a eatcher foil in 11 experiments except for the reaction $12 \mathrm{C}+252_{C} f$ in which a $0.83 \mathrm{mg} / \mathrm{cra}^{2} \mathrm{Pd}$ catcher was usect, In most cases, a very thin $20-40 \mathrm{ug} / \mathrm{cm}^{2}$ A1 stopper foil was inserted between the target and the catcher foll to stop target atoms knccked out of the target. A ccinparison of the spcitaneoys fission activity (identifled by half-1ife as $250 \mathrm{Fn}$ ) in the stopper and catcher foil indicated that greater than 958 of the beary recolling nuclet were caught in the I $\mathrm{mg} / \mathrm{cm}^{2}$ catcher foil. At the end of the bonbardments the growth and decay of the spontaneous fission activity in both folls was followed in imization chamber fission-alphe-beta counters.

Figure 2(a-d) shows plots of the spontaneous Fission count rate versus time after the end of pombardment for the reactions $15 \mathrm{~N}+249 \mathrm{Bk}$, For the reactions $160+2480 \mathrm{On}$ and $\mathrm{I}_{2} \mathrm{C}+2 \mathrm{C}_{\mathrm{C}} \mathrm{C}$, good decay curves are not available. Hower, spontanerous fission councing of the stopper folls fFon $160+248$ Gombardments suggest direct $250 \mathrm{~s}$ product ion. The rates of alrect fonnation of $256 \mathrm{jed}$ and $255 \mathrm{Fm}$ in those cases where decay curves were available were determined by a least square fit to the data using the standard growth and decay formulas. 2 Thus knowing the rates of formation, the bean intensities and target thickDesses, the cross sections to produce $256 \mu$ and 250 were caldulated.

Excitation functions have been measured for reactions $15 \mathrm{~N}+249 \mathrm{Bk}, 12 \mathrm{C}+252 \mathrm{Cf}$, and $11 \mathrm{~B}+253 \mathrm{Fg}$ (Fig. 3). However, an excitation function for 25 南 production in the reaction $160+2480 \mathrm{~m}$ has not been dneasured; thus the cross section corresponding to 94.5 ket could be $2-3$ times lower than the peak cross section.

Figure 3(a-c) shows a trend toward decreasing width in excitation function as the atoract nuber of the target nucleus approaches that of $\mathrm{Mt}$ and $F \mathrm{~m}$. A 10 $\mathrm{HeV}$ decrease in the width of the excitation finction jo observed in moving from a $249 \mathrm{Bk}$ target to an 25.Es target.

Figures 4 (a) and 4(b) display the angular

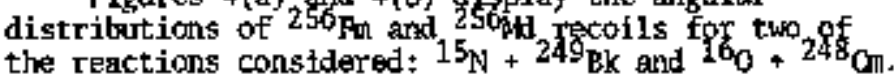
Considerable forward peaking is observed in each case. However, interpretation of these angular distributions is compligated by the fact that thick targets of $-0.5 \mathrm{mg} / \mathrm{chl}^{2} 249_{\mathrm{BK}}$ and $\sim 1 \mathrm{mg} / \mathrm{cm}^{2} 24 \mathrm{~g}_{\mathrm{Co}}$ were used.

Figure 5 is a plot of the total cross section for making $256^{\circ}$ spontapers $f$ ission activity (both by direct formation of $250 \mathrm{Frn}$ and direct formation of $250 \mathrm{~h}$ ) versus the distance in units of $z$ fron the target muclous $\left(z_{T}\right)$ to $k d$ (where $z_{p}$ a atomic number of the product). The epror bex for the point corresponding to the reaction $1 \mathrm{O}^{+}{ }^{248} \mathrm{Cm}$ is Iarge enough to include the possibility that all of the observed spontaneous fission activity is due to the direct formation of 256fut. Figure 5 shows that the cross section falls off at the rate of roughly an orier of mabnitude for each additional unft of $z$ away from $M$. The cross sections for these reactions used in Fiv. 5 , except $160+24 Q 0$ m, were those at the peaks of the respective excitation functions.

The ilmortance of the target and projectile thass nitbers, independert of $z_{p} \cdot z_{\tau}$, can be seen in the 256 min cross sections for the reactions $13 \mathrm{C}+249 \mathrm{Cf}$, $12_{C}+252_{C f}$, and $13_{C}+252_{C f}$. These cross section ratios are respectively $1 \times 10^{-} 3,1$, and 2 .

In the system $174 \mathrm{Mov} 22 \mathrm{Ne}+232 \mathrm{Th}$, Artukt et a1. 1 measured the yield of various projectile-like light fragments in the exit chamel after a multimuleon transfer reactiog had taken plece. In order to correlate the $2 \mathrm{Ne}^{2}+232 \mathrm{Th}$ results to tho 

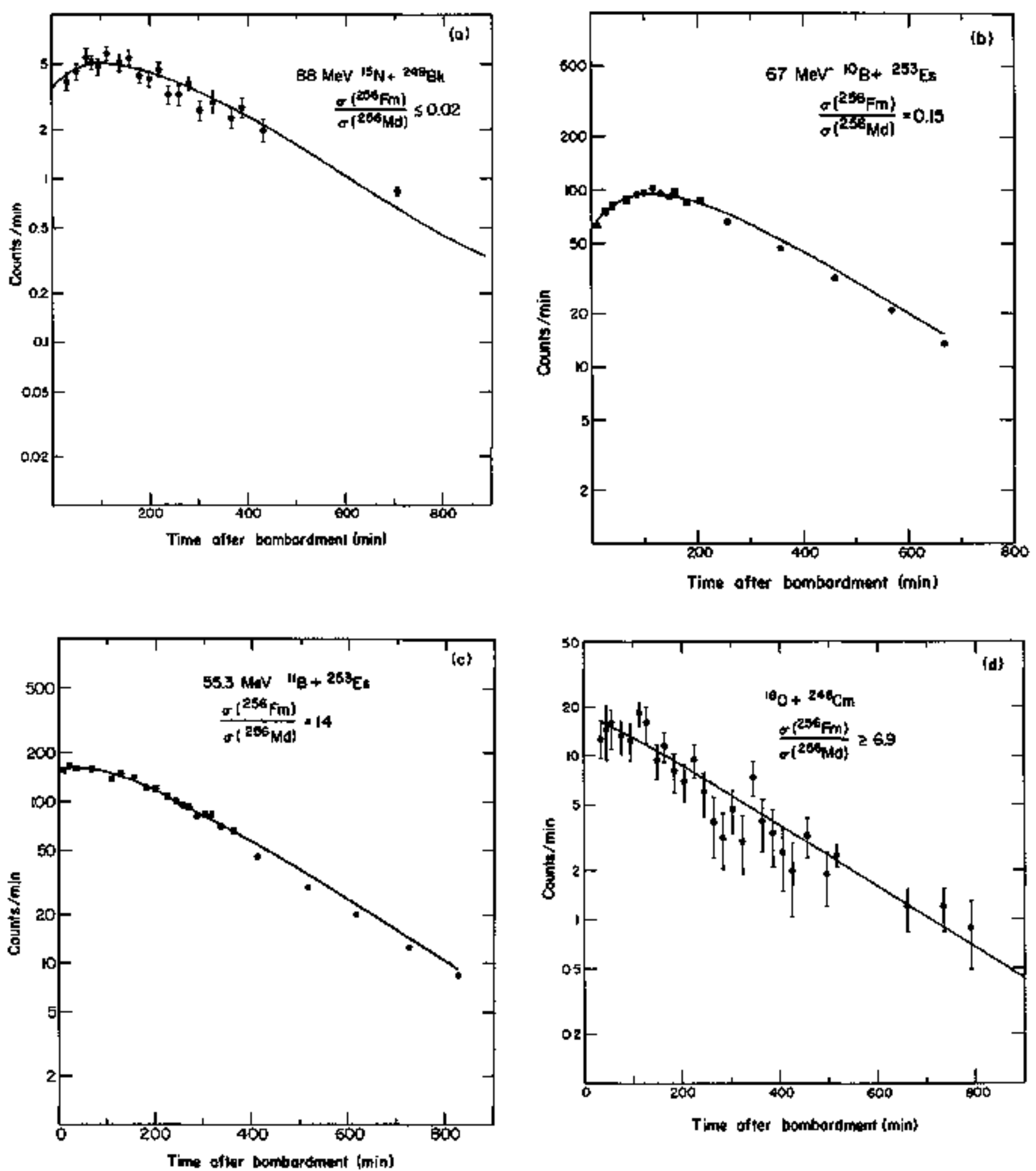

Fig. 2. Spontaneous fission decay cyrves for ${ }^{256} \mathrm{Fm}$ produced in the reactions (a) $15_{\mathrm{N}} \rightarrow 249 \mathrm{gk}$, (b) $10_{\mathrm{B}+}$ $25 \mathrm{E}_{5}$, (c) $1 \mathrm{1}_{\mathrm{B}}+25 \mathrm{~F}_{\mathrm{E}}$, and (d) $18_{\mathrm{O}}+24 \mathrm{8}_{\mathrm{Cm}}$.

(XBL $766-8580,-8226,-8227,-8228$ ) 

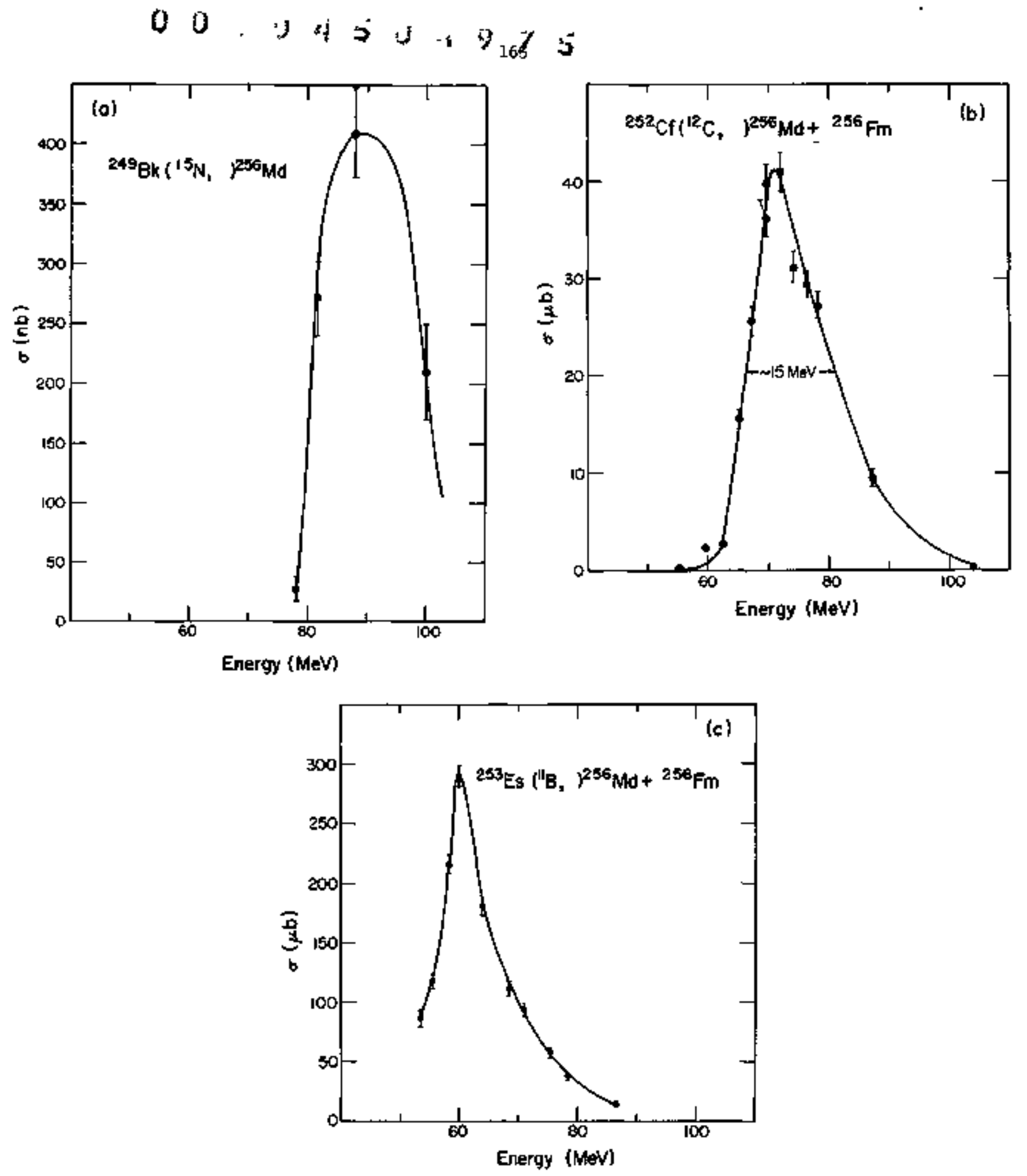

Fig. 3. Excitation functions for the restiong

(a) $1 \mathrm{~s}_{\mathrm{N}}+24 \mathrm{I}_{\mathrm{Bk}}$, (b) $\mathrm{1}_{\mathrm{C}}+2 \mathrm{2}^{2} \mathrm{CE}$, and (c) $1 \mathrm{H}_{\mathrm{B}}+25 \mathrm{E}_{\mathrm{Es}}$. 


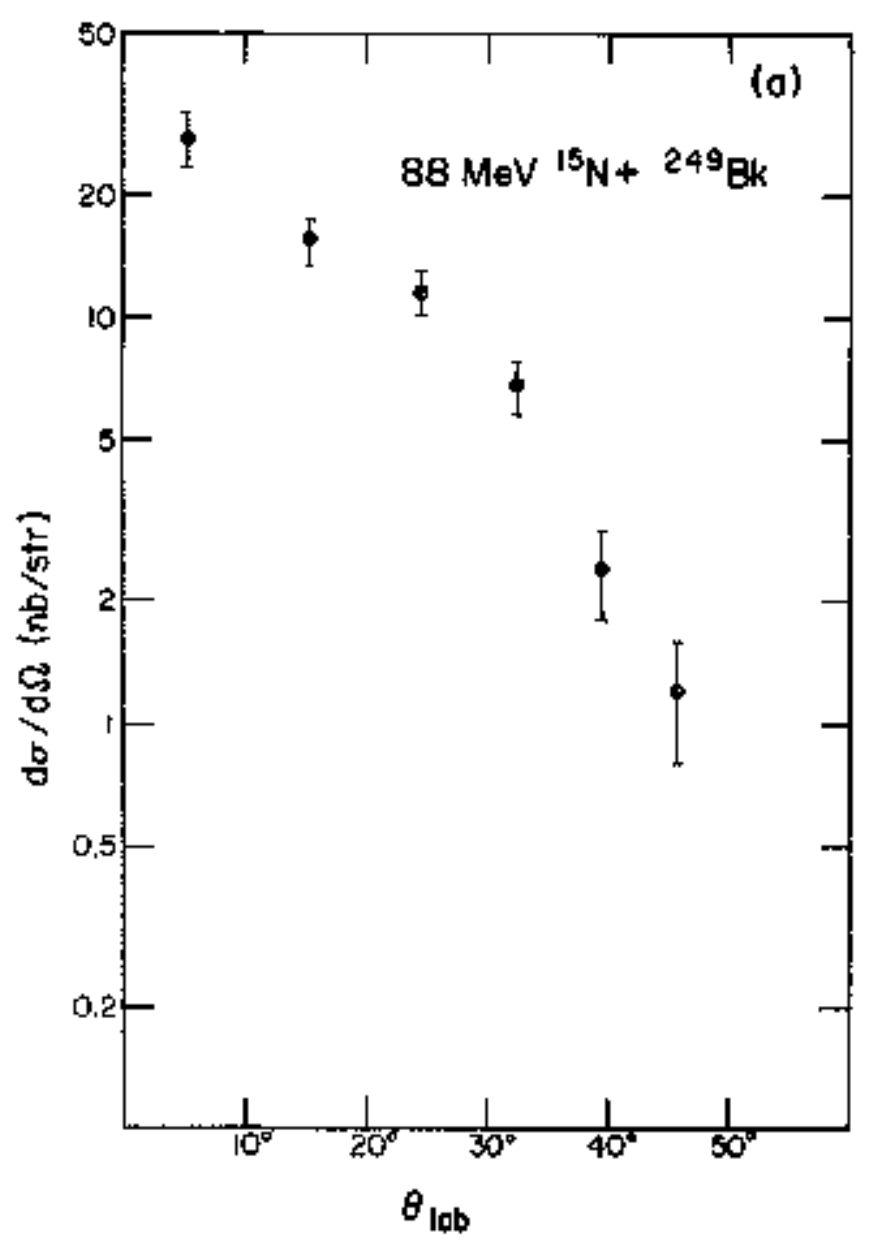

Fig. 4(a) Angular distribution of ${ }^{256} \mathrm{Fing}$ gontaneous fission activity from the reaction $15 \mathrm{~N}+249 \mathrm{Bk}$. Fig. 4(b) Angular distribution of $256 \mathrm{Fr}$ spontaneous fission activity from the reaction $160+240$.

(XBL 766-8330, -8331)

Fí. 5. Plot of production cross sections (represer.ted lo sokjd dots to make 25 hid and $256 \mathrm{Fra}$ in the compound systenl 264104 vs $\mathrm{z}_{\mathrm{p}}-\mathrm{z}_{\mathrm{T}}$, where $\mathrm{zp}_{\mathrm{p}}=$ I:Tojiact atonic nuglser $\left(Z_{p}=100\right)$ and $Z_{T}=$ target atomic number i left band scale in nanobarns). The rroduction cross sextions (represented by triangle shapal points) for the light fragment vs $z_{p}-2_{T}$ for the reaction $22_{\mathrm{Ne}}+232 \mathrm{Th}$ by Artukh ot al are also shown; for this reaction $Z_{p}-Z_{T}$ numbor of notons lost by the projectile, presumably the rumber of protons picked by the target (right hand scale in $1, b / s r)$.

(Ẍ) $766-8332$ )
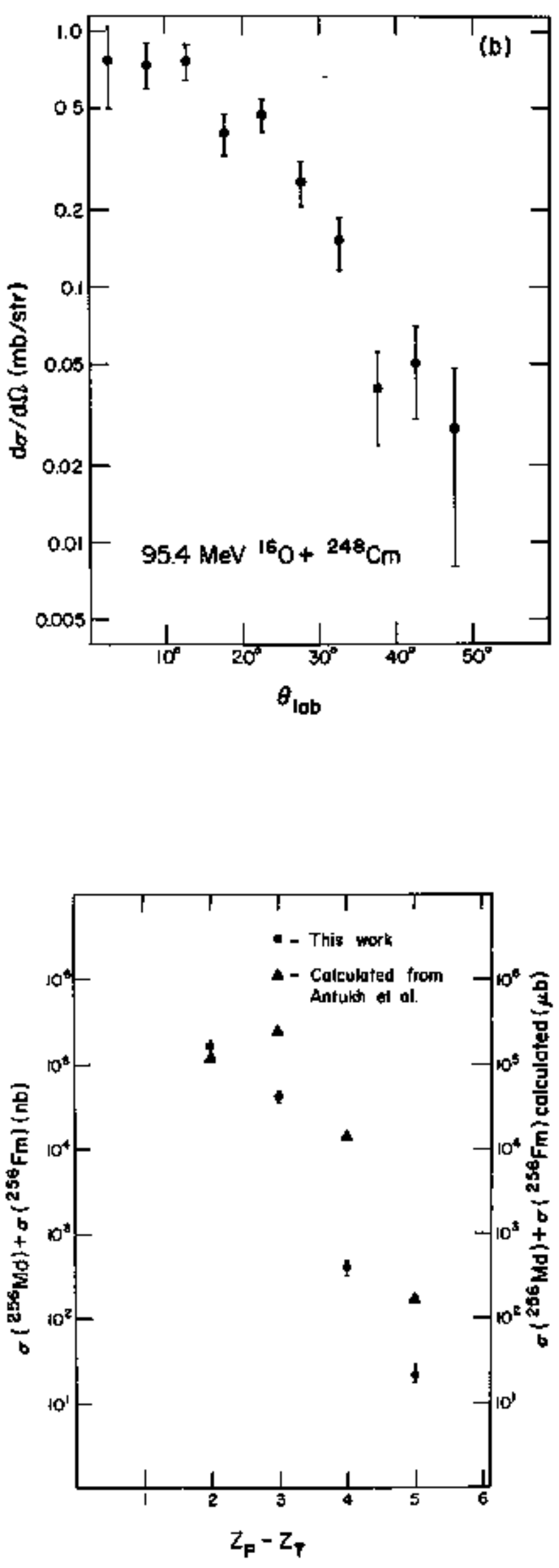


\title{
$00, y+j, y_{165} 7$
}

${ }^{256}$ Fu yield data, we deteruined the number of protons and seutrons which had to be picked tw by our target nuclei to produce $256_{4 d}$ and or ${ }^{2}{ }^{5} \mathbf{F}_{n+}$ The cross segtion (or cross sections when both 25 hkd and ${ }^{256}$ Fn were formed directly) for the light fraguent that corresponds to the same number of protons and neutrons lost by the light fragment,projectille was idontified in the data of the $2{ }^{2}$ ke $+{ }^{23}$ Th weartion. These cross sections (or the weighted average of two cross sections based on the relative production tates of 256 id and ${ }^{256} \mathrm{~F}_{\mathrm{n}}$ ) wers then plotted versus $\mathrm{z}_{\mathrm{p}}-\mathrm{z}_{\mathrm{T}}$ in Fig. 5. The rate of decrease of the yield of these light ions with $\mathrm{z}_{\mathrm{p}}-\mathrm{Z}_{\mathbf{T}}$ is simllar to the rate of decrease of the yleld for 25 bud and $/$ or $25 \mathrm{~F}_{\mathrm{F}}$ with $z_{\mathrm{p}}-z_{\mathrm{T}}$. This providos somo evidence that the mechenisn for producing the abserved 256 Fin activity is the save as the mechonism Artukh et al. observed for the production of nuclides lighter than the projectile in the reaction $22_{\mathrm{Ne}}+232 \mathrm{Th}$, which they called multimucleon transfer or deep inelastic processes.

From the magnitude of the cross sections observed here, one cm argue that tho nechanism involved in the production of $256_{F}$ and 256 m must be some type of transfer reaction. Cross sections for compownd rucleus evaporation reactions in this reaction of heavy nutlei are typically mach lower than for transfer reactions.

The large cross sectigns and the decreasing trend in the yield of the $2 \$ 6 \mathrm{k}$ and $256 \mathrm{gm}$ cross sections as $z_{p}=z_{T}$ increases, suggest that these products are prodiced in a transfer reaction mechanișn. The many kinematic studles of the light fragbents produced in similar heavy ion reactions have shown that there is a rapid transition frosa the quasi-elastic transfer mechanism to the multimacleon transfer nechanisu for products a few $Z$ units away from the target. Thus, wo might expect that the final ${ }^{256} \mathrm{Fin}$ act $11_{\mathrm{B}}+25 \mathrm{C}_{\mathrm{ES}}$ reaction is primarily fron the multi- nucleon transfer reaction. Consistent with this

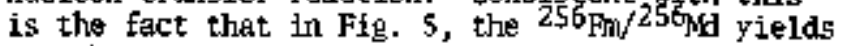
art about 1000 times less than tho corresponding Iight fragment yields from the $22 \mathrm{Ne}+232 \mathrm{Th}$ reaction,

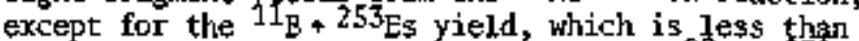
100 trues smaller than the corresponding $22 \mathrm{Ne}+232 \mathrm{Th}$ light fragment yield. This is consistent with the fact that the fisslon competition following multinucleon transfer is very large because of the low fission barriers and ligh excitation energies of the heary actinide fragments produced in these reactions.

Two factors could contribute to lower excitation errergy and thus less fission compecition in

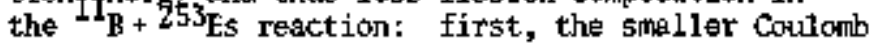
barrier with a lower $Z$ projectile and, second, the role of quast-elastic transfer as the most important transfer mechanism rather than deep inelastic scattering.

The increasing width in the excitation functions can be understood in terms of the nuber of nucleons transferred to the target. In these reactions to produce 256 Fu and 256N, the light projectiles such as $1 l_{B}$ and $12_{C}$ transfer only few mucleons, while heavier particles such as $15 \mathrm{~N}$ trinsfer thore mucleons. This is consistant with the observation that alpha particle induced reactions have narrow excitation functions while heavy ion induced reactions have broader excitation functions.

\section{References}

1. A. G. Artukh et a1., Nuclear Phys. A211, 299309 (1973).

2. Friedlander, Kennody and Miller, Nuclear and Radiochemistry, John Niley \& Sons, kew York, 2nd e्dition, Pp. $78-79$ (1964).

\section{A RADIOCHEMICAL STUDY OF THE MASS YIELD DISTRIBUTION FROM THE REACTION OF $25.2-\mathrm{GeV}{ }^{12} \mathrm{C}$ IONS WITH NATURAL URANIUM}

\author{
R $\mathrm{d}$ Otlo, $M$ M Fowler:" I Binder. \\ ¿ Lee, and $G \mathrm{~T}$ seabor
}

An analysis of the measwed partial cumblative and independent yiejld cross sections from the reaction of 25.2-Gev 12 $\mathrm{C}$ with wanim has been made. The bortbardment consisted of a four-hour 1rradtation of a stgck of three 5 -mil uraniun metal foils at $\sim 6 \times 10^{9}$ particles/minute. The intersected beam. area of the second foil was cut out, dissolved and a radiochemical group separation schene carried out. Sever chemtcal fractions were obtained. Ganmta-ray counting and analyşiș was done yielding cross sections for ower 60 muclides.

Iue to the combined target thickness, a Iarge fission product contribution ma have resulted from secondary particles inducing intermediate enorgy fission of the uratiun. The observed fission products were grouped tnto four regions and a two gaussian charge dispersian description fitted to the data.

In Figs. 1(a) through 1(d), the final two gattssian charge dispersion arrived at is shown. Independent yield croșs sections shown in Fig. 1(a-d) were calculated for all nuslides in this region using the charge dispersion description arrived at by an itorative fitting process and the growth and decay relationships for the observed nticlidies. This method works well for regions where the comulative yfelds represent 508 or less of the isobaric yield, as for the neutron-deficient nuclides. The procenture is less accurate for the neutron-excessive yields where greater than 90 of of the chain yield is represented in the cross section of a single nuclide. 

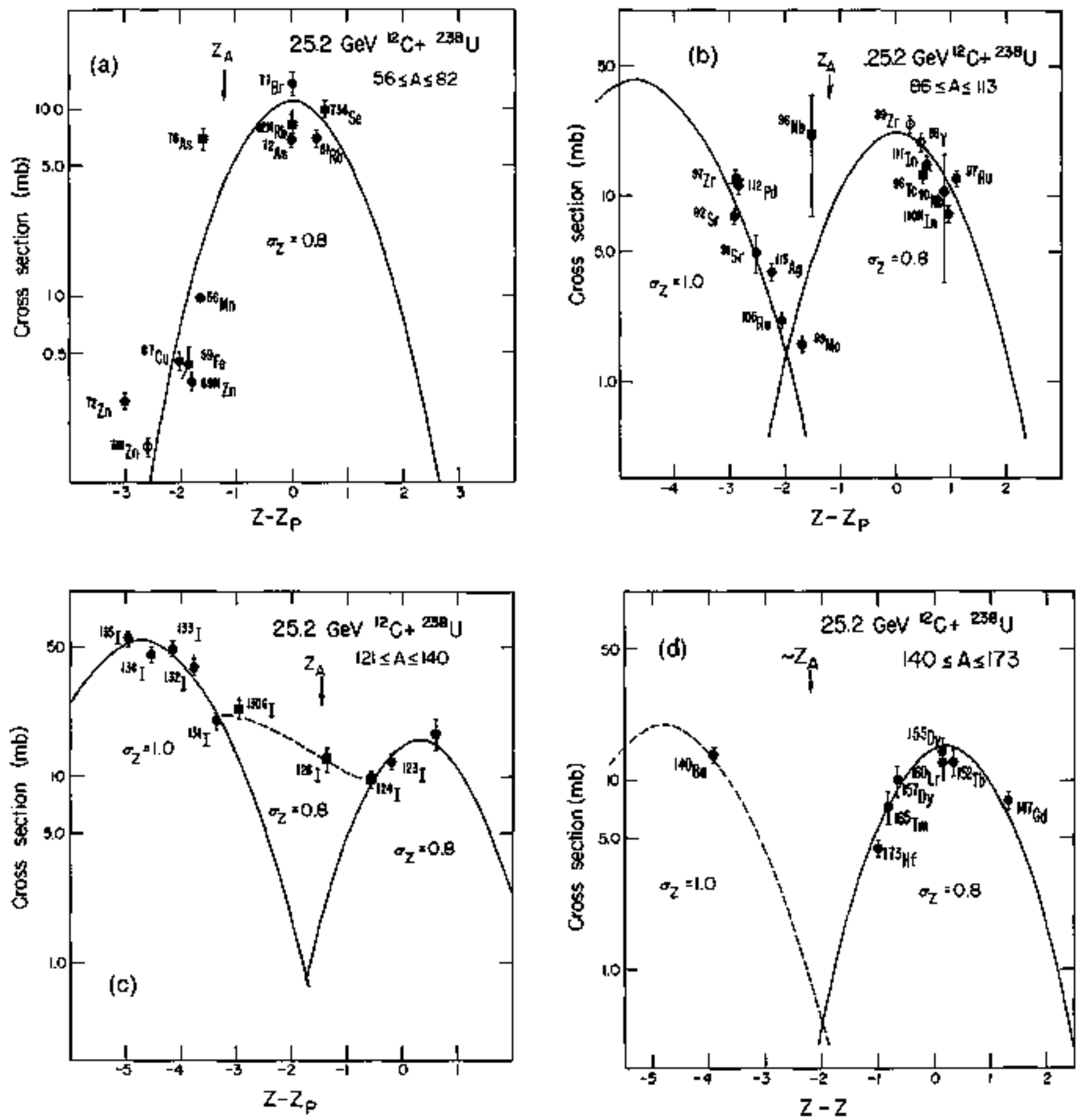

Fig. 1(a-d). Calculated and experimental independent yield cross sections in millibams for the mass regions sliow in the upper right plotted as a function $Z$ - $2 p$. Where $z p$ is the most probable charge for the mass chain $A$, and 2 is the charge of the experinentally observed nuclide. The cross sections show " as solid circles are independent yield cross sections calculated from the observed partial cumlativo yield cross section. The cross sections shuwn as solid squares are experimentally mansured independent yields. $\mathrm{Z}_{\mathrm{A}}$ indicates the positions of the valley of beta stability in each of the mass regions. Neutron deficient nuclides lie to the right of $z_{A}$ in this figure. The symbol $\sigma_{z}$ represents the geussion width parameter defining the charge dispersion curves (see Ref. 2)

(XEL 766-9248 to $766-8251$ ) 


\section{$05 ; 34 ; 3 ; 777$}

Figure 1 (c) shows the calculated independent yields for the jotine isotopes in the mass reglon $A=121$ to $A=135$. The two gausslan charge dispersion description in Fig. l(c) is not consistent with Gev proton results 20 this region. 1 Howerer, the cross sections for $130_{I}, 126 I$ and $124_{I}$ are experimentally detemined indefendent yields. The dotted line indicates a neutron excessive component thet hould not be the result of low energy fission from secondary reactions. If the dotted line is included, the overall shape of the charge disperșion may be consistent with the GeV proton on uranium results. In the mass region $A=56$ to $A=82$, Fig. 1 (a), $A$ single newtron-deficient charge dispersion curve was obtained. A gausstan width parameter ${ }^{2}$ of $\sigma_{2}$ of 0.8 best fit the neutron-deficient yields for all four mass regions. The neutron-excessive yields were fittest to a value of $\sigma_{z}=1.0$.

The final $z_{\mathrm{F}}$ functions for the region of nass number 56 to 176 are shown in Fig. 2. The neutron-

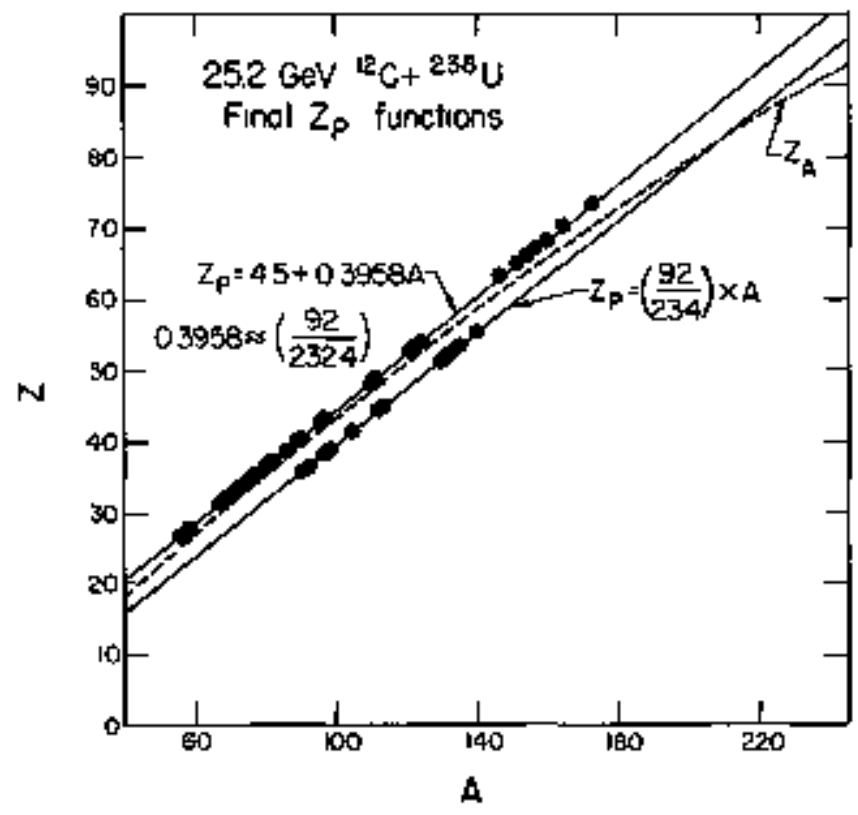

Fig. 2. plot of $\mathrm{Zp}$ functions for the reaction of $25.2 \mathrm{GeV} 12 \mathrm{C}+238 \mathrm{Uf} 2 \mathrm{z}$ is the most probable charge for each mass chain $A$. The dotted line labeled $Z_{A}$ corresponds to the cherge at the center of the valley of beta stability. The solid circles are the experimentally determined $z p$ values. The two Zp functions correspond to the neutron-deficient charge distribution (left of curves) and the charge distribution for the nextron-excessive products (right of curves).

(XBL $766-8252$ )

deficient yields rere fitted using the modified UCD rald whosn in Fig. 2 (upper left). Thet partial cumulative and independent yields for all nuclides except those identified as fission prohucts from secondary induced fission of uranium are show in Fig. 3. rields for products pear the mass of the target ( ${ }^{2} 3 \mathrm{~Pa}, 239 \mathrm{pp}$, $\left.236 \mathrm{kp}, 23 \mathrm{U}\right)$ probably were formed in secondary reactions.

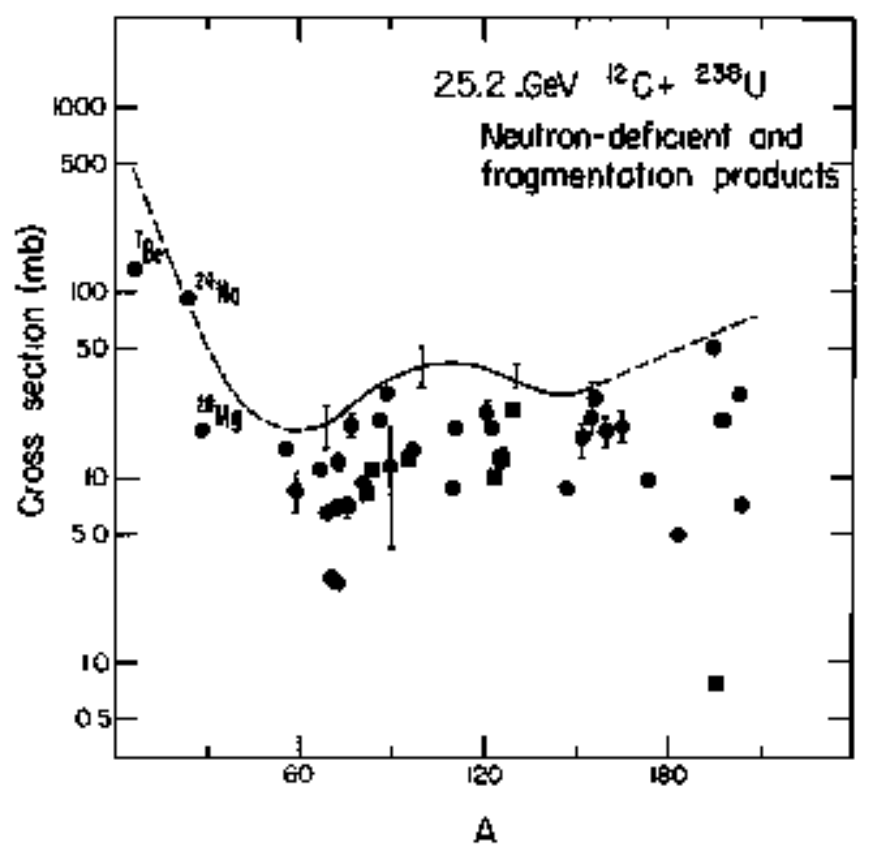

Fig. 3. The mass distribution for $25.2 \mathrm{GeV} 12 \mathrm{C}+$ $23 \mathrm{U}_{\mathrm{U}}^{+}$. The solid circles are the partial cumulative and independent yields of the neutron deficient and frapmentation products. Those ruclides identified as flssion or evaporation products from the reaction of secondary particles with uranium are not shown. The solid line represents the integrated isobaric yield in the mass region of $k=60$ to $A=150$.

(XBL $766-8253$ )

The solid line in this figure estimates the isobaric yields over the region $A=60$ to $A=150$ and was obtained by simming the four chare dispersion curvas for the neutron-deficient yields. The total cross section under this curvo is $2900 \mathrm{mb}$. Since a broad binary fission distribution is the dowinant feature in this region, part of the 2900 mb should be divided by 2 . Assuming a flat non-fission distribution of $10 \mathrm{mb}$ between $A=60$ and $A=150$, a total cross section of approxintely 2 barns would be divided evenly between binary fission and the nonfission mass distribution.

Nuclides were identified with mass above $A=180$, but their cross sections did not conform to the $z p$ function that fit most of the data. The mass distribution is uncertain in this region and sinown as a dotted line in Fig. 3.

Three product cross sections were measured in the fraguentation region: $7_{\mathrm{Be}}, 24 \mathrm{Na}_{\text {, and }} 28 \mathrm{H}$. Mithough the measured beam intensity ${ }^{3}$ is believed to be known gnily to within a factor of 2, the cross section for 24 production is much larger for the carbon on uraniwn reaction than for the GeV proton. on uranium reaction. 4 At $2.1 \mathrm{GeV} / \mathrm{macleon}$, the ratio of cross sections is thixty $\left[\sigma_{(\mathrm{C}+U)^{f o}}(\mathrm{P}+\mathrm{U})=30\right]$.

\section{Footnotes and References}

"Fresent address: Los Alamos Scientific Laboratory, Iniversity of Califomia, Los Alamos, New Mexico 87545 . 
1. Yu-Wen Yu and N. T. Porile, Phys. Rev. C7, 1597 (1973).

2. The charge dispersion curves were assumed to have the following functional form:

$$
P_{(z)}+\frac{P_{A}}{\sqrt{2 \pi} \sigma_{z}} \operatorname{ex\varphi }\left(\frac{-(z-z p)^{2}}{2 \sigma_{z}^{2}}\right)
$$

where ${ }_{2} p$ is the nost probable charge for the mass chain $A$ and $P_{A}$ is tine integrated chain yield for the isobar witll nass $A$, and $\sigma_{2-}$ is the aussian width parameter.

3. The bean was monitored using an ionization chamber. We would like to thank S. S. Narkowitz and D. Wrohy for their experimental determination of the average bean intensity.

4. A. M. Poskanzer, G. H. Butler, E. K. Hyde, Phys, Rev. C3, 882 (1971).

\title{
RECOIL RANGES OF HEAVY PRODUCTS IN THE QUASI-FISSION REACTION
}

\author{
A I OHo. M M Fowler'। Buder. \\ $D$ Lee. and $G T$ Seaboro
}

The recofl range distributions for the elements hafnituon to astatine produced fron heavy ion reactions of krypton and xenon with gold, bișulth and uraniom targets have been measured. These elements are part of the quasi-target mass distribution for the uranitum reactions, the mass distribution coliopuially itferred to as the "goldfinger." 11,2 They also include the quasi-elastic transfer products in the reactions with gold and bismuth targets. The results of these studies have lent support to the identification of the "goldfinger" as the remnant reass distribution of the quasi-uranium products.

Kinomatic analysis of the quasi-projectile projects in the quasi-fission or deep inelastic reaction of $\mathrm{Xr}_{\mathrm{T}}$ with $\mathrm{Au}^{3}$ and $\mathrm{Bi}^{4}, 5$ targets demonstrate several important characteristic features of the quasi-fission or the deep inelastic mechanism. First, the energies of the 1ight and heavy fragments correspond to the Coulont repulsion energy of two touching spheroids. Second, the angular distribution of the light fragments shows a definite sidetrays peaking. This sjdeways peaking effect disappears as the charge and mass of the 1ight fragment becomes increasingly farther aray from the original charge and mass of the projectile. Based on these observations, a tho-step reaction madel has been used to andlyze and correlate the measured recoil range distribution of the hedvy complementary elenents with the light particle kinomatic data on quasi-f1ssion and quasielastic transfer reactions.

A stacked foil arrangewent was used. $2^{\text {The }}$ targets consisted of approximately $2 \mathrm{mg} / \mathrm{cm}^{2}$ gold leaf, 3-4 $\mathrm{mg} / \mathrm{cm}^{2}$ bismuth metal on aluminm backing, and approximately $1 \mathrm{mg} / \mathrm{or}^{2} \mathrm{WF}_{4}$ on alumimm backing. These targets were placed directly onto and in front of a stack, typically ten or more, of 1.0 to $1.1 \mathrm{mg} / \mathrm{cm}^{2}$ aluminum recoil catcher foils. Following irradiation with $86 \mathrm{Kr}$ or $136 \mathrm{ke}$ ions at the SupertIILAC, the catcher foils were reroved, separated and individually counted for $x$-rays. The decay of the abserved $x$-ray peaks was followed in one of the more active foils.

Figure $I(a-c)$ chows typical $x$-ray spectra fopm experiments of ${ }^{80_{\mathrm{Kr}} \text { with }} 17_{\mathrm{Au}}, 209_{\mathrm{Bi}}$ and $23 \delta_{0}$ targets. Each of the $x$-ray peaks in the spectra is a combination of the $K a$ line of the element written above the peak in Fig. $1(\mathrm{a}-\mathrm{c})$ and the $\mathrm{K}_{\mathrm{r}}$ line of the element of the next higher 2. Since the relative intensity of the $\mathrm{Kox} 1$ and $\mathrm{Ka} \mathrm{a}_{2}$ lines is 2 to 1 , gamma-ray contributions in the $x-T a y$ peaks resulted in unacceptable intensity ratiog and were easily recognized.

The recoil range distributions were determined from the $x$-ray data by determining the area under each $x$-ray peak and correcting for decay. The results of this analysis are plotted in Figs. 2 and 3. The most striking feature is the similartty of these differential recoil range distrdbutions among the elements for a given bombarding energy and reaction. The simflarity spans a much broader region of elements than would be expected for the span of elements contributing to a single recoil range distribution by growth and decay. Thus, these curves are somewhat independent of the growth and decay relationships and rofloct the kinenatic properties of the fragments prodused and observed in these heavy ion reactions.

The majority $(50-805)$ of the activity of each of the reaction products for the krypton reactions was found in just two of the $i \mathrm{mg} / \mathrm{cm}^{2}$ recoil foils.

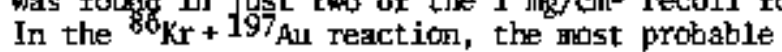
range of the products increases as the bombarding energy decreases. The have been able to reprofice these experimentally measured recoil range distributions and this trend of increasing renge with decreasing energy using the two-step quasi-fission model and the shift to larger angles of the peak in the angular distributions as the bombarding energy decreases, seen for the $\mathrm{Kr}+\mathrm{BI}^{5}$ and $\mathrm{Kr}+\mathrm{Pb}^{4}$ reaction. Since the focused angular distribution of the quasi-krypton also results in a complententary focused angular distribution of the quasi-targot prothist, the peak of the quasi-krypton sust move to larger angles in the center of mass system as the bombarding energy decreases. Thus, the quasttarget products most move to smaller angles (closer to $0^{\circ}$ ) resulting in longer axial ranges exactly as observed.

The recofl ronge distrlbutions from the $\mathrm{Kr}+\mathrm{U}$ reaction shows the same general shape as the $\mathrm{Kr}+\mathrm{Au}$ 


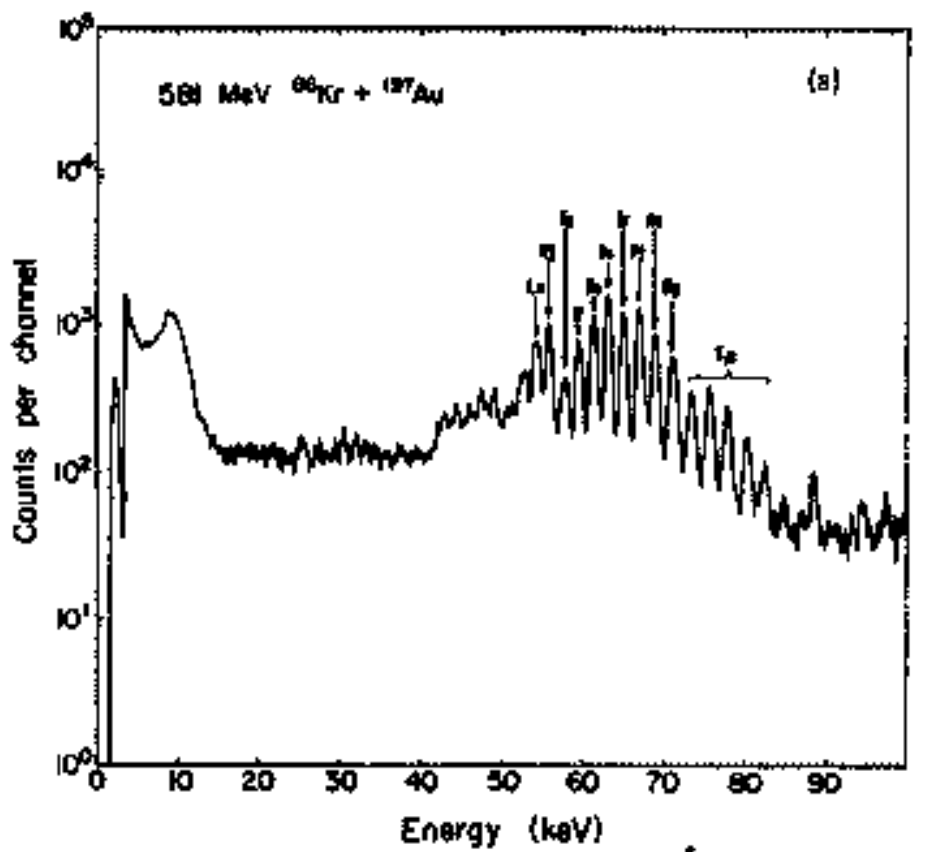

Fig. I $(\mathrm{a}-c) . X$-ray spectra from the $1 \mathrm{mg} / \mathrm{cm}^{2}$, nt recoil catcher fails for the reactions shown. Each peak is composed of the Kol line from the element witten above the peak and the $\mathrm{K}_{2}$ line of of the element one $z$ higher.
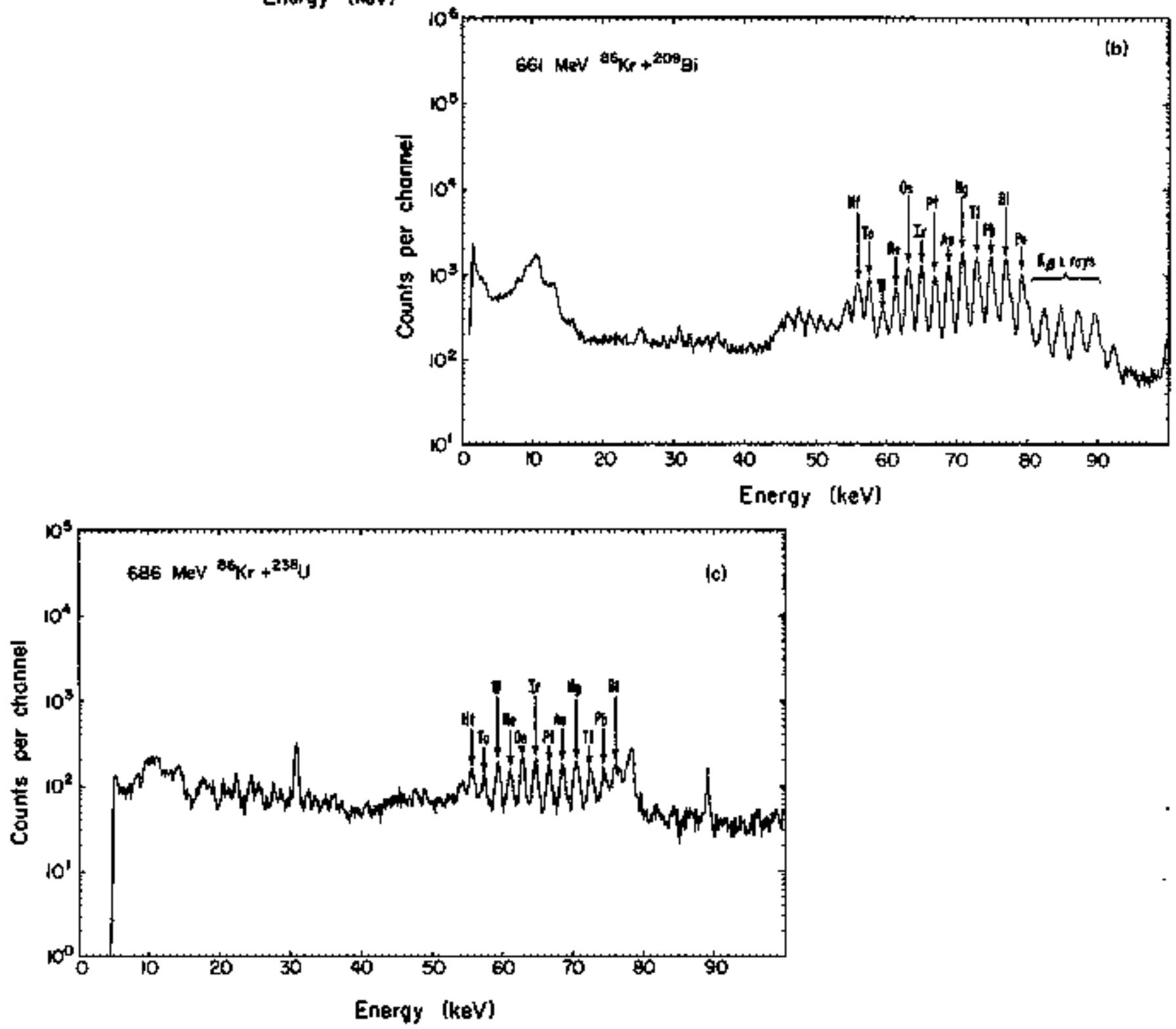


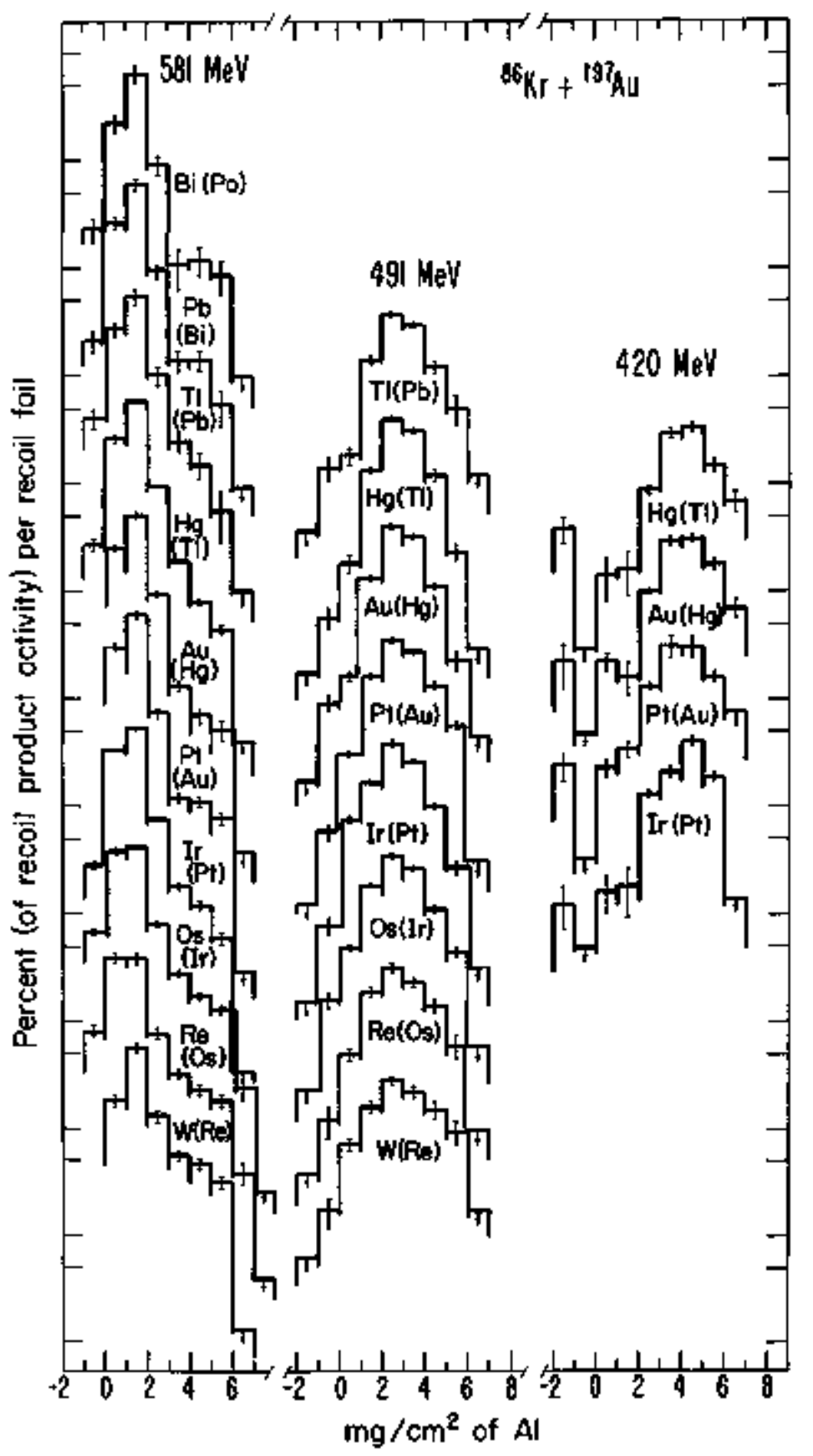

Fig. 2. Recoil range distributions from the analyzed $x$-ray spectra from the reaction of $86 \mathrm{kr}$ with $197 \mathrm{Au}$ at $581 \mathrm{MeV}, 491 \mathrm{MeV}$ and $420 \mathrm{MeV}$. (XBL 766-8286) 


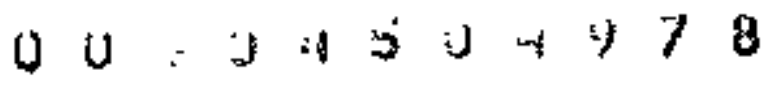

171

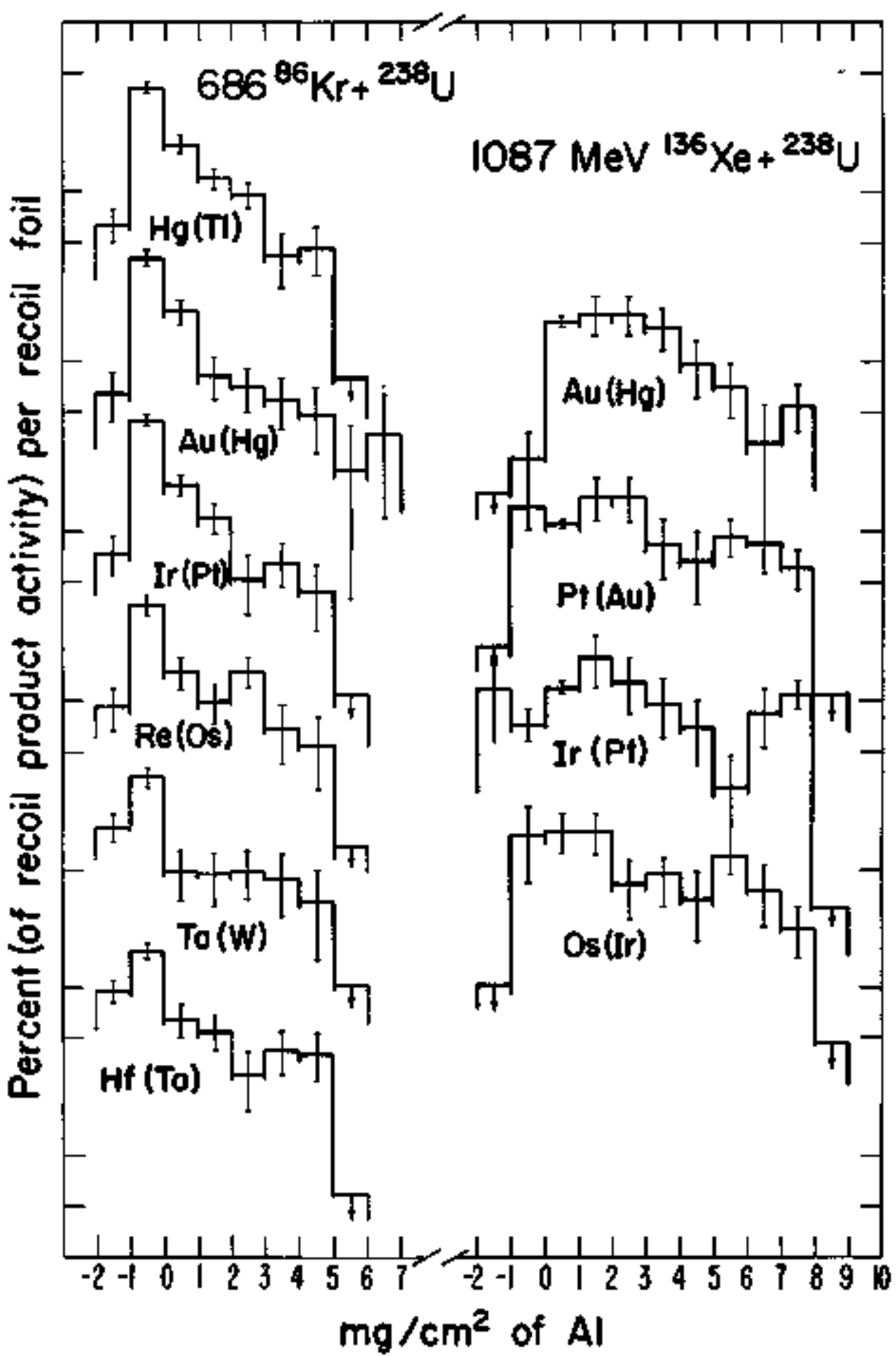

Fig. 3. Retoil range distributions from the analyzed $x+$ ray spectra from the resctions $686-\mathrm{kteV} 86 \mathrm{Kr}$ with

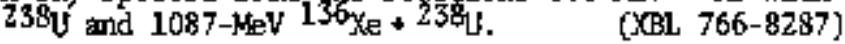


results. The quasi-uranium and therefore quasikrypton reaction products must have the same sideway's peaking in their angular distribution. Sideways peaked angular distributions, used to calculate the recoil distributions, reprohuced the observed recoil range distribution for the $686-\mathrm{MeV} 86 \mathrm{Kr}+238 \mathrm{U}$ reaction.

We found that our calculations pretict differential recoil range distributions that are distinctly different for different angular distributions. A sula11 $1 / \sin \theta$ component in the angular distribution causes a significant fraction of the activity to have the makimm range corresponding to alignntent of the center of mass and fission velocity vectors. No evidenge for such a component can be sem in the $586-\mathrm{MaV} 8{ }_{\mathrm{Kr}}+197_{\mathrm{Au}}$ reaction, although a difference in the long-Tange part of the recoil range distributions would have been seen with as little as 50 of the mechanisu having a $1 / \sin \theta$ conponent.

In the ${ }^{86} \mathrm{Kr}+{ }^{238} \mathrm{U}$ and ${ }^{136_{X e}}+{ }^{238} \mathrm{U}$ reactions, there is some evidence for a fractional part of the mechanism to have $\notin 1 / \sin \theta$ component, but at least 800 of the $K r+U$ recoil distributions can be accounted for by using quasi-fission angular distributions sigilar to the $\mathrm{Kr}+\mathrm{Au}^{3}$ and $\mathrm{Kr}+\mathrm{Bi}^{4}$ angular distributions. The assumption of total darping in the entrance channel is not strictly adhered to in xemon heavy 10 n reactions and this will affect the recoil range distribution for the $\mathrm{Xe}+\mathrm{U}$ reaction. We would like to point out that a 1/sine angular distribution iș a necesșary but not sufficient condition of conpound nucleus formation followed by fission, and it may only reflect noclear orbiting of the quasi-projectile fragment.

As a test of our model to proxtuce colsputersimulated recoil range distributions, we have used the neasured angular distributions of the complementary light fragment from a study of $620-4 \mathrm{kV}$ $80 \mathrm{~K}^{+}+197 \mathrm{Au}^{3}$ to predict the recoil range distributions of the corplententary quasi-gold products for the reartion of $586 \mathrm{Mov} 86_{\mathrm{Kr}}+197 \mathrm{Au}$. The measured angular distributions and calculated recoil range distributions are shown in Fig. 4, The agreenent between the experimental (Fig. 2) and the calculated (Fig. 4) range distributions is falrly good for the low ranges and exceptionally good for the longer range part of the distribution. It should also be pointed out that no nomalizations of the percent activity in each foil has been made. One question that arises is why the range distributions for elements with the same $Z$ as the target do not show strong evidence for quasi-elastic transfer. The answer, we believe, lies in the fact that were looking at $x$-rays relatively soon after the end of the bombardment (typically 2-4 hours). Our greatest sensitivity by this method is for the shorter-1ived neutron deficient nuclides that are primarily produced in the quasi-fission reaction.

\section{Footnotes and References}

*Present address: los Alamos Scientific laboratory. thiversity of Califormia, Los duamos, New Mexico 87545 .

1. R. J. Otto, M. M, Fowler, D. Lee and G. T. Seabors, Phys. Rev. Lett. 36, 135 (1976).

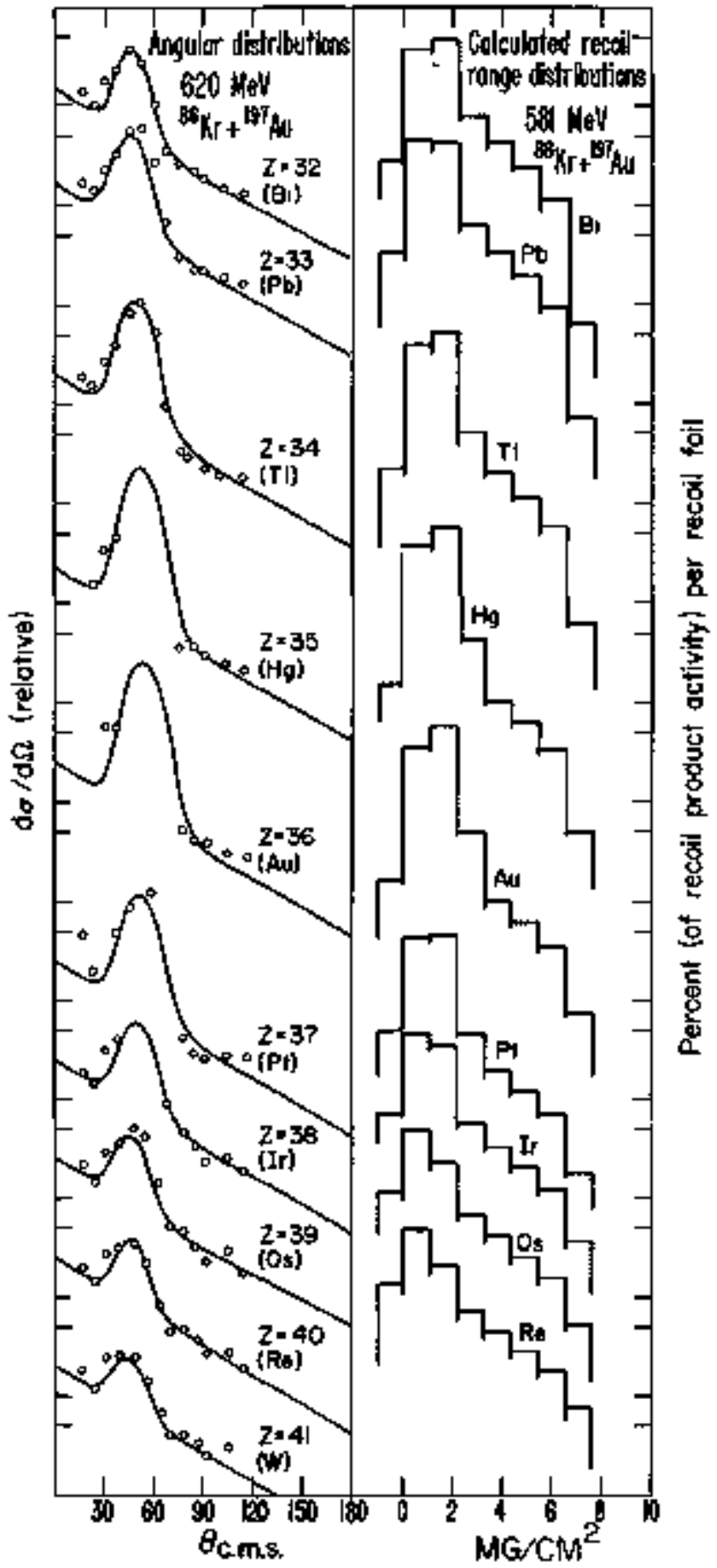

Fig. 4. Experimentally determined angular distributions for the quast-krypten produts from 620-MeV

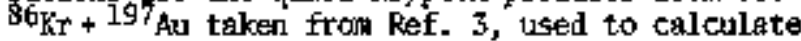
the corresponding recoil range distributions for the complenemtery guasingid products for the reaction 581 - KeV $86 \mathrm{Kr}+197 \mathrm{Au}$.

(XBL $766-8288)$
2. J. V. Kratz, A. E. Norris and G. T. Seaborg, Phys, Rev, Lett. 33, S02 (1974).

3. L. G. kbretto, B. Garvin, P. Glässel, R. Jared, P. Russo, J. Sventek and G. Wozniak, Phys. Rev. Lett. 36, 1069 (1976); and LBL-4362. 


\section{$00: 3453,179$ \\ 173}

4. R. Vandenbosch, M. F. Tebb and T. D. Thongs, Phys. Rev. Lett. $\underline{36}, 514$ (1976).

5. K. L, Folf, J. P. Unik, J. R, Huizenga, J. Birkelind, H. Freiosleben and V. E. Viola, Phys. Rev. Lett. 33, 1105 (1974).
6. $I+$ Binder, M. M. Fowler, D. lee, R. J. Dtto and G. T. Seaborg, in this Ansual Report

7. J. R. Birkelund, J. R. Huizenga, H. Freiesleben, K. L. Holf, J. P. Ghik and V. E. Viola, Phys, Rev. Cl3, 133 (1976). 


\title{
C. RELATIVISTIC HEAVY IONS
}

\section{Projectile Fragmentation}

\author{
MOMENTUM DISTRIBUTIONS OF ISOTOPES PRODUCED BY \\ FRAGMENTATION OF RELATIVISTIC ${ }^{12} \mathrm{C}$ AND ${ }^{16} \mathrm{O}$ PAOJECTILES ${ }^{n}$
}

\author{
D E Greaner, P J Lindstrom, H H Heckmen. \\ Busce Cork, and F S Bieser $\dagger$
}

Recently wave published meastrenents of the rementum distributions and production cross sections of the high velocity fragments protuced by beams of $12^{\mathrm{C}}$ at 2.1 and $1.05 \mathrm{Gew} / \mathrm{max}$ leon and $10_{0} \mathrm{at}$ $2.1 \mathrm{GeV} /$ muclecm on a variety of targets.

The momentum and cross-section measurenents were performed using a single-fostwing magnetic spectroneter with a half-angle acceptance of 12.5 III about zero degrees. 2 Targets were $\mathrm{Be}, \mathrm{CH}_{2}, \mathrm{C}$. $\mathrm{Al}, \mathrm{Cu}, \mathrm{Ag}$ and $\mathrm{Pb}$. The charge and mass of the fragments were obtained by measuring thefr rigidtty (Pc/Ze), energy loss in solid-state detectors, and time-of-flight. Particle trajectories were determined with multiple-wite proportional chambers. The longitudinal and projected transverse momenta, $P_{\|}$and $P_{1}$, were obtained from the rigidity and direction of the particle at the focal plene of the spectrometer. The rigidity range was scanned in 0.1 GV steps from 0.8 to $10.2 \mathrm{GV}$ for the $2.1 \mathrm{GeV} / \mathrm{h}$ $12 \mathrm{C}$ and 160 beans and 0.2 to 6.3 GV for the $12 \mathrm{C}$ beam at $1.05 \mathrm{GeV} / \mathrm{n}$. Because the velocities of the projectile fragments are near the beam velocity, these rigidity ranges allowed us to observe all particles produced having a laess to charge ratio, $A / 2$, between 0.2 and 3.4 .

For each isotope the longitudingl-momentum distribution, in the projectile rest frane, was fit to a Gaussiam dependersce or $P$. The fitted variables are arplitude, central momentum, $\left\{\mathrm{P}_{\|}\right\}$, and standard deviation $\mathrm{TP}_{\mathrm{F}}$. Figure 1 illustrates the Gaussian fit and the variables $\left\langle\mathrm{P}_{1}\right\rangle$ and $\sigma_{\mathrm{P}_{1}}$ for the case of $10 \mathrm{Be}$ produced by the fragmentation of $2.1 \mathrm{GeV} / \mathrm{n} 1 \mathrm{C} C$ on a Be carget. The fits were restricted to the interval $-400 \mathrm{MeV} / \mathrm{c}$ to $+400 \mathrm{MeV} / \mathrm{C}$ which cover typically 1 to 2 decades in the magnitude of the differential cross section. The spectra of all the observed fragnents exhibit properties similax to those show in Fig. 1, namely, the monentum distributions have standarf deviations of only 50-200 $\mathrm{HzV} / \mathrm{c}$, and the average monentum is slightly negatlve relative to the projectile.

We find that the Gausstan shape provides a good fit to the observed spectra for all isotopes regardless of beam, energy, or target except for the hydrogen isotopes. The ${ }^{2} \mathrm{H}$ and $3 \mathrm{H}$ spectra are fit by a Gaussian curve in the region $-300<\mathrm{P}_{\|} \mathrm{z}^{*}$ $400 \mathrm{keV} / \mathrm{c}$, but exhbit an onhancement for $\mathrm{P}_{\mathbf{H}}<-300$ $\mathrm{Ms} V / \mathrm{c}$. The $\mathrm{I}_{\mathrm{H}}$ spectrum cannot be fit by a Gaussian

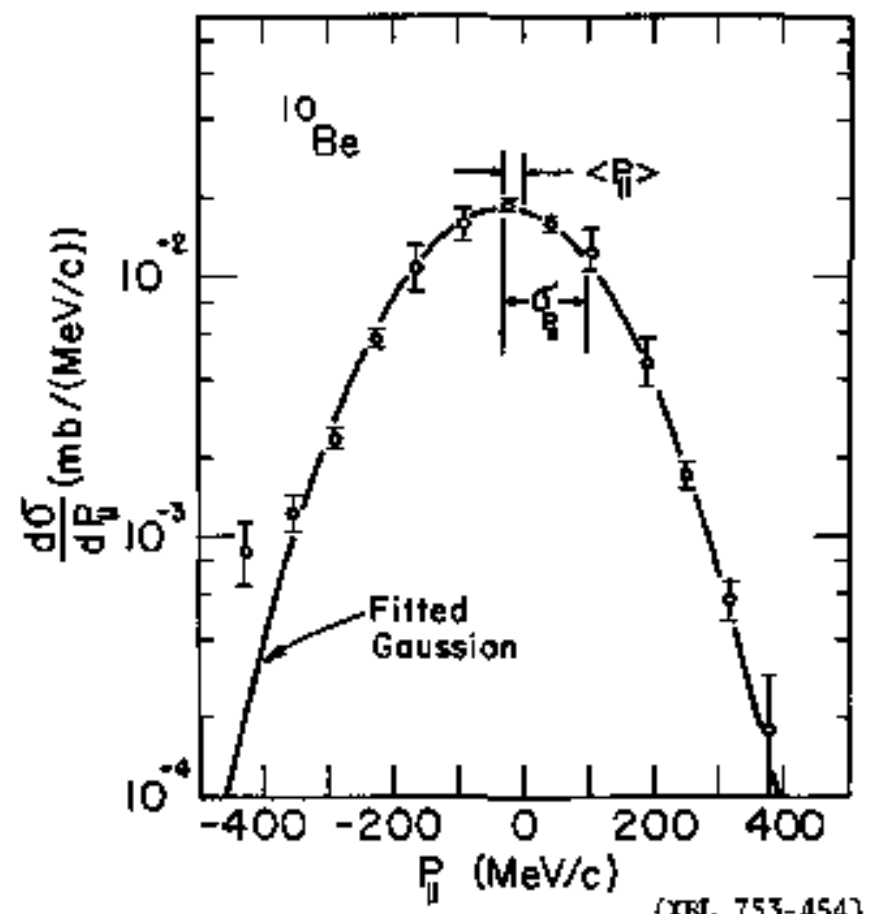

Fig. I. The projectile-frame parallel-momentum distribution for $10 \mathrm{Be}$ fragments from $12 \mathrm{C}$ at 2.1 $G \mathrm{GV} / \mathrm{n}$ on a Be target. The mean nonentum $t_{\mathrm{H}} /=-30$ MeV/c and standard deviation $\sigma_{F_{\|}}=129 \mathrm{MeV} / \mathrm{C}$ are

shape in the central region $\left|t_{1}\right|<150 \mathrm{MeV} / \mathrm{c}$. In this region a fit to the $1_{\mathrm{H}}$ spectrom is obtained with the exponential relation $\left.\mathrm{do} / \mathrm{dP}=\exp \left(-\mid \mathrm{P}_{0}\right] / 65\right)$. The regalt applies to the protons prohucest within our 12.5 mr acceptance cone and does not describe the spectra at larger proxhuction angles.

The $P_{1}$ distributions, projected onto the horizontal focal plane of the spectroneter, were heasured for $A$ b 2 fragments. We find $\sigma_{\mathrm{P}}=\sigma_{\mathrm{P}}$ to an accuracy of $10 \%$, consistent with the isotropic production of these fraguents.

If these reactions are exanples of limiting fraplentation, the large separation in rapidity, $y=\tanh ^{-1}\left(\mathrm{P}_{H} / \mathrm{E}\right)$, between the target and the fragmentation distribution requires the shape of the monentum distributions be independent of target 
and beam energy. ${ }^{4}$ For all reactions the target and energy dependence of the variables $\left\langle\mathrm{P}_{\|}{ }^{>}\right.$and $\sigma_{\mathrm{p}}$
were examined. Within the accuracy of this experiment we conclude there is no dependence on target mass above the $\$$ is level for $\sigma p$ and above the 10 \& level for $\left\langle\mathrm{F}_{\mathrm{g}}\right\rangle$. Because of this observed target independence we shall refer to the target-averaged values of ${ }^{\circ} p$ and $\left\langle\mathrm{P}_{\mathrm{p}}\right\rangle$ in the remainder of this paper. To determine if ${ }^{\circ} \|_{\|}$and $\left\langle\mathrm{P}_{p}\right\rangle$ are independent of energy we compare the measirements of these variables for the $12 \mathrm{C}$ bean at $2 . I$ and $1.05 \mathrm{GeV} / \mathrm{n}$. The weighted averages over all framents of the quontities $\sigma_{\mathrm{P}}(2.1 \mathrm{GeV} / \mathrm{n}) / \sigma_{\mathrm{P}}(1.05 \mathrm{GeV} / \mathrm{n})$ and $\left\langle\mathrm{P}_{\|}\right\rangle(2.1 \mathrm{EeV} / \mathrm{n})-\left\langle\mathrm{P}_{\|}\right\rangle(1.05 \mathrm{GW} / \mathrm{n})$ are $1.02 \pm 0.02$ ard $-1.0 \pm 2.0 \mathrm{WeV} / c$, respectively. This independence of beam energy and target leads to the conclusion that the $12 \mathrm{C}$ reactions satisfy the limiting fragmentation hypothesis and the limiting energy region is reached before $1.05 \mathrm{GeV} / \mathrm{n}$.

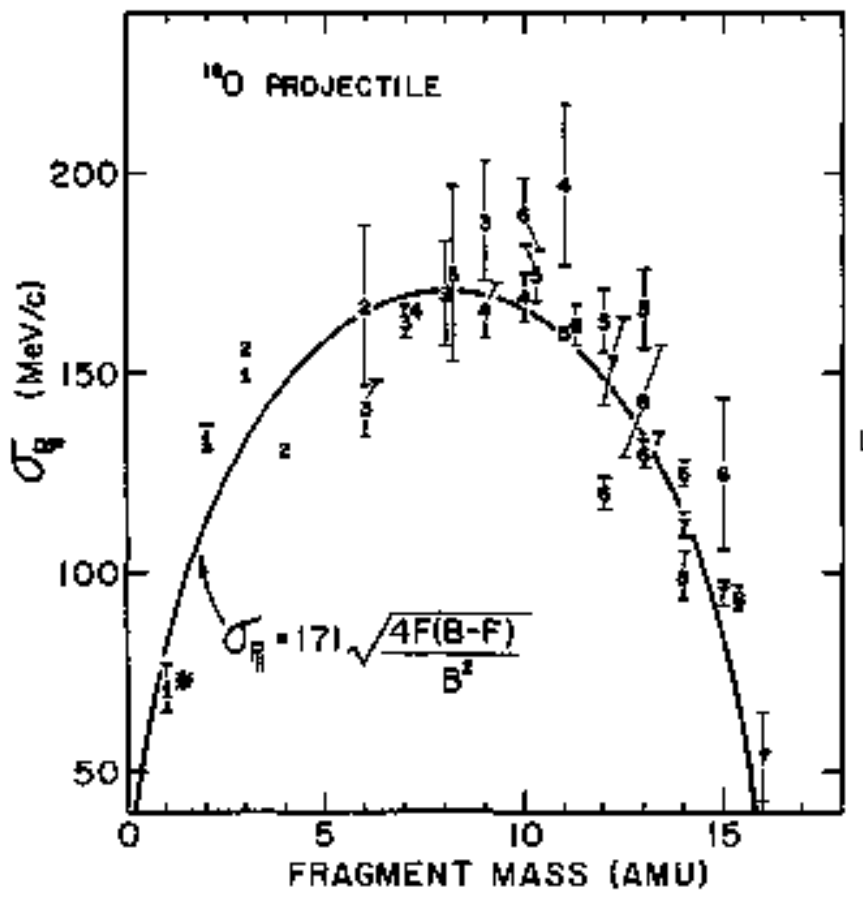

Fig. 2. Plotted are the target-averaged widths op of the projectile-frame parailel-momentur distributions, in $\mathrm{leW} / \mathrm{c}$, versus fraghent mass in amu. The plotted symbol indicates the charge of the fragment. These data represent fragments of 160 at $2.1 \mathrm{GeV} / \mathrm{n}$. The asterlsk denotes that the $\mathbf{1}_{\mathbf{H}}$ is a non-Gaussian monentum distribution and we have used the central region of this distribution to evaluate $\mathrm{opl}_{\mathrm{p}}$. This result applies to the protons produced within our $12.5 \mathrm{Jn}$ acceptance cone and does not describe the spectra at larger probuction angles. The parabola represents the best fit to the data.

(XBL 753-453)

In the liniting energy region the fragment distributions depend on the identity of the projectile and fraguent. 4 In Fig. 2 we have plotted the values of $\sigma_{\mathrm{K}}$ for 160 at 2.1 GeV/n versus the fragment mass in awes. The charge of each fragment is used as the plotting symbol. In an atterupt to parameterize the mass dependence we have fit the data to the function $\sigma_{\mathrm{P}}(\mathrm{B}, \mathrm{F})=4$ 3 $\mathrm{F}[\mathrm{B}-\mathrm{F} / \mathrm{B} 2$ where $B$ and $F$ are the mass numbers of the bean and fragment ruclei respectively, and $\sigma_{0}$ is the fitted variable. The best-fit curve for 160 is shown in Fig. 2. A1 though the parabola shape displays the general trend of the data, in no case does it provide a good fit to the observed values of $\sigma_{\mathrm{pu}}{ }^{5}$ The poor fit is denonstrated by the fact that 50 of of the data points are over two standard deviatlons from the curve. Particulerly striking is the observation that the same complex variation of op with fragkent mass is exhibited by both the $12 \mathrm{C}$ and ${ }^{16_{0}}$ fragnents, indicating nuclear strnctire effects are important variables Getermining $\sigma_{\mathrm{P}} \mid$.

The values of $\left\{\mathrm{p}_{0}\right\rangle$ have an approxinately 1 inear dependence on $\sigma_{0}\left(\left[\mathrm{P}_{\|}{ }^{3}=-0.5 \sigma_{\mathrm{P}}+30.0 \mathrm{MeV} / \mathrm{c}\right)\right.$. The general shifts in the monentum histribut fons toward velocities less than the bean correspond to snall energy transfers to the fraguent, typically $<130$ $\mathrm{keV}$ in the projectile frane. The obvious exceptions are reactions irrolving charge exchange, such as $12 \mathrm{C} \rightarrow 12 \mathrm{f}$, and charge exchange pIus loss of a nucleon, e.f., $160+15 \mathrm{C}_{+}$. The reactions involving charge exchange genera1ly have langer negative valles of $\left(\mathbf{A}^{\prime}\right)=100 \mathrm{MeV} / \mathrm{C}$. Calculation of the miss" In mass in these reactions gives values approxinktely $200 \mathrm{HoV} / \mathrm{c}$ after subtraction of the target mass. Thus the data are consistent with the assuption that the charge-exchange reactions proceed via pion productiom.

A parabolic dependence of $\sigma^{2}$, on fregment wass of the form of $* F(\mathrm{~B}-\mathrm{F})$, Fig: 1, was first predicted by Wenzel, ${ }^{6}$ T later by Lepore sond Ridde11,? and indirectly by Feshbach and thang as extended by Goldhaber. 9 In general the parabolic shape arises when one assumes: i) the fraggent monentum distributions are essentially those in the projectile nucleus, ii) that there are no correlations betwetn the ardenta of different nucleons, and iil) romentum is conserved. The validity and implications of these theories can be determined by couparison with the values of $\sigma_{0}$ measured by this experinent.

The work of Lepore and Riddell $1^{7}$ is a quantean mechanical calculation that eumloys the sudden approximation with shell-model wavefunctions to predict $a_{0}^{2}-1 / 8 \mathrm{mp}^{1 / 3}\left[4 \mathrm{~B}^{1 / 3}-25\right]$ (KeV/c). ${ }^{2}$ This expression, where the is the proton tass, gives qualitative agreemleft with the measured values as shown in Table 1. Feshbach and Huang assume sudden emission of virtual clusters and relate $\sigma_{0}$ to the Fermi momentwin of the projectile, $P_{f}$ and $\sigma_{f}$ is $\sigma_{f}^{2}=1 / 20 \mathrm{P}_{\mathrm{f}}^{2} \mathrm{~B}^{2} /(\mathrm{B}-1)$. The values of $\mathrm{P}_{\mathrm{f}}$ determined by quasìelastic electron scattering 10 give predicted values of $g$ that are generally 25 higher than the measured values as shown in Table 1 . An interesting point to note here is that through the predicted relationship between $\sigma_{0}$ and $P_{f}$, this experiment measures the profectile Femi monentum yla nuclear. fraguentation (șee Table I). By assuming the projectile has cone to thermal equilibrium at an excitation tenlperature $T$, Goldhaber ${ }^{9}$ has shown that the perabolic shape is again predicted and relates. $\sigma_{0}$ to $\mathrm{T}$ by the equation $\mathrm{kT}=40_{0}^{2} / \mathrm{m}_{\mathrm{B}} \mathrm{B}$, where $\mathrm{k}$ is Boltzmann's constant and in is the nucleon mass. The measured values of of then reflect excitation energies which we have listed in Table 1 along with the average binding energies per mxcleon as deter- 
Table 1. Comparison with theory and experiment of parameters related to a $\mathrm{o}_{\mathrm{P}}$ mass dependence of the forge $\sigma_{\mathrm{P}}^{2}=4 \sigma_{0}^{2} \mathrm{~F}(\mathrm{~B}-\mathrm{P}) / \mathrm{B}^{2}$. Derived quantities are Fellli momentum $\mathrm{P}_{\mathrm{f}}=2000^{2}(\mathrm{~B}-1) / \mathrm{B}^{2}$ and suerage excitation energy $\mathrm{kT}=4 c_{0}^{2} / \mathrm{m}_{\mathrm{h}} \mathrm{B}$. -

\begin{tabular}{|c|c|c|c|c|}
\hline \multirow[b]{2}{*}{ Parameter } & \multirow[b]{2}{*}{ Origin } & \multicolumn{3}{|c|}{ Projectile } \\
\hline & & $\frac{16 \mathrm{o}}{(2.1 \mathrm{GeV} / \mathrm{n})}$ & $\frac{{ }^{12} \mathrm{C}}{(2.1 \mathrm{GeV} / \mathrm{n})}$ & $\begin{array}{c}12_{\mathrm{C}} \\
(1.05 \mathrm{GeV} / \mathrm{n})\end{array}$ \\
\hline$\Phi_{0}(\mathrm{Mer} / \mathrm{c})$ & Thīs experiment & $171 \pm 3$ & $147 \div 4$ & $141 \pm 5$ \\
\hline$\theta_{0}(\mathrm{MeV} / \mathrm{C})$ & $\begin{array}{l}\text { Sudden } \\
\text { approximation }{ }^{7}\end{array}$ & 162 & 145 & $\mathrm{I} 45$ \\
\hline$\Phi_{0}(0, \omega / / c)$ & $\begin{array}{l}\text { Virtumi } \\
\text { clusters }\end{array}$ & 212 & 179 & 179 \\
\hline$P_{f}(\mathrm{HeV} / \mathrm{C})$ & This experiment & $185 \pm 3$ & $182 \pm 5$ & $174 \pm 6$ \\
\hline $\mathrm{F}_{\mathrm{f}}(\mathrm{l} / \mathrm{er} / \mathrm{n})$ & scattering $^{10}$ & 230 & 221 & 221 \\
\hline $\mathrm{kT}(\mathrm{H}+\mathrm{N} / \mathrm{n})$ & This experinent & $7.8 \pm 0.3$ & $7.7 \pm 0.4$ & $7.1 \div 0.5$ \\
\hline $\begin{array}{l}\text { Avexage binding } \\
\text { energy }(\mathrm{MeV} / \mathrm{h})\end{array}$ & $\begin{array}{l}\text { Mass } \\
\text { measurements }\end{array}$ & 8.0 & 7.7 & 7.7 \\
\hline
\end{tabular}

rined by the projectile mesșes. Because our measured excitation energles are essentially the binding energy per nuxleom of the projectiles, we infer that the fraglentation process which results in bound fraguents involves very little energy transfer between the target and fragment.

The frapment momentun distributions in the projectile rest frane are, typically, Gaussian shaped, narrow, consistent with isotxopy, depend on fragnent and projectile, and have no significant correlation with target mass or bean energy. The succlear temperature is inferred from the modentum distributions of the fragnonts and is approxinatily equal to the projectile nuclear binding energy. indicative of small energy transfer between target and fragpent.

\section{Footnotes and References}

*Condensation of LEL-3651, Physs, Rev, Lett. 35152 $(1975)$.

'University of California Space Sciences Laboratory, Berkley, Callfomia.

1. P. J, Lindytrack, D. E. Greiner, H. H. Hexkutan, Bruce Cork, and F. S. Bieser, LBL-36\$0 (stumitted to Ptrys. Rev, Lett.).
2. D. E. Greiner, P. J. Lindstran, F. S. Biesșer and H. H. Heckman, Muc1. Inst. and keth. 116, 21 (1974).

3. H. H. Heckman, D. E. Greiner, P. J Lizdstrom, and F. S. Bieser, Phys. Rev. Lett. 23, 926 (1972).

4. W. R. Frazer, et al., Rev. Mod. Phys. 44, 284 $(1972)$.

5. Prelininary results based on eight isotopes not including $\mathrm{l}_{\mathrm{H}}$ indicated oph $\approx 140 \mathrm{hteV} / \mathrm{c}$, independent of mass (H. H. Hechinan, 5th Intl. Conf. High-Energy Physics Nuclear Structure, Ijpsala, Sweden, Jume 1973,) Based on all the data presented here, a constont value of $\sigma_{F} \cdot 137 \mathrm{MeV} / \mathrm{c}$ gives a goodnessof-fit $x^{2}-600$ for the parabola with 32 degrees of freedom.

6. W, A. Wenzel, LBL Heavy Ion Serinar, 1973 (unpublished).

7. J. V. Lepore and R. J, Rdidell, Jr., LBL-3086, 1974 (umpublished).

8. H. Feshbech and $K$. Huang, Phys. Lett. 47B, 300 (1973).

9. Alfred S, Goldhaber, Phys, Lett, 53B, 306 (1974); The fragmentation problen has also be in studied by Fumiyo Ũchiyama, LBL-3318, 1974 (umpublished).

10. E. J. Moniz, I. Stck, R. R. Whitney, J. R. Ficenec, R. D. Kephard, and W.P. Trower, Phys. Rev, Lett. 26, 445 (197I). 


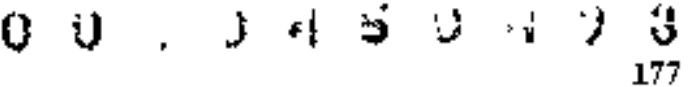

\title{
ISOTOPE PRODUCTION CROSS SECTIONS FROM THE FRAGMENTATION OF ${ }^{10} \mathrm{O}$ AND ${ }^{12} \mathrm{C}$ AT RELATIVISTIC ENERGIES"
}

\author{
P J Lindstrom, D E Greiner, H H Heckman,
} Bruce Cork, and F S Blesert

We have reasured at the Bevatron the singleparticle inclusive spectra of all isotope fragments of $16_{0}$ and ${ }^{12} \mathrm{C}$ at $2.10 \mathrm{GeV} / \mathrm{n}$ and of ${ }^{12} \mathrm{C}$ at 1.05 $\mathrm{GeV} / \mathrm{n}$. The targets were $\mathrm{Be}, \mathrm{CH}_{2}, \mathrm{C}, \mathrm{k1}, \mathrm{Ou}, \mathrm{Ag}$, and $\mathrm{Pb}$. The hydrogen target data were obtained by $\mathrm{CH}_{2}-\mathrm{C}$ subtraction. The measurenents were limited to secomdaries produced within a $\pm 12,5 \mathrm{mr}$ cone about $0^{\circ}$ from the direction of the prixary beam. Secondary momenta were linited to rigidities (pc/Le) less than $9 \mathrm{G}$. All secondaries with lifetimes greater than $10^{-8}$ seconds and production cross sections greater. than $I 0 \mu b$ were observed. The spectrometer systeml resolved charge, mass and momentem for all secondaries at any given spectrometer rigidity setting.

The observed longitudinal and transverse momentum spectra of the isotope fragmests, when transformed to the projectile rest frame, have Gaussign distributions centered near $0 \mathrm{l} / \mathrm{kW} / \mathrm{c}$, with standard deviations (S,D) $-60-200 \mathrm{MeV} / \mathrm{c}$. The cross sections were obtained by integrating these monentur distributions. 2 Since the observed fragnents were confined to a $12.5 \mathrm{mr}$ cone, it was necessary to extrepolate the transverse mosesntan distributions to obtain the total partial cross section. Except for the $2<2$ eragments, the 12.5 mr region accounted for $705-100$ of the total cross sections. Both observed momentua systematics and measured angular distributions in mxelear enulsion give confidence in this extrapolation for $A \geqslant 2$ secondaries. The proton nomentum distribution is non-Gaussian and no attempt was made to extrapolate this distributim to estinate total production cross section. The cross sections are corrected for bean and secondary fragmentation in the targets, vacum windows, and scintillators, as we11 as for interactions in the $\$ i(L i)$ detectors. The manentum distributions show the cross sections of target $\mathrm{T}$ to produce tragment $\mathrm{F}$, are measurements of frajments of the projectile and not frapnents of the target. No macleon-pickup isotopes were observed. Between $30 \%$ (Pb tanget) and 908 (H target) of 211 boson chare is accormted for by suming the of $\mathrm{F}$. The missing charge is principality in large moinentum transfer protons.

The $\sigma_{\mathrm{BT}}^{\mathrm{F}}$, for a hrdrogen target, car be compared with cross sections of proton-nucleus interactions at high energies. At proton energies $\geqslant 600$ HeV, 42 measured cross sections for 15 different secondaries have been compiled ${ }^{3}$ ito find agresmant to withtn 3 S.D. for all except $p+160+10 \mathrm{C}+\ldots$ at $\mathrm{T}_{\mathrm{p}}=1 \mathrm{GeV}$ and $p+160 \rightarrow 10 \mathrm{Be}+\cdots$ at $600 \mathrm{MeV}$, where the disagreement is 3-4 S.D. Comparison of our resilts çan also be made with the seni-emplicical model by Silberberg and Tsao ${ }^{4}$, which is based on the proton-nucleus data wet mentimed above, We find the experimental values of above $1 \mathrm{sb}$ are greater than those given by the Silberberg and Tsao model by an average of $22 \%$, with a S.D. of 373 .
We observe that the cross sections can be factored, $o_{\mathrm{B}}=\mathrm{YF}_{\mathrm{B}} \mathrm{Y}_{\mathrm{T}}$, where $\mathrm{\gamma F}_{\mathrm{B}}$ depends on the projectile and fragnent and $Y$ is the target factor (Fig. 1). Target factorization is expected both frum high-energy phenomenology and from an inpulse approximation mode1 of molear fragmentation. The exceptions to strict factorization are: 1) YT for a hydrogen target has a weak dependence on the mass of fragument $A_{F}, i . e ., \gamma_{T}(H)=0.66+0.028 A_{F}$ and 2) ${ }^{T}$ for single-nuxleon stripping is enthenced for heavy targets. The cross sections for singlerucleon loss on the heavler targets include a component for Coulomb dissociation, via the giant dipole resonence, in the target's virtual photon field. The Coulonb dissociation part of the cross sections can be computed and subtracted frosil the measured of $8 \mathrm{~T}$. The resultant cross sections are conslstent with the target factors and fit the data with a confidence level of 0.6 , and can be approxi-

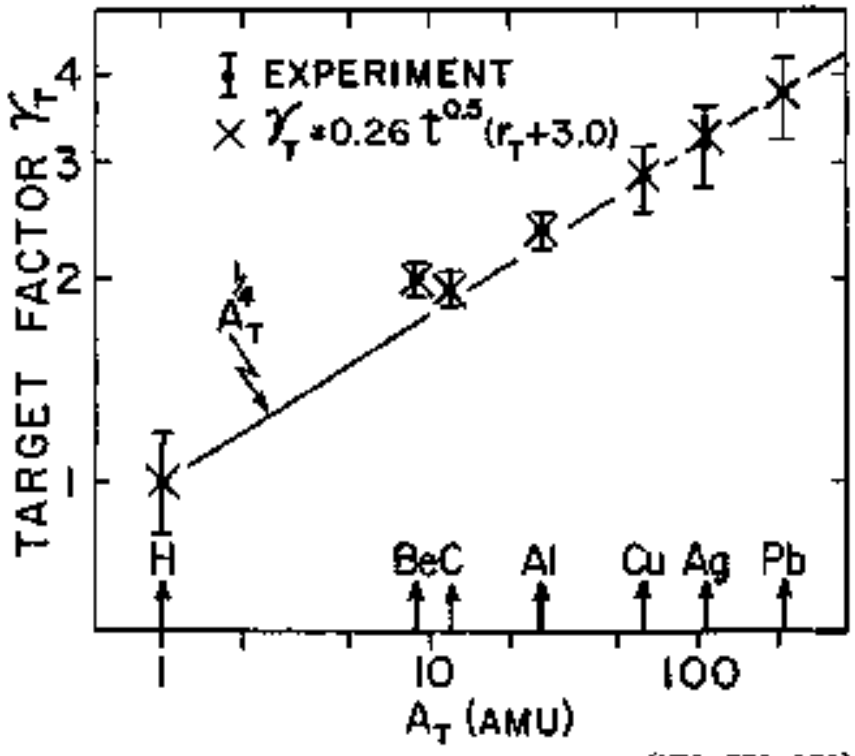

(XBL 7\$1-130)

Fig. 1. The cross sections for $\mathrm{B}+\mathrm{T}++-$ can be expressed as $\sigma \mathrm{F} T=\mathrm{YF}_{\mathrm{B}}$, where $\gamma_{\mathrm{T}}$ is the target factor. Plotted are the mean target factors versus the target mass $A_{T}$ (amu) for all cross sections measured in this experimint. The orror bars represent the error-wighted standard deviations and reflect the distribution of errors in the Individud cross sections of $\mathrm{T}$. The mean errors for $\gamma_{T}$ are approximately the dot size. The computed vatues of $Y T$ using the empirical fit are given by the symbol $x$. Physical parameters in the onpirical expresston for $Y T$ are $r_{T}$, the half-density charge radius and $t$, the charge-skin thickness of the target muclei. The line superimposed on the data points is an approxination for $\gamma_{\mathrm{T}}=\mathrm{A}_{\mathrm{T}} 1 / 4$. 
mately fitted by the expression $\gamma_{T}=A_{T}^{1 / 4}$ or $Y T \&\left(A_{B} T / 3-1.6\right)$. Both formulations for $Y T$ indicate the cross sections we observed are produced by peripheral interactions with the target. Neither fommlation for $Y_{T}$ explains the observed structure, however, particularly the result ${ } \mathrm{T}^{(\mathrm{Be})} \geqslant \gamma_{\mathrm{T}}(\mathrm{C})$, An accurate fit to the target factor is obtained by the expression $r T=k t^{n}\left(T_{T}+b\right)$ where $r_{T}$ is the measured half-density electric charge radius and $t$ is the measured charge skin thickness of the target. The three fitted variables are: the exponent $n=0.5$, $\mathbf{b}=3.0 \mathrm{fm}$, and norntizing constant $k=0.26$. Thts formula reproduces the structure in tho mean target factor to an accuracy bettor than 28 and with $a$ confidence level of 0.9. Since of factors and the momenta distributions are taret indpendent, 1 the partial differential cross sections factor $-a$ result expected by liaiting fragnentation models. Whether YT contains beam-dependent terms, e.g., the sun of radii of the bears and target muclei, as suggested above, carnot be determined with the present data.

The energy dependence of isotope production can be examined by: 1) comparing of ${ }^{12}$-bean energies, and 2) comparing ofs with values frow Ref. 3 meastret at different erergies. The cartom data for all fragulents are energy independent betheen 1.05 and $2.10 \mathrm{GeV} / \mathrm{n}$ and have an errorweighted mean ratio of $(2,10) / \alpha_{\mathrm{FT}}(1,05)=1,01 \pm 0.01$. Energy independence of of ${ }_{\mathrm{B}}$, above sume energy throshotd, is another result expected fror high-energy phencmenplogy. A chinparison of $\sigma_{\mathrm{BT}}^{\mathrm{F}}$ for the sagle fragments and targets but different beams, 160 and ${ }^{2} \mathrm{C}$, shows, in general, a weak beam dependence in the protuction cross sections of all fragments in comion, as long as a charge-exchange reaction is not recessary. The ratio of $\mathrm{F}_{\mathrm{T}}(160) / \sigma_{\mathrm{BT}}\left(1 \mathrm{C}_{\mathrm{C}}\right)=0.4-$ 1.35 , even though the individual cross sections vary over three orders of magniturte. It is noteworthy that more than 40 s of the ratios are in the interva1 1.0.0.15.

Production cross sections for Hirror nuclei should give insight into the mechanisms which produce the observed final state. If a neutron skin extends beyond the proton surface, a stripping process would result in $\sigma_{\mathrm{A}} / \sigma_{\mathbf{p}}<1$. We observe that, to the contrary, $1.0<$ of op $<4.1$, with most values of the ratio belng in the fnterval 1.1 to 1.7 .
That $\sigma_{N} / o_{P}>1$ is indicative of the effects of the bjading energy of the final state fragment. For example, inspection of the miss excess us o $F$ for isobars showg the fragnent with the lower mass excess has the higher production cross section. This dopinance of finsi state structure in of complicates the choice of any simple mechanisin describing the interaction process.

The pattems obserged in $\sigma_{\mathrm{BF}}^{\mathrm{F}}$ and in the momentu distributions ${ }^{1,2}$ indicate simplicity in the peripherat fragmentation process. Targot factorization, energy independence, and swalf transverse momenta are observed features of $\mathrm{F}_{\mathrm{KT}}$ and directly relate to 1 initing fragnantation nodels. The inclusion of a charge skin-thicksess term in the best fit for ${ } \mathrm{T}$, along with small parallel morenta widths in the beam rest frame, imply the observed fragments are the result of perfpheral interactions. Neution rich enhencenent of mirror. isotope cross sections, correlations in fragment binding energies, and a suprising degree of bean independerce in oF indicate a dowinance of fragtent nuclear structure in the production aplitudes.

\section{Footnotes and References}

*Condensed fron LBL-3650.

thiversity of California, Sace Siences Laboratory, Berkeley, Califontia.

1. D. E. Greiner, P. J. Lindstron, F. S. Bieser, and H. H. Heckman, Nucl. Instrum. Wth. 116, 21 (1974),

2. D. E. Greiner, P. J. Lindstrom, H. H. Heckmin, Bruce Cork, and F, S, Bieser, Phys, Rev, Lett, 35, $152(1975)$.

3. R. Silberberg and C. H. Tsao, KRL Report to. 7593, (1973). Also, F, Yiou, G. Raisbeck, C. Perron, and $P$. Fontes, 13th Internatimai Cosmic Ray Conference, Denver, Colorado, August 17-30, Vol. I, Paper ko. 251.

4. R, Silberberg and C. H. Tsao, Ap. J. Suppl. No. $220(\mathrm{I}), 25,315(1973)$.

\title{
FRAGMENTATION OF ${ }^{40}$ Ar AT $100 \mathrm{GeV} / \mathrm{G}$
}

\author{
P J Lindsirom, D E Breıner, H H Heckman,
} Bruce Cork, and $F \mathrm{~S}$ Biager'

Introduction. Relativistic mucleus-nucleus reaction cross sections are basic ingredients of many cosnic ray computations, without experimental data these cross sections have been largely a natter of guesswork. Relativistic heavy ion beams tn the Bevatron-Bevalac at Bexkeley now make these measurements possible. We present reaction cross sections of $4 b_{\mathrm{Ar}}$ at $1.8 \mathrm{GeV} / \mathrm{n}$, along with 160 and $12 \mathrm{C}$ at $2.1 \mathrm{GeV} / \mathrm{n}$, on a variety of targets, miasured in a simple transibission detertur system.
Experimental Procedures. The conputer controlled detector systen used to measure the cross sections consists of two detector modules containing forr one-inch diameter lithiln-drifted silicon detectors, $3 \mathrm{~mm}$ thick, in a telescope configuration (see inset Fig. 1). The leading detector Jopdule identified the charge of the incident bean by energy loss. The primary beam is confinsd to a one-forrth inch diameter spot in the center of the first modirle by an anticolncidence scintillator. Targets, 

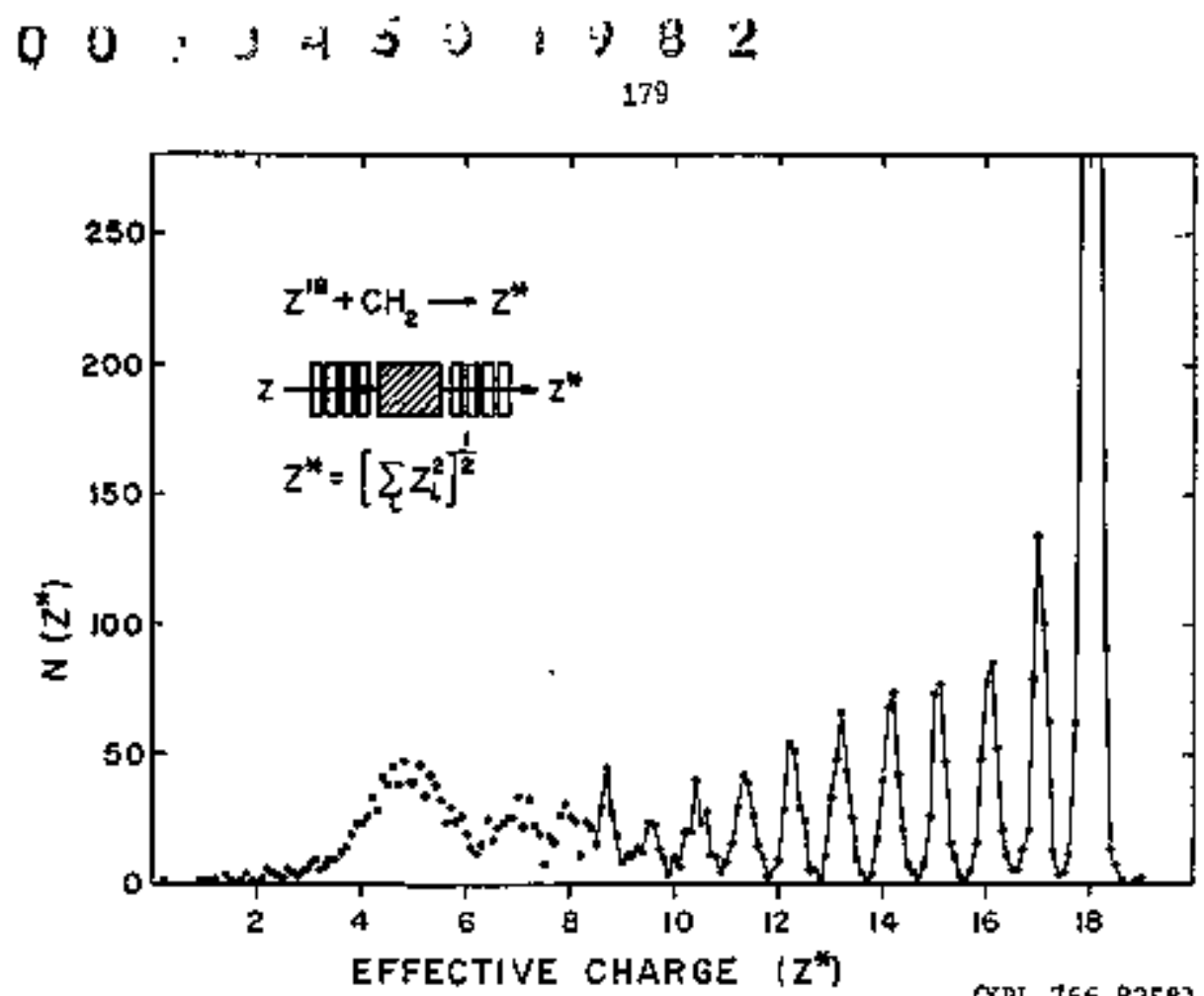

(XBL 766-8258)

Fig. 1. Effective charge spectrul of fragnents of $40_{\mathrm{Ar}}$ at $1.8 \mathrm{GeV} / \mathrm{c}$ on a $\mathrm{OH}_{2}$ target. The surviving primary bean is well resolvad from the fraguentation prodicts.

typically one-half of a man free path thick, are placed between the detector mochules. The sectind module of detectors serves to ideritify the interacting, as well as surviving, beam nuclei. The raw polse heights of all eight detectors were preserved or tape for further analysis. The targets were $\mathrm{CH}_{2}$, C, $\mathrm{S}, \mathrm{Ou}, \mathrm{Ag}, \mathrm{Pb}$, and $\mathrm{U}$. In the analysis the events were ordered by their effectiye charge $\left.z^{*}=[\text { (dE/dx) })_{\text {jeas }} /\left(d \mathrm{E} / d x_{p}\right)\right]^{1 / 2}$ and by their $x^{2}=\frac{n}{2}$ $\left(Z^{\star}-\bar{Z}^{\star}\right)^{2} / \sigma^{2}$ leach detector module. Figure ${ }^{i}$ is a typical spectrum of effective charge with $\mathrm{X}^{2}<5.0$ for $1.8 \mathrm{GeV} / \mathrm{n}^{40} \mathrm{Ar}$ on a $6.981 \mathrm{~g} / \mathrm{om}^{2}$ thick CH 2 target. The standard deviation of the 7.18 peak is $\sigma= \pm .11 \mathrm{e}$. The charge of the highest $z$ fragment is resolved down to $z=10$. Note that the effective charge peaks are shifted from unit charges, e.g., $Z^{*}$ (charge 10 ) $=\left[\Sigma Z_{j} \hat{9}^{*}=\left[10^{2}+\mathrm{B}\right] 1 / 2=10.4\right.$ implying the charge 10 frajgnts traveling at beash velocity. The cross section for $A 2>1$ of the primary bean is calculable from its survival. The partial proxhction cross sections for element with $2>10$ can be caloulated from theit peoks.

Reaction Cross Sections. The results are presented in Table 1 , The $B 231$ cross sections ${ }^{2} \mathrm{C}$ and 160 at $2.1 \mathrm{GeV} / \mathrm{n}$ from an earlier rum are also presented. By aditing the isotope production cross section for $\Delta Z=0$ to the $\Delta z a 1$ cross section we obtain

Table 1. Reaction cross sections (barms).

\begin{tabular}{|c|c|c|c|c|c|c|c|}
\hline \multirow[b]{3}{*}{ Target } & \multicolumn{6}{|c|}{ Eeam } & \multirow{3}{*}{$\begin{array}{c}{ }^{1} \mathrm{H} \\
\text { Reaction }\end{array}$} \\
\hline & \multicolumn{2}{|c|}{$40_{\mathrm{Ar}}$} & \multicolumn{2}{|c|}{${ }^{16} 0$} & \multicolumn{2}{|c|}{${ }^{12} \mathrm{C}$} & \\
\hline & $\Delta \geq>1$ & $\Delta Z>1$ & $\Delta 2>1$ & $\Delta A \geq 1$ & $\Delta Z>1$ & $44>1$ & \\
\hline H & $.58 \pm-04$ & $.68 \pm .05$ & $.32 \pm .02$ & $.34 \pm+02$ & $.22 \div .02$ & $.25 \pm .02$ & \\
\hline C & $1,36 \pm .03$ & $1.52 \div, 06$ &, $94 \pm, 02$ & $+98 \pm .02$ & $.75 \pm .02$ & $.81 \pm .02$ & $.23 \pm .01$ \\
\hline$\$$ & $1.94 t_{+}^{0.07}$ & $2.13 t .09$ & $1.31 \pm .05$ & $1,37 \pm .05$ & $1.15 \pm, 05$ & $1.22 \pm .05$ & \\
\hline au & $2.62++05$ & $2.85 \pm .08$ & $1.82 \pm .03$ & $1.90 \pm .03$ & $1.62 \pm+03$ & $1.71 \pm .03$ & $.71 \pm .02$ \\
\hline $\mathrm{Ag}$ & $3.20 \pm+07$ & $3.47 \pm .11$ & & & & & \\
\hline $\mathrm{Pb}$ & $4.19 \pm .13$ & $4.51 \pm .16$ & $3.10 \pm .07$ & $3.24 \pm .07$ & $2.82 \pm .06$ & $2.97 \pm$ & $1.78 \pm .05$ \\
\hline せ & $4.61 \pm .21$ & $5.00 \pm .24$ & & & & & \\
\hline
\end{tabular}

$a_{5 \$ 0 ~ \mathrm{MeV}}$ protons from Ref. 3. Targets not in conwon are also plotted in Fig.2. 
the reaction cross secticns for $\Delta A$ a 1, The isotope production cross section of Lindstrom et al. 1 for $A A=I$, two were tused for correcting ${ }^{12} \mathrm{C}$ and $10_{0}$. The caluputed isotope cross section for $\Delta z=0, \Delta A>1$ by Silberberg and Tsao, 2 corrected by the $\Delta Z=1$ production cross section of this $40_{\mathrm{Ar}}$ data, were used to coppute the $\triangle A>1$ cross sections are in Table 1 along with $\sigma_{T}$ for proton-nuclets from Renberg ot a1.

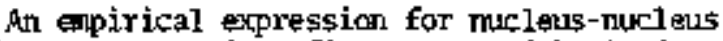
reaction cross section, fisst pToposed by Bradt and Peters, is $\sigma_{\mathrm{ET}}=-\pi r_{f}\left(A_{B} 1 / 3+A_{T} 1 / 3-b\right) 2$ where $A_{0}$ and $A_{T}$ are the mass mubers of the beam and target mulei

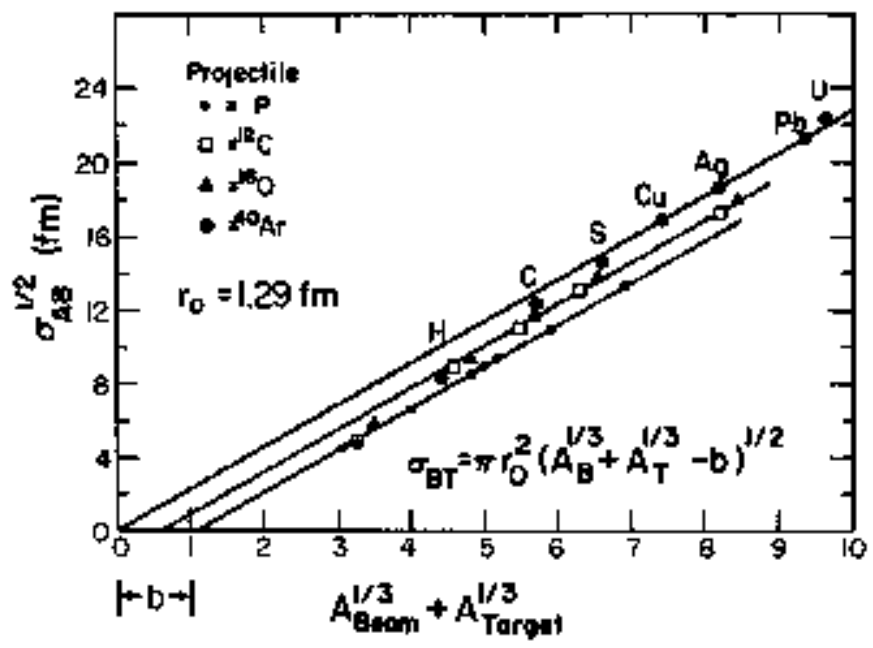

Fig. $Z_{+}$Plotted are $\sigma_{\mathrm{AB}} \mathrm{B}^{1 / 2}$ for $40 \mathrm{Ar}, 160,12_{\mathrm{C}}$, and $1_{\mathrm{H}}$ on targets of mass $\mathrm{AT}_{\mathrm{T}}$, where $\sigma_{\mathrm{AB}}=$ reaction cross section for nucleus $A$ on nucleus $B$ and $A T, B$. mass, number of the respective nuclei. If $\sigma_{A B}=\pi r_{0}$ $\left(A \mathrm{~A} / 3+A_{B} 1 / 3-b\right)^{2}$ then all of the data should fall on a single stralght line.

(XBL 766-8259) and $b$ is an ofeflap prameter. Figure 2 is a plot of $\sigma_{\mathrm{BT}} 1 / 2$ vs $\left.\left(A_{\mathrm{B}} 1 / 3^{2} \mathrm{~A}_{\mathrm{T}} 17\right)^{\prime}\right)$ of the roaction cross section in Table 1. The slope is $\pi^{1 / 2} T_{0}$ giving $I_{0}=1.29$, and the $x$ intercept is $b$. The deta can be roughly fitted by $b=1-0.028$ an if the mininum mass masber $A_{\text {min }}=\min \left(A_{\mathrm{B}}, A_{\mathrm{T}}\right) \times 36$ and $b=0$ if $A_{\min }>36$. This equation fits the experinental cross sections to within $10 \%$ in al1 but 3 cases; the worst excursion is $18 \mathrm{k}$. Theoretical corputations of the total reaction cross section $\sigma_{Y}$ by Barshay et al.4 using the Glauber model support a variable overlap and indicgte the miniman overlap is reached when $A_{\text {min }}$ *a 40 . We find when $A_{\pi i n}=40, b$ min $\neq 0$, consistent with black sphere geometrial cross sections. The Glauber model production of total reaction crops sections fit the data with accuracy canparable to the variable overlap model. Both the Glauber model and the modified Bradt and Peters formula are well outside of experimental errors. There is room for further refinement in reaction cross section rodels.

\section{Footrotes and References} *Utiversity of Califoria Space Sciences Laboratory, Berkeley, California.

1. P. J. Lindstran, D. E. Greìner, $H_{*} H_{+}$Heckpan, B. Cork, and F. \$. Bieser, 1975, LBL,-3650 (to be published).

2. R. Silberberg and C. H. Tsao, Ap. J. Sirpl, No. $220(\mathrm{I}), \underline{25} \quad 315$ (1973).

3. P. U. Renberc, D. G. Measday, M. Pepin, P. schwaller, B. Favier, and C. Rfchard-Serre, Nucl. Phys. A183 81 (1972).

4. S. Barshay, C. B. Dover, and J. P. Vary, Phys. Rev., Cli 860 (1974).

\section{FRAGMENTATION OF ${ }^{4} \mathrm{He},{ }^{12} \mathrm{C},{ }^{14} \mathrm{~N}$, AND ${ }^{16} \mathrm{O}$ NUCLEI IN NUCLEAF EMULSION AT $2 .{\mathrm{GeV} / N U C L E O N^{*}}^{*}$}

H H Heckman, D E Grenter, P J Lindelrom, and $\mathrm{H}$ Shwet

We have undertaken a study of the interactions of $2.1-\mathrm{GeV} / \mathrm{n} 4 \mathrm{He},{ }^{2} \mathrm{C},{ }^{4} \mathrm{~N}$, and 180 nuclei in nutlear research emutsion, with enphasis on the inter action mesn-free-paths and the properties of profectile fragmentation. The path-1ength data are satisfactorily interpreted in terms of a two parameter expression for the geometrical cross section. These parameters are $r_{0}$, defing by the expression for the muclear radius, $r=r_{0} A_{i} 1 / 3$, and $b$, the orerlap parameter. The quantity $b$ is equal to or $/ \mathrm{r}_{0}$, where $\Delta t$ is the geometrical overlap between the colilding nuclei. About 13 of the interactions of $C, N$, and O beam particles with eriulsion nuclei Ifad to "pure" projectile fraguentation, characterized by the fact that no target fragnentation, e.g., low energy, charged-particte evaporation, is detected in the interartion. Te have selected these interactions for specific study of projectile fragmentation.
The enulsion stacks were fabricated from Ilford G.5 pellicles, 600 microns thick. The stacks were exposed to the heavy ion beams parallel to the enulsion planes. The scanning technique for each bean was to select an incident ion 1 to $2 \mathrm{~mm}$ fro the entrance edge and scan along the track until the Iton interactet or left the pellicle. The scarning and measurements were perfomed with three-coprdinate digitized microscopes with one micron read-out accuracy. The interactions were classified as follows:

Type 1. Projectile fragnentation, no visible target fragmentation.

Type 2. Target and projectile fraguentation. Projectile may fragment, as in Type 1 , or suffer catastropic destruction. 


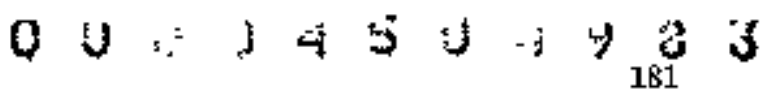

Type 3, Target fragmentation, no detectable change in charge of the fincident ian, i.e., the imverse of Type 1.

$$
\sigma_{\mathrm{BT}}=\mathrm{m}_{0}^{2}\left(\mathrm{~A}_{\mathrm{B}}^{1 / 3}+\mathcal{A}_{\mathrm{T}}^{1 / 3}-\mathrm{b}\right)^{2}
$$

Events of Type 1 ware selected for the sturty of projectile fragmentation. They were intensively examined for all socondary particles, for which ionization and angle measurements were raade $\left[ \pm 0.16^{a}\right.$ (S.D.) in the horfantal plane and $\pm 0.39^{\circ}$ in the vertical plane].

Table 1 sumarizes the data interacticti meanfree-path-length results of the experiment. The scanning efficiency was near 100 for events in which the difference between the charges of the beam and principal fragment $\Delta z=z_{B}-z_{F}>z_{+}$Fragneptation events for which $A 2=0$ or 1 tend to be missed, partiaularly when there is little or no evidence for excitation of the target macleus. A re-scan of about one-third of the $\mathrm{C}, \mathrm{N}, \mathrm{O}$-tract data contributed, out of 1059 events, 16 new events, all of which were Types $I$ and $3, A Z=0$, and $I$ only.

The isotope production cross section of Lindstrun et al. 1 were used to correct for the scanring losses of $\mathrm{o} z=0$ and 1 events. The corrections to Nobs (to give Ntotal) were: $12 \mathrm{C}, 16 \pm 25$; I 0 , $69 \pm 21$; and by interpolation of the $c$ and 0 cross section 14N, $31 \pm 33$. No corrections were mads to the He data. Given in Table 1 are the number of events $N_{h Z}>1$, for which the charges of the primcipal fragment differs from that of the incident fon by $A \mathrm{Z}>\hat{1}$. Table 1 concludes with the measured interactionmean-free paths $\lambda_{\text {total }}$ and $\lambda_{A Z} b_{1}$ in nuclear emul. sion for $4 \mathrm{He},{ }^{12} \mathrm{C}, 14_{\mathrm{N}}, 100 \mathrm{miclei}, \mathrm{E}=2.1 \mathrm{GeV} / \mathrm{n}$.

An empirical expression for the total reaction cross section that is useful to interpret the data given in Table 1 is the gemetrical formula first proposed by Bradt and Peters,?

were $A_{\mathrm{P}}$ and $A_{\mathrm{T}}$ are the mass numbers of the beam and target nuclei, respectively, the constant $b$ is the overlap parameter and $r_{0}$ is the constent of pro portionality in the expression for the genpetrical nuclear radius $\mathbf{r}_{i}=r_{0} A_{i}{ }^{1 / 3}$ : Because the parameters $r_{0}$ and $b$ are coupled, we find that consistent fits to our mean-free-path data can be obtained for $\Gamma_{0}$ and $b$ in the ranges $1.4<r_{0}<1.7$ fin and $1.26 b<1.9$. The fragmentation cross sections $(\Delta T 21)$ measured by Lindstron, et al. 3 for $12 \mathrm{C}$ and $100, E=2.1 \mathrm{GeV} / \mathrm{n}$, gave the finst evidence that the overlap $b$ is not constant, but depends upur the mas, i.e., radius, of the bean nucleus. The theoretical results of Bowman, Swiatecki, and Tsang 4 and Barshay, Dover, and Varys support this observation. Bowelan et al., describe heavy ion collisions under the conditions of i) abrasion, the shearing off of molear volme of the projectile by the target and li) ablation, further loss of meterial by the profectile dise to evaporated particles. From this model one obtains and expression for the overlap paraneter $b_{A z} \geqslant I$ that is appropriate for the shearing-off of at least one unit of charge. ${ }^{6}$ In their Investigation on the factorization of total cross sections in mucleusnucleus colfisions, Barshay et a1.5 calculated the total reaction cross sections using Glauber theory and a keometrical nuclear model. The monerical results given in Tables 3 and 4 of Barshay et al. 5 can be ordered in terms of the Bradt-Peters relation, Fig. 1. We have determined, by least squares fitting, the overlap parameter $b_{\text {total }}$ appropriate to these total reaction cross setions as a function of projectile mass $A_{B}$, i.e., the 1ighter of the two interacting nucle 2 .

Table 1. Interaction mean-free-path length data. Tabulated are: $N_{i}$, the nuber of observed interactions of type 1 , Nobs - $\Sigma_{i} N_{i}, N_{t o t a l}=N_{o b s}$ corrected for scanning losses of $\Delta Z=0$ and 1 events, and $v_{A}>1$, the number of charge-changing (at $\geqslant 1$ events. The interaction Iengths evaluated for N Notsl and $N_{\Delta Z>1}$ are $\lambda_{\text {total }}$ and $\lambda_{\Delta Z>1}$, respectively,

\begin{tabular}{|c|c|c|c|c|}
\hline Beam & ${ }^{4} \mathrm{He}$ & ${ }^{12} \mathrm{C}$ & $14 \mathrm{~N}$ & $16_{0}$ \\
\hline$N_{1}$ & 104 & 149 & 118 & 119 \\
\hline$N_{2}$ & 853 & 858 & 883 & 849 \\
\hline $\mathrm{N}_{3}$ & 54 & 88 & $\$ \$$ & 55 \\
\hline$N_{\text {obs }}$ & 1011 & 1095 & 1059 & 1023 \\
\hline$N_{\text {total }}$ & 1011 & $1111 \pm 39$ & $1090 \pm 47$ & $1092 \pm 38$ \\
\hline $\mathrm{N}_{\Delta Z}>1$ & 957 & $2044 \pm 39$ & $1031 \pm 45$ & $1040 \pm 36$ \\
\hline Pathlength $\left(c^{\prime}\right)$ & 22080 & 15302 & 14895 & 14174 \\
\hline$\lambda_{t o t a 1} \quad(0 \mathrm{n})$ & $21.8 \pm 0.5$ & $13.8 \pm 0.5$ & $13.7 \pm 0.6$ & $13+0 \div 0,5$ \\
\hline$\lambda_{\Delta Z}=1 \quad(0 \pi)$ & $23.1 \pm 0.8$ & $14.7 \pm 0.6$ & $14.5 \pm 0.6$ & $13.6 \pm 0.5$ \\
\hline
\end{tabular}




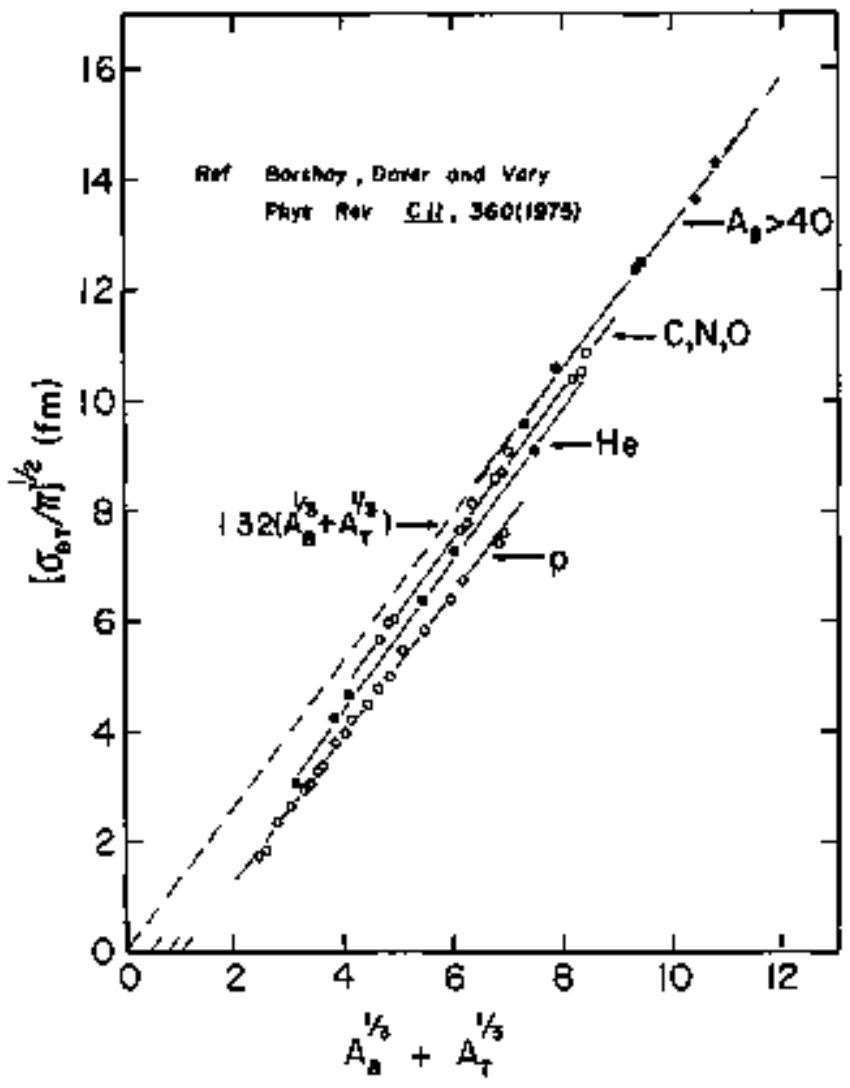

Fig. 1. Least squares fits to the total reaction cross sections computed by Barshay, Dover, and Vary (Ref. 5) ordered by $A_{B} \equiv A_{\text {glinin, }}$, the mass of the lighter of the two interacting nuclei in terms of the Bradt-Peters relation, $\mathrm{Bq}$. 1. The intercepts with the abseissa are the overlap parameters $b$ for the spectfic cases where $A_{B}$ is $1,4,[12,14,16]$ and $>40$. The valtes of $b$ versus $A_{B}$ are tabulated in Table 2.

(XRL 758-1914)

Table 2. Overlap paraneters b derived from theory and experinent used to calculate interection path lengths ${ }^{2} \Delta Z Z_{1}$ and $\lambda_{\text {fotal }}$ for canparison with this experiant. The ro radii are from least squares fits to the numerical results given in Ref. S.

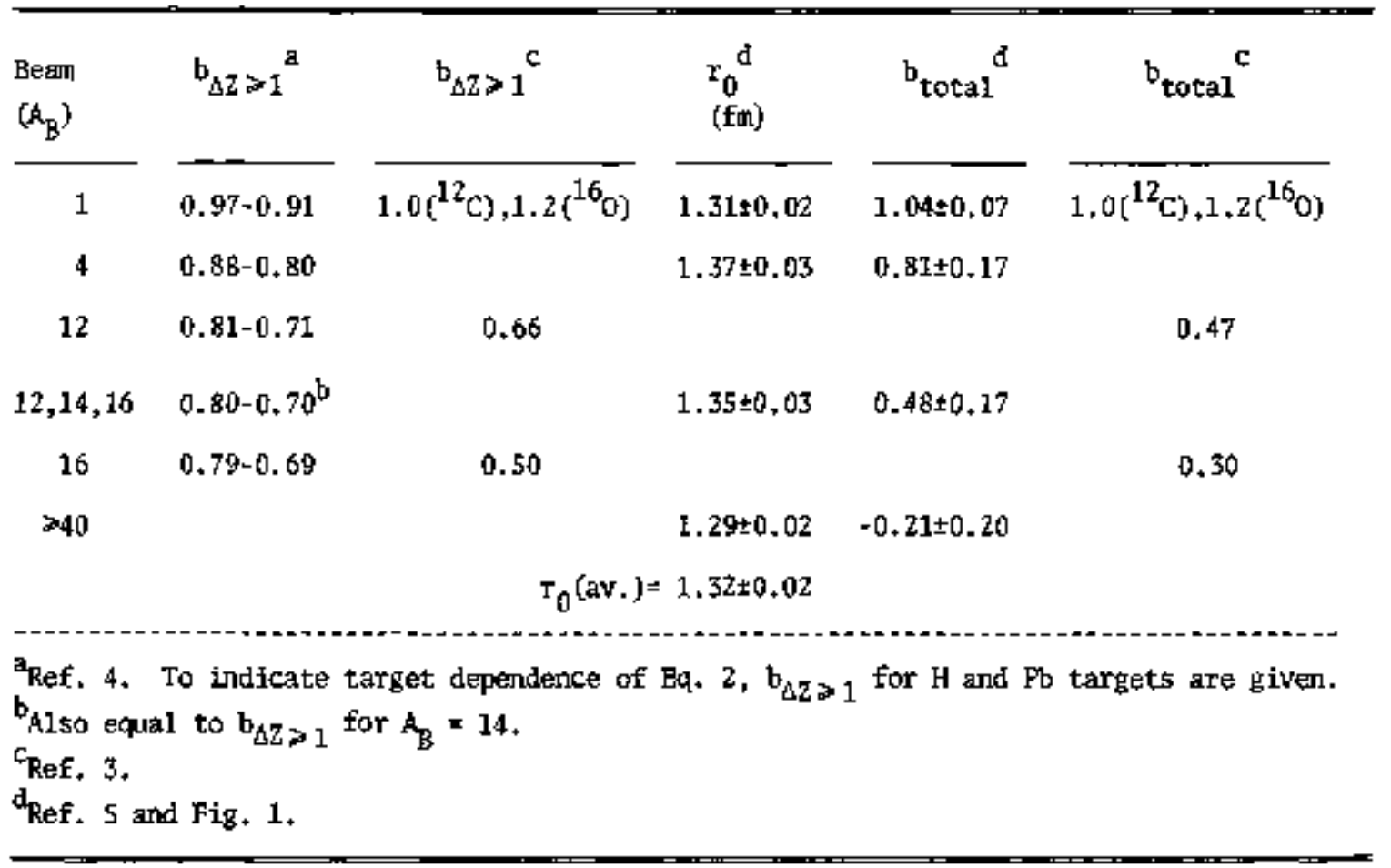




\section{0 ○

To interrelate the results of this experinent with those of lindstrom et al., Bownan et al. and Barshay et al., we take the following approach: 1) Select the appropriate value of the overlap paraneters b, given in Table 2, for each bean particle and nuclear constituents in emulsion. 2) Conpute $\lambda_{\text {calc }}=\left(E_{i} n_{i}(T) \sigma_{B P}\right)^{-1}$, where ni, $(T)$ is the number of nuгle1 $T$ per milliliter in emulsion. 3) Determine the best estimate of $r_{0}$ by the minimization of $x^{2}=\left(\Sigma_{i} \lambda-\lambda_{\text {calc }}\right)^{2} / \sigma_{\lambda}^{2}+$

The compoted path lengths are compared with experiment in Table 3 , wa find that the moan- freepath lengths of ${ }^{4} \mathrm{He}, 12_{\mathrm{C}}, 14_{N}$ and $16_{0}$ nuclei in

Table 3, Calculated interaction mean-free-path lengths (cm) using overlap paraneters $b$ derived from Refs. 3, 4, and 5 and values of $r_{0}$ that give miniman $x^{2}$ fits to data of this experiaent.

\begin{tabular}{|c|c|c|c|c|c|c|}
\hline & $b$ & $\begin{array}{c}\tau_{0} \\
\left.(\mathbf{f}){ }^{2}\right)\end{array}$ & ${ }^{4} \mathrm{He}$ & ${ }^{12} \mathrm{C}$ & $14 \mathrm{~N}$ & 160 \\
\hline \multirow{2}{*}{$\lambda_{\Delta Z \neq 1}(00)$} & Eq. (2) Ref, 4) & 1.24 & 22.3 & 15.2 & 14.3 & 13.6 \\
\hline & Tablo 4 (Ref, 3) & $I, 23$ & & 15.7 & 13.7 & 13.2 \\
\hline Experiment & & & $23.1 \pm 0.8$ & $14.7 \pm 0.6$ & $14.5 \pm 0.6$ & $13.6 \pm 0.5$ \\
\hline \multirow{2}{*}{$\lambda_{\text {total }}(a n)$} & Table 3 (Ref. 5) & 1.25 & 22.2 & 14.1 & 13.4 & 12.8 \\
\hline & Table 3 (Ref, 3) & 1.23 & & 14.7 & 13.4 & 12,4 \\
\hline Experiment & & & $21,8 \pm 0,7$ & $13.8 \pm 0,6$ & $13.7 \pm 0.6$ & $13,0 \pm 0,5$ \\
\hline
\end{tabular}

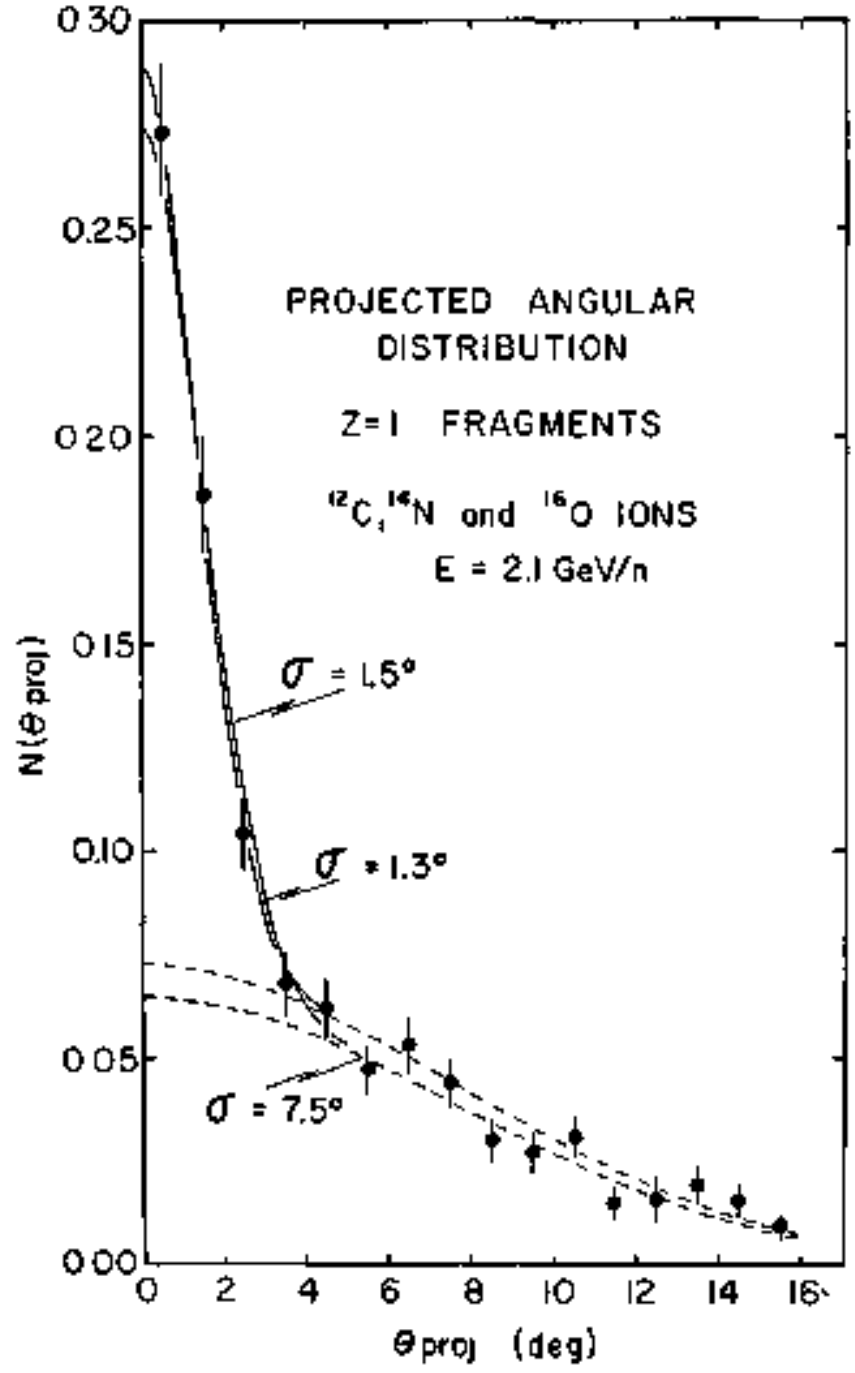

Fig. 2. The combined projected engular distribution for $2=1$ fragunents, $\mathrm{C}, \mathrm{N}$, and o projectiles, ${ }^{0}$ proj $<16^{\circ}$. The shaded area is bounded by curves thet are the stan of two Gaussian distributions, $0=1.3^{\circ}$ and $1,5^{\circ}$, and $7,5^{\circ}$, nomalized to unit areas. (XBL 758-195)

emulsion are well accounted for by the Bradt-Peters expression, Eq. (1). The observations are best explained when $r_{0} \neq 1.24 \mathrm{fm}$ and the overlap parameters $b$ is a variable dependent on $A_{B} \equiv A_{A n i n}$, the lighter of the projectile and target nuclei partaking in the collision, Fig. I. However, the possibility that b may depend upon both the projectile and target nuclei cannot be rule out by the present experiment.

Approxinately 104 of all the $c, N$, and 0 fragmentation is enulsion exhibit projectile fragmentation only, i+e., Type 1 . Figures 2 and 3 present, raspectively, the conposite projected angular distribution for $Z=1$ and $Z=Z$ secondaries from the fragmentation of $12 \mathrm{C}, 14 \mathrm{~N}$ and 160 nuclei at 2,1 $\mathrm{GeV} / \mathrm{n}$. The angular distribution data were combined because we observed no statistically signiflcant difference betweon them. The projected angular dis tribrtion for $Z=1$ particles, shows a peaked distribution at $0 \mathrm{deg}$, with about 99 \% of alI secondaries restricted to the fortard hemisplere. In Fig. 2 we show the structure of the $t=1$ distribution for Aproj < 16 deg. The projected argular distribution has a narrow central peak, superinmosed upon, and distinct from, a broader distribution. The observed projected angular distribution for $2=2$ secondaries is given in Fig. 3. This distribution is dofpinated by a narrog forward peak, having a characteristic width 1.0

In Table 4 we tabulate the average monentum widths (\$.D) for the CNO group measured by Greiner, 


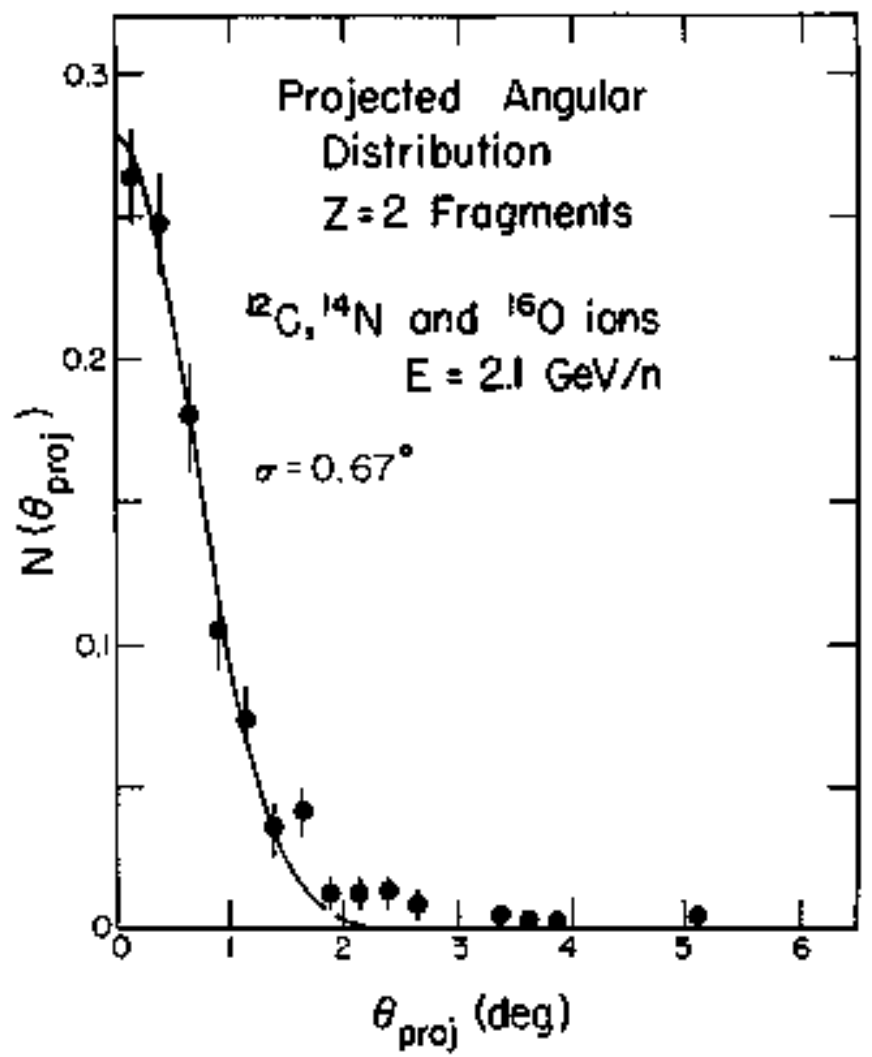

Fis. 3. Angular distribution, projected onto the emrlsion plane, for He $[z=2)$ fragments, from the fragmentation of $12_{\mathrm{C}}, 1 \mathrm{~d}_{\mathrm{N}}$, and $10_{0}^{\circ}$ projectiles. All interactions of Type 1 . Cune is a Gaussian distritution, $\sigma=0.67^{\circ}$, fitted to the data for $\sigma_{\text {proj }} x_{0} 1.5^{\circ}$.

(XBL 766-8257)

et al. ${ }^{7}$ and given by the theoretical work of Leporto and Ridde11, and the corresponding angular widths $\sigma\left(\theta_{\text {proj }}\right)$ for the $z=1$ and $z$ isotopes. The prodic- tion-weighted values of $\sigma\left(\theta_{\mathrm{proj}}\right)$, denoted as $\sigma_{2 \mathrm{~m}}$ and $\sigma_{z=2}$ are the quantities to be coupared with the experimental results.

Referring to Fig. 2, the narrow, forwand peak of the $z=1$ fragments can be fitted by a Gaussian distribution, with $\sigma_{2}=1=1.3-1.5^{\circ}$, in good agreerent with the results of Greiner, et al. 7 and the theory of Lepore and Riddell, s provided the largeangle background events can be described by a Gaussian distribution $a * 7,5^{*}$, whose armitturle is about $1 / 3$ of the central peak. Fignre 3 is the projected angular distributions for the $\mathrm{Z}=\mathrm{Z}$ (He) isotopes from the fragnentation of $\mathrm{C}, \mathrm{N}$, and 0 nuclei in emplsion. Dram through the distribution is a Gaussian curve Whose $\left.\square\left(S . D_{+}\right)=0.6\right\}^{\circ}$ is based on data restricted to $\theta$ prot $<1,5^{\circ}$. Correcting for measuretent error, we obtain $\sigma_{\mathrm{Z}=2}=0.65^{\circ}$.

The general agreenent between the values of $\sigma_{2=1}$ and $\sigma_{2}=2$ fron the data of Greiner, et al., (which pertain to longitudinal moventum distributions) and this experibent argues for the equality of the 1ongitudinal and transyerse monentum distributions inthe projectile frame for the respective $z=l$ and $z=2$ nuclei. Furthernore, within measurement errors, we also have verified that the transverse monentul distributions of the He nuclei projected onto orthogonal planes in the emulsion are equal. Thus, to about a 109 leve1, we have shown the momentur distribution of the He isotopes in the projectile frame of reference is consistent wth isotropy,

\section{Footnotes and References}

tCondensed fron LBL- 3656.

'East Stroutshourg State College, East Stroudspourg, Pa.

1. P. J. Lindstrom, D. E. Greiner, H, H. Heckman, Bruce Cork, and F. S. Bieser, LEL-3650 (1975).

Table 4. Standard deviation widths of the momentun and projected angular distributions for the $Z=1$ and $Z$ secondary isotopes from the fragmentation of the $\mathrm{C} \$ \mathrm{l}$ group at $\mathrm{E}=2.1 \mathrm{GeV} / \mathrm{n}$. The production-wighted angular widths of $Z-1$ and $Z=2$, respectively. Experimental data based updr Type 1 events.

\begin{tabular}{|c|c|c|c|c|c|}
\hline \multirow[b]{2}{*}{ Fragment (wt) } & \multicolumn{2}{|c|}{ Greiner, et al. ${ }^{7}$} & \multicolumn{2}{|c|}{ Ridde11 and Lepore } & \multirow{2}{*}{$\frac{\text { This experiment }}{\sigma_{\exp }}$} \\
\hline & $\begin{array}{c}\sigma(p) \\
\text { (leV/c) }\end{array}$ & $\begin{array}{c}\sigma\left(\theta_{\text {proj }}\right) \\
\text { (deg) }\end{array}$ & $\begin{array}{c}0(\mathrm{p}) \\
(\mathrm{MeV} / \mathrm{c}) \\
\end{array}$ & $\begin{array}{c}\sigma\left(\theta_{\text {proj }}\right) \\
\text { (der) } \\
\end{array}$ & \\
\hline$p(0.74)$ & $69+6$ & $1.38 \pm 0.11$ & 79 & 1.58 & \\
\hline$d(0.19)$ & $134 \pm 4$ & $1.34 \pm 0.04$ & 107 & 1.07 & \\
\hline \multirow[t]{2}{*}{$t(0.07)$} & $144 \pm 6$ & $0,96 \pm 0,04$ & \pm 26 & 0.84 & \\
\hline & \multicolumn{2}{|c|}{$\sigma_{z=1}=1.35 \pm 0.11$} & & 1.45 & $1.5-1.5$ \\
\hline${ }^{3} \mathrm{He}(0,24)$ & $150 \pm 6$ & $1.00 \pm 0.04$ & 126 & 0.84 & \\
\hline \multirow[t]{2}{*}{${ }^{4} \mathrm{He}(0.76)$} & $130 \div 1$ & $0.65 \pm 0.005$ & 138 & 0.69 & $0.64 \pm 0.02$ \\
\hline & & $=0.73 \pm 0.06$ & & 0.73 & \\
\hline
\end{tabular}




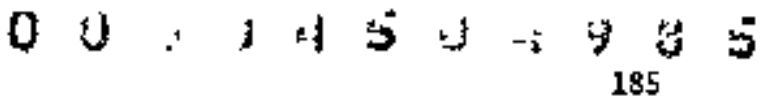

2. H. C. Bradt and B. Peters, Phys. Rev. 77, 54 (1950).

3. P. J. Lindstrok, D. E. Greiner, and $H . H$. Hecknan, Bu11. An. Phys, Soc. 17, 488 (1972). Also H. H. Hecknar, Relativistic He⿳亠丷厂,y Ion Seminar, Intemral LBL Report, 1973 (umpubished).

4. J. D. Bownan, W. J. Swiatecki, and C. F. Tsang, LBL-290B (1973),

5. S. Barshay, C. B. Dover, and J. P. Vary, Phys.
Rev, C 11, 360 (1975). Alsp private cammuication, J. P. Vary $(1975)$.

6. W. J. Swiatecki and C. F. Tsang, private commulcation (1974).

7. D. E. Greiner, P. J. Lindstron, H. H. Heckman, B. Cork, and F. S. Bieser, Phys, Rev. Lett. 35, $152(1975)$.

B. J. V. Lepore and R. J. Ridde11, Jr., LEL-3068, 1974 (tumpublished).

\section{COLLOMB DISSOCIATION OF RELATIVISTIC ${ }^{12} \mathrm{C}$ AND ${ }^{16} O$ NUCLEI}

Harry $\mathrm{H}$ Heckman and Peter J Lundstrom

Experirental evidence for the dissociation of Bevatrou/Bevalac beam nuclei in the nuclear Coulonb fields of target nuclei has cone from experiments. of the target dependence of the isotopic production eross sections for secondary nuclei produced by the fragnentation of $12 \mathrm{C}$ and 160 ruclel at energies $E=1.05 \mathrm{GeV} / n(12 \mathrm{C})$ and $2.1 \mathrm{GeV} / \mathrm{n}\left[{ }^{12} \mathrm{C}\right.$ and 160$){ }^{1}$ Ey use of photonuclear cross section data and the Helzsacker-Willians (wW) method of virtual quanta, 2,3 we are able to account for the measured cross sections and to determine the winiman impact parameters for coulomb dissociation of heavy-ion projectiles.

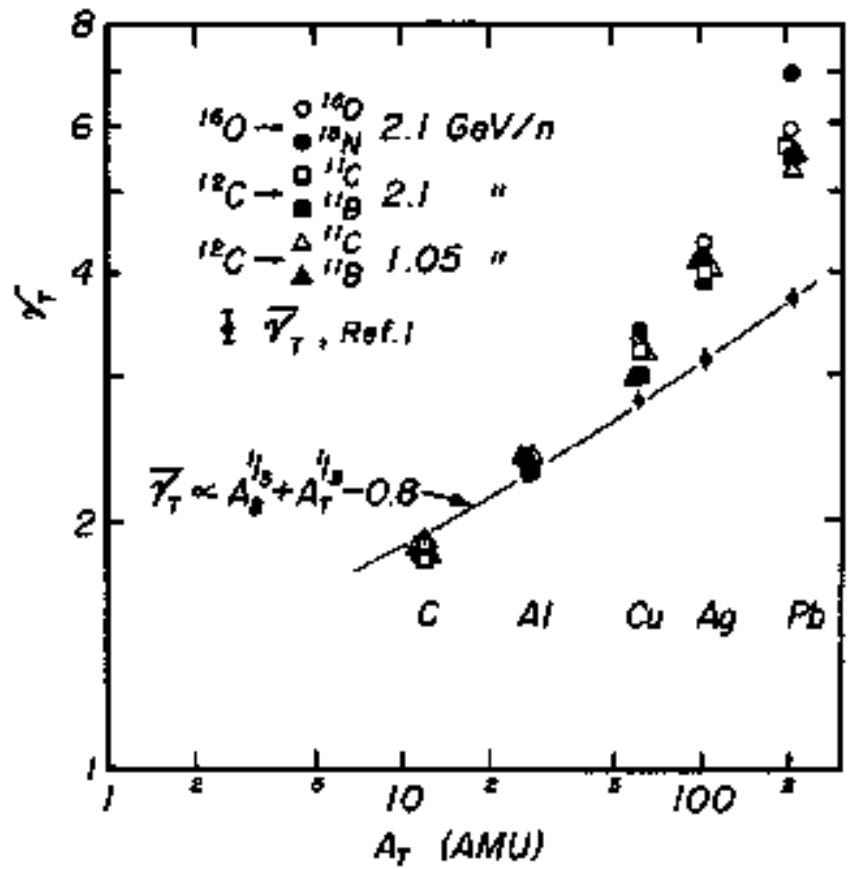

Fig. 1. Target factors $Y$ plotted versurs target mass $A_{T}$ (ANS), fron Lindstrom, et al. 1 Individual values of $\gamma_{T}$ are shown for the singlemukleon-Ioss cross sections tndicated. The curve $\bar{Y}_{T} \times A_{B} 1 / 3+$ $\mathrm{AT}^{1 / 3}-0.8$ is drawn through the mean tarfet factors, shown with error bars, for all cross sexions of where $A_{F}<A_{B}-2$.
Essential to our analys is is that the cross sections of for the reaction $\mathrm{B}+\mathrm{T}+\mathrm{F}+\ldots$, where $B, T$ and $F$ are the bear, tagget and fragment puclei, are factorable. That is, ofT = Y ${ }_{\mathrm{B}}^{\mathrm{F}} \mathrm{T}$, where of is deperdent on $B$ and $F$ only and $T_{T}$ is the target factor Plotted in Fig. 1 are the target factors $\gamma_{T}=\sigma_{\mathrm{KT}} / \gamma_{\mathrm{B}}$ versus target nass $\mathrm{A}_{\mathrm{T}}(\mathrm{AMU})$. For fragment nuclei with nass $A_{F}=A_{B}-2$, i.e., at Ieast tap nucleons are removed from the becon projectile, all isotoplc production cross sections, for a given tarket, are interrelated by a unique target factor, $Y_{\mathrm{T}}$. Striking doviation of $\mathrm{Y}_{\mathrm{T}}$ from $\bar{Y}_{T}$, U to 30 s in $\mathrm{Pb}$, are observed for those fragmentation cross sections that involve the loss of one nucleon from the projectile. The differences between the observed values of $Y_{T}$ and $\bar{\gamma}_{T}$ increase approximately as $z$ of the target, indicative of the Coulomb effect.

We have applied the Weizsäcker-Widliams method of virtual quanta to estimate the cross sections for Codlonb dissoclation of relativistic nuclej. $2-4$ To the extent that $N(\omega)$, the equivalent number of virtuel photons per Met, is the same for all electric and gnetic multipoles, the WeizsäckerWilliams cross section for the dissociation of a meleus, at velocity $B$, by the coulonb fiold of a target nucleus, etomic mumber 2 , is given by

$$
\sigma_{w W}=\int_{\omega_{0}} \sigma_{v}[(\omega) \mathbb{N}(\omega) d w \text {, }
$$

where $\sigma_{v}(\omega)$ is the measured photonuclear cross section at photrn energy w. The number density of virtual photons has the fimctional form $N(\omega)=$

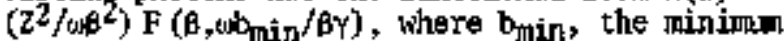
impact paraneter, is the only adjustable paraneter in $\sigma_{\text {WN. }}$.

By equating wy, $\mathrm{E}$. . (1), we have deternined that the irmact paranoter bmin appropriate for each cross section. The minimm inpact parageter is defined by the relation $b_{\min }=r_{0.1}^{8}+r_{0.1}^{T}-\mathrm{d}$, where the $x_{0,1}{ }^{\dagger} s$ are the $10 \%$ charge-density radif of the beand and target nuclei, 5 and $d$ is the radtal-overlap distance. The values of $b_{\text {min }}$ obtained in this experiment are, to within the accuracy of the data, confined to a limited rempe 


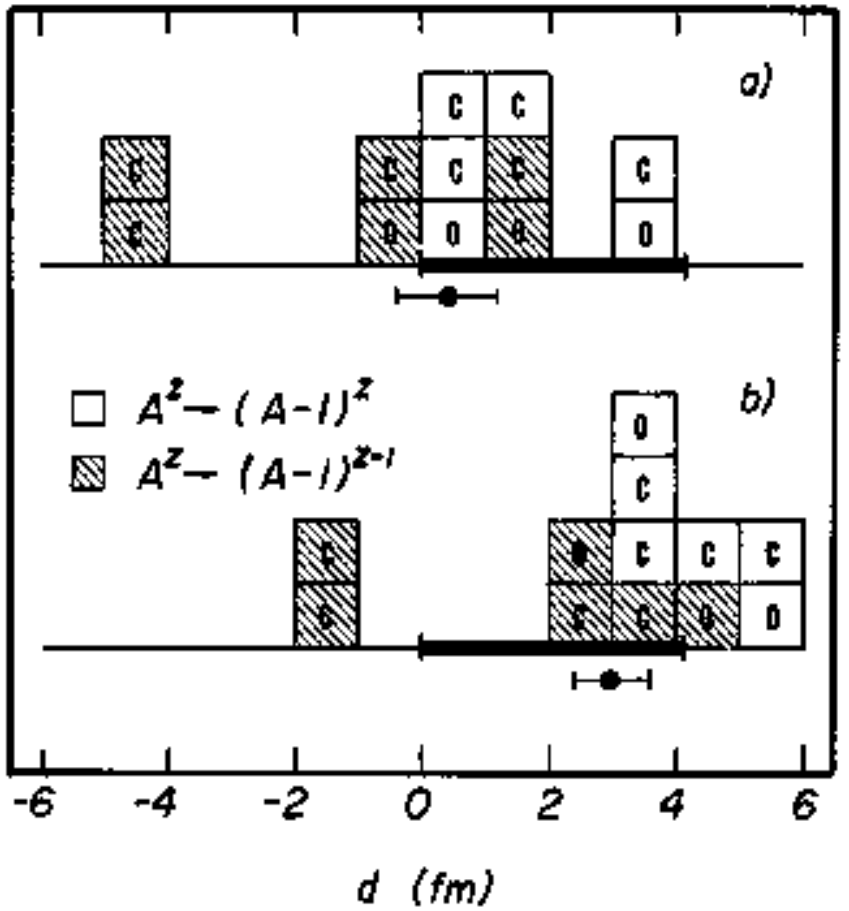

Fig. 2, Distributions of overlap distances $d\left(b_{\text {min }}\right)$, and their means, derived from ow (exp) when fittegd to the Heizșäcker-Williams cross sections own, as, given by a) Jackson ${ }^{2}$ and (b) Jackle and Pilkulu. 3 The dark horizontal bar delineates the overlap

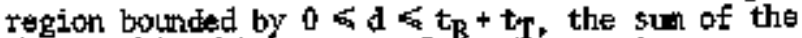
charge-skin thicknesses of the beam and target nuclei.

(XBL 763-881)

in d. Presented in Fig. 2 are histograms of the overlap-distances d that account for the experimental cross sections oww(exp) for $12_{C}$ and $1 b_{0}$ projectiles in $\mathrm{Ag}$ and $\mathrm{Pt}$ targets.

The standard deviatzons of the d-distributions are compatible with the statistical errors fth ow (exp). Principle systematic errors come from theory $(\mathrm{N}(\omega)$ ), the measure photonuclear cross sections ow $[w]$, and those inherent in the method used to extract $\sigma_{W W}(\exp )$ from of $F$ typical error in the ${ }^{\mathrm{BT}}$ photonuclear cross section data, leads to a 1-fin change in $₫\left(b_{m i n}\right)$.

Figure 3 presents the cross-gection date from

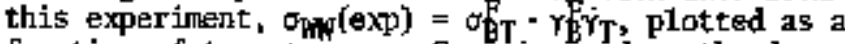
function of target mass. Superimposed on the data are curves of the computed cross sections $\sigma_{\mathrm{WW}}$ (Jäckle and Pilkuhn) evaluated for a constant over overlap distance $\bar{d}=3.0$ fin.

Following Lindstrom et ${ }^{a l}{ }^{1}$ we find that for $A_{T}{ }^{3} 12, \breve{Y T}_{T} \times\left(A_{B} 1 / 3+A_{T} 1 / 3=0,8\right)$ (see Fig. 1), which wher expressed in terms of $r_{0}$ becomes $\bar{Y}_{T} \propto\left(r_{0.1}^{R}+r_{1}-2.0\right)$. Thus, we find that an effective overlap distance in $\gamma_{T}$ of $d^{+}=2.0 \mathrm{fm}$ accounts for the nucleat (diract-interaction) part of the total fragnentation cross sections, a value that agrees well with the $\overline{\mathrm{d}}$ 's $[0.4$ and $3.0 \mathrm{fm}$, see $\mathrm{Fig}, 2$ ) deduced from the impact parameter's that account for the coulonb dissociation cross sections.

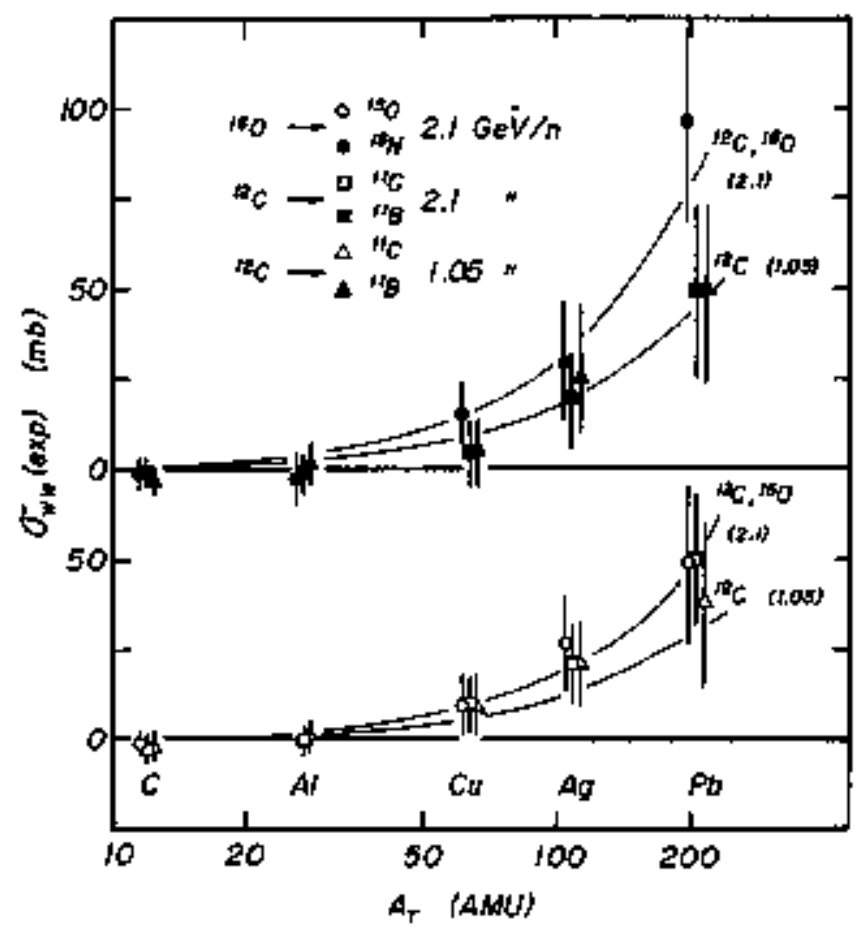

Fig. 3. Target dependence of the measured cross sections ouy (exp) for the Coulom dissoclation reactions indicated. The curves are corputed using the Jackle and Pilkuhn form of $\sigma_{\mathrm{T}}$ with $\overline{\mathrm{d}}=3.0 \mathrm{fm}$.

(XEL 763-883)

The salient features of own (exp), we conclude, are attributable to the fragnentation of projectile nuclei by the Coulomb field of the target musleus. The valute of $b_{\text {min }}$ dertved from ow (exp) lisaits the radial coverlap, a, of the colliding nuclei to distances comparable to their charge-skin thicknesses, $t$, a manifestation of the effects of nuclear absorption. The Coulcmb and nuclear fragnentation processes are related by the result that $\bar{d} \approx d$ ' which shows that the raximem overlap distance that accounts for Couldmb dissociation, in essence, tantsonount to the nuclear overlap afstance required to accotnt for muclear (direct-interaction) fragmentation.

\section{Foptrotes and References}

*Condersed from LEL-4380.

1. P. J. Lindstrom, D., E. Greiner, H. H. Hecknian, Bruce Cork, and F. S. Bieser, LEL-3650 (1975).

2. J. D. Jackson, Classical electrodynanics, 2nd ed., p 719, New York lility (1975).

3. R. Jäckle and Pilkuhn, Nhe. Phys. A247, 521 (1975).

4. X. Artru and G. B. Yodh, Phys. Letters 40B, 43 (1972).

5. R. Hofstadter and H. R. Collart, "Whclear radi determined by electron scattering," in Nerical Data and Functional Relationship in Science and Techology, vol. 2, H, Schopper, ed. (springer verlag, 1967, p.21. 


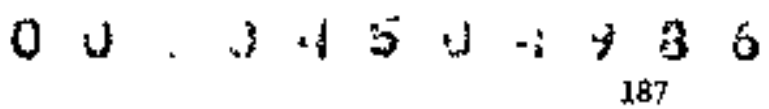

\title{
PFODUCTION OF LIGHT NUCLEI IN NUCLEUS-NUCLEUS COLLISIONS AT 1.6 AND 2.1 GeV/NUCLEON
}

\author{
J Papp." J Jaros.t L Schroeder, J Staples, \\ $H$ Steiner, A Wagner" and J Wiss
}

The avallability of heavy-ion beans at Berkeley with kinetic energies in the range of 1-2 GeV/ mucleon has opened up new areas of experimental investigation. In this experiment, measured the single-particle inclusive spectra of light noclei $\left(p, d, 3 \mathrm{He}, 3 \mathrm{He}\right.$, a) procuce at $2.5^{\circ}$ (1ab) by begals of 1.05 and $2.1 \mathrm{GeV} / \mathrm{n}$ deuterons and alphas, and by 1.05 GeV/n carbon netclei bombardtng a variety of targets $(\mathrm{Be}, \mathrm{C}, \mathrm{Cu}, \mathrm{Pb}$ ). The detection systen consisted of a magnetic spectroneter which measured $p / z$ from 0.50 to $5.0 \mathrm{GeV} / \mathrm{c}$, and plastic scintillation counters for measuring TOF and $\mathrm{dE} / \mathrm{dx}$.

There were several reasons for making these measurswents:

1. To obtain information on the nuclear structure of the incident projectile by studying projectile fragmentation.

2. To test whether high energy physics concepts, such as scaling and limiting fragmentation con be applied to nuclear processes at these energies.

Data ${ }^{1}$ for the prodxtion of light nuclei at $2.5^{\circ}$ (1ab) by $1.05 \mathrm{GeV} / \mathrm{n}$ incident derteron, alpha, and carbon nuclei on a carbon target are shown in Fig, $1,(a, b, c)$. The Lorentz invartant cross section $\mathrm{E} / \mathrm{k}^{2} \mathrm{~d}^{2} \sigma / \mathrm{d}$ aik is plotted against the monenttin (k) of the detected fragment. Fron these data we can make the following observations conserning fragments lighter than the profectile:

1. The alpha particle is seen to fragntent into protons, as well as signifiçant numbers of deuterons, tritons, and $3_{\mathrm{T}}$.

2. For each projectile, the manentura spectra for individual fragnents are strongly peaked, and extend over approximately 2 to 3 orders of magnitude in the cross

3. The value of the momentum at the peak of each of these fragentation distributions is approxinately equal to the momention/ nucleon in the incident projectile. This feature has been observed for heavier fragments from beans of carben, nitrogen, and oxysen by Heckman and collaborators, 2

4. Where overr1apping data points exist, the ${ }_{3} \mathrm{H}$ and $3_{\mathrm{H}}$ production cross sections agree reasomably well as expected for particles fron the salp 15ospin multiple.
For fragnents more massiye than the projectile (Fig. 1a) the spectra are observed to fall off as the monentum is increased. This is to be expected If these particles are either knocked out of the target, the result of pick-tp processes, or arise from conplicated flnal-state interactions.

Some insight into the production mechanism can be obtained by stuxdying the dependence of the pruduction cross section on the target mass. We have perametrized the production cross section in the form: $a$ a $A^{n}$, where $A$ is the mass of the target. In Fig. 2 we heve plotted the coefficient I versus the monentum of the produced particle for alpha particle collisions at $1,05 \mathrm{GeV} / \mathrm{n}$. For monenta in the projectiles frognentation region the cross section varies as $\mathrm{A} 1 / 3$, suggestive of a peripheral interaction. At lower momention the cross section exhibits a rapid growth. For the case of tritons the cross section starts to approach $\mathrm{A}^{2}$. The following picture of the production of light muclear particles at forward angles emerges. At high momenta, profectile fragentation dominates a11 processes and is a surface phenomena varying as $\sim \mathrm{Al} / 3$. As the momentimn is decreased other processes start to contribute to the cross sectical. The exact nature of these processes is not known at present, but will be the stbject of future investigations.

He are presently studying these data to sae if limiting fragmentation for processes like $d+A+p+X$ or $a+A+p+X$ holds at these energies.

\section{Footnotes and Reforences}

"Watkins-Johrson Corp., Redwood City, California.

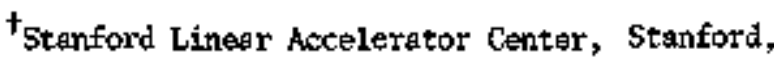
Calirornia.

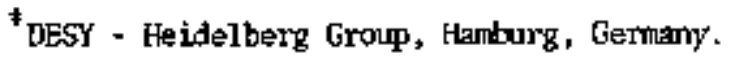

1. J. Papp, Single Particle inclusive Spectra Resulting from the Collision of Relativistic Protons, Deuterons, Alpha Particles, and Carbon Ions with Nurlei, LBL-3633 (unpubl ished thesis) .

2. H. H. Hectomen, D. E. Greiner, P. J. Lindstrom, and $F_{+}$S. Bieser, Phys, Rev. Letters, 28, 921 (1972); D. E. Groiner, P. J. Lindstrum, H. H. Heckman, B. Cork, and F, S. Bieser, Phys. Rev. Lett. 35, 152 (1975). 

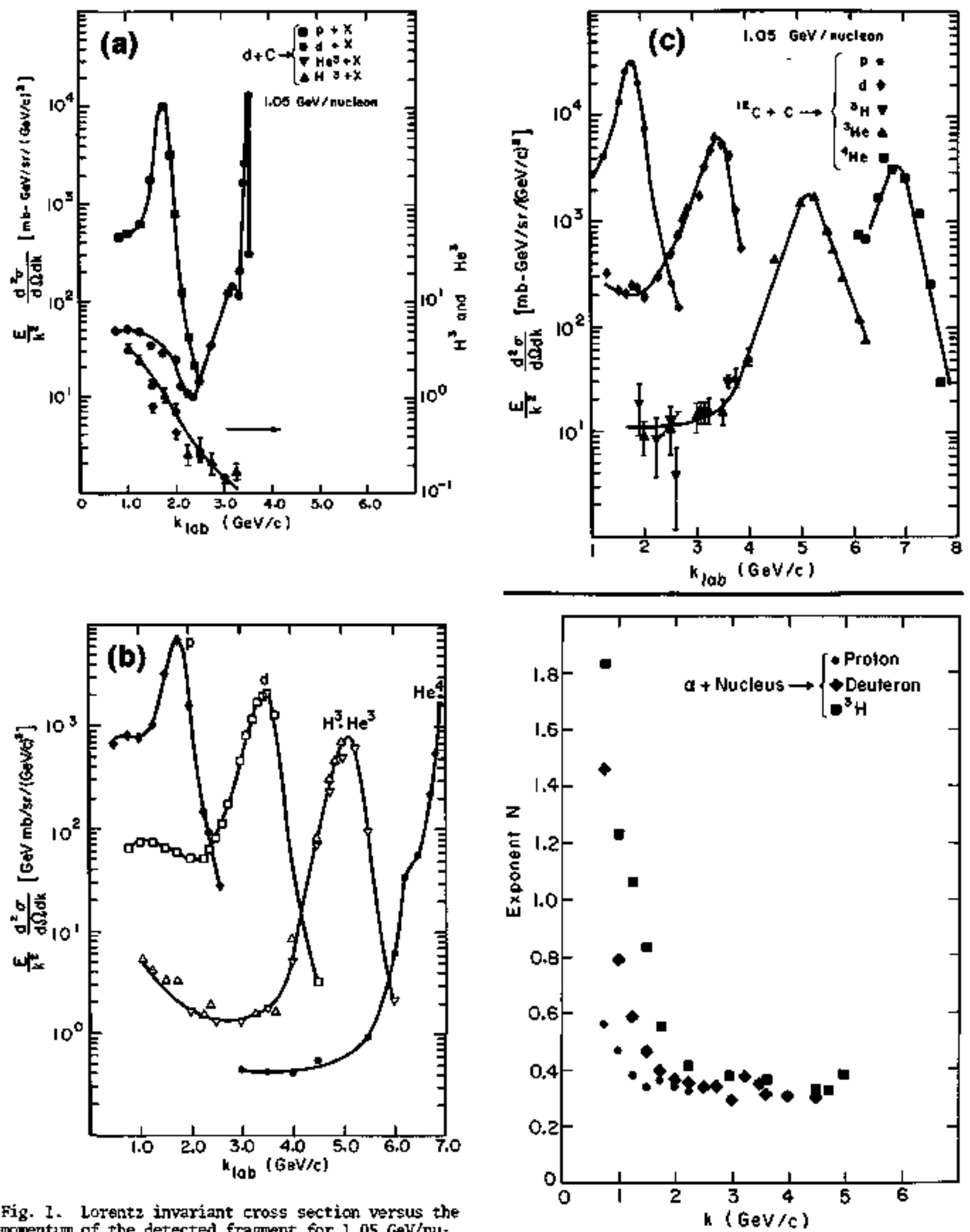

Fig. 1. Lorentz invariant cross section versus the momentum of the detected fragnent for $1.05 \mathrm{GeV} / \mathrm{nu}$ cleon (a) $\mathrm{dC}$, (b) $\propto C$, (c) $\mathrm{CC}$ collisions, Fragments detected at $2.5^{\circ}(1 a b)$.

CYBE 748-3790,

$-3803,-3797$ )

Fig. 2. Plot of the exponent $\mathrm{N}$ obtained from fitting the target dependence of variow cross sections with an $\mathrm{A}^{N}$ type function. (YRL 7411-8320) 


\title{
NUCLEUS-NUCLEUS TOTAL CROSS SECTIONS
}

\author{
John Jaroe, Duren Chambertan, Herber Steiner, Albrecht Wagner, \\ Faymond Fuzesy, Lee Schwoeder, Leonerd Anderson, and Gilpert Shapwo
}

The performed an experiment at the Bevatron in which we measured the total cross section for reactions initiated by protons, deuterons, alphas, and carbon malei on targets of hydrogen, deuterium, helium, and carbon. Measurements of proton-nucleus cross sections in the CoV/mucleon energy range have already been well explained in terms of Glauber's Inutiple scattering theory, 1-3 Using paraneters describing nuxlean-nuxleon scattering and the knowr nuclear sizes, Glauber theory has accurately predicted these total cross sections without invoking my special effects and, in fact, ignoring such genuine colplications as nuclear correlations and Fermi momentum. The formalism of the theory has been extended to encompass nueleus-nucleus collisions 4 and has been used by several authors $5-9$ to predict total and inelastic cross sections for various nucleus-tuclens reactions.

The theory is essentially geometrical and predicts that total cross sections are approxinately proportional to the square of an interaction radius that goes like the sull of the radii of the colliding objects. If $\mathrm{Ar}$ and $\mathrm{Ap}_{\mathrm{p}}$ are the atomic mass nubers of the target and projectile, $\sigma$ ToT $\sim\left(A_{T}{ }^{1 / 3}+A_{D} 1 / 3\right) 2$. Deviations from this simple behavior might indicate the presence of new procespspes in these collisions. In view of the mique conditions that occur in collisions of nuclei, we may hoge for some surprises. A carbon-carbon collision at energies presently available can produce states of roasomably high energy density over a volume an order of megnitude greater than ocours in pp collisions, The Coulgrb forces involved when large muclei interact are no longer smill compared to the nuclear forces. The interplay between the two should be irportant and interesting. The collision of tow nuclei provides a new envirument for nuxien-maleon collisions. Does a protion which has interacted in one part of a nucleus behave as a "regular" protor in subsequent colliskons?

An observation due to Gribov ${ }^{10}$ also motivated this experiment. He noted that a naive application of Regge factorization to nucleus-nucleus reactions leads to a very different $A$ dependence for ${ }^{0} \mathrm{~T}$ than that expected from gectaetrical grounds. The argument is as follows: The optical theoren relates $\sigma_{\mathrm{T}}\left(\mathrm{AB}^{\prime}\right)$ to the inaginary part of the elastic scattering amplitude $F$. If we assums this applitude is dominated by the Porweron, factorization lets us write it as $\mathrm{F} \sim \mathrm{gPA}_{\mathrm{BPB}}$, where $\mathrm{gPA}_{\mathrm{PA}}$ is the coupling of a Pomeron to mucheus $A$ at $t=6$, etc. for $\mathrm{F}_{\mathrm{PB}}$ Thus $\sigma_{T}(A B) \sim g_{\mathrm{PA}} \xi_{\mathrm{PB}}$; and similarly $\sigma_{\mathrm{T}}(\mathrm{BE}) \sim \mathrm{g}_{\mathrm{PB}}{ }^{2}$ and $\sigma_{T}(\mathrm{AA}) \sim \mathrm{g}^{2}$. Consequent1y, the "factorization relat $1 \mathrm{Cr}^{\prime \prime} \sigma_{\mathrm{T}}(\mathrm{AR})=\sigma_{\mathrm{T}}{ }^{2}(\mathrm{AB}) \sigma_{\mathrm{T}}(\mathrm{BB})$ obtains. If we use this to predict $T_{T}(A N)$ from the input $\sigma_{T}(p A)$ and put $a_{T}(\mathrm{pA}) \sim \mathrm{A}^{2 / 3}$ which roughly represents the data, we find $\sigma_{T}(A A) \sim A^{4 / 3}$. This differs radically from the behavior expected in a gegmetric picture where $\sigma_{T}(A A) \sim\left(A^{1} / 3+A^{1} / 3\right)^{2} \sim A^{2 / 3}$. Fl gure 1 shows

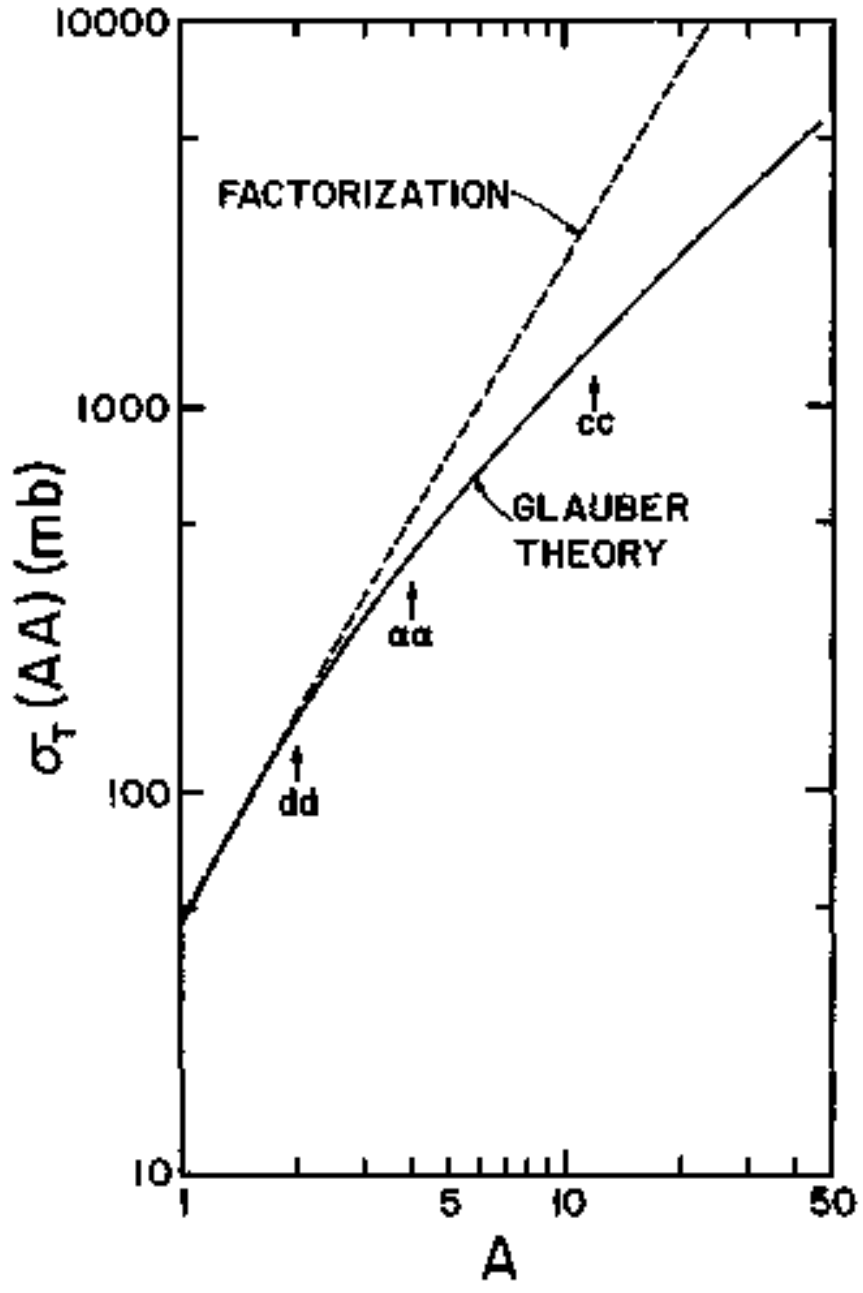

Fig. 1, Predictions for $\sigma_{T}$ (AA) vs $A$.

[X]-7511-9047)

the differences between geometrical and factorization relation predictions for $\sigma_{\mathrm{T}}(\mathrm{AB})$ as a function of $\mathrm{A}$.

We neasureo total tross sections for protons, deuterons, alphas, and ${ }^{2} \mathrm{C}$ on hydroger, deuterium, holium, and carbon targets at 1,55 and $2.89 \mathrm{GeV} / \mathrm{c}$ per nucleon using the "good geonetry" transnission method. In addition, we measured the inelest ic cross sections and elastic slope parameters for reactions initiated by deuterons, alphas, and $12_{C}$. The factorization relation $\sigma_{\Gamma}(A A)=\sigma_{T}(A B) / \sigma_{T}[B B]$ is violated for some of these reactions. Our restilts generally agree with Glauber theory predictions except in thejr detalled energy behavior. He find

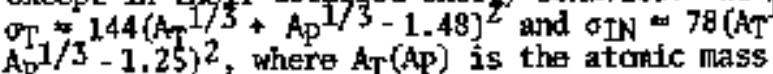
nuber of the target (projectile) and tho cross sections are given in mb (see Figs. 2 and 3 ), 


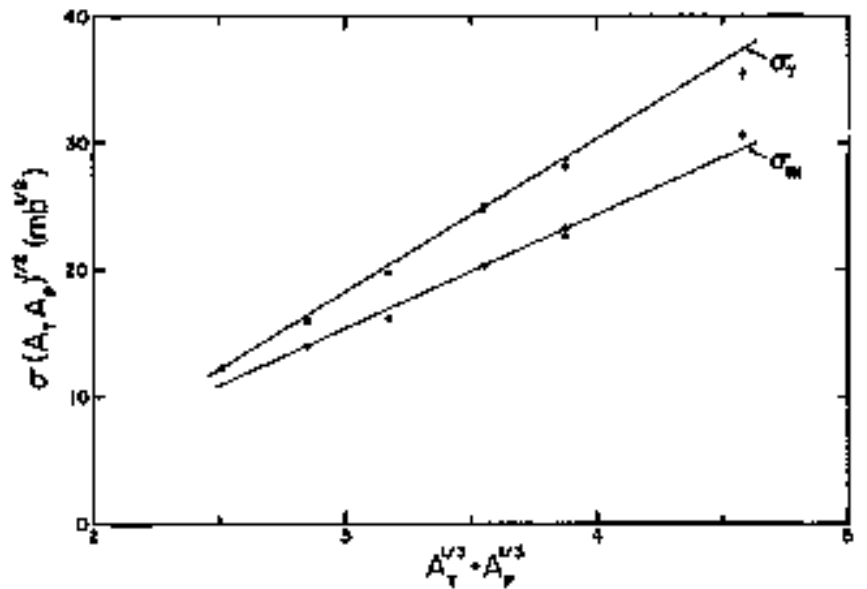

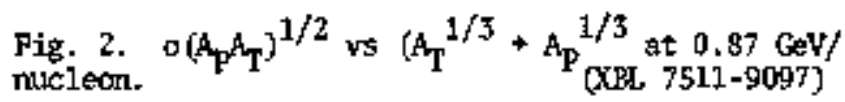

\section{References}

1. R. J. Glauber in Lectures in Theoretical Pbysics, Vol. 1 (Interscience, New York, 1959), p. 315.

2. R. J. Glauber in High Energy Physics and Nuclear Structure (North-Holland, Ansterdem, 1967), p. 311 .

3. R. J. GIauber in High Energy Physics and Nuclear Structure (North-Holland, Ansterdan, 1969), p. 207.

4. W. Czyz and l. C. Maximon, Annals of Physics 52, 59 (1969).

5. A. Tekou, High Energy Elastic and Quasi-E1astic Deuteron-holelus Scattering, International Center

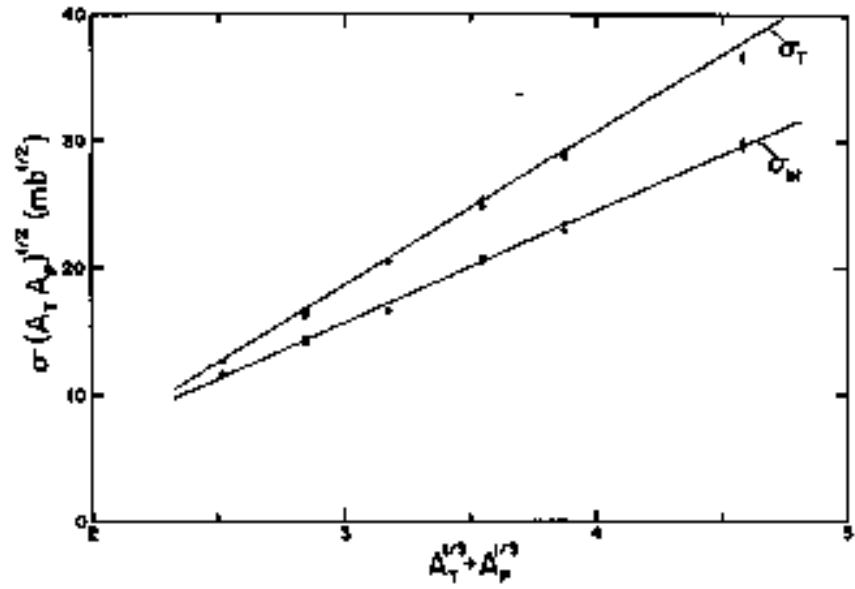

Fig. 3. $\sigma_{\mathrm{T}}\left(A_{\mathrm{P}} \mathrm{A}_{\mathrm{T}}\right)$ vs $\left(\mathrm{A}_{\mathrm{T}}^{1 / 3}+A_{\mathrm{P}}^{1 / 3}\right)$ at $2.2 \mathrm{GeV} /$ mucleon.

(KBBL. 7\$10-8499)

\section{(unpublished).}

6. Y. Pranco, Phys. Rev. Lett. 32, 911 (1974).

7. P. M. Fishbane and J. S. Trefil, Predictions for Nucteus-Nucleus Scettering at High Energies, University of Virginia preprent (umpublishes).

8. H. L. Kang, Ply's. Lett. B52, 142 [1974).

9. S. Barshay, C. B. Dover, and J. P. Vary, Phys. lett. BS1, 5 (1974); J. Vary, private comumication.

10. V. N, Gribov, Sov. J. Nuc1. Phys. 9, 369 (1969).

\title{
INCLUSIVE PION PRODUCTION IN COLLISIONS OF RELATIVISTIC PROTONS, DEUTERONS, ALFHAS, AND CARBON IONS WITH NUCLEI+
}

\author{
」 Papp, J Jaros, L Schroeder, ป Staples
}

H Steniner, A Wagners and J Wiss

Whe have meastred single-particle inclusive spectra of $\pi^{+}$and $\pi^{-}$nesons produced at $2.5^{\circ}$ (1ab) in the collision of $1.05-4.2-\mathrm{GeV}$ protons, $1.05-$ and $2.10 \mathrm{GeV} /$ nucleon deuterons and $\alpha$ particles, and 1.05 $\mathrm{GeV} /$ nucleon ${ }^{12} \mathrm{C}$ muclei with various targets at an angle of 2.5 (lab). The negative-pion spectra show scaling behavior. The results are coupared to a simple model in which individual nucleons in the projectile interact with the target to produce pions.

Our results on the yields of negative pions from collisions of 1,05-4,2-GeV (kinetic energy) protons with a carbon target are shown in Fig. 1 (a). The Loreritz-invariant cross section $\left(\mathrm{E} / \mathbf{k}^{2}\right) \mathrm{d}^{2} \mathrm{~d} / \mathrm{drdk}$, where $E$ is the energy of the outgoing $\pi^{-}$and $K$ is its monentur, is plotted against the scaling variable $\left.x^{\prime}=\mathbf{k}^{*} / \mathrm{k}_{\|}\right)_{\text {max }}$, where $k_{4}{ }^{\prime \prime}$ is the longitudinal momentum of the pion in the overall center-of- mass system. The nost striking feature of the data is that the spectra tend to lie on top of each other. Similar results are obtained for other targets. Higher-energy data $1,2(12-, 19-$, and $24-\mathrm{GeV}$ protons an Be) also fall on the sane cerrve. Scaling behavfor, where the pion yield does not depend on the जाergy but only on a scaling variable $x^{\prime}$ (at fixed $k_{4}$ ), is familiar in high-energy nucleon-nucleon interactions. The renarkable feature of the present data is that scaling behavior persists, at least approximately, down to 1 Gev. It wust be kept in mind that since this experiment performed at a fixed lab angle of $2.5^{\circ}, k_{1}$ is not quite constant. This effect is nost ibportant near $x^{\prime}=1$ where it could change our results by as much as a factor of 2 . However, since the scaling fit encompasses wore than 6 decades, a variation of even a factor of 2 near the end points nould not produce observable devia- 


\section{$00: 3450 \therefore 430$}

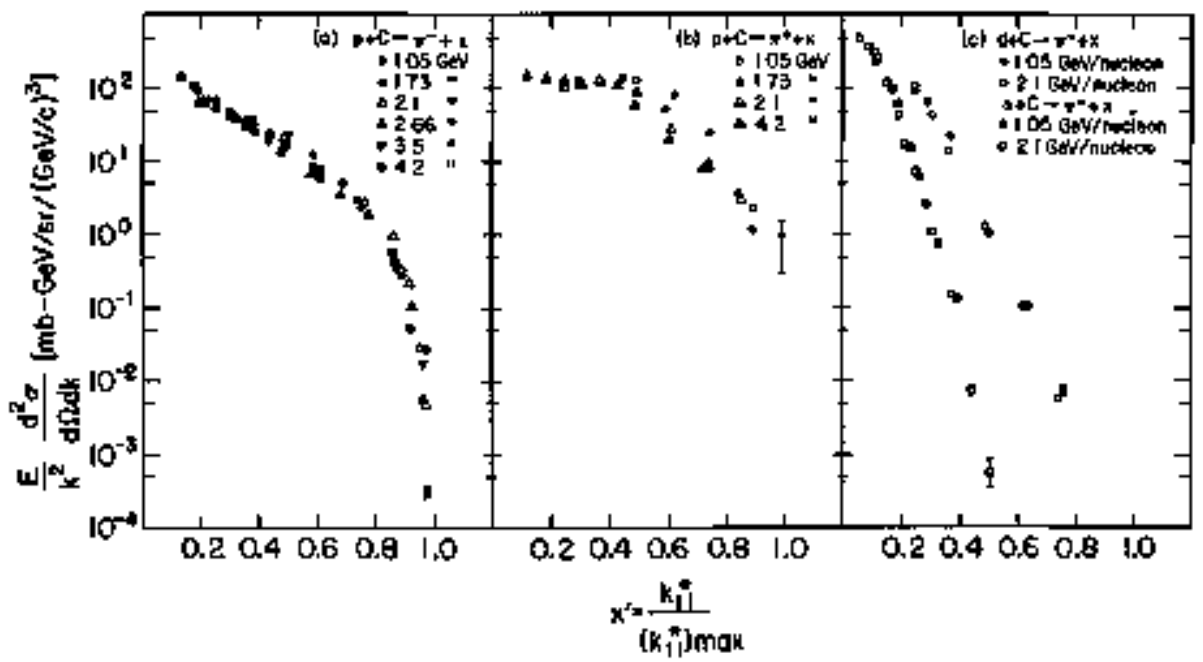

Fis. 1. Invariant cross section for pion production at $2.5^{\circ}$ (lab) from a carbon versus the scaling variable $x^{\prime}=k \mid * /(k \mid)_{\text {max }}$ : (a) Negative-pion production by 1.05-4.2-6eV protons, (b) positive-pfon production by 1,054.2-GeV protons, (c) negative-pion production by $1.0 \mathrm{~S}-$ and $2.1 \sim \mathrm{GeV} / \mathrm{nu}-$ cleon deuterons (circles) and $\alpha$ 's (squeres).

[XRL- 7411-8244]

tions from the trend of the data to scale. The ${ }^{+}$ production spectra are shown in Fig. 1(b). They do not scale nearly as well as those of negative pions, especially at low proton energies.

Invariant nogative-pion production cross sections for 1.05- and 2.1-GeV/nucleon deuterons and $\alpha^{\prime}$ 's incldent on carbon are shown as a function of $x^{\prime}$ in Fig. I(c). Again scaling is reasonably well satisfied. We see that the heavler the projectile the more rapid is the falloff as $x^{\prime}$ increases. This result indicates that nuclei, which are relatively loosely bound objects, tend not to transfer a large fraction of their kinetic energy to indivitual pions. In the case of deuterons, our $x^{\prime}$ distributions in the interval $0.5 \% \mathrm{X}^{\prime}<1 \mathrm{fa} 1$ much tore steeply than those of Baldin et al. 3 who measured $\pi$ production at $0^{\circ}$ by deuterons of about $\mathrm{g} G \mathrm{eV}$ on $\mathrm{Ou}$.

The pion production cross sections for 2,1 $\mathrm{GeV} /$ nuclecr protons, deuterons, and a particles on carbch are compared in Fig. 2. Tho features are evident: (1) The ratio of the cross sections for producing low-manentum negative pions $(\sim 1 \mathrm{GeV} / \mathrm{c})$ by Q's, deuterons, and protons is approximately 10:5*1. (2) The spectrun of observed pions exterkds to higher entrgies as the mass of the projectile is increased. The larger production cross sections in the case of deuterons and a's can be attributed to the presence of neutrons, which prodhce $\pi$ is more copicrsly than do protons, and to the increased energy of the system. Our measurements of $\pi$ production by beans of 1.05-GeV/nurleon ${ }^{12} \mathrm{C}$ show that the ratio of $\pi^{-}$produttion by ${ }^{12} \mathrm{C}$ to $\pi^{-}$proctuction by a particles is $3.0 \pm 0.3$ at a monemtum of $750 \mathrm{MeV} / \mathrm{c}$ for all targets.

In an attempt to deternine the inportance of mecharisms in which several mucleons in an energetic nuclear projectilo act cooperatively to produce pions. we have corpared our experimental ressults to calculations based on a model in which all pionns are produced in individual mucleon-nucleus collisions.

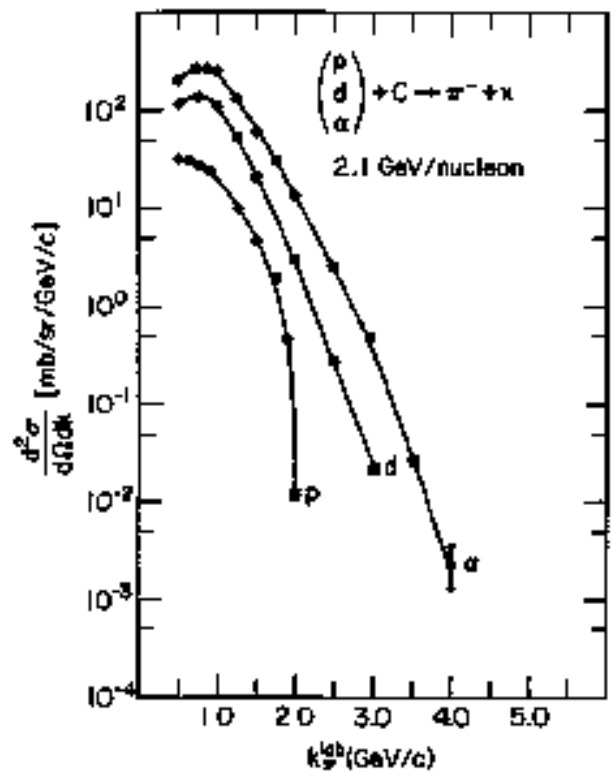

Fig. 2+ Laboratory cross section $\mathrm{d}^{2} \sigma / d$ sdk for negative-pion prothiction at $2.5^{\circ}$ (lab) by $2.1-\mathrm{GeV} /$ macleon proton, deuteron, and a beams on carbon tar. get versus the laboratory momenturu of the pion. The solid lines are hard-drawn and are to serve as a guide to the eye only. Only statistical errors are shown.

(XBL 748-3897)

We asșune that

$$
\begin{aligned}
\sigma_{a A} \pi\left(\vec{P}_{a}, \vec{k}_{\pi}\right)= & \sum_{N}^{a} \int_{W_{a N}}\left(\overrightarrow{\mathrm{P}}_{a}, \overrightarrow{\mathrm{P}}_{N}\right) \\
& \times \sigma_{N A}{ }^{n}\left(\vec{P}_{N}, \vec{k}_{\pi}\right) \mathrm{d}^{3} \mathrm{P}_{N},
\end{aligned}
$$


there a refers to the projectile and $A$ to the target rurcleus. $W_{a N}\left(P_{2}, P_{N}\right)$ is the mamentum distribution of the nucleon ${ }^{\mathrm{aN}}$ inside the projectile as transfonmed to the lab systen. By charge symmetry $\sigma_{1} C^{\pi+}\left(\hat{P}_{N}, k_{\pi}\right)=$

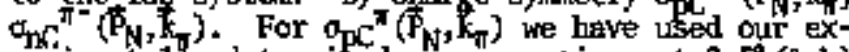
perinenta1 ly determihed crass sections at $2.5^{\circ}$ (1ab) and corrected them for the transverse-momentum variation by essuding that at al 1 momente corsidered in these measurements such a variation can be fitted with an exponential of the form $\exp \left\{-5\left|k_{\pi}\right| \sin \left(\theta_{k_{\pi}}\right.\right.$ $-\theta_{\mathrm{P}_{\mathrm{A}}}$ ]

In the case of the deuteron $w_{\mathrm{dA}}\left(\overrightarrow{\mathrm{F}}_{\mathrm{d}}, \overline{\mathrm{P}}_{\mathrm{W}}\right)$ was obtained fram the Lorent $z$ transfomation of a noma11zed bulthen wave function of the form $|\phi(q)|^{2}$ $\left[q^{2}+\alpha^{2}\right)^{-1}-\left.\left(q^{2}+\beta^{2}\right]^{-1}\right|^{2}$ with $\alpha=45.7 \mathrm{MeV}$ and $\theta=5.2$ MeV iq is the nucleon monentun in the rest frame of the deuteron). The predictions are shown together with the data points in Fig. $3(a)$. The fits reproduce quite well the general behavior of the measured cross sections for fast pions as a function of pion mamenttm. Thexe are no free parameters. These results disagree with the conclusions of Baldin et al, ${ }^{3}$ who clain to be unable to $\mathrm{f}$ t their data with such a model.

The case of a's is camplicated by the fact that the single-nucleon manentun distribution is not well known. From olectramagnetir-form-factor experiment 5 one can deduce a change distribution but it is difficult to translate this to the nowentien dis-

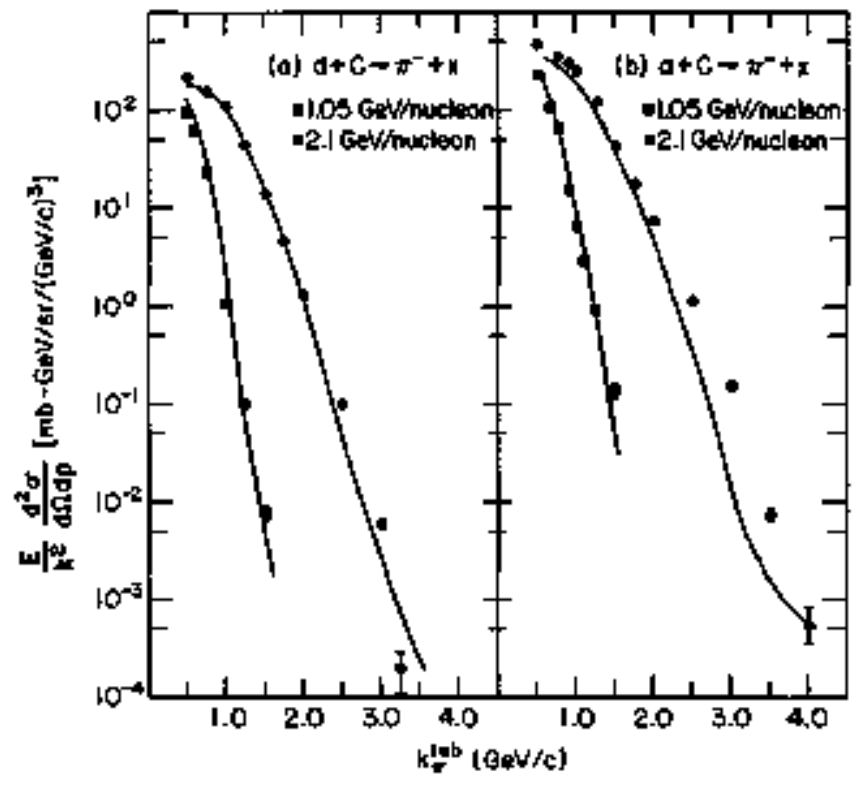

Fig. 3. Invariant cross section for negativo-pion production at $2+5^{\circ}$ (1ab) by 1.05- an $2.1-\mathrm{GeV} /$ nucleon (a) deuteron and (b) a beams yersus the laboratory mosemtum of the pion. The solid lines in each case represent the predictions of the nodel as described in the text.

(XBL 7411-8243) tribution of the individual nucleons $\left|\phi_{\alpha}(q)\right|^{C}$. As a first approxination to $\phi_{a g}$ (q) we Fourier transformed the square root of the nuclear-charge distribution.4 As before $\mathrm{N}_{\mathrm{CNN}}\left(\overrightarrow{\mathrm{P}}_{\mathrm{\alpha}}, \overrightarrow{\mathrm{P}}_{\mathrm{H}}\right)$ was then obtained by transforming $\left|\phi_{\alpha}(a)\right|^{2}$ to the lab system. The results are show in Fig. 3(b). Although the general trends of the data are reproduced by the model, quantitatively the agreenent is not good. At this point we are umable to say whether this is die to a poor ahoice for $H_{\text {oN }}\left(\mathrm{P}_{\mathrm{O}}, \mathrm{F}_{\mathrm{N}}\right)$ or to a breakdown of the model. Further work is in progress for both deuterins and $a^{\prime} s$.

Pion production from $\mathrm{Ee}, \mathrm{O}$, and $\mathrm{Pb}$ is quite similar to that frop carbon. The shape of the spectra is independent of the target material for $k>1 \mathrm{geV} / c$. The onanitude of these cross sections is proportionat to $A^{1 / 3}$, suggesting that fast pions are produced in peripheral collisions. For lowarmonentirin plans, the $A$ deppendence becomes nome pronounced $\left(e . g ., 0 * A^{1} / 2\right.$ for $k_{r}=500 \mathrm{keV} / \mathrm{c}$ ), suggesting that slow pions are produced in more central colisions.

For isosptn-zero muclel likke deuterons, a particles, and $12_{C}$ charge symatry predicts that in the reartions $d+C+\pi^{ \pm}+X$ and $a+C+\pi^{ \pm}+X$ the ratio of $\pi^{+}$to $\pi^{*}$ production should be unity. In every cage our results are consistent with this prediction to with In the experinental errors of about $10 \%$.

\section{Footnotes and References}

*Based on Phys. Rev. Lett. 34, 601 (1975).

${ }^{\dagger}$ Watkins-Johnson Corp, , Redwood City, Califormia.

\$Stanford Linear Accelerator Center, \$tanford, California.

\$Hidelbarg - LESY Group, Heidelberg, Germany

1. G. J. Marmer and D. Lundquist, Phys. Rev. D3, 1089 (1971); G. J. Henter, K. Reibel, D. M. Schwartz, A. Stevens, R. Winston, D. Kolfe, P. R. Phillips, E. C. Swallow, and T. A. Roranowski, Phys, Rev, 179, 1294 (1969).

2. D. Dekkers, J. A. Geibel, R. Mermond, G. Weber, T. R. Willitts, K. Winter, B. Jorden, M. Vivargent, N. M. King, and E. J. N, Milson, Phys. Rev. 137, B962 (1965).

3. A. M. Baldin, S. B. Gerasinov, H. Guiordenescu, V. N. Zuharev, L. K. Ivanova, A. D. Kirillow, V. A. Kuznetsov, N. S. Moroz, V. B. Ratomanov, V. N. Ramzhn, V. S. Stavinski, and $M_{+} I_{+}$Yatsuta, Yad. Fiz. 18, 79 (1973) [ Sov. J, NhucI. Phys. 18, 41[1974]].

4. R. F. Frosch, J. S. Hocartly, R. E. Rand, and M. R. Yearin, Plys, Rev. 160, 874 (1967). 


\title{
DIFFRACTIVE SCATTERING OF HEAVY IONS
}

\author{
A R. Clark, W. R. Holley, D. Keele, L. T. Kerlh, R. E Morgendo; \\ M. Richerdson, $G$ Scchnurmacher, L. S. Schroeder, and A. R. Znnghes
}

This experinent has measured $12 \mathrm{C}+\mathrm{p}+\mathrm{X}+\mathrm{P}^{\prime}$, $A_{\mathrm{H}}+\mathrm{p} \rightarrow \mathrm{X}+\mathrm{p}^{\prime}$ and $\mathrm{p}+\mathrm{p}+\mathrm{X}+\mathrm{p}$ ' 1nelastic scattering. the used \& Bevalac bean ${ }^{12} \mathrm{C}, 4 \mathrm{He}$, $\mathrm{p}$ at $4.2 \mathrm{GeV} / \mathrm{c}$ charge rigidity on a stationary liquid hydrogen target, observed the recoil proton $p^{\prime}$, and sumed over $X$ the unseen outgoing beam. We measured the double differertial eross section $\mathrm{a}^{2} \mathrm{o} / \mathrm{dtd} d \mathrm{x}_{\mathrm{X}}^{2}$ as a function of the nowentur transfer $(\sqrt{-t}=200-300 \mathrm{MeV} / \mathrm{c})$ and the excitation energ $\left(\mathrm{E}_{\mathrm{X}}-\mathrm{Hx}_{\mathrm{X}}-\mathrm{M}_{\mathrm{C}}, \mathrm{He}, \mathrm{p}=0-250 \mathrm{MeV}\right)$. This is equivalent to missing mass squared $A=b x^{2}$ $-M^{2}-0-6$ sel $V^{2}$ and $-t=.04$ to $.09(\mathrm{GeV} / \mathrm{c})^{2}$. This probes both the nuclear structure and the dymamics of diffractive scattering. It tests whether ideas of asyntotic scattering, particularly Reggetheller prguerth phencmernology, are relevant to heawy baryon. phystes. These ideas of a collective baryon scattering contrast with individual nutleon models such as the Glauber model.

This region of relatively smali mogentura transfer $t$ is expected to be dentinated by diffractive scattering. It is relatively easy to measure a snall momentur transfer to a stationary target. This is a major advantage over a proton benm on a carton target; there a small change in a large momentam would require a high-resoltution spectroneter. A snall disadvantage is that we are scmewhat sensitive to Coulonb scattering of the recoil proton. This region goes from elastic scattering, thru the nuxlear excitations and beyond the pion threstold, At these large missing masses (or large excitation), showld the cross section increase as the pion chamel opens? The corresponding situation at $F+N . A . L$. is $\mathrm{p}+\mathrm{p} \rightarrow \mathrm{X}+\mathrm{P}$ with large excitation end snall monentum transfer. Once the missing nass is large and nany channels are open $\left(e+g_{-}, X=p+m\right.$ many pions), then the double differentiai cross section approaches an asymptotic 1imit. Additional pion thresholds open wo at the expense of previous chameIs. This can be understood via the Regge-Mueller pateron phenomenology. Can relativistic muclei be effectively described by the same ideas? This picture contrasts with the Glauber model of nucleus-proton collisions as a num ber of individual mucleon-proton collisions. The latter model predicts that the double differential cross section should rise when the pion charmel opens up.

We have constructed a single arm spectrometer (Fig. 1) to look at the recoil protons. The spectrineter consists of three parts: a target, a quad. rupole doublet, and scintillators. First is a liquid hydrogen tareet with a very thin alumiman wali. This minimizes Coulamb seattering to allow us to look at low recoil proton monenta, and thus low monentum transfer. The second part is a magnetic quadrupole doublet which rotates around the target. It is between the target and the detectors, and it focuses parallet to point in the horizental plane, point to point in the vertical plane. This gives a large vertical acceptance with a precisely defined horizontal scattering angle. The third part is a sequence of elevern thin scintillators whích measure

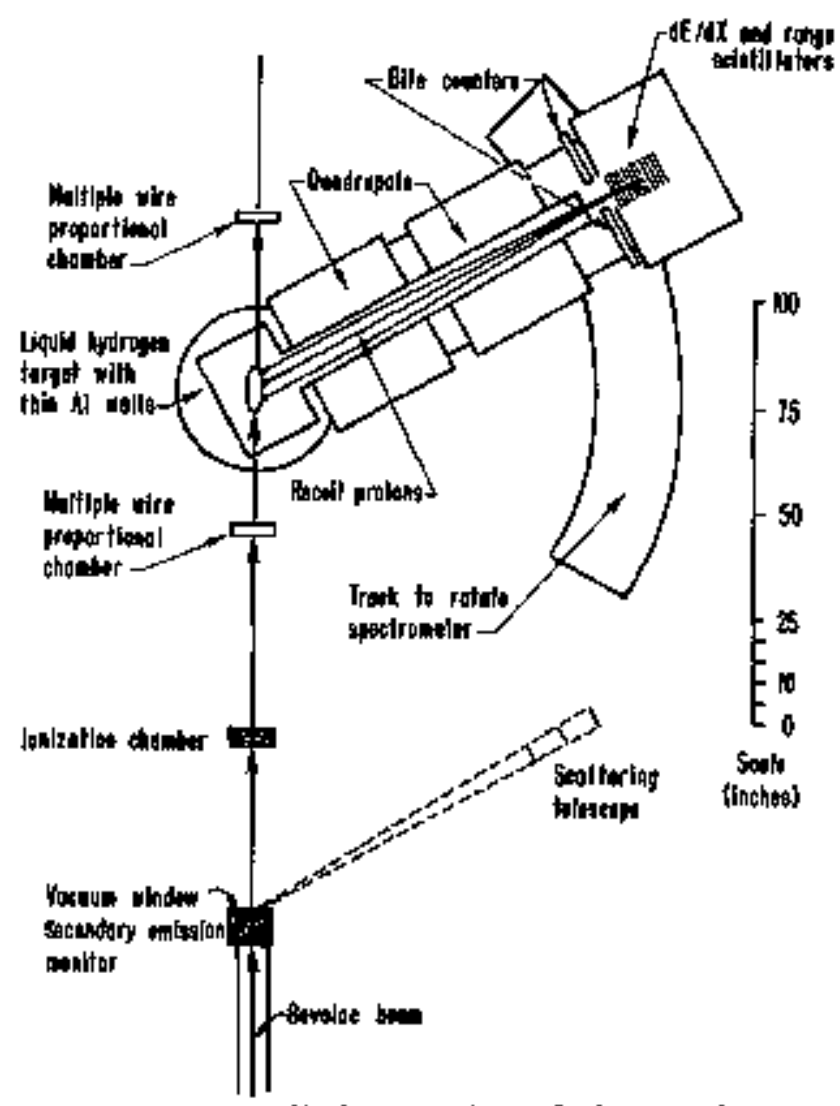

Fig. 1. A simpliffed plan viow of the recoil proton spectrometer for this experinent.

(XBL 766-8333)

the proton kinetic energy from its range in the scintillators. In addition, detailed pulse height measurements in each scintililator show the characteristic Bragg peak from valid stopping protons. This separated then fron the secondary pions, and from protons which interect in the scintillators or which go out the side. It is critical to avold such contaminations at large excitation. There, valid protons are only a snall fraction of the partictes produced from the target.

A necessary part of this exporiment was to calibrate absolutely the response of an ion chateber to these heavy ion beans. In conjunction with R. R. Snith and J. B. McCaslin we aIso measured the absolute eross section for ${ }^{1} \mathrm{C}+1 \mathrm{C}_{\mathrm{C}}+\mathrm{X}+1 \mathrm{~L}_{\mathrm{C}}$. These calibrations should be useful fur other experivents which use these techniques.

Our data are presently under apalyșis. A preliminary salp le is show in Fig. 2 . There are several different dynewpte reqjots. At the lowest excitation is a peak ${ }^{12} \mathrm{Cp}+12 \mathrm{Cp}$. This preliminasy sample cannot resolve $12 \mathrm{Cp} \rightarrow 12 \mathrm{C}$ (4.4 MeV) $p$ from trully elastic scattering: bat with further and lyş is of more data we will try to resolve it. The next 


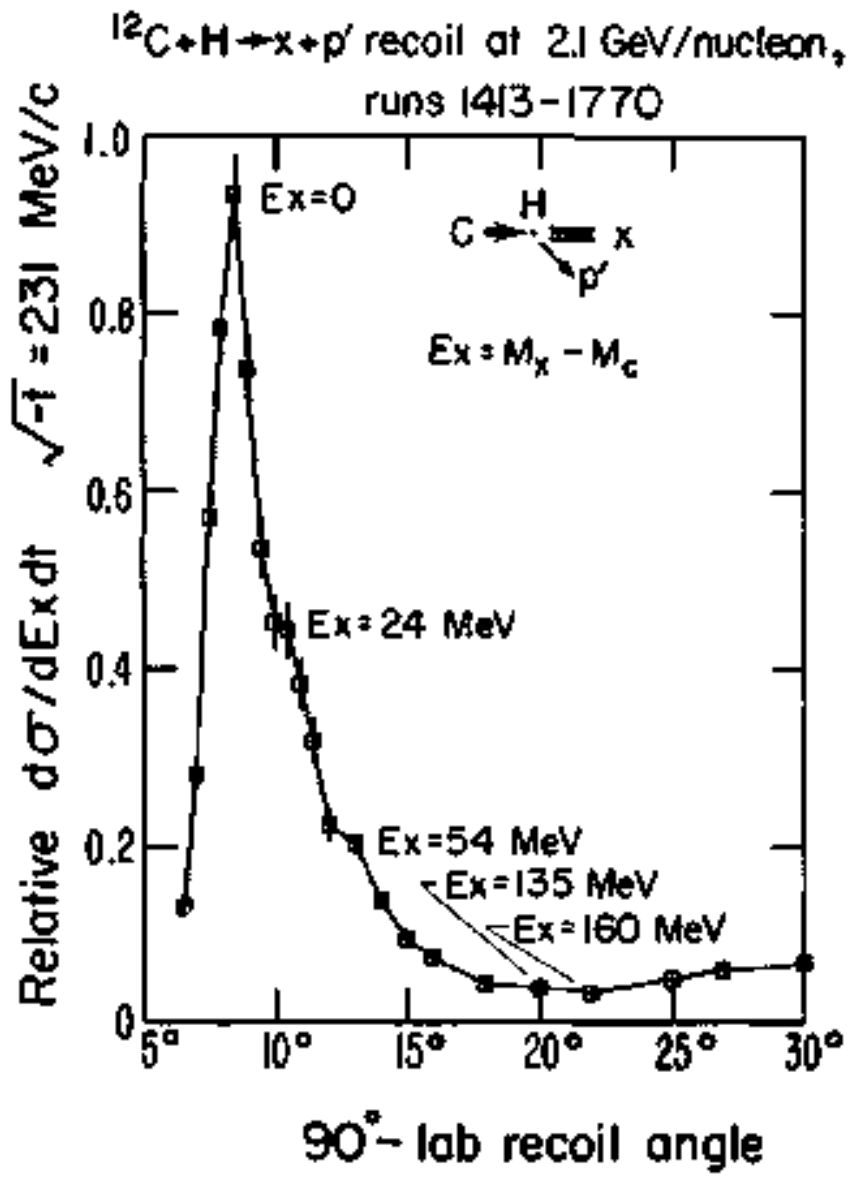

tho shotulders way be due to the giant dipole resonsuce, or quasi ap scattering, and quasi pp scattering. Other data show these structures more clearly, and no conclusions should be drawn until the data analysis is catplete. Beyond these peaks the cross section falls by an order of magnitude. This region puts new demands on Glauber calculations. Lisually these calculations assume negligibly snal1 energy transfer in the individual collisions, or else sum over all possible energy transfers. Here we megsure a large excitation energy, so a better calculation is required. The relevant theory was discussed above. Again, no reliable conclusion can be drawn untils the analysis is complete, and we are certain that there is no contamination. Dur ultimate resolution should be a few $\mathrm{MeJ}$ in excitation, above \pm 2 in monentum transfer $\sqrt{-t}$, and a few porcent uncertafinty in the cross section.

\section{Fogtnotes}

*Arizona Neqdical Certer, Tusçon, Arizona.

${ }^{\dagger}$ Prysics Dept., Harvard thiversity, Cambridge, Mass.

Fig. 2. Relattve double differential cross section $\mathrm{d}^{2} \mathrm{~J} / \mathrm{dm}^{2} \mathrm{dt}$ at fixed momentum transfer $\sqrt{-t}=231$ $\mathrm{HeV} / \mathrm{c}$ and variable laboratory proton recoil anglo. The abscissa is 90 dogrees minus the angle between. the laboratory recoil proton direction and the bean direction. This angle determine the excitation onergy, end some representatiwe values are shom. These excltations include a 1 degree overa 11 adjustment in the angular scale. The bars are nominal Poisson errors.

\title{
PROJECTILE FAAGMENTATION AND ASSOCIATED MULTHPLICITY DISTRIBUTIONS IN RELATIVISTIC HEAVY ION COLLISIONS
}

\author{
J Carroll, Y Perez-Mendez, E Whipple, M Gazzahy: J Gezga,
}

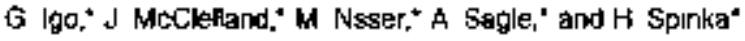

A large apertare, moveable magnetic spectrometer, instrumented with Hulti-wire proportional chambers and scintiflation counters has been set up in Chasme1 II at the Bevalac. The bașic properties of the spectraneter (originally installed to study forward alastic scattering) are listed below.

$$
\begin{aligned}
& \text { Aी } \Leftrightarrow 0.8 \mathrm{mst} \\
& \text { Tơ 31 feet } \\
& \text { theta: range }-1,14^{\circ} \\
& \mathrm{P} / \mathrm{Z} \text { : range } 0-7.5 \mathrm{GeV} / \mathrm{s} \\
& \begin{array}{rr}
\text { resolution } & 0.1^{\circ} \\
\text { bite } & \pm 1^{\circ} \\
\text { resolution } & 0.58 \\
\text { bite } & \pm 20
\end{array}
\end{aligned}
$$

A layout of the spectroneter is shrwn in Fig. 1.

A prelintnary parasitic rum with $1.05 \mathrm{GeV} / \mathrm{h}$ $16_{0}$ incident on a $12 \mathrm{C}$ target demonstrated that the spectroneter was suitable for measiring the fragmentation cross sections and was capable of identifying the 1ight isotopes up to He which doninate the flux of fragients scattered through more than a few degrees.
The spectrometer has since been augmented by an array of 30 lucite Cerenkov detectors surrounding the beam downstream from the target. These detectors aze in the fort of azimuthal seguents covering all of the arinuthal coordinate which is not ocaupied by the spectrometer acceptance. The anguler acceptance of the array can be adjusted to include the ranges $4-12^{\circ}$ or $5-16^{\circ}$ by varying the targetto-detector distance. The kimematies of the reaction ensure that this angular range will accept a large fraction of the fragments from the projectile which have received a modrentum transfer greater than the typical nuslear Fermi momentum. The lucite detectors have a velocity threshold Bth 260.7 , and are thus coxpletely insensitive to fraguents evaporated from the target.

Using this array we can study the multipllcity distribution of projectile fragnents (irktuding the Infrequent pions) in association with fragnents of various masses, charges, momenta, and angles detectod in the spectrometer. One can, of course, also 


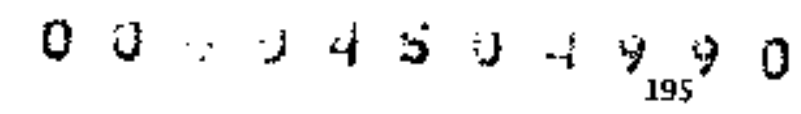

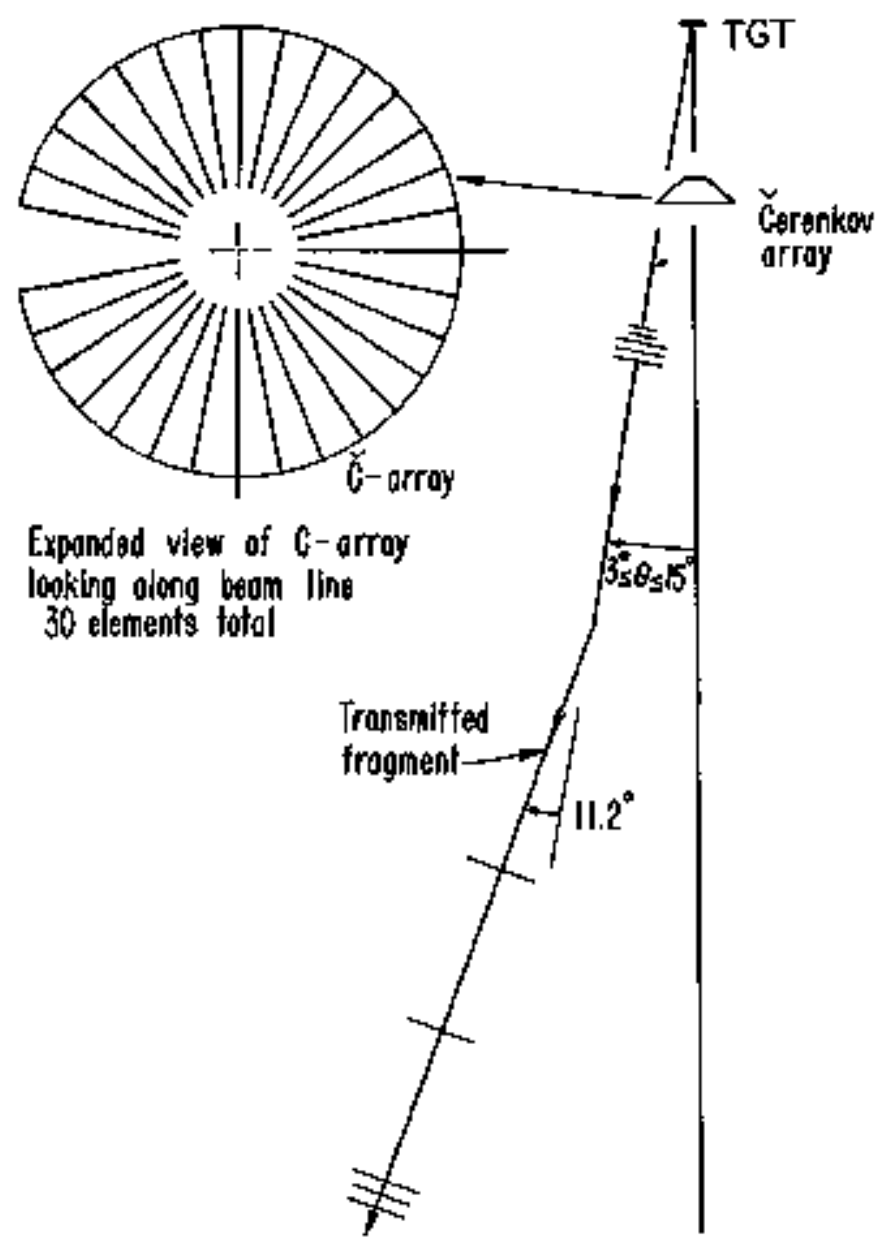

Fig. 1. Plan view of spectrometer for measuring multiplicities of projectile fragments associated with the transmitted fragment $A_{Z}$. (XBL 7611-4381)

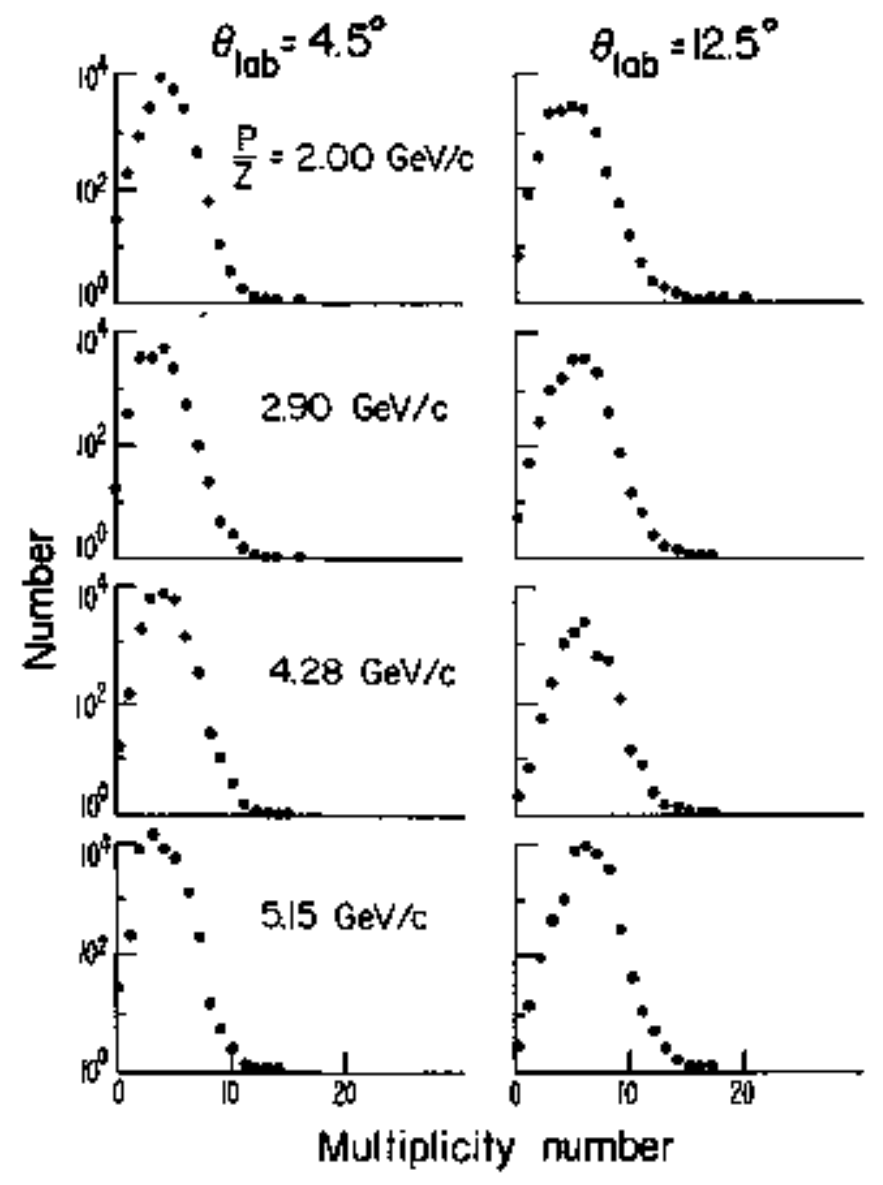

Fig. 2. Multiplicity distributions in the tag counters associated with fragments entering the spectrometer at $4.5^{\circ}$ and $12.5^{\circ}$ for the case of a $1.8 \mathrm{GeV} / \mathrm{n} 4 \mathrm{O}_{\mathrm{Ar}}$ bean on a ${ }^{\mathrm{Be}}$ target.

(XBL 766-85ㄴ) 


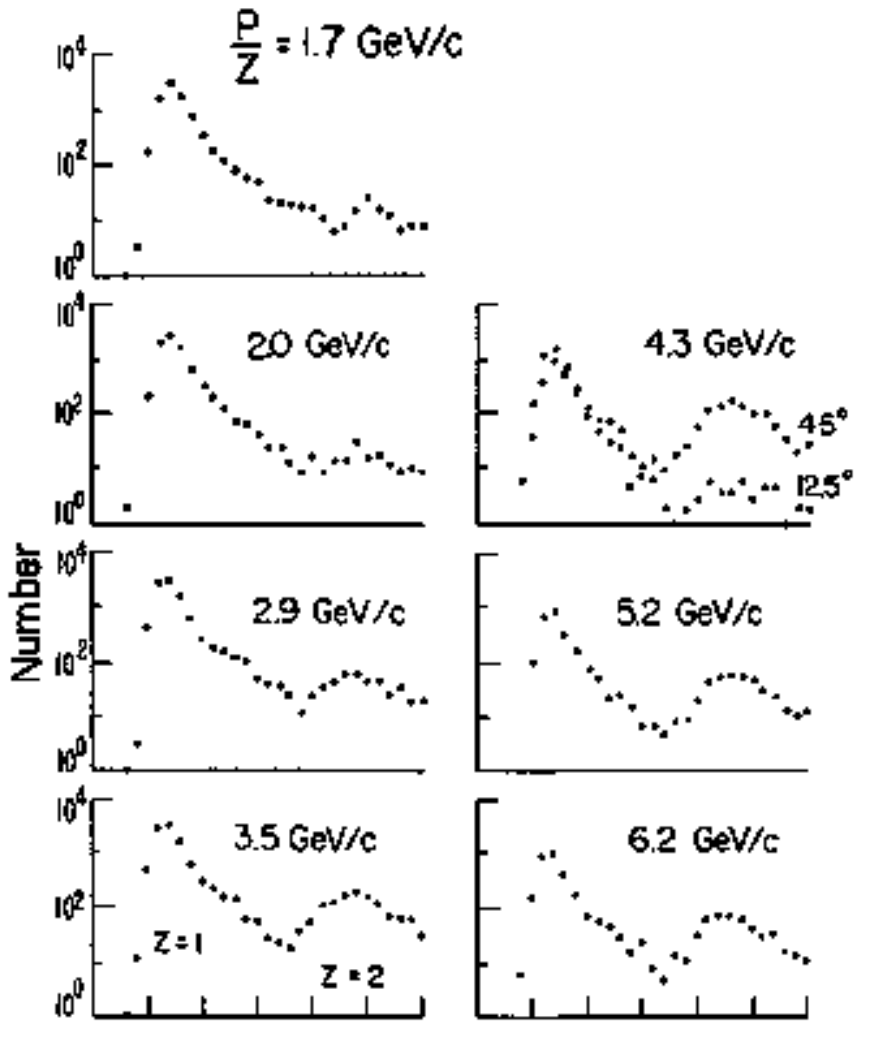

Chonnel

Fig. 3, Pulse height spectra from a scintillation counter in the spectrometer for $\mathrm{a} 1.8 \mathrm{GeV} / \mathrm{n} 40_{\mathrm{Ar}}$ beam on a 9 pe targets. The two different peaks in any given spectrum indicate the relative amount of $z=1$ and $z=2$ particle fragmentation.

[XEL 7611-4485] select for large multiplicity and study the effects on the distribution of particles in the spectronteter. Both spectroneter and Cerenkow array cover the range of angles at which one might expect to seor shock waves, and one can use the spectrometer to sae if larger multiplicities are associated with fragment momentun distributions that can be ascribed to an increased miclear temperature.

Data have been taken for $2.1 \mathrm{Gev} /$ nuclecr $12_{\mathrm{C}}$ and $1.8 \mathrm{GeV} /$ nateleon $4 \mathrm{AT}_{\mathrm{T}}$ beans on beryllitm and copper targets. While even a preliminary analysis is not yet canplete, certaîn observations can be made:

1. One can see a trend toward hither multiplicities associated with higher momentum transfers. This can be seen in Fig. ? for the case of the $1.8 \mathrm{GeV} /$ rucleon $40_{\mathrm{Ar}}$ beam or the ${ }^{9} \mathrm{Be}$ target. The average multiplicity varies frovn -4 to $\sim 8$ as the $P / 2$ and angle ere varied from $2.0 \mathrm{GeV} / \mathrm{C}$ at $4.5^{\circ}$ to $5.15 \mathrm{GeV} / \mathrm{c}$ at $12.5^{\circ}$.

2. The production of charge 2 fragnents rulative to charge 1 fragments decreases with scattering angle and scattared momentu明 as shown in Fig. 3.

3. The widths of the fragnentatich peaks in momentul are much broader than the Fermi mamentum even at $\theta_{1 \mathrm{ab}}=4.5^{\circ}$.

We are procepding with the analysis and hope to 5001 have momentum, angular, and multiplicity distributions for the different types of fragments.

\section{Fostnote}

Unfversity of California, Los Angeles, Califormia 90024 .

\section{Target Fragmentation}

\section{NUCLEAR GAMMA RAYS FOLLOWING INTERACTION WITH RELATIVISTIC CARBON PROJECTILES}

\footnotetext{
T Snibata," $H$ Eprn," F Anncil, $H$ Bowman, J G foannou-Yannou.
}

J O Rasmussen, E Rauscher, S Nagamna,ł ard K Nakał

Discrete nuclear ganua rays following interactions of relativistic 120 protectiles with ${ }^{12} \mathrm{C}, 19 \mathrm{~F}, 38 \mathrm{Sr}$ and $207 \mathrm{~Pb}$ were observed at $E(12 \mathrm{C})=3$ and $12.6 \mathrm{GeV}$, The lartest yields of the observed gama rays were from low-lying excited states of target nuclei, and there were also identified lines from muflei of nass nuber slightly leș than that of the target nuclei.

The work reported here mainly involves bombardments by ${ }^{12} \mathrm{C}$ at $1.05 \mathrm{CeV} /$ nucleon and $0.25 \mathrm{GeV} /$ mucleon, and the targets studied were "teflon" $\left(\mathrm{C}_{n} \mathrm{~F}_{2 \mathrm{I}}\right)$, natural $\mathrm{ST}\left(82.488_{\mathrm{Sr}}\right)$ and enriched $207 \mathrm{~Pb}(92.88)$. The gamma-ray energy range studied was from $80 \mathrm{keV}$ to $2.5 \mathrm{MeV}$.

The Bevatron prowided relattvistic carbon ${ }^{12} \mathrm{C}$ ion beams with energies 1.05 GeV/nucleon and $0.25 \mathrm{GeV} /$ nucleon. (Eariler, 4+8B GeV/n protons and $0.4 \mathrm{GeV} / \mathrm{n}$ çarbon ions were also used for survey runs.) The beam intensities were adjusted so as to get adequate peak counting rates of about $4,000 \mathrm{C} / \mathrm{sec}$ for gamia detectors and the proper live times (SO-7ót). They were about $0.25 \times 10^{7}$ carbon ions/burst for

$0.25 \mathrm{jeV} /$ nucleon. The accelerator was operated 


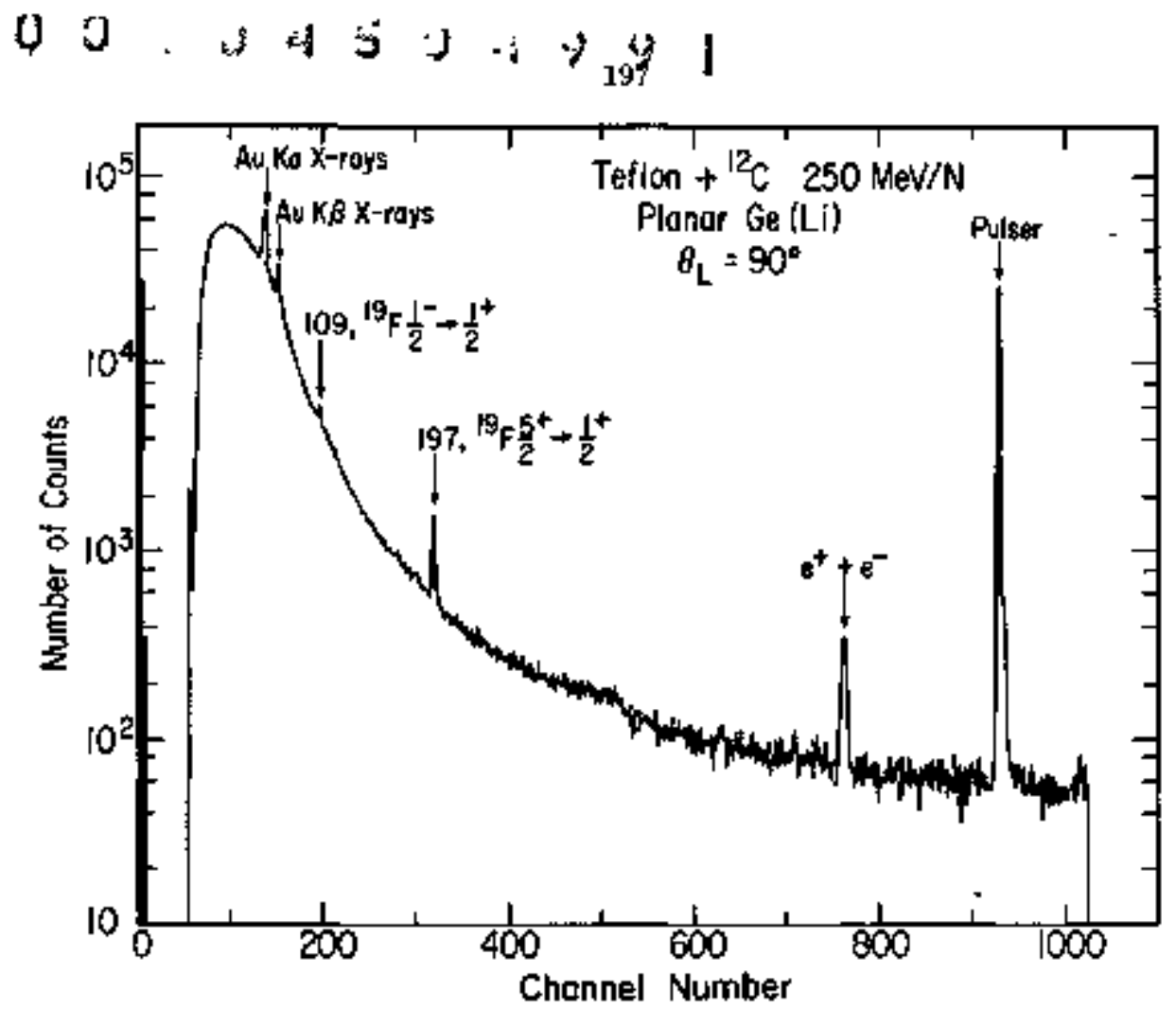

Fig. 1. Genme-ray spectrun for the 3 - $\mathrm{GeV}{ }^{12} \mathrm{C}$ interaction with the teflon target measured by the plarar $\mathrm{Ce}(\mathrm{Li})$ detector. (XBL 761-231)

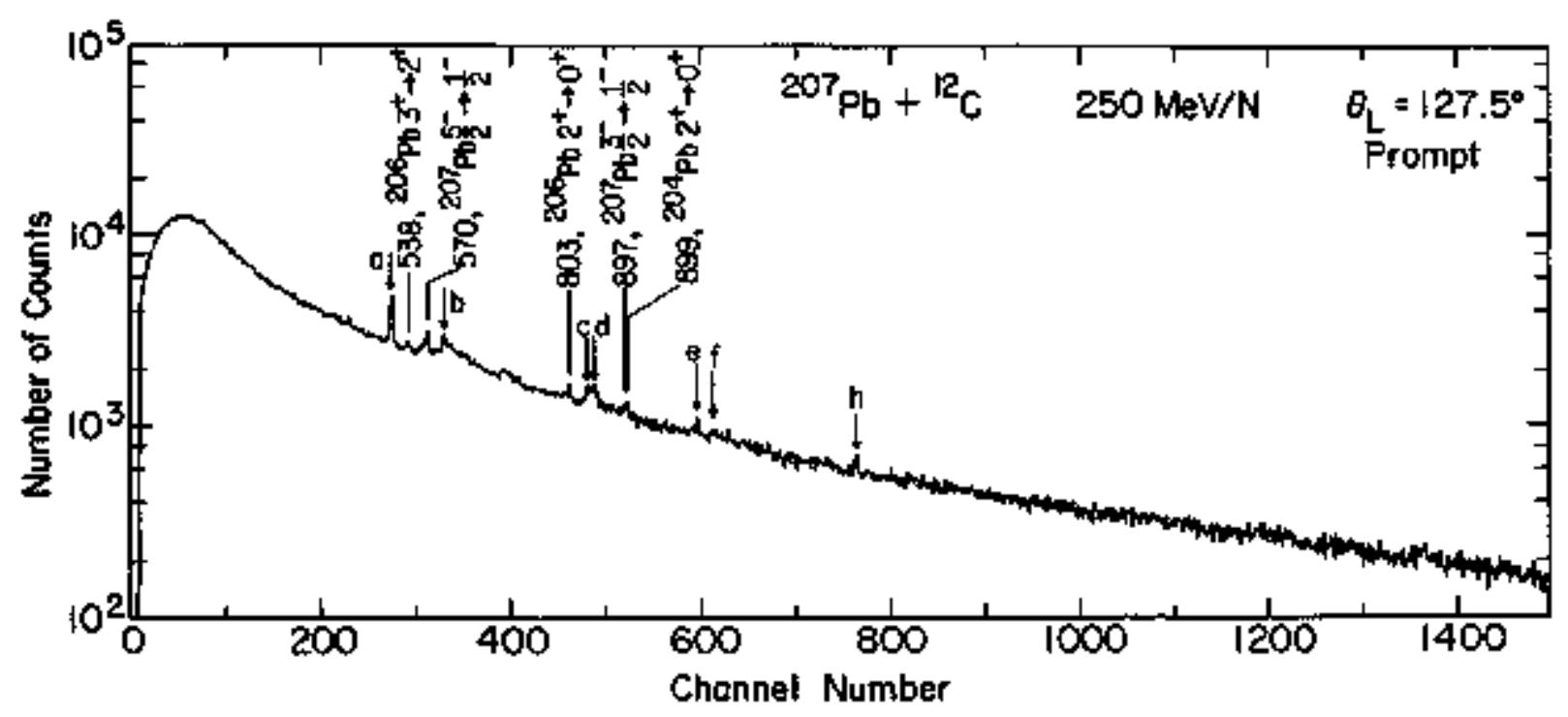

Fig. 2. Pronpt gapma-ray spectnm for the 3 Ger $12 \mathrm{C}+207 \mathrm{pb}$ measured by the coaxial Ge(Li) detector. The common lines dus to Ge of the detector, $k 1$ of the bean tube, ratioactivities,

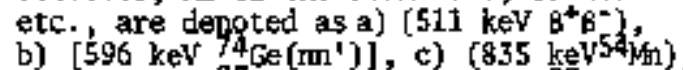

d) (844 keV $\left.27_{\mathrm{Al}}\right)$, e) (1014 keV $\left.27_{\mathrm{Al}}\right)$,

f) $\left.1040 \mathrm{keV} 70_{\mathrm{Ge}}\right)$, h) (1277 keV $\left.2 \mathrm{z}_{\mathrm{ka}}\right)$.

(XBL $761-227)$ 


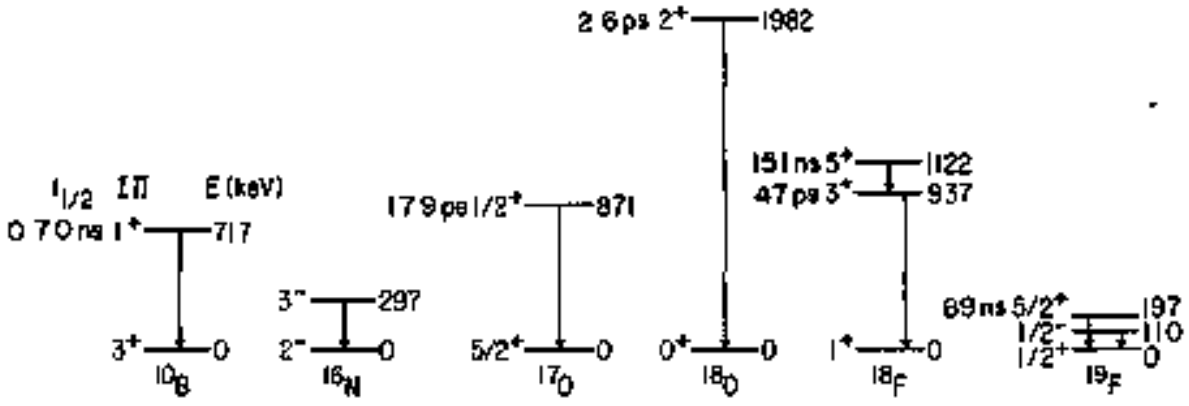

Fig. 3. Transition schemes for the proninent gammor rays observed with the teflon target. (XBL 761-235)

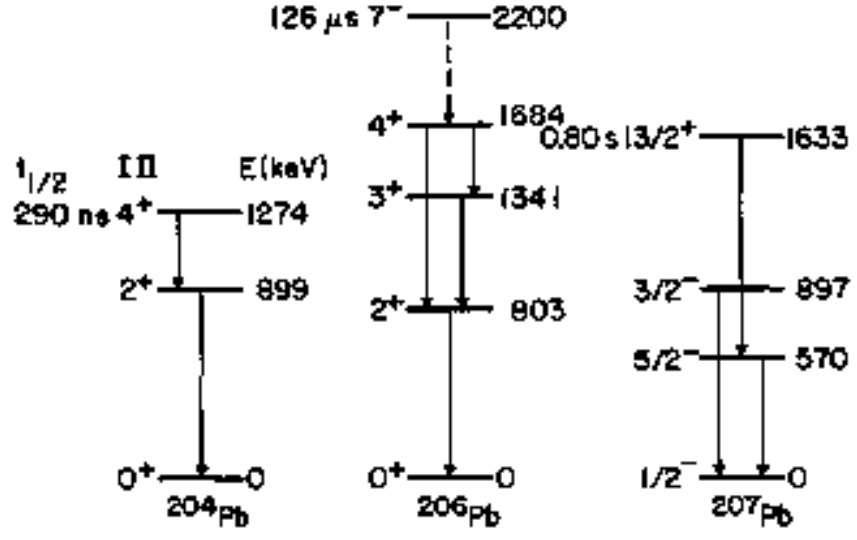

Fig. 4. Transition schenes for the prominent gama rays observed with the ${ }^{207} \mathrm{~Pb}$ target.

(X⿻1. 761-233)

in either of two modes, one with the rf power applied to the acceleration resonator and another without the rf power applief during the beam extraction from the accelerator. The former tode provides microscopic if bunched beam pulses with intervals of 455 nsec for $1.05 \mathrm{GeV} / \mathrm{n}$ and $653 \mathrm{nsec}$ for $0.25 \mathrm{GeV} / \Omega$. Since the macroscopic bear bursts of alxout 1 sec duration come every $\$$ or $6 \mathrm{sec}$ and each bear burst consists of a narrow $(\alpha 20 \mathrm{rsec})$ microscopic bear pulse avery 4500 nsec, the coincidence arrangement considerably reduced the gamma-ray backpround. The dead times were measured by comparing the muther of pulser pulses recorded in the multichansel PHA and the number of those fed into the preamplifier. Here the pulser pulse fed into one preamplifier was triggered by the scaled-down gamma ray pulses frcm another detector, and vice versa.

Gapme rays from the teflon and strontiun targets were measured at $\theta^{-1} 127,5^{\circ}$ and $\theta=90^{\circ}$. The ganra rays from the $207 \mathrm{~Pb}$ target were measured only at $127.5^{\circ}$

Nany discrete gamma rays following relativistic $12 \mathrm{C}$ - indixed nuclear reactions on ${ }^{12} \mathrm{C}, 19 \mathrm{~F}$, ratural $\mathrm{Sr}$ ( 837 of $885 \mathrm{r}$ ) and $207 \mathrm{~Pb}$ were observed. Some of the observed game-ray spectra from the Ge(Li) detextors are shown in Figs. 1 and 2. The transition schemes for most of the observed gamas rays are given in Figs. 3 and 4. The observed gamen rays come from excited states of the target nuxlet and nearby produsts derived by few-nucieon removal. The strong "continuain background" was established as largely target-originated, since it drops during target. out runs. The "continung background" is considered to be mainly due to inresolved gouma rays and their conpton tails from numerous low-yield products of nonperipheral processes.

The cross sections for the proninent ganma lines were obtained frow the observed $y$ itelds at. $\theta=127.5^{\circ}$, where the angular distribution effect is negligible because $P_{2}(\cos \theta) \approx 0$. The measured relative intensitles of the protuinent gamma rays are given in Table 1. The absolute cross sections for the strongest ganna lines, the relative intensities of which are given as 100 in Table 1 , are $39 \pm 15 \mathrm{mb}(24 \pm 12 \mathrm{mb})$ for the $19_{\mathrm{F}} \mathrm{5}^{+} / 2+1 / 2$ transition from the 3 Gev (12.6 Gev) $12 \mathrm{c}+$ teflon, $100 \pm 43 \mathrm{mb}(74 \pm 41 \mathrm{mb})$ for the $8 \mathrm{SSr}^{+}+0^{+}$ transition from the $3 \mathrm{GeV}(12.6 \mathrm{GeV}) 12 \mathrm{C}+\mathrm{Sr}$, and $220 \pm 130 \mathrm{mb}(157 \pm 100 \mathrm{mb})$ for the $20 \mathrm{Fb}^{5} / 2+$ $1^{+} / 2$ transition from the $3 \mathrm{GeV}$ (12.6 GeV) $12 \mathrm{c}$. $207 \mathbf{F t}$.

The following observations can be made from the measured gantra ray yields:

1. Target nuclei (bound states) are strongly excited. Thus, ganma rays from the low-1ying excited states are strong. The cross sections are of the ordet of $100 \mathrm{mb}$.

2. Gama rays from the low-lying states (the second $5 / 2^{+}$state in $19 \mathrm{~F}$, the first $2^{+}$state in $88 \mathrm{Sr}$, the fijst $5 / 2^{-}$state and the first $3 / 2^{-}$state in $207 \mathrm{Pbj}$ are always domingint. These low-lying states in $20{ }^{2} \mathrm{~Pb}$ are ${ }^{88_{\mathrm{S}}}$ are partly (half or more) populated by gavima cascades from the higher excited states.

3. Delayed gamma rays from the $13 / 2^{+}$state in $207 \mathrm{~Pb}$ are prominent for the ${ }^{207} \mathrm{~Pb}$ target (spin 1/2). This indicates considerable excltation of high spin particle-holo states. Such excltation is quite tulikely in inelastic scattering of low-energy heawy ions.

4. Gamm rays following few-nuxleon renoval from the target nucle1 are well observed with cross sections of $10 * 100 \mathrm{mb}$. Wha bound states 


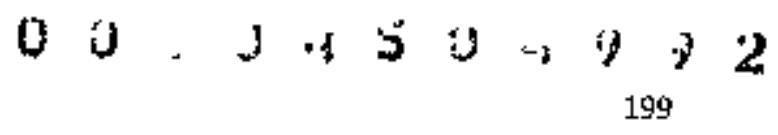

Table I. Relative interisities of $y$ rays following relativistic carbon-induced reactions. *

\begin{tabular}{|c|c|c|c|c|c|c|}
\hline Target & $E_{\gamma}(\mathrm{kV})$ & Transition & Reaction & $I_{Y}(0.25 \mathrm{GeV} / \mathrm{n})$ & $I_{Y}(I, \mathrm{geV} / \mathrm{n})$ & Prompt or Delayed \\
\hline${ }^{12} \mathrm{C}$ & $717.4 \pm 0.7$ & ${ }^{10} 1_{\mathrm{B} 1}+3^{+}$ & $-1 n,-1 p$ & $92 \pm 19$ & $116 \pm 23$ & Prompt \\
\hline \multirow[t]{7}{*}{${ }^{19} \mathbf{F}$} & 109.0 & ${ }^{19}{\frac{I^{-}}{2}}^{-} \frac{1^{+}}{2}$ & $D, 0 p$ & $24 \pm 3$ & & \\
\hline & $197.0 \pm 0.5$ & $19 \mathrm{~F} \frac{5^{*}}{2}+\frac{1^{+}}{2}$ & $O_{n}, O_{p}$ & $\underline{100} \pm 24$ & $100 \pm 31$ & De1ayed $\tau_{1 / 2}=89 \mathrm{~ns}$ \\
\hline & $1981, D \pm 1,0$ & ${ }^{18} 02^{+}+0^{+}$ & $0 \mathrm{r},-1 \mathrm{p}$ & $68 \pm 16$ & $83 \pm 27$ & Prompt \\
\hline & $936.5 \pm 0.5$ & ${ }^{18} \mathrm{~F}^{+} \rightarrow 1^{+}$ & $-2 n, O_{p}$ & $38 \pm 8$ & $42 \pm 23$ & Prompt only \\
\hline & $936.5 \pm 0.5$ & ${ }^{18} \mathrm{~F}\left(5^{+}\right) 3^{4}+1^{*}$ & $-1 \mathrm{n}, \mathrm{op}_{\mathrm{p}}$ & $16 \pm 5$ & 68 & Delayed $\tau_{1 / 2}\left(5^{+}\right)=151 \mathrm{~ns}$ \\
\hline & $869+0 \pm 0.5$ & $17_{0} \frac{1^{+}}{2}+\frac{5^{+}}{2}$ & $-2 n,-1 p$ & $30 \pm 8$ & $21 \pm 12$ & Promet \\
\hline & $297.2 \pm 0.5$ & $16 \times 3^{+} \rightarrow 2^{-}$ & $-\ln ,-2 p$ & $9 \pm 2$ & --- & Prompt \\
\hline \multirow[t]{5}{*}{$\begin{array}{l}\mathrm{n}_{\mathrm{Sr}} \\
(\mathrm{B} 2,4 \mathrm{Sr})\end{array}$} & $1836.4 \pm 0.7$ & ${ }^{88} \mathrm{ST} 2^{+}+0^{4}$ & $\mathrm{On}_{\mathrm{m}} \mathrm{Op}$ & $\underline{100} \pm 28$ & $100 \pm 36$ & Prompt \\
\hline & $898.0 \pm 0,7$ & ${ }^{88} \mathrm{Sr} 3^{-}+2^{+}$ & $\mathrm{On}_{\mathrm{n}}, \mathrm{p}$ & $65 \pm 18$ & $33 \pm 21$ & Proupt \\
\hline & $1049.4 \pm 0.5$ & $86_{5 T} 2^{+}+0^{+}$ & $-2 n, 0 p$ & $48 \pm 12$ & $55 \pm 23$ & Prompt \\
\hline & $1153+0 \pm 0.5$ & ${ }^{86} \operatorname{Sir} 4^{+}+2^{4}$ & $-2 n, 0 p$ & $36 \pm 18$ & $64 \pm 24$ & Prompt \\
\hline & $493.0 \pm 0.7$ & ${ }^{84} \mathrm{Sr} 2^{+}+0^{+}$ & $-4 n, 0 p$ & $36 \pm 14$ & $\$ 27$ & Prompt \\
\hline \multirow[t]{12}{*}{$207 \mathrm{~Pb}$} & $569.7 \pm 0.5$ & ${ }^{207} \mathrm{~Pb} \frac{\mathrm{s}^{-}}{2}+\frac{1^{-}}{2}$ & On, Op & $\underline{100} \pm 18$ & $100 \pm 46$ & Prompt enly \\
\hline & $569.9 \pm 0.5$ & ${ }^{207} \mathrm{~Pb} \frac{13}{2}+\frac{5}{2} \rightarrow \frac{1}{2}$ & $\mathrm{O}_{\mathrm{n}}, \mathrm{OP}_{\mathrm{p}}$ & $84 \pm 16$ & & Delayed $t_{1 / 2}\left(\frac{13^{+}}{2}\right)=0.85 \mathrm{~s}$ \\
\hline & $897.3 \pm 0.7$ & $207 \mathrm{pb} \frac{3^{-}}{2}+\frac{1^{-}}{2}$ & $0 \mathrm{n}, \mathrm{pp}$ & $43 \pm 14$ & $98 \pm 52$ & Prompt \\
\hline & $1063+640.7$ & ${ }^{207} \mathrm{pb} \frac{13^{+}}{2}+\frac{\mathrm{s}^{-}}{2}$ & $\mathrm{~m}, \mathrm{Op}$ & $92 \pm 28$ & & Delayed $\tau_{1 / 2}(13$ \\
\hline & $803.2 \pm 0.5$ & ${ }^{206} \mathrm{~Pb} 2^{+}+0^{+}$ & $-1 n$, Op & $56 \pm 13$ & $91 \pm 36$ & Protingt only \\
\hline & $803.2 \pm 0.5$ & ${ }^{205} \mathrm{~Pb}\left(7^{-}\right) 2^{+}+0^{4}$ & $-\ln , 0 \mathrm{p}$ & $24 \pm 8$ & 552 & Delayed $\tau_{1 / 2}\left(7^{*}\right)=126$ ps \\
\hline & $537.5 \pm 0.5$ & ${ }^{206} \mathrm{~Pb} 3^{+}+2^{+}$ & $-1 \mathrm{n}, \mathrm{Op}$ & $10 \pm 3$ & $<60$ & Prompt only \\
\hline & $881.0 \pm 0.7$ & $\left.{ }^{206} \mathrm{~Pb}_{\left(7^{-}\right.}\right) 4^{+} \rightarrow 2^{+}$ & $-1 \mathrm{n}, \mathrm{Op}$ & $20 \pm 8$ & $\$ 60$ & Delayed $\tau 1 / 2^{\left(7^{-}\right)}=126 \mu \mathrm{s}$ \\
\hline & $899.2 \pm 0.7$ & ${ }^{204} \mathrm{pb} 2^{+}+0^{+}$ & $-3 n, 0 p$ & $16 \pm 15$ & 460 & Prompt only \\
\hline & $899.2 \pm 0.7$ & ${ }^{204} \mathrm{~Pb}\left[4^{+}\right) 2^{+} \rightarrow 0^{+}$ & $-3 n, 0 p$ & $20 \pm 8$ & 560 & Delayed $\tau / / 2^{\left(4^{+}\right)}=0.29 \mu \mathrm{s}$ \\
\hline & $374.7 \pm 0.7$ & ${ }^{204} \mathrm{~Pb}\left(4^{+}\right) 4^{+}+2^{+}$ & $-3 n, 0 p$ & $17 \pm 11$ & & Delayed $\tau_{1 / 2}\left(4^{+}\right)=0.29$ us \\
\hline & $986.1 \pm 1.0$ & $205_{\mathrm{pb}} \frac{13^{+}}{2} \frac{9^{-}}{2}+\frac{5^{-}}{2}$ & $-2 \mathrm{r}, 0 \mathrm{p}$ & $19 \pm 7$ & & Delayed $\tau 1 / 2\left(\frac{13^{+}}{2}\right)=5$ us \\
\hline
\end{tabular}

Intensities are relative to the underlined 100 . Normalizations to absolute cross sections are given in the text. 
are probably exciter, and thus some particular ganua rays (mostly for the trartsition from the first excited state to the ground state] get the largest yield by feeding from the higher excited state.

5. The yield of gamm rays from resitual nuclei signifies that grotons as well as neutrons. are likely to be removed from the light target nuxlei $\left(19 \mathrm{~F}\right.$ and $\left.{ }^{2} \mathrm{C}\right)$. On the other hand gamma rays from nuclei of the same $z$ but lower reutron number than the target are predonirently observed with the heavier targets nucled ( 885 and $207 \mathrm{~Pb}$ ). The $86 \mathrm{Kr} 2^{+}+0^{*}$ gamma-ray cross section is less than onequarter of that for $86 \mathrm{Sr}$ for $0,25 \mathrm{GeV} / \mathrm{p} \mathrm{Cl2}$ on $88 \mathrm{Sr}$, and less than one-half for $1.05 \mathrm{GeV} / \mathrm{n}$.

6. Little dependerce of the ganma ray yield on the incident projectile energies is observed.

7. The total cross section for these peripheral processes amounts to an appreciable fraction $(10 \sim 15 \%)$ of the geonetrical cross section of $\pi\left(R_{1}+R_{2}\right)^{2}$.

The measured relative intensities for the $4.8 \mathrm{GgV} / \mathrm{c} 12 \mathrm{C}$ teflon target are $100 \pm 34,44 \pm 21$ and $29 \pm 9$ for the $19 \mathrm{~F} 5^{+} / 2+1^{+} / 2,1002^{+}+0^{+}$and $19 \mathrm{~F}$ $1^{*} / 2+1^{+} / 2$ transitions, respectively. The absolute value for the $19 \mathrm{~F} 5^{+} / 2+I^{+} / 2$ is $41 \pm 15 \mathrm{mb}$. The valves for the $4,8 \mathrm{~B} \mathrm{geV}$ protons on the teflon target are $100 \pm 8,47 \pm 10$ and $32 \pm 3$ for the $19 \mathrm{~F}^{+} / 2+1^{+} / 2$, $18_{0} 2^{+} \pm 0^{+}$and ${ }^{19} \mathrm{~F} 1^{-} / 2+1^{-} / 2$ transitions, ragpec. tively. The absolute cross section for the $19 \mathrm{~F}$ $5^{+} / 2+1^{+} / 2$ is $55 \pm 5 \mathrm{mb}$. It is thteresting to note that both the relafive and absolute cross sections for the $400 \mathrm{NeV} / \mathrm{n}{ }^{2} \mathrm{C}$ and $4,88 \mathrm{GeV} \mathrm{p^{+ }}$ projectiles are not very wuch different from those for the 250 $\mathrm{MeV} / \mathrm{n}$ and $1.05 \mathrm{GeV} / \mathrm{n}{ }^{2} \mathrm{C}$ projectiles.

Earlier gartha-ray work with relativistic protons 1 and pions $2-4$ on 1 ight target nuclei $(A<60)$ showed that alpha-rempoval products are prominent. It has been suggested that this abservation may be explained by ordinary cascadeevaporation theory, for cherpot-particle evaporation is comparable to neutron evaporation for light elements and binding energy considerations force large yields on even-oven products in the center of the valley of stability. There is no inconsistency in our observation of only small yields of alpha. removal products for strontium and lead targets, since at these higher atomic numbers neutron evaporation predoninates over charged-particle ewaporatich. Gur wery recent, partially analyzed results from $250 \mathrm{keV} / \mathrm{n}$ ${ }^{12} \mathrm{C}$ ions on sulfur and clacium targets show prominence of alpha-removal products, Just as in the cited proton and pion work. In the case of our teflon targets, ever if aipha-removal products are significant, would not expect to observe any ganma rays, as their energies would be beyond our range of observation.

In Fig. 5 are plotted the $2^{+}+0^{+}$gamma-ray relative yields for various even-even products, as a function of neutrons remover ficm the target. solid points are for 3 Gev ark open circles, for $12.6 \mathrm{GeV} 12 \mathrm{C}$ irrediation. The points show an exponential fall off of a factor of $1 / e$ for about four neutrons removed. If each neutron removal requires 7-8 $\mathrm{NeV}$, the exponential dependence impltes a characteristic energy of about $30 \mathrm{klov}$ in the monotonic fall-off of probability of prinary excitation energy.

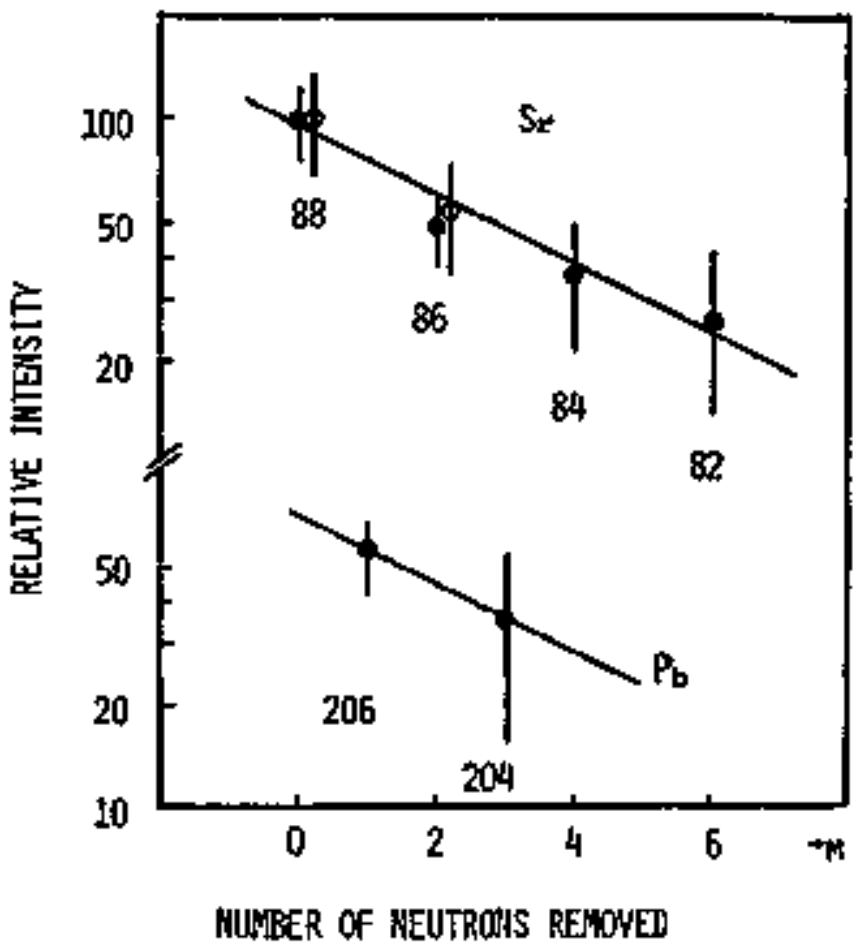

Fig. 5. Relative intensities of the $2^{+}+0^{+}$ transitions in even $\mathrm{Sr}$ and $\mathrm{Pb}$ ișotopes vs number [il] of neutrons removed. The closed and open circles are the values for the $3 \mathrm{GeV}$ and $12.6 \mathrm{GeV}$ $12 \mathrm{C}$, and mass numbers are also given. The solid lines represent the empirical expression

$I(\pi)=\exp [-\pi / 4.3\}$.

(XFl. 766-8091)

\section{Footnotes and References}

"Department of Physics, Osaka University, Toronaka, Osaka.

Tresent Address: Department of Physics, Sanford University, Sanford, $C A$.

"Department of Physies, Uhiversity of Tokyo, Bukyo-ku, Tokyo.

1. O. Artur, Y. Cassagnou, R. Legrain, N. Lisbonna, L. Rotussel, J. P. Alard, A, Baldit, J. P. Castilhes, J. Furgeix, G. Roche, and J. C. Taisin, Phys, Rev, lett. 35, 773 (1975).

2. H. E. Jackson, L. M. Schïtzmeister, T. P. hangler, R. P. Reclwine, R. E. Segel, J. Town, and J. P. Schiffer, Phys. Rev. Lett. 31, 1353 (1973).

3. V. G. Lind, H. S, Plendl, H. O. Fursten, i. J. Kossler, B. J. Lieb, H. F, Landford, and A. J. Buffa, Phys. Rev. Lett. 32, 479 (1974).

4. H. E. Jackson et a1., Physs. Rov, Lett. 35 641 (1975), ibid, 35, 1170 (1975). 


\section{$00 \therefore 4$ a}

\section{FRAGMENTS FROM C, Al, Ag, AND U IRRADIATED BY HIGH ENERGY PROTONS}

R G 5exiro, G D Westfal, A M Poskanzer, and A M Zebelman

Using a conter telescope consisting of a thin gas ionitation $\Delta \mathrm{E}$ counter and a $100 \mathrm{um}$ silicon $\mathrm{E}$ detector, 1 evergy spectra and angular distributions of fragments produced by the irradiation of $\mathrm{C}, \mathrm{Al}, \mathrm{Ag}$, and $\mathrm{U}$ with high energy protons have been measured. Previous measuremeints of fragments from these targets $2-4$ were restricted by the lower energy cutoff of the $4 \mathrm{E}$ detector with ranged from 1.5 MeV/rucleon for the lighter fraggnents to 2.S keV/nucleon for the heavier frapments. The present results extend down to $0.6 \mathrm{MeV} /$ nucleon for all fraguents observed.

The measurements comsisted of three sets of experiments. The first tised a $\mathbf{4 . 9} \mathrm{GeV}$ proton beam to bombard $\mathrm{C}$ and Al targets where energy spectra were measured for all products at 20, 45, 90,135 , and $160^{\circ}$. The second set of experinients used $2.1 \mathrm{GeV}$ protons on the same targets where measurements here made at 20,90 , and $160^{\circ}$. The third experiment used a $4.9 \mathrm{GeV}$ proton bean to irradiate the $\mathrm{Ag}$ and $\mathrm{W}$ targets where erergy spectra were observed at $90^{\circ}$ for $\mathrm{Ag}$ and at 20,90 , and $160^{\circ}$ for $\mathbf{~}$.

Figure 1 shows the energy spectra of He through $C$ fragments at $90^{\circ}$ from 2.1 GeV protons on a c target. The dashed tines in this figure are drawn to guide the oye. Using projectile frapinentation data, ${ }^{5}$ the observed energy spectra were extended to zero energy which allowed the integration of the energy spectra to obtain angulax distributions and total production cross sections for each element observed.

The energy spectra of He through wa fragnents observed at go frop 4,9 Ger protons on Al are shown in Fig. 2. The solid lines indicate previous data taken with silicon detector telescopes while the dashed lines represent the gas telescope data, Despite the lower energy cutoff of the gas $\mathrm{DE}$ colnter, the peak in the energy spectra of the higher-Z fragments from Al is not measured. Orrently, an attexipt is being made to calculate from available projectile fragmentation data this low energy part of the spectra in order to allow the integration of the energy spectra.

In Fig. 3 energy spectra for $F$ through Ar frapments neasured at $90^{\circ}$ produced by $4.9 \mathrm{BeV}$ protons incident on If are shown. Note that each succesșive element has been suppreșed by a factor of two. The data points are shown for Ne and $A r$ to demonstrate the energy range of the gas telescope data. Beyond these points the solid line signifies data taken with silicon detector telescopes. The energy spectra were fitted ușing an evaporation model and parameters were derived which were similar to those found for the lower-2 fragoents in previous works. The onergy spectra were integrated to obtain angular distributions and total production cross sections for each element observed.

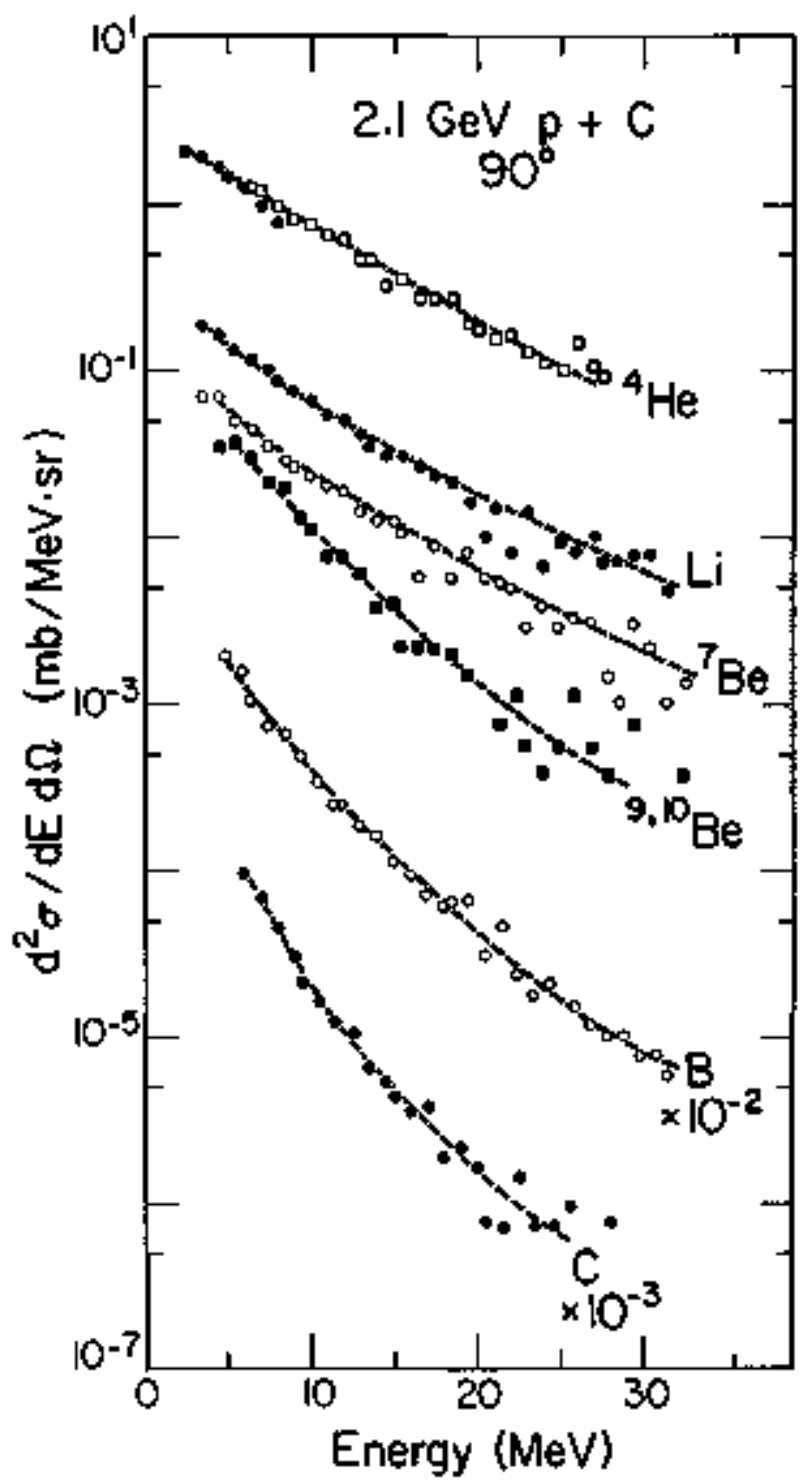

Fig. 1. Energy spectra at $90^{\circ}$ for fragnents from $2.1 \mathrm{GeV}$ protons on $C$. The dashed lines represent the trend of the data. (XBL 758-7906A)

Figure 4 shrws the energy spectra of $F$ through Ar frapuents produced by 4.9 jev protons incident on a $\mathrm{kg}$ target. A preliminary attempt was made to fit these spectra with a simple evaporation nodel, However, the paraneters derived were not consistent with previous data on lower- 2 frageints. This discrepancy may indicate the onset of soms mecharism other than evaporation. 


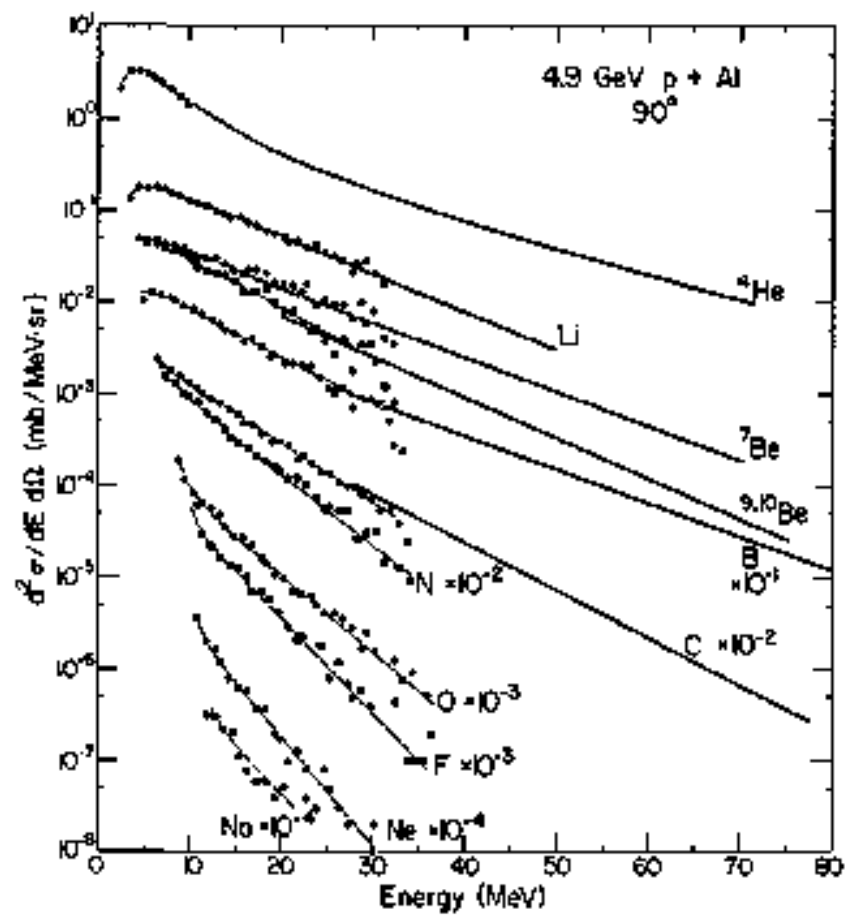

Fig. 2. Energy spectra at $90^{\circ}$ for fragments produced by the irradiation of al by $4.9 \mathrm{GeV}$ protons. The dashed lines reprosent the trend of the gas telescope data and the solid lines signify the previous data taken with silicon detector telescopes. (XBl 758-7907A)

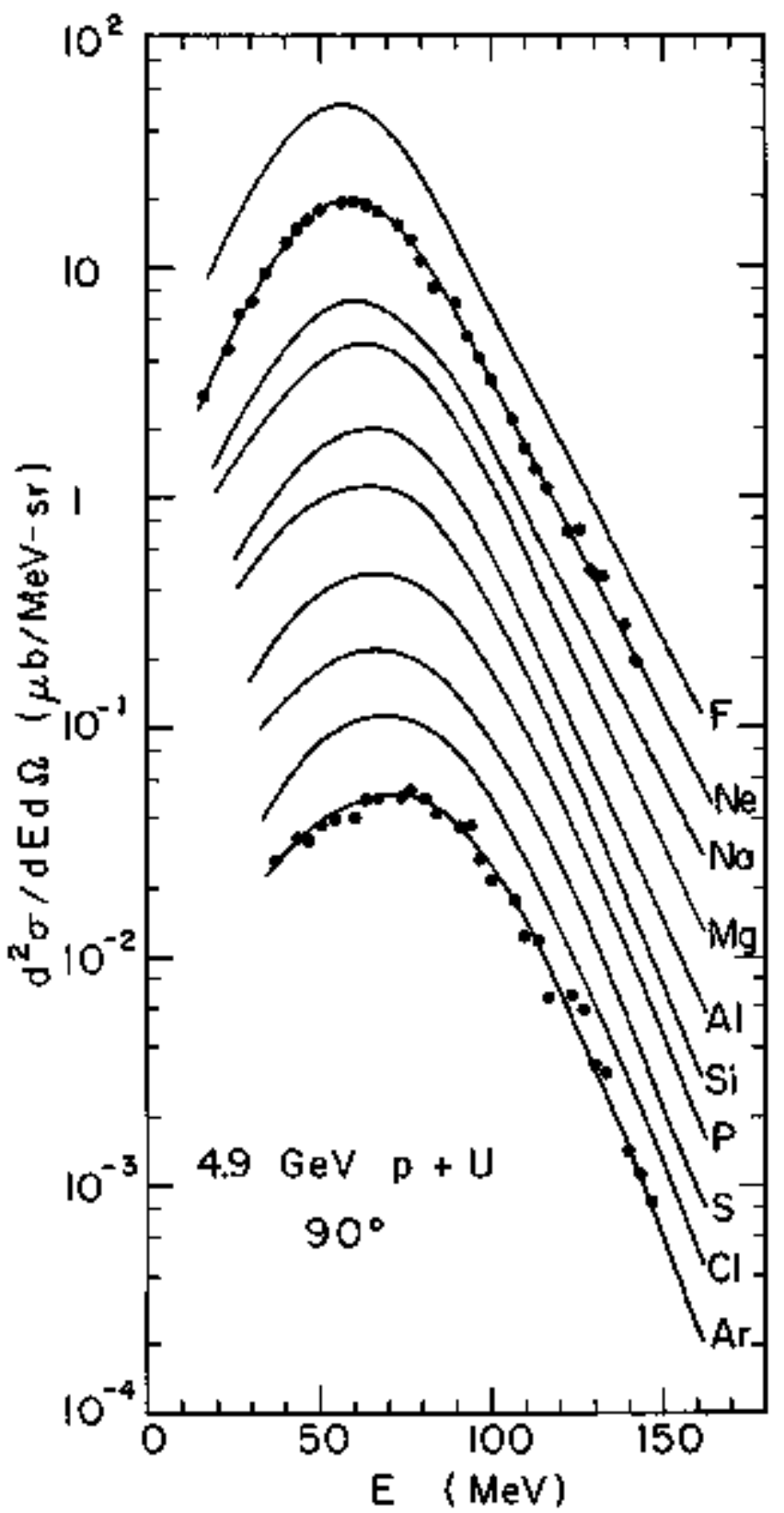

Fig. 3. Energy spectra at $90^{\circ}$ for fragments fron $\mathrm{Z}=\mathrm{g}$ to 18 resulting from the irradiation of $\mathrm{U}$ by $4.9 \mathrm{GeV}$ protons. Each successive element has been suppressed by a factor of two and the solid lines represent the trend of the data.

(XAL 7511-8676) 


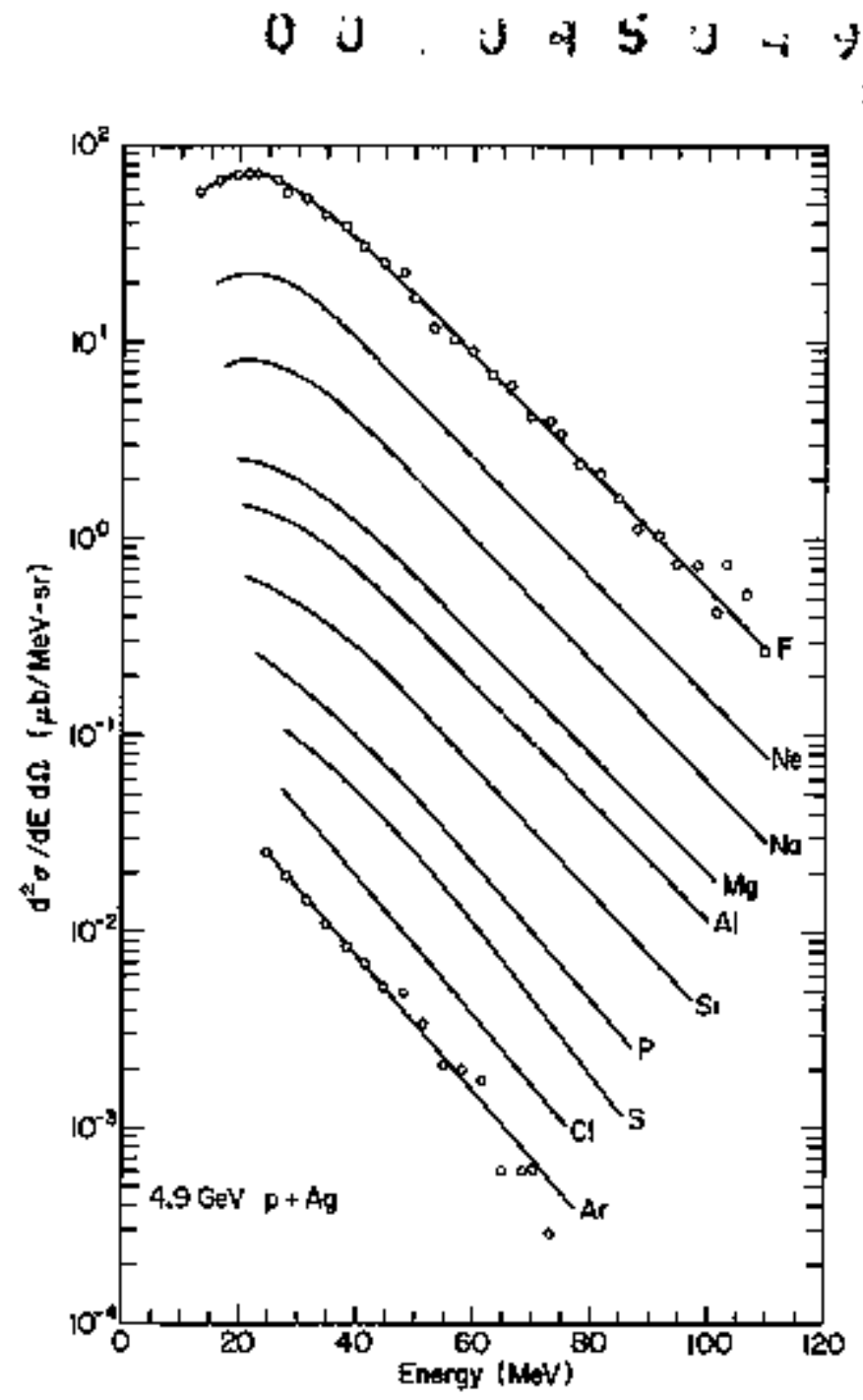

Fig. 4. Energy spectra for fraguents at $90^{\circ}$ from $z=9$ to 18 from Ag bombarded by 4.9 GeV protons. The solfd Iines represent the trend of the data and each successive element has been lowered by a factor of two.

(xBl 764-2620)

\section{Reforences}

1. R. G. Sextro, A. M. Zebelman, and A. M. Poskanzer, kuclear Chemistry Division Aruual Report, LBL-4000 (1974), p. 119.

2. A. M. Poskanzer, G. W, Butler, and E. K. Hyde, urpublished data (Al data).

3. E. K. Hyde, 6. W. Butler, and A. M. Poskanzer, Phys. Rev. C 4, 1754 (1971); R. G. Korteling, C. R. Toren, and E. K. Hyde, Phys. Rev, C $\underset{7}{ }$. 1611 (1973) (Ag data).

4. A. M. Poskanzer, G. W. Butler, and E. K. Hyde, Phys. Rev. C 3, 882 (1971) (U data).

5. P. Lindstron, D. Greiner, H. Heckman, B. Cork, and F. Bieser, $t, 6 l-3650(1975)$; D. Greiner,

P. Lindstrme, H. Heckman, B. Cork, and F. Bieser, Phys. Rev. Latt. 35, 152 (1975),

\section{COUNTER EXPERIMENTS IN THE THIN TARGET AREA AT LAMPF}

G W Guller, D G Perry: A M Poskenzer,

J B Natowntz, F Plassil.‡ and L P Remsbergs

The energy spectra of boron through mapnesium muclides produced in the interaction of $800 \mathrm{kgV}$ protons with a uranium target have been determined at $90^{\circ}$ (1ab) by $d E / d x$ and timeof-flight techniques with a silicon detector telescape. The experiment was done in the IAMPF Thin Target Area at a proton bear intersity of 10 uk. Fragrent flight tines were determined over the $25 \mathrm{am}$ distance between the $\Delta \mathrm{E}$ and $\mathrm{E}$ detectors and sibultanoously over the $4.3 \mathrm{~m}$ distarce from the target to the al detector, utitizing the $201 \mathrm{MHz}$ (5 nsec) Lingc of pulse as one of the tining signals. The maclear charge (z) of the fragnents was detemined from the
dF/dx Information and the mass (A) of the fragments was determined from both the time-of-flight and the energy sigmals via the equation $A=E \times T^{2}$. The tije resolution over the $25 \mathrm{~cm}$ flight path was 0.25 nsec (FH' for carbon ruclides. corresponding to a mess resolution of 5.2\%. The time resolution over the $4.3 \mathrm{~m}$ flight path was 0.9 nsec (FitM), correspounding to a mass resplution of 1.18 for $13 \mathrm{C}$. This dranatic improvement in mass resclution is obtained using the information from the short filght path reasurenent $t_{0}$ deterpine wich if pulse the fragment originated from, and then timing from the $T$ f over the long flight peth. 

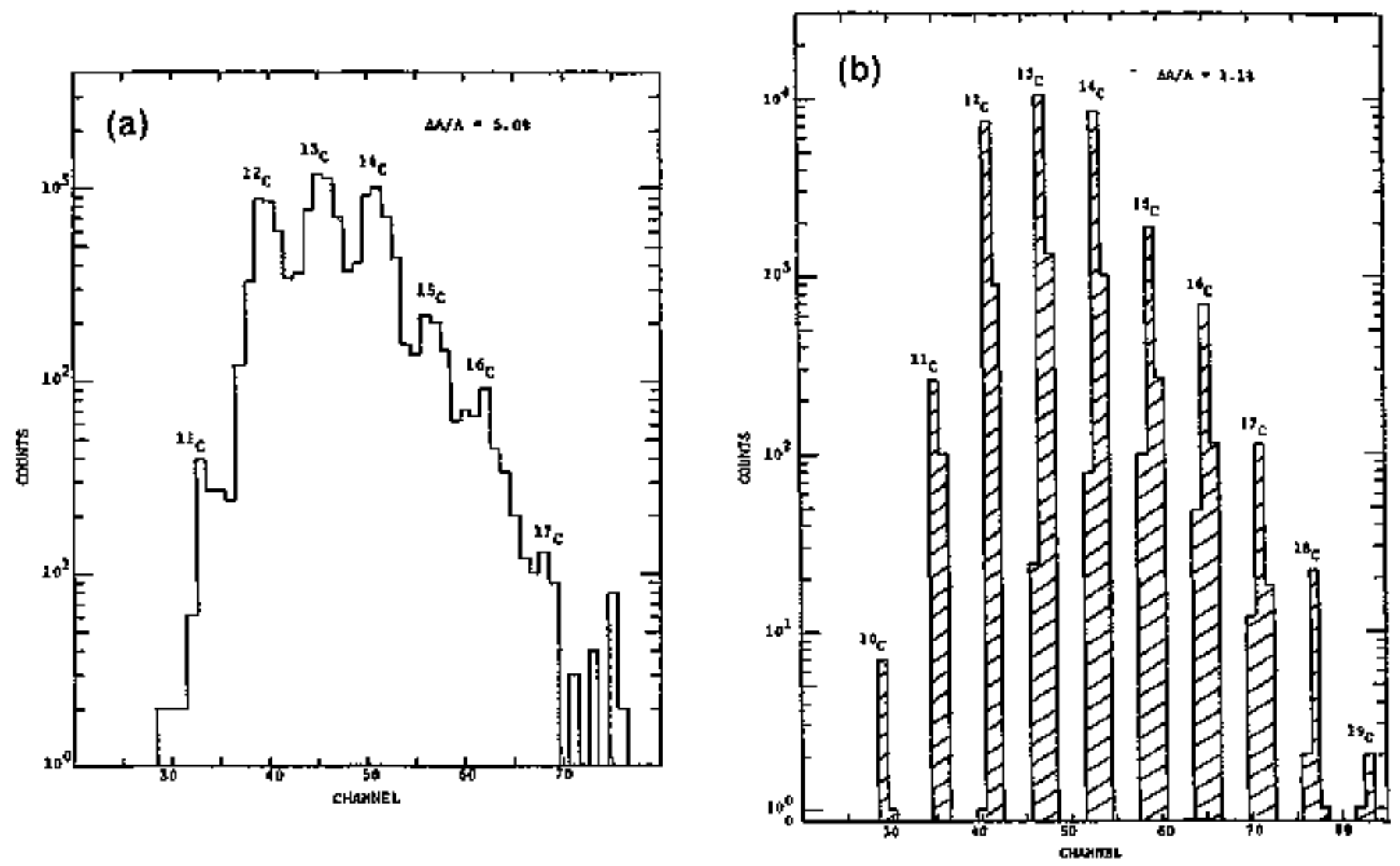

Fig. 1. Time-of-flight mass identification of carton isotopes. (a) Obtained with a 25-ch flight path between the $A E$ and $E$ colnters. (b) The same events tootstrapped to the 4.3-m flight path between the target (using the rf signa1) and the $\Delta E$ coumter. The four cycle semi-log scale shows the zero event level as well.

(XBL 759-7993) (XEL 759-7995)

\section{Footintes}

*Los Alanos Scientific Laboratory.

†Texas Agd University.

* Oak Ridge Natíonal Laboratory.

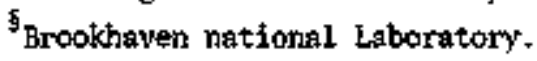

\section{EVAPORATION-LIKE FRAGMENTS FROM URANIUM IRFADIATED BY $2.1 \mathrm{GeV} /$ Nucleon ${ }^{12} \mathrm{C}$ AND ${ }^{20}$ Ne IONS}

A. M. Poskanzer, A. Sandoval, R. G. Sextro,

and $H \mathrm{H}$. Gulbrod

The stuty of low energy fragments from uranium irradiated by high energy protons 1 and atpha particles ${ }^{2}$ has been extended to heavier projectiles at the Bevalac. The plirpose of the expertiventel program is to study nuclear matter at high temperatures using the evaporation-like energy spectra of the light target fragments as a probe of highly excited nuclear matter.

The energy spectra for He through B fragments at $90^{\circ}$ in the lab are shown in Fig. 1 for the $20_{\mathrm{Ne}}$ irradiation. The shapes of the $4 \mathrm{He}$ and ${ }^{7} \mathrm{lt}$ fraginents are compared in Fig, 2 for all the projectiles used so far. It can be seen that the energy at the maximm, which is determined mainly by the conulomb barrier, remains fixed. However, as the mass of the projectlle increases, the spectra become considerably broader, the flattening on the high energy side being indicative of the high temperatures produced in the collision. (See Table 1). In Pig. 3 the 5 trong fncrease in production cross section with mass of the projectile is shown for 4 He fragrents. For the $2.1 \mathrm{GeV} /$ nucleon $20_{\mathrm{Ne}}$ fons the total $4 \mathrm{He}$ production cross section is estinated to be $\approx 29 \mathrm{~b}$, or an average of soven alpha particles enitted per 


\section{$05: 3$ a 5 .}
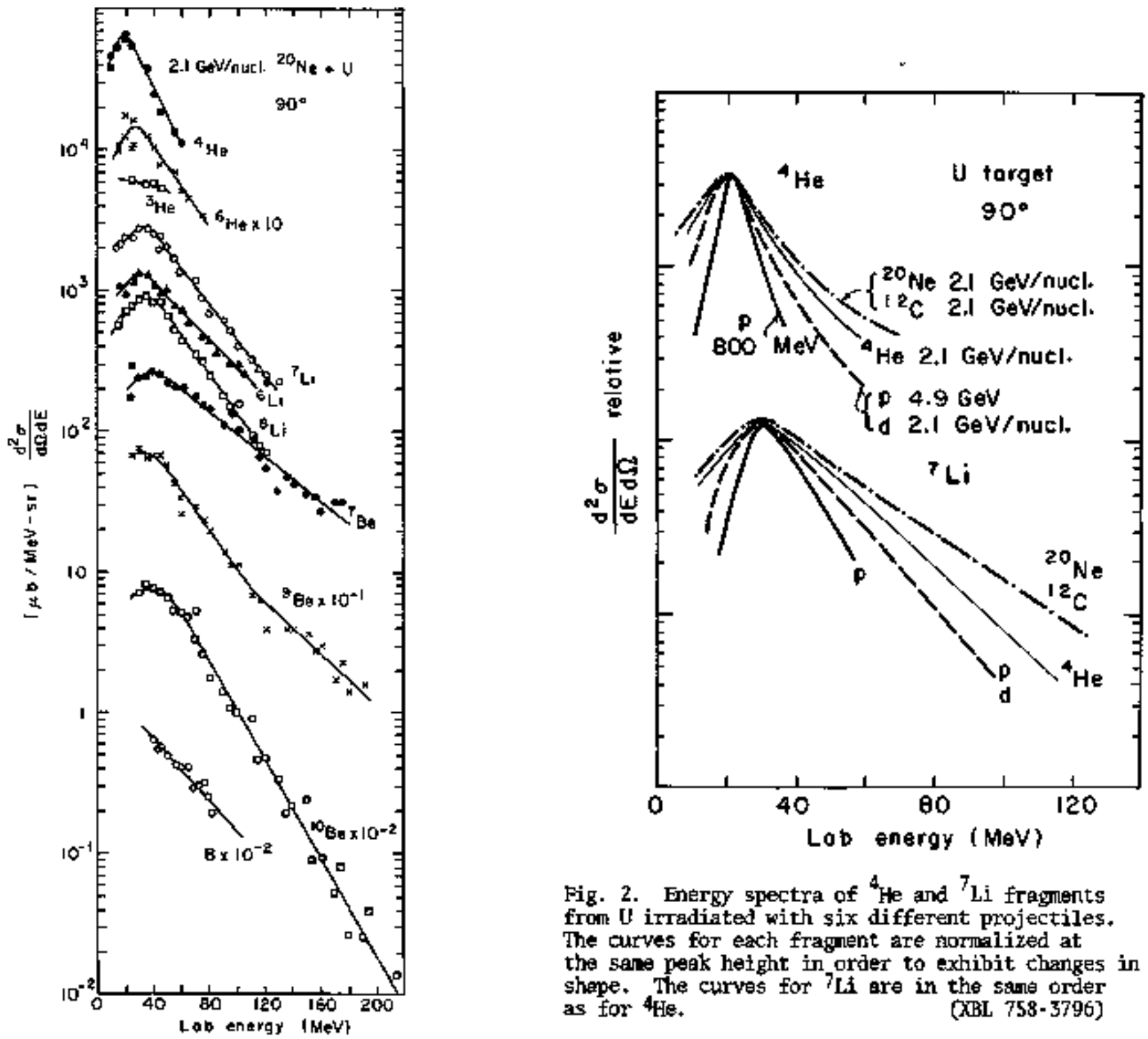

Fig. 2. Energy spectra of ${ }^{4}$ He and ${ }^{7} \mathrm{Li}$ fragments from $U$ irradiated with six different projectiles. The curves for each fragnent are normalized at the sane peak height in order to exhibit thanges in shepe. The curves for ${ }^{7} \mathrm{~L}$ are in the sand order as for ${ }^{4} \mathrm{He}$.

(XBL 758-3796)

Fig. 1. Energy spectra at $90^{\circ}$ in the lab for fragnents from $y$ irradiated with $2.1 \mathrm{GeV} /$ mucleon 20 No lons.

(XBL 758-3795)

Table 1. Apparent teleperatures and relative cross sectigns.

\begin{tabular}{|c|c|c|c|c|}
\hline \multirow[t]{2}{*}{ Projectile } & \multirow{2}{*}{$\begin{array}{c}\text { Temperature (MeV) } \\
{ }^{7} \mathrm{Li}\end{array}$} & \multicolumn{3}{|c|}{ Relative cross section } \\
\hline & & ${ }^{4} \mathrm{He}$ & ${ }^{7} \mathrm{Li}$ & ${ }^{7}$ Be \\
\hline S GeV protons & 10 & 1 & $\mathbf{1}$ & 1 \\
\hline $2.1 \mathrm{GeV} / \mathrm{mixl}$ eon alphas & 12 & 3 & 4 & 5 \\
\hline $2.1 \mathrm{GeV} /$ muxien 20 & 15 & 8 & 11 & 18 \\
\hline
\end{tabular}




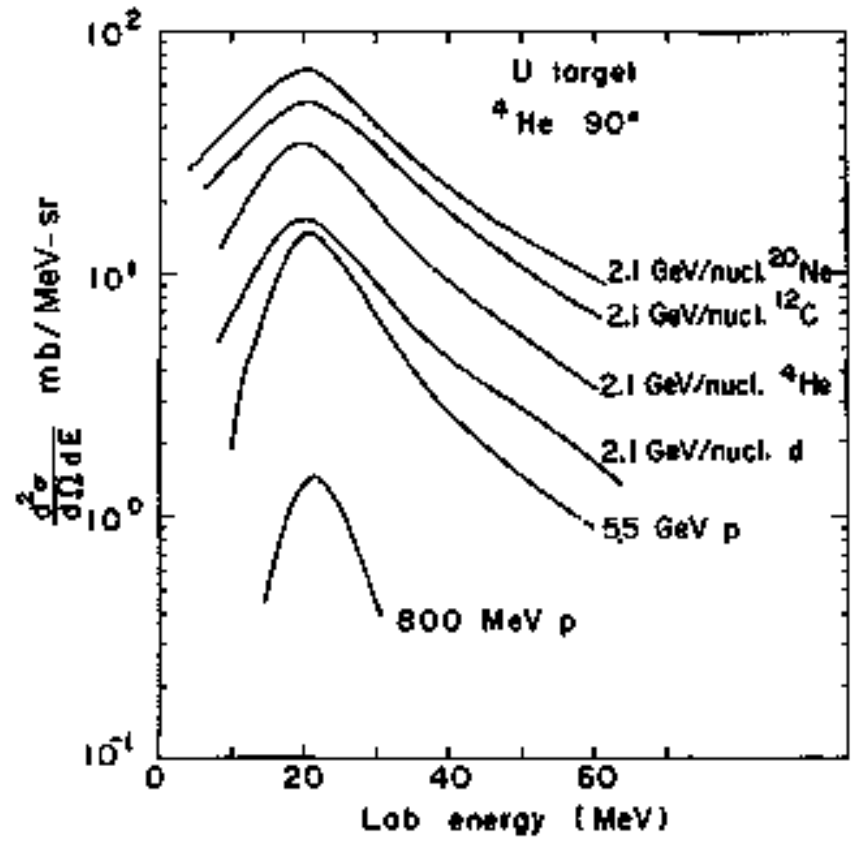

interaction. Lower-yield fragments, like ${ }^{7} \mathrm{Be}$, which are known to be produced only at high excitation energies, show an even rore dramat ic increase in relative cross section, as shown in Table 1 .

\section{References}

1. A. M. Poskanzer, G. W. Butler and E. X. Hyde, Phys. Rev, C ㅗ, 882 (1971).

2. A. M. Zebeluan, A. M. Poskanzer, J. D. Bowman, R. G. Sextro and V. E. Viola, Jr., Phys. Rev. C $\underline{11}, 1280(1975)$.

Fig. 3. Energy spectra of $4 \mathrm{He}$ fragments from $U$

(XBL 758-3795)

\title{
SEARCH FOR FRAGMENT EMISSION FROM NUCLEAR SHOCK WAVES*
}

\author{
A M Poskanzer, R G Sextro, A M Z pbelman, \\ H H Gulbrod, A Sendoval, and A Sock †
}

For central collisions of mulei at relativistic energies, recent theoretical investigations have focused on the question of how large amounts of energy and mementum are transferred from projectile to target nucleons, and on the early events in the evolution of hot, high-density ragions as thermal equilibrium is appropeched. In particular, the formation of squirts of muclear matter, or of nuclear shock waves carrying Iarge transverse momentuon and compressional energy, has been prodicted. These would be formed in central collisions if the projectile velocity exceeds the nuxlear soumd velocity which is about $0.2 \mathrm{c}$. The models are in disagreement about the angles in the lab system at which enission should occur, some predicting a narrow peak at angles ranging from $25^{\circ}$ to $45^{\circ}$ depending systematically on the incident energy while others anticipate a broad range of forward angles for the fragnents.

Baumgardt et al 1 studied the prong angular distributions of star events produced in AgCi crystals irradiated with $0.87-\mathrm{GeV} /$ mucleon 160 ions + They report the observation of narrow peaking in $\mathrm{do} / \mathrm{d} \theta$, with angular widths of about $20^{\circ}$ fult width at half-maxinam (FNM). The prongs anatyzed in that experiment are die to protons less than $28 \mathrm{kov}$ and he ruclei less than $200 \mathrm{MeV} / \mathrm{sucleon}$, with no further discrimination with respect to energy and isotope.
We have, therefore, undertaken a study at the Bevalac of target-fragment energy spectra and angular distributions with a $A E-E$ counter telescope that wold Identify He fragments with $155 \mathrm{E} \approx 150 \mathrm{MaV} /$ nucleon. The beans used were 2.7-GeV protons, 0,7 and 1.05-GeV/moleon alpha particles, and 1.05-GeV/micieon 100 ions. Targets of natural sflver and uranium, about 200 to 30 l) $\mathrm{mg} / \mathrm{cm}^{2}$ thick, were mourted in a scattering chamber equipped with a detector telescope consisting of a 1-mm-thick $5 i$ transmission counter as a $\Delta E$ detector, and a 5 -cm-thick plast ic scintillator (Pilot B) coupled to a $2.5-\mathrm{cm}-\mathrm{diam}$ phototube as an $\mathbf{E}$ detector. The telescope subtended a solid angle of 5 msr.

The energy calibration of the spectra was obtained for each kind of particle fron the $A E$ stgials in the surface barrier detector by use of the krown relation between energy loss in the of counter and total kjnetic energy. Due to the excellient resolution in $\Delta E$ and $E$, it was possible to identify the helium and lithium isotopes proxhred in the reaction. Spectra were taken in steps of $10^{\circ}$ or simetimes in steps of $5^{\circ}$.

Spectra are shom in Fig. 1 for ${ }^{3} \mathrm{He}$ and ${ }^{4} \mathrm{He}$ emission at $20^{\circ}$ in the lab systen from a $\mathrm{U}$ target bumarded with several projectiles and energies. For energies above $50 \mathrm{kbV} /$ nuclecm, the cross 


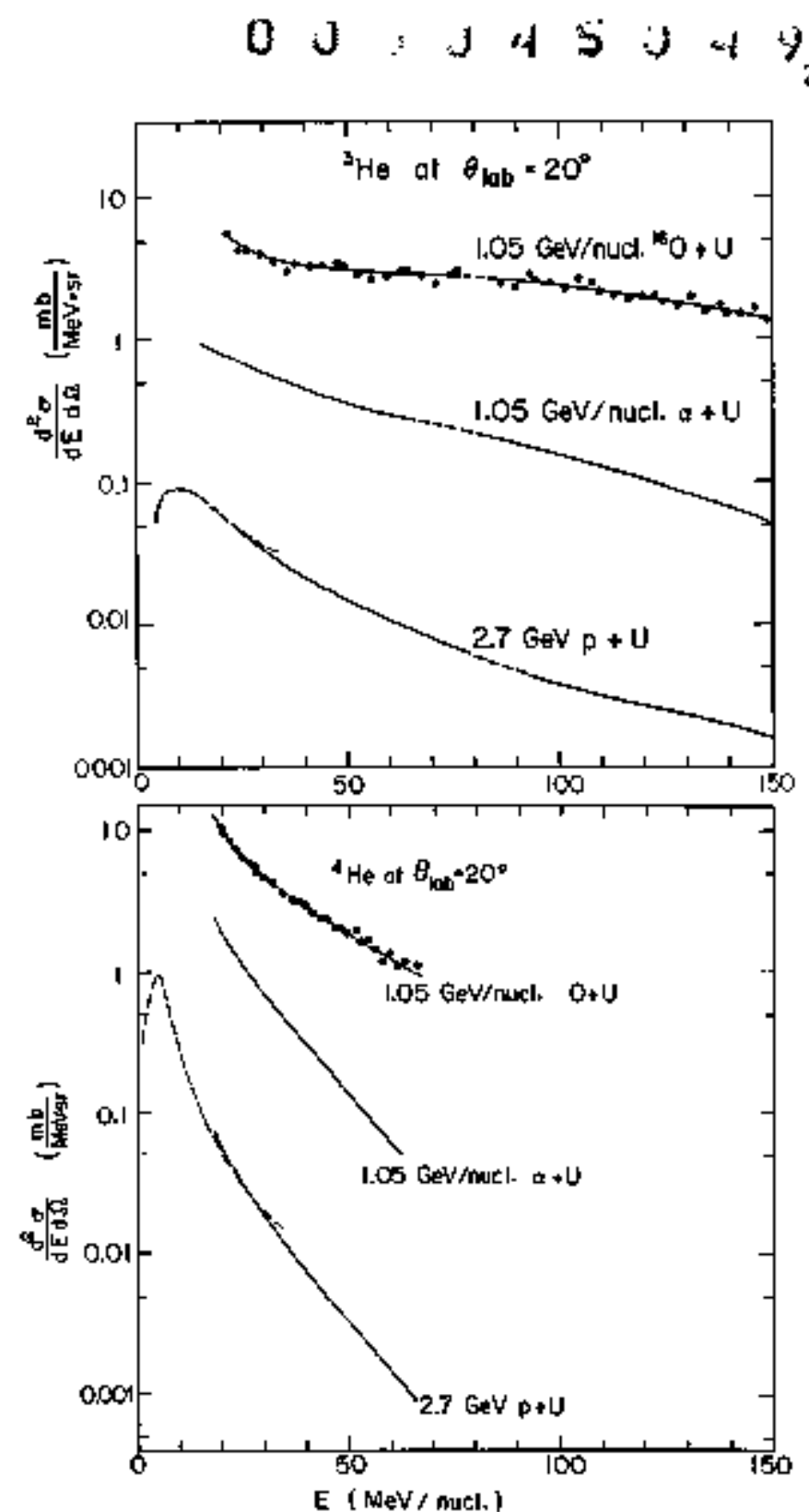

Fig. 1. Comparison of ${ }^{3}$ ile (upper part) and ${ }^{4} \mathrm{He}$ (lower part) spectra at $20^{\circ}$ (1ab) obtained upon bontbardment of a uranium target with $2.7-\mathrm{GeV}$ protons and with 1,05-GeV/nucleon of particles and 160 lons. The data points are shown for the ligo + I spectra. For $p+$ II dashed canves are shown for the low-energy evaporation spectra obtafned previously (Ref. 2).

(XRL 759-3896)

sections increase by more than an order of magnitude as the projectile changes fror $p$ to ${ }^{4} \mathrm{He}$, and from ${ }^{4} \mathrm{He}$ to $16 \mathrm{O}$. The ${ }^{3} \mathrm{He}$ spectrum from $100+U$ is renarkably flat, with cross sertions above $1 \mathrm{mb} / \mathrm{KeV}-5 \mathrm{r}$ even at $150 \mathrm{keV} / \mathrm{mucl}$ eon.

For ${ }^{3}$ He produced from ${ }^{16} 0+$ As, the angular distributions are shown in Fig. 2 for four successive bins of energy. The peak in do/do has a width of about $60^{\circ}$ Fhliti its position shifts from $58^{\circ}$ in the $1 \mathrm{cow}^{\circ}$ energy bin to about $30^{\circ}$ above $100 \mathrm{~W} /$ nucleon where the angular distributions show little further change in shape. The same

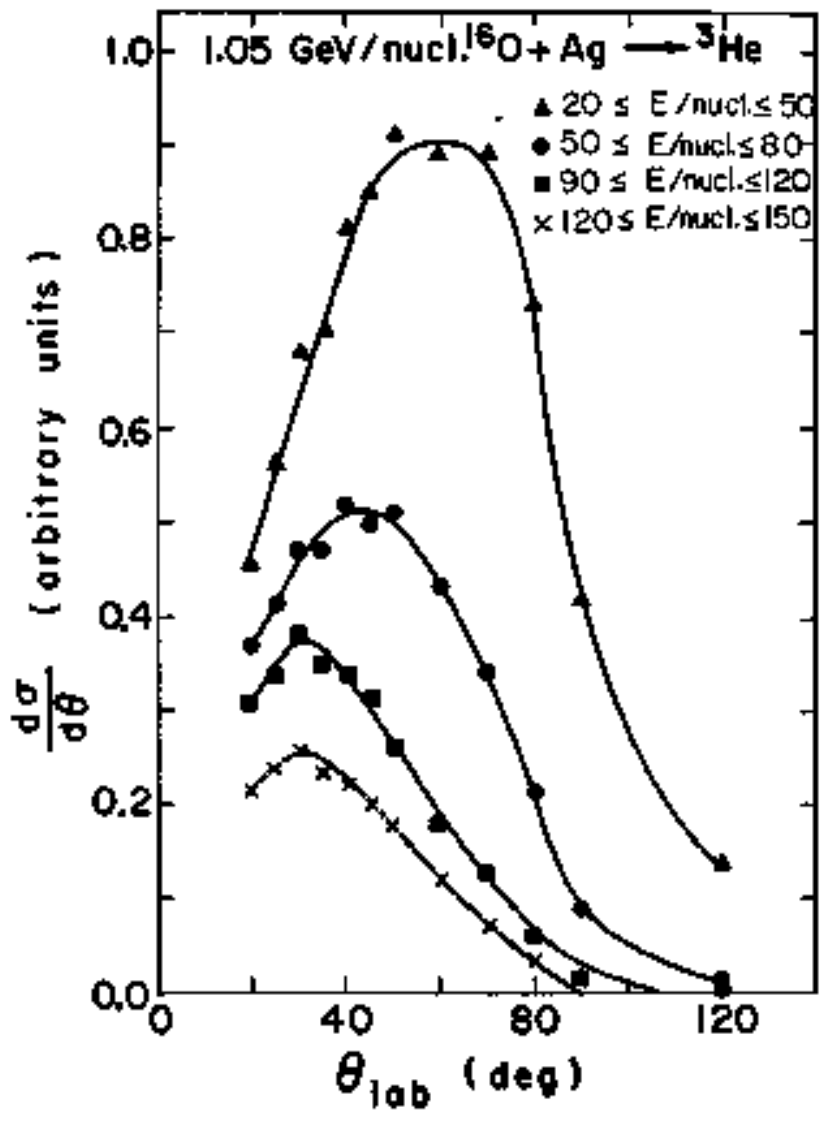

Fig, 2, Differential cross sections per unit angle, do/d $\theta$, of ${ }^{3} \mathrm{He}$ fragrents enitted ir various energy domains between 20 and ISD NoV/nucleon from 160 + Ag at $1.05-6 \mathrm{eV} / \mathrm{mucl}$ eon incident energy.

(XBL 758-3792)

behavior is observed for all ather combinations of target, projectile, and reaction produtt. No narrow peaking comparable with the $20^{\circ}$ width observed by Banmardt et al.1 is found.

Even thrugh our data are not selected for high multiplicity events we can compare our absolute cross section with those of Baungerdt et al. Their reported peak $\left(20^{\circ}\right.$ wide at $40^{\circ}$ width $\mathrm{d} \sigma / \mathrm{d} / 2=0.7 \mathrm{~b} / \mathrm{sr}$ ) camot be hidden under our smoth angular distributions for 160 on $\mathrm{Ag}$ at $1.05 \mathrm{GeV} / \mathrm{nucleon}$. We conclute that the events of Baungardt et al, 1 are not dine to high energy He nuclei.

Dur data present evidence for the nonevaporative enuission of ${ }^{3}$ He and $4 \mathrm{He}$. Their cross sections scale roughly with $A^{2}$ ( $A$, being the projectile mass). At l GoV/nucleon, a possible explanation for the production of high energy He and $\mathbf{4}_{\mathrm{He}}$ can be the final state interactions of cascade nucleons.

\section{Footnotes and References}

Condensed from A. M. Poskanzer, et al., Phys. Rev. Lett. 35, 1701 (1975).

${ }^{\dagger}$ thiversity of Narturg. 
1. Baumgardt et aI., 2. PTys. Az73, 359 (1975).

2. A. M. Poskarzer, A. Sandoval, R. G. Sextro, and A. M. Zebelman, unpublished data, 1975.

\section{CENTRAL COLLISIONS OF RELATIVISTIC HEAVY IONS}

H H Gutbrod, A Sandoyal. J Gossed, W G Meyer.

A M Poskanzer, G D Westfall, and $R$ slock"

A central collision of relativistic heavy ions with a target moleus is cheracterized by a high multiplicity of enjtted fragnents. En miclear track detectors 1 (AgCl and AgBr) star events with many prongs have been selected and andiyzed in rough energy bins (white, grey, and black tracks) obtaining angular distributions. Since a full scanning with the determination of charge, mass, and energy of the prong is nearly inpossible, conclusions drawn from this kind of data are very gross. To obtain more information regarding these central colliscions we have inproved our experimental setur ${ }^{2}$ by installing Is plastic scintillators (tag courters) connected to 2 in. diam photonultipliers around our scattering chamber. A special thin Al cover was built which served two puposes: (1) it increased the chamber volume by gos so that large volume detactor systems can be installed. (2) It serves as a thin $(3 / 8$ in, $)$ absorber so that
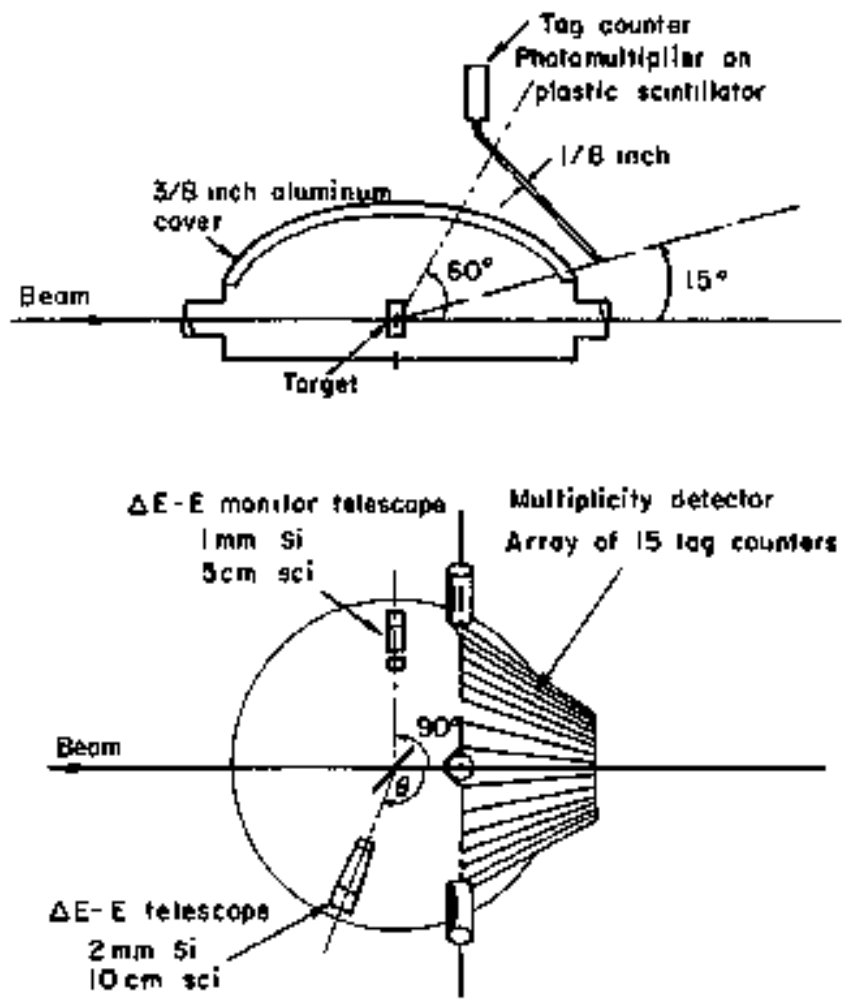

Fig. 1. 36-in. scattering chamber with telescopes and tag counters.

(XEL $7512-9933)$

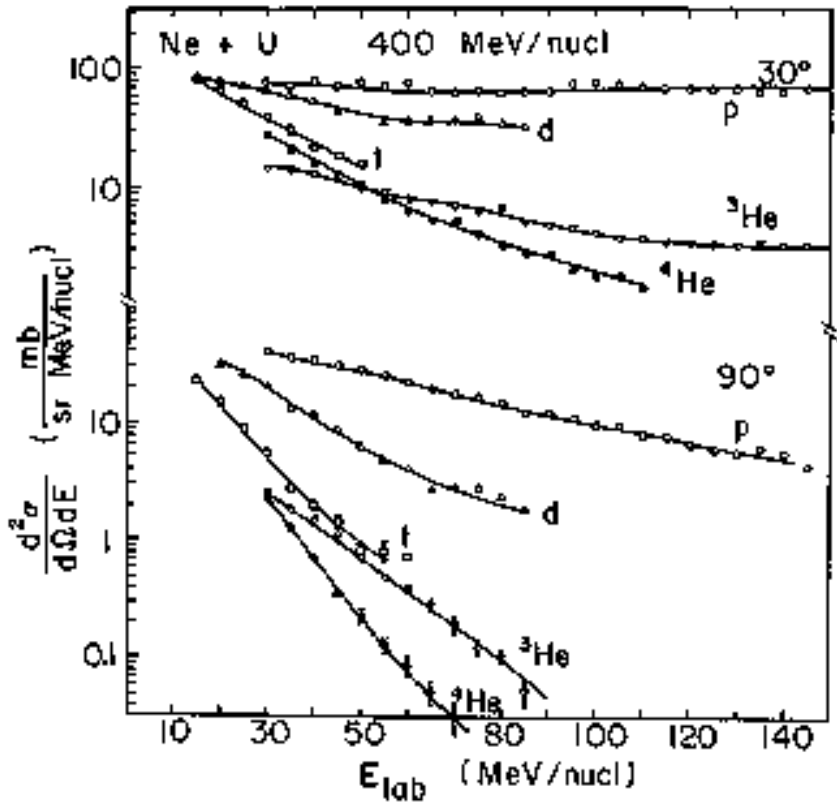

Fig. 2. Double-differential cross sections for hydrogen- and helíu-isotopes resulting from the interaction of $400 \mathrm{Bev} / \mathrm{nuxleon} 20 \mathrm{ke}$ with 238U,

(XBL $766-2954$ )

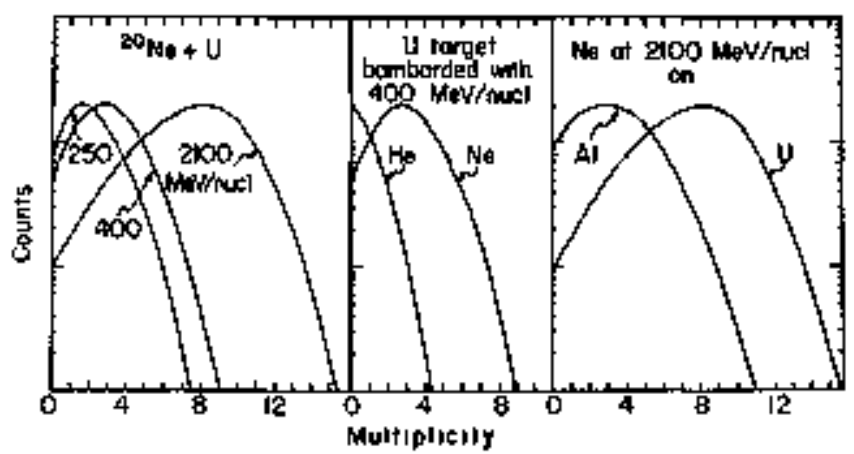

Fig. 3. Wutiplicity distributions for the systeras studied, as observed in the 15 tag counters.

(XBL 766-2956)

the tag colnters do not measure the targetevaporation products. Furthermore, the tag counters did not subtend the angle from $0^{\circ}$ to $15^{\circ}$ in order to arold any events due to break up of the projectile in a peripheral collision. The $A E-E$ detector described in Ref. 2 was used as 


\title{
$00 \therefore 3450 \therefore 997$
}

Table 1. Average multiplicity of particles (outside the chamber dome) with $E>45 \mathrm{MeV} / \mathrm{m}$ icleon for $\mathrm{p}$, $d, t$, and E.20 Mel for pions associated to the reaction products detected in the telescope. $\Omega$ was determined from the angular distributions of protons with $45 \times \mathrm{P}_{\mathrm{p}}<300 \mathrm{NeV}$.

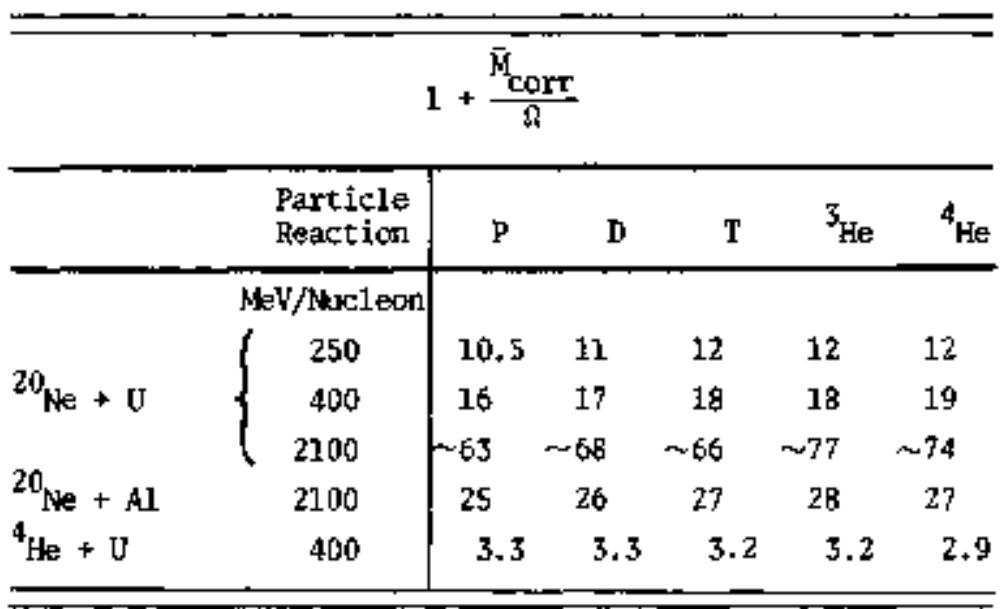

a monitor and a new silicon-scintillator telescope was built consisting of a 2 mil Si detector $A \mathrm{E}$ counter with an active area of $300 \mathrm{~mm}^{2}$ followed by a 10 an conically shaped plastic scintillator with a 2 in, phototube. A particle detected in this telescope opened a 30 -nsec gate in the l6-fold discriminator and coincldence latch of the 15 tag counters. Therefore, a particle detected in the telescope was identified by $A, Z, E, \theta$ and the relative mutiplicity of the star it belonged to, as well as by the information on which tag counters had fired. Thus, a rough spatial distrlbution of the multiplicity ovents could be detected.

Figure 1 shows the experivental layout. With this setup we studied 20 Ne induced reactions on $U$ at 250,400 and $2100 \mathrm{MkV} /$ nucleon and on Al at $2100 \mathrm{keV} / \mathrm{muk}$ loon, and $4 \mathrm{He}$ induced reactions on $U$ at $400 \mathrm{KeV} /$ mucleon. Hydrogen and helium fragments could be measured up to an energy of 260 and $300 \mathrm{HW} /$ mucteon respectively, and isotopic separation was obtained in hydrogen up to $100 \mathrm{MeV} /$ nucleon and in helium up to $130 \mathrm{kkV} /$ nucleon. Figure 2 shows a comparison of some spectra. Figure 3 shows the difference in the motitiplicity distribution for different target-projectile combinations at various energies. The single particle inelusive spectra of $d, t, 3_{\mathrm{He}}$ and ${ }^{4} \mathrm{He}$ could be related to the measured proton spectra using the Eutler-Pearson formalism for final state interaction between cascade nucleons, as modifled by Schwarzschild and zupancit. 3 The fingl state interaction as a mechanism for the production of the complex fragments is furthermore suggested by the observation that the complex fragrents are assaciated with a higher average mitiplicity than that associated with protons (Table 1 ).

\section{Footnotes and References}

"Eniversity of Marburg.

1. Baumgardt et al, 2. Phys, A273, 359 (1975);

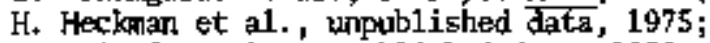

otterlund et al., wupublished data, $197 \mathrm{~S}$.

2. A. 付. Poskanzer, R. G. Sextro, A. M. Zebelman H. H. Gutbrod, A. Sandoval, and R. Stock, Phys. Rev. Lett. 35, 1701 (1975).

3. A. Schwarzschild and $\not{C}$. zupankiz, Phys. Rev. 129, $854(1963)$.

\section{FINAL, STATE INTERACTIONS IN RELATIVISTIC HEAVY-ION REACTIONS}

\author{
P. J. Johansen, J. Gosset. H, H. Gutbrod, W. G. Meyer,
}

A. Mogkenzor. A. Sandovat and G. D. Westiall

Experiments recently performed at the Beyalac by H. Gutbrod et al. 1 have beeg the study of this project. The projectiles were 20he at $250 \mathrm{MeV} /$ jucleon, $400 \mathrm{MeV} /$ nucleon and $2.1 \mathrm{GeV} / \mathrm{ntx}$ leon, and $\mathrm{He}$ 9 t $400 \mathrm{meV} / \mathrm{nu}$ leon. The target was in ati cases $230 \mathrm{U}$. From these experinents we have a series of double differential cross sections of the lighter fragments, $p$, $d, t$, 3 e and ${ }^{4} \mathrm{He}$ in an energy range from $15 \mathrm{keV} / \mathrm{ntul}$ ieon to $100 \mathrm{keV} / \mathrm{nucl}$ eon.

To explain these spectra, a theory by 5. T. Butler and $C$. A. Pearson has been applied. The 
theory was originally used to describe the denteron production in $25-6 e v, p$ on 238 veactions as a final state interaction. Here, the idea is to describe the spectra of the enitted composite particles, such as $d, t, 3 e, 4 t k$, in the relativistic heavy ion reactions on the basis of the monentum distribution of the cascade nuxloons. These nucleons con react with each other to form composite fragments with the surrounding nuclear matter acting as catalyst. In the phenomenological model this is described by assuming, thet particles closer together in moinentum space than a certain distonce $p_{0}$ will stick together and fokm heavier fragments.

A. Schworzschild and $x$. zupsinci ${ }^{3} 3,4$ simplifited the model of Butler and Pearson, and for the relativistic case get:

$$
\frac{d^{2} n}{p^{2} d p d 2}(N)=\frac{1}{N !} \frac{d^{2} n}{p^{2} d p d n}\left(\frac{d^{2} n}{p^{2} d p d 2} \frac{4 \pi}{3} \mu p_{0}^{3}\right)^{N-1}
$$

$N$ is the mass numer of the fragment, Po the radius in momentum space needed for the fraguent to coalesce, $\mathrm{d}^{2}{ }^{2} p^{2} \mathrm{~d} d \mathrm{~d}$ is the number of shower particles per eyent per volume element in momentim space, $\mathrm{d}^{2} \mathrm{n}$ (N)/ptpts is the number of conposite particles per event per volume element in momentilu space, and $Y$ is the Lorentz factor for a nucleon of momentixil $P$.

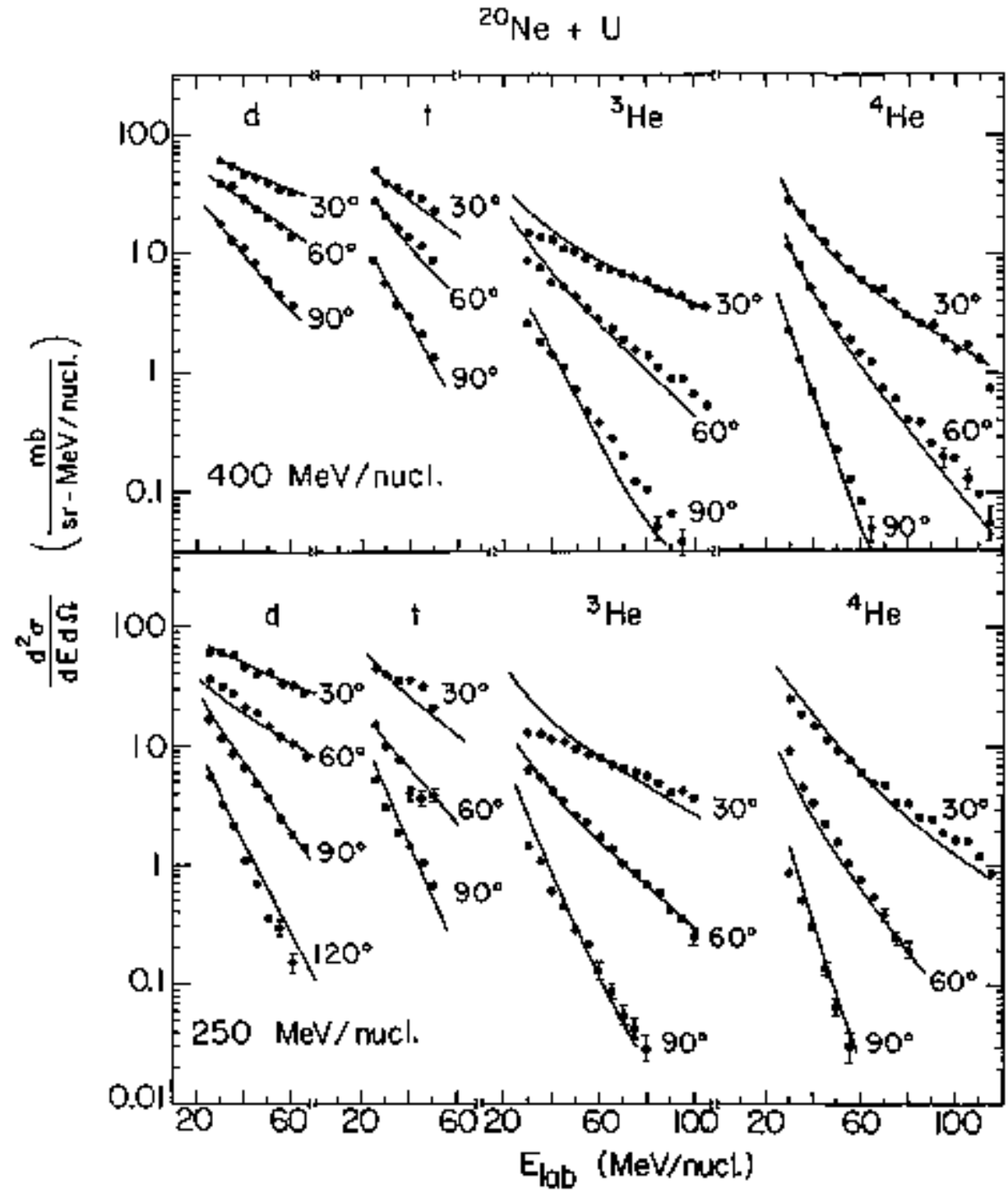

Fig. 1. Experimental and calculated double differential cross sections at vanious angles for 250 and $400 \mathrm{MeV} /$ nucleon $20 \mathrm{Ne}+\mathrm{U}$.

(XBI. 767-3109) 


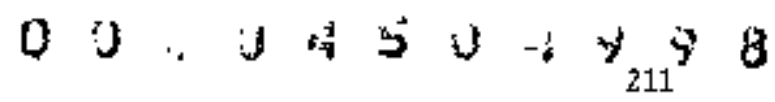

To test this theory, fits have been made for the different framents. Imput in this model is the experimentally determined distribution of protors. since the total cross section for cascade particle production has not been measured, Eq. (1) has been rewritten in terms of the proton cross section. Heglecting the differemes of $\sigma_{\mathrm{pp}}$ vs $\sigma_{\mathrm{pn}}$, we get:

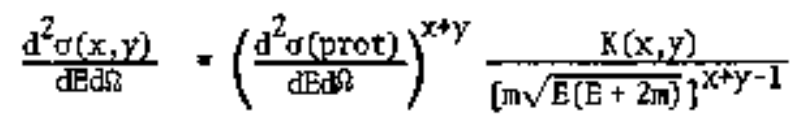

$$
K(x, y)=\left(\frac{4 \pi P_{0}^{3}}{3 \sigma_{0}}\right)^{K+y-1} \frac{1}{x ! y !}\left(\frac{N_{p}+N_{t}}{Z_{p}+Z_{t}}\right)^{y}
$$

Table 1. Radius $p_{0}(\mathrm{MeV} / \mathrm{c})$ of the micinentura sphere

\begin{tabular}{|c|c|c|c|c|}
\hline & $\begin{array}{c}p_{0}(d) \\
(A \in W / c)\end{array}$ & $\begin{array}{r}p_{0}(t) \\
(\mathrm{HeV} / \mathrm{c})\end{array}$ & $\begin{array}{l}\mathrm{P}_{o}\left({ }^{3} \mathrm{He}\right) \\
(\mathrm{p} b \mathrm{k} / \mathrm{c})\end{array}$ & $\begin{array}{l}\mathrm{p}_{\mathrm{o}}\left(\mathrm{L}^{4} \mathrm{H}\right) \\
\text { (Nter/c) }\end{array}$ \\
\hline $\begin{array}{l}20 \text { सye on } \\
23 \text { y }\end{array}$ & & & & \\
\hline $\begin{array}{l}400 \mathrm{kkV} / \\
\text { nucleon }\end{array}$ & 129 & 129 & 129 & 142 \\
\hline
\end{tabular}
for coalescence.
$X$ being the number of protons and $Y$ the number of neutrons in the composite particle.

So far we have been able to show, that the protiction of $d, t, 3_{\mathrm{He}}$ and $4_{\mathrm{He}} \mathrm{in}$ these reactions can te explained within a factor of 2 in the fimework of A. schwarsschild et al, 3,4 (Fig. I).

The value of $\mathrm{p}_{0}$ (Table 1) is very sensitive to the value of $\mathrm{a}$ ged [ see Eq. (2)]. Dur estimate comes around $130 \mathrm{MeV} / \mathrm{c}$ as compared to $100 \mathrm{MeV} / \mathrm{c}$ of Ref. 2, and $400 \mathrm{MeV} / \mathrm{C}$ of Ref. 3.

If contimation of this project more work is in progress to examine the possibility of describing also the heavier fragments like the $\mathrm{Li}$ and $\mathrm{Be}$ isotopes by this mechariom, and to introtuce a simple model to describe the energy and angular dependence of the shower particles.

\section{References}

1. H. Gutbrod, A. Sandoval, J. Gosset, W. G, Meyer, A. M. Poskanzer, G. D. Westfal1 and R. Stock, umpublished data, 1975.

2. S. T. Rutler and C. A. Persan, Phys. Lett. 1 , $77(1962)$.

3. R. Schwarzschild and $\stackrel{C}{C}$ Zuparcic, Phys. Rev. 129, $854(1963)$.

4. C. Zuponcic, Phys. Lett. 4, $182(1963)$. 


\title{
D. MESONIC ATOMS AND ATOMIC PHYSICS
}

\author{
MESONIC ATOMS
}

Clyde Whisgand, Gerry Godirey and Gary Lum

\section{Zu Dependence of Kaonlc X-Ray Intensities}

We completed a survey of the intensities of $x$ reys emitted when negative kaons were stopped in 9l targets ranging from $Z=2$ through $Z=92.1$ Some of the targets were pure isotopes and some were compounds. Included were $x$ rays from kacmic atons and $\Sigma^{-}$hyperonic atons. Kanonic $x$-ray intensities for low principal quantum number, $n$, transitions varfed from 0.1 to $0.5 \times$ rays $/ K_{s t o p}$ and showed a remarkable dependence on $\mathrm{Z}$ (see fily. 1). Peaks occurred near elentents with elosed electron she11s. There is no satisfactory explanation of this $Z$ dependence. A calculation of the kacms ${ }^{\dagger}$ cascade into the nuclei i was mode in an attempt to reproduce the measured intensities. Initial kaon distributions at $n=30$ proportional to $(2 x+1)$ out to certain $2 \max$ were used to begin the cascade. No kaons were assumed to be in angular momortum states higher than the assigned $x_{\max }$. Values of $l_{\max }$ were changed with 2 to fit the measured intensities. This scheme of arbitrarily trunceted distributions reasonably reprotuced the $\Delta=-1$ transition but gave an $=-2$ intensities high by a factor of two. It was suggested that $\mathrm{l}_{\text {max }}$ might be related to an impact partineter with a lever arm equal to one-half the distance between atoms, but this schenpe was unsatisfactory.

\section{Absence of Kam Decay}

There was a remote possibility that certain electronic configurations could result in wetastable orbital states for kaons due to suppression of Auger emission. If the cascade got "hung up", kaons could decay with their matural meanlife of $1.24 \times 10^{-8} \mathrm{sec}$. Dhuring scine of the $x$-ray messurements we searehed for nuwons from $\mathrm{K}^{-}+\mu^{+}+{ }^{2}$ and established an upper limit of 0.05 per $\mathrm{K}_{\text {stop. }}{ }^{2}$ Therefore, stopped kacons disappeared in nuclear reactions as had always been assumed.

\section{Effect of Hydrides}

We found that oxygen in a $\mathrm{H}_{2} \mathrm{O}$ target and $\mathrm{C}$ in a target of of (polyvinyltoluene) enitted raskedly fewer kaonic $x$ rays than oxygen or carbon alone, although it is known that only about 39 of the kachs interacted with free protchs. ${ }^{2}$ Several other hydrides have been tested to show that the presence of hydride bonkts reduced the $x$-ray intensities. The mechanisun that influences mesonic cascodes in the jresence of hydride tonds is probably related to the one that controls cascades in elements.
Loss of $x$-ray intensities from low in transitions is apparently caused by mesons being captured into predoninantly low $\&$ states where they react with ntcelei at high $n$ ievels. Or, atomic capture starts with statistical distributions that shift toward low \& states due to Stark mixing or sone other effect induxed by electric fields of partic" ulat electronde configurations.

Observation of Dymanic E2 Mixing Via Raonic X-Ray Intensities

A nuclear rosonance effect was observed between a kanic atom and a nuclear transition in

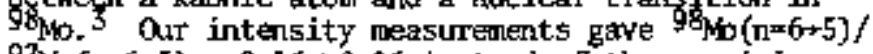
$92 \mathrm{k}(\mathrm{n}=6+5)=0.16 \pm 0.16$ instead of the no-mixing valuse of 1. Dynaraic E2 mixtng caused kaons to be strongIy absorbed from the $98 \mathrm{Ho}$ atomic state $n=6$, $\ell=5$, in agreentent with theoretical predictions. The effect occurs when the energy of a nuclear excited state nearty equals a kaonic atom de-excitation energ $\gamma_{*}$ In $9 B_{\text {ko }}$ niding of the | kaon $n=6$, $z=5$ ) |mucleus $0^{+}$ground state with the !kaon $n=4$, $t=3$ inucleus $2^{+}$excited is expected to cause kaons to be strongly absorbed frort the new $n=6,2=5$

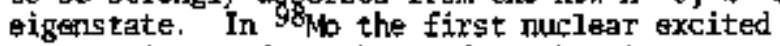
states lies at $787.4 \mathrm{keV}$ and the kaonic atcon transition $n=6+4$ is $798.2 \mathrm{keV}$. As a control wo compared $98 \mathrm{Ho}$ with $92 \mathrm{~kb}$ whose first nuclear excited state 11 es at $1540 \mathrm{keV}$. Figure 2 shows portions of the $\mathrm{k}$-ray spectra where the intensities are equal for the undisturbed $n=7+6$ transitions, The $n=6 \div 5$ tine is present in $92 \mathrm{Mo}$ and absent in ${ }^{9} \mathrm{MO}$. Studies of $E 2$ mixing, which is due to the quadrupole moment of the excited nuclear state, could lead to information on states not directly accessible in kaunle atoms. In our case, the kaonic $n=4, k=3$ state.

\section{Anisotropy of X-Ray Angttlar Distributions}

There is a possibility that the distribution of $x$ rays emitted by mesonic atans is not isotropic. Anisotropy relative to the direction of the inpinging beam coutd occur if the momenta of the mesons were sufficiently large at the instant of atomic capture. The axes of rotation of the mesonic atons would be confined to a plane perpendicular to the bean directicm. It was shown that theoretically the orientations should be preserved during Auger and dipole $\mathrm{x}$-ray enission. Existence of an anssotropy would give valuable information on the mech- 


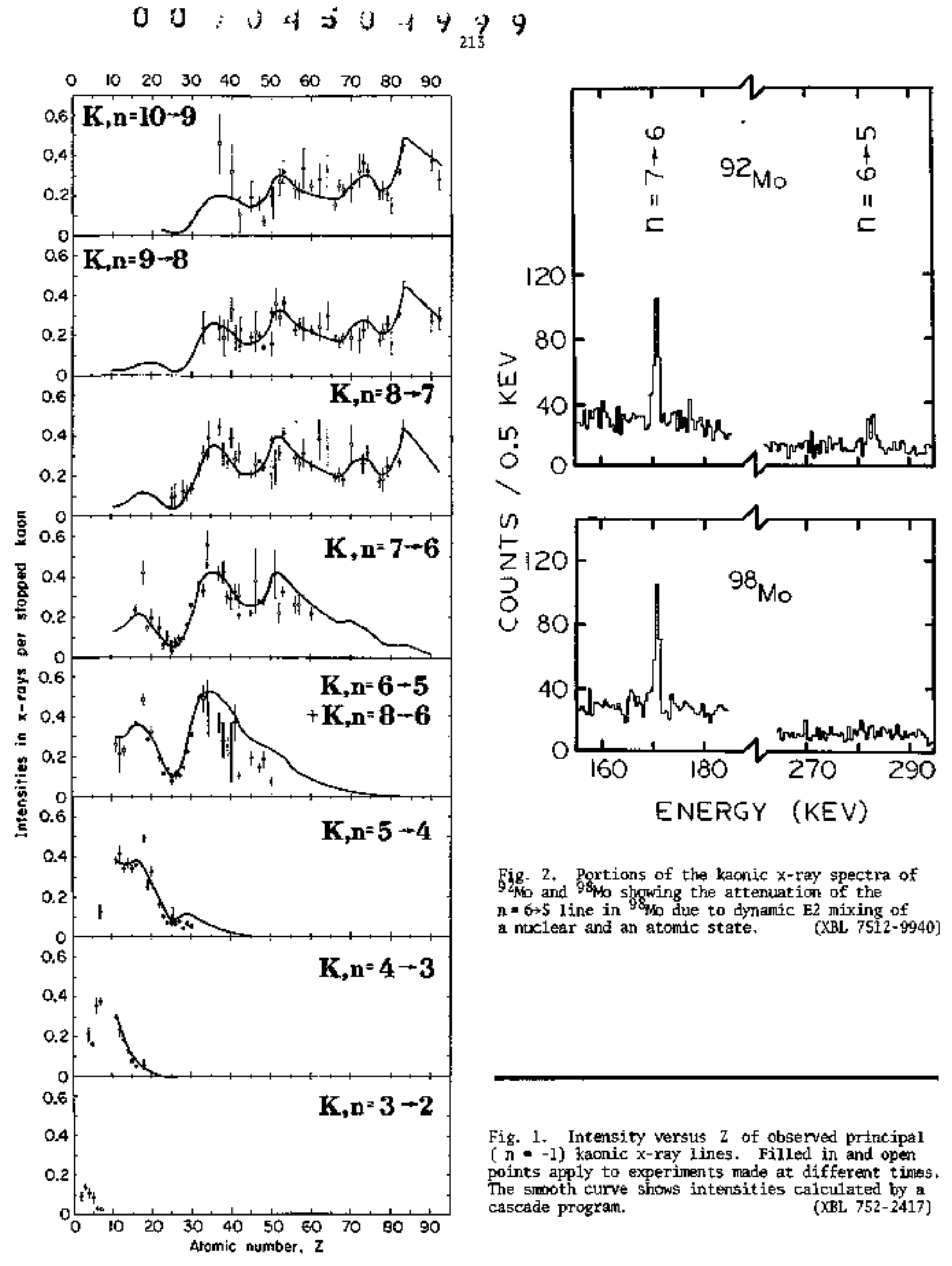


anism of formation of mesonic atons. In a recent experinent we measured the distributions of $x$ rays from pionic argon. Analysis of the results shows intensities in the beam direction and perpendicular to the beam direction to be equal within \& few percent where the maximo effect could have been 50 g.

\section{References}

1. Gary L. Godfrey, Larrence Berkeley Laboratory Report LAL-3857, Ph.D. Theșis, May 1975.

2. G. L. Godfrey and C. E. Wiegand, Phys. Lett. $\underline{56 \mathrm{~B}}, 255$ (1975).
3. G. L. Godfrey, G. K. Lum and C. E. Wiegend, Phys. Lett. 51B, 45 (1976).

4. 6. Ya. Korenman, Sov, J. Nuc1. Phys. 21, 398 (1975).

\section{Additional Publications}

Gary L. Godfrey, Strong Interactions, Zwieg's kule, and Weaker Interactions, LBL-3697, February 1975. R. Seki, C. Wiegand, Kaonic and Other Exotic Atoms, Annu. Rev. of threl, \$ci. (Ansual Revienss Inc., Palo Aito, California, 1975). Vol. 25, p. 241 .

\section{MULTIPLE SCATTERING AND ENERGY LOSS STRAGGLING OF HEAVY IONS}

a o Harwey and I Mahoneyt

The performance of instrments for the identification of heavy ions is often limited by moltiole scattering and the mergy loss straggling. For example, the resolution of angular measurentent made by double position measurments in a spectrometer focal plane detector can be 1imited by heavy ion multiple scattering in the gas space between two position-counter planes. The determination of atomic nuwber $Z$ depends upon the measurement of energy loss $\mathrm{dE} / \mathrm{dx}$, and in a well-designed instrunent the $d E / d x$ resolution should be almost entirely fixed by the energy loss straggting. instiument and experiment desion, therefore, require the accurate calculation of heavy im mitiple scattering and entrgy loss straggling.

\section{Wultiple Sçattering}

The theory of sultiple scattering $I, 2$ and the tables of Sigpimd and Winterbon ${ }^{2}$ are in good agreement with recent experinental work 3,4 The Rochester measurements, 5 however, appgar to give RAs multiple scattering angles that are too large by a factor of 2 or 3 . We have compared multiple scattering angles O1/2 (HitM) extracted from the Sigumd and Winterbon tables with values calculated frop the old formula, 6

$$
\begin{aligned}
\theta_{1 / 2} & =0.585\left[\frac{3.922 \times 10^{-8} \mathrm{t} z_{1}\left(z_{1}+1\right) z_{2}^{2}}{A_{1} E^{2}}\right. \\
& \left.\times \ln \left(\frac{211.9 t}{z_{1} / / 3+z_{2}^{2 / 3}} \cdot \frac{z_{1}+1}{A_{1} z_{1}}\right)\right]^{1 / 2} \mathrm{rat}
\end{aligned}
$$

Where ${ }_{1}{ }_{1} 2$ is the half width at half maximn, $A_{1}$ and $z_{1}$ refer to the scottering medium, $z_{2}$ to the heavy ion, $t$ is the thickness of the scatterer in $\mu \mathrm{g} / \mathrm{cm}^{2}$, and $\mathrm{E}$ is the heavy ion energy in $\mathrm{MeV}$. The empirical factor 0.585 brings the formula into agreenent with Sigrtund and Winterbon to within
10* over a very wide range of parameter values provided that $t$ is greater than $20 \mathrm{\mu g} / \mathrm{cm}^{2}$. Contrary to the findings of the Rochester group, agreentent with experinent is obtained even down to $1 \mathrm{MeV} / \mathrm{A}$ when $z_{2}$ Is used rather than the effective charge of the heavy ion.

Energy_Lasg Straggling

In contrast, the theory of energy loss straggling still needs improvement. Horeover, experimental measuremests in thin foils require a miformity of thichess on a microscogic scale that is very difficult to obtain, ${ }^{7}$ At low velocities, electron pick-up and loss contributes markedly to the energy toss straggling.

For the purpose of predicting the de/dx straggling that will be obtained in a gas-filled proportional couter or ion chanter, we find that the simple equation

$$
\S \mathrm{E}=\mathrm{KZ} Z^{\sqrt{\mathrm{t}}} \mathrm{keV} \text { FHM }
$$

is very useful. However, $\boldsymbol{K}$ is energy dependent. For 160 in propane it varies fram 1.13 ( $60 \mathrm{MoV}$ ) to 0.95 (300 Mev), the drop being perhaps due to the lesser inportance of electron plck-ip and loss at the higher ion velocity. In $\mathrm{Ar}^{-7} \mathrm{foH}_{4}$, the value of $K$ is also about 1 .

\section{References}

1. 1. Meyer, Phys. Status Solidi 44B, 253 (1971).

2. P. 5idwund and K. B. Winterbon, Nucl, Instrum. Woth. $119,541(1974)$.

3. B. W. Hootón, J. M. Freenan and P. P. Kane, Nuc1. Instrum. Keth. 124, 29 (1975). 


\section{$00: 243: 0$ i 0

4. G. Spahn and $K, 0$. Groenveld, Nac1. Instrum. Math. 123, $425(1975)$.

S. C. K. Cline, T. E. Pierte, K. H. Purser and M. B1arm, Phys. Rev. 180, 450 (1969).
6. E. J. Williams, Proc. Roy. Soc. 169, 531 (1939); Phys. Rev. 59, 292 (1940).

7. Preprint, VICKSI Group, Beriln.

\section{MEASUREMENT OF ATOMIC CHARGE EXCHANGE CROSS SECTIONS FOF HIGH ENERGY HEAVY IONS}

Fred Bieserr,' Eruce Cork, Hank Craurtord, Douglas Gremer,

Harry Heckman, Peler Lindstrom, Grant Ralsbeck,t and Lance wison

The effective charge of an fon passing thurugh matter is not a constant. The ion is cont inually picking u electrons from the medium, storing then in its vacsont orbitals, and then losing them again in subsequent collisions. The rate at which this happens depends on the $i$ in's nuctear charge $z_{I}$, its velocity $B_{I}$, and the electron distribution in the medium, which is characterized by the nuclear charge of the meditm $z_{n^{+}}$At low lon velocities, $B_{T}<z_{T^{t a}}$, the number of electrons localized on the ion, and hence its effective charge $z^{*}$, is a rapidly varying function of $B_{1}$. At vary high velocitles, $\beta_{I}>>z_{1} a$, the ion spends most of its time fully stripped of electrons, occasiona1ly attaching a singlo electron and losing it again before it has a chance to attach another. are using the high velocity ( $e \sim 0.65$ to 0.95 ), fully strippent 1 in betans of the Bevalac to investigate the rate at which single electron attachment and loss occurs for heavy ions ( $6 x_{0} Z_{I} x_{0} 26$ ) as they pass through various solid and gaseous targets $\left(4<Z_{m}<79\right)$. We present here sche results for Ne ions incident at $250 \mathrm{MeV} /$ mucleon and $2.1 \mathrm{GeV} /$ nucleon on various thick solid targets.

The experiment was originally motivated by an astrcphysical problem tonceming the propagation of cosmic rays which can only decay by capturing a $\mathrm{K}$-electron. 1 By measuring the relative abundance of these K-capture isotopes in the cosntic ray's, as a function of energy, and measuring the single electron attachment and 105s eross sections for then, we will have another way to probe the envirmonent in which the cosmic rays travel.

The problen of the effective charge of ions passing throuteh matter at low velocities [6 65.15 , (10 MoW/nucleon] has been investigated by many experimenters. kased on data for alpha particles and ions as heavy as ${ }^{84} \mathrm{Kr}$ at low B, $\mathrm{a}$ theoretical framework describing the attachment and loss process has evolved. This theory is most easily inderstood and applted to single electron systems; hovever, since even light ians like ${ }^{A T}$ have more than one electron attached, on the average, at $10 \mathrm{WeV} /$ mucleon, data to test the theories in their simple $1 e^{-}$form has not been available before this experiment. By reasuring the electron attachnent and loss cross sectich.5, $\sigma_{A}$ and $\sigma_{\mathrm{L}}$, as a function of $\mathrm{B}_{\mathrm{I}}, \mathrm{z}_{\mathrm{I}}, \mathrm{z}_{\mathrm{m}}$ we will provide input for the cosmic ray propagation calculation and be able to test atchatc-chargeexchange theories on simple systems at energies not previously investigated.

The experinent is performed in Bevalac Bean 33, using the spectroneter, by passing a bean of fully stripped ions, $z^{*}=z_{I}$, first through a target (gas or solid), then through a magnetic field and fito a charge-measuring solid state detector telescope (four Si(li) detectors in a row). The anglo through which the ion is deflected by the nagnetic field depends on the nowentun and effective charge of the ion. By placing a detector telescope at the angle corresponding to $2^{a}=Z_{T}-1$ and a secondary enission montor at the angle for $z^{*}=z_{T}$ we are $a b l e$ to count the number of ions emerging from the target with an electron attached $\mathrm{N}\left(\mathrm{Z}_{\mathrm{I}}-\mathrm{I}\right)$, and the number which emerge fully stripped, ${ }^{2}\left[z_{I}\right]$.

The ratio $N(z-1) / N(z)$ depends on the attachment and loss cross sections and on the target thichness, such that

$$
\mathrm{N}(\mathrm{z}-1) / \mathrm{N}(\mathrm{z})=\sigma_{\mathrm{A}} / a_{\mathbf{L}}\left(1-\mathrm{e}^{-\sigma_{\mathrm{L}} \mathrm{K}_{\mathrm{T}^{*}}}\right)
$$

Where $\mathrm{NT}_{T}=$ motuber of target atoms $/ \mathrm{cm}^{3}$ and $\mathrm{X}=$ target thickness (cm). For thick targets, $X>1 / \mathrm{N}_{\mathrm{T}}{ }_{\mathrm{L}}$, the ratio is independent of $X$ and represents

a dynaric equilibrium between attadhment and loss processes. We carl calculate of assuling that electrons are lost in collisions with target electrons and nuclei in which the energy transfer exceeds the attached electrons binding energy, and that $B_{I} \gg B_{\text {Tn, }}$ the velocity of electrons in the mediun. Thus, following Bohr, ${ }^{2}$ we find per target atom,

$$
\sigma_{L}=4 \pi a_{0}^{2} \frac{z_{\text {प }}^{2}+z_{\text {Et }}}{z_{I}^{2}}\left(\frac{\alpha}{B_{I}}\right)^{2}
$$

where $a_{0}=$ Bohr radius. For $20_{\text {Ne }}$ traversing $27_{A 1}$ at $B_{1} \leadsto 0.95$, we expect to find that an equilibriun will be reached in targets thicker than $1 \mathrm{mg} / \mathrm{om}^{2}$. As we go to higher $Z_{m}$, however, $B_{m} n_{\alpha} a Z_{M}$ is no longer snall with respect to B and our simple formpla for $\sigma_{L}$ is no longer valid. The loss cross section should decrease because low energy transfer collisions will become a larger fraction of the $e^{-}=e^{-}$interactions. Thus, at $z_{m}=80$ would expect the thickness in which equilibritm is reached to bo greater than $300 \mathrm{Hg} / \mathrm{cm}^{2}$.

There are two distinct processes that can lead to electron attachment: nonradiat ive (domingting at low energies) and radiative. The nomradiative process is a three-body process finvolving the transfer of the electron fros the target nucleus to the incident ion, Because Iittle work exists on the relativistic problem, for comparison with experiment we have used the nonrelativistic calculation of 
Brinlontan and kramers ${ }^{3}$ for a hydrogenic medium. After making corrections for attachment into higher states, and the streening of the nuclear charge, we find the formula (per target atom) becones,

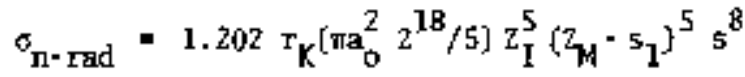

$$
\begin{aligned}
& \times\left[s^{2}+\left(Z_{I}+z_{M}-s_{1}\right)^{2}\right]^{-5}\left[s^{2}+\left(z_{I}-Z_{m}+s_{1}\right)^{2}\right]^{-5}
\end{aligned}
$$

where $s=a^{-1} \sqrt{2(\gamma-1)}$, a is the Bohr radius, $r_{K}$ is the rumber of $X$ shell electrons in the reditum and $s_{1}$ is the $K$-shell screenling constant $(0$ for hydrogen; 0.3 otherwise).

As the inverse to photoionization, the radiative attachment process is a two body process in which a "free" electron becomes bound to a murleus and simaltaseously enits a photon in order to conserve energy. In this context, the word free means that the orbital velocities of electrons in the medium are negligible when compared to the incldent ion's velocity. We have used the principle of detalled balance, the relativistic photoionization formula of pratk et al. 4 and corrected for attachenents into higher states to obtains the following radiative attachment forumla per atcm

$$
\begin{aligned}
& \sigma_{\text {rad }}=(1.202) 3 / 2 z_{I}^{5} z_{m^{a}} \sigma_{T} \frac{T^{2}}{\left(T+M c^{2}\right)^{2}-M_{u}^{2} C^{4}} \\
& x\left[\frac{M_{u} C^{2}}{T}\right]^{5}\left(B_{Y}\right)^{3}\left[4 / 3+\frac{Y(y-2)}{y+1}\right. \\
& \left.\times\left(1-\frac{1}{2 \operatorname{sr}^{2}}\left(\frac{1+\beta}{1-\hat{\beta}}\right)\right)\right] \mathrm{f}_{\cot }\left(2_{I}, \theta\right)
\end{aligned}
$$

where $\sigma_{\mathrm{T}}$ is the Thompson cross section, $M_{\mathrm{u}}$ is an atcouic mass unit, $T$ is the kinetic energy per nuxlem, and $f_{\text {cor }}$ is the correction function of Ref. 3 .

Note that for large $\gamma$, theory predicts $v_{n-\operatorname{rad}} n Z_{m}^{5} \gamma^{-6}$ and $c_{\mathrm{rad}}{ } z_{m} \gamma^{-1}$ so that for each target there should exist an energy above which radiative attachment dominates. Thus, by varying the target materlals and Ion energies, we can test for a change in the relative importance of the taro processes.

In June 1975 we made prelininary Jeasurements of the thick target exuilibrium ratio, $\mathrm{R}_{\mathrm{E}} \cdot \mathrm{N}\left(\mathrm{z}_{\mathrm{T}}-1\right) /$

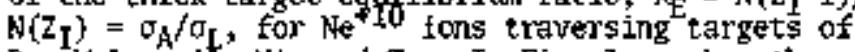
Ee, Mylar, Af, $\mathrm{Hi}$, and $\mathrm{Ta}$. In $\mathrm{Fig}$. 1 we show the equilibrius ratios for all targets relative to the equilibriu ratio for the Be target, $k E\left(z_{T,}\right) / R E(4)$, plotted against $Z_{\text {m }}$ calculated according to the theoretical formslas presented above. In Fig. 2 we show the same ratios calculated for the cases of only nomradiative attackinent process and only radiative attaclment process at $250 \mathrm{MeV} /$ nucleon. Our preliminary data show that we have clearly sepen a contribution from the radiative attachment of electrons.

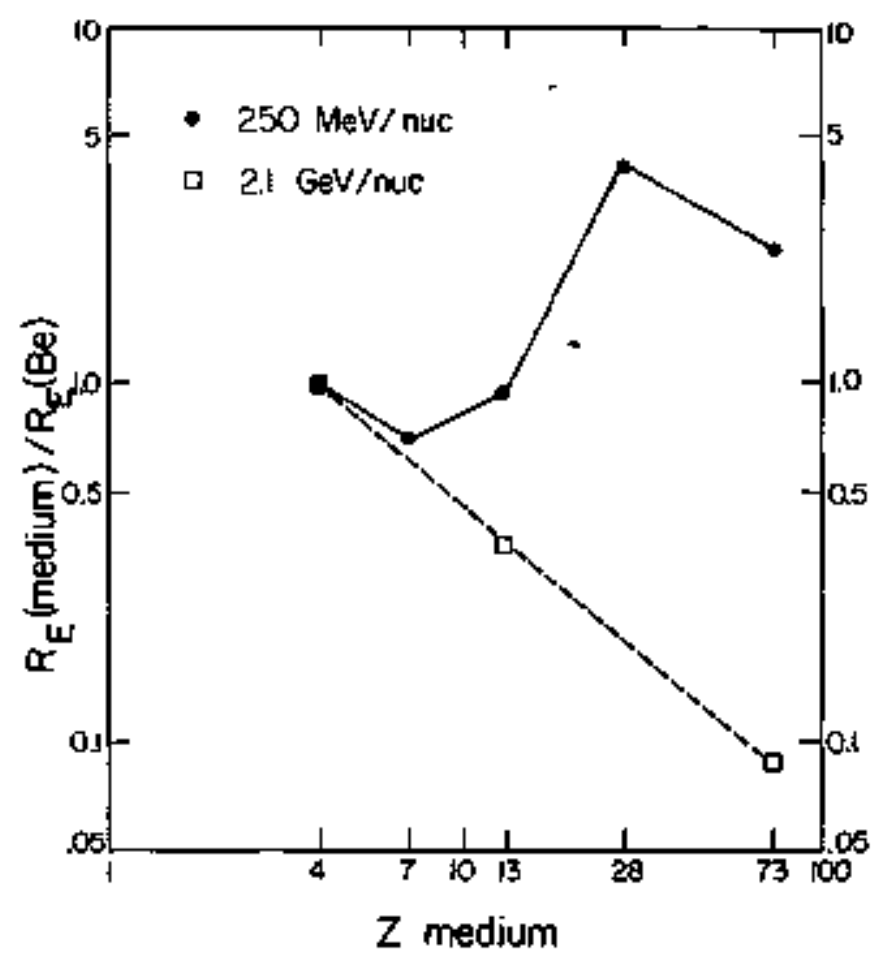

Fíg. 1. Calculated equilibring ratios $N\left(z_{I}-I\right) /$ N( $\left.Z_{I}\right)$ relative to the ratio in Be target v's nuclear charge of target medium.

(XBL 766-8269)

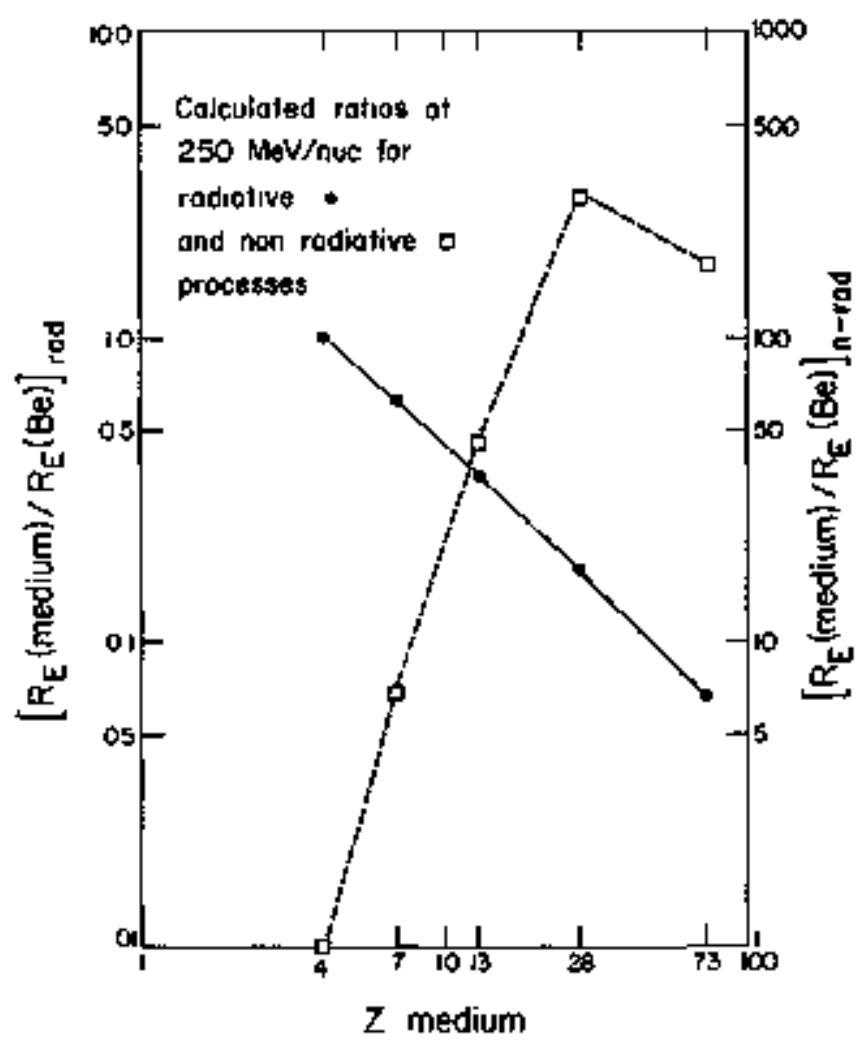

Fig. 2. Calculated equiflibrîm ratios relative to the ratio in Be assuming attarhment occurs by either nonradiative process (right hand scale) or radiative process (left hand scale) only, 
There are discrepancies between the theoretical calculations and the measured data. We are present ly exploring a relativisticalty correct theoretical description based on the two processes mentioned earlier which we hope will nore reasonably describe the measured data. Wo are continuing the experimental investigation in swomer 1976 to measure both $F_{4}$ and $\sigma_{h}$ (and hence $\sigma_{f}$ ) for $C$ and $A r$ beans at $250 \mathrm{HeV} /$ nuxleon and $2.1 \mathrm{GeV} /$ nucleon as well as for Ne at $500 \mathrm{Hew} /$ nueleon and $1 \mathrm{GeV} /$ nucleon.

\section{Footnotes and References}

* Lniversity of California Space Sclences Laboratory, Berkeley, California,

'Laboratoire Rene Bermas, Orsay, Fraxce.
I. G. Raisbeck, C. Perron, J. Toussaint and F. Viou, 13th Int. Cosmic ray conference, Denver (1973), Vo1. 1, p. 534.

2. N. Bohr, Kgl. Ianske Vidertskab. Selskab. Mat. Fys. Hedd. 18, 8 (1948).

3. H. C. Brinkman and H. A. Kramers, Proc. Acad. Sci. (Antsterdan) 33, 973 (1930).

4. R. H. Pratt, A. Ron and H. K. Tsang, Rev. Mod. Phys. 45, 273 (Ig73).

\title{
ATOMIC K-VACANCY PRODUCTION WITH 3 GEV CAFBON IONS
}

\author{
R Anholl,' J kannou-Yamow, H Bowman, E Pauscher,
}

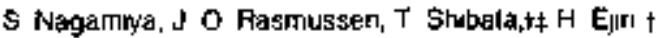

K-vacascy protuction by relativistic heavy charget particles is a campletely unexplored field. Measurements with relativistic protons, 2 indicated that an additional term mast be edded to the cross section given by the PLane Wave Born' (PWBA) and Binary Encounter 4 (BEA) Approximations. These theories only account for the interaction between the static coulorb fields of the projectile and electron. This contribution is called longitudinal excitation because the interaction exerts a force parallel to the momentum transfer. At relativistic energies, the curront-current interaction betaedn the tur particles is also important and gives rise to transverse exsitation. 5 For projectile energies greater than approxinately 2 GeV/amu ( $v_{1}$ constant कo c), the FWBA and BEA cross sections no longer change with projectile energy. The transverse contribution, however, causes the total cross
section to rise as the ln $\gamma^{2}+C$ where $y-\left[1-\beta^{2}\right]^{-3}$ $B=\forall_{1} / c$, and $C$ is a constant.

The experimental procedure was identical to the procedure described in Ref. 2 .

The measured cross sections are compared with various theories in Fíg. 1. The BEA and PWBA cToss sextions wero obtained from universol curves given by Garcia et al. 3 and Bastas et al.6 The reduced Ion velocity was taken as $6 \mathrm{C} / \mathrm{v}_{\mathrm{K}}$ where $\mathrm{v}_{\mathrm{K}}=\left(2 \mathrm{f}_{\mathrm{K}} / \mathrm{sl}\right)$ and $U_{K}$ is the $K$ binding energy.

Two relativistic modifications to these theories should be made. For large $v_{1} / v_{\mathbf{K}}$, the PWRA and BEA cross sections approach (but never squal) the integrated Rutherford cross section ( $\sigma_{R}$ ) for scattering an initially stationary electrop with an energy transfor $<* \mathrm{U}_{\mathrm{K}}$. For relativistic heavy ions $\sigma_{K}$ should epproach the integrated kKtnleyFeshbach cross section ( $0_{\mathrm{KF}}$ ) for relativistic electrons on nuclei.

The second relativistic correction factor comes from the inclusion of the transverse terso. ${ }^{2}$

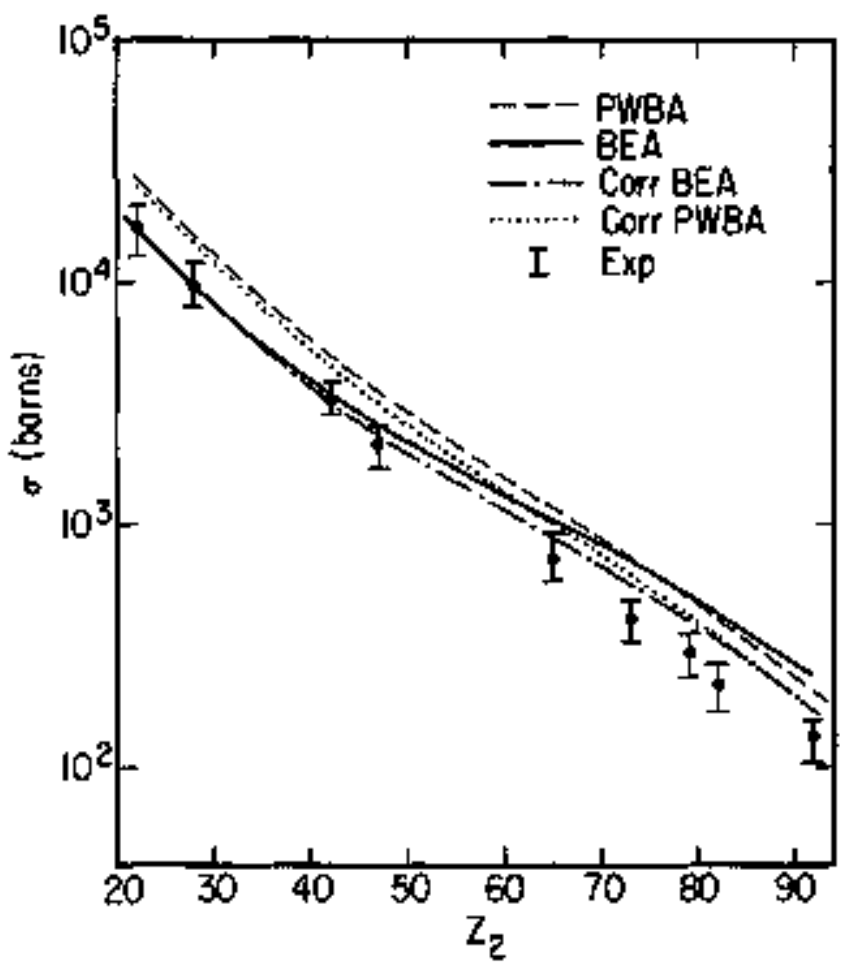

Fig. 1. K-vacancy production cross sections versus tarnet atomic nuwer. Comparison is made with the PWBA and BEA theories and relativistically corrected PWRA and BEA theories.

(XHL 761-449)

We have evaluated this contribution using the PWBA dipole approximation which is only valid when $2 v_{1} / v_{K} \gg 2$. In these cases $2 v_{1} / v_{K}$ lies between $1.6^{1}$ and 10 .

The measured cross sections generally lie lower than the predictions of the PWBA, BEA, and 
relativistically correted theories. For lomer atomaic numbers, the BEk theory works best; for higher atomic numer, all theories fail. The first relativistic correction becomes important at higher atconic nubers, lowering the cross sections, bringing then closer to experiment. The additive transverse contribution is not very important at these veloctties.

We have considered whether the polarization, 7 binding, ${ }^{6}$ and charge-exchange effects might account for the discrepancy between experibent and theory. For $2 v_{1} / v_{\mathrm{K}}>1$, the polarization effect is expected to be more important thon the binding correction and should give a positive contribution to the Kvacancy cross section. We evaluated the polarization contribution and found it adds only 2,4 , and 129 , respectively, for the $\mathrm{Ni}, \mathrm{Ag}$, and $\mathrm{Pb}$ targets. There are alsp negative contributions from the binding effect, so the net polarization plus binding correction is liable to be smaller than those factors, Oharge-exchange contributions are expected to give a positive contribution to $\sigma_{K}$ also.

Thus, while the measured cross sections clearty lie lower than the predictions of the first order PNBA and BEA theories, the polarization and charge exchange contributions to these cross sections appear to bo regligible ark, in any case, in the wrong direction to explain the discreparky.

\section{Footnotes and References}

*Present address: Department of Physics, Stanford University.

tLaboratory of Nuclear Studies, Osaka Hiversity, Toyonaka, osaka, Japan.

\#Supported by Mitsubtshi Foundation.

1. O. N. Jasvis, C. Thitehead and M. Shah, Phys. Rer. A 5, 1198 (1972).

2. $R_{+}$Anholt and the TOSABE Group, submitted to Phy's, Rev. A (1976).

3. J. D. Garcia, R. J. Fortuer and T. M. Kavanagh, Rev. Mad. Phys. 45, 111 (1973).

4. E. Merzbacher and H. Lewis, Hanbuch der Physik 34, 166 (Springer-Verlag, Berlin, I958),

5. U. Fano, Amur. Rev. Mucl. Sci. 13, 1 (1963).

6. G. Basbas, W. Brandt and R. Laubert, Phys. Rev. A $7,983(1973)$.

7. G, Easbas, ir, Brandt and R. Laubert, Phys. Lett. 34A, 277 (1971).

\section{K VACANCY PRODUCTION BY 4.88 GeV PROTONS"}

A Anhott, 5 Nagamya, H Bowman, J loannou-Yannou, $E$ Fauscher, and J $O$ Rasmussen

K. vacancy production cross sections were measured at the Berkeley Bevatron for $4.88 \mathrm{GeV}$ proton bombardments of $\mathrm{Ni}, \mathrm{Zr}, \mathrm{No}, \mathrm{Ag}, \mathrm{Tb}, \mathrm{Ta}, \mathrm{Pt}$, $\mathrm{Au}, \mathrm{Pb}$, and $\mathrm{U}_{+}$These cross sections were conpared with the Birary Encounterl (BEA) and PIarte Wave Born Aqproximations (PWBA) and were found to lie higher than theory by a Factor of 1.2 to 2.5 .

The BEs and FrEA calculations account only for the interaction between the static Coulomb fields of the projectile and target electron. At rolativistic energies, the interaction between the currents of the two particles is also important, since the projectile current has $B=v_{1} / c * 1$. The matrix element for this interaction is given by

$$
\begin{aligned}
& \frac{z_{1} e^{2}}{2 \pi^{2}} \int d \vec{k} \frac{\Sigma}{5} \\
& \frac{\left.\langle\vec{p} \cdot|\vec{a} \cdot \hat{A} \exp (i \vec{k} \cdot \vec{r})| p\rangle c_{n}\left|\sum_{j}^{*} \cdot \hat{A} \exp \left(i \vec{k} \cdot \vec{r}_{j}\right)\right| D\right\rangle}{k^{2}-\left(E_{n} / n c\right)^{2}}
\end{aligned}
$$

where $z_{1}$ is the projectile atomic mmber, $\vec{a}$ and $\vec{\alpha}_{j}$ are Dirac matrices operating on projectile wavefunctions $|\vec{p}\rangle$ and $\mid \vec{p}$ 's and electronic wavefunctions
Ins and I 0 > respectively, and $\mathrm{E}_{\mathrm{n}} \equiv \mathrm{E}_{\mathrm{n}}-\mathrm{E}_{0}+$. express | $\vec{p}>$ and $\mid \vec{p}$ 's as plane waves and followting Fano ${ }^{3}$, Eq. (1) is reduced and the cross section is obtained frojn

$$
d v_{n}=\frac{4 \pi z_{1}^{2} e^{4}}{v_{1}^{2}} \int_{q_{0}} \frac{\left|\vec{\beta}_{t} \cdot \vec{G}_{n}(q)\right|^{2} q d q}{\left(q^{2}-E_{n}^{2} / c^{2}\right)^{2}}
$$

where $\mathrm{q}_{0}=\mathrm{E}_{n} / v_{1}, \overrightarrow{\mathrm{B}}_{\mathrm{t}}=\overrightarrow{\mathrm{B}}-(\vec{\beta} \cdot \vec{q}) \hat{q}_{\text {, and }} \overrightarrow{\mathrm{b}}_{\mathrm{n}}[\mathrm{q}]=$ $\sum_{3 n}\left|\vec{a}_{j} \exp (i \vec{q} \cdot \overrightarrow{\mathbf{r}})\right| 0 \times$. No interference between the j longitudinal excitation anlitude (interaction between the static coularb fields) and the transverse amplitude (interaction between the currents) is present. It is seen that $G_{n}$ (q) is mothing mire than the fully retarded dipole velocity matrix element for photoelectric absorption. Following Merzbacher, ${ }^{2}$ we use nonrelativistic one electrom continum and 15 wavefunctions to caloulate $f_{n}$ (q). The cross section is integrated over continuma energies (excitation to bound states is neglected).

The cross section is unimportant at nonrelativistic projectile velocities. For relativistjs, projectiles, it rlses as $q_{0} \gamma^{2}$ ith $y=\left(1-\theta^{2}\right)-1 / 2$. 


\subsection{2

For redueed velocities $v / v_{K}=B C / v_{K} \gg 1$, it $1 s$ given by making the dipote approxinnation $(\exp (i \vec{q} \cdot \vec{r})=1): \sigma_{K}=1.00 \cdot 10^{4} Z_{1}^{2} / 2 z_{2}^{2}\left(\ln \left(\gamma^{2}\right)-\beta^{2}\right) / \beta^{2}$ bams.

Figure 1 compares theory with experiment. The transwerse term does not quite bring theory

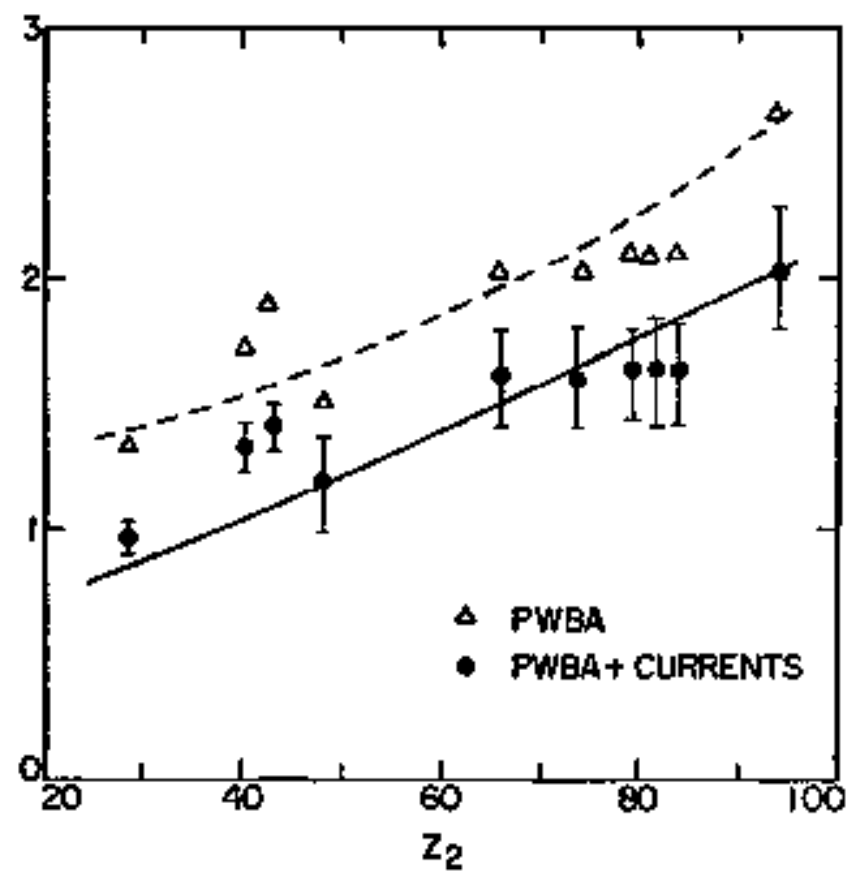

Fig. 1. Ratios of experimental to theoretically calcilated cross sections.

(XBL $766-8223$ )

\section{Footnotes and References}

"Abstract submitted to Fifth Int. Conference on Atomic Physics, Berkeley, Califomia, July 1976.

tPresent address: Department of Physics, Stanford University.

1. J. D. Garcia et al., Rev. Mod. Phys. 45, 111 (1973). into agreement with oxperiment, but clearly helps. We should mention that the Bethe approximationd agrees better with experiment: The Bethe approximation includes the transverse contribution, but is based on the dipole approxination whose validity is not whally justified for the case where $v_{1} / v_{K}$ is only slightly larger than unity.

Table 1.

K-vacancy cross sections frow $4.88 \mathrm{GeV}$ protons.

\begin{tabular}{cc}
\hline$z_{2}$ & $\begin{array}{c}\sigma \\
\text { (bains) }\end{array}$ \\
\hline Ni & $210 \pm 25$ \\
$z_{r}$ & $102 \pm 12$ \\
Ho & $94 \pm 12$ \\
Ag & $58 \pm 10$ \\
Tb & $31 \pm 7$ \\
Ta & $22 \pm 4$ \\
Pt & $18 \pm 4$ \\
Au & $17 \pm 3$ \\
Po & $15 \pm 3$ \\
U & $11 \pm 3$
\end{tabular}

2. E. Merzbacher and H. Lewis, "Encyclopedia of Physics," \$4, 166 (Springer-Verlag, Ber1in, 1958).

3. U. Fano, Annu, Rev. Nuxl. Sc1. 13, 1 (1963).

4. M. Inokati, Rev. Mod. Phy's. 43, 277 (1971); private communication.

\section{X-RAY CONTINUA OESERVED WITH 326-470 MEV Xe IONS*}

R Anhollt and W E Meyerhoit

The nonsharacteristic continum radiation observed between the $X$-ray transitions of the projectile and target atchls and the united atom is thought to come from transitions to the transiontly formed lso molecule orbital (ND) during the collision. In this paper we report the observation of continua in $\mathrm{X}_{e}+\mathrm{Sn}, \mathrm{Xe}_{\mathrm{e}}+\mathrm{Pr}$, and $\mathrm{X}_{\mathrm{f}}+\mathrm{Bi}$ borbardments with wited atom charges 104, 118 , and 137, respectively.
Figure 1 shows absolute thick target yields for these three systems. The experiments were done with intrinsic Ge $X$-ray and large volume intrinsic planar $(\mathrm{Xe}+\mathrm{Bi}$ only) detectors at the BerkeIoy SupertliLAC. For the case of $X_{e}+\mathrm{Sn}$, we show talculated yields due to Two Collision $\mathrm{MO} X$ rays ${ }^{1}$ (TCLO), nueleus-mucleus browstrabling (KNB), secondary electron bremsstrahlung ${ }^{2}$ (SEB), and" prinary bremsstrahlung or radiative ionization ${ }^{3}$ 


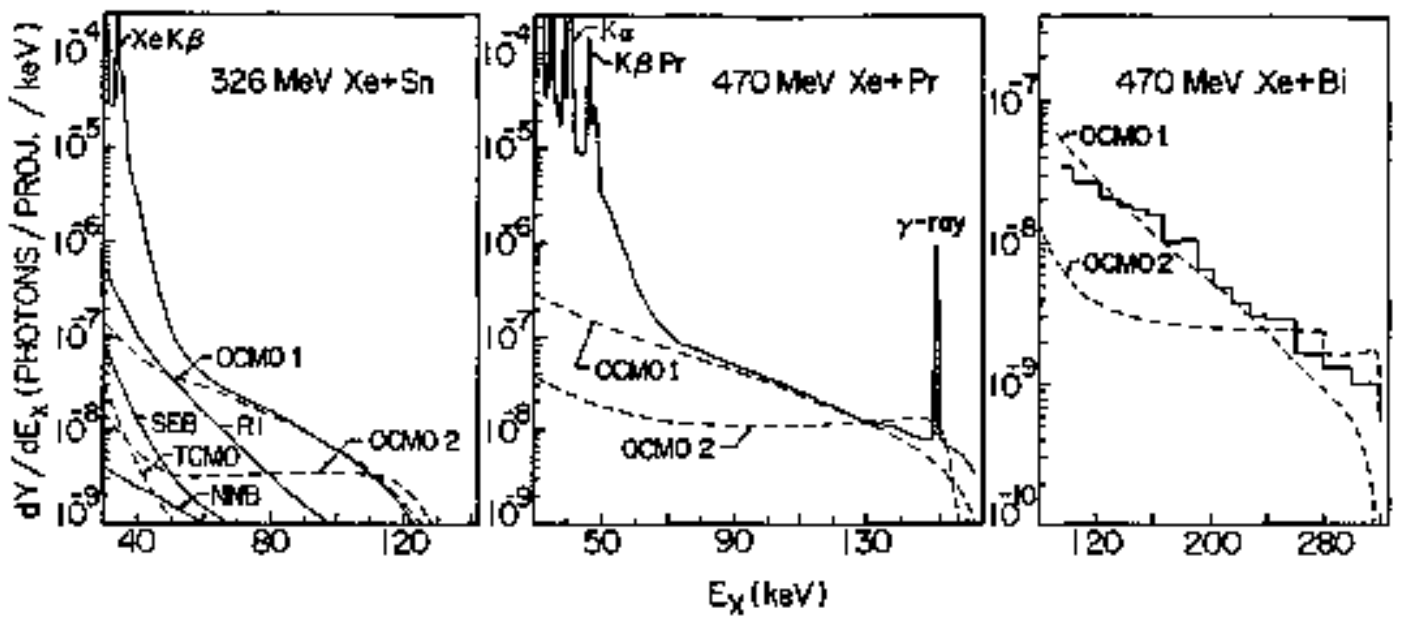

Fig, 1. Absolute thick terget yields. Various calculated intensities are described in text.

(XBL 766-8220)

(RI), The surn of all of these contributions does not account for the experifental continun yield, hence one has to invoke another process.

$\mathrm{MO} X$ rays may also be enitted in a one-colliston process in which a lso vacency made early in a collision is radiatively filled later in the same collision. In Ref. 1 the probability for this process is written as the product of the probability for making a lso vacancy $P(b) t$ tines the probability for $\mathrm{M}$, $X$-ray enission. Since $P(b)$ is not we11 know it has been paranetrized by the expresșion $\mathrm{P}(\mathrm{b})$ to $L\left[1+e^{b / a}\right]^{-1}$, With this parametrization the ls vacancy production cross section is given by $\left(\pi^{3} / 6\right) \mathrm{La}^{2}$. In Ref. 1 a was found to be approximately equal to the $K$ radius ak of the higher $z$ partner, whereas according to Ref. 4, it should be of the order of ${ }^{2 \theta} \mathrm{K}_{\mathrm{K}}\left(v_{1} / v_{\mathrm{K}}\right)\left(\mathrm{U}_{\mathrm{K}} / \mathrm{L}_{\mathrm{M}}\right)$ where $v_{1}$ and $\mathrm{v}_{\mathrm{K}}$ are the projectile and $\mathrm{X}$ electron velocities and $\bigcup_{K}$ and $V_{\text {lag }}$ are the $K$-shell binding energies of the heavier collision partner and of the united atom, respectively.

In the first calculation, we adjusted $L$ and a to give the best fit to experisent (OCMO1) and again found a $a_{K}$, but the computed value of $\left(\pi^{3 / 6}\right) \mathrm{La}^{2}$ exceeded the experirental 1so cross section by factors of 5 to 20 . In the second, we used a as suggested by $\operatorname{Ref}, 4$ and the experimental 1so cross sections (OCMOZ). While the shape is well represented by OCNO1, neither the shape nor intensity is we11 represented by $0 \mathrm{CMO2}$. Hence, the presentiy available prescription for the OCMO spectrun is not artequate to explain the observed continua.

\section{Footnotes and Refergnces}

*Abstract submitted to Fifth Int. Conference on Atcrinic Physics, Berkeley, Califormita, July 1976.

$\dagger^{\dagger}$ Present address: Department of Physics, Stanford University.

${ }_{\text {Department of Physics, Stanford Liniversity. }}$

1. W. E. Meyerhof ot al., Phys. Rev. Lett. 32, I279 (1974),

2. F. Folkmann et al., Nuxl. Instrum. Meth. 116, 487 (1974).

3. R. Nuholt and $T$. K. Saylor, to be published in Physics Letters A.

4. W. Brandt, K. W. Johes and H. W. Kramer, Phys. Rev. Lett. 30, $351(1973)$.

\section{A NEW WAY OF PREDICTING 1s VACANCY PRODUCTION BY HEAVY HONS"}

R Anthollt

Basbas ft al. 1 have previously shown that the cross section for the production by heary ions of $K$ vacencies in the is molecular orbital (MO) (or in the heavier collision partner) can be written as $\sigma_{\mathbf{K}}=z_{1} \sigma_{0} / \delta^{9}$. Here $z_{1}$ and $z_{2}$ are the projectile and tarpet atcmic charges and $\sigma_{0}$ can be either the theoretical or experimental $K$-vacancy production cross section by protans (or zero charged particles). We show that $\delta$ can be written as $1+\left(U_{U A} / U_{K}-I\right) g x$ $\left(1.3 \mathrm{~F}_{\mathrm{K}}\right.$ ) where tu and $\mathrm{V}_{K}$ are experiaentat binding energies of the corbined $\left(z_{1}+z_{2}\right)$ atom and the heavier atom respoctively and $\mathrm{g}$ is a numerical fluction of the projectile velocity divided by the K electron velocity of the heavier partner, EK - 


\section{$00: 34503003$

$2 v_{1} / v_{K}$. In the spirit of the approkimations made in obtaining this result (see Eq, (A, 10) of Ref. 1), it is also possible to write $\sigma_{\mathrm{K}}=Z_{1}^{2} \sigma_{0} / \Delta^{9} 8$ where $t_{0}=\mathrm{U}_{\mathrm{Ld}} / \mathrm{U}_{\mathrm{K}}$. A plot of the log $\sigma / z_{1}^{2}$ versus the log $U_{\text {ti }}$ should give a straight line whose slope depends on fust the reduce velocity $v_{1} / v_{K}$.

In Fig. 1 we show $\log -\log$ plots of assorted data for 0.5 to $3 \mathrm{keV} / \mathrm{m}_{\mathrm{j}} \mathrm{u}$. p, $\alpha, \mathrm{L}, \mathrm{C}, \mathrm{N}$, and 0 ions on $\mathrm{Ni}, \mathrm{Sb}$, and $\mathrm{Rb}, 2$ In Fig. 2 we show values of $\mathrm{n} \equiv 9 \mathrm{~g}$ obtained from this data and other data. While the clustering of $n$ values shown in Fig. 2 is not tight, $n$ values can be taken from this figure and applied to many combinations of projectiles, targets and projectille energies to obtain $\square_{K}$. CnIy the proton eross section is required, which can be theoretically predicted or obtained from experiment. Generally predictions using this method are better than those obtainod using "united ator"' scaling laws ${ }^{3}$ and better than the original binding correction rethod of Ref. 1. It is restricted to atoms with $20 \leq z \leq 50$, however, where screening and

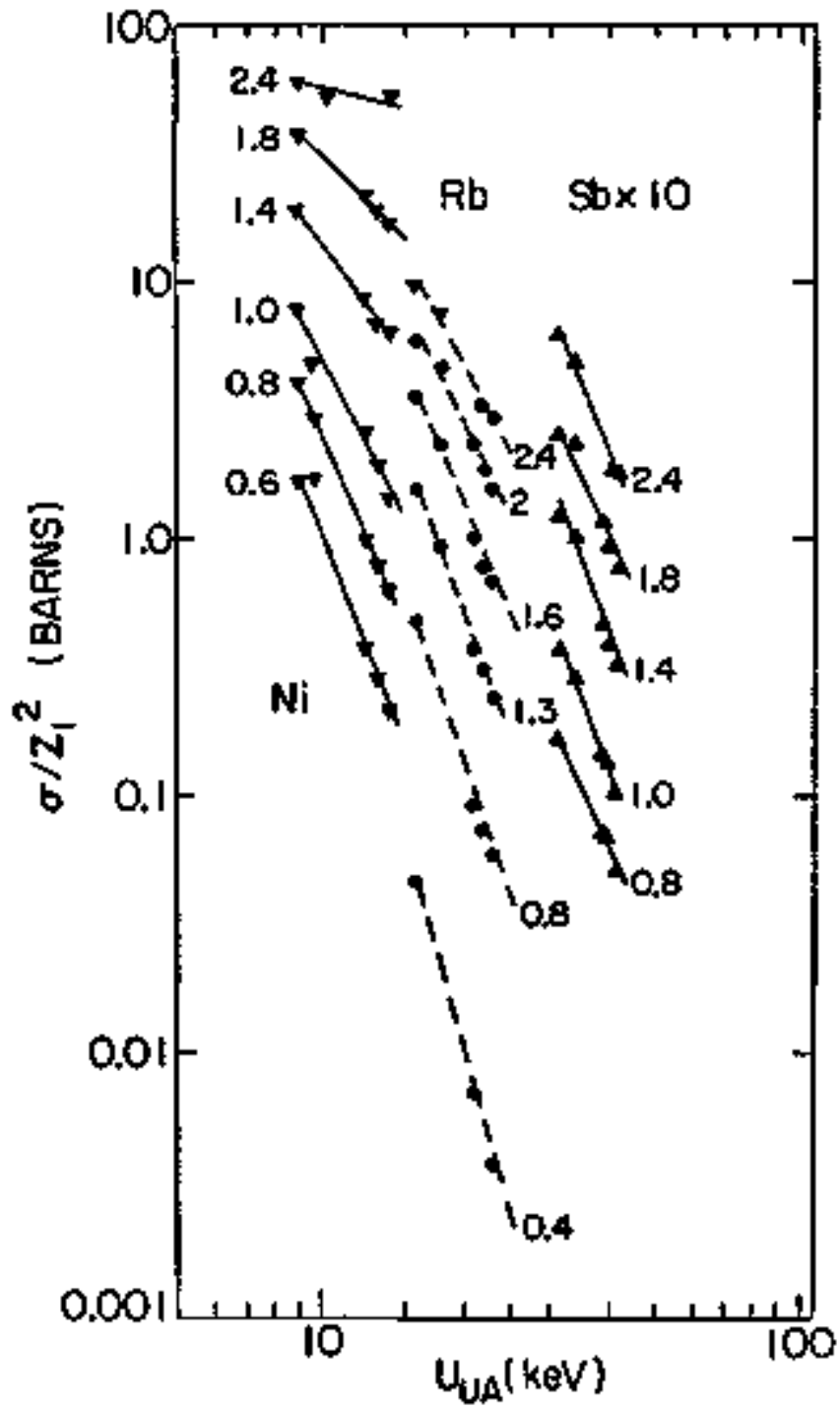

Fig. 1. $G / 2{ }_{1}^{2}$ vs UtA for $\mathrm{H} 1$, kb and Sb K-vacancy production by $p, l i, a, C, N$, and 0 heavy ions. khmbers indicate projectile energy per nucleon.

(XOBL $766-8221$ ) relativistic effects are unimportant. The relation also works no matter if the projectile is the heavier partner or the target is. one simply interchanges the definition of $z_{1}$ and $z_{2}$ such that $z_{1}$ is always the fower $z$.

A fundamental interest in the $n$ plot is that the binding-Correction prediction of Basbas et al. 1 (shown by the dashed tine in Fig. 2) does not

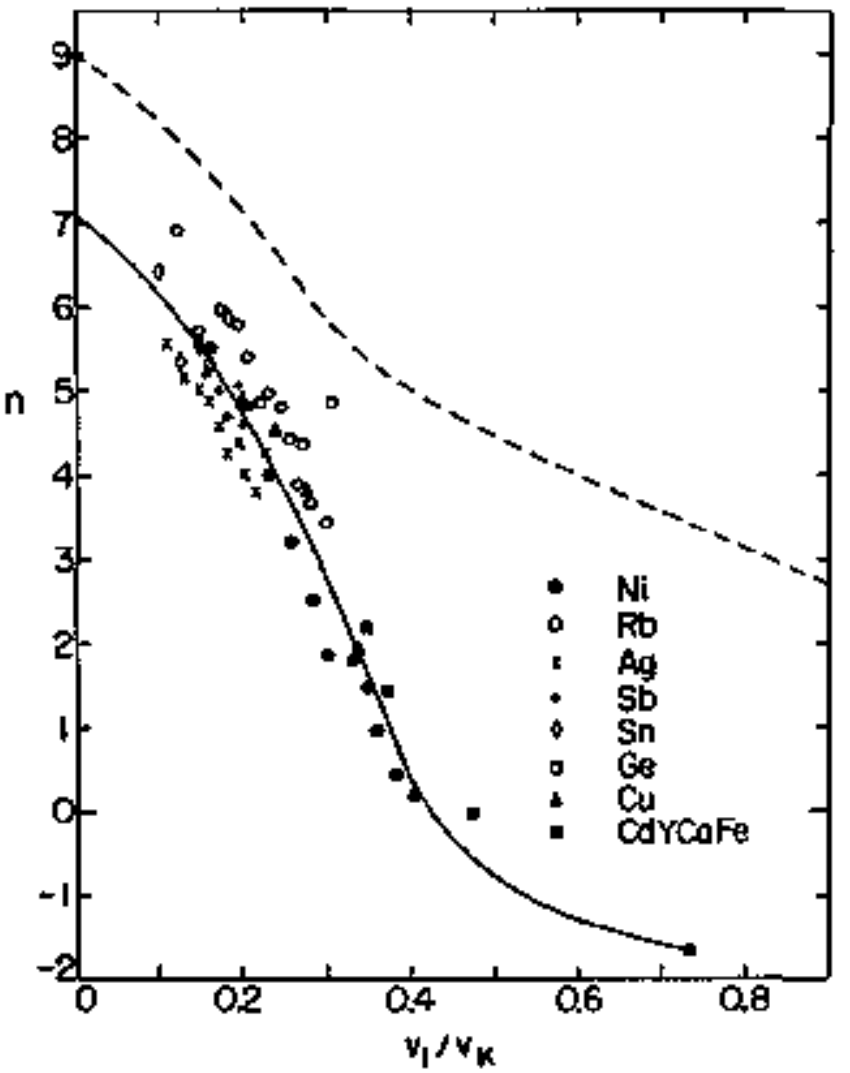

F1g. 2, $\pi$ vs $v_{1} / v_{K}$ for various targets. Solid line is an approximate vest fit. Dashed line is the prediction of fasbas et al. I

(XBL 766-8222)

accurately predict the $n$ values. This has been noted in the literature. Usually other corrections have been applied to specific pieces of heavy im data. Wo ab intitio theory of 1so vacancy production is currentiy available, however. Should such a theory becone available, the $\mathrm{n}$-plot will provide an excellent test of theory.

\section{Footnotes and References}

*Abstrart submitted to Fifth Int. Confexence on Atomic Ptysics, Berkeley, Califomia, July 1976.

Present address: Department of Ptysics, Stanford University.

1. B. Basbas, W. Brandt and R. Laubert, Mhys. Rev. A $7,983(1973)$.

2. Data taken from many sounces, principally

F. D. Mthoniel, T. Gray and co-workers.

3. C. Foster et al., J. Phys. B 7, 2563 (1975). 

$00.0450 \div 04$

II.

\section{THEORY}





\section{$00 \div 3450+0 \frac{15}{253}$}

\section{A. NUCLEAR REACTIONS}

\section{Microscopic}

\section{ENHANCEMENT OF FORWARD-ANGLE CROSS SECTIONS IN HEAVY-ION REACTIONS BECAUSE OF PROJECTILE EXCITATION*}

Norman K Gbendennung and Georg Wolschint

A remarkable feature in the spectnm of outpxygen nuclei from the reaction

$$
{ }^{122} \mathrm{~S}\left(16_{0}, 1{ }^{18}\right)^{120} \mathrm{Sn}_{\mathrm{S}} \quad \mathrm{E}=104 \mathrm{MeV}
$$

is that they are produced in the excited $2^{+}$state with a cross section which is about five tines larger than the ground state cross section. 1 A typical spectrum taken at the Berkeley 88-inch cyclotron is show in Fig. 1. Such a preference for excited states of the light outgoing nuxcleus has bejn observed for stoe other targets as well.

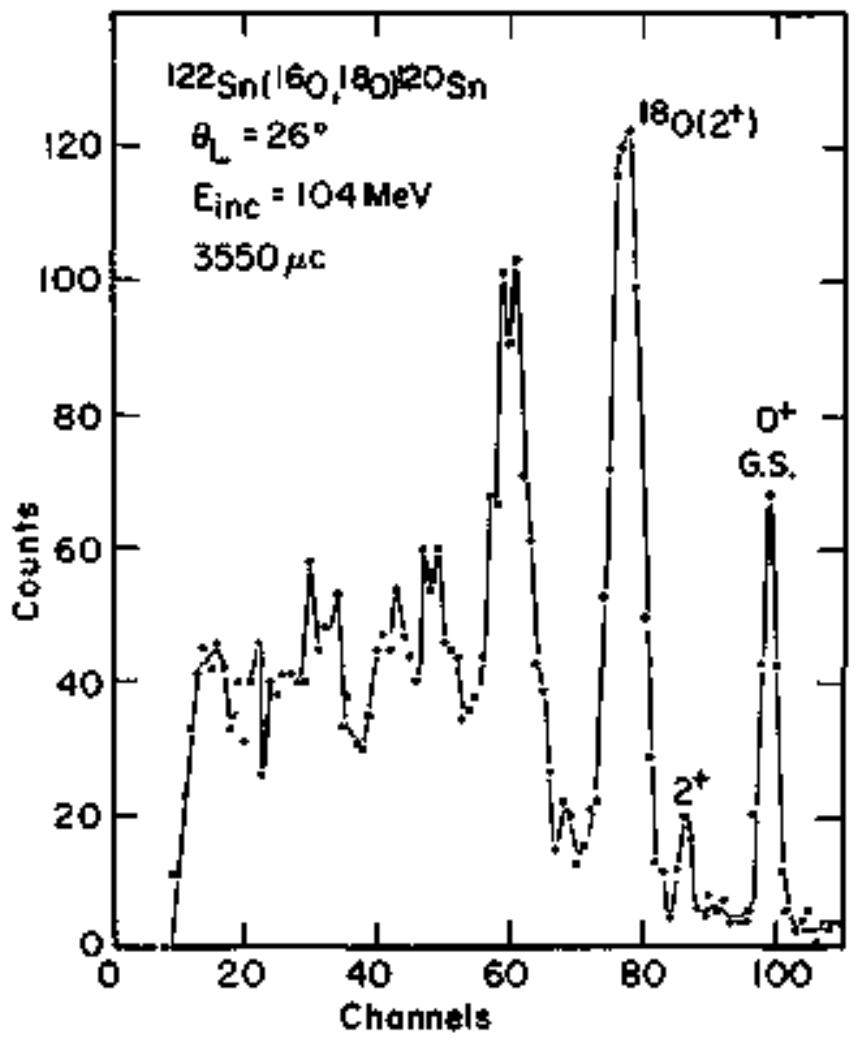

Fir. 1. Enorgy spectrom for the reaction indicated. Note large cross section for proxhicing 180 in its $2^{*}$ state (with $120 \mathrm{Sn}$ in its ground state.

(XKL 741-2191)

It is clear therefore that an accurate description of the reaction must include the coupling of the ground and excited states in oxygen, ${ }^{2}$ The results of such a coupling can be seen in Fig. 2 . Here we see a dramatic increase, by a factor of 10 , in the ratio of forward to grazing-angle cross section for the ground state, when the effect of excitation of 180 to its $2^{+}$state is included. Since the cross section to the $1 \mathrm{O}_{\mathrm{O}}\left(2^{+}\right)$state is adjusted to agroe with experident, and the stremgth of the inelastic transition in the $18_{0}$ rucleus is prescribed by the measured $e_{2}$ of this nucleus, this result is quite firm. We note that the sign of the interference changes between grazing angle and forward angles.

The significance of this result must be viewed in cormection with the interpretations of large for-

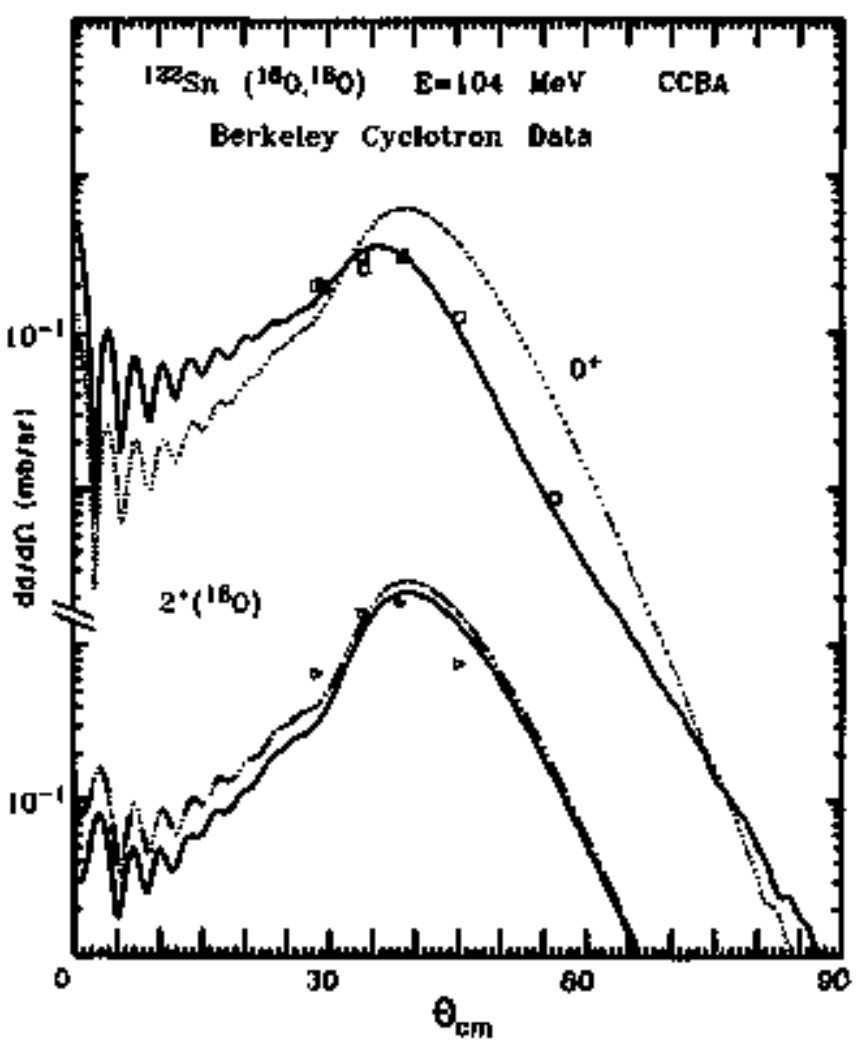

Fig. 2, Broken lines show direct cross sections for prodacing the ground state of 120 n with outgoing nucleus $18_{0}$ in its groud and excited state. Solid lines show effect of coupling these two levels. A combon nortualizing factor 2.7 is used for all ourwes. 
ward-angle cross sections by the Brockhaven group. he recall that in the $\mathrm{yfi}_{\mathrm{i}}\left(1 \mathrm{~B}_{0}, 160\right)$ reactions on a series of isotopes, the cross sections have normal grazing peaks for the heavier isotopes, while at the light end they have ancitalously large forward cross sections. 3 In an early work they varied the paraneters of a weakly absorbing potential to reproduce this trend.3 Recently, they have treated anly one of these cases using a surface transparent potential to obtain a large forward cross section. It appears, however, that these potentials nuy actualiy be simulating the physical process of indirect feeding of the ground state through excited states of the light mileus.

The indirect transitions have larger forward distributions compared to the direct. This can be understood as characteristic at onergies for which the classical deflection function has a rainbow angle, First we recall a general principle. \$ The angular monentude region that dominates a given process is deteruined by the competition of several factors. Two conpeting factors are the absorption, whtch reduces the cross section for small angular mamenta, and the preference for close colfisions when particles are transferred. Naturally a twostep process requites a closer collision than a direct one. The balance of these two factors leads to a preference of two-step processes for orbits with angular monenta somewhat smaller than the rair bow or grazing angular moneenta associated with the peak in the cross section of the direct process. Therefore, forward-angle scattering is onhanced for two-step processes conpared to direct ones when the energy is such that the deflection function possesses the rainbow or grazing angle.

A different behavior is observed hhen the energy is lower and closer to the Corlomb bartier. In this case there is no rainbow angle, and the closer orbits preferred by the two-step process scatter to larger angles then the slightly more distant orbits preferred by the direct process. This is the case for the sade reaction of Fig. 2 when the energy is just above the coulomb harriter, as in Fig. 3. The forward-angle cross section at this mergy is umaffected by the excitation of $180\left(2^{+}\right)$but the backangle cross section is rehoced by the destructive interference between direct and indirect transitions. The improvealent in the agreement is marked. The data are for the radetion inverse to the one for whith we did the computation. They are equal by time reversal invariance. We only chose to do the calculation for the plclam reaction because this is the one for uhich the important intermediate state, ${ }^{18} 0\left(2^{+}\right)$, is a direct observable.

To sumbarize our results, we have found that transitions through excited states in the projectile imolved in a transfer reaction can produce a large enhancement at forward angles in the ground state cross section, whem the energy is sufficiently high above the berrier so that the grazing angle is truly a rainbow effect. Such an enhancenent may occur when an excited state of one of the light muclei is produced in the transfer reaction with larger cross section than its ground state, and the two are strongly coupled.

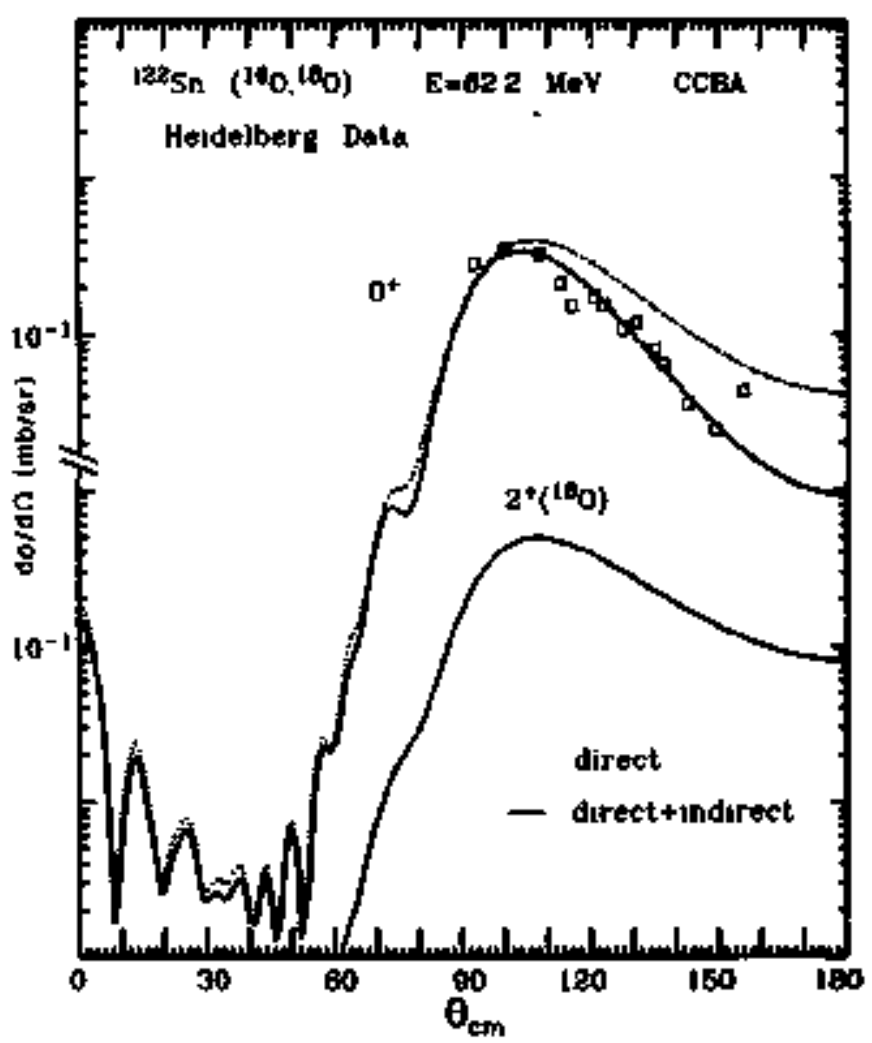

Fig. 3. Computed ground state cross section for pickup reaction is compered with data on the time reversed reaction (Ref, 6). The effect of indirect feeding of the ground state through the intermediate $2^{+}$state in $10_{0}$ is seen by carparing the dotted and solid gurve. Cross section conluted for the $2^{+}$ state in $10^{\circ}$ is also shom, but no data are avail. able. A comion jormalization factor 6.2 is used for all aurves, The calalation is as described for Fig. 2 optical parameters were derived from the $180+120 \mathrm{si}$ elastic scattering data at $60 \mathrm{MeW}$.

(XBL 753-757)

Clearly it is of interest to perform the (160, 180) reactions on the nickely isotoper so as to neasure the relative cross sections of $160\left(2^{*}\right)$ and $1 \mathrm{P}_{\mathrm{O}} \mathrm{gs}$ ). If the ratio is large, them it is very likely that a stubstantial part of the forward-peaked cross sections in the $16_{0}, 160$ ) reactions massured by the Broolhaven group is due to the second order effect of the 1ight-nuclets excitation.

\section{Footnotes and References}

Condensed fran 1EL-3472, Prys. Rev. Lett. 34,1642 (1976).

'Supported in part by the German National Fellowship Foundation.

1. J. K. Scott, private comtonication.

2. N. K. Glendenting and R. J. Ascuitto, Symposium on Heary-Ion Transfer Reactions, Argonne, I11. (1973) p. 513; Phys. Lett. 471, 332 (1973). 


\section{$00: 2 \leqslant 50+0_{227}^{5} 6$}

3. E. H. Auerbach, A+ J, BaItz, F, D. Bond, C. Chașıan, J. D. Garrett, K, W. Jones, S. Kahana, M. J. Levine, M. Schneider, A. Z, SchworzschiId, and C. E. Thom, Phys. Rev. Lett. 30, 1078 (1973).

4. M. J. LeVine, A. J. Baltz, P. D. Bond, J. B. Garrett, \$. Kahana, and C. E. Thom, Phys. Rev. C $10,1602(1974)$.
5. N. K. Glendeming in Intemational Conference on Reactions Between Complex Nuclei Nashville, Temmessee) eds. R. L. Robinson et al. (NorthHolland, 1974) Vol. II, p. 137.

6. H. G. Bohlen, A. Gobbi, K. D. Hildenbrand, and X. I, Kubo, Jahresbericht Mex Plarck Institut Heidelberg, 24 (1973),

\section{FHYSICAL BASIS FOR ENHANCED FORWARD CROSS SECTIONS IN $\mathrm{Ni}\left({ }^{18} \mathrm{O},{ }^{16} \mathrm{O}\right)$ REACTIONS}

Norman K Grendenning and Georg Wolschin †

At moderate energies above the Coltomb barrier, quasi-elastic heavy ion reactions are expected to exhibit a "grazing" peak in the differential cross section. 1 Brookhaven experinents ${ }^{2}$ produced the surpising result that several reactions of the type

$$
{ }^{60} \mathrm{Ni}\left({ }^{18} \mathrm{O},{ }^{16} \mathrm{O}\right){ }^{62} \mathrm{Nl}, \mathrm{P}=65 \mathrm{MeV}
$$

had a large cross section forward of the grazing engle. sl though findirect transfer can produce such offects, ${ }^{3}$ this was not at first suspected to be the explanation because the experiment did not reveal any likely çmdidates as intermeriate states.

Recently the Brookhaven group proposed a surface transparent optical potential with the property that the edge of the absorptive part is very sharp and 1 ies jnside the real part. 4

We now turn to what we consider to be the jingsical basis for the large forward cross section seen in the experiment. The clue was provided in a previous paper which took into account the fact that for the $\$ 1\left(160,10_{0}\right)$ reactions, the ejectile, 180 is produced in its excited $2^{+}$state more fre: quently than its ground state. 5 its subsequent deexitation through an inelastic interaction with the residual nucleus has the effect on the ground state cross section of: 1) shifting the grazing peak forward by a few degrees and 2) producing a forward angle yield that is ten times larger than calctilated from the direct process alone. The analogous process in the entrance chamel of the reac$t$ ion (1) above can explain the obsexved forward distribution when aIl relevant cross sections are determined to the extent possible by the avaitable experiments.

In Fig. 1 our complete calculation is shown for the inverse reaction to (1) which includes the direct transition to the ground state of both final maclei and the coherent indirect transition corresponding to particle transfer to the $2^{+}$state of 30 followed by inelastic deexcitation to the ground state. ne neglect tho explicit calculation of recoil effects but enlyloy the scaling factor $\alpha=1.02$ which as far as the direct transition is concemed reproduce the anisular distribution of the full recofl calculation. This point is not crucial, however, since with "normal" optical potentials the

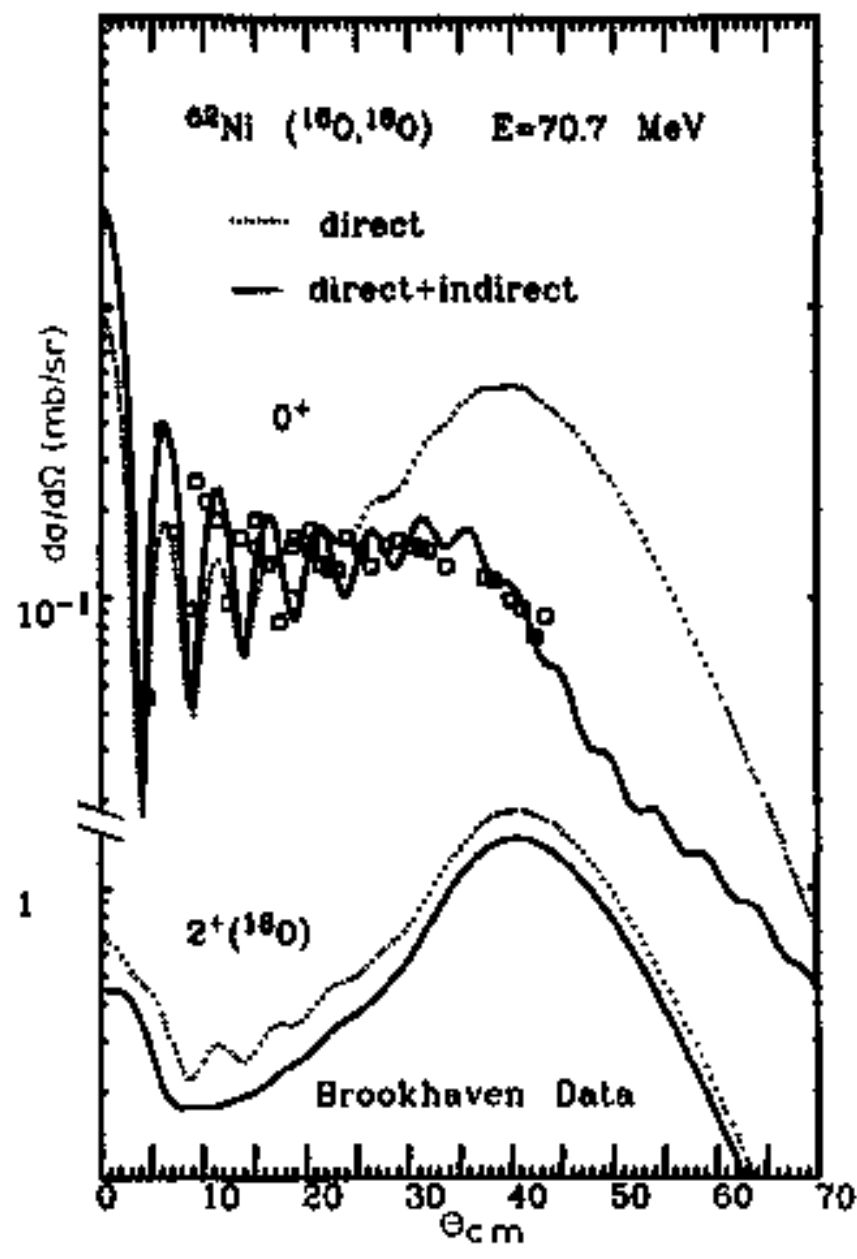

Fig. 1. The solid lines are cross sections based on a coupled calculation in which both states of oxygen, the $0^{*}$ and $2^{+}$are fed both by direct trassfer, and by indirect transfer through the other state. Dashed lines shww cross sections computed for each state when only the direct transfer contribute but the inelastic coupling is still included). Al1 cross sections are nonmalized by the same factor. The eround state data ${ }^{2}$ are for the time reversed reaction at the corresponding energy of $E=65$ Mfor (in the lab).

(XBL $7510-8436$ 
sensitivity to $a$ is quite sma11. A1so shown in Fig. 1 is the cross section for direct transfer alone, which by comparison allows us to see the large effect of the indirect transition on the ground state cross section. In an earlier publication we gave a simple classical explanation of why the two-step process is forward biased compared to a stngle-step process. In addition, it is broader becanse each scattering process introduces its ow dispersion on the previous one. (Thus, a delta funct Ion angular distribution centered at $\theta_{0}$ scatters to $\theta_{0}$ for a single scattering, and all angles betweepn $\theta$ and $2 \theta_{0}$ for a double scattering. Both effects are apparent in the figure. The ratio at the nuclear aurface of the two transfer ford factors in volying respectively the ground and excited state of 180 is consistent with the data of the neighboring reaction as concerts the relative cross sections, although the forward peaking of the $2^{*}$ cross section is not reproduced. It is quite possible that its angular distrlbution wold be modified by higher states just as trousitions through it modify the ground state cross section. Since however, neither the experimental data for the $2^{+}$nor any higher state is available for the reaction inverse to (1), we leave this matter as it stands.

In conclusion, we have exhibited a physica1 process that is capable of accounting for the large Gorward cross section in the Brookhaven exper iment $6 \mathrm{Nli}\left(1 \mathrm{E}_{0}, 1 \mathrm{60}^{6}\right)^{62 \mathrm{Ni}}$ at an energy where one would normally expect a grazing peak with sharply falling cross section on either side. This process consists of the inelastic excitation of the projectile 130 followed by the transfer of twa nucleons to form the ground states of 100 and ${ }^{62} \mathrm{Ni}$. The destructive interference of this process with the direct transfer causes a decrease of cross section in the region of the grazing angle where the two have comparable amplitudes. At angles forward of the grazing, the cross section is dominated by the second order process, since, es discussed above its distribution is ore forward blased and braader.

We note here tho additional features that can be introduced by indirect transitions which may be observable in experiment. i) The destruct ive interference between the peaked direct anplitude and the broad indirect one can lead to a minimum sodnewhere in the grazing region or beyond followed by a sec- ond peak. Sore indjcation of stach a phenonemon exists in the $62_{\mathrm{Ni}}\left(10_{0}, 100\right) 04 \mathrm{Ni}$ data $\mathrm{i}$ i) In a serjes of isotopes the relative contribution of direct and indirect processes can chango both because the structure of the nuclear wave fumctions change and because the $Q$ of the reactions change. It is worth noting that the reaction wher investigation is a badly Q-matched ore. Based on a semiclassical argument 3 one can understand that indirect processes are not attentated as strongly by Q-rismatch as direct ones. Thus the secund order processes may bo less important relative to the direct in other niclel isotropes where the Q-matching is better, unless the nuclear structure strongly favors the indirect. Asstrining that it does not, then there ought to be an evolution in cross sections from one end to the other of the nickel isotopes from forward distributions at the large-0 end to more grazing peaked at the shal1-Q end. The data suggest this trend,

\section{Footnotes and References}

\section{*Condensed from LEL-4334, published in Phys.}

Rev. Lett, 36, $1532(1976)$.

tSrpported in part by the Cennen National Fellowship Foundation.

1. N. K, Glendenning in Proc, of Int, Conf, on Reactions between Conplex Nuclef, R. L. Ronbinson et a1., eds. (North-Holland, Ansterdan 1974) vol. 2, p. 137 and Ref. Mod. Phys. 47, 659 (1975).

2. E, H. Auerbach et al, Phys, Rer, Lett, 30, $1078(1973)$.

3. R, J. Acuitto and $N, K$, Glenderning, Phys, Lett. $458,85(1973)$.

4. H. J. Levine, A. J. Baltz, R. D. Bond, J. D. Garrett, 5. Kahana, and C. E. Thoor, Phys. Rer. C 10,1602 (1974; A. J. Baltz, P. D. Bond, J. D. Garrett, and S. Kahand, Phys. Rer. C 12, 136 (1975).

5. N. K. Glendemning and G. Holschin, Phys. Rev. Lett. 34,1642 (1975).

6. D. L. Hedrdrie, I. Jabrke, C. F. Maguire,

J. Mahoney and D. K. Scott, private compunication.

\section{TWO-PARTICLE TRANSFER CONTRIBUTIONS TO ELASTIC AND INELASTIC HEAVY ION SCATTERING*}

Norman K. Glendenning and Georg Wolschin†

The coherent appearance of scattering and particle transfer can occur in heavy ion reactions when the two colliding muclei differ by only one or a few mucleons, leading to strong interferential oscillations in the angular distributions.

One aim of our andiysis is to show that by jncluding the indirect transfer processes in the $160+180$ reaction at energies near the barrier, the slight discrepancy between elast1c data and calculations 1,2 can be removed. Our second objective is to obtain a simultaneous fit of both elastic and inelastic data.

We consider the elastic scattering of projectìe A from target nucleus $\mathbf{B}$.

$$
\mathrm{A}+\mathrm{B} \rightarrow \mathrm{A}+\mathrm{B}
$$

and the inelastic scattering to excited states of $B$

$$
A+B+A+B \text {, }
$$




$$
00, \downarrow \text { 小 }
$$

where

$$
\mathbf{B}=\mathbf{A}+\mathrm{X} \text {, }
$$

so that the core of $B$ is identical to $A$, and the mass of $\mathrm{X}$ snall compared to the mass of $\mathrm{A}$, For simplicity, we assume A to have spin zero. Because of the identity of the cores, the transfer of particle(s) $X$ leads into the sane elastic and inelastic channels as the ordinary (direct) scattering, and therefore the two processes interfere.

The various processes that we include that lead to the elastic and inelestic channels are shown in Fig. 1. The method of calculation is the source term method. ${ }^{3}$

Couples channel caloulations for the ${ }^{16_{0}}{ }^{18_{0}}$ elastic and $180\left(2^{+}\right)$inolastic scattering without and with two-neutron transfer contributions are compared in Figs. 2-4 for the three energies $F_{L}\left(i_{0}\right)$

Fig- 1. Diagran representing the antilitudes considered in our calculatiof of elastic and inelastic $160+18_{0}$ scattering. Wavy lines indicate scattering interaction (treated to all orders], straight lines with arrows two-neutron transfer (first order treatnent). S)robols for the nuclear states are shown below the figure.

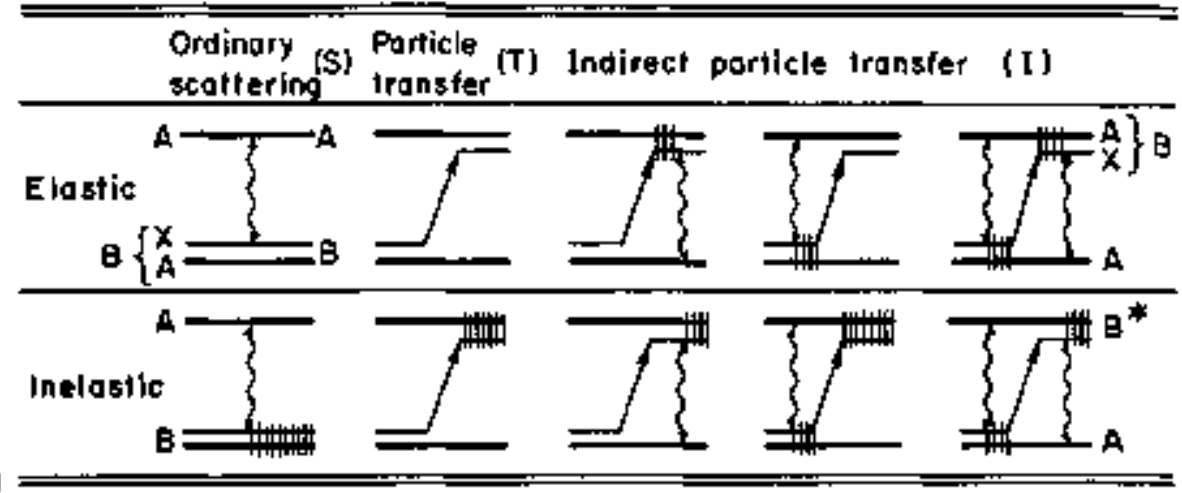

(XBL 7510-8562)

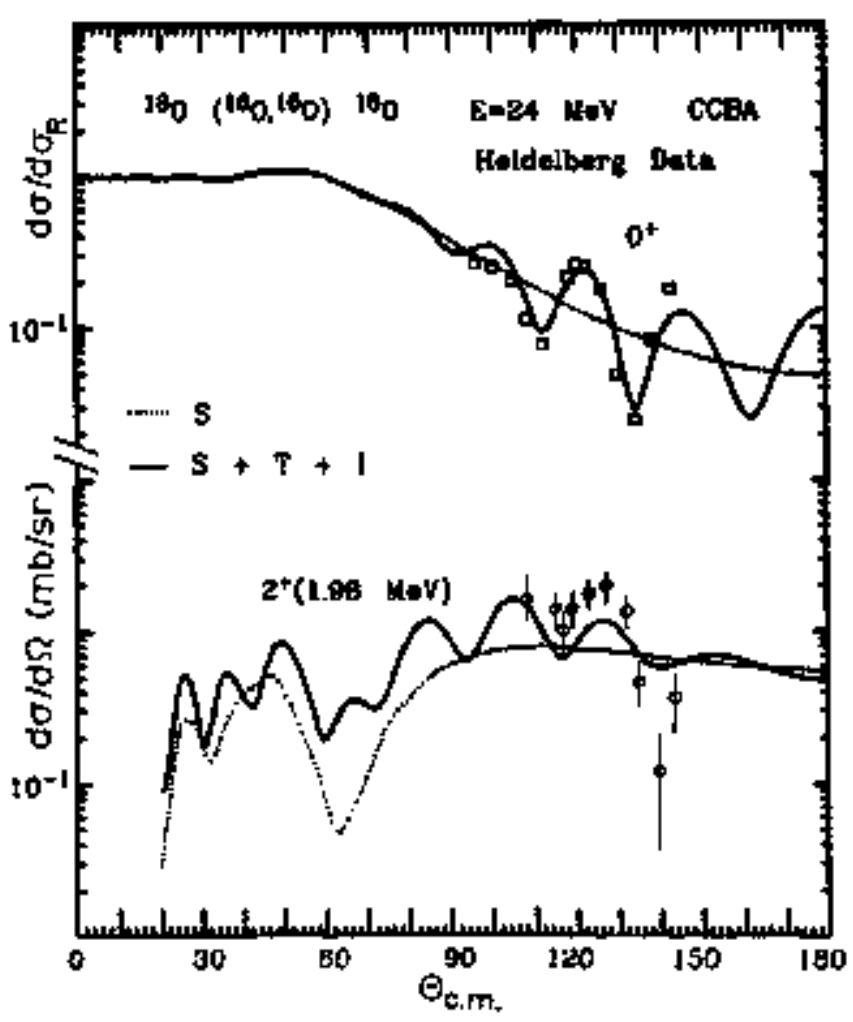

Fig. 2. Conputed elastic and inelastic $160+18_{0}$ cross sections for ordinary scattering (S) are comared with results where, in addition to the scittoring, particle transfer (T) plus indirect particle transfer (I) are included. The dats are from Refs. 7,8. Osciflations in both elastic and inelastic channel are identified as being die to the interference of scattering and transfer. Here and in all subsequent calculations, the transfer amplitudes are nortalized by a cormon factor of 6.3. The center-of-mass energy is $12.71 \mathrm{MeV}$. Exor bars on the inelastic data show statistical orrors only,

(XBL 7510-8440)

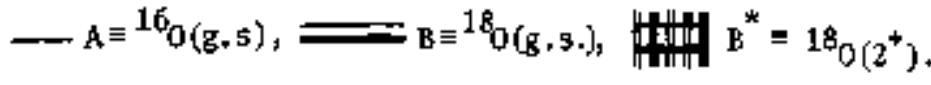

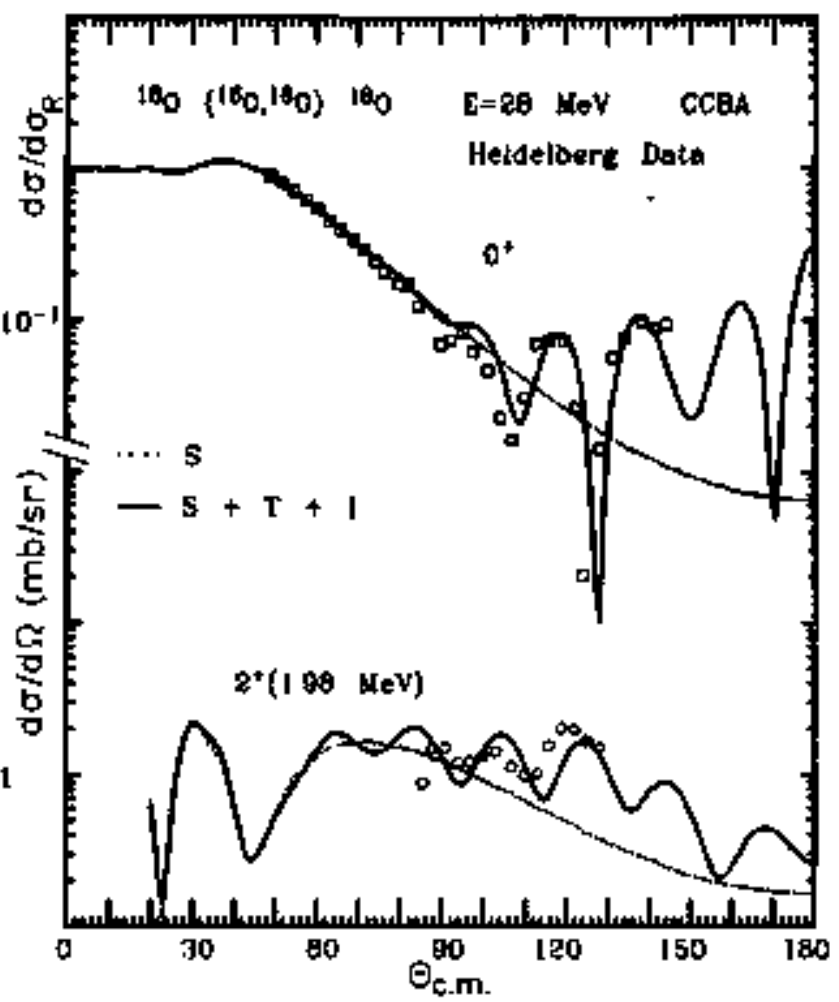

Fig. 3. As Fig. 2, but $E_{1}\left({ }^{16} 0\right)=28 \mathrm{keV}$, corresponding to $\mathbf{E}_{\mathrm{c} . \mathrm{m} .}=19.06 \mathrm{NeV}$. Error bars not shown.
(XRt $7510-8442$ ) 


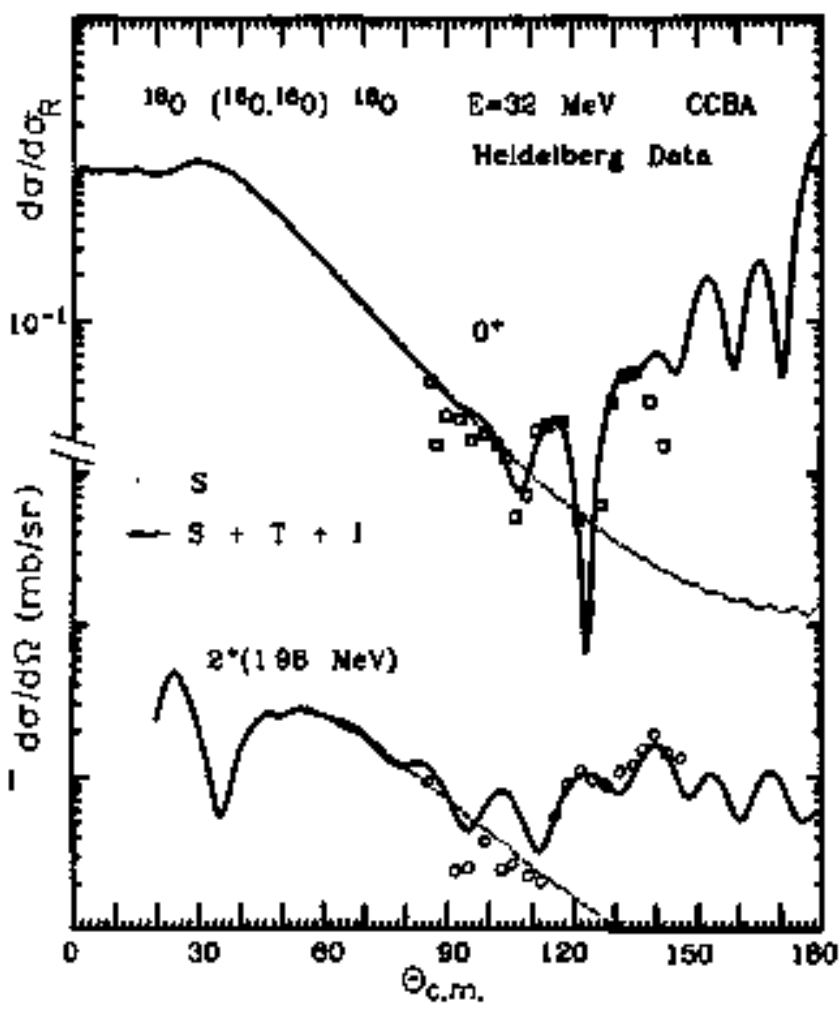

Fig. 4. As Fig. 2, but $\mathrm{E}_{\mathrm{L}}\left({ }^{16_{0}}\right)=32 \mathrm{MeV}$, carresponding to $\mathbf{E}_{\text {c.m. }}=19.06$ Nev. Error bars not shown.

(XBL 7510-8441)

$=24,26$, and $32 \mathrm{MeV}$ where data are available. Whereas, the ordinary elastic and fnelastic scatter- ing cross sections show no oscillations at backwand angles, the coherent addition of the transfer amblitudes reprotuces the oscillatory and backward-rising structure of the data, although the agreenent with the inelastic $24 \mathrm{kev}$ data is not good. Thus, the data for both elastic and inelast ic charnel find a consistent interpretation, if particle transfer is included.

In contrast, the INRA calculations for the elastic channel reported in Refs. I, 2 required different spectroscopic amplitudes for the transferred neutron cluster in order to account for the deglittude of the backward angle cross sections. The discrepancy is removed by means of the inclusion of indirect transfer mechanisas involving the $10_{0}\left(2^{+}\right)$ state.

\section{Footnotes and Referrences}

Condensed from LBL-4341, to be published in Muclear Physics.

${ }^{+}$Supported in part by the German National Fallowship Fourdation.

1. C. K. Gelbke, R. Bock, P. Braun-Manzinger, D. Fick, K. D. Hildenbrand, R. Richter, and W, weiss, Phys. Rev. Lett. 29, 1683 (1972).

2. C. K. Gelbke, R, Bock, and A. Richter, Phys, Rev., C 9, 852 (1974).

3. R. J. Ascuitto and N. K, Glondenning, Phys. Rev. 181, $1396(1969)$.

\section{TABLES OF NUCLEAR STRUCTL RE AMPLITUDES FOA TWO-NUCLEON TRANSFER INVOLVING LIGHT PROJECTILES (A $\leq 4)^{*}$}

Norman K Glenderinitng

Structure amplitudes for twomulion transfer reaction, denoted by

$$
A(a, b) B, \quad b=a-2, \quad B=A+2,
$$

are tabulated for light muclei, $A_{a}<4$ (denoted below by a 54 ). Soutewhat abbreviated tables were Thade evailable in umpublished report dated August 1968. The depletion of the supply of these reports and the contfntresi interest in two-mucleon transfer reactions are the occasion for the publication of the original tables, with the addition of some higher shell model configrations.

The structure tables facilitate the construction of the form factor for these reactions, which for a pure two-mucloon configuration $\left(\mathrm{n}_{I}{ }^{2}{ }_{1} j_{1}\right)$ $\left(n_{2}{ }_{2} j_{2}\right)$ is given by

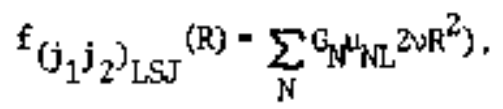

The structur anplitudes $G_{\text {N }}$ are talkulated for the quantum mubers appearing as subseripts on $f(R)$. The function thi is an oscillator function, defined 1ater. The size-parameter $v$ of the target nukleus is listed in the tables. For states more corplícated than pure configurations, the form factor is merely an appropriate linear corabination of the above.

In the distorted wave Born approximation (DWBA), the amplituds, when it is evaluated with a zero-range interaction, involves the following integrals orer the form factors $\mathrm{E}_{\mathrm{LST}}(\mathrm{R})$

$\mathrm{B}_{\text {LSL }}^{M L}=\frac{i^{-\mathrm{L}}}{[2 \mathrm{~L}+1]^{1 / 2}}$

$$
\times \int\left[\Psi_{b}^{(-)}\left(\frac{A}{\beta+2} R\right) f_{L S J}(R) \gamma_{L}^{M}(R)\right]_{a}^{*}{ }_{a}^{(+)}(R) d R .
$$


Tables for the structure anplitudes fros which $F(R)$ can be constructed are given for the various possible she11 model configurations appropriate to all regions of the periodic table. Since the allowed quantum numbers and size parameters deperd on the particular reaction, they are tabulated for $\left({ }^{3} \mathrm{He}, p\right)\left({ }^{3} \mathrm{He}, \mathrm{n}\right),(t, p)$, and $(\alpha, d)$ reactions.

\section{Foothote and References}

* * Condernsed fror Atomic Data and Nuclear Iata Tables 16, 1-134 ( $\{975)$.

1. N. K. Glendening, "Tables of Structure AnpIitudes for $(p, t)$ React lons," UCRL-1826.8; "Tables of Structure Arplitudes for (p, He ${ }^{3}$ ) Reactions, " UCRl18269 ' 'Tables of Structure Auplitudes for $(\alpha, \mathrm{d})$ Reactions," ICRL-18270, Berkeley, Calif. (1968).

\title{
POLARIZATION OF SINGLE-PARTICLE STATES INVOLVED IN HEAVY ION TRANSFER REACTIONS*
}

\author{
K Pruess, of Oelkc, L A Charlton, and N K Glondenniring
}

Direct reactions betwen heavy ions usually involve relatively 1ittle change in center-of-pass energy, angular nomentim, charge or mass of any of the reacting malei:

$$
\frac{\Delta E}{E}, \frac{\Delta L}{I}, \frac{\Delta Z}{Z}, \frac{A a}{A}<<1 .
$$

The strong absorption of close collisions out of the direct reaction channels also iaplies that the collision is peripheral. Inder all of these circtonstances it follows that the optical potential which describes the elastic scattering also plays a dominant role for the direct reactions. Taking account also of the fact that the wave number is 1arge, which inplies that the density of angular montentum states per mit impact parameter is Iarge, then classical conditions apply approximetely. In this cir cumstance the deflection function is a very useful vehicle for a discossion of the angular distribtion of mwleon transfer reactions. One need only supplement it with several additional relevant facts to understand the angular distribution of a quantal calcllation or experinent. One of these facts is that absorption out of the simple chomels occurs if the reaction partners come too close. A secand fact is that nucleons cannot be transferred if the reaction partmers are too distant. These too effects which reduce respectively the contribution of low and high partial wayes, imply the dominance of a band of grazing partial aves. Since nost of these scatter within a snall range of angles at or below the grazing angle, $\theta_{g}$, a one-step transfer cross section will have a peak in the neighborbood of the classical grazing angle of the real part of the optical potential that describes the elastic scattering.

There are many examples in the literature where the angular distribution has a grazing peak shape located near the angle defined by the elastic optica1 potential. This situtation is consistent with, but not a proof of the interpretation that the reaction is a one-step reaction and that the transfer occurs between normal states of the noninteracting nuclei. There are also a growing muber of observattons of angular distributions which do not have this form, but are biased toward the fonward angle, even though the grazing angle is well reuroved from the forward direction. There are also any cases where, al though the reaction has a peaked cross section, the peak is substantially shifted from the grazing angle. When the $Q$ of the reaction is snall, so that $\Delta \mathrm{E} / \mathrm{E} \ll<1$, the grazing angle can be read from the elastic cross section. When the $\nabla$ is large, the grazing angle can be substantially different in the entrance and exit charmels. A INRA calculation will locate the position of the erazing peak that is expected in the sense described above. In either of these two situations, where the cross section is either forward biased, or has a grazing peak substantially shifted from the expected location, we have a clear signal that the reaction cantot be described as a domirantly one-step reaction between asyoutotic states of the separated puclei. It is interesting nonetheless that the Dirk can be forced to fit the ancmalies rather consistently for a number of cross sections by the same change in optical modil par iseoters. 2,3 However, once having made this observation, it does not make sense to continue to try to force these reactions into that framework by changing the optical adol parameters so that they no longer describe the elastic scattering. This violates a sensible physical description and amounts to no more than a very elaborate (and costly) paremetrization for which the porameters have no physical meaning or applicebility beyond the isolated reaction.

When the angular distriftution of a reaction is andulous in either sense described above, we have the opportumity of learning smething new about the reaction.

In earlier work our theoretical calculations preadicted the first kind of ancinaly. ${ }^{4}$ A strongly biased forward cross section resulted from the interference of a one-step amplitude having a grazjng peak, and a two-step amplitude, which was more forward biased. The destructive interference forther resulted in a reduction of the grazing-angle cross section resulting in a forward-angle cross section with no evidemce of a grazing peak. Experiments 5 frovl Erookhaven were reported at the same time which had this fon of angular distribution and they have recently been interpreted successfully in the above way. 6

This paper is devoted to a posșible explanation of the second kind of andinaly where the angular distribution although having a grazing peak, is shifted substantially from the position expected from classical considerations, or in the case of 
large 0, from the position expected on the basis of a DhBA calculation.

The physical process that we consider in this paper is the following. As two muclel approach each other, the more weakly tound of the shell model states may be polarized by the field of the other mucleus. In the static case of two nuclei which are held stationary the polarization can be computed in the two-center shell model.7 The result is that a mucleon which is originally in one nucleus may have ar appreciable probability of being in the other when their sarfaces are close. Whether during the time of a typical roaction, the shell model states undergo an adiabatic polarization depends on the ratio of transic time to muclear period. This can be estimated as

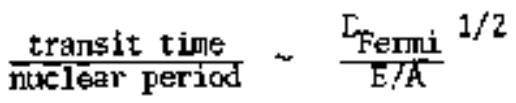

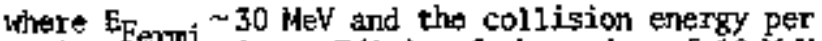
projectiflethucleon, $E / A$ is of the order of $10 \mathrm{MeV}$. If this ratio were very large compared to unity then we would expect the adiabatic polarisation to occur, whereas if it were very mall, we would not. For the conditions cited $\pm t$ is $\sqrt{3}$ which is indecisive, but it appears worth investigating the magnitude and trends of the effect.

The trends that we expect are the following. 1) Since transfer can take place for larger impact parameters if polarization occurs, then the effect on the angular distribution will be a shift to sua11er angles conpared to nomal [WAA. 2) the effect will increase for a sequence of states of decreasing binding in a micleus. 3) the polarization effect will ultimately vanish at sufficiently high bombarding energy.

The cross section for the phystcal process described above can be computed approximately in the DWBA framework. We propose to compute a possibly upper limit on the effect in the following way. We will replace the asymptotic shell model states used in nonp? DWEA calculations by the polerized sholl model states corresponiling to the distance of closest approach of the grazing orbit. In some sentse this is an upper linit on the magnitude of the effect, since the degree of polarization depends on the separation of the reacting nuclei. However, since the tronsfer is still expected to accur in a narrow anmulus around the nucleus (with radius somewhat increased over that expected for the asymptotic states\}, the use of a fixed polarization distance may be a good first approxination of the effect.

In order to use existing DWEk computer programs it is most convenient to express the polarized wave function of a spherical hanonic oscinlator basis. This a single-particle state wich, asymptoticaily, is described by the quantum numers nif $\Omega$ (with $r$ being the 3-projection of $j$ on the axis joining the centers of the nuclei) at a finite separation will appear as

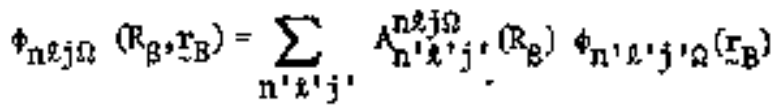

where $\mathrm{R}_{\mathrm{B}}$ is the distance separating the mass centers in the system $b+B$ and $r \mathbf{s}$ is the displacenent of the transferred nucleon from the mass center of $B(A \mathrm{~A}+1)$. For two colliding spherical nuclei, the axis joining their centers is a syumetry axis, Choosing it to detine the intrinsic 3-axis, the projection of $f$ on $i t$, which we call $\pi$, is conserved. For large separatios, $R_{\beta}$, the polarized state goes over to the normal shell model state; i.e.,

$$
A_{n^{\prime} \ell^{\prime} j^{\prime}}^{n \ell j \Omega}(\varphi)+\delta_{n^{\prime}} \delta_{l \ell^{+}} \delta_{j j^{*}} .
$$

In Fig. 1 we show a proton $f_{7 / 2}$ state in ${ }^{48} \mathrm{Ca}$ which is polarized by a nearby lop hucleus. We show the radial wave function along the line of centers of the two nucloi. One sees here clearly how the probability is distributed in part in the neighboring pxygen nucleus. A proton in the exygen nucleus can more easily be transferred to such a polarized state than to the asymptotic state, also shown,whlch overlaps with oxygen only in its tail.

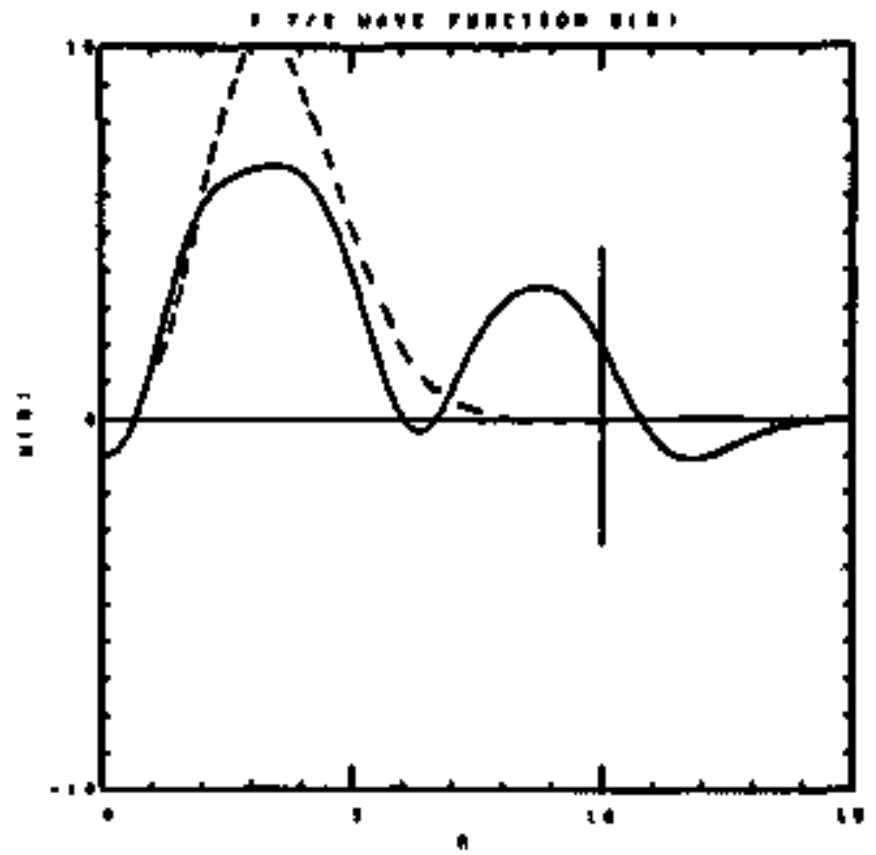

Fig. 1. The polarized vere function along the axis joining the centers of ${ }^{4} \mathrm{Ca}$ and $10_{0}$ is shom. For couparison the unpolarized kave function is shown. The center of ${ }^{48} \mathrm{Ca}$ is at the origin and the center of 1 for is at $10 \mathrm{Pt}$.

(XBL $764-1027$ )

The wave function (1) is expressed on a basis which has the symuetry axts connecting the line of centers of the two nuclei as the $\mathrm{Z}$-axis. Me have to relate this correctly to the laboratory fixed axis, which we may take to be defined by $\mathrm{k}_{\mathrm{a}}$ for the reaction

$$
A(a, b) B, \quad a=b+1, \quad B=A+1 .
$$




\section{$00 \div J$ औ}

The usual DWBA amp1itude we take to be

$$
\begin{aligned}
& \mathrm{T}_{\mathbf{1}_{1} \mathrm{~m}_{1}}+\mathrm{g}_{2} \mathrm{~T}_{2}
\end{aligned}
$$

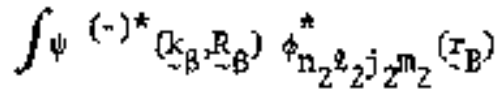

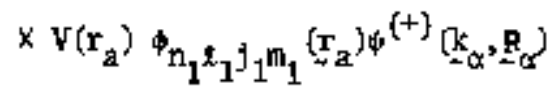

$$
\begin{aligned}
& \underset{\sim \alpha}{\mathrm{dR}} \mathrm{dR}
\end{aligned}
$$

where

$$
r_{a}=R_{1 a}, R_{\alpha}=R_{z A}, r_{B}=R_{1 E}, R_{B}=R_{B b} .
$$

The connection between wave functions expressed in the intrinsic frane and the laboratory frane is

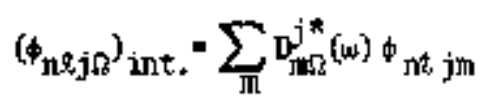

where $D$ is the rotation furction that rotates $\hat{\mathbf{k}}$ into the intrinsic axis (denote the Euler angles by w). Since

$$
\sum_{\Omega} T_{1 m 8}^{j} D_{10}^{j *}=\delta_{10 m}
$$

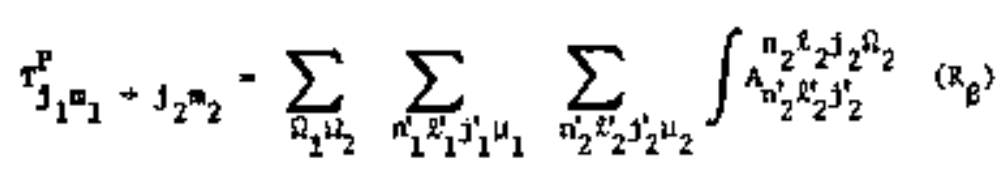

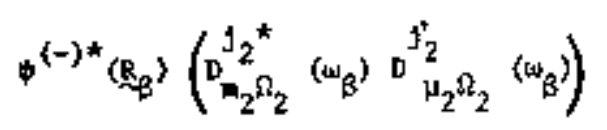

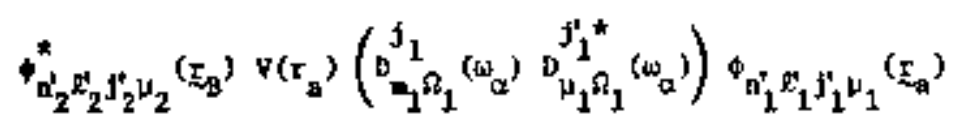

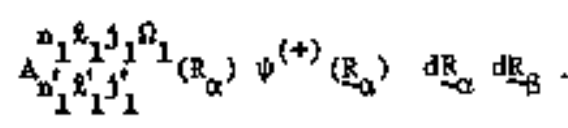

If the polarization of the single-particie states produces the expected trends, and an effect of the ragnitude comensurate with the ancmalies observed in experiment, then we would contemplate more refined calculations. There are a hisrarchy of improvements. The potential mode1 for the twocenter shell ropdel is based pol modified oscillator potentials. One could inprove the nodel by ustng, say Woods-Saxon potentials. Beyond this a dynamleal evolution of the polarization could be formulated.

\section{Foothotes and References}

Condensed froul LBL-5008. ${ }_{\text {Fellow of Deutscher Akademischer Austauschdienst, }}$
Bonn, Gerwany.

1. N. K, Glenderunsing, Rev, Mot. Phys. 47, 659 (1975).

2. H. J. Kömer, G. C. Morrison, l+ R. Greentood, and R. H. Siemssen, Phys. Rev, C 7,107 (1973).

3. $Y_{+}$Eisen, H. T. Fortune, W. Henning, D. G.

Kovar, s. Yigdor, and B. Zeidman, Phys, Rev, C 13. $699(1976)$.

4. N. K, Glendenning and R. J. Ascuitto, Argonne Symposium on Heary-Ion Transfer Reactions, Vol. II, 
513 (1973); Phys. Lett. 45B, 85 (1973); ibid. 47E, $332(1973)$.

5. Auerbach, Baltz, Bond, Chaspan, Garrett, Jones, Kahand, LeVine, Schnieder, Schwarzschild, and Thorm, Argonne Symposium on Heavy lon Transfer Reactions, Vol. II, 419 (1973).
6. N. K. Glendenting and G. Wolschin, LBL-4334 (1975), to be published by Phys. Rev. Lett.

7. J. A. Maruhn and W, Greiner, Z, Phys. 2\$1, 431 (1972).

\section{POLARIZATION OF SINGLE PAFTICLE STATES IN HEAVY ION INDUCED NUCLEAR REACTIONS WITHIN THE TWO CENTER SHELL MODEL: I. THE FORMALISH*}

K Pruesst

Two Conter She1l Madels (TCSN), using either progiven or self-consistent poteltials, heve been applist to problens of nuclear fission and nucleusnuclews collisions for quite some time. I-I0 They Were used to calculate total energies of nuclear configurations as a function of macroscopic parameters. These energies were taken as potentials goveming collective motions, such as the relative 敞tian of two colltding nuclei.

Underlying these mode1s is an adiabatic picture, with the single particle motion considered to be fast conpared to the collective motion. If this is to be taken seriously, and consistently, then not only the single particle energies but also the wave functions should adjust to the collective motion.

We put forward the hypothesis that, in "slow" nucleas-mucleus collisions, rearrangenent reactions will ocar between polarized [two center] states rather than between asymptotic states of the fres nuclei. 11

Polarized states contain a mixture of different angular monenta, In order to use these states in reaction calculations a decomposition in terms of asymptotic states is tequired. We have developed a formalism to achieve this. our methot, aIthough generally applicable, is formulated for the asymmetric two center shell model of Maruhn and Greiner 6 The potential is a tsodified two center oscillator, with the potential between the centers expressed by a polynamial of fourth degree to avoid the unphysical cusp. The usual $t+5$ and $t^{2}$ forces are included. In the case of axial ${ }^{2}$ symetry (collision of spherical nuclei) eigenstates may be written $(0>0)$

$$
\begin{aligned}
& \left.\left|E_{2}\right\rangle \equiv \mid \text { Rnlj } \Omega\right\}
\end{aligned}
$$

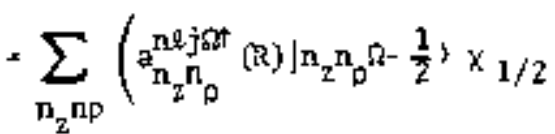

$$
\begin{aligned}
& \left.+a_{n_{2} n_{\rho}}^{n \ell j a t} \mid n_{2} n_{\rho}^{n+\frac{1}{2}}>x_{-1 / 2}\right) \text {. }
\end{aligned}
$$

The only integrals of motion are the energy $\mathbf{E}$ and $\mathrm{s}$, which is the projection of angular momentum on the $z$-axis (intrinsic syminety axis). nejo are the usual quartum numbers of the spherical oscillator shell nodel with $\ell \cdot s$-force, whlch are asymptotic quantin nubers as̃ the two center separation parameter $\mathrm{R} \rightarrow \infty$. The $\left.\mid \mathrm{n}_{2} \mathrm{n}_{\mathrm{f}^{\mathrm{fl}}}\right\}$ are eigenstates of the two center oscillator. ' $x_{u}^{\circ}$ is a spin function. The coefficients a are obtained by numerically diagona1izing the Hariltonian of the Marulm/Greiner model on the bașis of two center oscillator states. The 'asymptotic expansion' is written

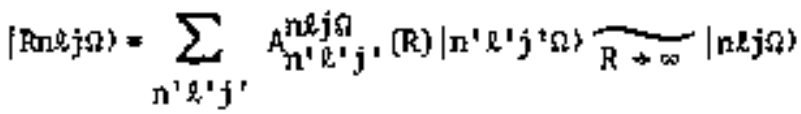

where $\left.\mid n^{+} 2^{1} j^{\prime} \Omega\right\}$ are states of the oscillator shell model, referring to either of the colliding nuclei.

To bring Eq. (1) into the form $\mathrm{Eq}$. (2) the states $\left(n_{z} n \pi r_{0}[(m+\mu) \Omega]\right.$ must be expanded in terms of states $\mid n \cdot \ell^{2} \Omega$. This is done in three steps. First, the z part of the two center oscillator states is expanded in terms of hamonic oscillator states

$$
\left.f n_{z}\right\}=\sum_{f}{ }_{h_{n}}^{n_{z}}\{\hat{f}\}
$$

Catculation of the $h$ involves integration over products of parabolic cylinder functions, which in part can only be done numerically. Then the states Iff $n_{\rho} m$, wich are eigenstates of the spherically symatric harmoric oscillator written in a cylindrin cal basis, are expanded in terus of eigenstates of 2

$$
\left.\left[\Omega_{p} n^{3}\right\}=\sum_{R^{\prime}} C_{Q^{\prime}}^{f}\left(N^{4}, \pi\right) \mid n^{+} \Omega^{\prime} m\right\}
$$

where

$$
N^{\prime}=\hat{n}+2 n_{p}+|m|=2 n^{\prime}+\ell^{\prime}
$$

is the phonon number. Calcuation of the $c$ involves, for each $N^{\prime}$, m separately, diagonalization of the matrix of 2 on the basis of the find? Finally, the $f^{\prime} t^{\prime} m{ }^{\prime} x_{\mu}$ are expanded in terms of totel angular monentum eigenstatos

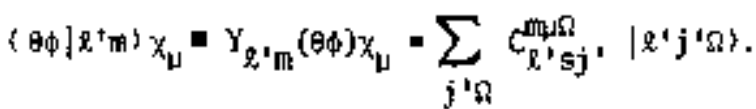




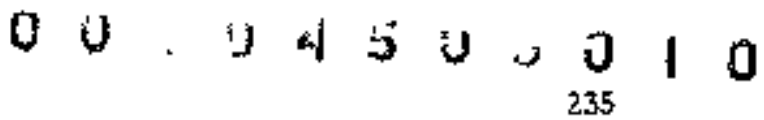

With the explicit forms for the Clebsch-Gordon coefficients we obtain for the polarization anplitudes in $\mathrm{Eq}$. (2) $(\Omega>0)$

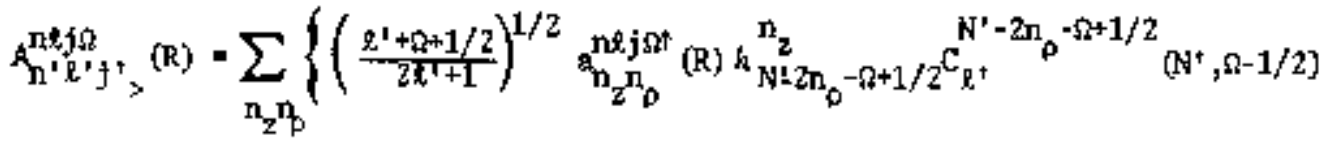

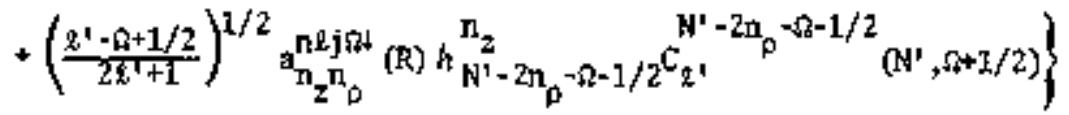

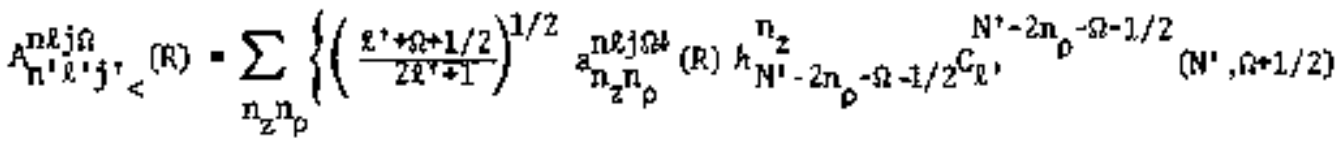

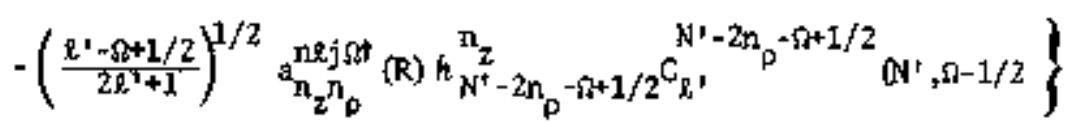

where

$$
N^{\prime}=2 n^{\prime}+\ell^{\prime} ; j j_{c}^{\prime}=\ell^{\prime}-I / 2 \text {. }
$$

As matrix elements of the TCSM-Hamiltmian are independent of the sign of $i$

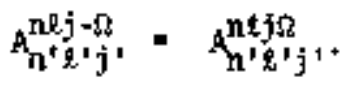

It is comvenient to write dom also a first-order perturbation expression for the $A s .12$ Denoting with $V_{R}$ the shell mode1 potential of the polarizing nucleus at distence $\mathrm{R}$ we have, taking only monenturindependent parts of $\mathrm{V}_{\mathrm{R}}$ into accomt

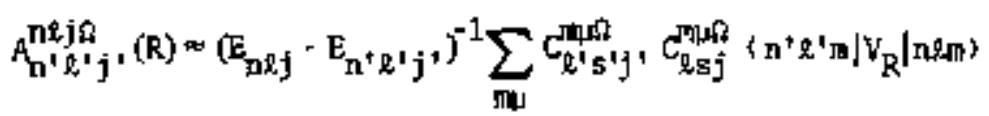

\section{Footnotes and References}

*Abridged version of 1BL-5066, to be published in Nuclear Phys. A.

${ }^{\dagger}$ FelIow of Deutscher Akadenischer Austauschdienst, Bann, Germany.

1. P. Holzer, U. MoseI, and W. Greiner, Nucl, Phys. AI38, 241 (1969).

2. K. Pruess and W. Greiner, Phys. Lett, 33B, 197 (1970); K. Pruess, Thesis, Frankfurt/Main 1972 (umpublished).

3. U. Mosel, T. D. Thomas, and P. Riesenfeldt, Phys. Lott. 333, 565 (1970).

4. D. Scharnweber, W. Gretner, and U. Moșel, Akrcl. Phys. A164, 257 (1971),

5. M. Rayet and G. Reidenteister, J. Physique 32, 259 (1971).
6. J. A. Marulm and W. Greiner, 2. Physik 251, 431 (1972); J. A. Menuhn, Diplone thesis, Franliurt/ Main 1971 (unqublished).

7. T. Morovic, Thesis, Frankfurt/Main 1973 (umptiblished).

8. H. C. Pau11, Pbys. Rep. 7C (1973) No. 1 (and references thorrein).

9. T. Tazawa, Progr, Theor. Phys, 51, 1764 (1974).

10. H. J. Specht, Rev, Nod, Phys, 46, 773 (1974) (and references therein].

11. K. Pruess, G. De1ic, 1. A. Char1tan, and N. K. GI endenting, lBL-5008, presented at the Symp. on Macr. Features of Heavy Ion Collisions, Argonne, I11., 1976, to be published.

12. A. S. Davydor, Quantum Mechanics, Perganon Press, Oxford 1965. 


\title{
POLARIZATION OF SINGLE PARTICLE STATES IN HEAVY ION INDUCED NUCLEAR REACFIONS WITHIN THE TWO CENTER SHELL. MODEL: II. NUMERCIAL RESULTS*
}

\author{
K Prusșt
}

We have prograntmed the calculation of polarization amlitudes, Bqs. (6) of preceding paper, on the CIC 7600 conputer at LBL. Table 1 gives a sumrary of the steps and times imolved. Careful checks of the numerical accuracy were made.

For collisions of spherical nuciel, our two center model has the following adjustable paraneters

$$
\begin{aligned}
& R \text {, the separation between the potential } \\
& \omega_{1}(i=1,2) \text {, the frequency parameters }
\end{aligned}
$$

Most of the calculations have made so far are for the systen $40 \mathrm{Ca}+16_{0}$. The parameters $w_{1}, \pi_{i}, \mu_{1}$ were adjusted to the asyotptotic values of the free muclei [ ${ }^{(t)}{ }_{1}=10.90 \mathrm{MeV}, 1 h_{\omega_{2}}=13.00 \mathrm{NeV}{ }^{2}$ $\left.x_{1,2}=0.08 ; \mu_{1,2}=0,0\right), 3$ Both rounded off $(\pi)$ and

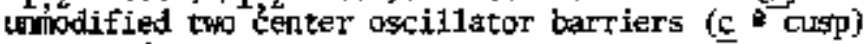
uere used.

It turns out that the polarization amplitudes

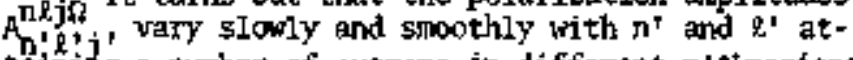
talining a number of extrenta in different $n ' \ell^{\dagger}$ regions. A compenient way to present and discuss the dats is in terms of contour maps (extending the A's to a continuous range of $n^{\prime} l^{*}$ through interpolation).
For each single particle state (asymptotic quantum numbers nijg), and each nucleus-nucleus distance $\mathbf{R}$, there are two such maps: One for the $j ;-x^{1}+1 / 2$ - states, and one for the $f^{\prime}=q^{\prime}=1 / 2$-states. Figure 1 shows polarization contow maps for the $1 f_{7 / 2} 1 / 2$-proton-state in $40_{\mathrm{Ca}}$, polarized from $16_{0}$ at various distances near the grazing distance $R=$ $10 \mathrm{fm}$. To abtain a general umderstanding of the polartzation effect consider also Fig. 2, which shows contorr maps of $\left.|\psi|\right|^{2}$ for the seine state. It is seen that the single particle states are slightly distorted from their asymptotic appearance within the polarized nucleus, and, nore important, have attained a wole new region of appreciable aniplitude near the center of the polarizing nucleus. It is this latter part of the wave furction which, when expanded on a ${ }^{4} \mathrm{Ca}$ basis 5 gme $10 \mathrm{fm}$ away, requires exansion coefflcients $\mathrm{A}_{\mathrm{T}}^{\mathrm{n}} \mathrm{i}, \mathrm{j}$, varying slowly and smoothly with $n^{\prime} \ell^{\top}$, as shown in Fig. 1 .

The polarization effect has a characteristic dependerte up the nucleus-mucleus distance $\mathrm{R}$, and the single particle quantum numbers $E$ and $\Omega$. Figures 1 and 2 show

\begin{tabular}{|c|c|c|c|c|}
\hline & Calculation & $\begin{array}{l}\text { Avaluated } \\
\text { quantities }\end{array}$ & Reanariks & $\begin{array}{l}\text { Calculation } \\
\text { tive (sec) }\end{array}$ \\
\hline (i) & $\begin{array}{l}\text { two center oscillator basis } \\
\text { states and diagonalization } \\
\text { of TCSM } \\
\text { Eq. }(I, 1)\end{array}$ & 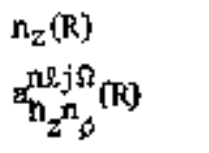 & $\begin{array}{l}100 \text { two center oscilla- } \\
\text { tor basis states } \\
\text { (E }<92.5 \mathrm{MeV})\end{array}$ & 8 \\
\hline (ii) & $\begin{array}{l}\text { expansion of z - part of two } \\
\text { center oscillator states } \\
\text { after hamonic oscillator sta } \\
\text { Eq. [I,3] }\end{array}$ & $h_{\mathrm{fes}}^{\mathrm{n}}$ & $\begin{array}{l}13 \text { two center oscillator } \\
\text { states; } 40 \text { hamionic os- } \\
\text { cillator states } \\
\text { Gaussian quadrature }\end{array}$ & 60 \\
\hline (iil) & $\begin{array}{l}\text { angular Jomentum eigen- } \\
\text { states in a cylindrical } \\
\text { hasis } \\
\text { Eq- (I.4) }\end{array}$ & $C_{Q}^{1},\left(N^{\prime}, n\right)$ & $\begin{array}{l}N^{+}=0, \ldots+39 \\
n=0, \ldots+, 6\end{array}$ & 10 \\
\hline (iv) & $\begin{array}{l}\text { asymptotic exparsion } \\
\text { Eq. }(1,6)\end{array}$ & $A_{n x^{\prime} j}^{n+j 8}(R)$ & 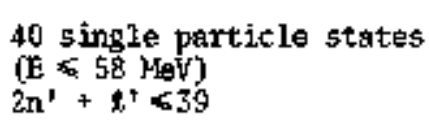 & s \\
\hline
\end{tabular}
that, while the polarization adnixtures becone stronger for decreasing distance $R$, their generat shape is nearly independent of $R$ in the grazing tegion. As a natural meagure of the overall strength of the polarization we introduce the total probability accouted for by the aduixed states

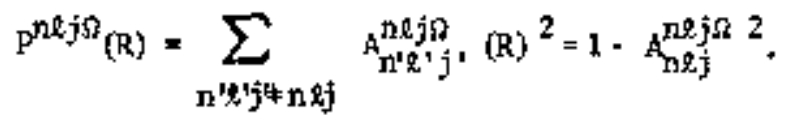

Table 1. Conputation of a typical case: ${ }^{40} \mathrm{Ca}+{ }^{16} \mathrm{o}$ at a grazing distance $\mathrm{R}=10 \mathrm{fm}$. 


$03,5+542$
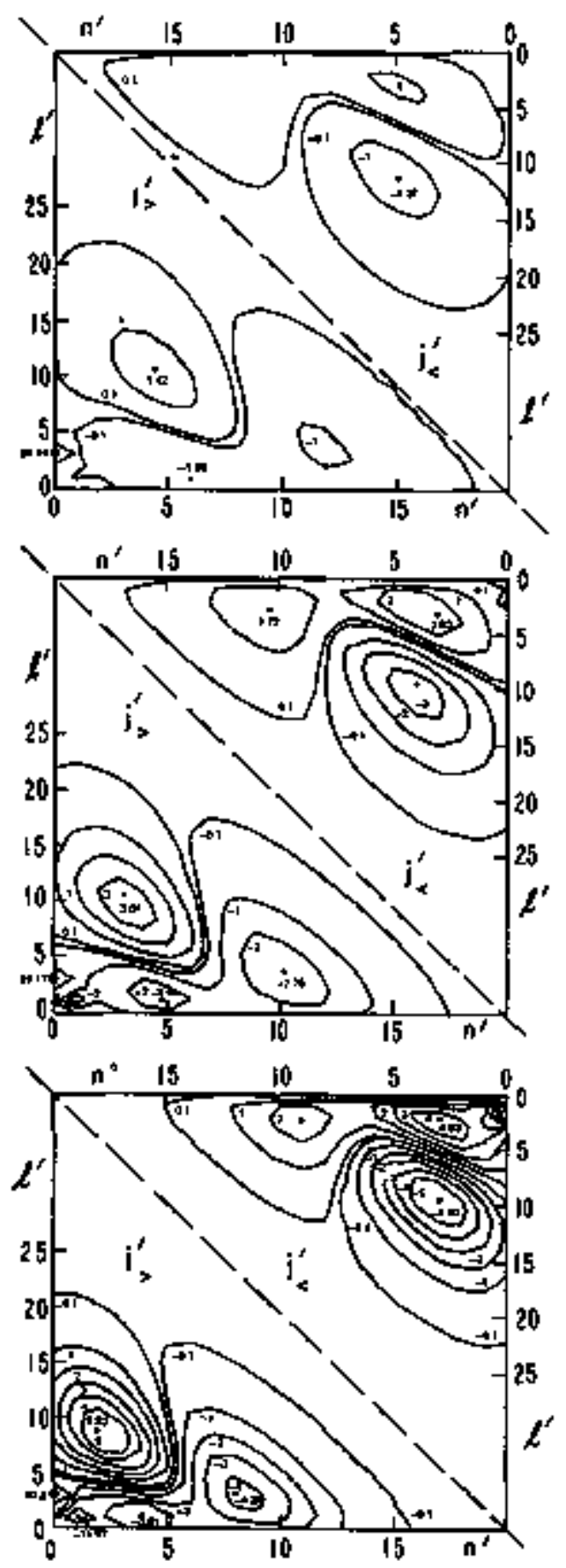

Fig. 1. Polarizgtion amplitudes for the $1 \mathrm{f}_{7 / 2} 1 / 2$ proton state in $40 \mathrm{Ca}$, polarized frool $\mathrm{H}_{0}$, as $\mathrm{cal}$ a. lated from Bqs. (6) of preceding paper for a two centar oscillator barrier (units ajong contours $\left.10^{-2}\right)$.

(XEL 766-2945)

Figure 3 shows that $P$ increases by roughly an order of magnitude if $\mathrm{R}$ decreases by 1 fu, From the sant figure it is also evident that the polarization decreases fast with increasing $|a|$. This feature is of purely geometric orisin, and can be understood from the first-order perturbation expression, Eq. (8) of the preceding paper. The state which is to be polarr ized has an angular dependenco $Y_{\text {gly }} \pm 1 / 2$. With increasing | ol it misses the polarizing potential or the 2-axis (around $\theta=0$ ) to an increasing extent.
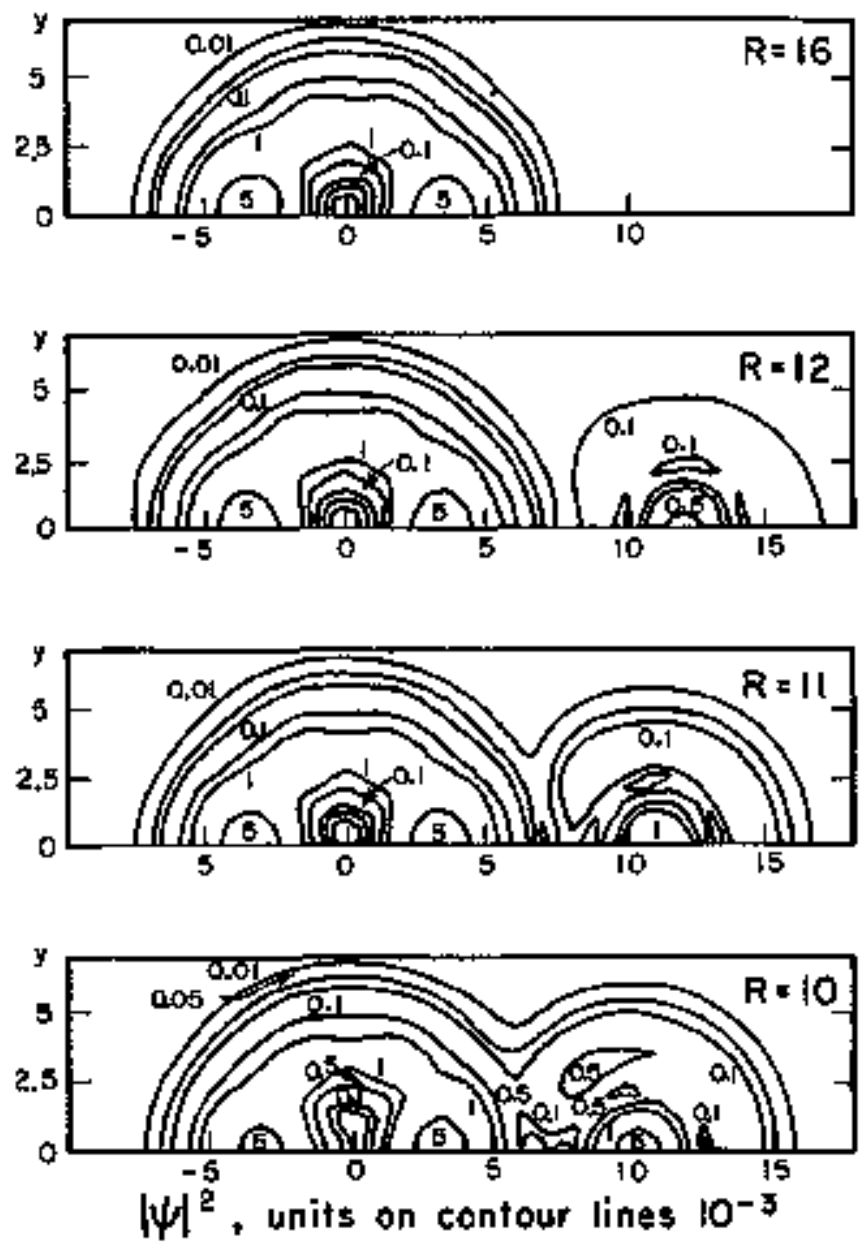

FIg. 2. Countour maps of $|\psi|^{2}$ for the same stato as calculated fron $\mathrm{Eq}$. (1) of preceding paper for a two center oscillator barrier ( $R$ in $\mathrm{fn}^{2}$ ).

(XBL 766-2944)

From Eq. (8) of the preceding paper it is also clear that the polarization generally will increase with increasing excitation energy (a longer tail) of the polarized state. Figure 4 shows the polarization as calculated fron Eq. (1) as a function of single particle energies at grazing distance $R=10$ fil for a realistic barrier $(\varepsilon=0.8 \mathrm{r})$. It increases by roughty an order of magnitude in going to the next major shell. Superimposed on this general trend are resouance effects. When the energy difference between single particle states in the colliding nuclei is snal1, these states are mutually strongly polarized. A. more careful analysis shows that the polarization effect essentially consists in adnixing a few states of the polarizing meleus which have neighboring energtes, Efforts are under way to observe "resonence polarization" experimentally, and to clarify its cor nection to the familiar resonance exchange scattering. 4

Investigations of the polarization effect for more realistic two center potentials (hoods-Saxon and corstraing fartfee-Fock), and for ather systems (especially $200 \mathrm{pb}+1 z_{\mathrm{C}}$ ) are in progress. 


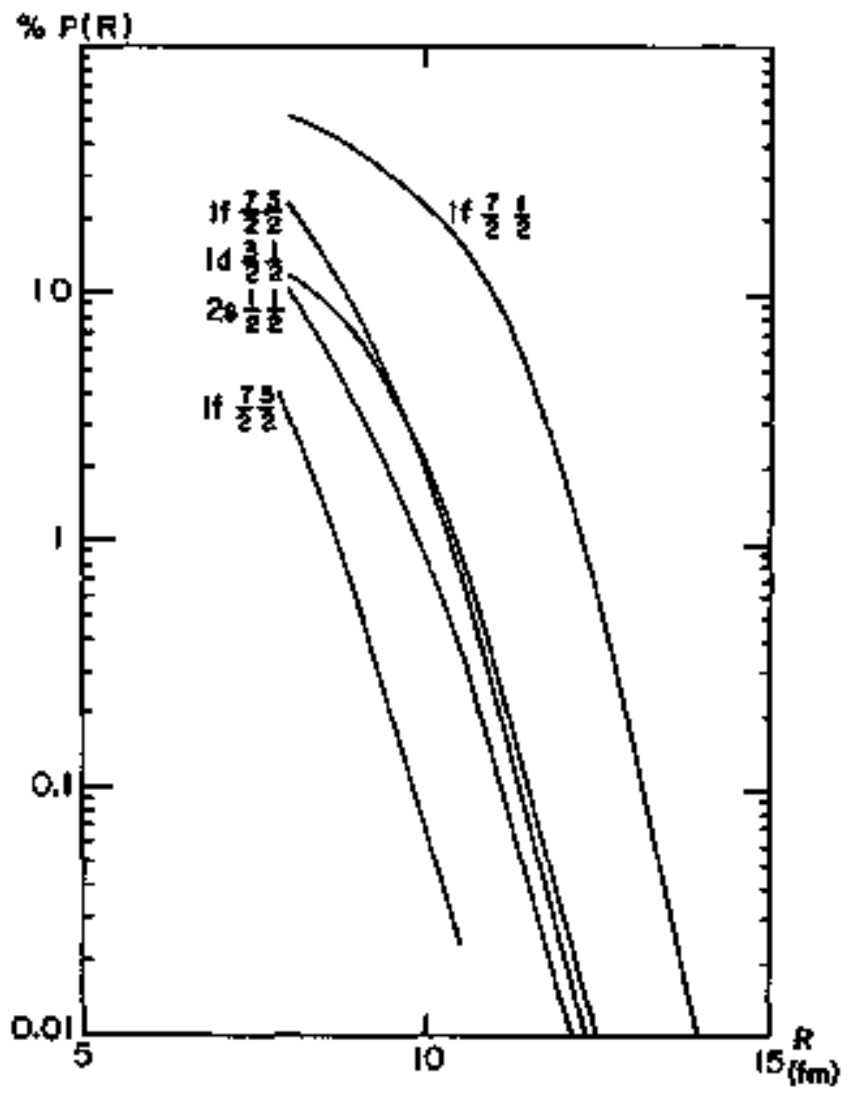

Fig. 3. Polarization for a muber of ${ }^{40} \mathrm{Ca}$ proton states, due to 100 , as calculated from Eq. (1) for a two center oscillator berrier.

(XEL 766-2943)

\section{Footnotes and Referencess}

*Abridged version of LBL-5066, to be published in Nhuclear Phys. A.

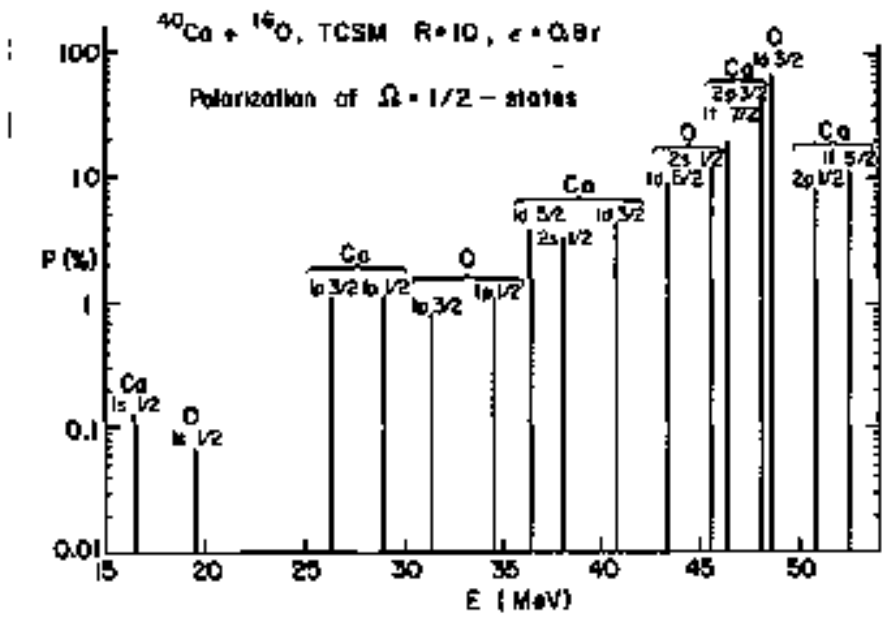

Fi ${ }_{\text {g. }}$ 4. Polarization as calculated from Eq. (1) for a realistite barries ( $\epsilon=0.8$, rounded).

(XII 766-2942)

\footnotetext{
${ }^{\dagger}$ Fellow of Deutscher Akademischer Austauschdienst, Bonn, Gerdanty.
}

1. T. W. DonnelIy and G. E, Walker, Phys. Rev. Lett. 22, 1121 (1969).

2. R. W. Shaw et a1., Proc. Phys., Soc. 86, 513 (1965).

3. C. Gustafsson, International Conference on the Properties of Nuxlei Far from the Region of BetaStability, Leysin, Switzerland, 1970, CERN Repport 70-30, p. 654; I. Ragnarsson, ibid., p. 847 .

4. W. von Oertzen, Nucl. Phys. A148, $529(1970)$; A. Gobbi, in Proceedings of the International Conference on Reactions Between Complex suclei Nasłrille, Tennessee (1974), Vol, 2, p. 211.

\title{
A NEW APPROACH TO ANTISYMMETRIZATION AND REARRANGEMENT IN NUCLEUS-NUCLEUS COLLISIONS*
}

\author{
K Prugest and P Lichtnert
}

We have iswestigated a new nicroscopic nodel for the description of maleus-rucleus collisions.

Consider a system of A nucleons, divided into two fragbent muclei $A_{a}, B_{a}$, The usubl microscopic description of their relative motion employs the vector

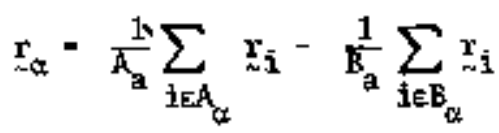

Which comects their mass centers. The definition of $r$ specifies what wo call a "partition" $a=A_{a}$, $\left.B_{0}\right), \hat{n}_{+} e_{*}$, a certain arbitrary but fixed distribition of the A nucleons anong the fraguents. With
I as given by Eq. (1) the kinetic energy separates éfactly into a relative and an internal part, and the mass associated with the $r_{\alpha}$ notion is coordinate independent and equal to the reduced nass $\mathrm{ma}$.

Becanse of the identity of nucleons actual physical states are always "fragmentations" a = $\left(A_{\mathrm{a}}, \mathrm{B}\right)$, i.e., only the nature of the fraguents is specified. A description in terrs of partitions as implied by $\mathrm{Eq}$. (1) is unnecessarily detailed and leads to well kow complications. $\mathbf{r}_{\text {ct }}$ being defined differently for different partitions, antisymetrization introduces nonlocal interactions. 1 The kinenatical description of rearragnement reactions is difficult, because different relative motion coordinates are involved. The incorporation of polarization effects (channels which depend pa- 


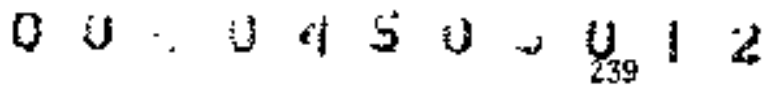

rametrically on the macleus-nucleus distance) requires complicated expansions and lengthy contiptitations (see foregoing papers).

To avoid thase difficulties, wo introduce symetrical relative motion coordinates, $i_{+} e$. coordinates which, beirg invariant with respect to nuxleon permutations, directly apply to physical fraglentations. 2-5 They are defined on the basis

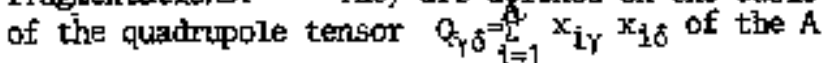
mucleon system. (Here $x_{i y}=x_{i}{ }^{*} y_{y}(Y=x, y, z)$ are the nucleon coordinates referred to a $c$.m.-frame tex, ey, ezz).

The principal axis of (hy define an intrinsic coordinate frathe $e_{11}(u=1,2,3)$ and three Euler angles $\mathrm{g}_{\mathrm{s}}$. They are clearly imvariant with respect to nucleon permutations and can be used to describe the relative angular motion of two muclei. A symmetrical relative aistance coovilinate nay be defined in a number of ways, due to the fact that the concept of relative distance is unambigupus only in the asyonptotic region of well separated nuclel. It is convenient to erploy the ths radius $Q=\Sigma, x^{2}$ of all mucleons and to define

$$
R_{a}=\sqrt{\frac{\pi}{m_{a}} Q}
$$

The couplete kinematical transfonmation to a redurdant set involving symetrical relative motion coordinates $R_{2} \theta_{5}$ is defined as

$$
\begin{aligned}
& x_{i \gamma}=\sum_{u=1}^{3} e_{\gamma u}\left(\theta_{s}\right) x_{i u} \\
& \xi_{u}=\sum_{i=1}^{A} x_{i v} x_{i w} \\
& s_{4}=\sum_{i \gamma} x_{i \gamma}^{2}=\frac{n_{a}}{\pi} R_{a}^{2}
\end{aligned}
$$

$\{i=1, \ldots, A ; \gamma+x, y, z ; s=1,2,3 ; u=1,2,3 ; u, v$, $w=1,2,3$ and cyclical permutations; equ ${ }^{*} e_{\gamma}{ }^{*} e_{u}$ is the rotation matrix).

In order to apply shell model ideas we want to keep the $3 k$ intrinsic coordinates $x_{i y}$ as independent coordinates, tagether with the four relative motion coordinates $\mathrm{R}_{\mathrm{a}}=\left(\mathrm{R}_{\mathrm{a}} \theta_{\mathrm{g}}\right)$. This is achieved with the help of four sperious coordinates $b_{c}(\mathrm{c}=1,2,3,4)$, They have to be integrated ott of matrix elements according to

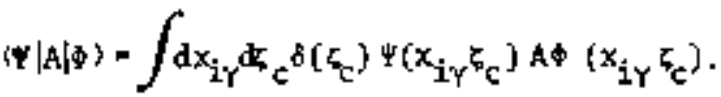

Practically, this can only be achieved in an approxinate fashiton. Calculations in the two center shel1 model indicete that matrix elements wil typically be averaged over a region of $\Delta R_{g} \approx 0.5 \mathrm{fm}$.

The $3 A+4$ coprdinates $\left(x_{i} R_{a} \theta_{S}\right)$ offer the folldwing advantages for a description of mucleusrucleos scattering:
- antisymetrization does not cause nonlocal interactions;

- relative distance coordinates for different two-body fragmentations are identical except for a trivial multiplicative factor

$$
R_{b}=\sqrt{\pi / m_{b}} \quad R_{a}
$$

- the Euler angles contain the total angular monentugn, so that no angular momentun coupIing of intemal and relative gotion is necessary.

We have explicitly transformed the nany-body Hanfltomian to the ( $x_{i}, R_{a}$ ) coordinates, With the usual volume olement

$$
\mathrm{dT}=\mathrm{dx}{ }_{\mathrm{ix}} \mathrm{dR} \mathrm{R}_{\mathrm{a}} \mathrm{R}_{\mathrm{a}}^{2}
$$

the radial part of the kinetic energy takes the form

$$
\begin{aligned}
& \mathrm{T}_{\mathrm{rad}}=\frac{\mathrm{p}_{\mathrm{a}}^{2}}{2 \pi \pi_{\mathrm{a}}}+\mathrm{T}_{\text {coupl }} \\
& T_{\operatorname{coup} 1}=\frac{1}{4 I_{a}} \sum_{i u}\left(x_{i u} p_{i u} x_{i v}\right)\left(\frac{1}{R_{a}} P_{a}+P_{a} \frac{1}{R_{a}}\right)
\end{aligned}
$$

where

$$
\begin{aligned}
& \mathrm{p}_{\mathrm{a}}=-\frac{\mathrm{I}_{\mathrm{H}}}{\mathrm{K}_{\mathrm{a}}} \frac{\hat{\partial}}{\partial \mathrm{k}_{\mathrm{a}}} \mathrm{R}_{\mathrm{a}} \\
& \mathrm{p}_{\mathrm{iu}}=-\mathrm{Ih}_{\mathrm{h}} \partial \mathrm{x}_{\mathrm{iu}} .
\end{aligned}
$$

$P$ is the ustual (Hemitean) radigl monentum. The metn result of this investigation is contained in Eas. (7), stating that $\tau_{\text {rad }}$ is by itself a Hemit ean operator. It is, except for the coupling between radial and particle motion, identical in form to the ratial part of the usual three-dimensionaI kinetic energy of a rass point. Angtular and particle part of the kinetic energy turn out to be identical to the well known expressions of tho unified model of nuclear rotations, and are not further discussed in this work, 6,9

The capling between radial and particle motion is of purely kinematscal origin. It is the price paid for the simplificatians introduced with symetrical relative motion coordinates. Such coordinates, depending upon all nucleon indices in a syumetrical way, inevitably oust contain a residual dependence upon the Internal motion.

We have formulated coupled chamel equations using the ( $\left.H_{1}, R\right)$ coortingtes. Simplifying the Harìtonian somewhat we obtain a free-motion solution in the form of an asymptotic series

$$
\begin{aligned}
& y_{\text {anem }}=\frac{e^{ \pm i k_{a} R_{a}}}{R_{a}} \sum_{v \lambda} \frac{k_{y}^{\lambda(t)}}{R_{a}^{\lambda}} \phi_{n+v e m t} \\
& \left(\lambda=0, \ldots, \infty ;-n<v<\infty ; k_{v}^{\lambda}=0 \text { if } \lambda<|v|\right)
\end{aligned}
$$


A reoursion telation is derived for the coefficimts $K_{\lambda}^{\lambda} / R_{a}^{\lambda}$ of kingnatical coupling. Mamerical exaleples indicate that in the grazing region successive orders decrease fast. Further investigatlons of the ccopling for $\mathrm{R}_{\mathrm{a}}+\mathbf{0}$ are recuired before actual reaction calculations can bo nade.

The dorivation of the two-center shell mode1 from the corpled apuations is a1so discussed.

\section{Fostmotes and References}

*abridged version of LBL-4381, in press in Nuclear Phys, A.

${ }^{\dagger}$ Fellow of Deutscher Aktadeuischer Austauschdienst, Borm, Gernany.

*Institut fuer Kemphysík der Universitaet Mainz, Gentary; Supported by Bundesninisteritum fuer Forschung und Technologie, Contract $N / 2002-L_{\text {. }}$.
1. E. yan der Spuy, Kuxcl. Phys. 11, 615 (1959).

2. K. Pruess, Thesis, Frankfurt, 1972 (umpublished).

3. K. Pruess, Proc. Buropean Conf. on Klacl. Phys., Aix -em-Provence, 1972, Yol. II, P. 46; K. Pruess, GSI-Bericht 73-6, p. 62, Dannstadt 1973.

4. P, Lichtner, Thesis, Mainz, 1974 [ungublished].

5. W. Scheid, Hab1litationsschrift, Frankfurt, 1970 (unpublished).

6. H. J. Lipkin, A. de Shalit, and I. Talni, Nuovo Cir. II (1955) 773 .

7. R. S. Kataf, Mucl. Phys. $\underline{2}(19 S 6) 497$.

B. F. Villars, Mucl. Phys, $\underline{3}$ (1957) 240 .

9. F. Villars and G. Copper, Ann. of Fhys. (N.Y.) 56 (1970) 224 .

\section{FINITE RANGE EVALUATION OF $[p-d, d-t)$ WITH MOMENTUM SPACE TECHNIOUES"}

L A Chartion

Recently a great deal of work has been done to investigate the contribution of a sequential transfer mechanism in $(p, t)$ reactions, $1-5$ This is usually done by adding a siriltaneous term, calculated using DwRA, and a sequential term using "second order" DwHA. Roth teros are aloost always evaluated using the zero-range approxination. This use of the zero-range approximation in the usual DimA calculations (firgt onder) does not allow the novnalization of the cross section to be found with any confidence. Further, both the shape and the nomma1izatiom of the sequential term (second order DWBA) can be uncertain when a zero range formalisan is used. In the paper frol which this report was condensed, the contributions to $(p, t)$ reactions from the simultaneous and the sequential mechanisns were considered using a full finite range evaluation of each term. This evaluation was performed by using ncanentwo spare techniques.

The teclmiques used for the first order term had been previously reported.8 The second order term required the calculation of two one-step matrix elewtents (p-d) and(d-t) and a Greens function to connect the two. The two one-step processes were evaluated as in Ref. 7. Since the Greens function is en inverse operator, an extension of the romentum space Fonmalism was required. A method suggested, in a different context, by Lane and Robsong proved suitable. This method allows an inverse operator to be evatuated as follows. By definition an operator $P$ satisfies

$$
\mathrm{P} \mathbf{P}^{-1}=1
$$

Forming a unit operator fron a set of nonorthogonal discrete states and taking a matrix element gives

$$
\sum_{n_{2}^{\dagger} n_{2}=1}\left\langle n_{1}^{+}|P| n_{2}^{*}\right) W_{n_{2} n_{2}}\left\langle n_{2}\left|p^{-1}\right| n_{1}\right\rangle-\left\langle n_{1}^{*} \mid n_{1}\right\rangle
$$

where the $\mathrm{N}^{+}$orthogoralizes the discrete states. Hriting the equation imediately above in matrix form gives

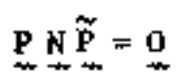

where $\widetilde{\mathbf{P}}$ contains the desired natrix elewent of $\mathrm{p}^{-1}$ and 0 contains the overlap of the discrete set. solving the matrix equation gives

$$
\tilde{\mathbf{P}}=\underline{\mathbf{N}}^{-1} \underline{\mathbf{P}}^{-1} \mathrm{~N}^{-1}
$$

where

$$
\underline{0}=\underline{\underline{N}}^{-1}
$$

has been used. Referme 6 gives a nore conplete description of the above procedme.

This stufy of the simuttaneous and sequential contributions to $[\mathbf{p}, t)$ reactions drew three conclustons: 1) finite range effects can be large 2) the shape of the calculated differential cross sections can be strongly dependent on the contribution fron a sequential txansfer mechanisn and 3) the postprior interchange usualiy used in the second order tem is probably the best realistic approximation. These conclusions wexe based on consideration of $20 \mathrm{~B}_{\mathrm{Pb}}(\mathrm{p}, \mathrm{t})^{206 \mathrm{pb}} \mathrm{s}$ only. Mork is now underway to astablish systerinitics for the effect noted by looking at a variety of nuclei. 


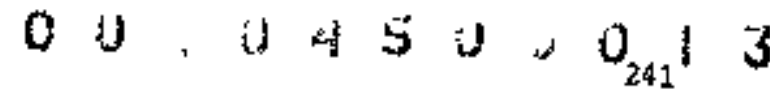

\section{Foptnotes and References}

*Condensed from Ref. 6.

1. P. D. Kunz and E. Kost, Bull. Anl. Phys. Soc. 17, $902(1972)$.

2. N. B, de Takacsy, Mucl, Ptrys. A231, 243 (1974).

3. V. Managoli and D, Robson, Nucl. Ehys. A252, $354(1975)$.

4. H. Segawra, K. I. Kuho, and A. Arina, Phys. Rer. Lett. 35,357 [1975).
5. N. Hashimoto and M. Kowa1, Phys. Lett. 59B, 223 (1975).

6. L. A. Char1ton, Phys. Rev, C14, 506 (1976).

7. L. A. Chariton, Phys. Rev. C 8, 146 (1973);

l. k. Charlton, Phys. Rev. C 9,413 (1974).

8. L. A. CharIton, Phys, Rev. C 12, 351 (1975),

9. A. M. Lane and D. Robson, Phys. Rev. 185, 1403 (1969).

\section{FINITE-RANGE EFFECTS IN MULTISTEP (p-d, d-n) REACTIONS*}

\author{
P D Kunz,t and L A Cinarton
}

Two step processes through reaction channels have been successful in explaining many pherromena. The nost striking example has been the disçrepancy in the population of high spin states in $\left({ }^{3} \mathrm{He}, \mathrm{t}\right)$ reactions. 1 Further, the drechanism has successfuily explained the $L=1$ angular distributions seen in ( $\mathrm{H}$ Het t) reactioms to anti-gralogue states. 2 For $(p, n)$ processes, however, a large remonalization of the direct charge exchange is needed in order to successfully fit shapes and gotudes of angular distributions. ${ }^{3}$ All the aboute calculations have used the zero-range approxination which, in part, is responsiblo for the renormalization problems in $p-d$, d-n) reactions. This may be seen calculationally by referring to $\mathrm{Fi}$. 1. The differential cross section for ${ }^{5} \mathrm{ki}(\mathrm{p}-\mathrm{d}, \mathrm{d}-n)^{5 \mathrm{~B}} \mathrm{Ca}$ is shown. The use of the zero range approximation overpredicts the two-step contribution by a large factor.

Scme understanding of the large finite-range effects can be gained by considering the first order flnite-range correctlon to the zero-range calculation. This first order correction contains a term in which the dewteron Laplacian acts on the intermediate state Greens function. The properties of the Grests function may then be used to get

$$
\frac{-n^{2}}{2 \bar{H}_{D}} \nabla_{D}^{2} G\left(R_{D}, R_{D}^{\prime}\right)=\left(E_{D}-V_{D}\right) G\left(R_{D}, R_{D}^{\prime}\right)-\delta\left(R_{D}-R_{D}^{1}\right)+
$$

The first term on the right hand side or the above equation is identical to the usual correction term found for (p-d) reactions. Since the zero range approxiration works very well in these single step processes it wald be expected to be sapgll. The second term, however, gives the off-shell contributions which exlst only in the (p-d, $d-n$ ) mechanism. This teno will collapse the ( $p-d, d-n$ ) tem with an equivalent $(p-n)$ term, Thus, when the single step contribution is large, finite range effects in the two step tern can be large. A rore complete explanation of the effect is contained in Ref. 4.

Work is now underway to establish systematics for finite range effects in ( $p-d, d-n)$ reactions. other tio-step reactions are also being exanined with a full finite range evaluation of the two step amplitude.

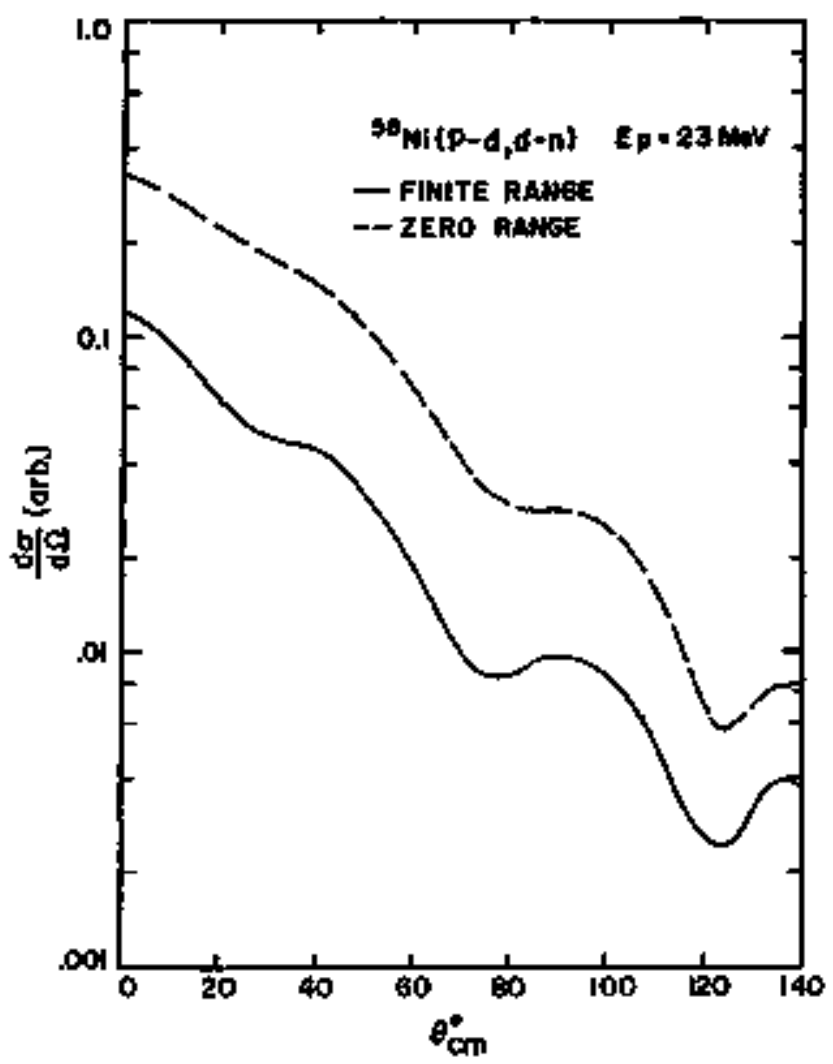

Fig. 1. The two-step differential cross sections with arbitrary overall nomalization. The dashed curve shows the zero-range result and the solid curve show the finite-range result for a model cat culation for $58 \mathrm{NE}(\mathrm{p}-\mathrm{d}, d-n) 58 \mathrm{Cu}$ to the aralogue state. The single-particle orbital involved was the $2 \mathrm{P}_{3 / 2}$ state.

(XHL 766-8274)

\section{Footnotes and References}

Condensed frón Ref. 4.

${ }^{\dagger}$ Dept. of Phrsics and Astrophsies, University of Colorado. 
1. M. Toyana, Phys. Lett. 38B, 147 (1972); Mucl. Fhys, A211, 254 (1973).

2. R. Schaefrer and G, F. Bertsch, Phys. Lett. 38B, 159 (1972); W. R, Coker, T. Udagawa and H. H. Wolter, Phys. Rev, Lett. 418, 237 (1972).
3. L. D. Rickertsen and P. D. Kumz, Phys, lett. $47 B, 1](1973)$.

4. P. D. Kunz ond l. A. Charlton, Phys, Lett, 61B, 1 (1976).

\section{CHEBYSHEV EXPANSIONS AND THE FINITE RANGE DISTORTED WAVE BORN APPAOXIMATYON FOA NUCLEAA TRANSFER REACTIONS*}

George Delic

The epithet "exact" was introduced by Delle and Robson 1 to describe corputation of the Distorted waves Horn approxination in a forthlisil for ouclear reactions which allowed both non-S state stripping from the incident projectile in the transfer reaction $A(a, b) B$, and an interaction of finite range in the DHBA transitfon matrixralplitude. These computations, perfortaed for d,p reactions, applied an approach suggested by Austern ot al.2 and did not introduce any further approxinations of either a mathematical or numerical nature, other than that of truncating the area integrated in the too-dimensionsl radial integrals which need to be evaluated. The method requires the mumerical evaluation of the Legendre series coefficients ${ }^{3} g_{K}$ at each point of the two-dinensional grid of the double radial integral. The Iargest value of $K$ is a few units larger than $\mathbf{L}_{\text {max }}$, the value of the Iargest partial wave re tained in the DW expansions describing the relative motions of a, A and b, B. Thus, the overall computation time of such calculations is dependent on the number of grid points used, as well as their distribution: within the area to be integrated. There are basically throe approaches to the problen of evaluating $n$-dimensional integrals numerical:4-6 (1) application of a prokuct mie, either of Newton-Cotes or Gauss type, (2) n-dimensional quahrature fortules based on construction of n-dimenstoral polynomials, (3) Nonte Carlo, or number theoretical technlques. frote Carlo techniques have been applied, with success, to nuclear reaction calculations by Bayman and Feng, 7 and a product Gaiss rule appears to be the ap. proach of Ref. 8 . In the present study approach (2) is taken becalyse the perfectly symetric two-dimensional quadrature formalas of kabinowitz and Richter ${ }^{\theta}$ have fewer points than the product Gauss rules of equitralent degree and because such quadratures, based on polymonial interpolation, 10 will display rapid convergence with increasing degree (or decreasing area), i,e, with increasing nuber of grid points.

The algorltim grogramed in MNDRY consists of defining squares of side length $S_{C}$ and packing the aree to be integrated with these squares. Then squares of side Iength $S_{s}$ and $S_{b}$ are defined so that $S_{a}=2 S_{b}=4 S_{c}$, and the 48 and 28 point formulas of Ref. 9 are applied to the squares $S_{9}^{2}$ and $S^{2}$ respectively, and the 12 point fomula of Tyler10 to the square $g^{2}$. Product Gatuss mules of equivalent degree would fequire 64,36 and 16 points, respec. tively, thus the method used in the code klltitri leads to substantial gains in coriputational speed without loss in accuracy. A comparison wath the code LOLA showed KINIRY to be typically four times faster in execution time, i.e., 62 versus 16 seconds.

The most important feature of the problem is determination of a criterion for trumeation of the two-dimensional space in the radial coordinates $r_{a A}$. $\mathrm{T}_{\mathrm{b}}$ (the arguments of the $\mathrm{D}$ (th radial waye functions) over which the double integral is performed. Assuming the bound state radial wave functions to be negligible when they have an asymptotic magnitude smiller than some nimber $c$ and defining the corresponding radil to be re and If $x$. two estimates for the truncation radius in the first DN cooxtinate may

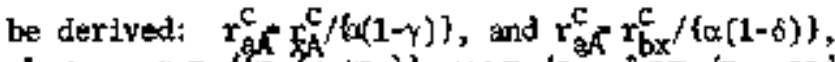

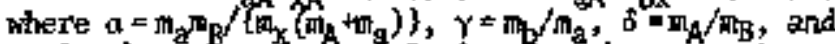
$m_{i}$ is the mass of particle $i$. For the range $\Delta \mathrm{r}_{\mathrm{bB}}$ of the socond variable $T_{b B}$ about the value $r_{b B}=r_{a d}$ two estimates can also be made $\Delta \mathbf{r}_{\mathrm{bB}}=\mathbf{r}_{\mathrm{X}} \mathrm{A} /(q \mathrm{r})$ r) and $\Delta_{\mathrm{bB}}=\mathrm{r}_{\mathrm{f} / \mathrm{\alpha}}, \mathbf{i} . \mathrm{e.}, \mathrm{r}_{\mathrm{bB}}=\mathbf{r}_{\mathrm{aA}}$ with only positive values of $\mathrm{r}_{\mathrm{bB}}$ allowed. The smaller of these tho estimgtes is used in each case. Thus truncation errors arising from either the physical assinptions (trencation of the bound state wave functions) or the nemerical assumptions (size of the area S?) may be explicitly investigated by computation. Experience with the code has shown the noed to take these trucation errors into serious consideration particularly for bound state wave finctions which decay slawly or in reactions at high energies.

The time taken to compute the two-dimensional radial integral is measured in seconds or fractions thereof, whereas the tine, taken to evaluate the twodinensional forl factor $h_{p}\left(L, f, t\left(r_{a A}, I_{b P}\right)\right.$ which is in the integrand rapidiy becthes orders of magnitude langer with increasing orbital angular nosuente $k$, '.' for $x$ bound to $A$ and $b$ respectively. This is shown in Fig. I for a case with 1944 grid pointsll with $2^{\prime}+1, j^{*}-1 / 2$ and $2=0$ to 13 and $L_{m}-42$, Thus the doninant anount of contputation tiaxie is spent in evaluating the expression 


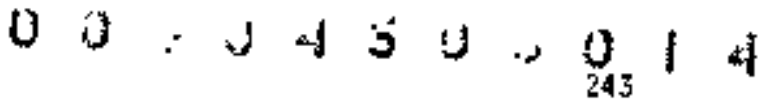

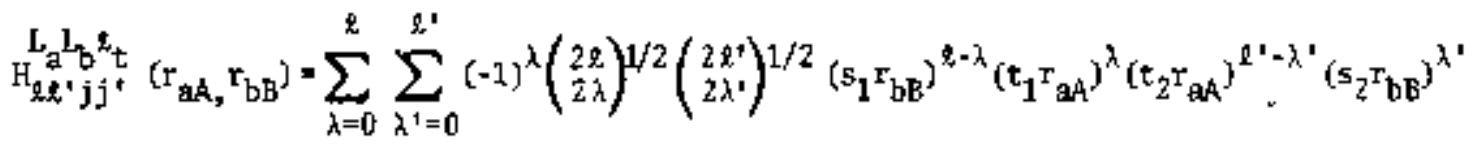

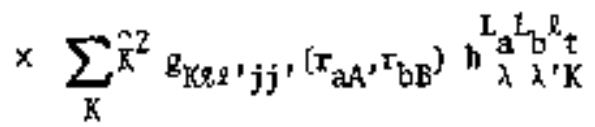

where

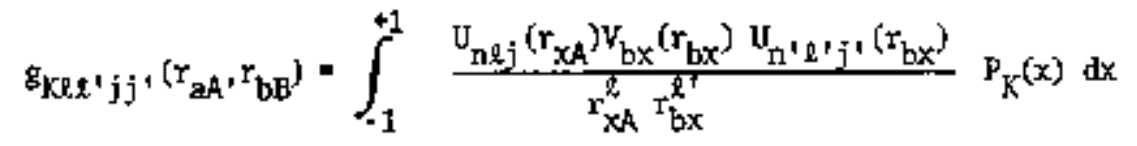

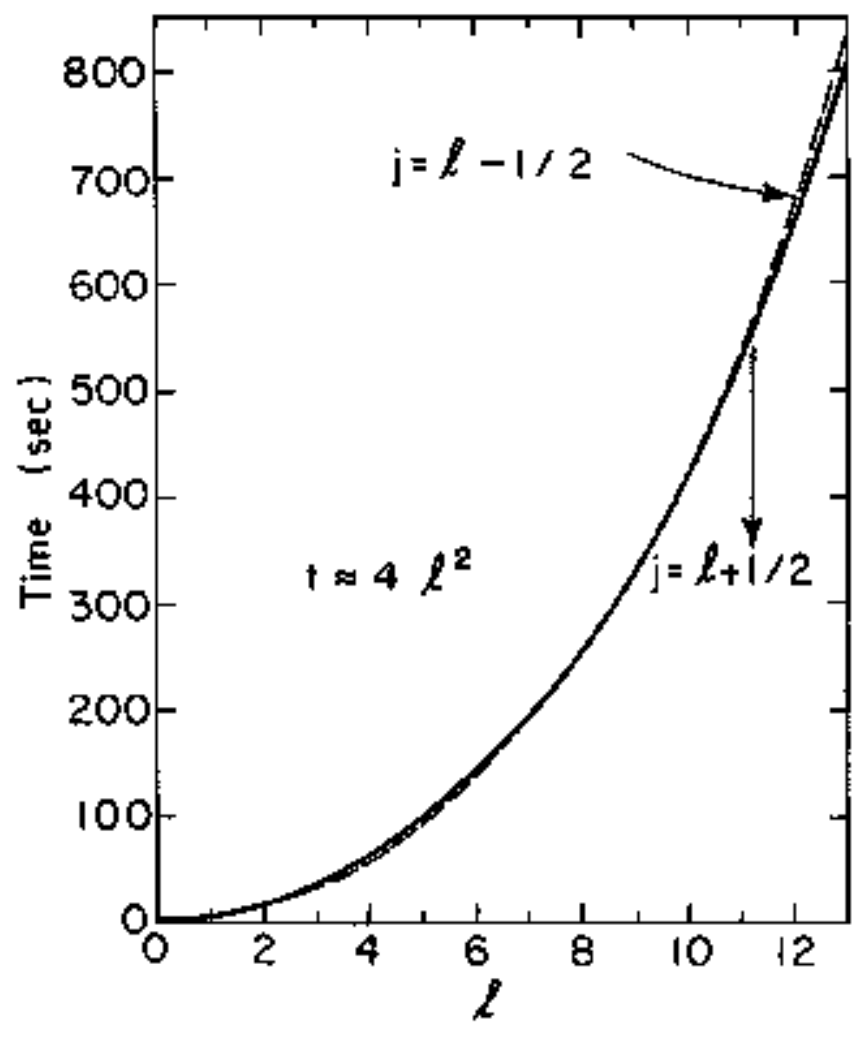

Fig. 1. Corputation tine (sec) for the form factor of Eq. (1) as a fumction of \& (see text). The shape of this curve is probably specific to the CDC 7600 and the remarkable properties of the IDC FIN4 Conpiler with OPT-2, For other nachines and compiters the curve could be steeper.

(XILL 766-8277)

and $\mathrm{h}_{\mathrm{a}} \mathrm{b}_{\mathrm{b}} \mathrm{l}_{\mathrm{t}}$ is a coubination of angular momentum couplitid' Eoefficients.

The wethod proposed here is to expand the bound state wave functions in series of shifted Chebysher polynomials

$$
u_{n R j}\left(r_{X A}\right) \times r_{X A}^{l} \sum_{m}^{M} a_{n} T_{m}^{*}(z)
$$

$$
V_{b x}\left(r_{b x}\right) U_{\pi^{+} l^{\prime} j^{\prime}}\left(r_{b x}\right)=r_{b x}^{q^{+}} \sum_{m^{+}}^{M^{r}} a_{m^{\prime}}^{\prime} T_{m^{\prime}}^{*},(y)
$$

where

$$
z=\left(\frac{r_{x A}}{r_{x A}^{c}}\right)^{2} \text { and } y=\left(\frac{r_{b x}}{r_{b x}^{c}}\right)^{2}
$$

Because $z$ (or $y$ ) and $x$ are 1 inearly related at each grid point $\left(\mathrm{t}_{\mathrm{aA}}, \mathrm{r}_{\mathbf{b B}}\right)$ it is possible to make the equivalence

$$
\begin{aligned}
& \sum_{j=0}^{M} b_{j}, T_{j},(x)=\sum_{\pi}^{M} a_{j 1} T_{m b}^{*}(z)
\end{aligned}
$$

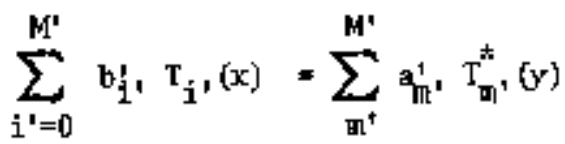

and it is straightforwand to derive explicit expressions for the coeffiecients $b_{j}$, and $b_{j}^{\prime}$, without the need to evaluate intergraIs. It follows that Eq. (2) reduces to

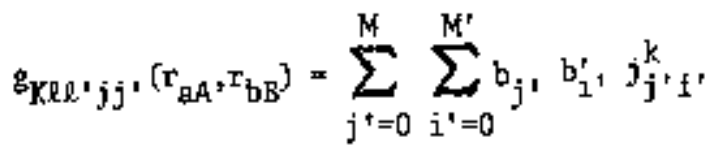

Where

$$
J_{j+i^{+}}^{X}=\int_{-1}^{41} T_{j},(x) T_{i},(x) P_{K^{\prime}}(x) d x
$$

is readily evaluated.

As in the Gaussian expension method of Payne and CarIson, 12 the sumpations of Eq. (7) need to be evaluated at each grid point $\left(\mathbf{r}_{\text {ad }}, \mathbf{r}_{\mathrm{p}}{ }_{\mathrm{B}}\right.$. Hower, because $J_{1}^{K}$, or Eq. (8) is the 1 月 nomial tin $x$, an important consequence of the Chebyshev expansion technique is that the sumnation over $K$ in Eq. (1) no longer needs ta be performed separately for each grid point. In particular, for the 
surmation over $K$ in $\mathrm{Eq}$. (1) it is now possible to write

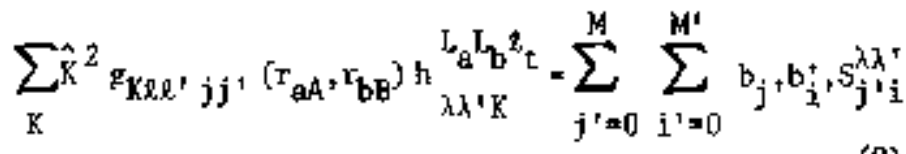

Where

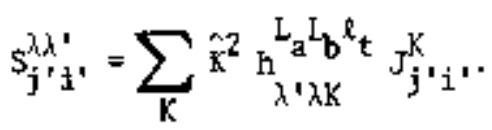

This reduces the computational effort in calculating Eq. (1) as well as machfre memory requirements, since the legendro series soefficients need not be stored in the computer.

\section{Footnote and References}

"Work in progress.

1. G. Delic and B. A. Robson, Muc1. Phys. A156, $97(1970)$.

2. N. Alstern, R+ M. Irisko, E. C. Halbert, and G. R. Satcher, Phys. Rey, 133, B3 (1964).
3. G. Delic, J. Corp. Phys. 14, 254 [1974).

4. A. H. Stroud, Approxinate-Calcilation of Akltiple Integrals, Prentice-Hall, Friglewod Cliffs, 1971.

5. I. Af. Sobol', Mrogonerniye Kvadraturniye Fontuly i Funktsii Ghaara otrlti-dimensional quadrature Formulas and Haar Functions), Izdat. "Nauka," Woscow, 1969.

6. I. S, Haber, SIAM Rev. 12, 481 (1970).

7. B. F. Bayman and D. H. Feng, Mur1. Phys. A205, 513 (1973).

8. J. L. Perrenoud and R. M. De Vries, Phys. Lett. 36B, $18(1971)$.

9. P. Rabinowitz and N, Richter, Math. Couput. $\underline{23}$, 765 (1969).

10. R. Franke, Math. Conput. 25, 803 (1971).

11. K. Ptuess, G. Delic, L+ A+ Charlton, and N. K. Glendenning, LBL-5008 and LBL-5074.

12. G. L. Payne and R. R. Carlsch, Phys. Rev. C 12, $1260(1976)$.

\title{
OPTICAL MODEL ANALYSIS OF $N+C$ AND C + C ELASTIC SCATTERING*
}

\author{
Gearge Delic
}

The data ${ }^{1-5}$ analyzed in the present work ware as follows: (all energies are quoted in center-ofJass uniess otherwise indicated elastic scattering of $14_{\mathrm{N}}$ by $12 \mathrm{C}$ at $9.230,9.922,10.84,11.54$, and 12.60 Mew, $14 \mathrm{~N}$ by ${ }^{13} \mathrm{C}$ at $9.649 \mathrm{MeV} ; 14_{\mathrm{N}}$ by ${ }^{14} \mathrm{C}$ at I0.00 and $12.50 \mathrm{Mev} ; 12 \mathrm{C}$ by $13 \mathrm{C}$ at $7.80 \mathrm{l}$ and $9.881 \mathrm{keV},{ }^{3} \mathrm{C}$ by ${ }^{14} \mathrm{C}$ at 7.778 and $9.852 \mathrm{keV}$; and ${ }^{12} \mathrm{C}$ by $14 \mathrm{C}$ at $\mathrm{B.078}, 9.694$ and $10.77 \mathrm{MV}$. In Table I the data are arranged in increasing order of $n$ (the Sormerfeld paranetser), and asymptotic wave number $k ; R=A_{a} 1 / 3+A / 3, A, \sigma_{A}$ (total absorption or reaction cross section) are also tebulated. Table 1 shows a selection of some of the optical model parameter sets which were found; those cases where the data did not cover a sufftciently broad range in angle were not searched upon. The definition of the optical model potential was the same as that of Ref. 6 with a surface type atsorbing potential. For the searches all six optical model parameters wore varied sintaltaneously. In the 500odd searches performed in the present work the absolute nomalization was not varied and a relative error of 54 was set on each data point. The comparison between experimental and theoretical ratio-to-futherford cross sections is show in Fig. $1 \mathbf{a}$ and $b$ for the paraneters of Table 1 . The potential found for the scattering of $12 \mathrm{C}$ by $14 \mathrm{C}$ at $9.694 \mathrm{keV}$ (18 MeV lab), which also gives a satisfactory description of the same system at $10.77 \mathrm{MkV}$ (20 MoV lab) was used to show how the structure observed in the angular distributions evolvad as a function of energy (cf. Fig. Ic). The optical model predicts that such structures change reIgtively rapidly for the axtreme backtard angles (> $120^{\circ}$ ) for energies above the Coulomb barrier. Another feature is that the optical motel predictions (especially in the backward henisphere) are sensitive to the cholce of the Coulonb radius $r_{c}$. The coulomb radius used throughout the searches was $I$. $45 \mathrm{fm}$, and the effects of changing this radius to 1.225 fin and 1.0 fu are shom in fig. lb by the solid and dotted curves, respectively, for $14 \mathrm{~N}+12 \mathrm{C}$ at $12.60 \mathrm{MoV}$, with the case corresponding to $\mathrm{r}_{\mathrm{C}}=1.45$ for shown in Fig. la,

Figure la and b together show 15 experimenta1 angular distributions; a careful comparison of these data reveals why it is inuoxtant to classify heavyion elastic-scattering data (and optical model potentials derived therefrom) according to the Sommerfeld parameter $n$, the asymptotic wave number $k$, and possibly sone interaction radius, e.g., $\mathrm{R}=$ $\mathrm{Aa}_{\mathrm{a}} / \mathrm{S}_{+} \mathrm{AlS}_{\mathrm{S}}$, defined by the systerm under consideratzon. In the following discussion the notation: $l_{\text {label }}$ vs label $I_{1} ; \delta n, \delta k, \delta R$, is used wher the labels are those of Table 1 , and $\delta_{n}$ is $\eta_{T}=\pi_{I}$ expressed as a percentage of $n_{I}$, etc. A variation of $\eta$ alone produces a shift of the structures in the experimental angular distributions toward small angles (for a decrease in $n$ ) or toward large angles (for an increase in $n$ )-compare C232 vs C243: -2.7,

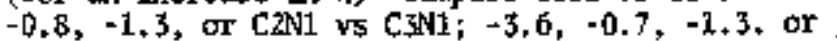
C242 vs C341: $-4.1,-0.3,1.3$, or C3N1 vs C342: 15.2, 


\section{$00: 34502913$}

Table 1. Optical nodel parameters for a $(A, A) a$,

\begin{tabular}{|c|c|c|c|c|c|c|c|c|c|c|c|c|c|}
\hline a & label & $\begin{array}{l}\text { EnerG }{ }^{\mathbf{a}} \\
\text { (MeV) }\end{array}$ & $\begin{array}{c}\mathbf{r}_{v} \\
\text { (fin) }\end{array}$ & $\underset{\text { (fin) }}{A}$ & $\begin{array}{c}V \\
(H+V)\end{array}$ & $\begin{array}{l}r_{w} \\
\left(f^{\prime}\right)\end{array}$ & (fm) & $\begin{array}{c}W \\
(M B V)\end{array}$ & $\begin{array}{c}{ }_{A} A \\
(m b)\end{array}$ & $\Delta^{\prime \prime}$ & $\pi$ & $\begin{array}{c}\mathbf{k} \\
\left(\mathrm{fm}^{-1}\right)\end{array}$ & $R^{b}$ \\
\hline $\mathrm{c}^{14} \mathrm{c}$ & 24 & $10.77(20.00)$ & no seams & ch (para & meter se & et $\propto 243$ & used) & & & & 4.391 & 1.825 & 4.700 \\
\hline $13{ }_{c}^{13} \mathrm{c}$ & c23e & $9.891(19.00)$ & 1.019 & 0.7010 & 67.79 & 1.077 & 0.1002 & 50.82 & 773.0 & 28.38 & 4.505 & 1.77 & $4.64+1$ \\
\hline${ }^{2} \mathrm{C}^{14} \mathrm{C}$ & $c 243$ & $9.694(18.00)$ & 0.9777 & 0.5256 & 88.26 & 1.027 & 0.1230 & 39.41 & 523.1 & 5.624 & 4.628 & 1.731 & $4.7 \infty$ \\
\hline 30140 & $\mathrm{CS}_{3}+2$ & $9.852(19.00)$ & no searc & ch (para & meter se & et $63 \mathrm{~N}$ & used ] & & & & 4.690 & 1.788 & 4.761 \\
\hline${ }^{4} y^{1}$ & cent4 & $12.60(27.30)$ & 0.9703 & 0.6636 & 85.47 & 0.9661 & 0.09149 & 92.87 & 653.7 & 12.50 & 4.737 & 1.973 & 4.700 \\
\hline & cerso & $13.54(25.00)$ & 1.034 & 0.5909 & 79.10 & 0.9940 & 0.07685 & 97.59 & 527.8 & 14.96 & 4.950 & 1.888 & 4.700 \\
\hline 4,1 & Clate & $12.50(25.00)$ & 1.053 & 0.5473 & 84.23 & 1.016 & 0.1027 & 67.82 & 675.6 & 21.35 & 4.950 & 2.046 & 4.820 \\
\hline 123 & ca32 & $7.801(15 . \infty)$ & 1.065 & 0.6220 & 69.91 & 1.109 & 0.1221 & 28.94 & 442.7 & 13.97 & 5.070 & 1.526 & 4.647 \\
\hline 21 & $\mathrm{c} 242$ & 8.078 $(15 . \infty)$ & 1.001 & 0.6771 & 80.23 & 1.063 & 0.1393 & 40.50 & 547.0 & 3.196 & 5.070 & 1.580 & 4.700 \\
\hline${ }_{n} 1 \varepsilon_{c}$ & cente & $10.84(23.50)$ & no searc & ch (pвara & queter вe & 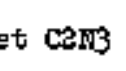 & LAsied) & & & & 5.105 & 1.832 & 4.700 \\
\hline & $\mathrm{Cs}+1$ & $7.778(35.00)$ & 0.9752 & 0.7456 & 80.10 & 0.8646 & 0.1255 & 33.06 & 523.9 & 30.73 & 5.278 & 1.584 & 4.761 \\
\hline & क्m & $9.922(21.50)$ & 0.9647 & 0.6266 & 83.55 & 0.9487 & 0.2036 & 33.29 & 449.6 & 25.67 & 5.337 & 1.751 & 4.700 \\
\hline $\pm 4{ }_{11}^{13} \mathrm{C}$ & c3in & $9.649(20.04)$ & 0.9751 & 0.6866 & 87.17 & 1.012 & 0.1389 & 43.15 & 510.1 & 13.73 & 5.528 & 1.754 & 4.761 \\
\hline $14_{3} 12 \mathrm{c}$ & $\cos 5$ & $9.430(20.00)$ & 0.9865 & 0.6251 & 82.18 & 0.9477 & 0.2101 & 28.88 & 367.0 & $5.0,32$ & 5.534 & 1.689 & 4.700 \\
\hline$-4 y^{24} c$ & $C^{\prime}+M$ & $10.00(20.00)$ & ap Ster & $9 n$ & noter & & ad) & & & & 5.534 & 1.830 & 4.820 \\
\hline
\end{tabular}

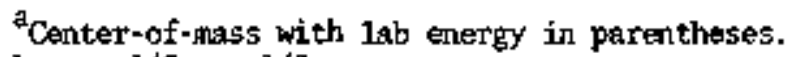

$b_{\mathrm{R}}=A_{\mathrm{a}}^{1 / 3}+A_{\mathrm{A}}^{1 / 3}$.

$-1.0,0$ or C243 us C2N1: $-15.3,-1.2,0$, or C2NA vs C232: $18.6,-1.7,1.3$. A variation of $k$ alone produces a shift toward snill angles (for an increase in $\mathrm{k}$ ) or toward large angles (for a decrease in k) - compare C243 vs C342: $-1.3,-2.9,-1.3$, or C231 vs C242: $0,-3.5,-1.3$, or C引1 vs C2NO: $-0.1,4.3$, 1.3 or C341 vs C2NI: $-1.1,-10.5,1.3$, or C342 v5 C2⿺辶4): $-1,0,-10.7,1.3$, or C242 vs C2N2: $-0.7,-15.9$, 0 . A variation in $n$ alone produces either a compression or decompression of the structures in the experimental angular distribution, while a variation of $\mathrm{k}$ alone produces a difference in phase of the observed angular distributions, which changes as $\theta$ approaches 180. The periodicity of this phase difference in. creases with increasing variation of $\mathbf{k}$ (cf. of $\sim 10$ ) intil (for $\delta k \sim 16$ ) the angular distributions are in phase throughout the measured angular range. Ar example where the variation of $\pi$ and $k$ act on the experimental angular distributions in an opposite sense to produce apparently little change in the positions of maxima and winina over the energy range 9230 to $12.60 \mathrm{Mk} 1 \mathrm{~s}$ the data for $14 \mathrm{~N}$ scattered by $12 \mathrm{C}$ where $\delta \mathrm{o}=14.4$ and $\delta \mathrm{k}=16.8$. That the optical madel is successful in reproducing such affects, qualitatively, is denonstrated by the four cases of Table 1 for which no searches were performed and another paranieter set was used, as well as the dotted curve for $14 \mathrm{~N}+13_{\mathrm{C}}$ at $9.649 \mathrm{MeV}$ in which the optical model parameters were the same as those used for $14 \mathrm{~N}+12 \mathrm{C}$ at $9.922 \mathrm{keV}$ (cf. Fig. Ia).

The optical maxel has proved to be successful in its abllity to reproduce the qualitative features of the $f$ ifter experimental angular distributions shom in Fig. $1 a$ and b. Therefore, such experimental angular distributions do rot find a unique interpretation in terus of a model wich requires the explicit inclusion of the elastic transfer charrel, The potentials of Table 1 shusld not be interpreted too 1iterally, however, as the experimental anguiar distributions analyzel are not of uniforn quatity; 9.8., excepting $14 \mathrm{~N}+{ }^{12} \mathrm{C}$ at 11.54 and $12.60 \mathrm{MeV}$, the points measured at forward angles are too sparse, or $14 \mathrm{~N}+12 \mathrm{C}$ at $10.84 \mathrm{MeV}$ where there seem to be problens of absolute nomalization of the data. A more thorough analysis should await more detailed neasure- 

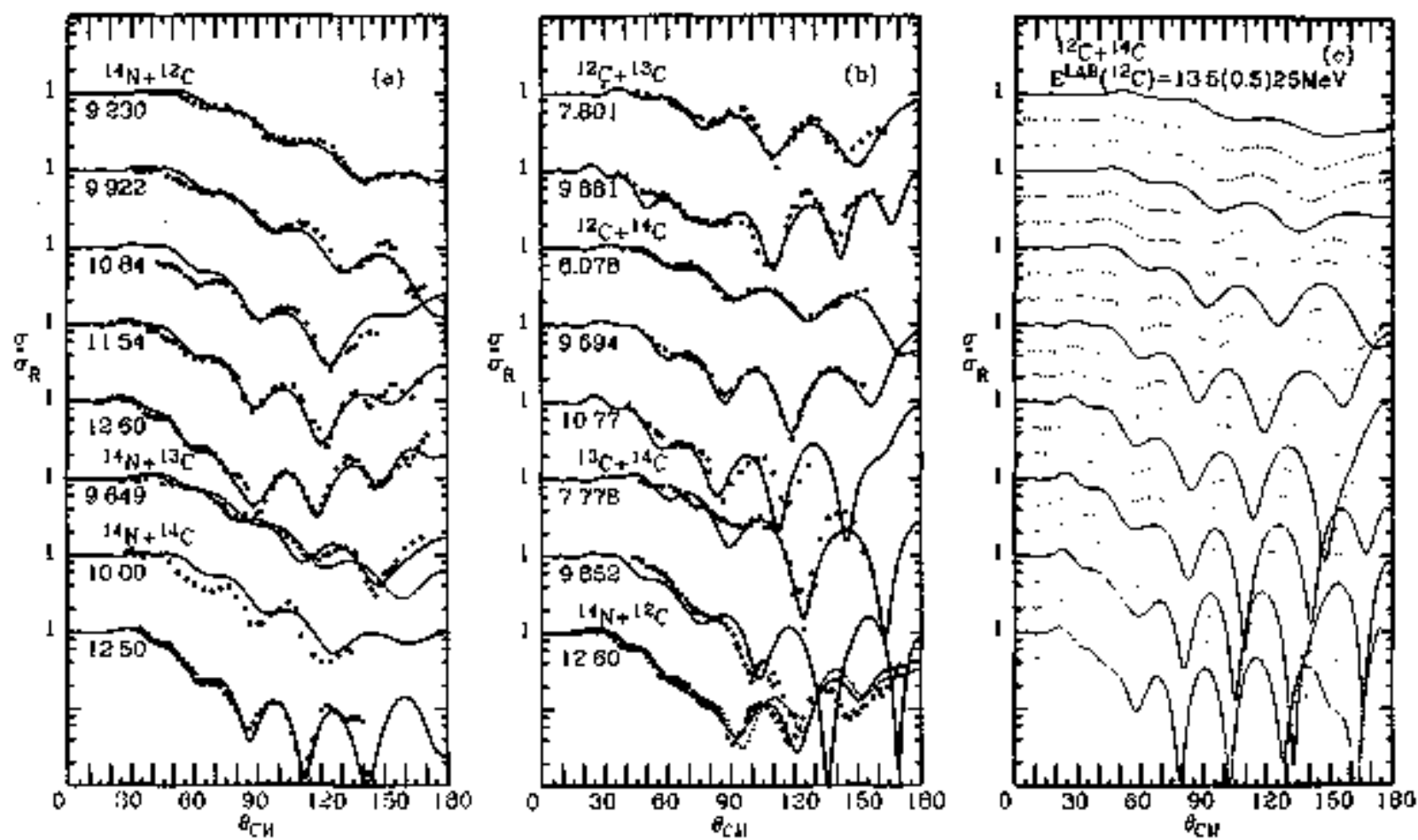

Fig. 1. Optical bodel predictions using the paraneters of Table 1 for (a) $N+C$, (b)

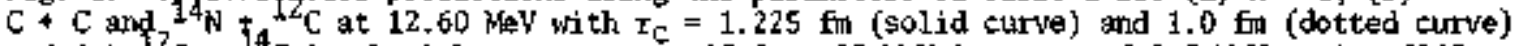
and $(\mathrm{C}){ }^{12} \mathrm{C}+{ }^{14} \mathrm{C}$ in the $\mathrm{lab}$ energy range 13.5 to $25 \mathrm{MeV}$ in steps of $0.5 \mathrm{HaV}$, using $\mathrm{C} 243$ parameters, with some curves dotted for clarity. In (a) and (b) the c.m. energy (in beV) is shown for each case-the data are trom Refs. l-4 (error bars are not shown) (KBL 754-870)

ments. An important qualitative result would seem clear, however: the heavy ion optical model potential for such systens has an inaginary part which produces sufficient reflected flux to allow for the possibility of interference with flux refracted by the real potential; this phenomenon provides a plausible interpretation for the stnuctures observed in the experinental argular distributions in heavy ion elastic scattering for systems such as those discussed here.

\section{Footnote and Referenceș}

"Condenșed from LBL-3492, publișhed in Phys. Rev. lett. 34, $1468(1975)$.
1. W. von Dertzen, Proceedings of the symposium on Heavy-Ion Scattering, Argonne National Laboratory, March 1971, ANL-7837.

2. W. von Gertzen, G. Bohlen, B. Gebauer, E. E. tabib, and N. Marquardt, Phys. Lett. 34B, 51 (1971).

3. H. 6. Bohlen and $H$, von Dertzen, Plys, lẹtt. $\underline{37 \mathrm{~F}}, 451(1971)$.

4. H. G. Bohlen, M. Feil, A. Gamp, B. Kohlmeyer, N. Marquardt, and W. von Oertzen, Phys. Lett. 41B, $425(1972)$.

5. W, von Oertzen and W. Wơnenberg, Nucl. Phys. $\underline{A Z B T}, 113(1973)$.

6. G. De1ic, Phys, Lett. $\underline{49 B}, 412(1974)$.

\section{COMPUTING THE FINITE RANGE DISTORTED WAVES BOFN APPROXIMATION FOR ${ }^{11} \mathrm{~B}\left({ }^{16} \mathrm{O},{ }^{15} \mathrm{~N}\right){ }^{12} \mathrm{C}$}

Georgen Delic

The FR DWRA code KNDDBY wap aptied to the study of the reaction $11_{\mathrm{B}}\left[\mathbf{1 6}, 1 \mathrm{~S}_{\mathrm{N}}\right) \mathrm{I} \mathrm{C}_{\mathrm{C}}$. This reaction is of particular interest in thet it can be interpreted in terms of two reaction mechenisms: proton transfer at forward angles and direct alpha cluster transfer at backward angles. The final cross sextion for the reaction would thus be expect. ed to include the effects of the coherent interference of the two react ton mechanisms. Previous studies 1,2 have analyzed scole of the available data 3,4 


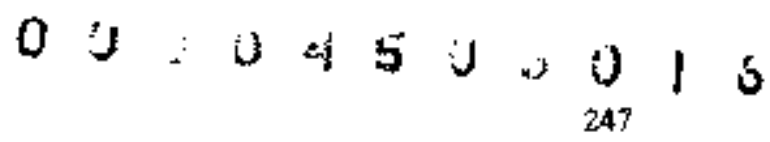

Table 1. Proton and alphe stripping for ${ }^{16} 0$ on ${ }^{11}$ B.

\begin{tabular}{|c|c|c|c|c|c|c|c|c|c|c|c|c|c|}
\hline $\begin{array}{c}E_{a d}^{a} \\
\text { OteV) } \\
\end{array}$ & $\begin{array}{l}15 \mathrm{~N}^{\mathrm{b}} \\
0 \mathrm{6} \\
\end{array}$ & $\begin{array}{l}1 \mathrm{c}^{\mathrm{b}} \\
\mathrm{rav} \\
\end{array}$ & $\mathbf{m}_{\mathrm{aA}}$ & $\begin{array}{c}\mathrm{k}_{\mathrm{ad}} \\
\left(\mathrm{m}^{-1}\right)\end{array}$ & $\begin{array}{l}a+h \\
a f\end{array}$ & tot & & $\begin{array}{l}b+b \\
\text { off } \\
\end{array}$ & $\mathbf{r}_{x d}^{d}$ & $\begin{array}{c}T_{b x}^{c} \\
\text { (fis) }\end{array}$ & $\begin{array}{c}\tau_{\mathrm{BA}}^{\mathrm{C}} \\
\text { (Af) }\end{array}$ & $\begin{array}{l}\Delta \mathbf{r}_{\mathbf{b B}} \\
\text { [fe] }\end{array}$ & $t_{t}^{d}$ \\
\hline 32.5 & 6.321 & B.s. & 4.419 & $2.0 \times 3$ & Do3z1 & $5.20 ?$ & 1.552 & c3in1 & $10.4 / 10.1$ & $10.0 / 9.5$ & $17.2 / 16.1$ & $1.42 / 4.3 t$ & $0,5,2,0$ or $2^{c}$ \\
\hline 50.0 & & & 4.598 & 1.953 & $\operatorname{Dos} 22$ & 5.473 & 1. Ito & C2nd4 & & & & & \\
\hline 27.0 & & & 4.848 & 1.955 & Bortz & 5.853 & 1.648 & Exinl & & & & & \\
\hline 27.0 & g.5. & 1.41 & & & $\mathbf{B O}_{2 J 1}$ & 5.796 & 1.821 & ¿tsi & $11.0,5$. & $11+2 / 8.2$ & $19.1 / 13.9$ & $1.59 / 3.69$ & $0,1,0,1,2,3,4$ \\
\hline 30.0 & t.s. & e-s. & 4.599 & 1.1553 & mozt & 4.261 & 2.263 & CEN4 & $10,4 / 5,4$ & $11.2 / 9.5$ & 19. $2 / 15.2$ & $1.57 / 4.28$ & $1,2 / 2$ \\
\hline
\end{tabular}

\footnotetext{
Bab बास्तr

Excitation.

Esat toxt.

dFor proton and alpha, respectivels.
}

in the FR DWBA but did not imvestigate the effects of coherent interference of the two regction mechanians. In a transfer reaction A $(a, b) B$ the DWEA transition mattrix amplitude at $\theta$ degrees (cmi is $T_{1}^{1}\left(M_{A}, M_{A}, M_{b}, M_{B}, \theta\right)$, where $M_{j}$ is the magnetic quantem muber associated with the spin projection of particle 1 . In the FR DWEA this anplitude contains a summetion over the alfowed values of the transferred orbital angular monentum $h_{t}$. For the transfer reaction $A(a, B) b$ at $\pi-B$ degrees $(m)$, the DWBA ampliturde is $T_{a_{B}}^{1}\left(M_{A}, M_{a}, M_{B}, t_{b}, \pi-6\right)$, both FR DWBA amplitudes contain a product of two spectroscopic amplitudes, ${ }^{5}$ specific to the transferred particle or cluster $x$ in the inttial and final boend state conflguration. Furthenore, where more than one type of configuration or cluster is allowed, either (or both) of the DHBA transition matriy amplitudes is a aum over such elusters $\mathrm{T}_{\mathrm{ab}}=\Sigma \mathrm{T}_{\mathrm{h}}$ and/or $\mathrm{T}-\Sigma \mathrm{T}_{\mathrm{k}}$; an example of the latter is the alpha cluster tookh to $\mathrm{Il}_{\mathrm{B}}$ to forn $1 \mathrm{FN}_{\mathrm{N}}$ in the $6.3 \mathrm{heV}$ state, where both $3 \mathrm{~s}$ and $2 d$ clusters are allowed. 6 The cross section for the coherent interference of the two reaction mechanims is the cross section formed fros the anpli-

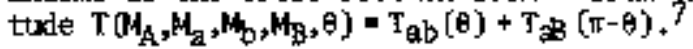

The trafsitions studied here were for the reactions induced by $160 \mathrm{om} 1 \mathrm{l}_{\mathrm{B}}$ for (i) the $6.324 \mathrm{MeV}$ state to ground state, (ii) g.s. to $4.44 \mathrm{MeV}$ state, and (iii)g.s. to g.s., respectively, in $1 \mathrm{~S}_{\mathrm{N}}$ and $1^{12}$. The first transition was studied at elab (160) $=32.5,30$, and $27 \mathrm{MeV}$, the second at $27 \mathrm{MeV}$, and the third at $30 \mathrm{MeV}$ boubarding energy. The proton bound strte configurations were the same as those of Ref. 3, and the bomd state alpha particle was asstmed to have the $p^{-s h e 11}$ cluster configurations of Kurath. ${ }^{2}$ The bound state paraneters for the proton were: radius $r_{0}=1.25 \mathrm{fm}$, diffuseness of $0.65 \mathrm{fm}$, spin orbit strength $12 \mathrm{Mel}+\mathrm{fo}^{2}$; for the alpha cluster the radius $\left(\mathrm{r}_{0}\right)$ and diffuseness paraneters were the same as those for the proton. The radius of the Woods-Saxon potential, binding the transforred particle $x$, was taken as $r_{0} A_{j}^{1 / 3}$, where $A_{i}$ is the mass number of particle $i$ to which $x$ is bound. The depth of the binding potential was adjusted to give the correct separation energy for particle $x$. The trur estion radii (cf, Table 1) corresponded to the bound state wave functions having a ragnitude $\sim 10^{-4}$. The elastic scattering of 160 on $11_{\mathrm{B}}$ has been discussed in Ref. 8 and that of $\mathrm{N}$ on $\mathrm{C}$ in Ref. 9. The 32.5 and $27 \mathrm{MeV}$ parameter sets of Ref. 8 are labeled here as $\mathrm{B} 0321$ and $\mathrm{B} 0271$. Two additional parameter sets yielding sonewhat improved fits, esperially forthe $32.5 \mathrm{HkV}$ data, were found with the optical model search code SOPHIE $32.5 \mathrm{keV}(B 0322), r_{V}=1.078$ fim, $a_{y} x 0.5660$ fin, $Y=70,42 \mathrm{MeV}, r_{W}=0.9810 \mathrm{fm}, \mathrm{a}_{W}=0.1509 \mathrm{fm}$. $\mathrm{w}=77.86 \mathrm{MeV} ; 27 \mathrm{MeV}(\mathrm{B0272}), \mathrm{r}_{\mathrm{Y}}=1.048 \mathrm{fm}, \mathrm{ar}_{\mathrm{Y}}=$ $0.6264 \mathrm{fm}, \mathrm{W}=75.34 \mathrm{HeV}, \mathrm{I}_{\mathrm{W}}=1.003 \mathrm{ftm}, \mathrm{t}=0.1269$ fin, $\mathrm{W}=71,49 \mathrm{MeV}$, whexe the definition of the potent tial is the same as that of Ref. 8. In the optical model the elastic scattering $A(a, a) A$ of any two $5 y^{\prime} s-$ tens for the same values of $n$ (Somerfeld parameter), $k$ (asymptotic wave number) and $R\left(-A_{a} 17 A_{A} 1 / 3\right)$ are the same. It therefore suffices to select potentials from Table 1 of Ref. 9 for the b-B chaniel in the reactions considered here. Since the effect of changes in $\pi$ and $k$ act on the elastic scattering angular distributions in an opposite sense, "where either perameter ( $n$ or $k$ ) in Table 1 of Ref. 9 is soballer (larger) thar that required here, the other is choser to be larger (다밀) by a similar percentage. The choice of owl parameters for the respective chamels together with the values of $n$ and $\mathrm{k}$ are given in Table 1 , the corresponding fits to the transfer reaction data as calculated by KIJDRY are show fi Fig. I. The unbroken curve corresponds to the cohsrent sum of the two reaction mechanisms; the dashed arves are protun transfer (at forward angles) and alpha cluster transfer (at backward angles). The bottan set of curyes for the transition from the $6.3 \mathrm{MeV}$ state in $15 \mathrm{~N}$ to the $\mathrm{g} . \mathrm{s}$, in ${ }^{12} \mathrm{C}$ at 27 WeV are calculated with the $\$ 1$ parameters of Ref. 3 for both channe1s: these parameters fitted the elastic $16_{0+11_{B}}$ date only at forward angles. A11 the calculated alphe cluster transfer cross șections contain the corresponding spectroscopic atpiftudes of Ref. 6 together with the center-of-mass correction factors of $\mathrm{Eq}+$ (9) of Ref. 6: no other nomalization factors are introduced. The proton transfer cross section is normalized to the data at forward angles; the factors by which the respective theoretical cross sections of Fig. 1 have been touttiplied are (in order from the top): $1.367,0.5252$, 


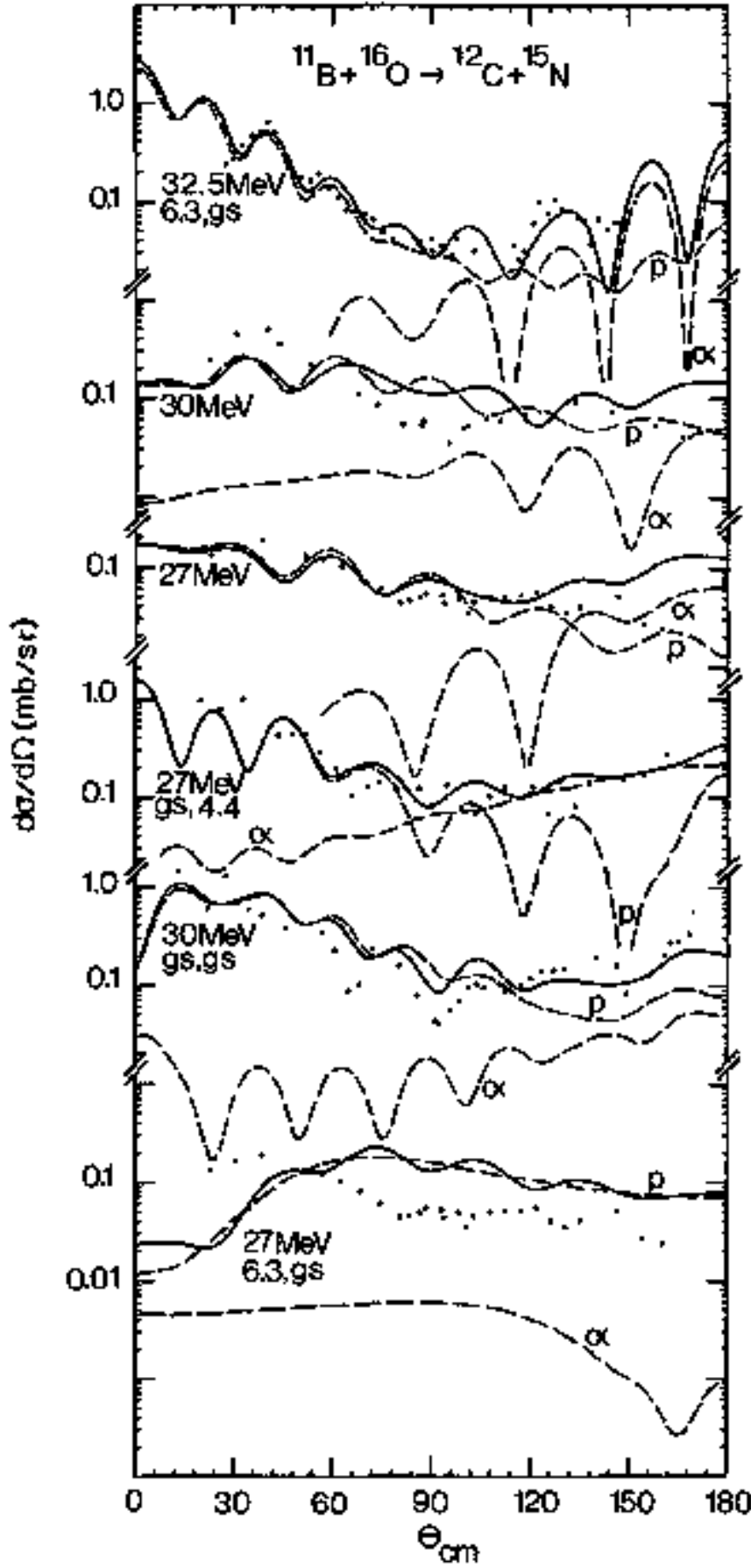

Fig. 1. Exact finite range DWBA calculations with KLWDRY for stripping of protons (at forward angles), and alphe clustors (at backward angles) are shown by the dashed arres denoted by $p$ and $a$, respective1y. The final cross section, formed from the coherent sum of the two reaction mechanisios, is show by the unbroken curve. laboratory ghergles of 160 and excitation energiess of ${ }^{1} \mathrm{~W}$ and ${ }^{2} \mathrm{C}$, respectively, are as shown. The bottom set of curves are for the parameters of Table 2 of Ref. 3. The data are those of Ref. 4; error bars are not shown.

(XBL 7511-9395)
$0.319 \mathrm{~g}, 0.2919,1.147,2.230$. Given the spectroscopic amplitudes (Ref. 10) for $11_{\mathrm{B}+\mathrm{p}}=12 \mathrm{C}$ (g.s.) )and $12 \mathrm{C}(4.4 \mathrm{MeV})$ respectively as -2.397 and $-1,048$, the presept analysis yields for $15_{\mathrm{W}}[\mathrm{g} . \mathrm{s})+.\mathrm{p}:-0.4821 \div 7$ ? and ${ }^{15} \mathrm{~N}(6.3 \mathrm{MeV})+\mathrm{p}$ : $-0.3634 \pm 35 \%$. These estimates igrore discrepancies betateen Ref. 3 and Ref. 4 in nomlalization of the data for the transitions to the excited states. It is seen from Fig. I that the ow paremetrization of Ref. 3 provides an inadequate description of these reactions. Calculations for the other two tronsitions with these paraneters (not shown) have forward peaked proton transfer cross soctions but underestimate the cross section for alpha cluster transfer by two orders of magnitude: no such discrepancy oocurs if ad parameters of the type discussed in Refs. 8 and 9 are used for the analysts of these transfer reactions.

Thus the present stuty shows that finite range Drah calculations successsulty predict the qualita tive feafures of the cross sections for the reaction

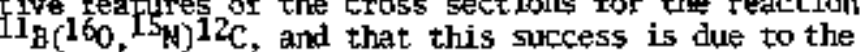
inclusion of coherent interferences between two reaction mechanisms, i.e., proton transfer at forward angles and alpha cluster at backward angles.

\section{Footrote and Reforences}

\section{Condensed from LBd-4343, published in Phys. Rev. Lett. 36, 569 (1976).}

I. T. Kanmuri and H. Yoshida, Nucl. Phys. A129, 625 (1969).

2. R, Bock and H, Yoshida, He1, Phys, AI89, 177 (1972).

3. U, C. Schlot thater-Voos, H. G, Bohlen, W, von Dertzen, and R. Bock, Nucl. Phys. A180, 385 (1972).

4. U, C. Schlotthauer-Voos, R, Bock, H. G. Bohlen, H. II, Gutbrod, and w. von Oertzen, Kuc1. Phys. Al86, $225(1972)$

5. D. Kurthth and I. S, Tower, Mucl. Phys. A222, 1 (1974).

6. D. Kurath, Phys. Rev. C 7,1390 (1973).

7. G. Delic, to be suluitted for publication.

8. G. Delic, Phys, Lett. 49B, 412 (1974).

9. G. De1ic, Phys. Rev. Lett. 34, 1468 (1975),

10. S. Cohen and D, Kurrath, Sucl. Phys. A101, 1 (1967). 


\section{$00 \because 4450 \omega 0+7$}

\section{FINITE RANGE DISTORTED WAVES BORN APPROXIMATION CALCULATIONS FOR ${ }^{13} \mathrm{C}\left({ }^{3} \mathrm{He}_{1}{ }^{8} \mathrm{He}\right)^{10} \mathrm{C}^{*}$}

George Delso and Ougter Kurathy

Experiments reported by Kachy et al 1 weasured angular distributions for the reaction $13 \mathrm{c}$ ( ${ }^{\circ} \mathrm{He}, \mathrm{o}_{\mathrm{He}}{ }^{10 \mathrm{C}} \mathrm{c}$ leading to the $\mathrm{J}^{\mathrm{T}}=0^{+}$, ground state and the $J^{\pi}=2^{+}, 3.35$ HeV state in 10 C for a 70.3 MeV 3 He bean. The experiments showed that both transitions, despite their low cross sections, were well resolved and displayed rather marked oscillatory structure indicative of a direct transfer reaction. The structures in the experinental differential cross setcions for the two transitions in phase at larger angles $\left(25-43^{\circ}\right.$ on) and were out of phase at maller angles $\left(7-25^{\circ} \mathrm{cm}\right)$. Furthermore, at the forward angles the observed strength to the $2^{+}$state in $10 \mathrm{C}$ was as much as 40 times greater than that to the $0^{+}$state. In an attent to describe these features of the dats Kachy et al.1 performed distorted waves Barn epproxination calculations and in their aralysis, to simp1ify the conputations, these authors assumed that. the interaction occurring in the expression for the DWBA transition matrix amplitute was of zero range. The results of the analysis showed a strong disagreement with experiment, particularly for the relative strengths of the transitions to the $0^{+}$and $2^{+}$states, respectively, in ${ }^{0} \mathrm{C}$.

Kashy et a1.1 concluded that nuclear structure considerations wold not sorrect the tiscrepancy with experiment predicted by the zerorrange DWBA anaIysis, and that the $1-\mathrm{C}\left(\mathrm{S}_{\mathrm{Hz}}, \sigma_{\mathrm{Hz}}\right]{ }^{1} \mathrm{C}$ reaction mechanism is probably not direct trinsfer. In the present study a fult finite range Dirk analysis, which included recoil effects exactly, was porformed and the results indicated that the reaction $13 \mathrm{c}\left({ }^{3} \mathrm{He}\right.$, ${ }_{\mathrm{He}}{ }^{10} \mathrm{C}$ can be interpreted as a direct cluster transfer to both final states in $10 \mathrm{C}$.

In the pick-up reaction $B(b, a) A$ the 3 transferred neutroms are assamed to be bound as a cluster to the mucleus b with orbital angular momentum $L$ and total angular maventum $J$, and to the nucleus $A$ with orbital angular momentum L and total angular momentum $J$. For the reaction ${ }^{13} \mathrm{C}\left({ }^{3} \mathrm{Ke},{ }_{\mathrm{He}}\right)^{10} \mathrm{C} \mathbf{J}=1 / 2$ and $\mathrm{I}$, the spin of the final mucleus ${ }^{10} \mathrm{C}$, is 0 or 2 . If $j$ denotes the total angular momentun of the cluster, and $Q$ and $\bar{Q}$ the number of oscillator quanta carricd in the relative wave functions for the cluster bound to ${ }^{10} \mathrm{C}$ and $3_{\mathrm{He}}$, respectively, then the cross section for the pick-up reaction $1{ }^{3}\left(^{3} \mathrm{He}, 6_{\mathrm{He}}\right)^{10} \mathrm{C}$, in the ab. serce of spin-orbit interactions in the optical model. potentials, is given by

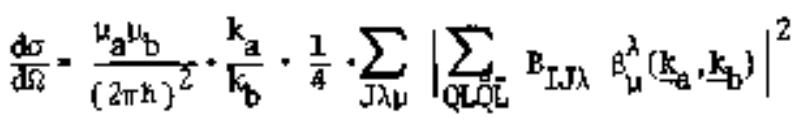

where the dependence of the cross section on the kinematics of the reaction is contained in the factor

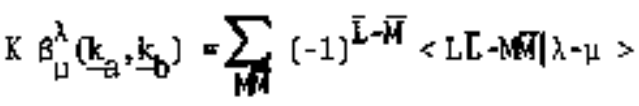

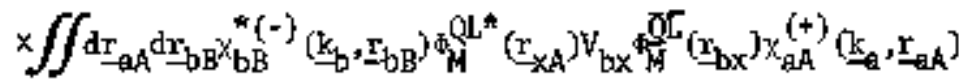

and the dependence on the nuclear structure in the factor

$$
\mathrm{B}_{\mathrm{IJ} \lambda}=\kappa \sum_{\mathrm{JC}} \mathrm{A}_{\mathrm{J}}^{*}(\mathrm{IjcQL}) A_{1 / 2}(\mathrm{JC} \overline{\mathrm{L}}) \omega\left(\mathrm{j} \frac{1}{2} \mathrm{~L} \lambda ; \mathrm{L} \mathrm{J}\right)
$$

The cross sections for the reaction can thus be expressed in terms of the $B_{\mu}^{\lambda}\left(Q L, \bar{Q},{ }_{L}\right)$, the form factor integrals of Eq. (2), by inserting the numer ical values of the $\mathrm{B}_{\mathrm{IJ}}$. For the $\mathrm{I}=0$ ground state of $1 \mathrm{~d} C$ only $\mathrm{J}=1 / 2$ with $\lambda=\mathrm{I}_{\mathrm{p}}=1$ contributes, whence

$$
\begin{aligned}
& \sum_{\mu}\left|\sum_{\mathrm{C} \mathrm{QL}_{\mathrm{L}}} \mathrm{B}_{\mathrm{D}} \frac{\mathrm{I}}{2} 1 \mathrm{~B}_{\mu}^{1}(\mathrm{OL}, \overline{\mathrm{OL}})\right|^{2}= \\
& 0.705 \sum_{\mu}\left|0.264 \beta_{\mu}^{1}(2 D, 1 P)-0.236 \beta_{\mu}^{1}(2 S, 1 P)+0.358 \mathrm{~g}_{\mu}^{1}(1 \mathrm{P}, 0 S)\right|^{2} \text {. }
\end{aligned}
$$


For the $3.35 \mathrm{MeV}, \mathrm{I}=2$ state, there is a contribution with $J=3 / 2$ and $\lambda=L_{p}=1$ and also contributions for $\lambda=\mathrm{L}_{\mathrm{p}}=2$ with $\mathrm{J} a \mathrm{~J} / 2$ and $\mathrm{J}=5 / 2$. Thus the swmmation of $\mathrm{Eq}$. (1) for this case becorees

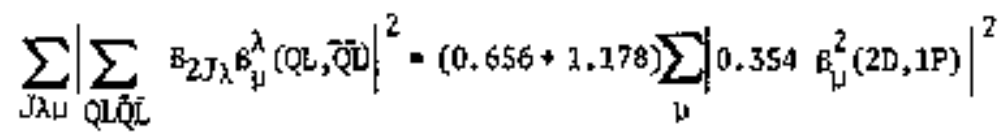

$$
\begin{aligned}
& 0.295 \sum_{\mu}\left|0.264 B_{\mu}^{1}(2 D, 1 P)-0.236 B_{\mu}^{1}(2 S, 1 P)+0.358 B_{\mu}^{1}(1 P, 0 S)\right|^{2} .
\end{aligned}
$$
In a zeromrange DrthA analysis only the
$\mathrm{B}_{4}^{\perp}(1 \mathrm{p}, \mathrm{OS})$ integral is nonvanisheng
(5). (5).

The optical model (ON) parameters deteruined for the ground state transition are given in Table 1 , and the corrosponding cross section is shown in Fig. 1. Also shown in Fig. 1 is the cross section calcuited for the $2^{+}$transition with the same or parameters. A1l finite range DWEA calculations reported in this study were performed with the code KURPRY, 2

In the parameter searches for the ${ }^{6} \mathrm{Hz+}{ }^{10} \mathrm{C}$ channel good fits to both transitions with the same on parameters were not found. However, it is passible to fit the shape and magnituda of the $2^{+}$cross section with the (D) paranetexs given in the last line of Table 1, as shown in Fig. 2. Also shown in Fig. 2 are the seperate contributions for the tho values of the transferTed orbital angular motentum, $\lambda$. As can be seen from Fig. 2 the $\lambda=2$ contribution, correspording to the $\mathrm{B}_{\mathrm{i}}^{\mathrm{L}}[\mathrm{2D}, 1 \mathrm{P})$ term of $\mathrm{Eq}$. (S), dor- inates the cross section. This feature of the finite range DhBA calculations is true for all our OM parameter variations. As is manifest in Fqs. (4) and (5) the shape of the grownd state cross section is the sane as that of the $h=1$ contribution to the $2^{+}$cross section. Furtherwore, the ragritude of the latter should equal 0.418 times the ground state cross section (this ratio is calculated to ber 0.55 rather than 0.418 due to the difference in Q-values). These relationships can be used to find the meak $\lambda=1$ contribution to the $z^{+}$cross section of Fig.1 or to find the $0^{+}$cross section from Fig. 2,

Thus, while the difference in 04 parameters between the last two lines of Table $I$ is greater than wovld normally be oxpected on the basis of differences in energy of the outgoing ${ }_{\mathrm{He}}$ in the two transitions, the analysis indicates that one could seloct a set of on paraneters in the range spanned by these limiting values, Stich a thoice would then give crude fits to cross section shapes for both transitions and still display the dominance of the $2^{4}$ transition magnitude which is found at both limits of the oN paranetrlzation.

Table 1. Optical model parameters for ${ }^{13} \mathrm{C}\left({ }^{3} \mathrm{He},{ }^{6} \mathrm{He}\right)^{10} \mathrm{C}$. The potential

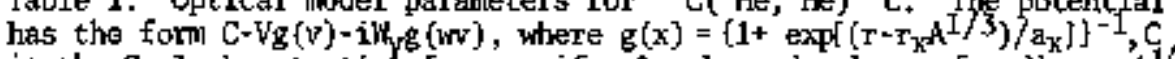
is the Coulonb potential for a umifomily charged sphere of radius $r_{c} A^{A} 1 / 3$ and $A=13$ and 10 , respectively, for the initial and flnal chamels.

\begin{tabular}{|c|c|c|c|c|c|c|c|c|}
\hline Nuclei & $\begin{array}{l}E^{\mathrm{Gl}} \\
\underline{(\mathrm{MeV})}\end{array}$ & $\begin{array}{c}\mathbf{r}_{\boldsymbol{v}} \\
(\mathrm{fm}) \\
\end{array}$ & $\begin{array}{c}\mathrm{a}_{\mathrm{v}} \\
\text { [fm }]\end{array}$ & $\begin{array}{c}V \\
(\mathrm{MeV}) \\
\end{array}$ & $\begin{array}{l}\mathbf{T}_{\text {WY }} \\
\text { (fim) }\end{array}$ & $\begin{array}{l}a_{W V} \\
\text { (EA }\end{array}$ & $\begin{array}{r}w_{v} \\
\text { (nev) }\end{array}$ & $\begin{array}{c}T_{c} \\
\text { (fm) }\end{array}$ \\
\hline $3_{\text {fes }}{ }^{13} \mathrm{C}$ & 57.07 & 1.240 & 0.6650 & 125,0 & 1.240 & 0.6400 & 20.00 & 1.260 \\
\hline${ }^{6} \mathrm{He}_{2}+{ }^{10} \mathrm{C}\left(\mathrm{O}^{4} \mathrm{~J}\right.$ & 41.82 & 1.650 & 0.9500 & 175.0 & 1.400 & 0.6400 & 35.00 & 1.260 \\
\hline$\left(2^{+}\right)$ & 38.47 & 1.460 & 0.7300 & 195.0 & 1.460 & 0.6400 & 50.00 & 1.260 \\
\hline
\end{tabular}




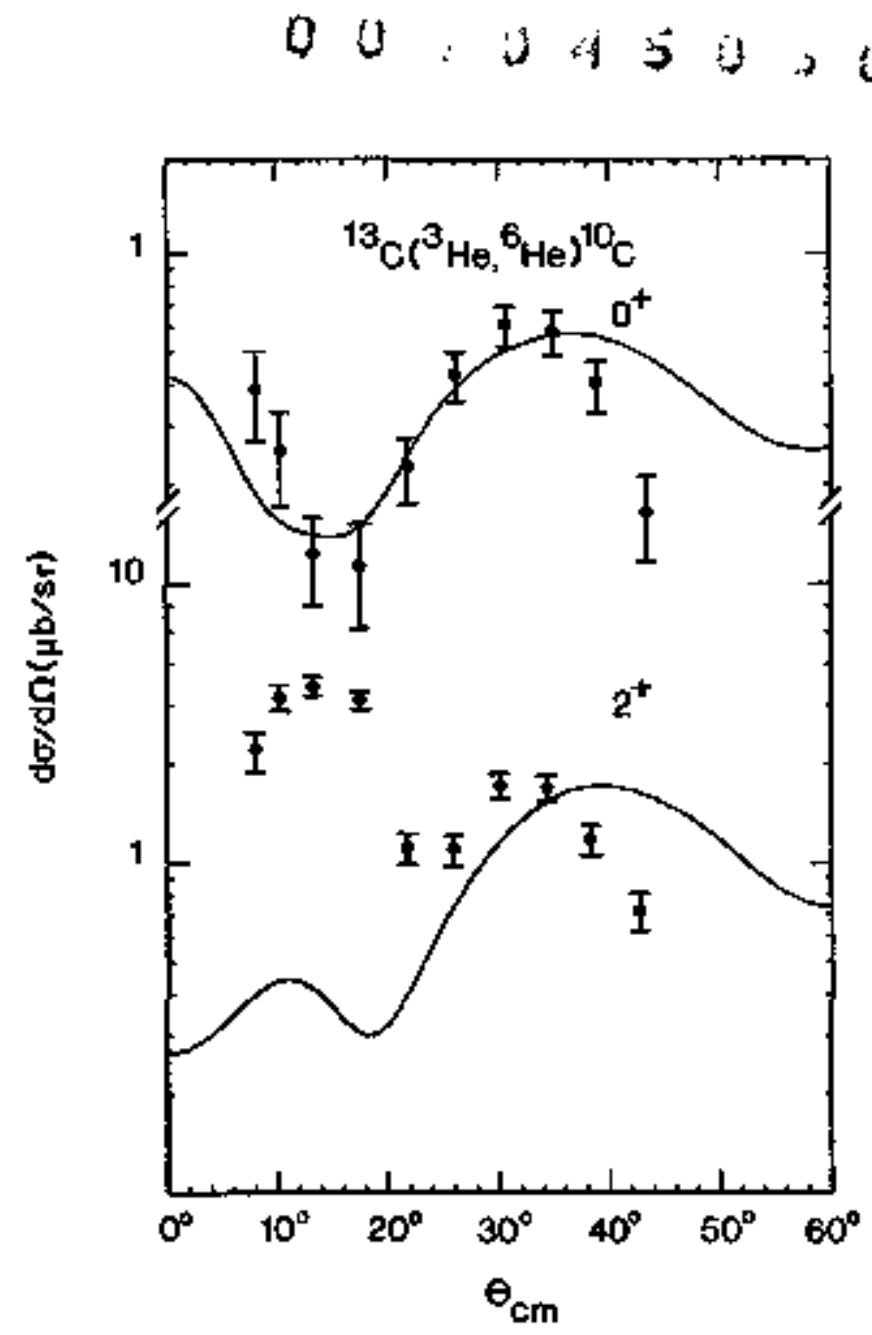

Footnotes and References

*Condensed from LEL-4364, published in Phys. Rev, Cl4, 519 (1976).

†hysics Division, Argonene National Laboratory, Argotrne, Ill.

1. E. Kashy, W. Benenson, I. D. Proctor, P. Hauge, and G. Bertsch, Phys, Rev, C7, 2251 (1973).

2. G. Delie, Phys, Rev. Lett, 36, S69 (1976).
Fig. 1. Exact finite range DWPA calculations for the reaction $13 \mathrm{C}\left({ }^{3} \mathrm{He}, \mathrm{H}_{\mathrm{He}}\right){ }^{10} \mathrm{C}$ for a $70.3 \mathrm{keV}{ }^{3} \mathrm{He}$ beam. The upper curve $1 \mathrm{~s}$ the transition to the $0^{+}$ ground state in $I 0_{C}$, and the lower curve the transttich to the $2^{+}, 3.35 \mathrm{MeV}$ state in $10 \mathrm{C}$ for the same set of optical paraneters in the $\mathrm{He}^{+}+{ }^{10} \mathrm{C}$ chanjel (the parameters are those given in the second line of Table 1). The data are those of Ref. 1.

[XEL 761-339]

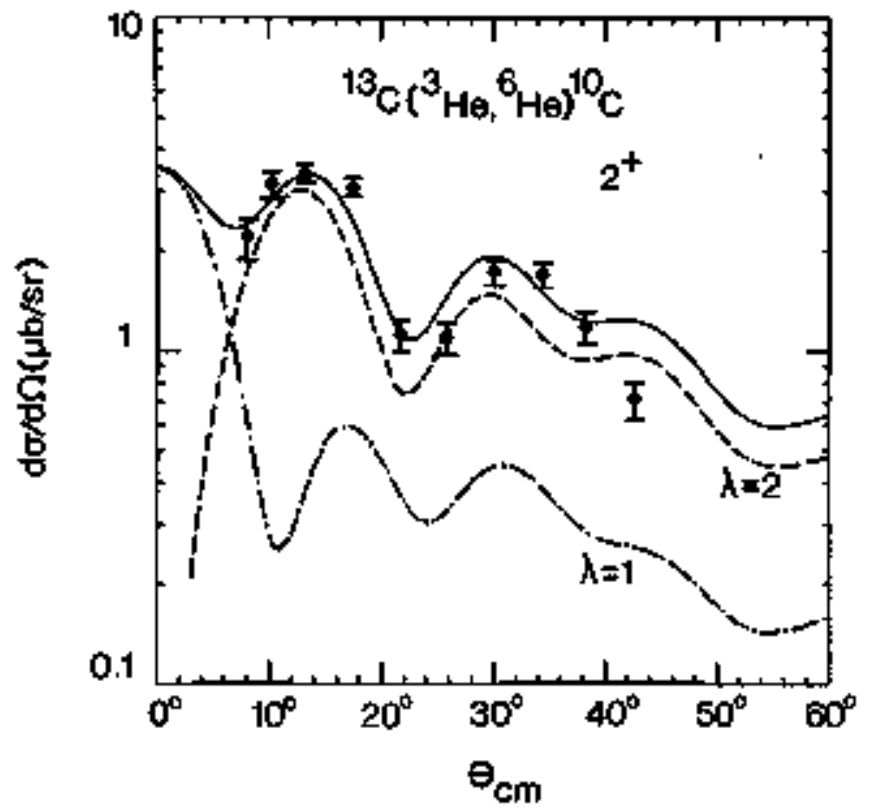

Fig. 2. Exact finite range DWFA calculaticns for the reaction $13{ }^{3} \mathrm{C}\left({ }^{3} \mathrm{He}_{\mathrm{e}},{ }^{6} \mathrm{He}\right)^{10} \mathrm{C}$ leading to the $2^{+}, 3.35$ NeV state in $10 \mathrm{C}$ for a $70.3 \mathrm{MeV} \mathrm{HF}_{\mathrm{Fe}}$ beall. The optical nodel parameters for the ${ }^{6} \mathrm{He}+{ }^{10} \mathrm{C}$ chamel are given in the thind line of Table I. The broken curve is the $\lambda=2$ component of the cross section and the dashed and dotted curve is the $\lambda=1$ compment. The final cross section ts the unbroken eltrve. The data are those of Ref. 1.

(XBL, 761-338)

\section{Macroscopic}

\section{A THEORETICAL APPROACH TO THE PROBLEM OF PARTIAL EQUILIBRATION IN HEAVY ION REACTIONS*}

L. G Morefloł and J S Svenlek

Many heavy ion reactions recently studied in our grop ${ }^{1-5}$ seem to indicate various stages of relaxation in the collective degrees of freadon excited in the process. In particular, a large fraction of the total cross section is associated with the production of fragments which, if have fully relaxed kinetic energy spectra; ii) portray a mass (charge) distributlon not wholly consistent with statistical equilibrium; iis) have angular distributions which are fornard peaked, with the forward peaking largest for $Z^{\dagger} s$ near the projectile and decreasing for $Z$ 's farther renoved from the projectilo. 
These general features appear to be consistent with the following qualitative picture: i] prompty after the initial collision, friction brings the two nucli into rigid contact with each othor, while the initial kinetic energy is dissipated into the interna1 degrees of freedon; a rotating intermediate conplex of well defined mass asymmetry is formed: ii) a diffusion process, corparable in rate to, or slower than, the rotation of the intermediate conplex, leads to the exchange of particles betwoen the two toukhing fragments, thus generating a timedependent distribution in the asymetry of the intermediate complex: $\mathbf{i i j )}$ the complex decays with a time constant comparable to or shorter than the rotational periof?.

The central festure of the present model is the diffirsion of the intermediate complex along the asylumetry degres of freeton. We use the Master Equation approach to describe the time-dependent population $\phi z[t]$ of systeras whose asymetry is characterized by the atomic numer 2 of ofe of the fragments:

$$
\phi_{z}=\Sigma_{z},\left(\Lambda_{z z}, \phi_{z},-\lambda_{z}{ }_{z} \phi_{z}\right)
$$

where $\dot{\phi}_{z}$ represents the time derivative of $\phi_{z}$ and $\mathrm{A}_{\mathrm{zz}}, \mathrm{A}_{\mathrm{z}^{\prime} \mathrm{z}}$ are the macroscopit transition probabilities between the systems whose asyntwetrles are deseribed by $z$ and $z+$.

The macroscopic transition probabilities can be wrikten in teruls of the nicroscopic transition probabilities and of the level densities of the Hacroscopic states:

$$
\begin{aligned}
& \lambda_{z z^{\prime}}=\lambda_{2 z^{\prime}} \rho_{z} ; \lambda_{2^{\prime} z}=\lambda_{z^{\prime} z} \lambda_{z^{\prime}} ; \\
& \lambda_{2 z^{\prime}}=\lambda_{z^{\prime} z^{\prime}}
\end{aligned}
$$

The symetry of the $\lambda^{\prime} s$ stems from microscopic reversibility. The quantities $\rho_{2}$ represent the level densities of the intersediate conplexes. These level densities can be written down in terms of the potential energy of the intermediate complex $v_{z}$ neasurad with respect to the rotating ground state,

$$
\rho_{z}=\rho\left(E-V_{z}\right)
$$

Where $\mathrm{E}$ is the enorgy of the systor also measured with respect to the rotating ground state and $\rho(x)$ is the functional form for the level density.

Since $\lambda_{z z}$, must be the order of $\frac{V_{F}}{D}$ (where $D$ is a typical linear size of the system and $V_{F}$ is the Fermi velocity of the muteons), the $\lambda_{2} z^{\prime}$ decrease as the level densities increase. For lack of better knowIedge, we assume:

$$
\lambda_{22}=\frac{\lambda_{0}}{\left[\rho_{z} \rho_{2}\right]^{1 / 2}} \text {. }
$$

The tevel densities can be expandet as follows:

$$
\rho\left(E-V_{z}\right)=\rho(E) e^{-V_{z} / T}
$$

where

$$
T^{-1}=\left.\frac{d \ln \rho(x)}{d x}\right|_{x=E-V_{z}}
$$

and the mastor equation can be rewritten as:

$$
\dot{\Phi}_{z}=\lambda_{0} \sum_{z^{\prime}} \mathrm{e}^{\left(V_{z}+V_{z}+\right) / 2 T}\left[\phi_{z^{\prime}} e^{-V_{z} / T}-\phi_{z} e^{-V_{z} / T}\right] \text {. }
$$

This equation is certainly correct in the long time limit when $\dot{\phi}_{2}=0$. A sufficient condition which satisfies $\phi_{\mathrm{z}}=0$ is that each term of the sum is zero. This gives:

$$
\frac{\Phi_{Z^{\prime}}}{\Phi_{z}}=\frac{\rho_{z}{ }^{\prime}}{\rho_{z}^{\prime}}=\mathrm{e}^{-\mathrm{V}_{z^{\prime}} / \mathrm{T} / \mathrm{e}^{-V_{z} / \mathrm{T}}}
$$

which expresses the equilibrium condition.

An important assulption in the present roodel is that the sim in Eq. (1) and Eq. (7) is extended only to $Z^{\prime}=Z \pm 1$. In other words, we assume that there is no correlation among transferred particles. We also assume that, at least for the rather 1ight systems which we have considered, the shape of the intermediate cormlex actually corresponds to a configuration of two touching spheres.

The angular momentum car in principal affect the shape of the intermediate complex and, consequently, the value of the potential energy. We write the potential emergy as follows:

$$
\begin{aligned}
\left.v_{\left(x_{i}\right.}\right)-v_{L D}\left(x_{i}\right) & -v_{L D}\left(x_{i}^{0}\right) \\
& +\frac{2^{2} h^{2}}{2}\left[\frac{1}{V\left(x_{i}\right)}-\frac{1}{g\left(x_{i}^{0}\right)}\right]
\end{aligned}
$$

where the $x_{j}$ is the set of coordinates chosen to describe the shape of the system; $x_{i}^{0}$ are the corresponding values for the ground state; $V_{1 D}$ is the liquid drop energy and of is the monent of inertia.

An example of the potential energies of the systend $\mathrm{Ag}+\mathrm{Ar}$ for various values of angular momentum is shown in Fig. 1 (a)

The diffusion constant $\lambda_{0}$ should also depend on the shape of the intermediate complex. We have considered it to be proportional to the interaction area of the two touching spheres, which, in turn, is proportional to their rediced raditis:

$$
\lambda_{0}=\text { 2r } \frac{R_{1} R_{2}}{R_{1}+R_{2}} k
$$

where $R_{1}$ and $R_{2}$ are the radii of the two touching
spheres. 


\section{$00 . y+5 y+319$}

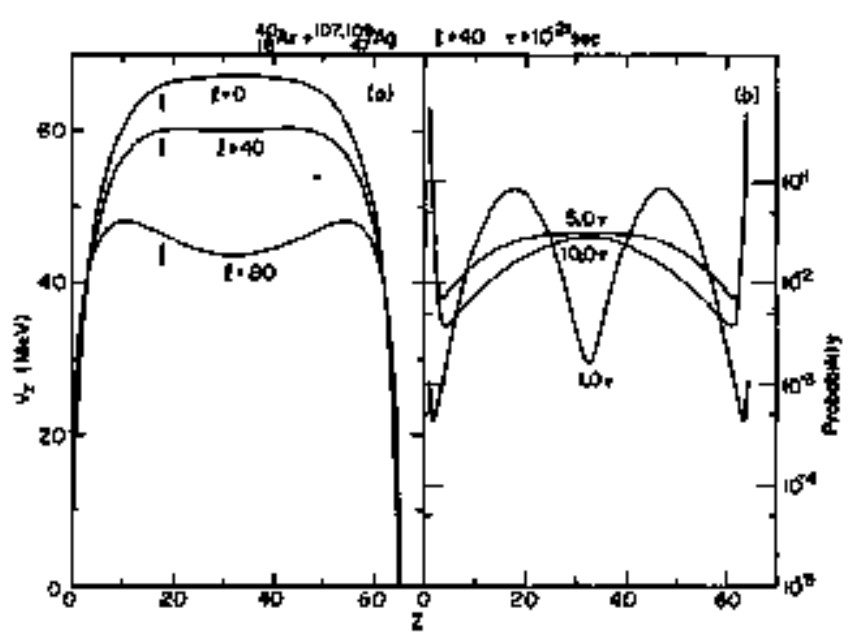

Fig. 1. (a) Potential energy of the intermediate complex as a function of $Z$ for three l-waves. (b) probabilities $\phi_{z}(t)$ as a function of $Z$ at three different times for the $\ell=40$ collision. For short tines the distribution reserbies a Gaussian as expected in a randion walk problen. For longer times, the drift is more visible.

(XEL 753-2475)

A calculation of the populations $\phi_{z}$ at various times after the beginning of diffusion is shown in Fig. I(b). The calculation of the cross sections and angutar distributions of the fragnents requires that at loast some essential part of the dynamics associated with the reaction be taken into accoumt. He assume that, after collision, target and projectile slip one over the other by an angle $\theta_{\mathrm{S}}$ proportional to tho initial tangential velocity at the time of collision. As the slippage takes place, the kinetic energy is dissipated. After the slfppage, the systen rotates with a rigid monent of inertia. Furthermore, we assime that, after a given contact time $t$, the fragment is emitted at an angle $\theta$ with a new $Z=Z_{\text {exit. }}$ This information allows one to calculate the sot of impact parameters $b$ which satisfy the above conditions. The final cross section is given by:

$$
\frac{d \sigma_{z}}{d n}=\int_{0}^{\infty} \frac{d t}{\tau} e^{-t / \tau} \frac{\sum_{b} \phi_{z}(b, t)}{\mid \frac{b P(b)}{\sin \theta \frac{d \theta}{d b}}}
$$

where $\tau$ is the mean life of the intermodiate cowplex and the sum contained in the integrand is carried over all the values of $b$ which, after $a$ time $t$, lead to the enission of a fragment of atomic number $Z$ at a center-of-mass angle $\theta$.

The quantity $P(b)$ or $P(x)$ at a given borbarding energy is another quantity that cannot be deternined on theoretical grounds as yet. It represents the probability $0<\mathrm{P}$ \& 1 that a given t-wave will lead to a reaction of the kind described in this paper. It seems safe to assume that $P(R)$ will be substantially different from zero for rather large l-waves, since the low knaves are certainly associsted with the for- thation of the compound nucleas and thus responsible at least for the cross section of the evaporation residue. On the other hand, the highest l-waves are associated with direct or quasi-elastit cross sections and, therefore, are not expected to be invalved in the relaxed cross section studied here to any great extent. It is possible to construct a fairly satisfactory expression for $P(x)$ from experiment.

In the present paper have trited to reprodice the experimental data obtalned for the reaction Ag + 288 WeV Ar. The function $\mathrm{P}(\mathrm{l})$ which we have used has a lower bound tain defined by 6 the evaporation residue cross section of $670 \mathrm{mb}^{6}$ and an upper bound inax defined by the sur of the evaporation residue cross section and the relaxed cross section, which is $\sim 1700 \mathrm{mb}$. Therefore,

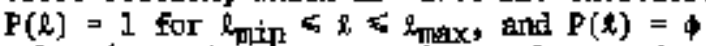
otherwise. At the same time we have made the rather bold assugmption that no sizable amount of fission is actually present in the cross section.

In Fig. 2 the calculated angular distributions of the fragments are plotted for atomic numbers of $Z=6$ to $Z=32$. The fragdents with $2 \times 18$ have a forward peaked angular distribution, the forwart peaking being more entanced for atonic numbers close to the projectile. The fraguents with $\mathrm{Z}>18$ show angular distributions which quickly become asymuetric about $90^{\circ}$ as the atomic number is farther removed fron $Z=18$, In fact, for atomic numbers close to symmetric division, the angutar distribution becomes indistinguishable from $1 /$ sing. This result suggests that, oven when particles are enitted with a $1 / \sin \theta$ distribution, one should not draw the inmediate conclusion that a compound nucleus has been formed.

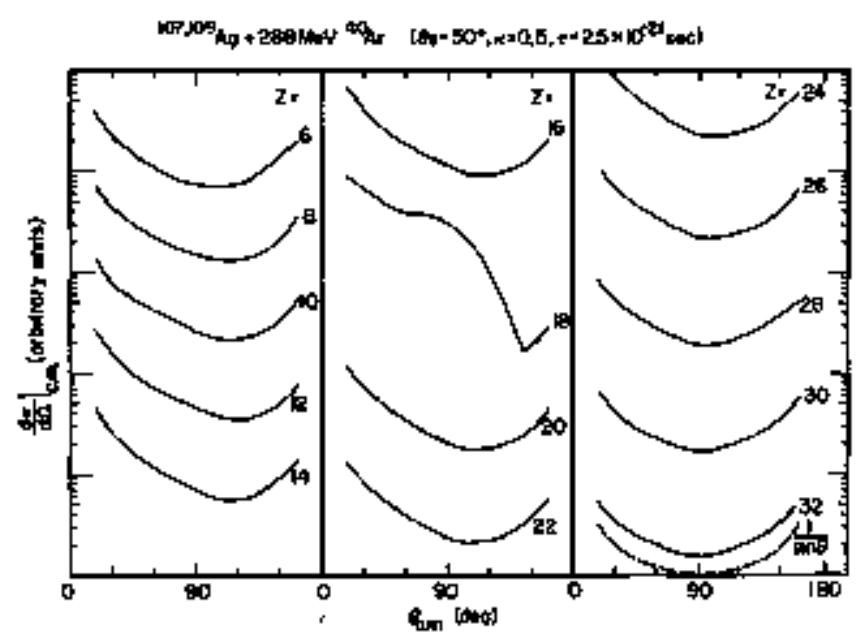

Fig. 2. Caloulated center-of-mass angular distribution for various oxtt chamels using the model described in the text. The nain feature is the non-negIigible forward peaking which decreases as the 2 of the exit particle is further retroved from the $Z$ of projectile. The anglular distributions approach 1/sine for exit changels far rewoved from the projectile.

(XBL 753-2472) 
In Fig. 3 the comparison betwen experiment and theory is show. It is important to notice that no normalization has been perr forms. The shapes of the angular distributions are reported with renarkable accuracy, while the absolute values are reporduced within better than 50 s. No allowance for shell effects has been made in the evaluation of the ridge line potential, nor has the effect of secondary decay been accounted for.

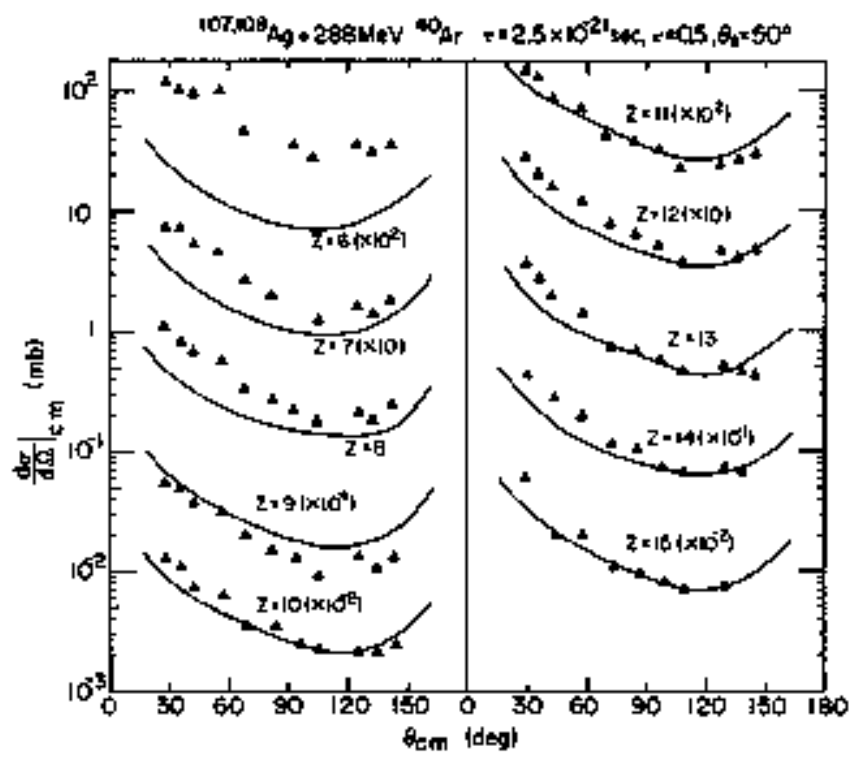

Fig. 3. Absolute comparison between the experimantal and the theoretical angular distributions using the mode1 described in the text. The values of the constants used are given in upper right hand comer of the figtre. The constant has the dimensions $\mathrm{fta}^{-1} 10^{21} \mathrm{sec}^{-1}$.

(XBL 753-2473)
A nore extensive analysis of the available experimental data is in progress. An attoupt is also being rogde to treat the thermalization of the kinetic energy and the particle diffusion oscurring prior to the thersolization by means of a generalized theory.

\section{Footnotes and References}

"Condensed frot LBL-3443.

t\$loan Fellow, 1974-1976.

1. L. B. Moretto, D. Hememarn, R. C. Jared, R. C. Gatti, and S. G. Thompson, Third Symposium on the Fhysics and Chemistry of Fission, August 1973, Rochester, NY, USA, IAEA-SA1-174/75.

2. S. G. Thonpson, L. G. kretto, R. C. Jared, R. P. Babinet, J. Galin, M. M. Fowler, R. C. Gatti, and $\rfloor$. B. Humter, Nobet Syaposium on Superheavy Elenents, June 1974, Ronneby Brun, Sweden, LRL-2940 (1974).

3. L. G. Moretto, S. S. Kataria, R. C. Jares, R. Schumitt, and S. 6. Thompson, Nusl. Phys. A255, $491(1975)$.

4. R, P. Babtnet, L. G. Mretto, J. Galin, R. C. Jared, J. Moulton, and S. G. Thompson, Mucl. Phys. A258, $172(1976)$.

5. J. Galln, L. G. Moretto, R. P. Babinet, S. Schmitt, R. Jared, and S. G. Thompson, Kuel. Phys. A255, 472 (1975).

6. H. H. Gutbrod, F. Plasil, H. C. Britt, B. H. Erkkila, R. H. Stokes, and M. Blann. Third Symposium on the Physics and Chemistry of Fisstion, August 1973, Rochester, NY, USA, IAEA-SM-174/59.

\section{DIFFUSIVE PHENOMENA REFLECTED IN THE CHANGE AND ANGULAR DISTRIBUTIONS OF N, Ne, Ar, Kr INDUCEO REACTIONS"}

A treatment which describes the time ewolution of a probability distribution along a given coordinate is offered by the haster Equation. Me have applied this equation to the problen of diffusion along the mass asymotry coordinate. 1 let $\phi\{z, t)$ be the probability distribution associated with a configuration of two touching fragnents, one of which has atovic mober $Z$ (we assume equilibration in the neutron-to-proton ratio and we label the asymnetry by $z$ since this is the quantity measure for each frapment). The Master Equation tan then bo written as:

$$
\phi(z, t)=\sum_{z}^{\prime \prime} \lambda_{z 2},\left(\phi_{2} p_{z}-\phi_{z} \hat{\rho}_{z}\right)
$$

where $\lambda_{z z 1}=\lambda_{z+z}$ is the microscopic transition probsility, $p_{z}$ and $p_{z}$ are the level densities associated with ${ }^{2}$ the asymotries $Z$ and $Z^{\prime}$.
Calculations of the probabilfty distributions have been performed for some of the reactions studied experínentally. ${ }^{2}$ An exald Fig. 1.

The angular distributions, being so sensitive to short interaction times, give the first indication of the noncomound nucleus nature of tho relaxed cross section. This was especially true for the reactions induced by Ar and lighter projectiles, or for that matter, for the reaction of $\mathbf{K r}_{\mathbf{r}}+\mathrm{Ag}$, were the charge distributions gave only an ambigucus answer regarding the degree of equilibration along the mass asyounetry coordinate.

In these reactions the center-of-miss angular distributions appear to be gemerally forward peaked, especially for fraglents close in $Z$ to the projectile. 


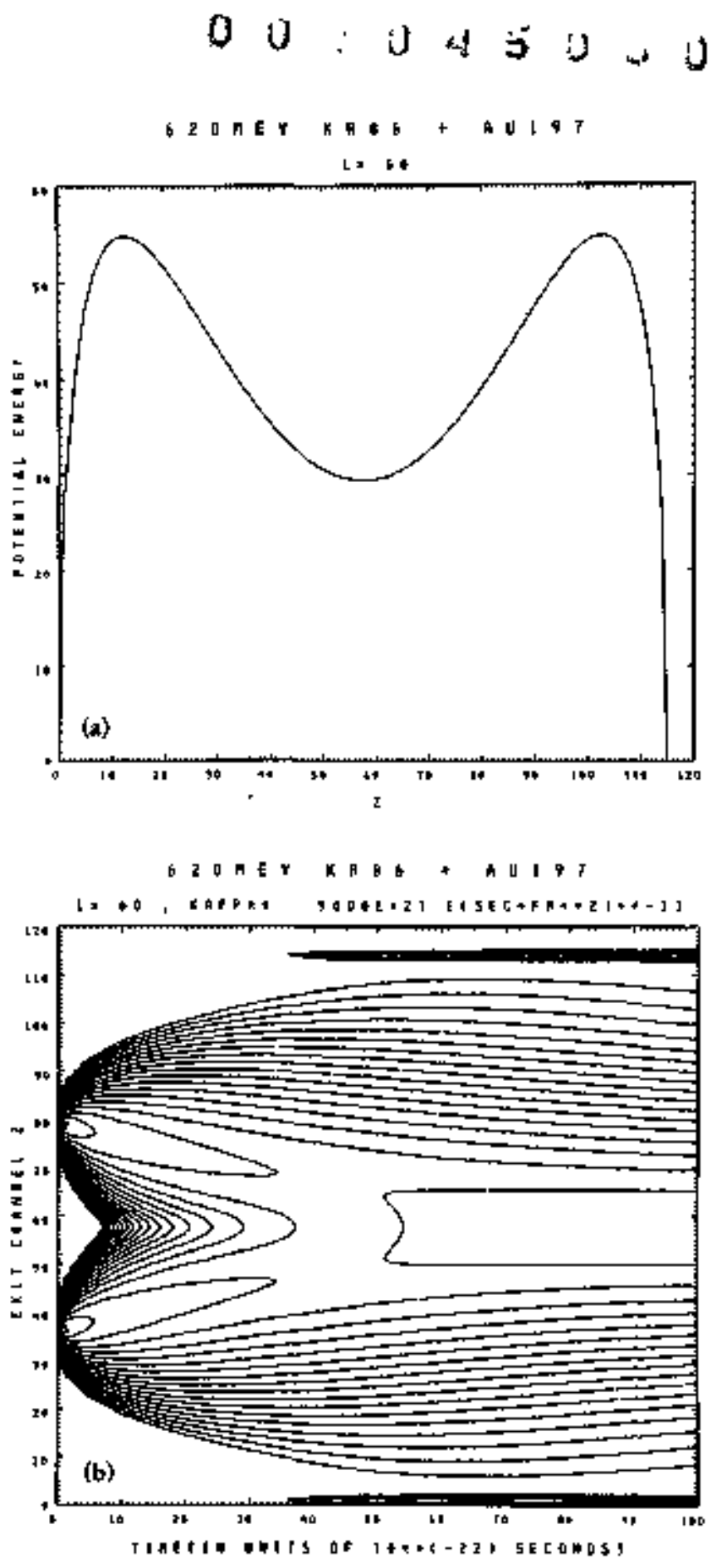

colrse, must be reletively short with respect to the mean rotational period; not too short though, otherwise the systen could not rotate enough to cross the $0^{\circ}$ line and would give rise to a side peak; not too long, otherwise the angular distribution wovld become symetrized about $90^{\circ}$.

In atz of these reactions, the forward peaking is stronger for fragments closer in $z$ to the projectile and decreases for fraguents substantially resooved fram the projectile. This phenomenon finds its qualitative explamation in the increasing time lag, introduced by the diffusion process, in the population of configurations farther and farther removed in mass asymetry from that associated with the target-projectile conbination (injection asymetry). In this way, fiagments close in $z$ to the projectile are rapidly populated by the diffusion process and cen rapidly decay, thus generating a substantially forwardpeaked angular distribution. Fragnents farther removed in $\vec{Z}$ from the projectile are populated on a longer time scale, so that their decay time is delayed. Such a delay aflows the system to rotate for a longer tine, which results in an increased tendency for the angtular distribution to become nore and more symetric at aboutt $90^{\circ}$.

When the reactions of $\mathrm{Kr}$ in heavy targets were first observed, the sharphess of the mass distributions associated with the side peaking of the gross angular distributions appeared to be so extraordinary that they were thought to be a completely new mechanisw, which was named quasi-fission. Yet, this process resenbles the deep inelastic processes described above in many respects. Howrerer, it seers that there is a continuous connection between the angular distributions observed in reactions induced by lighter projectiles (or by heavy projectiles on relatively light targets, $1 \mathrm{ke} \mathrm{Ag}+\mathrm{Kr}$ ], and the angular distributions observed in heavy projectile-heary target reactions. The side peaking in the gross angular distribution of the products reflects a very short interaction tine associated with a few $Z^{\prime}$ s about the projectile. However, if one were to look at the angular distributions of individual Z's, one should observe a progressive change townids forward peaking as one noves away from the $Z$ of the projectile. Examples of the cross sections and energles are given in Fig. 2 . The angular distributions for individual atomic mmbers, resolved up to $2=50$ are shown in Fig. 3. The effect we were looking for indeed appears with astounding clarity. Close to $z$ - 36 a very sharp side pealt is observed (for the 2 's closest to the projectile the separation of the quasi-elastic componemt appeared to be impossible so that soare of the points close to the maximum of the peak are actually skipped). Such a side peak implies an interaction time so short that the intermediate coaplex does not have time enough to rotate past $0^{\circ}$. However, as one Joves away in $Z$ from the projectile, the progressively longer time delay imposed by diffusion allows the intermediate complex to reach closef to $\sigma^{\circ}$, and eventually to reach past $0^{\circ}$. This results in a rapid filling in of the angles close to $0^{\circ}$, which slowly transforms the side peak first into a shoulder and later into a forward peak. In du+KI the side peak becomes a shoulder symmetrically about $z=36$, at $z \approx 30$ and at $z=41$. The shoulder disappears around $z=24$ and $z=46$ where the angular distribu- 


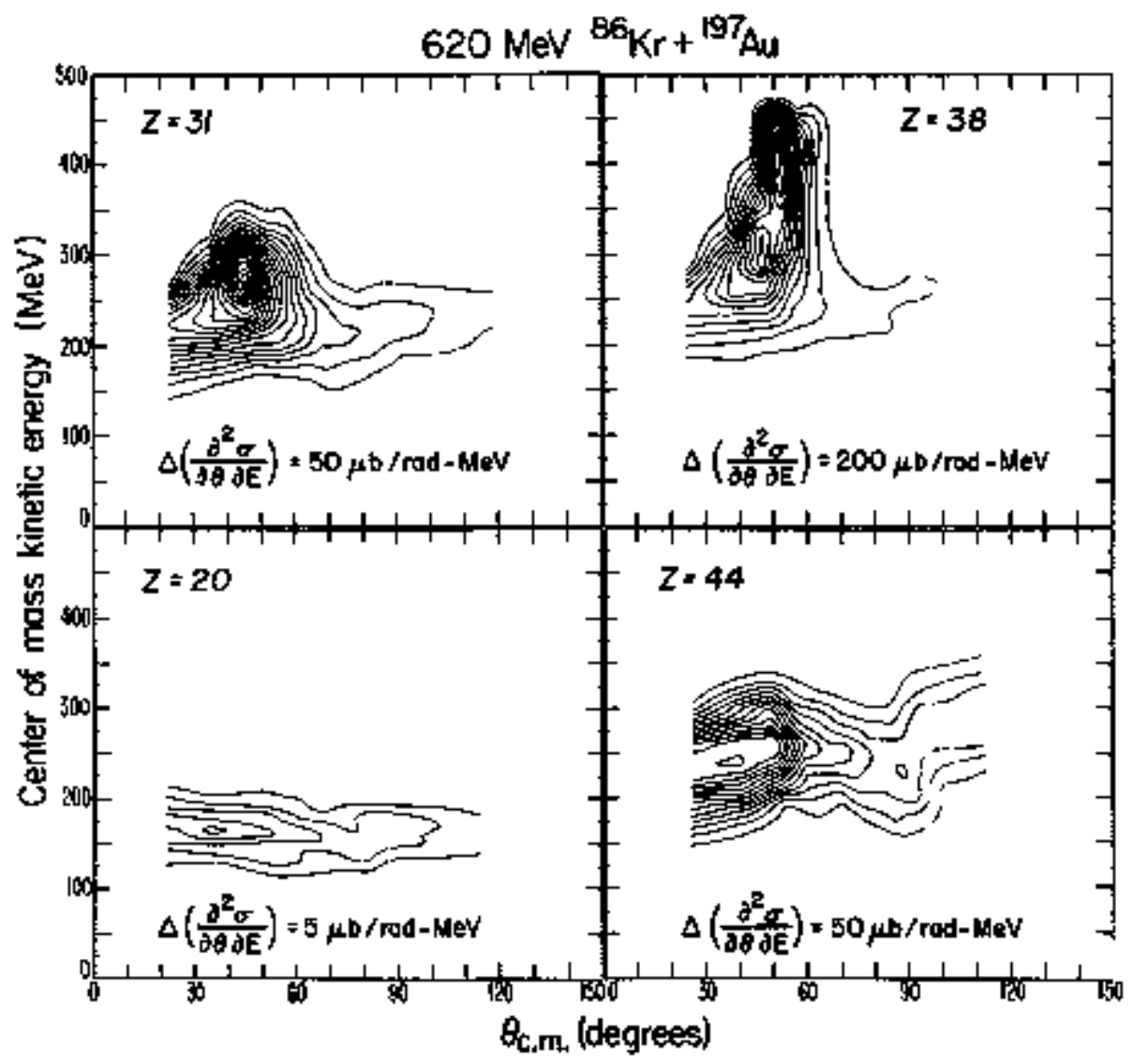

Fig. 2. Exaryles of contour plots of the center-ofmass cross section in the $E$, $\theta$ plane for various $2 ' s$ in the reaction Al $+86 \mathrm{Kr}$. (XBL 761-2089) 
$00: j a>0,01$

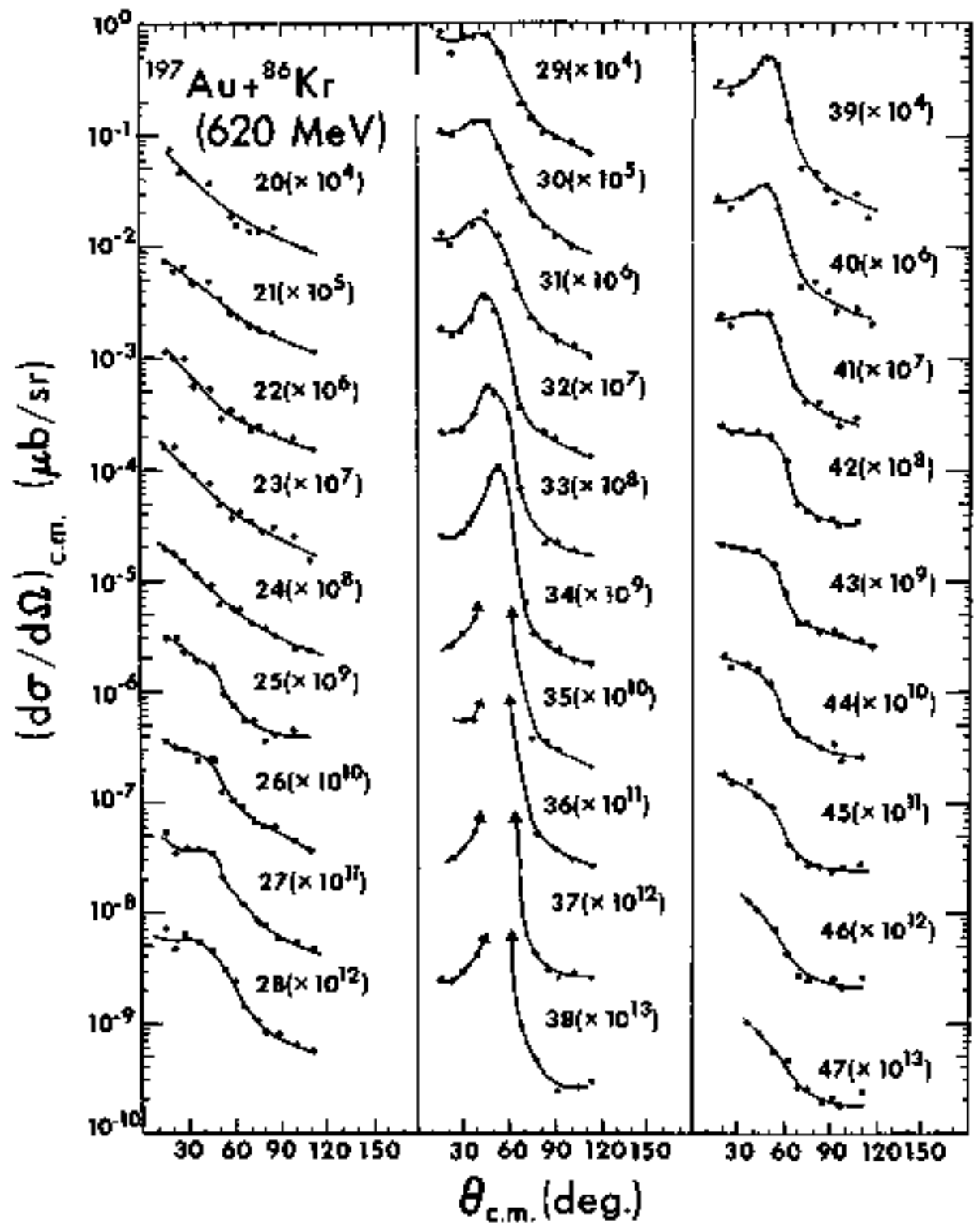

Fig. 3. Center-of-mass angular distributions for the reacticn $\mathrm{AaI}+86 \mathrm{Kr}$.

[XBL 7512-9891) 


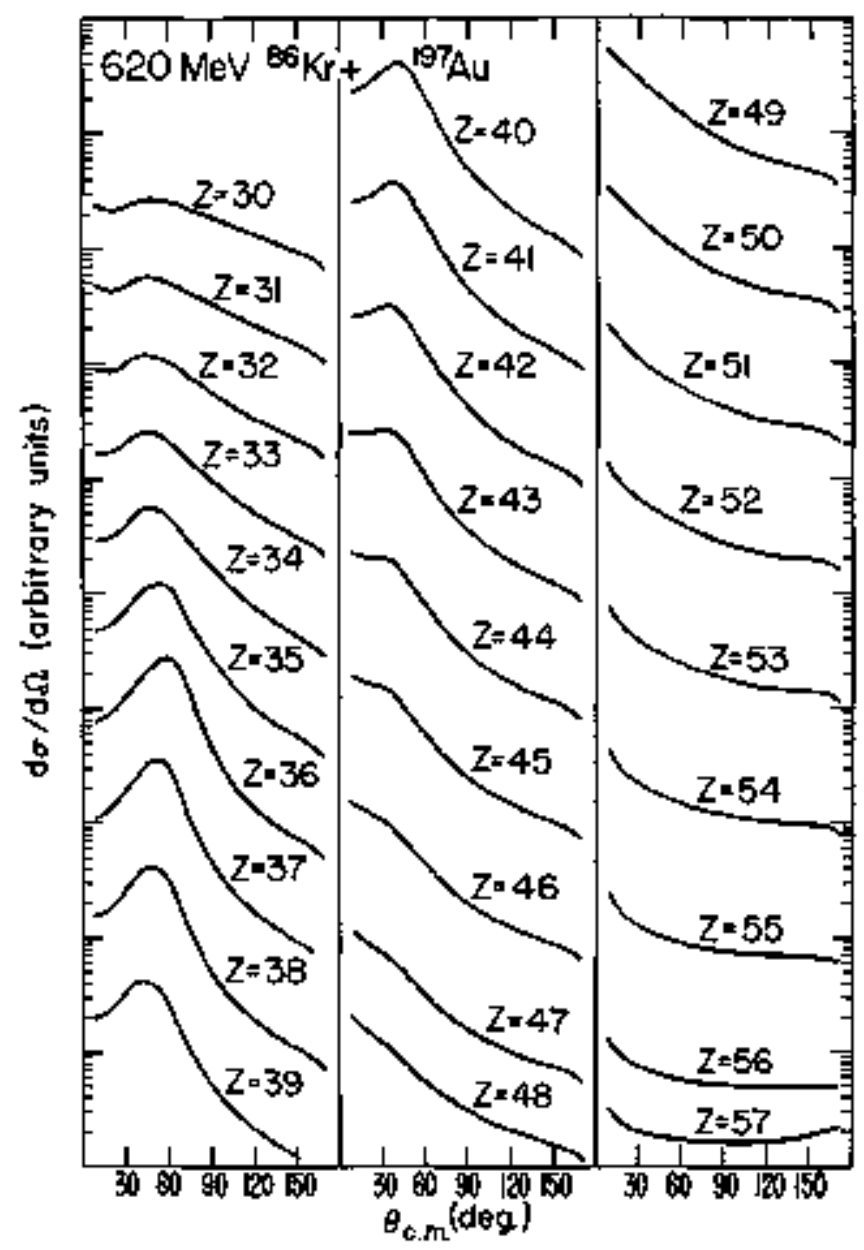

Fig. 4. Theoretical center-of-mass angliar distributions for the reaction $\mathrm{Au}+86 \mathrm{Kr}$ at $620 \mathrm{NeV}$.

(XBL 763-2504)

tions becone forward peaked. As expected, the side peak in the reaction of $\mathrm{Kr}+\mathrm{Ta}$, is less pronounced and diseppears earlier. The forward peaking then extends to the extreme $z$ 's, both high and low which were accessible in the present measurentent. The theoretical calculation shown in Fig. 4 reproduces in detail the experimental effects. Thus in a single reaction, one observes the desired connection botween the side-peaked and the forwart-peaked angular distributions.

\section{Footnotes and References}

"Condensed fram LBL-5006, Invited peper to the "Synposiun on Macroscopic Features of Heavy Ion Collisions" ANL, Argonne, IL, April 1-3, 1976.

$\dagger_{\text {Sloen Fellow 1974-1976. }}$

1. L. 6. Wretto and J. S, Sventeck, Phys, Lett. $58 \mathrm{~B}, 26(1975)$.

2. L. G, Moretto, R. P. Babinet, J. Galin, and

S. 6. Thompson, Phys, Lett. 58B, $31(1975)$.

\section{DIFFUSION MODEL FREDICTIONS FOF THE REACTION $620 \mathrm{MeV}{ }^{66} \mathrm{Kr}+{ }^{197} \mathrm{Au}$}

$\downarrow$ S Svenlęk and L G Morílto

In the Ref. I, we have described the Diffustion Model that was postulated to explain the experimental features of the relaxed (defp-inelastic) cross section in the reaction $288 \mathrm{MkV} 40_{\mathrm{Ax}}+107,109_{\mathrm{Ag}}$. Those experimental features are typical of most "light" target projectile systems that we have studied.

The purpose of this report is to extend the Diffusion Nodel to describe the experinental fea- 


\section{$00+0$; 30,0}

tures of the relaxed cross section in the reaction $620 \mathrm{beV} 86 \mathrm{Kr}+197_{\text {All. }}$. This reaction is representative of "heavy" target projectile systems in that the relaxed conponent displays the following features 2 :

1) The angutar distributions display z-dependent side peaking. The side peak is largest for the projectile $z$, dinirishes to a shoulder for $Z$ 's removed on either side of the projectile, and eventually becones a forward peak for $Z^{1} s$ sufficiently far removed from the projectile.

2) The width of the $Z$-distribution at a given center-of-mass angle $\theta$ increases as $\theta$ is farther removed fron the angle corresponding to the side peak in the projectile's angular distribution.

The fact that the angular distributions are side peaked as opposed to being forward peaked like the "Iight" systemsl is due to the difference between the average rotational period for the complex ${ }^{\prime} R$ and the average lifetine of the complex $\tau_{C} \mathrm{C}$. The large dass of the "heavy" sys tens serves to make $\tau_{R}$ mach larger than $\tau_{C}$, which prevents partial ortiting, and results in side-peaked distributims.

As in Ref. I, we have used the Master Equation to follow the time-dependent population $\phi_{\mathrm{z}}(t)$ of systems whose rass asymotry is characterized by the atomic number $z$ of one of the friments. Whe have assumed an equilibrated neutron to proton ratio, as shown to be true by Galin et al. 3 The potential energies used are those for ton towching spherical fraguents as in ref. 1. A plot of a typical potential energy orrve vs. asymetry can be seen in Pig. 1a.

The time dependerce of the populations $\phi_{z}$ is given by:

$$
\dot{\phi}_{2}=\sum_{z ; z z} \lambda_{z z},\left[\phi_{2}, \rho_{2}-\phi_{z} \rho_{z},\right]
$$

where $\lambda_{z z},=\lambda_{z^{\dagger} z}$ is the microscopic transition probability between systems spocified by the asymetries 2 and $2^{1}$, and $\rho_{2}$ is the Ievel density of the systen spectfied by $\mathrm{z}$. The level densities can be written in terms of the potential enorgy of the intermediate complex $\mathrm{V}_{z}$, discussed above, measured with respect to the roteting ground state, as $\rho_{2}=\rho\left(E-V_{Z}\right)$. The $\lambda_{z z}$ ' are the product of a diffusion constant $k$ and a nomalized forn factor. 1

The system of equations in Eq. (1) can be solved by standard matrix techniques to give the time-dependent populations $\phi_{z}(t)$ (normalized to 1). Fig. Ib is a map of cuntours of constant probability. calculated for the potential energy given in Fig. la, plotted as a function of mess asymutry $Z$ and the time $t$ (in wits of $10^{-22}$ seconds). Wote that the drift and spread of the distribution can bo easily discemed for short times, and the equilibration of the distribution can be seen for long times.

With the $\phi_{z}(t) ' s$, we can calculate the differential cross sections as

$$
\frac{d \sigma_{z}}{d z}(\theta)-\int_{0}^{\infty} d t\left\{\frac{b P(b)}{\sin \theta\left|\frac{d \theta}{d b}\right|} \phi_{z}(b, t) \pi(t ; b)\right\}
$$
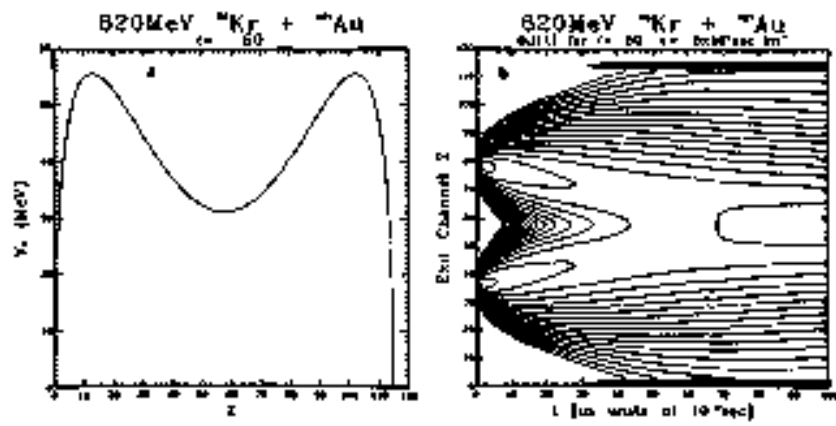

Fig. I. (a) Potential energy of the intermediate couplex as a function of 2 for $t=50$. (b) Probability distributions along the mass asymetry coprdinate as a function of time calculated for $2=50$.

[XIL $766-8131$ )

where $P(b)$ is the probability that a collision at impact parameter $b$ leads to a deep inelastic reaction, For this calculation, $P(0)=1$ for all impact parameters $B b_{1 i m}$, and $P(b)=0$ for b $>b_{1 i m}$. The quantity $b_{1 i n}$ is such that the cross section resulting fortol collisions at impact parameters in the range $b_{1}$ im $b<b_{\text {max }} b_{\text {max }}$ corresponds to a grazing colltsion) is equal to the quasi-elastic cross section reasured for $Z^{\prime}{ }^{\prime}$ near the profectili $Z$ at lab angles near the grazing angle. The sut in Eq. (Z) is carried over all ixpact paraguters b which result in particle $Z$ being enitted at the angle $\theta$ after the complex has Iived a time $t$. The quantity $n(t ; b)$ is the probability that the complex formed by a collision at Irpact paraneter $b$ will live a time $t$. In the previous work, we have assumed $\pi(t ; b)$ exp $(-t / \tau) / \tau$, independent of b with $\tau$ being the average lifetime of the intermediate complex. Hoteto et a $1^{2}$ have shom that the variation in the width of the 2 distributions with angle can be expleined by an averago lifetime for the complex which decreases with increasing $b$. In the light of these results, these calculations have been performed with:

$$
\exists(t ; b)=\frac{1}{N(b)} \exp \left[-(t-\tau(b))^{2} / \sigma^{2}(b)\right]
$$

where $N(b)$ is a monglization factor and $\tau(b)=$ T(0) $\left(1-b / b_{\max }\right)$. Since the dispersion in any random walk probess varies linearly with the elapsed time, 4 we have assumed that $\sigma^{2}(b)=\sigma^{2}(0)$ $\left(1-b / b_{\text {max }}\right)$. The quantity $T$ (b) represents the average Iifetise of the corplex formed at iupact parameter b, and $\sigma^{2}(b)$ represents the dispersion of the distribution of lifetimes about this average value. For these calculations, the values of $\nabla^{2}(0)$ and $\tau(0)$ are parameters but can in principle be deternined fron trajectory calculations.

The parameters used were: $k=0.25 \times 10^{21}$ $\mathrm{sec}^{-1}$. fal ${ }^{-2}, \tau(0)=3.5 \times 10^{-21} \mathrm{sec}$, and $\sigma(0)=$ $1.0 \times 10^{-21} \mathrm{sec}$. The calculations have not been optimized with respect to the value of $\kappa$, and the value used was chosen because of the success of the previous work on light systems. The position and width of the side poak depends very strongly upon $\tau(0)$ and $\sigma^{2}(0)$, as ane might expect. The position of the side peak in the experimental angular distribution detemines $\tau(0)$ very miquely, 
and $\sigma^{2}(0)$ has the effect of making the side peak broader or narrower. The value of $\tau(\theta)$ should be compared with the rotational periad of $8.6 \times 10^{-21}$ seconts for the average $\mathrm{l}$-value of 185.

The angular distrlbutions measured recently by our group for $620 \mathrm{kfV} 86 \mathrm{Kr}+197 \mathrm{Au}$ are displayed in Fig. 2a. Figure $2 b$ shows the angular distributions celculated for this systent using the mode1 described above. Note that the magnitute of the side peaking is greatest for $Z=36$ and decreases on either side of the projectile. The gradual disappearance of the side peak for 2 's above the projectile almost exactly parrots the experimental distributions. The distributions for $Z^{+} s$ below the projectile follow a similar pattern, but the side peaking disappears too qudckly (after too few Z's). This seems to be tue to the assumption of two toukting spheres for the shape of the intermodiate conplex.

Figure 3a shows the experimental $z$-distributions for constant center-of-ulass angles ineasured by our group. Note the increasing width of the distributions arodend $z=36$ as one moves both forward and backiward in the center-of-mass with respect to $60^{\circ}$. Figure 30 is an analogous plot of the calculated values. The lack of shift of the most probable value in the experimental results is probably due to the difficulty of separating the relaxpd component from the quasi-elastic conponent near the grazing angle.
Fig, 2. (a) Experimental center-ofmass argular distributions for the reaction $197_{\mathrm{Au}}+620 \mathrm{keV} 36 \mathrm{Kr}$.

(b) Theoretical center-of-mass angular distributions for the same re. action using model tescribed in text. (XBL 766-2905A)
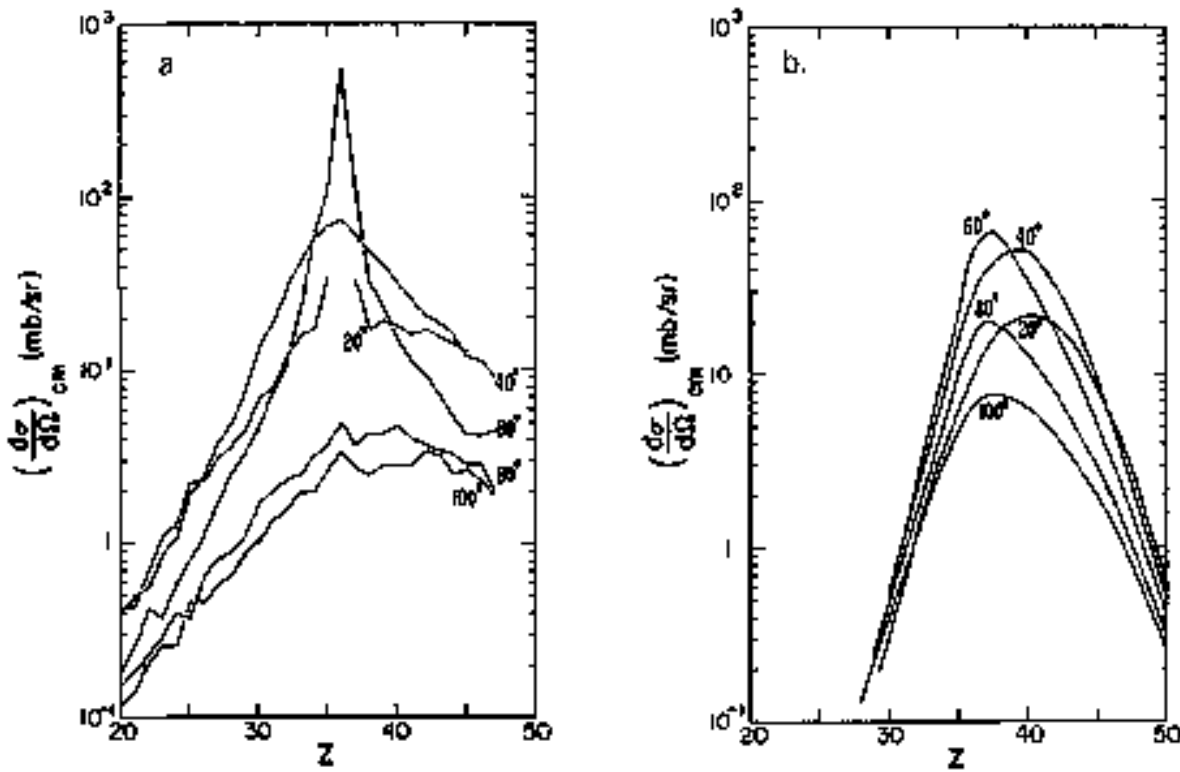

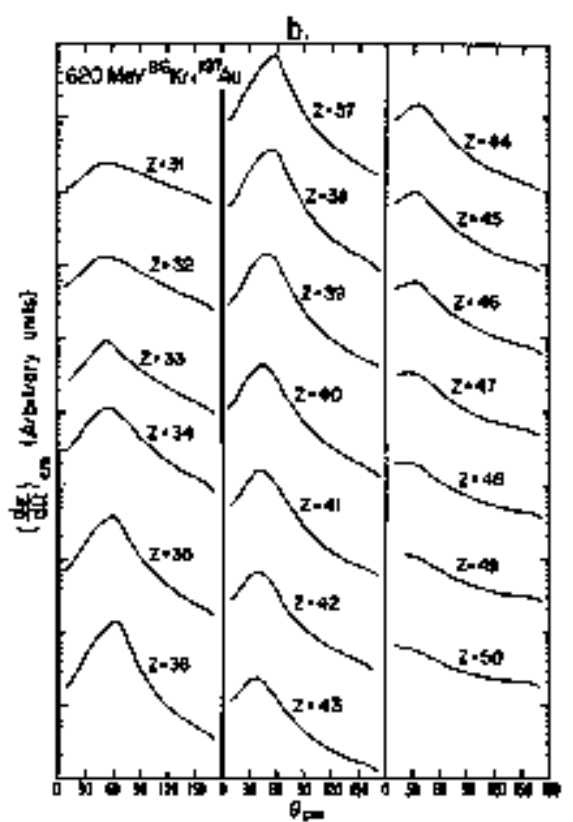

Fig. 3. (a) Experimental change of distributions for fixed center ofmass angle for $620 \mathrm{keV} 86_{\mathrm{Kr}}+19 \gamma_{\mathrm{Aul}}$. (b) Thooretical charge distributions for the same reaction using model described in text. (XEL 766-2904A) 


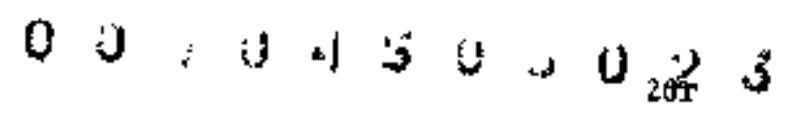

The absolute cross sections for $Z$ 's above the projectile are in agreement with a factor of 2 , but the cross sections for $Z^{\prime} ' s$ belur the projectile are in error by much more. The large difference between the experimental and theoretical cross sections seen for $Z$ 's Juch below 36 can again be attributed to our assumption concerming the shape of the complex. Relaxition in the shape of the tow touching fragments should aflow the 2 's below 36 to be populated on a mach larger scale.

\section{References}

1. L. G, Moretto ani J, 5. Sventek, Phys. Lett. $58 \mathrm{~B}, 26(1975)$.

2. L. G. Moretto, B. Cauvin, P, Glassel, R. Jared, F. Russso, J, Sventek and G. Whaniak, Phys. Rev. Lett. $\underline{36}, 1069$ (1976).

3. B. Gatty, D. Suerreau, M. Lefort, X. Tarrago, J. Galin, B, Cauvin, J. Girard and H. Nifenecker, Nukl. Phys. A253, 511 (1975).

4. S. Chandrasekhar, Rev. hod. Phys. 15, 1 (1943).

\title{
THE CLASSICAL-LIMIT \$MATRIX FOR COULOMB EXCITATION*
}

\author{
f Donangelo M W Gundry \\ $\checkmark P$ Boisson $t$ and $d O$ fasmussen
}

With the advent of very heavy nucleer projectiles there has grow a need for coulonb excitation theory applicable in such systems. Exact quantur rechenical calculations of the Coulomb excitation process are quite impractical. However, the shortness of the de Broglie wavelength for heavy ions brings the problenn more into the realm of classical mechanics. Seniclassical theories use this fact to find afproximate solutions to the problem, such as in the d1der-Winther method which is the foundation to the widely used Winther-dekoer code for Coulowb excitation. ${ }^{2}$ Recently a quite different approach, the biform Semiclassical Approximation (USCA) was applied to this problem. 3

We adapted and modified the formalism developed by Marcus 4,5 so as to study the Coulonb excitation process, and we show its relationship with the USCR as applied in Ref. 3.

We take as a target a deformed even-even nucleus in its ground state șo that its initial spin is zero and its firal spin quastum muber I is an even integer. For simplicity we restrict ourselves to the case of a head-on collision.

Let $F_{0}$ be the Haniltonian of the systen for distances large compared to the distance of closest approach, $\mathrm{H}_{\mathrm{O}}$ includes then the kinetic eneтgy and long-range coulomb interaction terms. Let $\left.\right|_{\phi_{T}}$ ) be the eigenstate of $\mathrm{H}_{0}$ in the exit charnel characterized by the spin quantum rumber I of the target. At short distances the Harailtonian is $\mathrm{H}=\mathrm{H}_{\mathrm{o}}+\mathrm{V}$, where in $\mathrm{V}$ we include the remaining terus of the electronagnetic interaction. We designate by $\left|\psi^{+}\right\rangle$the scattered part of the efeenstate of $H$ corresponding to the initial conditions given before and evaluated long after the interaction has taken place.

From the usual definition of the S-matrix,

$$
\left.\mathrm{S}_{\mathrm{I} \leftarrow 0} \equiv\left\langle\phi_{\mathrm{I}} \mid \psi^{+}\right\rangle=f\left\langle\phi_{\mathrm{I}} \mid \underline{\underline{q}}\right\rangle \mathrm{dq}\langle\underline{q}| \psi^{+}\right\}
$$

where $\mathrm{q}$ is a convenient representation and the integration is over all the g space. Since we do not know tha function $\psi^{+}(\mathrm{q})=\left\langle\mathrm{q} \mid \psi^{+}\right\rangle$, we approximate it with the generalized lWh wave function

$$
\bar{\psi}^{+}(\underset{\sim}{q})=A(\underline{q}) \exp \{i q(q) / h\}
$$

where, as seen in Ref. 5, a conserves probability flux and the phase $\phi$ satisfies Hamiltor-Jacobi equation.

We take the following coordinates to describe our system in the outgoing asymptotic regiom: $x$, angle between the syminetry axis of the target and the line jouning its center with the center of the projectile, and $r$, the distance betweer these centers. We denote by $L$ the angular momentim of the target, by $p_{T}$ the relative radial motion and by $\mathrm{v}$ the relative velocity. To obtain ita) trajectories are run with initial conditions: $r$ large, $x=\chi_{0}$ (arbitrary), $L=0$ and with $a$ given projectile energy. From the values of the dynamical variables in the final asymitotic region we can determine $A$ and $\phi$, which are in our case

$$
\begin{aligned}
& A=\sqrt{\frac{1}{2} \frac{\sin x_{0}}{\sin x} \frac{d x_{0}}{d x} \frac{v_{0}}{v}} \cdot \frac{1}{I} \\
& \phi=-\int\left(x[t) d p_{r}(t)+x(t) d L(t)\right)+I p_{Y}+X L
\end{aligned}
$$

Here quantities with subscript zero represent initial values, and those without subscript, the values at the end of the trajectory. In the asyototic region $\phi_{\mathrm{I}}$ is given by:

$\phi_{T}(q) \equiv\left\langle\underline{q} \mid \phi_{T}\right\rangle=$

$\frac{1}{2 \pi} \sqrt{\frac{v_{I}}{v_{0}}\left(I+\frac{1}{2}\right)} P_{I}(\cos X) \frac{1}{r} e^{i}\left[\frac{P_{r} r^{2}}{\hbar}-n \ln \left(2 \mathrm{P}_{T} r / \lambda\right)\right]$

where $v_{0}$ and $v_{I}$ are the asymptotic relative velocities for the values 0 and I respectively of the target spin quantum dumber, PI is the usual Legendre polynomial and $\eta$ is the Somnerfeld paraneter for the exit channel. 
Primarily because $x$ does not become constant in the asymptotic region it is not possible to define a good WRE wave function in this $q=(x, r)$ representation, so wo transform it to a representation analogous to the one in Rof. 5, that is,

$$
\bar{x}=x=\operatorname{sic}
$$

Here $w$ is the angular velocity of the target at the end of the interaction and $x$ is the time it would take the projectile to describe an elastic trajectory governed by the Haniltonian $\mathrm{H}_{0}$ for an energy equal to that of the projectile in the outgojing asymptotic brarch of its actual trajectory, measured from the point of closest approach on this trajectory.

In the same way as in Ref. 5 we can show that in this case the s-matrix is given by

$\mathrm{S}_{\mathrm{I} \rightarrow 0}>\jmath^{\prime} \phi_{\mathrm{I}}^{*} \bar{\psi}^{+} 2 \pi \mathrm{r}^{2} \sin \bar{x} \mathrm{~d} \overline{\mathrm{X}}=$

$$
\int \phi \overline{1}^{+} \bar{\Psi}^{+} 2 \pi r^{2} \sin \bar{x} \frac{d \bar{x}}{d x_{o}} d x_{o}
$$

or, more explicitly,

$$
\begin{aligned}
& S_{I+0}= \\
& \frac{\sqrt{2 I+I}}{2} \int_{0}^{\pi} P_{I}(\cos \bar{x}) \sqrt{\sin x_{0} \sin \bar{x} \frac{d \bar{x}}{d x_{0}} e^{i \phi}} d x_{0}
\end{aligned}
$$

where

$$
\begin{aligned}
\phi^{\prime}= & -\frac{1}{h} \int\left(r(t) d p_{r}(t)+x(t) d L(t)\right)+\frac{1}{h} \bar{x} l+ \\
& \eta \ln \left(2 p_{\Gamma} r / \hbar\right) .
\end{aligned}
$$

he will examine Eq, (7) in more detail. Let us first consider the gudden impact limit for the case in which onty the quadrupole morent of the target contributes to the excitation.

In this limit $\bar{x}=x_{0}$ and $\phi^{\prime}=2 q \sin ^{2} x_{0}$ where a is given by Eq. (S.11) of Ref. 1; therefore, Eq. (7) reduces to the expression found by Alder and winther for this same case. 1 This result is interesting, considering the conceptually different approach we have taken.

If we row take the asymptotic expression for the Legendre polymomials

$$
P_{1}(\cos \vec{x})+2 \cos \left[\left(1+\frac{1}{2}\right) \bar{x}-\frac{n}{4}\right] / \sqrt{\pi}(2 I+\overline{1}) \operatorname{sen} \bar{x}
$$

and make use of the fact that the initial orientation angles $x_{0}$ and $\pi-x_{0}$ result in opposite final angutar mamenta, we find?

$S_{I-0}=\frac{1}{\sqrt{\pi}} \int_{0}^{\pi} \sqrt{\sin x_{0} \frac{d \vec{x}}{d x_{0}}} e^{I\left[\phi^{\prime}-\left(I+\frac{1}{2} \bar{x}\right]\right.} d x_{0}$.

The evaluation of this integral by the stationary phase approximation results in the USCA as applied on Ref. 3. The weighting factors introduced through geometrical argunents in that paper and also its use of the $I+1 / 2$ spin. quantization condition appear straightforwardly here from the asymptotic expression for the Legendre function.

We have applied Eq. (7) to several cases. In Fig. 1 we compare our calculations with those abtained by means of the Coupled-Channel quantum. mechanical code AROSA. 6 The agreenent is good, especially considering that in these cases the systents are not very classical. The method entoloyed here should becone more accurate for heavy-ion systemis, No comparison with AROSA results is possible yet for very heavy ions, so that in Fig. 2 we have compared our results with the setriclassical winther de Boer code for a heavier projectile.

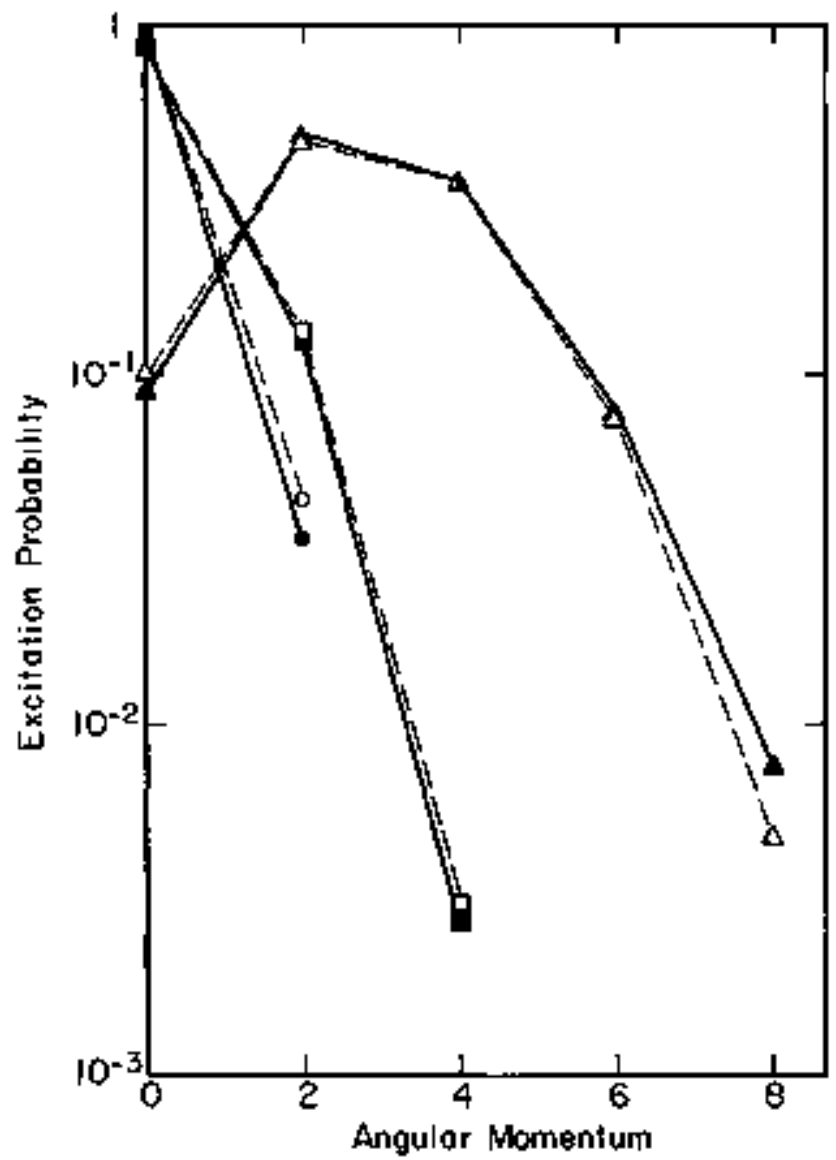

Fig. 1. Probabilities to Coulonb excite the rotational band of $168 \mathrm{Er}$ for backscattering of $7 \mathrm{MeV}$ deuterons, $14 \mathrm{MeV}$ a-particles and $45 \mathrm{MeV}$ 10 Be projectiles. The quantum mecharical results were obtained using the code AROSA, and are indicated by dark circles for ${ }^{2} \mathrm{H}$ dark squares for 4 ite and derk triangles for $10 \mathrm{Be}$, respectively, joined by solid lines. The results fron Eq. (7) in the text are shown, respectively, by open circles, squares and triangles joined by dashed lines. The quadrupole moment of $168 \mathrm{Er}$ is taken to be 7.67 barns, its energy levels are takent fros the rotational model, and no nuclear forces were considered in any of the calculations.

(XBL 766-8124) 


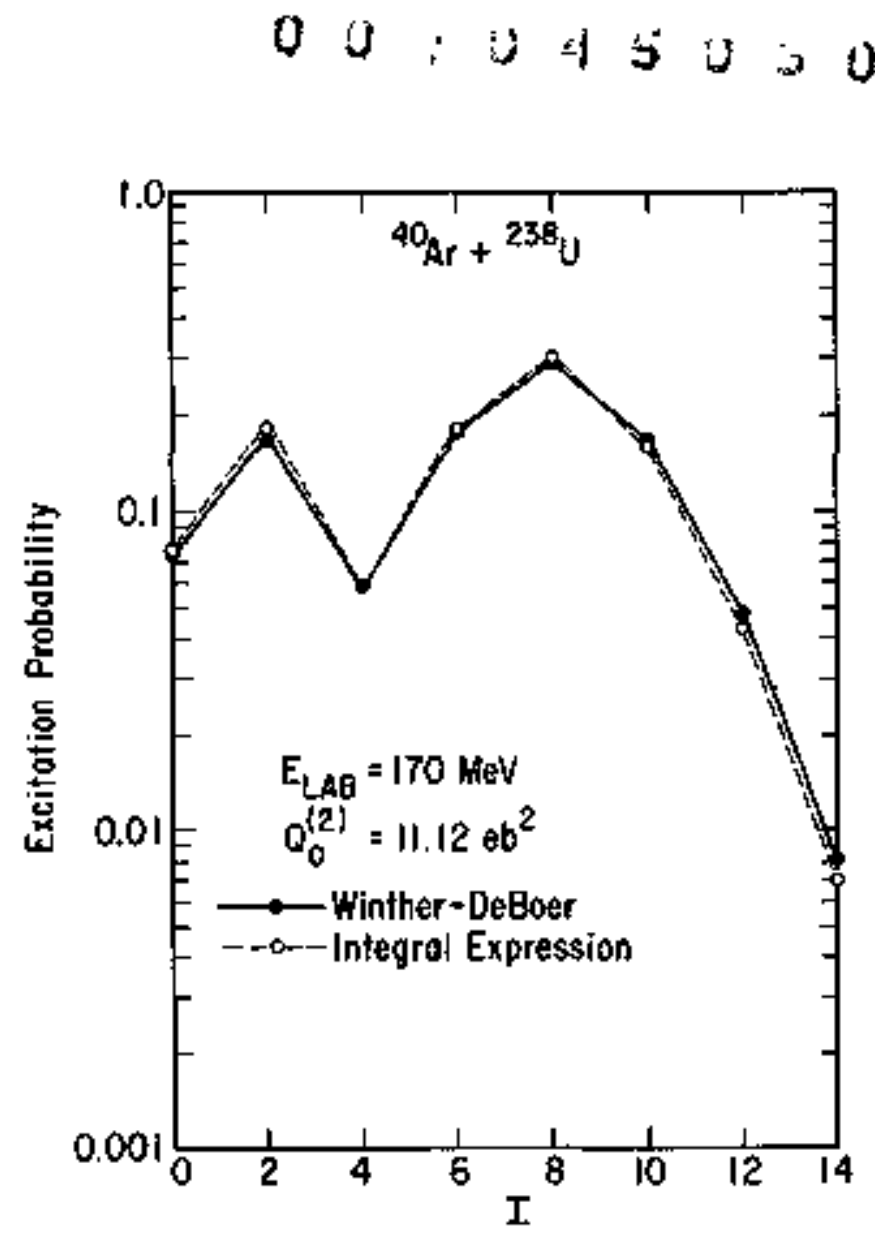

Fig. 2. Probabilities of Coulomb excite the rotationa1 ground band in 238U for backscattering of 40 ar at $170 \mathrm{keV}$. The results given by the Winther-deboer code are shown by dark tircles foirod by fulI lines and the results from Fq. in the text by open circles joined by dashed lines. The quadrupale moment of $23 \mathrm{BU}$ is taken to be 11. 12 barns; the energy levels are taken from the rotational model, and no muclear forces were considered in the calculations.

(XEL 7512-9896)

Whe should remark at this point that the validity of $\mathrm{Eq}$. (7) is limited to those ceses where the IKB wave function $\psi^{+}$is a good asylptotic approximation to the actual wave fumction $\psi^{*}$. This requires, in particular, that be a monotonic function of $x_{0}$; if not $\bar{\psi}^{+}$will break down at the points where

$$
\frac{d \bar{x}}{d x_{0}}=0
$$

These difficulties can appear, and Kreek et al. ${ }^{7}$ reported them in a reiated problen.

Equation (7) is applicable at energies around the Coulonb barrier if one includes the corplex nuclear potential in the equations of motion, in the Jannet of Ref. 8 .

The fact that the stationaxy phase approximation is not used makes Eq. (7) more straightforward to use than the ISCR in cases where the stationary phase points are not easy to locate, or where there are nore than _two such points. Since this approximation is not used, there is even reason to think that our results cculd be more precise than those of the USCR, especiatly in cases where the exponent in the integrand of Eq. (7) varies slowly. For comparison purposes we pive in Table 1 the rosults obtained by all the methods we have prentioned for a case where this exponent varies slowly. The USCA used there is the one based on tiry functions as considered in Ref. 3; for this case the Bessel uniforn approximation given by 5tine and Marcus 9 should be more appropriate. However, the results in Ref. 8 seern to Indicate that an integral expression such as Eq. (7) is thore accurate, even for these cases.

\begin{tabular}{|c|c|c|c|c|}
\hline Sprr & $\begin{array}{c}\text { This Nork } \\
\text { [Bq. T\} }\end{array}$ & $\operatorname{AROS}{ }^{\circ}$ & Ninther-deBoer ${ }^{2}$ & $u s A^{3}$ \\
\hline 0 & 0.1023 & $0.0 \$ 90$ & 0.1087 & 0.1267 \\
\hline 2 & 0.4615 & 0.4754 & 0.4375 & 0.1928 \\
\hline 4 & 0.3571 & 0.3522 & 0.3544 & $0.33 \times 0$ \\
\hline 6 & 0.0734 & 0.0375 & 0.0日的5 & 0.0531 \\
\hline $\mathrm{B}$ & 0.00468 & 0.00756 & 0.0103 & 0.00395 \\
\hline
\end{tabular}

\section{Foothotes and References}

*Condensed from LEL-4347, to be published in Phys, lett. B.

†Pemenent address: Institut des Sciences theleaires de Grenoble BP 257, 38044 Grenoble, France.

1. K. AIder and Aa. Winther, Mat, Fys, Medd, Dan. Vis. Selsk -32 (8) (1960).

2. Ae. Winther and J. DeBoer, in "Coulonb Excitation," K. Alder and Aa. Winther, eds. (Academic Press, NY, 1965), p. 303.

3. H. Massmarn and J. 0. Rasmussen, Nuc1. Phys. A243, 155 (1975).

4. R. A. Marcus, Chem. Phys. Lett. 7,525 (1970).

5. R. A. Marcus, J. Chem. Phys. 56, 3548 (1972).

6. F, Roesel, J. X, Saladin and $K$, Alder, Comp. Phys. Camts. $\underline{8}, 35$ (1974).

7. H. Kreek, R. L. Ellis and R. A. Marcus, J, them. Phys. 62, 313 (1975).

B. M. W. Guidry, H. Massmann, R. Donangelo, and J. O. Rasmussen, to be publișhed in Nucl. Phys. A.

9. J. R. Stine and R+ A+ Marcus, J. Chem. Phys. 59, $5145(1973)$. 


\section{THEORY OF COULOMB-NUCLEAR INTEFFERENCE FOA HEAVY-ION EXCITATION OF ROTATIONAL STATES*}

M W Gudr, $\mathrm{H}$ Massman,

F Donargelo, and J O Aasmussen

The Uniform Senaiclassical Approximation ${ }^{1}$ (USCA) has been applied to the problen of Coulombrnxlear interference for rotational states excited in heevy-ion interactions. 2 The classical model of the interference effect as a competition between electromagnetic and nuclear forces is tllustrated in Fig. 1. By solving the (complex) classical equations of motion the quantum number function of Fig. 2 is obtained, which relates the firat $5 p$ in of the rotor $I\left(B_{0}\right)$ to an initial orientation angle $\beta_{0}$.

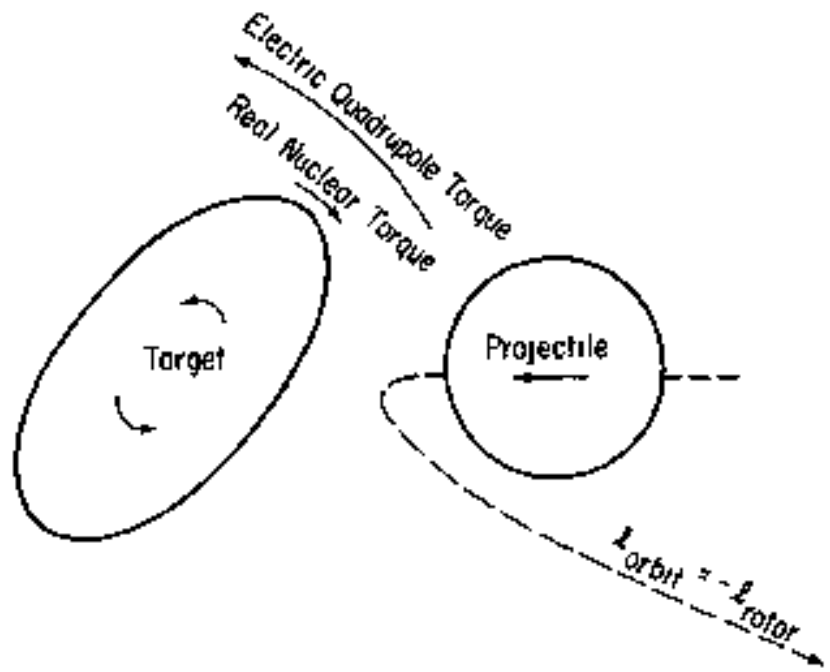

Fig. 1. The classical yodel for completition between the electromagnetic and nuclear forces.

(XBL $761-12 A)$

In this formalism, one finds that the excitation probability (the square of the S-matrix) is approximately given by

$$
\begin{aligned}
& P_{I+0} *\left|\vec{p}_{1}\right| e^{-2 \operatorname{Tm}\left(\Phi_{1}\right)}+\left|\overrightarrow{\mathbf{p}}_{2}\right| e^{-2 \operatorname{In}\left(\Phi_{2}\right)} \\
& +2 e^{1 / 2 \operatorname{Im}\left(\phi_{1}+\Phi_{2}\right)} \sqrt{\sqrt{\bar{p}_{1} \bar{p}_{2} I} \sin (\operatorname{Re}(\Delta \Phi)+\alpha)} \\
& \text { "allowed transitions" }
\end{aligned}
$$

$P_{I+0} e^{-2 I m 4}|\bar{p}| \quad$ "forbidden transitions"

where "allowed transitions" involved (approximately) those states below the maximen of the quantunnumber function in Fig. 2, and "forbidden transitions" involve (approximately) those states above it. The classical probabilities $\overline{\mathrm{P}}_{j}$ are defined by

$$
\dot{p}_{j}=\frac{2 \sin \bar{\beta}_{j}}{\left(\frac{\partial 1\left(\hat{\beta}_{j}\right)}{\partial \beta_{j}}\right)} \quad\left(\bar{\beta}_{j} \equiv\left(\beta_{0}\right)_{j} ; j=1,2\right)
$$

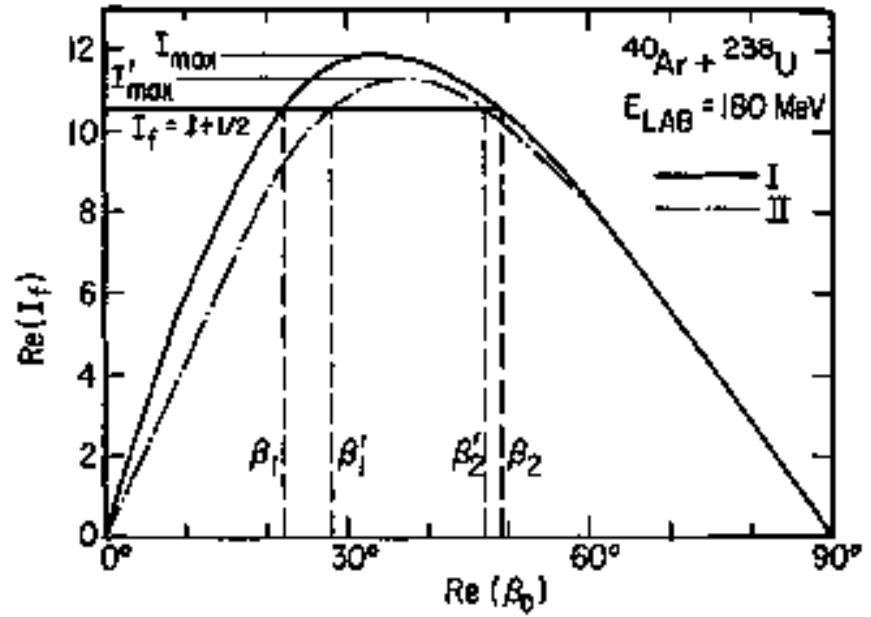

Fig. 2. The real part of the final spin vs the real part of the inltial orientation angle- the quantum number function. For the coaplex trajectory case the funtion is complex. The effect of the real nuclear potential is to lower the final spin relative to the case of no nuclear potential. Graphical solutions for the ID+ state are indicated for the Coulomb excitation case ( $B_{1}$ and $B_{2}$ ), and the couloub nuclear case ( $B_{1}$ and e') when the imaginary potential can be ignored.

(XBL 761-20)

where the derivatives are detergined from the function in Fig. 2 evaluated at the roots $\bar{\beta}_{1}, \bar{\beta}_{2}$, and the phase $\Phi_{k}$ is the classical action evaluated on the trajectory with an initial rotor orientation $\beta_{0}-\left(B_{0}\right)_{k}$. The guantity $\alpha$ is the phase of $-\vec{p}_{1} \bar{p}_{2}^{k}$ and is soll for the cases considered here. ${ }_{A}$ uniform expression impolving Airy functions was actually used for the calculations; but Eq. (2), which is an asyolptotic form of the uniform expression, is useful in illustrating two important concepts: (1) the interference between the amplttudes arising fron the different terms in the S-matrix, and (2) the exponential damping of the probability anplitudes by the imaginary part of the classical action $\Phi$.

For the systen ${ }^{40} 0_{A x}+238$ We have calculated excitation functions for excited states in the gromend state band for the zeroinqtact parameter case. These are shown in Fig. 3 for pure Coulom excitation (I), and for several representative conplex muclear potentials (II, III, IV) obteined from quosi-elastic heavyion scattering. 3,4 The deviation of the Coulonb nuclear probabilities from pure Coulonb excitation in these figures is easily expanded by the effect of the complex muclear potential on the interference torm of Eq. (la) and the damping factor of Ex. (1b). The interference effects are significant and depend on the form of the nuclear potential. 

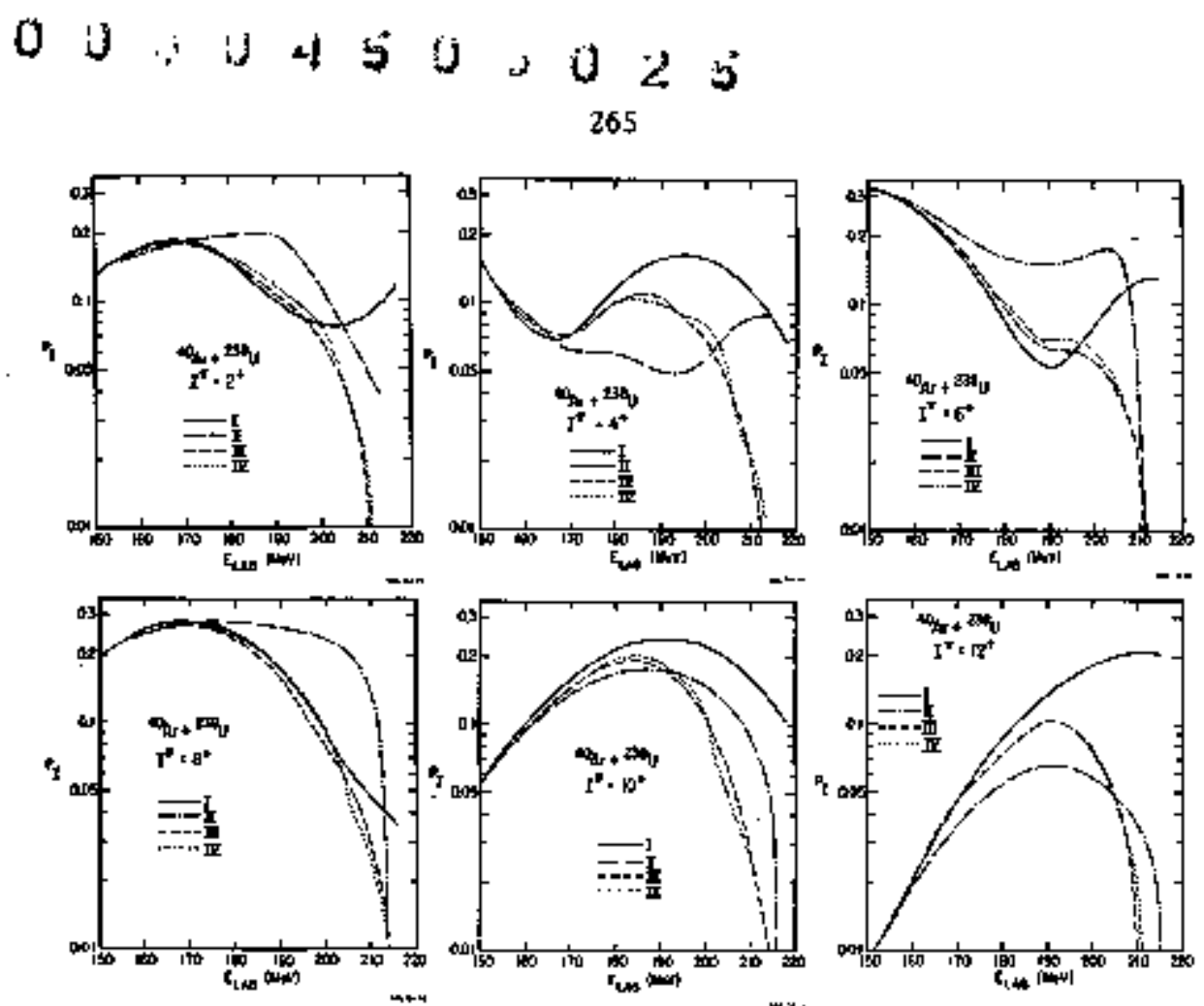

Fig. 3. Excitation functions for the $2^{+}-12^{+}$ states in the reaction $40_{\mathrm{Ar}}+23 \mathrm{U}_{\mathrm{U}}$. The calctulation was restricted to the zero impact parameter.

(XBL761-19A)

\begin{abstract}
Because the excitation probabilities are sensitive to Coulomb nuclear interference effects, we believe that heavy-ion rotational excitation patterns near Coufomb barrier energies could provide a detailed probe of the potential in the nuclear surface region. (The potential in the nuclear interior is probably inaccessible because of the strong absorption.) One nay speculate that the potential for a deformed nucleus may exhibit irregular variations with polar angle $B$. For example, the imaginary potential may be largely due at barrier energies to loss of flux into neitron transfer channels, and hence be strongest in the zones of the lightly-bound Nilsson neutron orbitals. If the concept of a classical trajectory has any physical sigatificance in these heary-ion systems, excitan. tion of different rotational states should probe different angular regions of the nuclear sunface. Referring to Fig. 2, we would infer that the excitation probability for spin 8 in the case displayed there nainly probes the miclear potential near $\beta_{1}^{+}=20^{\circ}$, since the large-angle root $\beta_{2}^{\prime}$ foels less influence from the nuclear potential. 'Excitation of lower spins should be sensitive to regions closer to the nuclear tips. At sufficiently higher energles the low angle roots should be
\end{abstract}

damped by the ingginary potential and the larger angle orientations, which now feel the nuclear force, should rake the dominart contribution. Therefore, one would expect the probabilities in this case to be sensitive to the nuclear potential noarer the belly of the classical nucleus.

\section{Fontrotes and References}

"Condensed from LBL-4357, to the published in thrcl, Phys, A.

${ }^{\dagger}$ Present address: Hahn-Meitner Institut, Berlin. on leave from Factulad de Ciencias, Lniversidad de chile, Santiago, chile.

1. W. H. Miller, J. Chen. Phys. 53, 1946 (1970); ibid 53, 3578 (1970).

2. H. Massyann ant J. O. Rasmissern, Nurl, Phys. A243, 155 (1975).

3. R. Vanderthosch, T. D. Thonas, and M. hebb, to be published.

4. J. R. Birkelund, et al, Phys, Rev. C 13, 133 (1976]. 


\title{
BARRIER PENETRATION THEORY IN MORE THAN ONE DIMENSION"
}

\author{
Peler Ringht لdohnt O Rasmussen, and Herber klassmanł
}

We have reviewed quantum mecharical tumeling theory in ware than one dimension.

Several systems from nuclear and molecular science were considered specificslly, such as, alpha decay of spheroidal nuxlei, spontaneous fission, and reactive collinear collisions of hydrogen atcins with hydrogen molecules. The range of validity of various approximations that reduce to one-dialensional path integrats or Frötan-Nosov matrices was examined, testing where possible against fully quartum mechanical coupled-channels solutions. The classical equations of motion methods using complex variables (uniform semclassical approximation] were explored for nonseparable fission-like model systems. Effects of varlable valley widths, curving valleys, and of variable inertial tensors were delineated.

In this brief report only one of the newr results is presented. More detail is contained in the paper frow which this report has been condensed. The generalized HIB integrand for barrfer penetration in spontaneous fission is

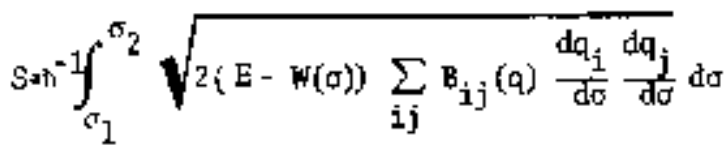

Where $\sigma$ is some arbitrary paraneter along a trajectory and $\mathrm{B}_{\mathrm{ij}}$ is the inertial tensor which may also depend of $\sigma$. Turing the tunneling process $W>E$, and the integrand is purely imaginary. The tumeling probability is given in the usual way by:

$$
p=|\exp (i 5)|^{2}=e^{-2|s|}
$$

In perhaps the most sophisticated fission barrier stuty of recent times Brack et al. 1 proceeded to find the trajectory which minimizes $|S|$. The trajectory so obtained usually did not follow the steepest descent of the potential and did not lead throltgh the extremal points of the deformation energy. It should still be noted that the entrance $\left(\sigma_{1}\right)$ and exit $\left(\sigma_{2}\right)$ points should be determined in such a way that the action integral is alșo stationary agkinst variation of these endpoints.

T. Ledergerber and H. C. Pauli ${ }^{2}$ using the same method (as the one described in Ref. I) have done calculations using three deformation parameters: an elongation coordinate, a constricticn or necking-in coordinate, and a parameter doscribing the left-right asymatry. The lifetimes obtaised from these calculations are in order-ofmagnitulite agreentent with the experimental data; they also show that mass-asymetric fission is favored and that the rost probable mass division (peak-to-peak ratio) agrees with the experimental data.
In this multidimensional approach, 1,2 however, they have still ignored the kinetic energy tied up in the motion orthogonal to the fisston path (which may change along the path). It has been customary in shell-corrected liquid-drop madel work (Strutinsky method) to ignore all zero-point vibrational energy corrections, since they would require assumptions about an inertial tensor. Thus, liquid drop model parameters are adjusted to fission barrier heights. With such a surface it is slearly incorrect simply to add zero-point energies. This wint is closely relaced to the point made by Marutur and Greiner; 3 for spontaneous fission from a sphervidal ground state the number of degrees of freedom for quadrupole vibrations is three, that is, tho ground state would have (3/2) hu of zero-point energy. At the saddle the frequency of the betavibrational mode in the fission direction has becoune imaginary, leaving only two wits of zeropoint energy, those associated with the gammavibrational (axial-symmetry-breaking) mode. In order to include these zero-point energy corrections (wthout sodifying the fission barrier heights) Marutin and Greiner readjusted sone of the liquid drop parameters of Myers and Swlatecki 4 to lower the groumd state potential energy (and that of the final fragments) by the zero-point emergy, which was obtained from the first $2^{+}$state experimentally observed.

Another way to consider this problem is in terms of tumneling and the Inverted Potential Energy Surface. Consider a system which noves toward a barrier and let time be the paraneter in the equations of motion describing the system. If the tine increments are kept reat, the system wtl1 nove toward the barrier and then reflect at the barrier and move back. If, however, one takes purely imaginery time increments when the system is at the tuming point, the system will start tunneling. In other words, during the tumeling process, the systerg follows the classical equations of motion but with purely imaginary time increments.

If at the classical turming point of the fission degree of freedom all the other degrees of freedmi are also at a turining point ( or in other words, if the system when it reaches tho barrier is at rest), then the tunneling process car be visuallzed by a very sinple picture. If the system is at rest at the turning point and if one uses purely inaginary time increments thereafter (until the turneling is completed), then all the coordinates will remain real, all quantities related to odd powers of the time (for example velocities, momenta, angular momenta, etr.) will be purely indeinary; quantities which depend on the square of momente (for example, the kinetlc energy, centriftigal force, etc.) will change sign. It is now tasy to see that the system car equivalently be described by using only real time increnents and changing 


\section{$0 y: 04540235$}

the sign of $\{\forall-E\}$. That is, the tumeling trajectories can be obtainet by finding how the systen moves on the inverted potential surface. 5 For the fission example, the entrance point of will now be close to the top of a hill and the fission valley will be a mountain ridge starting somewhere close to the hill (see Fig. 1 for a

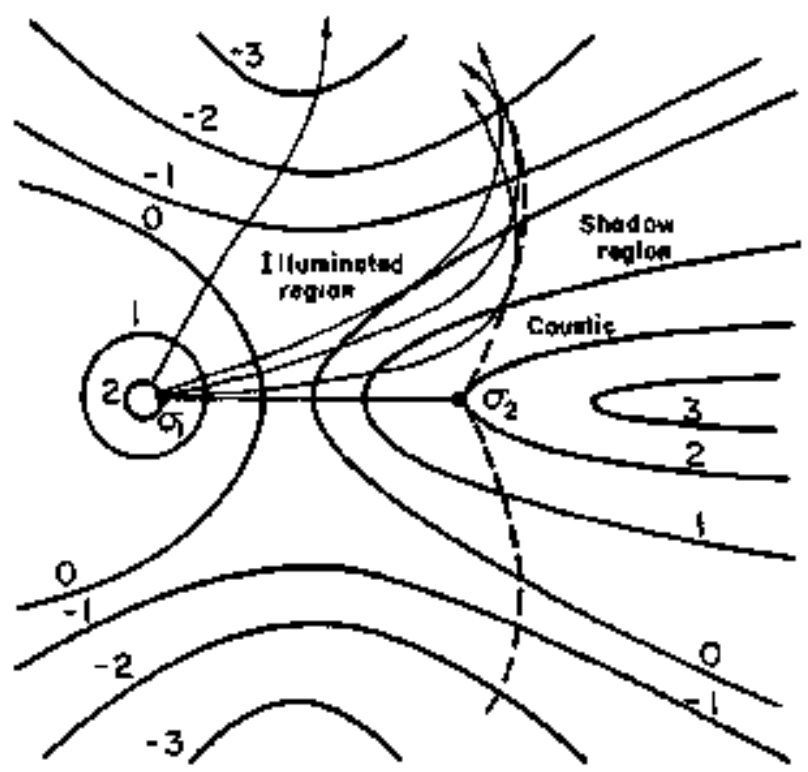

Fig- 1. Schenatic representation of a contour map of a potential energy surface turned aroumd to study the clessical motion below the barrier. The entrance and exit points, $\sigma$ and $\sigma_{2}$, 1 ie on the same equipotential. The hill at the left side corresponds to the potential well in which the nuclens sits before fissioning and the mountaln rarge at the right represents the fission valley.

(XBL 759-3977)

simple illustration of this idea). If after the system raaches $\sigma_{2}$ the time increments are kept purely imaginary (or equivalently, using real time one keeps the inverted potential surface), the systen will move toward of again. Finding the tunneling trajectory, then, is equivalent to firding the pertodic orbit on the inverted potential surface. In the exanple shown in Fig. I there is only one point (in general, it is a certain interval) on the entrance and one point on the exit onergy contour which are connected through classical equations of motion; therefore, when looking for the trajectory with the ninnisnm action $|\$|$ one also has to vary the endpoints $\sigma_{1}$ and $\sigma_{2}$ umtil the minisum is achieved.

We have alșo studied a two-dinensional model of a fission-like barrier system. In order to investigate the full two-dimensional dynamics and to test various approximations it is of value to study a model system with the potential given by an analytical function. An example studied by Hassmann et al. ${ }^{6}$ takes a Haniltonian with potential giving a Gaussian barrier in the $x$ direction ( $x$ being the fission coordinate) and a harmonic potentiaI in the $y$ direction. The mass tengor was taken to be diagonal and constant; the width of the valley, however, was variable. The Haniltonian considered was

$$
\begin{aligned}
H=\frac{p_{x}^{2}}{2 m_{x}}+\frac{p_{y}^{2}}{2 m y} & +v_{0} e^{-\left(\frac{x}{a}\right)^{2}} \\
& +\frac{1}{2} c\left(1+\alpha_{e}^{\left.-\left(\frac{x}{a}\right)^{2}\right) y^{2} .}\right.
\end{aligned}
$$

A non-zero value of the "coupling constant" a allows the width of the valley to vary over the saddle, and by doing so couples the two degrees of freedom. The numerical values were chosen so as to correspond to a typical fission case: $\mathrm{m}_{\mathrm{x}}=500 \mathrm{MeV}^{-1}, \mathrm{~V}_{\mathrm{g}}=7 \mathrm{MeV}, \mathrm{myy}=4.7 \mathrm{MeV}^{-1}$ $\mathrm{a}=0.18 \mathrm{~S}, \mathrm{C}=5.1 \mathrm{kV}$, Btot $=6.0 \mathrm{~km}$. This choice of $C$ and the gives a frequency of about $1 \mathrm{keV}$, typical of a garma vibrational mods. The coordinates $x$ and $y$ are dimensionless and correspond for instance to the deformation paremeters $\varepsilon_{2}$ and $\varepsilon_{4}$.

A fully two-dimensional quantum mechanical solution of this model is possible for not too strong coupling comstant $\alpha$. The method is analogous to the coupled channel calculations described for alpha-decay.

Figure 2 showis in its upper part the shape of the Gaussian barrier together with the adiabatic translational energies in the $x$ direction for the three $y$-vibrational charmels $n=0, n-2$, and $n=4$. Let us rember that the problem we are solving is typical of subbarrier fission, with $\sim 4.5 \mathrm{keV}$ of excitation and $-1.5 \mathrm{MeV}$ below the classical barrier threshold (the coupling is $a=0.1$ ). Higher In chamels are obriously closet, and ofd n charmels are not coupled for paraty reasons. The lower part of the diagran plots the square of the wave finctions for an incident wave from the left in chamel $\mu=2$ (i.e, $\left|u_{2}(x)\right|^{2}$ ). We note the standing wave in the channel $\mu=2$ on the left side of the barrier, as most of the flux is elastically reflected. About $10^{-5}$ is inelastically reflected in channel $\mu=4$ and $10^{-8}$ in ehaminel $\mu=0$. To the right of the barrier $v=0,2$ and 4 waves are transmitted at the $10^{-10}, 10^{-15}$, and $10^{-24}$ probability levels, respectively. There is a vibrational "cooling" effect on the passage through the barrier, with $\nu=0$ transmitted waves dominating regardless of vibrational state incident on tho barrier. only in the case of a very close to zero, a constant valley width, will the "cooling" feature not appear (see Fig. 3).

An obvious approxination to be tested by the coupled channel solution is that the $\gamma$ direction wave function, particutarly the Gaussian of the zero-point lowest state, adiabatically adjusts its width as the vallay changes along the path. That is, in this so-called vibrationaily adiabatic epproximation (WA) one assumes that during the fission process the systen always stays in the same oscillator state $\mu_{\text {. }}$ Then one carries out a one-dimensional calculation, taking into 


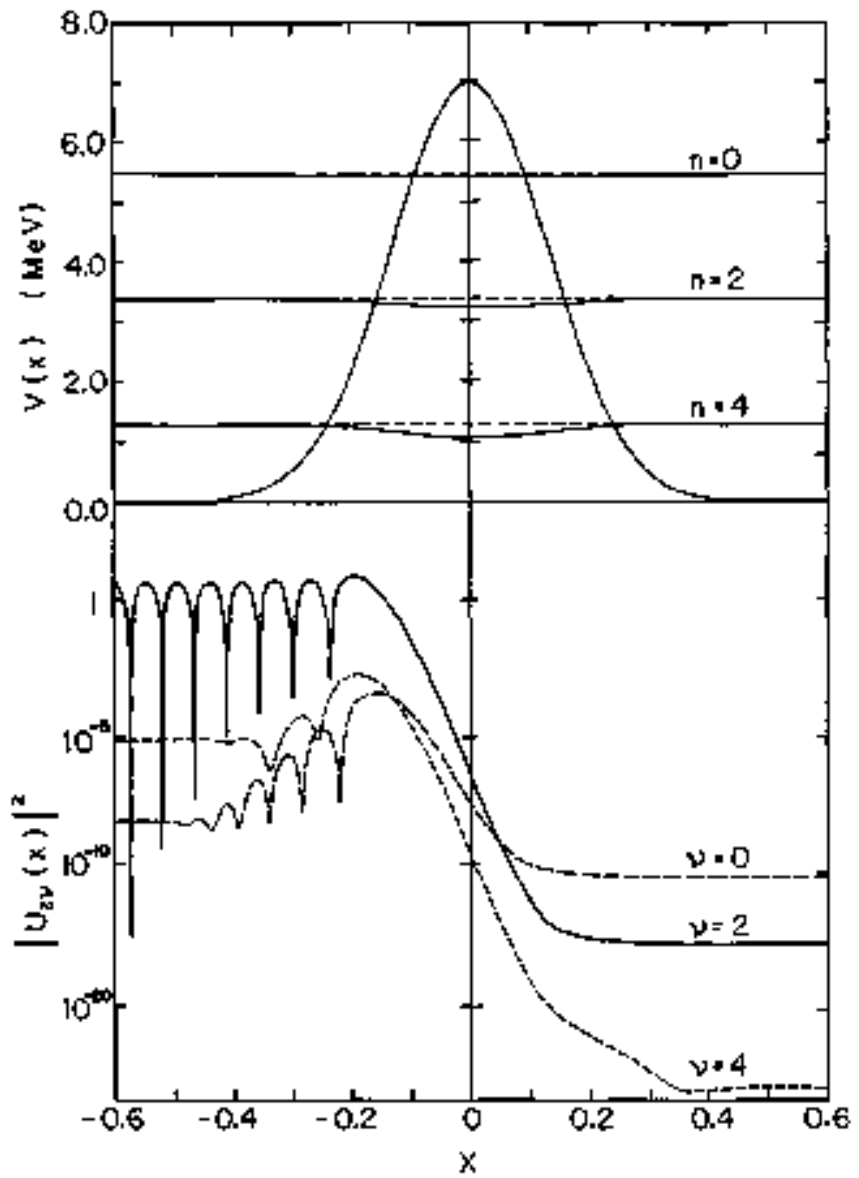

Fig. 2. Fission barrier and the square of the quintum mechanical channel function $U_{2 v}(x)$ for an incoming wave or the chamel $\mu=2$.

(XBL 755-2883)

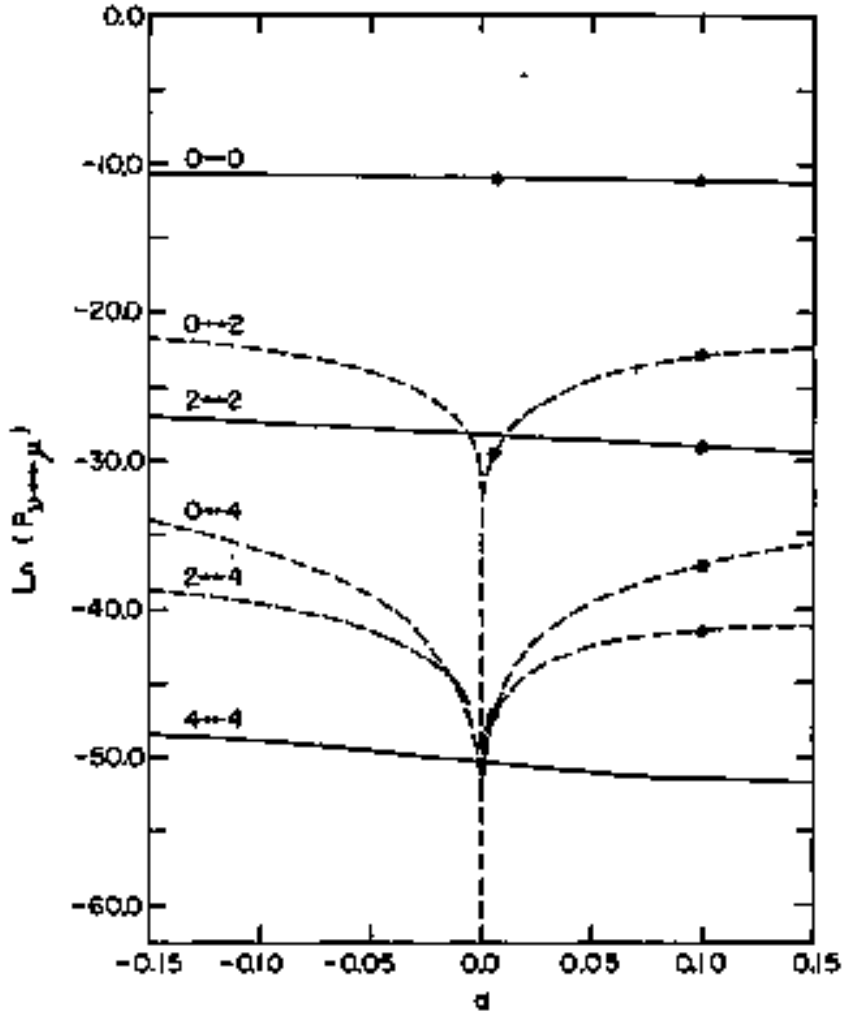

Fig. 3. Penetrabilities Puy for different values of the coupling constant $a$. The Iines correspond to the on coupled channel calculations (solid line for the diagonal and broken lines for the off-diagonal penetrabilities] and the dots. correspond to the USCA calculations.

(XBL 75\$-2884)

Table 1. Couparison betwen the oM and LSCA transition probabilities.

\begin{tabular}{|c|c|c|c|c|c|c|c|c|}
\hline & \multicolumn{5}{|c|}{$a=0.1$} & \multicolumn{3}{|c|}{$x=0.01$} \\
\hline & $D=0$ & $0-2$ & $0 \div 4$ & $2+2$ & $2+4$ & $0 \times[$ & $0+2$ & $0 \times 4$ \\
\hline$\alpha H$ & $1.40 \times 10^{-5}$ & $9.30 \times 10^{-11}$ & $1.03 \times 10^{-16}$ & $2.67 \times 10^{-13}$ & $9.86 \times 10^{-19}$ & $1.64 \times 10^{-5}$ & $1.22 \times 10^{-12}$ & $1.49 \times 10^{-20}$ \\
\hline USCA & $1.44 \times 10^{-5}$ & $9.49 \times 10^{-11}$ & $0.97 \times 10^{-16}$ & $2.52 \times 10^{-13}$ & $9.15 \times 10^{-19}$ & $1+56 \times 10^{-5}$ & $1.30 \times 10^{-12}$ & $1.42 \times 10^{-20}$ \\
\hline
\end{tabular}

account only the change of the oscillator energy. For this example the adiabatic approximation is gond for penetration in the lowest state.

We also tested the vatidity of the USCA (uniform semuclassical approximation) on this model problem. The underlying idea of the USCA is that one uses the analytical contimuation of the classical eqtations of motion to describe the dynamics of the system together with quantized boundary conditions and the quantun mechanical superposicton principle in adding anplttudes for different trajectories. The foundations of the
LSCA and many applications to molecular scattering and reartion problems have been given by Miller and others (see Refs. 7 and 8 ). Here we will, therefore, only give the main results of the USCA as applied to our model.

Once the 5-matrix is known the transition probabilitios $\mathbf{P}_{y+1} \cdot\left|\mathrm{S}_{n+1}\right| \overrightarrow{2}$ follow directly. There is very good agrement between those USCA calculations and the exact quantum mecharical coupled channel calculations, as can be seen froin Fig. 3 and Table 1, even though the model here considered is highly nonclassical. 


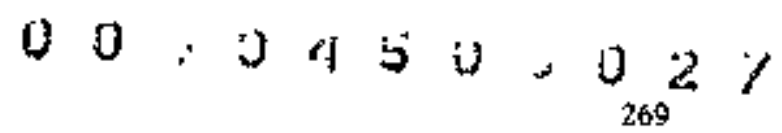

This very good agreenent gives confidence that the semiclassical method may also be applied to more realistic cases with strongex coupling, where the numerical effort does not change very much and where a quantum mechanical calculation would be unfeastble. Stnce coordinate-dependent inertial parameters introduce no additional difficulty, the USCA could be a useful tool to investigate the full dymamics of the coupling betaren the fission coordinate and the other degrees of freedom such as hexadecapole deformations, mass asymatries, and change in pairing correlation.

Recently attention has been focused on a barrier penetration method where one seeks a periodic orbit with time rufning in a purely imaginary direction. With imaginary time inserements the kinetic energy tersus of the classical equations of motion reverse sign and potential barriers invert into troughs. The approach is related to the more general semiclassical methods, except here the variables (except tise) may remain real. Already this picture affords a useful way of thinking about mulli-dimensional bartier penetration and the reciprocal behavior of positive and negative kinetic energy cases. One can apprecigte that penetration paths cut inside the curving barriers by virtue of a negative centrifugal potential, while over-the-barrier trajectories are forced to the outside of the curving valley. Penetration paths of negative kinetic energy are pulled toward lower values of the penetration thass tensor conponent, while at positive kinetic energy the paths seek higher values of the rass tensor ctrmosent. In a coupled channe1 situation there is a vibrational cooling effect for negative kinetic energies and a progressive exciting of vibrational rodes for positive kinetic energies.
We ma reasonably sumeise from the colpledchamel behavior that with inclusion of a friction tern in the classical equations of motion we gain kinetic energy for tunneling paths, just as we lose kinetic energy to heat for increasingly tapid real velocities.

\section{Footnotes and Referencess}

* 'Condensed fromo LBL-4028.

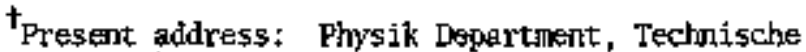
Universităt Minchen, Hest Gextmany.

* Present addreș: Hahn Meitner Institut für Kernforschung, West Berlin.

1. M. Brack, J. Damgaard, A, Jensen, H. C. Pauli, V. M. Strutinsky and C. Y. Wong, Rev. Mod. Phys, 44,320 (1972).

2. T. Ledergerber and h. C. Pauli, Mucl. Phys. $\mathrm{A} 207,1[1973]$

3. J. Waruhn and W. Greiner, Phys. Lett. 44B, $9(1973)$.

4. W. D. Heyers and W. J. Swiatecki, Ark. Fiz. 36, $343(1967)$.

5. W. H, Miller, J, Grem. Phys, 62, 1899 (1975).

6. H. Massmami, P. Ring, and J. 0 . Rasmussen, Phys. Lett. 57B, 417 (1975).

7. T. F. George and W, H. Miller, J. Chem. Phys $57,2458(1972)$

8. R. A. Marcus, J. Chem. Ptys. 54, 3965 (1972).

\title{
3. Relativistic
}

\section{NUCLEUS-NUCLEUS FAAGMENTATION PROBABILITIES ASSUMING EXACT ISOSPIN SYMMETRY}

\author{
Y Karent and R Warlbet
}

In nucleus-nucleus collisich in the $2 \mathrm{GeV} /$ nucleon (lab frame kinetłc energy) range, it has boen sugested by exporinental observations that the fragmentetion cross sections for peripheral collisions factorize into a target factor and a projextile factor, each bejng independent. 1 Sthce the abserved productiun cross sect ions for different isotapes show systematic behavior, it seereed of interest to see how wuch of an effect isospin syometry might play in these regularities.
To do this, we assined factorization and exact isospin symetry. He then considered the possible isospace statistical breakus of isospin 0 nuclides on the assumption that all allowed paths in isospace are equally probable. Using a reșut of Yeivin and de Shalit, ${ }^{2}$ we arrive at a formula for the relative probabilities of different states.

$$
\mathbf{P}_{\mathrm{II}_{3}\left(\mathrm{Z}_{\mathrm{F}}\right)}=\mathbf{F} \mathrm{P}_{\mathrm{II}_{3}}\left(\mathrm{~F}, \mathrm{Z}_{\mathrm{F}}\right)
$$




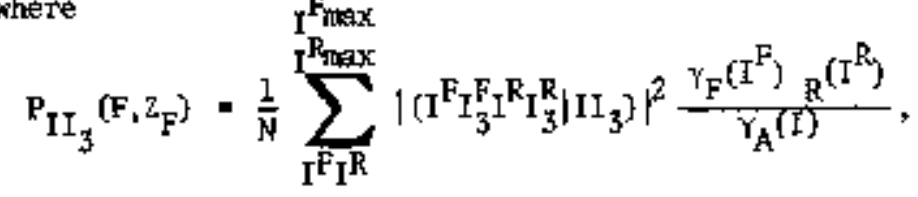

$$
\begin{aligned}
& \gamma_{a}\left(I^{\alpha}\right)=\frac{\left(21^{\alpha}+1\right) \alpha !}{\left(\frac{a t}{2}-I^{\alpha}\right) !\left(\frac{\alpha}{2}+I^{a}+1\right)},
\end{aligned}
$$

where a denotes $F, R$, or $A$, the nucleon ntanber in the fragment, Tesidue, or parent, respectively, and $N$ is the sun of unotmalized probabilities.

To compare this with experiment, we note that experimentally only the charge and mass of the fragments are cbserved; that is, $I_{3}$ and $A$ of the fragments are determined, but the actual I of the states is not observed. Hente, wat may identify an experimental stafe by examining $Z$ (which determines $\left.I_{3}\right)$ and $A$. For 160 , this gives the relative probabilities in Table $1_{\text {, with }}$ the experinental results beside thent.

While noting that relative rattos in selected parts of the table are in fair agreerent between experiment and this calculation, overall agreenent is not good. For example, the experimental ratio botwen ${ }^{3} \mathrm{H}$ and ${ }^{3} \mathrm{He}$ or ${ }^{8} \mathrm{LI}$ and ${ }^{8} \mathrm{~B}$ is not wnity as exact symetry would require, It is jinteresting to note that states such as $15 \mathrm{C}$ and $1 \mathrm{wN}_{\mathrm{N}}$, which ard forbidden by the theory, appear very weakly in the experisental results when compared with the allowed states near to them.

Wie attribute this disagreentent to two causes. First, there is the usuat effect from conlomb symnetry breaking. However, this is minnor connpared to the second problem, which is that wany of the states which are isospin allowed are not observed and are in fact urbound. Experimentally unobserved states were onitted as theoretical entries from Table $\lambda$, and the probabilities nomalized to only the included staras. Binding energy considerations were 1gnored in the calculation, and thus the calculated but unbservad states should really be intermediates, recotpled in isospece until only observed final states are fed. This was not done,

\begin{tabular}{|c|c|c|c|}
\hline$A$ & $z$ & $P_{\text {theory }}$ & $p_{\exp }$ \\
\hline $\begin{array}{l}1 \\
2 \\
3 \\
3 \\
4 \\
6 \\
6 \\
7 \\
7 \\
8 \\
8 \\
9 \\
9 \\
9 \\
10 \\
10 \\
10 \\
11 \\
11 \\
11 \\
12 \\
12 \\
12 \\
12 \\
13 \\
13 \\
13 \\
13 \\
14 \\
14 \\
14 \\
15 \\
15 \\
15 \\
16\end{array}$ & $\begin{array}{l}1 \\
1 \\
1 \\
2 \\
2 \\
2 \\
3 \\
3 \\
4 \\
3 \\
5 \\
3 \\
4 \\
6 \\
4 \\
5 \\
6 \\
4 \\
5 \\
6 \\
4 \\
5 \\
6 \\
7 \\
5 \\
6 \\
7 \\
8 \\
6 \\
7 \\
8 \\
6 \\
7 \\
8 \\
7\end{array}$ & $\begin{array}{l}5.32(-2) \\
8.04(-2) \\
3.72(-2) \\
3.72(-2) \\
5.57(-2) \\
1.58(-2) \\
4.70(-2) \\
2.87(-2) \\
2.87(-2) \\
1.55(-2) \\
1.55(-2) \\
7.02(-3) \\
2.87(-2) \\
7.02(-3) \\
1.57(-2) \\
4.70(-2) \\
1.58(-2) \\
6.59(-3) \\
3.11(-2) \\
3.11(-2) \\
1.70(-3) \\
1.84(-2) \\
5.57(-2) \\
1.64(-2) \\
5.32(-3) \\
3.72(-2) \\
3.72(-2) \\
5.32(-3) \\
1.66(-2) \\
8.04(-2) \\
1.66(-2) \\
0 \\
5.32(-2) \\
5.32(-2) \\
0\end{array}$ & $\begin{array}{l}5.63(-2) \\
2.46(-1) \\
8.69(-2) \\
8.08(-2) \\
2.85(-1) \\
1.13(-3) \\
1.94(-2) \\
1.50(-2) \\
1.27(-2) \\
1.37(-3) \\
7.85(-4) \\
3.12(-4) \\
5.19(-3) \\
2.41(-4) \\
2.23(-3) \\
1.12(-2) \\
1.59(-3) \\
1.16(-4) \\
1.51(-2) \\
1.13(-2) \\
2.41(-5) \\
1.48(-3) \\
3.58(-2) \\
3.70(-4) \\
2.70(-4) \\
1.55(-2) \\
4.74(-3) \\
1.59(-4) \\
2.94(-3) \\
2.66(-2) \\
8.98(-4) \\
2.55(-5) \\
3.23(-2) \\
2.55(-2) \\
4.33(-5)\end{array}$ \\
\hline
\end{tabular}

Table 1.

since it is a dynamic symnetry breaking not predicted by exact isospace invariance.

\section{References}

1. P. J. Lindstrom, D. E. Greiner, H. H. Heckonan, B. Cork and F. S. Beiser, LBL-3650.

2. Y. Yeivin and A. de Shalit, Il Nyovo Cjoento $1-6,1147$ (1955).

\title{
PERIPHERAL HIGH ENERGY COLLISIONS BETWEEN COMALEX NUCLEI
}

\author{
P Mallikes and $Y$ Karanl
}

Recently, soute attenlpts have been made to explast the observed cross sections for the produxtion of specific isotopes in a perfipheral heavy ion collision at relativistic energies (2 Gov/mucleon). As proposed by Bownan, Swiatecki and Tsang the process could be viowed as proceeding in two stages called abrasion (the scraping off of nuelear matter in the collision) and ablation (de-excitation of the remaining fragment). The abrasion part of the reaction has been reformulated by hifiner et al. 3 starting from Glauber's multiple scattering theory. Using a sum rule they calculate the corresponding excitation energles wileh then dictate the ablation part through nuclect and light ion ovaporation. Another model proposed by Lukyanov et a1. 4 asșunes a certain excitation energy distributed over the 


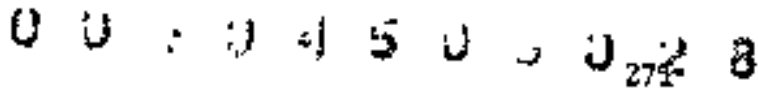

whole fragment which then decays into different sneller fragments with probabilities governed by the Q-values.

Both descriptions depend very juch on the amount of excitation energy released in the process and on the way in which it is distrfbuted over the fraguent. Therefore, a careftul calculation of the abrașion stages seems to be of primary importance. However, both models neglect certain effects, and neither is easily changed to incorporate these neglected effects. On the other hand, because the abrasion-ablation madel formally resembles the mulear cescade-evaporation approach used in light ion reactions, one would be tenteted to perform Honte Carlo type calculations. These are usulily very tedious and, moreover, the underlying physics is not very transparent. In order to circumvent all these objections we have choson a conpletely new exproach which is equivalent to the Monte Carlo one, but handles the physics in a clear manner and contains the Hijfner model as a very special case.

Our starting point is the classical Boltzmatu equation describing the evolution of the one particle distribution function $\mathrm{N}(\overrightarrow{\mathrm{r}}, \vec{v}, t)$ (postion $\overrightarrow{\mathrm{r}}$, velocity $\vec{v}$, time $t$ ) :

$$
\begin{aligned}
& \left\{\frac{a}{\partial t}+\vec{v} \cdot \vec{\nabla}_{r}+\frac{1}{\mathrm{~m}} \overrightarrow{\mathrm{F}} \cdot \overrightarrow{\nabla_{v}}\right\} \mathbf{N}(\overrightarrow{\mathbf{r}}, \vec{v}, t)=
\end{aligned}
$$

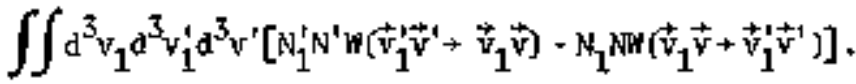

$F$ is the external force (which we will neglect further on) and $W$ denotes the transition probability for scattering of two particles. The right hand side of Eq. (1) Indicates the gain and loss due to collisions into or out of the $d^{3}$ v volume element. As a first application of the Boltzmann equation, we want to consider the case of proton scattering on a target nucleus. Thus the initial condition ( $t+-\infty$ ) for solving Eg. (1) is simpIy (in cylindrical coordinates for $\overrightarrow{\mathbf{r}}$ and spherical ones for $\hat{v}$ )

$$
\begin{gathered}
N(2, b, v, \Omega)=\delta\left(v-v_{0}\right) \delta\left(1+\cos \theta_{v}\right) \delta\left(\phi_{v}\right) \\
{[z+-\infty]}
\end{gathered}
$$

where $b$ is the impact parameter.

As it stands, Bq. (1) is a norlinear partial integro differential equat zon which presents a nontrivial numerical problem. However, we can incorporat由 certain reasonable and physicaly plausible assudations to reduce the complexity. These are: the so-called coherent approximation, which is to assume that the target muleus renains in its ground state whenever an interartion hes taken place; and there are no collisions between scattered particles conly between a scattered particle and ons out of the target density distribution). These approxinations should be reasonable in peripheral colltsions where not too many scattering events take place. The BoItzmann exquation now reauces to a transport equation:

$$
\begin{aligned}
& \left\{\frac{\partial}{\partial \mathrm{t}}+\vec{v} \cdot \vec{v}_{\mathrm{r}}\right\} N(\overrightarrow{\mathrm{r}}, \overrightarrow{\mathrm{v}}, \mathrm{t})=\int \mathrm{a}^{3} \mathrm{v}^{*} \rho(\overrightarrow{\mathrm{r}}) \\
& \times \quad\left[v^{\prime} w(\vec{r}, \vec{v}, t) \sigma\left(\vec{v}^{\prime}+\vec{v}\right)-w(\vec{r}, \vec{v}, t) \sigma\left(\vec{v}+\vec{v}^{\prime}\right)\right]
\end{aligned}
$$

where $\rho(\overrightarrow{\mathrm{r}})$ represents the target mass distribution, $\sigma$ denotes the cross section for nucleon-nucleon scattering, and $\mathrm{N}(\overrightarrow{\mathrm{r}}, \overrightarrow{\mathrm{V}}, t)$ describes the density distribution of the scattered particles. By defining new coordinate, velocity, and tine variables $\vec{t}_{s}, \vec{v}_{5}$, functions of a paraneter s such that

$$
\begin{array}{ll}
\frac{d \vec{r}_{s}}{d s}=\vec{v}, & \vec{r}_{s}(g-t)=\vec{r}(t), \\
\frac{d \vec{v}_{s}}{d s}=0, & \vec{v}_{s}(s-t)=\vec{v}(t) .
\end{array}
$$

we can transform the partial integro-differential Eq. (Z) into an integral eqution which is fosier to handle, and which we are currently solving.

If che would solve Eq. (2) in a probalistic manner, it is completely equivalont to a Monte Cerlo procedure, However, a direct (deterrinistic) solution of Eq. (2) has the advantage that special features like the Pauli exclusion principle, relativistic invariance, and production or absorption processes (for example, of pions) can be incorporated in a rather straightforward manner. A1so, we believe that the more conplex situation of two colliding heavy ichs has a better chance for solution in this framework. Another advantage of this method is that once one knows $N(\vec{I}, \vec{V}, t)$, it is possible to calculate averages, such as the average number of particles, average velocity, and average tenperature, giving one an important link with the vast kowledge developed in statistical mechanics. All the aforementioned aspects are currently under study and the results will be published elsewhere.

Finally, if one would set up an iteration scheroe in orders of scattering and one also restricts the cross sections $\sigma\left(\vec{\psi}^{\top} \rightarrow \vec{\forall}\right)$ to forward angle scattering only, Eq. (2) redices to (In an obvious notation) :

$$
\begin{aligned}
\frac{\mathrm{d}}{\mathrm{ds}} \mathrm{N}^{(n)}(s) & =\frac{1}{\lambda(s)} \mathrm{N}^{(n-1)}(s) \cdot \frac{1}{\lambda(s)} \mathrm{N}^{(n)}(s) \\
\mathrm{N}^{(0)}(s] & =\exp \left[\frac{-s}{\lambda(s)}\right] ; \\
\frac{\mathrm{s}}{\lambda(s)} & =\int v_{\nu}\left(\overrightarrow{\mathrm{r}}_{s}\right) \sigma_{\text {tot }}\left(v_{s}\right) \mathrm{ds}
\end{aligned}
$$

with has the following general solution

$$
N^{(n)}(s)=\left(\frac{s}{\lambda(s)}\right)^{n} \frac{1}{n !} N^{(0)}(s) \text {. }
$$


This Poissun distribut fon is equivalent to a binchl ial distribution and the result is identical to the Hifiner model for abration. 3

\section{References}

1. P. J. Lindstrom, D. E. Greiner, H. H. Heckrtan, B. Cork and F. S. Bieser, LBL-3650; D. E. Greiner,
P. J. Lindstron, H. H. Heckman, B. Cork and F. S. Bieser, Phys. Rev. Lett. 35, 152 (1975).

2. J. D. Bowmin, W. J. Swiatecki, C. F. Tsang, LBL-2908.

3. J. Hüfner, K. Schäfer and B. Schürmann, Phys. Rev. C $\underline{12}, 18 \$ 8$ (1975).

4. V. K. Lukymov, A. I. Titow, Phys. Lett. 57B, I0 (1975).

\title{
RELATIVISTIC HEAVY ION PERIPHERAL REACTIONS
}

\author{
J P Bolsson. T Shibela. J G loennou-Yaninou
}

and $\downarrow 0$ Rasmussen

Very recently peripheral reactions using relativistic heavy ions have been studied by observing particles $1-3$ and $\gamma$ rays. 4 The studies of projectile fragmentation have shown that the linear manentum trousferred from the target to tho profectile is small $(\leqslant 200 \mathrm{~kW} / \mathrm{c})$. We have developed two todels to try to explain isomeric ratios and cross sections for relativistic carbon beams on various targets.

In the first model (applicable to inolastic scattering) it is supposed that the target nucleus obeys a simple she11 model of noninteracting particles in a potential well of Saxon-koods type and that the grazting heavy-ion collision is reduced to an interaction between two peripheral nueleons. Furthermore, it is supposed that this interaction can be represented by the following time-dependent perturbing potential Gaussien in time fend delta function in space at the effge of the nucleus:

$$
V[t]=V_{1}\left(\vec{r}-\vec{R}_{0}\right) e^{-t^{2} / t_{0}^{2}},
$$

where $t_{0}=r_{o} / v$, where $r_{0}$ is the range of the interaction, $v$ is the speed of the projectile, $\mathrm{R}_{0}-\left(\mathrm{R}_{0}, \Omega_{0}\right)$ is the point where the interaction takes place, $V_{1}$ is the strength of the interaction which can depend upon the speed $v$ of the projectile. As a consequence of this interaction the struck nucleon of the target is scattered from a bound orbit labeled a elther to an unocoupied less bound orbit labeled $b$ or to an urbound orbit labeled $c$.

In the target's shell structure it appears as a particle-hole in the first case and a hole in the second case. We have restricted purselves to the first case for the calculation of isomeric ratios in the target-nucleus or to test the ISE (inelastic scattering followed by nticleon evaporation) mechanfsm for lighter-mass products. The transition probability lesfing to a final spin $I$ is

$$
p^{I}=\sum_{a, b} p_{a+b}^{i}
$$

with

$$
\begin{aligned}
& P_{a+b}^{I}=\sum_{m_{a}, H_{b}}\left|J_{a} M_{a} J_{b} M_{b}\right| I M_{a}+M_{b}>\left.\right|^{2} \\
& \times P_{\left(a J_{a} M_{a}\right]} \rightarrow\left(b J_{b} M_{b}\right)
\end{aligned}
$$

where $J_{3}$ and $J_{\text {p }}$ are the spins of the bound states $a$ and $b$. Furthermore it follows from the first order time- dependent perturbation thoory that

$$
\begin{aligned}
& P\left(\mathrm{aJ}_{\mathrm{a}} \mathrm{M}_{\mathrm{a}}\right) \rightarrow\left(\mathrm{bJ} \mathrm{J}_{\mathrm{b}} \mathrm{M}_{\mathrm{b}}\right)= \\
& \left|\frac{1}{\mathrm{~h}} \int_{-\infty}^{+\infty} \mathrm{dt} \mathrm{e}^{\mathrm{i}\left(\mathrm{E}_{\mathrm{b}}-\mathrm{E}_{\mathrm{a}}\right) \mathrm{t} / \mathrm{h}}<\mathrm{bJ}_{\mathrm{b}} \mathrm{H}_{\mathrm{b}}\right| \mathrm{V}(\mathrm{t})\left|\mathrm{aJ}_{\mathrm{a}} \mathrm{H}_{\mathrm{a}} 3\right|^{2}
\end{aligned}
$$

with

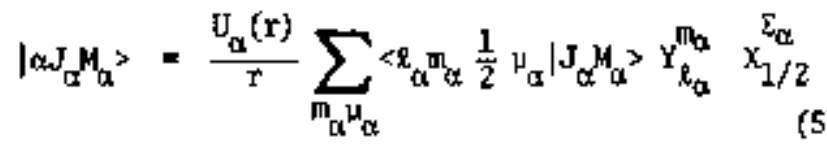

where $J_{a}(r)$ is as follows

$$
\begin{gathered}
\left\{\frac{d^{2}}{d r^{2}}+\frac{2 m}{\hbar^{2}}\left(E_{\alpha}+V(r)\right)-\frac{\ell_{a}\left(l_{a+1}\right)}{r^{2}}\right\} J_{a}(r)=0 \\
U_{\alpha}(0)=U_{\alpha}(\infty)=0
\end{gathered}
$$

In relations (6) and (7) a stands for $a$ or $b$. The potential $V(r)$ has been chosen according to Rof. 5. Froin relations (1) and $(3-5)$ it can be shown that

$$
\begin{aligned}
p_{a+b}^{J} & =\frac{\left(2 J_{a+1}\right)\left(2 J_{b+1}\right)}{3 / \pi}\left[\left\langle J_{a} \frac{1}{2} J_{b} \frac{1}{2}\right| 1 t>\frac{v_{1} t_{o}}{\hbar}\right. \\
& \left.\times \frac{U_{a}\left(R_{o}\right) v_{b}\left(R_{o}\right)}{R_{o}^{2}} \exp \left\{\left[\frac{\left(E_{b}-E_{a}\right) r_{o}}{2 f c}\right]^{2}\right\}\right]_{(8)}^{2}
\end{aligned}
$$

Using relation ( 8 ) we have calculated the probability $\mathrm{P}^{\mathrm{I}}$ so that the totat spin of the target mucleus, 


\section{$00 . \mathrm{J} A \mathrm{~S}+\mathrm{U}_{27} \dot{3}$}

just after the collision has taken place, is I, In these calculations $R_{0}$ was chosen equal to the radiug of the nucleus. The results obtained for ${ }^{208} \mathrm{pb}$ and ${ }^{4} \mathrm{Sr}$ are indicated in Fig. 1. First we conszider the experimental isomeric yield ratio $\sigma\left(13 / 2^{*}\right) /$ $\sigma\left(5 / 2^{-}\right)$in the $207 \mathrm{pb}$ target excited by inelastic scattering of relativistic $12 \mathrm{C}$ ions. The in-beem gamisa measurements of Shtbata et al 4 give a ratio of $0.84 \pm 0.20$. The $44 \mathrm{keV}$ proton $208 \mathrm{po}(\mathrm{p}, \mathrm{pn})$ measureurents of Kawakari et al. ${ }^{6}$ give $0.48 \pm 0.16$ and they concluded from various kinds of evidence that the reaction mechanism was predominantly clean knockout (CKO) and not inelastic scattering followed by evaporation (ISE). On Fig. 1b it is seen that the inelastic scattering (IS) gives a higher proportion of high spin states and thus explains the higher iscumeric ratio for the inelastic scattering case. The result qualitatively supports the idea of random colpling of the particle and hole angular mounentuda vectors. Now we consider the $\sigma\left(7^{*}\right) / \sigma\left(2^{+}\right)$ isomeric yield ratio tn $206 \mathrm{Fb}$. The $44 \mathrm{MeV}$ proton
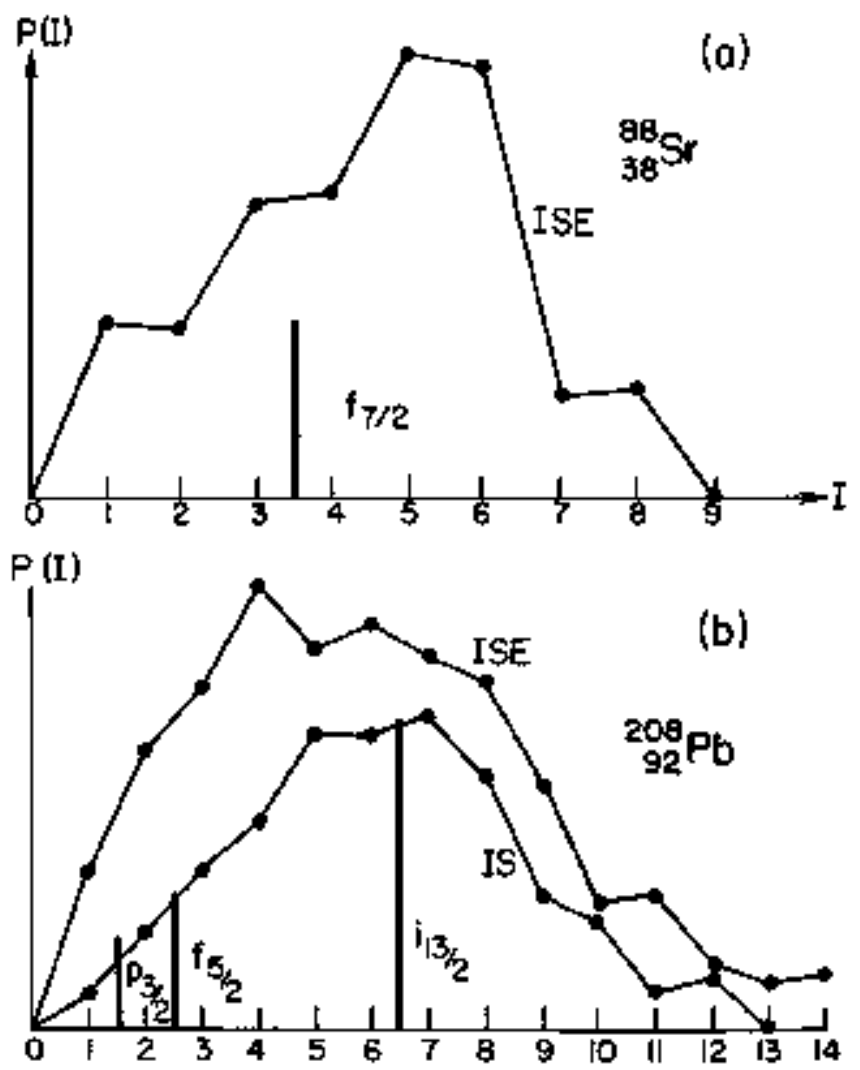

I

Fig. 1. Transition probability of a level of spin J. The curve of (a) corresponds to the transitions

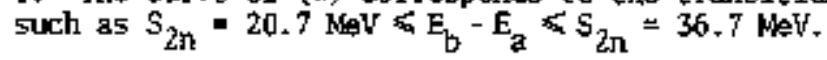

The lower curve (IS) of 1-b corresponds to the transitions such as o f $\mathrm{F}_{\mathrm{h}}-\mathrm{E}_{\mathrm{a}} \mathrm{F}_{\mathrm{j}} \mathrm{S}_{\mathrm{h}}=6.7 \mathrm{MV}$. The upper curve (ISE) of (b) corresponds to the transitions such as $S_{n}=6.7 \mathrm{MeV}<\mathrm{E}_{\mathrm{b}}-\mathrm{E}_{\mathrm{a}}<\mathrm{S}_{2 \mathrm{n}}$ 14.8 HeV. The length of the vertical lines are proportional to the weighting factor $21+1$ of possible states for clein knotk out process (CKO). The space between two different values of $I$ is proportional to $\mathrm{A}^{-1 / 3}$. results of Kawakani et a16, ${ }^{608} \mathrm{~Pb}(p, \mathrm{p} 2 \mathrm{n})$ yave $0.60 \pm 0.2$ for the ratio and they concluded that the dominant nechenism was knokk-out of one neutron followed by evgpration. In the high energy heavy ion studies 4 on the $207 \mathrm{pb}$ target the isomer ratio in $206 \mathrm{gb}$ was $0.4 \pm 0.2$. Examination of Fig. Ib indicates that the CKO and ISE mechanism should give about the same isomeric rat fos in the $P b$ region (this presumed insensitivity is similar to that observed by $\mathrm{Yu}$, Caretto and onurch for $197_{\mathrm{Au}}$ (p,pal) reactions). Within experimental error the low onergy proton and high energy heary-ion isomeric ratios are the same.

We considar now the $\sigma\left(9 / 2^{+}\right) / \sigma\left(1 / 2^{\top}\right)$ isomeric yield ratfo in $87 \mathrm{Y}$ formed by $8 \mathrm{~S}_{\mathrm{R}}\left(\mathrm{c}_{\mathrm{g}} \mathrm{n}\right)$ at 13 to $29 \mathrm{Mor}, 89 \mathrm{Y}(\mathrm{p}, \mathrm{p} 2 \mathrm{n})$ at 25 to $80 \mathrm{keV}$ and the $89 \mathrm{Y}$ $(12 \mathrm{C}, X){ }^{\prime} 87_{\mathrm{Y}}$ at 3 [eeV. on the $(\alpha, 2 n)$ study at 22 Nev the angular momentum distriburion in the $89_{Y}$ cropound nucleus should be higher than for our ISE calctulation plotted tn Fig. 1a. The experintentat isomeric ratio are $n 6$, indeed higher. In the low energy portion of the reaction the isomeric ratio levels off at $\approx 2$ between 50 and $85 \mathrm{MeV}$, and the 3 GoV 12 C results givo a stcill lower ratio of $1.4 \pm 0.3$. Kawakami et al, concluded that the (p, $\mathrm{p}^{2 \mathrm{n}}$ ) reaction on $20 \mathrm{BPb}$ went by $\mathrm{kCE}$ (knock out followed by evaporatiml. For a ${ }^{8} 8_{\mathrm{Sr}}$ target the only contributing neutron orbital for kole is the f $7 / 2$. Thus kos should give lower isomeric ratio than either twouneutron knock out or ISE (inelastic scattering followed by two-neutrons evaporation). Thus the $3 \mathrm{GeV} 12 \mathrm{C}$ ratio suggests dominance of KOE.

The second model involves a classical kinetic theory calculation of the probability of one and only one nucleon-mucleon collisicu. We consfder the terget and projectile nuclei as consisting of mucleon-gas clouds, interpenetrating each other. and we assune a Gaussian mass density profile, in the nucleouns rest frane, of the form:

$$
\rho \Rightarrow \rho_{0} e^{-r^{2} / 2 a^{2}}
$$

where in Eq. (9), Po and a are related through the normalizat in condition

$$
\int \mathrm{pd}^{3} \mathrm{r}=\mathrm{A}
$$

by

$$
a=\frac{1}{\sqrt{2 \pi}}\left(\frac{A}{\rho_{0}}\right)^{I / 3}
$$

with A being the atomic nomber of the nurleus. The values of $p_{0}$ and a can be then calculated using the experimental value of the rTs radius of the charge distribution of the nuclers and under. the assumption of the same mass and charge distribution in the nucleus. 10 the then proceed to calchlate the numer of grazing nucleor collisions by enploying classical kinetic theory argunents. We thus have that the nubber of collisions $\mathrm{dR}$, in a volume elearent $d+F$, and between time $t$ and $t+d t$, is given by:

$$
d R=\sigma_{0}\left(p_{T} d^{3} \cdot \vec{T}\right)\left(p_{p} v\right) d t
$$


where in $\mathrm{kq}$. (12) $\mathrm{T}, \mathrm{p}$ stand for target and projectile respectively, $\sigma_{0}$ is the nucleon-rucleon collision cross section, and $v$ is the velocity of the inconing projectile taken to be alonis the $x$-axis. Hence, we obtain an expression for the number of collisions $\mathrm{k}\left(\mathrm{b}_{0}\right)$ as a function of the inquat parameter $b_{\text {o }}$ that is, the distance between the centers of the target and projectile density profiles. Thus,

$$
R\left(b_{0}\right)=\sigma_{0} \frac{A^{4 / 3}}{2} D_{0}^{2 / 3} \exp \left[-b_{0}^{2} \pi / 2\left(\frac{p_{0}}{A}\right)^{2 / 3}\right] .
$$

We can therefore calculate the one nixleon collision cross section $\sigma$ (one nucleon collision) of ther by equating it, as a first approximation to the area between two concentric circles with radii equal to the impact paraneters corresponding to a ntwiber of collisions $\mathrm{R}=0.5$ and $\mathrm{R}=1 . \mathrm{S}$ respect assumfing a Polsson distribution of the collision events and proceeding accordingly, In the formes case, we have:

$$
\begin{aligned}
& \sigma[\text { ome nucleon collision })= \\
& {\left[\left[b_{0}(R=0.5)\right]^{2}=\left[b_{0}(R=1.5)\right]^{2}\right] .}
\end{aligned}
$$

In the latter case, the probability $P(m)$ of $\mathrm{m}$ collisimas is given by:

$$
p(m)=\frac{M^{m} e^{-H}}{m !}
$$

with $M=R\left(b_{0}\right)$, so that the cross section is given by:

$$
\int_{b_{0}}^{\theta(\text { one nucleon collision })=} 2 b_{0} d b_{0} p(m+1)=\frac{\pi}{C_{2}}\left[1-e^{-c_{1}}\right]
$$

where in Eq. (16)

$$
C_{1}=\sigma_{0} \frac{A^{4 / 3}}{2} p_{0}^{2 / 3}, \quad C_{2}=\frac{m_{0}}{2}{\frac{P^{2 / 3}}{A}}^{2 / .}
$$

Wh apply the above results in the case of ${ }^{12} \mathrm{C}$. $12_{C}$, since this is a well experimentally studied. process, so that we can compare results. Fros the iiterature 10 , li the pert inent values of $\sigma_{0}$ and rats charge radius are:

$$
\begin{aligned}
& \sigma_{0} \equiv 16 \text { thelativistic energies) } \\
& {\left[<\mathrm{r}^{2}>\right]^{1 / 2}=2.37 \times 10^{-13} \text { er for }{ }^{12} \mathrm{C} .}
\end{aligned}
$$

Thus we have:

Using Eq. (14), get: $\sigma=258$ nob

Using Eq. (16), we get: $\quad \sigma=235 \mathrm{mb}$

We compare Eqs. (18) and (19) with related experimental cross sections. 2,4 Table 1 gives for ${ }^{2} \mathrm{C}$ projectile on $12 \mathrm{C}$ the cross section for one and two-nucleon rerooval reaction. Clearly the experimental cross section for one- and two-renoval product is lower than that given by Eqs. (18) or (19). It may be argued that inelastic scattering
Table 1. Cross sections by $1.05 \mathrm{GeV} / \mathrm{N}$ carbon bombardment on carbon.

\begin{tabular}{cc}
\hline Product & Cross Section (jo) ${ }^{\mathrm{a})}$ \\
\hline $11_{\mathrm{C}}$ & $44.7 \pm 2.8$ \\
$10_{\mathrm{C}}$ & $4.44 \pm 0.24$ \\
$11_{\mathrm{B}}$ & $48.6 \pm 2.4$ \\
$10_{\mathrm{B}}$ & $27.9 \pm 2.2$ \\
$10_{\mathrm{Be}}$ & $5.34 \pm 0.29$ \\
$\mathrm{sum}$ & 131 \\
Ref. 2. &
\end{tabular}

should be included in the total, and it mity be around $24 \mathrm{mb}$, the cross gection for the $5 / 2+1 / 2$ (ground) transition in $19 \mathrm{~F}$ with $1.05 \mathrm{GeV} / \mathrm{n} 12 \mathrm{C}$ on teflon. Probably the theoretical cross section needs to be rexhred by a geometrical factor, so as to exclude collisions in which either nucleon enters the nucleus and causes a further reaction. These refinenents are being considered.

\section{Referances}

1. D. E. Greiner, P. J. Lindstrom, H. H. Heckman, B. Cork and F. S. Bieser, Phys. Rev. Lett. 35, 152 (1975).

2. P. J. Lindstran, D. E. Greiner, H. H. Heckman, B. Cork and F. S. Bieser, LBL-3650 (1975).

3. A. M. Zebelman, A. M. Poskanzer, J. D. Bownan, G. R. Sextro and V. E. Viola, Phys. Rev. C 11, $1280[1975)$.

4. T. Shibatg, H. Ejiri, R. E. Anholt, H. R. Bowosan, J. G. Ioannou-Ýanou, J. O. Rasmussen, E. A. Rauscher and S. Nageniya, in this Anmol Report (to be published in Nucl. Phys. A).

S. V. G. Soloviev, Theory of Complex Noxlei, translated by P. Vogel (Pergaten PTess, Ket York, 1976).

5. H. Kawakemi, M. Koike, K. Konnna, M. Sakai, N. Yoshikawa, R. Donangelo and J, O. Rasulussen, Nutcl, Phys, A262, $52(1976)$.

7. Y. H, Yu, A. A. Caretto, Jr, and L. B. Church, Nouc1. Phys. Al52, 295 (1970).

8. R. Von den Bosch, L. Haskin and J. C. Normon, Phys, Rev. B137, 1134 (1965).

9. G. B. Saha, N. T. Porilo and L. Yaffe, Phys. Rev. B144, $962(1966)$.

10. R. Hofstater, Am. Rev. Nuc1. Sci. 7, 231 (1957).

11. N. Metropolis et al, Phys, Rev, I10, 185 (1958). 


\title{
$00: 345 * 930$
}

\section{B. NUCLEAR STRUCTURE}

\section{Microscopic}

\author{
EVIDENCE FOR TRIAXIAL SHAPES IN Pt NUCLEI*
}

d Meyer-ter-tennt

From a theoretical standpoint, there are significant advantages in studying the motion of a high-j particle $\left(\mathrm{e}+\mathrm{g} \cdot i_{13 / 2}\right.$ or $\left.h_{11 / 2}\right)$ around a deformed core. First, these high J-orbitals have mique parity and hence the highri system may be treated to a good approxination by considering only a single t-shel1. Furthemore, positive (negative) parity states in odd nuclei in the mass region $150<\mathrm{A}<200$ hay be immediately associated with a predominant $v i_{13 / 2}\left(\pi h_{11 / 2}\right.$ or $\left.\pi h_{g / 2}\right)$ component. second, the large Coriolis force acting on the high-j particle tends to align its spin along the rotation vector, giving rise to characteristic decoupled level structures. 1 The degree to which the particle couples to or decotples from the intrinstc axes of the tore strongly depends on the shape of the core, not cilly on its deformatfor $B$ but also in a rather sensitive way on the $\gamma$-param. eter which distinguishes between prolate $\left(\gamma=0^{\circ}\right)$, oblate $\left(\gamma^{=60^{\circ}}\right)$, and yarious asyumetric, triaxial $\left(0<y<60^{\circ}\right)$ shapes. 2,3 This leads to striking variations of the odd-A spectum as a function of $\gamma+$ As one of the characterfstic features, second and third states of the same spin are considerably lowered in energy for triaxial shapes, paralleI to the $2+3,3_{1}^{+}, 42 \ldots$ states of the ever core. This is important for odd-A Pt nuclei.

A number of these features have previously beish noted 2,3 in the $h_{11 / 2}$ and $h_{g} / 2$ proton struc. tures of same odd-A Ir, ku, and thuclei. It thus appears that the shape change from the axially 5ymltric deformed rare-earths to the spherical $\mathrm{Pb}$ muclei proceeds through a triaxial region. The levels of the doubly even Pt nuclei which lie in this transitional reglon do indeed suggest triaxial shapes, with the $2 \frac{F}{2}$ levels Iying below the $4_{I}^{+}$levels.

In general, all the odd Pt nuclei we have studied have similar positive parity structures, a distinctive feature being the occurrence of decoupled $i_{13 / 2}$ bands with spacings very similar to those of the even-even neighbors. Many other positive parity levels are also observed, especially in 191Pt, which we have also studied in the radionctive decay of 191 Au. In fact, the combined 191 pt results provide the most cowplete set of data for an $i_{13} / 2$ family and therefore a new, inportant test of the model of a hifh-j hole or particle coupled to a triaxial core. It should be noted that the Fermi surface lies well within the $i_{13 / 2}$ shell in Pt nucle1, a situation not hitherto encountered in tests of this Joder.

A partial level scheme for 191pt containing mly the positive parity levels is shown on the left of Fig. 1 . Levels in colums $A, B$, and $C$ were observed in the $(a, 7 m)$ experinents; the levels in colum 5 plus the $13 / 2^{+}$and $11 / 2^{+}$levels of colums $A$ and $B$ were populated in the decay of $19 \mathrm{I}_{\text {Aus }}$. The right side of Fig. 1 shows levels expected fron coupling an $i_{13 / 2}$ hole to a triaxial core calculated

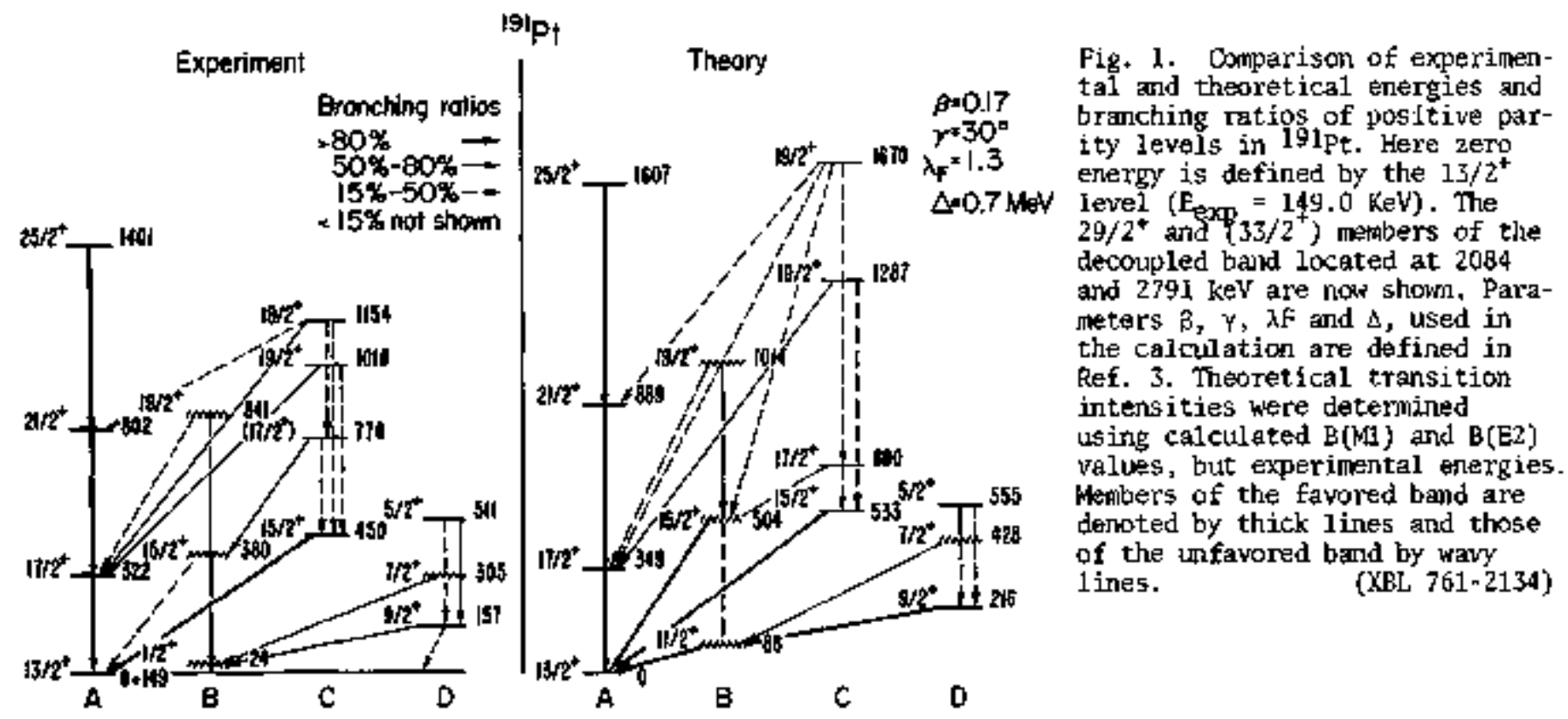


as described in Ref. 3. The parameters and $Y$ were determinet from the neighboring even cores. The Fermi level was located between the fifth and sixth menbers of the $i$ 13/2 multiplet $(v-5$ and $\psi=5$ levels, in the notation of Ref. 3) by exarining the single particle levels at $r=30^{\circ}$ of Larsson. ${ }^{\circ}$ The coriolis matrix elements were attenuated by jsing the modified pairing factor, $\left(u_{1} u_{2}+v_{1} v_{2}\right), 5$ following the ad hoc prescription of $\$$ tephens and Sinon. 5 This had the effect of reducing the matrix elements connecting states across the Fermi surface, a procedure previdusly foumd necessary for fitting $13 / 2$ bends in the rare-earth region.

The arrangement of levels in Fig. 1 has been guided by the main decay lines of the experimental spectron and the calculated results. Although the wavefurctions of the particle-triaxial core model consist of a highly complicated mixture of different Milsson bands and corresponding odd-A $\gamma$-bands, there are some approxinate features which ray give an intuitive pictire. (1) Columen A represents the decoupled yrast band corresponding to a maxtmum alignment of the $j=13 / 2$ spin with the core angular momentur $R$ : the $9 / 2$ and $5 / 2$ states in colum $D$ can be interproted as anti-alighed states of the same band, (2) Similarly, colum B and the onti-aligned $7 / 2$ state of colum D constitute a second (umfavored) decoupled band. It corresponds to incomplete alignment with a $J$ projection of approdinately $11 / 2$ on $R$. In the $h_{11 / 2}$ and $h_{9 / 2}$ level fanilies observed in neighboring Ir and tu nuclei, these unfavored levels appear much higher in energy. They are lowered in iflpt due to incomplete filling of the $j=13 / 2$ shell by core partleles and the resultant effect of the Pauli principle. This second decoupled band within the same $j$-shelI family is found here for the first timo. Similar bands have been observed in meighboring odd-A Pt isotopes and should also occur in $\mathrm{Hg}$ isotopes and in ather mass regions. (3) The levels in colum $\mathrm{C}$ reflest the additional rotational degrees of freedom due to triaxiality; levels with the same spin lie at much higher excitation $(1,7-4.7 \mathrm{Mar})$ in calculations with axially synuetrtc shapes. There are additional levels in the theoretical spectrum below the $21 / 2$ state which are not shown in Fig. 1: two 9/2 states at 367 and $624 \mathrm{keV}$, two $\$ 1 / 2$ states at 574 and $747 \mathrm{keV}$, and three $13 / 2$ states at 334,730 , and $875 \mathrm{keV}$. However, these are not expected to be populated with observable strengths in the experiments performed.

Figtre 1 shows clearly that the calculated level energies and transition brancheng ratios reprotuce very 11 the general trend of the data. The signs of the calculated $\mathbf{E} 2 / \mathrm{M}$ rixing ratios also agree with experiment, where measurable. Considering that the parameters entering the calculation were obtained from "external" data and not otherwise adjusted to produce detailod fits here, the agreesent between theory and experiment is excellent.

\section{Footnotes and References}

tCondensed from LBL-4349, subritted to Phys. Rev. Lett.

${ }^{\dagger}$ Present addrass: SIN, OH 5234, Villiger, Switzerland.

1. F. S. Stephens, Rev. Mod. Phys. 47, 43 (1975).

2. J. Meyer-ter-Vehn, F, S, Stephens and R. M. Diamond, Phys. Rev. Lett. 32, 1383 (1974).

3. J. Meyer-ter-Velm, Macl. Phys. M249, 111 [1975); Ibid, 141 (1975).

4. M. Pìparinen, J. C. Omnane, P. J. Daly, C. L. Dors, F. M. Bernthal and T. L. Xhoo, Phys. Rev. Lett. 34,1110 (1975).

5. S. E. larsson, Physica Scrlpte ㅇ, 17 (1973).

6. F, S. Stephens and R. S. Simm, thel, Phys. Als3, $257(1972)$.

\title{
SYMMETRY BETWEEN PARTICLE AND HOLE LEVELS SYSTEMS IN ${ }^{1 B 9} A U^{*}$
}

\author{
J $L$ wood, P w Fink,t and Meyerter-Venns
}

The $h_{11 / 2}$ and $h_{g / 2}$ level systems in $189_{\mathrm{Au}}$ have been studied both experinemtally and theoret. ically. These negative parity famflies are row know in a number of odd-A Ir, Au, and Tl lsotopes and are attributed to a hole in the $h_{11 / 2}$ sholl and a particle in the ha/2 shell, respectively. Going from 187 Ir to $195{ }^{2} 11$, for example, the ordering of levels in these families indicates that the coupling to the core undergoes smooth changes as follows:

$$
\begin{aligned}
& h_{9 / 2} \text { particle: decoupled } \rightarrow \text { strongly coupled } \\
& h_{11 / 2} \text { hole: }
\end{aligned}
$$

The different ordering of the levels has been explained in terms of the particle-rotor madel, I
Mbre recently this model has been generalized for triaxially deforted cores. 2 The corplementary behavior of the particle and the hole bands in 189 Au can be understood as a manifestation of the particle-hole symmetry underlying this model. The object1ve of this letter is to evaluate the available experimental data on the two level systems in 189. and to show that if scaled, they have almost identical energy spacings and therefore display this symmetry in a very striking way.

The excited states of ${ }^{189} \mathrm{Au}$ have been studied recent Iy at UNISOR, 3,4 and at Orsar 5 by population through the $\mathrm{e}^{+}$-decay of $189 \mathrm{ht}(9 \mathrm{~m})$ and $1890 \mathrm{Hg}(\mathrm{m})$ and at orsay by in-bean spectroscopy using the reaction 18 Ta $(12 \mathrm{C}, 4 \mathrm{n}) 189 \mathrm{AW}$, The levels schemes based on the Orsay work differ from Fig. 1 in that, with reference to the orsay schentes: the lovol at 


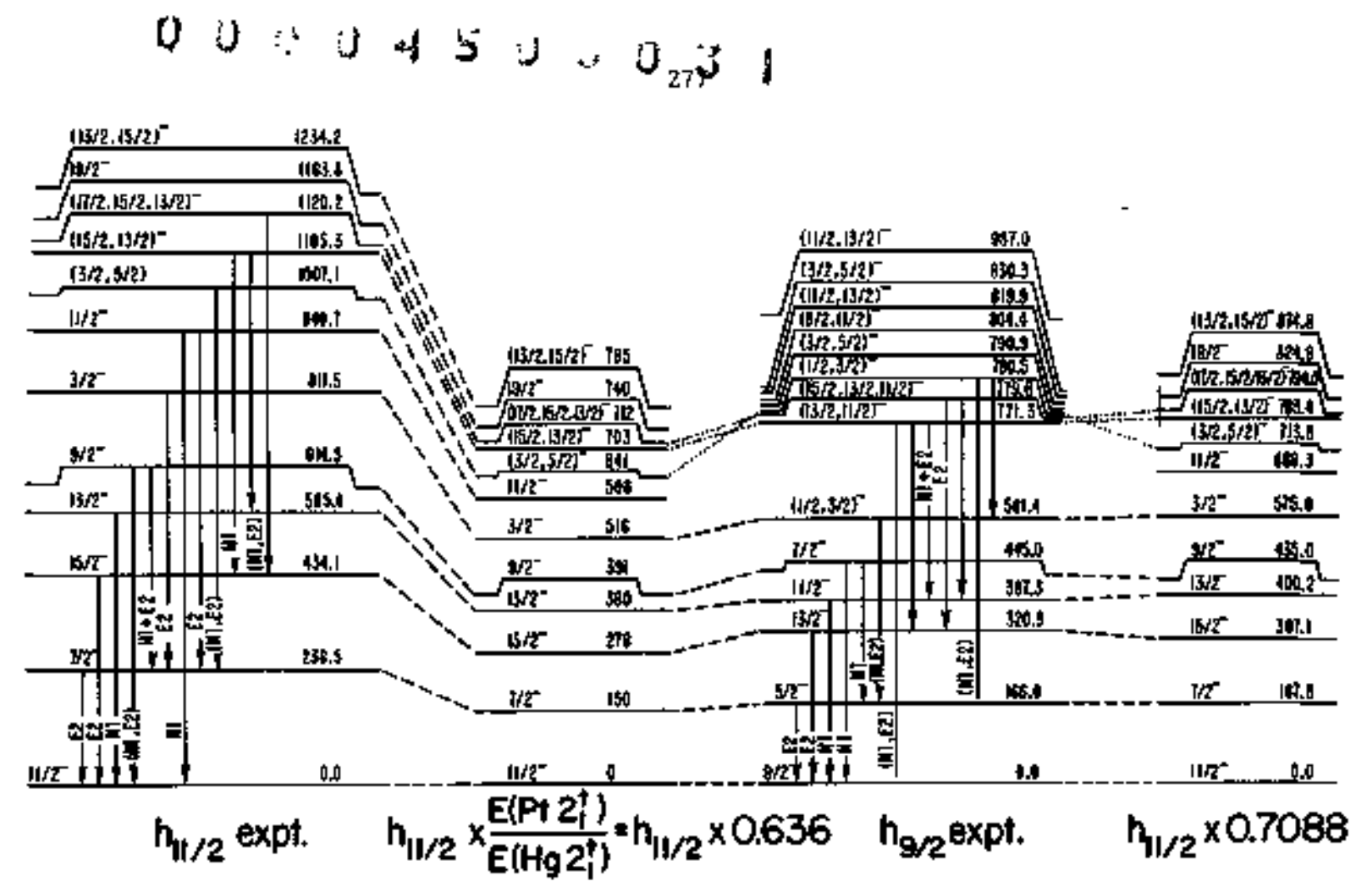

Fig. 1. The experimental $h_{1} / 2$ and $h_{g / 2}$ bands are based on the results of UNISt 2 experiments, topether with the $10 / 2^{-}$mopber assigned to the $h_{11 / 2}$ band in Ref, 6. Discrepancies betweer these data and those of Refs, 5 and 6 are discussed in the text. Multipolarities are included where krown, and if ambiguous, are denoted (MI,E2) to mean an wndetersined Ml/E2 admixture, the ambiguity generally being due to the complexity of the spectra. Fossible spin assigmants rely mainly on oultipolarityes, population systematics froin the decays of $189^{\prime} \mathrm{H}_{\mathrm{g}}\left(13 / 2^{+}\right)$ and $189 \mathrm{gHg}\left(3 / 2^{-}\right)$and for the $h_{1} / 2$ band, funther support from the strong $h_{11 / 2}$ band energy systematics through $189-195_{\text {Ala. }}{ }^{3}$ The mergy scale compression. factors $(0.636$ and 0.7088$)$ for the $h_{1} / 2$ band are determined by the energy ratio of the $2+$ state in the effective cores ( $190 \mathrm{Hg}$ for $\mathrm{h}_{11 / 2}$, $188 \mathrm{Pt}$ for $\mathrm{h} / \mathrm{l} / \mathrm{l}$ and by an ad hoc ratio that gives the closest anlogy for the two bands, respectively. In the region of the $j+4$ states, the particle-hole sylinetry is less clear and transitions are ondy included for levels that have tentatively identified analogs: these levels are comected by dots instead of dashes.

(XBL 76]-2135)

$1007.1 \mathrm{keV}$ in the $h_{11 / 2}$ band ts assigned $11 / 2^{\prime}$ (note that the energies of the levels within each band are specified relative to the band head); the $17 / 2^{-}$ nember of the $h_{11 / 2}$ band is placed at $1472.6 \mathrm{keV}$; and no spin assiguments are rade to the levels at $1105.3,1120,2$, and $1234.2 \mathrm{keV}$. Further, in the hg/ 2 band, the Orsay schemes do not contain the levels at $\$ 61.4,780.5,790.9,830.3$, and $987.0 \mathrm{keV}$; they have an assignuent of $17 / 2^{-}$to the $779.6 \mathrm{keV}$ level; and they have no spin assignment for the leve 1 at $771.3 \mathrm{keV}$. Firally, in the $9 / 2$ band, the $395.4 \mathrm{keV} \gamma-11 \mathrm{ne}$ (the transition between the $1 / 2^{-}$and $5 / 2^{-}$levels in the (NISOR scheme) is reported in the Orsty work to be M muttipolarity. other discreparcies include insufficient evidence in the UNISOR work to support the level reported in the Drsay measurements at $990.6 \mathrm{keV}$ in the $h_{9 / 2}$ band and minor ambiguities such as the assignment of $\gamma$-lines to more than me location. A detailed discussion of these differences is contained in the paper this report was condensed from.

The theoretical interpretaticn of these results is based on the model of a particle (ar hole) coupled to a triaxially defomed core. 2 It has been shron that the model describes well the low-energy mique parity states of transitional add- $A$ nuclei in the $A=190$ mass region. 2 The model spectrum $E_{I}\left(\beta, \gamma, \lambda_{F}\right)$ depends on the deformation $B$, the shape asyumetry $\gamma$, and the Fermi energy $\lambda_{F}$. 
(The subscript I is the nuclear spir.) Particle and hole spextra are connected by the symmetry relation

$$
\mathrm{E}_{\mathrm{I}}(\beta, \gamma, j \text {-particle })=\mathrm{E}_{\mathrm{I}}(\beta, \gamma, j-h \text { hle })
$$

where $h_{F}$ is located welI below the $f$-shell of the add nucleon for the particle spectrum and well above the $j$-she11 for the hole spectrum. This condition is essentially met in the Aul isotopes with respect to the $h_{9 / 2}$ shell ( $\lambda_{F}$ below) and the h11/2 shell ( $\lambda_{F}$ above). Taking into account that $E_{I}$ is weakly dependent on $j$ and depends an $B$ strongly only throutgh a scale factor $I / \mathrm{B}^{2}$, but much less otherwise, we have approximately

$$
\begin{aligned}
& \mathrm{B}_{h_{9 / 2}^{2}}^{2} \cdot \mathrm{E}_{\mathrm{I}}\left(\mathrm{Y}_{1} h_{9 / 2} \text { particle }\right)= \\
& \mathrm{B}_{h 11 / 2}^{2} \cdot \mathrm{E}_{\mathrm{I}+1}\left(60-\mathrm{Y}, h_{11 / 2} \text { hole }\right) .
\end{aligned}
$$

Based on this relation, the close reserblance of the , proportionally scaled $h_{9 / 2}$ and $h_{11 / 2}$ systems in 189 All (see Fig. 1) indicates that $\mathrm{F}_{9 / 2}=60^{\circ}-$

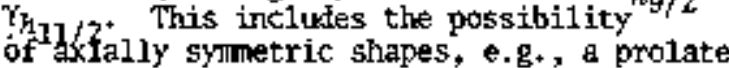
$\left(y=0^{\circ}\right)$ shape for the $h 9 / 2$ and an oblate $\left(y=60^{\circ}\right)$ shape for the $h 112$ system, and also permits an

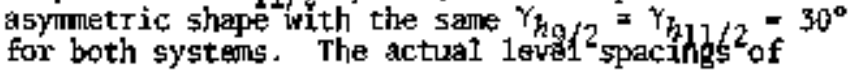
the h1/2 spertrum, which appear to bo almost identical for $189-195_{\mathrm{Au}, 2}, 3$ indicates $\gamma_{h_{11 / 2}}=$ $(37 \pm 2)^{\circ}$. Consequently, we deduce $\gamma_{\overline{7} 0 / 2}=(23 \pm 2)^{\circ}$ for $1 \mathrm{~s}_{\text {Aul }}$. These values should be 9 compared with $\gamma=(24 \pm 2)^{\circ}$ derived from the $158 p t$ spectrum and $\gamma=(38 \pm 2)^{\circ}$ derived from the $190 \mathrm{Hg}$ spectrum, specifically from the first and second $2^{+}$ energies. Also the ratio of the first $2^{+}$energies $\mathrm{E}_{2}\left(1 \mathrm{~B}_{\mathrm{Pt}}\right) / \mathrm{E}_{2}\left(\mathrm{lg} \mathrm{o}_{\mathrm{Hg}}\right) \cdot \mathrm{0.64}$ is close to the scaljing ratio $\mathrm{E}_{\mathrm{I}}[\mathrm{hg} / \mathrm{z}) / \mathrm{E}_{\mathrm{I}+1}\left(\mathrm{~h}_{11 / 2}\right)=0.7088$, which is found to give the best match in $189_{\text {Al }}$ (see Fig. 1 ).

From these results it is concluded that the $\mathrm{hg} / 2$ and $h_{11 / 2}$ systems in 189 Au are based on different shapes (asymmetric prolate and asynnetric oblate which are essentially those of the ${ }^{188} \mathrm{Pt}$ and ${ }^{190} \mathrm{H}_{\mathrm{g}}$ cores, respectively. In the onsay experiment $5^{5}$ it was abserved that the Mn transition between the $h_{g / 2}$ and $h_{11 / 2}$ band heods is retarded by a fastor I5000 relative to the Weisskopf singleparticle estimate, and the relationship of these bands to the reighboring doubly-even cores was recognized. Interpreted within the frame of the triaxial particle-core madel, the striking similarity in the level spacings of the two systems reflects a basic particle-hole syptretry and follows from tho fact that the $r$-values in $180 \mathrm{Pt}$ and $190 \mathrm{Hg}$ happen to Iie symmetrically about $\gamma=30^{\circ}$. (It should be noted that the triaxial rotor spectrum of a doubly-even nucleus has reflection symmetry about $\gamma=30^{\circ}$ and thus, the odit-A spectrum must be used to deternine whether the nuclear shape is prolate or obIats.) Conparing this nodel with the Alaga nodel, which also describes the $t_{11} / 2$ spectra well in odd-A Au isotopes, one notices that a corresponding symuetry relating the $h_{11 / 2}$ levels as 3 -proton hole clusters coupled to phonoms in closed-shell $\mathbf{P b}$ and should treat (not done so far) the hy/2 levels as 4-holesl-particle clusters. The microscopic model of Hecht, based upon the pseudo SU 3 coupling schene appears to have the same limitation. The particle-hole symatry arises under the assumption that the openshell nuklei $188 \mathrm{pt}$ and $190 \mathrm{Hg}$ are stabie encugh to be used as effective cores: this is done in the particle-core mode1 by treating the core correlations in terns of triaxisl shapes. The 189 kn spectrum provides strong evidence that such a treatment is justified and further, that the shapes of the even-A muclei in this mass region - or at leest their averaged parameters - are relatively stable, in contrast to general belief.

\section{Footnotes and References}

"Condensed from LBL-4350, subnitted to Phys, Lett.

'Present aditress: Georgia Institute of Technology, Atlanta, Georgia.

FPresent addres5: SIN, CH 5234, Vil1igen, Switzerland.

1. F. S. Stephens, Revs. Mod. Phys. 47, 43 (1975).

2. J. Meyer-ter-Vehn, F+ \$. Stephens, and R. M. Diamond, Phys. Rev. Lett. 32, 1383 (1974); J. Mkyer-ter-Velm, Mxcl. PH's. A249, 111 (1975); Ibid, 141 (1975).

3. E. F. Zganjar et al., Phys, Lett, 58B, 259 (1975).

4. J. L. Hood, E. F. Zganjar, and UNISOR coworkers, in preparation.

5. V. Berg, R. Foucher, and \&. Höglund, Nux1. Phys. A244, 462 (1975).

6. M. A, DelepIanque, C. Gerschol, N. Perrin, and

V. Berg, Nucl. Phys, A249, 366 (1975).

7. V. Panx, Ch. Vieus and J. S. Dionisio, preprint (1975).

8. K. T. Hecht, Phys. Lett. 58B, $253(1975)$. 


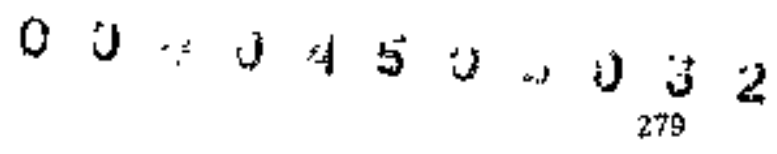

\section{CHOSSING OF ROTATIONAL BANDS IN THE CRANKING MODEL AND HIGH SPIN STATES}

H J Bank"

The calculations which are reported here have been performed within the framelork of the selfconsistent cranking thele1. 1 i Hevertheless the results can be interpreted as we11 as the consequence of the crossing of two bands, the ground state band, and the so called aligned band. 2

The basic equations are constrained HFB equations which in a condensed notation can be written as

$$
\begin{aligned}
& \delta\langle\phi|H| \phi\rangle=0 \\
& \langle\phi|N p| \phi\rangle=Z\langle\phi| \text { NQ }|\phi\rangle=A-Z \\
& \langle\phi|J x| \Psi\rangle=\sqrt{J(J+1)-\left\langle\phi\left|J_{Z}^{2}\right| \phi\right\rangle}
\end{aligned}
$$

Where $p$ refers to protons, $n$ to neutrons and $J$ is the total angular momentum. The quasi-particle operators B $\beta$ to which |\$> is the vacutm state are expanded in terms of particle operators $C_{k}$ Bogolyubov Transformation. The transformation which solves Eq. (1) is determined by iteration. First a $|\phi\rangle$ is constructed that fulfilis the constraints. Then in each step of the iteration a Thouless-Transformation ${ }^{3}$ is performed such that the energy decreases and the constratnts remain unchanged. Ho Lagranglan mult $i-$ pliers are introduced, Fig. 1 shows the results obtained for $164 \mathrm{Er}$. A pairing plus quadrupolequadrunole interaction was used and the configwation space was the same as in Ref. 1 . In order to account for the effect of a core of 40 protons and 70 neutrons a moment of inertia of the core $\theta$ core - $6 \mathrm{MeV}^{-1}$ was added. The characteristic features of the experimental data are woIl reproduced. Up to angular momentum 14 the yrast states belong to the ground band and from 16 on to the aligned band.

\section{Footnotes and References}

*Present address: Tecknische Universittät Nïnchen, D 8046 Garching, Gertuany,

1. H. J. Mang, Physics Reports Vol. 18, C No. 6 (1975).

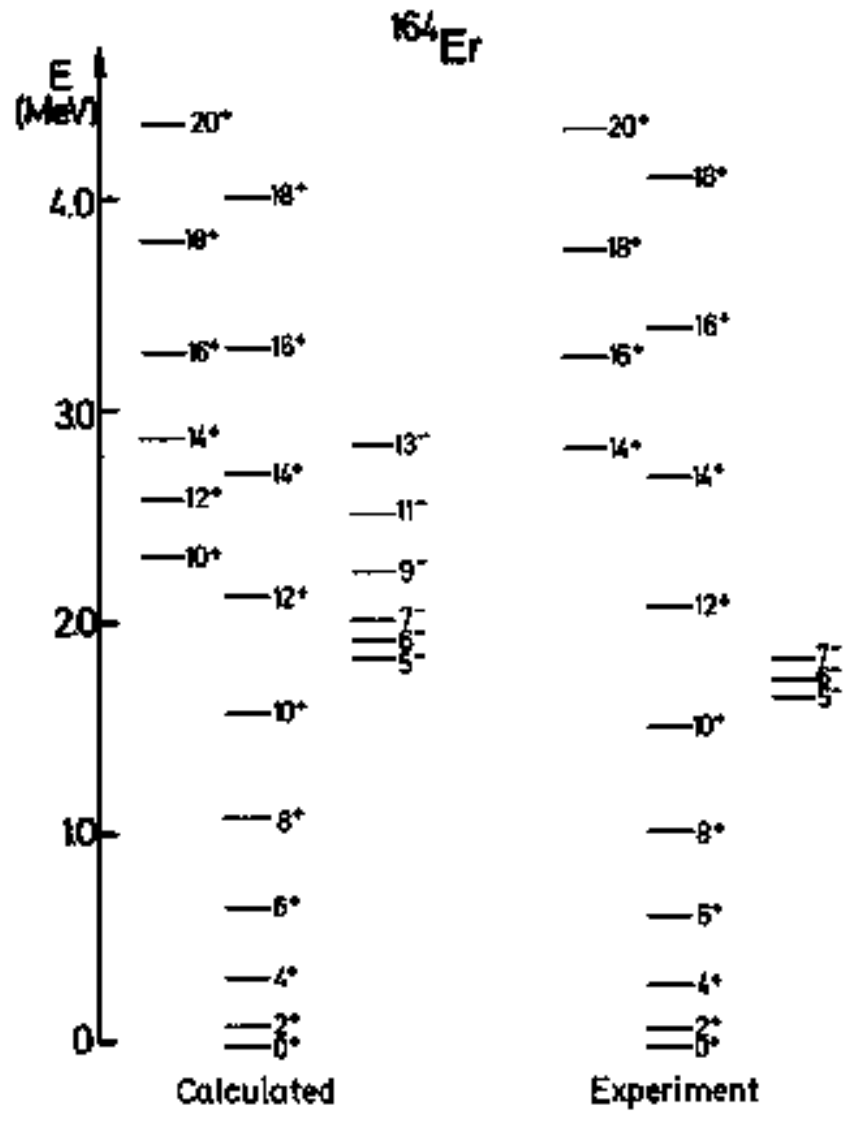

Fig. 1.

2. F. S. Stephens, in Proc. Intern. Conf, on Nuclear Physics, Mrnich, Vo1. 2 (1973), p. 367.

3. D. J. Thouless, The Quanturn Mechanics of Pany Body Systems (Acadenic PTess, New York and London, 1960 .

4. I. 5. Stephens and R. Diamond, private commication.

\section{SIMPLE DYNAMICAL MODELS INCLUDING PAIRING RESIDUAL INTERACTION*}

J Blockli and H Flocardq

The microscopic description of the dynamics of a many nucleon systen has been extensively inwestigated during recent years, I significant progress has ben achieved recently with the time dependent Hartree-Fock (TLFF) method that allows for a self-consistent description of the dymanics of the macleus. 2-4 However, in this method, the residual interattions are neglected. This together with the symuetries imposed in the calculations, leads to the unpleasant feature that a system oven moving infinitely slowly would not end ip in its ground state. This will appear each time two single particle levels with different quantum numbers (and therefore no possibility of transition between them) 
cross, Strin a Iinitation of the TIFF nethod could for exanple decrease noticeably tho calculated crosss section of the compound nucleus formation in heavy ion collisions and it is likely to have a more serious effect for a slower collective uotion like the one undergone by the nuclesus during the fission process.

In order to avoid these difficulties one could either lift all the symmetries imposed on the system which leads to a large cormputational problem or intraduce (in as simple as possible a way) a residual interaction that allowed for transitions betwegn levels with different quantum numbers.

It is our aim to show that the second alternative can be achieved by using the pairing residual interaction. We have selocted two models, which have been of great use in the static calculations including pairing: the constant gap $A$ model and the constant pairing strength $G$ model.

Starting from a variational principlo we arrive at sets of compled equations which contain the TDHF equations as a particular case plus simple equations goverming the occupation probabilities of each single particle level.

By applying the equations to a sinple two level model it is possible to show the cornection with the Landau-Zener theory. Going to the many level problem one can shuw that each level undergoes a landau-Zener type transition to a fictitious level symmetric with respect to the Fermi level and with a coupling strength determingt by the magnitude of the gap. Forkily the mly difference between the two pairing models lies in the fact that the gep is calculated self-consistently from the pairing tensor solution when one is working with the constant $G$ interaction.
From the solutions of the two level model it is atso possible to extract the quantitative features of the trousition induced by the two pairing hodels. Using the constant $g$ interaction the transition between two unperturbed levels is weaker (it requires a slower motion) and also it is easler to populate the lower perturbed level contrary to the Landau-Zener type (and crastant gap) coupling which gives equal probabilities of a jump from either perturted level to the other one, Both pairing residual interaction models can be used in a phenomenological way to allow for the interaction between levels with different quaritum numbers, however the constant gap formulation is Juch simpler. In this case the gap should be partly interpreted as a syumetry breaking satrix element of the field.

\section{Footnotes and References}

${ }^{*}$ Condensed from LBL-5016.

${ }^{\dagger}$ Permant address: Institute of Nuclear Research, 05-400 Swierk, Poland.

ҒPermanent address: Division de Physique Theorique (1aboratoire associé au ONRS) Inștitut de Physique Nucleaire BP no. 1, 91406 0nsay, France.

1. S. E. Koonin and J, R. Nix, Phys. Rev. CI3, 209 (1976), and references quoted therein.

2. P. A. H. Bonche, S. E. Koonin and J. W. Negele, Phys. Rev. $\mathrm{Cl} 3,1226$ (1976).

3. P. A. M. Bonche, S. E. Koonin, Proceectings from the Hirschegs Meeting $(1976)$.

4. S. E. Koonin, Proceedings from the Hirschegs Meeting (1976).

\section{TIME DEPENDENT HARTREE-FOCK CALCULATION OF NUCLEAR COLLECTIVE MOTIONS}

$\downarrow$ Blockl' ans $\mathrm{H}$ Flocerdt

The Tutr methodl provides a way to explore in a both microscopic and self-corsistent marner the dynamics of the nuclear motion. The wethod is microscopic because it uses as nuclear wrvefumction a Slater determinant and therefore exhibits all the individual nuclear degrees of freedom. The Pauli principle is also properly taken into account. The method is self-consistent because one treats an $\mathrm{N}$ boty problem and uses as a starting point a Hanil. tonian with a two-body intoraction. From this two-body interaction and the one-body denșity operator, associated with the Slater determinant, are constructs a one-body Hartree-Fock Hariltonian. This Heniltonlas in tum governs the motion of each individual orbit by simple one-body tíne dependent Schrobdinger equations. This method evidently relies on the validity of the nurlear shell model and assums that the nain effect of the mucteon-nucteon interactions is to generate a potential well which in turn deterjines the notion of the nucleons. The colmplicated $\mathrm{k}$ body problen is thus reduced to $a$ set of coupled norlinear equations whose solution appears feasible with the presently available coniputational facilities.

Because the indivinal nuclear degrees of freedon are explicitly taken into account in the TIHF equations, one can hope to describe both the coherent nuclear motion, also called collective modes, and the incoherent motions that are generally associated with the nome of heat. The study of the correlations between these twr types of nuctear motion, which introtures the notfon of viscous damping of the collective modes, is also evidently within the range of the TLHF model.

We solve the TIFF equations by expanding the Hartree-Fock wavefunctions on a basis. The partial derivative equations are thus reduced to coupled differential equations of first order in tims derivation. Presently our code uses an oscillator basis whose deformation can be adjusted to the 
particular motion that is studied. Fourteen oscillator shells have been fincluded in the basts wich appears stufficient to study vibrations and the fission of any known nuclear system. A further improwerient will be the introduction of a basis with a two center degree of freedom which will allow the study of heary-im reactions. The twobody interaction used is the Skywo interaction and direct and exchange Coulomb potentials have been included. The numerical method used to calculate the time propagation is the ustal Crank-Nicholson method. Particular attention has been devoted to the conservation of the total energy which is presently insured with a relat fve presision of $10^{-B}$, Finally a TIFF + BCS code has been constructed. one is able to staty the time evolution of a systen having a Bogolyubow wavefunction. This enlargentent of the TLPF method appears particularly use ful for heavy systems where a simple \$later deteminant is too restricted a wayefunction in view of the high density of individual levels near the Fermi surface.

Grrently the main enphasis is on the stuty of the vibrational nodes of the nuclei and the fission process. Whe are interested in the study of the viscous phonomens that could occur in such collective motions of the muclei. A prelininary stukty of the 1ight nuclei (A 5 80) has showm no trace of viscosity in the ronopole and quadrupole mades. Even when sorne trend appears indicating an exchange of energy betwem coherent and incoherent motions of the ruweleons such an exchange is periodic (and therefore reversible) with a period of about $10^{-21}$ sec. This is probably not too surprising since the systens that we have studied up to now have too few degrees of freedom and a statistical irreversibtlity is unlikely to occur. he have some hope that in heavier nuclear systens the typical tidne necessary to have the energy associated to the incoherent wotion of the nucleans converted in a collective motion will become much longer than the interesting muclear $t$ imes so that one could reasonably introduce the notion of irreversibility and viscosity. of particular interest will be the stuty of possible viscous phenomena in the fission process where their existence stiIl remeins ar open experimental and theoretical questicis.

\section{Footnotes and References}

"Permanent address: Institute of Nuclear Research. 05-400 Swierk, Poland.

†Permanent address: Divislon de Physique Theorique (laboratoire associe an ONRS), Institut de Physique hucleaire Bp no. 1, 91406 Orsay, France.

1. P. A. M. Dirac, Proc. Canb. Phil, 5oc. 26, $376(1930)$.

\section{ON THE SOLUTION OF CONSTHAINED HARTREE-FOCK-BOGOLYUBOV EQUATIONS*}

H J Mangt B Sarnadr,t and P Ringt

The interest in solutions of the HFB equations stens mainly fron the success of the theory in the calculation of energy surfaces 1 and properties of rotational states. 2 In the first case the expectation value of the quadrupole operator $\hat{Q}$, and if necessary of higher mulitipole operators, wust be constrained whereas in the second case the expectation value of the angular jomentum operator ntist be fixed at a given value. The conventional techloque for solving such problens is to introduce a Lagrasgian multiplier, $i+e_{1}$, to add a tem $-(j)_{x}$ or $-r 0$ to the Hamiltonian. Difficulties arise if the expectathon value is not a single valued function of the Lagrangian multiplier. They can be overcome by using a quadratic constraint like $c\left[\hat{Q}-\hat{Q}_{0}\right]^{2}$, which is added to the energy expression to be varied. In this paper a method is described which also circunvents such difficulties and has moreover the atwantage that the energy is only catculated for a single given value of the expectation value of the constraining operator. For instance, in the case of rotational states one obtains solutions which exactly fulfil

$$
\langle\hat{j} x\rangle=J(F+1)-\left\langle J_{2}^{2}\right\rangle
$$

and these are the only physically sipnificant salutions. There is no need to do a calculation for several values of the Lagrangian moltiplier and then interpolate between them.
For convenience the inqortant equations of HFB theory are given. Decails can be found in Ref. 2 and the literature cited there.

$$
\begin{aligned}
& \text { A Bogolyubov transformation is dofined by } \\
& \qquad d_{v}^{+}=\sum_{k} A_{k v} C_{k}^{+}+B_{k v} C_{k} .
\end{aligned}
$$

The coefficients $\mathrm{A}_{\mathbf{k}}$ and $\mathrm{B}_{\mathrm{k}}$ are only restricted by the requirement that the set of Fermi operators $C_{k}, C_{k}$ are transformed into a new set of Ferni operators $a, j, a_{v}$. To each set of Fermi operators

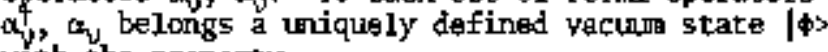
with the property

$$
\phi_{v} \mid \phi s=0
$$

Given a Hamiltonian $H$ one may now ask the question which vacund state $|\phi\rangle$ mininizes the expectation value $\langle\phi|H| \phi\rangle$. The equations of this variation principle are most converiently formulated by transforming the Haniltonian $H$ into the quasiparticle representation

$$
\text { H - } \mathrm{Ho}+\mathrm{H}_{11}+\mathrm{H}_{20}+\mathrm{H}_{22}+\mathrm{H}_{31}+\mathrm{H}_{40}+
$$

The equations to start with are

$$
\frac{\partial}{\partial C_{\mu \nu}}\langle\phi|H| \phi\rangle=\left(\mathrm{H}_{20}\right)_{\mu \nu}
$$




$$
\begin{aligned}
& \hat{\partial C}_{\mu \nu}\langle\phi|\hat{J}| \phi\rangle=\left(J_{20}\right)_{\mu \nu} \\
& \Delta E=\underset{\mu \nu}{\boldsymbol{\Sigma}} C_{\mu \nu}\left(H_{20}\right)_{\mu \nu}+O\left(C_{\mu \nu}^{2}\right) \\
& \Delta J=\sum_{\mu \nu} C_{\mu \nu}\left(J_{20}\right)_{\mu \nu}+0\left(C_{\mu \nu}^{2}\right) \text {. }
\end{aligned}
$$

From now only the terns linear in $\mathrm{C}_{\mu \mathrm{w}}$ will be taken into account. Clearly $A E$ is nogetive and has its greatest absolute value if one sets

$$
\mathrm{C}_{\mu \mathrm{N}}=-\mathrm{r}_{3}\left(\mathrm{H}_{20}\right)_{\mu \mathrm{v}} \quad \pi>0 .
$$

Then du is given by

$$
\Delta J=-\pi \underset{\mu v}{\mathbf{\Sigma}}\left(\mathrm{H}_{20}\right)_{\mu v}\left(\mathrm{~J}_{20}\right)_{\mu v} .
$$

An expression like $\sum_{\mu \nu}\left(\mathrm{H}_{20}\right)_{\mu v}\left(\mathrm{~J}_{20}\right)_{\mu v}$ can be interpreted as a scalar jroduct of two vectors $H_{20}$ and $\mathfrak{J}_{20}$ : lith this simplified notation $\Delta \mathrm{E}$ and $\mathbf{J}_{20}$ take the form

$$
\begin{aligned}
& \Delta E=-\eta\left(H_{20}\right)^{2} \\
& \Delta J=-\eta\left(H_{20} \cdot J_{20}\right) .
\end{aligned}
$$

of course, of should vanish provided the expectation value $\left\langle\phi\left\lceil J|\phi\rangle\right.\right.$ equals $J_{0}$ the desired value. If this is not the case on sts

$$
C_{\mu, U}=\frac{\left(J_{0}-c_{j} \hat{J}\right.}{\left(J_{20}\right)^{2}} J_{\mu v}
$$

and repeats this procedure if necessary until $\hat{\phi}\rangle$ equals $\mathrm{J}_{0}$. From now on one decreases the energr without chatging $<J>$ by setting

$$
\tilde{c}_{\mu v}=-n\left[\left(\mathrm{H}_{20}\right)_{k v}-\frac{\mathrm{H}_{20} \cdot \mathrm{J}_{20}}{\left[\mathrm{~J}_{20}\right)^{2}}\left(\mathrm{~J}_{20}\right)_{\mu v}\right] \text {. }
$$
are

The constraints used in applying HFB theory

$$
\begin{aligned}
& \left\langle\phi\left|\hat{\mathrm{w}}_{\mathrm{p}}\right| \phi\right\rangle=2 \quad \text { (proton number) } \\
& \left\langle\phi\left|\hat{\mathrm{N}}_{\mathrm{N}}\right| \phi\right\rangle=\mathrm{N}=\mathrm{A}-\mathrm{Z} \text { (neutron number) } \\
& \left\langle\phi\left|\hat{J}_{\mathrm{X}}\right| \phi\right\rangle=\mathrm{J}(\mathrm{JH})-\left\langle\mathrm{J}_{z}^{2}\right\rangle \text { (angular monentum) } \\
& \left\langle\phi \mid \phi_{0}\right\rangle=0 \text { (for exclted states }|\phi\rangle \text { ). }
\end{aligned}
$$

In the last case $\mid \phi_{0}>$ is the wavefunction of the onergetically lowost state with the sane quontwo numers as | $\phi>$. It should be noted at this point that the relation

$$
\left.\frac{\partial}{\partial C_{\mu \nu}^{-}}\left\langle\phi \mid \phi_{0}\right\rangle\right|_{\left[C_{\mu \nu}=0\right\}}=\left\langle\phi\left|\alpha_{\mu} \alpha_{\nu}\right|_{0}\right\rangle=0_{\mu \nu}
$$

holds. The requirenent on $H_{20}$ is in this case

$$
\left(0 \cdot \mathrm{H}_{20}\right)=0
$$

in every step of the Itsrative solution of the constrained HFB equations Calculations were performed for ${ }^{164} \mathrm{Er}$ and ${ }^{16 B} \mathrm{Yb}$. The single particle configuration space was the same as used earliar.? The strength constants of the quadrupole-quadrupole force (Q) plus pairing force (G) were slightly changed compared to the earlier work in order to give somewhat more qumtitative agroement with experimental data. Besides that, a moment of inertis of the core was introduced. The core consists of 40 protons and 70 neutrons in single particle statos not taken into account explicitly. The magnitude of this moment of inertia was chosen as $\theta_{\text {core }}=6 \mathrm{HeV}^{-1}$. It should be mentioned that the use of $\theta_{\text {core }}$ requires the knowledge of tho angular frequency w which is obtained from the relatión

$$
\omega=\frac{\partial E}{\partial \sigma_{\mathrm{K}^{3}}}=\frac{\left(\mathrm{H}_{20}^{\prime} \cdot \mathrm{J}_{20}^{\prime}\right)}{\left(\mathrm{J}_{20}^{\prime}\right)^{2}} \text {. }
$$

Hore $\mathrm{H}_{20}^{\mathrm{b}}$ and $\mathrm{J}_{20}^{\prime}$ are those parts of the vectors $\mathrm{H}_{20}$ and $\mathrm{J}_{20}$ that are orthogonal to $\mathrm{N}_{20}$ and $\mathrm{N}_{20^{+}}$

Calculated and experimental results are shown in Fig. 1. The results on $16 \mathrm{PO}_{\mathrm{M}}$ indicate that at

$$
{ }^{188} \mathrm{Yb}
$$

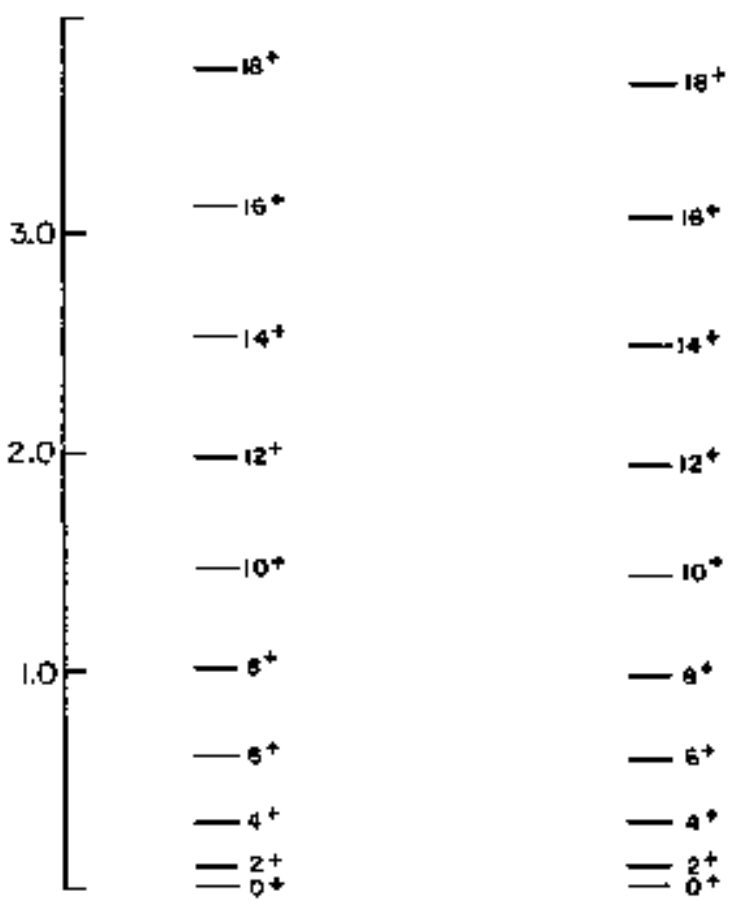

CALCULATED

EXPERIMENT

F1g. 1. Experimental and calculated entrgy levels of $163 \mathrm{rb}$.

(XELL 764-2776) 


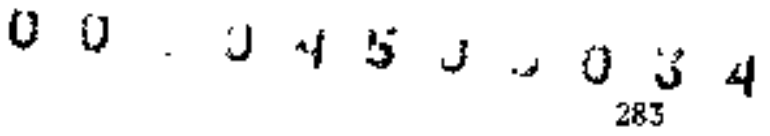

least within a limited range of mass numbers experimental data are reproduced by the same simple effective interaction. The results are encouraging onough to warrant the use of more scphisticated effective interactions in calculations of the type described here.

\section{Footnotes and References}

"Condensed from LBL-5015.
${ }^{\dagger}$ Present address: Technlsche thiversität Mtinchen, D8046 Garching, Germany.

1. P. Quentin, H. Flocand and D. Wautherin in Proc. Intern. Conf. on Nuc1. Phys., Minich, 1973, Vol. 1, p. 41.

2. H. J. Mang, Phys. Reports Va1, 18C, No, 6 (1975).

3. D. J. Thouless, The Quantum Mechanics of Mtiny Body Systents (Academic Press, New York and London, 1961).

\title{
TEST OF MANY-BOOY METHODS ON AN EXACTLY SOLUBLE MODEL WITH BACKBENDING AND GAPLESS SUPERCONDUCTIVITY
}

\author{
8 Y Chu, E R Marshalek,t P Ring.: \\ J Krumbnde, and $J$ Q Rasmussen
}

The exactly soluble $\mathrm{R}(8)$ nodel of Krutrlinde and $\$ z y m a f i s k i$ has bear used as a testing ground for thxe many-body wethods applied to the yrast band: (i) the self-consistent cranking model (SCC), (ii) self-consistent cranking plus RPA, and (iii) variation after projection of particle number. All three methods agreo very 11 with exact results outside of the critical region. The behavior in the critlca1 region has been exanined in detail.

The R(8) madel consists of 28 identical fenthons tnteratting via a palring force, distributed anong two 20-fotd degenerate single-particle levels separated by the energy $z e$ and coupled to an extemal rotor with fixed monent of inertia $a^{-1}$. The Haniltonian is

$$
\mathrm{H}=\left(\frac{1}{2} \mathbf{a}\right)|\overrightarrow{\mathbf{I}}-\overrightarrow{\mathbf{j}}|^{2}+\mathrm{H}_{\mathbf{s , p},}+\mathrm{H}_{\mathbf{p}},
$$

Where $\vec{I}$ is the total angular momentum of the systerd, and $\mathbf{j}$ is the particle angular momentum.

As discussepd in Ref, 1, $H$ is composed of generators of the group $\mathrm{R}(\mathrm{s})$. Exact eigenvaluss and eigenfinctions can be found by diagonalizing a matrix in the belfand basis, so that the exact solution corresponds to a prototype she11-model calculation.

In the case of a self-contained momy-fermion system with Hamiltonian $H$, the $S 0$; model is usually derived by applying Hartree-Bogolyubov factorization to the operator $\mathrm{H}-4 j_{X}$. With the $R(8)$ madel, there is no need to subtract wjy since the rotor al ready cranks the systerly. Instead, assure in the first approximation that the total angular monention is aligmed in the $x$-direction and has the classical c-number value I:

$$
I_{x} \approx I, \quad I_{y} \approx 0, \quad I_{z} \approx 0 \text {. }
$$

This form of the $\$ O C$ model, which shall be specifically referred to as $5 \mathrm{CC}$, is purely classicat, and as such, valid mainly for hisgh values of I. For low values of $I$, the rotational energy goes as $I^{2}$ rather than $I(I+1)$. However, the gddition of quantug fluctuations arising from the RPA, to be discussed later, gives the correct $I(I+1)$ dependence. If $R P A$ corrections are not incluted, the crarking energy may be improved for low I by some nodifications.

We now consider the corrections to SOC1 described by the RPA. The boson-expansion technique provides a convenlent way to introduce the additional corrolations. Boson expansions to all orders for the group $R(2 \pi)$ have been given in Ref. 2, In the present problem, $H$ murst first be expressed in terms of tho quasi-particles. Then, the quasi-particle pair operators are expanded in bosans to the RPA order.

In addition to the particles, the core must also be exponded in bosons, which physically corresponds to its wobbling motion about the steady rotation. A full description of the boson representation of a quantized rotar, with expressions valid to all orders, is given in Ref. 3. The follow here the lowest order treatment originally applied by Bohr and kottelson to the asymetric rotor, which is opplicable to the symmetric rotor as woll. Upom adding the RPA correlations, one finds that the total Hemlltonian breaks up into two disjoint quadratic bospin forms.

Stnce self-consistert cranking, or any HartreoBogolyubov approximation, in general violates syrmetries of the exact Hamiltonian, one nasy hope to inprove the situation by projecting out frow such wavefunctions the components with the correct symetry. The $R(B)$ model is not suitable for testing angular monentum projection, since the particle angular momentum is not a constant of motion of the exact Haniltonian. on the other hand, perticle numer is conserved by $\mathrm{H}$, but not by $\mathrm{H}$, and we ray therefore apply particle number projection, which has been used by several authors in the backbending region. 5 It is of interest to conpare the projection jethod with the FPA, since both introduce correlations, and the RPA conserves particle nimber approximately (to onder $\Omega^{-1}$ ) through the pairing rotation teru.

For optimum rosults, the variation is performed after projection (VAPN). 
It can be shown that in the limit + =0, WAPN repratuces the exact energies, as is the case for SOCl + RPA,

Because of the size of the matrices to be diegonalized, ${ }^{6}$ the maximm value of 0 for whith exact solutions could be practlcably obtained with our computing facilities was $n=4$. This means we were limited to not more then B particles.

The degree of backbending is controlled mainly by the ratio $\mathrm{\epsilon} /(\mathrm{G} \Omega)$ : the smaller the ratio, the weaker the band mixing and the greater the tendency to bend back. Whe have followed the procedure of keeping 6 fixed and varying $\epsilon$ to control the degree of backbending. Exact solutions were obtained for $0=3, G=0.1, a=0.02$, and $e=0.05,0.10,0.15$, and 0.20 , and $\Omega=4, G 0.075, a=0.019736$, and $\epsilon=0.05$, 0.10 , and 0.15 , which covers the range from sharp backtiending to no backbending and a smooth transition.

As show in Ref, 1, the critical angular monantim $I_{c}$ is giver by

$$
I_{C}=\Omega-\frac{1}{2}+\frac{1}{2}(G / a)(\Omega+I) \text {. }
$$

The ratio $G / a$ was fixed by the requirenent that the phase transition occur between $I=12-14$, in rough accord with experiment. The values of $G$ were atbitrarily chosen to maintain about the same magnitode of grourd state pair correlations as in Ref. 1 .

The salient features emerging from the calculations are sturarized in sone representative figures.

It is seen ficm the figures that the yrast trajectories cen have severai regions of interest. The sinplest picture is provided by the cratking mode1, in which the trajectories are composed of two segments, one with i 0 , which Jay or may not have a backbending region (depending on the value of $\epsilon / G(s)$, atong which $A$ decreases and 8 incroases contimuously with increasing $\omega^{2}$ until the intersection with the $\Delta=0$ segnent at some $\omega^{*} \omega^{*}$, corresponding to a cusp. For $w^{2}<\omega^{* 2}$, the $\Delta=0$ solution is unstable and is owitted. The situation becomes more complicated after adding the corrections dite to the RPA, which tests the stability of the selfconsistent cranking solutions.

The $\Delta^{2} 0$ segrent is always stable for $\omega^{2}>\omega^{* 2}$, but as $\omega^{*}+w^{*}$, the doubly degenerate pairing vibration drops to zero frequency, signaling the onset of the transition from a peir-vibrational to a pair-rotational scheme. As $w^{*}$ is approached along the $A \neq 0$ segment, one of the RPA frequencies approaches zero, in addition to the already present zero-frequency solution corresponding to the pair rotation. Also, as w $u^{\star}$ is approached along either segaent, the derivative of the zero-point energy with respect to angular monentum, becomes infinite. Therefore, the RPA correction breaks dowr completely at the cusp, accolnting for the hiatus in the RPA curves.

The 4 to sexment can show instabilities since the RPA quadratic form is not necessarily positive. An unstable regica occurs only along a portion of a backbending part, and only if the parmeter $\underline{a}$ is sufficiently sma11 compared to $02 / \epsilon$. That is, the rotor wust be sufficiently massive and the pairing correlations sufficiently strong. The tustable region is indicated by a dotted line in the figures. Figure 1, for excample, shows quite clearly that as $\epsilon$ is increased for fixed $\underline{a}$ and $m$, the unstable region decreases in size. This instability in the backbending ragion is the result of compettion between the particles, whlch favor increasing I with decreasing $|\omega|$ (the effect is proportional to $(0, / 6)$, and the rotor, which favors decreasing I with decreasing $|\omega|$ (the effect is proportional to $a^{-1}$. Figure 2 , which is a plot of $I$ Vs $|w|$, shows that in the unstable region the rotor wins out, producing the pecutiar "down bending." This is to be contrasted with Fig. 3, with another set of parameters, such that the yrast trajectories are stable everywhere. In this case, I increases with decreasing angulas velocity in the backbending region. Fortmately, in realistic calculations an externat rotor introduced to simulate a core would normally be swall enough to avold instability problems of this kind.

It has been seen that backbending per se does not necessarily imply instability (the criterion for which is the existence of an imaginary RPA frequency) as illustrated by Fig. 3. This is a counterexample to some claims trade in previous work.?

Since, as was already noted, the VAFN and the SCCl + RPA energtes are both exact in the 1 indit e-0, it is clear that these two methods will be superior for very stall $\&$ (very sharp backbending), except that the second method breaks doprom in a small neighborhood of the cusp. Figure 4 is a plot of the yrast excitation energies vs I for this case of i=4, $x=0.7$, while Fig. 5 is a similar plot for the case $\Omega=4, e=0.15$, in which the backbending disappears.

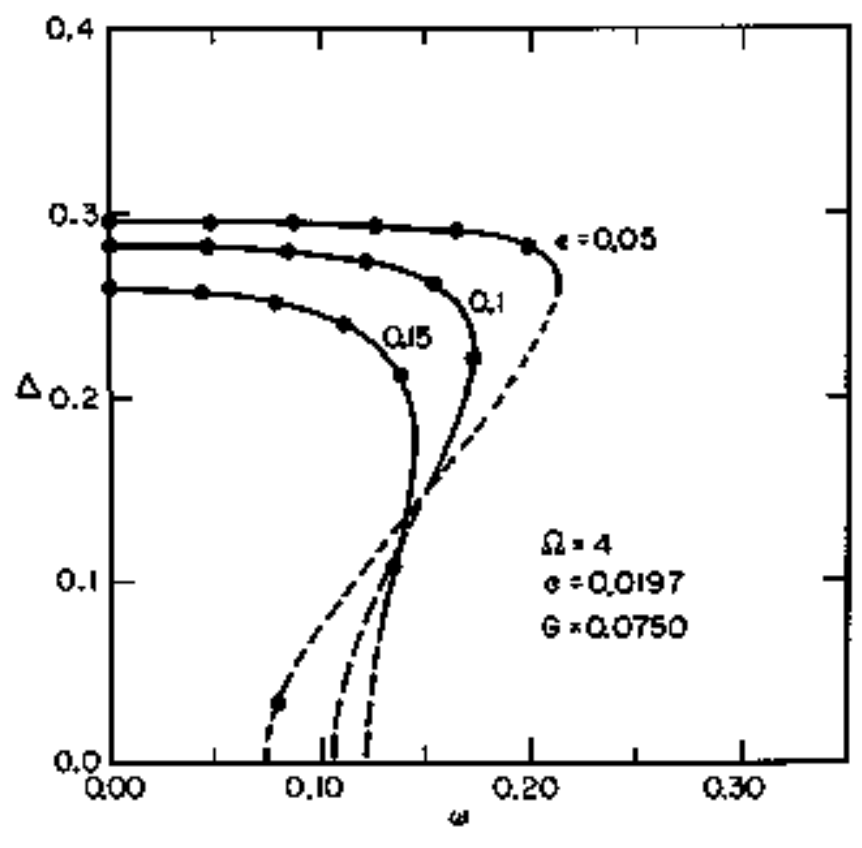

Fig. 1. The gap parameter $\Delta$ as a function of angular speed w for FCC2. The discrete points correspund to integer valuss of angurlar momentum. The cranking model solutions are unstable along the dashed portions of the curves. (XBL 753-2501) 


\section{$00.4450,00_{285} 3$}

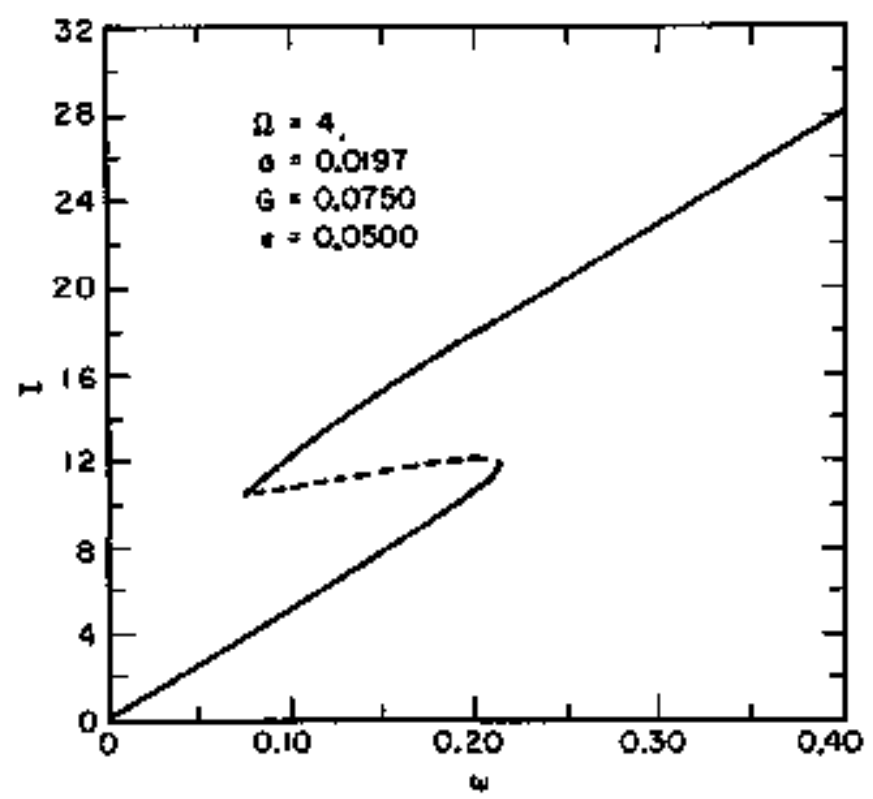

Fig. 2. The spin I as a function of angular speed $\omega$ for SOCZ. The cranking nodel solution is unstable along the dashed part of the curve. (XRL 753-2502)

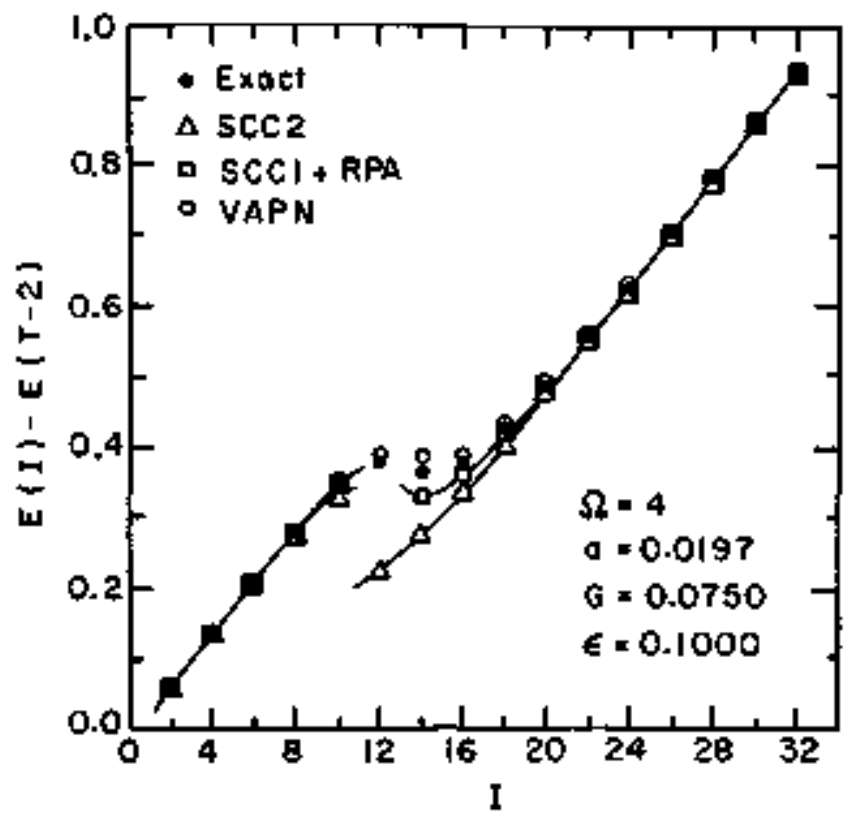

Fig. 4. Yrast excitation energies vs spin $I$.

(XBL $753-2610$ )

It is seen that all three methods, SCC2, $S Q C 1+R P A_{1}$ and WAPS are pleasingly accurate on the upper and lower branches outside of the trensition region. The least embellished method, 5002 , is also least accurate, especially in the transition region, although it is asymptotically exact in the classical linit of large 1 , where all the methods eventually converge. The transition region, however, only encoupasses three states or so. Clearly,

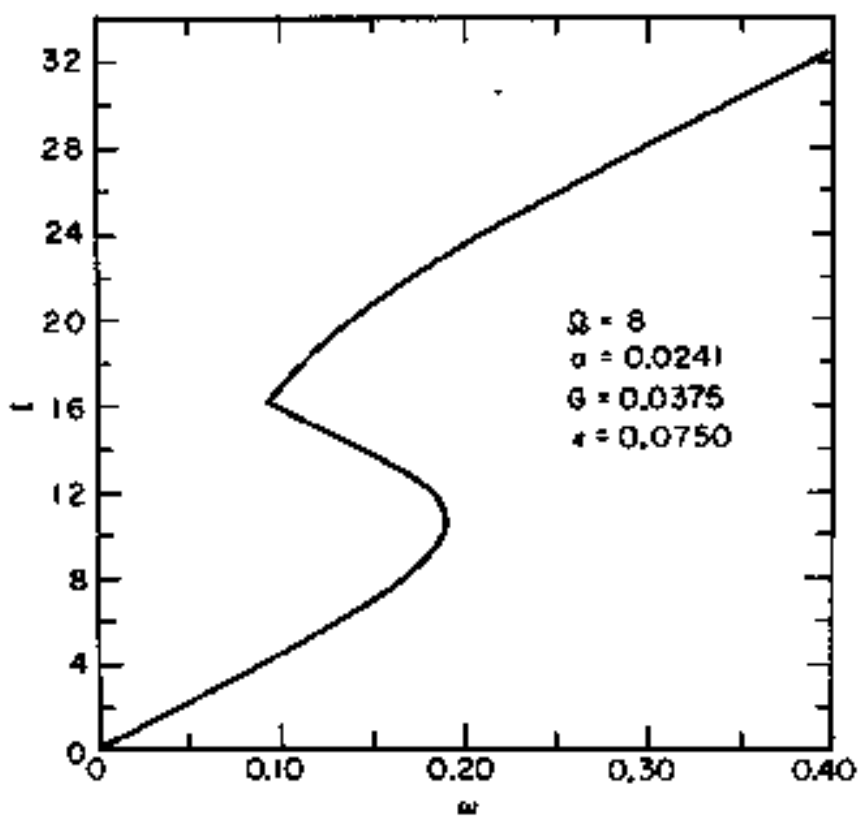

Fig. 3. The spin I as a function of angular speed $w$ for SCC2. The cranking model solution is stable everywhere.

(4BL 753-2593)

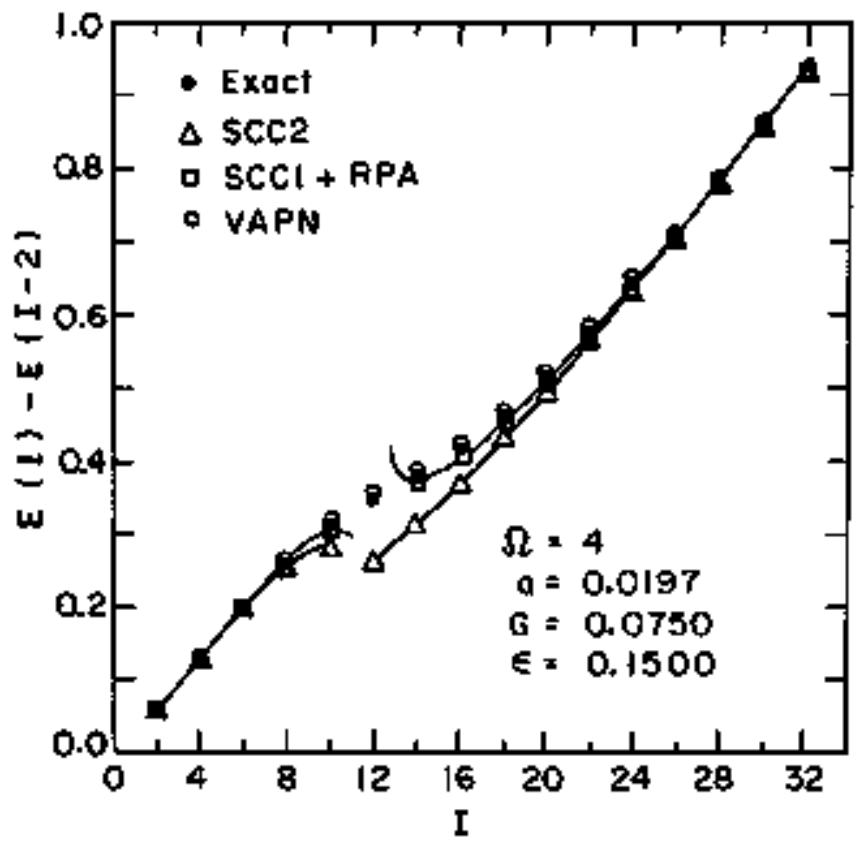

Fig. 5. Yrast excitation energies vs spin I.

(XFL 753-2606)

SCCI + RPA provides a nice improvement with only a bit more expenditure of time, except for the failure in the neighborhood of $\mathrm{I}=12$, due partly to the pernicious influence of the cusp and partly to the large amplitude of the pair fluctuations. These effects, however, present no problem for the VaPN method, which is quite good all the way through. For large I, the absolute energies given by SCCl + RPA are better than those given by VAPN. 
As e increases so that the backtending distapears, the VAFW method emerges as the best, SCC is also significantly less accurate with no backbending than with sharp backbending, which way be a general role since this result is a conseyuence of the Enlargenent of the transition region in which the two berids are strongly reixed and zero-point oscillations become ibportant.

\section{Footnotes and References}

${ }^{\star}$ Condensed from LBL-4021, subnitted to Phys. Rev.

${ }^{\dagger}$ Permanemt address: Lept. of Physies, University of Notre Dane, Notre Dame, Indiana 46556.

${ }^{\text {}}$ Permpanent address: Technische Universitä̆t Ninchen, 8046 Garchı̂ng, Neșt Genmany,

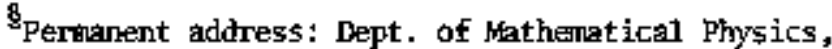
Lund Institute of Technology, Lund, Sweden.
1. J. KrumI inde and Z. 5zymański, Ans. Phys. (N.Y.) 79, 201 (1973).

2. E. R. Marshalek, NucI. Phys. A224, 221 (1974).

3. E, R. Marshalek, Phys. Rev. C11, 1426 (1975).

4. B. Mbttelson, "Rotational Motion in the Nucleus," in Proceedings Niclear Structure Sympositm of the Thousaind Lakes, Joutsa, 1970 (Nordita publication, (6. $4[7$ )

5. A. Faeşsler, F. Grïner, L. Lin and J. Urtang, Phys. Lett. 48B, 87 (1974).

6. The matrix disonsion $=(\Omega+5) ! /(120 \times \Omega !)$.

7. M. Smo, T. Takenasa and M. Wakai, Nhe1, Phys. Al90, 471 (1972).

\title{
ON THE SHELL EFFECT CONTRIBUTION TO THE WOMENT OF INERTIA AT VERY HIGH ANGULAR MOMENTUM
}

\author{
I Fagnarsson: D P Soroka, and F S Stephens
}

The yrast states of a nucleus are the states of lowest energy for each spin I. The internal degrees are not excited for these states, so they are especially easy to treat theoretically, and during the last few years scoue new approaches based on the shell correction method have been developed to describe then. 1,2 In addition, new experimental results have been obtained in this area. ${ }^{3}$ Here we report spone calaulations on shell effects at very large angular momenta. These offects will be exenplified by nuxlei in the Te region.

In the calculations presented here the pacroscopic-microscopic method was used. The generalization of this method to high spin states is described in detail in Ref. 1, so here only the most important features of the method are given. The single-partlicle wavefunctions are extracted from a rotating modifled oscillator (M.0.) potential corresponding to the Harpiltoniom

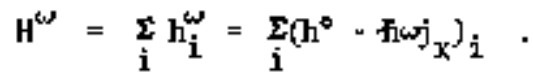

Here $h^{\circ}$ corresponds to the ustal non-rotating $M_{0} .0$. Hamiltonian, while wij can be regarded as the Hamiltonian form of the summed coriolis and centrifugal terms. The wavefunctions $x_{1}^{\Phi}$ are obtained fron the diagonalization of the one particle Haniltonian

$$
h_{i}^{4 x}=e_{i}^{\omega x} x_{i}^{\omega}
$$

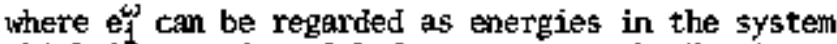
which is rotating with frequency w. The t'real enexgies" are calcutated as expectation walues

$$
\left\langle e_{i}\right\rangle=\left\langle x_{i}^{\omega}\left|h^{\circ}\right| x_{i}\right\rangle=e_{i}^{\omega}+k_{k}=n_{i}
$$

where $m_{j}$ is the expectation value of $j_{x-}$ The order of the leve1 occupation is determined from the order of the quantities $e_{i} \omega$, while the total energy is calculated as

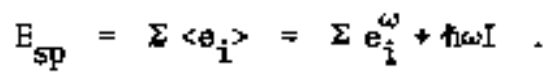

Here I is the total angular nonentum, i.e., I $=\Sigma m_{i}$. All these suns are carried out over the occipied states [i>.

By applying a generalized Strutinsky procedure, a snoothed energy E can be calculated in a sigilar why as in the non-rotating sase. The shell correction energy

$$
E_{\text {she11 }}=E_{s p}-\widetilde{E}
$$

is then calculated where all quantities are evaluated for the same I-value. Finally, the total energy is obtained as

$$
\mathbf{E}_{\text {tot }}(\epsilon, Y, I)=E_{\operatorname{mec} \Gamma}\left(E, Y, E_{4} I\right]+E_{5, h e 11}(e, \gamma, I) \text {. }
$$

The racroscopic ensrgy $E_{\text {macr }}$ is composed of the surface energy, the coulomb energy and the rotational energy correspming to a rigid rotation. For the calculations reported here the macroscopic energy was mininized with respect to $E_{4}$ while the shell correction energy was calculated for $e_{4}=0$.

The above formula for the shell correction energy was defined from a smearing over 'the energles" $\left\langle e_{j}\right\rangle$ in the Iaboratory system. How altemative definition is

$$
E_{\text {shel1 }}^{\prime}(\omega)=\Sigma e_{i}^{\omega}(\omega)-\Sigma e_{i}^{\omega}(\omega)
$$




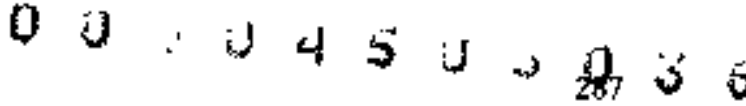

where all quantities axe defined for the sarie $\omega$ value and where the tilde denotes the sineared quantity. It is then possible, to show that, except for a small correction term, Eshell $(v)=E_{\text {shell }}(I)$. Due to the equality above and due to the generai features of the shell correction appraach, in a plot of $e_{i}$ vs $\omega$, the regions of a low level density can be inmediately identified as regions with a low value of $\mathrm{E}_{\text {shell. }}$. For the corresponfing $\mathrm{I}$-value there will then be a tendency to stabilize the nucleus to this shape.

In the calculations presented here pairing is completely neglected. For heavy and mediun-heavy nuclei, the pairing correlations are expected to disappear for $I$ - 20 - 30h, i.e., it is only for $I$ values larger than this limit that our calculattons can be expected to be realistic. However, even for lower I values it 1s perfectly reasonable to define the shell correction energy. In Fig. 1 the proton energy levels $e_{3}^{y}$ at the prolate deformation $=0.3$ are plotted as a function of $\omega$. In addition, sorae

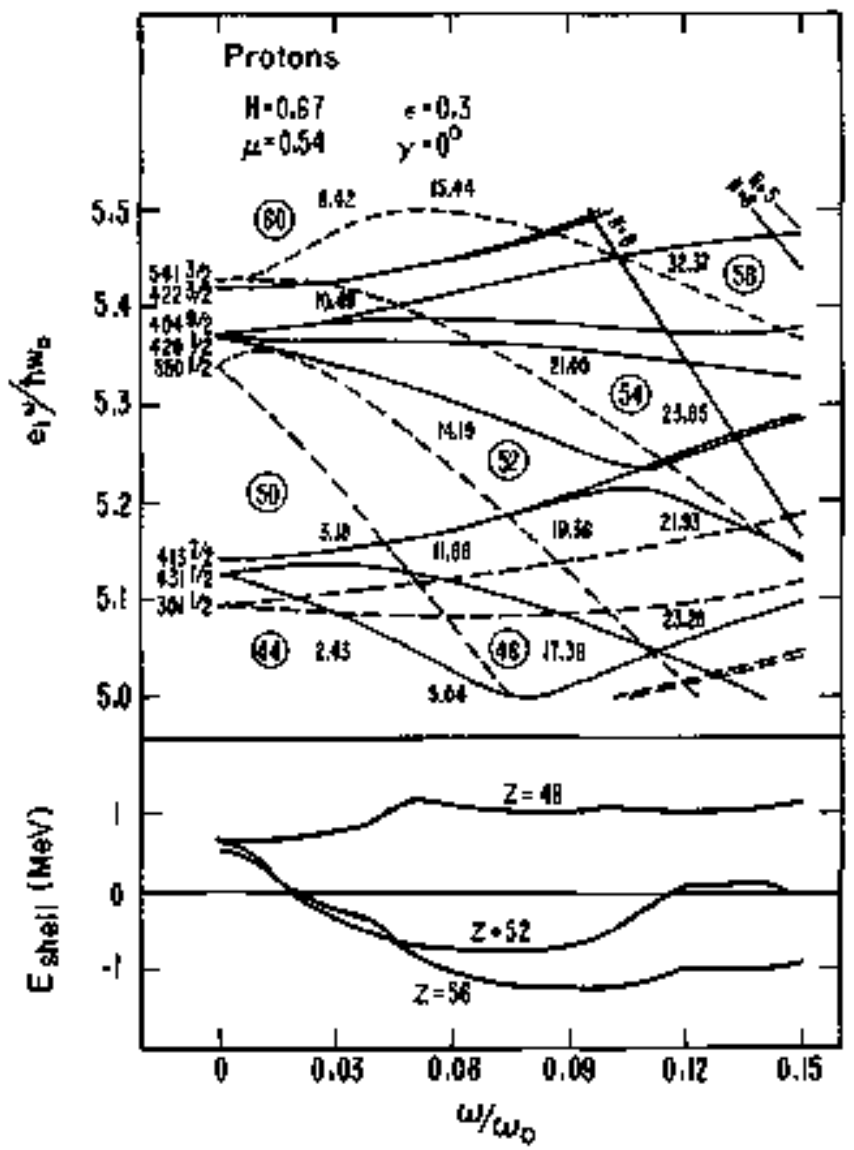

Fig. 1. In the upper part of the figure, the singleproton energies $e_{j}$ in the $Z * 50$ region are plotted as a function of the rotational frequency $\omega$. Both the quantities are expressed in oscillator units, to corresponding to the oscillator frequency, For different locations of the Femi level energy, the proton number and the sumed proton spin, $\mathrm{I}_{\mathbf{p}}=\boldsymbol{\Sigma}_{\mathbf{1}} \mathbf{n}_{\mathbf{i}}$, are given within circles and rectangles respectively. The total muclear spin in addition has a neutron cuntribution. In the 1ower part of the figure she11 correction energies corresponding to $z=48,52$ and 56 are exhibited.

(XBL 766-8216) corresponding shell correction energies are exhib. ited. In this figure orbitals with a positive value of $m_{i}$ will be dow sloping while up sloping levels have negative mi valtues; the larger is the slope, the larger is the absolute on -valine. For low $\omega$-values it is of particular interest to notice the influence on the shell correction energy on the alignment of the high $j$ states. The phenomenon of aligument was first discussed in Ref. 5. In the single-particle diagran of Fìg, 1 this aligment shows 4 in the fact that the tho first orbitals of $h_{11 / 2}$, labelled by [550 1/2] for $\omega=0$, becone steeply down sloping, i.e., large m-values are obtained for quite srall w-values. For $z=52,54$ and 56, they will open up a region of low level density and thus negative values of $\mathrm{E}_{\text {shell }}$ as seen in the bottom of the picture. This can then be expected to give rise to a more stable nuclear configuration.

Generally, the change in she1l correction energy is mainly detemined by the strongly sloping orbitals. Thus, in the very high spin region, the high $f$ orbitals are expected to give the fiatn effect. The reason is that these orbitals are most easy to align and in addition that they can carry the highest mi-values. In Fig. I surch orbitals, for $\mathrm{Z} \approx 52$, begin to chit the Fermi. surface at $w$. 0.10 . Consequently, the level density becomes higher and $\mathrm{E}_{\text {shel1 }}$ increases. General1y, in Fig. 1, there is a direct correlation between the level density and the shell correction energies.

For the nucleus ${ }^{118}$ Te the total energy was calculated on an $(e, y)$ mosh and for different spins. For each spin I the minimum energy $E_{\text {min }}$ (I) was calculated. Then, the transition energies, for stretched E2 transitions along the yrast Iine, were calculated as $E_{t}(I)=E_{\min }\left\{(I+2)-E_{p i n}(I)\right.$. These transition energies in the region 1020-70 are plotted in Ftg. 2. Around $I=50 h$ the lowest minimum changes its shape from prolate for $I \gtrless$ 30 h to oblate for I $x_{6}$ 3 prolate nininum with an energy close to that of the oblate miniman, In Fig. 2, for I < 30h, the transition energies conresponding to prolate shapes are indicated as crosses. The change around $\mathrm{I}=50^{\mathrm{h}}$ can be traced back to a general stretching of the mucleus. In this curve, due to the different approxinations involved, each different point should certainly not be taken too seriously. However, the gross structure is expected to be significant. The gross structure can be characterized by the average density of transition enercies per energy interval (essentially the slope in Fig. 2). This dersity corresponds to a diroctly observable feature in the experimental spectron, In Fig. 2, the average density is almost twice as large around $\mathrm{E}_{\mathrm{t}}=1.7$ WeV as it is just abcve $E_{t}=1 \mathrm{KeV}$. The aim of these calculations is to correlate the energies of Fig. 2 to the continuous $\mathrm{r}$-ray spectro following a heavy ion reaction with 11 Te as the final protuct. Hrwever, this question remains to be investigated as does also the question of which ortitals are mainly involved in the changes of shapers and shell correction energies giving rise to the structure of Fig. 2 .

Host of our computer codes were developed by the groute of S. G. Nilsson and we we thank 


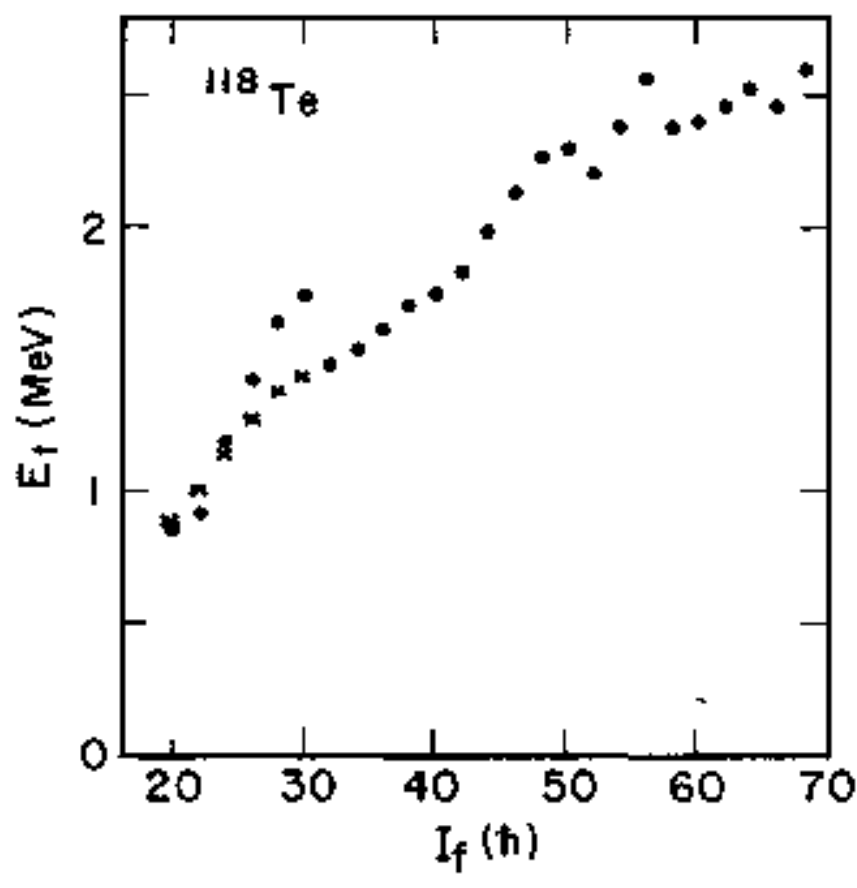

Fig. 2, The calcutated stretched E2 transition energies $E_{t}$ for ${ }^{118} \mathrm{Te}$ are shown as a function of the final spin $I_{f}$. For $I \neq 30$ f the deepest minimum changes its shape from proiate to oblate. In the $I=20$ - 306 region for the polnts the oblate (lowest) oining were used in calculating $\mathrm{E}_{\mathrm{t}}$ while for the crosses the prolate minisa were used.

(XBL $766-8217)$
R. Bengtsscr, S. E. Larsson, G, Leander, and S. Alberg for generuis access to these codes.

\section{Footnotes and References}

*on leave from the lund Insticute of Technology, Lind, Sweden.

1. R. Bengtsson, S. E. Larsson, G, Leander, P. Holler, S. G. Nilsson, S, Rberg and Z. Szymariski. Phys. Lett. 57B, $301(1975)$; G. Anders\$0m, S. E. Larsson, C. Leander, P. Holler, S. G. Nilsson, I. Ragnarsson, S. Aberg, R. Bengtsson, J. Dakiek, B. Nerlo-Ponorska, $K$. Ponerski and $z$. Szymáski, submitted to Nucl. Phys.

2. K. Nergaard, V. V. Pashkevich and S. Pratuerndorf, Nucl. Phys. A262, 61 [1976].

3. R. S. Simon, M. V. Banasclijk, P. Colombani, D. P. Soroka, F. S. Stephens and R. M. Diamond, Fhys. Rev. Lett. 36, $359(1976)$, Refs. quoted therein.

4. V. M. Strutinsky, Nucl. Phys. A95, 420 (1966) +

S. F. S. Stephens and R. S. Simon, Nutcl. Phys, AI $83,257(1972)$.

\section{SEMI-MICROSCOPIC ROTATIONAL BAND THEORY}

D Soroka, J Rasmussen, and K S Tanabe*

The have carried out four-dinonsional stretch model calculations of various properties of nuclear rotational bands-energies, quadrupole deformations (E), proton and neutron get parameters ( $v_{p}$ and $v_{0}$ ) and rotational g-factors. For each spin ralue the total energy minimm is found in a four-dimensional space of collective coordfnates; these are the three degrees of treedion $\left[e, v_{p}\right.$, and $v_{n}$ ) and the tri-axiality parameter $\gamma$.

Our code is a generatization of the one-dimensional beta-stretch mode1 of Diamond, Stephens and Swiateckil and the two-dinersional stretch model of tha and Rasurssen (proton and neutron pairing). Except for one adjustable parameter normalizing the roxuent of inertia, the calculations are corstrained by microscopic theoretical kork both for potential energy surfaces and for inertial properties.

our shape-potential surfaces are paranetrized least-squares fits of shell-corrected onergy surfaces calculiated by the Lund-Marsaw groups. 3 Spring constants for pairing changes are taken from the nuber-profected BCS continumus model formula from Ref. 4. Spring constants for shape changes are determined by the curvature of the shell-corrected energy surfaces.
The used an exponential dependence of mwant of inertia on pairing gap parameters, as daterutined by cranking iode1 calculations of Ref. 2. Indeed, we constrained ourselves to the theoretically calculated exponential periring coefficients $\gamma_{p}$ and $Y_{n}$ as given in Table 1 of Ref. 2 .

The dependence of the three moments of inertia on asymuetry $r$ was taken in the form given by Bohr from the hydrodynaric model.

$$
\partial_{x}=\partial[\epsilon] \sin ^{2}\left(\gamma-\frac{2 \pi_{k}}{3}\right)+j_{0},
$$

where $k=1,2$, or 3 for the primeipal axes of the ellipsoid. The mall constant $j_{0}$ is an effective monent of inertia for spherical muclei, based on the emergy of the giant quadrupoile state. The coefficient of $(e)$ is constrained to a roumded stairstep form stugigested by cranking calculations and tied to the level density dependence on deformation as the integral of the Nilsshr level denșity with respect to deformation:

In the overall norualization of $y(e)$ we allow one adjustable parameter, the only one in the calculation; it is adjusted to fit one rotationel transition $(4+2)$. 


\section{$00 \cdot 345 \%+0_{289} 37$}

With the help of Dr. Eric Leber, we have explored the shape dependence of Bp and $A_{1}$. hence, Nilsson level densities $\rho_{p}$ and $p_{n}$ fiear the Fermi surface. In this, $t, t_{4}$ and ${ }_{6}$ there varied. However, for the calaulations presented in this roport we heve held the values of $\rho_{p}$ and on as constants independent of deformation, but they are fixed through the continuous expression ( $\mathrm{Bq}$. (79) of Ref. 5) to the experimental odd-even mass differrences plotted in the comprehensive paper of Nílsson et al.

Table 1 lists experinontal and theoretical energies along with theoretical $A p, \Omega_{n}, e$, and $\mathrm{g}_{\mathrm{R}}$ values. In all cases calculated the equilibrium $\gamma$ remained at zero (prolate). Eviderntly the negiect of zero-point vibration causes a lack of the "ganna band mixing" effect of the band energies. The general agreement of transition energies is unusubl ly good for a one-parameter fit. The 2-0 transition energies are poor for $\mathrm{N}=90$ nuclet, but at this border of stable deformation it is felt that the $\mathrm{O}^{+}$state energies may be antualously low due to adnixture with spherical states. The calculated anowt of quadrupole stretching for $N=90$ ruclei is less than experiment. Our preliminary calculations show that a falling Nilsson level density with increasing deformation would produce

Table 1. Rotational band properties.

\begin{tabular}{|c|c|c|c|c|c|c|c|}
\hline \multirow[t]{2}{*}{ Nuxclẹus } & \multirow{2}{*}{$\begin{array}{c}\text { Spin } \\
1\end{array}$} & \multicolumn{2}{|c|}{$\begin{array}{l}\text { Ground band transition } \\
\text { energies }\left(\mathrm{H}_{\mathrm{I}}-\mathrm{E}_{\mathrm{I}-\mathrm{z}}\right) \text { keV }\end{array}$} & \multicolumn{2}{|c|}{$\begin{array}{l}\text { Gap parameters } \\
\text { (MkV) }\end{array}$} & \multirow{2}{*}{$\frac{\begin{array}{l}\text { Bquilibrius } \\
\text { deformation }\end{array}}{t_{2}(I)}$} & \multirow{2}{*}{$\frac{\begin{array}{r}\text { Gyromagnetic } \\
\text { coefficient }\end{array}}{g_{R^{(I)}}}$} \\
\hline & & $\left(\mathrm{E}_{\mathbf{I}}-\mathrm{E}_{\mathbf{I}-2}\right)_{\mathbf{t}}$ & $\left.-E_{1-2}\right)$ & $a_{p}(I)$ & $\Delta_{n}(I)$ & & \\
\hline $62^{152}$ & $\begin{array}{r}0 \\
2 \\
4 \\
6 \\
8 \\
10 \\
12 \\
14 \\
16 \\
18\end{array}$ & $\begin{array}{l}110.0 \\
244.6 \\
355.8 \\
440.5 \\
500.8 \\
540.7 \\
564.3 \\
575.0 \\
576.3\end{array}$ & $\begin{array}{l}121.8 \\
244.7 \\
340.7 \\
418.1 \\
483.5\end{array}$ & $\begin{array}{l}1.10 \\
1.08 \\
1.05 \\
1.01 \\
0.97 \\
0.93 \\
0.90 \\
0.88 \\
0.87 \\
0.88\end{array}$ & $\begin{array}{l}1.15 \\
1.13 \\
1.08 \\
1.01 \\
0.93 \\
0.84 \\
0.74 \\
0.63 \\
0.53 \\
0.43\end{array}$ & $\begin{array}{r}0.2224 \\
.2226 \\
.2230 \\
.2234 \\
.2240 \\
.2245 \\
+2249 \\
.2253 \\
.2257 \\
+2260\end{array}$ & $\begin{array}{r}0.405 \\
.397 \\
.386 \\
.370 \\
.349 \\
.324 \\
+295 \\
+264 \\
.233\end{array}$ \\
\hline $154 \mathrm{Ga}$ & $\begin{array}{r}0 \\
2 \\
4 \\
6 \\
8 \\
10 \\
12 \\
14 \\
16 \\
18\end{array}$ & $\begin{array}{l}111.4 \\
248.0 \\
361.2 \\
447.5 \\
508.7 \\
548.6 \\
571.3 \\
580.9 \\
580.9\end{array}$ & $\begin{array}{l}123.0 \\
247.8 \\
346.5 \\
426.7 \\
492.8 \\
547.7 \\
592.8 \\
627.2 \\
611.7\end{array}$ & $\begin{array}{l}1.15 \\
1.13 \\
1.10 \\
1.06 \\
1.02 \\
0.99 \\
0.96 \\
0.95 \\
0.94 \\
0.95\end{array}$ & $\begin{array}{l}1.08 \\
1.06 \\
1.01 \\
0.94 \\
0.85 \\
0.76 \\
0.66 \\
0.55 \\
0.45 \\
0.35\end{array}$ & $\begin{array}{l}.2319 \\
.2321 \\
.2325 \\
.2331 \\
.2338 \\
.2344 \\
.2349 \\
.2355 \\
.2359 \\
.2363\end{array}$ & $\begin{array}{l}.412 \\
.403 \\
.389 \\
.370 \\
.347 \\
.319 \\
.28 B \\
.256 \\
.226\end{array}$ \\
\hline${ }_{64}^{156} \mathrm{Gd}$ & $\begin{array}{r}0 \\
2 \\
4 \\
6 \\
8 \\
10 \\
12 \\
14 \\
16 \\
18\end{array}$ & $\begin{array}{r}88.4 \\
199.2 \\
295.4 \\
373.5 \\
433.5 \\
477.1 \\
506.7 \\
524.7 \\
533.5\end{array}$ & $\begin{array}{r}88.9 \\
199.2 \\
296.5 \\
380.7 \\
451.3\end{array}$ & $\begin{array}{l}1.05 \\
1.04 \\
1.01 \\
0.98 \\
0.95 \\
0.91 \\
0.89 \\
0.87 \\
0.85 \\
0.85\end{array}$ & $\begin{array}{l}I .00 \\
0.98 \\
0.94 \\
0.89 \\
0.82 \\
0.74 \\
0.65 \\
0.56 \\
0.47 \\
0.38\end{array}$ & $\begin{array}{l}.2513 \\
.2513 \\
.2515 \\
.2517 \\
.2519 \\
.2522 \\
.2524 \\
.2527 \\
.2529 \\
.2531\end{array}$ & $\begin{array}{l}.407 \\
.400 \\
.390 \\
.375 \\
.357 \\
.335 \\
.311 \\
.285 \\
.258\end{array}$ \\
\hline${ }_{64}^{158} \mathrm{Cd}$ & $\begin{array}{r}0 \\
2 \\
4 \\
6 \\
8 \\
10 \\
12 \\
14 \\
16 \\
18\end{array}$ & $\begin{array}{r}79.9 \\
181.9 \\
274.5 \\
354.6 \\
421.6 \\
476.1 \\
519.3 \\
553.0 \\
578.5\end{array}$ & $\begin{array}{r}79.5 \\
181.9 \\
277.6 \\
359.2\end{array}$ & $\begin{array}{l}0.83 \\
0.82 \\
0.80 \\
0.77 \\
0.75 \\
0.68 \\
0.64 \\
0.60 \\
0.56 \\
0.52\end{array}$ & $\begin{array}{l}0.83 \\
0.82 \\
0.79 \\
0.75 \\
0.69 \\
0.63 \\
0.60 \\
0.50 \\
0.43 \\
0.35\end{array}$ & $\begin{array}{r}.2617 \\
.2618 \\
.2619 \\
.2620 \\
.2621 \\
.2623 \\
.2625 \\
.2627 \\
.2629 \\
0.2630\end{array}$ & $\begin{array}{r}.404 \\
.403 \\
.400 \\
.395 \\
.391 \\
.385 \\
.378 \\
.369 \\
0.358\end{array}$ \\
\hline
\end{tabular}


an improvement in quadrupole stretching and retain good energy spacing, but we have not wished to involve more than one-parameter in the fitting to date.

The grrotagretic ratio always monotonically decreases with spin. This is a consequence of the fact that the neutrom pairing decreases more rapidiy with spin thim does proton pairing. In I5AGa the calculations show onset of backbending at spin 18 in accord with experintent, but there is no pairing collapss. In fact, the protoll gap parameter even increases silghtly from spin 16 to spin 18 .

\section{Footnotes and References}

*Present address: INS, Liniversity of Tokyo, Japan.
1. R. M. Diamond, F. S. Stephens and H. J. Swlatecki, Phys. Lett. 11, 315 (1964).

2. C. W. Na and J. O. Rasmusson, Phys, Rov, 69, $1083(1974)$.

3. S. E, Larsṣn, Physica Scripta 8, 17 (1973).

4. C. W. Ha and J. 0. Rasmussen, Phys. Rev. C2, $798(1970)$.

5. J, 0. Rasmassen, "ModeIs of Heavy kuclei", in 'Tuclear Spectroscopy and Reactions", Part D, , J. Cerry ed, Academic Press, New York, 1975.

6. S. G, Nilssm, C. F. Tsang, A. Sobiczewski, 2. Szymensty, S. Wycech, Ch. Gustafson, I. L. Lam, P. Moller and B. Nilsson, Nucl + Phys, AI32, 1 (1969).

\title{
GENERALIZED PARTICLE CORE COUPLING
}

\author{
G J Matherws
}

Here, results are presented from a simple, general model capable of describing the excitations in vibrational, transitional, or defonmed nuelei within a single spherical basis. The nethod has the advantages of a relatively stall set of basis states and that the tremsformation of the singleparticle states to a deformed basis is avoided.

Any model which addresses itself to so general a task as this uust acconmodate simutaneously the variation with deformation of the single-particle level spacings, and the pairing oscupation probabilities. Approaches to this problem in the past

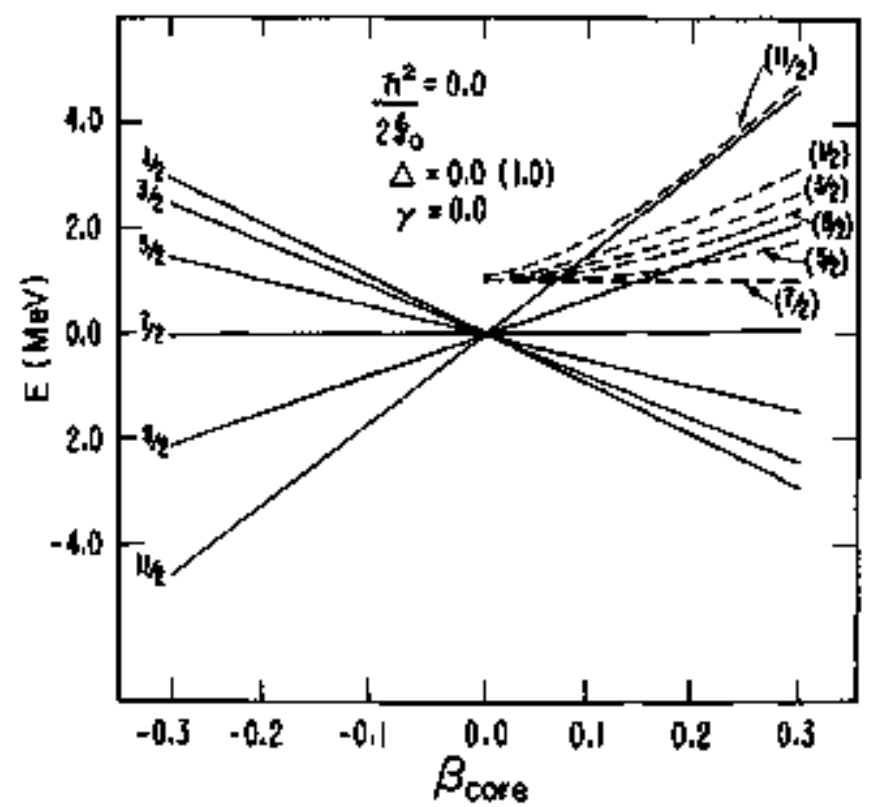

Fig. 1. Qeneralized particle core coulling for the $h_{1} 1 / 2$ shell with core energies - 0 . The dashed lines inclute pairing with $\lambda=0,4=1 \mathrm{Kev}$. The results are indistinguishable from a single-j shell Nilsson calculation.

(XBL 766-8298) have employed a man-body mictoscopic descripting such as the equations of motion method of Kleinl or Coswami et al.2 The disadvantage of these earlier formulations is that the microspopic basis becones too clumbersone away frod spherical nuclei.

The model discussed here is a generalization of the deshallt core excitation mode ${ }^{3}$ in that the malear core is described by a set of quadrupole matrix elowents and diagmal even-even core energies. An equation of motion formalism permits simultaneous description of the states of the odd particle subject to pairing and shape correlations, the latter Included as a "backwards" gmplitude.

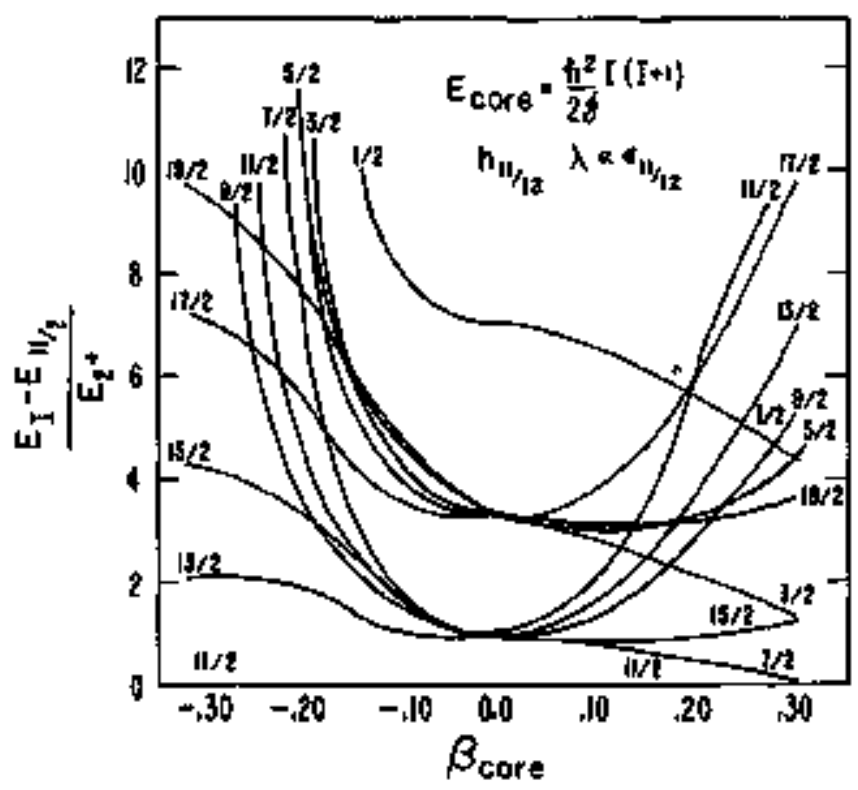

Fig. 2. Generalized perticle core coupling for the $h_{11 / 2}$ level with $\lambda=-\infty$, Rotational core energies ate assumed. The results are very sinilar to the coriolis coupling calculations of Ref. 4.

(XEL 766-8299) 


\section{$00,$. व}

Some results for a single-j shell calculation are presented in Figs. 1 and 2 . In Fig. 1 , the macroscopic core energies are set equal to zero, and the pairing has been turned off. One should expect the theory to then describe the singleparticle states as a function of deformation, analogous to a Nìisson calculation. In fact, Fig. 1 is indistirigulshable from an exact ivilsson calculation of the $h_{11 / 2}$ shell. The same exact axreement is obtained when the pairing is turned back on for both models as cam be seen by the dashed lines in Fig. I.

In Fig. 2, the Fermi surface has been set well below the shell. The core energies are assumed to be rotational. In this case, one would expect results similar to the coriolis coupling calculations of F. Stephens. 4 Figure 2 is, indeed, nearly identical to Fig. 12 of Ref. 4. The familiar coriolis decoupled band behavior is visible for positive defonation. The strongly coupled limit is approached for negative deformation.
In conclusion, it seems that this simple mode1 reproduces all of the familiar deformed wodel muclear structure, though it employs a spherical basis. The extension of the rodel to compute triaxial core shapes, electromagnetic moments, and transition probabilities is quite straightforward and currently undorway.

\section{Referencęs}

1. A. Klein, Ann. Phys, 66, 390 (1971).

2. A. Goswani, D. Malcioglu and A. I. Sherwood, Nucl. Phys. AI53, 433(1970).

3. A. deshalit, Phys. Rev. 122, 1530 (196I).

4. F. S. Stephens, Rev. Mod. Phys. 47, 43 (1975).

5. J. Weyer-ter-Vehn, Nucl. Phys, A249, 111 (1975).

\section{THEORETICAL. ESTIMATES OF MAGNETIC MOMENTS FOF EXCITED NUCLEI SHELL AND PAIRING EFFECTS}

L \$ Morello

The rotational constants, or moments of inertia, have been long recognized as valuable information regarding the flow of muclear matter in meclei. Spectroscopically detected rotational bands have produced evidence of moments of fnertia smllier than expected from the charge quadrupole sacment. Such a reduction has boen elegantly explained in tarms of the pairing interaction which makes the deformed raxlei superfluid, Variations in the moment of isertia with angular momentum have been observed auite recently and are particular1y inpressive in backbending tuclei. These variations sepen to be the rosult of a decolpled pair anot of the simul taneous disappearance of the pairing effects with angular nomentum. It is not settled as yet which of the two connected causes is the doninant one.

Variations in the moment of inertia along the yrast 1ine, now being observed experinentally, have also been consldered theoretically, with various aims in ufind. At lower angular monentum one is nore interested in the collapsing of the pairing correlation; at larger amgular momenta, the main interest is in gross changes in monents of inertia arising from radical changes in the nuclear shape (oblate to prolate). Calculations have been performed for excited spherical shell motel ruclei. The seniclassical rotational quantities like the angtular velocity and the momonts of inertia have been used in describing the variation of pairing and its eventual collapse.

Very much like the moments of inertia, the nuclear mggnetic noments reflect the intemal flow of nuclear matter and therefore can yield information related to that provided by the moments of inertia. Yot, there are substantial differences. Protons, for example, contribute with their orbital motion and spin to the magnetic mongat, while neutrons only contribute with their spin. Finthermore, the neutrun intrinsic magnetic moment is small and negative while that of the proton is small and positive. These observations raise the hope that the simultaneous determination of both moments of inertia and magnetic moments may allow ore to single out the contributions of each kind of particle to the overall peneration of both the megnetic moment and the moment of inertia.

A5 a begining, the most simple spherical case will be studled on the basis of the shell mode1 and the BCS Haniltonian. The expectation value of the BCS Hamsltorian, diagonel in quasiparticle space has the form

$$
\begin{aligned}
& \left\langle H^{\prime}\right\rangle=\langle H-\lambda N-Y H\rangle=\sum\left[E_{k}-\lambda-E_{k}\right) \\
& +\varepsilon_{n_{k}}^{*}\left(E_{k}-\gamma m_{k}\right)+\varepsilon_{n_{k}}^{-}\left(E_{k}+\gamma m_{k}\right)+A^{2} / G \text {. }
\end{aligned}
$$

In this expression $\mathrm{N}$ is the total particle rumber, $\lambda$ is the cotresponding Lagrange miltiplier, $i x$ is the total angular momentum $Z$ projection, and $r$ is the corresponding Lagrange mittiplier which assumas the reaning of angular velocity

$$
\gamma=\frac{\partial \varsigma \mathrm{H} s}{\partial \mathrm{H}} .
$$

We can thus generate the grand potential

$$
\begin{aligned}
\Omega & =-B E\left(E_{k}-\lambda-E_{k}\right)+\sum \ln \left[1+\exp B\left(E_{k}-\gamma m_{k}\right)\right] \\
& +\sum \ln \left[1+\exp \beta\left(E+\gamma m_{k}\right)\right]-B_{G} \frac{\Delta}{G}
\end{aligned}
$$


and from it the other thernoxymanic functions

$$
\begin{aligned}
& N=\Sigma\left\{1-\frac{{ }_{k}^{*}{ }^{-\lambda}}{2 E_{k}}\left\{\text { tanh } 1 / 2 B\left(E_{k}-\gamma q_{k}\right)\right.\right. \\
& \left.\left.+\tanh 1 / 2 \beta\left(E_{k}+m_{k}\right)\right\}\right] \\
& M=\Sigma_{m_{k}}\left[\frac{1}{1+\exp B\left(E_{k}-m_{k}\right)}-\frac{1}{1+\exp \theta\left(E_{k}+\gamma q_{k}\right)}\right] \\
& \text { E - }{ }_{\Sigma} \epsilon_{k}\left[1-\frac{\epsilon^{-\lambda}}{2 E_{k}}\left\{\tanh 1 / 2 \beta\left(E_{k}-17 m_{k}\right)\right.\right. \\
& \left.\left.+\tanh 1 / 2 B\left(E_{k}+m m_{k}\right)\right\}\right]-\Delta^{2} / G \text {. }
\end{aligned}
$$

The quantities $A, \beta, \lambda$, and $j$ are related to each other by the gap equation

$$
\frac{a 2}{a b}=0
$$

or

$$
\begin{aligned}
& \Sigma \frac{1}{2 \mathrm{E}_{\mathrm{k}}}\left[\tanh 1 / 2 \mathrm{~B}\left(\mathrm{E}_{\mathrm{k}}-\mathrm{m}_{\mathrm{k}}\right)+\tanh 1 / 2 \mathrm{~B}\left(\mathrm{E}_{\mathrm{k}}+\mathrm{y} \mathrm{m}_{\mathrm{k}}\right)\right] \\
= & 2 / 6 .
\end{aligned}
$$

In the extreme single particle mode1, a nucleus is characterized by a magnetic moment which results from the vector sum of the metic moments of the individual mucleons. A simple approach to stming the contribution of individual nucleons is to sum the projections of each single particle magnetic moment on the $Z$ axis, chosen 50 as to have the largest total angular momentim projection for the rucieus as $a$ whole. The $z$ projection of the singie particle mgnetic monent can be written as follows

$$
\mu_{z}=\mu \frac{\Pi_{j}}{j}=\left(g_{l} \frac{\ell+j}{j}+g_{s}=\frac{s+j}{j}\right) \frac{m_{j}}{j}
$$

where $g_{4}, g_{5}$ are the $g$ factors associated with the ortital and spin single particle angular momentwon 2 and $s_{i} j$ is the total single particle angular monentum and $m_{j}$ is 1 ts projection on the $z$ axis. Since

$$
\frac{\stackrel{2}{n} \cdot j}{j^{2}}=\frac{j(j+1)+k(l+1)-s(s+1)}{2 j(j+1)}
$$

and

$$
\frac{s+j}{j^{2}}=\frac{j(j+1)+s(s+1)-\ell(\ell+1)}{2 j(j+1)},
$$

we have for $j=2+1 / 2$

$$
\mu_{2}=\mu \frac{m_{j}}{j}=\left(1 / 2 g_{s}+l_{g_{l}}\right) \frac{\pi \theta_{j}}{j}
$$

and for $\mathrm{j}=\mathrm{\ell}-1 / 2$

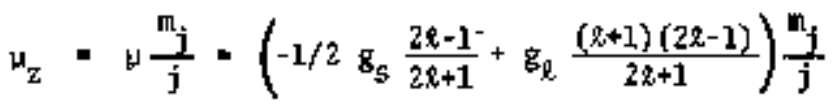

or

$$
p_{z}=\left[g_{Q} \pm \frac{1}{2 \ell+1}\left(g_{s}-g_{i}\right)\right] m_{j} .
$$

The same result ought to be obtained if we project separately the orbital and spin conponents on the $z$ axis. Than

$$
u_{z}=\sum_{l}\left(g_{\ell} m_{\ell}+g_{s} m_{s}\right) c_{l}^{2}
$$

where $\mathcal{C}_{l}$ is the relevant Clebsch Gordon coefficient. For $j=q+1 / 2$ we have

$$
\begin{aligned}
\mu_{z} & =\frac{j+m_{j}}{2 j}\left[g_{q}\left(m_{j}-1 / 2\right)+1 / 2 g_{s}\right] \\
& +\frac{j-m_{j}}{2 j}\left[g_{2}\left(m_{j}+1 / 2\right)-1 / 2 g_{s}\right] \\
& =g_{l} m_{j}+\frac{m_{j}}{2 j}\left(g_{s}-g_{q}\right) \\
& =\left[g_{q}+\frac{1}{22+1}\left(g_{s}-g_{q}\right)\right] m_{j}
\end{aligned}
$$

as was obtained before. Similar calculations can be performed for $j=\ell-1 / 2$.

Since $\mathrm{g}_{0}=1, \mathrm{~g}_{5}=5.586$ for protons, and $\mathrm{g}_{\ell}=0 ; \mathrm{g}_{\mathrm{s}}=-3.826$ for neutron, the following expressions for $\mu_{2}$ are obtained.

for protons:

$$
\begin{array}{ll}
\mu_{z}^{p}=\left(1-\frac{2.293}{j+1}\right) m_{j}^{p} & \text { for } j=\ell-1 / 2 \\
\mu_{z}^{\mathrm{P}}=\left(1+\frac{2.293}{j}\right) \mathrm{m}_{j}^{\mathrm{p}} & \text { for } j=\ell+1 / 2
\end{array}
$$

For neutrons:

$$
\begin{array}{ll}
\mu_{z}^{n}=\frac{1.913}{j+1} m_{j}^{n} & \text { for } j=\ell-1 / 2 \\
\mu_{z}^{n}=\frac{1.913}{j} m_{j}^{n} & \text { for } j=\ell+1 / 2
\end{array}
$$

The nuclear magnetic moment for a single nigutron or proton component can now be written as follows:

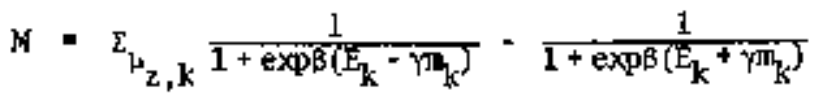




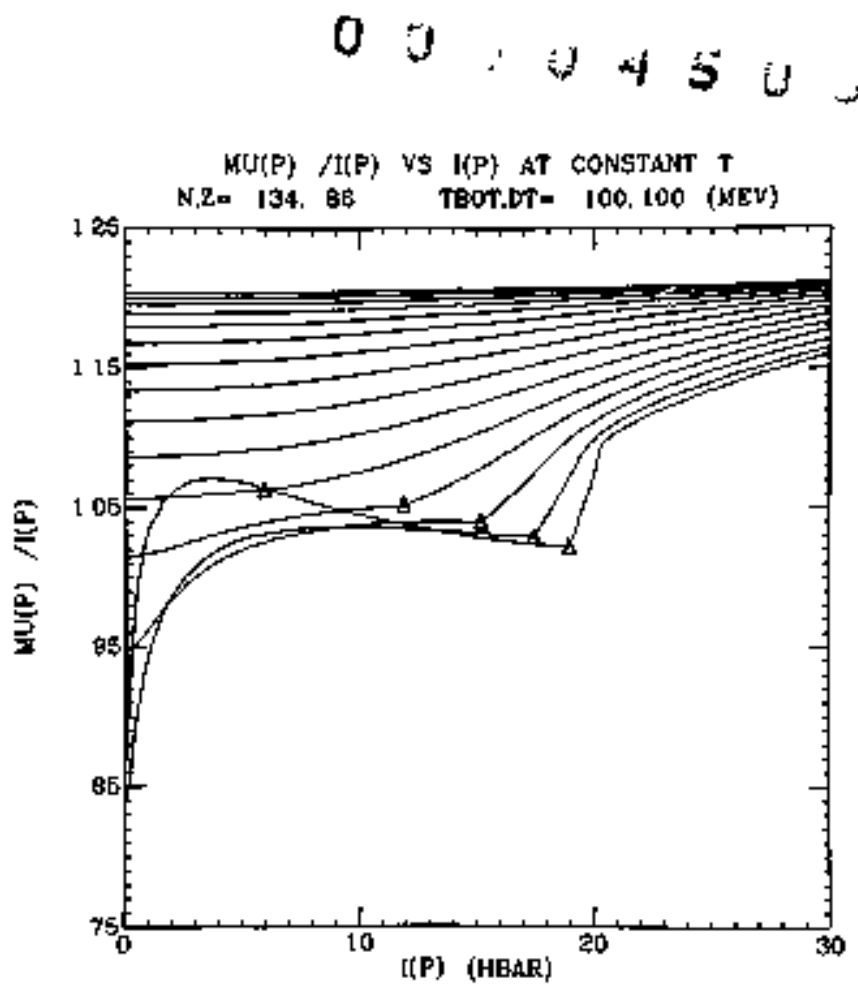

Fig. 1. Proton component g factor as a function of the proton component angular momentum for various teraperatures, The triangles on the lower isotherus indicate the disappearance of the proton pairing correlation. The nucleus in stuty is 220 Ron.

(XBL 7612-10861)

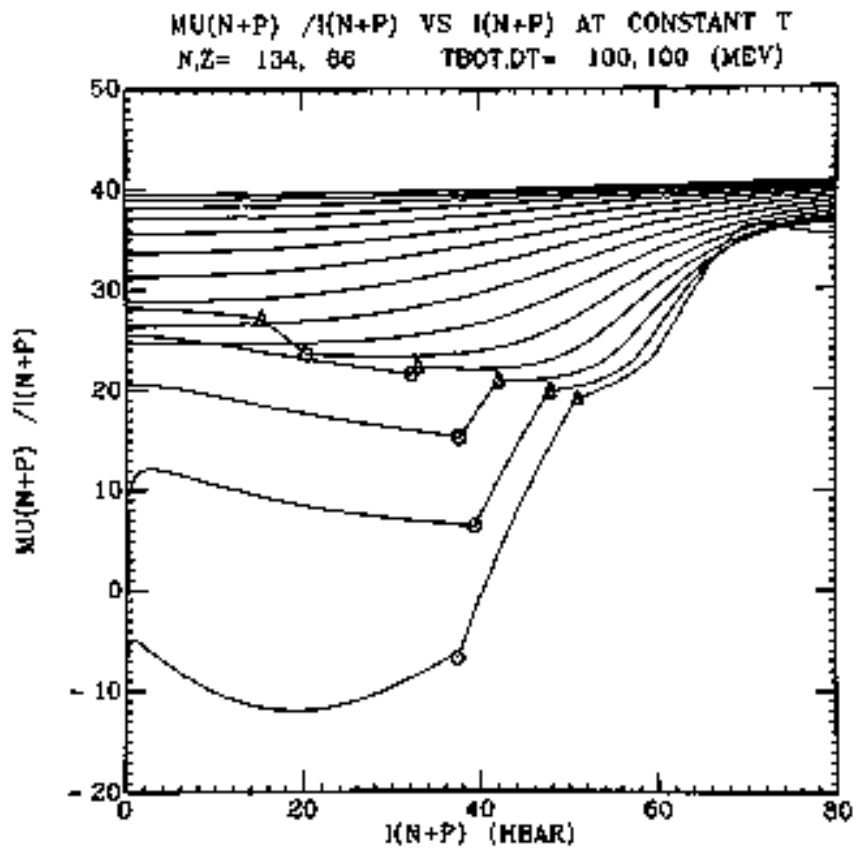

Fig. 3. Overall g factor as a function of total angular momentum for various tenperatures in the nucieus 220 Rin. The triangles and ppen circles indicate the disappearance of proton and neutron pairing correlation respectively. (XEL 7612-10867)

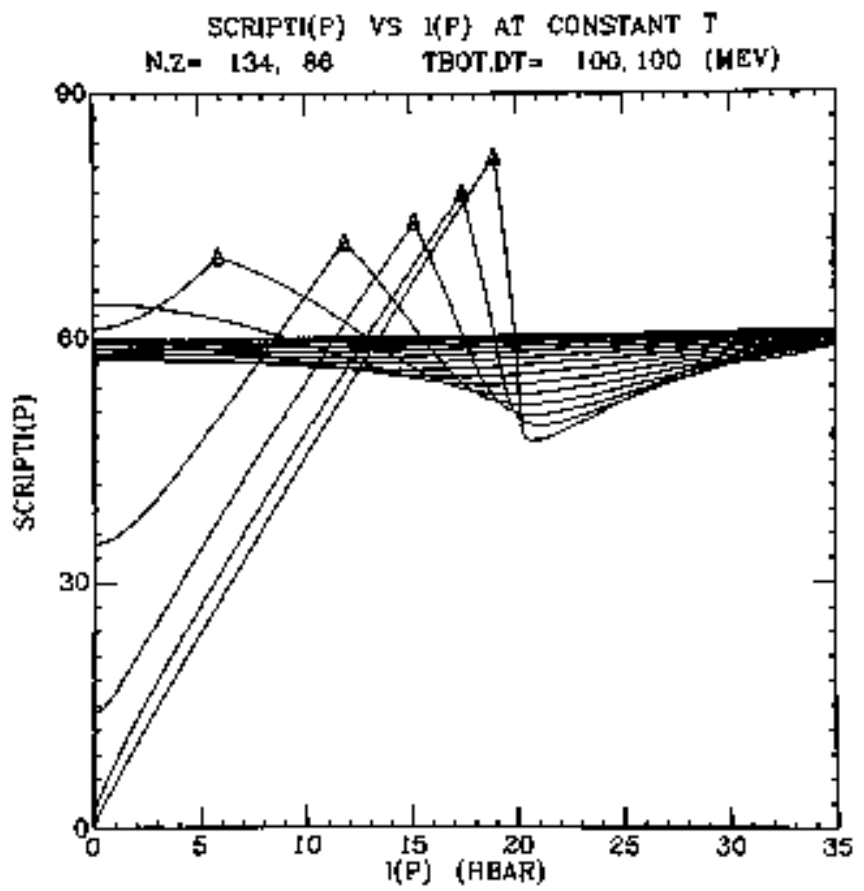

Fig, 4. Proton cormonent of the moments of inertia as a function of the proton component angular monentum for various termeratures. Notice the effect of pairing in the lower isotherms.

(XBL $7612-10856$ ) 


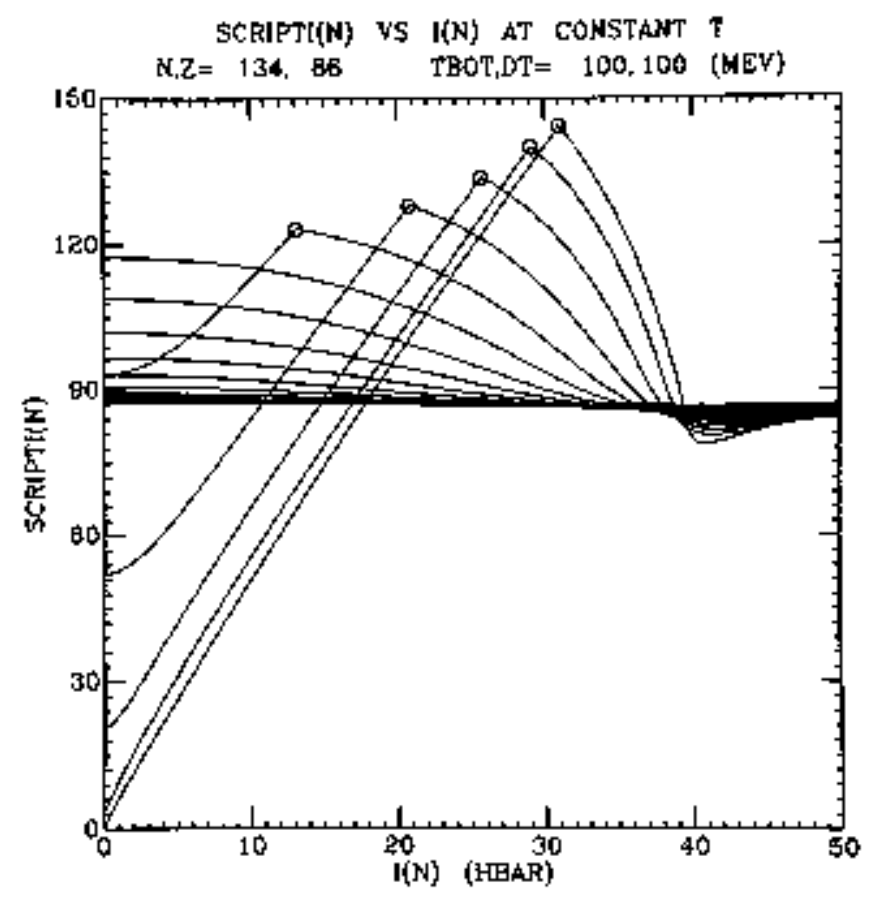

Fig. 5. Same as in Fig. 4 for the neutron conponent.

(XBL $7612-10864$ )

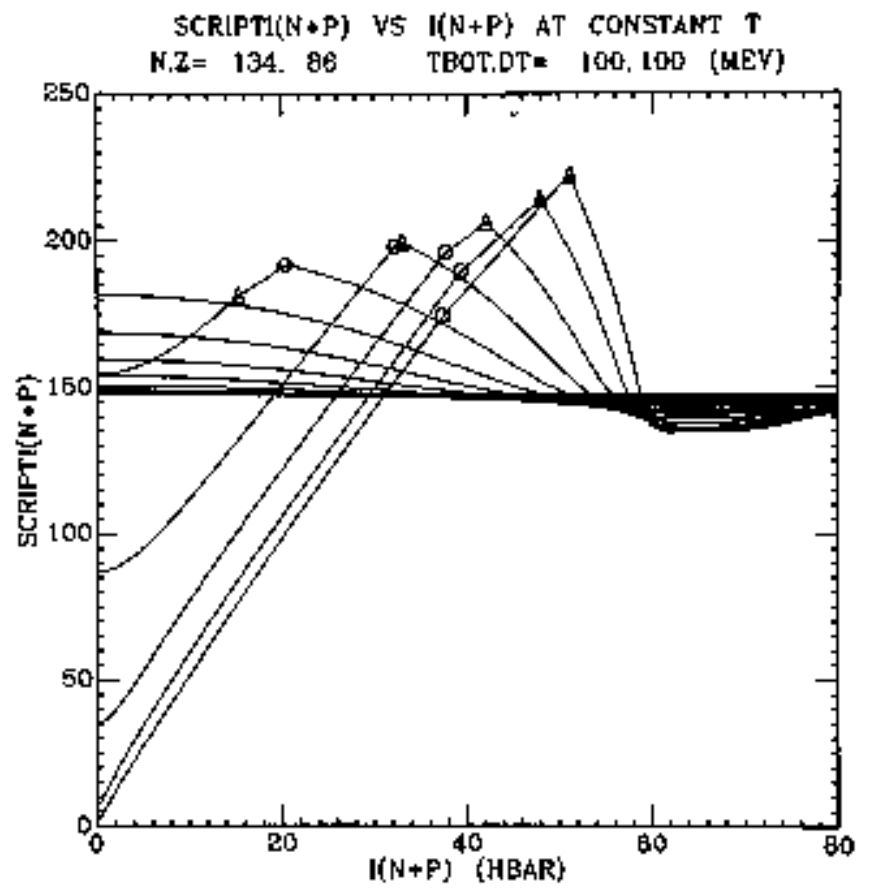

Fig. 6. Overall morents of inertia of $220 \mathrm{mon}$ as a fumction of total angular morentum for various temperatures. Notice the effect of neutron and proton pairing in the lower 1sotherms.

(XBL 7612-10855)

strongly affected by pairing at small angular momenta. At higher angular nonenta the pairing effects varish.

The total magnetic moment obtained by combining both components is shown in Fig. 3. It can be seen that at low temperatures the magnetic mionent is nogative, thus indicating the dominence of noutrons, At bigher temperatures the magnetic moment is mainly controlled by the proton component. In Figs. 4 and 5 the neutron and proton contributions to the moment of inertia are shown and in Fig. 6 the total moment of inertia is shom. Notice that both conponents contribute substamtially to the moment of inertia. Also notice how the pairing effects substantially redice the morent of inertia.

\section{NORMALIZED SHELL MODEL ALPHA DECAY THEOHY APPLIED TO UNFAVORED DECAY*}

T Fllessbach, $\mathrm{H} J$ Mang.ł and $J O$ Rasmussen

Antisymotrization is usually taken into account in the sholl model theory of alpha decay. 1 It enforces the requirements of the Pauli principle, preventing double occupation of states. However, ant isymetrization between two conposite particles leads to ron-normalized states. The proper norjulization may be hidden in the furction of relative
Jotion between the alpha particle and the daughter nucleus. We are not able to calculation this function (incluting the nomalization) exactly; instead we introdxce a distorted wave approximation for the wavefunction of relative motion, and then it is vital to take the proper normalization expIicitly into account. 


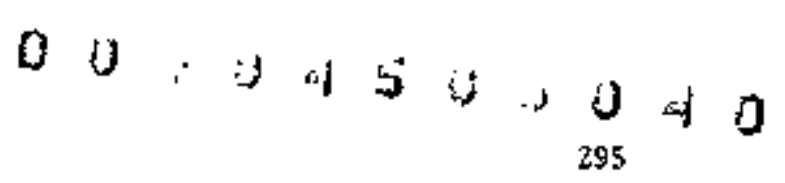

This normalization results $s^{2,3}$ in a comsiderable enhancenent of the reduxed width for alpha decay. Retuced width means here $\delta^{2}=\hbar\left(\mathrm{P} \cdot \mathrm{T}^{2}\right)$, with $\tau$ the mean life and $P$ the barrier penetration factor.

Since the new theoretical absolute ground- toground state decay widths are strongly enhanced 2 over the previous version of the theory, we felt it inportant to check also on various cases of hindered alpha decay to see to what extent relative alpha transition rates would be affected by the normalization.

Tables $1-3$ give the results for the relative and absolute alpha decay rates for ${ }^{11} \mathrm{Po}_{\mathrm{p}}$ and $2 \mathrm{il} \mathrm{BI}_{\mathrm{I}}$.

The reșutts for ${ }^{212} \mathrm{Po}$ are given in a different form: in Table 4 we listed the spectroscopic factors and the hindrance factors (reciprocal of $\$$ normalIzed to the ground state transition).

Frran exardinatich of Table 1 it appears that the alpha branching ratios by the old and new theory are not inuch different. That is, the substantial enhancement of alpha decay rates by the new theory

Table 1. Alpha brouching ratios for $211_{\mathrm{Fo}}$ and $211_{\mathrm{Bi}}$. The results of the old and new theory are displayed vs the exporiment. The numbers in the luper linte are from a charmin ladius dependont formelle, the loner line is calculated wth spectroscopic factors.

\begin{tabular}{|c|c|c|c|c|}
\hline \multirow{2}{*}{$\begin{array}{l}\text { Parent } \\
\text { nucleus }\end{array}$} & \multirow{2}{*}{$\begin{array}{l}\text { Laughter } \\
\text { nucleus }\end{array}$} & \multicolumn{3}{|c|}{ Alpha branching retios } \\
\hline & & Experiment & Old theory & New theory \\
\hline \multirow{3}{*}{${ }^{21 I_{P_{0}}}$} & $\mathrm{pI} / 2$ & 99 & $\begin{array}{l}98.0 \\
98+0\end{array}$ & $\begin{array}{l}96.8 \\
97.8\end{array}$ \\
\hline & $\mathrm{f5} / 2$ & 0.5 & $\begin{array}{l}1.6 \\
1.6\end{array}$ & $\begin{array}{l}2.7 \\
1.7\end{array}$ \\
\hline & $\mathrm{p} 3 / 2$ & 0.5 & $\begin{array}{l}0.4 \\
0.4\end{array}$ & $\begin{array}{l}0.5 \\
0.5\end{array}$ \\
\hline \multirow{2}{*}{${ }^{211}{ }_{\mathrm{Bi}}$} & $\mathrm{sl} / 2$ & 83 & $\begin{array}{l}32 \\
82\end{array}$ & $\begin{array}{l}78 \\
81\end{array}$ \\
\hline & $d 3 / 2$ & 17 & $\begin{array}{l}18 \\
18\end{array}$ & $\begin{array}{l}22 \\
19\end{array}$ \\
\hline
\end{tabular}

Table 2. Relative angtlar montum nixing aplitaxles. To

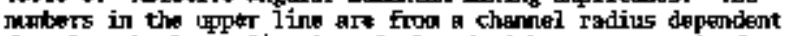
formale, the lower lifhe is chlsulated with spactrosopic factors.

\begin{tabular}{|c|c|c|c|c|}
\hline \multirow{2}{*}{$\begin{array}{l}\text { Parent } \\
\text { iucleins }\end{array}$} & \multirow{2}{*}{$\begin{array}{l}\text { \$tive of } \\
\text { daupherer } \\
\text { nucletus }\end{array}$} & \multicolumn{3}{|c|}{ Pelative angular monentm ining amplitudes } \\
\hline & & Experinent & old thesory & New theory \\
\hline & $f 5 / 2$ & $1:-0.96: 0.55$ & $\begin{array}{l}1:=0.88: 0.58 \\
1:=0.88: 0.58\end{array}$ & $\begin{array}{l}1:=0.85: 0.52 \\
1:=0.85: 0.56\end{array}$ \\
\hline & $\mathrm{p} 3 / 2$ & $1:-0.14$ & $\begin{array}{l}1:-0,33 \\
1:-0,33\end{array}$ & $\begin{array}{l}1=-0.32 \\
1=-0.33\end{array}$ \\
\hline $\mathbf{2 l k}_{\mathrm{Bi}}$ & $d 3 / 2$ & $1:-0.27$ & $\begin{array}{l}1:-0.35 \\
1:=0.33\end{array}$ & $\begin{array}{l}1:=0 . \$ 2 \\
1:=0.33\end{array}$ \\
\hline
\end{tabular}

Table 3. Alpha decay width in $10^{-22} \mathrm{kzV}$. The last line gives the ratio of the total decay width for ${ }^{21}$ Po and
gil.

apply rather foifomly to the various state combinations of $211_{P_{O}}$ and $211_{\mathrm{Bi}}$ decay. This result is reassuring in that it suggests that extensive previous calculations in the literature with the old shell-model alpha deray theory are not all invalidated, likewise, in Table 2 we see that the relative anplitudes for various $\&$ values are not inxch altered, also there seems to be some systematic lowering of higher 2 values by a few percent.

From Table 3 we see that the absolute decay widths are increased by two orders of magnitude in the new theory, giving good agreetent with experiment. The ratio of widths for the two mulei differs by a considerable amount for the old and new theories, with the result of the new theory in better agreedent with experiment.

It is when we conte to the spectroscopic factors for $212 \mathrm{Po}$ in Tablo 4 that find striking differences between the old and new theory, In particular the considerable hfndrance factors predicted for the second $0^{4}$ and second and thire $2^{*}$ states are lowered by ar order of magnitude in the new theory.

As far as the absolute decay widths are concemed, the new theory can reproduce the ground state decay width (see also Ref. 2), the old theory falls short by tho orders of magnitude. The ratio $\Gamma\left(18^{4}\right.$, assumed to be the isomer at $\left.2930 \mathrm{keV}\right) / \Gamma\left(0_{1}^{+}\right)$ comes out too Iarge by a factor of 30 in the new theory and a factor of 150 in the oid one.

\section{Footnotes and References}

Condensed from LBL-4335, Phys, Rev. C13, 1338 (1976).

PPresent addre55: Tecflnische Џniversität Mtinchen, Physikdept., 8046 Garching, W. Germany.

${ }^{\ddagger}$ Permanent address: Technische loniversität vincher Physikdept., 8046 Carching, W. Germany.

1. H. J. Mang, Ann. Rev. Nucl, Sc1, 14, 1 (1964) +

2. T. Fliessbach, Z, Phyșik A272, 39 (1975).

3. T. Fliessbach and H. J. Mang, proprint (1975).

\begin{tabular}{llll}
\hline $\begin{array}{c}\text { Alpha } \\
\text { enitter }\end{array}$ Experiment & old theory & Nent theory \\
\hline $211_{\mathrm{Po}}$ & 8.8 & $9 \times 10^{-2}$ & 12 \\
$211_{\mathrm{Bi}}$ & $3.5 \times 10^{-2}$ & $5.4 \times 10^{-4}$ & $4.4 \times 10^{-2}$ \\
$\Gamma_{\mathrm{Po}} / \mathrm{T}_{\mathrm{Bi}}$ & 250 & 167 & 273 \\
\hline
\end{tabular}


Table 4, Alfola decay theoretical propertics from ground and excited states.

\begin{tabular}{|c|c|c|c|c|c|}
\hline \multirow{2}{*}{$\begin{array}{l}\text { Stare } \\
\text { of } \\
212 \mathrm{Po}\end{array}$} & \multicolumn{3}{|c|}{ Spect roscopic factors S } & \multicolumn{2}{|c|}{$\begin{array}{l}\text { Hindrance Cactors } \\
\text { /5 (0, }) / 5 \text { (excited)] }\end{array}$} \\
\hline & $\begin{array}{l}\text { old theory } \\
\left(10^{-5}\right)\end{array}$ & $\begin{array}{c}\text { New theory } \\
\left(10^{-2}\right)\end{array}$ & $\begin{array}{c}\text { new to old } \\
\left(10^{2}\right)\end{array}$ & $\begin{array}{l}\text { old } \\
\text { theory }\end{array}$ & $\begin{array}{l}\text { New } \\
\text { theory }\end{array}$ \\
\hline$o_{1}$ & 6.0 & 2.6 & 4 & 1 & 1 \\
\hline $\mathrm{O}_{2}$ & 0.71 & 3.1 & 44 & 8 & 0.8 \\
\hline$D_{3}$ & 0.17 & 0.16 & 9 & 35 & 16 \\
\hline 2) & 1.6 & 0.56 & 3.5 & d & 5 \\
\hline$z_{2}$ & 0.09 & 0.40 & 44 & 66 & 7 \\
\hline $2_{3}$ & 0.17 & 0.47 & 3 & 35 & 6 \\
\hline $2_{4}$ & 0.08 & 0.04 & s & 71 & 68 \\
\hline$z_{5}$ & 1.6 & 0.29 & 2 & 4 & 9 \\
\hline 4) & 0.79 & 0.20 & 2.5 & 8 & 13 \\
\hline 101 & 2.8 & 0.76 & 3 & 2 & 3 \\
\hline 163 & 0.79 & 0.39 & 5 & 8 & 7 \\
\hline 181 & 6,0 & 0.50 & $\mathbf{]}$ & $\mathbf{I}$ & 5 \\
\hline
\end{tabular}

\title{
APPLICATION OF THE COUPLED CHANNEL ALPHA DECAY THEORY TO OOD-MASS NUCLEI*
}

\author{
A. J. Soinski, E. A. Hausther, \\ J. O. Rasmussen, and D. G. Raich
}

The alpha decay of spheriodal nuslei in which coupting exists between the enitted alpha particle and the intemal parent nuxlear states, can be treated with a set of coupled equations. The priflary noncertral coupling is the collectively enhanced rotational E2 transitions. Wh set up and numeric. ally integrated a set of coupled secand-order linear differential equations in the radial separation variable for the penetration through an anisotropic barrier.

Nithough other researchers hate performed the nunerical integration of coupled channel equations for the alpha decay of even-even spheriodal auclei, only thassman and Rasmussenl have comsidered alpha decay of odd-mass nuclei. In the latter case the conservation of angular momenturn pernits an alpha particle partiat wave of a given orbital argular momentum, o, to branch to more than one energy leve1 of the daughter mucleus. Thus, for the favored alpha decay of $233_{\mathrm{I}}$ chasman and Rasmussen considered the decay of the 5 -wave to the $5 / 2$ level and the branching of the $d$-wave to the $5 / 2,7 / 2$ and $9 / 2$ levels of $229 \mathrm{Th}$. Ohannel coupling effects are most significant for the case of a relatively weak wave, such as the highly hindered g-wave, coupled to a strong wave such as the s-wave. Therefore, have extended the work of thasmen and Rasmussen by considering the branthing of the s-, d-, and g-upaves in the favored alphin decays of both 253 Es and $255_{\text {fal. }}$ Favored alpha decays are those in which the quantum numbers of the odd nucleon are the same for both the parent and daughter. Both $253_{\mathrm{Es}}$ and $255_{\mathrm{Fm}}$ are spin 7/2 nuclei and are ideal for applying an exact numerical trestment because alpha transitions to the favored bands of the daughters have been well studied. 2 and angular distribution data from low temperature nuclear alignnent experiments are available. 3 If we include the $\downarrow=0,2$, and 4 partial waves, then nine coupled second-order differential equations Jaust be solved for favored decays to the five Iowest levels $(7 / 2,9 / 2, I 1 / 2,13 / 2,15 / 2)$ of the daughter rotationsl band. Contribution from the $x=f$ was found to be small, as it has a large hindrance factor.

The formalism for the alpha decay in the presence of a noncentral field is given by Perlman and Rasmussen.4 A multipole expansion is made for the Coulombic potential energy outside the nuclear strface where the zero ordar term is the central couldrabic terto. The E2 interaction contributes the only important coupling term for these nuclei. The coupled differential equations in the radial separation variable can be written

$$
\begin{aligned}
& u_{I_{d}, t}^{\prime \prime}=\frac{2 M}{\hbar^{2}}\left(\frac{2 Z e^{2}}{r}+\frac{\pi^{2} x[2+1]}{2 v r^{2}}-Q_{I_{d}}\right) u_{I_{d}, k}
\end{aligned}
$$

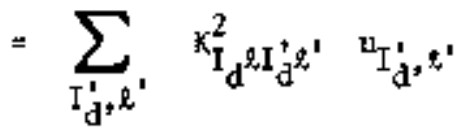




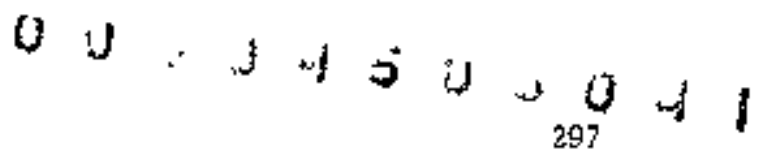

Where $y$ is the reduced mass, $z$ is the charge of the daughter nucleus, $Q_{\mathrm{I}}$ is the effective $Q$ value for the alphe particle "with electron screering and daughter recoil energy corrections), and the $K_{\mathrm{A}^{2} \mathrm{I}_{\mathrm{d}} \mathrm{d}^{\prime}}$ are matrix element of the quadrupole coupling operator which is proportional to the product of the intrinsic nuclear quadrupole moment of the daughter times $\mathbf{P}_{2}(\cos \theta)$ divided by $\mathbf{r}^{3}$, and $\mathbf{r}$ is the radial separation variable.

Explicit expressions for the quadrupolo copp1ing matrix element were given for alpha decay by Perlman ard Rasmussen ${ }^{4}$ and for optical mode1 scattering applications by Tamura. ${ }^{6}$ The general method of solution of coupled equations is given by $\mathrm{E}$. $A$. Rauscher et aI. ${ }^{7}$ solutions of the coupled differential equations approach the Coulonb functions asyutotically at large radius.

We construct a set of nine second-order coupled differential equations which is transformed into a set of eighteen first-order coupled differential equations having oighteen linearly independent solutions or, equivalently, nine complex solutions, Because the physically meaningfu1 solutions are exponentially decreasing going outward through the barrier, it was not possible to obtain stable solutions by outward minerical integretion. Insteart we used coulomb functions as starting conditions at a radius sufficiently large (150 fu) such that the coupling forces were shali and integrated inward to $10 \mathrm{fin}$. The solutions of interest then increased in the direction of integration and thus were stable. In general the phase of an oscillating coupled-channel solution in the far region will differ from the phase of the correspond? ing coulonb function. This phase difference wiIl be referred to as the quadrupole phase shift, Q $_{\mathrm{d}}$ + Although phase shifts do not affect intensity data, they do affect angular distribution data through the interference terms between different alpha waves going to the same final state $I_{d}$.

The experinental intensities are sensitive only to the relative partial wave intensities whereas the alpha particle angular distributions are sensitive to both the relative partial weve intensities and phases. The angular distribution data yield directly a maximm of two nembers to compare with theory whereas the intensity date yield four. For odd-rass nuclei there are insuffictent relative intensity data to provide the required boundary conditions. For the problem considered here, eight boundary conditions are required. For the five levels of the daughter there are only four relative intensities, and there exist no direct experimental measurements of the mix and the relative phases of the partial waves. The low temperature angular distribution data provide two experimental numbers (the coefficients of the $P_{2}(\cos \theta)$ and $P_{4}(\cos \theta)$ terms in the angular distribution), but they do not determine uniquely relative partial wave amplitudes and phases, Because there are insufficient experinental data to completely fix the boundary conditions, we ușed nuclear nodel constraints. The remaining two boundary conditions were left as free parometers and are denoted by $\alpha_{2}$, the ratio of total d- and s-wave anplitude at $\mathrm{F}_{0}$ the nuclear surface and $\mathfrak{a}_{\mathbf{4}}$, the ratio of total $\mathrm{g}$ to s-wave amplitude at $\mathrm{R}_{\mathrm{O}}$.

The numerical integration of the coupled differential equations permits us to tost two assimptigns of the strong coupling model as usually applied. The first is that near the nuclear surface only $m_{t}=0$ alpha partial waves occlr. Subject to this $m_{p}=0$ constraint, we wish to determine if the coupled channel treatment can reproduce both the experimental relative intensities to the five lowest rotational levels (with spins $7 / 2,9 / 2$, $11 / 2,13 / 2$ and $15 / 2$ ) of the daughter and the angular distribution data.

The coupled chiminel treatonent can also improve the usual approximate handling of anisotropic barrier penetrability by setting the relative intensity of a given wave to a glven level equal to (the square of a Clebsch-Gordan coefficient) times (a calculated spherical barrier penetratility factor for the alpha group). (This approxination is exact only in the limit of infirite mowent of inertia or vanishing nuclear quadrupole moment.)

The most general way to present the results of the nunerical integration is in the form of the complex matrix $\mathbf{U}^{-1}$ (the inverse of a complex $9 \times 9$ nestrix Uf\}. The matrix elements of $\mathrm{U}$ are the anplitudes of the linearly independent solutions on or near the mulear surface. Those matrices rechice to wit matrices for vanishing nuclear quadrupole moment. Operation with either complex matrix on any boundary conditions on a sphere near the nuclear surface will yield partial wave amplitudes and quadrupole phase shifts at large distances. If boundary conditions are fixed on the spheroidal nuclear surface, then a Frönan matrix cas be used to transform them to boundary conditions on a sphere at $10 \mathrm{fm}$. The real and imaginary comporents of our matrix are givon in Table 1 for $25{ }^{\mathrm{Es}}$. In Rof. 9 , the presented the percentage intensities for favored rotational band alpha decay for the two odd pass nucleì discussed here.

It is still not clear why it is insufficient: to have only $k=0$ components of alpha waves to fit the experimental alpha population pattern to the groumd basis. This question was discussed in roore detail in Ref. 8. 
Table 1. Inverse of ${ }^{253}$ Es surface alplitude matrix,

\begin{tabular}{|c|c|c|c|c|c|c|c|c|c|}
\hline$I_{f}^{\prime}, t^{\prime}$ & $7 z^{2}+0$ & \multicolumn{7}{|c|}{ Real Components } & 1512,4 \\
\hline $7+2$ & $2+\operatorname{tin} 4[-13$ & $-4+5 \sin 7 E-14$ & $1+91$ QBE- 15 & $4,36045+14$ & $-3,7 \bullet 28 E+1 \$$ & $+2.1475 E+14$ & $4-2719[-13$ & $-3,22172+15$ & $1.46312+13$ \\
\hline 72,2 & $-4,3547 E-14$ & $7 \cdot 3829 \overline{5}-15$ & $-2.39628-14$ & $1.90 B$ QR-1屯 & eै ،594! E-16 & $1.16108-16$ & $-1 .+053 E-14$ & $-6+94215-5 b$ & $-0.9471 E-14$ \\
\hline $7 / 8,4$ & $1.4277 \mathrm{E}-15$ & $-2+42140-19$ & $1+11+b[-1]$ & $+8.5 \div 2 \%-15$ & $1.4567 E-14$ & $-1+7649 E-13$ & 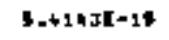 & 9.1 blse-1b & $1.14025-16$ \\
\hline $3 r 2,2$ & $3.97045-10$ & $1+507$ se-14 & $-6 .+1.57 E-15$ & $1.0553 E-23$ & $-4.7 \$ 57 F=15$ & $2+2526 E-14$ & $2.013 z e-14$ & $-1 . \$ 144 E-110$ & -1.96 3at -1.5 \\
\hline $9 / 2$ & $-2.2250 t=15$ & 1. Boyse-14 & $1.194 t E+19$ & $+J .7263 E+15$ & $1.09156-13$ & $+2+720$ de-13 & trandte-14 & $4.28075-15$ & 9. $2149 k-18$ \\
\hline $11 / 2,2$ & $+1,29] 4[-14$ & $7,4147 E=15$ & $-2+36976-15$ & $1+7 E S \bullet E-1 \downarrow$ & $-2.5409\{-1\}$ & $1.59106-13$ & $1.04 a b E=15$ & 1. ATTLE-14 & $-1,50$ WaE - 14 \\
\hline $11 / 7,0$ & $\therefore, 953 B E-15$ & $-5+50325-13$ & $3.54096-13$ & $1-34 t+E-14$ & 9. 42 t2E-15 & $1 .+10095-16$ & 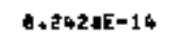 & $1,0484 E-15$ & $7,1049[-19$ \\
\hline $15 / 2,4$ & $+1.0550 E-13$ & $-2.5902 E-t 6$ & $w .06416=16$ & $-7.51968=15$ & $2.67992[-15$ & $6.7963 \varepsilon-15$ & $6,07765=15$ & $\$ .15446-14$ & $t-95 \pm 66-15$ \\
\hline \multirow[t]{2}{*}{$15+2,4$} & $3.1896 \mathrm{E}-16$ & $-2, A 337 E-16$ & $5+19096-17$ & $-5+62 t a c=16$ & $1.94586-\$ 6$ & $-t, 7 \in 64 t-15$ & $1+51 J 7 E=15$ & $2.835 T E-15$ & $3.71+35-16$ \\
\hline & \multicolumn{9}{|c|}{ Indatrary Components } \\
\hline it 2, & $-3+0000 t-15$ & $7+182 I E-15$ & $-6.10935-26$ & $-5+2612 E+15$ & $1+436 \%=-15$ & $1,0,1215-15$ & $-1.034+6+15$ & $6,+430 E=16$ & -3 \\
\hline $7+2+2$ & $9.0654 E=15$ & $4,03158-18$ & $z+40640-15$ & $+2,0274 E-13$ & $-2.175 \varphi E+15$ & $-1.9257 c-15$ & $3.0429 t-14$ & $9.3705 E-17$ & $2+04764-15$ \\
\hline 712,4 & $-7+61265 \cdot 16$ & $5+0526 E+15$ & $+7+1092 \mathrm{E}=16$ & $2+16 \theta Z E-15$ & $-4.7015 E+15$ & $6+1921 E-16$ & $=0.07462+15$ & $+2.7953 E+16$ & $-7,27564+17$ \\
\hline $9+2+2$ & $-0.4778 E-25$ & $-2+745 E-15$ & $1.6148 E-15$ & $=3+0096 E=15$ & $1.5162 E-16$ & $-2+4673 E-14$ & $-1.07215=15$ & $+.32665-15$ & $1.4119 E+16$ \\
\hline 913,4 & $1+31 \geq 9 E-15$ & $-5+21$ yse -55 & $-2+31355+15$ & $7.6912 \mathrm{E}-26$ & $-5,492=C-15$ & $4+325 J 6-56$ & $-2 . \$ 5 J \xi E-15$ & $-5.6217 \varepsilon-16$ & $-1+3+000-16$ \\
\hline แみZ, & $3+9[A+E-15$ & $-2.4771 E-53$ & $\forall+a b$ QE $-1 b$ & $-J .8 \div 9+E-15$ & $3+27[r e-16$ & $-1.40018-11$ & $=J .77+6 E-26$ & $-6.6739 E+16$ & $0,5652 E=16$ \\
\hline $11+2,+$ & $-1+2899 t-13$ & $1.7539 E-15$ & $-1.04525-15$ & $=++2994 E-15$ & $-2,1287 E+15$ & $\rightarrow 138+16-16$ & $-1,0708 E=15$ & $+8.2390 E-16$ & $+1, d \theta e d-16$ \\
\hline $13 \times 2,4$ & $7.0633 E-16$ & $1+40326-16$ & $-2+9500 E-15$ & $2,7+2 \leq 1-19$ & $-6+2 \sin 5-16$ & $-2,4551 E=15$ & $-4.20735-15$ & $-4+2$ \& 7 GE - 16 & $-2,62616-16$ \\
\hline $15,2+4$ & -2.150 AE-16 & $1.4442 E=16$ & $-4.7929 E-17$ & $-4+3255 E-16$ & $+1.23 .92 \dot{z}= \pm 5$ & $2-967 x-13$ & $+7,5+11 t=1 t$ & $+5.9705 E+26$ & $+3.79018-17$ \\
\hline
\end{tabular}

\section{Footnotes and References}

${ }^{*}$ Condensed froun 1BL-5075.

${ }^{\dagger}$ Presently at LFE Environgental Ansilysis Laboratory, Richmond, California.

1. R. R. Ghașan and J. O. Rasmussenn, Phys. Rev. 115,1257 (1959).

2. I. Ahrad, F. T. Porter, M S. Froednar, R, F. Bames, R. K. Sjoblom, Phys. Rev. C2, 390 (1971).

3. A. J. Soinski, R. B. Frankel, W. O. Navarro and D. A. Shirley, Phys. Rev. C2, 2379 (1970).
4. I. Perlman and J. O. Rasmussen, Handbuch der Physik, ed. S. Flügge, 42, 169 (1957).

5. J. 0, Rasnussen, Alpha-Beta and Germa-Ray Spectroscopy, $K$, Siegbakn, ed. (Northriolland Pub. Co,, The ketherlands, 1965), Vol, 1, p.701.

6. T. Tanura, Rev. Mod. Fhys, 37, 679 (1965).

7. E. A. Rauscher, J. O. Rasmussen and K. Harada, Nucl. Phys, A94, 33 (1967).

8. A, J. Soinski, LBL-3411, Septenber 1974 (Thesis, unpublished).

9. A. J. Solnski, I. G. Raich, J, 0. Rasmussen and E. A. Reuscher, Muclear Themistry Atrulual Report, LBL-2366 (1973). 


\section{$0 \mathrm{U}: y=\mathrm{s} \mathrm{J}=\mathrm{J}$ a 2}

\section{Macroscopic}

\section{MODIFIED THOMAS-FERMI APPROXIMATION FOR MOMENTUM-DEPENDENT INTERACTIONS*}

$\sqrt{ }$ Randrup广

A nodification of the standard Thomes-Fermi approxination has been suggested for the treatment of interactions having a quadratic momentum dependence. 1 While of general theoretical nature this subject has particular interest to muclear physics, where morical studies are carried out with phenomenological Jacrentum-dependent interactions,

Suppose, in a many-particle systion, a two-body interaction is present which depends quadratically on the relative momentun $\vec{p}_{12}$, Let the spatial dependence be given by the function $g\left(\dot{T}_{12}\right)$. where ${ }_{12}$ is the particle separation. For the following discussion the monentumindependent part of the interaction can be disregarded. Stuch interaction way then have the form $1 / 2\left(p^{2} g+g^{2}\right)$, or $\vec{p} g \vec{p}$, or a 1inear combination of these two forns. This group constitutes the cless of interactions to which the suggested nodification applies.

The standard Thomas-Fermi treatment is briefly recalled. Suppose that the local Fermi monentum $p_{F}(\vec{t})$ is a klown function of position. At each point the particles are assuned to be distributed uniformily within the available mowentum sphere. This implies that the local density $\mathrm{p}(\overline{\mathrm{r}})$ is given by

$$
\rho(\vec{r})=\frac{t}{h^{3}} \int^{P_{F}(\vec{r})} d_{p}^{3} \cdot \frac{4 \pi}{3} t \frac{P_{F}(\hat{r})^{3}}{h^{3}}
$$

where $t$ denotes the degeneracy in each singleparticle orbit. The (anly) kinetic density is given by

$$
r(\vec{r})=\frac{t}{h^{3}} \int^{P_{F}(\vec{r})} p^{2} a^{3} \vec{p}=\frac{3}{5} P_{F}(\overrightarrow{\mathbf{r}})^{2} p(\vec{r})
$$

Any two-body interaction of the form $\overrightarrow{\mathrm{p}}^{2} \mathrm{~g}(\overrightarrow{\mathrm{T}})$ gives rise to the same effective static potential $v=p^{2}(r)+G(x)$ irrespectiwe of the particular algebraic form of the interaction. Here the functions of and $Q$ are obtained by folding $p$ and $Y$ by the spatial function g. Furthermore, the associated irteraction energy density is given by $v=1 / 2(v R+p \xi)$.

Thus, in the standard Thonas-Fermi treatment, no distinction ts made between the different interactions rentioned above. However, at least in the nuclear case, different algebraic forms may lead to simificantly different results for the self-consistent many-particle solution.
Therefore, it is desfrable to have available, within the simple Thomas-Fermi franework, a tool for the general analysis of such models.

The modification suggested may bo formulated as follows. Atry of the momentudi-dependent interactions considered can be rewritten in the general form.

$$
\begin{aligned}
W_{12}(\eta)-1 / 2\left\{\overrightarrow{\mathrm{p}}_{12}, 1 / 2\left\{\overrightarrow{\mathrm{p}}_{12} \mathrm{~g}_{12}\right\}\right\} \\
-\pi 1 / 2\left[\overrightarrow{\mathrm{p}}_{12}, 1 / 2\left[\overrightarrow{\mathrm{p}}_{12}, \mathrm{~B}_{12}\right]\right]
\end{aligned}
$$

where $\mathrm{g}_{12}=\mathrm{g}\left(\overrightarrow{\mathrm{r}}_{12}\right)$. The square brackets denote the conmutator and the curly brackets the anticomputator. The parameter $n$ is a reat nuber characterizing a given particular interaction. For exarule, the first of the two forms nentioned above corresponds to $\eta=-1$ and the second one corresponds to $\pi=1$.

The double-anticomutator algebraic fort has been exhibited because this particular form tends to average put the uldulations in the density distributions due to the quantum phase correlations in the single-particle wave functions. The presumption is now made that the Thomas-Fermi approwimation, which completely neglects phase correlations, is most accurate for the quantities represented in terms of the double-anticonmutator form. The modification suggested thus anoumts to adding to the standard Thoulas-Ferui formulas the contribution arising fros the refratning $\pi$-dependent terml.

Consequently, the effective single-particle potential $v$ takes on the form $V_{n}=V_{0}+n \delta V$, where $V_{0}$ is the effective potential given by the standard Thomas-Fermi approxination. The madification potential (which is momentum independent) is given by

$$
\delta \mathrm{V}(\overrightarrow{\mathbf{r}})=\mathrm{n}^{2} \mathrm{~s}(\overrightarrow{\mathrm{r}})
$$

where the fumction $\boldsymbol{f}$ is obtained from the density distribution $\rho$ by folding with the spatial part of the interaction, g.

Correspondingly, the interaction-energy density $v$ takes on the form $v_{-}=v_{0}+n$ t $v$, where $v_{0}$ is the standard Thomas-Ferihn result and the modification term is given by

$8 v=1 / 2 n^{2} 1 / 4\{0 \Delta \theta+27 \rho \cdot 7 a+\Delta p A\}$ 


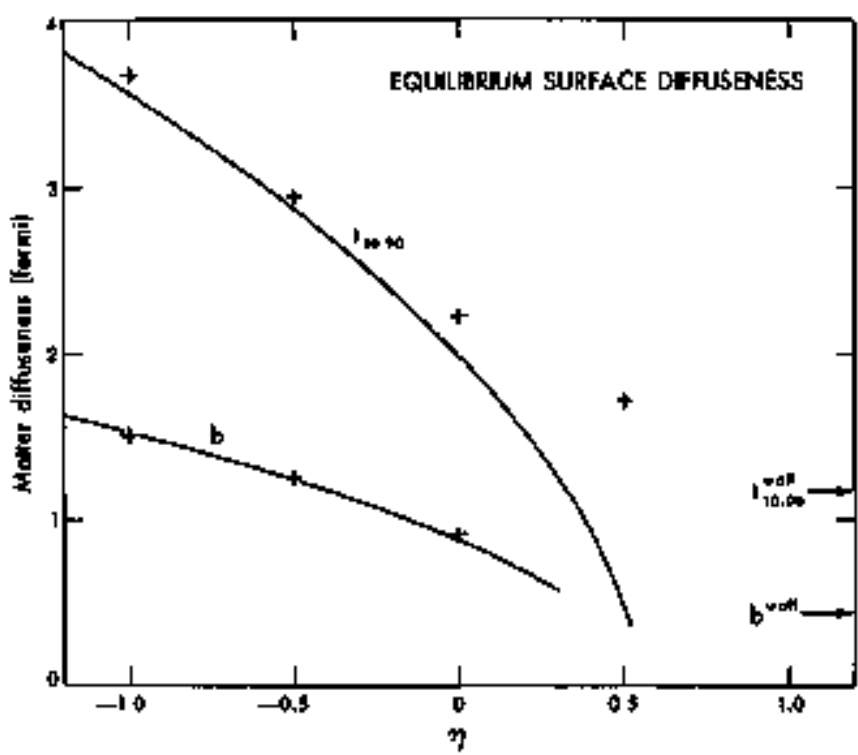

Fig. 1. Surface diffuseness. As a function of the parameter $\eta$ is plotted the surface diffuseness for the self-consistent solution obtained with the modified Thomas-Fermi approxiTation. The quantities displayed are the 10-908 diffuseness, t10-90, and the second surface moment $b$. The crosses indicate restuls obtained in the Hartree approxilation.

(XPL 759-3871)

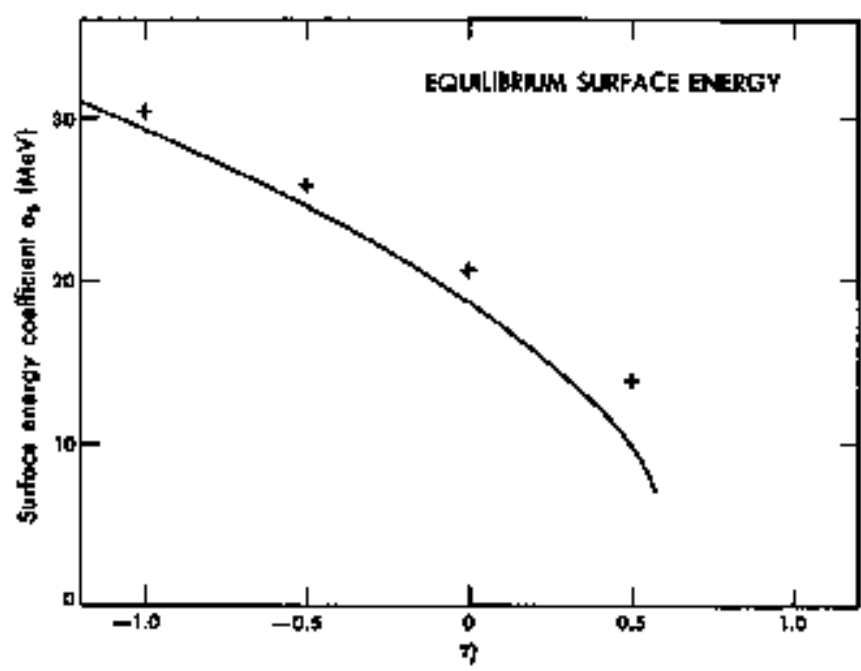

Fig. 2. Surface energy. As a function of the parameter $n$ is plotted the surface onergy coefficient $a_{5}$ for the self-consistent solution obtainet with the modified Thoms-Fermi approximation. The crosses indicate resurts obtained in the Hartroe approximation.

(XBL $759-3870)$
It is noted that in regions of constant $p$ and $s$ there is no modification.

As an illustration, the case of the inclear surface is consideret. For this, a SeylerBlanchard type nodel is used.

$$
w_{12}^{S B}=-C g\left(\frac{r_{12}}{n}\right)\left(1-\frac{p_{12}^{2}}{b^{2}}\right)
$$

where $g(r)=\exp (-r) / r$. Following byers and Swiatecki ${ }^{2}$ the three parameters entering are taken as a $=0.62567$ fm, $b=372.48 \mathrm{HeV} / \mathrm{c}$, and $C=328.61 \mathrm{kgV}$. Numerical calctilations have been cerried out for the fouily of interactions corresponding to the above form.

The self-consistent solution was calculated by use of nodified Thomas-Fermi aptroximation, as a function of the partmeter $\eta_{+}$Figures 1 and 2 display the results for the surface diffuseness and the surface energy. Furthermore, "exact" values were obtained by use of the Hartree approxination, which mintains the independence of the particles while treating the quantum mechanics in an exact manner.

It is seen that the general $\eta$ dependence of the results, as given by the Hartree values, is well reproduced in the modified Thrmas-Fermi approxination. The systenatic underestleation by the Thomeds-Fermi approxtmation can be mainly accouted for by the lack of a density tail and the neglect of phase correlations near the surface.

When judging the value of the modification is should be borme in mind that without the modification all the different Hartree results would be approximated by the Thomes-Fermi results obtained for $n=0$.

The suggested modified Thoinas-Fermi approxination is currently being employes in a study of the muclear surface. 3

\section{Footnotes and References}

"Condensed from LBL-4308.

${ }^{t}$ on leave from the Iniversity of Aarhus, karhus, Denmark.

1. R. G. Seyler and C. H, BIanchard, Phys, Rev. 124, 227 (1961); 131, 355 (1963).

2. W. D. Myers and W. J. Swiatecki, Arn, Phys. $55,395(1969)$.

3. J. Rendrup, Nucl. Phys. A259 (1976) 253. 


\title{
$00 \because 1450,043$
}

301

\author{
THE NUCLEAR SURFACE \$TUDIED WITH A MOMENTUM-DEPENDENT \\ INTERACTION IN THE THOMAS-FERMII AND HARTREE APPROXIMATIONS*
}

$\downarrow$ Randrup t

Further studies of the muloar surface have beon carriend out with momentura-dependent effective interactions which in the classical linit reduce to the form

$$
v_{12}=-C_{g}\left(\frac{r_{12}}{a}\right)\left(1-\frac{p_{12}^{2}}{b^{2}}\right)
$$

Here the separation between the two interacting particles is denoted by $\overrightarrow{\mathbf{r}}_{12}{ } \overrightarrow{\mathbf{r}}_{1} \cdot \overrightarrow{\mathrm{F}}_{2}$ and their relative memeritum by $\vec{p}_{12}=\mathrm{p}_{1}=\mathrm{p}_{2}$ (1) is assumed that all parttcles have the same jeass m). The spatial dependence, assuned to possess spherical syluetry, is confained in the function $\mathrm{g}$. (The Seyler-Blanchard model corresponds to the special case of $\mathrm{g}$ being a Yukawa fumction.)

For the development of the quanturn-Jachanictal mode1 the interaction-energy operator corresponding to the classical interaction (1) rust be spectfied. Acoording to the standard rules of quantization, this is accouplished by replacing the momentum variable $\overrightarrow{\mathrm{p}}$ in the classical interaction by the differential operator - ih to the requirement that the emerging operator be Hermitean. As is readily seen, there is no unique way of "quantizing" the classical product $p^{2} g(r)$. Rather, there exists a whole fanily of quantummechantcal operators which all have the same classical 1init. This family of operators may be parametrized by one real parameter since all of the possible algebraic constructions may be reduced to the form

$$
\begin{aligned}
\mathrm{p}^{2} \mathrm{~g}(\overrightarrow{\mathrm{r}})+1 / 2\{\overrightarrow{\mathrm{p}}, I / 2\{\overrightarrow{\mathrm{p}}, \mathrm{g}(\boldsymbol{r})\}] \\
-\eta 1 / 2[\overrightarrow{\mathrm{p}}, 1 / 2[\overrightarrow{\mathrm{p}}, \mathrm{g}(\overrightarrow{\mathbf{r}})]] \text {. }
\end{aligned}
$$

Here, the sharp brackets denote the commitator, $[a, b]=a b-b a$, and the curly brackets the anticommtator, $\{a, b\}=a b+b a$.

This unified formolation makes it possible to discuss in parallel the whole family of different quentum interactions. In pessing it should be noted that $\eta=-1$ leads to $1 / 2\left[p^{2} g+p^{2}\right)$ and $\eta=+1$ leads to pg.

The two-particle interaction-energy density is given by

$$
\begin{array}{r}
w_{\eta}\left[\vec{r}^{\prime}, \vec{r}^{\prime \prime}\right)=-C g(r / a) \rho\left(\vec{r}^{\prime}\right)\left(\rho\left(\vec{r}^{\prime \prime}\right)+2 \pi\left(\vec{r}^{+}\right) \cdot \vec{\pi}\left(\vec{r}^{\prime \prime}\right)\right. \\
-\rho\left(\vec{r}^{\prime}\right) \gamma\left(\vec{r}^{\prime 1}\right)-\gamma\left(\vec{r}^{\prime}\right) \rho\left(\vec{r}^{\prime \prime}\right) \\
\left.-\eta \frac{h^{2}}{b^{2}} \frac{1}{4} \Delta\left(\rho\left(\vec{r}^{\prime}\right) \rho\left(\overrightarrow{\mathbf{r}}^{+1}\right)\right)\right)
\end{array}
$$

Where the Laplace operator s acts with respect to F. The one-particle density djstributions entering are the matter density $\rho$, the monpentur density $\vec{i}$, and the kinetic density $\gamma$ (which is not the standard kinetic-energy density but is defined in terms of the double-articomutator construction).

The corresponking effective Haniltonian may be written on the form

$$
\mathrm{H}_{\mathrm{J}}^{\text {eff }}=1 / 2|\overrightarrow{\mathrm{p}}, 1 / 2| \overrightarrow{\mathrm{p}}, \frac{1}{2 \mathrm{~B}}||+\mathrm{U}_{n}
$$

1.e., in terms of a variable wass $B$ and a static potential $U_{n}$. The effective alass B is given by the relation

$$
\frac{1}{2 \mathrm{~B}(\overline{\mathrm{T}})}=\frac{1}{2 m}\left(1+\frac{\mathrm{C}}{\mathrm{b}^{2} / 2 \mathrm{~m}} \mathbb{R}(\overrightarrow{\mathbf{r}})\right)
$$

The renormalization of the particle nass arises from the quadratic momenturn dependence of the interaction. The effective mass $B$ does not depend on $\eta$, i,e, on wich actuel algebraic quantization form is chosen. The effective potential is is given by

$$
U(\vec{r})=-c\left(a(\vec{r})-\mathscr{G}(\tau)-n \frac{h^{2}}{b^{2}} \Delta a(\vec{r})\right) .
$$

(In the above. expressions the functions o and $\mathcal{G}$ are obtained from the densities $\rho$ and $\gamma$ by folding with the spatial interaction g.) It should be pointed out that while the choice of another algebraic form of the kinetic term in the effective Hanil tonian would not affect the effective mass $B$ as giver above, it wold lead to an modification of the effective potential $v$. a simple feature of the effective Hamiltonian is that it does not depend on which particlo is considered; the functions $B$ and $U$ are cormon to all the particles.

The n-dependent tem introduces an explicit senstivity to density inhomogenities. For negative values of $n$ sixh inhomogenelties will raise the energy and the term will have the effect of smearing out the intomgenities. For positive values of $\eta$, however, the energy will be lowered by inhomogenities and the $n$-dependent term will tend to enhance then. This may prevent the existence of a self-consistent solution.

Studies were carried out for the case where the spatial dependence of the two-body interaction is a Yukawa function, $g(r)=\exp [-r) / r$. This corresponds to the type of interaction introduced by seyler and Blanchard, 1 For the three model 
parameters entering are chosen the vilues detormined by Myers and Swiatecki ${ }^{2}$ in their study of average nuclear properties. It should be added that the substance of the conclusions is not expected to depend either on the specific choice of a Yukgus spatial dependence or on the actual parameter values, as long as they are reasonable.

The properties of the mucloar sirface have been studied by considering a seni-infinite system. It is exsy to understand the general behavior of the self-consistent solution, considered as a function of the parameter $n$ :

For a semi-infinite systen the essential degree of freedcin is associated with the surface diffuseness. As a function of the diffuseness, the part of the energy arising from the $n$-independent part of the interaction has a minimum at a certain diffuseness, the equilibrium diffuseness in the case of vanishing $\eta$. For the norranishing values of $n$, there is an additional energy contribution, proportional to $n$ for a given systent. The term multiplying $n$ is regative; it tends to zero for very diffuse systems and to minus infunity for systems with very sharp surfaces. Hence, for negative in values, the additional torm gives rise to an increase of the surface energy. Furthermore, it follows that the diffuseness equilibrium value is shifted towards larger values. It is worthwile noticing that an equilibrium always oxists for negative $n$. In the apposite case, $n>0$, there is a negative contribution from the r-dependent tertr. For sufficiently smsil $n$ values, the addition of the abovementioned diverging function to the original function pertaining to $n=0$ will still result in a function having a local minimm (as well as unaximutu). The value at the nitnimum as at1 as the corresponding diffuseness will be decreased relative to the case of $n=0$. However, as is evident in this simple picture, for positive a values above a certain critical value the minisam disappears and no self-consistent solution exists.

These simple qualitative considerations are borme out by actual celculations, as is illustrated in Fig. 1, which is a plot of the surface energy vs the surface diffuseness. The circles indicate the locations of the equilibritur solutions obtained with the modified Thomas-Femni approxistation described in the previous contribution to this report. The figure also displays the two curves referred to in the preceding qualitative discussion, namely the n-independent part of the surface energy, $a_{g}^{\circ}$, and the contribution $\delta a_{g}$ from the term multiplying $\pi$. The first curve is to a very good approxingtion identical to what would result from a simple scaling (stretching or conpression' from the equilibrita solution corresponding to $\eta=0$; this fact underscores the close reiation between the surface diffuseness and the freedom associated with the $n$ parameter. In this figure is also shown parts of the surfaceenergy curves for a few nonvanishing $\eta$ values. Finally it should be added that the results for $n$ values larger than 0.5 are less accorate and are indicated by dashed lines.

The self-consistent solution has also been

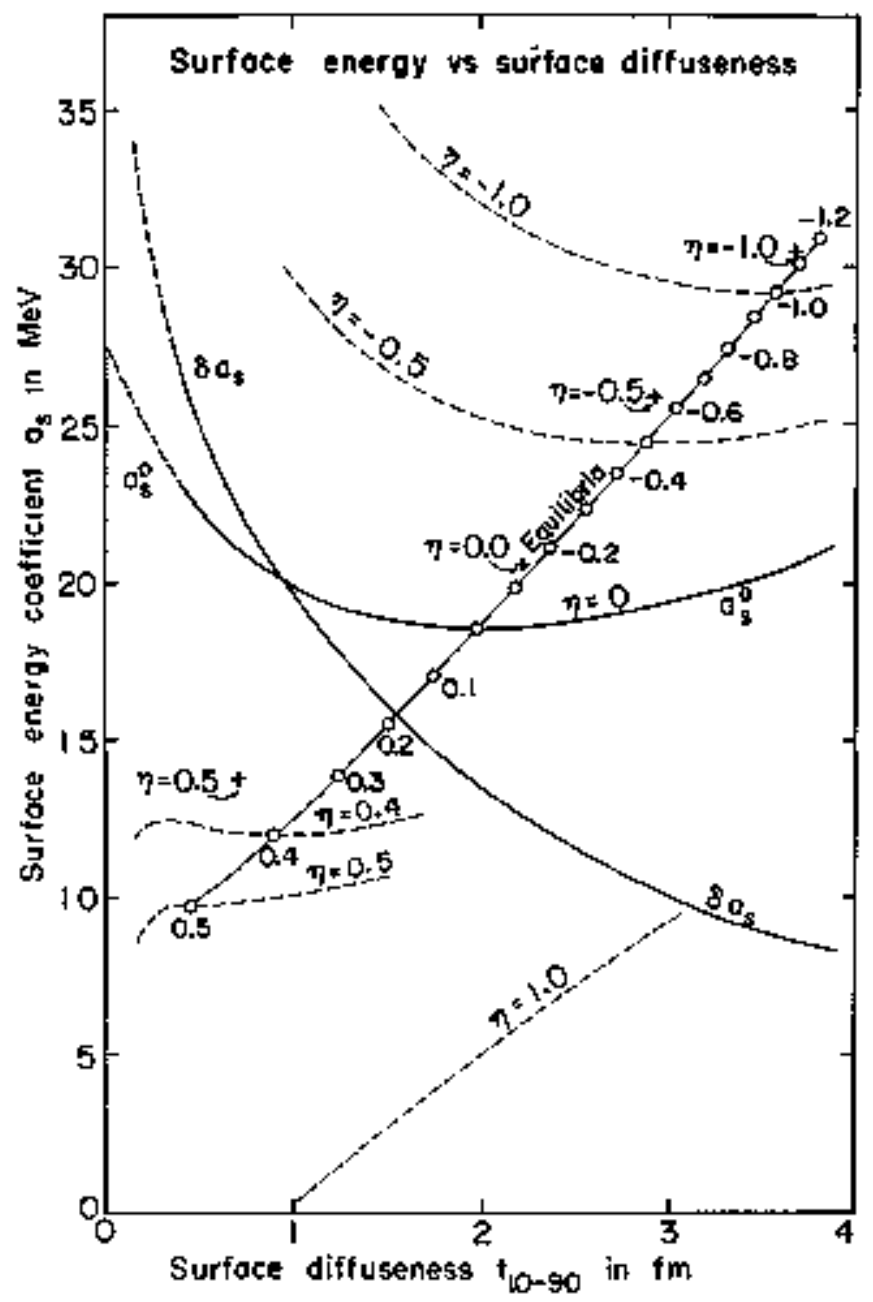

Fig. 1. 5urface eneryy ws surface diffuseness, The crrve labeled "exuilibria" joins the locations of the Thamas-Fermi equflibriun solutions obtained for a series of values of the parameter $n$. The abscissa is the 10-90o matter diffuseness $\mathbf{t}_{10-90}$ and the ordinate is the surface energy coefficient $a_{s}$. The arve tabeled "go" is the contribution to the surface mergy originating from the $n$-independent part of the Hanlitonian; it is to a very good approximation identical to what would result from simply stretching or conqressing the equilibriutm solution corresponding to $\eta=0$. The curve labeled " $\delta a_{5}$ " is the contribution to the surface energy due to the $n$-dependent term (with the $n$ divided away). The total surface energy $a_{5}-a+n \delta a_{s}$ is plotted as a function of the diffuseness for some values of $i$ (dashed curves). The crosses indicate the self-consistent Hartree solutions calculated for four selected values of $\pi$. (XBL 759-3876)

calculated in the Hartree approximation, for $\eta=-1.0,-0,5,0.0$, and 0.5 . Figure 2 displays the resulting matter density distributions; the corresponding Thomas-Fermi densities are indicated by dashed lines. The extracted values for the surface diffuseness and the surface energy aro indicated in Fig. 1 by crosses. 


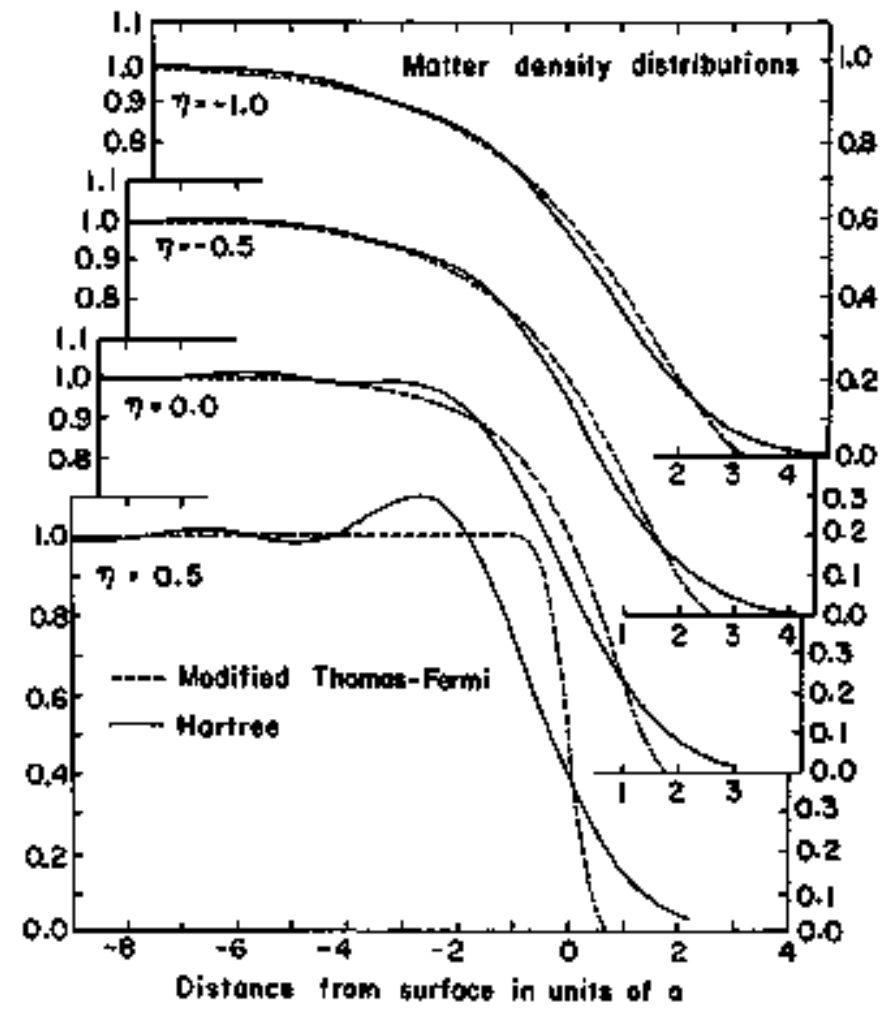

Fig. 2, Matter density distribution (in umits of the bulk value) for four selected values of the parameter $n$. The dashed curves aTe the results obtained with the modified Thrmas-Fermi approximation while the Hertree results are show by the fuxl curves.

(XBL 759-3874)

The general trend given by the modified Themas-Farmi analysis 15 well reproduced on the whole by the Hartree calculation, In particular, the results indicate that no Hartree solution exists for 17 values appreciably above 0.5 . The 10-90s surface diffusenesses obtained in the Hartro calculations are somewhat larger than the corresponding Thonas-Fermai values. This is mainily due to the density tail extending into the classically forbidden region outside the surface. This effect becomes particularly noticeable for positive $i$ values when the ThonasFenni diffuseness approaches zero. For large negative values of $\eta$, where the syster becimes more and more diffuse, the geometric properties of the surface proflle will scale with the diffuseness. Hence, the absolute discrepancy between the Thomas-Fermi value and the Hartree value for $t_{10-90}$ will incroase while the ratio will tend to a constant valus, For $\eta=0$ the relative underestination is 10.4 and for $n=-1$ it is 3.28 .

For the surface energy there is also gentral agreement as to the dependence on $n$. The
Table 1. Results for the surface diffuseness $t_{10-90}$ and the surface energy $a_{5}$, for various values of the parameter $\eta_{\text {. }}$ The upper results are obtained in the modified Thomas-Fermi approxination while the lower resuIts are obtained in the Hartree approxination.

\begin{tabular}{crrrr}
\hline$n$ & -1.0 & \multicolumn{1}{c}{0.5} & 0.0 & \multicolumn{1}{c}{0.5} \\
\hline & 3.58 & 2.88 & 1.99 & 0.53 \\
$t_{10-90}$ (fin) & 3.70 & 2.95 & 2.22 & 1.71 \\
$a_{5}(\mathrm{MeV})$ & 29.25 & 24.48 & 18.56 & 9.77 \\
& 30.34 & 25.85 & 20.55 & 13.75 \\
\hline
\end{tabular}

modified Thonas-Fertai approximation underestimates the surface energy, and increasingly so with increasing $n$. For $n=-1$ the discrepancy amounts to 3,68 and for $n=0$ to 9.78 .

A sumbary of the results for the surface diffuseness $t_{10-90}$ and the surface energy $a_{5}$ is presented in Table I. Generally, the results obtained with the modifled Thronas-Fermi approximation agroe relatively weIl (typically to within 109) with the Hartree results. The surface diffuseness as well as the surface energy are somewhat inderestintated by the Thomas-Fermd treatment. For the surface diffuseness, this is mainly a consequence of the absence of a density tail. The underestimation of the surface energy can (for the main part) be traced to the neglect of the phase correlations in the surface region.

In conclusion the following can be stated. In the nuclear case, the particular algebraic form of the momention dependence of the two-body interaction has a large bearing on the surface properties of the corresponding self-consistent many-particle solution; this is underscored by the fact that for certain (not extreme) forns no solution exists. With a simple nodification, the Thromas-Fermi approxigation can be used for the treatment of many-particle systens with (quadratically) roomentum-dependent interactions. This extends appreciably the applicability of the Themlas-Femni approximation for the study of nuclear properties.

\section{Footnotes and References}

"Condensed from LEL-4302, Nuxe1. Phys. A257 (1976) 253. ton leave from Aarhus University, Aarhus, Dennark.

1. R. G. Seylet and C. H. Blanchard, Phys. Rev. 124,227 (1961); 131, 355 (1963).

2. W. D. Myers and W. J. Swiatecki, Ann. Phys. 55,395 (1969). 


\section{THE NUCLEAR INTERACTION POTENTIAL IN A FOLDED-YUKAWA MODEL WITH DIFFUSE DENSITIES*}

$J$ Fendrup $\dagger$

The study of the interaction between two nuclei has becone of increasing interest in recent yoars. Several models have been suggested for the calculation of the nuclear interaction potential. For example, krappe and Nixl have proposed a model in which the interaction energy is cafculated as the Yukawa interaction between two muclear distributions with sharply defined surfaces and uniform interior. The Yukrwa interaction is supposed to contain the combined effect of two diffuse matter distributions interacting via some short range interaction. This procedure leads to a simple analytic potential.

A different approach is represented by the Froximity Formula, 2 which expresses the force between two gently curved leptoderiours surfaces as a product of a seonetricel factor proportional to the mean radius of curvaturo of the gap between the surfaces and $\mathrm{a}$ universal function equal to the interaction energy per unit area betamen two parallel surfaces. This 1atter approach approach is very general and has the advantage of being simple to use, once the problen inwolving the parallel surfaces has been solved.

In this work an analytical wodel has been studied which enables one to gain insight into the accuracy of som of the various approaches, including the two mentioned above. In the model studied, each of the two interacting objects has a diffuse surface which is generated by folding a yukara function into a generating sharp-surface distribution. The interaction eneтgy is subsequently abtained on the basis of a two-body Yukawa interaction.

This model can be considered a generalization of the Krappe-kix 1 model. Hence it peruits a test of the idee that the Interaction can be representod as a single effective Yukawa interaction acting between sharp-surface distributions. Moreover, the model is sufficiently realistic to present a good test case for the Proximity Formula. 2 so far, such tests have orly been carried out for the extrene cases of zerom diffusemess distributions (the Krappe-Nix model) or a zero-range interaction between diffuse surfaces. 2

The interaction enexgy $v$ between two matter distributions $\rho_{1}$ and $\rho_{2}$ is given by

$$
V=-\cos \rho_{1}\left(\mathrm{r}_{1}\right) \mathrm{Y}_{\mathrm{a}_{0}}\left(\mathrm{r}_{12}\right) \rho_{2}\left(\overrightarrow{\mathrm{r}}_{2}\right) \mathrm{d}^{3} \overrightarrow{\mathrm{I}}_{1} \mathrm{~d}^{3} \overrightarrow{\mathrm{r}}_{2}
$$

where the notation

$$
Y_{a}(r) \equiv \frac{1}{4 \pi a^{3}} \frac{e^{-T / a}}{r / a}
$$

has been introduced. The strength of the intoraction is governed by the constant $C$ which is positive for an attractive interaction. The matter density distribution $p_{i}(i=1,2)$ is obteined by folding a Yukawa function of sone range a into a generating sharp distribution $\hat{p}_{i}$,

$$
p_{i}\left(\vec{r}_{1}\right)=\int y_{a_{i}}\left(r_{12}\right) \hat{p}_{i}\left(\vec{r}_{2}\right) d^{3} r_{2} \text {. }
$$

The starting point for the analytical treatment of this model is the observation that the interaction energy may be calculated as the interaction between the two sharp generating densities arising from a composite two-body interaction $-C \mathscr{C}$. This colposite interaction is given as the folding product of the three entering Yukawa interactions,

$$
\mathscr{Y}=\mathrm{Y}_{\mathrm{a}_{0}} * \mathrm{Y}_{\mathrm{a}_{1}} * \mathrm{Y}_{\mathrm{a}_{2}}
$$

(the syrabol * denotes the folding).

It should be stressed at this point that the above observation implies that the formulated generalized folded"yluam mode1 is conceptually similar to the standard folded-Yuksta nodel due to Krappe and $\mathrm{Nix}^{1}$ in that it calculates the energy by folding some kermel into generating sharp densities. The generalized rode1 thus applies to atl cases covered by the Krappe-N $1 x$ model. In particular, the modified surface-onergy prescilption stiggested by Krappe and $\mathrm{kix} 1$ can be generalized by ermploying the composite kerpel If rather than a single Yukawa furction.

The final formula for the interaction potential may be written in the form

$$
\begin{aligned}
& V(d)= \\
& -C \sum_{i=0}^{2}\left(1-\frac{a_{j}^{2}}{a_{i}^{2}}\right)^{-1}\left(1-\frac{a_{k}^{2}}{a_{i}^{2}}\right)^{-1} \Gamma_{a_{i}}\left(R_{1}\right) r_{a_{i}}\left(k_{2}\right] Y_{a_{i}}(d) .
\end{aligned}
$$

This formula is valid in the case where the two generating spheres are positioned outside each other (Which is the case of interest in the present study); otherwise more complicated expressions will result (they may be obtained by the seine method). Furthermore,

$$
\Gamma_{a}(R) \equiv 4 \pi^{3}\left(\frac{R}{a} \cosh \frac{R}{a}-\sinh \frac{R}{a}\right)
$$

is a geometrical factor depending on the size of the sphere. It has the limiting forms, $\Gamma<\frac{4 \pi}{3} R^{3}$ for $R<$ a and $\Gamma=2 \pi a^{2}(R-a) \exp (R / a)$ for $R \rightarrow$ a. 


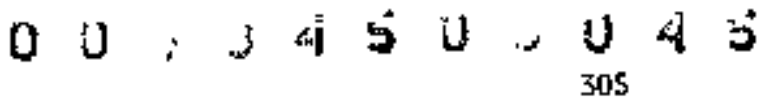

The above formula applies to the case of different Yukawa ranges, $a_{0} \neq a_{1} \neq a_{2} \neq a_{0}$. The various degenerate cases can be obtained by taking the appropriate linit. This leads to relatively complicated expressions. However, for most numerical purposes it is satisfactory to use the above formula with a sinall arbitrary splitting of the degenerate ranges; this has been done in the present stuky. It should be noted that if ore of the Yukawa ranges tends to zero the corresponding term simply drops out.

The general formulation piven above covers a number of special cases of interest, in particular the case of zero density diffuseness and the case of zero interaction range. These two particular modeis may be discussed relative to a more realistic reference case having diffuse densities and a finite interaction range.

The reference case, which shall henceforth be noted as the standard model, is characterized by the following parameter values:

$$
\begin{aligned}
& \gamma=1 \mathrm{ks} / \mathrm{fm}^{2} \\
& a_{0}=1 \text { fin } \\
& a_{1}=a_{2}=1 / \sqrt{2} \text { 俋. }
\end{aligned}
$$

Here $y$ is the nominal surface onergy coefficient defined as minus half the interaction onergy per unit area for two parallel seraijnfinite systeru at contact. It is more convenient to specify $Y$ than the interaction strength $C$. With the above values for $\gamma$ and the ranges a 0 , al, and $a_{2}$, the value of the interaction strength follows as

$$
\mathrm{C}=1 /\left(1-\frac{7}{16} \sqrt{2}\right) \mathrm{HeW}
$$

The value of $1 \mathrm{MeV} / \mathrm{fm}^{2}$ chosen for the rominal surface energy is realistic.2 Furthertore, it is noted that the surface diffuseness inplied by the values chosen for $a_{1}$ and $a_{2}$ is given by $b$ * $1 \mathrm{fm}$ which is also a realistic value. 3 As an illustration, Fig. 1 shows a comparison of the above standard model and the zero-diffuseness (Krappe-Wix) models (the quantity $b$ is the second surface moment), with the following set of parameter values:

$$
\begin{aligned}
& \gamma=1 \mathrm{kkV} / \mathrm{fm}^{2} \\
& a_{0}=\sqrt{2} \mathrm{fm} \\
& a_{1}=a_{2}=0 .
\end{aligned}
$$

It should be noted that the value of the range parameter an is close to the value of $1.4 \mathrm{ft}$ employed by Krappe and Wix. I It is seen that while agreeing on the whole quite well when close to contact (which is a consequence of the demand thet the nominal surface energies be the sama) the zero-diffuseness model gives rise to a longer tail. This is because the longrange behavior is governed by the largest of the
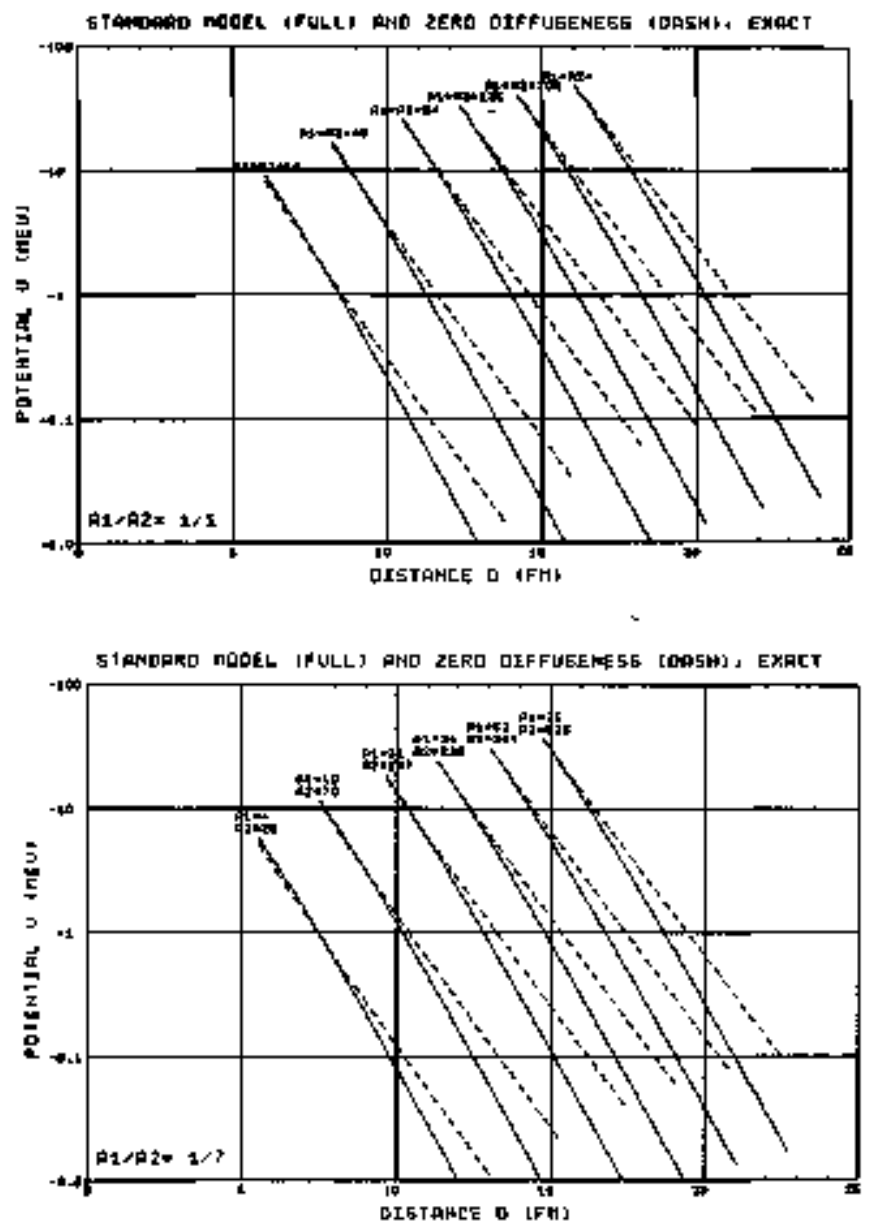

Fig. 1. The potential obtained by the standard model (full lines) and the zero-diffuseness (doshed lines), (b) for two different values of the mass ratio.

(XBl 759-8226)

(XBL 75\$-8238)

three Yukawa ranges entering in each model.

The Proximity Formula ${ }^{2}$ reads

$$
V_{p}(s)=2 \pi \bar{R} \int_{s}^{\infty} e\left(s^{1}\right) d s^{+}-2 \pi R \mathscr{Q}(s) .
$$

Here $s$ is the separation between the effective surfaces which are located at the radii $C_{1}$ and $C_{2}$. The geometrical factor $\vec{k}$ is given in terms of the effective radi by the relation $\mathbf{R}^{-1}=\mathrm{C}_{1}^{-1}+\mathrm{C}_{2}^{-1}$. Furthermore, the fumction e(s) is tho interaction enery per unit area betwen two jarallel sequiinfinite surfaces with a separation equal to $\mathrm{s}$.

In the generalized folded-Yukawa model, the expression for the integrated function $E(s)$ is

$$
\varepsilon(s)=-c \sum_{i=0}^{2} a_{i}^{3}\left(1-\frac{a_{j}^{2}}{a_{i}^{2}}\right)^{-1}\left(1-\frac{a_{k}^{2}}{a_{i}^{2}}\right)^{-1} \frac{1}{2 a_{i}} e^{-s / a_{i}} .
$$

As in the preceding, the proper limit rast be taken if some of the Yukawa ranges $a_{1}$ are equal. 
The veonetrical factor $\bar{R}$ entering in the Proxipity Formula is given in terms of the effective radius $C_{i}$ wich is taken as the average of the density profile location and the potential profile location, 2

$$
c_{i}=R_{i}-\frac{a_{0}^{2}+2 a_{i}^{2}}{R_{i}} .
$$

The separation $s$ between the effective surfaces is given by $s=d-C_{1}+C_{2}$ where $d$ is the distance between the two conters.
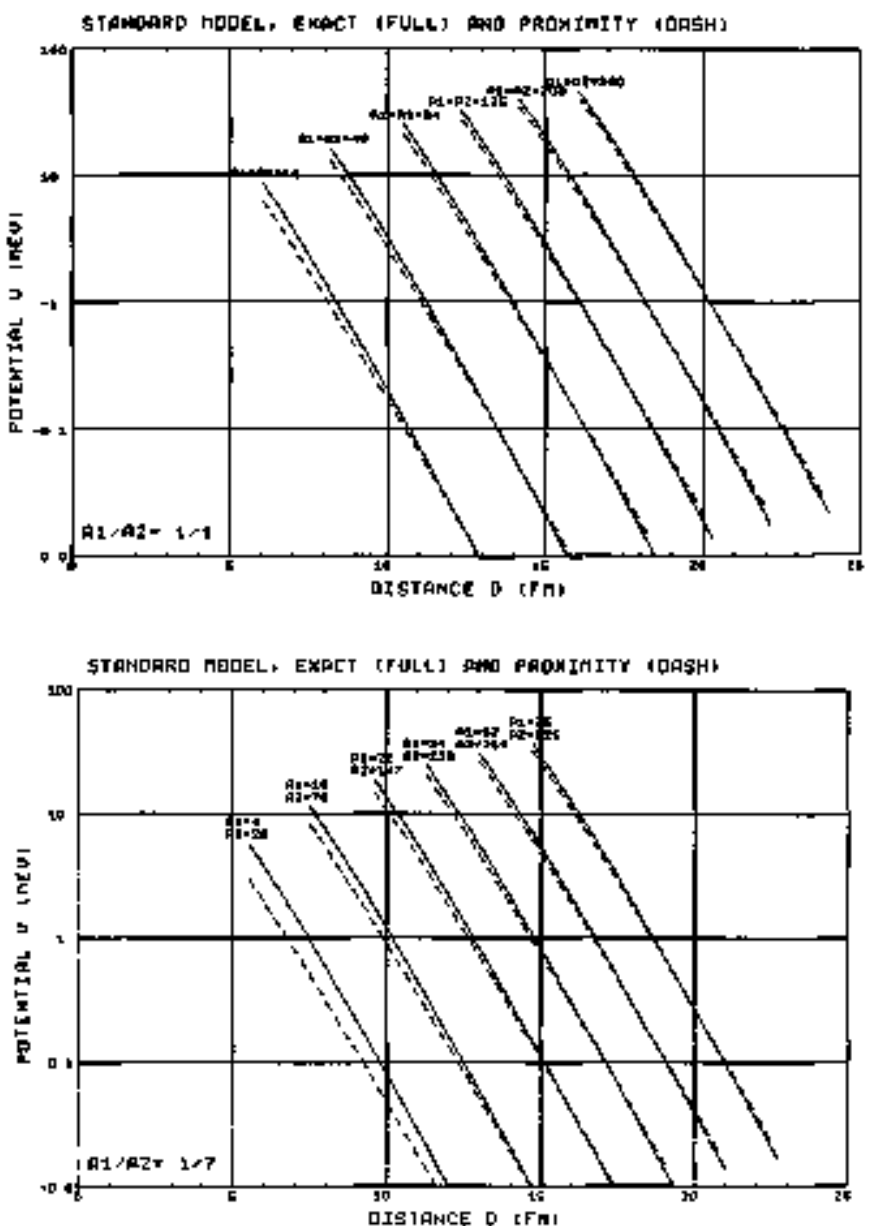

Fig, 2. Standard model. The exact interaction potential is given by the full curves while the proxindty expression is indicated by the dashed curves. Results for two different mass ratios are displayed.

(XBL. 759-8221)

(XBL 759-8233)
The Proximity Fordunta has been compared mmerically with various exact models. As an example, Fig. 2 shows a comparison made for the standard rodel. On the whole the reproduction of the exact results is good, except for systems containing very light nuclei. It should be stressed that the absolute as well as the relative agreenent is good, over the wide range of nuclear combinations considered and over distances where the potential changes by more than a factor of a hundred. This result provides support for the applicability of the Proximity Fonmula to the stiuly of heavy-ion interactions.

The folded-Yukawa model ${ }^{1}$ has been generalized to the case where the two interacting objects have a diffuso surface, generated by folding a Yukata function into a sharp generating distribution. While only slightly more complicated to treat thar the usual folded-rukstwa model, the general ized model ts conceptually more appealing. Furthernore, it incltales the folded-Yukawa modol as well as the s-interaction model as special cases. This facilitates the analytical stuhy of effects associated with the density diffuseness and the interaction range.

Woreover, the generalized folded-yukawa model provides a realistic case for testing approximative representations of the interaction potential. In this study the Proximity Formula ${ }^{2}$ was imvestigated; its quanitative validity was generaily supported.

Finally, it should be recalled that the possible applicability of the generalized foldedYukawa model exterds beyund the muclear interaction potential. For exalple, the modified-surfacemergy prescription suggested by Hrappe and Nix can be easily generalized along the sane lines.

\section{Foothotes and References}

"Condensed from LBL-4317.

ton leave from the Lhiversity of Aarhus, Aarhus, Dermark.

1. H. J. Krappe and J. R. Nix, Proc. Third IAEd Syuposium on the Physics and Chemistry of Fission, Fixchester, NY, IAEA-SM-174/12 (1973) 159.

2. J. Blocki, J. Randrup, W. J. Swiatecki and

c. F. Tsang, LBL-5014, to be published.

3. W. D. Myers, Mucl. Phys, A204, 465 (1973).

\title{
MACAOSCOPIC ASPECTS OF NUCLEUS-NUCLEUS INTERACTIONS*
}

\author{
J Bibskı J Randrup, $\neq$ W L Swladockl, C F Toangs
}

The foltowing qualitative classification of nucleus-nicleus interactions has been fot to be useful in sorting out the great variety of experi- mental data (see Fig. 1), Nuclei that do not Touch undergo tistant Collisions, where only electromiagnetic interactions, rodifled by the tail of the 


\section{0 ป ‘.}

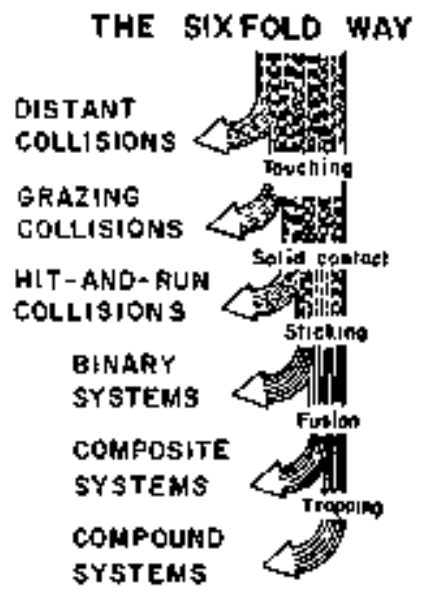

Fig. 1. An atterget to classify collisions between two nuclei into six main categories. (XBL 759-4071)

nuclear potential, tome into play, (Touching could be defined approximately as tourhing of the Fermi levels of the two systens.) Muclei that touch but do not make Solid Contact undergo Grazing collisions. (Solid Contact inlght be defined approximately as begiruing when the half-density contour acquires the topology of a single object.) Nuclei that make sol id contact but do not Stick make Hit-and. Pan Collisions. (Sticking might be defined approximately in terms of the sharing of linear momentum.) Nuclei that stick but do not Fuse form a binary Systen. (Fusion might be defined in terms of the 10ss of identity of the pieces, associated with a filling-in of the neck.) Nuelei that fuse but ate not Trappea form a Cppmposite System. The remainder are trapped in a potential energy hollow, and form a Compound System.

The first problem in describing the above processes, whether quantally or classically, is to map the potential energy as a function of the shape of the systean,

With certain qualificaticus it is possible to prove the following theorem about the functionat form of the potential energy $V$ (shepe) of a thinskimed system of volume $V$ whose shape is specifies by an (effective sharp) surface $E_{\text {. }}$

$$
V(\text { shape })=V_{\text {Bulk }}+V_{\text {Surface Layer }}
$$

where

$$
\mathrm{V}_{\text {Bulk }}=\mathrm{c}_{1} 2^{\prime} \text {, }
$$

and

$$
\begin{aligned}
& v_{\text {Surface Layer }}-c_{2} f+c_{3} \not+c_{4} g+c_{4} d \\
& + \text { corrections that tend to zero as } 2 \\
& \text { grows + Proximity Energy } v_{p} .
\end{aligned}
$$

Here $c_{1} \ldots c_{4}$ are, for a fixed $\mathcal{W}$, constants independent of shape. The shape dependence is given by the Proximity Energy $y / p$ and by the following functionals of shape: $f^{2}$ - area of surface $\bar{L}$, $K=$ integral over $\Sigma$ of $k\left(=R_{1}^{-1}+R_{2}^{-1}\right)$, $\xi=$ integral over $\Sigma$ of $\left(\mathrm{k}_{1} \mathrm{k}_{2}\right)-1, q=$ integral over $\Sigma$ of $k^{2}$. Here $R_{1}, R_{2}$ are the principal radii of arvature at a point on $F_{5}$. Fir a nucleus the terms in $C_{j}$ are of order $A\left(A^{-1} / 3\right) i-1$. The last term in Eq. (2) is less familiar and is the subject of the next subsection.?

The expansion in $\mathrm{A}^{-1 / 3}$ giver above is an asymptotic ${ }^{2}$ exparsion and fails if there are violent contortions in $\Sigma$, for example, such that slemsents such that elements of the surface face each other across a gap or crevice whose width is of the order of the surface thicloess. If this width $D$ is only a slowly varying function of position the additional onergy may be approxinated by

$$
V_{p} \approx \iint e[\mathbf{D}) d x d y .
$$

Here e[D] is the interattion energy per unit area between tho flat parallel surfaces at separation $D$ and the integral is ovet the gap or crevice, assumed to lie approximately in the $x-\gamma$ plane.

For the farily of gap or crevice functions $\mathrm{D}\left(\mathrm{x}, y^{\prime}\right)$ defined implicitly by

$$
\sum_{n=0}^{N} c_{n} d^{n}=\alpha x^{2}+\frac{1}{\alpha} y^{2} \quad\left(\equiv r^{2}\right) .
$$

where $\alpha$ is an area-preserving transverse stretching parameter, Eq. (3) leads to the simple result (independent of $a$ )

$$
v_{p}=\pi \stackrel{N}{L} \text { n } c_{n} I_{n-1} \text {. }
$$

where

$$
I_{n}=\int_{s o r}^{\infty} D^{n} e(d) d D,
$$

are moments of the universal interaction function $\theta$ (d). The fower limit 5 or 0 , distinguishes between gaps (in which case D has a least value $s$ and, for negative $s$, the two objects overlap) and crevices (in which case $D$ is zero at a finite value of $r$, given by $\sqrt{c_{0}}$, and there is no density doubling.) The case $\mathrm{N}=2$, for which the relation between $D$ and the "stretched" cylindrical radius vector $i$ is a conic section, gives

$$
V_{p}=\pi c_{1} I_{0}+2 \pi c_{2} I_{1}
$$

where, for gaps, $I_{0}, I_{1}$ are two "tsilversal" functions and, for crevices, two "miversal" constants, characteristic of the material of which the surfaces are made. Gaps or crevices corresponding to the following shapes are covered by Eq. (4). (For stevices the overlapping portions are orased.) (i) For two juxtaposed coaxial paraboloids w1th radii of curvature at tips $c_{1}, c_{2}$ and tip distance $s$ (which may be negative): $c_{1}=2 \mathrm{R}, \mathrm{t}_{2}=0$, where $R=C_{1} C_{2} /\left(C_{1}+C_{2}\right) \cong$ C. (ii) For two equal coaxial spheroids with semi-axes $C, B$ (C along the line of 
centers and $t i p$ distance $s: c_{1}=B^{2}(C+s / 2) / C^{2}$, $c_{2}=-B^{2} / 4 C^{2}+$ For spheres $C \cdot B$. (iii) As above but with one spheroid infinitely large [i.e., a plane : $c_{1}=2 B^{2}(C+5) / C^{2}, \quad c_{2}=-B^{2} / C^{2}$.

(iv) For two equal juxtaposed hypertoloids with axes $C, B$, (C along the line of centers) and tip distance $s: c_{1}=B^{2}(C-5 / 2) / C^{2}, C_{2}+B^{2} / 4 C^{2}$. (v) As above, with one byperboloid degenerating into a plane: $c_{1}=2 \mathrm{~B}^{2}(\mathrm{C}-5) / \mathrm{C}^{2}, \mathrm{c}_{2}=\mathrm{B}^{2} / \mathrm{C}^{2}$. (vi) Stingle hyperboloid of one or two sheets: $\mathrm{C}_{1} \mathrm{O}, \mathrm{C}_{2}=\mathrm{B}^{2} / 4 \mathrm{C}^{2}$. (vii) Coakial juxtaposed circular cones with semi-opening angles ot, 12 and tip distance s: $c_{2}=\left(\text { cot } \alpha_{1}+\cot \alpha_{2}\right)^{-2}$, $c_{1}=-2 \mathrm{sc}_{2}$. (viii) In addition, for the case of a gap between two coaxial eliptic paraboloids with tip distance $s$, with radii of curvature $A_{1}$ and $B_{1}$ in the principal plines of curvature through the tip of paraboloid 1 , and $A_{2}, B_{2}$ for paraboloxd 2 , and azimuthal angle 4 botween the principal planes of curvature of 1 and $2: c_{1}=2 \bar{R}, c_{2}=5$, where

$$
\begin{aligned}
\frac{l}{\mathrm{R}^{2}} & =\frac{1}{\mathrm{~A}_{1} \mathrm{~B}_{1}}+\frac{1}{\mathrm{~A}_{2} \mathrm{~B}_{2}}+\left(\frac{1}{\mathrm{~A}_{1} \mathrm{~B}_{2}}+\frac{1}{\mathrm{~B}_{1} \mathrm{~B}_{2}}\right) \sin ^{2} \phi \\
& +\left(\frac{1}{\mathrm{~A}_{1} \mathrm{~B}_{2}}+\frac{1}{\mathrm{~A}_{2} \mathrm{~B}_{1}}\right) \cos ^{2} \phi .
\end{aligned}
$$

By taking the negative derivative of $V_{p}$ with respect to 5 in case (i) (which is a lowest order approximation to the general case of arbitrary gently curved surfaces with least separation s] we arrive at the following Proximity force Theorem: 'The force between two gently curved surfaces as a function of the separation degree of freedon is equal to the interaction potential per wit area between two ilat surfaces, times 2 in into the geonetric mean of the principal radii of curvature of the gap width function," Thes

$$
F(s) \equiv-\frac{\partial V_{p}}{\partial s}=2 \pi \bar{k} e(s) .
$$

The (universal) function $\mathrm{e}$ (s) vanișes for $s$ beyond the range of the surface width, has a minimum of about minus twice the sturface energy coefficient $Y$ when the surfaces are in solid contact at $s \approx 0$ (and the two bodies have conbined to make approximately uniform bulk matter), and then rises linearly to infinity as the bodies pilp tp. with $s$ going to nogative values, It folluws that the maximug attraction between two gently curved bodies is $\mathbf{F}_{\text {max }}$ they.

Introducing a unit of length b proportional to the width (diffuseness) of the surface, the proximity potential may be written in terms of a dimensionless universal function $\phi(\zeta)$ :

$$
V_{P}=4 \pi \% \mathrm{Rb} \cdot \Phi(\zeta),
$$

where

$$
\Phi(\zeta)=(2 r b)^{-1} \int_{s}^{\omega} \theta(s) d s, \quad \zeta=s / b .
$$

Using W. D. Ifyers' version of the 5eyler-

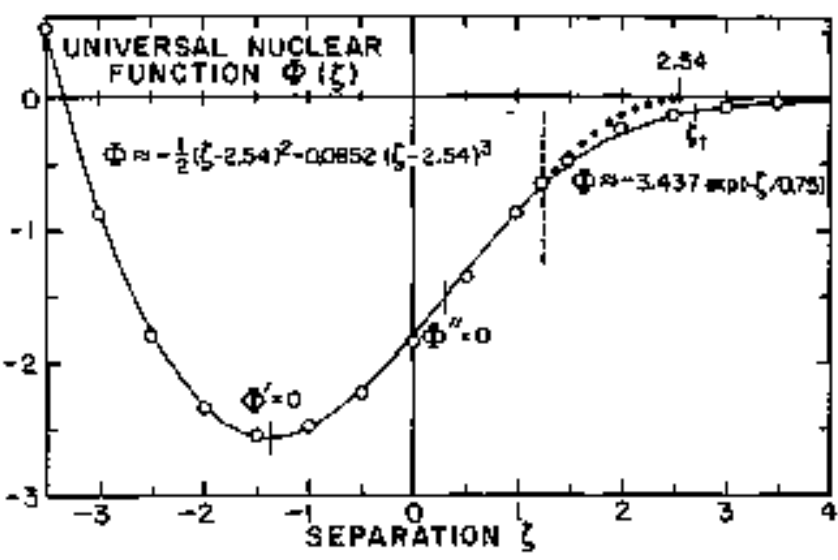

Fig. 2. The universal nuclear fumction $\Phi(5)$. The circles give the cubic and exponential approximations joined smoothly at the dashed line. The dots are the continuation of the cubic to where it touches the $\zeta$ axis at 2.54 . The Ferpic leve1s touch at $\zeta_{t} \div 2.71$.

(XBL 769-4073)

Blanchard nuclear Thonas-Fermil method ${ }^{3}$ we have calculated the universal nuclear fumction $\$$, to which an excellent anatytic approximation is ssee Fig. 2):

$$
\Phi(\zeta<1.2511)=-\frac{1}{2}(\zeta-2.54)^{2}-\Omega(\zeta-2.54)^{3},
$$

with $n=0.0852 \approx 1 / 12$.

$$
\Phi(\zeta>1.2511)=-3.437 \exp (-\zeta / 0.75) .
$$

The following valuss of the parameters entering $V_{p}$ would be reasonable for a nurlear system with total neutron, proton, and mass numbers N, Z, A:

Surface energy, 4

$\gamma=0.9517\left[1-1.7826\left(\frac{N-2}{A}\right)^{2}\right] \approx 1-2\left(\frac{N-2}{A}\right)^{2} \nLeftarrow \mathrm{HV} / \mathrm{fm}^{2}$

Surface width, 5 b $\approx 1$ fu,

Nuclear Central ${ }^{5,6}$ Radius $C=R-b^{2} / R$,

whero $R=$ Effective Sharp Radius

$$
-1.284^{1 / 3}=0.76+0.84^{-1 / 3} \mathrm{fm} \text {. }
$$

The accuracy of the Leptodermous Theorem has been tested against the exactIy soluble Krappe-Nix model of interacting nuclei ${ }^{7}$ and against computergenerated models based on the "energy-density formalism. "18 Very recently a coimparison of the Proximity Potentlal was made with 57 experimental interaction depths deduced from elastic scattering data. These congarisons suggest that the Leptodermous Theoren, lncluding the Proximity Fotential, should be useftul down to quite șnall systens, with mass nubers of about 15 or even lower.

Using the Proximtty Patential for the nucleat interaction and freezing all degrees of freedom 


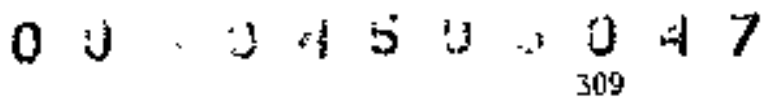

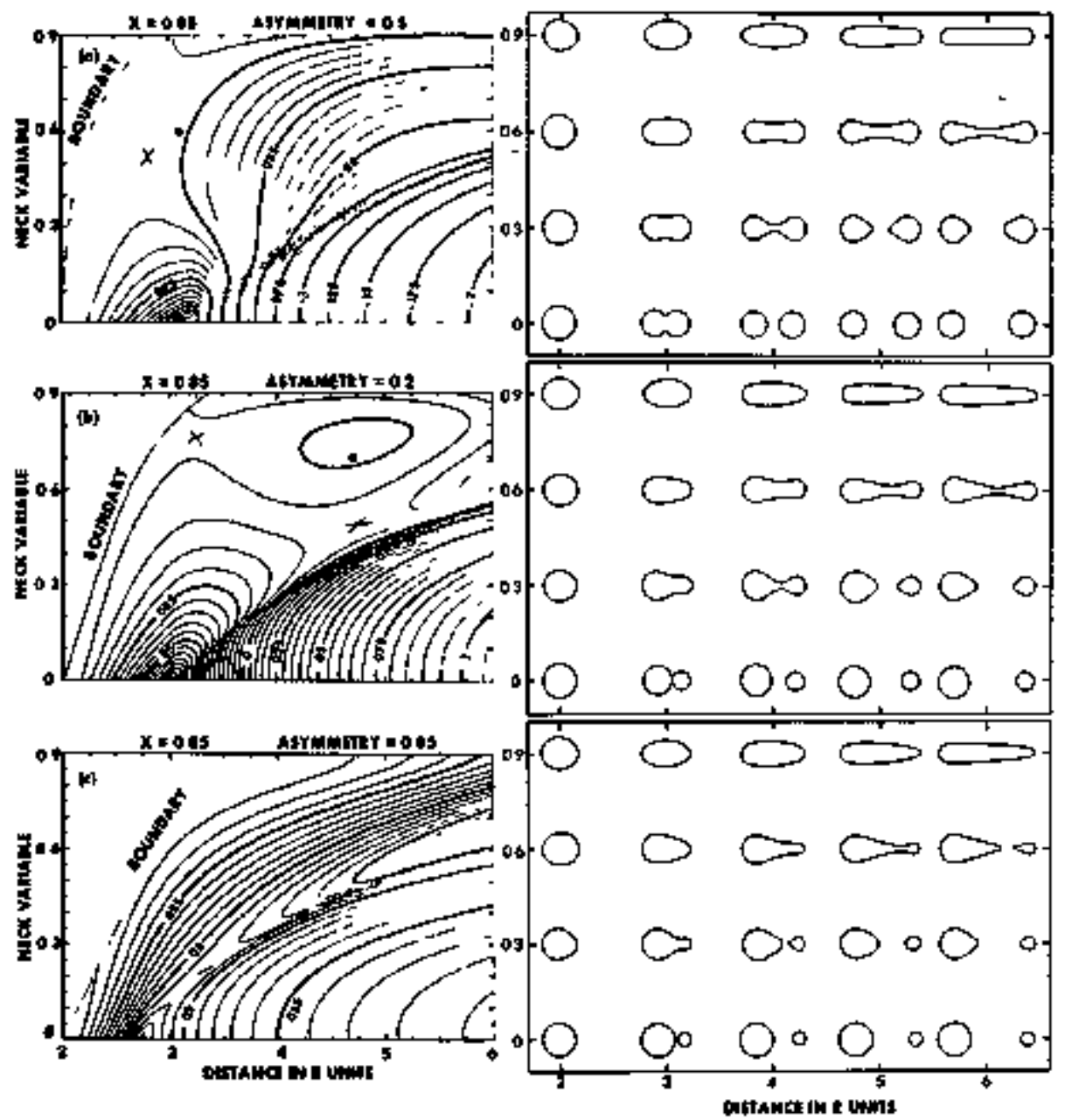

Fig. 3. Potential energy corresponding to families of shapes shown on the right, (The maps stop at the line marked "Boundary",) The defomation energy is m units of the surface energy of the standard sphere.

(XBL 759-8199)

except the separation between the nuclei, an approxinate but wholly analytic descriptim of nucleus mucleus scattering may be derived. One consequence of this is the isolation of 5 foling paraneter $X_{\text {, }}$

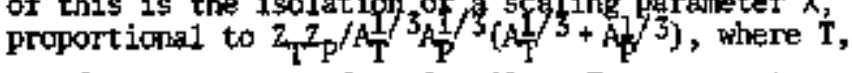
$P$ refer to target and projectile. For tyo systews with the same valte of $X$ (e.g., $180_{\mathrm{Hg}}+4 \mathrm{CO}_{\mathrm{Ar}}$ a्वा $8 \mathrm{Kr}_{\mathrm{K}}+3 \mathrm{~B}_{\mathrm{Kr}}$ ] the plots of properly scaled cross sections against properly scaled boubarding energies would be identical if the frozen 1dealization were vatid. This is talogous to macroscopic fission theory, where properly scaled fission barriers, fragnent energios, etc., should be primartly universal functions of a scaling parameter $x$. proportional to $Z^{2} / A$. The ordaring of experimental scattering data according to $\mathrm{X}$ might serve a similar purpose as the ordering of fission data according to $\mathrm{x}$; the assessment of the validity of the uderlying ideallzations and the isolation of the expected deviations.

The expected deviations from the frozen idealization are associated with unfreezing: (a) Other shape degrees of fregdon, (b) Fragmentorientation degrees of freedon, 9 (c) Intemal degres of freedorn (this Ieads to disslpation). In connection with (a) we have prepared an extensive atlas of muclear potential maps (primarily sums of Coulchb and surface energies). This parametrization is two spheres joined by a conic; each map is for a fixed mass asymutetry of the spheres and displays the deformation energy as function of elongation and neck degrees of freedom. Figure $3 a$ shows stuch. a family of shapes and the associated energy for a mass tetio 1:1, F1g. 3o for $1: 4$, and Fig. $3 c$ for 1 i19. The fissility parameter $x$ for the whole systen is 0.85 in each case $(2 \pm 102)$. Colliding nuelei would come in from the right, toush at the top of the ridge, and either be trapped near the sphere (left lowex comer) or re-separate, making use - as in fission - of the neck degree of freedom. The atlas covers $x^{+} s$ fron 0 to 1.5 and mass ratios l:1 to $1: 49$.

\section{Footnotes and References}

*Condensed from LBL-4296.

† Permanent address: Institute for Nuciear Research, 05-400 Swierk, Poland. 
FPermanent address: Institute of Physics, Universtty of Aarbus, Aarhus, Denuark.

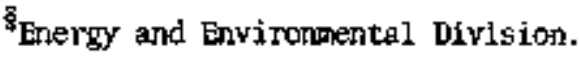

1. J. Randrup, W. J. Swiatecki and C. F. Tsang, LBL-3603 (December 1974); and J. Blockf, J. Randrup, W. J. Shiatecki and C. F. Tsang, LBL-5014, to be published.

2. H. Krappe, private commulcation.

3. W, D, Myrars and W. J. Swiatecki, Annals of Phys. 5\$, $395(1969)$,

4. W. D. Nyers and W. J, Swiatecki, Arkiv $f$, Fysik 36, 343 (1967).
S. W. D. Myers, Muclear Physics A204, 46S (1973).

6. G. Süssmann, LBL-1615 (May -1973).

7. H. J. Krappe and J. R, Nix, Proc. Third LAEA

Symp. on Physies and Chemistry of Fission, Rochester, 1973, Vol. 1, p. 159, IkEk, Vienna 1974.

B. C. Ngo et a1., "Properties of Heavy Ion Interaction Potentials Calculated in the Energy Bensity Formalisa", Preprint IPJo/TH 75-13 (Aprif 1975), submitted to Nucl. Phys.

9. For example, J. P. Bondorff, M. I. Sobel and

D. Sperber, Phys. Report 15C, 83 (1974);

C. F. Tsang, Physica Seripta 10A, 90 (1974) +

\title{
PROXIMITY FORCES
}

\author{
J Blockı + J Fendtup, \& W J Swalackl.
} and $C$ F Tsing

For certain physical systems such as homogeneous solids, fluids or the heavier atonic muclei, made up of elements interacting by short-range forces and possessing a surface region which is thin compared to the size of the object under consideration (leptodermous systems), the potential energy of the system may be decomposed into a bulk term and a surface-layer term. The surface-layer term is associated with the surface region and is, therefore, approximately proportional to the area of the surrface bourding the obfect. For a simply cormected system the above decomposition is accurate if the principal radii of curvature of the surface are everywhere much larger than the thickness of the surface region. Moreover, when this condition is satisfied, cortections to ths leading areaproportional term in the surface-layer onergy (such as the curvature correction) hay be derived by expansions in powers of the ratio of the thickness of the surface to the size of the system, thus making the expression for the potential energy even more accurate. Such a series expansion bas been useful in discussing the average binding energies (masses) of atomic mulei, and one might have thought that, apart from offects associated with the discreteness of nucieons (shell offects) there nes no more to the problew of average nuclear energies than the calculation of the abova series exparisions to a sufficiently high order. This is not the case. Thus, when the surface of the s)stem becomes contorted into features whose characteristic dimensions are of the order of the thickness of the surface ragion itself, the above series expansions become useless. This failing is by no means of merely academic interest: it may be serious for a system with a thin neck, on the verge of dividing into two fragments (as in nuslear fission), or in the case of two sub-systers about to come into contact (as in collisions between heavy nuclei). In the latter case in particular, when the system is not sinply connected, a calculation of the suxface layer energies of the two pleces, no matter how accurately they are corrected for the curvatures of the thip surfaces, can never give rise to the (strong) attraction that in pract ice appears when the two strfaces approach to within a distance comparable with the surface thickness.

Various attempts to remedy these fallings have been made in the past. They range fron micro5copic colputer calculations on indivitual pairs of nuclei-3 through various folding prescriptions where a potential well is folded into a density distribution, $4=6$ to direct estimates of certain aspects of the nucletus-nucleus force in torts of the experimentalty know surface-energy coefficient. 7,8

In line with the latter developsent we have found it possible to derive simple expressions for the additional potential energy (or Forces) assoc. iated with certain of the more important types of violently contorted surfaces, which should entable one to complement is a usefuf way the ustal series expansions of the molear energy. We sha11 call these aditional forces "Proximity Forces" because they arise from the proximity of elements of the contorted sirface, the contortion being such that different pieces of the surface actualiy face each other across a (small) gap or crevice. In particular we have re-derived and extented a theoren that makes it possible to relate (approximately) the interactín between two finite nuclei to the interaction between two flat parallel slabs of semi-infinite mulear matter - a problem that is simpler, and can be solved (in a suitable approximationj once and for al1.

\section{Footmotes and References}

*Condensed front LBL-5014.

${ }^{f}$ Permerient addross: Institute for Nuclear Research, 


\section{U $:$ J}

311

05-400 Swlerk, Poland.

${ }^{\ddagger}$ Permanent address: Institute of Physics, Uhiversity of Aarhus, Aarhus, Denrark.

1. W. Greiner, Proceedings of theavy-Ion Sumber Study at Cak Ridge National Laboratory, Jine 12July 1, 1972, p.i.

2. K. A. Brueckner, J. R. Buctuler, 5, Jorng and

R. J. Lonbard, Phys. Rev. 171, 1188 (1968),

3. For exarmle, C. Ngô et al , Nucl. Phys. A240, 353 (1975); J. Galin et al., Phys. Rav, C9, 1018 (1974).
4. H. J. Krappe and J. R. Nix, Proceedings of the Third IAEA Symposium on Physfes and Themistry of Fission, Rochester, New York, August 1973, p.159.

5. D. M. Brink and N. Rowley, Nucl, Fhys. A215, 79 (1974).

6. R. A, Broglia and A. Winther, Phys. Reports 4 , $153(1972)$.

7. J. Wilezynski, Nucl. Fhys, A216, 386 (1973).

8. R. Bass, Phys, Lett, 478, 139 (1973).

\section{HEAVY-ION POTENTIAL DERIVED FROM A VELOCITY-DEPENDENT NUCLEONHC INTERACTION*}

$\checkmark$ Randrupt

Recently, ${ }^{1}$ a simple expression for the nuclear interaction potertial has been derived on the basis of the prosinnity formla, In that treatment the matter distributions of the two interacting nuclear systems were superinposed without readjustsents while the nucleonic momenta were redistributed in order to comply with the exclusion principle. However, when the tio nuclei are in relative motion the importance of the exclusion principle is decreased and eventually for velocities higher then and twice the Fermi velocity (whith correspords to a kinetle energy of around $100 \mathrm{MeV}$ per nucleon), it coases to be in effect. In concert with this gracual disappearance of the effects of the exclusion principle, the velocity dependence of the baste two-nuclecr interaction is expected to become of increased significance in the ion-ion potential.

In order to obtain a simple tool which may yield rough but not too unrealistic estimates of the expected dynamical implications of such velocity dependence, the following approach has been taken. At first the exclusion principle is neglected all together (as stated above, this is strictly valid oniy for sufficiently high velocities) and the ionion interaction is derived on the basis of an effective nucleon-nucleon interaction. It should be pointed out that the assumption of no tynamical readjustment of the colliding density distributions is not decisive for the present approach to be applicable. The essential point is the assunption that the two nuslei preserve their identity such that, at any stage, the situation may be described as an interaction between two distinct 5ysteres. The exclusion prixciple may be taken approximately into account subsequent ly by an appropriate interpolation between this noadfus ment limit and the full-adjustment limit treated in Ref. 1 .

The effective muleon-ntucleon interaction employed is of the following form

$$
v_{12}=-C_{g}\left(\frac{T_{12}}{a}\right)\left(1-\frac{p_{12}^{2}}{b^{2}}\right) \text {. }
$$

This type of momentum-dependent interaction was first introducod by Seyler and Blanchard ${ }^{2}$ and has proved very useful for the discussion of gross nuxtear properties, see for example Ref, 3 . The parameter a goveris the spatial range of the interaction while the parameter b is that "critical" mamentum at which the interaction changes from attractive to repulsive. The strength constant $\mathrm{C}$ is here assumed to be the same for all nucleon pairs, as is justified for systens with identical neutron and proton distribution; the extension to asymmetrite systents is straightforward. An approximate amount of the nuclear asymmetry may be taken by scaling the emerging potentials in proportion to the surface energy of the actual system under stuky.

The interaction energy $U_{v}$ of the two nuclei can be written as

$$
u_{v}=u+\frac{1}{2} w w^{2}
$$

where the velocity-independent functions IJ and $W$ are given by

$$
\begin{aligned}
& \mathbf{u}=-\left[\int \int \left(\rho_{a}\left(\vec{r}^{\prime}\right) \rho_{b}\left(\vec{r}^{\prime}\right)+2 \vec{\pi}_{a}\left(\vec{I}^{-1}\right)\right.\right. \\
& \text { - } \left.\vec{n}_{b}\left(\overrightarrow{r^{\prime}}\right)-p_{a}\left(\vec{r}^{\prime}\right) \gamma_{b}\left(\overrightarrow{\mathbf{r}^{\prime}}\right)-\gamma_{a}\left(\vec{r}^{\prime}\right) \rho_{b}\left(\overrightarrow{\mathbf{r}}^{\prime}\right)\right) \\
& g\left(\frac{5}{a}\right) a^{3} \dot{r}^{\prime} d^{3} \vec{r}^{-1}
\end{aligned}
$$

and

$$
\left.w=m \frac{C}{b^{2} / 2 m} \iint a_{a}\left(\vec{r}^{\prime}\right) o_{b}\left(\overrightarrow{r^{\prime}}\right) g: \frac{r}{a}\right) d^{3 \vec{r}^{\prime}} \mathrm{d}^{\overrightarrow{3} \vec{r}^{\prime}}
$$

It follows that the total energy of the two-ion system may be written in the form

$$
\text { E } y \mathrm{E}_{\text {C.m. }}+\frac{1}{2} \mathrm{Bv}^{2}+\mathrm{U}
$$


whew $E_{c}, m$ is the energy of the overall center-ofHass motion. Thus the relative motion is described in terns of a static potential $U$ (in which the Coulomb contribution should be included) and an effective nass $B-\mu+1$ where $\omega$ is the usual reduced mass, $1 / \mu=1 / \mathrm{m}_{\mathrm{a}}+1 / \mathrm{k}$.

As stated earlier, tho potential U derived atcove applies to large relative velocities only, In the intemodiate region a partial readjustment of the nuxleoric momenta mast take place because of the exclusion principle. This adds an implicit velocity dependence of the interaction to the explicit veloclty dependence represented by the variable effective mass $B$. The question conceming the proper form of the interpolation between the two extremes is currently boing pursued in a separate studidy.

The general formulas may be transformed into a practical tool for simple analyses by use of the proximtty formula. 1 This formila expresses a shortrange overlap integral botween curved surfaces as a gemetrical factor containing the actual gecratry (such as size and defornation) tônes a "universal" function evaluated (once and for all) for a seriinfirite configuration. For a detafled discusston see Ref. 1. The application of the proximity formula leads to the following simple expressions,

$$
\begin{aligned}
& \text { U } \approx 2 \pi R \tilde{\mathrm{u}}(\mathrm{s}) \\
& w \approx 2 \pi \mathrm{R} \tilde{w}(\mathrm{~s})
\end{aligned}
$$

Here the geometrical factor $\bar{k}-c_{a} \hat{b}_{b} /\left(C_{a}+c_{b}\right)$ measures the radius of curvature of the gap between the surfaces. As discussed in Ref. 1, the effective surface locations $C$ are given approximately by $C \Rightarrow R-b^{2} / R$ where the nuclear radius $R$ * $1.15 \mathrm{~A}^{1 / 3}$ fin and the strrface diffuseness $b \approx 1$ for. The variable $s$ denotes the fective surface separation, $s=R-C_{\mathbf{a}}-C_{b}$.

A muserical calculation of the "universal" functions $\tilde{t}$ and $\tau$ has been carried out. The parameter values are those detemined in Ref. 3: $\mathrm{a}=0.62567 \mathrm{fm}, \mathrm{b}=372.48 \mathrm{kkV} / \mathrm{c}$ and $\mathrm{C}=328.61$ 14ev. The results are displayed in Figs. 1 and 2 . Two parallel sets of calculations have been perfomed, one based on the Thonas-Fermi approximation and one based on the Hartree approximation (see Ref. 4 for the formulation of the model in the Hartree approximation). The overall shift towards larger separation for the Hartree results is a consequence of the extended density tails, which are not preșent in the Thoms-Ferui treatment. As discussed in Ref. 1, the associated worertainty is greatly reduced by scaling the separation according to the surface diffuseness. Included in Fig. 2 is the potential obtained in Ref. $I$ based on a total monentum readjustment, $\vec{v}_{p^{+}}$

A simple approximate representation of the elastic ion-ion interaction has been sugrested. The interaction contains an expltcit velocity dependence in the form of a variable reduced mast; this is due to the velocity dependence of the basic nucleonic interaction. In addition an inplicit

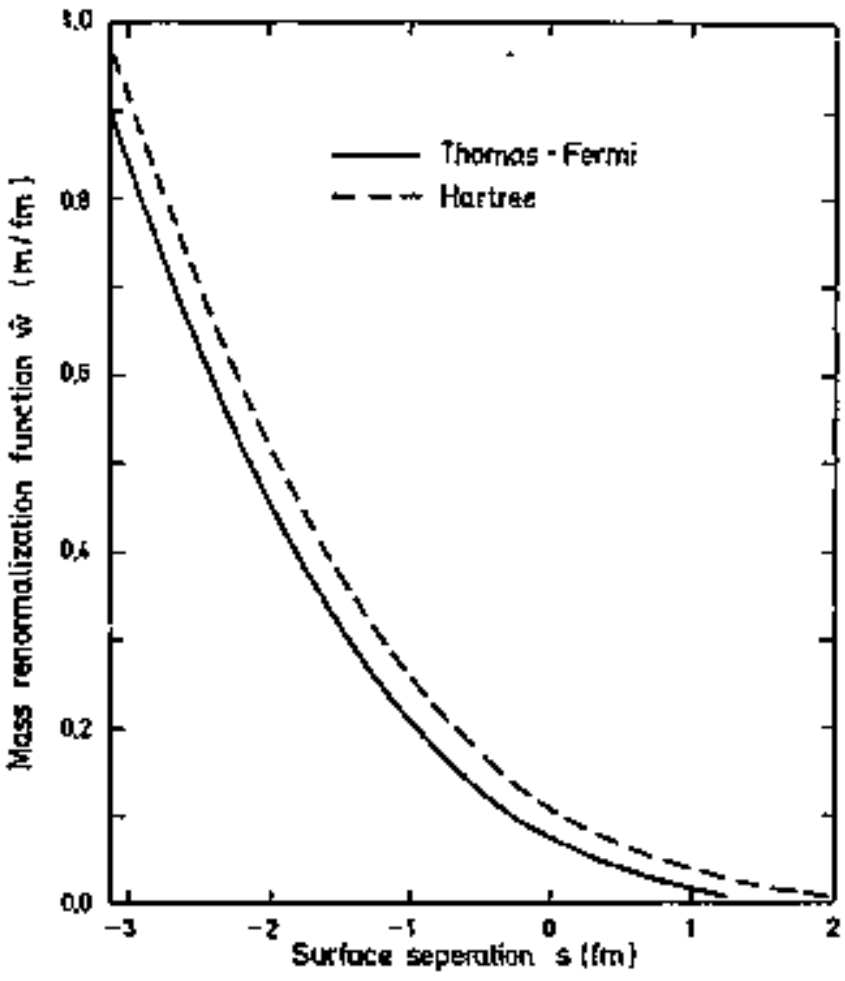

Fig. 1. The 'miversal' mass-renomalization function as calculated on the basis of the velocity-dependent effective interaction in the Thonas-Fermi (full curve) and Hartree (dashed curve) approxinations.

(XBL 766-8275)

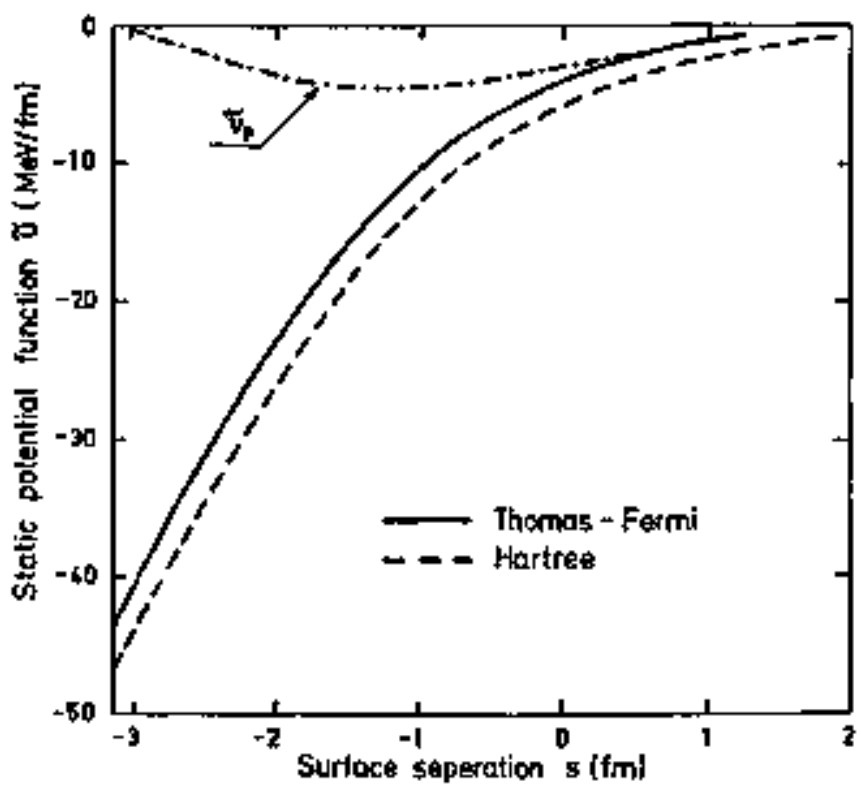

Fig. 2. The "miversal" static-potential function น. Sinilar to Fig. 1. The praximfty function tip derived in Ref. 1 is indicated by the dot-dashed curve.

(XBL 766-8276) 


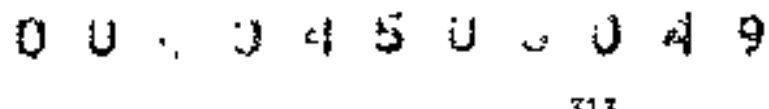 \\ 313
}

velocity dependence eppears because of the exclusion principle. A major simplification is accomplished by erploying the proximity formula for the evaluation of the overlap integrals. The corresponding "tuiversa1" mass and potential functions have been calculated mumerically. The very simple form of the final forrulas makes the rode1 a practical tool for discussing the gross features assoclated with molear collisions. A study of heavy-ion dynamics based on this model is currently underway.

\section{Footnotes and References}

"Condensed from LBL-4376. ton leave from laiversity of Aartus, Aantus, Demark. Present address: The Nieis Bohr Instituto, Coperhagen, Dermark.

1. J. Blocki, J. Randrup, W. J. Swiatecki and C. F. Tsang, "Troximl ty Forces", LBL-5014, to be published.

2. R, G. Seyler and C. H. Blanchard, Phys, Rev. 124,227 (I961).

3. W. D. Myers and W. J. Swiatecki, Ann, Phys. 55, 395 (1969).

4. J. Randrup, LBL-4302, hucl. Phys. A, 257 (1976) 253.

\section{CONYOLUTION AS A GEOMETRICAL ANSATZ*}

\author{
Whillam D Myers
}

In a detailed study of the geometrical properties of nucleit it was pointed out that a number of problems arise when one attent ts to generate optical model potential wells by folding a short range interaction into the density distribution. However, there are a number of applications where simple folding is an extremely useful approach. one of these applications concerns a nodified definition of the 1iquid-drop model surface energy ${ }^{2}$ that is free of sprrious sensitivity to highmaltipolo wiggles in the shape of the drop. Another application whose significance is just beginning to be realized is the use of corrolution as a geometrical ansatz for creating diffuse surface distributions. In virtually all of the literature of nuclear physics, diffuse surface distributions (of density or potential) are represented by Fermi functions. This choice has a ntunber of awkward features which are easily overcune by creating the diffuse distribution from one with a sharp surface by convolution.

One of the more obvious advantages of using the convolution method for generating the surface diffuseness concerns the question of normalization. When a Ferma function is used to represent a nuclear density distribution its woluno integral wist be chosen to contain the correct number of nucleons. This isn't a sirple task even for spherical muclei. The integral must be done nuslerically and the halfradius $c$ chosen by successive approximations. For a deformed nucleus this becones a two or threedimensional numerical integration and iteration procedure, for the çan use analytic approximationss of various kinds if it isn't iuportant to have the numer of particles exactly right.) By contrast, the nomalization problem conpletely disappears when the diffuse density distribution is generated by folding. The volume of a distribution generated by folding two other distributions together is the product of their separate volunes. If the comvolution function is normalized to unit volume, then the diffuse surface function has the same volume as the sharp distribution it is derived from and no calculation whatever is required.
Another afvartage of using convolution to generate diffuse surfaces is that the diffuseness nornal to the surface is nearly the same at any point on the surface. This is true as long as the local curvature of the surface is not too great. When Fernil functions are waed, the diffuseness normal to the surface is proportional to the cosine of the angle between the radius vector and a nowral at that point. This kind of angular ependence of the surface diffuseness can generate spurious multipole momonts that have no basis in reallty.

Perhaps the most useful feature of diffuse distributions created by convolution is that their multipole moments are identical to the moments of the sharp surfaced generating functions they are based on. 3 This neans that only sharp distributions need be ewployed in the discussion of any physical phenomena that is concemed with multipole moments. Becanse of the identity of the roments, such a discossion applies equally 11 to diffuse distributions created by convolution. This is certainly not the case when Fermi functions are used to create the diffuseness.

Papers have been written and tables of ntmerically calculated comversion factors have been compiled, for relating the muitipole morments of a Fermi function to those of the generating shape. The need for such conversions is not a general feature of diffuse distributions, but simply an artifact created by the poor choice of method for creating the diffuseness.

Another idportant application of the convoIution method is in the calculation of moments of inertia. A number of nuclear phencowena depend opon the moments of inertia of nuxiei whose deformations are sometimes simple (muclear grovend states) and sonetimes complex (fission barriers, for exalale, or colliding heavy-ions,) Questions often arise as to the effect of diffureness or these phenconena which, up until now, have usually been disoussed only in terms of sharp-surfaced density distributions, of course, if Fermi functions are used to 
create the diffuseness, numerical integrations would be required to calculate the conversion factors for every case of interest.

If convolution is used to create the diffuse. nes5, then the moments of inertia of the diffuse and sharp distributions are related by the simple expression, 3

$$
I_{2}=I_{1}+2 \mathrm{Mb}_{\mathrm{c}}^{2}
$$

In this expression $I_{2}$ is the moment of inertia of the diffuse distribution about an arbitrary axis and $I_{1}$ is the corresponding moment of inertia for the sharp-surfaced function it is derived frort. The quantity $M$ is the total mass of the object and $b_{0}$ is the "width" of the convolution function, where $b$ \& $=1 / 3<r^{2}>c$.

Actually generating the density distribution itse1f (or the diffuse surface potential well) by folding may be complicated, depending on the generating shape. Eut, since one is usully interested in the properties of the distribution such as radial moments, multipole monemts, moments of ivertia, etc., it is often iơnecessary to generate the distribution. If the diffuseness is thought of in terms of convolution as a geometrical ansatz, then only sharp distributions neod actually be enployed in the calculations.

\section{Footnotes and References}

*ondensed from Ref. 3 .

1. H, D, Ngrers, Nuc1. Phys. A204, 465 (1973).

2. H. J. Krappe and J. R. Nix, Proceedings of the Third Syuposium on the Physics and Chemistry of Fission, Rochester (Angust 1973).

3. W. D. Myers, Mukleonika 21, 3(1976).

4. L. W. Owen and G. R. Satchler, Muci. Ptys. 51. 155 (1964).

\section{THE NUCLEAR SURFACE DIFFUSENESS*}

Wh $D$ Myers and $H$ won Grolls $\dagger$

The nuclear radius constart $r_{0}$ is a fundamental constant of nuclear physics which is defined in terms of the equilibrium density of infinite nuclear netter by the expression $\rho_{0}-\left(4 / 3 \pi r_{0}^{3}\right)^{-1}$. The "effective sharp radius" (the terminology and notation of Ref. 1 is used] of a nucleus is not simply $r_{0} \mathrm{l}^{1 / 3}$, but is smaller for light nuclei which are squeezed by surface tension, and larger for lieavy nuclei where dilation (due to Coulomb and neutron excess effects) beglns to doninate. In addition the neutron excess in heavier muclei is expected to produce a neutron skin of thickonss $t=R_{1}-R_{2}$, where $R_{n}$ and $R_{2}$ are the effective sharp radil of the neutron and proton density distributions respectively. 2 A model that fncludes these features can be used to infer the fundamental quantity $\mathbf{r}_{0}$ frow the experimental measurements of the radii cf actual roxlei.

In a similar way the diffuseness of semiinfinite nuclear matter $b_{0}$ can be inferved from experimental measurements of the diffuseness of actural muclei by using a model that describes the deviations to be expectud because of the Coulomb repulsion.

Both the surface energr and Coulonis onergr of a nucleus depend on the surface diffuseness. Consequently, variatioms in diffuseness effect the total energy through the two terpls in the expression,

$$
\begin{aligned}
\mathbf{E}_{\text {diffuseness }} & =\mathbf{E}_{\mathrm{s}}^{0}\left[\mathbf{1}+\frac{1}{2}\left(\phi_{1} \Gamma_{n}^{2}-2 \phi_{2} \Gamma_{\eta} \eta_{z}+{ }_{1} \eta_{z}^{2}\right)\right. \\
& +\ldots]+E_{\tau}^{0}\left[1-\gamma_{2} \beta_{z}^{2}+\gamma_{3} \beta_{z}^{3}\right. \\
& \left.+\gamma_{4} \beta_{z}^{4}+\ldots\right]
\end{aligned}
$$

In this expression $\mathrm{E}_{5}^{0}=a_{2} / \mathrm{A}^{2 / 3}$, the quantity $a_{2}$ being the equilibrium surface energy coefficient of senti-tnfinite nuclear matter. The second term in the brackets describes the increase in surface energy associated with deviations of the neutron and proton diffusenosses from their semi-infinite nuclear matter values. The quantities $n_{n}$ and $n_{2}$ are defined by tho expressinn

$$
\left.\left.n_{\frac{n}{2}}=\underset{z}{\left(b_{n}\right.}-b_{0}\right) / b_{0}\right)
$$

where $b_{n}$ and $b_{z}$ are the diffusenesses of the neutron and proton density distributions and $b_{0}$ (the fundamental quantity whose value we seek) is theit value in the absence of outside influences. A ThomasFerui calctuation (similar to the ones described in Ref. 3) was performod, 4 which yielded the following estimate for the coefficients appearing in Eq. (1):

$$
\begin{aligned}
& \phi_{1}=0.45, \\
& \phi_{2}=0.23
\end{aligned}
$$

The second term in Ed. (1) represents the Coulond energy and its dependence on the diffuseness of the proton distribution. The coefficient Ec $=$ $\mathrm{c}_{1} \mathrm{Z}^{2} / \mathrm{A}^{1 / 3}$, where $c_{1}$ is the Droplet Model Coulanb energy coefficient. The expansion paraneter $B_{2}$ is defined by the expression,

$$
B_{2}-b_{z} / R_{z}
$$

where $R_{z}$ and $b_{z}$ are "the equivalent sharp radius" and "surface width", respectively, of the proton 


\section{$00: 9 \times 30 ; 0 ; 0$}

density dłstribution. For a Fernd function depsity distribution the coefficients have the values,

$$
\begin{aligned}
& r_{2}=5 / 2 \\
& r_{3}=3.02168 \\
& r_{4}=1 .
\end{aligned}
$$

For the Broplet Model coefficients an and $c_{1}$ we will use the values of $a_{2}$ and $r_{0}$ from the latest droplet model fit to nuclear masses, fission barriers and radii, which are

and $\quad \begin{aligned} & a_{2}=20.69 \mathrm{kV}, \\ & \mathrm{r}_{0}=1.18 \mathrm{fm}, \\ & \text { hence } c_{1}=\frac{3}{5} \frac{\theta^{2}}{\mathrm{r}_{0}}=0.7322 \mathrm{MeV} .\end{aligned}$

For purposes of 11lustrating the consequences of $\mathrm{Eq}$. (1) it is sufficient to set $\mathrm{R}_{2}=\mathrm{R}=7 \mathrm{~T} \mathrm{~A} / \mathrm{3}$ in Eq. (4), to retain only the terps in $\gamma_{2}$ and $\gamma_{3}$ in the cowlont energy, and to expand the coulond energy to second order in $\pi_{z}$. The resulting energy expression is

$$
\begin{aligned}
E & =\mathrm{E}_{\mathrm{s}}^{0} \phi_{1}\left[\text { constant }+\frac{1}{2} \mathrm{r}_{\mathrm{n}}^{2}-2 \operatorname{en}_{n} \eta_{z}+\eta_{\mathrm{z}}^{2}\right. \\
& \left.-\mathrm{c}(1-\delta) \eta_{2}-\mathrm{c}(1-2 \delta) n_{2}^{2}+\ldots .\right],
\end{aligned}
$$

where

$$
\begin{aligned}
& \varepsilon=\Phi_{2} / \phi_{1}, \\
& s=\frac{3}{2}\left(\gamma_{3} / \Upsilon_{2}\right)\left(b_{0} / r_{0}\right) A^{-1 / 3}, \\
& c=\left(S_{c_{1}} / a_{2} \phi_{1}\right)\left(b_{0} / r_{0}\right)^{2} 2^{2} A^{-5 / 3} .
\end{aligned}
$$

Minimization with respect to $n_{n}$ and $n_{z}$ results in the following expressions for the equilibrikm values:

$$
\begin{aligned}
& n_{z}=C(1-\delta) /\left[\left(1-e^{2}\right)-C(1-2 \delta)\right], \\
& n_{n}=\pi_{2} .
\end{aligned}
$$

In order to estimate the value of bo we have calculated the values of $b_{2}$ for nucles along betestability [using Green's approximation that $\left.N-Z=0.4 \mathrm{~A}^{2} /(200+\mathrm{A})\right]$ assuting various values of $b_{0}$. These predictions are connared with the experimental results ${ }^{8}$ in Fig. 1a, from which we estinate that

$$
b_{0}=0.82 * 0.05 \mathrm{ft}
$$

In the lowerpart of Fig. 1 the predicted diffusenesses ( $b_{1}$ for neutrons and $b_{z}$ for protons) are plotted against the mess nuber $A$ for nuctei along beta-stability. At the bottom of the figure the neutron skin thickness predicted by the Droplet
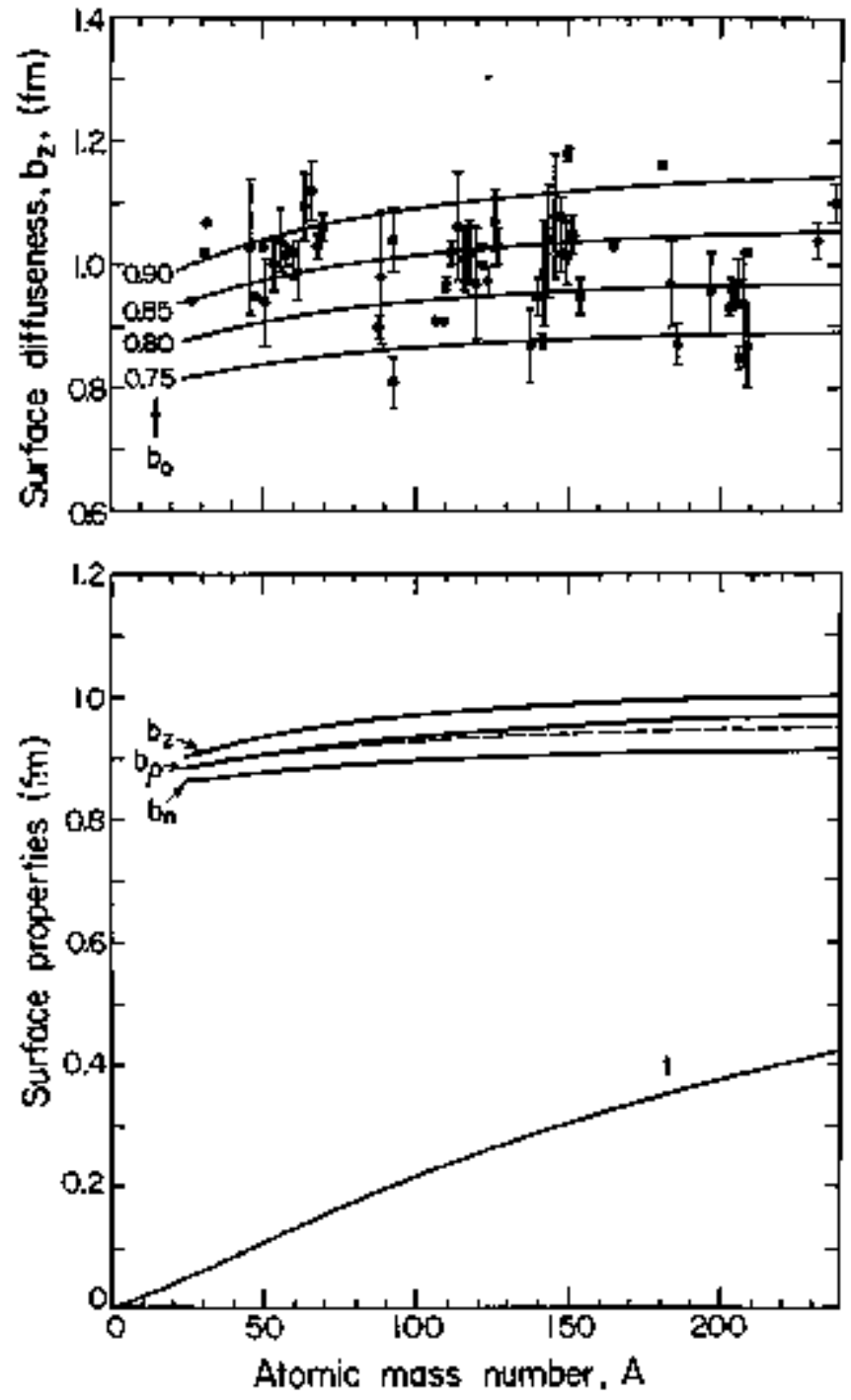

Fig. 1. In the upper part of the figure calculated and experimental values of the diffuseness of the proton distribution $b_{z}$ are plotted against the atonic nass number $A$. The different curves corrospon to different values of the fundamental cristant bo. In the lower part of the figme the calculated vallues of the neutron skin thickness $t$ and the diffusenesses $b_{p}, b_{z}$, and $b_{p}$ are given for nuclei along beta-stability.

(XBL $7510-6557 \mathrm{~A}$ )

Hodel is plotted.6 If it were not for the neutron skin the diffiseness of the total matter distribution $b$ (neutrons plus protoris) would siruply be the weighted nean of $b_{n}$ and $b_{2}$ that is shom by the dashed line. The actual velue of $b_{p}$ is always larger since the separation between the neutron and proton surfaces contributes to the apparent diffuseness. The relationship between $b_{0}, b_{n}, b_{2}$ and the skin thickness $t$ is

$$
b_{\rho}^{2}=\left(\frac{N}{A}\right) b_{n}^{2}+\left(\frac{Z}{A}\right) b_{z}^{2}+\left(\frac{N \cdot Z}{A^{2}}\right) t^{2} .
$$

Recogrition of the nuclear diffuseness degree of freedon also has inportant implications in the 
fleld of collective nuclear excitations. In principle, both $T=0$ and $T=1$ nuclear diffuseness vibrations are posstble and these may be of monpole, quadrupole, or higher muItipale order. The excitation energies are probably quite high because the restoring force is largo and the inertial parameters are sudil. In adrition these vibrations are probably strongly mixed with the corresponding bulk vibrations because of the inertial coupling between thet.

\section{Footnotes and References}

*ondensed from Physics Letters 61B, 125 (1976).

'Institut für Kermphysik, Dakmstadt, Germany.

1. W. D, Myrers, Nucl. Phys. A204, 465 (1973).

2. W. D. Myers, Phys, Lett. 30B, 451 (1969).
3. W. I. Myers and W. J. Swiatecki, Ann, Phys, (N.Y.) 55, 395 (1969).

4. H. won Groote, Proceedings of the First International Workshop on "Gross Properties of Nuclei and Nuclear Excitations", Hirschegg (Jan, 1973),

5. H. C. Carlson, Iowa Stat J. of Sci. 35, 319, (1961), and more recently, J. Janecke, $\sqrt[3]{u c 1}$. Ptys. Al 81,49 (1972).

6. W, D, Mysts, LBL-3428 (Noverder 1974),

7. A. E. S. Green, Nuclear Physics (McGraw-Hill, New York, 1955].

8. H. R. Collard, L. R. B, Elton and R, Hofstadter, in "Murlear Radii", Vol. 2, Grou I; landolt-

Bornstein, Numerical Data and Functional Rolationships in Sclence and Technology (Springer-Verlag, Berlin, 1967); C. W. deJager, H. deWries and $C$. deWries, Atomic Data and hucl, Data Tables 14, 479 (1974); and R. Engfer, et al., ibid., p.509.

\title{
THE DROPLET MODEL. OF THE GIANT DIPOLE RESONANCE'
}

\author{
Willam D Myers
}

Since the early work of Goldhaber and Teller (GI) ind the related work of Steinwedel and Jensen ${ }^{2}$ (SI) most of the progress in our understanding of the nuclear Giant DipoIe Resonance (GIR) has been concemed with the microscopic basis of the phenomenta. There have also been attenpts to inclube coupressibility effects or surface diffuseness effects but there seems to have been no recognition of the fact that the macroscopic nature of the GDR is probably best described as a superposition of the two modes (GT and SD) discussed ear1ler.

In addition to considering the coupling of these two modes (both inertial and potential) we chose to calculate the restoring forces from the Droplet Madel.3 This choice puts both the GI And SJ calculations on a solnder footing since the Droplet NodeI has the important feature that it includes the netutron skin thichness as a degree of freedom.

The actural catculations were performed with the vector $\vec{a}$ whose two components $a_{1}$ and $a_{2}$ repre. sent the relative magnitudes of the GT and $S J$ nodes respectively.

The GT node is simply a rigid displacement of the protons with respect to the neutrons by an amount $\mathrm{d}=\pi_{1} R$. Actually, since the center-of-mass remains fixed the relative proton and neutron: disptaceplents are

$$
d_{2}=\frac{N}{A} d \quad \text { and } \quad d_{n}=-\frac{Z}{A} d
$$

The \$J mode corresponds to a vibration of

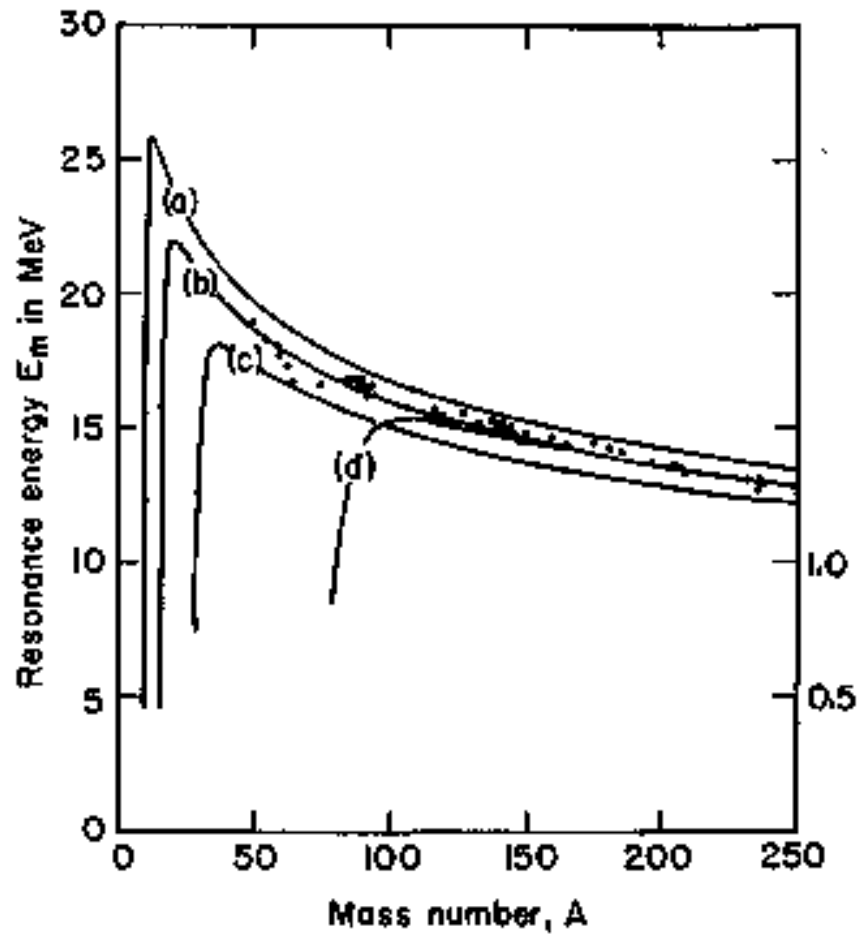

Fig. 1. The energy of the GDR in $\mathrm{MtV}$ is plotted against the mass number $A$, for nuclei throughout the Periodic Table, The curve labeled (b) corrosponds to the set of Droplet lode1 coefficients given in the text. The experimental points are from Ref. 4. 


\title{
$00: 94502031$
}

protons against the neutrons, with the total density held constant, corresponding to the lowest acoustic mode in a spherical cavity. For this mode the proton afit neutron density variations are given by

$$
\delta \rho_{2}=\frac{N}{A} p_{z} \delta n \text { and } \delta p_{n}=-\frac{Z}{A} \rho_{n} \delta n \text {. }
$$

where

$$
\text { on }=a_{2} \mathrm{Cj}(\mathrm{kr}) \cos \theta .
$$

The constant $\mathrm{C}-2 \mathrm{a} / \mathrm{f}_{0}(\mathrm{a})$ is chosen so that equal displacenents in a and a produce equal dipolo morments. The quantity $\mathbf{a}=\mathrm{kR}=2,081576$ is determined by the boundary conditions and $j_{1}$ and $j_{0}$ are spherical Bessel fametions.

In tertas of these degreas of freodon the inertia matrix $B$ is

$$
B=\left(\begin{array}{cc}
1 & 1 \\
1 & \frac{a^{2}-2}{2}
\end{array}\right) \cdot m r_{0}^{2}\left(\frac{N Z}{A^{2}}\right) A^{S / 3}
$$

and the components of the potential matrix aro

$$
\begin{aligned}
& c_{11}=\frac{2}{3} \mathrm{HA}^{4 / 3} \\
& c_{12}=C_{21}=\frac{4}{3} \mathrm{PA}\left(\frac{\mathrm{NZ}}{\mathrm{A}^{2}}\right) \mathrm{a}^{2}, \\
& C_{22}=\mathrm{J}\left(\frac{\mathrm{NZ}}{\mathrm{A}^{2}}\right)^{2} 4 \mathrm{a}^{2}\left(\mathrm{a}^{2}-2\right)-\frac{8}{3} \mathrm{aA}^{2 / 3}\left(\frac{\mathrm{N} 2}{\mathrm{~A}^{2}}\right)^{2} \mathrm{a}^{4} .
\end{aligned}
$$

The quantities $r_{0}, H, P, J$ and $G$ are Droplet Mode1 coefficients.

The eigenvalue equation, Det $\left|\omega^{2} \mathrm{~B}-\mathrm{C}\right|=0$, can be solved and the corresponding energies crapared with the experinental values for the GDR. Figure 1 shows the results (which have been scaled using an effective mass $\boldsymbol{n}^{*} * \overline{0} .7 \mathrm{n}$ ) for nuclei throughout the Periodic Table. The different curves correspond to different sets of values for $H, P$ and G. These coefficients are not uniquely deterained by the Droplet Hodel fit to masses because they only appear in the mass equation in the conbination represented by the synbol $Q$, where

$$
\mathrm{Q}-\frac{\mathrm{H}}{\left(1-\frac{2}{3} \frac{\mathrm{F}}{\mathrm{J}}\right)} \text { and } \quad \frac{G}{\overline{\mathrm{F}}}=\frac{3}{2} \frac{\mathrm{J}}{\mathrm{Q}} \text {. }
$$

For the curve 1abeled (b) the values used were

$$
\begin{aligned}
& r_{0}=1.18 \mathrm{fm} \quad H=12 \mathrm{NeV} \\
& \mathrm{J}=36.8 \mathrm{MeV} \text { and } \mathrm{P}=16.235 \mathrm{MeV} \\
& \text { Q }-17 \mathrm{HoV} \quad \text { G }=52.727 \mathrm{MeV} \text {. }
\end{aligned}
$$

The A dependernce of the GIR energy is well reproduced (which is not possible with the GT or SI modes alone).

\section{Footnotes and References}

"In collaboration with L. J. El-Jaick and T. Kodana of Centro Brasileiro de Pesquisas fisteas, Rio de Janerto, Braztl.

1. M. Goldhaber and E. Telier, Phys, Rev. 74, $1046(1948)$.

2. H. Steintedel and J. H. D. Jensen, Z. Naturf. $\underline{5 a}, 413$ (1950).

3. W. D. Myers, 1RL-3428 (November 1974).

4. B. l. Bermien and S. C. Fult2, Rev. Mod. Phys, 47, $713(1975)$.

\section{PROXIMITY FISSION}

\author{
J S Smieck. L G Moretio and W J Sweteck।
}

Recently, we have investigated the theoretical possibility for a new process, which we have terned Proximity Fission (PP). This process should occur as follows [see F1g. 1 for the relevant shapes at each stop of the process):

1) A target nucleus, with a reasonably large spontameous fission barrier, is struck by a projectile which has just enough energy to overcone the coulowit barrier.

2) While the short range nuclear force maintains the contact between target and projectile, the target deforms and, if the atomic nimber of the projectile is large erough, fissions along the incident direction.

3) The projectile is re-mitted in the backward direction.

The experimental earmark for such a process would be the coincidence of fission fragments from a "target-like" nucleus in the forward direction with a "projectile-1ike" nucleus in the backward direction. This process should be distinguishable from Coulonb Fission (CF) since the most probable direction of emission of $\mathrm{CF}$ fragments is perpendiculst to the incident diroction, while the most probable direction of emission of PF fragnents is 
Shape Sequence

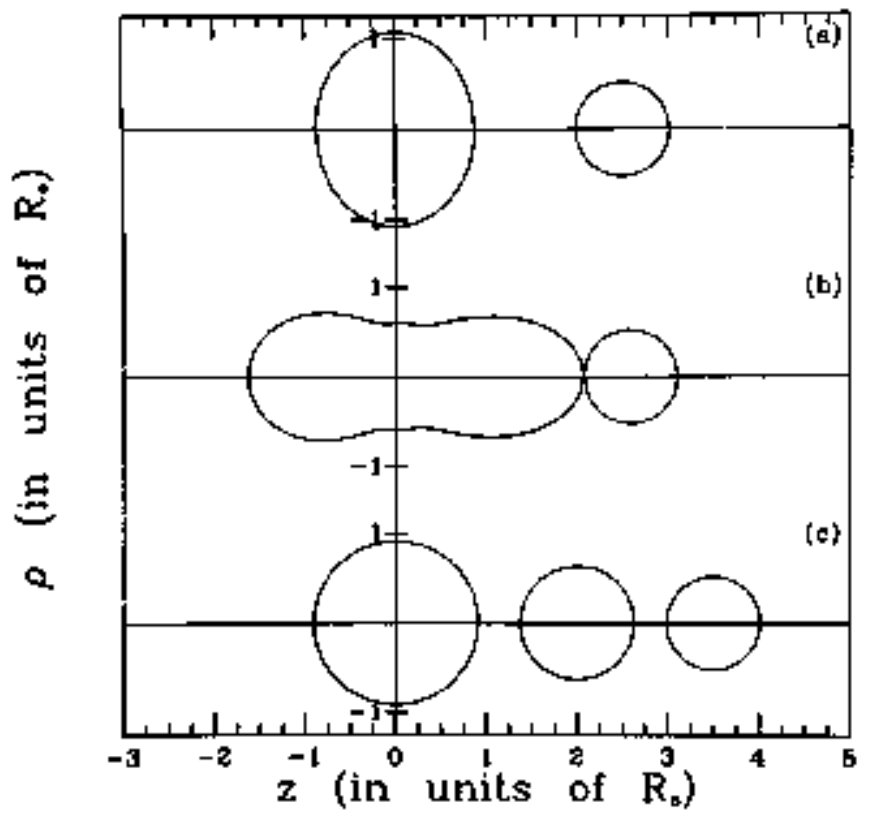

Fig. 1, a) Shape prior to and just as the collision takes place. b) Deformed shape of target while in the proximity of the projectile. c) Projectile re-emitted in the backward direction. (XBL 766-8289)

Shape for $Z_{p}=13+A u$

$Q$

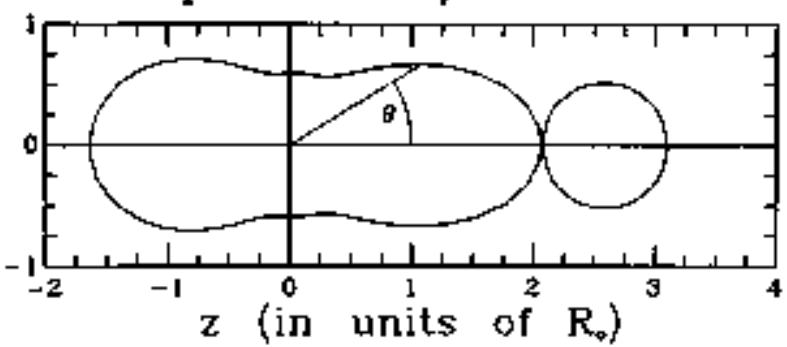

Fig. 2 . A typical shape encountered in the calculation. The angle $\theta$ is measured counter clockwise from the positive $z$-axis.

(XBL 766-8292)

in the incldent direction.

The potential energy calculations were performad by expanding the radius of the target in Legendre polynondals, i.e.,

$$
R_{t}(\theta) \cdot=u\left[1+\sum_{i} \alpha_{i} P_{i}(\cos \theta)\right]
$$

where the $\alpha_{i}$ 's are expansion parameters and $u$ allows volute normalization. The projectile, which throughourt the process is assumed to remain spherical with radius $\mathrm{R}_{\mathrm{p}}$, is placed at a distance $R_{t}(0)+R_{p}$ (see Fip. 2). The liquid drop potential energy (strrface + Coulonb) is then calculated for the shape described by the paraneters $\left\{a_{i}\right\}$, and
Proximity Barrier for ${ }^{19} \mathrm{Au}$

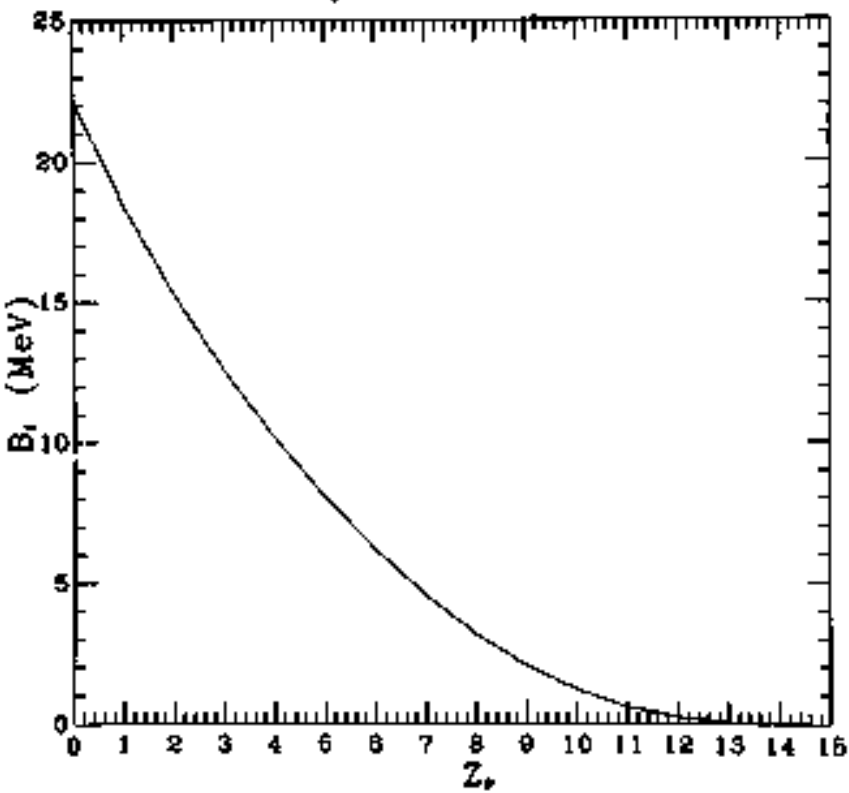

Fig. 3. Proximity barrier (in $\mathrm{MeV}$ ) as a function of $z_{p}$ for $x=0.67$ (a gold target nucleus),

(XBL 766-8293)

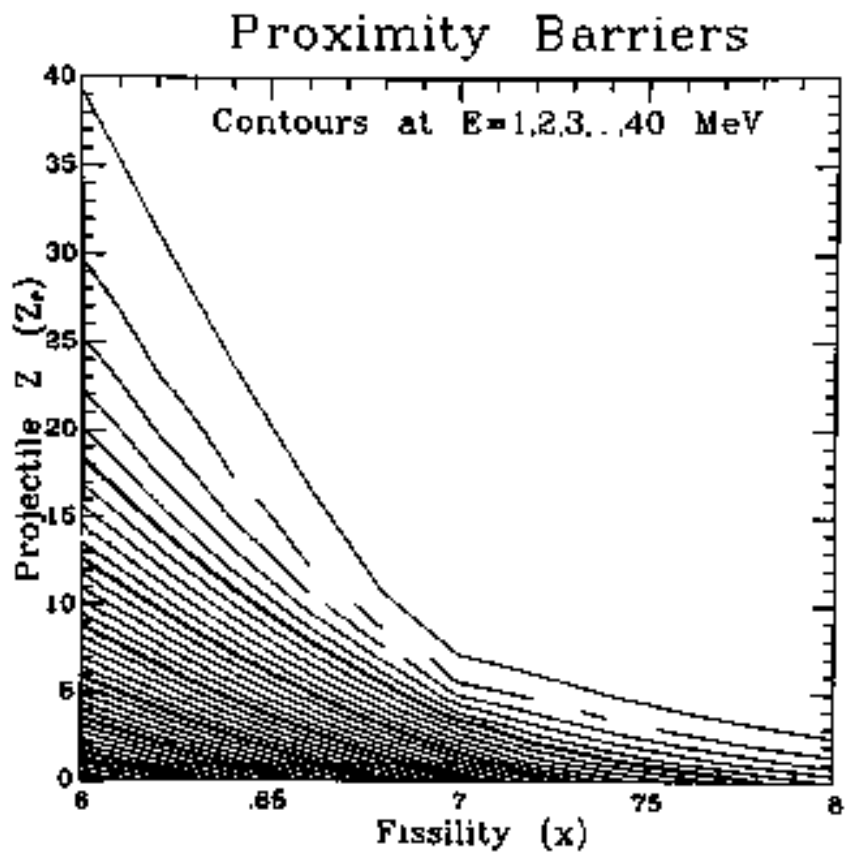

Fig. 4. Contoors of constant proxinity barrier (in Hev) as a function of $z_{p}$ and $x$. The solid line at which the contours end is the line $z_{C r}(x)$.

(XRL 766-8294)

the rasulting potential energy surface is searched for the perturbed ground state and sadifile point. The proximity fission barrier is then the difference in energy betwen the perturbed ground state and saddle point.

Prior to considering the problen dynamically, we have here stuktied the static problen, 1.e., the 


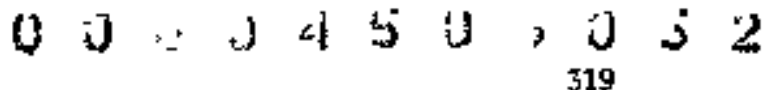

potential energy only. Infortunately, the stationary points th an inlinited paranotrization are the trivial solutions cortesponding to the regular ground state and saddle point shapes of the target comected to the projectile by an infinitely long, infinitesimal thread of zero volum and surface area. It is conceivable that dymanical considerations way prevent such instabílities from developIng. On this basis, we have proceeded to evaluate the ground state and saddle point shapes and energies in the restricted parametrization of $\mathrm{P}_{2}, \mathrm{P}_{3}, \mathrm{P}_{4}$ and $\mathrm{P}_{5}$ deformations.

In general, the fission barrier of the target is reduced as the atonic number $Z_{p}$ of the projectile is increased framb) (It is asstoled that $A_{p}=2 z_{\text {p }}$, and $R_{p}=1.2249 A_{p} / 3$.) This behavior for a target in the gold region of the Periodic Chart is displayed in Fig. 3. For a critical atonic number $Z_{e r}$, the perturbed ground and saddle shapes coalesco, with the result that the fission barrier of the target vanishes. There is also no barrier for projectiles with $z_{p}>z_{c r}$. This behavior is enalogous to thet displayed by the spontaneous fission berrier for rotating, charged liquifd drops, 2 As the rotational parameter $y$ increases fron the value 0 , the barrier for spontmeous fission decreases, and ultimately disappears for $y y_{C T}$, where $y_{c r}$ represents the maximm arount of angular monentum that the liquid drop can tolerate.

Like $y_{c r}$, the value of $z_{c r}$ is a function of the fissility paranter $x$ of the target. A contour map of the barrier for proxiaity fission of the target (in heV) as a function of the fissility parareter of the target and the atoric number of the projectile com be seen in Fir. 4. The solid line at wich the contours end is the Iine $z_{c r}(x)$. As one would expect, $z_{\mathrm{cr}}$ is a monotonically decreasing function of $x$, and vinishes for $x=1$, since the 1iquid drop is mstable to spontameous fission (or proxinity fission with $z_{p}=0$ ) at $x=1$.

The situation of nost interest to us occurs for target nuclei in the gold regton $(0,65 \% \times$ $0.70)$. Since the spontaneous fission barriers in this region are in the neightorhood of $20 \mathrm{bev}$, true fisssion probabilities reingin negligible until several ters of MeV of excitation energy are deposited in the target. The expected $y$ teld of fission products for interactions near the Coulonb barrier is essentially zero. On the othor hand, as can be seon in Fig. 4, the proxinity fission barrier for targets in this region vanishes for projectiles with $\mathrm{z}_{\mathrm{p}}>13$. Therefore, projectiles with $z_{p}>13$ coufd induce fission at energies just slightly above the Coulomb barrier.

Wore precisely, we mould expect to see a dramatic increase in fission products as the energy of the projectile [with $z_{p}>13$ ) is varied from energies below to energies above the Coulonb barrier. For very high energies, the fission products could be attributed to an highly excited gold nucleus fissioning after the projectile has been re-emitted, but the yifeld near the barrier, where no products can be expected from the fission of an oxcites? gold nuxieus, world be the solely to the proxinity of the perturbing charge. ife are presently preparing experinents to try to detect such products.

\section{References}

1. E. Guth and L. Wilets, Phys, Rev. Lett. 16, 30 (1966).

2. S. Cohen, F. Plasil and W. J. Swiatecki, Annals of Phys. 82,557 (1974). 


\section{DAMPING, FISSION, GENERAL RELATIVITY}

\section{SCHRÖDINGER EQUATION FOR \$CATTERING WITH ENERGY LOSS"}

A Lipperhelde†

An attermpt has been made to establish a model Schrödinger equation for the description of heavy ion scattering with energy 1oss, This equation is linear and cam be related to the quantal coupled chanmels approach. A possible parametrization of the madel Familtonian is considered. Energy nonconservation in the Schrodinger pictare can be connected with an explicit time dependence of the scattering potential between tho heavy ions:

$$
\left(-i 3 / x-t^{2} / 2 \pi+v+v^{1}(t)\right) d(r, t)=0
$$

To describe energy loss in the relative motion owing to internal excitation, the energy-losing potential vi must contain onty positive frequencies,

$$
W^{L}(t)=\int_{0^{+}}^{\infty} \vec{V}^{\tau} L(\omega) e^{j \omega t} d \omega .
$$

Transforning Bq. (1) to the energy dependent form

$$
\left.\left(E+\frac{\nabla^{2}}{2 m}-V\right) \tilde{\phi}(r, E)=\int_{E^{+}}^{\infty} \tau^{L}\left(E^{\prime}-E\right) \underset{\phi}{(r}, E^{\prime}\right) d E^{\prime},
$$

we see indeed that the term $\hat{\nabla}^{\mathrm{L}}(\omega), \omega>0$, appears in a source tem via which current frou wavefunction camponents $\bar{\phi}(T, E ')$ with energy $E^{\prime}$ is fed into components $(r, E)$ with the lower energy $E=E^{t}=\omega_{\text {, thas }}$ describing tenergy loss.

The initial condition for the solution of $\mathrm{Bq}$. (1) is that it starts out as a free normalized wave packet with mean momentum $\mathbf{k}_{\hat{f}}$. The corresponding bondary condition for $\mathrm{Fq}$. (3) is 1 that the solution $\tilde{\phi}(r, E)$ must consist of an incident free ways at energy $\mathbf{E}_{0}$

$$
\phi_{\mathbf{k}_{0}}^{0}(\mathrm{r}, \mathrm{E}) \cdot\left[\Delta\left(E-E_{0}\right)\right]^{1 / 2} \mathrm{e}^{\mathrm{ik}+\mathbf{T}}
$$

plus terms at all energies which asymptotically are purely outgoing. Here $h(x)$ is a narrow distribution about $x=0$, nornalized to mity. Equation (3) autconatically restricts the solution to energios $E . \leq E_{0}$, as is most easily seen by a perturbation theory argument. Asymptotically have

$$
\phi[\mathbf{r}, \mathrm{E})+[\Delta[\mathrm{E}-\mathrm{E}]]^{1 / 2} \mathrm{e}^{i \mathbf{k}_{0} \cdot \mathbf{r}}+\mathrm{F}(\Omega, \mathrm{E}) \frac{\mathrm{e}^{i \mathbf{k r}}}{\mathbf{r}}
$$

where the scattering amplitude $F(\Omega, E)$ detemines the differential cross section per unit energy for final energies $E<\mathrm{E}_{0}$ :

$$
\frac{d^{2} \sigma}{\text { didit }}=\frac{k}{k_{0}}|F(\Omega, E)|^{2} .
$$

The requirenent that during scattering the waye may lose energy but not probabi] ity current, implies

$$
\int_{0}^{E_{0}} d E[\tilde{\phi}(E), \operatorname{Im} V \tilde{\phi}(E)\}+\operatorname{In}\{\tilde{\phi}(E),
$$

The second ters in Eq. (7) receives gontributions onty froil those components in the bra $(\phi(E)$ which have energy $\mathrm{E}<\mathrm{E}_{0}$, end contain only outgoing waves; this

$$
\int_{0}^{E_{0}} d E(\tilde{\phi}(E), I m v \tilde{\phi}(E))=-\int_{0}^{E_{0}} d E\left(\int _ { 0 } ^ { E _ { 0 } } d E ^ { \prime } \left(\tilde{\phi}\left(E^{\prime}\right),\right.\right.
$$

where $G_{+}(E)=(E+i n-T-V)^{-1}$, Since the r.h.s. of Eq. (8) is negative, we see that the introduction of the energy losing potential $\mathrm{W}(r, w)$ requires the presence of absorption in the energy conserving potential, Im $\mathrm{V}<0$. This has a matural interpretation (cf. Eq. (3)): the wave component $\phi(E)$ at enerRy E receives current influx $v$ ia the source tem fról cc"monents $\phi\left(E^{\prime}\right)$ at higher channel energies $E^{\prime}>E$; but since in tarn this wave cortponent $\phi(E)$ appears again in the sarre term for components $\phi(E)$ with $\mathbf{E}<\mathbf{E}$, feeding current into the Iatter, this current loss for $\phi(E)$ must be accomted for $b y$ an absorptive part in the energy conserving potential $\mathrm{V}$. Condition (7) reads in the time dependent description

$$
\int_{-\infty}^{D} d t\{\phi(t),[\operatorname{In} V+\operatorname{Im} V(t]) \phi(t)\}=0,
$$




\section{$0 U: j$ \&}

probability:

$$
\{\phi(t), \phi(t)\}_{t=-\infty}^{t=\infty}=0 .
$$

The condition of probability cinservation thus gives rise to a relation between the energy losing potential $\mathrm{V}^{\mathrm{L}}$ and the absorptive part of the energy conserving "optical" potentalal. This relation is rather indirect, however, as it involves the wavefunctions and an integral over chamel energies or equitalently, time. An analogous relation between the imaginary part of the optical potential and the friction force appegrs in the perturbation theor' $\gamma$ of $n$ clear friction."

It is interesting to note that Eq. (3) is closely analogous to the urual system of cauled elastic and inelastic channels,

$$
\left(E_{0}=\varepsilon_{\mu}-T-v_{\mu v}\right) \psi_{\mu}=\sum_{\nu=0} v_{u v} \psi_{v}
$$

where the $\psi_{\mathrm{j}}$ are the channel wavefunctions and $\varepsilon_{\mu}$ is the internal excitation energy of the fraguents. Ordering the charnel labels according to fncreasing excitation energy $\Sigma_{\mu}$ (with dogenexacies taken care of appropriately), we can eliminate, in the equation for a given channel $\mu$, the coupling to all channels with lower channel energy $E_{\psi}=E_{0^{-}} E_{y}, E_{v}<E_{\mu}$,i.e. , hligher excitation energy $\varepsilon_{4}>E_{\mu}$ and obtain

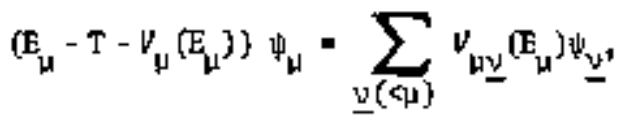

with

$$
\begin{aligned}
& v_{\mu}(E)=v_{\mu \mu}+\sum_{\substack{v, v^{\prime} \\
(>\mu)}} v_{\mu v}\left(\frac{1}{E^{*}-E+\varepsilon_{\nu}-H}\right)_{\bar{v} \nu} v_{v^{\prime}}
\end{aligned}
$$

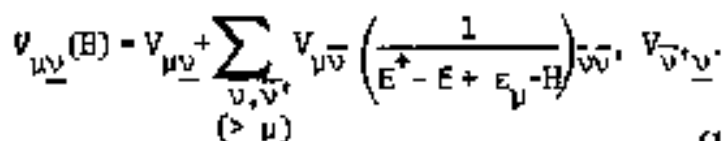

In Eq. (11) the channels are coupled via a triangular coupling matrix. The elastic chanel $\mu=0$ is uncoupled, and is described by the opticat-model oquation

$$
\left(E_{0}-T-v_{0}\left(E_{0}\right)\right) \psi_{0}=0
$$

Where the optical potential $v_{0}$ is given by Eq. (12) with $\mu=0$ [Geshbach's generallzed optical potential, but would in practice be constructed phenomenologically. The first inelastic chamel is explicitly coupled only to the elastic channel, but loses current to the second, third, etc, channels via the inaginary part of $v_{1}$. So it goes dom to the last open channel with $E_{\mu}=0$. The optical potential $V_{\omega}(E)$ in channal $\mu$ [ Eq. (12)] at energy $E_{\mu}-\mathbf{E}$ is quite similar in structure to the optical potential in the elast ic chansel, $v_{0}[\mathrm{E}\}$. In the coupling terms $v_{\mu \nu}$ we neglect the sum on the r.h.s. of Eq. (13) since,
In contrast to the analogous sum in Eq. (12), it consists of terns with indefinite sign. Thus we write $V_{\mu v}(E)=V_{\mu \underline{y}}$ in $\mathrm{Eq}$. (11).

In typical inelastic heavy ion scattoring situations the muber of thamels to be included in the system is very large. Going over to a continuous distribution of channel energies we may write

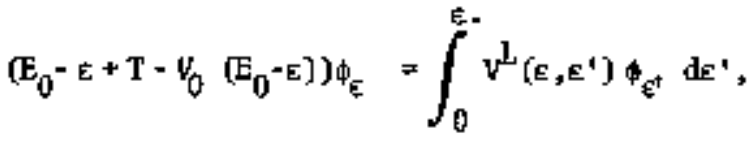

where the "chanme1 density wavefunction"

$$
\phi_{E}=\sqrt{\vec{D}(\epsilon)} \bar{\psi}_{\mu}(r)
$$

is the average 4 of the usual chamel wavefunction in the interval $\varepsilon-d s<\epsilon_{1}<\varepsilon+d \varepsilon$ raltiplied by the sqtuars root of the average number of channels per unit excitation energy. The integral kemel on the 1.h.s, of Eq. (15) is

$$
\begin{aligned}
& V^{L}\left(\varepsilon, \bar{\varepsilon}^{\prime}\right)=\sqrt{\bar{\phi}(\varepsilon)} \nabla\left(r ; \varepsilon, \varepsilon^{\prime}\right) \sqrt{\bar{\rho}\left(E^{\prime}\right)}, \varepsilon>\varepsilon^{\dagger}, \\
& =0, \quad \varepsilon<\varepsilon^{+},
\end{aligned}
$$

where $\nabla(r ; E, E)$ is soane average matrix element of the intermulitar interactlon taken between internal states with excitation energles $E$ and $E^{\prime}+$ If one simply sets $V^{L}\left(\varepsilon, \varepsilon^{\prime}\right)=V^{L}\left(\varepsilon-\varepsilon^{\prime}\right)$, then with $E_{q^{-\varepsilon}}=E$, $E_{0}-E^{1}=E^{\dagger}$.

$$
\left(E+T+V_{0}(E)\right) \phi_{\varepsilon}=\int_{E^{+}}^{E_{0}} \mathbb{V}^{L}\left(E^{\prime}-E\right) \phi_{\varepsilon^{\prime}} d^{\prime} .
$$

Identifying $\phi_{E}(x)$ with $\tilde{\phi}[\mathbf{r}, \mathbf{E})$, we find that Eq. (18) is the same as Eq. (3) (the integral in Eq. (3) is effectively cut off at $E^{\prime}=E_{0}$, owing to condition (4)]. The square of the outgoing part of the channel density watefunction (16) yields the scattering cross section per tuit excitation energy, as in Eds. (5) and (6). The energy conserving optical potential $V_{0}(E)-V_{U}(E)$ is complex and $t$ ive reveral nonjuvariant because in rearranging the original time reversal invariant systeal to obtain $\mathrm{Eq}$, (11), the boundary condition of outgoing waves in all chantels with lower channel energy has been incorporated in $v_{\mu}(\mathrm{E})$. The aurrent conservation relation (7) follows from the hemiticity of the original coxpled charmels problem $(10)$. We see that in physical content, the "smoothed-out" coupled channels problem (18) is closely analogors to a linear (as opposed to Ref. \$) Scturodinger equation with a tire dependent potential.

\section{Footmotes and Refiorences}

Condensed frum lBL-4333, subaitted to Phys. Rev. Lett.

ton leave from the Haln-Moitmer-Institut fir Kextiforschnng Rerlin, Berelch Kem- und Strahlenphysik, and Freie Universität Berlin, Fachbereich Physik, Bertir-West, Germany. 
1. A. Hessiah, Qunatum Mechanics, North-Folland Publ. Co, Ansterdan (1964), ch. $X$, Sec. S.

2. H. H. E. Gross, luecl. Phys. A240, 472 (1975).

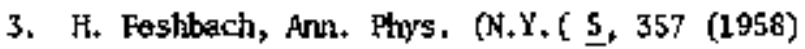

4. A. K. Kentan and S. E. Koonin, Physica Scripta 10A, 118 (1974).

5. X. K. Kan and J. J, Griffin, Phys. Lett. 50B, 241 (1974). K. Albrecht, Phys. Lett. 56B, 127

\title{
ONE BODY VISCOSITY; COMPARISON BETWEEN QUANTUM MECHANICAL AND CLASSICAL DESCRIPTIONS
}

\author{
ل Blockı, $Y$ Goneh, $†$ and $\mathbf{J}$ Randrupt
}

Recent calculations have shown that a one-body type of viscosity can play an extremely important rols in the dynamics of collective processes, 1 In this siturtion it seemed reasonable to make sconle fundamental studies in order to obtein a better understanding of the physical picture of the phenonencm we are dealing with. We decided to study the simple case of quadrupole vibrations and calculate how much intermal excitation is prohuced by the motion. In the quantum mechanical calculations we solve the time dependent single-particle Schrôdinger equatín using a hoods-Saxon potential adapted to the deformed shapes. The excitation energy is calculated simply as a difference betreen the actual time dependent energy at a given deformation and the ground state energy at this point.

In the classical calculations we assuned the model of independent particles colliding with boundaries chrmging in time (surface of the nucleus). In each collision the ehange of the velocity (or energy) of the particle can be calculated. Assuaing the initial distribution of the velocities of the particles to be thet of a Fermi gas and the initial density to be toniform within tho boundaries, we calculated tho energy gained by the particles during the motion of the boundaries using a bonte Car1o oumerical technique. On the other hand, on the assumption that the initial distrubution does not change too nuxh during the motion of the bondaries, w arrived at a simple analytic fonjala for the dissipation energy [see $J_{+}$ Blocki, J. Ranktrup, N. Robel, and it. J. Swiatecki, Ome-Body Dissipation and a New Dymamics, in this Annual Report). The fommia, apart from a constant factor depending on density and the sverage velocity of the particles, consists of an integral over the surface of the square of the nommal surface velocity. Once the time evolution of the surface is fonon the dissipation energy can be easily calculated with this expression. It seans wortinhile to establish on the one hand the correspondence between the quantum mechanical and classical picture and on the other the applicability of the analytic expression. We have done calculations of the quadrupole vibrations for six different values of the frequency, starting from the frequency, estimated for the Liquid Lrop Model and then increasing the frequency by doubling.

The comparison shows that the analytic formula cannot reproduce the Uonte Car1o and quantum mechanical results at low frequencies, beculise of their reversibility. At high frequencies the agreentent between all thres cases is wuch better; however, in the quantum mechanical calculations the dissipated energy is lower because of shell effects. We have also conpared the Monte Carlo results with the analytic expresston for low frequency vibrations of hìgher mtltipolarity. It was found that begineing from multipolarity eocual to six both curves are almost indistinguishabie, The reșutt indicates that the simple analytic formula can be applied in such processes like fission, where shapes of the nuclei are that of the higher multipolarity.

\section{Footnotes and Reference}

"Permanent address: Institute of Nuclear Research, o5-400 Swierk, Poland.

tPresent address: Nuclear Research Center, Negov, Beor-Sheva, P.O.B. 9001, Israel.

*Present address: Niels Bohr Institute, DK-2100, Copenhagen, Dennark.

1. W. J. SW Iatecki, in Proceedings of the International School Seminar on Reactions of Heavy Ions with Nuclei and Synthes is of New Elenents, Dubna, ЏडSA [Oct. 1975].

\section{ONE-BODY DISSIPATION AND A NEW DYNAMICS*}

J Blockı, J Randrup.t M Hobel, and W J Sinaleckı

Ordinary viscosity in liquits is the result of tho-body molecular forces which degrade nomiforifor collective flows into heat. A different, one-body nechinișth for exchanging energy betwen collective and intermal degrees of freedan is the collision of particles with a moving container wall. This energy 


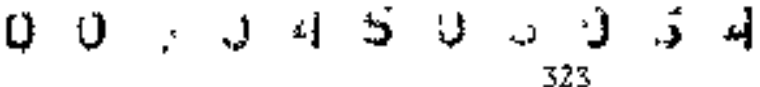

exchange between wall and particles, calculated to lowest order in the ratio of perticle to wall velocities, is responsible for the Ideal Gas Law pW = RT. By going to the next order in the ratio of velocities we arrive at the following 'wall formula" for the rate of energy flow $\mathbf{E}$ into a gas [with mass density o) composed of independent particles with average speed $v$, and contained in a vessel of fixed valume, whose watls deform with slow normal velocities it with respert to the buik of the gas

$$
\dot{E}=0 v \& \dot{n}^{2} \mathrm{~d} \sigma .
$$

\section{[For a degenerate Fenmi gas v $=(3 / 4)$ (Fenni velocity.]}

To illustrate the order of magnitude of the dissipation predicted by the above formula we added such a damping to the equations of motion of an idealized muclear droplet vibrating irrotationally around the spherical shape in its nth noltipole mode. One may define a factor of overdemping $\omega$, which gives the number of times the dissipation term exceeds the walue for which critical damping would occur for the given mode. The formula for $\nu$ is

$\left.\mu=\frac{3 \sqrt{3}(9 \pi)^{1 / 3}}{16} \sqrt{\frac{h^{2} / m r_{0}^{2}}{4 \pi r_{0}^{2} \gamma}\left\{\frac{n-1}{n}\left(n+2-\frac{20 x}{2 n+1}\right\}\right.}\right\}^{-1 / 2} A^{1 / 6}$,

where $m$ is the muclean mass, $\gamma$ the surface energy coefficient and $r_{0}$ the nixilear radius constant. The following table illustrates the degree of overdaruping for three idealized nuclei and four hampon-

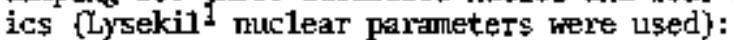

\begin{tabular}{|c|c|c|c|c|}
\hline & $\Omega=2$ & $\mathrm{n}=4$ & $\mathrm{n}=\$$ & $\Omega=16$ \\
\hline $\begin{array}{l}20 \mathrm{Ne} \\
10^{\mathrm{Ne}}\end{array}$ & 1.50 & 0.97 & 0.69 & 0.50 \\
\hline $1200^{5 n}$ & 2.62 & 1.44 & 0,97 & 0,69 \\
\hline${ }^{238_{8}}{ }^{2} \mathrm{~J}$ & 4.73 & 1,79 & 1.14 & 0.79 \\
\hline
\end{tabular}

The damping appears to be large (as with ordinary viscosity when applied to small systess'), but note that contrary to conventionally daniped drows the deroping is largest for long-wavelength modes fand large systems). A high ruclear viscosity is indeed implied by deep inelastle scattering, which suggests that nuclei often behave like two pieces of tar that get stuck but do not flow together. However, high two-body riscosity is inadmissible in fission ${ }^{3}$ where it would predict stretched out scission shapes leading to mwch too low fissfon "fragment kinetic energies. The stretching out is due to the stronger damping of short-wavelength modes (1ike necking) compared to a general elorigation. Since with one-body danping the situation is just the reverse, it is quite possible that in a fission process dominated by one-body demping the scission shape would be moderately cantpact and would reproduce the observed frapment energies.
To test this possibility Níx \& Sierk have made dymanacal sadale-to-scission calculations with tho one-boty dissipation replacing-ordinary (two-body) viscosity in the equatinns of motion. The resulting calculated fission-fragment kinetic energies for elewents throughout the forfodic table are compared with experiment in Fig. I. The solid curve shows that the calculation (which has no adjustable parameters) is not in drastic disagreement with experjment, even though the descent from saddle-toscission is overdamped (creepy). In contrast, an overdamped system with ordinary viscosity would correspond to the dashed curve, giving energies as much as a factor of 2 too low. The reason for the difference is as anticipated, nemely the greater compactness of the scission shapes in the case of the caloulation with the one-body dissipation.

In order to test whether the one-boty dissipation con indeed account for deep inelastic scattering as well as fission-fragnent energies, we have begun a dyramical study of rucleus-mucleis collisions in the presence of such dissipation. The relevant dissipation formula for the geometry of two mulei beginting their interaction by way of a neck or window of area $\Delta \sigma$ is the following "window for而星la"

$$
\vec{F}=-\frac{1}{4} \rho v[\hat{t}+(\hat{U}+f i] n]
$$

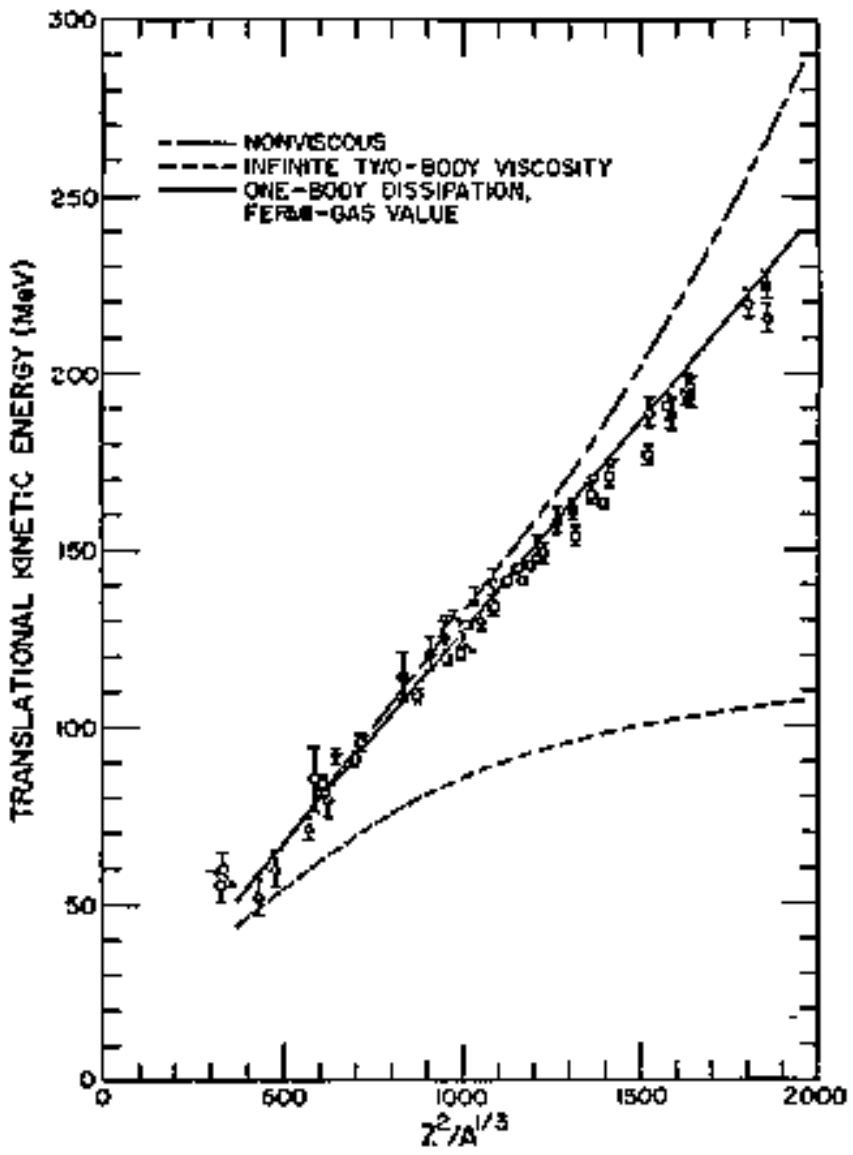

Fig. 1. Kinetic energies of fission frapments calalated without dissipation (dot-dash curve), with a large two-body viscosity (dashed curve) and with one-body dissipation (solid arre).

(XRL 766-8224) 
Here $\mathbf{F}$ is the dissipative force resisting the relative motion of the two nuclei, whose relative (vector) velocity is U. The symbol fi stands for a tuit vector normal to the window do. If the two nuclei are in grazing motion if is at right angles to $\mathrm{h}$, the term (U-î) $f$ is zero, and the result reduces to the window forwula of Ref. 2 ,

The order of magnitude of the frictional force predicted by the window formula appears to be sufficient to account for the damping of the kinetic energy in deep inelastic scattering. The remaining crucial question is whether one vill find at the same time that the mass transfer between the nuclei rentins smal1, as required by experiment.

In addition to canfronting the "one-body dissipation dynamics" with experiment we are testing the analytic thall formula" against

a) Meroscopic canputer caloulations of classical particles boumeing inside a deforming container,

b) The corresponding quantizod problem (work with $Y$. Boneh, et al. A Microscopic Method for Calculating the Intrinsic Excitation occurring in Mulear Fission in this Annual Report),

c) Self-consistent time dependent tartreeFock calculations (see J. Bdocki and $H$. Flocard, Tine Dependent Hartree-Fock Calculation of the Muclear Collective Motions, in this Anmulal Reporty.

The indications so far are that: (i) quantization does not irvalidate tho wall formula in any catastrophic way (as one might have feared) and (ii) that tho symetries of the nuclear potential well may crucially affect the fissipation, both in the quantal and classical calculations. (Konnin and Rardrup have recently derived a quantal wail formula wich reduces exactly to our expression in the appropriate 1 imit].

The emerging picture of the "New Dynanics" for macroscopic nuclear processes seems to be analogous to the Two-Part approach to nuclear statics:

\section{NEH DYAAMICS =}

\section{SHOOTH BACKCROURT + EFFECTS DF SMMETRIES.}

The "Smboth Rackgrand" is a type of dymanics gaversed by the batance of conservative and dissipative forces, the latter given approximately by the wall formula. Inertial effects, apart from translations and rigid-body type rotations, are often dopthated by the dissipation and may be disregarded in a first approximation. In that case the intrinsic shape of the macroscopic nuclear system changes according to a normal surface displacement to (at each point on the surface) whose time evolution is given by the following simple first-order differen tial equation (free of adjustable or unkown parameters].

$$
\delta q=-\frac{1}{\rho v}(\phi-\bar{\phi}) \delta \mathrm{t} .
$$

Here $\phi$ is the surface potential due to conservative and centrifugal forces and $\bar{\phi}$ is its surface average.

In cases when the topology of the system is about to change fas in the last stages of fission, or in tho initial stages of a nucleus mucleus collision) the wall formula for dissipation gives place to the window formula. (The transition ficm one to the other rengins to be worked out.) This is analogous to the need for a special Proximity For for the static nuclear potential energy, associated with changes in the topology of the systeta.

The secoond aspect of the New Dymanics, the "Effects of Symetrles," is analogous to major shell effects in ruclear statics, which have been shown in the past few years to be associated with symmetries in the nuclear potential well.

The working out of the New Dynamics and 1ts confrontation with relevant macroscopic nuclear exoeriments is our current objective.

\section{Footnotes and References}

*Ir collaboration with J.R. Nix and A. J. Sierk, LASL.

TPermanent address: Institute for Muclear Research, 05-400 3wierk, Poland.

* Permanent address: Niels Bohr Institute, DK-2100, Coperihagen, Dervark.

1. W, D. Nyers and W. J. Swiatecki, Axk, Fys. 36, $343(1967)$.

2. W. J. Swiatecki, J. Phys. Suppl., No. 8-9, 33 45 (1972).

3. J. R, Nix and A. J. Sierk, Physica Scripta 10A, 94 (1974). 


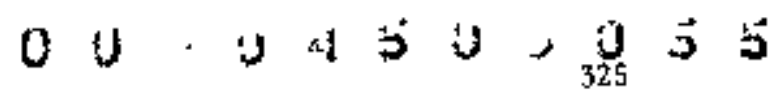

\title{
A MICAOSCOPIC METHOD FOR CALCULATING THE INTRINSIC EXCITATION OCCURRING IN NUCLEAR FISSION*
}

\author{
$Y$ Boneh $\uparrow J P$ Bboki : and W O Myers
}

One of the often observed fegtures of atomic muclei is the demping of large scale collective motion into intrinsic excitation. 1 (An excellent list of references related to the work described here is contained in Ref. 2.) The fact, that dipole and quadrupole vibrations are heavily damped while rotations seem to be maffected, indicates that the damping is associated with chonges in shape of the nuclear density distribution. The evaporation of neutrons from the separating fragments in nuclear fission is another indication that collective motion hes beem converted to internal excitation. The origin of this energy has usualiy been assoctated with vibrations of the separating riwclei as a consequence of their deformation at scission. This view is now bejng questioned because microscopic calculations ${ }^{2}, 3$ (1ike those described here) have show that considerable intemal excitation is to be expected at an earlier stage in the fission process during the descert from saddile to scisşion.

While the familiar phencmena fust montioned give clear indications of the joportance of nuclear damping none of them is quite so dranatic as thrt seen in the collisims of very heary lons (Refs. 1242 of our Ref. 2). The so-called "deep inelastic'" processes seen in reactions such as $\mathrm{Kr}$ on $\mathrm{Bj}$ are characterized by almost total damping of the relative motim of the colliding nuclei. The separating fragments seen to have started from rest and have only the nergy gained from Coulcmb repulstion.

Efforts to understand the damping of nuclear collective motion in terms of a classical hydrodynamic viscosity have not been very successful. calculations have shown that the measured asyplptotic kinetic energy release in fisslon ${ }^{4}$ is only conpatible with a relatively small viscosity coefficient while the "deep inelastic" processes in heavn ion reactions seem to require a large value. The idea of a hydrotynanic viscosity is also questionable on fundamental grounds. Such a daruping mecharism requires that the constituent particles have a rather short mean-free-path which seens to be incompatible with the fact that many nuclear phenomena are consistent with a completely independent particle Hodel.

One way to determine the inportance of the "single-particle" danping of collective motion into Intrinsic states is simply to calculate the excitation produced in a system of indopendent particles when the shape of the potential well is changed as a function of time. In this paper, which is a con. timation of kef. 5, we want to describe a particularly simple approach and some pretiminary applications that have been hade to the flsslon of 2360 .

We have solved the time dependent Schrödinger equation in two sequences of potential we115 whose shopes correspond to those deterribied by following the classical hydrodynamical evolution of the nucleus in the course of its descent frod saddle to scission 4 (see Fig. 1),

The Schrodinger equation was solved in a fixed basis corresponding to a shape irtomediate between the saddle and scission shapes using the method of Ref. 6. The time dependent equations were solved numerically with an inprowed version of the predictor-corrector nethod. 7 only neutron levels were considered in this preliminary work and we we did not include oither spin-orbit compling or resituml interactions.

The results gbtained in the damped bydrodynamic calculations for the fassion of 23 in indicate that of the $60 \mathrm{MeV}$ that becones available in the course of the motion ( $1 \mathrm{MkY}$ of kinetic energy in the fission direction and $59 \mathrm{MkV}$ from the saddle-toscission change in potential energy) approxinetely $21.3 \mathrm{HeV}$ is dissipated as a consequence of the viscosity assumed. Another $38.7 \mathrm{HeV}$ appears as collective kiretic energy. The time develoyment of these

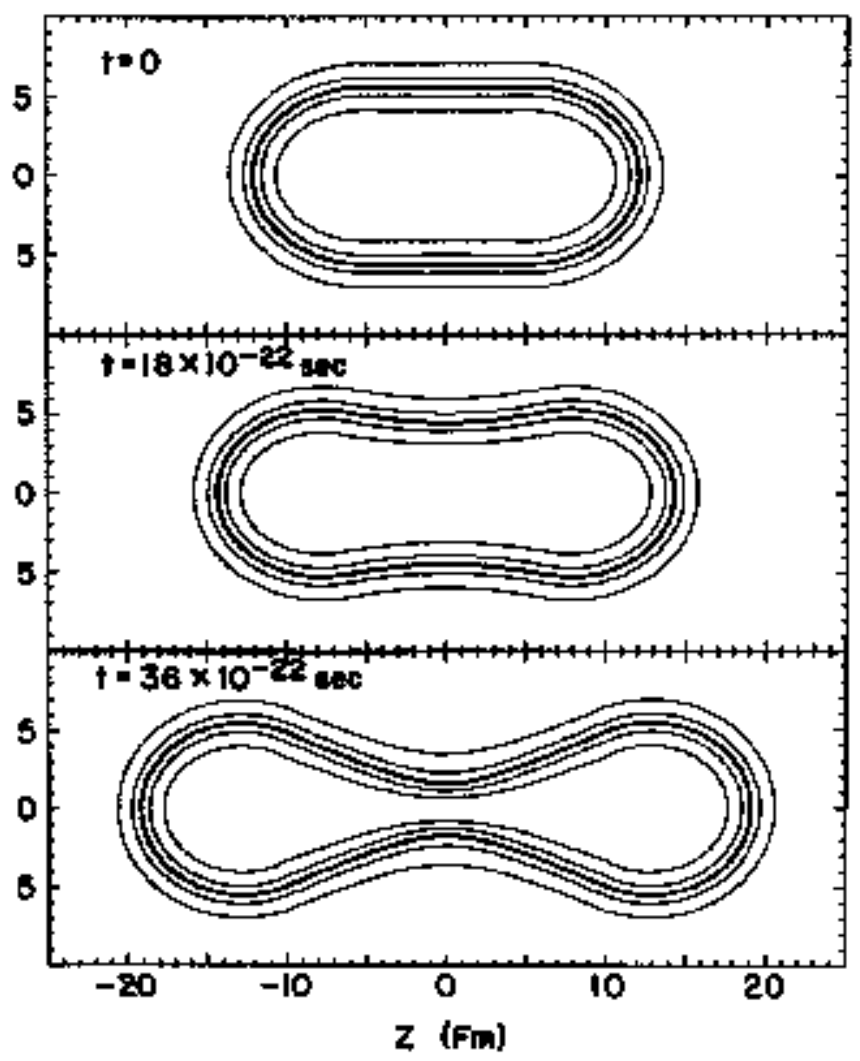

Fis. 1. The 10, 30, 50, 70 and 908 contours are shoum for three shapes in the sadile-to-scission sequence used in the calculations described here.

(XBL 763-2374) 
quantities is shown in Fig. 2. The dashed line indicates how the collective kinetic energy increases during the $38.5 \times 10^{-22}$ sec period necessary to move to scission. The solid line is obtained by adding the dissipated energy. [Both of these curves are scaled down by a factor of 144/236 so they can more easily be corpared with the microscopic calculation which was done for the neutrons alone.)

For croparison with the coliective kinetic erergy found in the classical hydrodyamic calculation we calculated the microscopic guantity $\mathbf{E}_{\mathrm{coll}}$, Where

$$
E_{\operatorname{coll}}(t)=\int \frac{1}{2} \rho v^{2} d r .
$$

The local flow velocity is calculated from the expression $\vec{v}=\vec{j} / p$. Where $\rho$ and $\vec{j}$ are, respectively, the quantum mechanical donsity and conrent.

The values we caloulated for $\mathbf{E}_{\mathrm{Coll}}$ all lit above the curve of hydrodynamic values in Fig. 2. This result might have been anticipated since the hyidrodynamic calculations assure almost irrotational flow which ylelds the lowest possible kinetic energy. The microscopic calculations are presumedty more turbulent.

The corparism of the dissipation energy in the two cases is not so straightforward. Because of the axial symmetry of the system the magretic quan-

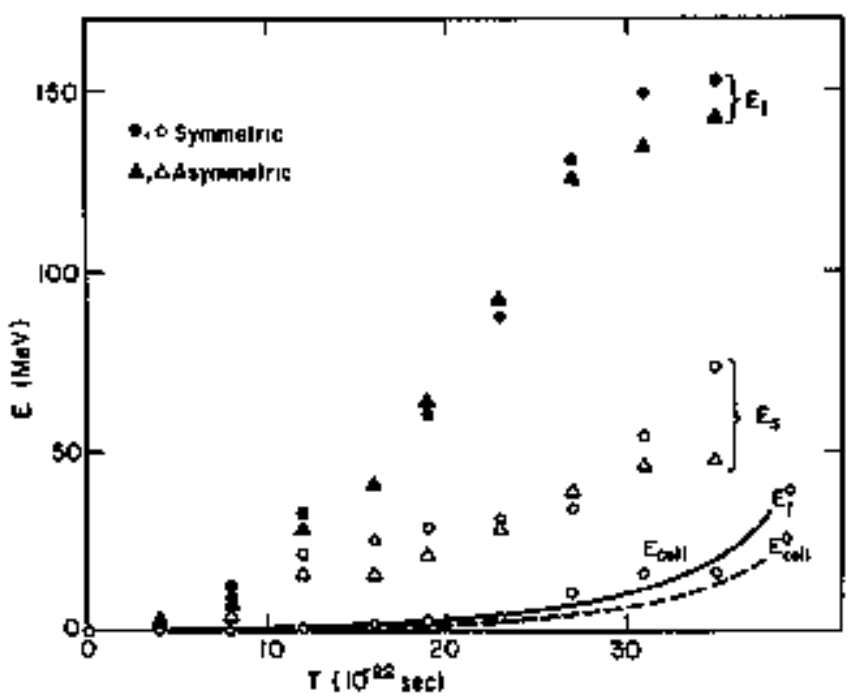

Fig. 2. The results of our microscopic calculations for the intrinsic excitation of the reutrons in 236, for a hydrotymamically determined sequence of shapes from saddle to scission. The dashed tine is the corresponding hydrodynamic collective kinetic energy (irrotational flow assmed) and the solid line is obtained by adding the intemal energy arising from the viscous damping of the motion. The circles represent the results of our calculation of $E_{\text {cot }}$ the collective kinetic energy, $E_{5}$ the total excitation and $E_{5}$, which is that part of the total excitation connected with the syumetry of the potential. The triangles represent these same quantities in the case when reflection asylfotety is introduced. tum number m of a given level must remain the same in the course of the motion. The sang is true for the parity $\pi_{z}$ in the case of reflection symmetric shapes. Consequently, even if the motion of the potential is extremely slow (adiabatic) the systen may ond un in an excited state since there is no way for a particle to change to an enpty fevel that moves down through the Fermi surface if its quantum mubers $m$ and $x_{z}$ are different from the levels being crossed. In our caiculatiors a substantial part of the apparent excitation energy is of this type. This part of the energy $\mathrm{E}_{\mathrm{s}}$ (where the subscript $\mathrm{s}$ indicates that it has its origin in symmetry effects] is simply the difference between the ground state of the system $\mathbf{P}_{0}$ (filling the lowest levels) arrd the "adiabatic" energy $\mathrm{E}_{\mathrm{a}}$ (whare the quantum nubers appropriate to the systen are conserved).

$$
\begin{aligned}
& E_{5}=E_{a}-E_{0} \\
& E_{0}=\sum_{i=1}^{N} E_{i}(\beta), \text { lowest N levels, (2) } \\
& E_{a}=\sum_{i=1}^{N} E_{i}(\beta), \begin{array}{l}
\text { lowest N levels having the } \\
\text { appropriate quantum nitubers. }
\end{array}
\end{aligned}
$$

In this expressions $B$ iș a one-dinensional deformatlon parameter measuring the distance along the dynamical path.

The total excitation energy $\mathrm{E}_{\mathrm{t}}$ is defined as the difference between the total energy $E$ of the systen described by the tire dependent Schrobinger equation and the ground state at the same value of 6 , where

$$
\begin{aligned}
& E_{t}=E^{*}-E_{0^{\prime}} \\
& E^{*}=\sum_{i=1}^{N} E_{1}[B(t)],
\end{aligned}
$$

and the $\left.E_{i} l \beta(t)\right)$ are the time dependent energy expectation values, of course, $E_{t}$ contains $E_{s}$ (the apparant excitation arising purely from symmetry), as well as $\mathbf{E}_{\text {coll }}$.

In Fig. 2 both the total excitation energy $\mathbf{E}_{\mathbf{t}}$ and that part arising from symetry $E_{s}$ are plotted against time for the two cases we have considered. one is the purely symmetric case and the other is a similar case where an asymuetry was grantually introduced along the trajectory so that the mass ratio was 1,4 to 1 at scission. The reason for comparing these two calculations was to determine whether the microscoplic dysamics would give preference to asymmetric scission shapes as has of ten been speculated.

The results of this work serve to draw atcention to the inportance of single particle damping for large scate collective motion. They show that the energy dissipation profile is quite different than that associated with a hydroct)mamic (or "twobody") viscosity. They also show (as has been frequently speculated) that asyumetric fission is preferred aver symetric fission on the basis of microscopic dynanical considerations. Another interesting result is that the microscopic collective kinetic energy is greater than that for irrotational flow indicating that sone turbalence is generated by the 
collective motion. Consequently, we are inclined to question the applicability of the hydrodynamical saddio-to-scission trajectories, Both the dissipation and intermal flow may well prefer a different sequence of shapes.

\section{Footnotes and References}

"Condensed from LsL-4374.

${ }^{\dagger}$ Present address: huclear Research Cester-Hegev, Beer-Sheva P,O.E. 9001, Israel; partially supported by an IAEA fellowship.

*Present address: Institute of Muclear Research, 02-400 Swijerke, Poland; partially supported by a Fulbright-Hays senior fellowship.

I. R, Wieczorek, R. W, Hasse and G. Sussinzan, in "Proceedings of the Third International Atonic Energy Agency Symoposium on Physics and Chenistry of Fission", Rocheșter, N.Y., 1975 (International Atomic Energy Agency, Viemna, 1974).
2. 5. E. Koonin and J, R. Nix, Phys. Rev, C13, z09 $(1976)$.

3. G. Schutte and L. Willets, in "Procedings of the Thiod Intermational Atonic Enorgy Agency Smposium on Physics and Chersistry of Fission", Rochester, $N$. Y. 1973 (Intemationa1 Atonic Energy Agency, Vienna, 1974).

4. K. T. R. Lavies, A. Sierk, and J, R. Nix, Los Alamos Scientific laboratory Report LA(Feb, 1976).

5. Y. Boneh and z. Fraenkel, Phys. Rev. C 10, 893 (1974).

6. H. C. Pauli, Phys, Rep. 7C, 35 (1973).

7. R. W. Hamming, J. Assoc. Comp. Mach, 6, 37 (1959).

8. According to a suggestion of S. E. Koonin.

\title{
MICROSCOPIC ANO MACROSCOPIC CALCULATIONS OF ONE-BODY DAMPING IN FISSION"
}

\author{
$Y$ Boneh,,$J P$ Blockl, f and W D Myers
}

As a part of a contiming study of the damping of large-scale collective notion into intrinsic excitation, we have solver the time-dependent schrödinger equation for a se्cguence of shapes corresponding to the fission of 230 t. These calculations, which are described in detail in Ref. I, were mot1vated by the fact that classical hydrotymanical calculations of the fission process sem to be incompatible with the strong damping observed in heavrion collisions. We wanted to compare the actual flow of energy into single-particle states with that calculated using a classical viscosity.

The inicroscopic calculations that have beell perfonad so far are not self-comsistent, and two mejor defects remain even when the viscosity has been varied in the hydratynamical calculations until the total energy absorbed is the same as that foumd in a microscopic calculation for the same sequence of shapes: ${ }^{2}$ (1) The calculations no longer agree with experiment, and (2) the energy absorption proflles differ along the trajoctory. These calcula. tions served to drandize the fact that the concept of hydrodynamic viscosity poorly represents the darping mechanism that creates the internal excitation in the single-particle caloulations.

In order to obtain results fran the hydrotysamical calculations that would be more nearly comparable to the microscopic calculations it was necessary to find a classical damping mechanism analogous to that sean in the microscopic calculations. Such a mechanisn is obtained ${ }^{3}$ from classical kinetic theory by carrying the system energy expressions, for volune conserving systems, to second orfer in the appro- priate expansion paraneter (the ratio of the wall velocity to the average velocity of the particles). We have used this method to calculate the damping to be expected from such a mechanism and have compared it with our earlier microssopic calculations, which are described elsewhere in this report.

Some difficulty is encolntered in interpreting the microscopic calculations because the special symmetries of the sequence of potentials that are enployed led to apparent excitation even in the case of adiabatic motion. In Fig. 1 have plotted the total vicroscopic excitation energy (along the saddleto-scission trajectory) minus the microscopic collective kinetic energy as the quartity Egax. Whan the contribution to the total energy from sommetcy effects is renoved the points labeled engit are obtained. In both cases we have we also considered another sequence of shapes derived from the symnetric ones by introducing a mass asymetry that increases 1 in. early from the saddle to a value of 1.4 at scission.

For comparison we have plotted the calculated value of the energy dissipation from the hydrodynamcal calctulations on which the sequence of shapes is based. For comparisgn with the microscopically determined energy flow into intrinsic states we also calculated the dissipation to be expected on the basis of the classical one-body damping expression mentioned earlier. ${ }^{3}$ The rate of energy absorption is given, in this theory, by the slifface integral

$$
\dot{\mathrm{E}}=\rho_{m} \overline{\mathrm{v}} \mathrm{g}_{\mathrm{n}}^{2} \mathrm{~d} a
$$




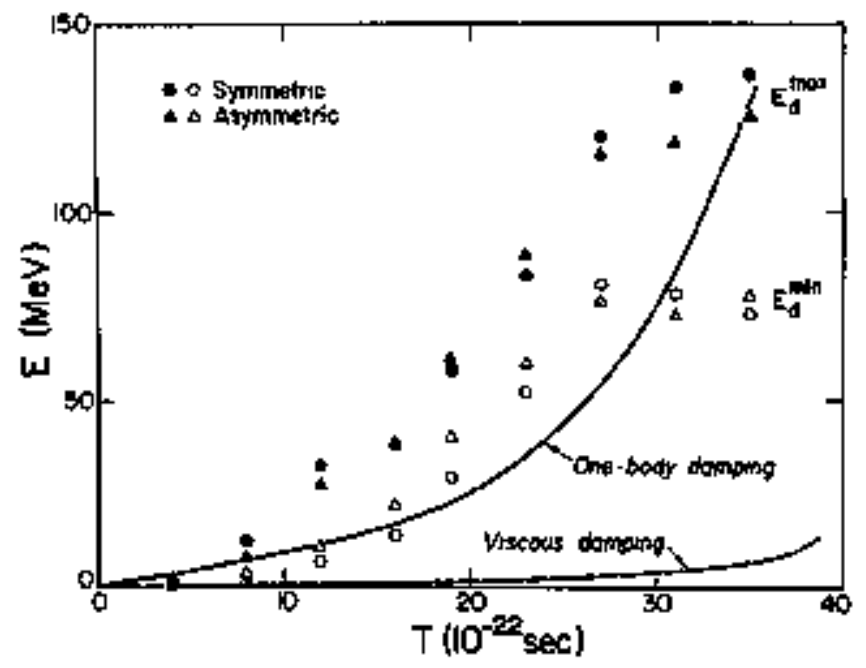

Fig. 1. The largest and smallest amouts of internal excitation consistent with our microscopic calculations are plotted as Egex and Bin for the two cases considered (symatrie and asymetric). For comperison the one-body damping fram Eq. (1] is showr as a solitd lire and the viscous damping (from the caloulation used to generate the shapes) ${ }^{2}$ is shom as a dashed line.

(XEL 764-2731)

where $a_{\mathrm{g}}$ is the mass density, $\bar{v}$ the average velocity of the particles $\left(3 / 4 v_{F}\right.$ in the Fermi gas model), and $\dot{n}$ is the normal velocity of the nuclear surface. Hote that unitke ordinary viscaus domping, this expression contains no adjustable parameters.

The results of this work serve to draw attencion to the importance of single particle damping for large-scale collextive motion. They show that the energy dissipation profile is quite different than that associated with a hydrodymemic (or "itwoboty") viscosity.
They also show that the results for one-body dissipaticin treated quantum mechanically and classically are very close and it seems that this type of dissipation is more neary comparable to that witeh takes place in muctei where an independent particle description certainly applies.

Classical hydrodynamicat calculations performed recently with this new type of damping give rise to a distinctly different sequence of shapes than those used here. 5 An important step the continuation of this work would be to perform the micros. copic calculations in potential wells following the new shape sequence obtained with the use of one-body damping to see if the darping along the path to sclssion is more nearly like that of the classical prediction.

\section{Footnotes and Roferences}

"Condensed fror lBL-4397.

†present address: Muclear research Center-Neger, Beer-Sheva P.0.B. 9001, Israel.

Permanent address: Institute of khaclear Research, 05-400 Swierk, Poland.

1. Y. Boneh, J. P. Bdocki, and W, D. Myers, lewrence Berkeley Laboratory Report, LBL-4374 (Feb, 1976).

2. S. E. Koonin and J. R. Nix, Phys. Rev. C 13, 309 $(1976)$.

3. W. J. Swiatecki, in "Proceedings of the Intert. Schno1 Semjuar on Reactions of Heavy Ions with Muclei and Synthesis of New Eletents", Dubma, USsR [0ct. 1975).

4. K. T. R. Davies, A. J. Sierk, and J. R. Nix, los Alamens Seientifle Laboratory Report LLA-UR-76361 (Feb, 1976).

5. J. R. Nì and A. J. Sierk, private cutrinication.

\title{
FURTHER STUDIES ON SPONTANEOUS-FISSION HALF-LIVES FOR EVEN NUCLEI WITH $Z \ni 20^{\circ}$
}

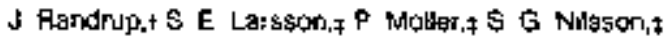 \\ K Pomorski,5 and A Sobuczewski*"
}

Since an earlier semi-empirical study of the spontaneous-fission process for transthoriun elements, inproved theoretical deformation-energr surfaces heve been obtained, 1 based on the ModifiedOscillator single-particle model and the macroscopic Droplet Mode1. 2 In those caloulations the effect of axial asymetric distortims as well as reflection asymetrfic distortions is included in a more accurate way,

On the basis of new calculational material, we have considered the even-ever elements beyond thorium, for the purpese of increasing the accuracy of the theoretical calculations of the spontaneous fission half-lives.

The fisston process is treated as a penetration through a one-dimensional potential barrier, calculated along an effective path in the multidinensional deformation space. The penetrability is calculated in the NKB approxination. Thus one reeds the action integral $K$ along the fission path, 


$$
x=\frac{2}{h} \int_{r_{1}}^{r_{j}} \frac{}{\sqrt{2 B(r)\left(V(r)-E_{v i b}\right) d r} .}
$$

The path is described by the fission coordinate $r$. $V(r)$ is the deformation energy of the shape described by $T$, and $B(r)$ is the effective inertial mass function corresponding to a wotion along the fission path. Moreover, Evib denotes the fission mode zero point energy in the initial state; it is taken to $0.5 \mathrm{MeV}$. The integral limits $r_{1}$ and $r_{2}$ are the entrance and exit point, respectively. From the integrated astion $K$ the half-life $T 1 / 2$ is given by the relation

$$
T_{1 / 2}=10^{-28,04}\left(1+e^{K}\right) \text { years. }
$$

The fission barrier potential $V(t)$ is calculated theoretically. In the present study $V(x)$ is based on recent calculations presented in Ref. 1 .

At present the theoretical calculations of the effective fission inertial-mass function $B(r)$ are less accurate them the calallations of the potential $V(r)$. Therefore we introduce one overaIl massrenomalization parameter which is subsequently determinet by fitting to the known halfilives. In this paper two trial forms of $B(\mathbf{r})$ are studied. One is based of macroscopic (hydrodymamical) irrotationalflow calcolations and is similar to what was previously studied. In didition to this, we have calcu1ated microscopic inertial-plass parameters in the cranking approximation and used these for the determination for the effective fission inertia.

According to the somi-emperical method, the fission barrier potential is generated from the statínary points on the deformation-energy surface (i.e., the uninina and saddle points) together with one (or more) additionaI point(s) in the exit region beyond the last saddle point.

We have included the effect of axially asym ${ }^{2}$ metric muclear shapes as described by the $y$ defommtion coordinate. A detailed study of the $\gamma$ deformation in the actinide region is performed in Ref. 3 and a similar study for the supor-heavy region in Ref. 4. Here we put the emphasis on the most recent results employing a three-dimensional variation in the $\left(\varepsilon \varepsilon_{\mu} \gamma\right)$-space.

In the nuchear region consiteres here, reflectìn asymetric shapes play an important role for large nuclear distortions. For our present study we have employed the reflection-asymenetry corrections to the second saddle given in Ref. 5. They were obtained by calculating a two-dimensiomal mp using a a conbined symetrical coordinat $E_{24}$ and a cchhived asymetrical coordinate $\varepsilon_{35}$.

Whe have also statied the effect of $P_{6}$ deforma$t$ inon at the grourd state minimu. The $P_{6}$ deforma tion energies used are those given in Ref. 1. Most of the ruclei considered hare some $P_{6}$ deformation in the grornd state and it turns out that the ineIusion of the correction inproves the overall fit to exper- iment by a factor of around five. For this reason we have inctuded this effect in the fission barriors.

The explicit form of the macroscopic inertial mass function is

$$
\mathrm{B}_{\mathrm{r}}^{\operatorname{macro}}(\mathrm{r})=1+k_{\frac{17}{15}} \exp \left(\frac{c}{\pi}\left(\frac{3}{4}-\mathrm{r}\right)\right)
$$

Here $I$ is,measured in units of the nuclear radius $R=r_{0} A^{1 / 3}$ and $B_{T}^{\text {macro }}$ is measured in toits of $k$, the reduced mass of the final two-fragment systeml (which we asgeme to be symuetrical). Mrreover, $\lambda$ is a numerical constant equal to $1 / 2,452$ deternined from a fit to the exact curve for $B_{r}$ irrot

In addition to the renomalization parameter $\mathrm{k}$ we have introducad a second parameter o governing the slope of the mass function. For $k=c=1$ the irrotational-flow inertial-mass function is approximately reproduced.

As an altemative, we have calculated the spontaneous fission half-lives on the basis of inertialmass parameters calculated in the cranking approxination.

One adfustable parameter is introduced in this case in the form of the overall renomalization factor $p$. One should bear in mind that becausa of the approximate treatment of the inertial matrix one carmot expect o to come out exactly equal to unity, even if the basic inertial-mass parameters were correct. On the other hand, since the adopted treatment is expected to include the main part of the inertia, the factor $p$ should not be too different from unity, Recalling the enpirical finding that the microscopically calculated mass parameters in the rotational case tend to cone out somewhat too large. it appears satisfactory that the best fit is obtained for $\rho=0.80$.

With the figsion barrier potentials established as described, we have, using the different sets of trial inertia1-mass furctions, calculated the spontareous=fission half- 1 ives for the known eveneven nuclei ranging from $232_{\mathrm{U}}$ to $25 \mathrm{~N}_{\mathrm{NO}}$. Mininiza. tion of the average logarithoic deviation $\Delta$ of the calculated half-lives frcm the experimental ores determines, for each set of intertial functions, the adjustable parameter(s) enterisg in the trial functíons.

For the hydroctynamic type inertial mass fucc tion with $c-1$ the best reproduction of experimental ha1 $f-1$ ives is obtained for $k=11,5$. This value is lareer than the previously employed value of 10.0. Thts change is mainly due to the inclusion of the $\epsilon_{4}$ dependence of the $r$ coordinate. The average logarithuic deviation is $\Delta=1.7$, which corresponds to a factor of around 50 .

The half-1ives obtained with this type of effective mass function are shown in Fág. I together with the experimental data.

The iscrueric fisstion tests a nore 1 imited part of deformation space as it only explores the second barrier region and this serves to favor one solution. 
In Fig. 1 he have included some calculated (with $k=11.5$ and $c \times 1.0$ ) and experimental isomerichalflives. For the calculated isomeric half-lives there is a pronounced erroneous trend in going from $U$ through $\mathrm{Pu}$ to $\mathrm{Om}$. For the two measured $\mathrm{l}$ iscmers $(236,228 \mathrm{U})$ the calculated values are around five or ders of megnitude too long. This may indicate thet the second barriers of these jsotopes are too large. one should notice that for $234 \mathrm{~J}$ the calculated value is of the same order as the two experimental nubers

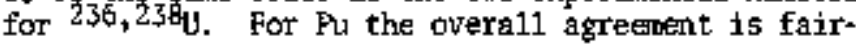
Iy good but the isotopic variation is in error. This may reflect the sensitivity to the more detailed structure of the second barrier, which we treat here in a rather crude manner. Finally, the calculated values for 242,244 Co come out around seven orders of ragnitude too short as connluard with experiment. The similar calculations with $k-6.5$ and $c=0.5$ yield isomeric half-lives which are one to two orders of magnitude longer (this change can be estimated). Although the present results for the isomeric halflives are far fion satisfactory, the resutts do seen

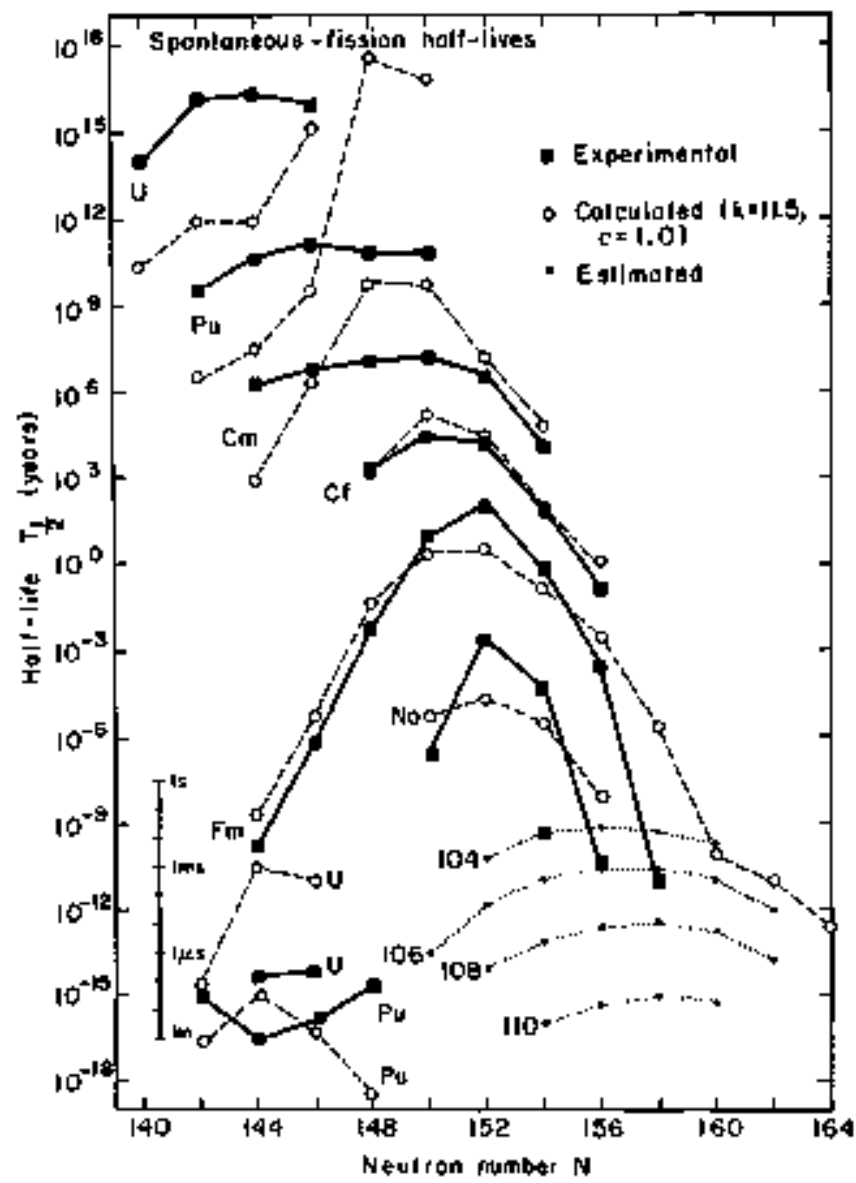

Fig. 1. Spontaneous-fission half-lives calcollated with the macroscopic inertial-mass functions having $\mathbf{k}=11.5$ and $c=1.0$ (open circles joined by dashed lithes). The experinental values are indicated by full squares joined by full lines. In the lowerleft corner are included some iscmeric half-1ives. Also included in the figure are the half-Iives calculated with the estimated approximate barriers for the transuranium elements (dots joined by dotted lines].

(XEL 756-3150) to point in fawor of the irrotational-type slope, i.e., a value of the slope parameter $c$ not far fros unity.

Sipilarly, for the microscopic inertial-mass functions the renomalization factor $p$ is determined by fit to experinent. The optimm fit obtains for $p=0.80$, corresponds to $b=1.3$, which is a factor of aroumd twenty. One should thus note that the aicroscopic calculations with one overall nomalitation factor leads to a fit which is equally goot as that obtained with two parameters in the case of a macroscopic type inertial function. Figure 2 shows the corresponding results for the spontaneousfission half-lives. Also in this figure we have included iscineric half-lives.

We have otade preliminary estimates of the spontaneous-fission and alphe decay half-lives for the olments with $Z$ between 104 and 11D. For this purpose we have estimated the fission barrier potentials by calculating the deformation energy in five deformation points fixed a priori.

Beyond nobelium the theoretical fission barriers have only one peak. Consequently, the calculated fission half-lives are relatively short (at most milliseconds) and, moreover, exhibit a smooth variation wh neutron numer. It follows from our

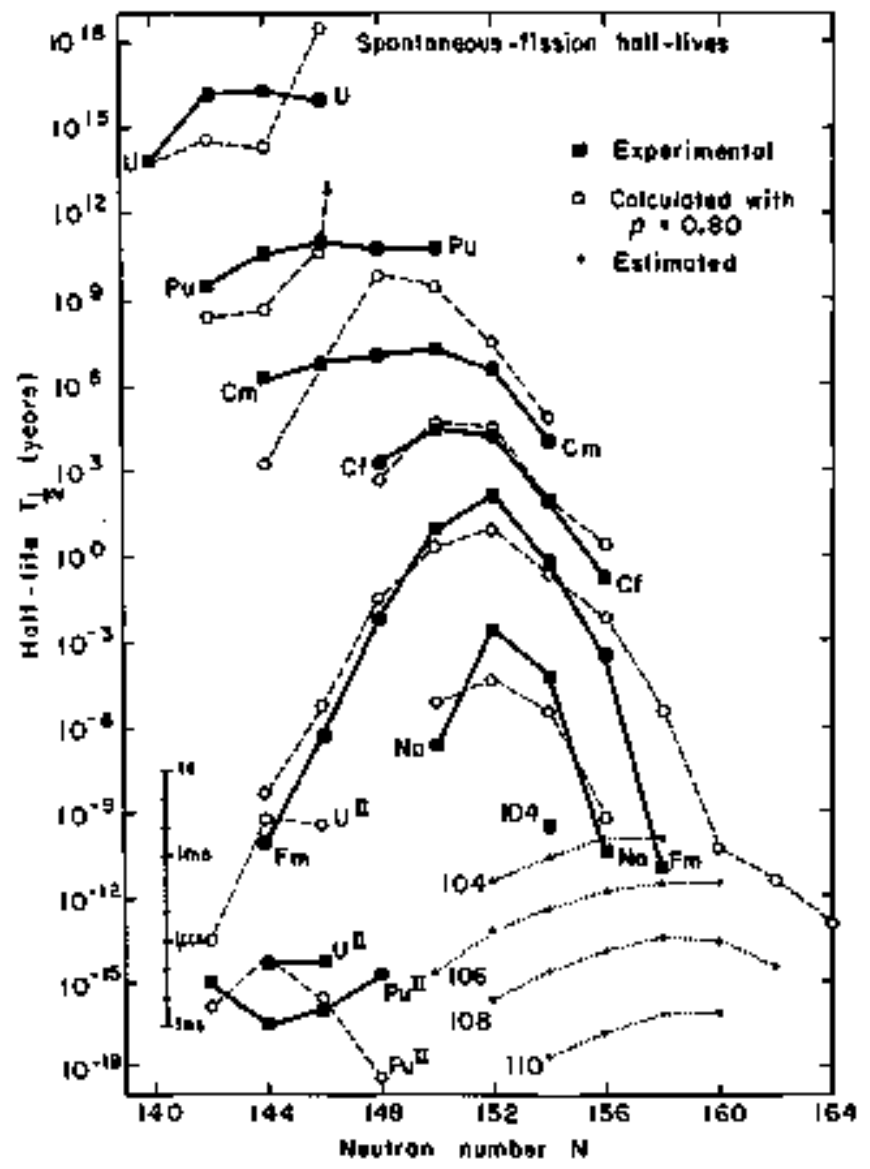

Fig. 2. Spontanecus-fission half-lives calculated with the microscopic inertial-mass functions having $\rho=0.80$. Similar to Fig. 1 .

(2सI. $756-3149$; 


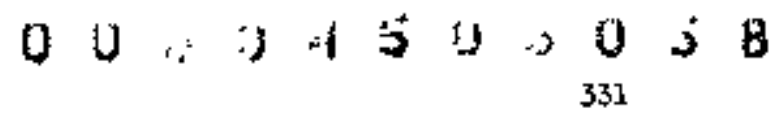

remarks above that the existence of a shell at $N$ 152 could drastically change this behavior in the neighborhood of that neutron number. A possible experisental measurement of the spontaneous-fis. sim half-Iife of the isotope 25604 wold add a valuable piece of information concerning this crucial point.

Taken at their face value, the pressent calculations indicate that the isotopes of the element 106 generally have spontaneous-fissich half-lives which are shorter by a factor of exound 3n thar the 104 isotopes with the stime neutron nuber, This suggests that even 106 isotopes may exist with a spontanegus-flssion half-life not much shorter than that of $25 \mathrm{Fm}$. Because of the uncertajity arising from the $N=152$ problem it is not possible to predict with confidence which neutron nuber will give the largest stability.

The calculated half-1ives for the element 108 show the same trend as thos for 106 but are generally a factor of around $10^{2}$ sharter. The halt-lives of the 110 isptopes are further reduced by a factor of $10^{3}$, approximately.

It appears that more accurate theoretical predictions for this particular region of muclei cannot be made tontil more accurate defomation energy surfaces have been established.

In our previous calculations ${ }^{7}$ of the spontaneous-fission half-1ives for the superheavy elements the $E_{4}$ dependence of the $r$ coordinate nos not included. A consistent inclusion will lead to longer half-life predictions for muclei with less exterkded barriers. We have therefore reexamined the superheavy region, with the present more complete treatment.

On the whole, the resulting effect is small, particularly so for the most stable nuclei near the certer of the superheavy island. For nucloi with half-1fves of around one year the increase in the stability only anounts to less than a half order of matitude. 'The general conclusions of our previous study in the superheavy region are this not significantly altered by the present investigation.

\section{Footnotes and Referefices}

"Condersed frán L.EL-4036.

$t^{\prime}$ on leave from the University of Aarhus, Aarthus, Detriark.

"Department of Mathosmatical Physics, Lurd Institate of Technology, Lund, Sweden.

${ }^{5}$ Institute of Physics, Marla Sklodowka-Carie Univer" sity, Lublin, Foland.

** Institute for Nuclear Researeh, Hoza 69, PL-0D681 Harszawa, Poland.

1. S. E. Larsson, P. MB1ler, S. G. Nilsson, I.

Pagnarsson, J. Randrup, C. F. Tsang, Nuc1. Data, to be sutuitted.

2. N, D, Myers and w. J. Sviatecki, Ans, Fhys, 84, 186 (1974).

3. S. E. Larsson and G. leander, Proceedings of Third IAEA Symposium on Physics and Chemistry of Fission, IAEA, Rochester, N. Y, (1973), p. 177.

4. 5. E, Larsson, P. Möller, and S. G. Nilsson, Physica Scripta tod, 53 (1974).

5. P, Míller, huc1. Physs. A192, 529 (1972).

6. E. D. Figet and J. R. Nix, kuc1, Phys. Al93, $647(1972)$.

7. J. Rartrup, S. E. Larsson, P. hbller, A. Sobiczewski, and A. Kukgsiak, Physica Scripta 10A, $60(1974)$.

\title{
INTERSTELLAR DEUTERIUM ABUNDANCEE AND THE CLOSED VERSUS OPEN UNIVERSE
}

\author{
Elvabeth A Pauscher
}

With the advent of Einstein's general relativity theory in $1915^{1}$ the distribution of matter ard energy becane describable in tens of the gecmetric structure of space. The spatial distribution of matter-energy in a localized region of space determines the local curvature of space in that region. In 1919 with the occurrence of a coiplete solar eclipse in Chili, Einstein's theory appeared to be tested. ${ }^{2}$ Light from distant stars is bent in the gravitational space of the sun or to say this another way, the space next to the sun is curved due to the mass of the sum, and light from distant stars follows the shortest pathlength which is a geadesic of the curved space. 3,4
Within the context of this curved space description, one can ask what is the natrre of the carrvature of space of the entire universe, If the curvature due to the universe matter energy content is everywhere and always positive (and enough matter is present), thon the universe will be finite and closed. From Einstein's field equations, (which describe the dynamical evolution of the universe and the marmer in which matter and energy curve spacce) we car define three possible miverso wode1s, 5 We can define these three models in terJas of the solutions of Einstein's field equations where have the curvature constant, $k$, derived from the curvature in the field equations. ${ }^{6}$ Then, we have three possible values 
of the curvature of $k \cdot 0, \pm 1$ which corrosponds respectively to Euclidian (flat space), closed, or open universe models.

Before proceeding further, should mention several assumptions. They are that the universe is isotropic and honogenexts (called the cosmological principle) in which stars and galaxies are just

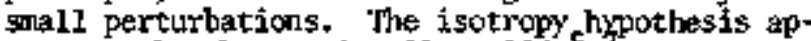
pears to be observationally valid. $\mathrm{S}$,

Two other observations are of particular intorest. The füst is that made by E. P. Fubble in the early $1920^{\circ} \mathrm{s}$ that the universe was dynamite and expanding. In 1915 it was thought that the miverse whs fixed and static so that, although einstein's field equations describing the maner in which the matter-energy content determined the aurvature of space, he added the "cosnolfgIcal constant" to give static solutions to his equations. 7 (Later, einstein was to have said that his was the bijgest mistake of his tife!)

Another stonfficant prediction and observation was the hypothesis of the "bIg bang" cosmology theories of A.G.E. Letraitre and G. Gantow, 10 in observation of the $3^{\circ} \mathrm{K}$ black body radtationl in the $1960 \mathrm{~s}$. The bis hang model assumes that all the matter in the universe originated from a bighly condensed, singular state and that it exploded and is expanding at the rate Hubble predicted. 8

The Huble recession is determined from the red shifting of the spectra observed from stars. Independentiy, a distance scale can be set.12

The Hubble's constant is then defined as $H=(k / R)$ or the velocity of recession over the distance anay from us. The inverse of $\mathrm{H}$ or $\mathrm{t}_{0}<1 / \mathrm{H}$ sets an upper limit on the age of the universe. From the solutions to Einstein's fie]d equations we can derive the relationship of the eritical density for a just closed undverse and the Hubble's constant, 5 or $p_{C}=3 H^{2} / B \pi G$, where $G$ is the gravitational constant.

Since the field equations do not specify $k$, the curvature, how can it be determined? one observation may be a critical and sensitive tost and that is the amount of interstellar deuteriun 13,14 In the early stages of the evolution of the universe, the temperature was very large; initially (according to sore nodels), the big bang temperature was, $1 \sim 10^{32}{ }^{\circ} \mathrm{K}$. The onset of nucleo synthesis occurs around $\mathrm{T} \rightarrow 10^{\circ}{ }^{\circ} \mathrm{K}$ (called the $\mathrm{T}_{\mathrm{g}}$ era) in which the temerature is cool enough for nucleons to stick together. Since stellar processes appear to destroy denterium, it is then assumed that all the present deuterium abundance was created in the $T_{9}$ era. 5 (Stellar creation occurs aroumd the $\mathrm{T}_{4}$ (or $\mathrm{T} \sim 10^{4} \mathrm{~K}$ era.) There are approxinately 144 reactions which detennine the deuterlin abundance but there are six primary ones that we need to ccinsider. 13 We can relate the decay constant of those six reactions to the nuelear species concentration and express these in ternis of the baryon nunber density and the temperature in $T_{9}$ enits. 13

Deuterium is created by the reaction $n+p+D+\gamma$. Most muclear processes "bun" or destroy deuterliki. (See Ref. 5 and Teble 1 for the five other reactions.) Also in Ref. 5 is given scre observed values of neutral interstellar deuterium ratio to neutra1 hydrogen is about $\mathrm{D} / \mathrm{H} \sim 10 \mathrm{~s}$. Analysis of the six equations gives an 8th power law of the mass
Table 1. Six basic reactions for the alumbance of desterium.

\begin{tabular}{|c|c|c|}
\hline & Reaction & 8 \\
\hline (1) & $\mathbf{n}+\mathbf{p} \rightarrow \mathbf{D}+\mathbf{Y}$ & $+2.22 \mathrm{MeV}$ \\
\hline (2) & $D+D+{ }_{H}+p$ & $+4.03 \mathrm{seV}$ \\
\hline (3) & $\mathrm{D}+\mathrm{D}+{ }^{3} \mathrm{He}+\mathrm{n}$ & $+3.25 \mathrm{MeV}$ \\
\hline [4] & ${ }^{3} \mathrm{H}+\mathrm{D} \rightarrow{ }^{4} \mathrm{He}+\mathrm{n}$ & $+17.60 \mathrm{MeV}$ \\
\hline$\{5)$ & ${ }^{3} \mathrm{He}_{\mathrm{a}+n}+{ }^{3} \mathrm{H}+\mathrm{p}$ & $+0.76 \mathrm{MeV}$ \\
\hline (6) & ${ }^{3} \mathrm{He}+\mathrm{D}+{ }^{4} \mathrm{He}+\mathrm{p}$ & $+18,35 \mathrm{KeV}$ \\
\hline
\end{tabular}

Note that $\mathrm{D} \Xi^{2}{ }_{\mathrm{H}}$, and $\mathrm{t} \in{ }^{3} \mathrm{H}$, and $\mathrm{p} \equiv \mathrm{H}$. Equation creates $D$ and all others bum $D$ to ${ }^{4}$ He essentially.

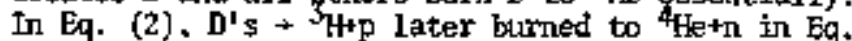
(4), $\mathrm{p}^{\prime} \mathrm{s}$ and $\mathrm{n}^{\prime}$ 's produced are binned in $\mathrm{Eq}$. (1). In Eq. (1) the only tie to radiation as $y^{+} s$ is created.

fraction of dectorium near the critical density, or $D / H \infty p_{b}^{8}$ near $p_{0}{ }^{*} p_{c}$. Recalling that the critical density for a just closed universe depends on the Hubble's constant squared, we then have a range of possibte values. The recessional red shift is quite well deterinined but the distance scale is not so well known. Althotuh previous values of $\mathrm{H}$ and hence $p_{C}$, conpared to the observed D/H ratio Implied an open cospology, recent observations and determinations of $\mathrm{Ft}$ imply that a cloced cosmoIogy $(\mathrm{k}-+1)$ is also possible. See Fig, 1, 5,6 For $\mathrm{H} \times 50 \mathrm{~km} / \mathrm{sec}+1 \mathrm{pc}$,

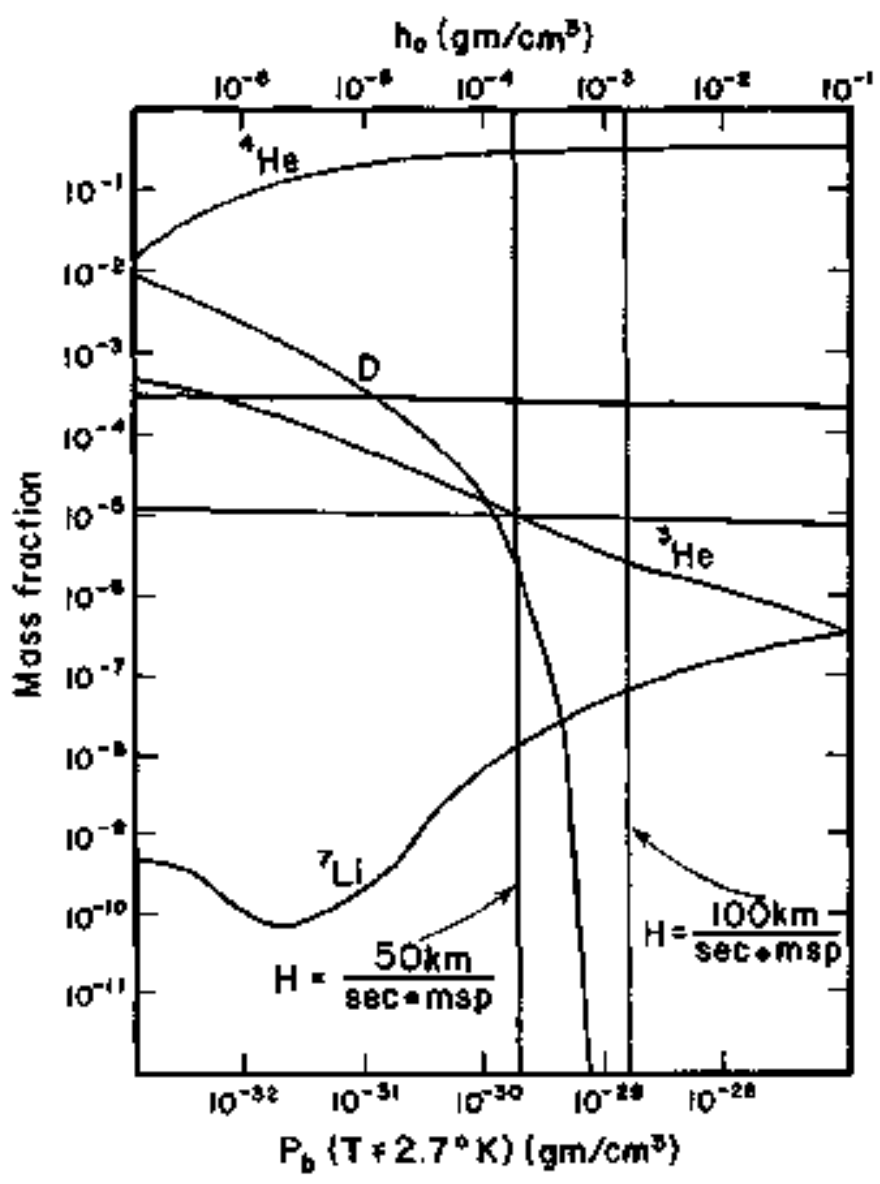

Fig. 1, Ratio of deuterium to hydrogen abundance us nuclear density.
(XRL $760-8225)$ 


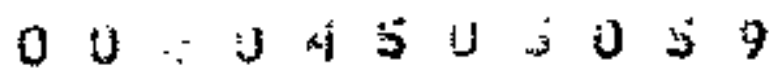 \\ 333}

Pc $4 \times 10^{-30} \mathrm{gnd}^{\mathrm{m}} \mathrm{cm}^{3}$ ibteh is consistent with the $\mathrm{D} / \mathrm{H}$ ratio. 16 (A parsec is 3.26 light years, where one light yoar is 5.8 trillion miles and Spe stands for mega parsect.)

The theoretical determination of the curvature constant is given in Ref. 17 in which an additional constraint on Eistein's field equation is given in terus of gecoetrical constaint which are expressed in terms of universal constants. The value of the curvature constant is found to be positive, giving closed cosentoglcal solutions.

\section{References}

1. A. Einștein, Annalen dẹr Physsik. 49, 169 (1916).

2. C. W. Misner, K. S. Thorne, and J. A. Wheeler, Gravitation, (W. H. Freedman, 1973).

3. W. Pauli, The Theory of Relativity, (Pergaton Prest, N. Y, 1958).

4. A. Einstein, The Meaning of Relativity, (Princeton University Press, New Jersey, 1955).

5. E, A, Rauscher, LBL-4353, Decenber, 1975.

6. E. R. Harrison, Amn. Phys, 35, 437 (1965).
7. E. A. Rauscher, UOPL-71435, October 1968 and Bul1, Am, Plyrs. Soc. 13, 1643 (1968).

8. E. P. Hubble, Proc. Nat. Acad. Sci. 15, 169 (1929).

9. A. G. E. Lenaitrs, The Prineral Aton, Wan Nostrand Press, N,Y, 1\$50).

10. G. Ganow, Nature 162,680 (1948).

11. R. H, Dicke, P. T. E. Peebles, R, G, Roll, and D. T. Wilkenson, Astrophys, I. 142, 4I4 (1965),

12. Edited by Hogr-Yee Chiu and Amadur Mriel, Stellar Evolution, MIT Press, Canbridge, Mass., 1972).

13. R, V. Wagoner, W. A. Fowler, and F. Hoyle, Ast rophys, J. 148, 3 (1967).

14. R. V. Wagoner, Astrophys. J. 179, 343 (1973).

15. P. J. E. Peebles, Astrophys. J. 146, 542 (1966).

16. R. P. Kirgher and J. Kwan, Astrophys. J. 193, $27(1974)$.

17. E. A. Rauscher, Nwow Cirsento lett. ㅁ, 925 (1972). 
$00: 0450 \div 060$

III. APPARATUS 


\title{
$00,0430>3951$ \\ A. ACCELERATOR OPERATIONS AND DEVELOPMENT
}

\author{
88-INCH CYCLOTRON OPERATION, DEVELOPMENT AND STUDIES
}

J Bowen, [. J Clark, P E Frazer, L Glasgow, R A Gougti.

D L Hendree, W A Holley, A bann, and D Morns

During 1975 and the first half of 1976, the cyclotron was scheduled for 20 ight-hour shifts per weak for experinents in muclear science, isotope production, and bean developant. Distribution of beam time for 1975 is shown in Table 1, while an taplated particle distritution bistory is shown in Fig. 1. Table 2 lists extermal beam intensitios of ions which have been accelerated through bay 1976 along with the energy range available with each jon. New,

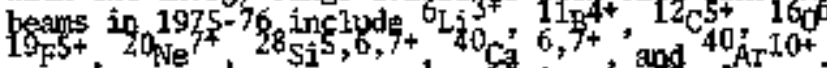
Now spectal-isotope beans of $\mathrm{ISN}^{4+}$ and $22 \mathrm{Ne}^{5+}$ have alșo been developed.

Table 1. 88-inch cyclotron operation tine distribution 1975.

Tune-ury

Beap optics

Experingents

4

Bear derelopuent

Operating time (5663 hours) 699

Planned majntenance

24

Inplanmed maintenance

$\begin{array}{cc}\text { Total maintenamed (2545 } & \overline{318} \\ \text { hours) } & \\ \text { Total work time ( } 8208 \text { hours) } & 1008\end{array}$

A new PIG source design with larger elearances and a smaller bore dimmeter has greatly increased the availability and reliabjilty of high charge state beams. The $172 \mathrm{MoW}{ }^{40} \mathrm{Ca}^{7 *}$ beam for example, had nevor been obtained. yith earlier source deslgis. The recentIy developed $16_{0}{ }^{77}$ bear at $-27 \mathrm{MeK} /$ muleon, believed to be the first verified $0^{7+}$ beam extracted from a PIG source, is now in use by experinental groups.

Another 10n source design, similar to the above but with provision for filament hoating of the cathades, has been fabricated and is undergoning testing. This new feature is intended to provide a simple means of controlling the source iutput.

A new bending magnet was designed, fabricated, and installed in an experimental beam life. This magnet is used to steer the bean into one of the three different experimental caves. It replaced an older magnet and has greatly inproved the beam optical characteristics. A regulated $1000 \mathrm{~A}$ de power supply has been built for the new nagnet. High aurrent "hockey"puck" diodes and Darlington power transistors are used to simplify its cirauit design and construetion.

A f1annable-gas venting system has bẹen installed in scrle of the experimental caves to safely provide for experiments that require these geses.

A continuing strong effort is being made to improve day to day operating retiability. Hinor changes are being made in corponents as wealnesses occur. There are infications that we are making progress towards increased reliability. A considerable amount of evailable manpower goes into this aspect of cyclotron irprovemeint.

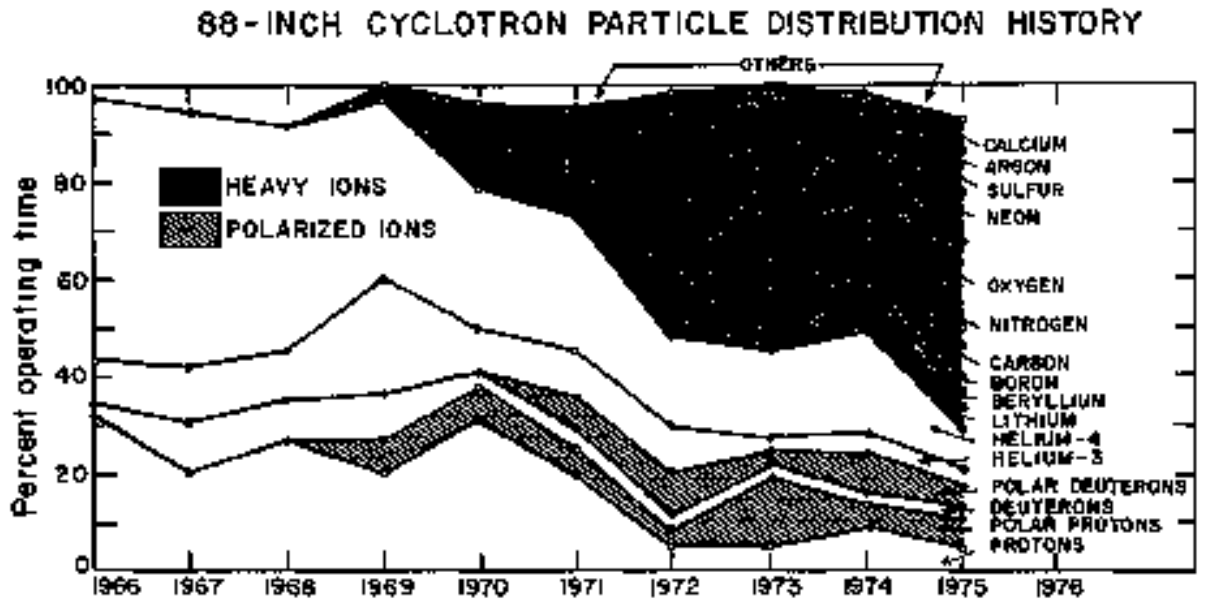

Fig. 1. 88-inch cyclotran particle distribution history, (XBL 7512-9594A) 
Table 2. 88-Inch cyclotron beans, to May 1976.

\begin{tabular}{|c|c|c|c|c|c|c|}
\hline $\operatorname{Ian}$ & $\begin{array}{l}\text { Enerery } \\
\text { eMtert } \\
\end{array}$ & 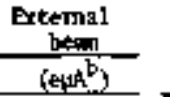 & I $\boldsymbol{m}$, & Energy & & 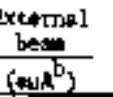 \\
\hline $\mathrm{p}$ & 0.2 .55 & $100-20$ & & 63.112 & & 10 \\
\hline p(polurized] & $6-55$ & 0.40 & & $\mathrm{t} 1 \mathrm{z} \cdot 17 \mathrm{~s}$ & & 5 \\
\hline \multirow[t]{2}{*}{ d } & $0.5 \cdot 65$ & $300-20$ & & $175-252$ & & $\mathbf{l}$ \\
\hline & - & - & & $252-343$ & & 0.014 \\
\hline \multirow[t]{8}{*}{ d (polarized) } & $10-65$ & 0.40 & & $100=159$ & & 5 \\
\hline & $1-140$ & $100-10$ & & - & & - \\
\hline & $3: 130$ & $100 \cdot 10$ & & 50.5s & & 2 \\
\hline & 2.23 & 10 & & $95-146$ & & 0.2 \\
\hline & 23.95 & 5 & & $50-90$ & & 2 \\
\hline & $93-195$ & 1 & & $4 B-86$ & & 2 \\
\hline & $20-80$ & 5 & & - & & - \\
\hline & & & & 80.125 & & 1 \\
\hline \multirow{3}{*}{$\begin{array}{l}9 \mathrm{Be}^{2+} \\
9 \mathrm{Be}^{3+}\end{array}$} & $15 \cdot 62$ & 5 & & $1 \geq 5-150$ & & 0.2 \\
\hline & $6 \overrightarrow{2}-140$ & 2 & & $190-245$ & & 0.08 \\
\hline & & & & $100-158$ & & 2 \\
\hline \multirow{5}{*}{$\begin{array}{l}10 \mathrm{~B}^{2+} \\
10 \mathrm{~s}^{3+}\end{array}$} & $24-56$ & 10 & & $158-214$ & & 0.2 \\
\hline & $56-126$ & ID & & $55-14$ & & $0,0^{5}$ \\
\hline & $12-51$ & 10 & & $3.5-126$ & & 4 \\
\hline & $51-I 15$ & 10 & & $126-172$ & & 2 \\
\hline & $115-204$ & 0.3 & & $172-224$ & & 0,5 \\
\hline \multirow{4}{*}{$\begin{array}{l}12 \mathrm{C}^{3 *} \\
12 \mathrm{C} \\
12^{5+4}\end{array}$} & $45-145$ & 20 & $x^{10 *}$ & $200-350$ & $10^{3}$ & Fant/sec \\
\hline & JUS-287 & $s$ & & - & & ; \\
\hline & $187-292$ & 0.05 & & $85-126$ & & 1 \\
\hline & & & & $126-172$ & & 0.2 \\
\hline \multirow{6}{*}{$\begin{array}{l}14 \mathrm{~N}^{4+} \\
14 \mathrm{~N}^{5+} \\
14 \mathrm{~N}^{6 *} \\
15 \mathrm{w}^{4+}\end{array}$} & $10-40$ & 20 & ${ }^{2}$ & - & & - \\
\hline & $10-90$ & 15 & $\mathrm{Fe}$ & $280-250$ & 1 & part $/$ /sec \\
\hline & $90-1.60$ & 15 & $\mathbf{k T}^{2+}$ & $5+19$ & & .001 \\
\hline & $160-250$ & 78 & $\mathrm{x}_{r}{ }^{12 *}$ & $200-240$ & 1 & part $/$ sec \\
\hline & $250-360$ & $10^{3}$ pert/sec & - & - & & - \\
\hline & $130+\frac{1}{2} 33$ & 15 & - & $\cdot$ & & - \\
\hline \multirow{5}{*}{$\begin{array}{l}160^{3+} \\
160^{4+} \\
160^{5+} \\
160^{6+} \\
160^{7+}\end{array}$} & $35-79$ & 20 & - & - & & - \\
\hline & $79-140$ & 20 & - & . & & . \\
\hline & 140.819 & 2 & - & - & & - \\
\hline & $219-315$ & 2 & - & , & & . \\
\hline & $315-429$ & $=50 \operatorname{egh}^{a}$ & - & - & & $\cdot$ \\
\hline $180^{4+}$ & $70-124$ & 10 & - & $\cdot$ & & - \\
\hline \multirow{2}{*}{$\begin{array}{l}19_{F} 4 * \\
19_{F} 5+\end{array}$} & $66-118$ & 10 & - & - & & - \\
\hline & 218-184 & 2 & . & - & & - \\
\hline
\end{tabular}

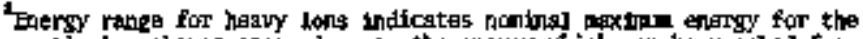

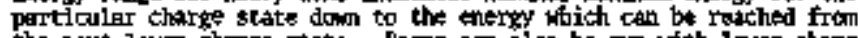
the aext lower cherge state. Beans cam also be run with lamer charge than slxpra at energies below $1 \mathrm{MeN} / \mathrm{kkch}$ loon.

Electrical nicreaperes except ag ooted.

Gel hamknif.

15th hamenic.

A new servo control has been desgined and buitt and will soon be installed to replace the existing if trinmer control unit. A I6-chemel digital readout unit has beem built and installed. Four of these channels are used to display center regjon parameters. More of these channels will be usod to monitor vital parmeters in the center region and plans are being made to interface this unit to the TI $960 \mathrm{k}$ commuter. A new program for the TI $960 \mathrm{~A}$ is now being written and it will be ready shortly.

The use of additional dee-to-ground capacity to extend the lower linit of the if frequency toward $4 \mathrm{Mfz}$ has been show to be feasible. A disconnect switch is being designed so the additional capacitance can be added or removed without having to let the cyclotron tank to atrospheric pressure.

A project was undertaken to develop the cycIotron for ninth harmonic acceleration. The object

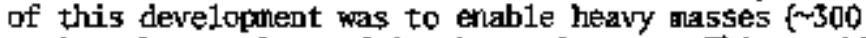
ariu to be accelerated in the cyclotron. This would make possible certain experiments like searching for the existence of winute traces of the so-called "surperheavy elenents" in nature with a high resolution and sensitivity. Extracted beams have been observed up to 15th harmonic. Sone center region modiftcations mere necessary to correct problems associated with orbit centering and the long source to pullex transit tine. On 9th harmonic, $370 \mathrm{nA}$ of $4 \mathrm{Ar}^{2+}$ were seen as extermal beam and ' lnA of $84 \mathrm{Kr}^{2+}$ was observed on 15 th harmonic. The basic capability has been developed for acceleration of mass $300^{8+}$ ions in the cyclotron with substantial intensities.

A project was undertaken to study systanatics in the control settings of the $\$ 8$-inch cyclotron. The motivation was to prodict the control settings of a new beard with sufficient accuracy to avold time consuning beam developplent and calculations. Alout 60 paroueters need adfustant $(\theta+g .$, maln coil current, frequency, 17 trim coil conrents, center region and deflector parameters, valley coils, etc.) for the acceleration of a bean of specified energy/ mucleon and charge to moss (e/m) ratio. Eqpressions have been derived describing the phase history of a particle $(\mathrm{e} / \mathrm{s})_{\mathrm{b}}$ accelerated in a magnetic field tuned for another particle $(e / m)_{a} \neq(\theta / m)_{b}$, By placing constraints on the gllowed phase excursions, limiting values of (e/m) have been obtained as a function of $(\mathrm{e} / \mathrm{m})_{a}$. Similar calculatiors are in progress to detemine by how much the energy/mucleon can be changed without modifying the basic cyclotron solution. It appears that about 200 model beams wil1 adectuately spark the entire enerky/nurleon and $\mathrm{e} / \mathrm{m}$ spectrum of the cyclotron on such a tro-dimensional grid. Other new beans could then be accelerated by interpolating or "stretching" the control settings of the nearest model boam. A capilation of the 60 control settings for each of these 200 chosen model bears (about $60 \times 200=12,000$ significant numbers) has therefore been udertaken. Wherever possible, control settings of model beans were taken from previously nim beams. For model beams which had not been run, trim coil parameters were calculated using the conluter code CYDE deslgned for this purpose. Other parameters were obtained by scaling laws. This vast conpllation is row nearing completion and shall be helpful in accelerating beams of new energy/nucleon or e/m.

A project has been started to redesign the ion optics of the axial injection system for the future addition of an external heavy ion source. In this 


\title{
$00:$ if $\overrightarrow{5} 0 ; 0$ i 2
}

design the acceptance of the axial injection line is matched with a 200 mol -ाIrad emittance from a PIG heavy ion source to pentit efficient transfer of heavy ions from the source to the machine center. The matching is done with the aid of an electrostatic quadrupole triplet and a 90 bending magnet with edge focusing. The optimization of the ion optics was done with the calputer code BELIN (e version of TRANSPORT) and a very satisfactory solution of the bean transport element parameters was found.
Experimental tests of the performance of this and other optics designs are being planed on a test bench.

\section{Footnote}

On leave from tho Bhabha ktamic Research Center, India.

\section{MEV PROTON MEDICAL CYCLOTRON DESIGN STUDY ${ }^{n}$}

\author{
R J Burleagh, D J Clark, and w 5 Flood
}

A brief design studiy has been done for a 150 Mei proton sector cyclotron. The object was to minimize cost but maintain good reliability and easy maintenance. The use of the proton beam would be therapy, radiography, and isotope production.

Protons have the advantage over $x$-rays and neutrons of having a fixed range and a high rate of energy loss near the end of the range. This makes possible the radiation of small internal tumors with 10 dosage to the surface tissue. Heavier ions and * mesons also have fixed ranges and advantage over protons of a low OER (oxygen enhancentent ratio) which reduces damage to normal tissue, but they require considerably larger accelerators to produce beans with enough range to penetrate the fruman body. So we present this preliminary design study in the hope that there is an area of therapy which can utilize the high definition of protons at a cost which is Jakch less than for heavier ions or ${ }^{\prime}$ Jiesons,

For a 150 hey proton cyclotron for the modest current of 0.1 WA external bean we have a choice between a sector-focused and a synchro-cyclotron. Srme may like the synchro-cyclotron for its stimilic. ity of thagnetic field design and loose tolerance on dee voltage regulation. We prefer the sector focused design because there is no rotating capacitor and high currents of $100 \mathrm{uA}^{\mathrm{A}}$ are easily available for op ${ }^{-}$ tional isotope production. The thagretic field design is similar to many such cyclotrons and the magnetic fiold, frequency and dee voltage stabilization are rout ine engineering design jabs now.

A new design option has arisen in the past several yearș: normal conducting vs superconduct ing main coil. Although the superconducting design has a pole diameter about $40 \%$ that of the normal dosign, the higher cost of the superionducting technology makes the overall costs about the same, for this size. At larger $K$ values the superconducing design becomes cheaper than the normal one. Pecause some development still is necessary for the superconducting design such as deflection, and becanse a medical envirorant requires high reliabllity and proven desiep, decidod in a normal coil magnet.

Another design choice is that of the dee configuration. The dee or dese can extend over both hill and valleys, as in most of the sector cyclotrons. Or two dees can lie in two oppostte valleys of a.4-sector magnet as ith the UCLA design. In the first case (dee-over-hili) the magnet gap is determined by the dee-ground clearance over the hills. In the second case [dee-in-valley] it is determined by the dee-ground clearance in the valleys, and the mapnet gap car be considerably srialler. In couparisor of these two options it was found that if other factors are left constant (such as average field. current density, dee-ground clearance, etc.) the dee-in-valley design requires only 60 of of the copper, $60 \%$ of the magnet powrer, and 805 of the steel, compared to the dee-over-hlli design. 1 so the deein-valley design was chosen for this sturty.

The paramoters thosen are shomn in Table 1.

Table 1. Specifications.

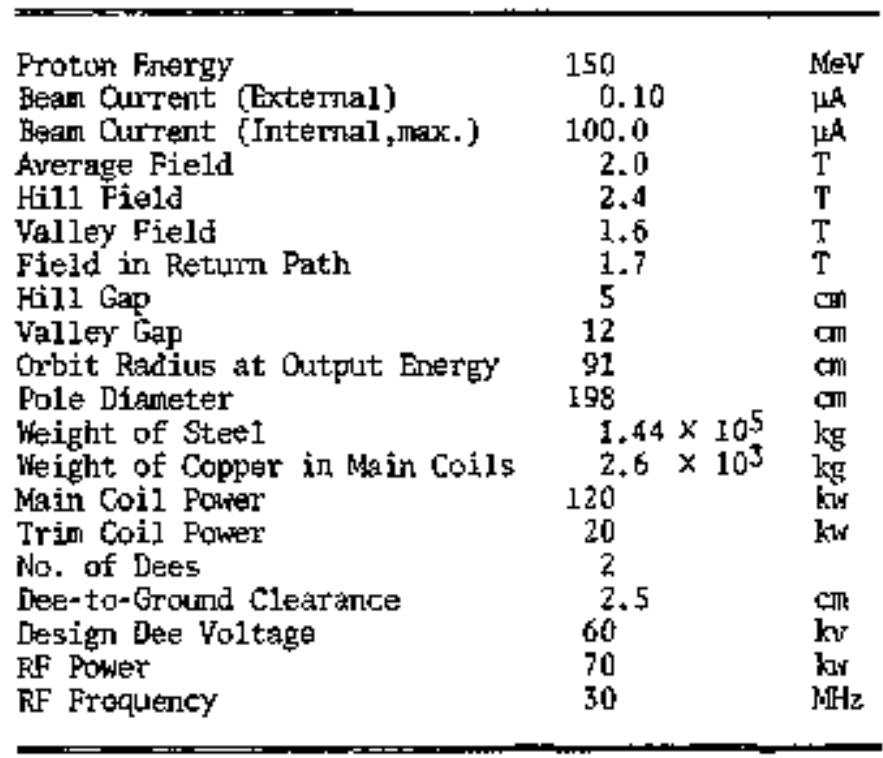

The high field level of $2.0 \mathrm{~T}$ average is based on siniliar values used at UCLA. For a fixed energy design such as this the steel can be run into saturation in the gap region, but $1.7 \mathrm{~T}$ is used in the return yoke to conserve coil power. These values were not optimized. The smali average gap allows 


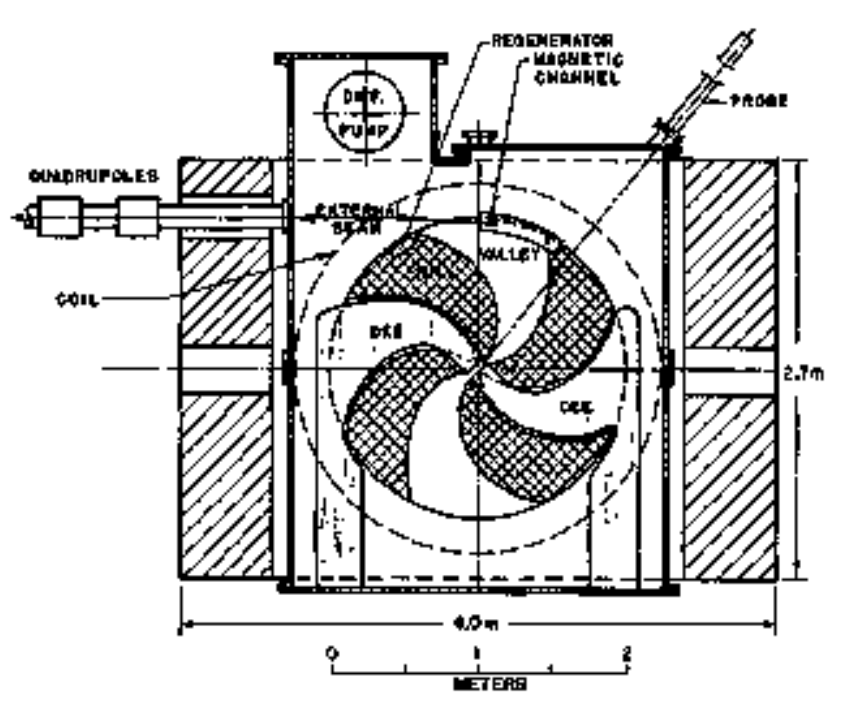

RLAH VIFW OF GYCLOTROH

Fig. 1. R1an view of magnet and if system at Median plane.

(XBL 757-3580)
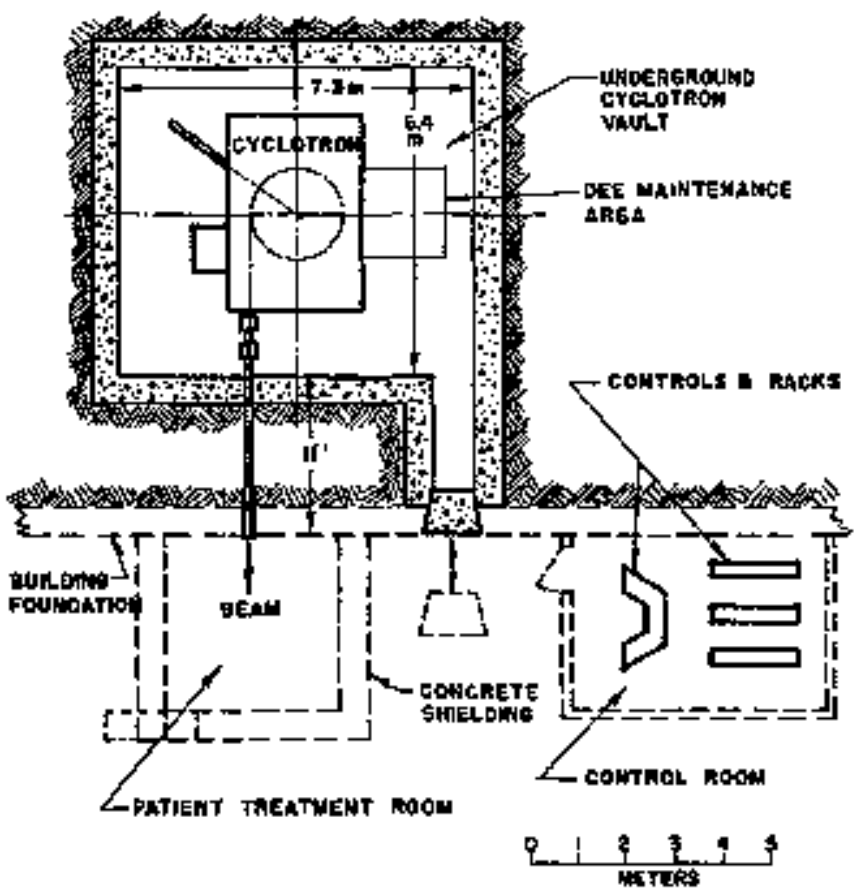

\section{PLAN YIEW OF VAULT}

Fig, 2. Plan view of vault, control rocm, and one treatment rocm.

(XBL 757-\$576) the outer orbit to cone close to the pole edge for ensler extraction, and also requires only a salk main cofl power. The doe voltage of $60 \mathrm{kV}$ across a 2.5 cin gap is consistent with previous cyclotron experience.

The Iayout of the magnet and dees is show in Fig. 1. The side return legs of the magnet are quite close to the dee tark and are wider than the pole diameter to retuce cost at the expense of accessibility. The larger size makes the $1 / 4$ wave dee stems end at the tank wall, glving a unit stoucture which can be rolled away from the minget for minintenance. The ion source is inserted axially through the pole and is not show in this figure. Alternatively it could cathe in radially on the deflector side, if space above the roof ware not available. One probe for bearl daeasurements is shown. This could be specially cooled for isotope production. Molem couputer programs are so acarate that a centered orbit gecmetry can be designed in advance, so other probes should not be necessary. Extraction is shom lising a regenerator.

Dee support structure ts simplifled and if power minimized by using separate grounded dee stems. The total rf power to dees, stems and Iiners is $70 \mathrm{kN}$ at $60 \mathrm{kH}$ dee vol tage, $30 \mathrm{kmz}$. This requirement is we11 within ratings of a single forced-air cooled power tube (4C 35000$)$.

No detalled beam dymanics calculations have been done on this design. The inner section out to $50 \mathrm{MeV}$ is very similar to the UClA cyclotron as far as center region, magnetic field level and flutter, and dee system are concerned. The spira1 angle necessary to obtain vertical focusing out to $150 \mathrm{MeV}$ wgs calculated using the approxinate formula: $v^{2}=1-y^{2}+F\left(1+2 \tan ^{2} \alpha\right)$, where $v^{2}$ is the square of the vertical frequency, $Y=\mathrm{J} / \mathrm{m}_{\mathrm{n}}, \mathrm{Z}_{\mathrm{F}}$ is the manetic field flutter and $a$ is the spiral angle (angle between hilledge and a radial line).

The 1ayout of the cyclotron in a vault is shown in Fig. 2, This is a rwinimm cost configuration, us ing earth shielding around concrete walls. space is provided in the vault to rencove the if systeal for maIntenance. One or more patient treatrient romis can be provided in the building. It is assumed that space for the vault is available at ground level next to the building where treatments take place. This makes installation inexpensive. Also any serius repair on the cyclotron which requires taking apart the magnet can be done easily by removing the roof blocks with a compercial crane.

\section{Footrote and Reference}

`Condenșẹd frow LBL-4085.

1. R. Burleigh, LBt, Engineering Note M4582, Feb. 1973. 


\title{
$00, y 490 ; 063$
}

\section{HEAVY-ION BOOSTER CYCLOTRON DESIGN STUDIES AT BERKELEY*}

\author{
A J Burkegh, D J Clark, and L A Glasgow
}

Design studies on four booster cyclotrons for hoavy ions are descrited. Couparisons are made of nonial vs suppercondheting main coils, and $\mathrm{K}=400$ and 800 sizes. Performance and cost estimitos are given.

The present heavy ion accelerators at LBL are the SuperHILAC, the Bevation and the 88 -inch cyclotron. In Fig- 1 the solid lines show the performance of these existing accelerators when developed to their full potential. The regions inacessfble to our present machines are the energy region above the 88-inch cyclotron and the SuperHILAC but below the Betalac, and the heavier mass region at Bevalac energies. We explore some of the options for using singIe pole cyclotrons as boosters (post accelerators, for the SuperHIlAC and the 8B-inch cyclotron. The cyclotron has the desirable characteristic af 1009 macroscopic duty factor. Since this cyclotron study was done it was decided that the highest priority for a proposal at LBL for heavy ion bean expansion is in the extersion of the Bevalac to heavier ions and lower energies ("Improved Bevalac," Fìg. 1) and increasting the superHIlAC intensity for heavier masses.

There are several types of sector-focused cyclotron designs which can be usext. These are iI. lustrated schematically in Fig. 2. The four cases show relative sizes of cyclotrons with either a sinFie pole or separate sectors, and with either normal condteting or supercondurting coils. These cases all have the same $\mathrm{K}$ or maximum bending strength. The pole diameter is about twice as large for the separate sector as for the corresponding single pole design, because of the suatlor average fieid

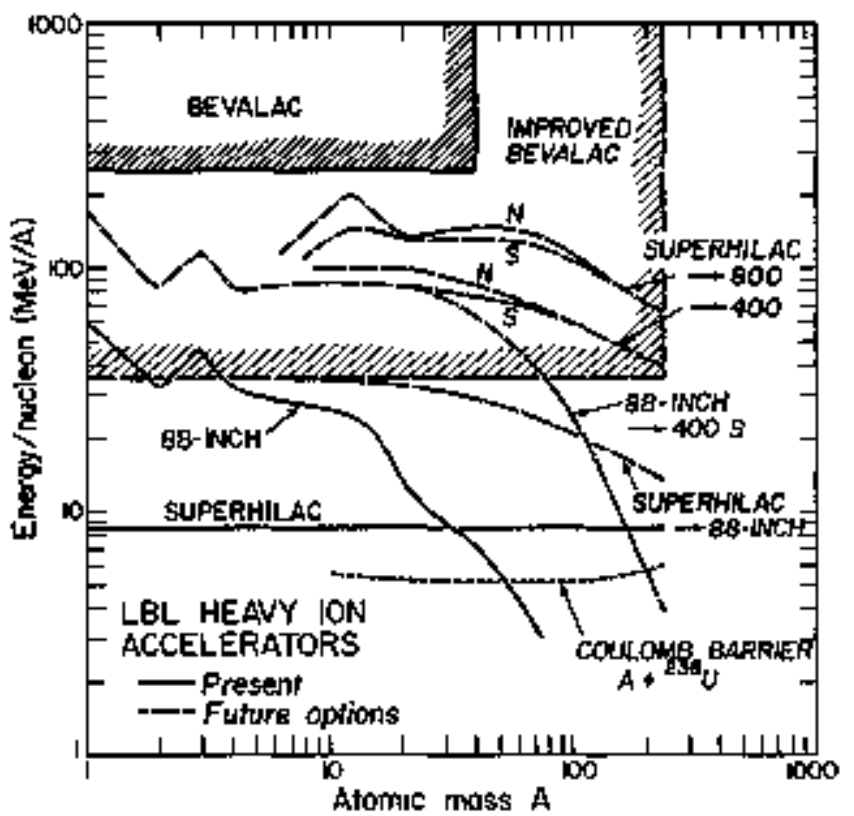

Fig. 1. Performince predictions for present and possible future lBL heavy-ion accelerators.

(XEI 757-3584) around the orbit. The pole dianeter is about 3 times as large for the normal conducting as for the corresponding superconducting nachine, 5ince the field is 3 times lower. The peak field for the normal confucting cases is $1.7 \mathrm{~T}$. The field in the rettirn yoke is $1.7 \mathrm{~T}$ for all cases. The beam injection and extraction would be easier in the separate sector Cases 1 and 3 , but they are more expensive than Cases 2 and 4 . Injection would be by a stripping foil to get the beam across the magnetic field in Cases 2 and 4.

For this study Cases 2 ard 4 were chosen because of their lower cost compared to Cases 1 and 3 . Case 4 is interesting because of its sulall size and resultant low cost. The extraction is a spectal challenge because of the $5.0 \mathrm{~T}$ field level in the poIe. The sizes thosen are $K=400$ and $K=800$ for each case, where $K$ is the energy constant in the
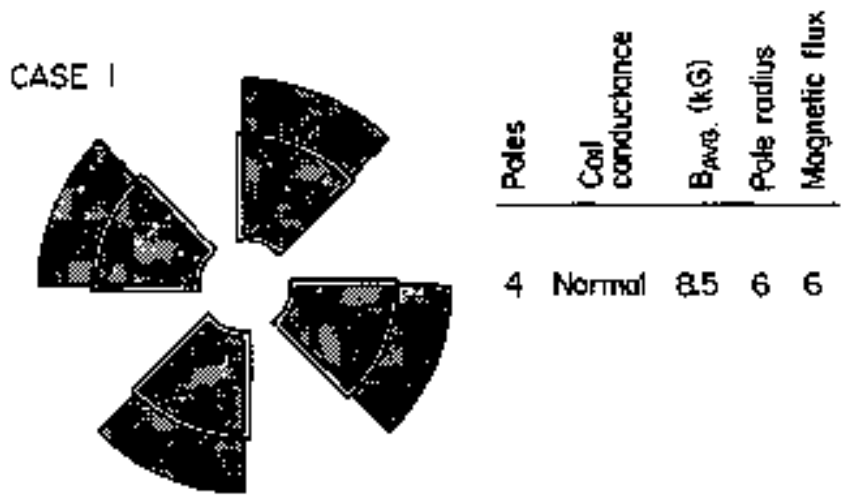

4 Normal $85 \quad 6 \quad 6$

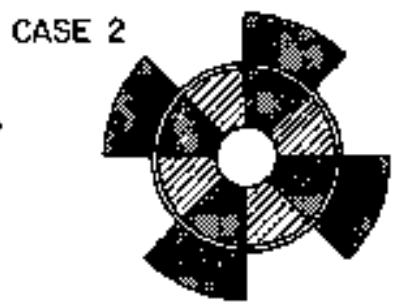

1 Nomal 143.63 .6

CASE 3

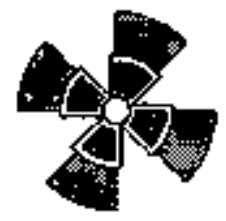

4 Super 2522

CASE 4

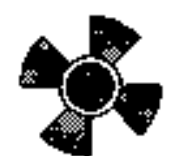

I Super 50 I 1

\section{CYCLOTRON STRUCTURES WITH SAME K}

Fig. 2, Four cyclotron structures with the sane $k$ value or bending strength.

(XBL 757-3586) 


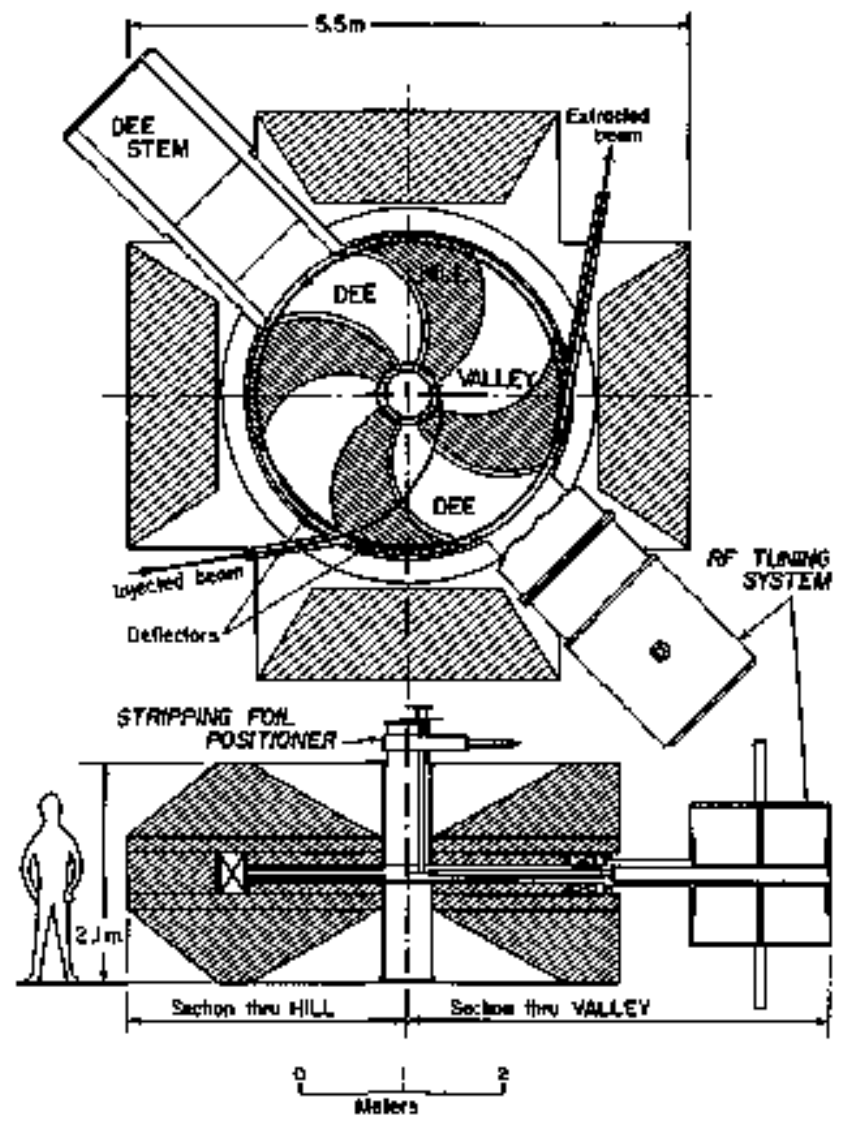

$400 \mathrm{~N}$ CYCLOTRON

Fig. 3. The $400 \mathrm{~N}$ booster cyclotion.

(XEl 757-3590)

exuation $\mathrm{E}=\mathrm{KQ}^{2} / \mathrm{A}$ where $\mathrm{E}$ is maximum treargy, $\mathrm{Q}$ and $A$ are particle charge and mass in proton units. In the following sections the letters $S$ and $N$ follow the $K$ to denote strperconalucting or normal conducting main coils.

A schertatic design for a $400 \mathrm{~N}$ booster cyclotron is shown in Fig. 3. Four magnet return legs are used to reduce the amount of steel. A high average field of $2.0 \mathrm{~T}$ is used to minimize the size and cost.

The rf system has 2 dees in the valleys to rinimize magrietic gap. The hill gap is 5 cm-large enough to withdraw the dee system without raising the upper magnet woke. Hamonics are 2-4 which give high energy gain/tum with 45 degree wide dees. The energy range $\overline{15}$ then covered with an rf frequency range of 2 to 1 .

The coils are tape wound with slots for the dees and injection and extrartion channels. This construction gives good magnetic fiepld out to large radius, and fast fall-off for easier extraction, A Iifting systen for the upper yoke would be used for installation and maintenance of the deflection sys. ter.,

A Schematic elevation view of a $400 \mathrm{~s}$ cyclotron is show in Fig. 4. The superconducting coil provides $5,0 \mathrm{~T}$ average field in the bore. It has a

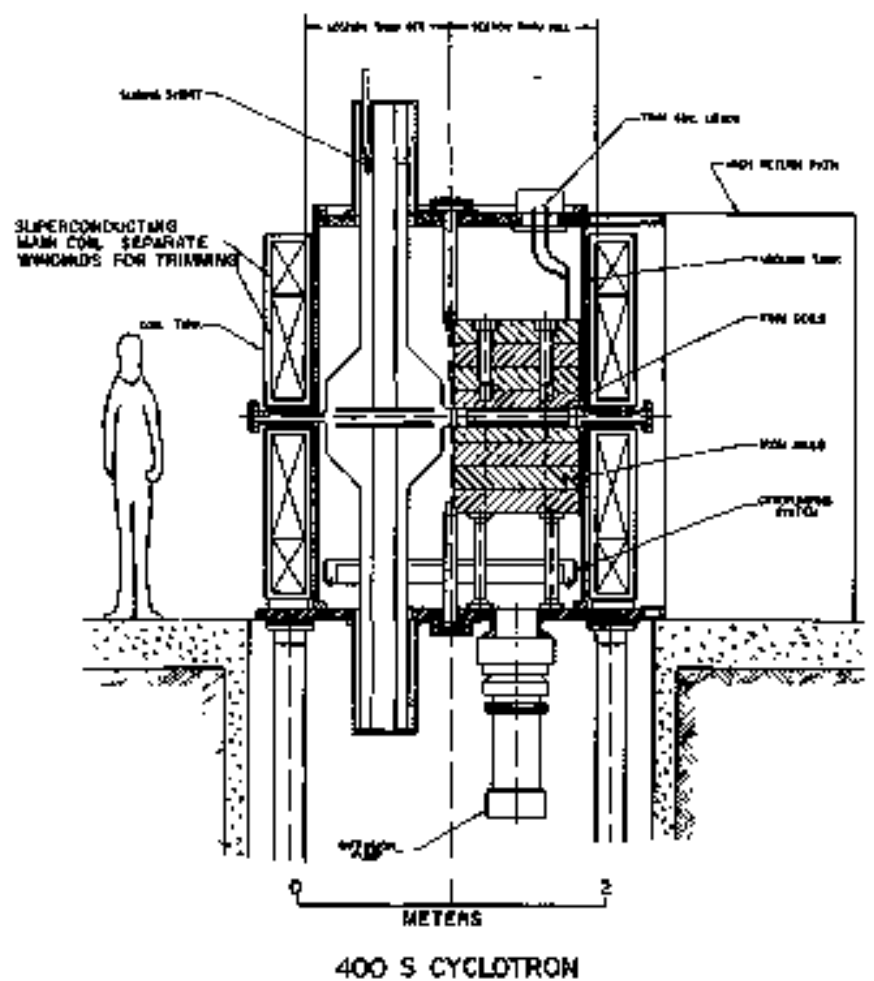

Fig. 4. Elevation viơ of the 400 \$ cyclotron. (XBL 757-3589)

gectuetry similar to that of large bubble thamber coils. Arronue National Laboratory, U.S.A. has built a "15 foot" bubble chamber coill with an inside diameter of 14 feet $(4.3 \mathrm{~m})$, a central field of $3.0 \mathrm{~T}$ and a maxinum field at the coil of $5.0 \mathrm{~T}$, The coil in the $400 \mathrm{~S}$ syclotion is $1.9 \mathrm{~m}$ inside diameter and has a maximum field at the coil of 5.0 $T$ with some iron just inside the coil for shielding. Thus it is well within current engineering practice. Each coil is show split into an upper and lower section for triming the radial field profile.

Flutter in the magnet is provided by saturated steel sectors supported inside the superconducting coil bore. The field in the saturated steel is 2.0 $T$ higher than in the space between sectors. But in a practical gentuetry the hill-valley difference on the median plane is typically $1,0 \mathrm{r}$. Calculations of the flutter from several gap configurations were made using the program TRM.

The dees are supported by dee stens coming in axially in tho of the valleys. This is a natural configuration since the valieys are orpty and there is little space for the dee stems between the main coils, which need to be close together for a good magnetic profile.

The vackndm systexl is a cryopunp, which is supplied with cold helium gas by a small fraction of the refrigeration used for the main coil. A stall diffusion pum or turbo-punp can be used to purtpp hrdrogen and helium.

The estimated performence of each option with injection frum either the SuperHIlAC or the 88 -inch cyclotion is stown in Fig. 1. Since the Superfrtac 


\title{
$00 \because 4 \times 30 \div 064$
}

produces higher energies for Ions with $A>30$, it Hakes a better injector than the $88-$ inch. The gain in going from a $K$ of 400 to 800 is not great. The sumerconductling and normal conducting versions have similar performance. An interesting option is injecting the 88-inch with the superHildC. The machines are assuned not to be liaited by extractlon.

Table 1, Cost sumbary, U.S. k $\$ 12 / 74$

Total minimum cost $\quad \frac{400 \mathrm{~S}}{4,900} \frac{400 \mathrm{~N}}{\mathrm{~J} 0,300} \frac{800 \mathrm{~S}}{7,600} \frac{800 \mathrm{~N}}{18,000}$

Cost estinates for the 4 designs studied are shown in Table 1. Thery inctude engthering and construction of the cyciotron, bat not shielding, bean transport or building. The costs are based an standard LEL construction account rates. The lowest cost option is the $400 \mathrm{~S}$, and its perfordunce is very good in Fig. 1.

\section{Footnote and Reference}

*Condensed from LBA-40B6.

1. J. Purce1l et a1., AN/HEP 7215, February 1973.

\section{A FAST-CLOSING VACUUA FOR THE 88-ENCH CYCLOTRON}

\author{
F. A Gough, R Lam, D Morris, and C Marlinez
}

A fast-closing vacun valve was recently doveloped to protect the 88-inch cyclotron against contamination during bombardments of highly radioactive targets. Such a "slammer" valve is particularly necessary during bomberdments of trensuranic targets monted on the vacum foil window of a gas jet system. 1 Should the foil break dise to mecharical or thermal stresses, the radioactivity could be carried into the acceleration chamber of the $\mathrm{cy}$ clotron by a sonic expansion of gas into the cyrlotron vacuns. The development of this vatie and as' sociated trigger efrculty is an outgrowth of eartier work at this laboratory. 2 Commercial explosives in the form of squibs were chosen as a power source for the valve. Among the advantages of using an electro

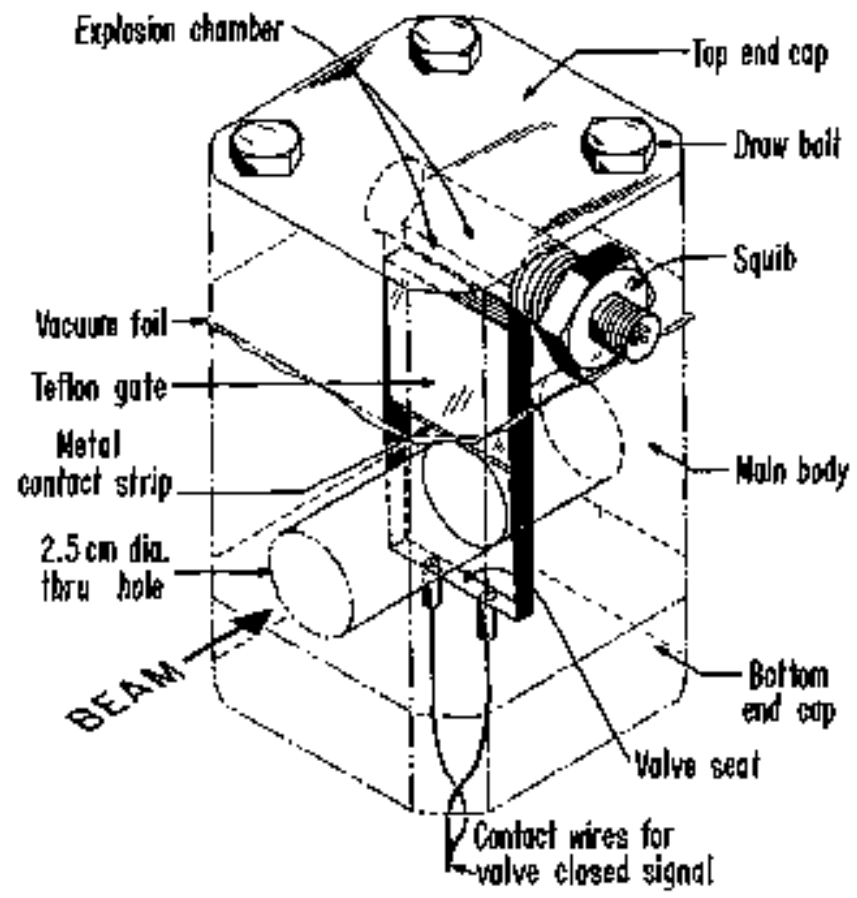

Fig. 1. An isometric view of the slandmer valvo. (XBL $766-2960$ ) explosive device are tts very hijh degree of reliability, the reproducibility, unifomity, and magmitude of the force it creates, and its compatibility with fast electronic firing mechanisms.

The valve is pictured in Fig. 1 . It has a very light replaceable teflon gate and a case hardened steel body which, wlike mechanical prototypes tested, and has shown no signs of distortion even after many firings. A 2.5 on dianeter hole is provided for passage of the beam. Only 2 msec are required to move the teflon gate from fully open to the fully-closed, vacunm-sealed position. $550 \mathrm{Jag}$ of Bullseye pistol powder, chosen for its fast-burmins qualities, was found to be an adequate and reliabile charge for the squib. A metal contect strip on the bottom surface of the teflon gate prowides an electrical indication when the valve is fullyclosed. To reload the valve after each firing it is necessary to reforve ard replace the squitb, the teflon gate and a vactatul foil, all of which takes abcut 10 Itis.

Sone experimentation was necessary to deter mine the optimm compression of the tefilon by the groove in which it travels. A $3^{\circ}$ standard lorse taper was cut on both the teflon gate and the groove. Wien the valve is fired and the gate moves across the hole toward the valve seat, the leading edge of the teflon makes a vacurm seal $\sim 5 \mathrm{~mm}$ before reaching the end of its trave1. During the last 5 mo of trav el the wedging action serves to decelerate the gate. About 5\% conpression on the teflon is sufficient for a good seal. One gate having $\sim 10 \%$ compression failed to close fully with the same 550 Jog charge, Too 1ittle corppression could result in a poor vaculun seal or in the gate bouming fron the valve seat and settling in a partially open position.

There are two independent thannels from which the trigger signal con be derived [see Fig. 2). Each channel can accormodate up to 8 inputs from some appropatate sonsor such as an ion gauge or vaculn analyzer, At present, two ion gauges (one for each channe1) are used to monitor the beam line pressure near the entrance to the target chantber. A Granyille 


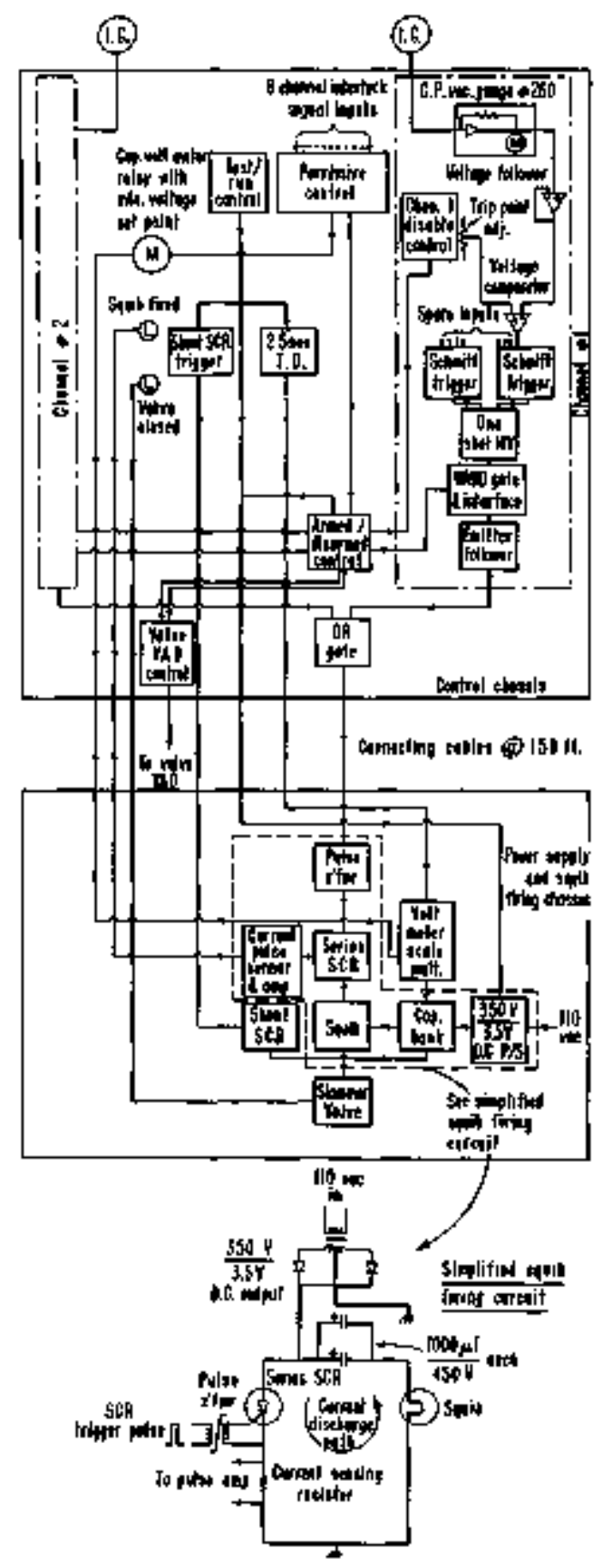

Fig. 2. An electrical schenlatic of the triggering and firing circuitry.

(XBL 766-2959)

Phillips (G.P.) power supply (sertes 260) is used because its output is corpatible with the TTL (transistor transistor logic) infut requirements. However, a voltage follower has been edded to the output of the electroneter anplifier for better isolation and impedance natching. The output of the voltage follower, whtch has the same voltage level as the meter reading on the G.P. tonit, is condpared with an adustable voltage derived from a 10-turn helipot whose output voltage is such that the dial reading of the holipot is the stare as the meter reading of the G.P. power supply. This correspondence simplifies the setting of the trip-point of each chamel. The comperator output is fed into a Schoitt trigger and a one shot mutivibrator to produse a fast rising trigger pulso with a minimm width of 5 msec. The trigger pulse is then fed into a NAND gate, used for aroling or disaming each chamel, followed by an OR circuit which peruits either channel to independontly trigger the gate of the SCR. The response time of the trigger circuit to a sudden pressure rise is $\sim 1$ as and is completely dominated by the response time of the ion gange.

The squib is fired by a stimple capacitive discharge frous a $2000 \mu \mathrm{F} / 450 \mathrm{~V}$ capacitor bank in series with an Sck which acts an an electronic switch. The capacitor bark is charged to (and maintained at) 350 $V$ by a hiph voltage de power sugply while the SCR is biased off. When the trigger puIse turns on the SCR as described above, the mergy stored by the caparitor [ 10 joules] is discharged through the fuses of the squitb, and the heat thus generated innites the gumpowder. A current pulse of $140 \mathrm{~A}$ peak (exponentially decaying to 504 in $4 \mathrm{~J}$ seel caluses the gunponter to be coopletely burned in less than 0.1 Jasec.

The electrical cont inuity of the firing tirait and of the squib can be readily verified by lowering the capacitor volcage to $3,5 \mathrm{~V}$ and discharging the capacitor into the squib. The cerrent pulse through the squib is then sufficiently reduced that it will not cause the squib to fire. A return signal from the current sampling resistor (see Fig. 2) serves to confican the circuit contimity. If the trigger signal is initiated from on ion genge, the entire trigger and firing chain can be simmltanecousty tested. This can be done by turning on the "degas" switch on the G.P. power supplies thereby checking each chanueI independently.

A control unit for the slander valve systen has been built and installed in the cyclotron control room but, in order to minimize the attemtation of the current pulșe, the capacitor bark is loceted near the squatb. The valve itself is located where the beam is well focused in a bean line leading to the high level cave area (see Fig. 3) sone 9 ot upstream of the target chamber, Both air driven vacunm valves-designated in Fig. 3 as VAO and VExit wordd nomially close automatically if the beam line pressure exceeds $50 \mathrm{ir}$ but have a relatively slow closing time -1 sec. Whan the slanmer systen fires, both these valves will also close for adied protectlon in case the sloumer fails to completely seaI. Ar interlock feature prevents opening valve VA0 until the slanmer is armed and similarly prevents disaming the siammer until the valve VAO is closed again. The system cannot be ammed if the firing capacitors lose voltage or if either ion gauge fi1ament is not drawing eurrent. Located on the control unit are a series of diagnostic 1ights which indicate if the SCR gate has been openosd, if the squib has fired, and if the gate is fully closed. Thisy also indicate which channel (s) cansed the valv' to be fired.

A sinple sifilator has been built to substitute for the electrical interaction with valve VAO and Verit: It is also possible to substitute a sinple fuse for the squib. This simulation has proven very useful for debugging and pperator training. In this way, for example, the trigger circuit was desensitized to randon electrical nolse, a problem 

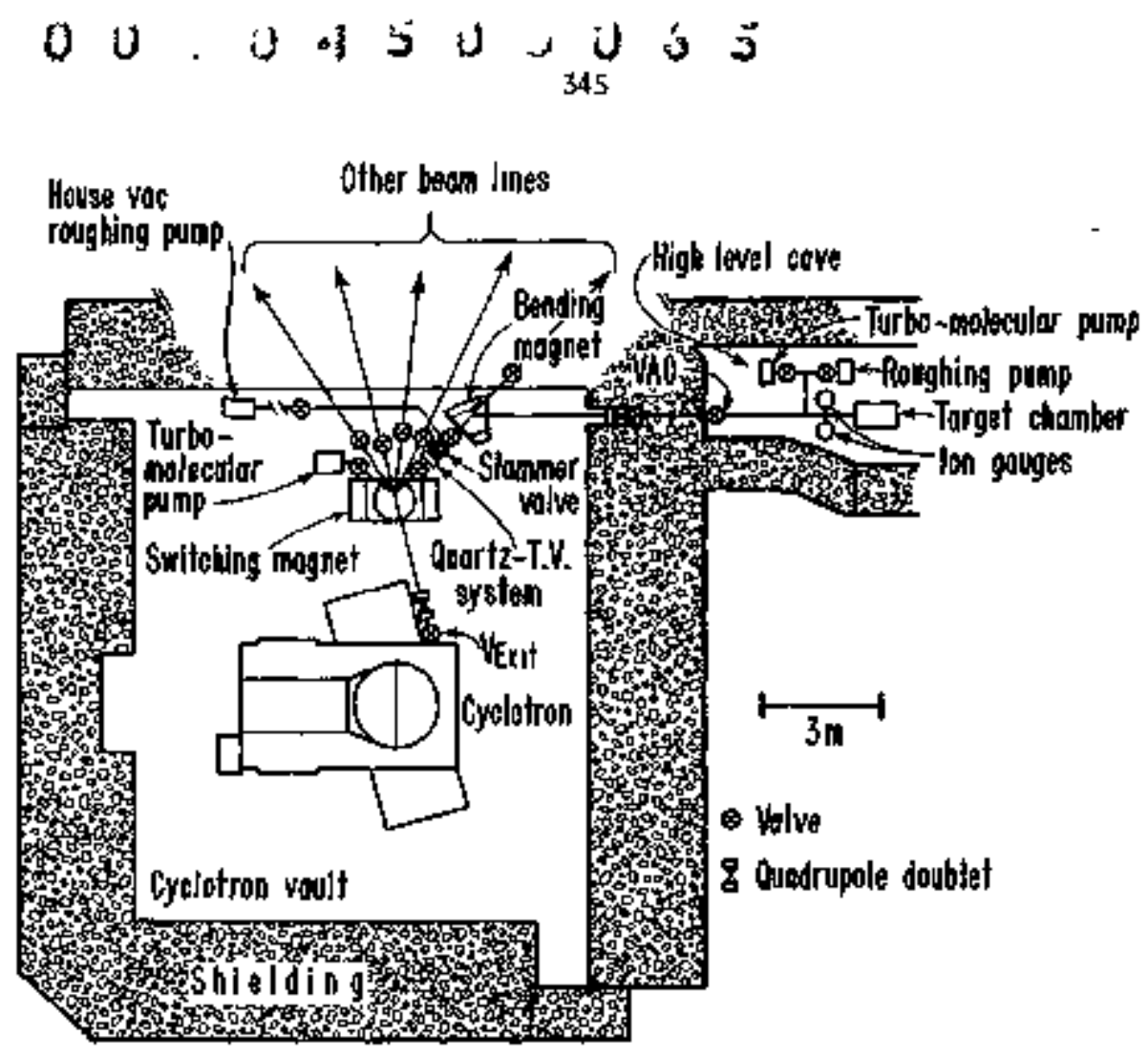

Fig. 3. A schernatic layout of the slander valve bean 2 ine.

XBL. 766-2958)

Referonce

which caused several misflrings in the eardy stages of developnent. The slamer has now been successfully operated for $\sim z$ months on the simllator and louger than 100 hours of real tine operetion without incident.
1. A. Ghiorso et al., Phys. Rev, Lett. 33, 1490 (1974),

2. R. Aume et al., Second Internationa1 Conference on Accelerator Dosisetry and Exarience, stantord, Califormia, 886 (Nov. 5-7 (1569).

\title{
HEAVY ION SOURCE DEVELOPMENT
}

\author{
Daurd \ Clark
}

The objective of this project is to tmprove the perforuance of the ion sources used on the LBL heavy ion accelerators: the SPertiLAC, the Bevatron, and the 88-inch cyclotron. The conon goals required by all the accelerators are to produce beans of new ions, or beams of higher intensity and better quality, and sourcess with longer lifetioe and better reliability. The achievement of these goals opens up new fields of research in nuclear science and medicine, and makes more of the scheduled time available for research.

In 1975 the sulall groups working at each accelerator on ion source development wero consolidated into the central group. This has greatly inproved commuication between groups. The mowing of the scattered test stand work into a central byilding is now in progress. All accelerators use the same type of source: the Penning Ion Gauge or "PIG" source, which has proven over the years to produce the highest intensities of high charge state heary ions. The comfigurations vary for the different accelerators, but the sharing of experience and usedil techinues is proving of great vałue.

Developitent work is done both at test stands and at the accelerators. It is important to have some people working on the sarpe source at both the test stand and the accelerator so that iuprovements developed at the test stard can be applied quickly at the accelerator, and problents arising at the accelerator can be solved on the test stand to save valuable accolerator time. For this roason a strong effort is being made to holicate the accelerator conditions as closely as possible at the teșt stand.

The SuperHILAC compact Adam ion sorrce $\{2,5$ wh for the heaviest ions was developed for produc. 
tion of new solid material beams of iron, calcium and gold. A special source insert for calcium (Ca ${ }^{3+}$ will make possible the recovery of most of the calciun when fuming beans of the very expensive isotope ${ }^{4 B_{\mathrm{Ca}}}{ }^{1}$ the output of gold ions Aulot and At11+ or the test stand was increased by a factor of 3 by splitting the sputter electrode into 2 rings, Calcium and iron were accelerated through the SuperHILAC to full energy. Studies are mderway to improve the Adam source by lengthening the arc thamber.

The SupprHILAC Eve source $(750 \mathrm{kV}$ ) produces ions up to argon for the SuperHIlAC and Bevalac. studies were doge on using a duoplasnatron for high currents of $\mathrm{Ne}^{3+}, 2$ They denonstrated a serious space charge blow-up when analyzing beams of $20 \mathrm{~mA}$ at low energy. Plans are underway to study acceleration of the beain to $750 \mathrm{kV}$ in a spare jnjector before analyzing, and to use the prowising new geometry of Darmstadt in the source.

The PIG scurce used at the Bevatron has improved transmission resulting frosn improved vacum primpirg and gas pulsing. Beam intensities of carbon and axygen have doubled.

The hot cathode PIG source used by the 88 inch cyclotron was developed both on a test stand ${ }^{3}$ and on the cyclotron. The emphasis is on new higher charee states, since the cyclotron energy is proporthona1 to charge squared. New beans accelerated include $\mathrm{Ar}^{9+}$, $\mathrm{Ar}^{\mathrm{OO}}{ }^{+}$, and $\mathrm{Ne}^{{ }^{+}}+$+ Careful emittance measuroments have been made on the test stand source showing emittarce to be nearly independent of charge state, but dependent on arc density.4 A new type of tathode mounting is working well on the test stand and will be tried soon on the cyclotron.

The autonatic enittance reasuring system became fully operational on the test stajk, and is being used to study dependence of source enittarce on various operating parameters. It is important that the enittance area of any somre be sma1l enough to be accepted by the accelerator.

The second International Conference on Heavy Ion Sources was held in Gatlinterrg, Tennessee, October 27-30, 1975. This provided an excellent opporturity to discuss recent developments with de1egates from most of the heavy ion source groups in the world.

Under construction this year are two new test stand facilities in the central fon source building. One is an exact durplicate of the Adam accelerator source system. This will make possible the testing and debugging of sources for the accelerator offline, saving valuable accelerator time. Also inprovements in structure and power supplies can be tested before installation at the accelerator. The other new facility is a 180 degree PIG source test magnet with a new pulsed orc supply. Here all the LEL PIG sources can be tested, compared, and devreloped. Also prototype sources incorporating a11 the best features of the existing sources will be tested. These will be used at the existing accelerators and in the proposed 3rd injector for the SinerHILAC. AIso In the design stage is an inproved Adan source which will have lerge cathode-cathode spacing, fil-

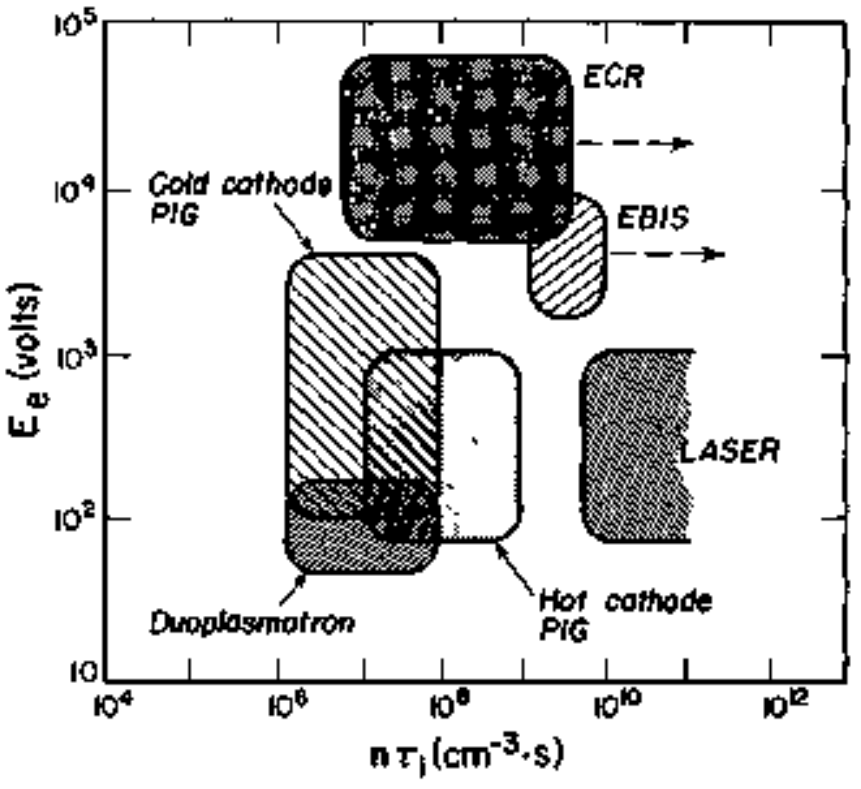

Fig. 1. Plasma parameters of positive ion sources. $E_{e}$ is electron temperature, $n$ is electron density. $t_{j}$ is ion confividant time.

(XBL 7512-9583)

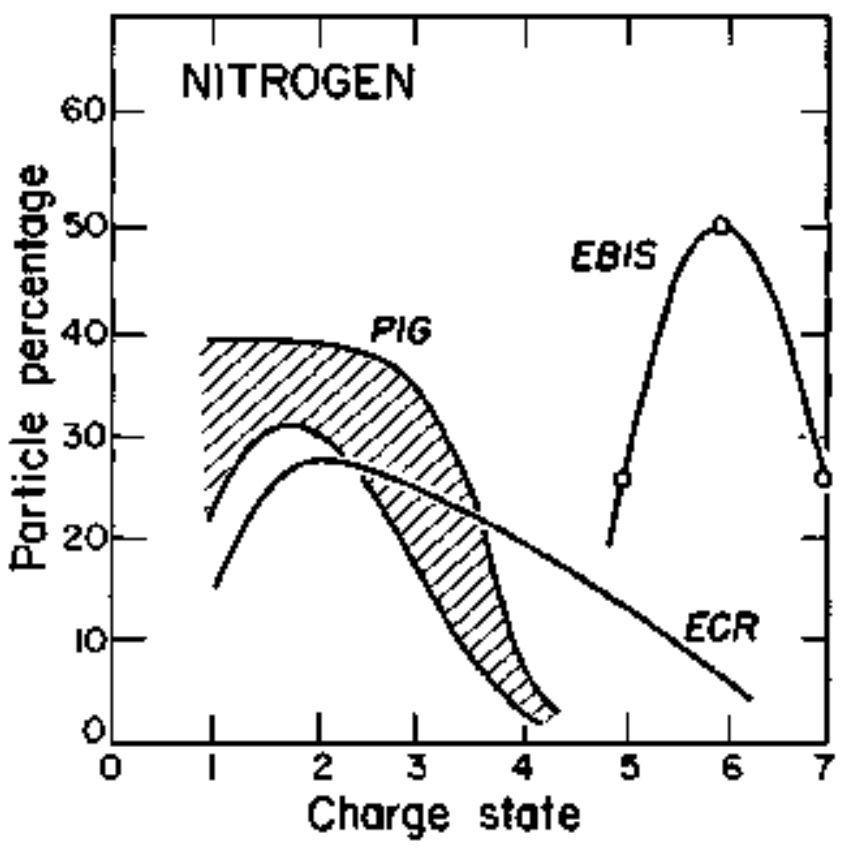

Fig. 2. Charge state fistributions of three types of positive ion sources

(XEl 7512-9582)

ament heating of one cathode, and a nirror magnetic field. Evaluation is boing completed on new concepts in heavy ion sources so that a choice can be made on which type of advanced source development to intplement. Couparisciss of some adranced sources are given in Figs. 1 and 2 (Ref. 5).

The heavy-ion sorrce group has menbers from the accelerators, who are listed as authors in the reports below. Another nember is D. Spences. 


\section{$00 ; \quad$\begin{tabular}{lllllll}
\hline & 0 & 0 & 0 & 0 & 0
\end{tabular}}

\section{References}

1. Basil Gavin, Ancde Syuttering Characteristics of the Berkeley 2.5 MS Source, Proc. Int, Conf, on Heany Iom Sorrces, Gatlinburis, Tennessee, October 27-30, 1975, IEEE Trans. Nuci. Sci. N5-23, 2 (1976).

2. R. M. Richter and E. Zajec, Heavy Ion Source Development at the Bevatron, Proc. Int. Conf. or Hegry Ion 5ources, Gatlinbrig, Temessee, October 27-30, 1975, IEEE Trans. Mucl. SeI. NS-23, 2 (1976).

3. L. Bex, D. J. Clark, C. E. Ellsworth, H. S. Flood, R. A. Gough, H. R. Holley, J. R. Heriwether, and D. Morris, High Charge State Hagry Ion Prochu- tion from a PIG Source, Proceedings of the Particle Accelerator Conference, Washington, D.C. March 12-14, 1975, IEEE Trans, MucT, Sci. N5-22, 3 (1975).

4. L. Bex, D. J. Clark, C, E. Ellsworth, R. A. Estrella, R. A. Gough, and H. R. Holley, Enittance Measuranents of High Charge State Argon Beans from a PIG Source, Proc. Int. Conf. on Heary Ion Sources, Gatlinburg, Ternessee, October 27-30, 1975, IEEE Trans. Nuc1. Sci. N5-23, Z (1976).

5. D. I. Clark, Conference Sllmary, Proc. Int. Conf. on Heavy Im Sources, Gatlinburg, Termessee, Detober 27-30, 1975, IEEE Trans. Aucl. 5ci. NS-23, 2 (1976).

\section{SUPERHILAC - 1975}

The year an eventful one for the SuperHIIAC. An extensive effort to increase the reliabt1ity and voltage holding capabtlities of Adam, the 2.5 W injector, was completed in December 1974, and Adam used to deliver krypton beams successfully in January. Also in January, the first wodConil computers ware delivered, the first (visible) indication at the SuperHILAC of the conturter control hartware destined to play such a prominent part in machine operation in the coming months.

A new "puffer" value systert was installed on the Adarp ion source early th February, with the objoct of more easily attaining the very low gas flow rates needed for rimning heavy ions. This was very successful. In February modifications were begun to make the SuperHILAC ready for couputer control and timeshare operation. Computer interface hardhare was installed for control and ponitoring of if gradient and phase in the eight accelerating tanks and for the frulsod magrets. The control roout changes needed were so extensive that a decision was made not to attelupt a conplete conversion at this time, but to modify the existing control roor, primarily an enlargement, so as to permit two control stations to be menned, while postponing the major rebuilding until later.

The machine was turned on, primarily for test pupposes, for three weeks in April. Two beams were accelerated together for the first time. After this, the shritdown work was completed, and late in May the accelerator was ready for delivering bear to experinenters. The remaining six morths of the year the accelerator ran for experiments. From the start, it was clear that the tine-sharing operation was much more difficult than any previous operations of the SuperHIlac. The operators had to keep abreast of many more parameters than before, and tuning was much sharper, with less room for error. For these reasons beam intensity was frepuently low for some beams in the first week of operation; however, by Septenber it had been dentrustrated conclusively that beams of krypten and neor, both of adequate zitensity, could be time-shared together.

Operations for 1975 are sumarized in Figs. 1-4. The number of actual research hours devoted to the ion species studied (Fig. 1) shows a general

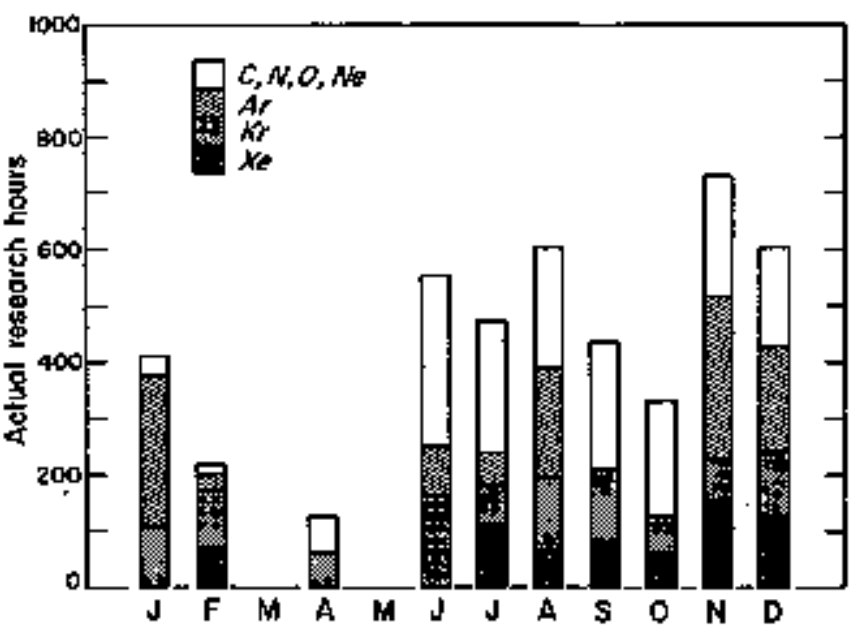

Fig. 1. Ions used for research at the SupertrIAC in 1975. (XBL 761-2091)

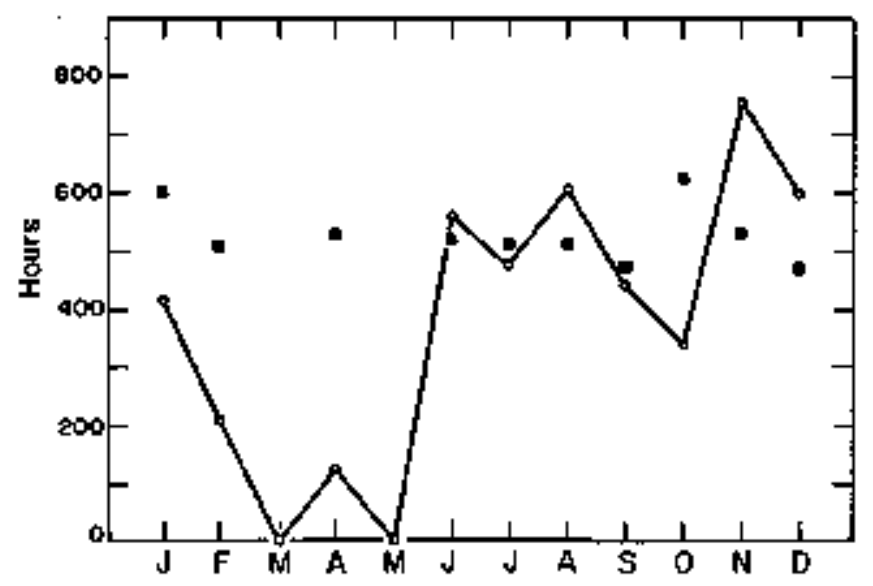

Fig, 2. Wuthly accounting of research hours in 1975. Open circles are total actual research hours; closed squares are total scheduled crew tinte. Warch, part of April, and May were shutdown nonths.

[XRL 761-2092] 


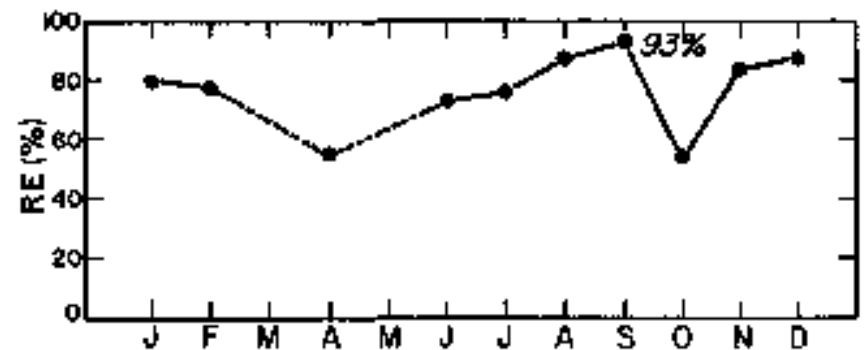

Fig. 3. The research efficiency (RE) of the superHILAC in 1975, expressed as the ratio of actual research hours to scheduled research hours.

(XBL-761-2093)

increase dring the year. In Fig. 2 both actual research hours and scheduled crew time are presented for comparison. The crew time includes all of the tine that the machine is available for use and includes scheduled maintenance.

The month of Japuary thus represents "best" operation before the introduction of tineshare. Berm was delivered to experisenters for 709 of the available machine time, which is good "innage" time for a research machine such as the superHILAC, considering the necessity for source changes and the setups required for new ich species, etc. In contrast, in June, the first month with futl tijer-share operation, the ondmber of research hour's exceeded the available machine time. This is sirply a result of the rultiplicity of beans available with timeshare, permitting two experinents to be done sinultannousiy.

The ratio of actual research hours to scheduled research hours (Fig. 3) is here called research efficiency. If there were no breakdowns, unsched uled batintenance, or source changes, this ratio would be $100 \%$. Nost months it ranged between 70 and 90\%, with two wnisually poor wonths below 608 .

Sone of the ressearch hours are spent in setup including such processes as getting equipment ready, switching wonitoring from one beam line to another, and tunirg. Thus the proportion of research hours In which the bean is on target, called here the target efficiency, is of interest as a useful indication of accelerator performance. It can be seen in Fig. 4 thatmonth by month, during the year, this target efficiency remained relatively constant within the range of $60-70 \%$.

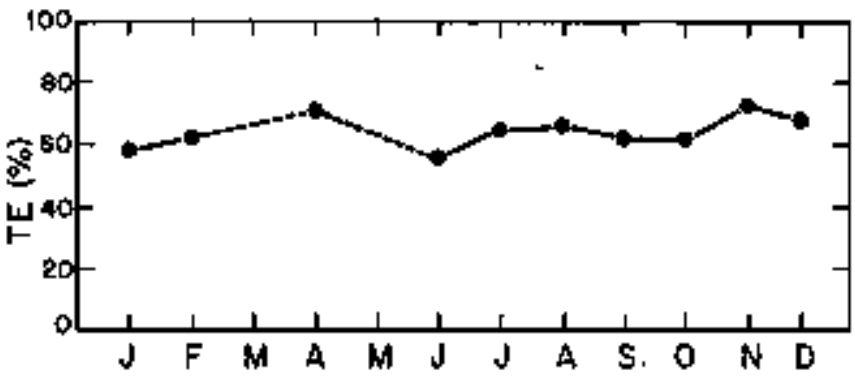

Fig. 4. The proportion of research hours in 1975 in which the beam was actually on tarpet-known as the Target Efficiency (TE).

(XBL 761-2094)

\section{SuperHILAC Dperations Stmary for Decenber}

. Sceldulued time

Percent of

Research hours, 686

Machine studies, 26

Machine operation for research

Machine studies and toning

Maintenance

Total schotuled

$385 \quad 78.6$

$66 \quad 13.5$

$399 \quad 7.9$ crew time

$490 \quad 1008$

Actual time

\begin{tabular}{|c|}
\hline $\begin{array}{l}\text { Setup } \\
\text { Trme-up } \\
\text { Target time }\end{array}$ \\
\hline \\
\hline
\end{tabular}

Parasftic time, 64.50

Machine tume

Machìne studies

27.25

7.50

5,6

Maintentance

190.25

$\begin{array}{r}1,5 \\ 38.8 \\ \hline\end{array}$

Total support time

225.00

$45.9 \%$ 


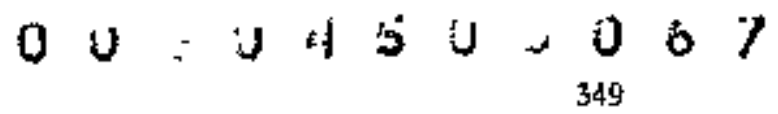

\section{B. NUCLEAR INSTRUMENTATION}

\author{
A LARGE SOLID ANGLE ${ }^{\text {BBe IDENTIFIER* }}$
}

G J Woznık, N A Jelly.t D P Sahel, and Joseph Cerny

A counter-telescope systen with a large effective solid anple ( $\sim 1 \mathrm{msr})$ for detecting ${ }^{6} \mathrm{Be}$ events and incorporating kinematic compensation ${ }^{1}$ is a doscribed below. A position-sensitive $\mathbf{E}$ detector is uged to measure both the energy and the direction of the ${ }^{8 \mathrm{Re}}$ events. Thus the detaction efficiency and the energy resolution can be optimized concurrently with inimal restrictions on the ${ }^{3}$ es accoptance angle.

The distribution of the breakm $\alpha$ particles is sharply peaked at the surface of the breakm cone (apex angle $-6^{\circ}$ ); thurs, in order to detect a substantial fraction of the ${ }^{8} \mathrm{Be}$ events, a detector should subtend an angle larger than the opendig arcle of the cone. For a large anglilar acceptance (10 ), a considerable variation in the detection angle $\left(\theta_{1,}\right)$ of the ${ }_{\mathrm{Be}}$ events is possible. On light targats (A $\div$ 16), a typical value of $\mathrm{dE} / \mathrm{d \theta}$ noar $25^{\circ}$ (lab) for the $\left(\alpha, 8_{B e}\right)$ reaction at $\mathrm{E}_{\alpha}-65 \mathrm{MeV}$ is argund $500 \mathrm{keV} / \mathrm{deg}$. By Heasuring the direction of a "He event and kinematically correcting its evergy, the energy resolution can be greatly inproved.

A particle striking a position-sensitive detector generates both an energy signal $E$, and a signa1 XE proportional to the probuct of its energy $\mathbf{E}$ and lts distance of impact $X$ fron one side of the detector (see Fig. 1). For high emergy 'Be efents, the breakup $Q$-value is stall compared to the ${ }^{B} \mathrm{Be}$

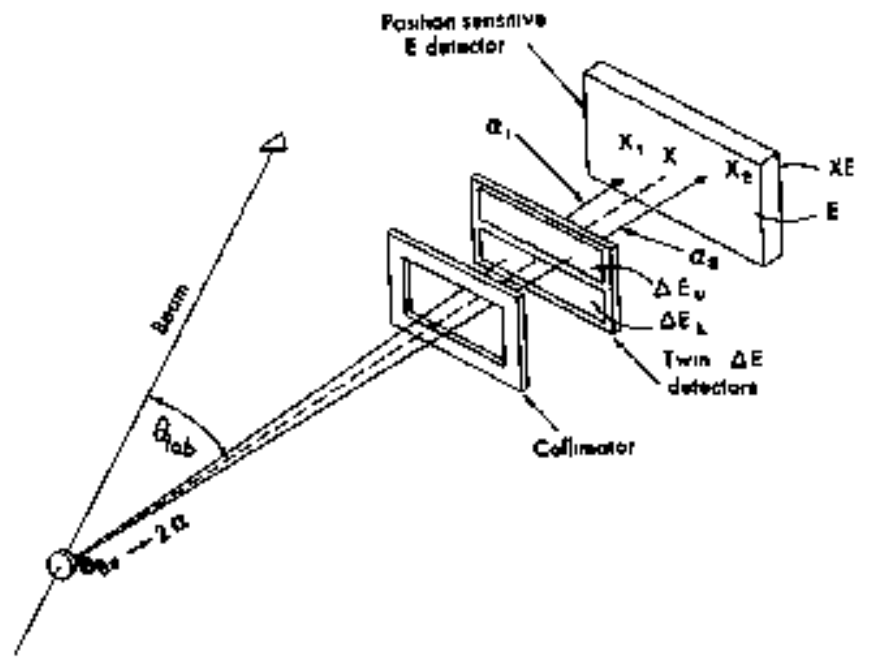

Fig. 1. A. schematic diagran of the ${ }^{8}$ Be identifier showing the twin transmission detectors, the PSD, the trajectories of the breakp a-particles (solid lines), and the measured direction $X$ of tho ${ }^{8} \mathrm{Be}$ evert.

(XEL $7511-8675$ ) energy, and so the two a particles have, to a good approxiration, equal energies. Since both a particles arrive within a fraction of a nanosecond of one another, the individual $\mathrm{E}$ and $\mathrm{XE}$ signals are automatically sended in the detector and the resultant $E$ signal gives the energy of the Be event. The position signaI $X$ obtained by dividing out the energy dependence is given by:

$$
\mathrm{X}=\left(\mathrm{X}_{1} \mathrm{E} / 2+\mathrm{X}_{2} \mathrm{E} / 2\right) /(\mathrm{E} / 2+\mathrm{E} / 2)=\left(\mathrm{X}_{1}+\mathrm{X}_{2}\right) / 2
$$

In addition to having equal energies, the two a particles are detected at approximately equal distances fran the axis of the ${ }^{B}$ Be breaky cone, which cor'responds to the direction of the original $\delta_{\mathrm{Be}}$ event as show in Fig. 7 . This average position $K$ establishes the direction of the "Be event and can be used to kinematically correct the be event's energy.

thile good efficiency and energy resolution can be obtained with a PSD alone, nuterous particlestable nuclei would also be detected and obscrre ${ }_{\mathrm{Be}}$ everits except when they happaned to be more energetic. To select only ${ }^{b}$ e events, a twin transnissfon detector is placed in front of the PSD as shown in Fig. 1. This detector consists of a singIe silicon wafer wilh two $\Delta E$ comters diffused side by side. Because the two breakip a particles have approximately the same velocity, their time-offilight difference ( $(\mathrm{TOF}$ ) to the upper and lower $\Delta E$ detectors is approximately zerp. The full width at the base of the peak in Fig. $2 \mathrm{a}$ (2 $\mathrm{ATOF}=0.85 \mathrm{~ns})$ illustrates the similiar filght thoes of the two a particles and the central dip is the effect of collimation on their velocity aistribution. By performing a subnamosecond cosncidence between the lupper and lower halves of the twin $4 \mathrm{E}$ detectors, $8_{\mathrm{Be}}$ everits can be selectively observed and the intrabeam-burst chance background rotuced by a factor of ten. Some further reduction in background and additional selection of $\mathrm{B}$ e events is obtained by performing particle identification with the somed $\Delta E$ and $\mathrm{E}$ signtals as show in Fyg. $2 \mathrm{~b}$ ("Be identifies as 1 í 1 t were a ${ }^{7} \mathrm{~L}$ i event). ${ }^{2}$

Since comercially awailable PS's give position information along their longest dimensim, the largest effective solidd angle and kinematic compensation are obtained by orienting the twin transmission detector as show in Fig, 1, In this configuration Be events can be detected over an angular spread of several degrees whth an almost constant detection efficiency. Charactoristics of this particular geonetry (V) and the other geonetries (I-IV) previously amployed are given in Fig. 3 and Table 1. 


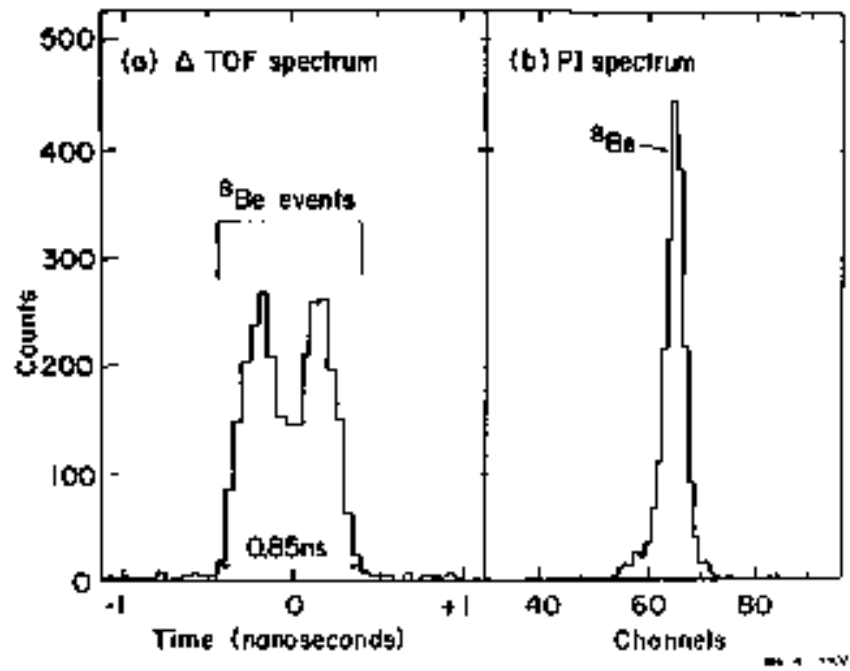

Fiy. 2, (a) Differential time of flight $\triangle T O F$, (b) particle identificaticn PI spectra obtained with the 8 Be identifier.

(XBL 745-33)2)
Fig. 3. The effective solid angles $\Omega_{\text {eff }}(I+V)$ which were used to study solid targets (solid lines) and gas targets (dashed line). See Table 1 for a description of the geonatries of these identifiers.

(XBL 7511-8673)

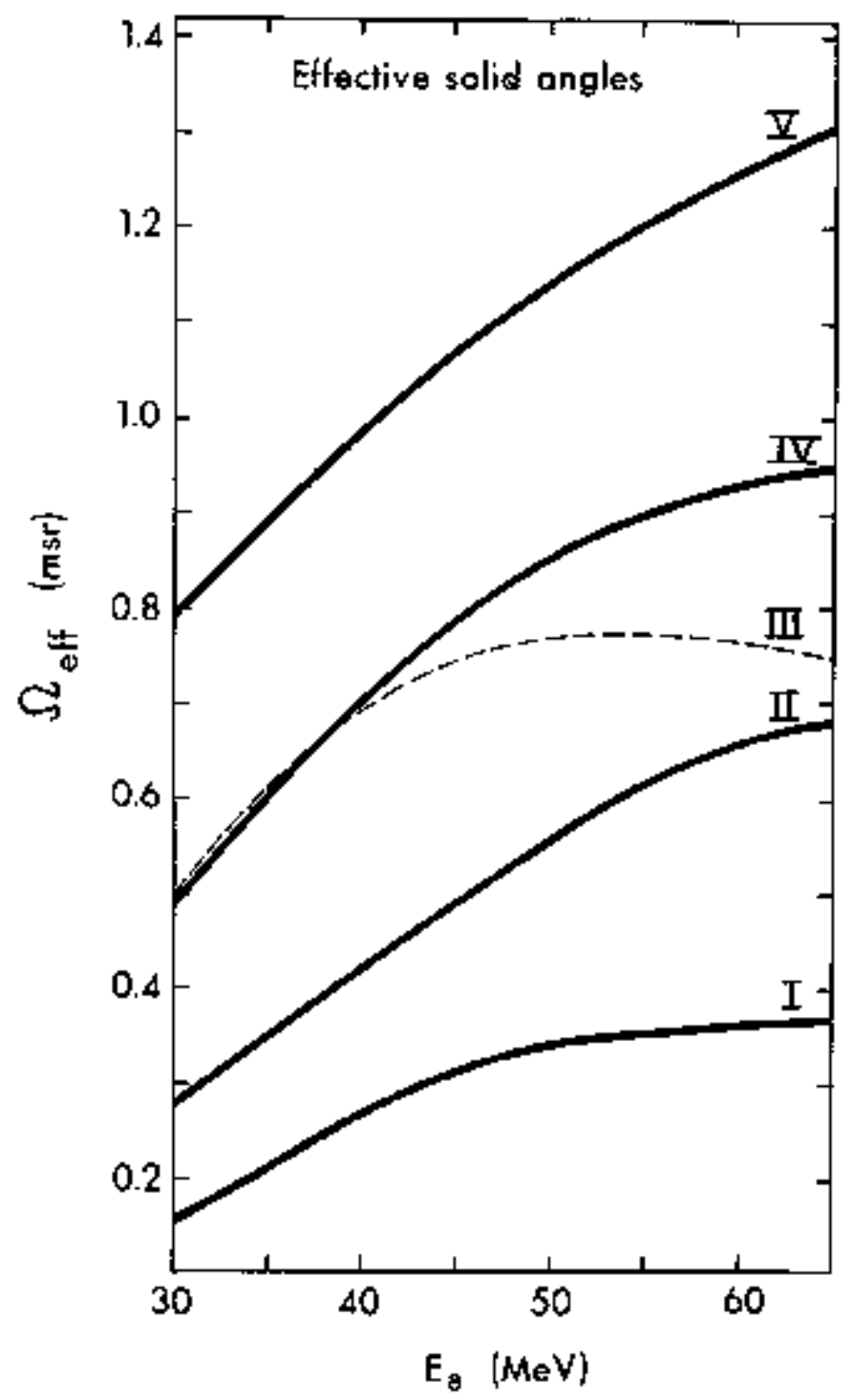

Table 1. ${ }^{8}$ Be Identifler geometries.

\begin{tabular}{|c|c|c|c|c|c|c|c|c|}
\hline $\begin{array}{l}\text { Exper - } \\
\text { irent }\end{array}$ & $\begin{array}{l}\text { Target to } \\
\text { PSD distance } \\
\text { (명) }\end{array}$ & Collinator ${ }^{a}$ & $\begin{array}{l}\text { Diameter }{ }^{a} \\
\text { (ou) }\end{array}$ & $\begin{array}{l}\text { Width } \\
\text { (om) }\end{array}$ & $\begin{array}{l}\text { Height }{ }^{a} \\
\text { (cm) }\end{array}$ & $\begin{array}{l}\text { Post or b } \\
\text { gap-width } \\
\text { (coll) }\end{array}$ & $\begin{array}{c}\text { Positione } \\
\text { gate-width } \\
\text { (col) }\end{array}$ & $\begin{array}{c}\text { Acceptance } \\
\left.\text { angle ( }{ }^{8} \mathrm{Be}\right) \\
\text { (deg) }\end{array}$ \\
\hline I & 8.00 & circular & 0.83 & - & - & $0.28 \mathrm{~V}]$ & 0,12 & 0.9 \\
\hline II & $13+00$ & rectangular & - & 1.12 & 1.25 & $0.30(\mathrm{~V})$ & 0.25 & 1.1 \\
\hline III & 7.45 & $\operatorname{gas} s^{d}$ & - & 0.78 & 0.81 & $0.24(V)$ & 0.26 & 2.0 \\
\hline$T V$ & 13.35 & rectangular & - & 1.51 & 0.98 & $0.19(\mathrm{~V})$ & 0.53 & 2.3 \\
\hline$V$ & 13.05 & rectangular & 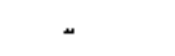 & 2.00 & 0.99 & $0,09(\mathrm{H})$ & 1,07 & 4.7 \\
\hline
\end{tabular}

AProjected dinensions of the collimator on the PSD.

Erojected dimension on the PSD of the divided collinator post-width or the gap-width of the undepleted region between the twin transmissiom detectors. The letters $\mathrm{V}$ or $\mathrm{H}$ indicate whether the gap was vertical (V) or horizontal (H).

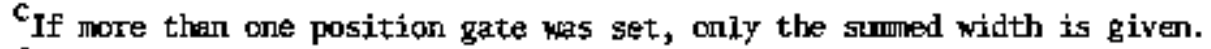

The distances frow the target to the gas collimator $\left(\mathrm{L}_{1}\right)$ and from this collimator to the second one $\left(\mathrm{L}_{2}\right)$ were $3.60 \mathrm{~cm}$ and $3.85 \mathrm{~cm}$, respectively. Only the dinensions of the second collimator are listed abowe. The finensions of the width, height, and post of the front gas collinator were $0.38 \mathrm{~cm}, 0.38 \mathrm{~cm}$, and $0.12 \mathrm{~cm}$, respectively. 


\section{$00 . y 450 ; 560$}

Shown in Fig. 3 are the effective solid angles ofeff for these gecmetries as a function of the ${ }^{8}$ Be energy; Table 1 gives the gecometric parameters empIoyed. The effective solid angle decreases at lower energies due to the increasing size of the breakup cone ( $\Omega_{\text {eff }}$ - $\varepsilon \Omega_{\text {acc }}$ whore hacc $_{\text {is }}$ is the acceptince solid angle and $E$ is the detection efficiency). By exploying a $1 \times 2$ an PSi, >1 msr effective solta andes (gectetry V) have been obtatned for detecting $\mathrm{Be}$ nuxlei. Furthemore, high selectivity for ${ }^{\text {B }}$ be events and kinematic conpensation have been incorporated into thas detection systeplo which has been successfully used to study the $\left(c,{ }^{8} \mathrm{Be}\right)$ reaction on $\mathrm{IP}_{\mathrm{p}}$ and is-1d sheil targets.

\section{Footnotes and Reforences}

“Condensed from LR1-4368, Phys. Rev, C14, 81S (1976),

${ }^{\dagger}$ Present adiress: Puclear Physics Labpratory, thiversity of Oxfort, England.

1. G. J. Hozniak, N. A. Jelley, and Joseph Cextry, Nacl. Instrum. Meth. 120, 29 (1974).

2. G. J. Hozniak, N. A. Jelley, and Joseph Cerny, Phys. Ret, lett. 31, 607 (1973).

\section{CONPLETION OF A FECOIL ATOM MASS ANALYZER (RAMA)}

D M. Moltz F. A, Gough, M. 5. Zisman,

O. J. Vieira. and J. Cerny

Decay studies of neutron-deficient nuclei become increasingly difficult the further one explores frow the line of $\beta$-stability. Coupeting reactions create a formidable background interfering with the study of muclei with low production cross sections and making conventional experimental techniques inadequate. To overcone this obstacle he have coupled the helftw $j$ et recoil transport method 1,2 with mass spectronetry techniques, we can now report the completion of a Recoil Atcol Mass Analyzer (RAkA) with this on-itne mass separation capability.

Figure $I$ is en overajl schematic of the tusk system. The operation of the system throph the wien filter has been previously described, 3 In an effort to increase the ion source efficiency, the auxiliary support gas inlet was changed to a point nearer the arc. The total extracted bean remained approximately constant, but a factor of five less arc support gas was peeded. Atterpts to detect the B-delayed $a$-Eitter 20 wa were made difficult by a large sea of electrons and neutrals downstrean of the Wlen filter. With the addition of the electrostatic quadruole and dipole unget, these electrons should no longer be a problem.

Fhysically the Iempinder of the system is in place and aligned. At present off-ling bean calibrations are beginning (using ions from the RAWA ion source) in order to map out the focal plane of this QSDS system (quadrupole, sextupole, dipole, and sextupole). The two sextupoles aro needed to rotate the focal plane perpendiallar to the beard axis as we11 as to reduce second-orfer aberrations, thereby improving the rass resolution to an expected 1:180 at 50 t transiassiom froal the ion source. The focal plane has a mass renge of approximately \pm 10 from the central mass. To separate the variots isotopes

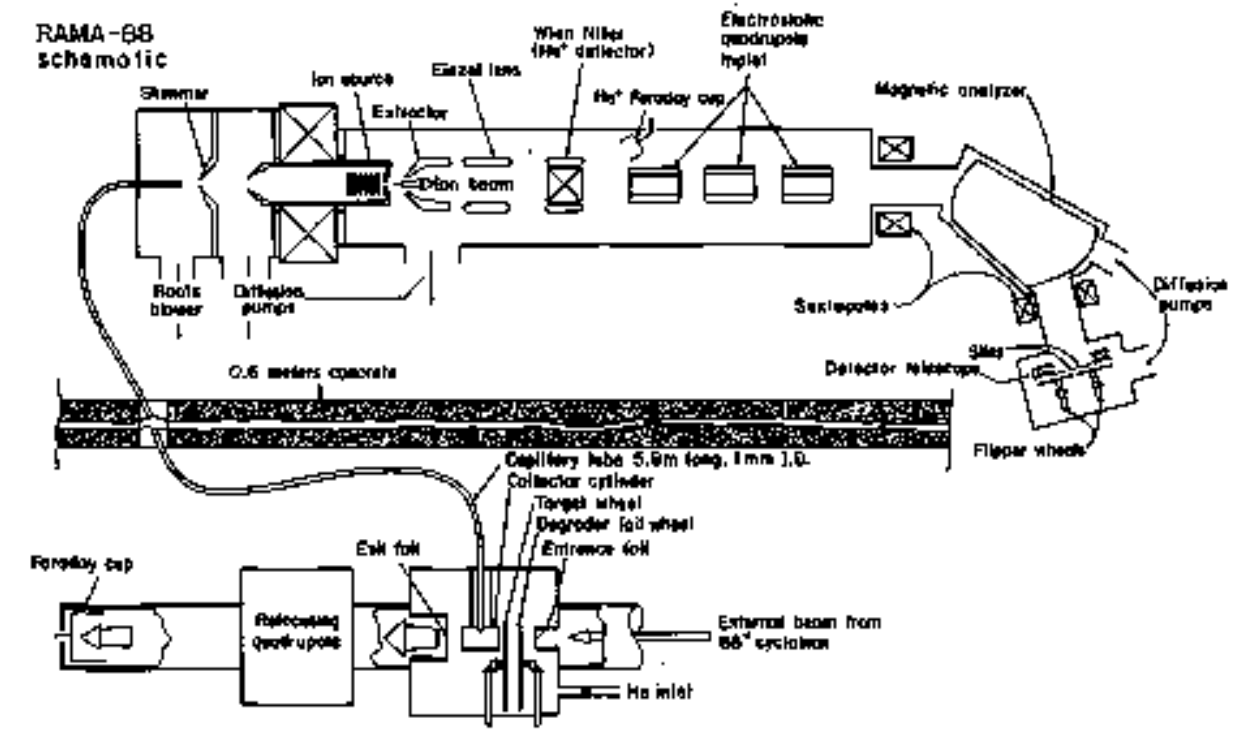

Fig. 1 .

(XBL $758-3797 A)$ 
in the mass region of interest, a pair of sysmetrically opening slit systens have been built. The opening width and the position of the slits can be adjusted to within a few mils. Behind each stit is positioned a collector foll attached to a $180^{\circ}$ flipper wheel driven by a ratary solenold. Rotational bounce is greatly damped by means of a mapnetic clutch-brake. The flipper wheels remove the activity fron the slit area to the side of the detector box for coknting, thereby providing a large physiçal separation of the collected sample. Provisionshave been made for solid-state detector telescopes, a plastic scintillation telescope, and ganms-ray de- tectors for studying the decays of the exotic nuclides under investigation.

\section{References}

1. R. G. Sextro, R, A. Gough, and J. Cemy, Phys, Rer. C 8, 258 (1973).

2. Puclear Chestistry Division Annual Report for 1972 , LBL-1666, p. 361 .

3. Puclear Cheristry Division Anmal Report for 1973, LEL-2366, p. 443.

\title{
A PROPORTIONAL COUNTER TIME-ZERO SYSTEM
}

\author{
G KeKe⿻ls, G Gabor, D K Scolt, and C K Gelbke
}

A new system has been developed to perform tine-of-flight messurenents in the 88 -inch cyclotrol magnetlc spectronter. With the present tiste-zero systen, which consists of an NE111 foll coupled to two photomultipliers with a Iucite light cone, it is not possible to measure cross sections at angles smaller than $10^{\circ}$. This is because taking data at such sma11 angles requires that the beam be stopped in a Faraday capp which is located inside the scattering chanber. Since a photomultiplier time-zero system is sensitive to the gandu radiation and large neutron fluxes protuced at the Faraday cup, there is considerable "in" and 'between'" beam-burst background.

The new time-zero systen is a parallel plate jroportional counter [see Fig. 1), It consists of three electrodes which are located symetrically between two gas contaiment windows. the electrodes are each seperated by 1 cn and the central plane. which is used as the anode, is conpletely shielded frcol all ground surfaces except for the ground plane electrodes. A propane gas fill is used at ID Torr with approximately +1000 volts on the anode. Electrodes and gas containment wirdows are made of aliminized mylar, and the active area is 15 al 2 .

The signal resulting from the passage of a charged particle consists of primary ionization which is enhanced about 100 times by electron multiplication. Signal rise times are limited by the electron collection and are typically $\sim 10$ nsec. Associatad positive lon collection times are 5 to 8 usec and are the lifmiting factor when counting at high rates. The fast output of a fast-sIow preapp is used for the timing signal.

Using the alowe detector at the entrance of the magnetic spectronster time resolution between. the gas counter and a large plastic scintillator has been linated to about $2 \mathrm{nsec}$, although resolution of bettor than 300 psec has been reported between a very thin gas counter of this type and a solid state corter. 1

Currentiy, the above detector is being used to measure ( $\mathrm{H}_{\mathrm{He}}, 8 \mathrm{He}$ ) reactions at migles between $4^{\circ}$

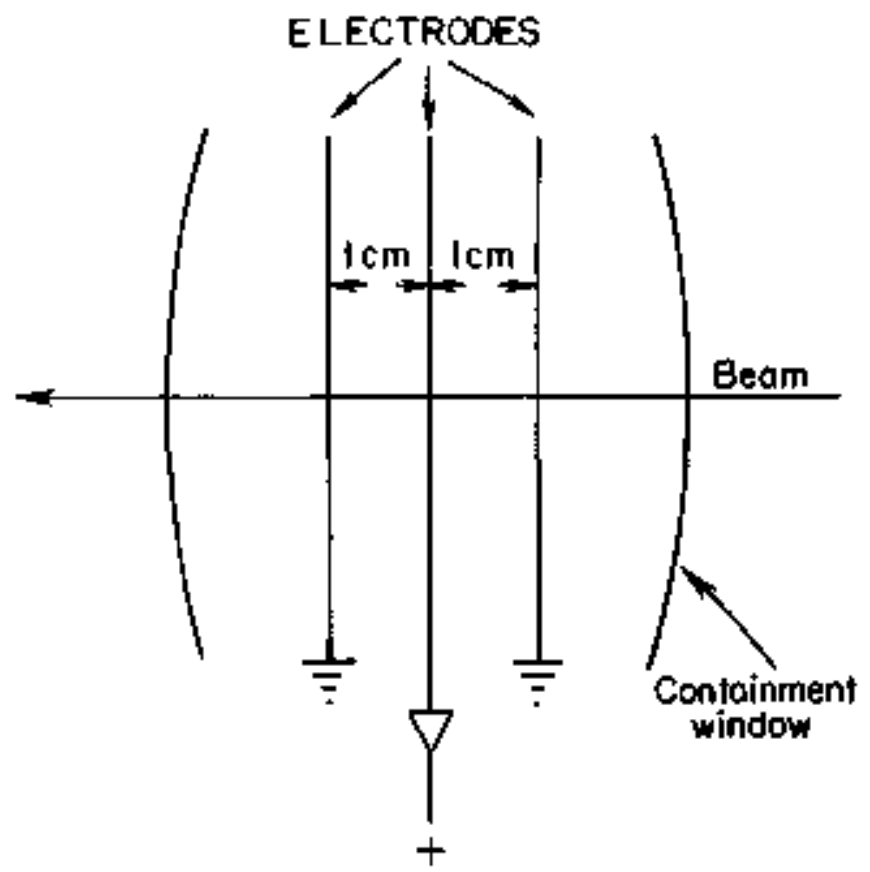

Fig. 1. Schematic of the parallel plate, gas proportional counter.

(XPL $766-8312$ )

and $10^{\circ}$. The detector has proven to be relatively insensitive to gamma rays and neutrons, making it: ideal for the present application. Detection officiency is 100 for charged particles which lose at Ieast $5 \mathrm{keV}$ ( $100 \mathrm{HeV}$ \% $\mathrm{He}$ ) when counting at less than $1 \times 10^{5} \mathrm{~Hz}$. In $\left({ }^{4} \mathrm{He},{ }^{6} \mathrm{He}\right)$ experiments efficiencies of at least 700 have been attained at a count rate of $1 \times 106 \mathrm{~Hz}$. The ultinate goal of this develofment progran is to produce a time-zero detector whichmay also be used for hoavy-ion measurements.

\section{Reference}

1. H. Stelzer, submitted to Nucl. Instrum, Meth., January 1976. 


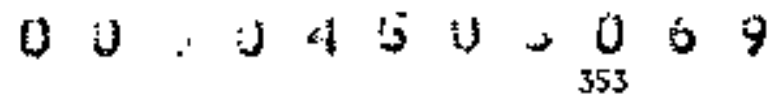

\section{RESPONSE OF PILOT U SCINTILLATOR TO HEAVY IONS*}

M Evernerd, D L Hendne, U Jahnke.t J Mahoney.

A Menchaca-Rocha,t C Olmer, and O K Scoll

The measurement of the time-of-flight (TOF) and of the specific energy Ioss (dE/dx) of particles detected and localized in the focal plane of a magnetic spectroseter, permits an identification of $Z$ and $A$ for futly ionized particles ( $Z$ and $A$ are the atconic muber and the nass of the fonj. Infortunately, this nethod leaves an ambiguity in the idemtification arising from different charge states $q$ of particles of given 2 , because both $T O F$ and $d E / d x$ depend on $\mathrm{A} / \mathrm{q}$. The response of a scintillator stopping the detected particles in the focal plane of a magnetic spectrometer depends on the energy $E$, the charge $Z$ and the pass $A$ of the ion through a function $L(E, Z, A)$. Since $E a q^{2} / A$, the parameter $L\left(q^{2} / A, 2, A\right)$ can remore the $A / q$ ambitgutty if the dependence of $L$ on $E$ and $Z$ are sufficiently distinct. For that purpose the response of a pilot $U$ scintillator has been measured for various ions at several incident errergies.

The experinents were carried out at the 88 -inch cyclotron and the measurements pere permed with the detection system and the OSD spectrometer; the geometry of the detector is shown in Fig. 1 .

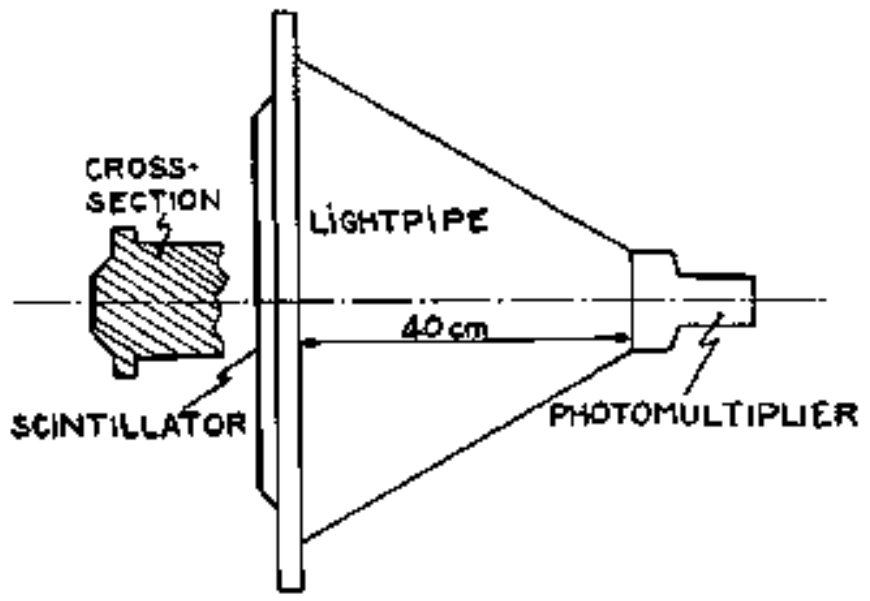

The scintillatur was placed at the focal plane and a $5 \mathrm{~mm}$ wide slit was placed in front of it. The measurements were made with particles elasticalify scattered from a thin gold target. For each incident beam, a set of different particle energies was obtained by degrading the energy of the scattered particles with aluminu absorbers placed in the scattex. ing chamber before the entrance aperture of the spectrameter. For each particle enerzy in the focal plane, the spectrometer was tumed so as to center the elastic peak on the slit in front of the selntillator, envuring a constant positioning of the particles in the focal plane. The energies were detemined from the field setting of the magnetic spectroneter.
The 1ight outpart was collected with a XPl040 photoImuliplier through a lucite light pipe (Fig. 1). The signal proportional to Iight output was taken from the ninth dymode of the photomultiplier, and fed to an ORTEC type I13 scintillator preaplifier.

The data were aralyzed in such a way as to provide experimentalists with a means of dedtcing with reasmable accuracy the $Z$ of a particle from the lowoledge of the scintillation response and energy. To that end, wo used a comvenient parmotxization to reproduce the data.

The set of data points obtained from the measurements are shown in Fig. 2 . For simplicity, the

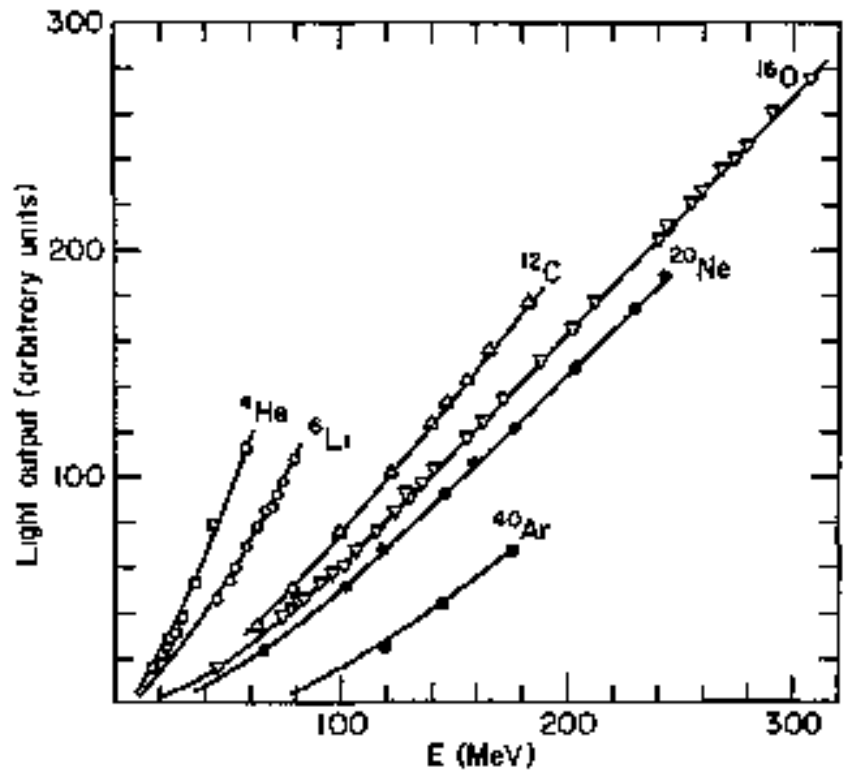

Fig. 2. Experinental resolts obtained in this work. The errox bars are less than the size of the data points. The curres are obtained from a paranetrization of the tight output $L$ as a function of energy using Eq. 1 in the text.

(XBL 762-2313)

data were fitted with a simple analytical parametri2ation suggested from the shape of the experimental curve, Good results were obtained with the trial function (see Fig. 2):

$$
L(E)=\gamma \hat{\mathbf{E}}+B\left(e^{-(\mathrm{CE}}-1\right)
$$

where $Y$ Is the slope of the light oxtput in the region linear with energy. The values of $Y$ for the different particles are plotted as a function of $2^{-1}$ in Fig. 3a; they are we1l reproduced by the first order equation:

$$
Y=0.58+3.87 z^{-1} .
$$




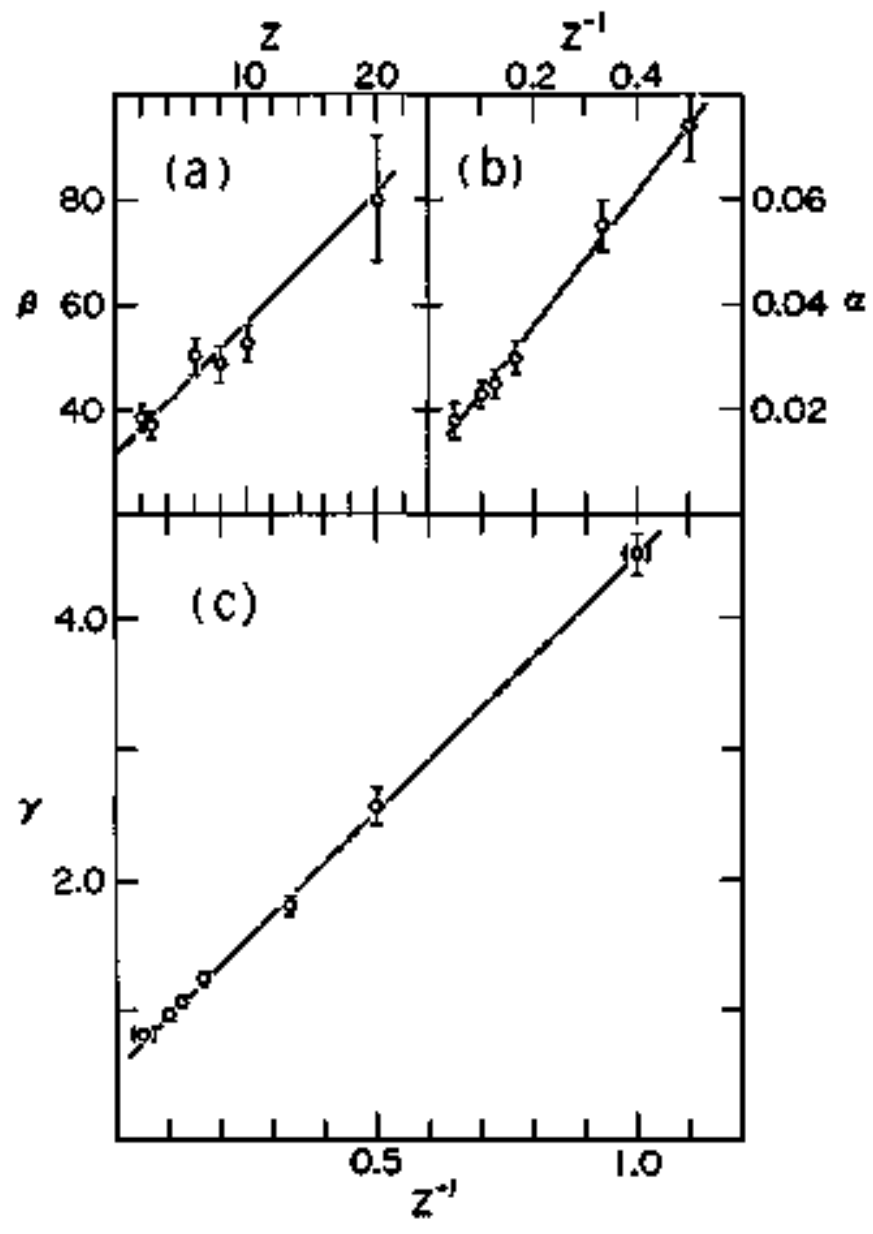

Fig. 3. The values of the paraneters $\gamma$, B, a fran Eq. 1, For each particle, $\beta$ is plotted as a function of $z$, and $\alpha$ and $y$ as a function of $z^{-1}$. The equations of the straight lines are given in the text (Eqs. 2, 3, 4).

(XBL 762-2311)

The value of $\gamma$ for $z=1$ was extracted from the proton data of Becchetti et a1. 1 The values of $L(E)$ for the two experiments were nomalized with the date for $\alpha$-particles, Although the measurements of Becchetti ${ }^{1}$ are for NE102 scintilletor, the proper- ties of NElO2 and PIlot $U$ scintillators are close encugh to justify the assurption of similar responses,

The values of the paraneter $\beta$ wero deduced from extrapolation of the asyuptote (YE-B) to the $\mathrm{E}$ axis, and the values of $o$ from the data points in the curved region of the response. The $Z$ dependence of the $B$ and $\alpha$ parameters is shom in Figs. 3b ard $3 \mathrm{c}$. Their empirical values are:

$$
\begin{aligned}
& \beta=32+2.6 z \\
& \beta=0.01+0.13 z^{-1} .
\end{aligned}
$$

Note that L(E) in Eq. (1) is always positive for $y>a \beta$. In practice $\gamma$ was obtained slightly smaller then $\alpha \beta$ for the cases studied here, and thus relation 4 is not valid for $\mathrm{E}$ tower than the root $\mathrm{E}_{0}$ of $\mathrm{Eq}$. (1) The value of $E_{0}$ is lower than $10 \mathrm{MfV}$ from 4 He to 160 ; it is about $20 \mathrm{keV}$ for $20 \mathrm{Ne}$ and about $60 \mathrm{HeV}$ for $40_{\mathrm{Ar}}$. As show by Fig. 2 this description is not expected to be accurate for energies below those of our experiments, especially for $40 \mathrm{Ar}$ ions lower than $100 \mathrm{MeV}$. For this latter projectíle, a rore compticated parametrlzation is probably called for.

Collecting all the previous results, one can predict the response of a pilot U scintillator to an ion (between ${ }^{4} \mathrm{He}$ and $4 \mathrm{D}_{\mathrm{Ar}}$ ) of given $\mathrm{z}$ by using the relation:

$\mathrm{H} L(\mathrm{E})=\left(0.58+\frac{3.87}{2}\right) \mathrm{E}+(32 .+2.62)\left[\mathrm{\theta}^{-\left(0.01+\frac{0.13}{2}\right) \mathrm{E}}-1\right]$

where $\mathrm{N}$ is a normalization coefficient, accounting for the effects of the photmultiplier, of the lightpipe atteruation and of the experinental gecmetry.

\section{Footnotes and Reference}

*Condensed fron LBL-4872.

†Hahn-bleitmer Institute, Ber1in, Gormany.

F Instituto de Fisica, Mexico $20 \mathrm{DF}$, Hexico

1. F, S. Becchetti, private commication.

\section{SPECTROMETER FOCAL PLANE DETECTOR SYSTEM}

B G Harvey, J Mahoney and $R$ F Burlon

Identification of heavy ions in a magnetic spextronetor requires the measurement of several parameters. The focal plane detector that has been in use or the spectraneter at the 88 -inch cyclotron for several years measures, for each particle, position (i.e., $\mathrm{B}_{\mathrm{p}}$ ), time-of-flight (TOF), energy loss $\mathrm{dE} / \mathrm{dx}$, and the cesidual energy (with rather poor regolution)

Resolution in TDF is 1imited to about 1-2\% by the dispersfor in flight paths caused by the finite radial angle of acceptance into the spectrometer. We have therefore built a new detector that measures the position of a particle in two Borkowski-Kopp resistive wire proportional comters ${ }^{2}$ placed 11 on apart, thits detemining the angle at which the particle arrives at the focal surface and the angle of emission fron the target. TOF can thereby be adjusted for flight path variation. Hopefully it will now be possible to increase the spectrometer solid angle by a factor of two or nore while retaining TOF resolution at close to the zero-solid-angle value of 0. 5 s. 


\section{$00: 5$ a is $0 \begin{array}{ccc}0 & 0 & 0\end{array}$}

The angular resolution of the double position reasurement depends upon the intrinsic resolution of the two position-sensitive counters and upon the mu1tiple scattering in the gas-filled space between them. As a comprorise betwem these two effects, a seperation of $11 \mathrm{~cm}$ was chosen, although the nultiple scattering unst still be uinimized by the use of a 1ow- 2 counter gas such as propane.

The space between the two position counters is used as an ion chamber with a cylindrical rod as arode, shielded by a tumgsten wire mesh "grid" as shom in Fig. 1. Electron collection times for par. ticle tracks at the top or botton of the ion chamber differ by about 2 us, thus peninitting the vertical position of a track to be determined by measurement of the tine difference betwen signals from the stopping plastic scintiliator and the ion chamber signals,

Resolution in the $\mathrm{de} / \mathrm{dx}$ measurement appears to be 1 intited almost entirely by energy loss straggling, although calculations of thisquantity for comparison with experinertally measured resolutions are not very reliable. For 104 Met 160, the measured (calculated) FoW is $2.58(2,3 \%)$ with 200 Torr of propane. For 315 HeV 160 , the experimental resolution is $5.4 \%$ Fill.
The whole detector is constructed in a matular fashion so that configurations most suitable for a specific experiment can be asșembled, For exarple, a second $\mathrm{dE} / \mathrm{dx}$ ion thamber or a stopping fon chamber can be inserted between the second position counter and the plastic scintillator.

Resfdual energy resolution in the scintillator is a strong fimction of particle energy, varying from $20 \%$ (104 $\mathrm{keV} 160)$ to $6 \%$ (315 $\mathrm{MeV} 160)$, where the energies are those of the particle at the entrance Wirdow of the whole system. Due to energy loss straggling in windows, the position counters and the $\mathrm{dF} / \mathrm{dx}$ ion chamber, a stopping lon chamber would be expect ed to give a resolution of at best about 2.5 .

\section{Refertices}

1. B. G. Harvey, et al., Nucl. Instrun, Meth. 104, 21 (1974); B. G. Harvey, H. Honeyer, and $J$. Mahoney, Nuc1. Instrum, Meth. 118, 311 (1974),

2. C. J. Borkowsk1 and M. K. Kopp, IEEE Trans. Nucl. Sci. NS-17, 340 (1970).

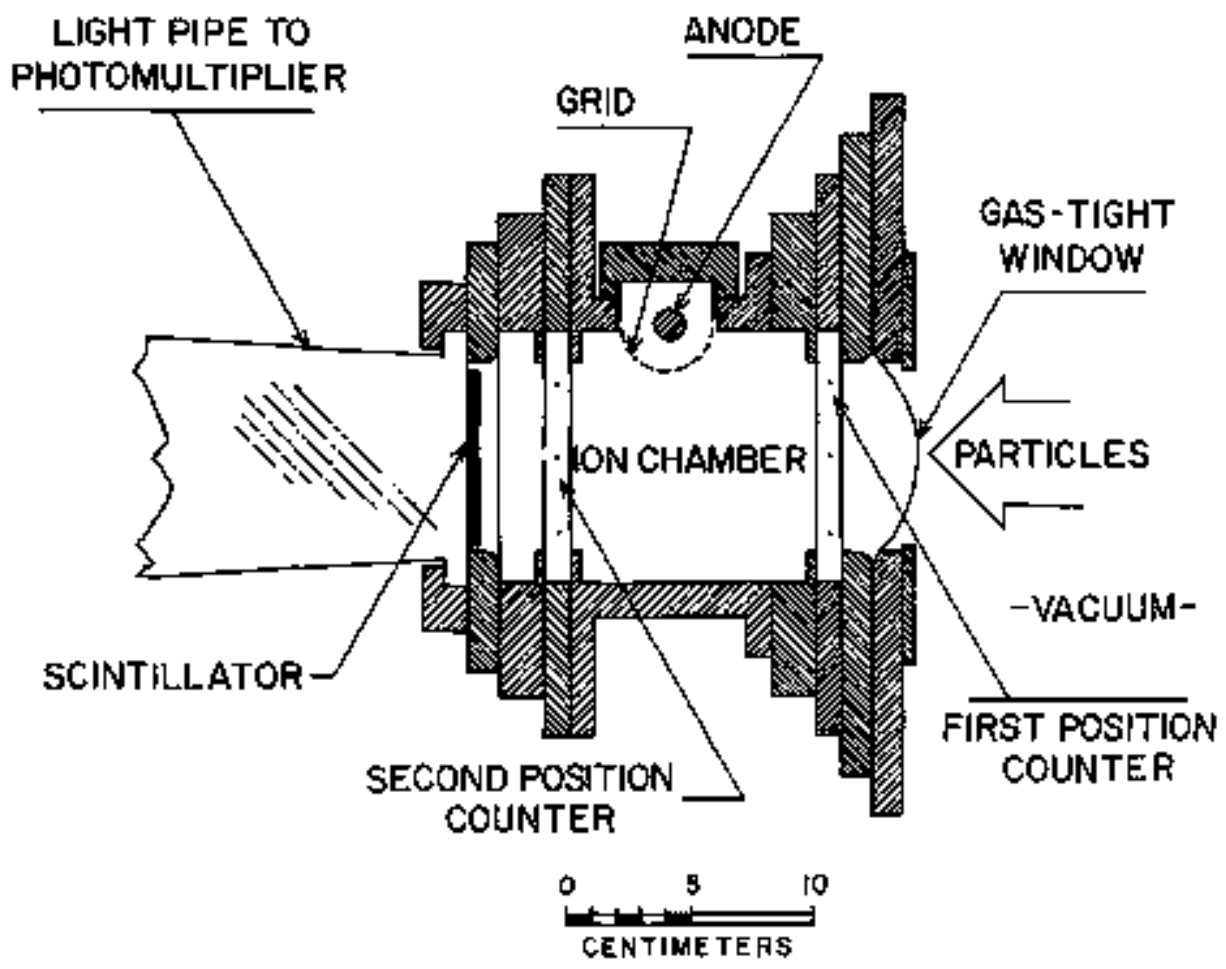

Fir. 1. Spectrometer focal plane detector systenu. Gas is usually 200 Torr propare.

(XBL 766-8219) 


\author{
A Jared, 9 Glassel, R Schmilt. \\ $P$ Fusso. and L G Moreytro
}

The stculy of heavy ion reactions has stimulated the developient of hardwre and software took for the identification of the products. A telescope ccilposed of a gas ionlzation of conter and a solid state $E$ counter which has been developed in ours group allows us to identify the fragment atomic norbers ( 2 ) over more than half of the periodic table. Unfortmately, the comonly used particle identification algorithes to not work rellably if used over a large range of atomic mmbers $(Z=4$ to 60$)$. In response to this need for a better algorithm, a data reduction system has been devised which uses tho experifental oE-E two dimensional maps to determine the location of the individual 2 ridiges.

This system has been used to reduce data ob tainet frop reactions îwolving many $2-4$ different beam-target-combinations at the $\$ 8$ ' cyclotron and at the SumerHILAC. Typically the data are taken in a scattering chanber with two to four counter telescopes. The signals from the counters are staject to normaI amplification and coincidence requirenents. The experinental data in digitizwd forms are stored on magnetic tape in an event by event form.

The data so collected are then assembled into $A E$ - E arrays $(E=100, A E=960$ channels) on a PDP-9 corputer. A simple array is shown in Fig. 1. This map shows that ridges associated with individual atamic numers cross the array with weIl-beheved slopes. The average slope is used to compress serera1 adjacent colums into a few new conpreșesed coluns with inproved statistics. Figure 2 is a colum obtained from the $A \mathrm{E}, \mathrm{E}$ map of reaction $600 \mathrm{MaV}$ $\mathrm{Xe}_{\mathrm{e}}+\mathrm{Ou}$. The peaks in the hastogram due to individual $Z^{t} s$ are nearly equidistant.

In principle, the peaks in the reduced colmos corld be fitted to find the centroid of each $z$, but this approach is ineffective at very high $2^{\prime} \mathrm{s}$ because of statistical difficulties and poor resolution. In order to exploit the periodicity of the spectrum a convolution intogral of the corpressed colum with a three peaked mask $\mid M(2)\}$ is employed-

$$
\begin{aligned}
& C(z)=\int_{0}^{\infty} 6\left(z^{\prime}\right) M\left(z-z^{\prime}\right) d z^{1}, \\
& M(z)=\sum_{i=0}^{z}[r(z-i-1)-2 r(z-i-0,5)+r(z-i)] .
\end{aligned}
$$

$r(Z)$ is a unit rant, and $f(Z)$ is the data

to determine the ridge paints; this method requires little computer time. Three pieces of in- formation are tised in the program: the average slope, the average $z$ spacing in a $\mathrm{E}$ and the muber of columlus to be compressed. The convolution stm is evaluated for corpressed colums and a triangular peak rask with a base width of one average 2 spacing. The significint peaks in the corrolution sum are used to define a new $Z$ spacing. This is then used to inmprove the $z$ spacing as a function of $\Delta E$ and $F$. Three adjacent triandular wave shapes with a base width of one $Z$ trit for each peak are then comoluted with each compresset colum, and normalized to one (Fig.3). The peaks in each convolution sun are then stored along with the statistical signiflcarce of each peak. The standard deviation is shown for several peaks in Fig. 3. The figure also shows the valley-to'peak ratio and the FWifN. These wore cal. corlated fron the convolution integral of several unit area Goussfans one 2 wit apart with a triple triangle wave forn. The two cirves are for the envelope of the maximata and ininimm of the convolution sim. It should be noted that these two curves apply to the compressed colum. The $Z$ regolution is affected by the slope used. The slope was optimized for large atomic numbers where the resolution is more critical. The maxima of the convolution sum generate an array of ridge points essociated with the various 2's. A fourth order polynomial is fitted to thespe points by raeans of an interactive program.

Mese ridge equations, for each $z$, the energy calibration equations, the dead layers, the constants necessary to deteruine absolute cross sections and the $\mathrm{Z}$ of one of the ridges are then used to produce energy spectra for each atonic number. These spectra are also interactively deconposed on the PDP-9 computer into the various components of the mergy spectrun. The center-of-mass spectra are also used to produce Wilczynski-type contour plats on the CDC 7600 computer.

\section{References}

1. M. M. Fowler, R. C. Jared, Nucl. Instrtan. Meth. $124341(1975)$.

2. F. Rosso, et al., Binary Breakre (and Secondary Fission) of a Heavy leformed System: $979 \mathrm{MeV} \mathrm{X}_{\theta}+$ Au, in this Armul Report.

3. R. P. Schnitt, et at., Nuctear Relgxation Phenoment 时 Ifffusion in the Reaction 107 , $109 \mathrm{Ag}+$ $84 \mathrm{Kr}$ at $7.2 \mathrm{MfeV} / \mathrm{rut}$ leon, In this Amual Report.

4. P. G1ässel et a1, Stady of Fragplents Enttted in the Interaction Between $18 \mathrm{I}_{\mathrm{Ta}}$ and $8 \mathrm{TTS}_{\mathrm{T}}$ at 620 MeV Bombarding Energy, in this Anmal Report. 


\section{$0 \cup \div: 45 \cup 507$}

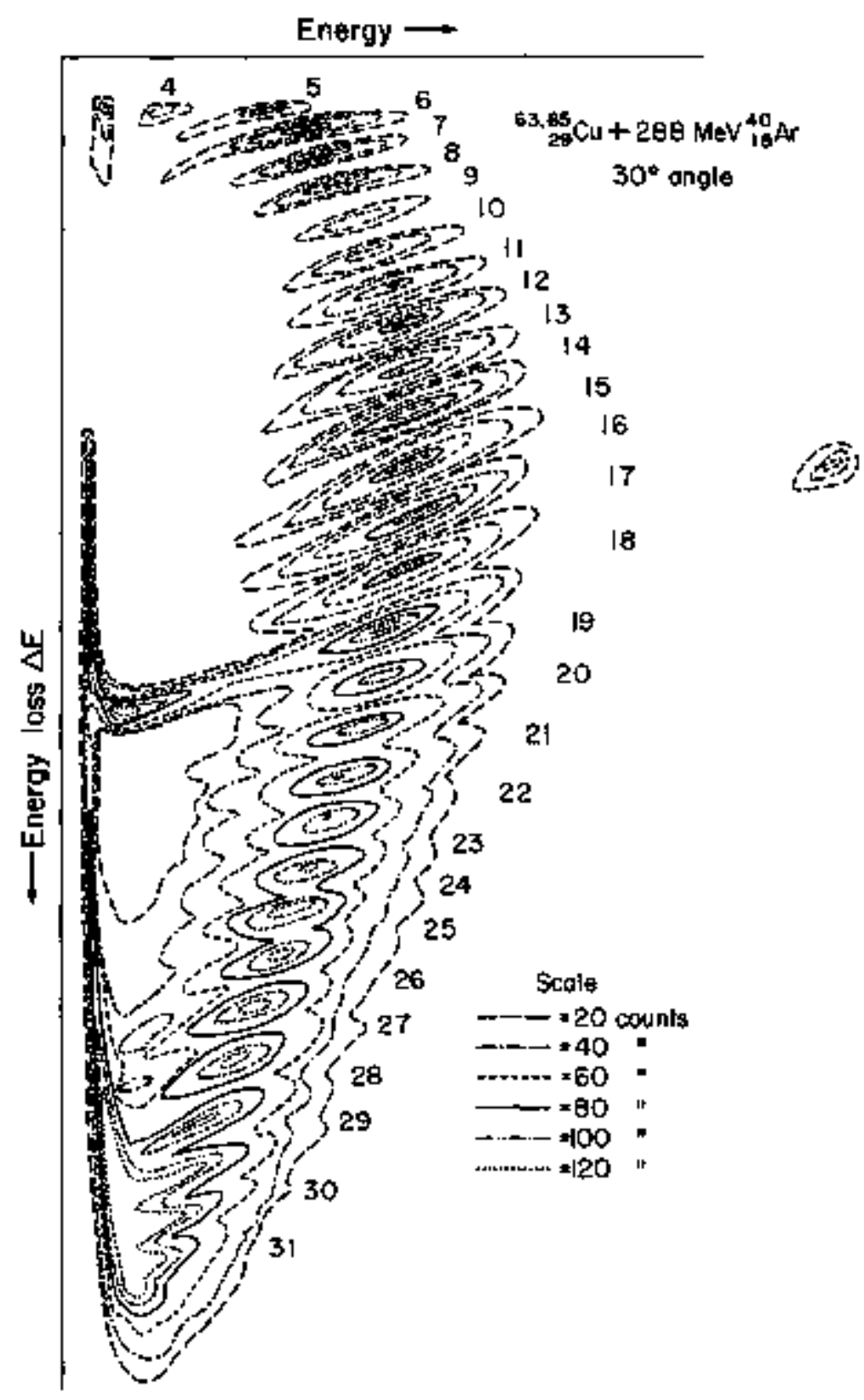

Fig, I. Map of the energy deposited in the gas counter ws that observed at the energy detector. (XBL 7411-8233) 


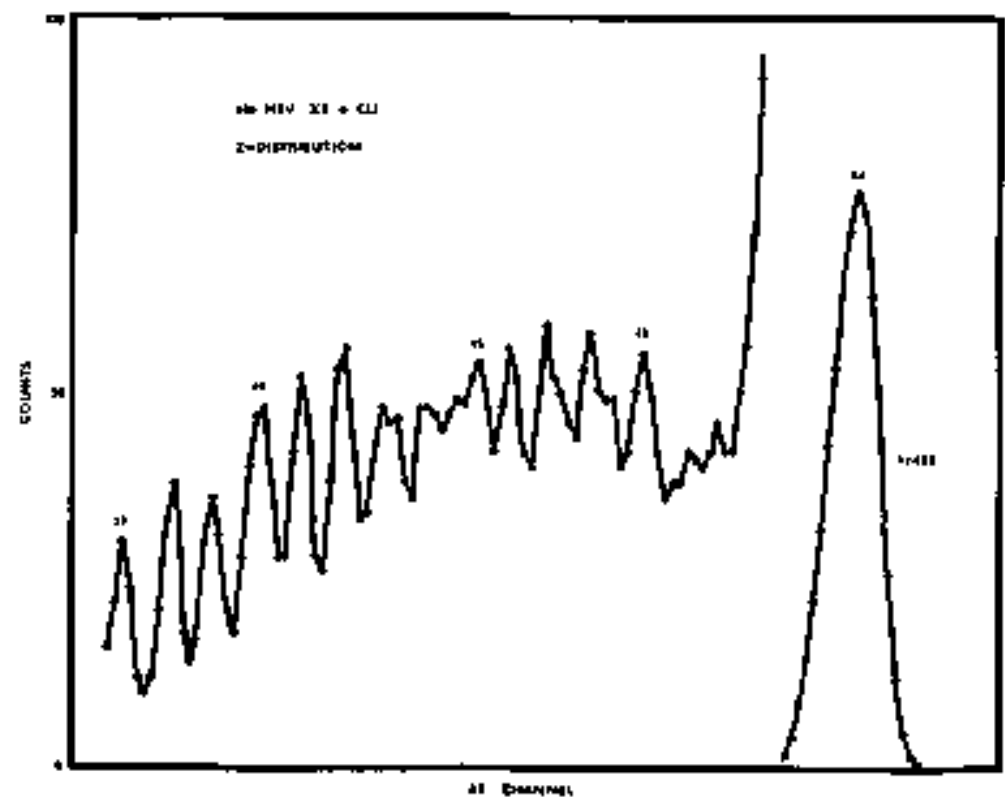

al beringat

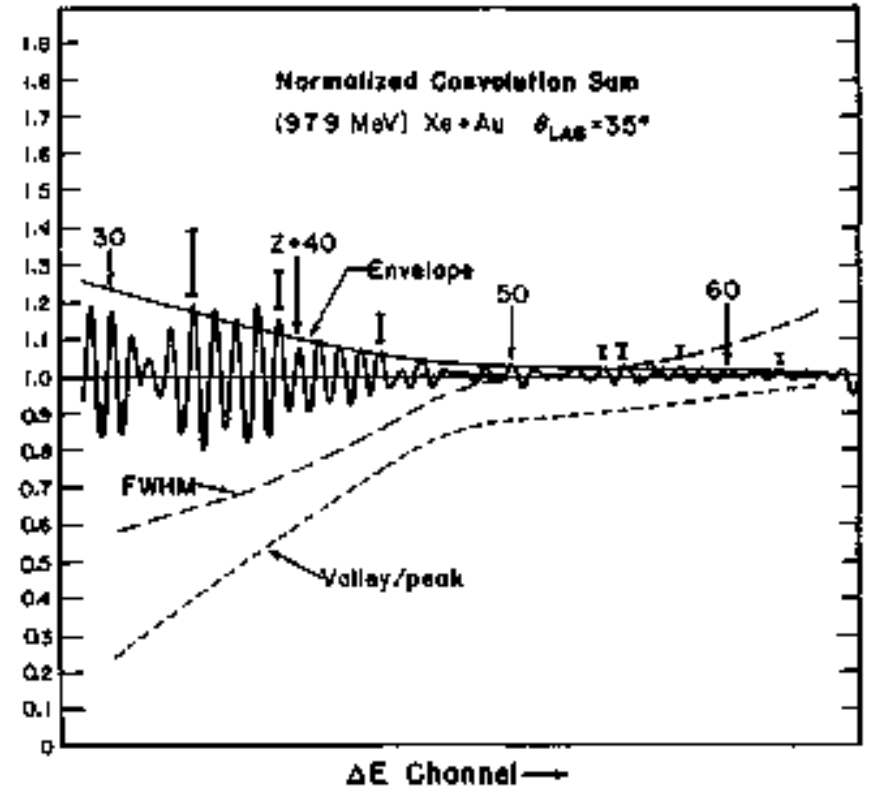

Fig. 2. Spectrum of $\mathrm{AE}$ conts obtained by adding adjactent columins of a tE-E map with an average sIope.
(XBL 706-8281)
Fig. 3. Composite diagram showing the result of the convolution sum. The calculated full width at half raximu (dashed line) and calculated valley-to-peak ratio (dotted lines) for several adjacent equal area Gaussians are shown for the envelope of the convolution șum.

\section{ENERGY CALIERATIONS OF SOLID STATE COLNTERS FOR HEAVY ION EXPERIMENTS}

P Fusso, $R$ Jared. R Babined. and L G Moretio

A new class of heavy ion experiments investigates processes in which the break-lp of the intermediate system occurs in a time scale which is some fraction of the total time required for the equilibration of aIl degrees of freedom. Accurate measurements of the laboratory energies and angles of the two correlated heavy fragments originating from the binary break-up of the heavy complex are necessary to determine suxch quantities as the masses of the two fragments at the time of breakup and the number and type of particles ewitted by the fragments. Details such as these reflect the froperties of the partially relaxed complex on its way to oquilibrikm, and so it is compelling to porform such meastrements as a probe for understanding relaxation phenomena in nuclear matter.

Critical to the success of heavy-ion coincidence measurenents in predicting the masses of the heavy fragments at the time of breakum is an accu- 


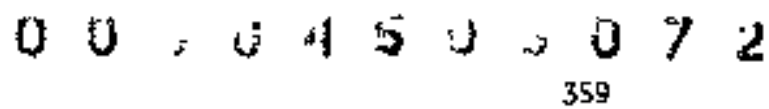

rate determination of the energies and angles in the laboratony and of the 2 for at least one of the fragments. Heavy-ion measurements are couplicated by unusually high ionization densities and a broad range in the $\mathrm{z}$ of the protuct. The use of a telescope which consists of a gas ionization ( $A E)$ detector wfth a solid state silicon surface barrler $(E)$ counter allows for resoluticrs in $z$ up to $Z=60.1$ A salal1 thin window on the telescope permits accorate determination of the argle of the detected fraguents. Masured in coincidence with the telescope events are fragnents which are stopped in a hight-resistivity silicon position sensitive detector which defines the position of the detected fragJnert of measured energy to within $1 \mathrm{~mm}$, It remains to obtain a precise caldbration of the analog re-

I sponse of the solid state coldenters to the ionization produced by the stopping heary froginents.

The pulse height defect (PFD) denotes the apparent energy defect of a charged particle detected by a solid state counter. While the effect is very small for low 2 fons, detected in 511 icon, the pulse height of the signal praduced by $a$ heavy ion of the same kinetic energy will be less than that for the light ion due primarily to the effects of recanbination which octurs increasingly with ionization densłty and is enthansed in high-resistivity silicon.

The PHD in the PSO and the telescope E counter has been determined from measurements of elastically scattered argon in coincldence with recoll gold, sllver and copper target nuclei. A bear of $163 \mathrm{MoV}$ argon from the 88 -inch cyclotron was incident on thin (200 to $350 \mu \mathrm{g} / \mathrm{cm}^{2}$ ) self-supporting foils of natural gold, silver, and copper. The incident projectlle energy is below the Coulomb barier for gold and very near the Coulomb berrier for silver elininating the possibility of contanination of the elas tic peak whth transfer products. The observed energies of detected elastics and recoils were corrected for energy defects the to dead layers and target and window thicknesses using the range-energy calaulated elastic and recoil energies, differences being attributed to the PHD. The PFD for rold, silver and copper ions in the high-resistivity silicon of the PSD is plotted in Fig. 1 as a function of the energy of the ion as it enters the silicon. The dimensionless energy units (LSS units) defined ${ }^{3}$ by Lindhard, Scharff and Schiott have been formulated in order to express in terus of a single function of ion energy the pulse height defect of any ion in the same medium. The PFD is approximately linear with the stopping power so that the rolationship between energy in MeV and LSS units is a fumction of the $z$ and $A$ of the ion in the stopping nediun. This relationship for an ion stopping in silicon is:

$$
\mathrm{E}_{\mathrm{L} 55}=6.525 \times 10^{4} \frac{\left(\mathrm{z}^{2 / 3}+5.808\right)^{-1 / 2}}{\mathrm{z}(\mathrm{A}+2 \mathrm{~B} .09)} .
$$

A single, eapirically-determined ${ }^{4}$ function of the form:

$$
\mathrm{PHD}=\frac{6 \mathrm{E}}{\mathrm{E}+\mathrm{B}_{1}}+\frac{\mathrm{B}_{2}}{1+500 \mathrm{E}^{-1,4}}
$$

where FHD and E, the ton energy, are in LSS umits can then be fitted to all of the PHD data obtanned

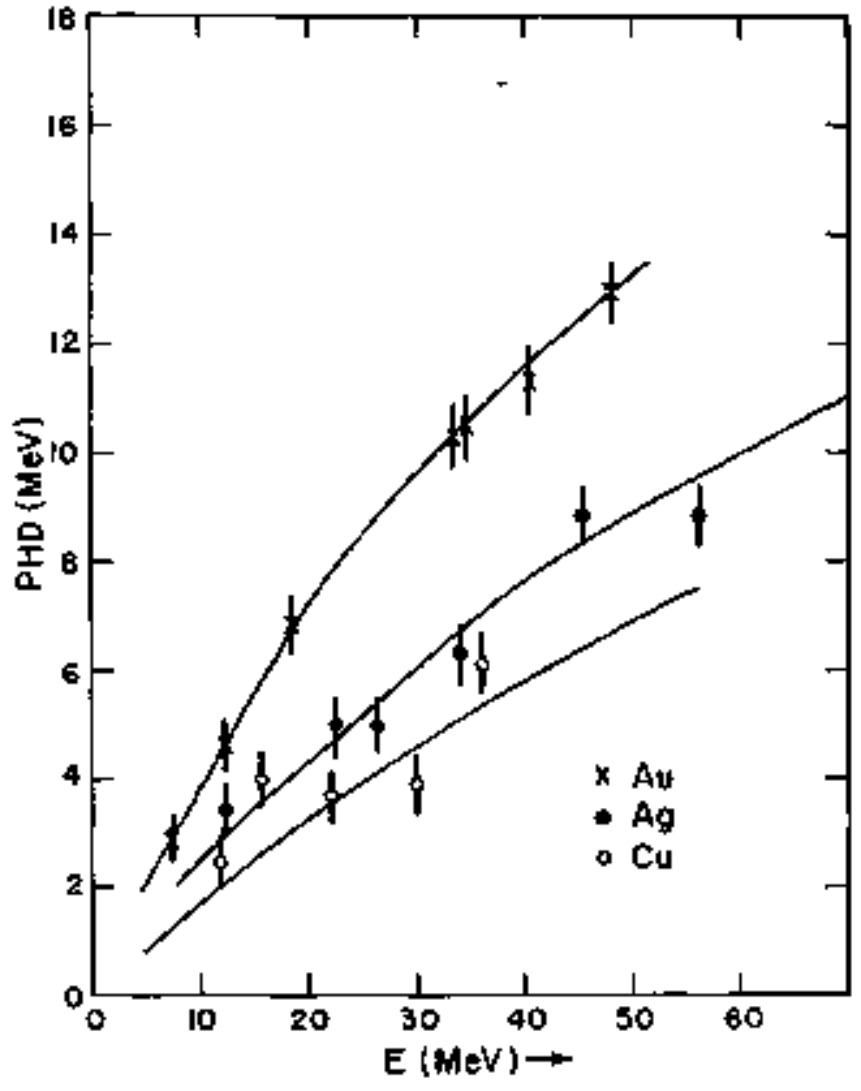

Fig. 1, Experimentally deteruined pulse-height defocts for gold, silver, and copper ions of various eneryies detected by a high-resistivity (25000 ohw/ot position sensitive silficon counter. (KBL 766-8211)

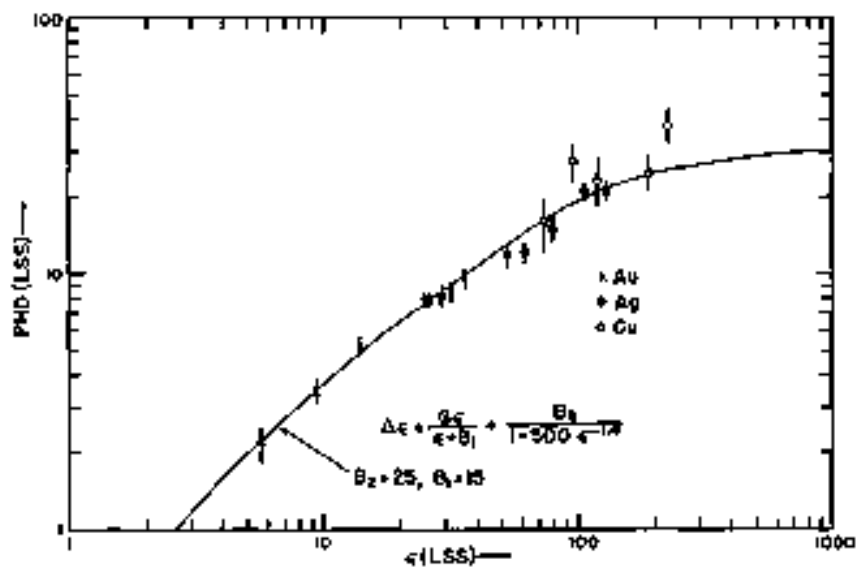

Fig. 2. The pulse tright defects of gold, sllver, and copper ions converted to LSS units and plotted as a function of ion energy in LSS units. The solit line is a fit to the data allowing the coefficients $\mathrm{B}_{1}$ and $\mathrm{B}_{2}$ to vary.

(XBL 766-8212)

with a given detector. Figure 2 is a plot of the PHD data from Fig. 1 comperted to LSS units. A fit to the data with the ofmpirically-deternined fumction gives values of 25 for $\mathrm{B}_{2}$ and 15 for $\mathrm{B}_{1}$. $A$ similar technique applied to the silicen surface bar- 
rier detector gives values of 18 for $\mathrm{B}_{2}$ and $B$ for $B_{1}$, in the agregnent with previously determined paranetars for detectors of this type. The resistivities of the PSD and the surface barrier detectors are approximate $1 \mathrm{y} 25,000 \mathrm{ohm}$ cm and $4000 \mathrm{ohm}$ cur respectively.

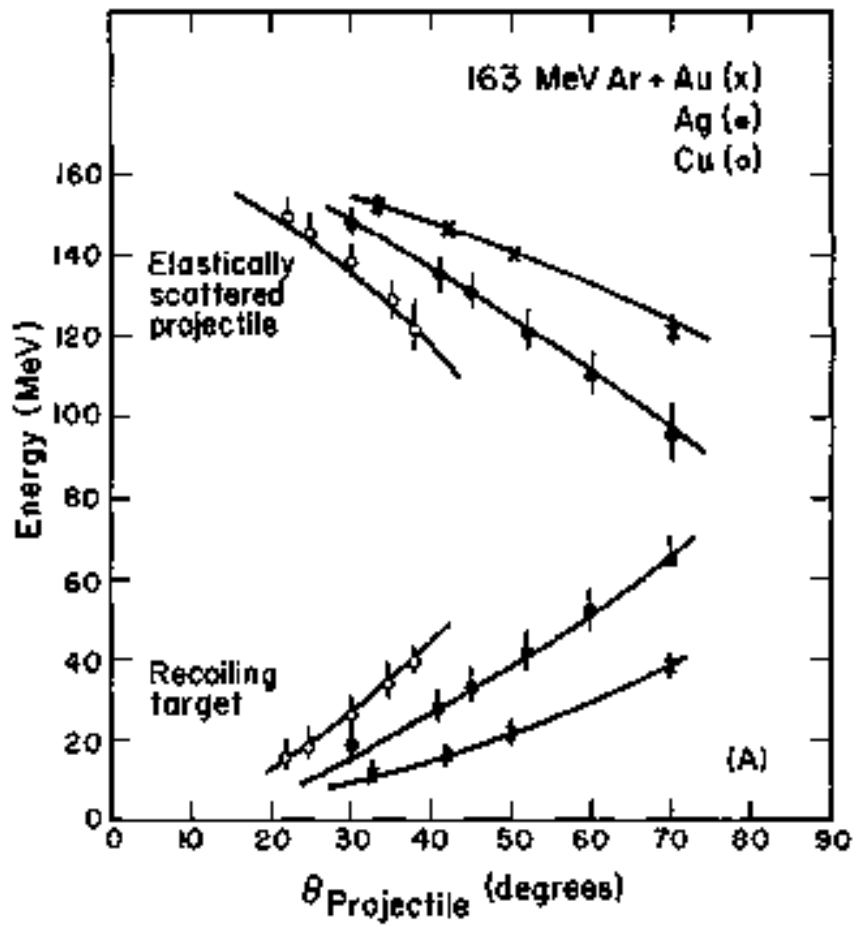

Fig. 3. Results of elastic-recoil coinciderce measurements for $163 \mathrm{keV}$ argon incident in copper, silver, and gold targets. (A) Experimental laboratory energies of the elastically scattered projectile and the recoil target plotted vs angle for the elastic projectile. The solfd lines are the calculated results for elastlc scattering. (B) Experimental laboratory angles for target recolls plotted vs angle for the elastic projectife.

(XBL 766-8213)

(XEL 766-8214)

cases, the PSD was the recoil detector. The agresement with elastic scattering (solid lines) is good except when the recoil energy drops below about 15 MeV. At such low entergles, the net energy correction appraaches the recoll energy for the heaviest ions. Table 1 gives the values of the recoil energies and the sumed c,m, angles for the coincident fragerents. Again, deviations from $180^{\circ}$ occur whon the recoil energy falls below $15 \mathrm{MkV}$.

The coincidence data obtained in the experinemt with a silver target and 340 Wev argon projectiles requires a precision of 28 in the enorgy calibration of the PSD in orter that a determination of the masses of the two fragmenits be accurate to within one mass tnit. The accarracy of the neasurement is within this linit since the frappent energies are approximataly $50 \mathrm{MeV}$ in the laboratory frane.
A critical test of the accuracy of the energy corrections for heavy ions is the ability to reproduce elastic-recoil coincidence kinematics. The results of correlated elastic-recoil measurements meade with $163 \mathrm{kleV}$ Argon incident on gold, silver and copper targets are shown in Figs. $3 \mathrm{~A}$ and $3 \mathrm{~B}$. In all

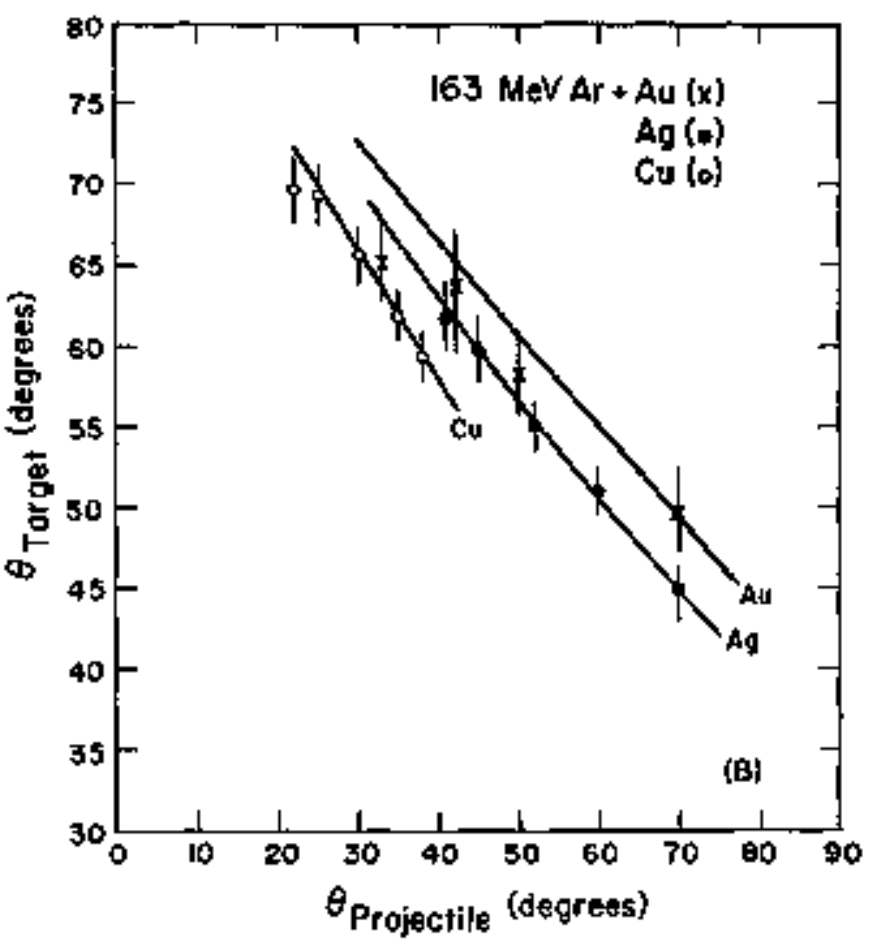

Table 1 .

\begin{tabular}{|c|c|c|c|c|}
\hline${ }^{z_{\text {Proj }}}$ & ${ }^{2}$ Tgt & $\theta_{\text {Prot }}^{\text {Scatt }}$ & $\mathrm{E}_{\mathrm{Recoil}}^{*}(\mathrm{MeV})$ & $\begin{array}{l}\text { Observed } \\
\left(\theta_{1}+\theta_{2}\right) \text { on }\end{array}$ \\
\hline \multirow[t]{5}{*}{18} & 29 & $38^{\circ}$ & 39.1 & $179.2^{0}$ \\
\hline & & $35^{\circ}$ & 33.9 & $179.5^{\circ}$ \\
\hline & & $30^{\circ}$ & 25,6 & $179.7^{\circ}$ \\
\hline & & $25^{\circ}$ & 18,2 & $179.2^{\circ}$ \\
\hline & & $22^{\circ}$ & 14.3 & $142.3^{\circ}$ \\
\hline \multirow[t]{5}{*}{18} & 47 & $70^{\circ}$ & 64.7 & $180.6^{\circ}$ \\
\hline & & $60^{\circ}$ & 51.7 & $180,2^{6}$ \\
\hline & & $52^{\circ}$ & 41.2 & 180.00 \\
\hline & & $45^{\circ}$ & 32.3 & $179.3^{\circ}$ \\
\hline & & $41^{\circ}$ & 27.5 & 178.80 \\
\hline \multirow[t]{4}{*}{18} & 79 & $70^{\circ}$ & 38.6 & 179.9 \\
\hline & & $50^{\circ}$ & 22.1 & $179.5^{\circ}$ \\
\hline & & $42^{p}$ & 16.2 & $179.3^{\circ}$ \\
\hline & & $33^{\circ}$ & 10.4 & $175.8^{\circ}$ \\
\hline
\end{tabular}

Compeited from elastic scattering. 


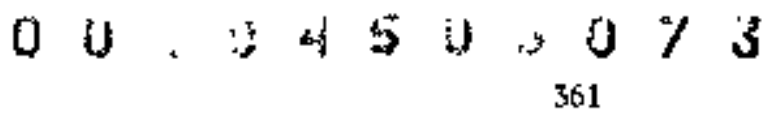

\section{References}

1. K. Jared, ot al., Identification of Atom Nembers Up to $z=60$ in Fragnents fron Heavy-Ion Reactions, in the Anrual Report.

2. L. C. Northeliffe and R. F. Schilling, Nuclear Data Tables $7,322(1970)$.
3. J. Lindhard, M. Scharff, and H. E. Schtott, Mat. Fys. Metd. Dan. Vid, Selšk. 33, 14 (1963):

4. S. B. Kaufran, E, P. Steinberg, B, D. Kilkins, J. Inik, and A. J. Gorski, Me1. Instrtm. Meth. 1ㅗ, 47 (1974).

\section{WILCZYNSKI PLOTS: AN EFFECTIVE WAY TO DISPLAY SINGLES DATA}

J S Sventek and L. G Morelto

In order to provide a more powerful means of displaying the large amount of 2-resolved singles data taken by our grouy for various reaction systems, we have developed a set of interactive computer prograns which allow us to draw Wilczynski plots 1 (maps of contours of constant double differential cross

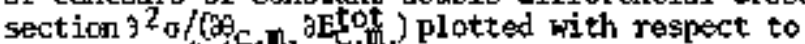
$\theta_{c}$ and $\mathrm{Etol}^{\mathrm{C}}$. for each 2 resolved in these reactions. A quick perusal of Figs, 1-4 will indicate to the reader the amount of information which can be conveyed in such a plot.
The initial results of our data reduction axe kinetic energy spectra for each $z$ in the 1ab system, $\left.{ }^{2} 25 / 0 \mathrm{~S}_{1} 3 \mathrm{El}\right)$. By making an assumption about the mass of the fragoment and assuring a binary breakII of the complex, these spectra can be converted to the center-of-mass systen giving $a 2_{\sigma} /\left(\partial \Omega_{\mathrm{c}}, \mathrm{m}, \mathrm{E}_{\mathrm{E}, \mathrm{m}}^{1}\right)_{+}$ Conservation of linear monentum gives

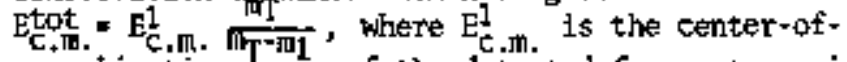
mass kinetic onergy of the detected fragment, $m_{1}$ is Its mass, and tor is the total mass. Since the reaction is binary, there is no aziruthal dependence

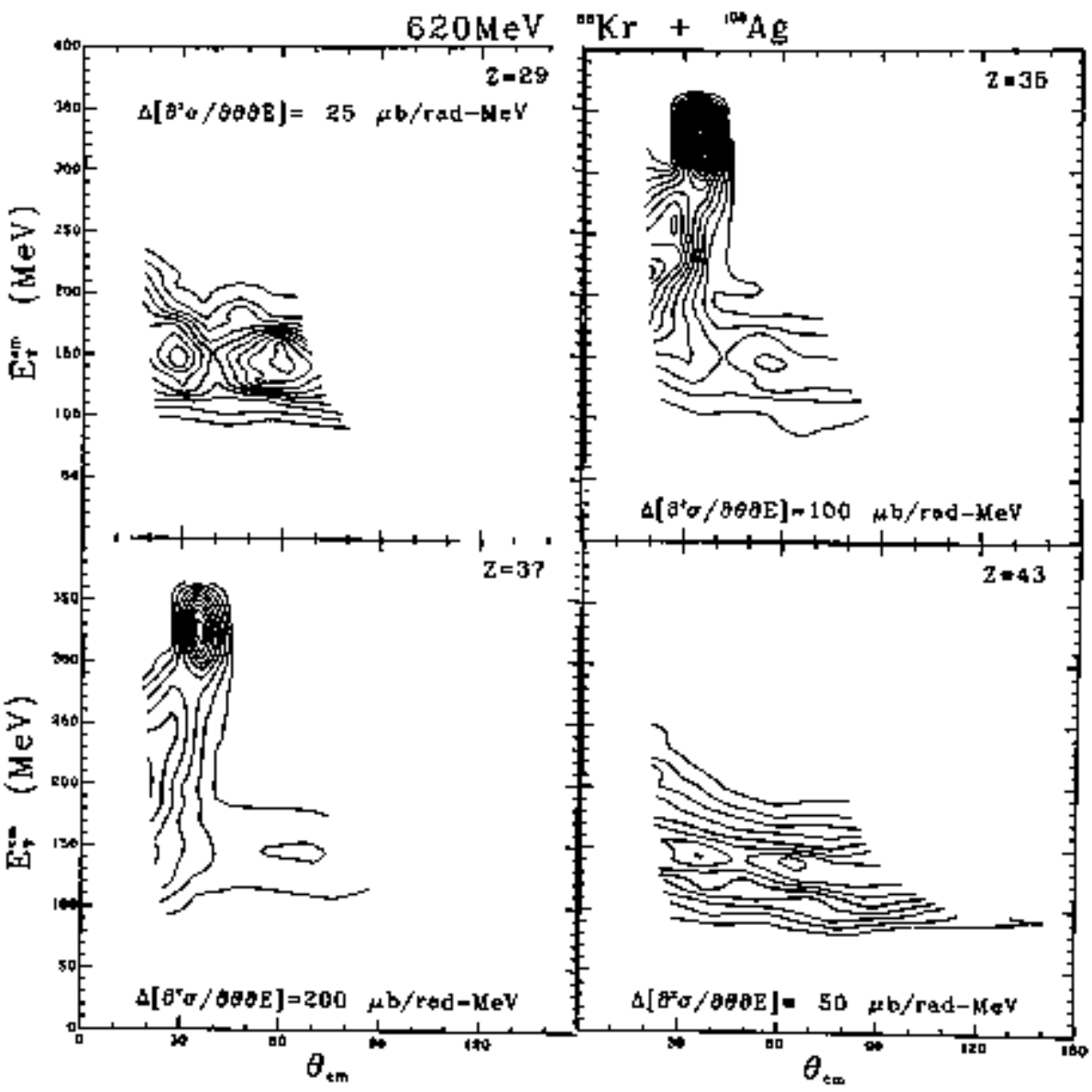

Fig. 1. Wilczynski plots for four repressentative 2 's for the reactlon $620 \mathrm{MeV} 8 \mathrm{~b}_{\mathrm{Kr}}+107.109 \mathrm{Ag}$. QBL 766-8167] 


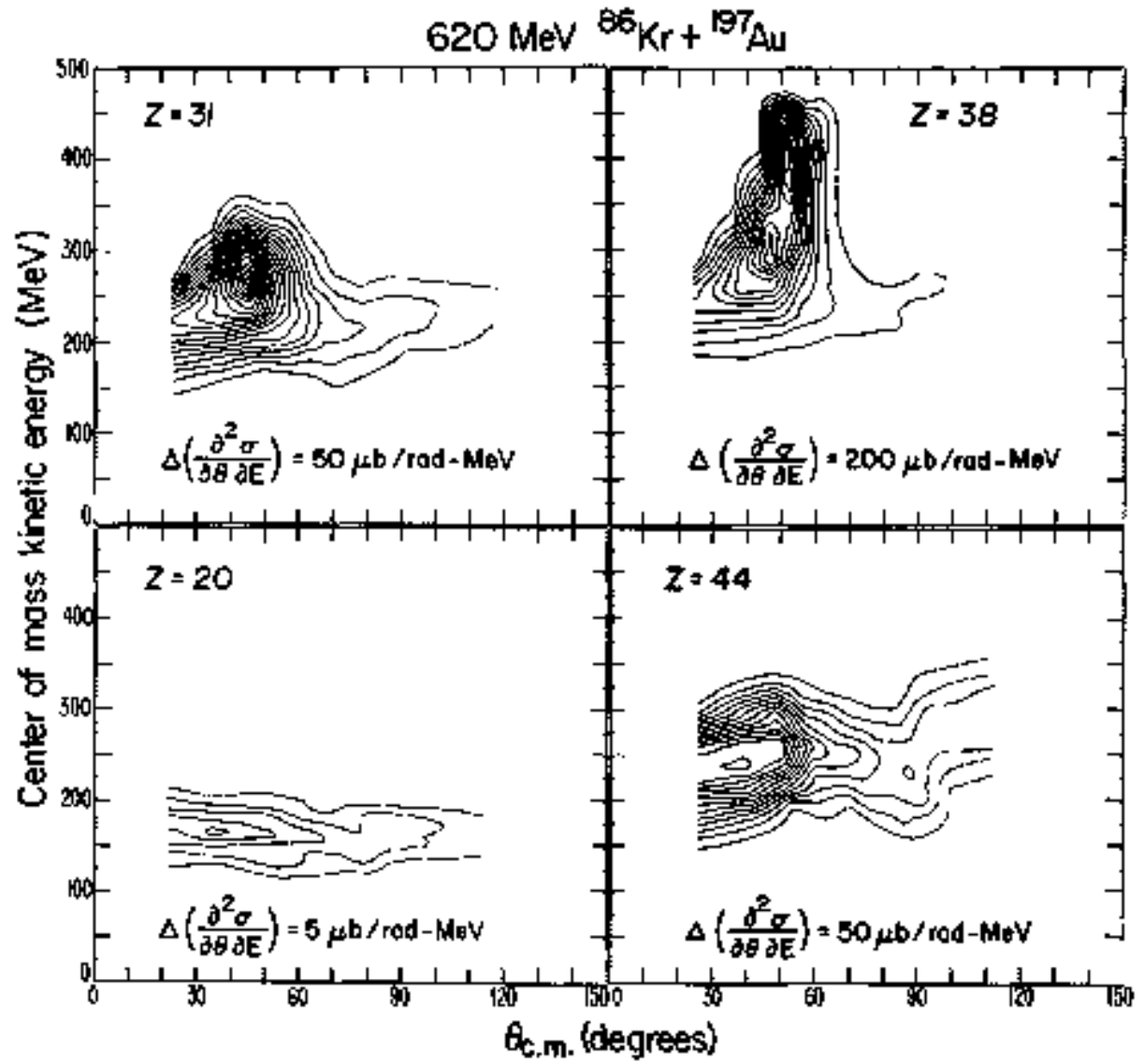

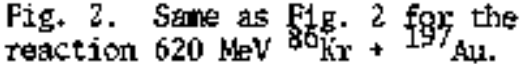
(XBL 766-2089)

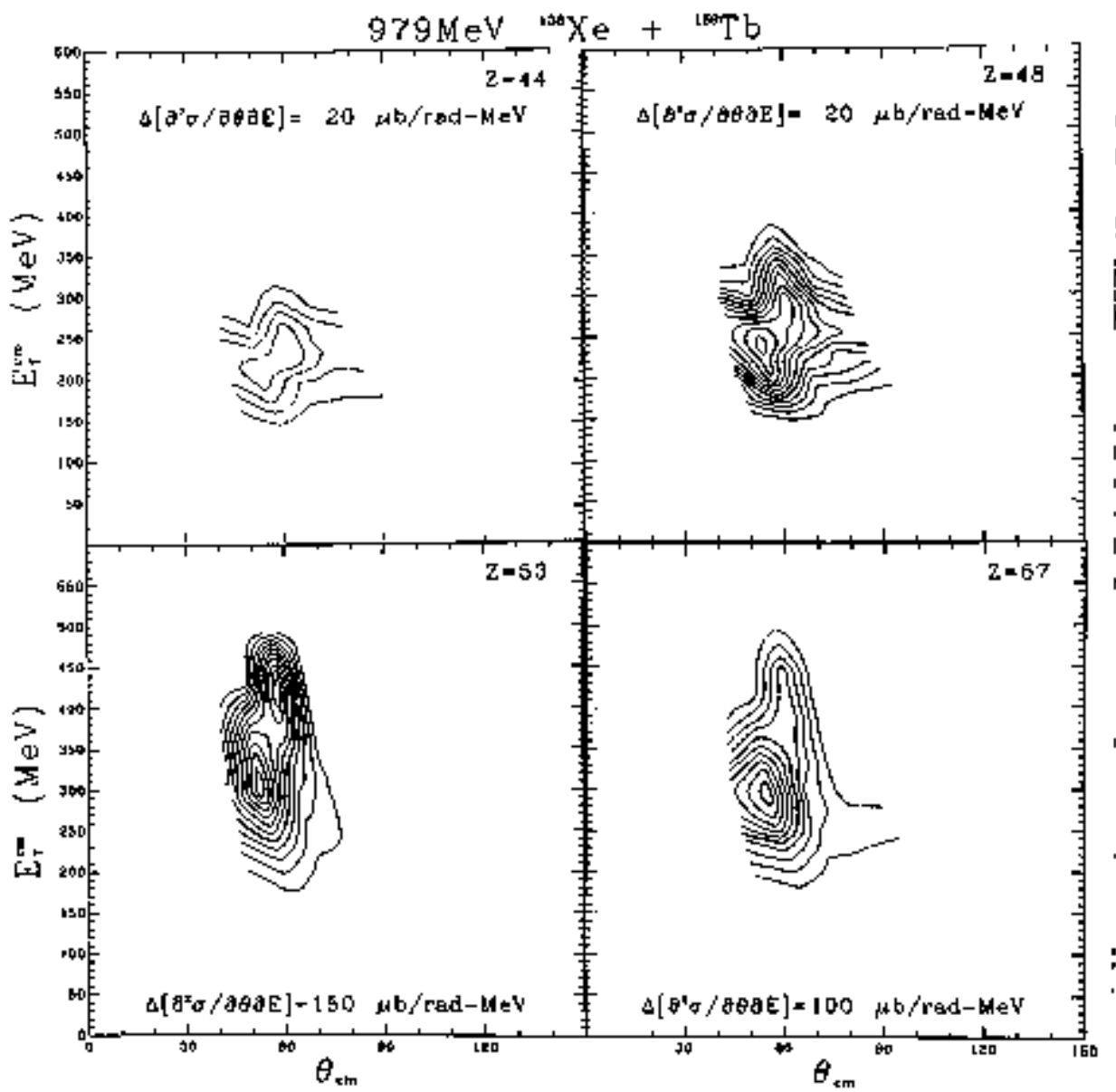

Fig. 3. Same as Fig. 1 for the reaction $979 \mathrm{KeV} 136 \mathrm{Xe}+159 \mathrm{Jb}$. (XEL 766-81S3) 

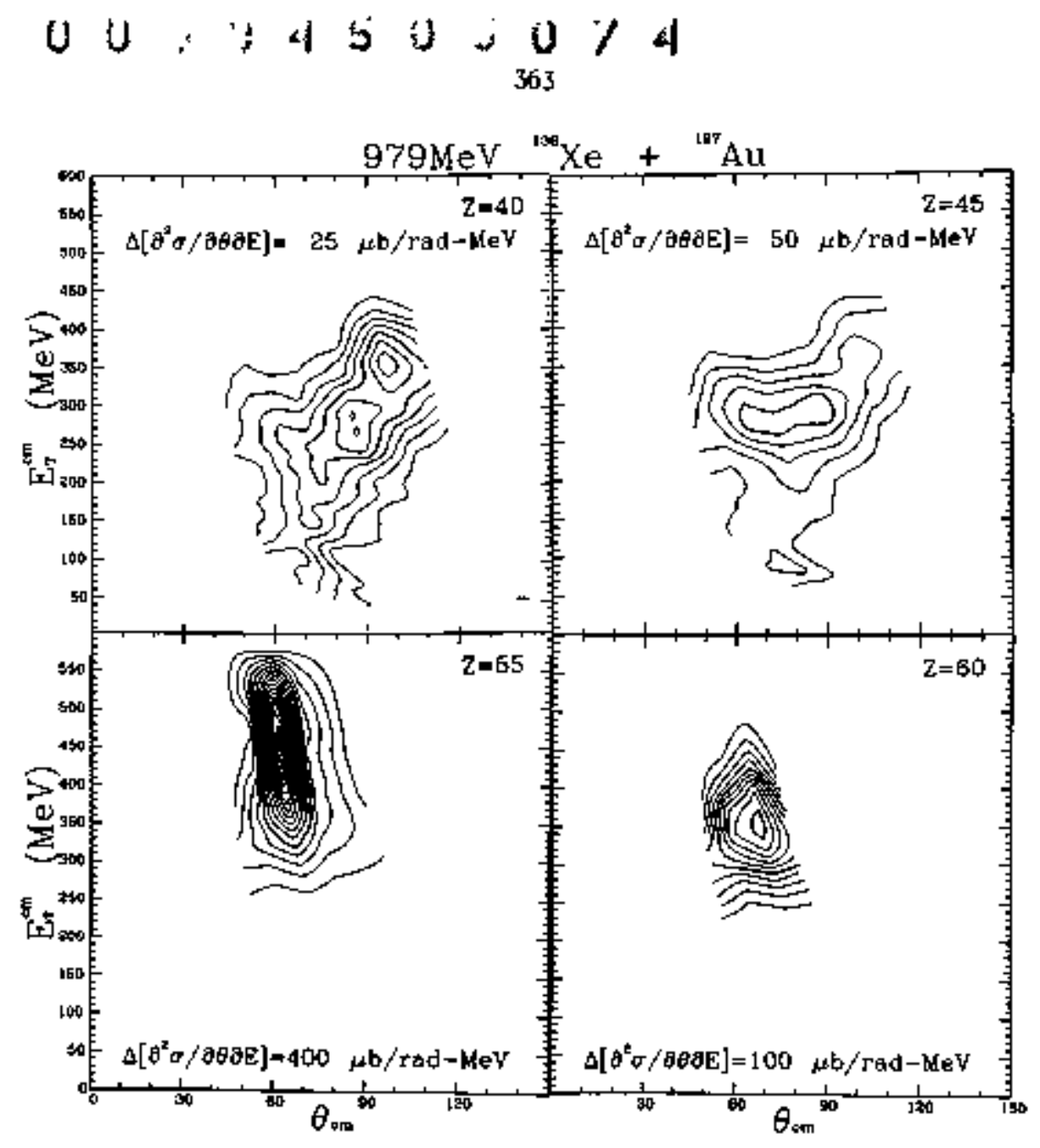

Pig. 4. Somle as Fig. 1 for the reaction 979 Mev $130 \mathrm{Xe}_{\mathrm{C}}+197 \mathrm{Au}$.
(XBL ?66-8152)

of the cross section, so $d_{c_{c}}=2 \pi \sin \theta_{c} m_{+} d \theta_{c, m_{+}}$. The transformation of the cross section to

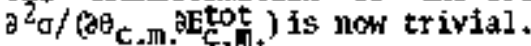

We assune that the neutron to proton ratios of the two fragments are equilibrated, as has been shown to be true experimentally by Galin et al.2 This means that for a given division of the total rass, the atconic number $Z$ of one of the fragments takes the value which minimizes the liquid drop potential onergy for the shapes involved. For very large target-projectile systens (such as $\mathrm{Kr}+\mathrm{Au}$ ), such an assumption differs negligibly from assoming the $N / Z$ ratio to be the same as that for the hypothetical contpound nuclets. The rassses have not been corrected for neutron evaporation, but, even though such a correction will only cause small shifts in the features of the maps, such corrections are being considered.

Automation was employed to draw the maps since hand-draying all the maps would have been very similar to requiring a medieval monk to transcribe 20 copies of the bible. (Consider the fact that in re action systems joestired recently, we hove had to draw 30 to 40 maps for ench system.) The computer also has the advantage of total lack of bias. Hand- drawn maps suffer from a subconscious smbothing algarith which is extrenely nonlinear and device (person) dependent. The method is extremely sensitive to calibration problems; it is this inability to subjectively comproise that has holped us to discover problems wich would not have manifested themselves otherwise.

Figures 1-4 display sets of 4 maps fram 4 different reaction systens. As you can see, ar enormous amount of information can be had at a glance. The real power of this method of display, though, is the ability to quickly scan fram one 2 to the next through a whole sequence of $z$ 's frum a single reaction. The $Z$-dependerce of certain topographical features of the maps then become ovenwhelmingly obvious, such as the gradual appearance and then diseppearance of the quasi-elastic component as one scans from $Z$ 's far below to $Z$ 's far above the projectile.

The computational method can be easify modified to display any experimental quantity which depends upon tuo variables, of course, the plotting subroutines are sfte-dependent, but one only needs a local subroutine which draws straight lines between two points. We are presently plaming to compile a 
paper which consists solely of these contour diagrams for all the singles date we hrve collected over the past few years.

\section{References}

1. J. Wilczynșki, Phys. Lett. 47B, 484 (1973).

2. B. Catty, D. Guerreau, M. Lefort, X. Tarrago, J. Galin, B. Caurin, J. Girand and H. Nifenecker, Nucl. Phys, A253, sil $\{1975\}$.

\title{
A HIGH INTENSITY HEAVY-ION RECOIL TARGET SYSTEM
}

\author{
Jogechum Mf Nilschke
}

At present the power density of beaml windiows and recoil targets at heavy ion accelerators is 1 inited to approxinately 10 to $20 \mathrm{H}^{2} \mathrm{kn}^{2}$. In typical experiments to produce transfermium elerents this corresponds to a production yield of about 10 atoms hr per namobarn. Since the cross section for the produstion of new elentents including stperheavy $s l-$ enents might well bo in the picobarn range, a target systen was developed which increases the present cepabilifties substantially.

The operation principle of the new target systen can best be understood by referring to Fig. 1. The conventional single target which also acted as a window to separate the recoil stopping gas from the machine vacung is replaced by a double window, A similar arrangenent is used for the exit of the beam. A high velocity pas stream passes between each of the two foils and renoves the heat generated by the beam in the entrance and exit windows, the gas itself, the target backing, and the target. It is inportant to realize that the differential pressure across the target can be nade zero if the cooling gas has the same pressure as the stopping gas. This greatly reduces mechanical stress on delicate targets. Even a slight "leak" in the target is of no consequence if the cooling and stopping gas are of the same species (in genoral $\mathrm{He}$ ).

For vacung spectrcrueters, etc., a single "tua1 windor" can, of course, be used. A detailed account of the experimental and theoretical investigations

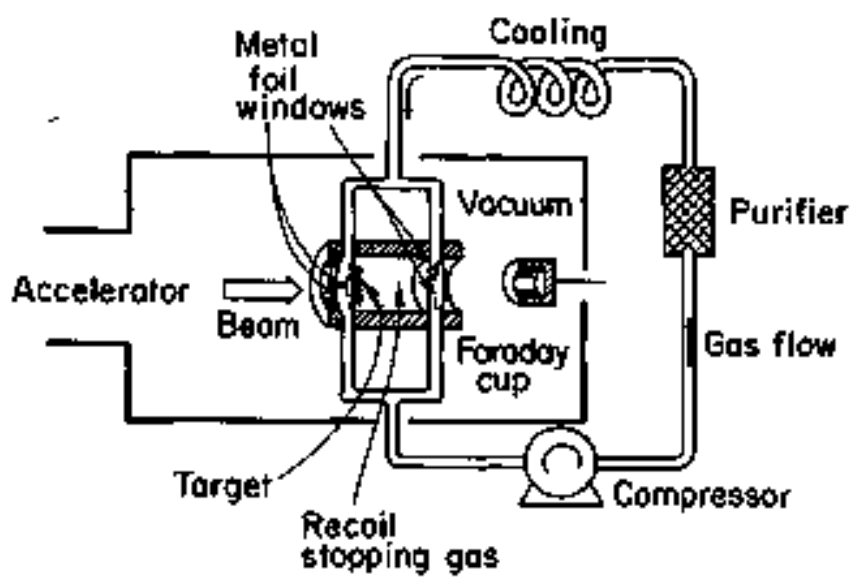

Fig. 1. The operating principle of the gas-cooled target system. of this target system is givern In Ref, 1 . A theoretical expression for the flim transfer coefficient o ls obtained:

$$
\alpha=\text { const. } \frac{\lambda}{l}(\operatorname{Re})^{0.8} \cdot(\mathrm{Pr})^{0.3} \text {. }
$$

Here $\lambda$ is the themal conductivity of the gas, \& a characteristic longth, Re the Reynolds number and Ps the Prandl numbr. From this expression it is ca]culated that hydrogen and heifum are the best candidates for a cooling gas.

The experimental investigations are carrled out with an electron gun and an infrared TV systen which is used to deternine the terperature distribution of the windows. The terperature of the cooling ras can be lowered to $77^{6} \mathrm{~K}$. A gas circulator syster is capable of delivering up to $34 \mathrm{cfm}$ of gas at this terperature and one atmosphere pressure.

The goal of the mersurmants was to find the optisnom cooling conditions by varying the following parameters:

$$
\begin{aligned}
& \text { - the Window materlal } \\
& \text { - the nature of the cooling gas } \\
& \text { - the gas flow } \\
& \text { - the gas tolperature. }
\end{aligned}
$$

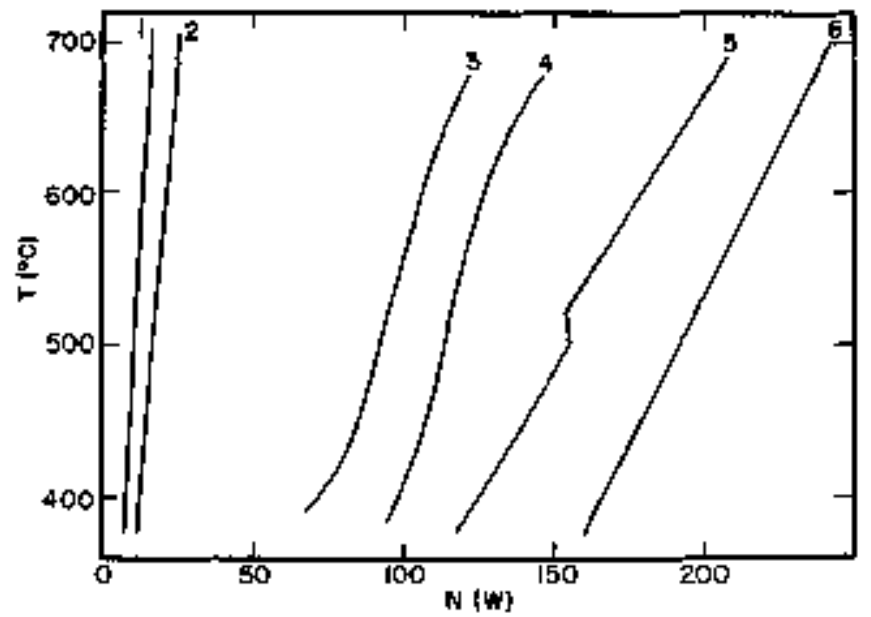

Fig. 2. Window temperature $T$ as a function of bean polver $N$ for different cooling conditions. (1) vac on both sides, (2) I atm, $290^{\circ} \mathrm{C}$, stagnant he on one side; vac on the other side, (3) 12.5 scim He flow at $290^{\circ} \mathrm{K}$, (4) 25 scfin He flow at $77^{\circ} \mathrm{K}$, (5) 10, I scfin $\mathrm{H}_{2}$ flow at $290^{\circ} \mathrm{K}$, (6) 18.2 scfin $\mathrm{H}_{2}$ flow at $77^{\circ} \mathrm{K}$.

(XBL 766-8232) 


\title{
$0 \because 245 \% 50$
}

Figure 2 shows an excmple of the results obtained. With hydrogen as a cooling gas more than che order of raginitude inproventent of the beam power capability is achieveri.

\section{Reference}

1. J. M. Nitschke, LBL -4387 (to be published in Nucl. Instrum. Meth. ).

\section{A HEAVY-ION SPECTROMETER FOR THE SUPERHILAC}

\author{
Joschim M Nitschke
}

For the inwestigation of heavy-ion mucleax reaction mechanisuts and the identification of reaction products, a magnetic spectrometer can be of great berefit.

The proposed spectrameter for the SuperHILAC would have the following principal features:

(1) Physical separation between the primary beam and the recoil products if these differ in one or more of the following parateters: velocity $(v)$, energy to charge $(\mathrm{E} / \mathrm{q})$, or mass to charge ratio (sta). The suppression of unwanted particles will depend on the extent to which thoy differ frum the desired particles in these three quantities.

(Z) Mass detemination up to the superheavy eletent region with unit mass resolution.

(3) Physical separation of masses according to their $\mathrm{w} / \mathrm{q}$ values for further stuxy of decory modes.

(4) Z-identification. The Z-resolution will depend on the detials of the reaction and the construction of the spectrometer. In favorable cases unit Z-resolution up to $Z=100$ can be expected.

(5) The full angular range from of to approxinately $160^{\circ}$ can be studied.

(6) All particles which can be accelerated by the SuperHIlAC can be analyzed. limitations imposed by target thickness and mifomly, scattering and straggling, begn quality and bean intensity, high coulmb berriers, kinematic broadening, etc. rake it advisable to consider $-a 5$ the first spectroneter for the SupertILAC-an instrument with moderate energy xesolution but gret flexibility as a kinematic tool.

The basic concept is therefore a velocity filter corpled with a magnetic spectrometer (Fig. 1). The whole instriment is dispersionless in energy and constitutes a recoil mass spectrometer. Noderate energy information will however be obtained from the focal-plane detsctor and/or time-of-flight measurements.

The following is a brief description of the principle of the spectrometer. The electrostatic deflection in the velocity filter can be expressed g5

$$
F_{\rho}=\frac{m^{2}}{q},
$$

and the magnetic deflection as

$$
\mathbf{B}_{\boldsymbol{q}}=\frac{\pi \mathrm{N}}{\mathrm{q}} \text {. }
$$

$P$ is the electric fleld, all other letters hove the usual meaning.

The principal canonents of the spectrometer consist of one electrostatic followed by two nagnetic deflectors.

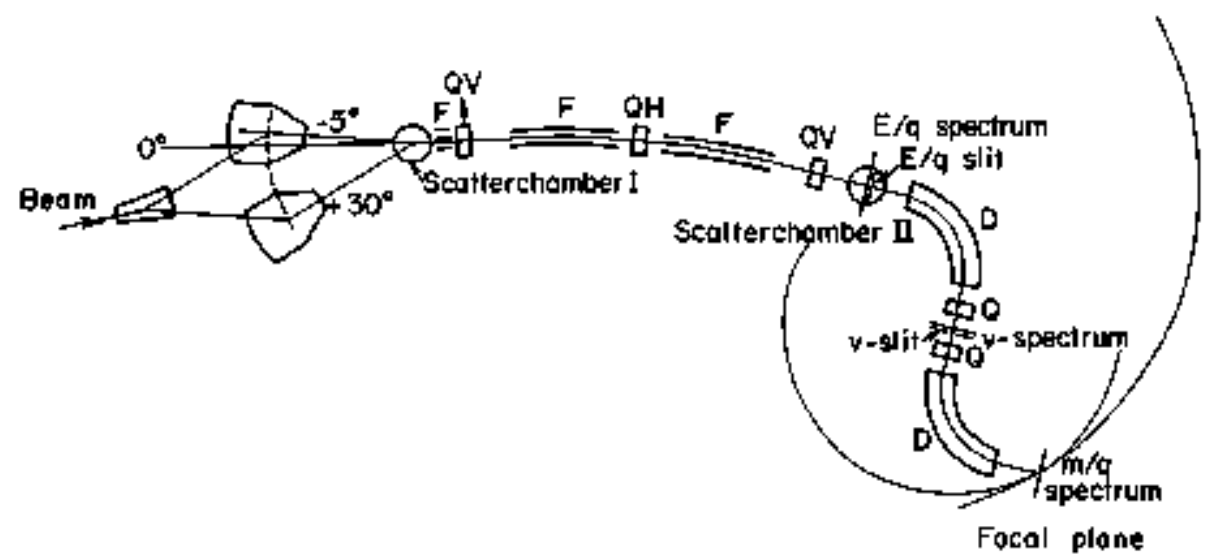

Fig. 1. Heavy ion spectroneter. 
A variation in the olectric rigidity can be expressed to first order as:

$$
\frac{\Delta(F \hat{F})}{\mathrm{F}}=\mathrm{K}_{\mathrm{el}}\left(\frac{\Delta \mathrm{m}}{\mathrm{m}}+\frac{2 \Delta v}{v}\right)
$$

$K$ is the dispersion constant. This is followed by a magnetic fleld. Its variation in rigidity is expressed as:

$$
\frac{\Delta(B \rho)}{B}=K \operatorname{mag}\left(\frac{\Delta m}{m}+\frac{\Delta V}{V}\right) .
$$

If the magnetic is opposite and equat to the electric dispersion $K_{\text {e }}=-K_{\text {mas }}$ one obtains a pure velocity spectrum (the $\mathrm{dm} / \mathrm{m}$ - terms cancels). The second magnetic dipole is identical to the first one and coupensates the rentining velocity dispersion so that a pure mass spectrum results. Schematically this can be written as:

$$
\frac{\Delta\left(\mathrm{F}_{\rho}\right)}{\mathrm{F}}-\frac{\Delta\left(\mathrm{B}_{\rho}\right)_{1}}{\mathrm{~B}_{1}}-\frac{\Delta\left(\mathrm{B}_{0}\right)_{2}}{\mathrm{~B}_{2}} m-\frac{\Delta \mathrm{d}}{\mathrm{H}_{1}} .
$$

All rass lines in the focal plane will however be split into "moltiplets" according to their ionic charge. In sate cases these lines will overlap. To remove this tharge anbigulty a time-of -flight systerl forms an integral part of the spectrometer. Start detectors can be located behind the E/q-slit and/or the velocity slit. The focal-plane detector acts as a stop-detector. In addition the focalplane detector allows a coarse energy neasirement. This coarse chergy measurentent (E) and the velocity raeasurement from TOF result in a coarse mass determination (m) which allows to resolve the $\mathrm{N} / \mathrm{q}$ anbiguities. Schenatically:

$$
\begin{aligned}
& \tilde{E}+v(T O F) \Rightarrow \tilde{m} \\
& \tilde{m}+\mathrm{m} / q \Rightarrow m
\end{aligned}
$$

The "exact" energy of the particle can then be obtained from

$$
n+v(T O F) \Rightarrow E \text {. }
$$

Two nodes of operation of the spectrometer are anticipated. For compound nucleus reactions and other reactions near zero degress a beam inflection systetm will be used which scans a moderate angle of $-5^{\circ}$ to $+30^{\circ}$. For studies at angles Iarger than $30^{\circ}$ the first part of the spectroneter (the electrostatic deflector) wirl be bypassed and the second paxt used as an "ordinary" magnetlc analyzing systen. The angle over which this magnetic part of the spectromleter can turn will be as large as practical: $-10^{\circ}$ to approximatety $+160^{p}$. The idea bere is that for large angles the bean separation afforded by the electrostatic deflector is not jecessery because the intensity of the scattered bean falls sharply with fncreasing angle.

\title{
A VERSATILE SPUTTERING SYSTEM
}

\author{
S W Eckroad. A E Leter, and A Gherso
}

hell-controlled materlal removal and deposition have been achleved with a sputtering system constructed from components readily evailabie in the laboratory environment.

Fabrication of unifon films (here, targets for nuclear reaction studies) from high-melting-point or refractory substances is often difficult to realize with standard evaporators, in that the requisite high temperatures may donage the physical and/or chemical integrity of substrate and deposit. Additionally, the need to provide (accelerated) ions of specific and woll-resolved energies frequently dictates the availability of miform degrader foils of varying thickness. A sputtering syster recently doveloped at this Laboratory has a breadth of application inclusive of these purposes.

The sputtering-electrode assembly (Fig. 1) is housed in a bell jax (the inverted base of a vacum desiccator] evacuated by a (21 cful mechanical rotary pump with a liquid-ritrogen cold trap. As shown in the block circuit diagram (Fig. 2), a $3 \mathrm{kV} /$ $40 \mathrm{mk}$ regulated power supply is used in this systetr in conjunction with meters to monitor voltage and arc current and a strip-chart recorder, which provides the history of the discharge.

Interchangeable parts in the cathode allow for considerable latitude in appliçation. Selective conturtive shielding within the discharge dark zone will erable differoitial sputtering of the cathodic material and may provide a degree of collimation to enhance the lime-of-flight (ws diffusion) component of deposition. With the discharge thus restricted, a defined portion of the cathode foil is preferertially removed.

Sprttering progress is monitored in situ by measuring with a surface-barrier silicon detector the energy loss of alphr particles of known incident energy. Information on cathode and for deposit thickness way thereby we conveniently obtained without having to reflove the material for wighing (with the conconitant risk of breaking the folls).

Argon-the preferred sputtering gas-is metered into the bell far through double needle ralves at a rate sufficient to maintain an ambient pressure of 20 to 200 mTorr. At these pressures and a discharge potential of fron 0.5 to $3.0 \mathrm{kr}$, the cathode fall (dark zone) attains a maximum length of approximate ly one inch and the arc current varies between 1 and $40 \mathrm{MA}$. Industrial-gradte argon is of sufficient pur1ty in nost cases. Although at the higher pressures, contaminants in the gas show a propensity to react with-generally oxidize-the more reactive sputtered materials. Such chemistry (in-flight or surfacecatalyzed) is a potential means by which to obtain oxide or halide films frar elerental cathodes. 


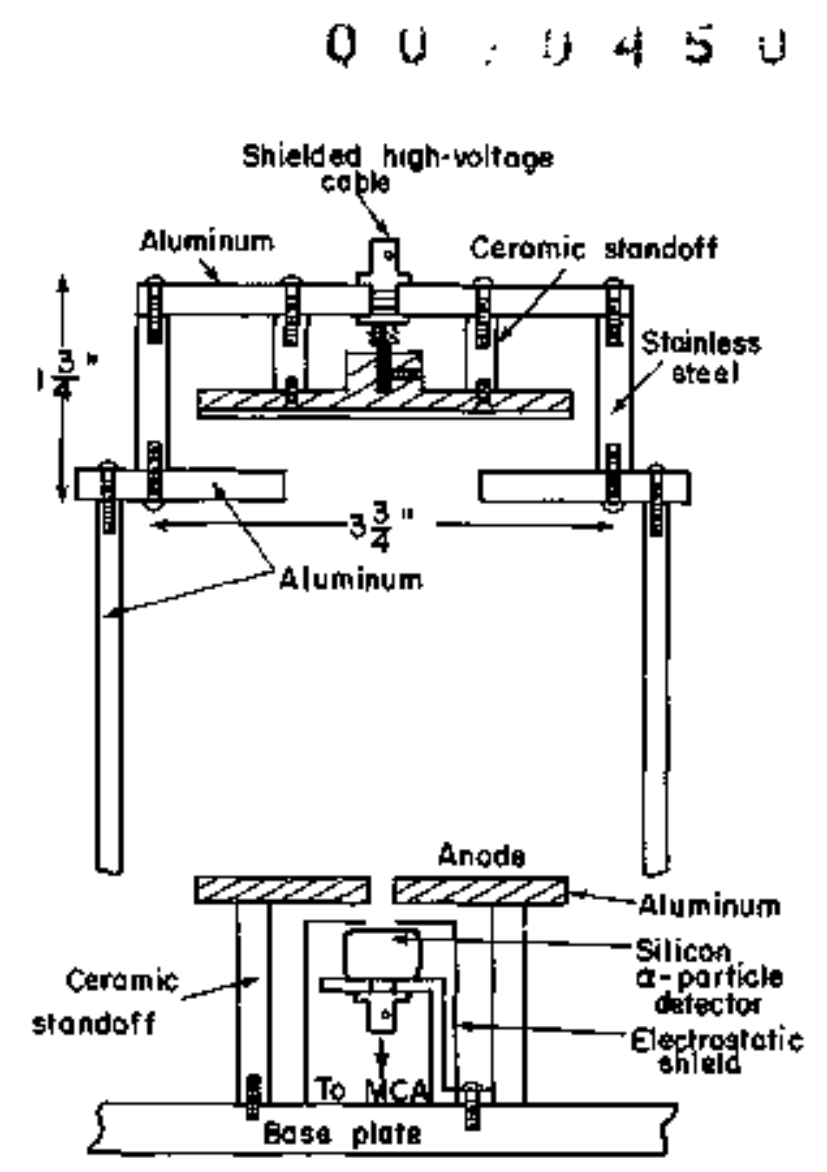

Fig. 1. Full-scale mechonfcal drawing of the sputtering-electrode assembly.

(XBLl, 766-3009)

Crystal structure has been observed to be an important factor in producing quality films through sputtering. Insofar es the sputtered material wil tend to conform in lattice structure to the substrate (epitaxial growth), it is advantageous from the mechandeal stability standpoint to choose a substrate whose tonit celi shape and size closely reseruble those of the crystalline deposit. limitations to the quality of films nade by thioning commercial foils are the unifomity and crystal-lattice integ n

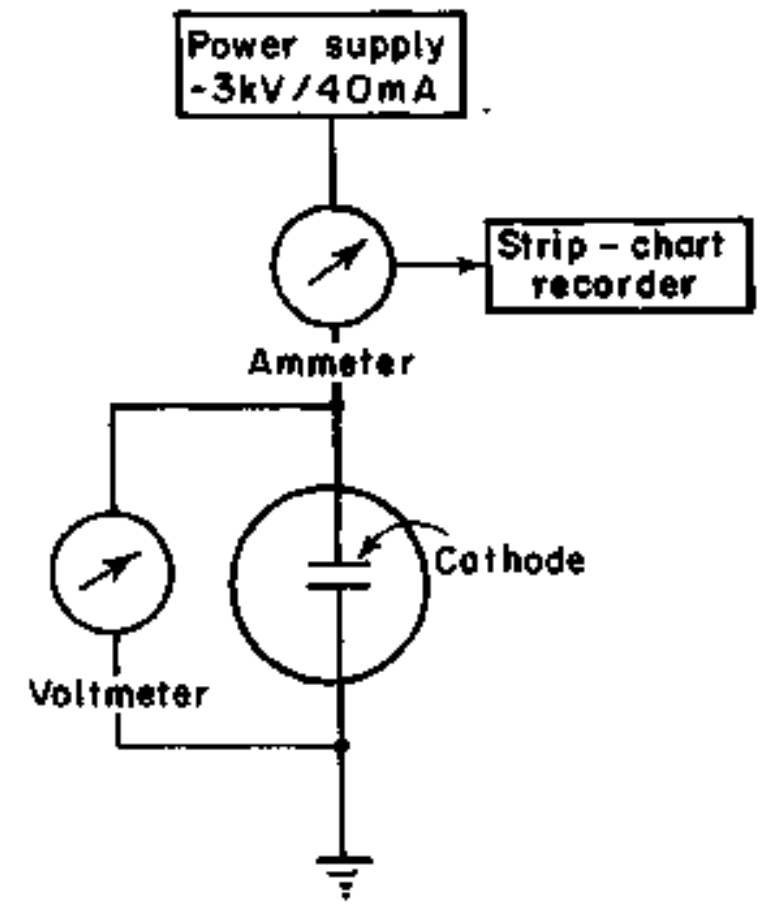

Fig. 2. Block diagram of sputtering circuit.

(XEL $766-3010)$

rity of the original foll. Initial surfare discontinuities and unequal sputtering susceptibilities due to lattice defects for example, from stress hardening will be manifested in the induction of pinholes, (This situation ray be improved to same extent by annealing the foils prior to sputtering.)

Built from low-cost, off-the-shelf ccamponents, this sputtering system has been valuable in making thin filins of gold, molybdettum, and platimum, and

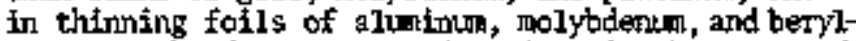
lium. In the future, consideration will be extented to new cathode-substrate systents with work continwing on present conbinations and the functional dependence of operating parameters: pressure, temperature, voltage, and current will be established.

\title{
A TIMING DETECTOR UTILIZING ISOCHAONOUS TRANSPORT OF SECONDARY ELECTRONS
}

\author{
A M Zebelman, W G Meyer, K Haibach, A M Poskanzer.
}

$R$ G Sexiro, $G$ Gabor, and D A Lendus

A timing detector has been developed for use in reaction product $3 a 5 s$ identification, utilizing the time-of-flight technique. The detector, as schematically pictured in Fig. 1, has as its noweI feature an isochronous electron transport system.

Secondary electrons produced when particles pass through a thin foil (approximately $10 \mathrm{wg} / \mathrm{cm}^{2}$ of carbon) are accelerated to approximately $2 \mathrm{kV}$ by
0.0005 inch stainless steel wires arranged in the form of a harp of 99 trarsmission. The accelerated electrons are then transported $180^{\circ}$ in a uniform field of a permanent megnet, through a $90^{\circ}$ collinator and a suppression grid. The electron detector is conprised of two micro-channel plates which are oper. ated in a tandun configuration. The charmel plates act as an electron multiplier and the electrons are collected on a 50 otm anode, from wich a fast tining signal is obtained. 

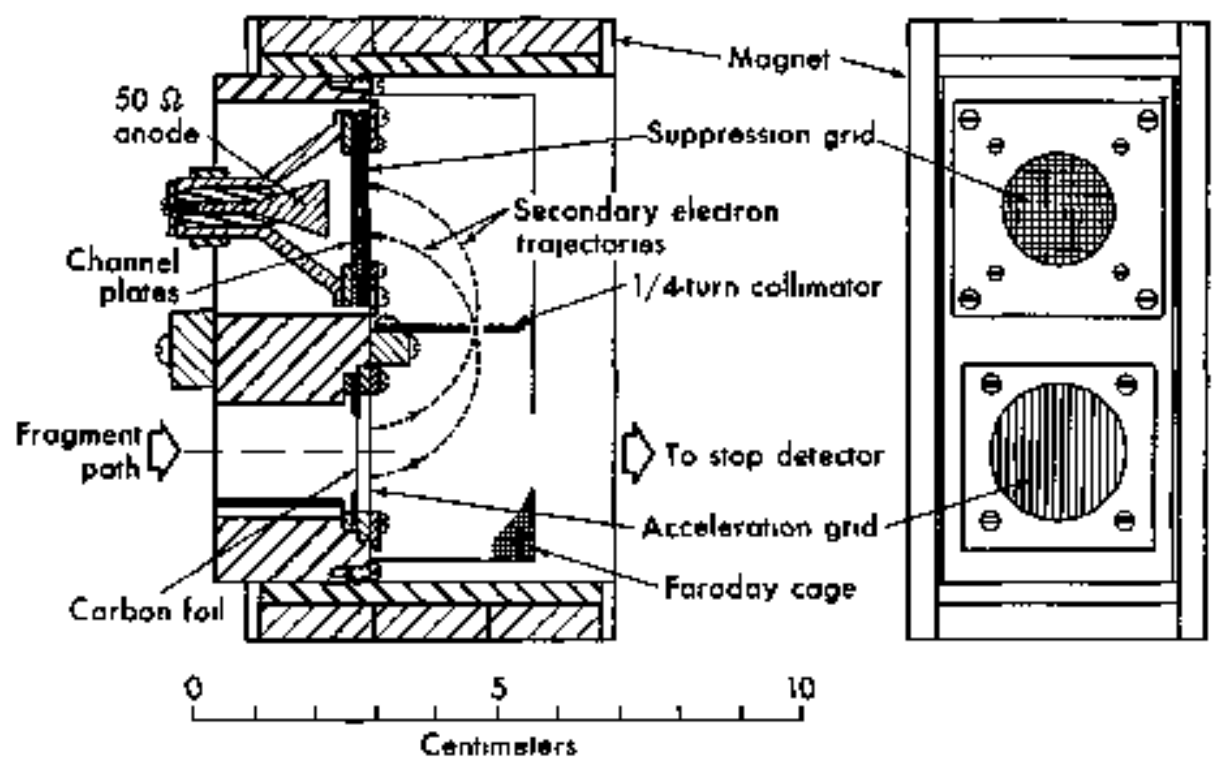

Fig. 1. Scheslatic view of tiving detector.

QBBL 757-3468)

The basic concept for this timing device arose from a desire to use a thin foll which was oriented perpendicular to the fragment flight path and to remove and shield the chamel plates from the beam and reaction products. The concept of a $1800^{\circ}$ magnetis transport was chosesn because it is isochronous, independent of changes in the lateral position of the origin of the elextrons and their energy. However, the transport tims is sensitive to the andle of entission of the electrons in the plane perpendicular to the magnetic field. The divergence in this angle is salall because of the $2 \mathrm{kV}$ acceleration and because the harp structore produces a unifonm accelerating potential in this plane. The affect of this anglilar divergence on the electron tramsport time is minfinized further by the quarter-turn collimator. The sugpression grtd serves to elininate background originating from interactions at the surface of the chaninel plate.

Tine-of-flight reasuresents have been made between this timing detector and a $120 \mu \mathrm{m}$ Si detector with $100 \mathrm{MeV}$ loo ions (frum the 88-anch cyclotron) and $8.78 \mathrm{HeV}$ alpha particles; the results are tabulated in Table 1. Figme 2 shows a tijne spectrum obtained with 100 MeVto ions.

TabIe I. Time resolution and detection efficiency

\begin{tabular}{|c|c|c|c|}
\hline & FWM pic & sec.) & (8) Eficiancy \\
\hline $100 \mathrm{kev}^{16} \mathrm{o}$ & 90 & 31 & 95 \\
\hline $8.7 \mathrm{KeV}^{4} \mathrm{He}$ & 156 & 96 & 56 \\
\hline
\end{tabular}

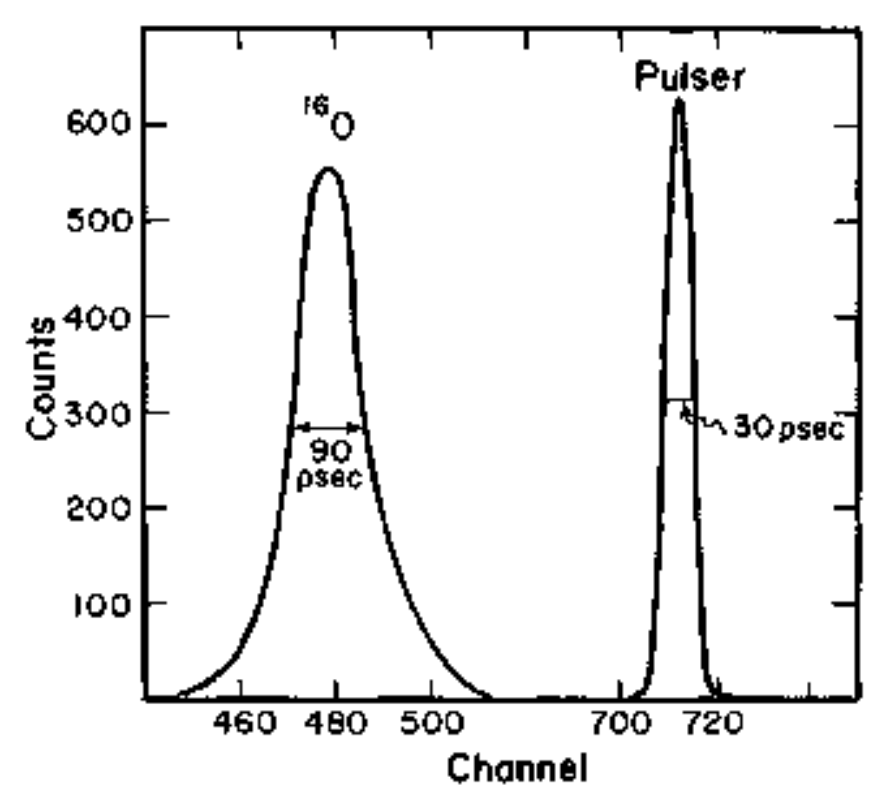

Fig. 2 Tine spectra for the reaction $197_{\mathrm{Al}}$ (160, i6o). (XEL 766-9256)

The authors wish to express their greatful appreciation to D. J. Wieira, F. S. Goulding, C. E. Fiartsock, M. Maier, G. D. Westfal1, W. S. Cooper, and $M$. I Isan for their help in different phases of this work. 
$00: \forall 450>076$

\title{
AN EASY-TO-BUILD WIAE CHAMBER WITH GOOD POSITION AND PULSE-HEIGHT RESOLUTION
}

\author{
J C Lugor and F S Bleser $t$
}

A simply constructed rultiwire drift chamber has been developed. The left-right ambiguity in herent in all muttiwire chembers is solved using two staggered planes per axis (Fig, 1). The cathode planes (double-sided aluminied mylar) and field wires (75 micron $\mathrm{Be}-\mathrm{Cu}$ ) are held at negative high voltage while the anodes (20 micron tungsten) are dC coupled to amplifiers at ground potential.

Using P-10 gas (90* Argor, 108 methane), the correlation betmeen drift time and position can be approximated by a single straight line with $< \pm 0.5$ mum exror (Fig. 2). The spatial resolution is approxinately $\sigma=0.1$ r.m. The pulse-height resolution for $6 \mathrm{keV} g$ almas fion $55 \mathrm{Fe}$ is $<20$ a (Fig. 3).

\section{Footnotes}

*On leave from Centre D'Etude Muleaires de Saclay, gl Gif-sur-Yvette, Paris, France.

tUniversity of Califomia Space Sciences laboratory, Berkeley, Callformia.

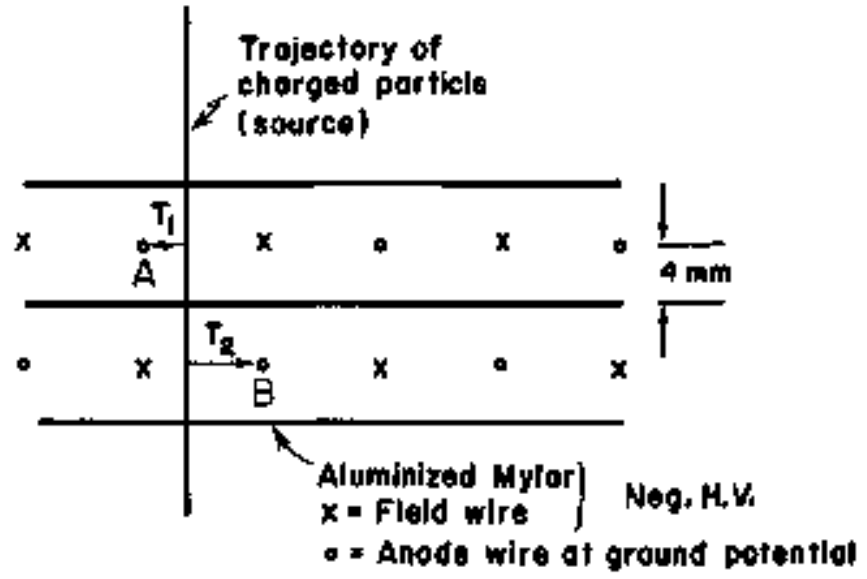

Fig. 1. Construction of a 1-axis pair of nultiwire pIanes, Drift times to anode vires $A$ and $B$ are $T_{1}$ and $\mathrm{T}_{2}$.

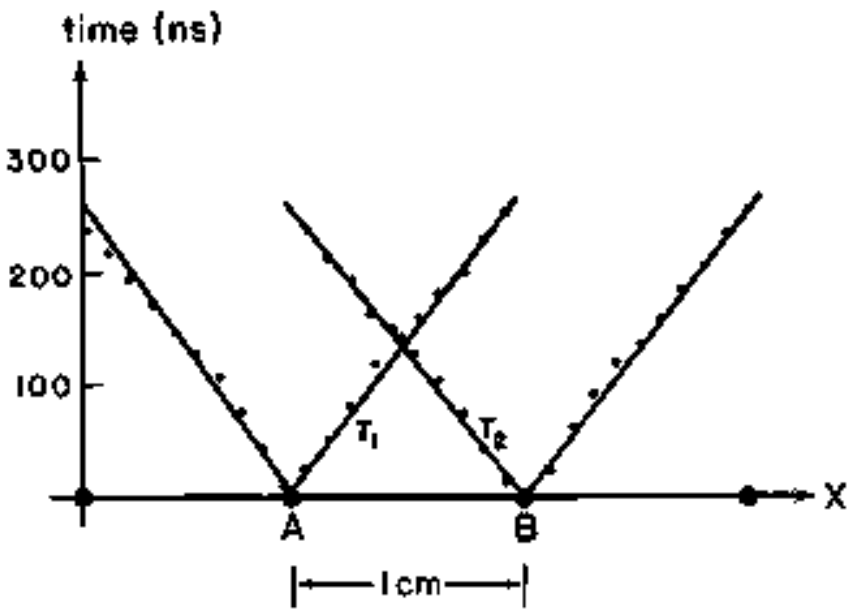

Fig. 2. Irift times $\mathrm{T}_{1}$ and $\mathrm{T}_{2}$ as a fumction of source location $(x)$ to anode wires $A$ and $B$.

(XBL, 766-8261)

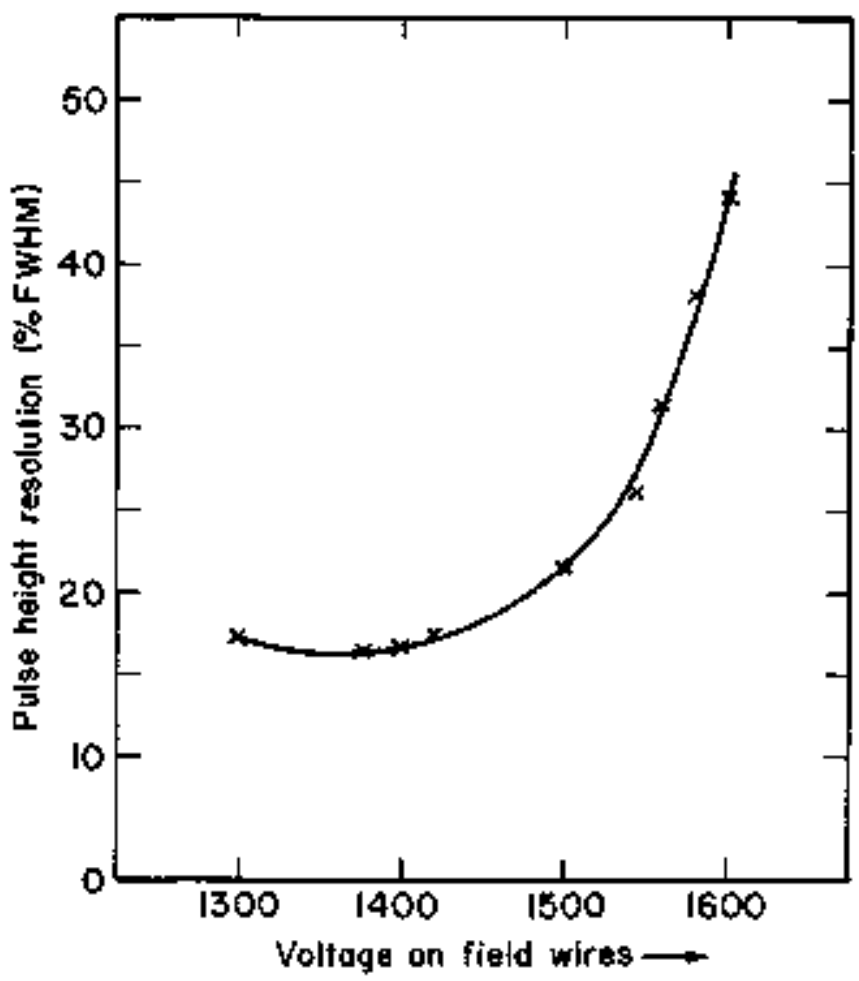

FIg, 3. Pulse-height resolution of $6 \mathrm{keV}$ gammes from $55_{F e}$ as a function of high voltage,

(XBL $766-8262$ ) 


\section{DRIFT CHAMBERS FOR HEAVY-ION COSMIC RAY EXPERIMENT}

Fred Bieser' and Bouglas Greiner

An array of six single wire proportional drift chambers has been developed for use in the UCSSL/LBL High Energy Cosmic Ray Experiment on the NASA ISEE-C SATELlITE. Three $X-Y$ pairs of chmbers are used to measure the angle of incidence of energetic heavyIon cosmic rays entering a "telescope" of ten lithiumdrifted silicon detectors. Stngle-wire drift chambers chosen over muItitire systems for several reasons: 1) the readout systen for nany wires requires many amplifiers [too mach power) or a large delay line (too heavy). 2) femer wires reduces the probability of failure dte to wire breakage. 3) a single-wire system Iends itself to a simple solution to the problen of Iesolution degradation caused by delta rays.

Saudinos, et al., have constructed single wite drift chanbers up to 50 on $X 50$ oा with resolutions (Fin) of 0.6 mo at 11 con drift length and 1.6 men at 50 col. 1 The task at hand was to squeeze six of these chambers (each being $\sim 1$ inch thick) into thves inches, orient thein into orthogonal peirs without distorting the electric fields in the drift region, and learn how to deal with the copious delta-ray sigmaIs.

The chavber thickness was reduced to $6 \mathrm{~mm}$ by scaling down the width and spacing of the parallel field-shaping metalized strips, and increasing the sensitivity of the amplifier-discriminator. Unless the electrtc field is very precisely formed the drifting electron cloud will diffuse and never reach the collecting anote. "Cross-talk' between the electric fields of adjacent chambers can be elininated by staggering the metalization on the two sides of the kepton (Fig. 1), This results in a pattern that

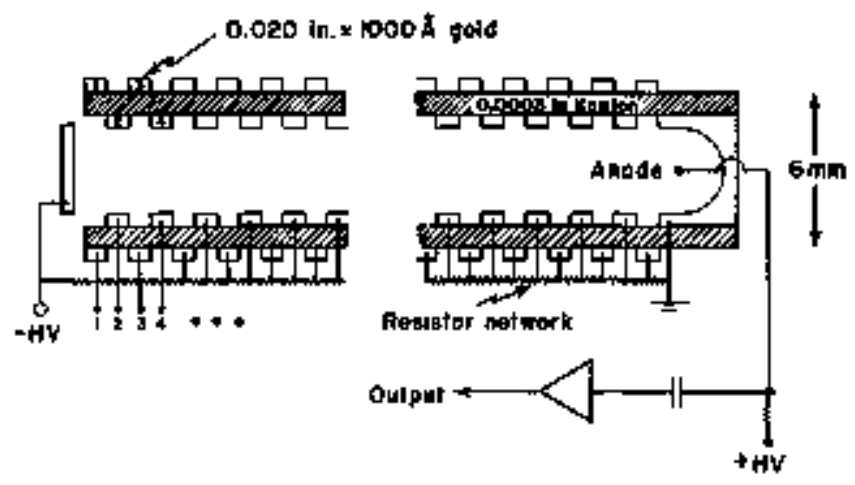

Fig. 1. Scherpetic structure of single-wire drift chamber showing a cross-section view of the kepton metalization schene for the elimination of cross talk between adjacent chambers.

(Xt) $766-8263$ ) gives 100 o electrostatic shielding while allowing the substantial field gradient ( $50 \mathrm{~V} / \mathrm{cm}$ ) along the drift axis. This gradient is established by a thickfilm resistor network connected to both sides of the kapton by pressaure contact.

While the chamber and electronics utist be sensitive to all particles $(z=1$ to 26$)$, relativistic hecary ions produce hundreds of delta rays in the windows and gas which often make larger signals than the lighter cosmic rays, 2 If a large delta-ray signal occurs on the anode side of the central core ionization, the observed drift tine and hence the deduced position will be incorrect. A log-compressed integrating constant-fraction discrivinator has been developed which can pick-off the core ionization of any particle $(z=1$ to 26$)$ regardless of attendant delta rays (Fig. 2.)

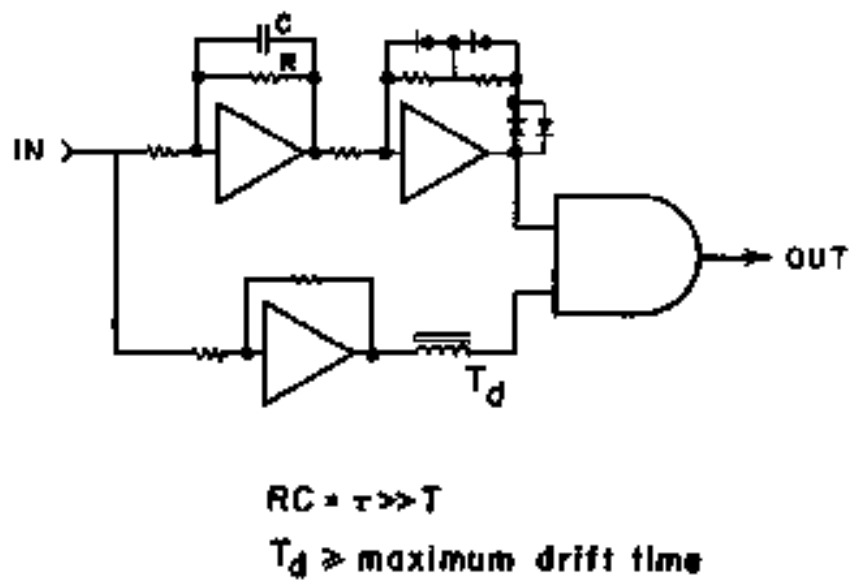

Fig. 1. Schomatic diagram of discriminator. (XBL 766-8264)

\section{Footnote and References}

"University of California Space Sciances Laboratory, Berkeley, California.

1. J. Soudinos, et al., Nucl, Instrum. Meth. 111, 77 (1973).

2. E. Beleal, A Nonte CarIo Calculation of \&-ray Effects on Drift Chamber Resolution, in this Annual Report. 


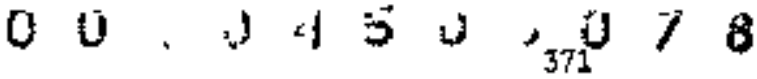

AN INTELLIGENT MULTICHANNEL PDM TRANSMITTER

A Ross Harrower " Chuck McPerland," and A \& ZınX"

An IMTEL 8080 Microprocesşor PLM (Pulsọ Dẹlay Modulation) Controller with 8 addressabie line drivers was developed to control up to 8 magnets for the Bevalac Experinent 162H. The controller can operate in conjumetion with its companion modwle, an eight-chameI PIM Receiver, in eithor an autconated feedback 1opp controlled by a PGP 11-45 or in OFF-LDE mode Independent of the computer.

The block diagran is ghown in Fig + 1. The module contains 2k bytes of PRMM (Progammable Read Only Menory) and $2.5 \mathrm{k}$ bytes of RAM, of which $2 \mathrm{k}$ bytes are used as a WCS [Writable Control Store)during the initial debug stages. These $2 \mathrm{k}$ bytes are made to appoar in the PRoM address space during debugging. After the softwere has been debugged and the FRON programmed, this $2 \mathrm{k}$ of RAM is freed and can be used as satellite memtory and processor to the PDP-11 via CANAC. 1

The oDT (Octal Debugger) and Binary Loader programs from the Livenore MCS-8 system were installed in the lowest $\mathbf{5 1 2}$ words of PROM. For ease of soft ware development, an external teletype interface is provided. Then a teletype is connected, the 3080 microprocessor is able to sense its presence and thus pourer up in the ODT mode. When no teletype is connected, the microprocessor automatically goes in- to the magnet controller progrgm. CAMAC initialization (2) and module pororup are treated by the 8080 microprocessor as separate starting procestures so that processor restarts are possible without destroy. ing volatile RAM.

The controller has various control modes depending on the restrictions imposed on the various magrets: a) slewing control, b) instantaneous control, and c) battery cortrol. Each of the eight magrets can simultaneously be slewing to either higher or lower currents at one of two slewing rates. Battery changes required in any of these operations are handied gutanatica11y. A visual display of mag net values (nanually selectable) is avaliablo on the front pane1. Iaternuts for the 8080 Microprocessor are provided to allow for CAMAC cometenication in the ON-LTNE mode or for communication through the Front Panel INCREMEN-DBCRAMENT Iever in the OFF-LIKE mode.

\section{Footnote and Reference}

University of Callforsia Space Scíences Laboratory, Berkeley, Calfformia.

1. Cakte, A modular instrunemtation system for Data Handing, USAEC Doctment HTID-25875, 1972.

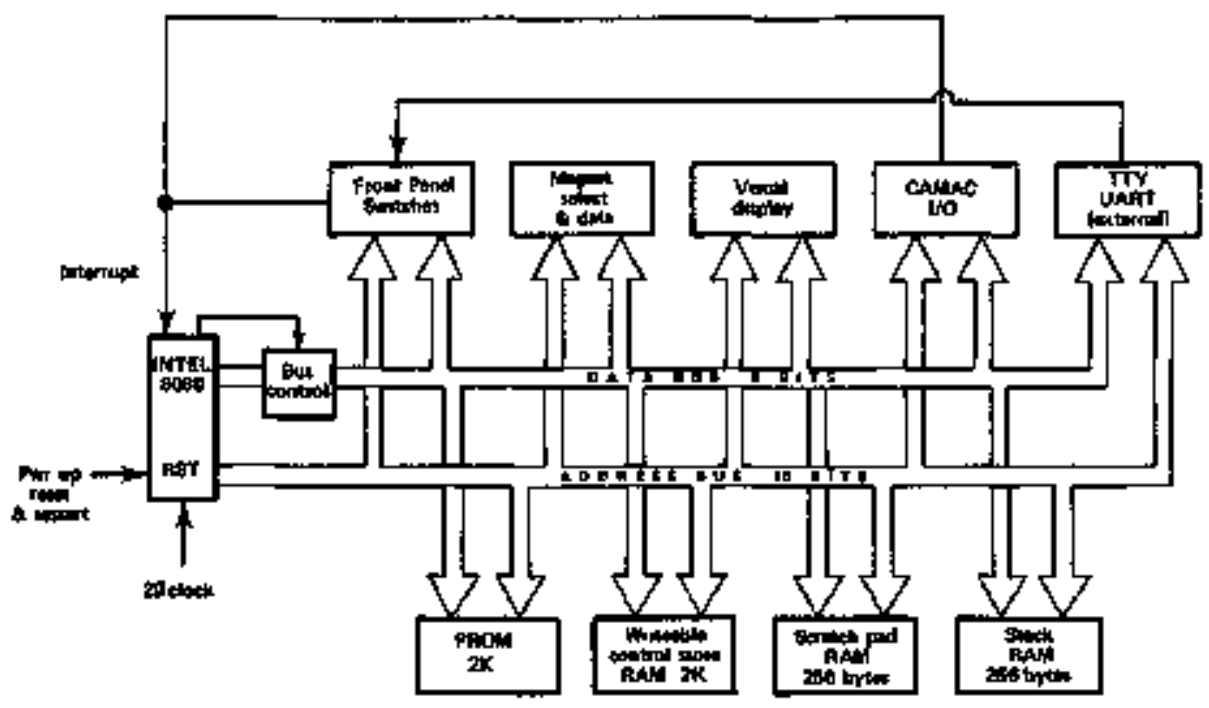

Fig. l. Block diagran of an INTEl Microprocessicr PuM Transmitter. XEL $766-3266$ ) 


\section{HIGH INTENSITY FLUX MEASUREMENTS USING CURAENT TO FREQUENCY CONVERTERS}

A Ross Harrower

A tweIve channel chrrent-to-frequency convert. er module (double width NDM) has been designed and built to facilitate high-intensity bean flux toeasurenents, By integrating the currant output from a plotomaltipliex tube viewing a plastic scintillator, fluxes up to $10^{10}$ pps or more can be reasured and digitized with high acouracy ltsing inexperisive scalers.

A sipplified schendic diagram of the circuit is shom in Fig. 1. By renoving the anode bleeder resistor from the base, all the MT output cturrent flows into the charge sliming note of $\mathrm{OA}-1$. The extretenely low imput bias current $(\sim 10 \mathrm{pA})$ of the CA3130 elininates all critical codponenits except one feedback resistar $\left(\mathrm{B}_{f}\right)$. $\mathrm{R}_{\mathrm{f}}$ determines the amount of charge $Q=$ vef $\Delta t$ fed back to the suming node per outjout pulse. ${ }^{2}$ The output is thus a pulse rate sufficient to mairtain a charge balance of zero at the sumiaing node,

The integral 1 insarity over any 5 decades of count-rate is $<0.28$ from $2 \mathrm{nA}$ input (1 $\mathrm{Hz}$ output) to $200 \mu \mathrm{A}$ input (max. output frem RCA $8575 ; 100 \mathrm{kHz}$ output). The $100 \mathrm{kHz}$ clock frequency allowg time" incresental flux measurements with 18 resolution in I millisecond windows. Note that while the converter has a linited dynamic range, any particle flux can be measured by simply adjusting the PMT gain, the scintillator-to-PMT light transmission, or the feedback resistor $R_{f}$.

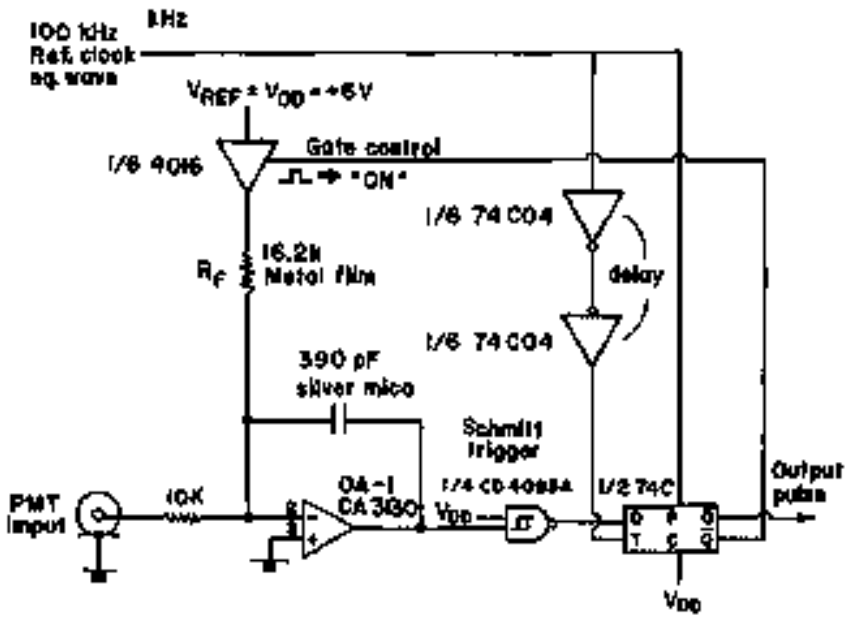

Fig. 1. Si.plified 1ogic schenatic for arrent-tofrequency comerter. (XBL, 766-8265)

\section{Footnote}

"University of California Space Sciences laboratory, Berkeley, California.

\section{A MONTE CAFLO CALCULATION OF $\delta$-RAY EFFECTS ON DFIFT CHAMEER RESOLUTION}

Emestine Beleal, Douglas E Greiner, and Fred Beser*

A signfficant problem associated with any sensitive partfele position measuring instmment is the distortion introduced in the stgral of the particle of interest by \&-ray production along the particle's trafectory through the instrument. In the worst cass, the additional signel introduced by $6-$ rays can mask the true signal enough to makg subsequent inalysis irpossible. The character of such distortion is directly related to the design of the instrument. Therefore, detalled knowledge of the signal and inhorent distortions in it is necessary both for developing optimum instrumernt design and planning analysis procehres.

The handing of this problem becomes especially critical for the exporimenter considering instruments to be used with high- 2 beans such as those probuced at the bevalac, Since the number of 8 -rays produced varies approximately as the square of the charge of the incident particle, the resolution of the signal given by a hoavy ion becomes increasingly difficult as the charge increases. In the case of a maltifire chamber, a large nimber of $\delta$-rays accompanying the primary fon can cause so manty trires to fire that an intolerable area of uncertainty will surround the true coordinate location. by crintrast, a drift chanber may give better results for high-Z beans becaluse the signal of the primary is generally large mough to overtide the accusulated contribution of its accompanying backsromen. In order to develop a design which will mininize distortion and inaprove resolution, it becmes necessary to study, not only the ranifications of different instrumental configurations, but also the influence of varyn ing charges and energies on the instrument. 
Our first motivation for developing a comeuts simulation of $\mathrm{d}$-ray production cane as the result of designing drift chapbers for use in a space flight experiment. In this experimental situation an instrument was needed which could supply signal infor mation, clear enough to distinguish betwen particles sampling the whole range of costic radiation (from protons to very high- 2 ). Using lonte Carlo teclmiques to simulate s-ray production by particles treversing the drift chimber system, we were abIe to study the signal generated by ions of various charge and energy incident on the detector at either specific or randon angleg. the were also able to simulate thanges in design of the instruncht with respect to materials, scale, and configuration. Having generated those signtis typical of the sxpected final experimental sitution, we were then able to work backwards and analyzo our sin.ulated dat with knoledge of whet the answer should be, thus supplying a powerful tool for eraluating the design and developing future analysis programs.

This computer porgran, initially intended to create a motel for the specific study of $\delta$-Tay ef-

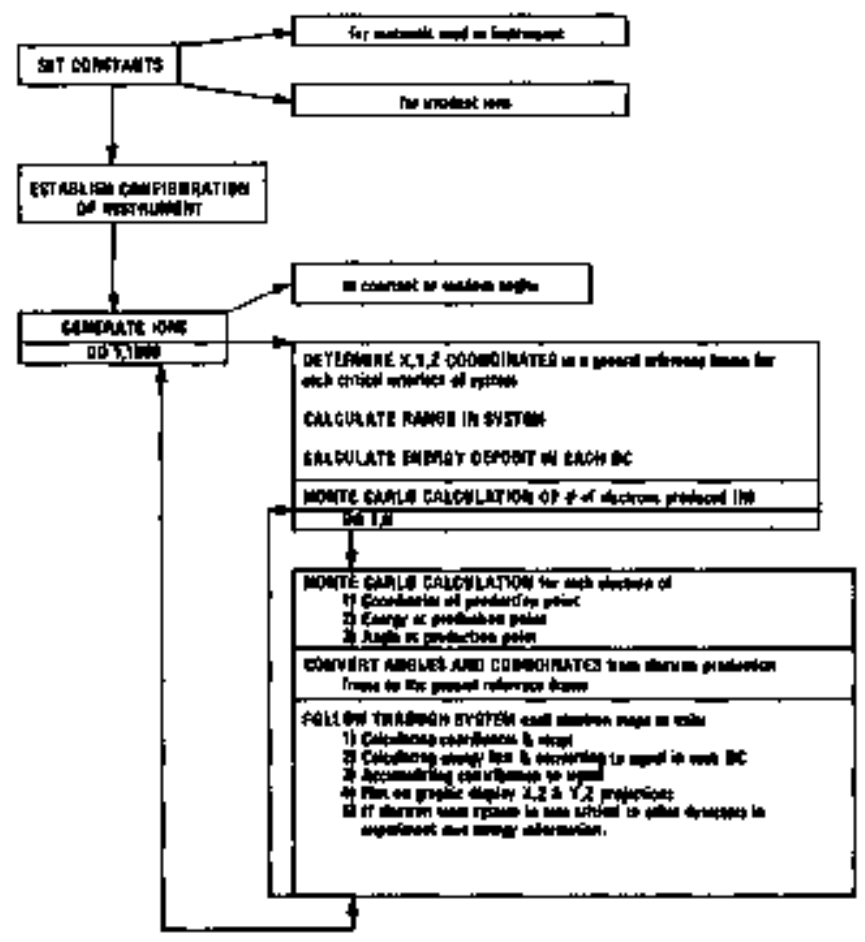

Fig. 1. Skeletal framework of progran showing establishent of instrunent configuration, generation of ions, protuction, and trace through system of i-rays.

(XBL $766-8267$ ) fects on drift chmber resolution has now evolved as a more general tool. It will be possible to sirentate other detection instrumetts and other experimenta1 situations by the introduction of $\mathrm{Tel}-$ atively winor changes in the general program such as a subroutine which establishes the experinental configuration and variable paraneters containing the pertinemt specifications. Figure 1 shows a biock diagran detalfing organfzation of the program.

The graphic display of a particle and its accompanying 6 -rays in both $x-z$ and $y-z$ coordinate systems was found helpful in debugging the program and in giving a visual understanding of the physical situation. Figure 2 shows a plot in $x-2$ projection of a typical heary jon and the $\delta$-rays it probuced th traversing the drift chanber. Eech 8 -ray wa plotted fran its origin to that point where it either stopped or exited the configuration.

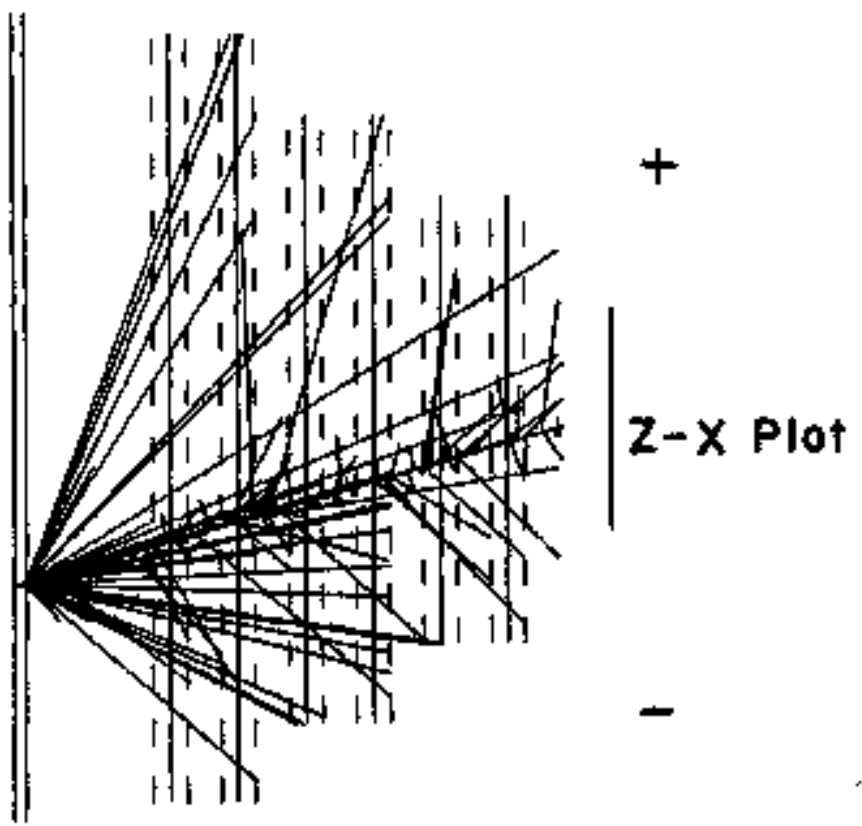

F1g. 2. Computer protuced plot in in $x-2$ coordinate frame, of an ${ }^{36} \mathrm{Fe}_{26}$ ion at $455 \mathrm{MNV}$ and the electrons protuced along 1ts path through the drift-chamber syster.

(XEL 766-8268)

\section{Foothote}

"University of Califomia space sciences Laboratory, Berkeloy, Califormia. 
1.

$+$ 


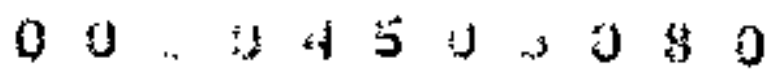

IV.

THESIS ABSTRACTS 



\section{$00: 0450 \% 50$

K VACANCY PEOUUCTION IN MEDITA-Z FEAVY ION COLLISIONS

R. Anholt

(LBL-4312)

During slow encounters between projectile and target atoms, the electronic atomic wefunctions of the two collision partners distort and form Molecular orbitals (nos). Th1s thosis considers $K$ vacancy formation in slow heavy-ion collisions which ocaur when electrons are excited ont of the zpo and 1so Mos.

Experinkental work using $200 \mathrm{MeV} \mathrm{Kr}$ ions is described. Thick target yìtlds of projectile and target $K x$ rays coning from $2 p o$ and $15 \sigma$ excitation are identified.

Our rain interest is the excitation of electrons from the 1so $\mathrm{mD}$. This cannot be observed in symmetric or near symetric collisions since Derkov coupling between the projectile and taxget $K$ shells at large intermaclear distances calse vacancies, which are created in the 2po orbital, to be transferred into the 150 orbital giving an apparent 1so yield many orders of magnitude laxger than for direct excitation. In collisions iwolving $\mathrm{Kr}$ ions, Iso vacancy production is only observed in very asylunetric colli" sions: as $K r K \times$ rays in encounters with taxget atcons between $H$ and $A r$, and as target $K \times$ rays in encounters with atcins between Ce and $\mathrm{t}$.

Theoretical approximate calculations of 150 ionization cross sections are described. The caloulations indicate that when the experinental proton $+z_{2}$ cross section ( $\sigma_{p}$ ) is known (where $z_{2}$ is the target atcmic nuber), the $z_{I}+z_{2}$ cross section can be calculated from the relation:

$$
q\left(w_{1}\right)=\sigma_{F}\left(v_{1}\right) z_{1}^{2}\left[\begin{array}{l}
U_{\left.K^{(2}=z_{2}+1\right)} \\
\varpi_{K^{(2}=z_{1}+z_{2}}^{(z)}
\end{array}\right]^{n}
$$

Where $z_{1}\left(<z_{2}\right)$ is the projectile atonic number, $u_{K}$ ts the $K$ binding energy, and $n$ is a constant depending on the reduced projectile velocity $v_{1} / v_{K}$ ( $v_{K}$ is the velocity of the $K$-shell electron). This formula may also be used where $z_{2}<z_{1}$ by interchanging $z_{1}$ and $z_{2}$ everywhere.

Data from experinents where the target $K$ vacancy cross section is measured as a function of the proJectile charge (at the sathe velocity) are disctussed. The above relationshfp fits the data but $n$ is not gs large as theory indicates. Finally we compare our $\mathrm{Kr}$ data and other heavy ion data with this relation.

\section{APPRICATION OF GENERALIZED CLASSICAL TRAJECTORIES IN MILCLEAR PHYSICS}

Herbert Massman leser

(LBL-4316)

A new semi-classical method, the so-called uniform semic1assical approxínation, is described briefly and then applied to tho nuclear physics problens. The baste features of this method are that the dyrimics of the problem is treated coupletely classicatly (that is, one solves classical equations of motion), but the quantum raechanical superposition principle is retained by exaluating a phase along the classicaI 
trajectory and adding probability anplitudes for indistinguishable processes rather than probabilities themselves.

The first problea considered is the backstattering from a deformed nucleus and the excitation of rotationgl states in the target at energies up to the Coulasb barrier. The unl tiple Coulonb exchtation calculations are in quantitative agreenent with a very different pethod (the de Boer-Winther code). A nuelear optical potential is also considered and the nuclear-Coulonb interference for heavy ions is studied,

The second problen considered is the turmeling through a top-dimensional barrier. This problen (which is supposes to simlate the penetration through a two-dimensional fission barrier) is investigated by a fully quantum-mechanical coupled-channel calculation and by the unifom semiclassical approximation. A quantitative agreement is found.

\title{
THE EFFECT OF FREE-PARTICLE COLLISTONS DN HIGH ENERGY PRDTON AND PIOA-INDLCED NUCLEAR REACTIONS
}

\author{
Norman P. Jacob, Jr.
}

(LBL-4067)

The effect of free-particle collisions in simple "knockont" roactions of the form (a,aN) and in more complex nuclear reactions of the form $(a, X)$ was irvestigated in a two part study by using protons and pions as the incident projectiles. In the first part of this study, cross sections for the ${ }^{48} \mathrm{Ti}(p, 2 p){ }^{47} \mathrm{Sc}$ and the ${ }^{74} \mathrm{Ge}(\mathrm{p}, 2 \mathrm{p})^{73} \mathrm{Ga}$ reactions were measured from 0.3 to $4.6 \mathrm{GeV}$ incident proton energy. The results indicate a rise in $(\mathrm{p}, 2 \mathrm{p})$ cross section for each reaction of about (25 \pm 3 ) correlated to a large increase in the total free-particte pp scattering cross sectlons over the same onergy region. The experimental results are compared to previous $(p, 2 p)$ excitation functions in the Gey energy region and to $(p, 2 p)$ cross section calculations up to $1 \mathrm{GeV}$ incident proton energy based on a Monte Carlo intranuclear cascade-evaporation model. This model yields cross sections that are generally a factor of 2 greeter than the experimental values.

In the second part of this thesis, cross section measuregnents for $\pi^{4}, \pi N$ ) and other more canplex pioninfuced spallation reactions were measured for the light target guklei $14_{\mathrm{N}} 1{ }^{16} \mathrm{O}$, and $19 \mathrm{~F}$ from 45 to 550 incident pion energy. These measurements indicate elearly a broad peak in the excitation functions for both $(\pi, \pi \mathbb{N}$ and $(\pi, X)$ reactions negr $180 \mathrm{keV}$ incident pion energy. This corresponds to the large resgances observed in the free-particle $\pi^{+} p$ and $x^{-} p$ cross sections at the same energy. Striking differences in $(n, n k)$ cross section nagnitudes are observet among the 1ight nuclei targets. The experimental cross section ratio $R_{\pi^{-} / \pi^{+}}=\sigma\left(\pi^{+}, \pi^{*} n\right) / \sigma\left(\pi^{+}, \pi N\right)$ at $180 \mathrm{MeV}$ is $1.7 \pm 0,2$ for all three targets. The experimental results are compared to previous pion and analogous proton-induced reactions, to Monte Carlo intranuclear cascade-evaporation calculations, and to a semi-classical nucleon charge exchange nodel in an effort to understand the mechanism of plon reactions, particularly of the $(\pi, \pi N)$ reaction, 


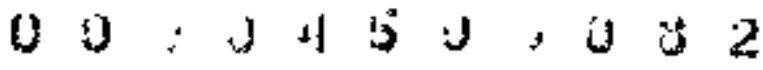

MASS MEASUROAENS OF HIGFLY NEITRON-EXCESS NLCLEI TN THE

LIGHTI ELEMENTS

Kenneth Hugh Vilcox

(LBL-4029)

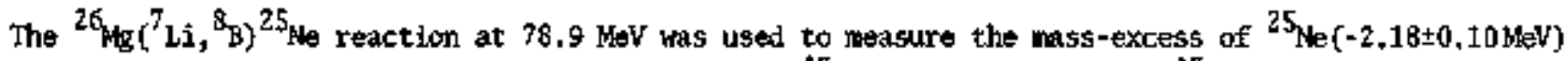

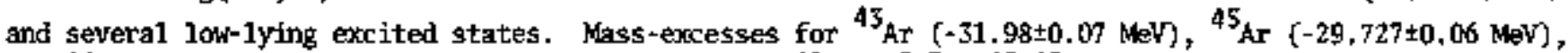

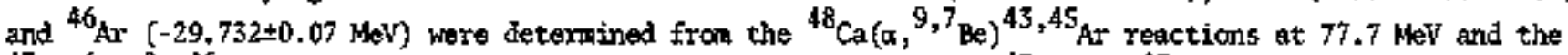
${ }^{47} \mathrm{Ca}\left({ }^{6} \mathrm{Li},{ }^{8} \mathrm{~B}\right){ }^{46} \mathrm{Ar}$ reaction at $80.1 \mathrm{MeV}$. Several excited states of ${ }^{43} \mathrm{Ar}$ and ${ }^{45} \mathrm{Ar}$ were observed. These and the excited states of ${ }^{25} \mathrm{Ne}$ are compared with theoretical predictions. Ground state differential cross sections are given for all reactions, ranging from 0.1 to $1 \mu \mathrm{b} / 3 \mathrm{r}$ at forward angles. tpper limits of about 1 to $25 \mathrm{nb} / \mathrm{sT}$ were determined for the grand state cross sections of the reactions ${ }^{48} \mathrm{Ca}\left(a,{ }^{\left.8,10,11_{\mathrm{B}}\right)}\right.$ $44,42,41_{\mathrm{C} l}$ and ${ }^{48} \mathrm{Ca}\left(a,{ }^{10-13} \mathrm{C}\right)^{42-39} \mathrm{~s}$ at $110 \mathrm{MeV}$.

Recoil coincidence techniques were used to measure the mass-excess of the particle-unbound nucleus ${ }^{10} \mathrm{Li}(33.8340 .25 \mathrm{MeV})$ by the ${ }^{9} \mathrm{Be}\left({ }^{9} \mathrm{Be},{ }^{8} \mathrm{~B}\right){ }^{10} \mathrm{Li}$ reaction at $121 \mathrm{MeV}$; the ground state cross section was 30 $\mathrm{rb} / \mathrm{sr}$. The ${ }^{14} \mathrm{C}\left({ }^{9} \mathrm{Be},{ }^{8} \mathrm{~B}\right)^{15} \mathrm{~B}$ reaction at $120 \mathrm{MeV}$ was also investigated using coincidence techniques, Contamination probleas only permitte determining a ground state cross section upper linit of 50 ng.sr.

A simple mass relation is derived, sinilar in approsch to the Garvey-Kelson mothod burt taking more explicit account of she1l effects. Compartson is made with the Garvey-Kelson relation, and predictions of masses and of the stability of neutron-excess light nuclei are given for both methads. The modified mess relation is showl to often better account for highIy noutron-excess nuclei, including the values reported here for ${ }^{25}$ the and $43,45,46 \mathrm{Ar}$. 
$0 \cup \div 3450.533$

V.

PUBLICATIONS 



\section{$05: 44$ b}

\section{PAPERS PUBLISHED AND LBL REPORTS ISSUED}

ANHOLT, R., S. Nagamiya, J. 0. Rasaussen, H. Rowman, J. G. Inannow-Yannou, and E. Rauscher K Vacancy Production by 4.88 GeV Protons LBL-4307 Rev., April 1976

ANHOLT, R.

$K$ Vacancy Production in Medinm-2 Heavy Ion Collisions lol-4312, Decenber 1975

$\mathrm{Ph}$. D. Thesis

AWHDLT, R., J, Ioamou-Yarucal, H, Bownan, E. Rauscher, S, Nagamiya, J. O. Rasmussen, $T$. Shibata, and H. Ejiri

Atoric R Vacancy Production with 3 Gevf Carbon Ions

LBL-4359, January 1976

AWHLT, R, and T. K. \$aylor

Radiative Ionization in Slow Fon-Aton Collisions LBL-4360, Jantuary 1976

Phys. Letters $\mathrm{A} 263,500$ (1976)

ANSOLT, Robert (See Neyertof, N. E., LBL-4078)

ANHDLT, R. (See Meyerhof, N. E., LBL-4019)

ANOLT, R, and T, K. Saylor Ancualous $X$ Ray Continua in Heavy Ion Collistons LBL-4066, Juty 1975

Phys. Rev. Letters

AHOLT, R,

On the Separated Aton Lorentzian Contribation to the Molentalar Orbital X-Ray Yield 1.BL-4301, August 1975

Journal of Physics B

ANEOLT, R. and the TOSALE group

$K$ Vacancy Production by $4.88 \mathrm{GeV}$ Protons

LBL-4307, September 1975

Phys. Rev. A

ART2Y, Micha1, F. Asaro, and I. Perlnan Alastya of the Amasma Letters LBL-2375, June 1974

J. of Near Easterm Studies 35, 171 (1976)

ARTZY, Michal, I. Perlman, and F. Asaro Theel-Nade Pottery of the M,C. III and L.C. I in Cyprus Identified by Neutron hctivation Analysis

LEL-2975, August 1975

Report of the Department of Antiqulties of Cyprus
ARTZY, MAchal

An Archeologist Looks at X-Ray Fluorescence ve. Neutron Act Lvation Analysis LBL-5D17, April 1976

Applications of Science to Metieval Ceramics, Lawrence Berkeley Laboratory, Berkeley, CA March 18-22, 1975

ARTZY, Michal

Alasia I

LBL-5024, May 1976

ASARO, F. (See Artzy, Michal, LAL-2975)

ASKERY, D, (Soe Hilcox, K. H+, LBL-40B9)

ASHERY, D., M. S. Zimman, G. W. Goth, G. J. Wozniak, R. B. Weisemilier, and Joseph Cerryy Study of $\mathrm{T}=2$ States in $1 \mathrm{C}^{\mathrm{C}}$ and $12_{\mathrm{B}}$ L.PL-4315, Nowenber 1975

Phys. Rev. C 13, 1345 (1976)

A2JENBERG-SELOVE, P. (See MaguiTe, C. F., LBL-4338)

BABCHET, R. (See Nifenecker, H, C., LBL-1950)

BABIKIET, R. P. (See Thompson, 5. G., LBL-2940)

BABDET, R. P. (See Noretto, L. G., LBL-3444)

BABENET, R. (See Galin, J., LBL-4064)

BABDET, R., L. G. Woretto, J. Gal in, R. Jared, J. Bbulton, and S. C. Thompson

Diffusion Processes in the Relacked Cross Section for the Reaction $107,109 \mathrm{Ag}+20$ the

LBL-4080, August 1975

ict. Phys. A258, 172 (1976)

BABINET, R. (See Noretto, l. G., LBL-4084)

BANASCHIK, M. V., R. S. Stmom, P. Colombani, D. P. Soroka, F. S. Stephens, and R. M. Diamond Trensitions Betweem High-Spin Muclear States LEL-3456, January 1975

Phys. Rev, Letters 34, 892 (1975)

BANASCHIK, M. V. (See Bertschat, H. LFI-4039)

BANASCHIK, M. V. (See SimCr, R. S., LBL-4313) 
BECK, F.

Angular Focussing in HEAVILY Dauped Heavy Ion Collisions

LBL-4300, August 1975

Plys. Letters B

EERTSCIAT, H. (See Matd, D., LBL-3446)

RERTSCHAT, H. (See Butter, F+ A., LEL-3483)

BEX, L., N. J. Clark, C. E. El1sworth, w. \$. Flood, R. A. Gough, W. R. Holler, J. R. Meriwether, and D. Morris

High Charge State Heavy Ion Production from a PIG Scrure

LBL-3433, 1975

Presented at the 1975 Particle Accelerator Conf. and pubtished in the Proceedings, page 1702

BEX, L., D. J. Clark, C. E. Elswoth, R, M. Estrella, R. A. Gough, and W. R. Holley Enittance Measurements of High Charge State Argon Beans from a PIG Source LBt-4075, October 1975 International Conference on Heavy Iọn Saurces, Gatlinbrr, TN, October 27-39, 1975

BIRCHaLL, J., H. E. Conzett, F. N. Rad, S. Chintalapudi, and R. M. Larimer The Depolerfyation Parameter in $\overrightarrow{\mathrm{p}}-10_{\mathrm{B}}$ elastic Scattering and the Spin-Spin Interaction LBL-4054, June 1975

4th Intemational Syuposium on Polarization Phenamena in Nuclear Reactions, Ziirich, Switzer. 1a्यd, August 24-29, 1975

BEOCKI, J. (See Boneh, Y., LBL-4374)

ByockT, J. P. (See Boneh, Y., LBL-4397)

BYOCKI, J., J. Randnup, W. J. Swiatecki, and C. F. Tseng

Proximity Forces

LBL-5014, Aptil 1976

BDoCKI, J, and $\mathrm{H}$. Flocard

Simple Dynanical Models Includint Pairing Residual Intoraction

LBL-5016, April 1976

BoISSON, J. P. (See Donangelo, R., LBL-4347 Rer.)

RONEH, $Y_{+}$, J. P. BJocki, and W. D. Myers A Witcroscopic Wethod for Calculat ing the Intrinsic Excitation Occurring in Nuclear Fission LEL-4374, Fobruary 1976
BOAEE, Y., J. P Blocki, and W. D. Myers

Microscopic and Macroscopic Calculations of OneBody Dawping in Fission .

LEL-4397, April 1976

BCND, P. D. (See Meyerhof, W. E., LBL-4079)

BOHAW, J. D. (See Zebelnan, A. M., LBL-3427)

BOMAN, H. (See Arholt, R., LEL,-430? Rev.)

Bowdik, H, (See Anholt, R., LBL-4359)

BUCFER, J, J. and R. M, Diamond

Backbernding and other Neviations from Ifeality in Extraction Systems

LRl-3453, Jamuary 1975

J. Cheml. Phys. 79, 2259 (1975)

BUENED, M., D. L. Hendrie, U. Jalmke, J, Mahoney, A. Menchaca-Rocha, C. Olmer, and D. K. Scott Response of Pilot $\mathrm{y}$ scintiliator to Heavy Ions 1.BL-4372, Јатинагу 1976

Nucl. Instr, and Meth.

ENRLEIGH, R, J., D, J, Clark, and W. S. Flood $150 \mathrm{keV}$ Proton Medical cyelotron Design Study LBL-4085, August 1975

7 th Intexpational Conference on Cyclotrons and Their Applications, Züirich. Switzerland, August 19-22, 1975

BUTLE, P. A. (See Nard, D., LEL-3446)

FULLLR, P. A., J. Meyer-ter-Velm, D. Ward, H. Pertschat, P. Colonbani, R. M. Diampond, and S. S. Stephens

Triaxial Shapes in Light La Nuclel

LBL-3483, tharch 1975

Phys. Letters 56B, 453 (1975)

BUTLER, P. A, (See Guidry, M. W., LEL-403B)

ItTTLER, P. A. (See Ward, D., I,BL-4375)

BUTLE, P. A. (See Colorbani, P., LBL-5027)

GULER, P. A. (See Lee, I, Y, LBL-5031)

CAIVIN, B. (See thretto, L. G., LBL-4362) 


\section{$00, \sqrt{1} 50, \frac{j}{385} 85$}

CAVELL, Ronald G.

Core Photollectron Spetroscopy of Scme Acetylenic Molecules

LBL-2941, Warch 1974

J. Electron spectroscopy and Related Phenomenta 6, $281(1975)$,

CAVEll, Ronald G.

Core Photoelectron Spectroscopy of Some Volatile Phosphorus thalides

LEL-4095, Hay 1975

Inorg. Chem. 14, 2830 (1975)

CERNY, Joseph (See Je11ey, N. A., LRL-3414)

CEAWY, Joseph (See Hileox, K. H*, LBL-4089)

CERNT, Joseph (Set heisermiller, R, B,, LRL-4304)

CERNY, Joseph (See Ashery, D., LikL-4315)

CERRY, Joseph (See Vieira, D. J., LRL-4321)

CERNY, Joseph (See Wozniak, G. J, , LRL-4368)

CENAY, Joseph

Studies of Exotic Nuclei

LBL-5054, Nay 1976

$3 x$ International Conference on Nuclei Far fros

Stability, Cargese, Corsica, Italy, May 19-23,

1976

GHARLTON, L. A. (SEe Kunz, P. D., LBL-A345)

GHARLTON, L. A.

Finite Range Evaluation of $[p-d, d-t)$ with Manentim Space Techniques

LEL-4356, January 1976

Phys, Re्षr. C

CHARLTON, L. A. (Sruess, K., LBt-5008)

GHARLTON, L. A. (Set Glendenring, N. K., LBL-5009)

CHASE, L. F., JT. (See Meyorhof, W. E*, LEL-4078)

GIINLALAPLDI, S. (See Birchat1, J., LBL-4054)

CHI, S. Y., E. R. Marshalek, P. Ring, J. Knumlinde, and J. 0 . Rasmussen

Test of Mary-Body Mothods on an Exactly Soluble Model with Backbending and Gaples Superconductivity

L.FI-4021, May 1975

Phys. Rev. C 12, 1017 (1975)

CIARK, D. J. (See Bex, 1., LBL-3433)
CLARK, D. J. [See Bex, l., LBL-4075]

CIARK, D. J. (See Burleigh, R, J., LEL-4085)

CLARK, D. J, (See Burleigh, R. J., LAEL-4086)

CLARK, D. J. [See Burleigh, R. J., LEL-4086]

CLARK, David J.

Conference Sulmary

LBL-4348, October 1975

International Conference on Heavy Ion sources,

Gatlingburg, TN, October 27-30, I975

CLENE, D. [See Colambani, P., LEL,-5027)

CLINE, D. (5ee Lee, I. Y*, LBL-5031)

COLOMRANI, P. (See Hard, D., 1.BL-3446)

COLOMEAN], P. (See Banaschik, M. V., LBE-3456)

COLORBANI, P. (See Butler, P. A., LBL-3483)

COLONEAN], p. (See Guidry, M. W., LBE-4038)

CoLARAkI, P, (See Sìnon, R, S. LBL-4313)

COLOMANI, P. (See Wart, D., LBL-4375)

ColamanI, P., P. A. Butlet, I. Y. Lee, D. Cline, R. H. Diamond, F. S. Stephens, and D. Mard Sulccoulanb Fission Intuced by Xe and Kr Ions LBL-5027, May 1976

COLOMBaNI, P. (See lee, I. Y., LBL-5031)

CONZETT, H. F. (See Seİlet, F, LBL-3496)

CONZHTT, H. E, (See Rad, F. N. LBL-4040)

CONZETT, H. E. [See Roy, R. LEL-4041]

CDNZETT, H. E., Dahone, Ch. Lemman, J. A. Macilonald, and J. P. Merilders

The Vector Analyzing Power in d-4 Scattering Between 15 and $45 \mathrm{MkV}$

LRL-4042, June 1975

4th International Symposium on Polarization

Phenomena in Nuclear Reactions, Zürich,

Switzerland, Aurgust 25-29, 1975

CONZETT, H. E. (See Seiler, F., LAl-4043) 
GWZEIT, H. E., F. Seiler, F. N. Rad, R. Roy, and R. M. Lariner

Measurement of the Vector Analyzing Power Nesr the Value $\mathrm{Ay}=1,0$ in d-4He Elastic Scattering

LBL-4044, June 1975

4th Internationa1 Symposium on Polarization

Phengnens in hucleat Reactions, zürich,

Switzerland, August 25-29, 1975

CONZETT, H. E. (5ee Seiler, F., LEI,-40\$5)

COAZETT, H, E. (See Seiler, F, LBL-4047)

CONZETT, H. E. (See Seiller, F*, LBL-4048)

CONzETT, H. E., W. Dabne, R. U. Lariner, Ch. Leenam, and J, S. C. MeKee

The Vector Aralyzing Power in Elastic DeuteronDeuteron Scattering Between 20 and $40 \mathrm{keV}$ LELL-4049, Jume 1975

4th International Symposiuen on Polarization Phencitina in Nuclear Reactions, Zïrich, Switzerland, August 25-29, 1975

CONEET, H. E., J. S. C. NcKee, R. M. Larimer, and Ch. Leenonn

The Vector Analyzing Power in the ${ }^{2} \mathrm{H}(\mathbf{d}, \mathrm{p})^{3} \mathrm{H}$ Reaction at $30 \mathrm{MeV}$

LEL-4050, Junt 1975

4th International \$yppositu on Folarization

Phembnena in Nuclear Reactions, Zürich,

Switzerlard, Albust 25-29, 1975

CONzETT, H. E., R. M. Lariner, F. N. Rad, R. Roy,

and F. Seiler Reaction Between 15 and $25 \mathrm{keV}$ LBE-4051, Jume 1975

4th International Sympositen on Polarization

Fhenumene in Phxlear Reactions, Zïrich, Switzerland, Axpust 24-29, 1975

CONZETT, H. E. (See Roy, R., LEL-4052)

CONzEIT, H. E. (See Roy R., LBL-4053)

CONZETT, H. E. (See Birchall, J., LBL-4054)

CONZETT, H. E.

Polarization Symmetcries in Direct Reactions:

$3_{\mathrm{He}}(\mathrm{t}, \mathrm{d})^{4} \mathrm{He}$ and ${ }^{2} \mathrm{H}(\mathrm{d}, \mathrm{p})^{3} \mathrm{H}$

LRL-4087, August 1975

4th Intermational Symposium on Polarization

Phenomena in Muclear Reactions, Zïrich, Switzer

land, August 25-29 (1975)

CONZEIT, H. E. (See Rad, R, N, , 1EL-4091)
CONZETT, H. E.

Polarization Phencmena in Few Body Systems

I.EL-4351, Decenter 1975

VII Internations1 Conference on Few-Body Problems in Nucleat and Particle Physles, Delhs, Intia, Decauber 29, I975 - January 3, 1976

DAHE, W, (Soe Conzett, H. E., LEL-4042)

DAHUE, W. (See Conzett, H. E., LEL-4049)

đẹ BOER, J. (See Grosse, E, , LBL-4037)

DELIC, George

Optical Model Analyses of $\mathrm{NHC}$ and $\mathrm{C}+\mathrm{C}$ Elastic Scattering

LBL-3492, Karch 1975

Phys. Rev. Letters 34, 1469 (1975)

DELIC, George

Conputing the Finite Range Distorted-Weve Approximation for $11_{\mathrm{B}}(160,13 \mathrm{~N})^{12} \mathrm{C}$

LBl -4343 , Hovember 1975

Phyrs. Rev. Letters 36, 569 (1976)

DEdIC, George and Dieter Mrath

Finite Range Distortet Waves Borm Approxination Calculations for ${ }^{13} \mathrm{C}\left({ }^{3} \mathrm{He},{ }^{6} \mathrm{He}\right\} 1 \mathrm{C}$

1.8I-4364, January 1976

Phys, Rev, $\mathrm{C}$

DELIC, 6. (See Prtiess, K., LBL-500B)

Del.IC, G. (See Glenderring, N. K., LkL-5009)

DER MATES5IAN, E. (See Rezanka, I., LEL-2965)

Dincts, R. M. (See Newton, J. O., LRL-2333)

DIAMOHD, R. M. (See Ward, D., LEL-3446)

DIADN, R. M. (See Bucher, J. J., LEL-3453)

DLAMTO, R, M, [See Banaschik, M, Vi, LEL-3456]

DLWOND, R. M. (See Butler, P. A., LBL-3483)

DIALAND, R. H. (See Grosse, E,, LBL-4037)

DLANDW, R. N. (See Guitry, M. W, LBL-4038)

DLAMOND, R, M, (See hard, D., LBL-4375) 


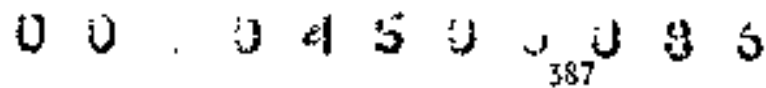

DIdupD, R, M. (See Cologuanl, P. LBl-5027)

DIANCND, R. H. (See Lee, I, $Y_{+}$, LBL-503I)

DIAWOND, R. M.

Continum: Gamte Rays Following (IHI, xnY) Regctions

LEL-4081, August 1975

Eighth Sumer School on Nuclear Physics, Mikotajki, Poland, Alfust 24-5eptenber 6, 1975

DIAMCAD, R. H. (See Simon, R. S. LBL-4313)

DOtর 将ELO, R. (See Kawakani, H. , LBL-4303)

DOMANGELO, R., M. W. Guidry, J. P. Bolsson, and

J. O. Rasmusgen

The Classica1-Linit S-Matrix for Coulomb Excitation

LEL-4347 Rev., Jtme 1976

DORAHGELO, R. (See Guidry, M. W., LBL-4357)

ExGERS, R, C. $C_{+}$W. 5. Ribbe, and $J_{+} 0_{+}$Rasmussen Argon Ion Reaction Studies or Tantalum and Gold Near Coulamb Barrier Enerries

LEL-4097, August 1975

Nuxlear Physics S6A, 455 (1976)

EICHLER, R, (SeA Ouidry, M. W., LBL-4038)

EICHLER, E. (See lee, I. Y, , LBL-5031)

EJIRI, H+ (See Aniolt, R, , LBL-4359)

ELLSWORTH, C. E. (See BeFK, L., LBL-3433)

ELLSWOKTH, C. E. (See Bex, L., LBL-4075)

ESrRElJA, R. M. (See Bex, L+ LEL-4075)

FDK, R. W. (See Wood, J, L., LBL-4350)

ELIESSRACH, T. and P. Manakos

Alpha-Spectroscopic Factors for Light Nuclei

LBL-4094, Algust 1975

Nuc1. Phrs. A

FLIESSEACH, T., H. J. Nary, and J. O. Rasmussen Normalized Shell Model Alpha Decay Theory Applied to Infayored Decay

LAL-4335, Oct tuber 1975

Fhys, Rev, C 13, 1318 (1976)
FLOCARD, H. (See Blocki, J., LBL-5016)

FLOOD, w. S. (See Burleigh, R. J., LBL-4085)

FOMLER, M. M. (See Thompson, 5. G., L.BL-2940)

FowLER, Malcolm $M_{+}$and Richard $G_{+}$.Jared

A Gas Ionization Counter for $P$ article Identi fication

LBL-3435, October 1974

Nucl. Instr. Methods 124, 34I (1975)

FONLER, M. M. (5ee otto, R. J+, LBL-4322)

FRAENKEL, 2. (See Noretto, L. G,, LBL,-4084)

GALIN, J. (See Thanpson, S. G., LEL-2940)

GALIN, J. (See Moretto, L. G, , LBL-3444)

GALEN, J., L, G. Moretto, R, Babinet, R, Schmitt, R. Jared, and S. G. Thampson

Evidence of Incimplete Relexation in the Reaction $\mathrm{Ag}+40_{\mathrm{Ar}}$ at 288 and $340 \mathrm{MeV}$ Bcmbarding Energ ies

LEL -4064 , July 1975

Necl. Phys. A255, 472 (1975)

GALIN, J. (See Habinet, R., LBL-4080)

GALIN, J. (See Moretto, L. G+, LBL-4084)

GATTI, R. C. (5ee Thampon, S、G., LBL-2940)

GAVTN, Basil

Anode Sputtering Characteristics of the Berkeley 2.5 MV Source

LBL-4291, October 1975

IEEE Conference on Plasma Science, Gatlinburg,

$\mathrm{TN}_{+}$, October 27-30, 1975

GHIORSO, AIbert.

The Berkeley New Element Progran

LBL-4346, September 1975

International Summer School Sentinar on Reactions of Heavy Ions with NucleI and Synthesis of New Elements, Dubna, Moscor, ЏSSR, Septenber 3october 4, 1975

GLASGON, L. R. (\$ee Burleigh, R. J., LBL-40B6)

GLÄSSEL, P. (See Noretto, l. G., LBL-4362) 
GLEDEENTA, N, K.

Classiçal and Quantum Machanical Descrlptions of Heavy-Ion Interactions

LBL-2905, June 1974

Invited paper for the International Conference on Reactions Between Complex Nuclei, Nashville, Tennessee, June 10-14, 1974

GLENDENINIG, N. $K_{+}$

Interplay of Classical and Quantal Description of Heavy-Ion Interactions

LEl- 2905 Rev. . Jurse 1974

Rev, Mod. Phys, 47, 659 (1975)

GLENDENANG, N. K. (See Scott, D. K, LEL-3415)

GLEOENANG, Nonthan $k$. and Geong Wolschin

Enhancentent of Forward-Anele Cross Sections in Hiavy-Ion Reactions Because of Projectile Excitation

LEE-3472, I975

Phys, Rey, Letts, 34, I642 (1975)

GLEMDENDNG, Norman $\mathrm{K}$.

Tables of Muclear Sturcture Amplitudes for TwoNhicleon Transfer Involvinb Light Nuclides (a $\times 4)$

LEL-3490, March 1975

Muclear Data Tables 16, I (1975)

GLENDENING, Noman K. and Geory Wolschin

Physical Basis for Enhanced Forward Cross Soctions in Heavy Ion Reactions

1,BL 4334, October 1975

Phys. Rev. Letters 36, $1 \$ 32$ (1976)

GLENDENING, Noman $K$. and Georg Wolschin

Two-Particle Transfer Contributions to Elastic and Inelastic Heavy Ion Scattoring

LEL-4341, Noventber 1975

Nuct. Phyș, A

GLENDENING, Norman $K$, and Georg Molschin

Physical Basjs for large Forward Cross Sections in $60 \mathrm{Ni}\left(18_{0}, 10_{0}\right)$ React ion

LEL-5007, March 1976

Synposium of Macroscopic Features of Heavy Ion Collisions, Argonne, $\pi$, April I-3, 1976

GLENDENAING, N. K. (See PTuess, K., LLL-5008)

GLENDEWING, N. K., L, A. Charlton, G. Delic, and M. A. Nagarajan

A New Recoir Effect in Mucleon Transfer Reactions between Heavy Ions

L8L-5009, March 1976

S)mpositra on Macroscopic Features of Heavy Ion

Collisions, Argonne, IL., April 1-3, 1976

GOTH, G. W. (See Ashery, D., LBL-4315)
GOUGH, R. A. (See Bex, L., LBL-3433)

GOHGH, R, A. (See Ber, 1., LBL-4075)

GIJGH, R. A. (Se Vieira, D. J., LEL-4321)

GOULDING, F. S. and B. G. Harvey

Identification of Muclear Particles

LBL-2952, February 1974

Arr. Rev. of Nucl. Sci. 25, 167 (1975)

GROSSE, E., J. de Boer, R. M. Diamond, F. S. Stephens, and $P$. Tjofta

Couldo Excitation of High-Spin States in 23.0

LBL-4037, Jame 1975

Phys. Rer. Letters 35, 565 (1975)

GUTHY, M. W., P. A. Butler, P. Colombani, I. Y. Lee, D. Ward, R. M. Diemond, F. S. Stephens, E. Eichler, N. R. Johnson, and R. Sturm Reduced Transition Probabilities for High Spin States in $232_{\mathrm{Th}}^{\mathrm{y}}$

LRL-4038, Jartuary 1976

Nutc1. Phys, A

GIDfY, M. Y. (See Donargelo, R., LBL-4347 Rev.)

GIDRY, M. W., H. Massmenn, R. Dhrangelo, and J. 0 , Rasmussen

Theory of Coulonb-Nuclear Interference for HeavyIon Excitation of Rotational States LEL-4357, January 1976

GIIDY, M. W. (See LEe, I. Y., LBL-5031)

GUTBRCD, H, H. (See Poskanzer, A. M., LBt, 4309 Rev.)

HAL.BACH, Klaus

A Simple Class of Beam Transport Systems with Dptically Axisymetric Transfer Properties LBL-5026, May 1976

HALBACH, K. and F, R. Holsinger Superfish--A Camprter Progran for Evaluation of Rf Cavities with Cylindrical syllietry LBL-5040, Jume 1976

HARVEX, B. G. (See Goulding, B. G., LBl-2952)

HARVEY, B. G, (See Scott, D, K., LBL-341S) 


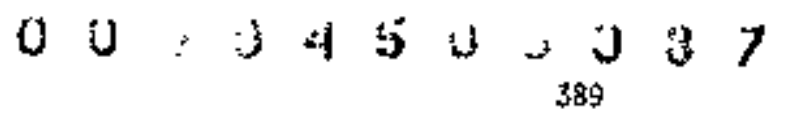

HARVEY, B. G.

Recent Multinuxleon Transfer Experiments with Heavr Ions at Berkeley

LBL-34 87, 1975

Presented at Second International Conf. on Clustering Phengene in Nutciei, College Park, ND, Apri1 21-25 I975

HARVE, Bernard G.

Radioactivity

LRL-5030, Hay 1976

Encyelopedis of Physics

HECONAN, Harry H. and Peter J. Lindstrom Coulomb Dissaciation of Relativistic $12 \mathrm{C}$ and 160 Nuciei

LBL-4380, Mrch 1976

Phys. Rev. Letter's

HFDRIE, D. L. (Se Yagi, K., LBL-2982)

HENDRIE, D. L. (See Scott, D. K., L.hL-3415)

HRMRIE, D. L. (Seg Scott, D. K., LBL-3445)

FEMDRIE, D, L. (See Scott, D. K., LBL-4059)

HFNDRIE, D. L. (See Shemant, Joseph D., LBL, -4306)

FENDRIE, D. L. (See Maguire, C. F., LBL-433B)

HENDRIE, D, L. (See Bunerd, M., LBL-4372)

HEDDIE, D. l. (Se日 Yag 1, K., LBL-4382)

HENDRIE, D. L. (Ser stock R., LEL-504J)

HOLLEY, W. R. (See Bex, L., LBL-3433)

HOLLEY, W, R. (See Ber, L., LEL-4075)

HOLSIXIFR, R. F. (See Halbach, K, , LBH-5040)

HUNTER, J. B. (\$̧ee Thorpson, S. G., LEL-2940)

TOANOUI-YANAOU, J. G. [Se Anholt, R., LPL-4307 Rev.']

FOANNOU YANALU, J, (See Anholt, R. LBL-4359)

JAHNKE, U. [See Scott, D. K,, LEL-3415]
JAJEVE, U. (See Scott, D. K., LEL-344S)

JAYWRE, U, [See Buenerd, M. LBL-4372]

JAHNE, IJ. (See Yagi, K., LRL-4382)

JAHNKE, U. (See Stock, R., LBL-5DA1)

JAH, R., G. J. Wozniak, D. P. Stahel, and Joseph Cerriy

The $\left(\alpha,{ }^{2} \mathrm{He}\right)$ Reaction as a Spectroscopic Tool for Investigating High Spin States

LBL-5038, Jume 1976

JARDINE, L. J, and Shihab-Eldin, A.

Alpha Decay of 210 At to Levels in $206 \mathrm{Bj}$

LBL-2943, October 1974

Nuxl. Phys, A224, 34 (1975)

JARDTNE, L. J. and A, A. Shthab-Elatg

The Electron-Capture Decay of ${ }^{200}$ po

LEL-3447, Jarmuary 1975

Phys. Rev. C 13, 348 (1976)

JARDINE, K. J. (See Shihab-EIdin, LBL-3409)

JARED, R. C. (See Thempson, \$. G+, LBL-2940)

JARED, Richard G. (See Conway, John LBL-3435)

JARED, R, C. (See Moretto, L. G., LEL-4063)

JARED, R. (See Galin, J., LBL-4064)

JARD, R. (See Babinet, R., LBL-40B0)

JARED, R. (See Moretto, L. G., LBL-40B4)

JARED, R. (See Moreptto, L. G., LBL-4362)

JEHlEY, N. A., Cerny, Joseph, Stahe1, D. P. and Wilcox, $K . \mathrm{H}$.

Predictions of the Masses of Yery NeutronExcess Light Nuclei

LBL-3414, Noterber 1974

Phys. Rev. G11, 2049 (1975)

JELLEY, N. A. (See HHlcax, K. H., LBL-4089)

JELLEY, N. A, (See Weisermiller, R. B. LBL-4303)

JELLEY, N, A. (SEe Wozniak, G. J., LBL-436B) 
JOHNSON, N. R. (See lee, I. Y., LBL-5031)

JOH+KSON, N, R. (Sen Gukdry, M. W., LBL-4058)

KATARIA, S. S, (See Moretto, L. 6., lBL-4063)

KRHAKAMI, H., M. Kolke, K. Komura, M. Sakai, N. Yoshikawa, R. Donangelo, and J. 0. Rasmussem Cross Section and Reaction Mechanions of (p.pxn) Reactions on $200 \mathrm{~Pb}$ in the 24-52 MeV Range LBL-4305, Angust 1975

Nuclear Physics $\mathrm{A}$

KOMRA, K. (See Kawakami, H. [B,-4303)

KOIKE, M. (See Kalrakanl, $H_{+}$, LBL-4303)

KDLBE, T. (See Church, D. A., LBL-3464)

KRAl', J. V., J. O. Liljenzin, A. E. Norris, and G. T. Seaborp

Charge and Mass-Distributions in the Reaction of $40 \mathrm{Ar}$ Ions with $230 \mathrm{~J}$

LEL-4339, Noverber 1975

Phys. Rev. C 13, 2347 (1,976)

KRALIS, L. (See Yagi, K., LBL-2982)

KRAIUS, L. (See Scott, D. K., LBL-3415)

IRAUS, L, (See Scott, D, K+, [EL-3445)

KRALIS, L. (See Scott, D. K., LBL-4059)

KRAMLTNDE, J. (See Chu, S. Y., LBL-4021)

HUNZ, P. 0. and l. A. Charltan

Finite-Range Effects in Sulti-\$tep (p-d, d-n) Reactions

LBL-4345, Deçember I 975

Phys. Letters

MLRATH, Dieter (See Delic, Gerrge Lal-4\$̧64)

LADENBGUER-BELLIS, I. M. (See Rezanka, I., LBL2965)

LARIMER, R. M. (See Conzett, H. E, , LBL-4044)

LARDER, R. M. (See Conzett, H. E., LBL-4049)

LARIMER, R. M. (See Conzett, H. E., LBL-4050)
LARDME, R, M. (\$e Conzett, H. E. , LBL-4051)

LAPMIER, R. M. (Ser Roy, R., LRL-4052)

LARIMER, R. M. (See Roy, R., LBL-4053)

LARIMER, R. M. (See Bircha11, J., LEL-4054)

LARSOSN, S. E. (See Randrup, J., LBL-4036)

LAZARUS, \$. M. (See Meyerhof, N. E., LRL-4D78)

LEE, D. (See Otto, R. J., L.BL-4322)

LEE, I. Y. [See Guidry, M. พ., LBL,-4038]

LEE, I. Y, (See Ward, D. , L.BL 44375 )

LEE, I. Y. (See Colopbani, P., LBl,-5027)

LEE, I. Y,, B. Cline, R. S, Simon, P. A, Butler, P. Colcubani, M. W. Guidry, F. S. Stephens, R. M. Dianond, N. R. Johnson, and E. Eichler Couldo Excitation into the Backbend Region of LBL-5031, May 1976

LEBMANN, Ch. (Seę Conzett, H. E., LBL-4042)

LEEMAN, Ch, (See Conzett, H. E., LBL-4049)

LERIAW, Clt. (See Corzett, H. E., LBL-40S0)

LESER, Hortert Mașsman

Application of Generalized Classical Trajectories in Nurlear Physics

LEL-4316, Septentber 1975

Ph.D. Thesis

LICHINER, P. (See Ptuess, K., LBL-4381)

LILJENZIN, J. 0. (See Kratz, J. V., LEl-4339)

LIMDSTRON, Peter J. [See Heckman, Harry $\mathbf{H}$. LEL-4380 j

LIPPERHEIDE, R.

Schrödinger Equation for Scattering with Energy Loss

LEL-4333, october 1975

Phys. Rev, Letters 


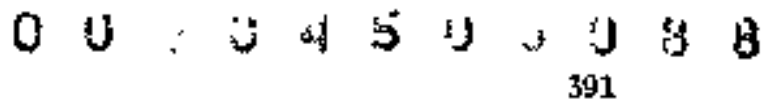

LIPPERHEIDE, R.

Quantal Description of Inelastic Heavy-Ion Scattering

LBL, -4336, October 1975

Muclear Physics A260, 292 (1976)

LITTLE, A. (See Meyerhof, H. E., LBL-4078)

LOW, K. S. (Se日 Yei $i, K$, LBL-2982)

McKEE, J. 5. C. (See Conzett, H. E. LBL-4049)

MckKE, J. S. C. (See Conzett, H. E., Lkd,-4050)

MaCDONALD, J. A. (See Conzett, H. E., LBL-40AZ)

MGUIRE, C. F. (See Yapi, K., LRA-2982)

MAOUIRE, C. F. (See Scott, D, K., LBL-3415)

MAGIIRE, C. F. (See Scott, D. K., LEL-3445)

MAGIIRE, C. F. (See Scott, D. K., LFl-4059)

MAGJIRE, C. F., D. L. Hendrte, D. K. Scott, J. Mahoney, and $F_{12}$ Ajzenberg-Selove

LBL-4338, October 1975

Physs. Rev. C 13, 933 (1976)

MAGJTRE, C, F. (See Yag i, K., LBL-4362)

MAGUIRE, C. F. [See Stock, R., LEL-5041]

MAHONEY, J, (See Yagi, K, , LBL,-2982)

MAHONEY, J. (SE Scott, D. K., LBL-3415)

MAFINEY, J. (See Scott, D. K., LBS,-344S)

MAFONEY, J, (See Scott, D. K, LBL-4059)

MAFTREY, J. (Se Mguire, C. F., LBL-4338)

MAHONEY, $J$, (See Busnerd, M., LBL-4372)

MAHONEY, J. (See Yagi, K., LBL-4382)

MAHONEY, J. (See Stock R., LBL-S041)
MANAK0S, P. (See Fliessbach, T, L LLL-4094)

MANG, H. J. (See Fliessbach, T, LBL-4335)

MARSFALEK, E, R, , (5ee Chu, S. Y,, LBL-402!)

MASSANN, Herbert and Racnussen, Jahn o.

Uniform Sentclassical Orbital Calculations of Heavy Ion Coulomb Excitation

LBL-3440, November 8, 1974

Nue1. Phys. A243, 155 (1975)

Massuan, H., P. Ring, and J. O. Rasmussen

Quantum Mechanical and Seniclassical Description of a T wo-Dimensional Fission Hodel LEL-4023, May 1975

Phys, Latters $\underline{57}$, 417 (1975)

MASSAN, Herbert (See Ring, Peter LBL-4028)

MASSMANN, H. (See Guidry, M, W, , L.Bl,-43557)

MENGHACA-RKCHA, A. (See Buenerd, M., LBL-4372)

MERIWETHER, J, R. (SEe Bex, L., LBL-3433)

HEIDERS, J. P. (See Conzett, H. E., LBt-4042)

MEYERHFF, W. E., T. K. Saylor, S. M, Lazarus, A. Littile, B, B. Triplett, L, F. Chase, Jr., and Robert Anho'st

Nolecular-0rbital K X-Ray Fomation in HeavyIon Collisions

LAL- 4078

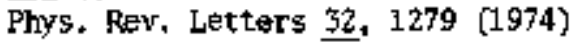

MEYERHOF, W. E., R. Anholt, T. K. Saylor, and P. D. Bond

K X-Ray Yield from $100 \mathrm{HeV} \mathrm{Pb}+\mathrm{Pb}$ Collisions LBL-4079

Phys. Rec. A 11, 1083 (1975)

MEYER-TER-VEFN, ড,

Rotational Band-\$tructures in Trensitionel odd-A Nuclei

LBL-3416, Septenber 19, 1974

Phys + Rev. Letters 55B, 273 (1975)

MEYER-TER-VEHN, J.

Collective Model Description of Trensitional Odd-A Nuctei I. The Triaxial-Rotor-PlusParticle Madel

LEL-3458, 1975

Macl. Phys. A249, 111 (1975) 
AEYER-TER-VRN, J.

Collective Hotel Description of Transitional odd-A Nuclei II. Camparison with thique Pority States of Nuclei in the $A=135$ and $A=190$ phass Region

LPL - 3459, 1975

Nucl. Phys. A249, 141 (1975)

MEYER-TER-YEHN, J. (SEe But1eT, P. A, , t,RL-3483)

MEYER-TER-YTH, J. (See Mood, J. L., LBL-4350)

MOLER, P. (Se Randrup, J., LBL-4036)

MORETTO, L. G. (See Thompson, S. G., LEL-2940)

MORETTO, L. G. and J. S. Sventek

A Theoretical Approach to the Problean of Partial

Equilibration in Heavy Ion Reactions

LBL-3443, Novelber 1974

Phys. Letters 58B, 26 (1975)

MORETTO, L. G., R. P. Babinet, J. Ga1in, and S. G. Thampson.

Experimental Evidence of a Diffusion Process Associated with the Mass Asymetry Degree of Freedon in Heavy Ion Reactions

LBL-3444, Novenber 1974

Phys. Letters 58B, 31 (1975)

WORETTO, L $\mathrm{L}$.

Statistical Bnission of Large Fragenents: A General Theoretical Approach

LEL-3454, January 1975

Niscl. Phys. A247, 211 (1975)

MARETTO, L. G.

Antipairing Effects in the Collective Nuclear potion and Their Relevance to the Saddle to Scission Descent in Fission LBL-3499, 1975

Phrs, Letts. S7B, 317 (1975)

MORETTO, L. G., S. S. Kataria, R. C. Jared, R. Schnitt, and S. G. Thonpson

Intemedfate-Mass Fraggnents Bnitted in the Reaction $\mathrm{Ag}+1 \mathrm{~d}_{\mathrm{N}}$ at $100,160,250 \mathrm{HeV}$ Bapharding Enengy

LBL-4063, July 1075

Nucl. Phys. A255, 491 (1975)

MORETTO, L. G. (See Galin, J., LRL-4064)

MORETTO, L. G. (See Babinet, R., LBL-4080)

MERTTO, L. G. (See Nardi, E., LBL-4083)
MARETTO, L. G., J. Galin, R. Babinet, 2, Fraenkel,

R. Schitt, R. Jared, and S. G. Thinjpson

Potential Energy Effects and Diffusion in the Relaxed Colaponents of the Reaction $197_{\mathrm{Au}}+4 \mathrm{O}_{\mathrm{Ar}}$ at 288 and $340 \mathrm{MeV}$ Bonbarding Enong ies

LEL-4084, September 1975

Nhiclear Physics A259, 173 (1976)

MRETTO, L. G., B. Catvin, F. Glässe1, R. Jared, P. Russo, J. Sventek, and G. Wozniak

Diffusive Phentranta in the Charge and Angular Distributions for the Reaction ${ }^{197} \mathrm{Au}+620 \mathrm{MeV}$ ${ }^{B} \sigma_{X_{Y}}$

LBL-4362, January 1976

Phys. Rev. Letters 36, 1069 (1976)

MKRETTO, L. G. and J. \$, Sventek

Diffusive Phenomena Reflected in the Charge and Argular Distributions of $\mathrm{N}, \mathrm{Ne}, \mathrm{Ar}, \mathrm{Kr}$ Induced Reastions

LEL- 5006 , Parch 1976

Symposiun on Macroscopic Features of Heavy Ion

Collision,ANL, Argone, I1., April 1-3, 1976

MOREITO, l. G. (See Sventek, J,, LBL-5012)

MARRIS, D. (See Bex, L., LBL-3433)

MOULTON, J. (See Babinet, R., LAt,-4080)

MYERS, W. D. and H. von Groote

The Nuclear Surface Diffuseness

LBL-4327, October 1975

Phys. Letters 61b, 125 (1976)

MERS, Williana D.

The Droplet Kodel Atamic Mass Predictions

LBL-4332, Oetober 1975

Atanic Data and Nhelear Data Tables

MYRS, Willem D.

Lectures on Geometrical Properties of Muclei

L,RL-4342, Novenber 1975

Ejghth Senmer School on threlear Physics,

Mikolajki, Poland, August 24-September 6, 1975

MERS, H, D. (See Boneh, Y., L.BL-4374)

MYERS, W. D. (5ee Eoneh, $Y_{*}$, LBL-4397)

NAGAMIYA, S. (See Anbolt, R., LBL-4307 Rev.)

NAGAMIYA, S. (See Antolt, R., LEL-4359)

MAGAarAIAN, M. A. (5ee GlendennItn, N. K., LBL-5009) 


\section{$0 \mathrm{j}, 3435+393$}

NARDI, E., L. G. Moretto, and S. G. Thampson

Statistical Decay of Ganda Rays in $\left(\pi_{t h}, \gamma\right)$ Reactions

LRL-4083, April 1974

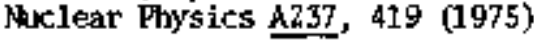

NEhTaN, J. 0., F. S. Stephens, and R. M. Dianond

Rotational Bands in the Light Odd Mass Thallium Nuclei

LBL-2333, January 1974

Nuc1. Phys. A236, 225 (1974)

NIFENECKER, H., C. Signarbietx, R, Babinet, and J, Poitou

Neutron and Camma Emission in Fission

LBL-1950, Ju1y 1973

(Third Symposium on the Phystes and Chenistry of Fission, Rochester, New Kork, August 13-17, 1973), Vol. II, 117, (1974)

NILSSON, S. G. (See Randrup, J., LBL-4036)

NITSCHXE, Joachim M.

A High Intensfty Heavy-Ion Recoil-Target System LBL-4387, May 1976

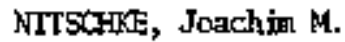
Heavy Ion Spectrometer at the SuperHILAC LEL-5044, Nay' 1976

NCRRIS, A. E. (Se Iratz, J. V., LBL-4339)

OLMER, C. (See Buenerd, M. LBL-4372)

OTTO, R. J., M. M. Fowiler, D. Lee, and G. T. Seaborg Mass Yield Distutbutions in the Reaction of $136 \mathrm{Xe}$ Ions with 238

LBL-4322, October 1975

Phys. Rev. Letters 36, 135 (1976)

PERUMAN, I. (See Artzy, Michal, LBL-2975)

POITOU, J. (Sge Nífentecker, H. C., LBL-1950)

PQMORSKI, K. (See Rarkirup, J., LEL-4036)

POSXANZER, A. M. (See Zebelman, A. M., LEL-3427)

POSKANZER, A. M., R. G. Sextro, A. M. Zebelmar, H. H. Gutbrod, A. Sandova1, and R. Stock Search for Fragment Buission fran Nuclear Shuck vaves

LBL-4309 Rev, , October 1975

Phys. Rev. Letters 35, 1703 (1975)
PRIESS, K. and P. Lichtner

A New Approach to Antisymetrization and Rearrangerent in Thelets-lhuclets - Collisions

LBL-4381, March 1976

Nucl. Phys.

PRUESS, K+, G. Delic, L. A. Char1ton, and N. K. Glenterming

Polarization of Singla-Particie States Irvolved in Heavy Ict Transfer Reactions

LBL-5008, March 1976

Symposiura on Macroscopic Features of Heavy Ion

Collisions, Arigome, IL., April 1-3, 1976

RAD, F. N. (Sese Seiler, F+, LBL-3495)

FAD, F. N., H. E. Conzett, R. Roy, and F. Seiler Heasureant of the Proton Analyzing Power in the $p^{-d}$ Breaku Reaction

LBl-4040, Jume 1975

4th International 5ymposium on Folarization Phernmena in Nuclear Reactions, Z̈̈rich, Switzerland, August 25-29, 1975

RAD, F. N. (See Roy, R. LRL-4041)

RAD, F. N. (See Seiler, F,, LBL-4043)

RAD, F. N. (See Conzett, H. B. LBL-4044)

RAD, F. N. (S甲e Seīlex, F., LBL-4045)

RAD, F, N, (See Seiler, F, 18Li-4047)

RAD, F. N. (See Seller, F. LBL-404B)

RAD, F. N. (See Conzett, H. E., LBL-4051)

RAb, F. N. (See Roy, R., LEL-4052)

RAD, F. N, (See Roy, R+, LRL-4053)

RAD, F. N. (See Bircha11, J., LBL-405A)

RAD, F. N., H. E. Conzett, R. Roy, and F. Sefler Polarization Effects in the Final-State Interaction Region of the p-d Breakap Reaction LBt-4091, August 1975

Phys. Rev, Letters 35, 1134 (1975) 
RANORIP, J.

The Nuclear Surface Studied with a MonentunDeperndent Interaction in the Thumas-Femi and Hartree Approxination

LBL-4302, August 1975

Nuclequ Physics A

RANERUP, J., S. E. Larsson, P. Moller, S. G. NH1sson, K. Powarski, and A, Sobiczewski

Spontaneous-Fission thlf-Lives for Even Nuclei with $z$ \& 92

LBL-4036, Noventber 1975

Phys. Rev, Letters 36, 569 (1976)

RANDWNP, J,

Modjfied Thamas-Fepni Apprarimation for MamentunDependent Interactions

L.BL,4308, Septenber 1975

Physics Letters B

RANDRUP, $\mathbf{J}$.

The Nuclear Interaction Potential in a FoldedYukawa Model with Diffuse Densities

LBL-4317, Septenter 1975

N.F.P.

RARDRIS, J.

Heavy-Ion Potential Derived fras a VelocityDependent Nucleonic Interaction

LBL-4376, Narch 1976

Phys. Letters

RARDRiJP, J. (See Blqcki, J., LEL-5014)

RASMSSEN, J. 0., (See Rezanka, I., LBL-2965)

RASWISSEN, J. O. [See Shihab-Eldin, LBL-3409]

RAOMUSSEN, John 0. (See Massmann, Herbert, LBL-3440)

RAMUSSEN, J. O. (See Chu, S. Y., LBL-4021)

RASAISSEN, J. O. (See Massaam, H., LBL-4023)

RASMUSSEN, Joln O. (\$Se Ring, Peter LEL-4028)

RAKUUSSEN, J. 0. (Soe Eggers, R. C., LBL-4097)

RASMUSSEN, J. O. (See Kawakani, H., L.BL-4303)

RAMUSSEN, J. 0. (See Anholt, R., LBL-4307 Rev.)

RASISSSEN, J. O. (See Fliessbach, T., LEL-4335)

RASMISSEN, J. O. (See Druange1o, R, L.BL-4347 Rev.)
RASMUSSEN, J, D. (Sae Guidry, M. W., LBL-4357)

RASAJSSEN, J. O. (See Anho1t, R. LBL-4359)

RASMUSSE, J. O. (See Raich, D. G., L LEL-5049)

RAUSCHER, E. (Se Anholt, R., LEL-4307 Rev.)

RAUSCHER, E. (See Anho1t, R., LEL-4359]

REZANKA, I, I, M, Ladentauer-Bellis, J, 0 . Rasuussen, $W$. Ribbe, and $E$. đer luteosian High Spin Rotational States in $16 \mathrm{~S}_{\mathrm{Hf}}$ from the January 26, 1975, LPL-2965)

Phys, Rev. C 11, 1767 (1975)

RALSCHER, E. A.

Speculations on the Evolution of a Sthrarzchild Unituerse

LBL-4353, Deceanber 1975

RIBEE, W. (See Rezanka, I., LEL-2965)

RIBEE, W. S. (See E⿱宀女 gers, R. C., LBL-4097)

RING, P. (See Chu, S. Y, LEL-4021)

RING, P. (See Nassamin, H. LBL-4023)

RING, Peter, John O. Rasoussen, and Herbert Massmenn Rarrier Penetration T heory in Wore Than one Dimension

LBL, -4028, Septenber 1975

Fizika Elenentarnyleh Chast1ts 1 Atonnogo Yadra (Soviet Journal of Particlos and thelei)

For), R. (See Rad, F. N., (LBL-4040)

ROY, R., F. N. Rad, H. E. Conzett ang F. Seilerr Vextor Analyzing Power in the ${ }^{3} \mathrm{He}(0,3 / 6) n p$ Reaction

LBL-4041, June 197S

4th International 5ymposium on Polarization Phenanena in Muclear Reactions, Zürich, Switzerland, August 25-29, 1975

BOY, R. (See Conzett, H. E., 1.BL-4044)

ROY, R. (Se Seiler, F., LEL-4045)

ROY, R. (See Seller, F., LBL-4047)

ROY, R. (See Seiler, F,, LBL-4048) 


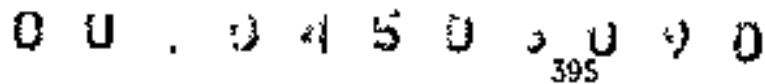

RW, R. (See Conzett, H, E., LEL-40\$I)

ROY, R., H. E. Conzett, F. N. Rad, F. Seiler, and R. M. Larimer Cross-Sections and Vector Analyzing Ptwers in d-3e Elastic Scattering Between 15 and 40 MeV

LEL-4052, June 1975

4th Intexnational 5yspositun on Polarization

Phencunena in Nuclear Reactions, Zürlch, Switzerland, Algust 24-29, 1975

ROY, R., F. Seiler, H. E. Conzett, F. N. RAd, and R. M. Lariner

Cross-Section and Vector Analyzing Powers in the $3_{\mathrm{He}}(\mathrm{d}, \mathrm{p})^{4} \mathrm{He}$ Reaction Between 15 and $40 \mathrm{MkV}$ LEL-4053, June 1975

4th International Sysposium ch Polarization

Phencraena in Noxlear Reactloris, Zurich, Switzerland, Aupust 25-29, 1975

ROY, R. (See Rad, F. N., LBL-4091)

RUSSD, P. (See Noretto, L. 6., LEL-4362)

SAKAI, M. (See Kawakemi, H., LBL-4303)

SANDOVAL, A. (See Posksnzer, A+ M., LRL-4309 Rev.)

SAYLOR, T. K, [hee Anto]t, R, , LBL-4066)

SAYLOR, T. K. (See Meyerhof, W. E., LEL-407\$)

SAYLOR, T. K. (See Meyerhof, W. E., LBL-4079)

SAYLOR, T. K. (See Anholt, R, LEL-4360)

SCHITT, R. (Seo Moretto, L. G., LBL-4063)

SCHITT, R. (See Noretto, L, G, LBL-4084)

SCWITT, R. [See Ga1fn, J., LBL-4064]

SCHNILER, W. F. W. (Soe Stock, R., LEL-5041)

SCOTT, D. K. (See Yag $i, K .$, LRL-Z982)

SCOTT, D. K., B. G. Harvey, D. L. Hendrie, U. Jahnke, L. Kraus, C. F. 萔 Uire, J. Mahoney, Y. Terrien, K. Yagi, and N. K. Glendentive

The Interference Between Direct and Indirect Modes in Two-Nuгlem Transfer Reactions with Heavy-Iors

LBL-3415, Jaruary 1975

Phys. Rev. letters 34, 895 (1975)
ScorT, D. K. , D. L. Hendrie, U. Jathnke, L. Kraus, C. F, Maguire, J. Mahoney, Y. Terrien, and K. Yag 1

The Energy Variation of Multinucleon Transfer Reactions with Heavy-Ions

LEL-3445, July 1075

Phys. Rev. Letters

SCOTT, David $x$.

New Aspects of High Brergy Heavy-Ion Transfer Reactions

LBL-3495, March 1973

(Presented at the International conference on Cluster Structure of Muclei and Transfer Reactions Indused by Heaty Ions, Tokyo, Japan, March 17-22, 1975)

SCOTT, D. K., D. L. Hendrie, L, Kraus, C. F. Maguire, J. Mahuney, Y. Terrien, and K. Yagi Seraiclassical and pusantum Hechanical Comparison of one and two Nucleon Transfer LBL-4059, May 1975

Phys. Rev. Letters 36, 226 (1976)

SCOTT, D. K. (See Meguire, C. F., LBL-4338)

SCOT, D. K. (See Buenerd, M., LBL-4372)

SCOTT, D. K. (Gee Yag i, K. , LBLL-4382)

SCOTT, D. K. (See 5tack R, , LBL-5041)

SEABDR, Glenn T.

The Search for New Elerents: The Projects of Today in a Larger Perspective

LEL, -3423

Physica Scripta 10A, $5(1974)$

SEABCRG, 6. T. (See Dtto, R. J., LBL-4322)

SEARORG, G. T, (See Kratz, J. V., LBL-4359)

SEILER, F., F. N. Rad and H. E. Conzett

Extreme Yalues $A_{y}= \pm 1$ of the Analyzing Fower for Spin-1 Polarization.

LEL-3496, September 1975

Phys. Rev. Letters

SEILER, F, [See Rad, F. N., LBL-4040)

SEILER, F. (See Roy, R., LBL-4041)

SEILER, F., F. N. Rad, and H. E. Conzett

Extrenle Values of the Analyzing Power for Spin-1 Polarization

LeL-4043, June 1975

4th International Symosium on Polarization

Phenomena in Nuclear Reactions, Zürich, Suitzerland, August 25-29, 1975 
SEILER, F. (See Conzett, H. E., LBL-4044]

SEILER, F+, H. E. Conzett, F. N. Rad, and R. Roy Points of Maxinum Polarization Efflciency in ${ }^{4} \mathrm{He}(\mathrm{a}, \mathrm{d})^{4} \mathrm{He}$

LEL-4045, June 1975

4th International Sympositu on Polarization

Phengue ona in Nuclear Reactions, Ztirich,

Switzerlani, Algust 25-29, I975

SEILER, F.

Maximum Tensor Analyzing Power Ay $=1$ in the ${ }^{3}$ te $(\dot{d}, p)^{4}$ He Reacticit

LBL-4046, hate 1975

4th International Smposium on Polarization

Phonomena In Nuclear Reactions, Zürich,

Switzerland, August 25-29, 1975

Phys, Letters 61.B, 144 (1976)

SEILER, F., R. Roy, H. E. Contett, and F, N. Rad Points of Maximum Analyzing Power in the ${ }^{3} \mathrm{He}(\mathrm{t}, \mathrm{p})^{4} \mathrm{He}$ Reaction

LBL-4047, June 1975

4th International Symposium on Polarization

Phenonena in Nuclear Reactlons, Zlirich,

Switzerland, August 25-29, I975

SEILER, F., F. N. Rad, H. F. Conzett, and R. Roy Extrene Values of the Analyzing Power in tho Li, (d, con) ${ }^{4}$ He Reaction

LBL-4048, June 1975

4th International Symposiun on Polarization

Phencmena in Nuclear Reactions, Zilirich,

Switzerland, August 25-29, 1975

SEILER, F. (See Conzett, H. E., LEL-4051)

SEILER, F, (See RoY, R., LBL-4052)

SEILER, F. (SOe Roy, R, LAL,-4053)

SEnER, F. (See Rad, F. N., LBL-4091)

SEILER, F.

Analysis of Reactions Induced by Polarized Particles

LBL-4096, August 1975

4th Internationat Symposium on Polarization

Phenament in Muclear Reactions, Zirich,

Switzerland, August $25-29,1975$

SEXTRO, R. G. (See ZebeTman, A. M. , LRL-3427)

SEXTRO, R. G. (See Foskanzer, A. M. , LBL-4309 Rev.)

SHEFHA, Joseph D., D. L. Hentrie, and M. \$. Zisnan Spectroscopy of the $(\alpha, 2 \alpha)$ Reaction at $\mathrm{F}_{\alpha}=90$ $\mathrm{MeV}$

LBL-4306, Septenther 1975

Phys, Rev. C 13, 20 (1976)
SHEFEAAN, D. F. (See Vieira, D. J., LEL-4321)

SसIBATA, T. (See Arholt, R., LEL-4359)

SHTHAB-ELDN, A. (See Jardine, I. J., LBL-2943)

SHIHAB-ELIA, A, A., L. J. Jardine, and $\$, 0$. Rașustsen

Extension of Relative Alpha Decay Rate Theory to Spherical odd-odd huclei (2I0)

LBL-3409, Soptember 1974

Noc1. Phys. A244, 435 (1975)

SHIHAB-ELDIN, A+ A. (See Jardine, L, J., LAl,-3447)

SIGNARBIE $U$, C. (See Nifemecker, H. C., LBL-19SD)

SDDN, R. S. (See Baraschik, M. V., LEL-3456)

SDWON, R. S., M. W. Banaschìk, P. Colanbeni, D. P. Soroka, F. 5. Stephens, and R. M, Diamturd Moments-of-Inertia in $162 \mathrm{Yb}$ at Very High Spíns LBL-4313, Septenber 1975

Phys, Rev, Letters 36, 359 (1976)

STMON, R. S, (See Ward, D., LEL-4375)

SIMON, R. S. (See Lee, Y, Y,, LBL-5031)

SOBICZEISKI, A. (Sel Randrup, J. LEL-4036)

SOROKA, D. P. [See Banaschlk, M. V., LBL-3456)

SUROKA, D. P. (Se Simon, R. S. , LRL-4313)

STAHEL, D. P. [\$Ee Jelley, N. A., LBL-3414]

STAHEL, D. P. (See hozriak, G. J., LBL-4368)

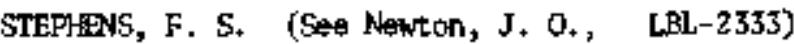

STEPHENS, F, 5 .

Coriolis Effects and Rotation Aligmaent in Nix:lei

LBL-2352, Jamuary 1974

Rev, Alod. Phys, 47, 43 (1975)

STEPHE1S, F, S. (See Hard, D., LBL-3446)

STEPHENS, F. S. (Sed Panaschik, M. V., LFl-3456)

STEPHENS, F. \$. (\$ee Butler, P. A., LBl-3483) 


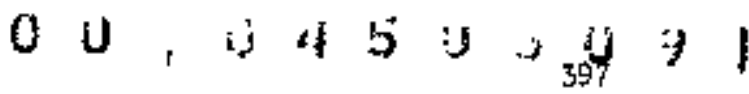

STEPHENS, F. S. (See Grosse, E., LBL-4037)

STEPHENS, F. 5. (See Guidry, M. W., LBL-4038)

STEPHENS, F. S.

Experimental Status of High-Spin States LBL-43I1, September 1975

Conferente on Highly Exc1ted States of Nuclei, Jülich, Germany, September 22-27, 1975

STEPHENS, F. S. (See SimG1, F. S., LBL-4313)

STEPHENS, F. S. (Seo Ward, D., LEL-4375)

STEPHWNS, F. S. (See Colmbani, F, , LBL-5027)

STEPHANS, F. S. (See Lee, I. Y., LEL-S031)

STEPHENS, F, S.

Nuclei at High Angular Hmenta

LBL-5034, Jue 1976

SIOCK, R. (See Poskanzer, A. M., LEL-4309 Rev.)

STOCK, R., U. Jahnke, D. L. Herdrie, J. Mahoney, C. F. Maguire, w. F. W. Sctmeider, D. K. Scott, and G. Wolschin

On the Contribution of Alphe Cluster Exchnges to Elastic and Inelastic $160+20$ Ne Scatter ing LBL-5041, Ju्य, 1976

SIUFA, R. (See Guidry, M. W., LBL-4038)

SVETEK, J. S. (See Noretto, l. G., LBL-3443)

SWFTEK, J. (See Horetto, L. G., LRL-4362)

SVENTEK, J. S. (See Noretto, L. G., LEL-500ई)

SVEVIEK, J, S. and L. G. Moretto

Diffusion boxlel Predict-on for Heavier 5ystems: The Reaction $197 \mathrm{Au}+620 \mathrm{MeV} 86 \mathrm{Kr}$ LEL-5012, April 1976

SWIATECKI, W, J, (See Btocki, J., LBL-5014)

TAMLIR, T. [See Yagi, K., LBL-2982)

TERRIEN, Y. (See Yagi, K., LBL-2982)

TERRIEN, Y., (SNe Scott, D. X., LBL-3415)
TERRIEN, Y. (See Scott, D. K., LBL-3445)

TERRIEN, Y. [See Scott, D. K. , LBl-4059)

THOMPSON, S. G., L. G. Moretto, R. C. Jared,

R. P. Babinet, J. Galín, M. M. Fowler,

R. C. Gatti, and J. B. Hunter

Macroscopic Aspects of Heavy Ion Reactions LBL-2940, June, 1974

(Presented at the Nobel Symposium on Superheavy Elements, Ronneby Brumn, Sweder, June 11-15)

Physica scripta I0A, 36 (1974)

THOLPSOA, S. G. (See !toretto, L. G., LBL-3444)

THLNeSTA, S. G. (See Mretto, L. G., LBL-4063)

THOMPON, S. G. (5ee Galin, J., LBL-4064)

THOUPST, S. G. (See Babinet, R., LBL-4080)

THOAPSON, S. 6. (See Nartit, E., LBL-4083)

THOMPSON, S. G. (See Horetto, L. G+, LBL-40B4)

TJWM, P. (See Grosse, E., LBL-4037)

TRIPLETT, B. B. (Sọe Myerhof, H. E., LBL-4078)

TSAKG, C. F. (See Blocki, J., LBl-5014)

UDAGAHA, T + (Gee Yagi, K., LBL-2982)

von GROOTE, H. (See Mevers, W. D., LBL-4327)

VIEIRA, D. J., D. F. Shermin, M. S. ZISmin, R. A. Gough, and Joseph Cerry

Extemsion of the $T_{2}=-3.2$ Beta-Delayed Proton Precurtor Series to $57 \mathrm{Zn}$

LBL-4321, Novenber 1975

Phys, Letters 608, 261 (1970)

VIOLA, V. E., Jr. (See ZebeIman, A. M, , LBL-3427)

WARD, D., H. Bertschat, P. A. Butler, P. Colonbani, R. M, Dianoted, and $\vec{F}, S$. Stephens

High-spin states in $127,129 \mathrm{ga}$; A Test of Earkbending in the Even $\mathrm{Ba}$ and $\mathrm{Ce}$ Nuclei LBL-3446, Decentber 1974

Phys. Rev. 56B, 139 (1975)

WARD, D. (See Butler, P. A., [RL-34B3) 
WARD, D. (See Guidry, M. W, , LBL-4038)

WARD, D., P. Colnuban1, I. Y. Lee, P. A. Butler, R. S. Simon, R. M. Dijenond and F. S. Stephens High-Spin States of 174,176 yb Studied in Coulomb Excitation with Ke and Xe Beans LEL-4375, March 197\%

WARD, D. (See Colombani, P., LRI,-5027)

WEISEMLLLER, R. B. (See Wilcox, K. H., LBL-4089)

WEISENIILLER, R. B., N. A. Jeller, K. H. Hilcox, G. J. Wozniak, and Joseph Cerny

The Spectroscopic Utility of the Two-Proton Pick-Uf ( $\left.{ }^{6} \mathrm{i},{ }^{8} \mathrm{~B}\right)$ REACTION

LEL-4304, Octaber 1975

Phys. Rev. C 13, 1330 (1970)

HEISEMILLER, R. B. (See Ashery, D., LBL-4315)

WILOOX, K. H. (Se Jelley, N. A., LBL-3414)

WILCOX, Kenneth Hugh

Mass Measurentents of High Neutron-Excess Muclei in the Light Elements

LBL-4029, May 1975

N.F.P. (Ph.D thesis)

WILOOX, K. H., R. B. Heisenrailler, G. J. Hozniak, N. A. Jelley, D. Ashery, and Joseph Cerry

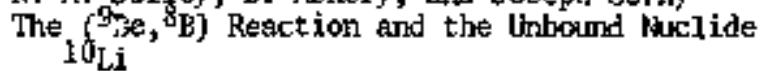

LAL-4089, Aapust 1975

Phys. Letters 59B, 142 (1975)

WILCOX, K. H. (See Weisenmiller, R. B+, LBL-4304)

WOLSCHIN, Georg (See Glenderming, Norman K., LBL-3472)

WOLSOHN, Georg (See Glendemzing, Nonnan k., LBL- 4334)

WOLSGIIN, Georg (See Glendenning, Norman x., L.EL-4341)

WOLSOHIN, Georg (See Glendenning, Horman K., LEL-5007)

WOLSCHIN, G. (See Stock, R., LBL-5041)

WOOD, J. L., R. W. Fink, and J. Meyer-ter-Vehn Symmat chattween particle and Hole level Systens in $189 \mathrm{Au}$

L.BL-4350, October 1975

Phys. Letters
WDENIAK, G. J, (See Wilcox, K. H., LBL-40\$9)

MOZNLAK, G. J. (See Weisenniller, R, B., LBL-4304)

WOZNIAK, G. J. (See Ashery, D., LBL-4315)

WOZNIAK, G. (See Moretto, l. G. , LEL-4362)

WOZNIAK, G. J., D. P. Stahei, Joseph Cerny, and N. A. Jelley

The $\left(\alpha,{ }^{8}\right.$ ) Reaction in the 1p \$hell

LBL-4368, April 1976

WOZNIAK, G. J. (See Jahn, R., LBL-5038)

YAGI, K. B., L. Krauts, 1. Maguire, C. F. Mehoney, D. K. Scott, Y. Terrien, T. Ldagawa, K. S. Low, and T. Tamura

One- and bulti-Step Processes in the Reaction $144 \mathrm{kd}(12,1400)$

LBL-2928, December 1974

Phys. Rev. Letters 34, 96 (1975)

YAGI, K. (See Scott, D, K., LBL-3415)

YAGI, K. (See Scott, D. K., LBBl-3445j

YAGI, K. (See Scott, D. K+, LBLL-4059)

YaGI, X., D. L. Hendrie, U. Jamke, C. F. Maguire, $J$. Mahoney and D. K. Scott

LBL-4382, Narch 1976

Phys. Rev, C

YOSHIRARA, N. (See Kawakani, H., LEL-4303)

ZERELMAN, A. M+, A. M. Poskanzer, J. D. Bownan, R. G. Sextro, and V, E. Viola, Jr. Fragnents from Uranium Irradiated by $2.1 \mathrm{GeV} /$ NocIeon Deuterons and Alpha Particles LBLl-3427, October 1974

Phys. Rev. ClI, 12Bo (1975)

ZEREILAN, A. H. (See Poskarzet, A. M, LBL-4309 Rev.)

ZISWN, A. S. (See Sherman, Joseph D., LBL-4306)

ZISMAN, M. S. (See Ahsery, D., LBL-4315)

ZISWAN, A. S. (See Vieira, D. J., LBL-4321) 


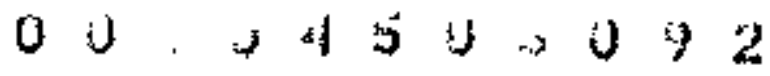

VI.

AUTHOR INDEX 


\section{\begin{tabular}{lllllllll}
\hline & 0 & 0 & 1 & 5 & 0 & 0 & 0 & 3
\end{tabular}}

AUTHOF INDEX

ANDERSON, L. $\quad 189$

ANHOLT, R. 196, 217, 228, 219, 220

ARVIEUX, J. 67

ASHERY, D. 82, 83, 97

AZJPNBERG-SELOWE, $F 、 3,5$, B8

BABINET, R, 132, 134, 136, 358

BAISIFN, P. A. 40

BANSOHTK, M. V. 29, 31 .

BELEAL, E. 372

BEMIS, C. E. 40

EERTSOHAT, H. 16,19

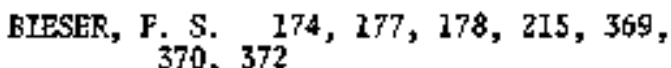

BINDER, I. 40, 153, $158,165,168$

BIROHALL, J. 67,70

HYockI, J. $325,327,280,306,310,322$,

BOISSON, J. P. 261, 272

BONEH, Y. 322, 325, 327

BOMEN, J. 337

BCMMAN, H. $196,217,218$

BOMNW, J. D. 8

BUENERD, $M_{+} 88,89,91,102,121$, $115,117,353$

PURLEIEG, R. J. 339,341

BURTON, R. F. $\quad 354$

BUILER, G. W. 203

BITLER, P. A. $16,19,22,26$

BUITLE, P. J. A. 67

CARROLL, J. 194

CAININ, B. 138, 140, 123, 126, 147 127,129 ,

CERNY, J* ${ }_{83}^{11}, 13,74,78,74,79,81,82$,

OHAMEERLATN, 0. 189

OHARLTOW, L. A. 231, 240, 241

OHINTALPUDT, $5 . \quad 70$
aN, s. Y. 283

CLARK, A. R. 193

CLRRK, D. J. $337,339,341,345$

DOLOMEANI, P. $16,19,22,26,29,31$

CONZETT, H. E. $44,46,47,50,51,52,53$, $70,58,59,61,64,65,66,67,68$,

DORK, B. $174,177,178,215$

GARE, J. G. 107, 109

CRAFFORD, H. 215

DAHE, W. 50, 58, 67

de BOER, J. 24

DELIC, 6. 111, 231, 242, 244, 246, 249

DeVRIES, R. M. 107, 109

DIAMONO, R. M. $14,16,19,22,24,26$, $29,31,34$

TONANKELD, R. 72, 261, 264

ECXROAD, S. W. 366

EQGEPS, R. C. 98

EICHLER, E. 22

ENRT, H. 196,217

EPPLEY, R. E. B

FINK, R. W. 276

FLIESSBAOH, T. 294

FLOCARD, H. 279,290

FLOD, พ. S. $\quad 339$

FOHLAR, M. M. $40,153,158,165,168$

FRAENRELL, 2. 134

FRAZIER, P、 E. $\quad 337$

FULESY, R. 199

GABOR, G. 352,367

GALD, J, 132, 134

GAZZALY, M. 194

GEACA, J. 194

GEF.BKE, C. K. $102,115,352$

GHORSO, A. 36, 39, 40, 155, 160, 366 
GLASCOW, L. R. 337, 341

GLäSSEl, $P$, 144, 147, $356,138,140,142$,

GLENDENAING, N. K. 225, 227, 228, 230, 231

GODFREY, G. 222

COLDBERG, D. A. 107, 109

GOSSET, J. 208, 209

GOUGH, R. A. $11,337,343,351$ TREINER, D. E. 174, 177, 178, 180, 215,

GROSSE, E. 24

GIIDFY, M. W. 22, 261, 264

GITAROD, H. H. 204, 206, 208, 209

GITENAN, A. 97

HALBAOH, K. 367

HARROWRR, A. R. 371,372

HARWEY, B. G. 1.T5, 214, 354

HECMAN, H. H. $174,177,178,180,185,215$

HENRIE, D. L. 3, $88,89,91,93,100,102$, $110,111,115,117,337,353$

HOLIEY, N. R. 193, 337

HALT, E. K. 40

IGO, G. $\quad 194$

IOAKNOU-YANROU, 3. G. 196, 217, 218, 272

JAHN, R, $74,81,85,88$

JAHNKE, J. 93, 110, 353

JAIN, A. 337

JARED, R. C. 119, 120,123,126,127, 128, $129,132,134,136,138,140,142$, $144,147,356,358$

JAROS, J. $187,189,190$

JELLEY, N. A. $77,83,349$

JELTENA, B. D. 85

JOHALSEN, P. J. 209

JOHNSON, N. R. 22

KARANT, Y. 269,270

KAYAKAMI, H. 72

KEEFE, D. $\quad 193$
XEXELIS, G. B1, 88, 89, 352

KERTH, L. T. 193

KEI,INHEINZ, P. 14

KOMRA, K. 72

KOIKE, M. 72

KRAT2, J. Y. 150

KRATS, L. $\quad 100$

KRARINDE, J. 283

KUNZ, P. D. 241

RURATH, D. 249

LAM, R. 343

LANDIS, D. 367

LANDRTA, J. H. 40

LARLMER, R. M. $50,51,52,53,59,64$, 67,70

LARSSTN, S. E. 328

IEBER, R. E. $36,40,366$

LEEE, D. $\quad 40,153,158,165,168$

LEEE, I. Y. 22, 26

LEPMAN, Ch. 50, S1, 58

LEEMANN, B. T. 59

LIOGTINER, P. 238

LILJENZIN, J. $0 . \quad 150$

LINISTRIM, P. J. $174,177,178,180,185$,

LIPPERFEIDE, R. $\quad 320$

LOUGHEED, R. K. 40

LUGGI, J. 369

LLM, G. 212

MCCLELLAND, J. 194

NCKEE, J. \$, C. 50,51

MEPARLAND, C. 371

MACDONALD, J. A. $\quad 58$

MAQUIRE, C. F. 3, 5, 93, 97, 100, 107, 109,11

MAHONEY, J. $3,5,89,93,100,102,110$,

MAIER, M. R. I4 


\section{$00 \because y 450 \begin{array}{llll}5 & 0\end{array}$}

MALFLIET, R. 269, 270

MANG, H. J. $279,281,294$

MARSHALEK, E. R. 283

MARTINEZ, C. 343

MASSMANN, H. 264, 266

MATHEWS, G. J. 119, 123, 127, 128, 290

MENOHACA-ROOHA, A. 89, 91, 102, 111, 115, 117,353

MEILIRS, J. P. 58

MEYER, W. G. 208, 209, 367

MEYERHOF, W. E. 219

MEYER-TER-VAH, J. 16, 275, 276

MLLER, P. $\quad 328$

MOLT2, D. M. 13,351

MORETTO, L. G. 119, 120, 123, 126, 127, 128, $129,132,134,136,138,140,142$, $144,147,251,254,258,291,317$, $356,358,361$

MRRGADO, R. E. 193

MORRIS, D. 337,343

MORRISSEY, D. $\quad 40,155,160$

MOLLTON, J. 126, I27

MTERS, พ. D. $313,314,316,325,327$

NACAMTILA, S. 196, 217, 218

MAKAI, $K_{+} \quad 196$

NASSER, M. 194

NATONITZ, J. B. 203

NEWAN, M, 120

NiLsSCON, S, G. 328

NITSOHE, J. M. 36, 40, 364, 365

NORRIS, A. E. 150

NURMIA, M. J. $38,39,40$ OLER, C. $117,88,89,91,102,111,115$,

OTO, R. J. $40,153,155,158,160,165,168$

PAPP, J. 187,190

PEREZ-MENTEZ,$V . \quad 194$

PERPY, D. G. 203
PLASIL, F. 203

FOMORSKI, X. 32B

POSKANZER, A. M. 201, 203, 204, 206, 208, 209, 367

PRUESS, K, 231, 234, 236, 238

RAD, F. N. $44,46,52,53,57,61,64$, $65,66,70$

RAKRARSSOCN, I, 286

RAICH, D. E. 8, 296

RAISBECK, G. 215

RANDFUP, $\frac{J .}{322,328}, 301,304,306,310,311$,

RASNUSSEN, J. 0. B, 72, 98, 196, 217, 218, $261,264,266,272,283,288,294$, 296

RAIFGER, E. A. $196,217,218,296,331$

PEMEERS, L. P. 203

PEZANWK, I. 8

RIBBE, भ. S. 98

RICHAPDSON, M. 193

RING, P. 266, 28I, 283

ROAEL, M. 322

BCXY, R. $\quad 44,46,52,53,57,59,64,65,66$

Fils 50, P. A. $\quad 119,120,126,129,136,138$, $140,142,144,147,356,358$

SAGLE, A. $\quad 194$

SAKAI, M. 72

SAMRDI, B. 281

SAWLOVAL, A. 204, 206, 208, 209

SOHITT, R. $123,127,128,129,132,134$, 136. $138,140,144,147,356$

SONEIDER, W. F. W. 93

SOANTIAACHER, G. 193

SOIROEMER, L. $187,189,190,193$

SCOTT, D. K. $3,5,81,88,89,91,93,97$, $100,102,110,111,115,117,352$,

SEABORG, G. T. ISD, $153,155,158,160$, 165,168

SEILER, F. $44,46,52,53,56,57,61$, $64,65,66$ 
SEXTROG, R. G. 201, 204, 206, 367

SAPIRO, G. $\quad 189$

SHELINE, R. K. 14

SHERMAN, D. F. 11

SHIBATA, T. $196,217,272$

STHE, H. $\quad 180$

SILWA, R. J. 40

SIMAN, R, S. 26, 29, 3I

SCRICZBNSKI, A. 328

SOINSKI, A. J. 296

SOMERVILLE, L. P. $\quad 39,40,160$

SOROKA, D. P. 31, 286, 288

SPINIKA, H. 194

STAFEL, D. P. $74,77,79,85,349$

STAPLES, J. 187,190

STEINER, H. $\quad 187,189,190$

STEPHENS, F. S. $14,16,19,22,24,26$,

STEPHENSON, E. J. $\quad 59$

STOCK, R. $\quad 93,206,208$

SIURM, R. 22

SVENTEK, J. S. 142, 251, 254, 258, 317, 361

SHIATECKI, N. ง. 306, 310, 317, 322

TANABE, K. S. 288

TERRTEN, Y. 100

THONAS, K. 39

THOMPSON, S. G. 132,134
T.JPA, P. 24

TSAst, C. F. 306, 310

VONGROOTE, H. 314

VIEIRA, D. J. $11,13,351$

NACNER, A. $187,189,190$

WARD, D. $16,19,22,26$

WEISENILUER, R, B. $\quad 82,83,97$

WESTFALL, G. D. 201, 208, 209

WHIPPIE, E. 194

WIEGAND, C. 212

witcor, K. H. 82,83

WIL, J. F. 40

WTLLIANS, K. E. 38,40

WILSTN, L. 215

NISS, J. 187,190

MOLSOHN, G. 93,225, 227, 228

Wob, J. L. 276

WOLNIAK, G. J. 74, 77,79,82,83,85, 119, $126,128,138,140,142,144,147$, 349

YAGI, K. D. 100

YASHITA, S. 155

rosIKAMA, N. 72

ZEBELMAN, A. M. 201, 206, 367

2INGFE, A. R. 193

ZINK, A. C. $\quad 371$

2ISMAN, $M_{+} S_{+}, 107,11,13,79,81,85,88,97$, 